\title{
DISSERTATIONSSCHRIFT
}

\section{Die Bewahrung und \\ Förderung der Biodiversität als theoretisch-praktische Herausforderung für den modernen Natur- und Umweltschutz}

Erstbetreuer:

Prof. Dr. Holmer Steinfath

Lehrstuhl für praktische Philosophie Georg-August-Universität Göttingen

Zweitbetreuer:

Prof. Dr. Konrad Ott

Lehrstuhl für Philosophie und Ethik der Umwelt

Christian-Albrechts-Universität zu Kiel

Drittbetreuer:

Prof. Dr. Thomas Potthast

Lehrstuhl für Ethik, Theorie und Geschichte der Biowissenschaft Eberhardt-Karls-Universität Tübingen

\section{SILKE LACHNIT}

Promotionsstudiengang Biodiversität und Gesellschaft

Philosophisches Seminar

Georg-August-Universität Göttingen 



\title{
Die Bewahrung und Förderung der Biodiversität als theoretisch-praktische Herausforderung für den modernen Natur- und Umweltschutz
}

\author{
Dissertationsschrift \\ zur Erlangung des philosophischen Doktorgrades \\ an der Philosophischen Fakultät der \\ Georg-August-Universität Göttingen
}

vorgelegt im August 2017

verteidigt im Februar 2018

von

Silke Lachnit,

geboren am 03.07.1983

in Berlin 



\section{Inhaltsübersicht}

I. ABSCHNITT I: EINLEITUNG.

1. Fokus, Arbeitsthese, Methodik und Zielstellung der Arbeit

2. Präliminarien

3. Aufbau und Fortgang der Arbeit

II. ABSCHNITT II: GRUNDLAGENREFLEXION $.41-90$

1. Biodiversität als biowissenschaftliches Konzept

2. Biodiversität als politischer Symbolbegriff

3. Biodiversität als Wert- und Normbegriff

4. Konklusion: Biodiversität als Grenz- und Hybridkonzept

III. ABSCHNITT III: WISSENSCHAFTSTHEORETISCHE DETAILREFLEXION $.92-128$

1. Das realistische Wissenschaftsverständnis hinter dem Biodiversitätsdiskurs

2. Die kritische Reflexion des wissenschaftlichen Realismus

3. Die Rekonstruktion der kulturellen Konstitutionsbedingungen des Forschungsprogramms Biodiversität

4. Fazit und Überleitung zum nächsten Kapitel

IV. ABSCHNITT IV: ETHISCHE DETAILREFLEXION $.129-255$

1. Die argumentationstheoretischen Grundlagen der Rede von 'guten Gründen '

2. Kritische Reflexion der gängigen Gründe und Begründungen die Erhaltung der Biodiversität

3. Die theoretische Neuausrichtung der Umwelt- und Naturschutzethik hin zu einer anwendungsorientierten Biodiversitätsethik

4. Fazit und Überleitung zum nächsten Kapitel

V. ABSCHNITT V: POLITIKWISSENSCHAFTLICHE DETAILREFLEXION $.256-430$

1. Die Grundlagen und Bedingungen der Good Biodiversity Governance

2. Die Spezifizierung von Good Governance vor dem Hintergrund demokratietheoretischer Überlegungen

3. Die Spezifizierung von Good Governance vor dem Hintergrund governancetheoretischer Überlegungen

4. Darlegung der Anforderungen, Defizite und Entwicklungsmöglichkeiten der deutschen Biodiversitätspolitik

VI. ABSCHNITT VI: SCHLUSSBETRACHTUNG $.432-458$

1. Bilanz der inhaltlichen Reflexionen

2. Bilanz der normativen Reflexionen

3. Bilanz der politischen Reflexionen 



\section{Inhaltsverzeichnis}

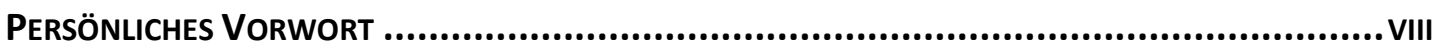

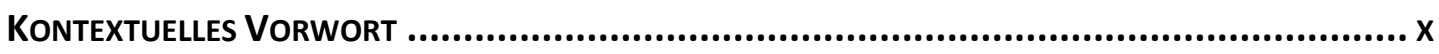

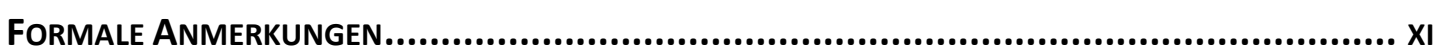

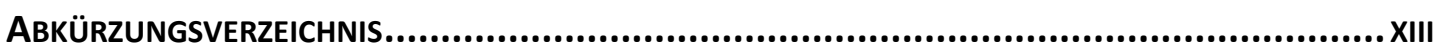

I. EINLEITUNG:

DAS LEITBILD DER BEWAHRUNG UND FÖRDERUNG DER BIODIVERSITÄT ALS THEORETISCH-PRAKTISCHE HERAUSFORDERUNG FÜR DEN MODERNEN NATUR- UND UMWELTSCHUTZ................................. 17

1.1 Fokus, Arbeitsthese, Methodik und Zielstellung der Arbeit.......................... 19

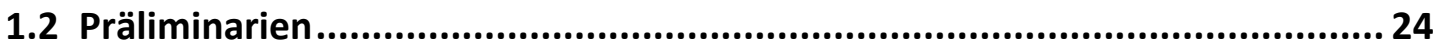

1.2.1 Das Konzept der gesellschaftlichen Naturverhältnisse zum Verständnis der Biodiversität ....................................................... 25

1.2.2 Eine anwendungsorientierte Umwelt- und Naturschutzethik zur Beurteilung des Umgangs mit der Biodiversität

1.2.3 Eine "Good Biodiversity Governance« als Modell zur politikstrategischen Ausgestaltung der Bewahrung und Förderung der Biodiversität 30

1.3 Aufbau und Fortgang der Arbeit 34

II. GRUNDLAGENREFLEXION:

DAS DREIDIMENSIONALE VERSTÄNDNIS VON BIODIVERSITÄT ALS GRENZ- UND HYBRIDKONZEPT................................................ 41

2.1 Biodiversität als biowissenschaftliches Konzept ........................................ 41

2.2 Biodiversität als politischer Symbolbegriff für einen modernen, umfassenden und integrativen Natur- und Umweltschutz 51

2.2.1 Der internationale Kontext: Die CBD und der ökosystemare Ansatz ........ 54

2.2.2 Der nationale Kontext: Die NBS und das Bundesnaturschutzgesetz......... 56

2.3 Biodiversität als Wert- und Normbegriff...................................................62 62

2.3.1 Die »'Fakten `Argumentation« für die Erhaltung der Biodiversität.......... 63

2.3.2 Die »'Eigenwert'-Argumentation« für den Schutz der Biodiversität ........ 68

2.4 Zusammenfassung und Fazit: Biodiversität als Grenzobjekt und Hybridkonzept zur Erschließung gesellschaftlicher Naturverhältnisse

2.4.1 inhaltliche Ebene: Die Ausblendung der kulturellen Konstitutionsbedingungen des Forschungsprogramms Biodiversität....

2.4.2 normative Ebene: Die problemindizierte Neuausrichtung der Umwelt- und Naturschutzethik hin zu einer problem- und anwendungsorientierten Biodiversitätsethik

2.4.3 politische Ebene: Die 'blinden Flecken` beim Versuch der theoretischen Explikation und Beurteilung der "Good Biodiversity Governance».... 84 
III. WISSENSCHAFTSTHEORETISCHE DETAILREFLEXION: DIE KULTURELLEN KONSTITUTIONSBEDINGUNGEN DES FORSCHUNGSPROGRAMMES BIODIVERSITÄT

3.1 Das realistische Wissenschaftsverständnis hinter dem Biodiversitätsdiskurs 93

3.2 Die kritische Reflexion des wissenschaftlichen Realismus 100

3.2.1 Die externe Kritik am wissenschaftlichen Realismus:

Die Ausblendung der kulturellen Konstitutionsbedingungen

der Biodiversitätskonzeption

3.2.2 Die innertheoretische Kritik am wissenschaftlichen Realismus:

Die defizitäre Verteidigung der Korrespondenztheorie der Wahrheit und der Zielbestimmung wissenschaftlicher Praxis

3.3 Die Rekonstruktion der kulturellen Konstitutionsbedingungen des Forschungsprogramms Biodiversität

3.3.1 Die Erschließung der methodologischen Konstruktionsbedingungen der biologischen Vielfalt....

3.3.2 Die Erschließung der geistes- und ideengeschichtlichen Konstitutionsideen der biotischen und abiotischen Vielfalt

3.4 Fazit und Überleitung zum nächsten Kapitel

IV. ETHISCHE DETAILREFLEXION:

DIE THEORETISCHE NEUAUSRICHTUNG DER UMWELT- UND NATURSCHUTZETHIK HIN ZU EINER ANWENDUNGSORIENTIERTEN BIODIVERSITÄTSETHIK

4.1 Die argumentationstheoretischen Grundlagen der Rede von guten Gründen

4.2 Kritische Reflexion der gängigen Gründe und Begründungen für die Erhaltung der Biodiversität

4.2.1 Die 'Argumente' der NBS: ökologische, ökonomische, soziokulturelle und ethische Gründe für die Erhaltung der Biodiversität

4.2.2 Die umwelt- und naturschutzethischen Begründungsansätze:

Der Eigenwert und die direkten (Schutz-)Pflichten

4.2.2.1 Die Reflexion der Wertedebatte........................................................148

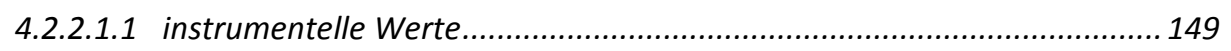

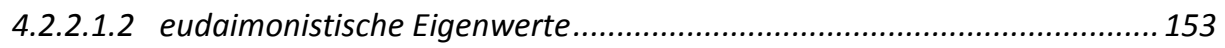

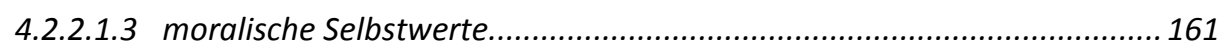

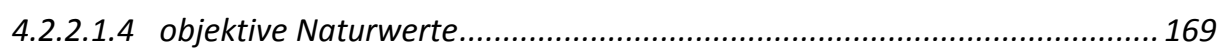

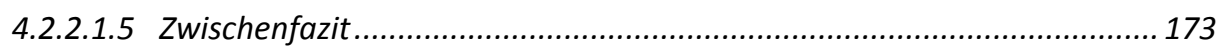

4.2.2.2 Die Reflexion der physiozentrischen Begründungsansätze

für den Schutz der Biodiversität 'um ihrer selbst willen `....................176

4.2.2.2.1 Reflexion der holistischen Position zum Inklusionsproblem ..................... 177

4.2.2.2.2 Reflexion der ökozentrischen Position zum Inklusionsproblem ................. 181

4.2.2.2.3 Reflexion der biozentrischen Position zum Inklusionsproblem .................. 185

4.2.2.2.4 Zwischenfazit. 190 
4.3 Die theoretische Neuausrichtung der Umwelt- und Naturschutzethik hin zu einer anwendungsorientierten Biodiversitätsethik

4.3.1 Die relationale Perspektive: Die Ansätze und Schwierigkeiten einer inklusiven Umwelt- und Naturschutzethik.

4.3.1.1 Die Relationen zwischen Mensch, Natur und Gesellschaft und die wesensmäßige Naturbeziehung in der relationalen Perspektive

4.3.1.2 Die Relevanz eudaimonistischer Ansätze für die relationale Umwelt- und Naturschutzethik und die Bedeutung der Biodiversität für das gute Leben

4.3.2 Der biodiversitätsethische Argumentationsraum zur Integration der Biodiversitätszieltrias und zur Überwindung des deontischen Fokus der Umwelt- und Naturschutzethik

4.3.2.1 Das konzeptionelle Problem des biodiversitätsethischen Argumentationsraumes

4.3.2.2 Die Rekonstruktion der diskurstheoretischen Fundamente der Argumentationstrias

4.3.2.2.1 Zum pragmatischen Gebrauch der praktischen Vernunft .....

4.3.2.2.2 Zum eudaimonistischen Gebrauch der praktischen Vernunft...................227

4.3.2.2.3 Zum moralischen Gebrauch der praktischen Vernunft.

4.3.2.3 Ein mehrdimensionales Ethikverständnis als Grundlage der gesamten Argumentationstrias

4.3.3 Die Akzeptabilitäts- und Akzeptanzbedingungen für die Kommunikation und Aushandlung der Biodiversitätsziele und -maßnahmen

4.4 Fazit und Überleitung zum nächsten Kapitel .253

V. POLITIKWISSENSCHAFTLICHE DETAILREFLEXION: DIE THEORETISCHEN GRUNDANNAHMEN, ANFORDERUNGEN SOWIE CHANCEN UND RISIKEN DER GOOD BIODIVERSITY GOVERNANCE .... 256

5.1 Die Grundlagen und Bedingungen der Good Biodiversity Governance ........ 259

5.1.1 Das Politikfeld Biodiversität und dessen Charakteristika ....................... 260

5.1.1.1 Vertikale Koordination im Mehrebenensystem ..................................260

5.1.1.2 Horizontale Koordination im Mehrebenensystem ...............................263

5.1.1.3 Prinzipien und Instrumente der Umweltpolitik ....................................267

5.1.2 Good Governance als Leitbild des Regierungshandelns und als normative Grundlage für die GBG

5.1.2.1 Das Standardkonzept von Good Governance und dessen Problematiken

5.1.2.2 Die alternative Good Governancekonzeption

5.2 Die Spezifizierung von Good Governance vor dem Hintergrund demokratietheoretischer Überlegungen zu Partizipation, Deliberation und Diskurs

5.2.1 Partizipative Governance vor dem Hintergrund kontextbezogener Demokratietheorien.... 
5.2.1.1 Das theoretische Grundgerüst der kontextbezogenen

Demokratietheorien

5.2.1.2 Das Modell der »Empowered Participatory Governance»

nach Fung und Wright

5.2.2 Deliberative Governance vor dem Hintergrund prozeduralistischer Demokratietheorien

5.2.2.1 Das theoretische Grundgerüst der kontextbezogenen

Demokratietheorien in ihrer diskursiven Variante.....

5.2.2.2 Die Modellierung »deliberativer Governance« nach John Dryzek

5.2.3 Zusammenfassung und Zwischenfazit 307

5.3 Die Spezifizierung von Good Governance vor dem Hintergrund governancetheoretischer Überlegungen

5.3.1 Der Begriff der Governance und die theoretischen Gehalte der politikwissenschaftlichen Governanceforschung

5.3.2 Der Wandel von staatlicher Steuerung hin zu kooperativer Regelung:

Von der Steuerungstheorie zur Governancetheorie

5.3.2.1 Die theoretischen Verschiebungen beim Übergang von Government zu Governance und von Steuerung zu Regelung.....................................326

5.3.2.2 Die Kontinuitäten zwischen Steuerungs- und Governancetheorie ........327

5.3.2.3 Der akteurszentrierte Institutionalismus als theoretische

Hintergrundfolie zur Explizierung von Governance

5.3.2.4 Zwischenfazit 336

5.3.3 Die »komplexe Demokratietheorie» als demokratietheoretisches Pendant der Governancedebatte 338

5.3.3.1 Demokratietheorie zwischen Utopie und Anpassung ... 340

5.3.3.2 Die 'Erweiterungen' der komplexen Demokratietheorie unter den Voraussetzungen der zunehmenden Internationalisierung der Staaten und der Globalisierung der Wirtschaft .................................................349

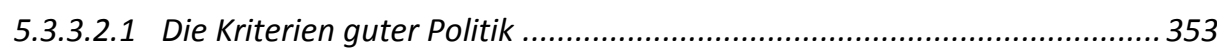

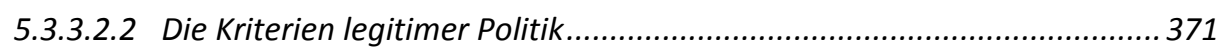

5.3.3.3 Zusammenfassung der komplexen Demokratietheorie mit Fokus auf die Konkretisierung der theoretischen Grundannahmen der GBG 400

5.4 Fazit: Die Anforderungen, Chancen und Risiken der deutschen Biodiversitätspolitik 408

5.4.1 Die Anforderungen an die deutsche Biodiversitätspolitik 408

5.4.2 Die Defizite und Entwicklungsmöglichkeiten der deutschen Biodiversitätspolitik.

VI. SCHLUSSBETRACHTUNG

Bilanz der inhaltlichen Reflexionen.....

Bilanz der normativen Reflexionen

Bilanz der politischen Reflexionen .448 


\section{PERSÖNLICHES VORWORT}

Alle größeren wissenschaftlichen Arbeiten haben ihre eigene Entstehungsund Verlaufsgeschichte. In diesem Fall wurden die Eckpfeiler dieser Arbeit bereits im Vorfeld der Dissertation eingeschlagen, maßgeblich beeinflusst durch die vielen aufschlussreichen, interessanten und inspirierenden Erfahrungen, die ich in der Doktorandenarbeitsgruppe Umweltethik, im Rahmen meiner studentischen Arbeit für Prof. Dr. Konrad Ott, damals noch an der Universität Greifswald, bei Prof. Dr. Thomas Potthast am Internationalen Zentrum für Ethik in den Wissenschaften und in dem gemeinsamem BfN-Forschungsprojekt zusammen mit Dr. Uta Eser sammeln durfte. Insbesondere diese Hintergründe führten mich, die ich nicht Philosophie, sondern Politikwissenschaft, Kommunikationswissenschaft und Psychologie studiert habe, in das spannende Feld der Umweltethik, zeigten mir aber zugleich auch die Grenzen und Probleme einer rein disziplinären Betrachtungsweise von Umwelt- und Naturschutzproblematiken. Daraus ergab sich die Motivation, eine interdisziplinär angelegte Arbeit zu schreiben, die disziplinäre Selbstverständlichkeiten hinterfragt, sprich grundlagenreflektorisch ausgerichtet ist, dabei einen differenzierten, aber auch konstruktiven Blick auf die komplexen Verhältnisse zwischen Politik, Moral, Natur und Umwelt als auch den Menschen und sein Handeln in diesen Zusammenhängen wirft und dabei gleichzeitig stärker an der problem- und lösungsorientierten Analyse von gesellschaftlichen Naturverhältnissen orientiert ist, als an fachlich-disziplinären Grenzen. Eben dies veranlasste mich dazu im Rahmen des Promotionsstudiengangs Biodiversität und Gesellschaft, eine solche Arbeit anzuvisieren.

Diesen Anspruch habe ich im Laufe der Arbeit nie aufgegeben, auch wenn mir mehr und mehr bewusst geworden ist, dass eine ganz eigene Anstrengung und wissenschaftliche Leistung darin liegt, diesem auch gerecht zu werden. Ein Desiderat dieser Arbeit - auch wenn dieses in der Arbeit selbst nicht niedergeschrieben ist - ist daher, dass das interdisziplinäre und anwendungsorientierte wissenschaftliche Arbeiten eine ganz eigene Qualität und Schwierigkeit besitzt, der man sich (leider) erst dann in Gänze bewusst wird, wenn man sich auf dieses Unterfangen eingelassen hat. Auch habe ich innerhalb der Promotionszeit meine Tochter - Mia - bekommen, was für mich und meinen Mann ein großer Wunsch, aber für die Fertigstellung der Arbeit nicht immer ein Segen war. Auch hier musste ich die Erfahrung machen, dass es nicht einfach ist, beides unter einen Hut zu bekommen. 
Trotz oder gerade wegen dieser Umstände bin ich froh und dankbar, dass mir mein Erstbetreuer, Prof. Dr. Holmer Steinfath, die Chance sowie die Geduld und Zeit gewährt hat und mich bei meiner Aufholjagd der ethiktheoretischen Grundlagen nicht allein gelassen hat. Für mich war es eine ungemein anstrengende, aber auch spannende und lehrreiche Erfahrung, die ich lieber mit einem Dr. int. (eben für eine interdisziplinär ausgerichtete Promotion) honoriert sähe. Dass ich behaupten kann, die Arbeit für mich zu einem zufriedenstellenden Ende gebracht zu haben, verdanke ich in hohen auch meinen beiden anderen Doktorvätern Prof. Dr. Konrad Ott und Prof. Dr. Thomas Potthast, die ihre Betreuungsaufgaben sehr ernst genommen haben und mich immer wieder dazu motiviert und befähigt haben weiterzumachen und Sackgassen auszubügeln bzw. zu vermeiden, indem sie mir vor allem hinsichtlich der philosophischen und metaethischen Debatten immer wieder wichtige, kleinteilige und konstruktive Anregungen gegeben haben, mit denen ich gut weiter arbeiten konnte. Auch haben sie mir in schwierigen persönlichen Phasen die nötige Zeit, das nötige Vertrauen und die nötige Ruhe gelassen, damit ich die Arbeit zu Ende bringen konnte. All das war unverzichtbar auf dem langen Weg bis zur Fertigstellung der Arbeit, und ich bedanke mich recht herzlich dafür.

Hinter Ihnen stand indes noch ein ganzes Netzwerk an Kommilitonen aus dem Promotionsstudiengang, mit denen ich mich manchmal fachlich, viel öfter aber - und nicht weniger wichtig - auch persönlich austauschen konnte sowie die Organisatoren des Promotionsstudiengangs - Prof. Dr. Rainer Marggraf, Dr. Jan Barkman und nicht zuletzt Dr. Bettina Roß - die die organisatorischen und finanziellen Grundlagen dafür geschaffen haben, dass ich diese Arbeit schreiben konnte. Neben dem dreieinhalbjährigen Exzellenzstipendium der Lichtenberg-Stiftung habe ich zwei halbjährige Abschlussstipendien der Graduiertenschule für Geisteswissenschaften (GSGG) und der Graduiertenschule Gesellschaftswissenschaften (GGG) erhalten. Alle drei Stipendien haben mir eine intensive Arbeit an der Dissertation erst ermöglicht. Nicht zuletzt dafür bedanke ich mich recht herzlich.

Auch - und nicht zuletzt - meine Freunde und meine Familie, die mich aktiv begleitet, mich immer wieder motiviert, aufgebaut, unterstützt, aber auch wenn nötig abgelenkt und zerstreut haben, waren tragende Pfeiler dieser Arbeit. Ihnen gilt mein besonderer Dank! 


\section{Kontextuelles Vorwort}

Die vorliegende Dissertationsschrift ist in den institutionellen Rahmen des Promotionsstudienganges Biodiversität und Gesellschaft unter dem Dach der Göttinger Graduiertenschule Gesellschaftswissenschaften (GGG) an der Georg-August-Universität Göttingen eingebunden. Hier hat man es sich zur Aufgabe gemacht, die bereits etablierte und weit verzweigte naturwissenschaftliche Biodiversitätsforschung mit geistes- und sozialwissenschaftlichen Forschungsschwerpunkten zu flankieren. Der Fokus des Promotionsstudienganges liegt daher auch auf den gesellschaftlich relevanten Fragestellungen der Erhaltung der Biodiversität, so dass danach geforscht wird, durch welche rechtlichen, wirtschaftlichen, normativen, sozialen, kulturellen, politischen, institutionellen, prozeduralen etc. Faktoren die Erhaltung der Biodiversität bestimmt ist, und wie sie vor diesen Hintergründen am besten bewahrt werden kann und soll. Ein besonderes Augenmerk aller Arbeiten im Promotionsstudiengang soll dabei auf der wissenschaftlichen Auseinandersetzung mit den Biodiversitätszielen und-maßnahmen liegen, wobei dieser theoretische Fokus wiederum mit der praktischen Zielstellung verbunden werden soll, über den Einzelfall hinaus Wege aufzuzeigen sowie Instrumente und Verfahren zu entwickeln, die zur Verbesserung der gesellschaftlichen und individuellen Entscheidungsfindung in Bezug auf die drei, mit der Biodiversität verbundenen Zielstellungen des Schutzes, der Nutzung und der Verteilung der Biodiversität dienen können.

Die im Promotionsstudiengang vertretenen Forschungsvorhaben teilen sich dabei in verschiedene Cluster auf. Die hier vorliegende Dissertationsschrift ist schwerpunktmäßig im dritten Cluster angesiedelt, das sich auf Forschungen zu den gesellschaftspolitischen Aktivitäten zur Erhaltung der Biodiversität in Deutschland mit Blick auf die Internationale Biodiversitätskonvention (CBD) sowie die Nationale Biodiversitätsstrategie Deutschlands (NBS) fokussiert. Die hier angesiedelten Forschungsvorhaben sollen das Grundlagenwissen zur allgemeinen Akzeptanz und Wertschätzung der Zielstellungen und Maßnahmen sowie der gesellschaftspolitischen Praxis der Bewahrung der Biodiversität in Deutschland erweitern. Dies ist nicht als ein rein strategisches Unterfangen zu verstehen, sondern soll dem Umstand Rechnung tragen, dass die Grundlagenforschung, trotz des Vorhandenseins politisch gewollter und kodifizierter Biodiversitätskonventionen und -strategien, weiterhin notwendig und von praktischer Relevanz ist. 


\section{FORMALE ANMERKUNGEN}

Jede Arbeit hat, auch aufgrund ihrer disziplinären Hintergründe, ihre eigenen Konventionen, die im besten Fall unbemerkt und unkommentiert bleiben können, im schlimmsten Fall aber zu Verwirrung führen, wenn diese nicht erläutert werden. Um diesem Fall vorzubeugen, sind die folgenden Formalien zu beachten:

\section{Genderkonvention und Personenbezeichnungen:}

Alle Personenbezeichnungen gelten sowohl für männliche als auch für weibliche Formen. Es wird ein großes Binnen-I verwendet: bspw. AkteurInnen, BürgerInnen, ForscherInnen. Auf eine vollkommen genderneutrale Schreibweise wie bspw. Akteur_, Akteur(x), Bürger ${ }^{\lambda} / q$, Forscher* wird aufgrund mangelnder Lesbarkeit und Verständlichkeit verzichtet.

Wenn Positionen, Hypothesen, Konzepte etc. von bestimmten AutorInnen im Text wiedergegeben werden, dann erfolgt dies - bei erstmaliger Nennung - unter Verweis auf den Vor- und Nachnamen, damit keine Verwechslungen mit gleichnamigen Autorlnnen entstehen. Im weiteren Verlauf wird dann nur noch der Nachname genannt.

\section{Schlüsse/wörter und Begriffskonventionen:}

Wenn es im Text Schlüsselwörter bzw. zentrale Aussagen gibt, werden diese kursiv markiert. Dies geschieht zur besseren Lesbarkeit auch in den Fußnoten.

Bestimmte Terminologien, die im Satzzusammenhang als erst einmal als näher zu spezifizierende Eigennamen dastehen wie bspw. „Good Biodiversity Governance» oder aber die Titel von Aufsätzen, Monographien oder Sammelbänden sowie die Namen politischer Konventionen wie bspw. "Nationale Strategie zur Biologischen Vielfalt« oder "United Nation Conference on Climate Change« werden in Doppelpfeile eingerahmt. Ansonsten werden die Pfeile auch in Zitaten übernommen, wenn diese bereits dort vorhanden sind.

Begriffe oder Wendungen, die zwar umgangssprachlich gebräuchlich sind, aber einen stark metaphorischen Gehalt haben, wie bspw. 'blinder Fleck' oder 'Mangelware', Wendungen die terminologisch für problematisch erachtet werden, wie bspw. 'ökologische Ethik' und näher erläutert bzw. kontextualisiert werden müssen, werden bei der ersten Nennung in einfache Anführungsstriche gesetzt. 


\section{Literaturverweise im Text:}

Begriffe, die theoretisch eingebettet sind, wie bspw.

„Politikverpflechtungsfalle“ sowie Formulierungen oder ganze

Textpassagen, die von anderen AutorInnen übernommen worden sind, werden zitiert, indem sie bei der ersten Nennung in doppelte

Anführungsstriche gesetzt und mit einer Quellenangabe versehen werden.

Die Quellenangabe erfolgt gemäß der Harvard-Zitierweise direkt im Text: „Zitat" (Name/n Jahr:Seite/n). Erfolgen Zitationen hintereinander, die sich auf dieselbe Quelle berufen, werden diese mit: (ebd.) oder aber bei unterschiedlicher Seitenzahl mit: (ebd., Seite/n) abgekürzt.

Zitate, die länger als drei Zeilen sind und sich nicht in das Satzgefüge einbetten, werden gesondert hervorgehoben und als Textblock abgesetzt und eingerückt:

„Zitat." (Name/n Jahr:Seite/n)

Weiterführende Referenzliteratur wird mit: (vgl. Name/n Jahr:Seite/n) gekennzeichnet.

Bei zwei bzw. drei AutorInnen werden diese durch ein \& verbunden:

(Name, Name \& Name Jahr:Seite/n). Bei mehr als drei Autorlnnen erfolgt eine Abkürzung durch: (Name et al. Jahr:Seite/n).

Bei abgeschlossenen Zitaten wird nach der Quellenangabe kein Punkt mehr gesetzt: „Zitat.“ (Name/n Jahr:Seite/n)

Bei unabgeschlossenen Zitaten, die in den Satz eingefügt sind erfolgt: „Zitat“ (Name/n Jahr:Seite/n) und es wird direkt im Anschluss die Quelle genannt, danach geht entweder der Satz weiter oder nach der Quellenangabe wird ein Punkt gesetzt.

Bei Aufzählungen erfolgt der Praktikabilität halber am Ende des Satzes die Quellenangabe: „Zitat“ Satz „Zitat“ Satz „Zitat“ Satz (Name/n Jahr:Seite/n).

Zitate in englischer Sprache werden mit doppelten Anführungszeichen oben ein- und ausgeführt: "Zitat." (Name/n Jahr:Seite/n)

Eigene Hervorhebungen in Zitaten werden im Anhang an die Quellenangabe deutlich gemacht: (Name/n Jahr: Seite/n; SL). Ist dies nicht vermerkt, so findet sich die Hervorhebung bereits im Original.

Einfügungen in Zitate, veränderte Schreibweisen oder Einschübe, die im Originalzitat an anderer Stelle zu finden sind, werden durch eckige Klammern gekennzeichnet: [...].

Auslassungen in Zitaten jedweder Art wie Literaturverweise, Einschübe, Sätze oder Textpassagen sind durch runde Klammern gekennzeichnet: (...). 


\section{Abkürzungsverzeichnis}

\begin{tabular}{|c|c|}
\hline AA & Auswärtiges Amt \\
\hline AAP & Addis Abeba Prinzipien \\
\hline ABS & $\begin{array}{l}\text { Access and Benefit Sharing-Mechanism/ } \\
\text { Regelungen zum Zugang und gerechtem Vorteilsausgleich }\end{array}$ \\
\hline B.B.N & Bundesverband beruflicher Naturschutz \\
\hline BBR & Bundesamt für Bauwesen und Raumordnung \\
\hline BfN & Bundesamt für Naturschutz \\
\hline BfE & Bundesamt für kerntechnische Entsorgungssicherheit \\
\hline BfS & Bundesamt für Strahlenschutz \\
\hline BDI & Bundesverband der Deutschen Industrie e.V. \\
\hline BHU & Bund Heimat und Umwelt in Deutschland \\
\hline BMBF & Bundesministerium für Bildung und Forschung \\
\hline BMEL & $\begin{array}{l}\text { Bundesministerium für Ernährung und Landwirtschaft } \\
\text { (ehemals Bundesministerium für Ernährung, Landwirtschaft } \\
\text { und Verbraucherschutz - BMELV) }\end{array}$ \\
\hline BMF & Bundesministerium der Finanzen \\
\hline BMG & Bundesministerium für Gesundheit \\
\hline BMI & Bundesministerium des Inneren \\
\hline BMUB & $\begin{array}{l}\text { Bundesministerium für Umwelt, Naturschutz, Bau und } \\
\text { Reaktorsicherheit } \\
\text { (ehemals Bundesministerium für Umwelt, Naturschutz und } \\
\text { Reaktorsicherheit - BMU) }\end{array}$ \\
\hline BMVI & $\begin{array}{l}\text { Bundesministerium für Verkehr und digitale Infrastruktur } \\
\text { (ehemals Bundesministerium für Verkehr, Bau und } \\
\text { Stadtentwicklung - BMVBS) }\end{array}$ \\
\hline BMVg & Bundesministerium der Verteidigung \\
\hline BMWi & $\begin{array}{l}\text { Bundesministerium für Wirtschaft und Energie } \\
\text { (ehemals Bundesministerium für Wirtschaft und Technologie) }\end{array}$ \\
\hline BMZ & $\begin{array}{l}\text { Bundesministerium für wirtschaftliche Zusammenarbeit und } \\
\text { Entwicklung }\end{array}$ \\
\hline BNatSchg & Bundesnaturschutzgesetz \\
\hline BUND & Bund für Umwelt und Naturschutz Deutschland \\
\hline CBD & $\begin{array}{l}\text { Convention on Biological Diversity/ } \\
\text { Internationale Biodiversitätskonvention }\end{array}$ \\
\hline CHM & $\begin{array}{l}\text { Clearing House Mechanism/Informations-, Kommunikations- } \\
\text { und Kooperationssystems der CBD }\end{array}$ \\
\hline
\end{tabular}


CITES Convention on International Trade in Endangered Species of Wild Fauna and Flora/ Washingtoner Artenschutzabkommen

CMS Convention on Migratory Species/

Bonner Konferenz über wandernde Arten

COP Convention of Parties/Vertragsstaatenkonferenz

DAV Deutscher Alpenverein

DB Deutscher Bundestag

DBU Deutsche Bundesstiftung Umwelt

DNR Deutscher Naturschutzring

DUH Deutsche Umwelthilfe

EBS Europäische Biodiversitätsstrategie

eSr epistemischer Strukturrealismus

EU Europäische Union

EU COM Europäische Kommission

FFH Richtlinie 92/43/EWG des Europäischen Rates zur Erhaltung der natürlichen Lebensräume, der wildlebenden Tiere und Pflanzen/Fauna-Flora-Habitat-Richtlinie

FN Fußnote

GBG Good Biodiversity Governance

GEF Globale Umweltfazilität

GG Grundgesetz

IMA NBS Interministerielle Arbeitsgruppe zur Umsetzung der Nationalen Strategie zur Biologischen Vielfalt

IPBES Intergovernmental Science-Policy Platform on Biodiversity and Ecosystem Service/Weltbiodiversitätsrat

ISOE Institut für sozial-ökologische Forschung

Kk Kulturkonstitutionalismus

KR Konstruktiver Realismus

MEA Millennium Ecosystem Assessment

MAT Mutually Agreed Terms and Conditions/ gemeinsam festgelegte Regeln

mK methodischen Kulturalismus

NABU Naturschutzbund Deutschland

NBS Nationale Strategie zur Biologischen Vielfalt

PIC Prior Informed Consent/ vorherig informierte Zustimmung

PIK Potsdam Institut für Klimafolgenforschung

RNE Rat für Nachhaltige Entwicklung 
SBSTTA Subsidiary Body on Scientific, Technical and Technological Advice/ wissenschaftlicher Beirat der CBD

SRU Sachverständigenrat für Umweltfragen

SUP Strategische Umweltprüfung

UBA Umweltbundesamt

UN United Nations/Vereinte Nationen

UNESCO United Nations Educational, Scientific and Cultural Organization/ Organisation der Vereinten Nationen für Erziehung, Wissenschaft und Kultur

UNFCCC United Nations Framework Convention on Climate Change/Klimarahmenkonvention

USA United Staates of America/Vereinigte Staaten von Amerika

UVP Umweltverträglichkeitsprüfung

WBGU Wissenschaftliche Beirat globale Umweltveränderungen

WGRI Working Group on the Review of Implementation of the CBD/ Arbeitsgruppe zur Überprüfung der Umsetzung der CBD

WWF World Wide Fund for Nature

VDA Verband Deutscher Automobilhersteller 
ABSCHNITT I

EINLEITUNG 
I. EINLEITUNG:

\title{
Das Leitbild der Bewahrung und Förderung der Biodiversität als theoretisch-praktische Herausforderung für den modernen Natur- und Umweltschutz
}

\begin{abstract}
"Natur ist uns zum Problem geworden, und dies gleich in mehrfacher Hinsicht. Ökologische Themen wie die anthropogenen Klimaveränderungen (...), die Ausdünnung der stratosphärischen Ozonschicht oder der Verlust der biologischen Vielfalt beschäftigen schon seit Jahren die politische und wissenschaftliche Öffentlichkeit. Doch solche Themen, die überwiegend unter dem Oberbegriff Umweltprobleme verhandelt werden, werfen auch die Frage auf, ob es denn Sinn macht, sie überhaupt unter Rekurs auf den Begriff der Natur erfassen zu wollen. (...) Offensichtlich ist nicht die Natur 'an sich', sondern das menschliche Verhältnis zu ihr das Problem, d.h. die immens gestiegenen technischen Fertigkeiten und die wissenschaftlichen Interpretationen im Umgang mit der Natur. Es ist das Eingriffs- und

Transformationspotenzial des Menschen ihm selbst zum Hindernis, zum Problem geworden." (Görg 2003:9)
\end{abstract}

Mit diesem Zitat und speziell dem Verweis auf das Problem der menschlichen Verhältnisse zur Natur und Umwelt ist ein prägnanter Einstieg in die Thematik um das Leitbild ${ }^{1}$ der Bewahrung und Förderung der Biodiversität ${ }^{2}$ und die theoretisch und zugleich praktische Herausforderung des modernen Umwelt- und Naturschutzes als gesellschaftspolitische Gestaltung der menschlichen Naturverhältnisse gefunden. Das Zitat macht deutlich, dass die Vernichtung ${ }^{3}$ der Biodiversität einerseits zu den drängendsten mo-

1 Leitbilder werden in diesem Kontext als kognitiv-motivationale Orientierungshilfen für das Denken und Handeln von Menschen verstanden, die aber nur vorläufige und unvollständige Bestimmungen erfahren können, weil diese sowohl zeit-, situations- als auch wissensabhängig sind. Das bedeutet, von einer Idealvorstellung auszugehen, die eine Orientierung für einen langfristigen Such-, Erfahrungs- und Lernprozess darstellt, weil über das Leitbild bereits bestimmte Grundprinzipien formuliert werden, deren Nichtbeachtung zu gesellschaftlichen Entwicklungen führen würden, die den grundlegenden Gedanken des Leitbildes zuwiderlaufen.

2 Biodiversität ist ein vielschichtiger und oftmals schillernder Begriff, dessen inhaltliche Bezüge und Bedeutung(en) nicht einfach als gegeben vorausgesetzt werden sollten. Vielmehr müssen und sollen diese in der Arbeit, im Rahmen der Grundlagenreflexion auf das dreidimensionale Verständnis der Biodiversität als Grenz- und Hybridkonzept (siehe Abschnitt II) und die Detailreflexion auf die kulturellen Konstitutionsbedingungen des Forschungsprogramms Biodiversität (siehe Abschnitt III), untersucht werden.

3 Im Anschluss an Károly Henrich wird hier (anders als im Zitat) nicht von Biodiversitätsverlust (»biodiversity loss") oder Biodiversitätsreduktion gesprochen, sondern von Biodiversitätsvernichtung, -reduzierung oder -verminderung, womit die Biodiversitätsproblematik von vornherein (auch) auf menschliches Handeln verweist. Eben dieser Handlungsbezug ist relevant, da alle passiven Ausdrucksweisen ein „Moment verbaler Problemvernebelung und Beschwichtigung" enthalten (Henrich 2003:195): „Die biologische Vielfalt ist kein Objekt, das der Menschheit schlicht verlorengegangen ist und vielleicht auf irgendeinem kosmischen Fundbüro wieder abgeholt werden könnte. Es handelt sich vielmehr bei der neuzeitlichen Biodiversitätsreduzierung eindeutig um einen anthropogenen Prozess der Vernichtung von Arten und Biotopen." (ebd., 195-196) 
dernen Umwelt- und Naturproblemen gerechnet wird und anderseits, dass dieser Problemzusammenhang selbst Ausdruck einer Vielzahl an problematischen Umgangsweisen mit der Natur und Umwelt und den menschlichen Verhältnissen zu diesen ist. Beides zusammen führt dazu, dass sowohl die Wissenschaft als auch die Politik auf die, mit dem Begriff der Biodiversität sowie mit den Begriffen Artenvielfalt, Ökosystemvielfalt und genetische Vielfalt verbundenen ökologisch-sozialen und politischen Problematiken auf je eigene Weise reagiert haben: Für das politische Bemühen um Problemlösungen ist es symptomatisch, dass vor allem der vordergründigen Herausforderung der Vernichtung der Biodiversität begegnet wird, so dass es mittlerweile zahlreiche Dokumente, Abkommen und Verträge gibt, in denen die Erhaltung der Biodiversität als Ziel anvisiert und politisch geregelt wird. Dem wissenschaftlichen Bemühen um Problemlösungen ist demgegenüber immer auch daran gelegen, die Vernichtung der Biodiversität sowie deren Erhaltung selbst als Problematik bzw. als Lösungsansatz zu thematisieren und zu reflektieren. Auch hier kann auf zahlreiche wissenschaftliche Abhandlungen zum Begriff, zu den Funktionen und Leistungen und zum Wert der Biodiversität sowie zur politischen Umsetzung der mit der Biodiversität verbundenen Ziele und Maßnahmen zurückgegriffen werden, in denen es um die Problematisierung und Beurteilung der Vernichtung und Erhaltung der Biodiversität geht.

Allerdings zeigt sich an der breit geführten wissenschaftlichen und politischen Debatte um Biodiversität und deren Bewahrung und Förderung, dass häufig gar nicht klar ist, ob alle ProtagonistInnen überhaupt über den gleichen Gegenstand oder über das gleiche Problem reden. Dies liegt vor allem daran, dass der Begriff und das Konzept der Biodiversität einer der schillerndsten, weil inhaltlich flexibelsten Begriffe des modernen Natur- und Umweltschutzes ist. Konrad Ott und Ralf Döring konstatieren für den Begriff der Nachhaltigkeit, was auch für Biodiversität vollends zutreffend ist:

„Diese Entwicklung führt dazu, dass es scheint, als könnten höchst unterschiedliche politische Ziele und Programme unter Rekurs auf diese Begrifflichkeit gleichermaßen gut begründet werden. Unterschiedliche Akteure sind bestrebt, den Ausdruck gemäß ihren Interessen zu besetzen. (...) [A]uf der Ebene verbaler Bekundungen [hat der Begriff] daher auch keine Gegner, sondern ist gleichsam von Freunden umzingelt.“ (Ott \& Döring 2004:18)

Zwar ist es so, dass Begriffe, deren Umfang (Extension) recht groß ist, zwangsläufig an spezifischer Bedeutung (Intension) verlieren, dies kann aber nicht bedeuten, dass solche Begriffe willkürlich definiert werden dürfen. Gleichzeitig muss bei allem Bemühen um terminologische Klarheit und plausible begriffliche Differenzierung berücksichtigt werden, dass terminologische Abgrenzungsversuche kein Selbstzweck sind. Hierbei geht es vor allem um die Frage, ob Begriffe adäquat oder zweckmäßig zur Gewinnung von Informationen über die Realität sowie für leistungsfähige Erklärungen relevanter 
Phänomene sind (Henrich 2003:162). Insofern liefert die Problematik der inhaltlichen Flexibilität der Biodiversität sowie der Umgang damit die Grundlage, den Fokus, die Arbeitsthese, die Methodik und die Zielstellung der Arbeit zu präzisieren.

\subsection{Fokus, Arbeitsthese, Methodik und Zielstellung der Arbeit}

Gemein ist dabei sowohl den wissenschaftlichen als auch den politischen Abhandlungen über Biodiversität, dass sie sich zumeist - implizit oder explizit - an der theoretischpraktischen Aufgaben- und Zielstellung ausrichten, die Biodiversität sowohl zu bewahren als auch zu befördern. Eben diese übergreifende Aufgaben- und Zielstellung ist seit der Verabschiedung der »Internationalen Biodiversitätskonvention « (»Convention on Biological Diversity«, CBD) mit den drei integrativen ${ }^{4}$ Zielstellungen des möglichst umfassenden Schutzes, der möglichst nachhaltigen Nutzung sowie der möglichst gerechten Verteilung der genetischen und ökosystemaren Vielfalt sowie der Artenvielfalt verbunden (UN 1992: Art. 1). ${ }^{5}$ Sowohl politische als auch wissenschaftliche Beschäftigungen mit der Biodiversität haben daher die, die Biodiversitätszieltrias als Ganzes umfassende, Bewahrung und Förderung der Biodiversität zum Ziel. ${ }^{6}$ Gemäß dieser Zielstellung wird die Arbeit auf das Leitbild der Bewahrung und Förderung der Biodiversität ausgerichtet, die als eine eigenständige, spezifische Aufgabenstellung des politischen und wissenschaftlichen Umwelt- und Naturschutzes verstanden wird, deren besondere Herausforderung vor allem darin besteht, Schutz-, Nutzungs- und Verteilungsaspekte gleichermaßen zu berücksichtigen:

4 Das, was hierbei mit integrativ gemeint ist, kann und wird unterschiedlich ausgelegt (Theobald 1998: 7-8): Aus der naturwissenschaftlichen Perspektive meint integrativ zumeist „medienübergreifend“ (ebd., 7), sprich Gene, Arten und Ökosysteme umfassend, womit die Gegenstände der Bewertung erfasst werden - „die zu bewertende Wirklichkeit (Umwelt), aufgefasst als ökosystemares Modell.“ (ebd.) Was die Wertesysteme angeht, wird integrativ aus der Perspektive der PhilosophInnen, SoziologInnen, der TheologInnen und/oder ÖkonomInnen auch als Aggregierung oder Synthetisierung verschiedener (bspw. ethischer, rechtlicher, ökonomischer, äs-thetischer, sozialer, kultureller, religiöser) Bewertungsaspekte verstanden (ebd.). Für die Erreichbarkeit integrativer Zielstellungen ist es dabei wichtig und unverzichtbar, dass Bewertungs- und Begründungsmaßstäbe auf der jeweiligen Ebene loalisiert werden (ebd. 12) und dass hierbei die Frage gestellt und beantwortet wird, wer etwas in Hinblick auf welches Ziel unter Verwendung welcher Kriterien bewertet. In einem dritten Sinne erscheint integrativ im Kontext der Politik und Politikwissenschaft, wenn von einer sektorübergreifenden Integration die Rede ist. Hier geht es darum die Querschnittsthematik in allen relevanten Politikbereichen zu etablieren und die Kooperation und Zusam-menarbeit zwischen den politischen Ressorts auszubauen (Töller \& Böcher 2012:96). Alle drei Varianten von 'integrativ` sind im Laufe der Arbeit relevant.

5 Auf die drei Biodiversitätszielstellungen wird an späterer Stelle noch einmal ausführlicher eingegangen (siehe 2.2.1).

6 Es gibt derzeit keinen einheitlichen Sprachgebrauch zur Bezeichnung der Biodiversitätszieltrias. Übergeordnet ist zumeist von Erhaltung die Rede, wobei damit mal alle drei Zielstellungen, sprich Schutz, Nutzung und Verteilung gleichermaßen, mal Schutz und Nutzung in Kombination und mal nur Schutz gemeint sind. In dieser Arbeit wird terminologisch einheitlich von Bewahrung und Förderung gesprochen, wenn alle drei Zielstellungen gleichermaßen gemeint sind. Die Bezeichnung Erhaltung soll demgegenüber nur für die Kombination aus Schutz- und Nutzungsziel gebraucht werden. 
Der Terminus der Bewahrung ist im Kontext des sogenannten ökosystemaren Ansatzes zu verstehen: Der ökosystemare Ansatz ist ein System von Prinzipien und Managementregeln im Umgang mit Umwelt und Natur, ${ }^{7}$ über den im Kern die Ansicht zum Ausdruck gebracht wird, dass die Biodiversität nur dann erfolgreich bewahrt und gefördert werden kann, wenn man die Wechselwirkungen von Menschen mit und in den Ökosystemen betrachtet (Stadler 2007:177). Aus diesem Ansatz heraus ergeben sich bestimmte Annahmen und Konsequenzen, zu denen u.a. die Integration von Umweltund Naturschutzanliegen unter dem Dach der Bewahrung und Förderung der Biodiversität zählt. Hierbei ist wichtig, dass der Schutz, vor allem der belebten Natur, als die zentrale Aufgabenstellung des Naturschutzes, die nachhaltige Nutzung der Umweltmedien Wasser, Boden und Luft hingegen als zentrale Aufgabenstellung des Umweltschutzes angesehen wird. Dies ist relevant, da sich Handlungen zum Schutz der Umwelt bereits begrifflich auf die Umwelt des Menschen und die Nutzungsinteressen des Menschen an der Natur als Ressource für das menschliche Wohlergehen beziehen, so dass hierbei vor allem die Bedürfnisse des Menschen im Vordergrund stehen. Bei Handlungen zum Schutz der (belebten) Natur steht indes die Natur bzw. die menschlichen Verständnisse von Natur im Vordergrund, die sich nicht per se als Umwelt des Menschen verstehen lassen müssen. Vielmehr kann Natur, bspw. als Gegenbegriff zu Kultur, auch das nicht vom Menschen Gemachte und Geschaffene bedeuten, so dass beim Naturschutz - erst einmal nur rein begrifflich betrachtet - von Bedürfnissen und Nutzungsinteressen des Menschen abstrahiert werden kann. Daher stellen sich im Kontext des Naturschutzes immer auch die Fragen, ob und inwieweit Menschen die Natur auch jenseits von menschlichen Interessen an ihr schützen können und sollten.

Die Thematisierung der Bewahrung bzw. Erhaltung der Biodiversität reicht indes noch nicht aus, denn Leitbilder beinhalten immer auch 'proaktive' Momente, bei denen es darum geht, die bestehenden Verhältnisse so zu verändern, dass diese sich dem Ideal annähern. Wie wichtig dabei die Perspektive auf gesellschaftspolitische Veränderungsprozesse ist, wird vor allem in solchen Fällen ersichtlich, wo bspw. Schutz- und (nachhaltige) Nutzungsbemühungen nicht automatisch positiv zusammenwirken und zur Erhaltung der Biodiversität beitragen (können). Da die nachhaltige Nutzung eine bestimmte Form der Nutzung ist, die es anzustreben gilt, die aber bei weitem kein Nutz-

\footnotetext{
Zu diesen Prinzipien gehören, dass die Managementziele gesellschaftlichen Entscheidungen obliegen sollen (Deliberationsprinzip), dass die Gestaltung des Managements möglichst dezentral sein sollte (Subsidiaritätsprinzip), dass bei der Ausgestaltung die aktuellen und potenziellen Folgen und Nebenfolgen beachtet werden sollen (Umsichtigkeitsprinzip), dass der Schutz der Strukturen und Funktionen des Ökosystems ein Hauptziel darstellen sollte (prioritärer Schutz von Ökosystemleistungen), dass die Zielsetzungen für das Management langfristig ausgelegt und ausgerichtet sein sollten (Prinzip der Zukunftsverantwortung), dass Veränderungen in Ökosystemen unvermeidbar sind (Prinzip des Wandels), dass einschlägige Informationen jeglicher Art in der Praxis Berücksichtigung finden sollen (Prinzip der Praxistauglichkeit) und dass alle einschlägigen Bereiche der Gesellschaft und der Wissenschaft einbezogen werden sollen (Integrationsprinzip) (Sekretariat der CBD 2000:Abschnitt 6).
} 
ungsstandard ist, können Schutzbemühungen und Nutzungsformen immer nur unter günstigen Umständen bei der Erhaltung der Biodiversität zusammenwirken. Daher muss, vor dem Hintergrund der weiterhin andauernden und stellenweise auch stetig steigenden Vernichtung der Biodiversität, immer auch die gesellschaftspolitische Ausgestaltung und gesetzliche Regelung der Umwelt- und Naturschutzanliegen thematisiert werden. Diese Thematisierung kann wiederum auf unterschiedlichen Abstraktionsebenen geschehen: Die niedrigste Abstraktionsebene ergibt sich über die Betrachtung konkreter Fälle, Situationen und Konflikte, die wiederum einen konkreten, fallspezifischen Problemlösungsansatz erfordern. Auf der mittleren Abstraktionsebene geht es um konkrete Instrumente, Maßnahmen, Verfahren und Prozesse, die hinsichtlich ihrer Tauglichkeit und Zweckmäßigkeit zur Lösung allgemeiner und fallspezifischer Problematiken untersucht werden. Auf der höchsten Abstraktionsebene geht es wiederum um die Rolle politischer, ökonomischer und sozialer Problematiken sowie um dazu passende Problemlösungsmechanismen und -ressourcen, bei der, bspw. die Rolle des Politischen im weitesten Sinne und die Funktion politischer Institutionen und des Staates im engeren Sinne im Vordergrund stehen. Die zentrale Frage, die sich auf dieser Ebene stellt, lautet, welche Art und Weise der Umsetzung der Bewahrung und Förderung der Biodiversität politisch-strategisch angestrebt wird und werden sollte. Dazu bedarf es einer Gesamtstrategie zur Koordination und Umsetzung der damit verbundenen Ziele und Maßnahmen. Eben diese politikstrategischen Aspekte sollen hier über den Terminus der Förderung erfasst werden.

Um diesen Gesamtzusammenhang zu erschließen, kann bereits auf zahlreiche Arbeiten zurückgegriffen werden, die aus unterschiedlichen Perspektiven zentrale Erkenntnisse auf Biodiversität sowie deren Bewahrung und Förderung generieren. Der Großteil der bisherigen wissenschaftlichen Arbeiten zur Biodiversität ist dabei fachwissenschaftlich ausgerichtet, so dass es bisher nur sporadisch um die Beleuchtung der wechselseitigen Einflüsse von biowissenschaftlichen Erkenntnissen, normativen Werte- und Moralvorstellungen im Umgang mit der Biodiversität sowie politisch-strategischen Fragen der Umsetzung der Biodiversitäzsziele und -maßnahmen ging. Des Weiteren sind nur wenige dieser Arbeiten auch um die Herausstellung praktischer Desiderate bemüht, die mit der Bewahrung und Förderung der Biodiversität einhergehen. Vor diesen Hintergründen ist es der Anspruch und die Zielstellung dieser Arbeit, eine interdisziplinäre und zugleich, mit Blick auf die Hindernisse und Potenziale bezüglich der Bewahrung und Förderung der Biodiversität, problem- und lösungsorientierte Arbeit vorzulegen:

Das Modell von Interdisziplinarität, das hierbei zur Anwendung kommt, richtet sich an der interdisziplinären Bearbeitung disziplinenübergreifender Problemfelder aus, wie sie durch Heinrich Parthey am Modell der persönlichen Interdisziplinarität dargelegt worden ist (vgl. Parthey 1999). Parthey hinterfragt dabei die gängige Annahme, dass 
Interdisziplinarität erst durch die Zusammenarbeit von VertreterInnen verschiedener Disziplinen beginnt (Bobach 1999:119), indem er anmerkt, dass „Interdisziplinarität im Denken von Wissenschaftlern mit Problemen und Methoden (...) dann beginnt, wenn Neues zu erfahren mit dem Risiko verbunden ist, die disziplinäre Forschungssituation zu verlassen." (Parthey 1999: 244) Parthey zur Folge ist folglich die persönliche Bereitschaft Probleme disziplinenübergreifend anzugehen und vor dem Hintergrund unterschiedlicher disziplinärer Ansätze zu reflektieren das entscheidende Kriterium für Interdisziplinarität. Interdisziplinarität ist somit eine spezielle Reflexionsmethodik, bei der wiederum entscheidend ist, dass es verschiedene Ebenen der Reflexion gibt und dass sich Reflexion als Methodik gerade dadurch auszeichnet, dass diese unterschiedlichen Reflexionsebenen auch unterschiedliche Ansätze nach sich ziehen und verschiedene theoretische Fertigkeiten verlangen:

Die Reflexion als Selbstbeobachtung (Reflexion erster Ordnung) bedeutet, dass die Bewahrung und Förderung der Biodiversität, die ja bereits als Lösungsstrategie für das praktische Problem der Vernichtung der Biodiversität angedacht ist, selbst als theoretisch-praktischer Problemzusammenhang begriffen und rekonstruiert werden muss. Allgemeiner ausgedrückt: Es wird davon ausgegangen, dass Problem- und Lösungszusammenhänge nicht einfach vorhanden sind, sondern diese werden als "Interpretationskonstrukte“ verstanden (Lenk 1993:606-608). Das bedeutet, dass Probleme je nach Perspektive unter anderen Gesichtspunkten interpretiert werden und damit nicht nicht wertneutral beschrieben werden können, weil sie erst unter bestimmten Wertestandards überhaupt als Probleme erkannt werden und dass Probleme eine substanzielle Herausforderung an das Handeln darstellen, weil die betreffenden Schwierigkeiten gezielt in wünschenswerter Weise verändert werden sollen (Kettner 2014:27-29). In diesem Sinne dreht sich jeder Problemzusammenhang, allgemein betrachtet, um die Frage, wie man von einem gegebenen Ausgangszustand (dem Ist-Zustand) zu einem erstrebten Zustand (dem Sollzustand) gelangen kann (Deppert 1998:36).

Sobald etwas als Problem oder auch als Lösung festgestellt wird, stellt sich die Frage, warum die, für die Feststellung verwandten Ansätze die richtigen bzw. adäquaten sein sollten. Die Reflexion erster Ordnung zieht daher ein reflexives Begründungsverfahren (Reflexion zweiter Ordnung) nach sich (Ricken 2003:15). Ein solches Begründungsverfahren ist dabei bereits kommunikativ verankert, da dieses „nicht mehr eine Sache des Erkenntnissubjekts [ist], das sich objektivierend auf sich selbst bezieht." (Habermas 1985:375) Wer etwas begründet, begibt sich in einen argumentativen Diskurs mit anderen, in dem es entweder um die Wahrheit von Tatsachenbehauptungen oder um die Richtigkeit von normativen Aussagen als Wertaussagen oder als vorschreibende (präskriptive) Aussagen geht. Die Wahrheit bzw. die Richtigkeit von Aussagen erweist sich indes immer erst über eine intersubjektive, d.h. von allen Teilnehmern einer Gemein- 
schaft anerkannte Begründung, wobei Begründungen selbst immer nur insoweit als legitime Ausweisungen für die Wahrheit und Richtigkeit von Aussagen, Argumenten und Urteilen angesehen werden können, wie zugleich davon ausgegangen werden kann, dass es intersubjektiv geteilte Urteilspraxen gibt (Kannetzky 1999:227).

Dies wiederum zeigt, dass auch die Reflexion zweiter Ordnung ein weiteres Mal auf der Ebene sprachlich-semantischer Reflexion (Reflexion dritter Ordnung) reflektiert werden kann und ggf. sogar muss, wenn in Frage steht, ob Aussagen überhaupt als wahr oder richtig begründet werden können (Ricken 2003:15). ${ }^{8}$ Auf dieser Reflektionsebene geht es darum, welche Bedeutungen die Begriffe Wahrheit und Richtigkeit haben und welche Grundannahmen hierbei zugrunde liegen.

Die Beachtung dieser drei Reflexionsebenen führt dazu, dass ausgehend von der Bestimmung der Zieltrias in der »Internationalen Biodiversitätskonvention« und darauf aufbauend den Spezifizierungen dieser Zielstellungen in der "Nationalen Strategie zur Biologischen Vielfalt « eine Reihe von Grundlagen- und Detailreflexionen vorlegt wird. ${ }^{9}$ Die Reflexionsebene erster Ordnung wird hierbei als Grundlagenreflexion und die Reflexionsebenen zweiter (und ggf. auch dritter Ordnung) als Detailreflexion bezeichnet. Grundlegend wird hierbei von der Annahme ausgegangen, dass die Ziele und Maßnahmen zur Bewahrung und Förderung der Biodiversität durch eine ganze Reihe von Problemen zu einer praktisch-theoretischen Herausforderung sowohl für die Wissenschaft als auch für die Praxis werden. Diese Problematiken, die es zu analysieren gilt, stehen dem Ziel entgegen, zu einer Praxis gelingender gesellschaftlicher Naturverhältnisse zu gelangen. Wenn diese Probleme indes detailliert dargelegt und dafür konstruktive Lösungsmöglichkeiten aufgezeigt und erarbeitet werden, dann besteht die Möglichkeit, dem Ideal einer gelingenden Praxis zumindest entgegenzukommen. In diesem Sinne geht es in dieser Arbeit darum, einen konstruktiven Beitrag dazu zu leisten, das Leitbild der Bewahrung und Förderung der Biodiversität zu bekräftigen und zu bestärken, indem aufgezeigt werden soll, dass es bei weitem nicht nur darum geht, dem vordergründigen Problem der Vernichtung der Biodiversität Herr zu werden, sondern dass man es bei der Bewahrung und Förderung der Biodiversität mit einer Reihe an untereinander verbundener Metaproblematiken zu tun hat:

„Die Menschheit hat nicht nur ein Biodiversitätsproblem - sie hat offensichtlich auch ein Problem mit dem Biodiversitätsproblem. (...) Solange sich die Wissen-

8 Wenn hier gesagt wird, dass die Bedeutungsanalyse eine notwendige Bedingung für die Einlösung der Begründungsanforderung ist, dann bleibt dabei offen, ob sie auch hinreichend ist.

9 Da sich die Arbeit oft auf die NBS beziehen wird, haben die Reflexionen einen spezifisch deuschen Kontext, obwohl speziell die allgemeinen theoretischen Überlegungen, die anhand der NBS lediglich konkretisiert und veranschaulicht werden sowie die daran anschließenden Lösungsansätze sicherlich auch auf andere Länder übertragbar sein dürften. Dies ist indes nicht die Zielstellung der Arbeit und daher lediglich als Nebenprodukt, denn als explizites Desiderat der Arbeit anzusehen. 
schaft nicht einigen kann, was Biodiversität ist, welchen gesellschaftlichen Wert sie darstellt und welche Maßnahmen zur Erhaltung der Biodiversität notwendig sind, solange fehlt auch die Grundlage für eine rationale Biodiversitätspolitik." (Hoffmann, Hoffmann \& Weimann 2005:20; SL)

In Bezug auf die Zielstellung eine problem- und lösungsorientierte Arbeit vorzulegen, geht es daher darum, die der Bewahrung und Förderung der Biodiversität 'im Wege stehenden`Problematiken in Bezug auf das Verständnis, den Wert und die Umsetzung der Biodiversität erst einmal zu identifizieren und als Probleme auszuweisen und daraufhin nachvollziehbare und praktisch relevante Lösungen aufzuzeigen. Daher soll in der Grundlagenreflexion (Abschnitt II) geklärt werden, welche Problematiken sich bei der Beschäftigung mit der Biodiversität und ihrer Bewahrung und Förderung ergeben und in den, sich daran anschließenden Detailreflexionen (Abschnitte III, IV und V) wird dann wiederum auf ganz bestimmte, sich aus der Grundlagenreflexion ergebende, Detailproblematiken eingegangen (zur Darlegung dieser spezifischen Problematiken siehe $2.4 .1 ; 2.4 .2$ und 2.4 .3$)$.

Die Erkundung der Metaproblematiken mit dem Biodiversitätsproblem ist bereits in gewisse Kontexte eingebettet. Wenn es also darum gehen soll, zu erkunden, was Biodiversität ist, welcher gesellschaftliche Wert ihr zukommt und wie die Bewahrung und Förderung der Biodiversität umgesetzt werden kann und soll, dann generieren diese drei Fragstellungen bereits gewisse Präliminarien (Setzungen), die grundlegend und damit richtungsweisend für die gesamte Arbeit sind und die im Folgenden ausgeführt werden sollen.

\subsection{Präliminarien}

Die Präliminarien der Arbeit werden im Folgenden dargelegt, so dass danach der Aufbau und Fortgang der Arbeit expliziert (1.3) und anschließend mit der Grundlagenreflexion (Abschnitt II) begonnen werden kann. Im Fall dieser Arbeit sind dies:

- die wissenschaftlichen Kontexte um das Konzept der gesellschaftlichen Naturverhältnisse zur Beantwortung der Frage, was Biodiversität ist (1.2.1),

- der wissenschaftliche Kontext einer auf Anwendungsorientierung ausgerichteten Umwelt- und Naturschutzethik zur Beantwortung der Frage, welchen gesellschaftlichen Wert die Biodiversität hat (1.2.2) sowie

- der wissenschaftliche Kontext um eine "Good Biodiversity Governance« zur Beantwortung der Frage, wie die Bewahrung und Förderung der Biodiversität realisiert werden kann und soll (1.2.3). 


\subsubsection{Das Konzept der gesellschaftlichen Naturverhältnisse zum Verständnis der Biodiversität}

Das Konzept der gesellschaftlichen Naturverhältnisse (vgl. insb. Görg 1999d; Becker \& Jahn 2003; Brand \& Görg 2003; Görg 2003; Becker \& Jahn 2006; Becker, Hummel \& Jahn 2011) geht auf die ältere kritische Theorie und hier insbesondere die Arbeiten von Max Horkheimer und Theodor Adorno zurück (vgl. Görg 2003:14). Bereits hier wird die Untersuchung der Entwicklung der Naturverhältnisse zu einer zentralen Aufgabe der Gesellschaftswissenschaften erklärt, bei der die Wechselwirkungen und Vermittlungen zwischen Natur und Gesellschaft sowie die wechselnden Konstellationen von Individuum, Gesellschaft und Natur zentral sind (ebd., 27-28). ${ }^{10}$ In der Umweltdebatte wurde der Begriff seit den 1980er Jahren vor allem von der Forschungsgruppe "Soziale Ökologie« eingebracht, die als praxisbezogene Forschung zum einen nach Lösungen für gesellschaftliche Probleme sucht, während sie als theorethische Wissenschaft zum anderen das methodisch erzeugte Wissen begrifflich zu ordnen versucht (Becker \& Jahn 2006:7). Insofern geht es ihr auf beiden Ebenen um die inter- bzw. transdisziplinäre Auslotung der in sich vielschichtigen und zudem wechselseitigen Beziehungsstrukturen zwischen individuellen Lebensformen, sozialen Handlungsstrukturen und ökologischen Zusammenhängen (ebd., 70-85), so dass es beim Begriff der gesellschaftlichen Naturverhältnisse um die gesellschaftstheoretische Erschließung der ökologischen Problematik (Görg 2003:14-15) sowie um die Auslotung der damit verbundenen Problembereiche (Reproduktions-, Risiko-, Abgrenzungs- und Normativitätsprobleme) geht (ebd., 15-22). Nach Görg wirft die Gestaltung der gesellschaftlichen Naturverhältnisse zum einen die Reproduktionsproblematik auf, sprich die materialen Probleme des 'Stoffwechsels' mit der Natur sowie den Disput um den Zugang zu Natur. Des Weiteren stellt sich die Risikoproblematik, worunter der Umgang mit Unsicherheiten und Nichtwissen fällt, die wiederum in engem Zusammenhang mit der Abgrenzungsproblematik steht, sprich, dass wissenschaftliche Beschreibungsmuster, durch die wiederum in Frage stehenden Grenzziehungen von Umwelt und Gesellschaft, Natur und Technik sowie Mensch und Natur verschwimmen. Alle drei Problematiken werden wiederum von der Normativitätsproblematik überlagert, bei der es um die Beurteilungsmaßstäbe bezüglich des richtigen Umgangs mit der Natur geht.

In Bezug auf alle vier Problematiken muss im Besonderen betont werden, dass sich das Konzept der gesellschaftlichen Naturverhältnisse von vornherein gegen ein Naturverständnis sperrt, das nur die Natur als für sich seiende Einheit und nicht von vornherein das menschlich-gesellschaftliche Verhältnis zur Natur meint. Demnach kann weder Ge-

10 Das Konzept der gesellschaftlichen Naturverhältnisse ist, wie an dieser Charakterisierung bereits ersichtlich wird, sehr viel umfassender als es hier dargelegt werden kann. Daher werden nur die für die Arbeit relevanten theoretischen Bezüge dargelegt. Expliziert werden diese Bezüge dann an verschiedenen Stellen in der Arbeit. 
sellschaft unabhängig von Natur thematisiert werden, da „der soziale Prozess konstitutiv mit Natur vermittelt ist“, noch zielt der geschichtliche Prozess auf „eine immer umfassendere Kontrolle der Natur." (Brand \& Görg 2003:17) Gesellschaft kann also den Abhängigkeiten im Verhältnis zur Natur nicht entkommen, weil der gesellschaftliche Prozess auf den 'Stoffwechsel' mit der Natur angewiesen ist. So wird darauf verwiesen, dass der Prozess der Moderne auf einem Anwachsen der 'Naturbeherrschung 'beruht. Diese führte aber nicht zu einer wachsenden Kontrolle, sondern zeigt sich vielmehr an der Zerstörung der Natur und in einer immer größer werdenden Abhängigkeit von den Folge- und Nebenwirkungen der Versuche der Beherrschung der Natur, die keine vollständige Beherrschbarkeit ist. Aber auch die Natur ist gesellschaftlich 'überformt' und das nicht nur in der Hinsicht, dass es spätestens seit Beginn des 21. Jhd. keine vom Menschen 'unberührte' Natur mehr gibt. Vielmehr muss die gesellschaftliche Überformung der Natur dahingehend verstanden werden, dass das Verständnis davon was Natur ist, kulturell, situativ und historisch kontingent ist, so dass es die Natur als Singular oder als eigenständiges, vom Menschen, seinen Wahrnehmungen und Beschreibungen unabhängiges Erkenntnisobjekt nicht gibt. In diesem Sinne wird Natur hier als sozial konstruiert verstanden, ${ }^{11}$ auch wenn dabei nicht bestritten wird, dass es materielle und stoffliche Bedingungen menschlicher Existenz gibt, die durch menschliche Aktivitäten respektiert oder aber ignoriert werden können - mit positiven oder eben negativen Folgen in Form der Verminderung oder aber der Vermehrung ökologischer Risiken. Da diese Konstruktionen von Natur eng verbunden sind mit den vorherrschenden Weltund Menschenbildern und zudem von den technischen und ökonomischen Strategien der Nutzung von Natur geprägt sind, gibt es verschiedenartige Konstruktionen von Natur, so dass es auch eine unhintergehbare Pluralität gesellschaftlicher Naturverhältnisse gibt, in die Macht- und Dominanzverhältnisse eingeschrieben sind (ebd., 18). In diesem Sinne geht es darum, welche Formen der Vergesellschaftung von Natur sich durchsetzen und behaupten können, welche dadurch marginalisiert und ausgeblendet werden und wie diese sich wiederum im Laufe der Zeit wandeln.

Wenn Biodiversität in den Kontext gesellschaftlicher Naturverhältnisse gestellt wird, dann geht es folglich zum einen darum, die hinter dem Begriff und Konzept der Biodiversität stehenden, kulturell vermittelten Ideen, Vorstellungen und Ansichten von der Natur einerseits und dem Menschen andererseits sowie deren Verhältnis zueinander

11 Wenn hier von 'sozialer Konstruktion' bzw. Sozialkonstruktivismus die Rede ist, dann soll dies nicht Missverständnisse ontologischer Art implizieren: Soziale Konstruiertheit meint mitnichten, dass die sozial konstruierten Gegenstände (in diesem Fall Natur) keine Entsprechung in der Wirklichkeit besitzen würden, es wird nur bestritten, dass die Realitäten, die wir erkennen (Naturvorstellungen), vollständig deckungsgleich mit einer solchen Wirklichkeit sind. Zudem sei gesagt, dass das Konzept der gesellschaftlichen Naturverhältnisse auf der epistemischen Ebene nicht automatisch einen sozialen Konstruktivismus impliziert, allerdings ist dieser mit sozialkonstruktivistischen Annahmen umstandslos vereinbar. 
zu bestimmen. Wird zudem die Bewahrung und Förderung der Biodiversität in diesem Kontext betrachtet, dann erscheint die Problematik der Vernichtung der Biodiversität von vornherein als Veränderung der individuellen und kollektiven menschlichen Umgangsweisen mit dem, was sie als Natur begreifen bzw. was sie davon ausschließen. Dieser Fokus ist vor allem deshalb zentral, weil die Konflikte, die sowohl zwischen den Zielen und Maßnahmen der Bewahrung und Förderung der Biodiversität als auch mit anderen gesellschaftspolitischen Zielen und Maßnahmen entstehen können, nicht als Konflikte zwischen Menschen und der Natur betrachtet werden dürfen (was allerdings, wie sich an verschiedenen Stellen in der Arbeit zeigen wird, oftmals der Fall ist). Vielmehr müssen sie unter dieser Perspektive als Konflikte zwischen Menschen mit unterschiedlichen Vorstellungen von Natur, Interessen und Bedürfnissen an der Natur sowie unterschiedlichen Überzeugungen und Haltungen, warum Natur wichtig, notwendig und wertvoll für den Menschen ist und wie Menschen sich in Bezug auf die Natur verhalten sollten, verstanden werden (vgl. insb. Potthast 1999; Eser 2003b; Eser \& Müller 2006). Daher geht es zum einen um die Auslotung der kulturellen Interpretationen und der damit verbundenen Wissensformen und Praktiken im Umgang mit der Biodiversität, zum zweiten um die Analyse der in das Konzept der Biodiversität sowie der damit verbundenen Zielstellungen eingeschriebenen Werte- und Normvorstellungen mit dem Ziel etwas substanzielles zum richtigen Umgang mit der Biodiversität sagen zu können sowie drittens um die Auslotung der Möglichkeiten und Grenzen der Gestaltung gesellschaftlicher Naturverhältnisse und genauer um die Frage, wie die Bewahrung und Förderung der Biodiversität gesellschaftspolitisch umgesetzt werden kann und soll.

\subsubsection{Eine anwendungsorientierte Umwelt- und Naturschutzethik zur Beurteilung des Umgangs mit der Biodiversität}

Der umfassende Fokus auf die gesellschaftlichen Naturverhältnisse ist - wie sich im Rahmen der Grundlagenreflexion noch zeigen wird (siehe Abschnitt II) - in allen Bereichen des Umwelt- und Naturschutzes und im Besonderen für den wissenschaftlichen Bereich relevant. Hierbei ist entscheidend, dass Handlungen zum Schutz der Natur und Umwelt nicht nur beschrieben und analysiert, sondern auch bewertet und beurteilt werden müssen, was wiederum auf einen weiteren relevanten Kontext für die Arbeit verweist - eine anwendungsorientierte Umwelt- und Naturschutzethik, ${ }^{12}$ die "nach den Gründen und den aus ihnen gewonnenen Maßstäben (Werte und Normen) fragt, die

12 Im Folgenden ist nicht - wie sonst üblich - von praktischer oder angewandter Ethik ("applied ethic«) die Rede, sondern von anwendungsorientierter Ethik, da hierüber besser zum Ausdruck kommt, dass sich die allgemeine Ethik als Begründungswissenschaft nicht ohne weiteres auf praktische Fragen anwenden lässt. Vielmehr verdeutlicht die Rede von der Anwendungsorientierung, dass es zwar das erklärte Ziel solcher Ethiken ist, einen Beitrag zur Lösung konkreter gesellschaftlicher Probleme zu leisten, dass eben dies aber eine ganz eigene Herausforderung ist, die mit bestimmten Schwierigkeiten für das Selbstverständnis der Wissenschaftsdisziplin Ethik verbunden ist. 
unser individuelles und kollektives Handeln im Umgang mit der außermenschlichen Natur bestimmen sollen." (Ott 2010:8) Die für die (Umwelt-)Ethik zentrale Frage nach den Gründen (Argumenten) für Umwelt- und Naturschutzanliegen, setzt ein grundlegendes Verständnis davon voraus, warum und wofür Menschen einander überhaupt Gründe schulden und wie Menschen sich selbst und anderen gegenüber für oder gegen etwas argumentieren (ebd., 8). Insofern ist eine Umwelt- und Naturschutzethik, die das Konzept der gesellschaftlichen Naturverhältnisse aufnehmen und integrieren will, bereits im menschlichen Sprachgebrauch fundiert.

Daher bietet es sich an die ethische Beschäftigung mit Natur und Umwelt in der geteilten Argumentationspraxis zu verankern (ebd., 13). ${ }^{13}$ Dies wiederum legt eine diskurstheoretische Fundierung der Umwelt- und Naturschutzethik nahe (vgl. Ott 1993, 1996, 2004, 2010), deren Grundlage die Habermasche Theorie des kommunikativen Handelns ist (vgl. Habermas 1981a, 1981b). ${ }^{14}$ Die Grundlegung eines solchen diskurstheoretischen Fundaments bedeutet in praktischer Hinsicht, dass Menschen sich darüber austauschen und verständigen müssen, was sie meinen, wie sie etwas meinen und warum sie etwas meinen. Dies ist gerade in Bezug auf Biodiversität und das Leitbild der Bewahrung und Förderung relevant, denn diese sind Paradebeispielw dafür, auf wie vielen Wegen verständigungsabträgliche Missverständnisse entstehen, obwohl doch alle (scheinbar) über dasselbe reden, aber damit ganz unterschiedliche Dinge vor ganz unterschiedlichen Hintergründen meinen. Mit anderen Worten: Wer über Biodiversität und über deren Bewahrung und Förderung redet, muss immer auch klarstellen, worüber er oder sie eigentlich redet. In jedem Diskurs müssen die Begriffe und Verwendungsweisen der Begriffe erklärt und ggf. geklärt werden, die in den Argumenten auftauchen. Dies ist bisweilen eine trockene und mühselige Aufgabe, sie ist aber notwendig, denn sonst laufen die Diskursteilnehmer Gefahr mit ihren Argumenten aneinander vorbei zu reden und gerade das ist eben kein Diskurs.

Allerdings kann und darf es dabei nicht nur um begriffliche Deutungsanalysen gehen, da auch eine diskurstheoretisch verfasste Ethik der zentralen Herausforderung aller an-

13 Dies ist weder notwendig, noch zwingend. Es ist schlicht eine Setzung, deren Für und Wider an dieser Stelle nicht diskutiert wird.

14 Damit wird vorausgesetzt, dass es die Möglichkeit einer gemeinsamen intersubjektiven Verständigung sowie die Möglichkeit gemeinsamer Einsichten darüber gibt, was 'wahr' und 'richtig' ist (siehe die Ausführungen im Text zur Reflexion dritter Ordnung). Dies wiederum setzt, ohne die dahinterstehenden diskurstheoretischen Annahmen an dieser Stelle weiter explizieren zu können, voraus, dass die Sprache das spezifische Medium der Verständigung ist und dass jede verständigungsorientierte Sprechhandlung auf einem gemeinsamen Hintergrundkonsens der Sprecher untereinander basiert, bei dem bestimmte Geltungsansprüche (Verständlichkeit, Wahrheit, Richtigkeit, Wahrhaftigkeit) implizit erhoben und gegenseitig unterstellt werden müssen, von denen zumindest Wahrheit und Richtigkeit in dem Fall, in dem der Hintergrundkonsens gestört ist, in einem Diskurs, der wiederum bestimmten Kriterien unterliegt (ideale Sprechsituation), argumentativ geprüft werden können (vgl. Habermas 1981a). 
wendungsorientierten Wissenschaften begegnen muss. Diese Herausforderung besteht vor allem darin, dass die anwendungsorientierte Ethik als Wissenschaft zur Reflexion normativer Problematiken in der unauflösbaren Spannung steht, einerseits Grundlagen- und allgemeine Begründungswissenschaft zu sein und andererseits ihr Ziel in der praktischen Anwendung normativer Maßstäbe sieht. Dies bedeutet speziell für die ethische Analyse - aber grundsätzlich auch für alle weiteren Analysen - dass diese sich in der unauflösbaren Spannung zwischen Abstraktem und Konkreten bewegen müssen, wenn auf der einen Seite die allgemeinen begrifflichen und theoretischen Grundlagen und auf der anderen Seite auch die speziellen Themen, Fälle und Problemlagen von Relevanz sein sollen. Mit diesem Spannungsverhältnis wird wie folgt umgegangen:

Es wird davon ausgegangen, dass anwendungsorientierte Ethiken sich an den praktischen Grundfragen orientieren, was Menschen in ganz bestimmten Kontexten und konkreten Situationen tun sollten (Bayertz 1994:8). Über diesen Fokus werden zwar die Lebensumstände von Menschen und das gesellschaftliche Zusammenleben stärker in den Blick genommen, als dies innerhalb der allgemeinen Ethik der Fall ist, trotzdem wird auch die anwendungsorientierte Ethik primär als ein theoretisches Projekt angesehen, in dessen Zentrum die Begründung normativer Maßstäbe und nicht die praktische Lösung konkreter Probleme steht, da das 'Kerngeschäft' der Ethik in der Diskussion, Interpretation, Systematisierung, Entwicklung, Reflexion und ggf. Revision ethischer Kriterien gesehen wird, selbst wenn sich dies an konkreten Problemlagen orientiert (Nida-Rümelin 1994:315). Insofern wird das praktische Element als sekundär begriffen, da das spezifisch Praktische an der anwendungsorientierten Ethik nicht in der Vorgehensweise, sondern in der Zielstellung gesehen wird:

Normative Problematiken sollen nicht einfach nur um ihrer selbst willen theoretisch reflektiert werden, sondern es soll etwas Substantielles zu ihnen beigetragen werden (Bayertz 2004:53). Das bedeutet wiederum Allgemein, dass in allen anwendungsorientierten Wissenschaften - und so auch in der Umwelt- und Naturschutzethik - versucht werden muss, über rein theoretische Analysen, die dennoch das Kernstück ihrer Arbeit bleiben, hinauszugehen, indem danach gefragt wird, wie die theoretisch gewonnenen Maßstäbe praktisch umgesetzt werden können. Hierbei ist speziell für die Umwelt- und Naturschutzethik entscheidend, dass Menschen - dies ist eben ihr Spezifikum - handelnde Wesen sind, die auf Eingriffe in die Natur und die Transformation der Natur festgelegt sind. Daher können Eingriffe in die Natur nicht per se als problematisch angesehen werden (was aber oft der Fall ist), weil Umwelt- und Naturschutz immer schon ein handelndes Tun ist, obgleich das primäre Ziel der Handlungen (insbesondere wenn es um Schutzhandlungen geht) das Unterlassen von unnötigen Transformationen und das Zulassen von Nicht-Eingriffen ist. Dies versetzt den Umwelt- und Naturschutz in die unauflösbare Spannung zwischen den Möglichkeiten und Beschränkungen des Tun- 
Sollens und Zulassen-Könnens, so dass es gar nicht um das ob, sondern immer nur um das wie des Handelns und um die Bewertung und Beurteilung bestimmter Handlungsoptionen gehen kann (ebd., 42).

\subsubsection{Eine «Good Biodiversity Governance» als Modell zur politikstrategischen Ausgestaltung der Bewahrung und Förderung der Biodiversität}

Wenn es im Kontext der Theorie der gesellschaftlichen Naturverhältnisse immer auch um die Frage geht, wie die Bewahrung und Förderung der Biodiversität gesellschaftspolitisch umgesetzt werden kann und soll und wenn es im Kontext der anwendungsorientierten Umwelt- und Naturschutzethik auch um die Bewertung und Beurteilung von nicht rein individuellen, sondern gesellschaftlichen Handlungsoptionen zum Schutz der Natur/Umwelt/Biodiversität geht, dann muss es immer auch um die stärker politikwissenschaftlichen Fragen gehen, was eine 'Politik der Biodiversität' ausmacht, welche Maßnahmen zur Bewahrung und Förderung der Biodiversität notwendig und ratsam sind und wie die Umsetzung der Biodiversitätziele und-maßnahmen politikstrategisch ausgestaltet werden kann.

In der, für das Forschungsfeld 'Politik der Biodiversität' in vielen Aspekten vergleichbaren Nachbarschaftsdisziplin, die als Politik der Nachhaltigkeit bezeichnet wird, stehen diese Fragen im Zentrum und hier wird zudem der Anwendungsorientierung wissenschaftlicher Studien bereits stärker entsprochen als dies im Bereich der Biodiversitätspolitik der Fall ist, wo selbst in vielen politischen oder politikberatenden Berichten vornehmlich „die physischen Merkmale sowie die Veränderungen des Biodiversitätszustandes im Vordergrund stehen." (Laws 2014:16) ${ }^{15}$ Daher können die Arbeiten zur Politik der Nachhaltigkeit hier konstruktive Hinweise für die, unter dem Aspekt der Förderung relevante, politikstrategische Ausgestaltung der Bewahrung der Biodiversität liefern: Eine am Leitbild nachhaltiger Entwicklung orientierte Politik muss, so konstatieren Karl-Werner Brand und Volker Fürst, „den funktionalen Erfordernissen einer präventiver (sic!) Langfrist- und integrativen Querschnittsorientierung entsprechen, die Formulierung und Umsetzung konkreter Nachhaltigkeitsprogramme ermöglichen und die globale Dimension nachhaltiger Entwicklung und sozialer Gerechtigkeit berücksichtigen.“ (Brand \& Fürst 2002:43) Als wesentliche Voraussetzungen für ein erfolgreiches Agieren seitens der professionellen politischen Akteure im Umwelt- und Naturschutz werden dabei die Schaffung gesellschaftlicher Akzeptanz für Umwelt- und Naturschutzanliegen sowie die Beteiligung betroffener und interessierter Akteure an Planungs- und Entscheidungsprozessen genannt (Heiland 2008:231). In der NBS findet

15 Vergleiche in etwa die aktuellen "Living Planet Reports» des World Wide Fund of Nature (vgl. WWF 2012, 2014a, 2014b) oder der aktuelle »Indikatorenbericht zur Nationalen Strategie zur Biologischen Vielfalt« (vgl. BMUB 2014). 
sich dieser Anspruch wie folgt wieder:

„Die nationale Strategie spricht (...) alle gesellschaftlichen Akteure [an]. Ziel der Strategie ist es, alle gesellschaftlichen Kräfte zu mobilisieren und zu bündeln. Weiteres Ziel ist es, dass Deutschland seiner Verantwortung für eine weltweit nachhaltige Entwicklung verstärkt gerecht wird. (...) Eine nachhaltige Entwicklung kann nicht einfach vom Staat verordnet werden. Nur wenn die Akteure in Wirtschaft und Gesellschaft das Thema zu ihrer eigenen Sache machen, kann die Strategie erfolgreich umgesetzt und können die darin enthaltenden Ziele erreicht werden.“ (BMUB 2011:17)

Mit dieser recht vagen Formulierung klingen bestimmte Annahmen bezüglich der politischen Umsetzung der Biodiversitätsziele und -maßnahmen in Deutschland an, die hier wiederum als Präliminarien für die Diskussion um die Umsetzung der Biodiversitätsziele und -maßnahmen zu verstehen sind:

Zum einen klingt hierbei die basale, aber auch immer wieder strittige Annahme an, dass eine demokratische Ordnung zum einen fähig und zum anderen unersetzbar bei der Lösung umwelt- und naturschutzpolitischer Probleme ist (vgl. insb. Buchstein 2012; Ott 2013). Die in diesem Zusammenhang immer wieder vorgetragenen Kritikpunkte der Demokratieskeptiker bzw. der Anhänger und Verfechter ökoautoritärer Positionen sind im Kern, dass „der innere Zeittakt der Demokratie (...) generell viel zu kurz [sei] für nachhaltige politische Vorhaben wie den Erhalt der natürlichen Umwelt“ (Buchstein 2012:53), dass „die Kultur der liberalen Demokratie (...) verzichtsfeindlich und damit umweltzerstörend [sei]“ (ebd., 54) und dass „die freiheitliche Demokratie ein Koordinationsproblem hat, das im Bereich der Umweltpolitik zu fatalen Auswirkungen führt.“ (ebd.) Alle drei Argumente lassen sich nicht ohne weiteres von der Hand weisen und haben in gewisser Hinsicht auch ihre Berechtigung, sie führen aber, wenn überhaupt nur zu einer demokratieskeptischen Position, nicht aber auch dazu, dass Demokratien gegen Autokratien ausgespielt werden können (ebd., 54-56): So ist das bemängelte Kurzzeitdenken der Demokratie „gleichsam eine Halbwahrheit, die die tieferen Kontinuitäten - zumindest in der deutschen Umweltpolitik - seit den 1970er Jahren ignoriert." (Ott 2013:8) Auch entzünden sich in vielen, gerade demokratischen Ländern, anhand von Umweltthemen Debatten um gesellschaftliche Fragen und es bilden sich zunehmend auch gesellschaftliche Konsenszonen um die Grenzen des Wachstums heraus, an denen sich das „Koordinatensystem der Politik weg von der BIP-Fixierung hin zu der Frage [verschiebt], wie soziale Sicherungssysteme auch ohne hohes BIP-Wachstum funktionstauglich bleiben." (ebd., 6) Die Kultur der liberalen Demokratie ist folglich nicht per se umweltzerstörerisch. Das, der Demokratie innewohnende, Koordinationsproblem ist schlussendlich keine spezifische Demokratie-, sondern vielmehr eine allgemeine Koordinations- und Kooperationsproblematik (vgl. Scharpf 2006). Freiwilli- 
ge Kooperationsbereitschaft bei individuell zu erwartenden Vorteilen und Kooperationsverweigerungen bei individuell nachteiligen Ergebnissen sowie Kooperations- und Koordinationsprobleme wie Trittbrettfahren, Abwärtsspiralen und Einigungen auf dem kleinsten gemeinsamen Nenner statt auf die, im normativen und empirischen Sinne, bestmögliche Lösung, sind Herausforderungen, die in Demokratien angegangen werden müssen, vor allem dann, wenn diese in zunehmendem Maße durch Kooperationen über die politischen Ebenen hinweg (Mehrebenenpolitik bzw. Multi-Level-Governance) und durch pluralistische und neokorporatistische Verhandlungen zwischen öffentlichen und privaten Instanzen geprägt ist (ebd., $281 \mathrm{ff}$.). Diese Problematiken stellen sich in einer vernetzten, globalisierten Welt auch für Autokratien, die Lösungsstrategien hier sind indes Zwang statt freier Entscheidung, Anweisung statt Aushandlung und bloße Hinnahme statt Akzeptanz. Alternativen sind es, es stellt sich nur die Frage, ob es legitime und effektive Alternativen sind und ob der Zweck hierfür die Mittel heiligt.

Zum zweiten basiert die Vision in der NBS auf der empirisch bestätigten, aber theoretisch nicht einheitlich konzeptualisierten Prämisse, dass gerade im Politikbereich des Umwelt- und Naturschutzes, wo es seit den 1970er Jahren zunehmend auch um grenzüberschreitende Problematiken ging (Ozonloch, Verschmutzung, Abholzung, Klimawandel, Biodiversität etc.), die Reichweite des separierten einzelstaatlichen Handelns den zu bearbeitenden Problemen immer weniger gerecht wird. Dies ist dem Umstand geschuldet, dass solche grenzüberschreitenden Umwelt- und Naturschutzproblematiken immer auch einen transnationalen Ansatz zu ihrer Eindämmung verlangen. Gerade am Beispiel der Umweltpolitik kann daher der "Wandel von National Government zu Global Governance « exemplarisch besonders eindrucksvoll beobachtet werden. ${ }^{16}$ Dirk Messner hält diesbezüglich fest, dass sich „die monozentrische Machtstruktur rivalisierender Nationalstaaten (...) in eine polyzentrische Machtverteilung " transformiert und dass darüber die „Trennungslinien zwischen Innen- und Außenpolitik immer unschärfer werden." (Messner 1998: 17-18) Allerdings impliziert dies mitnichten, dass die Bedeutung von Staaten und Staatlichkeit dadurch zwangsläufig abnimmt. Die Europäisierung und Internationalisierung ehemals nationalstaatlicher Aufgaben, die eine Antwort auf die zunehmend globalen wirtschaftlichen, natürlichen und sozialen Problematiken darstellt, schafft zwar eine „neue Architektur von Staatlichkeit“ (vgl. Grande 1993), die eine Herausforderung an traditionelle Konzepte und Denkmuster des Politischen darstellt, diese "neue Architektur" ist aber nicht zwangsläufig gleichbedeutend mit einer generellen oder gar negativ zu bewertenden Einschränkung nationalstaatlicher Hand-

16 Es muss von vornherein gesagt werden, dass diese Wandlungsprozesse (siehe auch im Folgenden den Wandlungsprozess von staatlicher Steuerung zu kooperativer Regelung) im Sinne eines Trends oder eines Veränderungsprozesses und keineswegs im Sinne eines Ablösungs- oder Ersetzungsprozesses verstanden werden sollten. Nationale Regierungen gibt es nach wie vor, genauso wie staatliche Steuerung. Beide Modi werden aber durch das Hinzukommen globaler Governance und kooperativer Regelung in einem politischen Mehrebenensystem herausgefordert und verändert (siehe 5.3). 
lungsmöglichkeiten. Fest steht vielmehr, dass dort, wo sich alternative Spielräume des Politischen eröffnen, neue, in komplexer Weise verwobene, Politikebenen und Politikarenen entstehen (politisches Mehrebenensystem), die den Nationalstaat zu einem Akteur unter vielen werden lässt, wenn auch nach wie vor zu einem Wichtigen und Besonderen. Deshalb ist und bleibt der Nationalstaat der zentrale „Dreh- und Angelpunkt unseres politischen Koordinatensystems.“ (ebd., 14).

Vor diesen Hintergründen geraten drittens auch die etablierten Institutionen, Regelwerke und Strukturen der nationalstaatlichen Politik unter enormen Anpassungs- und Veränderungsdruck, da sich die Staatlichkeit nicht nur der Form nach und nach außen wandelt, sondern auch inhaltlich und nach innen. Der damit verbundene Wandel von einer hoheitlich-staatlichen, hierarchischen Steuerung hin zu stärker kooperativen, verhandelnden und dialogischen Formen des Regierens, kann als „Wandel von staatlicher Steuerung hin zu kooperativer Regelung ${ }^{17}$ beschrieben werden und steht immer dann im Fokus, wenn vom „kooperativen Staat“ (vgl. Ritter 1979), dem „verhandelnden Staat“ (vgl. Scharpf 1991), von „Verhandlungsdemokratie“ (Czada 2000) oder von Politik als "Interdependenzmanager" (Walk 2008:15) die Rede ist, da hierbei den politischen Akteuren die Aufgabe zugeschrieben wird, zwischen den unterschiedlichen gesellschaftlichen Interessen und politischen Ebenen zu vermitteln. Damit steht die Annahme des innerstaatlichen Politikwandels im Fokus, wenn in der NBS davon die Rede davon ist, alle gesellschaftlichen Akteure anzusprechen, zu mobilisieren und zu bündeln, damit Deutschland seiner Verantwortung für eine weltweit nachhaltige Entwicklung gerecht werden kann.

Die Beachtung dieser drei Kontexte, die hinter der in der NBS formulierten Vision stehen, führen dazu wiederum dazu, dass - erst einmal theoretisch noch gänzlich unbestimmt- von "Good Biodiversity Governance» (GBG) als politikstrategischen Modus zur Ausgestaltung der Bewahrung und Förderung der Biodiversität in Deutschland gesprochen wird. Dieser 'Titel` ergibt sich wie folgt: Von »Governance« wird gesprochen, weil alle drei hier darlegten Aspekte - die Fokussierung auf eine demokratische Ordnung, der Wandel von Government zu Governance sowie von staatlicher Steuerung zu kooperativer Regelung - mal mehr, mal weniger explizit über das Governancekonzept erfasst und zugleich durch die politikwissenschaftliche Governanceforschung beschrieben und analysiert werden. Des Weiteren ist von »Biodiversity Governance» die Rede, weil in der Vision der NBS zum Ausdruck kommt, dass die formellen politischen Instan-

17 Hinter dem, hier erst einmal unscheinbar daherkommenden Begriff der "kooperativen Regelung" verbirgt sich ein wichtiger theoretischer Zusammenhang: Renate Mayntz und Fritz Scharpf haben den Begriff der „Regelung“ als prozedurales Konglomerat für den oftmals rein strukturell ausbuchstabierten Governancebegriff eingeführt (Mayntz \& Scharpf 1995a:16). Die beiden hier benannten Wandlungsapekte (von Government zu Governance auf der Strukturebene und von Steuerung zu Regelung auf der Prozessebene) sind folglich zugleich für Governanceforschung relevant (siehe 5.3). 
zen, wie Parteien, Regierung und Verwaltungsapparat weniger die Ausgangspunkte, als vielmehr die strategischen Einsatzpunkte der Politikformulierung zur Bewahrung und Förderung der Biodiversität sein sollen, was das Governancekonzept im Gegensatz zum klassischen Topos des Government ebenfalls auszeichnet (Demirović \& Walk 2011:7). Zu guter Letzt ist vor den Hintergründen des (inner-)staatlichen Wandels immer auch von den 'New Modes of Governance' (oder neuen Regelungsinstrumenten) die Rede. Hierbei geht es um einen kooperativen, verhandlungsbasierten und dialogischen Politikstil, an den wiederum die Hoffnung geknüpft ist, dass speziell durch ein 'Mehr' an Partizipation, Deliberation und Diskurs zu einer zugleich legitimeren als auch effektiveren Politik zum Schutz der Natur gelangt werden kann (Bäckstrand et al. 2010: 3). Da dies einen starken normativen Bias hat und vielfältige normative Implikationen birgt, ist dies der Grund, warum von »Good Biodiversity Governance» gesprochen wird. ${ }^{18}$

\subsection{Aufbau und Fortgang der Arbeit}

Neben der Einleitung (Abschnitt I), in der es um den Fokus, die Arbeitsthese, die Methodik, die Zielstellung und die soeben dargelegten Präliminarien der Arbeit ging und die mit der nun folgenden Darlegung des Aufbaus der Arbeit abgeschlossen wird sowie der Schlussbetrachtung (Abschnitt VI), in der die Erkenntnisse der Arbeit noch einmal in der Zusammenschau und mit Bezug zueinander dargelegt werden, wird die, in der ganzen Arbeit im Fokus stehende, Problemreflexion über eine umfassend und systematisch angelegte Grundlagenreflexion auf die Biodiversität sowie ihre Bewahrung und Förderung eröffnet und diese wird dann, probleminduziert in den jeweiligen Detailreflexionen vertieft:

In der Grundlagenreflexion (Abschnitt II) geht es erst einmal darum, den bisherigen Forschungsstand zur Biodiversität festzuhalten. Hierbei sollen die oftmals gar nicht als Problem erkannten und damit unreflektierten Präsuppositionen und Konsequenzen des Leitbildes der Bewahrung und Förderung der Biodiversität ersichtlich gemacht werden, so dass Wege aufgezeigt werden können, wie mit diesen möglichst konstruktiv umgegangen werden kann. Die zentrale Annahme hinter der Grundlagenreflexion lässt sich dabei wie folgt formulieren: Sowohl über die Konzeptualisierung der Biodiversität, die primär durch die Biowissenschaften geprägt ist, als auch über die mit dem Leitbild der Bewahrung und Förderung verbundenen politischen Ambitionen und nicht zuletzt über die, in das Konzept hineinlegten, Wert- und Normvorstellungen, wird ein spezifi-

18 Bei der hier vorgenommenen Charakterisierung von Good Governance muss von vornherein beachtet werden, dass diese in wesentlichen Aspekten von dem abweicht, was die "Weltbank und der »Internationale Währungsfond " als entwicklungspolitischen Terminus Technicus geprägt haben, indem es hier um kooperative, verhandlungsbasierte und dialogische demokratische Strukturen und partizipative, deliberative und diskursive demokratische Verfahren geht. Dies wird in Abschnitt 5.1.2 genauer ausgeführt. 
sches Problemgefüge generiert, das die Bewahrung und Förderung der Biodiversität zu einer eigenständigen theoretisch-praktischen Herausforderung werden lässt, der man sich stellen muss, soll die Bewahrung und Förderung der Biodiversität keine reine Vorstellung der Vernunft sein, sondern auch eine annähernde Entsprechung in der Realität finden. In diesem Sinne wird zuerst in inhaltlich-konzeptioneller Hinsicht geschaut, welche Problematiken sich über das Verständnis von Biodiversität als biowissenschaftliches Konzept ergeben (2.1). Zweitens wird die Biodiversität als politisches Schlagwort für einen modernen und umfassenden Natur- und Umweltschutz untersucht (2.2). Drittens wird Biodiversität als Wert- und Normbegriff analysiert (2.3). Abschließend werden die Querverbindungen zwischen den inhaltlich-konzeptionellen, politischen und normativen Aspekten der Bewahrung und Förderung der Biodiversität aufgezeigt. Dies geschieht über die Charakterisierung der Biodiversität als Grenzobjekt und Hybridkonzept (2.4). Hierbei werden ausgehend von den Problemen der Biodiversität als Wertund Normbegriff Rückschlüsse zu Biodiversität als politisches Schlagwort (Grenzobjekt) und als wissenschaftliches Konzept (Hybridkonzept) gezogen. Im Anschluss an diese, dem bisherigen Forschungsstand entsprechenden, Ausführungen wird die oftmals vernachlässigte Frage aufgeworfen, wie mit dem Grenz- und Hybridcharakter der Biodiversität im Rahmen einer problemorientierten, anwendungsorientierten und interdisziplinär angelegten Biodiversitätsforschung umgegangen werden kann und sollte. Hierfür wird zwischen drei Ebenen unterschieden, die Biodiversität als Grenz- und Hybridkonzept kennzeichnen (a. inhaltlich; b. normativ; c. politisch), die wiederum mit bestimmten Reflexionsweisen auf diese drei Ebenen (a. wissenschaftstheoretisch; b. umwelt- und naturschutzethisch; c. politiktheoretisch) zusammengebracht werden, indem bestimmte Detailproblematiken auf der inhaltlichen (2.4.1), der normativen (2.4.2) und der politischen (2.4.3) Ebene selektiert werden. Hierbei wird herausgestellt, welche speziellen Problembereiche im weiteren Verlauf der Arbeit detailliert reflektiert und konstruktiv rekonstruiert werden und es wird begründet, warum gerade diese Problematiken relevant für die übergeordnete Zielstellung der Arbeit sind, die Probleme mit dem Biodiversitätsproblem konstruktiv anzugehen und Antworten auf die ökosozialen Fragen zu finden. Den drei selektierten Detailproblematiken wird dann im Rahmen der Detailreflexionen in den Abschnitten III, IV und V nachgegangen.

Im dritten Abschnitt der Arbeit wird, im Anschluss an die Problemselektion in Abschnitt 2.4.1 wissenschaftstheoretisch auf den Forschungsgegenstand der Biodiversität und dessen kulturelle Konstitutionsbedingungen reflektiert. Dabei wird, ausgehend von der in Abschnitt 2.4.1 festgehaltenen Detailproblematik um den methodologischen Kurzschluss der Gleichsetzung von biologisch und biotisch, auf das, hinter der Detailproblematik stehende realistische Wissenschaftsverständnis eingegangen (3.1). Im Anschluss daran wird dargelegt, welche Aspekte dabei jeweils für das Verständnis der Biodiver- 
sität als Grenz- und Hybridkonzept problematisch sind (3.2). Hierbei wird einerseits aus einer externen, d.h. konstruktivistischen und wissenssoziologischen Perspektive auf die pragmatischen Kritikpunkte am wissenschaftlichen Realismus als wissenschafts- und erkenntnistheoretische Grundlage für den Forschungsgegenstand der Biodiversität eingegangen (3.2.1). Hierbei wird argumentiert, dass das realistische Verständnis von der naturwissenschaftlichen Praxis als Produktion wahrer und wirklichkeitsnaher Theorien durch 'objektive Naturbeobachtung' die teilnehmenden Aspekte von Wissenschaft als sozialer und kultureller Praxis der Gegenstandskonstitution und konzeptionellen Konstruktion nur ungenügend ins Blickfeld rückt, womit aber viele Aspekte, die für die Entgrenzung und Hybridisierung der Biodiversität aus der wissenschaftstheoretischen Perspektive relevant sind, nur ungenügend berücksichtigt werden können. Andererseits wird der wissenschaftliche Realismus aus einer innertheoretischen Perspektive kritisiert, indem auf die realistischen Annahmen bezüglich der Wahrheitskonzeption und der Zielstellung wissenschaftlichen Handelns eingegangen wird (3.2.2). Hier wird argumentiert, dass sich die Begründung des wissenschaftlichen Realismus an theorieentscheidenden Stellen in Zirkelschlüsse verfängt, womit genau das bereits vorausgesetzt wird, was zu beweisen versucht wird. Die mit dem wissenschaftlichen Realismus verbundene Zielstellung, möglichst wahre und der Wirklichkeit entsprechende Theorien zu produzieren, ist damit zu hoch gegriffen, weil das theoretische Fundament, auf dem dieser Anspruch basiert, nicht tragfähig genug ist, um diesen Anspruch auch plausibel begründen zu können. Daher wird in Anschluss an Imre Lakatos' „Methodologie der wissenschaftlichen Forschungsprogramme» (vgl. Lakatos 1982) im dritten Schritt eine alternative Zielbestimmung wissenschaftlicher Praxis vorgenommen, auf deren Basis die kulturellen Konstitutionsbedingungen des Forschungsprogramms Biodiversität rekonstruiert werden (3.3). Dazu werden einerseits die methodologischen Konstruktionsbedingungen des Forschungskonzeptes der biologischen Vielfalt mittels des methodologischen Konstruktivismus herausgestellt (3.3.1). Andererseits werden die geistesund ideengeschichtlichen Konstitutionsideen der Forschungsobjekte der abiotischen und biotischen Vielfalt mittels des Kulturkonstitutionalismus beleuchtet (3.3.2). Über beide Ausführungen soll überprüft werden, ob die dabei zur Anwendung kommenden Ansätze zu einer progressiven Problemverschiebung, sprich zu einem theoretischen und empirischen Gehaltsüberschuss gegenüber der realistischen Betrachtung der Biodiversität als Grenz- und Hybridkonzept führen. Die wichtigsten Ergebnisse der Analyse werden abschließend in einem Fazit zusammengefasst, welches zugleich eine Überleitung auf die normative Ebene sowie die ethische Reflexion der Gründe und Begründungen für die Bewahrung und Förderung der Biodiversität ermöglicht (3.4) 
Im vierten Abschnitt der Arbeit wird aufbauend auf der Problemselektion in Abschnitt 2.4.2 sowie stellenweise auch aufbauend auf den Ergebnissen der wissenschaftstheoretischen Detailreflexion auf die normative Ebene übergegangen, auf der die theoretische Neuausrichtung der Umwelt- und Naturschutzethik hin zu einer speziellen Biodiversitätsethik im Detail reflektiert wird. Dabei wird - als notwendige Vorbereitung - sowohl die in der Ethik zentrale Rede von den 'guten Gründen` argumentationstheoretisch beleuchtet (4.1) als auch eine umfangreiche Bestandsaufnahme und kritische Reflexion der gängigen Gründe sowie der Begründungen für die Bewahrung und Förderung der Biodiversität vorgenommen (4.2). Dabei werden zum einen die Argumente in der NBS kritisch beleuchtet (4.2.1) als auch die umwelt- und naturschutzethischen Begründungsansätze für den Eigenwert und die direkten Pflichten gegenüber der Natur reflektiert (4.2.2). In Bezug auf letzteres wird einerseits auf die Wertedebatte in der Umweltethik eingegangen (4.2.2.1), indem die instrumentellen Werte (4.2.2.1.1), die eudaimonistischen Eigenwerte (4.2.2.1.2), die moralischen Selbstwerte (4.2.2.1.3) als auch die objektiven Naturwerte (4.2.2.1.4) hinsichtlich ihrer Begründungsansätze betrachtet werden und indem diese Betrachtung in einem Zwischenfazit (4.2.2.1.5) ausgewertet wird. Andererseits werden die physiozentrischen Begründungsansätze für den Schutz der Biodiversität um ihrer selbst willen reflektiert (4.2.2.2), indem die holistische (4.2.2.2.1), die ökozentrische (4.2.2.2.2) und die biozentrische (4.2.2.2.3) Position zum Inklusionsproblem dargelegt wird. Im Anschluss daran wird zusammenfassend argumentiert, dass es einer theoretischen Neuausrichtung der Umwelt- und Naturschutzethik bedarf, wenn es darum gehen soll die Bewahrung und Förderung der Biodiversität ethisch zu begründen (4.2.2.2.4).

Der theoretischen Neuausrichtung der Umwelt- und Naturschutzethik hin zur einer anwendungsorientierten Biodiversitätsethik (4.3) stehen indes drei spezielle Detailproblematiken entgegen (zu starker Fokus auf das Inklusionsproblem, auf Schutzpflichtbegründungen und auf Akzeptabilitätsbedingungen, siehe 2.4.2). Um zu einer Lösung der drei Problematiken zu gelangen, wird mit Bezug auf die erste Problematik auf die relationale Perspektive und die Ansätze und Schwierigkeiten einer inklusiven Umweltund Naturschutzethik eingegangen (4.3.1). Hierbei werden zum einen die Relationen zwischen Mensch, Natur und Gesellschaft fokussiert sowie die wesensmäßige Naturbeziehung als Grundlage der relationalen Perspektive spezifiziert (4.3.1.1). Zum anderen wird auf die Relevanz eudaimonistischer Ansätze für die relationale Umwelt- und Naturschutzethik sowie die Bedeutung der Biodiversität für ein gutes Leben eingegangen. (4.3.1.2). Im Anschluss daran wird, mit Bezug auf die zweite Problematik, ein biodiversitätsethischer Argumentationsraum zur Integration der Biodiversitätsziele und zur Erweiterung des deontischen Fokus dargelegt (4.3.2). Diesbezüglich wird bezogen auf das Ansinnen, der Integrativität der Biodiversitätszielstellung gerecht zu werden, ein kon- 
zeptionelles Problem des Ansatzes herausgestellt (4.3.2.1). Diesem Konzeptionsproblem wird begegnet, indem die diskurstheoretischen Fundamente der Argumentationstrias aus Klugheit, Glück und Gerechtigkeit rekonstruiert werden (4.3.2.2). Bezogen auf den zu einseitigen Fokus auf Pflichtenargumente werden dann die drei Argumentationstypen in einem mehrdimensionalen Ethikverständnis verortet (4.3.2.3). Daran anschließend wird die dritte Detailproblematik behandelt, indem die Akzeptanzund Akzeptabilitätsbedingungen für die Kommunikation und Aushandlung der Biodiversitätsziele und-maßnahmen ausgeführt werden (4.3.3). Dies ermöglicht wiederum eine passgenaue Überleitung in die politik-wissenschaftliche Detailreflexion (4.4).

Im fünften Abschnitt der Arbeit wird aufbauend auf der Problemselektion in Abschnitt siehe 2.4.3 und wiederum aufbauend auf den Ergebnissen der wissenschaftstheoretischen und ethischen Detailreflexion, auf die politische Ebene übergegangen, wo auf die Anforderungen, Chancen und Risiken zur Bewahrung und Förderung der Biodiversität durch eine "Good Biodiversity Governance" (GBG) reflektiert wird. Dabei werden erst einmal die Grundlagen und Bedingungen der GBG dargelegt (5.1), indem zum einen auf das Politikfeld Biodiversität eingegangen wird (5.1.1), das hinsichtlich seiner vertikalen Koordination zwischen den politischen Ebenen (5.1.1.1), seiner horizontalen Koordination speziell auf der nationalstaatlichen Ebene (5.1.1.2) und hinsichtlich der umweltpolitischen Prinzipien und Instrumentarien dargelegt wird (5.1.1.3). Zum anderen wird das in dieser Arbeit zur Anwendung kommende Verständnis von Good Governance als Grundlage der weiteren theoretischen Reflexionen spezifiziert. (5.1.2). Dazu wird erst einmal auf das entwicklungspolitische Standardkonzept von Good Governance und dessen Problematiken eingegangen (5.1.2.1) und im Anschluss daran werden die relevanten Unterschiede zwischen der Standardkonzeption und der anvisierten kooperativen, verhandlungsbasierten und dialogischen Good Governance Konzeption erarbeitet (5.1.2.2), indem aufgezeigt wird, dass das alternative Verständnis von Good Governance durch seine theoretische Nähe partizipativen, deliberativen und diskursiven Ansätzen in der Demokratietheorie gekennzeichnet ist, so dass das Good Governance Konzept anknüpfend daran anhand der Annahmen der kontextbezogenen und prozeduralistischen Demokratietheorien zu Partizipation, Deliberation und Diskurs expliziert wird (5.2). Dafür werden, vor dem Hintergrund kontextbezogener Demokratietheorien, die Zusammenhänge zwischen Good Governance und demokratischer Selbstbestimmung erarbeitet (5.2.1), indem das theoretische Grundgerüst der kontextbezogenen Demokratietheorien skizziert (5.2.1.1) und dann beispielhaft auf das Modell der „Empowered Participatory Governance« eingegangen wird (5.2.1.2). Dann werden vor dem Hintergrund der Ansätze der prozeduralistischen Demokratietheorie die Zusammenhänge zwischen Good Governance und diskursiver Verständigung sowie deliberativer Politik aufgezeigt (5.2.2). Auch hier wird erst der theoretische Hintergrund 
skizziert (5.2.2.1), bevor beispielhaft auf das Modell "deliberativer Governance» eingegangen wird (5.2.2.2). In einem Zwischenfazit (5.2.3) werden die bis dato erbrachten Überlegungen zu einer auf diesen Wegen konkretisierten Good Biodiversity Governance zusammengefasst und bilanziert. Dabei wird aufgezeigt, dass es aufgrund der theoretischen Defizite beider Herangehensweisen der Berücksichtigung governanceund policytheoretischer Erkenntnisse bedarf.

Daher wird die Good Biodiversity Governance im nächsten Schritt vor dem Hintergrund governancetheoretischer Überlegungen spezifiziert (5.3). Hierfür wird erst einmal auf den Begriff der Governance und die theoretischen Gehalte der Governanceforschung eingegangen (5.3.1), indem dargelegt wird, wie der Übergang von Government zu Governance und mithin von Steuerung zu Regelung auf der theoretischen Ebene vollzogen wird (5.3.2). Dabei wird sowohl auf die theoretischen Verschiebungen (5.3.2.1) als auch auf die Kontinuitäten zwischen Steuerungs- und Governancetheorie eingegangen (5.3.2.2) und das Governancekonzept wird über den Ansatz des wakteurszentrierten Institutionalismus« analytisch erschlossen (5.3.2.3). Diese Überlegungen werden erneut bilanziert, wobei darauf verwiesen wird, dass die Berücksichtigung der governancetheoretischen Annahmen zu einem qualitativ veränderten Demokratieverständnis führen (5.3.2.4), so dass die governancetheoretischen Überlegungen in einem nächsten Schritt um demokratie- und legitimationstheoretische Überlegungen zum Modell der Verhandlungsdemokratie erweitert werden. Diese Erweiterung leistet die »komplexe Demokratietheorie» von Fritz Scharpf (5.3.3): Ausgehend von der 1970 erschienenen Grundlegungsschrift "Demokratietheorie zwischen Utopie und Anpassung" (5.3.3.1), werden die, lediglich in Artikeln dargelegten 'Erweiterungen` der komplexen Demokratietheorie unter den Voraussetzungen der zunehmenden Internationalisierung der Staaten und der Globalisierung der Wirtschaft herausgearbeitet (5.3.3.2), indem in Rückgriff auf den Ansatz des akteurszentrierten Institutionalismus einerseits auf die Kriterien guter Politik (5.3.3.2.1) und andererseits auf die Kriterien legitimer Politik (5.3.3.2.2) unter verhandlungsdemokratischen Bedingungen eingegangen wird. Schließlich wird die komplexe Demokratie zusammengefasst und bezüglich der theoretischen Annahmen hinter der GBG expliziert (5.3.3.3). Daraufhin kann aufgezeigt werden, vor welchen Anforderungen, Chancen und Risiken die deutsche Biodiversitätspolitik steht (5.4), indem die Anforderungen expliziert werden, die sich vor dem Hintergrund der theoretischen Annahmen der GBG ergeben (5.4.1) und indem aufgezeigt wird, welche Defizite und Entwicklungsmöglichkeiten sich vor dem Hintergrund dieser Anforderungen ergeben (5.4.2). 


\section{ABSCHNITT II}

\section{DIE GRUNDLAGENREFLEXION}

\section{AUF DAS DREIDIMENSIONALE VERSTÄNDNIS \\ DER BIODIVERSITÄT ALS GRENZ- UND \\ HYBRIDKONZEPT}




\title{
II. GRUNDLAGENREFLEXION: Das dreidimensionale Verständnis von Biodiversität als Grenz- und Hybridkonzept
}

\begin{abstract}
"Biodiversität existiert zwar, aber man ist sich nicht einig, was sie ist. Biodiversität ist endlich und verschwindet stetig, aber man ist sich nicht einig, wie man sie messen soll, Biodiversität ist wichtig, aber man ist sich nicht einig, wie wichtig und für wen. Mit anderen Worten: Über das Ziel sind sich alle Experten einig, über den Weg dorthin keineswegs. (...) Gerade in Mitteleuropa ist Biodiversität eher ein abstraktes Gebilde, von dem man bestenfalls weiß, dass sich einige Wissenschaftler damit beschäftigen. Im Herzen und im Bewusstsein der Gesellschaft ist Biodiversität jedoch kaum verankert trotz aller Bemühungen von Wissenschaft und Politik, genau das zu erreichen." (Hoffmann, Hoffmann \& Weimann 2005:16, 17- 18)
\end{abstract}

Andreas und Sönke Hoffmann sowie Joachim Weimann bringen in ihrem Buch »/rrfahrt Biodiversität " prägnant zum Ausdruck, welche Problematiken bei der Beschäftigung mit der Biodiversität offenkundig werden. Eben diese sollen im Folgenden im Vordergrund der weiteren Betrachtung stehen. Die zentrale Hintergrundthese lautet dabei, dass sich Biodiversität als Begriff zwischen wissenschaftlicher Konzeptualisierung (2.1), politischer Symbolisierung (2.2) und normativer Imprägnierung (2.3) bewegt. Alle drei Aspekte prägen gleichermaßen die Bedeutung des Begriffes und die Zielstellung der Bewahrung und Förderung der Biodiversität und ergeben erst zusammen einen geeigneten Ansatz zu deren Verständnis. Wenn indes nicht alle Aspekte zugleich beleuchtet werden, dann führt dies zu einem unterkomplexen, einseitigen und in sich nicht stimmigen Verständnis von Biodiversität, weil hierbei entscheidende Elemente ausgeblendet bleiben.

\subsection{Biodiversität als biowissenschaftliches Konzept}

Obwohl die Frage, was Biodiversität ist, erst einmal einfach zu beantworten klingt, beginnen die Schwierigkeiten bereits bei der Nachfrage, worauf sich Biodiversität inhaltlich bezieht, wie Biodiversität in den Biowissenschaften terminologisch, theoretisch und methodisch eingeholt wird und wie die biowissenschaftlichen Grundlagen der Biodiversitätskonzeption außerhalb der Biowissenschaften angewandt werden. Ausgangspunkt der folgenden Analyse sind folglich die Definitionen, Begriffsbestimmungen und Erklärungen, die zur inhaltlichen Bestimmung der Biodiversität angeführt werden können. ${ }^{19}$

19 Hierzu muss sicherlich gesagt werden, dass die CBD-Definition zwar die gebräuchlichste Definition ist, sie ist aber keine Standarddefinition, da es viele verschiedene Definitionen von Biodiversität gibt, die 
Bezüglich der Definitionen ist der Umstand relevant, dass sich die gebräuchlichste Definition der Biodiversität in der Internationalen Biodiversitätskonvention (CBD) wiederfindet. In der CBD-Definition wird Biodiversität als die Vielfalt der Lebensformen (biotische Vielfalt) und ihrer Lebensbedingungen (abiotische Vielfalt) verstanden:

„»(B)iologische Vielfalt« [bedeutet] die Variabilität unter lebenden Organismen jeglicher Herkunft, darunter unter anderem Land-, Meeres- und sonstige aquatische Ökosysteme und die ökologischen Komplexe, zu denen sie gehören; dies umfasst die Vielfalt innerhalb der Arten und zwischen den Arten und die Vielfalt der Ökosysteme." (UN 1992:Art.2; SL)

In der NBS wird auf diese Definition rekurriert, es wird aber ein deutlich umgangssprachlicheres Vokabular gewählt, das einen noch größeren Interpretationsspielraum eröffnet, als die obige CBD-Definition. Biodiversität wird hier - zumindest was den Gegenstandsbereich angeht - mit belebter Natur gleichgesetzt, allerdings fallen darunter auch alle Lebensbedingungen, die biotische Vielfalt ermöglichen, womit die Unterscheidung zwischen natürlicher und anthropogener Vielfalt unscharf wird:

„Biologische Vielfalt oder Biodiversität ist letztlich alles das, was zur Vielfalt der belebten Natur beiträgt." (BMUB 2011:9;SL)

Zwei weitere wichtige Aspekte finden sich in der Definition von Timothy Farnham, der herausstellt, dass Biodiversität primär auf die biotische Vielfalt ausgerichtet ist und die abiotischen Bedingungen nur insofern relevant sind, als das sie zur Aufrechterhaltung dieser beitragen:

„Biological Diversity is more than simply a new name for nature; its definition requires a certain perception of the structure of the natural world. First, the central interest of biological diversity is the variety of all life on earth (...). Living things are primary. Any interest in geologic, chemical, and physical attributes of the natural world is placed in the context of their impact upon or connection to biological life. Second, biological diversity is most commonly considered at three distinct levels of organization: genetic diversity, species diversity, and ecosystem diversity. (...) The definition thus establishes a specific framework for how we are to consider the phenomenon of life and our impact on it." (Farnham 2007:2)

Außerdem wird Biodiversität bei Farnham als ein wissenschaftliches Modell verstanden, welches über die drei biologischen Ebenen der genetischen Vielfalt, der Artenvielfalt und der ökosystemaren Vielfalt versucht, der unüberschaubaren Vielfalt des Lebendigen eine naturwissenschaftlich erforschbare Struktur zu geben. Damit zeugt der Begriff der Biodiversität von dem (bio-)wissenschaftlichen Interesse ein übergeordne-

unterschiedliche Aspekte fokussieren und thematisieren. Eine Übersicht solcher Definitionen und ihrer inhaltlichen Variabilitäten findet sich bspw. bei David Takacs (vgl. Takacs 1996) 
tes Konzept zur Erschließung der Vielfalt des Lebens und deren Lebensbedingungen zu generieren. Hierbei kommt es indes darauf an, was genau unter Diversität zu verstehen ist und wie die Begriffe Art, Gen und Ökosystem bestimmt werden. Mit Otto Solbrig kann (bio)divers als Eigenschaft lebender Systeme verstanden werden, voneinander spezifisch verschieden zu sein. Diversität verweist folglich auf Variabilität und das Vermögen von Organismen, sich voneinander zu unterscheiden:

„Biodiversität wird hier definiert als die Eigenschaft von Gruppen oder Klassen von Einheiten des Lebens, sich voneinander zu unterscheiden. D.h. jede Klasse biologischer Entitäten - Gen, Zelle, Einzellebewesen, Art, Lebensgemeinschaft oder Ökosystem - enthält mehr als einen Typ. Biologische Systeme sind hierarchisch strukturiert. Biodiversität zeigt sich auf allen Ebenen der biologischen Hierarchie, von Molekülen bis zu Ökosystemen." (Solbrig 1994:9; SL)

Obwohl diese Definition eine explizit fachwissenschaftliche ist, werden auch hier mehr Fragen aufgeworfen als Zusammenhänge erhellt (Potthast 1999:143): Zum einen stellt sich die Frage, was es bedeuten soll, dass Biodiversität eine Eigenschaft von Einheiten des Lebens oder lebender Systeme ist, denn es bleibt ungeklärt, was genau es ist, was diese Gegenstände zu Einheiten des Lebens oder lebenden Systemen macht. Des Weiteren muss danach gefragt werden, worin die Eigenschaft biodivers zu sein, genau bestehen soll, was genau es bedeuten soll, dass Einheiten des Lebens oder lebende System unterschiedlich voneinander sind und anhand welcher Merkmale eine solche Unterschiedlichkeit festgemacht werden soll. Mit diesem Nachfragen rutscht man bereits tief in die biowissenschaftlich theoretischen Debatten hinein. Ohne auf diese hier im Detail eingehen zu können und zu wollen, soll hier vielmehr folgender Aspekt für die weitere Betrachtung fokussiert werden:

Das Biodiversitätskonzept erscheint als eine "neuartige biowissenschaftliche Matrix" (Potthast 1999:149), in der alle Einheiten des Lebens als hierarchisch geordnet verstanden werden, vom Gen bis hin zum Ökosystem. Hierüber wird der Eindruck erweckt, als könnten alle biowissenschaftlichen Disziplinen, innerhalb eines einheitlichen Konzepts, ihren Beitrag zur Erfassung und Analyse der Vielfalt des Lebens und ihrer Lebensbedingungen leisten. Es zeigt sich jedoch, dass vor dem Hintergrund der Vielzahl an widerstreitenden Konzeptionen und Ansätzen in der Biologie und Ökologie diese schlicht additiv und damit beziehungslos aneinander gereiht wurden (Takacs 1996:51), ohne dass die funktionalen Zusammenhänge sowie die Mechanismen, Übergänge und wechselseitigen Beziehungen zwischen den biologischen Ebenen spezifiziert wurden:

„Im Grunde genommen verweist der Biodiversitätsbegriff - biologietheoretisch auf ein ungelöstes Problem der Integration von Systemtheorie, Evolutionstheorie und Ökologie. Entweder geht die Richtung hin zu einer konsequenten Fortführung des systemtheoretischen Gedankens zu selbstreferentiellen und autopoieti- 
schen Systemen. Lebewesen sind dann nur wenig besondere Fälle im Rahmen allgemeiner - in weiterem Sinne - physikalischer Gesetze zur Selbstorganisation aller Materie (...). Oder man betont den Organismus als besonderes System, denn genau solche 'Lebewesen` bilden die unhintergehbare Spezifität der Biologie und der Ökologie als einer ihrer Subdisziplinen (...)." (Potthast 2007a:78)

Solange dieses biologietheoretische Problem besteht, ist indes fraglich, ob die Diversität der Lebensformen oder das Ausmaß der Vielfältigkeit überhaupt einheitlich konzeptualisiert, erfasst oder gar gemessen werden kann oder ob dieser Anspruch schlicht verfehlt ist, weil Biodiversität für Untersuchungen und Messungen immer schon auf bestimmte Aspekte 'heruntergebrochen' werden muss. Ein Blick auf die Begriffe Art, Gen und Ökosystem, die mitnichten einheitlich zu definierende Konzepte sind und an denen sich zudem keine rein biologischen Kontroversen entzünden, zeigt weitere Problematiken hinsichtlich des Anspruchs, dass Biodiversität ein einheitliches biowissenschaftliches Konzept zur Erfassung der Vielfalt des Lebendigen sein soll und erhärtet den Verdacht, dass eben dieser Anspruch nicht einlösbar ist:

Der Artbegriff, der sowohl in den Biowissenschaften als auch im politischen Natur- und Umweltschutz eine zentrale, wenn nicht sogar die zentralste Rolle spielt (Gutmann \& Janich 2001a:282), wird über die Biodiversitätsdebatte noch einmal aufgewertet, mit der Konsequenz, dass die Artenvielfalt zum einen als der zentrale Indikator für die Biodiversität im Ganzen betrachtet wird und andererseits Biodiversität in der Öffentlichkeit oftmals auf die Vielfalt von Tier- und Pflanzenarten reduziert wird (BMUB 2012: $60)$. Die Gründe dafür werden in der (vermeintlich) allgemeingültigen Systematik zur Erschließung der Artenvielfalt gesehen:

„Von den drei Vielfaltstypen der Konvention gibt es nur für die Artenvielfalt eine allgemein gültige und verständlich erscheinende systematische Einteilung. Daher erhielt dieser Biodiversitäts-Typus den Vorzug und sogar ein Übergewicht mit der Folge, dass fast alle Diskussionen um die biologische Vielfalt auf die Artenvielfalt beschränkt sind." (Haber 2009:25)

Die vermeintliche Allgemeingültigkeit und Relevanz des Artbegriffs in den Debatten täuscht indes darüber hinweg, dass dessen Definition in den Biowissenschaften kontrovers diskutiert wird (vgl. Gutmann 1998), weil es hier ganz unterschiedliche Artkonzepte und -kriterien gibt, nach denen Arten identifiziert und Artzugehörigkeiten festgelegt werden können (Gudo \& Steininger 2001:36). ${ }^{20}$ Von den vorliegenden Definitionen

20 Die gängigen Bestimmungen des Artbegriffes in den Biowissenschaften sind das morphologische, das biologische, das ökologische, das evolutionäre, das phylogenetische, das nischenbezogene, das Rekognitions- sowie das Kohäsionskonzept (vgl. Henrich 2003:212-215 nach Bispy 1995: 41-44): In der morphologischen Artdefinition wird bspw. eine Gruppe von Individuen als Art bezeichnet, die sich in morphologischer oder komparativer Hinsicht von anderen Gruppen unterscheidet. In der biologischen Artdefinition hingegen ist eine 'Art' eine Gruppe tatsächlich und potenziell kreuzbarer Indivi- 
des Artbegriffs ist keine für sich allein genommen geeignet, alle heute bekannten Arten taxonomisch zweifelsfrei abzugrenzen (Piechocki 2010:67). Hieran wird deutlich, dass es selbst in den Biowissenschaften keine unkontroversen Kriterien der Artzuschreibung gibt, was bedeutet, dass es auch kein umfassendes Artkonzept gibt, das in die Biodiversitätskonzeption eingehen könnte, da die Merkmalsauswahl immer relativ zu einem vorgegebenen Konzept erfolgt und auch nur in diesem Kontext operationalisierbar und kritisierbar ist. Daher ist der Artbegriff immer schon eine Beschreibungskategorie und eine "wissenschaftliche Abstraktion." (Haber 2002:26) Real sind hingegen nur die einzelnen Organismen, die im Vergleich zueinander bestimmte Ähnlichkeiten und Unterschiede aufweisen, die je nach Systematik wechseln. Hierbei wird zugleich vorausgesetzt, dass es Lebensphänomene sind, die die Biologie studiert (Schark 2005:5). Damit bauen alle Artkonzepte bereits auf einem vorwissenschaftlichen Verständnis dessen auf, was Leben ausmacht und was das Lebendige vom Nicht-Lebendigen unterscheidet, die ihrerseits nicht biowissenschaftlich, sondern ontologisch-metaphysisch bestimmt werden (Reuter 2014:30). In diesem Zusammenhang spricht Marianne Schark von einem "lebensweltlichen Apriori der Biologie“ (Schark 2005:5). Auch Katrin Reuter verweist in ihrer Doktorarbeit auf diesen Punkt und konstatiert,

„dass die gegebenen Definitionen von Biodiversität nicht weiterhelfen, wenn es darum geht, „Gegenstände biologischer Vielfalt als solche zu identifizieren oder zu verstehen, was sie sind, da sie erst einmal nicht über die Tatsache der Unterschiedlichkeit von Gegenständen, die wir bereits als mit Leben zu tun habend identifiziert haben, hinausweis(en). In dieser Weise (geben) sie jedoch nicht mehr an, als dass die entsprechenden Gegenstände in irgendeiner Weise mit Leben zu tun haben." (Reuter 2014:19)

Im Kontext des Natur- und Biodiversitätsschutzes ist des Weiteren entscheidend, dass Artenschutz aus verschiedenartigen Gründen heraus betrieben wird, die zuweilen in Konflikt miteinander geraten können, indem dadurch ganz unterschiedliche Arten zu priorisierten Schutzgütern werden (Piechocki 2010:68). So umfasst ein ästhetisch motivierter Artenschutz andere Arten (bspw. sogenannte Flaggschiffarten wie Papageien, Tiger, Elefanten oder besonders schöne Schmetterlinge) als ein Artenschutz aus ökologischen (wo es um funktionelle Aspekte oder Schlüsselfunktionen der Art im Naturhaushalt oder im Nahrungssystem geht), aus ökonomischen (Schonung 'nützlicher' Arten, wie bestimmte Heilpflanzen oder Nahrungsmittel) oder aus kulturhistorischen Gründen (Schutz heimatlicher Arten, wie bspw. Rotwild, Wölfe oder Seeadler). Des Weiteren ist hier die Unterscheidung zwischen einheimischen und gebietsfremden Ar-

duen, die sich mit Individuen anderer Gruppen unter natürlichen Bedingungen nicht fortpflanzen, während die ökologische Artdefinition davon ausgeht, dass eine 'Art' eine Population oder eine Gruppe von Populationen ist, deren Mitglieder eine definierte ökologische Nische nutzen und an diese in charakteristischer Weise angepasst sind (Lönnig 1993:Abschnitt III). 
ten von besonderer Bedeutung, was fachwissenschaftlich eingefärbt, auch in der Sprache von endemischen Arten sowie Neophyten (Pflanzen) und Neozoen (Tiere) daherkommt. Was indes an der Ausbreitung 'fremder Arten' aus der Naturschutzperspektive problematisch ist und ob bzw. mit welchen Gründen Bekämpfungsmaßnahmen gerechtfertigt sind, ist höchst strittig (Eser 2004:168) und weniger eine Frage biologischer und ökologischer Fakten, als vielmehr unterschiedlicher emotionaler und sozialer Projektionen auf die Natur (ebd., 170-188), denn ob Menschen der Natur und genauer bestimmten Arten gegenüber positive oder negative Haltungen entwickeln und einnehmen, hängt zu einem großen Teil davon ab, welche Bedürfnisse sie an diese haben und nicht selten auch, welche Sehnsüchte und Ängste sie auf diese projizieren (ebd., 165). Eben dies bleibt aber oftmals aus den Debatten ausgeblendet bzw. wird wenn dann nur als Argument gegen die Gegner der eigenen Position verwandt, indem diesem vorgehalten wird, „aus ideologischen Gründen die Tatsachen zu verkennen." (ebd., 169)

Analog zum Artbegriff, sind auch die in den Biowissenschaften üblichen Verwendungen des Wortes Gen mehrdeutig. Auch wenn innerhalb der Genetik durchaus einheitliche Definitionen für den Begriff des Gens gebildet werden (Gutmann \& Janich 2001a:323) und der Begriff gegenüber den Begriffen Art und Ökosystem mit der (vermeintlich) größten wissenschaftlichen Autorität auftritt, sind dessen inhaltliche Bedeutungen genauso kontextvariabel, sprich abhängig vom jeweiligen theoretischen Hintergrund, so dass auch der Begriff des Gens innerhalb der Biodiversitätskonzeption keineswegs so einheitlich definiert ist, wie gemeinhin angenommen wird (ebd., 323). Diese Kontextgebundenheit hat, genau wie beim Artbegriff, zur Folge, dass die unterschiedlichen biowissenschaftlichen Disziplinen, die diesen nutzen, mit ihren jeweiligen Theorien und Methoden zu unterschiedlichen Beschreibungssprachen kommen (Hiekel 2012:192), so dass sich die Explananda genetischer Theorien nicht auf einen einzigen Genbegriff reduzieren lassen. Hier stellt sich die Frage, was genau die (biologische) Grundeinheit lebender Systeme sein soll.

Im Kontext des Natur- und Umweltschutzes und genauer der Bewahrung und Förderung der Biodiversität ist die genetische Vielfalt ganz wesentlich in den Kontext der Inwertsetzung natürlicher Ressourcen und der internationalen Regelung des Zugriffs auf diese eingebunden. Hier eröffnet sich ein breites und komplexes Feld rechtlicher, politischer und nicht zuletzt ökonomischer Zusammenhänge. So legen Christoph Görg und Ulrich Brand in zahlreichen Studien dar, dass mit dem immer stärker werdenden und von machtvollen Interessen gesetzten Imperativ der internationalen Wettbewerbsfähigkeit sowie gefördert durch die neuen Bio- und Gentechnologien auch der Umgang mit den genetischen Ressourcen bzw. deren Inwertsetzung den Rentabilitätskriterien des Kapitals unterworfen wird (Görg \& Brand 2000:237). Hierbei spielen ökonomische 
Kalküle und technische Innovationen eine ebenso große Rolle wie politisch-rechtliche Regelungen der Zugangsermöglichung und -beschränkung sowie soziokulturelle Bewertungen von Eigentum und Besitz. Da die genetische Vielfalt in diesem Kontext vor allem im Bereich der Pflanzensorten von entscheidender Bedeutung ist und hier wiederum die gentechnische Entwicklung zur Nutzbarmachung genetischer Ressourcen betrachtet werden muss, ist mit der Inwertsetzung der genetischen Vielfalt nicht nur gemeint, dass sie zum Gegenstand eines warenförmigen Austauschs wird, sondern dass neue internationale Regulierungen mit einem international gültigen System an Eigentumstiteln entwickeln werden, mit dem die ökonomisch interessanten Aspekte und Teile der genetischen Vielfalt in kalkulierbare Investitionsentscheidungen in einem globalen Markt überführt werden können (ebd., 243). Die in der CBD festgelegten Regelungen zur Problematik der Zugangsregelungen (access) und zur Gewinnverteilung (benefit sharing) aus der Nutzung der genetischen Ressourcen (siehe 2.2.1) und die im Vorfeld und im Nachgang der Konvention bestehenden Kontroversen zwischen den Ländern des Südens (biodiversitätsreich und technologiearm) und des Norden (biodiversitätsarm und technologiereich) sowie den Anhängern und Bedürftigen traditioneller Nutzungsformen (indigene Völker, Bauern) und internationalen Großkonzernen (Patentinhabern auf Saatgut, Pflanzenbestandteilen, Medikamenten etc.) zeigen, dass gerade in Bezug auf die genetische Vielfalt ein spannungsreiches Konfliktfeld eröffnet wird, bei dem die Nutzung der Biodiversität als solche nicht das eigentlich strittige Problem ist. Vielmehr geht es hierbei um grundlegende Fragen, wie bspw. ob Nahrungsmittel in Patentregelungen einbezogen werden können und dürfen, ob Gene überhaupt unter den materialistisch geprägten Eigentumsbegriff fallen und damit Gensequenzen als solche patentiert werden dürfen oder ob nur die technische Innovation zur Erzeugung oder Nutzbarmachung dieser patentfähig ist und schließlich unter welchen sozialen und ökologischen Bedingungen und mit welchen Konsequenzen (für Staaten, die Bevölkerung, internationale Konzerne, traditionelle Nutzer) diese Patentierungen einhergehen.

Die, weit über biologische und ökologische Fakten, hinausgehende Kontroversen, die bereits in Bezug auf die Begriffe Art und Gen konstatiert wurden, treffen vor allem auf den Begriff des Ökosystems zu. Hier allerdings mit dem wesentlichen Unterschied, dass die theoretischen Kontroversen über die Fragen, was Ökosysteme sind, ob sie zur Selbstregulation fähig sind, ob sie sich in einem Gleichgewicht befinden können, ob sie sich durch Stabilität auszeichnen und wenn ja inwiefern, welche Funktionen und Leistungen Ökosysteme erbringen und für wen, ob und ab wann Ökosysteme zerstört sind und wie man Ökosysteme schützen kann, innerhalb und außerhalb der Biowissenschaften rege geführt werden (vgl. Jax 2004). Dies wiederum erklärt sich daraus, dass der Begriff des Ökosystems über die Grenzen der Biowissenschaften hinaus in der Po- 
litik und in der Alltagssprache etabliert ist und hier unzählige Bedeutungen annehmen kann, die in Grenzfällen mit dem biowissenschaftlichen Begriff des Ökosystems, als einem bereits vage definierten „Beziehungsgefüge der Lebewesen miteinander und mit ihrem Lebensraum" (ebd., 137) nur noch wenig zu tun haben: Kurt Jax führt aus, dass der Begriff des Ökosystems in einer Auseinandersetzung über die 'Natur' von Ganzheiten bzw. Systemen in der Ökologie entstanden ist und dass der Begriff von Beginn an im Detail sehr unterschiedlich ausgelegt werden konnte (Jax 2004:137). In einer Art Minimaldefinition bezeichnet »Ökosystem« ein „Beziehungsgefüge der Lebewesen miteinander und mit ihrem Lebensraum.“ (ebd.) Die Unterschiede ergeben sich dabei darüber, ob die Grenzen von Ökosystemen funktional oder topographisch festgelegt werden, welche Art von Wechselwirkungen der Lebewesen untereinander fokussiert werden, wie diese verstanden und empirisch untersucht werden und welche Komponenten als Bestandteile des Ökosystems berücksichtigt werden (Piechocki 2010: 99). Jax hält daher fest, dass es „keine triviale Aufgabe [ist], die Charakterisierung dessen, was mit 'Ökosystem ' jeweils gemeint ist, so genau zu fassen, dass zwei Beobachter tatsächlich den gleichen physischen Gegenstand damit aus der Natur heraus kristallisieren können." (Jax 2004:138) Die Geläufigkeit und Allgegenwärtigkeit des Wortes Ökosystem täuscht folglich darüber hinweg, dass es sich hierbei selbst innerhalb der Ökologie um einen offenkundig undifferenziert verwendeten Ausdruck handelt (Gutmann \& Janich 2001a:311), bei dem die theoretischen Problemlagen offensichtlich werden, wenn holistische gegen reduktionistische, organizistische gegen individualistische und ontologisierende gegen nominalistische Ökosystemkonzepte gestellt werden (Potthast 1999:57).

Im Kontext des Natur- und Umweltschutzes, aber auch der Bewahrung und Förderung der Biodiversität, werden diese Kontroversen ersichtlich, wenn geschaut wird, auf welche Gegenstände bzw. Gegenstandsbereiche der Begriff des Ökosystems angewandt wird und welche Rückwirkungen dies wiederum auf die Bedeutung der Arten innerhalb dieser Ökosysteme hat. Hierbei stehen jeweils unterschiedliche Betrachtungsweisen von Ökosystemen im Fokus, aus denen sich wiederum ganz unterschiedliche Schutzimperative in Bezug auf das Ökosystem selbst und die darin vorkommenden Arten ergeben. Reinhard Piechocki macht deutlich, dass Ökosysteme im Natur- und Umweltschutz vor allem als Wildnis-, (ökosystemare) Dienstleistungs-, Landschafts- und Heimatobjekte ausgedeutet werden (Piechocki 2010:99): Ökosysteme, die als Wildnis betrachtet werden, beinhalten den Schutzimperativ, 'Natur Natur sein zu lassen', wobei der Schutz spezifischer Arten dabei nicht relevant ist. Hierbei wird das Ökosystem als ein System aus Stoff- und Energieflüssen betrachtet, das aus bestimmten funktionalen Kompartimenten besteht, die ins sich austauschbar sind (Jax 2004: 138). Das bedeutet wiederum, dass solange bestimmte Basisfunktionen innerhalb des Systems erhalten 
bleiben (bspw. eine bestimmte Primärproduktion oder ein bestimmter Wasserkreislauf), das Ökosystem als 'intakt' angesehen wird, selbst wenn bestimmte Arten wechseln oder das Ökosystem Arten beinhaltet, die - in welcher Hinsicht auch immer - problematisch für den Menschen oder das von Menschen als ästhetisch empfundene Landschaftsbild sind (ebd.). ${ }^{21}$ Werden Ökosysteme als Landschafts- und/oder Heimatobjekte betrachtet, dann geht dabei primär um die Pflege und Kultivierung der typischen Eigenart des (Kultur-)Landschaftsbildes (Piechocki 2010: 100). Speziell bei Heimaten geht es zudem nicht nur um die Erhaltung eines bestimmten Landschaftsbildes, das zumeist als ein Ökosystem aus ganz bestimmten Arten verstanden wird, die in einem betrachteten Gebiet leben (Jax 2004:138), sondern auch um die Bewahrung der emotionalen und sozialen Bezüge von Menschen zu einer bestimmten Gegend, wobei es hierbei insbesondere um den Schutz besonders typischer, für die Wahrnehmung der Heimat repräsentativer Arten und Landschaftselemente geht, die der jeweiligen 'Heimat' bestimmter sozialer Gruppen ihre spezielle Charakteristik verleihen (Piechocki 2010:100). Insofern wird sowohl beim Landschafts- als auch beim Heimatschutz der Wechsel von Arten als eine signifikante Veränderung oder gar Zerstörung des Ökosystems betrachtet, wenn es sich dabei um charakteristische Schlüsselarten handelt, die als zugehörig zur Eigenart des Ökosystems angesehen werden (ebd.). Wiederum andere Aspekte werden betont, wenn Ökosysteme primär unter der Perspektive ihrer Leistungsfähigkeit betrachtet werden, da der Fokus hierbei darauf liegt, die Ökosystemleistungen ${ }^{22}$ qua Schutz und Nutzung zu erhalten. In Bezug auf das dabei relevante Verständnis von Ökosystemen kommt es darauf an, welche 'Maßstabsebene` gewählt wird (Haber 2009:27), denn je nachdem, welche „Naturraum-Einheit“ (ebd.) hierbei betrachtet wird, stehen andere Funktionen im Vordergrund und man erhält andere Ergebnisse. Der Artenschutz speielt bei der Leistungsbetrachtung auch nur insofern eine Rolle, als dass bestimmte Arten eine der angestrebten Funktionen des Ökosystems begünstigen und daher geschützt werden sollen oder dass andere (invasive) Arten diese schädigen oder beeinträchtigen (Piechocki 2010:100).

21 Die in Umwelt- und Naturschutzkreisen hitzig geführte Debatte um die 'Borkenkäferplage 'im Nationalpark »Bayrischer Wald« verdeutlicht diese Zusammenhänge exemplarisch. Die Kernfrage des Disputs zwischen NaturschützerInnen und AnwohnerInnen lautetete: „Darf sich im dichtbesiedelten Deutschland ein Stück Wildnis entwickeln? Unweigerlich fällt im Gespräch mit Protestlern der Satz: "Wir sind doch hier nicht im Yellowstone!" Wildnis müsse da erhalten werden, wo es sie noch gibt; der Bayerische Wald jedoch sei schon seit Jahrhunderten eine Kulturlandschaft, und das solle er auch bleiben." (DER SPIEGEL 1997/47)

22 Unter Ökosystem(dienst)leistungen werden die vielfältigen Funktionen verstanden, die Ökosysteme oder lebende Organismen haben und die dazu beitragen, dass die Natur in einem (für Menschen) lebenswerten Zustand bleibt. Solche Leistungen umfassen bspw. die Erhaltung der Qualität und Zusammensetzung der Atmosphäre und Biosphäre, die stabilisierende Einflussnahme auf das Klima, die Reinigung von Boden und Wasser, der Transport von Nährstoffen, die Abfallbeseitigung, die Pflanzenbestäubung u.v.m. (MEA 2005:39). Hier soll im Folgenden von Ökosystemleistungen die Rede sein, weil das volkwirtschaftliche Konzept der Dienstleistung bereits zu viel präjudiziert. 
Zusammenfassend zeigt sich vor allem anhand der Ambiguität und Kontextgebundenheit der, für die Biodiversität so zentralen, Begriffe Art, Gen und Ökosystem, dass weder die Bedeutung noch die Parametrisierung der, für das Biodiversitätskonzept relevanten Begriffe der Art, des Gens und des Ökosystems in den Biowissenschaften einvernehmlich geklärt sind. Stattdessen findet sich unter dem 'Label' Biodiversität eine Fülle biowissenschaftlicher Ansätze mit höchst verschiedenen Definitionen, Theorien und Modellen, die additiv zusammengesetzt und keineswegs integrativ aufeinander bezogen sind. Vielmehr sind die biowissenschaftlichen Fachsprachen zur Bestimmung der zentralen Begriffe Art, Gen und Ökosystem sowie die dabei zum Tragen kommenden nicht fachspezifischen Ausdrücke wie System, Ordnung, Hierarchie, Wandel etc. in den Biowissenschaften selbst kontrovers, so dass die Spezifizierungen der biologischen Einheiten im Biodiversitätskonzept ebenfalls nicht einheitlich sein können. In diesem Sinne wurde die Biodiversität zwar als neuartiges biowissenschaftliches Konzept eingeführt, aus ihm folgt aber kein theoretischer Neuansatz über den die biologischen und ökologischen Disziplinen integriert werden könnten. Die gängige Forschungspraxis besteht vielmehr aus einem eher multi- als interdisziplinäres Gefüge aus biowissenschaftlichen Disziplinen, die ihre Forschungen, trotz der Einführung des Biodiversitätsbegriffes, weiterhin getrennt voneinander verfolgen, so dass das Konzept der Biodiversität höchst unterschiedliche und sich auch widersprechende Ansätze subsumiert, wie die Vielfalt theoretisch verstanden und empirisch erfasst werden soll und ausgehend von welcher Ebene (Gene, Arten oder Ökosysteme) dies am besten geschehen kann. Daher bringen alle Definitionen der Biodiversität in je unterschiedlicher Weise Teilaspekte zur Anwendung, die für weitere Interpretationen offen sind. Christine Hertler fasst dies pointiert zusammen, wenn sie fragt, ob die Rede von der Artenvielfalt, der genetischen Vielfalt oder der Ökosystemvielfalt überhaupt gleichgesetzt werden könne, mit der Rede von Biodiversität:

"In den einzelnen biowissenschaftlichen Disziplinen werden die vorgestellten Forschungsprogramme schon seit unterschiedlich langen Zeiträumen getrennt verfolgt. Dort wird schlicht weiter das untersucht, was als jeweiliger Forschungsgegenstand bestimmt wurde. Heute wird es jedoch als Biodiversitätsforschung bezeichnet. Der Titel `Biodiversitätsforschungı soll also eine Klammer für ausgesprochen heterogene Forschungsfelder zur Erschließung eines Forschungsgegenstandes bilden (...), den es jedoch so überhaupt nicht gibt.“ (Hertler 1999:28-49)

Das Zitat macht deutlich, dass im Grunde genommen alles, was irgendwie mit Arten, Genen und Ökosystemen, aber auch mit Vielfalt, Natürlichkeit, Hierarchie, Komplexität etc. in Verbindung gebracht wird, auch als Biodiversität bezeichnet werden kann. Das wiederum bedeutet, dass jedwede biowissenschaftliche Forschung immer auch Biodiversitätsforschung ist. Insofern kann aber konstatiert werden, dass über die politischen 
Dokumente sowie über die biowissenschaftlichen Fachbegriffe lediglich der Eindruck suggeriert wird, dass hinter der Rede von der Biodiversität ein einheitlicher biowissenschaftlicher Forschungsgegenstand stehen würde, zu dessen Erschließung einzelne biowissenschaftliche Disziplinen einen gemeinsamen Beitrag leisten würden. Vor diesem Hintergrund kann in gerechtfertigter Weise von einer methodisch-theoretischen Unterbestimmtheit des Biodiversitätskonzeptes gesprochen werden, da es weder eine einheitliche Biodiversitätsforschung, noch einen einheitlichen Forschungsgegenstand gibt, der aus den Biowissenschaften herausgenommen und in anderen Kontexten 'angewandt' werden könnte (Gutmann \& Janich 2001b:1-27; Potthast 1999: Abschnitt 6, 140-153), denn alle Anwendungen des Begriffes müssen bestimmte Objekte, Eigenschaften, Zustände oder Beziehungsmuster herausgreifen und fokussieren, da es die Biodiversität als solche nicht gibt. Dementsprechend wird über den Begriff Biodiversität folglich nur suggeriert, dass der Biodiversitätsdebatte ein durch „wissenschaftliche Forschung hinreichend geklärtes Konzept von Biodiversität zugrunde liegt." (Janich 2001:XXI)

Daher ist die Relevanz der Biodiversität nicht so sehr wissenschaftlicher, als vielmehr politischer Natur. Dies zeigt sich vor allem daran, dass gerade die sich in den 1960er und 1970er Jahren ausbreitende Krisenwahrnehmung bezüglich der drastischen, industriell verursachten Vernichtung von Arten und ihrer natürlichen Lebensräume einen maßgeblichen Einfluss auf die Herausbildung des Begriffes Biodiversität hatte (Werner 2003:41): Insbesondere das Artensterben durch die Übernutzung von tropischen Ökosystemen, aber auch das zunehmende wissenschaftliche und nicht zuletzt das ökonomische Interesse an der Verfügbarkeit und Nutzung genetischer Ressourcen, machten die Vernichtung der natürlichen Ressourcen zu einem Problem, für das ein politischer, rechtlicher und institutioneller Rahmen ausgehandelt werden musste. Auf eben diese politische Dimension der Biodiversität wird im Folgenden genauer eingegangen.

\subsection{Biodiversität als politischer Symbolbegriff für einen modernen, umfassenden und integrativen Natur- und Umweltschutz}

Das »National Forum on BioDiversity« war die 'Geburtsstunde' des Begriffes Biodiversität im politischen Kontext. Die hier beschriebene „Biodiversitätskrise“ schaffte die Rahmung der Vernichtung der Biodiversität politisch zu begegnen:

"The human species came into being at the time of greatest biodiversity in the history of earth. Today as human populations expand and alter the natural environment, they are reducing biodiversity to its lowest level since the end of the Mesozoic era, 65 million years ago. The ultimate consequences of this biological collision are beyond calculation and certain to be harmful. That, in essence, is the biodiversity crisis." (Wilson 1989:61; SL) 
Eine solche Darlegung der Biodiversitätskrise ${ }^{23}$ zeigt bereits, dass der biowissenschaftliche Diskurs einen bedeutenden Einfluss auf das Verständnis der Biodiversität im politischen Raum hatte und nach wie vor hat, indem die Synthese der genetischen, ökosystemaren und Artenvielfalt zum Begriff Biodiversität - so problematisch deren wissenschaftliche Grundlagen auch immer sein mögen - zu einer Neustrukturierung der Umwelt- und Naturschutzpolitik führte (Brand \& Görg 2003:55). Dies zeigt sich vor allem daran, dass der Begriff und das Konzept der Biodiversität von Beginn an nicht nur mit dem innerwissenschaftlichen Ziel und dem Vorhaben kreiert worden ist, die Vielfalt der Lebensformen und Lebensbedingungen auf der Erde empirisch zu erforschen und zu beschreiben, sondern auch, um der Öffentlichkeit und der Politik die Relevanz der Artenvielfalt, die Erhaltung ihrer Lebensräume sowie die Gefahren des weltweiten Verlustes an genetischer Vielfalt deutlich zu machen (vgl. Takacs 1996; Potthast 1996). Sofern dies der primäre Zweck des Biodiversitätsbegriffes außerhalb (und vielleicht sogar innerhalb) der Biowissenschaften zu sein scheint, hat die Diagnose des methodisch-theoretischen Defizits nur bedingt Folgen für die Abschätzung des Potenzials der Biodiversität im politischen Raum, da über den Begriff Biodiversität, unabhängig davon, wie dieser in den Biowissenschaften konzeptualisiert wird, versucht wurde zu einer substanziellen Veränderung der Praxis des Naturschutzes und zu einer Sensibilisierung der Gesellschaft für naturschützerische Belange zu gelangen (Hertler 1999:50).

Biodiversität wurde vor diesem Hintergrund zum politischen Schlagwort, um die Problematik der Vernichtung der Arten und ihrer Lebensräume sowie die damit einhergehende Reduktion menschlicher Optionen und Handlungspotenziale möglichst sachlich, faktisch und unabweisbar zu verdeutlichen und zu untermauern. Die Biowissenschaften mit ihrem naturwissenschaftlichen Selbstverständnis erschienen hierfür ideale 'Partner'. Es ist daher auch nicht weiter verwunderlich, dass die Aufzählung von Daten zum 'Artensterben` die gebräuchlichste Strategie ist, um die Vernichtung der Biodiversität politisch zu verdeutlichen und dass insbesondere über die ökologische und nicht zuletzt auch ökonomische 'Nützlichkeit' der Biodiversität - was immer darunter im Einzelnen gefasst wird - dafür argumentiert wurde, wie wichtig, wertvoll und unersetzbar diese in Hinblick auf andere 'Umweltproblematiken` (bspw. Klimawandel, Bodendegration, Dürren, Überflutungen aber auch Hungersnöte und Verschmutzungen der Landschaft) sowie als 'Naturkapital' für die Aufrechterhaltung und Entwicklung ökonomischer und sozialer Prozesse ist. Dabei ermöglichen es gerade die Breite und die Un-

23 Die Problematiken, die über den Begriff der Biodiversität eingefangen und anvisiert werden, stellen wie bereits in der Einleitung ausgeführt (siehe 1.2) - keine rein ökologischen, sondern umfassender und tiefgreifender sozialökologische Problematiken dar. Eben diesem Umstand wird die Charakterisierung der 'Biodiversitätskrise' durch das "National Forum on BioDiversity« nicht gerecht, da diese den Anschein erweckt, als ginge es hierbei um durch die Natur endogen hervorgebrachte Symptome und nicht um eine umfassende Krise der gesellschaftlichen Naturverhältnisse (siehe auch 1.2.1). 
schärfe des Begriffes, dass unter dem 'Label' Biodiversität sowohl Interessen des Naturschutzes mit, unter dem Begriff der nachhaltigen Nutzung firmierenden Umweltschutzinteressen und zugleich Interessen an einer weitreichenden, andauernden und kommerziellen Ressourcennutzung sowie Interessen an allgemeinen oder exklusiven Zugangs- und Nutzungsrechten dieser Ressourcen zusammenkommen. Mit anderen Worten: Wenn nicht beachtet wird, dass es sich bei der Biodiversität nicht lediglich um eine biowissenschaftliche Beschreibungskategorie, sondern um ein Terrain handelt, in dem unterschiedliche Akteure um die Durchsetzung ihrer Interessen kämpfen (Brand \& Görg 2003:53), dann hat die Breite und Unschärfe des Begriffes zur Folge, dass die, auf verschiedenen politischen Ebenen angesiedelten, Konflikte um die Regulation gesellschaftlicher Naturverhältnisse verdeckt und ausgeblendet werden können.

Daher lässt sich im Folgenden die These aufstellen, dass es in der Biodiversitätspolitik nicht nur um die Lösung des vordergründigen Problems der Vernichtung der Biodiversität geht und gehen kann, das, umso besser es erfasst ist, auch umso besser politisch gehandhabt werden kann, sondern dass es dabei um eine politisch-strategische Neuausrichtung des Umwelt- und Naturschutzes unter dem Kerngedanken geht, dass 'die Natur' nicht gegen 'den Menschen ' geschützt werden darf, sondern dass es um die Auslotung der Möglichkeiten und Grenzen einer nachhaltigen Entwicklung der gesellschaftlichen Naturverhältnisse geht. Damit wird von vornherein anerkannt, dass die Problematik der Vernichtung der Biodiversität immer Konflikte bezüglich der Fragen mit sich bringt, wie die Vernichtung verursacht wird, wer bzw. was dabei als Ursache in Frage kommt, wie tiefreichend die Vernichtung ist und was sie für Folge- und Begleitprobleme mit sich bringt, welchen Stellenwert die Vernichtung der Biodiversität (auch im Vergleich zu anderen ökologischen und sozialen Problematiken) hat und wie dem Problem am besten begegnet werden kann. Insofern kommt dem Leitbild der Bewahrung und Förderung der Biodiversität eine strategische Relevanz hinsichtlich der Modernisierung des Umwelt- und Naturschutz zu, indem es stärker um die Berücksichtigung globaler Apekte des Umwelt- und Naturschutzes geht, Fragen der nachhaltigen Ressourcennutzung und des Zugangs und der Verteilung dieser Ressourcen ebenfalls adressiert werden können und indem Biodiversitätspolitik als Querschnittsaufgabe nahezu alle Politikfelder berührt und somit eine Politikintegration auf der vertikalen und horizontalen Ebene notwendig macht.

Diese strategische Relevanz wird im Folgenden einerseits über die Auseinandersetzung mit der »Internationalen Biodiversitätskonvention« (CBD) und ihrem ökosystemaren Ansatz (2.2.1) als auch über die Konkretisierung der CBD-Zielstellungen im nationalen Rahmen anhand der »Nationalen Strategie zur Biologischen Vielfalt» (NBS) (2.2.2) und deren Einbettung in das Bundesnaturschutzgesetz dargelegt. 


\subsubsection{Der internationale Kontext: Die CBD und der ökosystemare Ansatz}

Die CBD ist das Ergebnis eines, seit den 1970er Jahren anhaltenden, zunehmend verstärkten, politischen Handeln auf der internationalen politischen Ebene. Auf der Konferenz der Vereinten Nationen für Umwelt und Entwicklung (UNCED) 1992 in Rio de Janeiro mündeten diese Anstrengungen u.a. in der Verabschiedung der Internationalen Biodiversitätskonvention. Die CBD ist eines von drei völkerrechtlichen Abkommen der UNCED. Es handelt sich dabei um "soft law«, da die CBD zwar von den nationalen Regierungen unterschrieben und in den nationalen Parlamenten ratifiziert werden muss, die Konvention beinhaltet aber keinen eigenen Sanktionsmechanismus. Genau wie die "Klimarahmenkonvention " (UNFCCC) handelt es sich auch bei der CBD um eine Konvention, die in nationales Recht umgesetzt werden muss und deren Ausgestaltung auf der internationalen Ebene Gegenstand weiterer Verhandlungen ist. ${ }^{24}$

Die CBD war ursprünglich als »umbrella-convention « angedacht, die die bereits bestehenden internationalen Artenschutzabkommen aufeinander abstimmen (ebd., 52) und zugleich über den etablierten Ansatz des Artenschutzes und der Regulierung der Nutzung einzelner Arten hinausgehen sollte (Wolfrum 2001:418). ${ }^{25}$ Im Laufe der Verhandlungen gewannen vor allem die Verteilungsfragen um den Zugang und die Regulierung genetischer Ressourcen mehr und mehr an Gewicht (Görg 1999c:301). Dadurch vollzog sich ein Wandel weg von etablierten Naturschutzstrategien, die höchstens für Teile der Biodiversität, wie bspw. für den tropischen Regenwald oder die Korallenriffe, eine Lösung darstellten, hin zu einer Aufwertung nachhaltiger Nutzungsstrategien, die mit ökonomischen Interessen vor allem an den 'genetischen Ressourcen` und geostrategische Zielsetzungen und Verteilungsfragen einhergehen.

24 Dies geschieht über alle zwei Jahre stattfindende Vertragsstaatenkonferenzen („Conference of the Parties", COP), das wissenschaftliche Beratungsgremium der CBD ("Subsidiary Body for Scientific, Technical and Technological Advice«, SBSTTA) und ein Sekretariat in Montreal. Ein wichtiges Element der Umsetzung der CBD ist darüber hinaus der Finanzierungsmechanismus (»Globale Umweltfazilität«, GEF), der bei der Weltbank angesiedelt ist.

25 Die internationalen natur- und umweltpolitischen Maßnahmen erfassten bis in die 1990iger Jahre hinein nur bestimmte zumeist besonders repräsentative oder ökonomisch besonders relevante Arten [Abkommen zum Schutz der Pelzrobben (1911, 1957); der Wale (1931, 1946); von Langusten, Krebsen und Hummern sowie Thunfischen im Atlantik (1966), der antarktischen Robben (1972), der Eisbären (1973) und der Vicuñas (1969, 1979 und 1981) sowie das umfassendere Washingtoner Artenschutzabkommen um den internationalen Handel mit gefährdeten, freilebenden Arten (CITES 1973) und die Bonner Konvention zur Erhaltung der wandernden Tierarten (1979)] sowie Gebiete und Landräume [neben regionalen und kontinentalen Regelungen, die teilweise bereits vor der CBD bestanden (u.a. amerikanische Konvention 1942, afrikanische Konvention 1969, Amazonas-Konvention 1980, Berner Übereinkommen über die Erhaltung der europäischen wildlebenden Pflanzen und Tiere und ihrer Lebensräume 1982), betrifft dies eine Reihe internationaler Abkommen zum Schutz wichtiger oder gefährdeter Lebensräume und Gebiete (u.a. Ramsar Konvention 1971, Madrider Protokoll 1994, UNESCO-Naturerbe-Abkommen 1975)]. 
Diese 'Erweiterung' zeigt sich vor allem an den drei integrativen Biodiversitätszielstellungen - die einschließlich notwendiger Präzisierungen ${ }^{26}$ - hier wie folgt aufgeschlüsselt werden:

1) Das Ziel des möglichst bestandserhaltenden Schutzes der genetischen Vielfalt, der Artenvielfalt und der ökosystemaren Vielfalt zum Schutz aller Lebensformen (und ihrer Lebensbedingungen).

2) Das Ziel der möglichst nachhaltigen Nutzung ihrer Bestandteile, Funktionszusammenhänge und Leistungen auf all diesen Ebenen.

3) Das Ziel der möglichst gerechten Verteilung aller potenziellen und tatsächlichen Nutzen und Lasten, die aus der Erhaltung aber auch der Vernichtung der Biodiversität entstanden sind und entstehen können.

Über die drei Zielstellungen wird versucht, die ökologischen, ökonomischen und sozialen Aspekte beim Umgang mit der Biodiversität zu berücksichtigen (WCED 1987:57, Absatz 15) und die Ziele und Maßnahmen in Einklang miteinander zu bringen (Stadler 2007:175). Hieran wird im Besonderen deutlich, dass die CBD unter dem Tenor der nachhaltigen Entwicklung stand, worunter nach der Brundtland-Definition eine Entwicklung verstanden wird, „die die Bedürfnisse der Gegenwart befriedigt, ohne zu riskieren, dass künftige Generationen ihre eigenen Bedürfnisse nicht befriedigen können." (WCED 1987:51; Absatz 49 und S. 54, Absatz 1) Der dahinterliegenden Idee der 'Versöhnung 'von Ökologie und Ökonomie, von Nord und Süd sowie von Schutz und Nutzung wird über den sogenannten ökosystemaren Ansatz entsprochen, über den die Überwindung der Gegenüberstellung von Natur und Kultur sowie Umwelt und Gesellschaft vorangetrieben wird (vgl. Sekretariat der CBD 2000:Abschnitt 6): Hierüber werden vor allem die strukturellen und prozeduralen Aspekte natur- und umweltschutzpolitischer Handlungsoptionen (als 'Natur'-Schutz und 'Umwelt'-Nutzung) zur Erhaltung der Biodiversität fokussiert, da nur dann, wenn die wirtschaftlichen und sozialen Bedingungen in den CBD-Beitrittsstaaten dies auch zulassen, die Biodiversitätsziele und -maßnahmen verwirklicht werden können. Insofern geht es in der CBD bezüglich der Förderung der Biodiversität um Fragen der gerechten Aufteilung und Verteilung von Ressourcen, Optionen und Lasten (vgl. Katz et al. 1996). Um hierbei überhaupt von einer gerechten Verteilung sprechen zu können, bedarf dies immer schon entgegenkommender und bereitstehender Strukturen, Institutionen und Verfahren, so dass für

26 Die Präzisierungen betreffen hierbei insbesondere die erste und die dritte Zielstellung: Anders als in der CBD soll beim ersten Ziel nicht von Erhaltung der biologischen Vielfalt sondern ganz explizit von Schutz gesprochen werden, da Erhaltung als Kombination aus Schutz und Nutzung verstanden wird. Des Weiteren wird nicht von gerechter Aufteilung, sondern allgemeiner von gerechter Verteilung, nicht nur der Nutzen, sondern konsequenterweise auch der Lasten aus dem Schutz und der Nutzung, aber auch aus der Vernichtung der Biodiversität gesprochen. Überdies hinaus wird das Verteilungsziel hier auf alle drei biologischen Ebenen der Biodiversität bezogen und nicht nur auf die genetische Vielfalt, wie das in der CBD der Fall ist. 
die Bewahrung und Förderung der Biodiversität immer auch die politischen Strukturen und ihre Kontextbedingungen berücksichtigt werden müssen. Daher werden die, in den Millennium-Entwicklungszielen fokussierten Ziele zur Überwindung der Armut auch zum Anliegen in der CBD:

„[I]n der Erkenntnis, dass die wirtschaftliche und soziale Entwicklung und die Beseitigung der Armut die ersten und vordringlichsten Anliegen der Entwicklungsländer sind, in dem Bewusstsein, dass die Erhaltung und nachhaltige Nutzung der biologischen Vielfalt für die Befriedigung der Nahrungsmittel-, Gesundheits- und sonstigen Bedürfnisse einer wachsenden Weltbevölkerung von ausschlaggebender Bedeutung sind und dass dazu der Zugang zu genetischen Ressourcen und zu Technologien sowie die Teilhabe daran wesentlich sind, (...).“ (UN 1992:Präambel;Art. 1)

Insofern wird aber auch ganz deutlich, dass das Ziel der gerechten Verteilung einen anderen Status besitzt als das Naturschutz- und das Umweltnutzungsziel, da es hier darum geht, wie Schutz und Nutzung umgesetzt werden, damit die Potenziale genutzt und die Hindernisse abgebaut oder verhindert werden können.

\subsubsection{Der nationale Kontext: Die NBS und das Bundesnaturschutzgesetz}

Die drei Zielstellungen sowie die damit verbundenen politischen Ansprüche spiegeln sich (mehr oder weniger) in den jeweiligen nationalen Biodiversitätsstrategien wider. Die Bundesrepublik Deutschland hat die CBD bereits ein Jahr nach ihrer Implementierung, also 1993 ratifiziert, hat aber erst 2007 mit der »Nationalen Strategie zur Biologischen Vielfalt “ (NBS) Artikel 6 der CBD erfüllt, der besagt, dass „jede Vertragspartei (...) nationale Strategien, Pläne oder Programme zur Erhaltung und nachhaltigen Nutzung der biologischen Vielfalt entwickeln oder zu diesem Zweck ihre bestehenden Strategien, Pläne und Programme anpassen (soll)." (ebd., Art. 6) Die NBS bildet mit ihren umfangreichen naturschutzpolitischen Aussagen und ihren ca. 330 Ziele und rund 430 Maßnahmen den zentralen Bezugsrahmen für die gegenwärtigen und zukünftigen Biodiversitätspolitiken der deutschen Bundesländer und des Bundes im Kontext der europäischen und internationalen Umweltpolitik (vgl. BMUB 2011). ${ }^{27}$ Im Folgenden werden die nationalen Konkretisierungen der drei Ziele (a. Schutzziel; b. Nutzungsziel; c. Verteilungsziel) dargelegt, wobei deutlich werden soll, auf welche Bereiche sich die Ziele jeweils beziehen und welche „Visionen“ und Maßnahmen diesbezüglich existieren, um die formulierten Ziele umzusetzen. Im Anschluss daran, d) wird dann auf die (mehr oder weniger konsequente) rechtliche Integration der Biodiversität im Rahmen der 2010 erfolgten Novellierung des Bundesnaturschutzgesetzes eingegangen:

27 Die NBS wurde 2011 zwar mit neuem Cover, aber ansonsten inhaltlich unverändert, nach dem Original von 2007 nachgedruckt. Die Ausführungen beziehen sich dennoch auf die aktuelle Ausgabe (vgl. BMUB 2011). 
a) Das Schutzziel wird in der NBS mit der „Vision“ verknüpft, dass es „in Deutschland eine für unser Land typische Vielfalt von natürlichen sowie durch menschliches Handeln geprägte Landschaften, Lebensräume und Lebensgemeinschaften gibt (...). Die diesen Lebensräumen zugehörigen Arten existieren in überlebens- und langfristig anpassungsfähigen Populationen." (BMUB 2011:26; SL) Das Schutzziel wird dabei auf allen drei Hierarchieebenen (Artenvielfalt, Ökosystemvielfalt und genetische Vielfalt) ausbuchstabiert und ist an die Erreichung des 2010-Ziels ${ }^{28}$ geknüpft: So gilt das Schutzziel in Bezug auf die Vielfalt der Arten dann als erfüllt, wenn bis zum Jahr 2010 der Anteil der vom Aussterben bedrohten und stark gefährdeten Arten verringert ist (ebd., 27). In Bezug auf die Vielfalt der Lebensräume sieht die NBS das Schutzziel als erfüllt an, wenn bis 2010 der Rückgang von gefährdeten Lebensraumtypen (Wälder, Küsten und Meere, Seen, Weiher, Teiche und Tümpel, Flüsse und Auen, Moore, Gebirge und Grundwasserökosysteme sowie Wildnisgebiete aber auch Kulturlandschaften und urbane Landschaften) aufgehalten ist (ebd., 27-43). In Bezug auf die genetische Vielfalt von wildlebenden und domestizierten Arten soll der Verlust der genetischen Vielfalt durch ex- situ- und in-situ/on-farm Erhaltung bis 2010 aufgehalten sein, wobei insbesondere die regionaltypische Vielfalt von Nutztierpflanzen und Kulturpflanzensorten erhalten bleiben soll, so dass diese nachhaltig genutzt und als Lebens- und Zuchtgrundlage verfügbar bleiben sowie das Landschaftsbild prägen können (ebd., 30). Des Weiteren soll hier die 'Verfälschung' bzw. 'Verarmung' der genetischen Vielfalt der wildlebenden Tier- und Pflanzenwelt einerseits durch Ansiedlung und Ausbreitung von nicht heimischen bzw. 'invasiven gebietsfremden 'Tier- und Pflanzenarten verhindert werden, andererseits sollen in Zukunft keine gentechnisch veränderten Organismen mit Auskreuzungs-, Verwilderungs-, Etablierungs- und Ausbreitungspotenzial zugelassen werden. Um diese Schutzziele zu erreichen wird die Minimierung von Zerschneidungseffekten, die Vermeidung von Konflikten mit dem länderübergreifenden Biotopverbund bei künftigen Planungen und Projekten der Siedlungsentwicklung sowie von Verkehrswegen, die Reduzierung der wesentlichen Gefährdungsfaktoren, die zu einer Degradation von Lebensräumen führen sowie die Regeneration gefährdeter Biotoptypen und Biotopkomplexe und die Erhaltung und Vermehrung von extensiv genutzten Lebensräumen angestrebt (ebd., 29). Des Weiteren soll das länderübergreifende Biotopverbundsystems "Natura 2000« bis 2010 auf mindestens 10\% der Landesfläche ausgeweitert und bis 2020 soll ein funktionierendes Managementsystem für alle Großschutzgebiete und Natura 2000-Gebiete etabliert werden (ebd., 28).

28 Das (europäische) 2010-Ziel wurde 2001 in Göteborg von den EU-Staatschefs auf dem Gipfeltreffen der Europäischen Union mit der Absicht verabschiedet, den Rückgang der Biodiversität bis zum Jahr 2010 zu stoppen. 2002 verpflichteten sich die Unterzeichnerstaaten der CBD auf das (internationale) 2010-Ziel, allerdings mit der Einschränkung den Biodiversitätsverlust auf globaler, regionaler und nationaler Ebene als Beitrag zur Armutsbekämpfung und zum Wohle allen Lebens auf der Erde signifikant zu reduzieren. 
b) Das Nutzungsziel wird in der NBS mit Verweis auf die, auf der 7. Vertragsstaatenkonferenz in Kuala Lumpur im Jahr 2004 verabschiedeten »Addis Abeba-Prinzipien für nachhaltige Nutzung« konkretisiert, die wiederum im Kontext der UN-Millenniumsziele formuliert wurden. ${ }^{29}$ Dabei wird in der NBS auch hier ein sehr komplexer Aufriss des Nutzungsziels gegeben, um deutlich zu machen, dass die Zielsetzungen der nachhaltigen Nutzung immer auch die Schutz- und Verteilungsziele beeinflussen. Dabei wird sowohl auf die Vorbildfunktion des Staates, die Auswirkungen deutscher Aktivitäten auf die Biodiversität weltweit sowie die Rolle der Landwirtschaft als auch auf die kritischen Faktoren der Bodennutzung, des Rohstoffabbaus, der Energiegewinnung, der Flächeninanspruchnahme für Siedlung und Verkehr, der gestiegenen Mobilität, der flächendeckenden diffusen Stoffeinträge sowie des Klimawandels eingegangen (ebd., 43-57). Es erfolgt allerdings keine dezidierte Unterscheidung der sich daran anschließenden Ziele und Maßnahmenbündel auf ihren jeweiligen Einfluss auf den drei Hierarchieebenen, da hier immer zusammenfassend von biologischer Vielfalt oder Biodiversität die Rede ist. Dementsprechend ist in der NBS formuliert, dass „die wirtschaftlichen Tätigkeiten in Deutschland im Einklang mit dem Schutz der biologischen Vielfalt erfolgen [sollen], wobei Gewinne aus der Nutzung der biologischen Vielfalt angemessen aufgeteilt sind." (ebd., 43) Des Weiteren sollen alle Produkte und Dienstleistungen, die zu einer Belastung der Biodiversität führen, ebenso wie wirtschaftliche Aktivitäten, die die Biodiversität fördern, für die Menschen besser erkennbar sein. Die Nachfrage nach umweltverträglichen Produkten und Dienstleistungen soll zudem kontinuierlich verstärkt, als auch ihr Angebot deutlich verbessert werden und es sollen immer mehr wirtschaftliche Aktivitäten einen Beitrag für die Erhaltung der Biodiversität leisten, so dass Biodiversitätsanliegen bis 2020 umfassend in die Welthandelsordnung integriert sind und die Biodiversitätsbelastungen der Produktion zurückgehen (ebd., 43).

c) Schließlich geht die NBS auch auf das Verteilungsziel näher ein. Gerade hier gab es bei den Verhandlungen zur CBD und über diese hinaus die meisten und nach wie vor hartnäckigsten Kontroversen. ${ }^{30}$ Kernstück des Verteilungszieles sind dabei die Regelungen zum Zugang und gerechtem Vorteilsausgleich (»Access and Benefit Sharing",

29 Die »Addis Abeba Prinzipien« (AAP) bestehen aus vierzehn aufeinander aufbauenden Prinzipien, operativen Regeln und Implementierungsinstrumenten, die die nachhaltige Nutzung der Biodiversität regeln und bestimmen sollen (Sekretariat der CBD 2004:Artikel 1). Dabei wird die nachhaltige Nutzung als wertvolles Instrument verstanden, um den Schutz der Biodiversität zu gewährleisten und Schutzimperative werden immer auch als Teil der nachhaltigen Nutzung angesehen (ebd., Artikel 2).

30 Anhand des Umfangs der Ausführungen in der NBS wird deutlich, dass die Konkretisierung des Verteilungsziels bis 2007 am wenigsten fortgeschritten war. Dies ist insofern verständlich, als dass eine intensive Weiterentwicklung der ABS-Verhandlungen erst auf der 9. und 10. Vertragsstaatenkonferenz 2008 in Bonn und 2010 in Nagoya erfolgte, auf denen es um einen gemeinsamen Weg zu einer international verbindlichen Vereinbarung zur gerechten Aufteilung der Vorteile aus der Nutzung der Biodiversität ging, die von der NBS (Auflage 2007) nicht berücksichtigt werden konnten, aber leider auch in der Neuauflage 2011 nicht berücksichtigt wurden. 
kurz $A B S)$, die in der CBD und damit auch in der NBS selbst nur auf genetische Ressourcen bezogen sind. Der ABS-Mechanismus betont das souveräne Recht der einzelnen Nationalstaaten auf ihre 'natürlichen` Ressourcen, mit dem gleichzeitigen Verweis darauf, dass der Zugang zu diesen Ressourcen auch für Nutzer außerhalb des Territoriums, in dem diese Ressourcen vorkommen, ermöglicht werden soll. Im Gegenzug dazu sollen die daraus entstehenden Vorteile fair und gerecht verteilt werden. ${ }^{31}$ Aufbauend auf diesen Regelungen wird der gerechte Vorteilsausgleich in der NBS in den „konkreten Visionen“ wie folgt bestimmt: „Die Herkunftsländer, die indigenen und lokalen Gemeinschaften profitieren gleichberechtigt von den Vorteilen, die sich aus der nachhaltigen Nutzung genetischer Ressourcen ergeben. Gleiches gilt für die Nutzung des mit den genetischen Ressourcen verbundenen traditionellen Wissens. Unsere Vision für die Zukunft ist: Die Nutzer genetischer Ressourcen befolgen die Zugangsregelungen des Übereinkommens über die biologische Vielfalt. Dabei werden insbesondere die Rechte indigener und lokaler Gemeinschaften berücksichtigt.“ (ebd., 57)

d) Da die NBS keine Rechtsgültigkeit besitzt, da sie als Strategiepapier weder ein Gesetz noch ein Rechtsverordnungstext ist, ist von Relevanz, inwieweit 2010, im internationalen Jahr der Biodiversität, das Bundesnaturschutzgesetz (BNatSchG) novelliert und um Biodiversitätsaspekte ergänzt worden ist:

"Natur und Landschaft sind auf Grund ihres eigenen Wertes und als Grundlage für Leben und Gesundheit des Menschen auch in Verantwortung für die künftigen Generationen im besiedelten und unbesiedelten Bereich nach Maßgabe der nachfolgenden Absätze so zu schützen, dass 1. die biologische Vielfalt, 2. die Leistungs- und Funktionsfähigkeit des Naturhaushalts einschließlich der Regenerationsfähigkeit und nachhaltigen Nutzungsfähigkeit der Naturgüter sowie 3. die Vielfalt, Eigenart und Schönheit sowie der Erholungswert von Natur und Landschaft auf Dauer gesichert sind.“ (DB 2009:Paragraph 1; SL)

31 Die internationalen Zugangs- und Teilhaberegelungen für genetische Ressourcen sind in den Artikeln 15 bis 19 der CBD festgeschrieben: In Art. 15 wird das souveräne Recht der Staaten in Bezug auf ihre Ressourcen anerkannt (Abs. 1). Den Regierungen der einzelnen Staaten wird dabei die Befugnis zugewiesen, den Zugang zu genetischen Ressourcen anhand ihrer innerstaatlichen Rechtsvorschriften so zu bestimmen, dass deren umweltverträgliche Nutzung über einen ausgewogenen und gerechten Vorteilsausgleich gewährleistet wird (Abs. 3), sprich deren Nutzung auch durch andere Vertragsparteien erleichtert wird und diesen keine Beschränkungen auferlegt werden die den Zielen der CBD zuwiderlaufen (Abs. 2). Vorgesehen ist dabei eine Beteiligung der Ressourcenstaaten an der Forschung, vor allem durch ihre Verlagerung in das jeweilige Land (Abs. 4) Demensprechend hat die Zugangsgewährung zu einvernehmlich festgelegten Bedingungen (»Mutually Agreed Terms and Conditions", MAT) zu erfolgen, wobei der Zugang einer vorherigen informierten Zustimmung (»Prior Informed Consent«, PIC) bedarf (Abs. 5). Das soll dem Staat ermöglichen eine informierte Entscheidung über den Zugang zu treffen, womit die Verhandlungsmacht der ressourcengebenden Staaten gestärkt werden soll. Außerdem soll eine ausgewogene und gerechte Aufteilung der Ergebnisse und Vorteile aus Forschung und Entwicklung stattfinden, die sich aus der kommerziellen und sonstigen Nutzung der genetischen Ressourcen ergeben (Abs. 7). Art. 16 sieht des Weiteren einen Technologietransfer in die Entwicklungsländer vor und enthält verschärfte Kooperationspflichten zu ihren Gunsten. 
„Zur dauerhaften Sicherung der biologischen Vielfalt sind entsprechend dem jeweiligen Gefährdungsgrad insbesondere 1. lebensfähige Populationen wild lebender Tiere und Pflanzen einschließlich ihrer Lebensstätten zu erhalten und der Austausch zwischen den Populationen sowie Wanderungen und Wiederbesiedelungen zu ermöglichen, 2. Gefährdungen von natürlich vorkommenden Ökosystemen, Biotopen und Arten entgegenzuwirken, 3. Lebensgemeinschaften und Biotope mit ihren strukturellen und geografischen Eigenheiten in einer repräsentativen Verteilung zu erhalten, wobei bestimmte Landschaftsteile der natürlichen Dynamik überlassen bleiben sollen.“ (ebd., Paragraph 2)

In Kombination mit der, im Grundgesetz festgehaltenen Staatszielbestimmung Umweltschutz (Art. 20a GG), die fordert, dass „(d)er Staat (...) auch in Verantwortung für die künftigen Generationen die natürlichen Lebensgrundlagen und die Tiere (...) [schützt]“, wird der Schutz der Biodiversität zum zentralen Leitbegriff des deutschen Naturschutzes erhoben, der Bewahrung und Förderung der Biodiversität wird hier aber nicht im Ganzen entsprochen, da hier weder die nachhaltige Nutzung noch die gerechte Verteilung angesprochen werden und zudem auch nur von Arten und Ökosystemen, nicht aber von der genetischen Vielfalt die Rede ist. ${ }^{32}$ Dieser Umstand lässt sich so interpretieren, dass der Schutz der Biodiversität in das BNatSchG als begrifflicher Stellvertreter für den Arten- und Biotopschutz aufgenommen worden ist, ohne dass den, mit der Bewahrung und Förderung der Biodiversität einhergehenden konzeptionellen Neuerungen, die insbesondere über den ökosystemaren Ansatz im Rahmen der CBD formuliert worden sind, Rechnung getragen wurde. Damit wird indes auch das konzeptionelle Problem der NBS ersichtlich:

Die Betrachtung der CBD zeigt, dass die dort anvisierte Biodiversitätspolitik - zumindest ihrem politischen Anspruch nach - weit über eine reine Naturschutzpolitik hinaus geht, indem hier der innovative politische Anspruch formuliert wird, eine Rahmenkonvention zur Bewahrung und Förderung der gesamten natürlichen Vielfalt zu schaffen, die nicht allein durch Schutzbemühungen, sondern gleichsam durch nachhaltige Nutzungsund gerechte Verteilungsanstrengungen erreicht werden soll. In diesem Sinne kann die CBD als der bisher umfassendste politische Ansatz des internationalen Natur- und Umweltschutzes betrachtet werden, dem ein hohes Innovationspotenzial zur Modernisierung des politischen Umwelt- und Naturschutzes zukommt. Dieses Innovationspotenzial wird in Deutschland, wo die NBS in den Kontext einer reinen Naturschutzgesetzgebung gestellt wird, allerdings nicht eingeholt, weil das eigentlich Innovative an der Be-

32 An dieser Stelle könnte natürlich eingewandt werden, dass unter das Schutzziel bereits nachhaltige Nutzung subsumiert werden kann, weil natürliche Ressourcen nicht effektiv geschützt werden können, ohne dass sie auch nachhaltig genutzt werden. Nach dem Motto: 'Was weg ist, ist weg, da braucht es dann auch keinen Schutz mehr'. Schutz impliziert aber nicht in derselben Weise auch gerechte Verteilung, insofern ist vor allem das Verteilungsziel hier zu wenig berücksichtigt. 
wahrung und Förderung der Biodiversität, nämlich die Integrativität der Zieltrias, immer wieder auf den bestandserhaltenden Schutz der Biodiversität verengt wird. Dies ist indes in zweierlei Hinsicht problematisch:

Zum einen weil die Fokussierung auf das Ansinnen möglichst alle Lebensformen und Lebensbedingungen so zu erhalten, wie man sie kennt, implizit davon ausgeht, dass die Natur so bleiben soll, wie sie gerade vorgefunden wird, womit erneut das statische Naturschutzdenken betont und der Grundfehler des klassischen Naturschutzes wiederholt wird, der eigentlich über die Zielstellung der Bewahrung und Förderung der Biodiversität behoben werden könnte (vgl. Haber 2009). Zum anderen weist sich die Strategie damit zwar dem Namen, den Ambitionen und den Zielen nach als eigenständige Biodiversitätsstrategie im Sinne der CBD aus, in ihrem rechtlichen Kontext gestellt ist sie aber eine klassische Naturschutzstrategie. Denn auch wenn die Erhaltung der Biodiversität, laut NBS, ganz ausdrücklich nicht auf Naturschutzaktivitäten beschränkt sein soll (BMUB 2011:6), so ist sie hinsichtlich der damit verbunden Maßnahmen im BNatSchG auf die klassischen Formen des Naturschutzes (Biotop-, Arten-, Landschafts- und Prozessschutz) festgelegt. Damit wird aber das Innovationspotenzial, was über die Bewahrung und Förderung der Biodiversität in den politischen Natur- und Umweltschutz hineingebracht wurde, im deutschen Kontext nur selten ersichtlich und bleibt ungenutzt, weil der Fokus auf die nachhaltige Nutzung und die gerechte Verteilung, die im Naturschutz wenn überhaupt dann nur marginal thematisiert worden sind, nicht als wichtige und konstituierende Aspekte der Biodiversitätspolitik berücksichtigt werden (können).

Zum anderen ist problematisch, dass wenn die Integrativität der Zieltrias nicht in ausreichendem Maße beachtet wird, auch die beiden anderen Innovationsmomente - der Modernisierungsaspekt und der Koordinationsaspekt - nicht in Gänze zum Tragen kommen können, womit wiederum die strategische Relevanz des Biodiversitätskonzeptes nicht eingeholt wird. Dies zeigt sich insbesondere anhand des Verteilungsziels, das, wenn es denn überhaupt eine Rolle spielt, nur auf politische und darüber hinaus lediglich globale und internationale Kontexte bezogen wird. Dies reicht aber nicht aus, da darüber zum einen intertemporale, nationale, lokale als auch interpersonelle Verteilungs- und Gerechtigkeitsfragen in Bezug auf die Erhaltung der Biodiversität aus dem Fokus geraten, als auch die normativen Grundlagen solcher Gerechtigkeitsforderungen nicht in ausreichendem Maße thematisiert werden (können). Die normative Frage, welchen individuellen und gesellschaftlichen Naturverhältnissen der Vorzug gegeben werden sollte, ist indes für den Umwelt- und Naturschutz nicht erst seit der Biodiversitätsdebatte elementar, so dass das Verteilungsziel als umfassende Gerechtigkeitsthematik verstanden werden sollte, weil die gesellschaftspolitischen Konflikte zwischen Schutz- und Nutzungsinteressen, die es sowohl innerhalb als auch außerhalb des Natur- und Umweltschutzes gibt sowie zwischen den Ansprüchen jetziger und zukünf- 
tiger Generationen am Naturerleben einerseits und der Ressourcennutzung andererseits, nicht in ausreichendem Maße berücksichtigt werden können, wenn das Verteilungsziel primär als politisches (nicht aber auch als normatives) Ziel verstanden und dabei lediglich auf internationale und globale Kontexte beschränkt wird. Wenn das Verteilungsziel hingegen im Kontext einer generellen Verteilungstheorie mit Blick auf gesellschaftliche Naturverhältnisse verstanden wird - was ja im Biodiversitätskontext bereits angelegt ist - erweitert sich der politische Fokus ganz automatisch. Hieran anschließend ist im Folgenden die Beleuchtung der Biodiversität als Wert- und Normbegriff relevant.

\subsection{Biodiversität als Wert- und Normbegriff}

Jede Beurteilung des Umgangs mit der Natur, die in politische Entscheidungen eingeht, ist mit der Entscheidung verbunden, welcher Teil, welche Aspekte, welche Zustände und/oder Prozesse von Natur/Biodiversität dabei relevant sein sollen. Einmal abgesehen von der bereits ausgeführten Problematik, dass zur Beantwortung aller praktischen Fragen im Umgang mit der Biodiversität immer eine Vielzahl biowissenschaftlicher Begriffe und Konzepte mitverhandelt wird, die oftmals einfach unhinterfragt übernommen werden (siehe 2.1), ist die notwendige, aber oftmals gar nicht als problematisch reflektierte Selektion bestimmter Elemente aus der gegebenen Vielzahl der Möglichkeiten, die Biodiversität begrifflich umfasst, immer schon ein Urteil darüber, welche Aspekte der Biodiversität als wertvoll und damit als schützenswert angesehen werden. Biodiversität ist indes bei Weitem nicht nur auf dieser niederschwelligen Ebene normativ imprägniert, sondern fungiert auch ganz explizit als Wert- und Normbegriff, der als Chiffre für den Wert der Vielfalt sowie für die Pflicht und die Verantwortung des Menschen steht, diese zu erhalten. Gerade in dieser Funktion als Wert- und Normbegriff spielt die Biodiversität auch in der Ethik eine zunehmend wichtige Rolle.

Gerade die ethische Auseinandersetzung mit der Biodiversität als Wert- und Normbegriff verdeutlicht die Problematik, dass die 'normative Imprägnierung`der Biodiversität auf verschiedenen Wegen geschieht, obgleich jeder dieser Wege für den Wert der Biodiversität oder die Norm der Bewahrung und Förderung der Biodiversität zu argumentieren, mit je eigenen Begründungsproblematiken behaftet ist, die vor allem daraus resultieren, dass nicht alle diese Versuche aus einer ethischen Perspektive heraus auch gleichermaßen geeignet sind, dies zu tun. Wie weit die normative Imprägnierung im Fall der Biodiversität geht, kann anhand von Argumentationsstrategien für die Erhaltung der Biodiversität aufgezeigt werden: $:^{33}$

33 Stefan Körner und Ulrich Eisel verweisen darauf, dass die Argumentationsschwerpunkte wechseln und sich stellenweise überlagern. Obwohl diese sich im ehrenamtlichen, amtlichen und wissenschaftlichen Umwelt- und Naturschutz gleichermaßen wiederfinden, soll hier primär auf die Umwelt- 
Wenn die Erhaltung der Biodiversität möglichst faktisch, sprich a) naturwissenschaftlich oder b) ökonomisch gerechtfertigt wird, wobei zumeist auf die ökologischen Zusammenhänge oder aber die Funktionen, Nutzen sowie instrumentellen Werte der Biodiversität rekurriert wird, kann von einer "'Fakten '-Argumentation" gesprochen werden, wobei Fakten hierbei bereits in kritischer Absicht als vermeintlich feststehende und gültige Tatsachen zu verstehen sind und kritisch darauf reflektiert wird, dass über Faktenargumentationen oftmals Wert- und Normvorstellungen transportiert werden, die als solche nicht explizit thematisiert werden. (2.3.1). Wenn hingegen argumentiert wird, dass der belebten Natur oder aber Teile von ihr, ein wie auch immer gearteter Eigenwert zukommt, woraus auf die Handlungsnorm geschlossen wird, dass die Biodiversität 'um ihrer selbst willen` erhalten werden sollte, dann kann die zweite Strategie als "'Eigenwert'-Argumentation« bezeichnet werden. Hier wird die Erhaltung der Biodiversität zwar explizit normativ gerechtfertigt, aber es wird hier von vornherein in kritischer Absicht die Frage aufgeworfen, was genau damit gemeint sein soll, wenn von einem Eigenwert der Biodiversität die Rede ist (2.3.2).

\subsubsection{Die »'Fakten`-Argumentation « für die Erhaltung der Biodiversität}

a) Historisch betrachtet, lässt sich die naturwissenschaftlich abgestützte "'Fakten 'Argumentation « auf die, in den 1960er bis 1980er Jahren gewonnenen Erkenntnisse bezüglich des Zusammenhangs zwischen ökosystemarer Stabilität und Artenvielfalt zurückführen, die sich vor allem in den verschiedenen Varianten der Diversitäts-Stabilitäts-Hypothese niederschlagen. ${ }^{34}$ Wie eingängig diese Hypothese zu Zeiten der Genese

und Naturschutzforschung fokussiert werden, weil man hier gemäß der guten wissenschaftlichen Praxis sowie der ihnen zugewiesenen Aufgabe der Bereitstellung von Erkenntnissen, Methoden und Kriterien für die Verwirklichung der gesellschaftspolitischen Zielstellungen am ehesten erwarten kann, dass die Biodiversitätsziele begründet werden. De facto ist dies selten der Fall. So weist nicht nur Kirsten Meyer in ihrer Arbeit zur „Begründungsvielfalt im Naturschutz« darauf hin, dass lange Zeit die einhellige Meinung im Umwelt- und Naturschutz herrschte, dass diese sich „ökologisch“ begründen ließen, wobei dabei angenommen wurde, dass das Aufzeigen der ökologischen Zusammenhänge als Begründung für die Umwelt- und Naturschutzziele ausreichend sei (Meyer 2003:45). Dies wird hier als »naturwissenschaftliche Faktenargumentation« bezeichnet. Weiter führt Meyer aus, dass erst seit den 1980er Jahren in den Publikationen der Umwelt- und Naturschutzforschung vermehrt darauf hingewiesen wurde, dass sich Umwelt- und Naturschutzziele nicht rein naturwissenschaftlich begründen lassen, da die Ökologie nur Zustandsbeschreibungen geben und prognostizieren, diese aber nicht bewerten kann (ebd., 46). Als Reaktion darauf wurde dann versucht, die Umwelt- und Naturschutzziele über den Wert der Natur/Umwelt zu begründen - entweder über deren instrumentelle Werte, was hier als »ökonomische Faktenargumentation « bezeichnet wird oder über den Eigenwert, was als »Eigenwertargumentation« bezeichnet wird - wobei aber die Spezifika der Ethik als wissenschaftliche Reflexionstheorie nicht in ausreichenden Maße ernst genommen wurden und werden (siehe 2.3.2).

34 Bezüglich der Bedeutung der Diversität für die Stabilität von Ökosystemen gab es schon vor dem Beginn der Biodiversitätsdebatte kontroverse Debatten in der Ökologie, die mit dem Beginn der Biodiversitätsdebatte erneut Konjunktur bekamen (Trepl 1999:92). Die Diversitäts-Stabilitäts-Hypothese, die, anders als ihr Name suggeriert, oftmals als naturwissenschaftliche Gesetzmäßigkeit betrachtet wurde, wird seitdem immer wieder angegriffen und kritisiert, anderseits aber auch immer wieder partiell bekräftigt. Die Bedeutung, die der Diversitäts-Stabilitäts-Hypothese zugeschrieben wird, er- 
des Konzepts der Biodiversität war und es noch immer ist, zeigt sich nicht zuletzt an der NBS, in der die Zusammenhänge zwischen Artenvielfalt und der Pufferkapazität ökologischer Systeme unter dem Stichwort „Versicherungshypothese“ (BMUB 2011:10) aufgenommen werden:

„Es wird angenommen, dass Ökosysteme mit einer natürlichen Vielfalt an Arten Störereignisse (...) besser abpuffern können als Ökosysteme, in denen viele Arten bereits ausgestorben sind. Eine hohe genetische Variabilität der Arten macht es wahrscheinlicher, dass zumindest Teile von Populationen dieser Arten in der Lage sind, sich an verändernde Umweltbedingungen anzupassen. Zudem nimmt mit zunehmender Anzahl der Arten die Wahrscheinlichkeit zu, dass zumindest einige dieser Arten in der Lage sind, unterschiedlich auf äußere Störungen und Änderungen der Umweltbedingungen zu reagieren. Außerdem erhöht eine größere Anzahl an Arten die Wahrscheinlichkeit, dass in dem Ökosystem zwei Arten sich funktionell weitgehend decken, so dass die eine bei Wegfall der anderen deren Rolle im Ökosystem übernehmen kann." (ebd., 10-11)

Über diese Zusammenhänge wird indes nur 'zwischen den Zeilen` deutlich, warum es gerechtfertigt sein sollte, die ökosystemare Stabilität zu erhalten: Implizit wird hier die Einsicht vermittelt, dass die Natur durch den Menschen und sein Handeln, wenn dieses sich nicht drastisch ändert, unwiederbringlich und unrevidierbar zerstört werden kann, womit wiederum die Gefahr verbunden wird, dass der Mensch sich gerade aufgrund seines Eingreifens in die Natur seiner natürlichen Lebensgrundlagen beraubt. Um eben dies zu verhindern, sollen Biologie und Ökologie dazu beitragen, die Grenzpunkte und Schwellen der ökosystemaren Belastbarkeit zu bestimmen und festzulegen. Biologie und Ökologie werden damit zu Leitwissenschaften des politischen Natur- und Umweltschutzes erhoben. ${ }^{35}$ Wenn aber die biowissenschaftlichen Systemvorstellungen nicht

lebt in der Wissenschaft und insbesondere in der Ökologie, im fachlichen Naturschutz sowie in der Naturschutzpolitik daher ein stetiges auf und ab. Fest steht mittlerweile, dass das Bild, das die 'rivet Hypothese`1981, basierend auf der, in den 1960iger Jahren gängigen Annahme, dass (Arten-)Diversität die ökosystemare Stabilität generell begünstige, gezeichnet hat, viel zu einfach gedacht ist: Es gibt keine einfachen positiven, linearen Zusammenhänge zwischen Diversität und Stabilität, es stimmt aber auch nicht, dass diese Zusammenhänge überhaupt nicht vorhanden sind (ebd., 116). Daher muss detailliert gefragt werden, „auf welche Eigenschaften welcher Arten sich welche Art von Stabilität genau bezieht (ebd., 121).

35 Auch wenn bis heute viele berechtigte und ernstzunehmende Argumente angeführt wurden, die das (vermeintlich) solide Fundament der Biowissenschaften als Leitwissenschaft des politischen Naturund Umweltschutzes erschüttern (müssten), ist die von Ludwig Trepl konstatierte Diagnose, die er bereits 1988 formuliert hat, nach wie vor zutreffend: „Es sinddie Figuren der Ökologie, die das Denken über die Natur und über die Stellung des Menschen in ihr organisieren: Ein Bild der Natur als Systemzusammenhang ist populär geworden, worin nichts geschehen kann, ohne Wirkung auf das Ganze, und das Ganze weist den Teilen die Richtung und setzt ihnen Grenzen." (Trepl 1988:164-165) Diese Grundannahme der Ökologie ist - vor allem außerhalb der Biowissenschaften - zur Selbstverständlichkeit geworden, so „dass diejenigen, die nicht nur feststellen, dass die Ökologie eine Leitwissenschaft geworden ist, sondern darauf die wissenschaftspolitische Forderung aufbauen, sie sollte es 
nur dazu benutzt werden, die Grenzen der ökosystemaren Belastbarkeit festzulegen, sondern wenn die Ergebnisse der empirisch-analytischen Biowissenschaften auch dazu benutzt werden, um aus ihnen bestimmte 'ökologische', aber in diesem Sinne gerade nicht rein beschreibende Zusammenhänge, sondern normative Lebenseinstellung und Handlungsanweisungen zur Erhaltung der Natur/Biodiversität zu gewinnen, dann werden speziell die BiowissenschaftlerInnen zu Experten und Anwälten für die gesellschaftlich wertgeschätzte Vielfalt des Lebens (gemacht), auch wenn dies weit über die eigentlichen Gegenstands- und Kompetenzbereiche der Ökologie und Biologie hinausreicht: ${ }^{36}$

Werden die Biowissenschaften in einer solchen Weise 'entgrenzt', wobei Uta Eser hier durchaus passend von Selbstentgrenzung spricht, da diese Versuche vor allem aus den Biowissenschaften selbst heraus erfolgt sind (Eser 2001:144), so transportieren sie, als Fundament des modernen Natur- und Umweltschutzes, eine Vielzahl an politischen Einstellungen, Überzeugungen sowie Wert- und Normvorstellungen, die sich nicht aus ökologischen und biologischen Fakten, sondern aus den Bewertungen der Beziehungen von Menschen zu ihrer Umwelt ergeben. Der Versuch, die Biowissenschaften auf diesem Weg zu entgrenzen - führt indes in eine 'Sackgasse', da Biologie und Ökologie dazu in der Lage sein müssten selbst die Ziele zu begründen, die es zu verfolgen gilt, sprich Biologie und Ökologie müssten zugleich deskriptiv-empirische und normative Wissenschaften sein. Dass dem Versuch eine solche deskriptiv-normative Leitwissenschaft zu kreieren, bestimmte Grenzen gesetzt sind, zeigt sich schon allein daran, dass der oftmals erfolgte Versuch, die Normativität direkt aus der Natur heraus zu gewinnen, indem alles was natürlich ist zum normativen Orientierungszusammenhang gemacht wird, die Gefahr der Begehung von naturalistischen Fehlschlüssen und SeinSollen Fehlschlüssen hervorruft:

Damit wird der Fokus auf die Problematiken gelenkt, dass in der Rede von der Bewahrung und Förderung der Biodiversität von zahlreichen normativ gehaltvollen, aber deskriptiv verwendeten Begriffen (wie bspw. Funktion, Leistung, Vernichtung, Nachhaltigkeit, Entwicklung,) Gebrauch gemacht wird und dass von den Ergebnissen biologischer und ökologischer Forschungen direkt auf bestimmte Handlungsnormen geschlossen wird, die dann als Handlungsgrenzen im zivilisatorischen Umgang mit der Natur erscheinen (Birnbacher 1980:103). Insofern wird der Fokus auf die Problematik gelenkt, dass in der Biodiversitätsdebatte die Tendenz besteht, Begründungen für präskriptive Urteile (die Natur/Biodiversität soll geschützt werden) in Form naturwissenschaftlicher

sein, auf einem sehr soliden Fundament stehen: Von wissenschaftlicher Kritik ist es kaum zu erschüttern." (ebd., 165)

36 Auf eben diese Gefahr weisen seit langem zahlreiche Autoren hin (vgl. insb. Takacs 1996; Eser 2001, 2007, 2009; Piechocki 2005, 2007; Potthast 1999, 2005a, 2005b, 2007b, 2007a; Haber 2009). 
Hypothesen zu formulieren, wobei die normativen Prämissen aus denen sich die Normen eigentlich ergeben, oftmals weggelassen werden. Solche unvollständigen Argumente (Enthymeme) finden sich zwar in der Alltagssprache und im politischen Raum unentwegt (siehe auch 4.1), ${ }^{37}$ sie sind aber sowohl im formalen argumentationstheoretischen Rahmen, wenn es um die gültigen Logiken und die Struktur von Argumenten geht, als auch im politischen Raum, wenn es um die Aushandlung von Interessenskonflikten geht, problematisch, da präskriptive Urteile nur dann eine wissenschaftliche Relevanz beanspruchen bzw. ihre handlungswirksame Überzeugungskraft entfalten können, wenn es sich dabei um plausible und nachvollziehbare Aussagen und Urteile handelt. Daher gilt, dass im Einzelfall immer nach der Leitunterscheidung zwischen deskriptiven, evaluativen und präskriptiven Aussagen 'gefahndet' werden muss, ${ }^{38}$ so dass sich hier die Aufgabe stellt, die über den Begriff der Biodiversität transportierten Wertund Normvorstellungen sowie die dahinterstehenden Prinzipien und Maximen sowie Interessen und Präferenzen derer, die sich der Biodiversität bedienen, als angreifbare und kritisierbare Bewertungen und Beurteilungsmaßstäbe kritisch zu reflektieren, damit diese nicht als naturwissenschaftlich legitimierte Prämissen stillschweigend vorausgesetzt werden.

b) Der ökonomische Strang der "'Fakten'-Argumentation « vermeidet die Problematiken des naturalistischen Fehlschlusses und des Sein-Sollen-Fehlschlusses, denn hier wird die Erhaltung der (belebten) Natur aufgrund menschlicher Interessen an ihr gerechtfertigt. Hierbei wird i.d.R. das Argument bemüht, dass die Biodiversität deshalb im Rückgang begriffen ist, weil den Menschen noch nicht hinreichend bewusst sei, wie grundlegend und weitreichend die Bedeutung der Biodiversität für die Befriedigung menschlicher (Grund-)Bedürfnisse eigentlich ist. Daher geht es in erster Linie um die Rechtfertigung der Erhaltung der (belebten) Natur aus menschlichen Eigeninteressen an ihrer Nutzung. ${ }^{39}$ In diesem Sinne konstatiert auch die CBD in ihrer Präambel, dass

37 Die Unvollständigkeit solcher Argumente ist nicht per se problematisch, sondern immer dann vollkommen problemlos, wenn die implizite normative Prämisse ganz eindeutig und unstrittig ist. Dies ist i.d.R. sogar der Fall, kann aber dann nicht mehr als gegeben betrachtet werden, wenn es zu Konfliktsituationen kommt.

38 Die Aufforderung nach der Unterscheidung zwischen deskriptiven, evaluativen und präskriptiven Aspekten zu fahnden, soll hier nicht bereits die epistemische Stellungsname implizieren, ob dies auch immer zweifelsfrei möglich ist. Frido Ricken verweist indes auf die Möglichkeit, dass dies selbst bei 'dicken ' moralischen Begriffen, wie dem der Verantwortung oder des Versprechens, möglich ist und expliziert dies exemplarisch (vgl. Ricken 2003:63-65).

39 Thorsten Galert stellt diesbezüglich die weitverbreitende Annahme heraus, dass es, um jeder Person begreiflich zu machen, dass es im Sinne des Eigennutzes jedes Menschen ist, die Biodiversität zu erhalten, keiner moralischen Begründung und damit keiner Ethik bedarf (Galert 1998:14). Hier ist anzumerken, dass der Verweis darauf, dass der Eigennutz kein genuin moralischer Grund sei, sicherlich zutreffend ist, es bleibt aber zu fragen, ob derartige pragmatische Erwägungen als solche normativ neutral sind, so dass es sich hierbei um Aspekte handelt, die außerhalb ethischer Erwägungen stehen können (siehe 4.3.2.2.1). 
die Biodiversität auch aufgrund ihrer zahlreichen instrumentellen Werte für den Menschen erhalten werden soll: ${ }^{40}$

"[C]onscious (...) of the ecological, genetic, social, economic, scientific, educational, cultural, recreational and aesthetic values of biological diversity and its components (...)“ (UN:Präambel, Abs. 1)

Ohne hier bereits darauf eingehen zu können, was instrumentelle Werte überhaupt sind und durch welche Annahmen sie von anderen Werttypen unterschieden werden können (siehe 4.2.2.1.1), kann bereits herausgestellt werden, dass der Verweis auf die instrumentellen Werte der Biodiversität bedeutet, dass es hierbei um die Erhaltung der (belebten) Natur geht, die dem Menschen potenziell nützlich ist oder sein könnte. Diese Argumente finden sich vor allem in den einflussreichen Debatten um die ökosystemaren Leistungen der Biodiversität. Solche Potenzialitätsargumente, dass man nie genau wissen kann, ob man bestimmte Aspekte der Biodiversität nicht doch irgendwann brauche und deshalb besser alles erhalten solle, rekurieren auf das Vorsorgeprinzip (»precautionary principle«). Dieses besagt, dass bei Entscheidungen unter Unsicherheit und begrenztem Wissen, die mit den geringsten Risiken behaftete Option zu wählen ist, so dass selbst dann, wenn man nicht von jedem Element der Biodiversität weiß und wissen kann, ob und wozu man es braucht, man es sicherheitshalber doch erhalten sollte. Dies scheint auf den ersten Blick geeignet, um die Beweislast zugunsten der gesamten Erhaltung der Biodiversität umzukehren, ist aber an die (schwierige) Explizierung dessen gebunden, welche Risiken hier genau gemeint sind und wie genau festgestellt werden kann und soll, welche Handlungsoption die geringsten Risiken birgt.

Zudem kann hierüber nur die Erhaltung derjenigen Aspekte der Biodiversität argumentiert werden, bei denen wir, aus einer Nutzenperspektive heraus, Gründe haben, deren Vielzahl und Vielfalt möglichst zu erhalten. Dies schließt bspw. Krankheitserreger, aber auch fortpflanzungsfähige Chimären und stellenweise auch Neophyten aus, vor allem dann, wenn diese eine Gefahr für die Gesundheit des Menschen oder die Existenz von Nutzpflanzen darstellen. Des Weiteren muss dieser Nachweis in jedem einzelnen Fall erneut geführt werden, so dass natürliche Entitäten nie ein für alle Mal als schützenswert ausgewiesen werden können, weil gerade der technische Fortschritt die Möglichkeit schafft, den Nutzen, den die (belebte) Natur erbringt, möglicherweise auch schneller, handhabbarer und/oder preiswerter zu ersetzen. In einem solchen Fall spräche nichts mehr gegen das Ausrotten einer Art oder die Zerstörung von Ökosystemen, es sei denn, Umwelt- und NaturschützerInnen brächten Argumente ein, warum die Erhaltung der gesamten Biodiversität 'um ihrer selbst willen`zu rechtfertigen sei.

40 In der Präambel der CBD ist ebenfalls davon die Rede, dass die Biodiversität aufgrund ihres Eigenwertes ( „intrinsic value“) erhalten werden soll. Auf diesen Aspekt, wird im Kontext der Eigenwertargumentation detaillierter eingegangen. 


\subsubsection{Die »'Eigenwert'-Argumentation« für den Schutz der Biodiversität}

Gerade aufgrund der dargelegten Problematiken und 'Mängel' der faktischen Argumentationsstrategien werden biowissenschaftliche oder ökonomische Argumente von vielen Umwelt- und NaturschutzaktivistInnen, PolitikerInnen und WissenschaftlerInnen skeptisch betrachtet und als nicht ausreichend für die Begründung der Erhaltung der Biodiversität angesehen. Genau aus diesen Gründen ist die Eigenwertargumentation im Umwelt- und Naturschutz so relevant: Da mit biowissenschaftliche Argumenten nur beschrieben, nicht aber auch gefordert oder aufgefordert werden kann und die Nutzenargumente im Rahmen des ökonomischen Paradigmas immer wieder in die 'missliche Lage` führen, dass die Relevanz der Biodiversität immer wieder aufs Neue plausibilisiert werden muss, kann die Eigenwertargumentation als Hoffnung darauf verstanden werden, die Beweislast umzukehren: Wenn der Biodiversität ein - wie auch immer gearteter - Eigenwert ${ }^{41}$ zukommt oder zugesprochen werden kann, der den Schutz der Biodiversität 'um ihrer selbst willen`als allgemeine Pflicht rechtfertigt, dann wäre Biodiversitätsschutz der Normalfall, so dass Ausnahmen davon einer besonderen Begründung bedürften. Gerade weil das Anliegen und der Wunsch nach einer solchen 'Superbegründung mit eingebauter Beweistlastumkehr' aus der Perspektive von Umweltund NaturschützerInnen so nachvollziehbar ist, ist der Eigenwert der Biodiversität eines der wichtigsten Argumente, um die Erhaltung der Biodiversität zu begründen.

Dies zeigt sich bereits an den zahlreichen politischen Dokumenten und Abkommen, die zumeist an erster Stelle auf den Eigenwert der Biodiversität verweisen:

"Conscious of the intrinsic value (...).“ (UN 1992: Präambel, Abs. 1)

„By 2050, European Union Biodiversity (...) are protected, valued and appropriately restored for biodiversity`s intrinsic value (...)." (EU COM 2011:2)

"Natur und Landschaft sind auf Grund ihres eigenen Wertes (...) zu schützen, zu pflegen, zu entwickeln und (...) wiederherzustellen." (DB 2009:Paragraph 1)

„Natur und Landschaft sind auch auf Grund ihres eigenen Wertes zu erhalten." (BMUB 2011:9)

41 Die Rede vom Eigenwert wird hierbei in der Regel begrifflich unsauber geführt, weil mal von Selbstwert, Eigenwert, inhärentem Wert (»inherent value«, »inherent worth«), intrinsischem Wert (»intrinsic value«), nicht-instrumentellem Wert (»non-instrumental value«) oder auch nutzenunabhängigem Wert (»non-use value«), absoluten Wert, objektivem Wert, unabhängigen Wert, nicht-anthropozentrischen Wert, Existenzwert (»existence value«) etc. die Rede ist. Eine solche begriffliche Vielfalt wäre nicht weiter problematisch, wenn im Einzelfall immer expliziert werden würde, was einen solchen Eigenwert überhaupt zum Eigenwert macht und welche Annahmen und Prämissen hinter der Eigenwert-Typologisierung stehen würden. Genau dies ist aber eher selten und wenn dann zumeist nur recht oberflächlich der Fall, so dass die gesamte Wertedebatte, die außerhalb und innerhalb der Umwelt- und Naturschutzethik geführt wird, eingehender reflektiert werden muss. Eben dies wird in Abschnitt 4.2.2.1 geschehen, da dafür noch weitere Vorarbeiten notwendig sind. 
Die Rede vom Eigenwert, vom Selbstwert oder intrinsischen Wert spielt aber nicht nur in den politischen, sondern auch in den umwelt- und naturschutzethischen Debatten eine zentrale Rolle, womit die Eigenwertargumentation wiederum zu einem zentralen Bindeglied zwischen der Umwelt- und Naturschutzpolitik und der Umwelt- und Naturschutzethik wird. So zentral das Eigenwertargument in Politik und Ethik ist, so unterschiedlich wird die Eigenwertdiskussion hier jeweils geführt, da die Diskussion unter gänzlich anderen Vorzeichen und unter anderen Voraussetzungen geschieht: Die Ethik ist gerade keine bestimmte Moralposition, sondern sie ist die Wissenschaft oder Reflexionstheorie der Moral, die versucht die gelebten Sitten und Moralvorstellungen argumentativ zu prüfen und zu bewerten sowie Handlungsanweisungen zu begründen. Damit wird nicht danach gefragt, wie Menschen sich faktisch verhalten, sondern wie sie sich verhalten sollten, so dass die Unterscheidung von Moral und Unmoral bewertend (evaluativ) und vorschreibend (präskriptiv) gemeint ist, was nicht ohne eine Rechtfertigung qua Begründung beurteilt werden kann. Daher wird der Eigenwert in der Ethik nicht einfach als gegeben und geltend vorausgesetzt, sondern es geht darum, die Annahmen, die hinter der postulierten Eigenwertigkeit stehen sowie die daraus abgeleiteten Pflichten kritisch zu reflektieren und plausibel zu begründen.

Genau dieses Spezifikum der Ethik wird indes oftmals gar nicht in Rechnung gestellt, wenn - vor allem von Seiten vieler NaturwissenschaftlerInnen, aber auch PolitikerInnen - daran gezweifelt wird, dass die Ethik überhaupt ein Gebiet ist, in dem sich ein Standpunkt vor einem anderen als besser begründet auszeichnen lässt (Galert 1998:18). Dementsprechend werden ethische Begründungen oftmals einfach mit religiösen, spirituellen oder ideologischen Sichtweisen und Positionen gleichgesetzt, was nicht heißt, dass deren Bedeutung für Naturschutzanliegen deshalb auch gering geschätzt wird. Vielmehr ist es so, dass das eigentliche 'Wesen' ethischer Begründungen und Argumente vollkommen verkehrt wird, weil Ethik bereits mit bestimmten Moralpositionen gleichgesetzt wird und eben nicht im wissenschaftlichen Sinne als (kritische) Reflexionstheorie aller Moralpositionen verstanden wird. Wie verbreitet solche grundsätzlichen (Miss-)Deutung von Ethik sind, zeigt die Aussage von Anne und Paul Ehrlich, zwei der führenden wissenschaftlichen Umwelt- und NaturschützerInnen der USA:

"Our own view, and that of many biologists and environmentalists, is that, as the dominant species on the planet, Homo sapiens has an ethical responsibility to preserve biodiversity. This means opposing intentional exterminations of other species and supporting conservation efforts. One cannot assert this ethical responsibility on scientific grounds. It clearly arises from essentially religious feelings; we believe that our own living companions in the universe have a right to exist." (Ehrlich \& Ehrlich 1992 in Galert 1998:18) 
Indem Ethik hier mit moralisch gleichgesetzt und auf diese Weise 'entwissenschaftlicht`wird, gleichzeitig aber den moralischen Haltungen gegenüber der Natur eine große Relevanz zugeschrieben wird, wird die Ethik zum Legitimierungsinstrument funktionalisiert (Potthast 1999:35):

„In einer moralphilosophischen Diskussion um Werte und Normen mutet es merkwürdig an, wenn unmittelbar nach der Praktikabilität oder der Durchschlagskraft ethischer Argumente gefragt wird. Ethische Forderungen können nur dort berechtigterweise erhoben werden, wo Werte und Normen plausibel und nachvollziehbar begründet sind. Trifft dies nicht zu, ergeben sich auch keine ethisch legitimierten Handlungsweisen." (ebd., 37)

Hierbei wird indes übersehen, dass die ethische Prüfung normativer Argumente nicht direkt mit der Frage der politisch-praktischen Durchsetzung der natur- und umweltschützerischer Ziele verknüpft werden kann, weil es vor der instrumentellen Anwendung normativer Argumente auf der politischen Ebene für die Ethik immer darauf ankommt deren Prämissen zu begründen. Hieran wird ersichtlich, dass die 'Tücke' der Eigenwertargumentation in einem, aus der ethischen Perspektive, problematischen Verständnis von Ethik und Begründung liegt: Ungeachtet der terminologischen Probleme, wie Eigenwert im Einzelnen betitelt wird und den werttheoretischen Fragen, welche Aspekte den Eigenwert dabei zum Eigenwert machen und welche Annahmen im Einzelnen dahinterstehen sowie der pflichtenethischen Frage, welche ethischen Implikationen bezüglich der Ableitung und Rechtfertigung von Pflichten damit ggf. einhergehen, ergeben sich aus der Strategie, die Erhaltung der Biodiversität als eine Pflicht 'um ihrer selbst willen 'zu begründen, in Rückgriff auf die bereits herausgestellten Problemlagen bezüglich der biowissenschaftliche Konzeption der Biodiversität (siehe 2.1) sowie ihrer politischen Symbolisierung (siehe 2.2) vor allem zwei Probleme:

Zum einen ist aufgrund der methodisch-theoretischen Unterbestimmtheit der Biodiversitätskonzeption unklar, worauf genau sich der Eigenwert der Biodiversität genau beziehen soll, da das Biodiversitätskonzept grundsätzlich zwei Ebenen integriert, die dafür in Frage kommen (Ott 2007:91): Einerseits die Ebene der unterschiedlichen Entitäten (und ihrer möglichen Gruppierungen) und zum anderen die Ebene der Unterschiede und Verhältnisse, die zwischen diesen Entitäten postuliert werden (Wood 1997:253). In diesem Sinne ist gar nicht klar, ob sich der Eigenwert auf die einzelnen Entitäten, die Gruppen von Entitäten, das Beziehungsgeflecht dieser Entitäten bzw. Gruppen unter- und zueinander oder auf die Unterschiedlichkeit und Vielfalt als solche ${ }^{42}$ beziehen sollen (Ott 2007:96). Je nachdem welche Ebene gewählt wird, ergeben

42 Für Konrad Ott macht die Bewertung der Unterschiedlichkeit als solche indes keinen Sinn, da er nicht nachvollziehen kann, warum die Differenz zwischen etwas als solche wertvoll sein soll. Exemplarisch führt er dies an unterschiedlichen Sprachen aus (vgl. Ott 2007:96-97). 
sich unterschiedliche Bewertungs- und Begründungsansätze. Zumeist wird die Bewertung auf die Gegenstandsebene bezogen (ebd., 91-92). Dies ist indes eine pragmatische Entscheidung, theoretisch induziert ist sie nicht.

Zum zweiten ist nicht klar, warum sich aus dem Eigenwert der Biodiversität auch schon eine gesellschaftliche Norm zum möglichst umfassenden Schutz der gesamten Biodiversität ableiten sollte, wie dies gemeinhin politisch über das Eigenwertargument gefordert wird. Zwar wird mit dem Verweis auf den Eigenwert der Biodiversität gemeinhin die Annahme verbunden, dass sich aufgrund des Eigenwertes eine moralische Verpflichtung des Menschen zur Erhaltung der belebten Natur oder sogar Rechte nichtmenschlicher Lebewesen rechtfertigen lassen, dies ist aber nur dann schlüssig, wenn auch klar ist, was den Eigenwert überhaupt zum Eigenwert macht, sprich auf Grundlage welcher werttheoretischen Annahmen dieser zugeschrieben wird ob eben diese Annahmen stichhaltig und plausibel sind. Des Weiteren wird hierbei oftmals die Kluft zwischen der, auf der axiologischen Ebene liegende Frage, ob es so etwas wie Eigenwerte der Natur bzw. Eigenwerte in der Natur geben kann und wenn ja, was diese ausmacht und der, auf der moralphilosophischen Ebene relevanten Frage, was daraus für unser Handeln jeweils folgt, übersehen. Hier steht folglich in Frage, ob man, wenn man der Biodiversität oder Teilen bzw. Aspekten der Biodiversität einen Eigenwert zuschreibt bzw. diesen anerkannt, auch notwendigerweise folgt, dass daraus moralische Pflichten gegenüber jemanden oder etwas resultieren. Daher fehlt bei dem Schluss von Eigenwert auf die moralische Berücksichtigungswürdigkeit der Schritt von der werttheoretischen Annahme, dass Eigenwerte in der Natur entdeckt oder der Natur zugeschrieben werden können hin zur pflichtenethischen Annahme, dass Eigenwerte plausible Gründe dafür darstellen, dass die Biodiversität als eine moralische Pflicht zu bewahren und zu fördern ist.

Zusammenfassend lässt sich festhalten, dass jede der Argumentationsstrategien mit je eigenen Problematiken und Schwierigkeiten behaftet ist, die vor allem daraus resultieren, dass hierbei auf verschiedenen Wegen versucht wird, die Zielstellung der Erhaltung der (gesamten) Biodiversität zu rechtfertigen, wobei nicht alle diese Versuche auch gleichermaßen geeignet sind, dies zu tun: Die naturwissenschaftlich abgestützte Argumentationsstrategie zur Erhaltung der Biodiversität führt ökologische Gründe an, deren Kennzeichnung als ökologisch den Anschein nahelegen, es handle sich dabei um rein wissenschaftliche und in diesem Sinne sachliche, objektive und wertfreie Gründe. Bei genauerer Betrachtung sind indes viele der ökologischen Gründe normativer Natur, was aber über die faktische Argumentationsweise als solche aber nicht deutlich wird. Die ökonomisch ausgerichtete Argumentationsstrategie setzt von Beginn an die Prämisse, dass (nur) das für den Menschen Dienliche und Nützliche einen Wert hat und damit schützenswert sei. Je nach Argumentation sind damit nur ganz bestimmte Teil- 
aspekte der Natur instrumentell wertvoll, was wiederum der, nicht nur in Naturschutzkreisen selber weitverbreiteten Intuition entgegenläuft, dass die Natur auch ganz unabhängig von menschlichen Nutzungsinteressen an ihr einen Wert hat. Die Eigenwertstrategie, die diese Intuition bedienen kann und die sich als ethische Argumentationslinie ausweist, krankt indes an der oftmals zu funktionalistischen Verwendung normativer Argumente sowie an der problematischen Gleichsetzung von Ethik und Moral.

\subsection{Zusammenfassung und Fazit: Biodiversität als Grenzobjekt und Hybridkonzept zur Erschließung gesellschaftlicher Naturverhältnisse}

In Bezug auf Biodiversität als biowissenschaftliches Konzept wurde im Vorangegangenen die methodisch-theoretische Unterbestimmung der Biodiversitätskonzeption als Metaproblematik herausgestellt, da über die biowissenschaftlichen Fachbegriffe, aber auch über die alltagssprachlichen Formulierungen zur Bestimmung dessen, was Biodiversität ist und ausmacht lediglich der Eindruck suggeriert wird, dass hinter der Rede von der Biodiversität ein einheitlicher biowissenschaftlicher Forschungsgegenstand stehen würde, der aus den Biowissenschaften herausgenommen und in anderen Kontexten 'angewandt' werden könnte.

In Bezug auf Biodiversität als politischer Symbolbegriff wurde des Weiteren herausgestellt, die Neuausrichtung des Umwelt- und Naturschutzes, die sich über das Leitbild der Bewahrung und Förderung der Biodiversität ergibt, darin besteht, dass es um die Auslotung der Möglichkeiten und Grenzen einer nachhaltigen Entwicklung der gesellschaftlichen Naturverhältnisse geht. Insofern kommt dem Leitbild der Bewahrung und Förderung der Biodiversität ein hohes Innovationspotenzial zur Modernisierung des klassischen Umwelt- und Naturschutzes zu. Als Metaproblematik wurde herausgestellt, dass dieses Potenzial in Deutschland, wo die NBS in den Kontext einer reinen Naturschutzgesetzgebung gestellt wird, nicht in ausreichendem Maße eingeholt wird, weil das eigentlich Innovative an der Bewahrung und Förderung der Biodiversität, nämlich die Integrativität der Zieltrias, immer wieder auf den Schutzaspekt verengt wird.

Schließlich wurden in Bezug auf Biodiversität als Wert- und Normbegriff die Metaproblematiken festgehalten, dass sich die normative Imprägnierung der Biodiversität bereits über die Selektion bestimmter Elemente aus der gegebenen Vielzahl der Möglichkeiten ergibt, die Biodiversität inhaltlich zu bestimmen, da diese Selektionen immer schon ein Urteil darüber sind, welche Aspekte der Biodiversität als wichtig und damit mittelbar auch als wertvoll und schützenswert angesehen werden. Die explizite Ausweisung der Werthaftigkeit der Biodiversität und der Schutznorm geschieht wiederum über verschiedene Argumentationsstrategie, wobei jede dieser Strategien mit je eigenen Schwierigkeiten behaftet ist, die vor allem daraus resultieren, dass hierbei versucht wird, die Erhaltung der gesamten Biodiversität zu rechtfertigen. 
Über die nun folgende Charakterisierung der Biodiversität als Grenz- und Hybridkonzept werden die drei Einordnungen der Biodiversität als biowissenschaftliches Konzept, als politischer Symbolbegriff sowie als Wert- und Normbegriff sowie die damit verbundenen Metaproblematiken in Verbindung zueinander gesetzt. Wenn Biodiversität dabei, wie von Uta Eser, als Grenzobjekt oder wie von Thomas Potthast als Hybridkonzept bezeichnet wird, dann wird darüber auf ähnliche Phänomene fokussiert: Im Falle des Grenzobjektes geht es um die Bezüge zwischen Biodiversität als Wert- und Normbegriff und Biodiversität als politischer Symbolbegriff. Hierbei steht die wissenssoziologisch relevante Entgrenzung der, die Biodiversität erforschenden Wissenschaften im Fokus. Im Falle des Hybridkonzeptes geht es um die Bezüge zwischen Biodiversität als biowissenschaftliches Konzept und Biodiversität als Wert- und Normbegriff, wobei die wissenschaftstheoretisch relevante Hybridisierung der unterschiedlichen Blickwinkel auf Biodiversität (bspw. TheoretikInnen und PraktikInnen, NutznießerInnen und Geschädigte, ExpertInnen und Laien etc.) fokussiert wird:

Uta Eser, die Biodiversität als Grenzobjekt (»boundary object«) charakterisiert (vgl. Eser 2001, 2003a, 2003b, 2007, 2009), weist über diese Charakterisierung insbesondere auf die Schwierigkeiten der wissenschaftlichen Beschäftigung mit der Biodiversität als Grenzarbeit (»boundary work«) hin. Damit macht sie darauf aufmerksam, dass es ganz unterschiedlichen Arten des Zugangs und der Nutzung von Informationen durch unterschiedliche soziale Gruppen gibt, die wiederum gänzlich unterschiedliche Potenziale und Möglichkeiten haben, diese für ihre Zwecke zu gebrauchen. Eben diese Asymmetrien schlagen sich auch in der Begriffsbildung und -rezeption nieder, so dass hierüber 'Grenzen ' etabliert, aber auch ständig revidiert und hinterfragt werden. Eben dies geschieht in besonderem Maße über das Konzept der Biodiversität, indem hier Gefühle, Emotionen, Werte und Normen als Aspekte naturwissenschaftlicher Argumente anerkannt werden (Takacs 1996:165), womit das für die empirischen Wissenschaften konstitutive Prinzip der Wertneutralität implizit in Frage gestellt wird (siehe 2.3.1):

Gefühl und Geist sollen offenbar nicht länger aus der wissenschaftlichen Beschäftigung (...) ausgeschlossen sein. Das sonst aus der Wissenschaft systematisch ausgegrenzte Gefühl wird programmatisch eingeschlossen, die Grenze zwischen Wissenschaft und Gesellschaft (...) bewusst überschritten. Zugleich wird das Ideal wissenschaftlicher Wertneutralität verabschiedet und die klassische Trennung von Fakten und Werten damit in Frage gestellt." (Eser 2007:45)

Dies zeige sich, so Eser, vor allem an den kryptonormativen Beschreibungssprachen, die für die politische Biodiversitätsdebatte prägend und vor allem in Bezug auf die naturwissenschaftlichen Argumentationsstrategien zur Erhaltung der Biodiversität von Bedeutung sind (siehe 2.3.1a): Indem Begriffe wie Leben, Lebewesen, Vielfalt, Vielfältigkeit und Unterschiedlichkeit auf Alltagserfahrungen rekurrieren, wohingegen die 
fachspezifischen Begriffe wie Diversität, Variabilität, Gen, Art, Organismus oder Ökosystem (siehe 2.1) biowissenschaftliche Kenntnisse und naturwissenschaftlichen Sachverstand erfordern, werden die mit der Biodiversität verbundenen Alltagserfahrungen sowie die damit verbundenen Wert- und Normvorstellungen im 'Deckmäntelchen ' biowissenschaftlicher Begriffe und Konzepte mitgeführt und invisibilisiert, da über die vermeintlich objektive, sachliche und wertfreie Sprache, kulturell geprägte Vorstellungen davon, was Natur ist und was Leben ausmacht, sowie Wertvorstellungen und Moralpositionen als tatsachenlegitimiert ausgegeben werden (ebd., 43). Darüber wird zum einen der Eindruck erzeugt, dass sich 'ökologische' Haltungen sowie Wert- und Normvorstellungen über den Naturumgang aus Faktenargumenten ableiten lassen. Zum anderen werden darüber ganz bestimmte Antworten auf die Fragen vorgelegt, was genau das Problem bei der Biodiversität ist, für wen es ein Problem ist und warum es ein Problem ist. Eben die Reflexion auf die Art und Weise der Problematisierungen zieht nicht nur die Frage nach sich, wie und mit welchen Zwecken bestimmte Begriffe gebildet und gebraucht werden, sondern führt auch direkt ins Zentrum des sozialökologischen Problemfeldes Biodiversität als eine Problematik der gesellschaftlichen Naturverhältnisse:

„Nach einem verbreiteten Verständnis von Politik wie auch sozialwissenschaftlicher Forschung haben sich Politikerinnen wie Sozialwissenschaftler die Beschreibung der Problemlage von Natur- und Biowissenschaftlern vorgeben lassen. Dies gilt weitgehend ungeteilt selbst noch bei sozial-ökologischen Problemen oder bei einem interdisziplinären Vorgehen. Und wie sollte es auch anders sein, sind doch diese allein dafür zuständig, eine präzise Definition des Problems und seiner Ursachen zu liefern. Erst in den letzten Jahren ist in Verbindung mit einer verstärkten Beachtung konstruktiver Ansätze in der Wissenschaftstheorie diese eindeutige Aufgabenzuweisung in Frage gestellt worden (...). Damit rückt die Frage, um welches Problem es eigentlich geht, wie es am besten zu beschreiben ist und wie sich im am besten zu nähern sei, ins Zentrum des Interesses. Gibt es keinen a priori privilegierten Zugang zum Problem, dann ist es aussichtsreicher, zunächst auf die jeweiligen disziplinären Zugangsweisen und ihre Grenzen zu reflektieren." (Görg 2003:220-221)

Eben diese Reflexion auf die disziplinären Grenzen und deren Etablierung und Verschiebung wird folglich fokussiert, wenn Biodiversität als Grenzobjekt charakterisiert wird. Nimmt man dies ernst, dann muss bereits die Problematisierung des Biodiversitätsproblems auf interdisziplinäre 'Beine' gestellt werden, wobei ein Standbein davon die Ethik sein muss, denn nur sie kann eine wissenschaftliche Reflexion auf die Normativitätsproblematik sozialökologischer Probleme im Allgemeinen sowie die normative Imprägnierung der Biodiversität im Speziellen leisten. 
Zu eben diesem Ergebnis kommt auch Thomas Potthast, der mit seiner Charakterisierung der Biodiversität als epistemisch-moralisches Hybridkonzept (vgl. Potthast 1999, 2000, 2005a, 2005b, 2006, 2007a) einen weiteren Aspekt deutlich macht. Indem er Biodiversität als epistemisch-moralischen Hybrid charakterisiert, fokussiert er die (problematische) Verbindung von naturwissenschaftlichen und genauer biowissenschaftlichen Erkenntnisinteressen mit dem moralischen Gebot zum Schutz der belebten Natur. Potthast macht deutlich, dass sich im Begriff bzw. im Konzept der Biodiversität bestimmte Annahmen darüber, was Biodiversität ist und wofür die Biodiversität gut oder wichtig ist, mit dem Appell vereinen, dass die Biodiversität erhalten werden soll und problematisiert darüber den Umstand, dass aus den empirischen Zusammenhängen, die die Bedrohung und den Verlust der Biodiversität quantifizieren sollen (die ja selbst unter empirischen Vorbehalten stehen) über den Verweis auf die Natürlichkeit als Orientierungszusammenhang sowie den Eigenwert der Biodiversität auf die moralische Pflicht aller Menschen zum Schutz der gesamten Biodiversität geschlossen wird, so dass der Begriff der Biodiversität bereits die moralische Pflicht diese zu erhalten impliziert. Hierbei kritisiert er, dass über die Ausblendung und Verschleierung der normativen Gehalte von Natürlichkeit sowie der Prämissen des Eigenwertes oftmals nicht genug in Rechnung gestellt wird, dass normative Überzeugungen sich über die ethische Reflexion zwar mehr oder weniger stark plausibilisieren lassen, dass dies aber nicht zwangsläufig der Fall sein muss (Potthast 1999: 35).

Insofern verweisen beide Charakterisierungen auf die Wichtigkeit und Notwendigkeit einer bereits bei der Problemformulierung beginnenden interdisziplinären Auseinandersetzung mit der Biodiversität. Im Kern wird dabei verdeutlicht, dass die biowissenschaftlichen und ethischen Erkenntnisse über die Biodiversität sowie die Art und Weise ihrer politischen Umsetzung in einem wechselseitigen Bedingungszusammenhang stehen, so dass die Rede von der Biodiversität automatisch die Rede von ihrer Bewahrung und Förderung nach sich zieht - wenngleich die Frage danach, was Biodiversität ist, eine andere Antwort verlangt, als die Frage, was mit der Bewahrung und Förderung der Biodiversität gemeint ist. Dass auf beide Fragen indes oftmals die gleichen Antworten gegeben werden, liegt vor allem daran, dass die aufgeführten Metaproblematiken über den Grenz- und Hybridcharakter der Biodiversität vermittelt sind, da über diesen viele verschiedene Betrachtungsebenen und Zugangsweisen auf und zu Biodiversität zusammenkommen und vermengt werden. Dabei ist entscheidend, dass die Biodiversität als Wert- und Normbegriff als 'Brücke' zwischen den inhaltlichen und den politischen Aspekten fungiert, gerade die normativen Aspekte aber häufig gar nicht reflektiert werden. 
So wichtig die Feststellung der Entgrenzung- und Hybridisierung in Bezug auf Biodiversität und deren Bewahrung und Förderung ist, so dringlich muss in Anschluss daran die oftmals vernachlässigte Frage aufgeworfen und beantwortet werden, wie mit dem Grenz- und Hybridcharakter der Biodiversität im Rahmen einer anwendungsorientierten und interdisziplinären Biodiversitätsforschung, wie sie in dieser Arbeit anvisiert wird, umgegangen werden kann und soll. Gemäß der in Abschnitt 2.3.2 dargelegten und eingeforderten strikten Trennung von Sach- und Wertungsebene, wird auch in Bezug auf Biodiversität als Grenz- und Hybridbegriff zwischen einer auf der Sachebene angesiedelten, aber dennoch normativ imprägnierten inhaltlichen Ebene, auf der es um den Forschungsgegenstand der Biodiversität geht, einer explizit normativen, mit Wert- und Normfragen beschäftigen Ebene und einer wiederum auf der Sachebene liegenden, aber auch mit normativen Fragen beschäftigten politischen Ebene unterschieden werden, bei der es um die gesellschaftspolitische Umsetzung und die politischstrategische Ausgestaltung der Bewahrung und Förderung der Biodiversität geht. Mit Blick auf das Verhältnis der drei Ebenen zueinander - und in Erinnerung der in Abschnitt 1.2 festgehaltenen Präliminarien - wird im Folgenden dargelegt, auf welche speziellen Detailproblematiken auf der inhaltlichen (2.4.1), der normativen (2.4.2) und der politischen (2.4.1) Ebene die Arbeit überhaupt eingehen wird. Die im Folgenden darzulegenden Detailproblematiken sind somit die Fokuspunkte der weiteren Arbeit (siehe 1.1) und diese gilt es, nachdem diese in den Detailreflexionen eingehend behandelt wurden, noch einmal in der Schlussbetrachtung mit Bezug zueinander zu bilanzieren (siehe Abschnitt VI).

\subsection{1 inhaltliche Ebene: Die Ausblendung der kulturellen Konstitutions- bedingungen des Forschungsprogramms Biodiversität}

Auf der inhaltlichen Ebene steht die, in nahezu allen wissenschaftlichen, politischen und journalistischen Abhandlungen zur Biodiversität vorgenommene Gleichsetzung der Begriffe der abiotischen und biotischen Vielfalt einerseits und der biologischen Vielfalt anderseits über die Kurzform Biodiversität im Fokus der Analyse, die hier, im Anschluss an die wissenschaftstheoretischen und -philosophischen Arbeiten von Peter Janich und Mathias Gutmann (vgl. Janich \& Gutmann 2000; Gutmann \& Janich 2001a) als methodologischer Kurzschluss charakterisiert werden soll. Diese Gleichsetzung, so die hier vertretene These, ist sowohl in wissenschaftstheoretischer als auch in praktischer Hinsicht in Hinblick auf Biodiversität als hybrides und grenzüberschreitendes Forschungsprogramm relevant und wird daher als spezififische Detailproblematik auf der inhaltlichen Ebene herausgestellt. Der Hintergrund der Problematik des methodologischen Kurzschlusses ist dabei folgender: 
Das was Biodiversität bezeichnet, kann sich zum einen auf die Vielfalt der Lebewesen und ihrer Lebensbedingungen, sprich die biotische und abiotische Vielfalt beziehen, die methodologisch betrachtet, als Referenzgegenstände der Biodiversitätsforschung fungieren. Zum anderen verweist Biodiversität aber immer auch auf die Vielfältigkeit der Zugänge und Perspektiven zur Beschreibung, Erfassung und Erschließung der Artenvielfalt, der genetischen Vielfalt und der ökosystemaren Vielfalt, sprich der verschiedenen Forschungskonzepte, die unter dem Oberbegriff biologische Vielfalt subsumiert werden. Eben diese Unterschiedlichkeit zwischen abiotischer und biotischer Vielfalt einerseits und biologischer Vielfalt andererseits, als methodologischer Unterschied zwischen Referenzobjekten und Forschungskonzepten, wird immer dann nicht beachtet, wenn Biodiversität synonym mit biologischer Vielfalt gesetzt wird und wenn die biologische Vielfalt wiederum als Konglomerat aus der genetischen Vielfalt, der Artenvielfalt und der ökosystemaren Vielfalt definiert wird, die aber nicht als biologische Beschreibungskonzepte ausgewiesen werden, sondern als Spektrum der biotischen und abiotischen Vielfalt definiert wird.

Bei der umgangssprachlich fest etablierten Gleichsetzung von (a)biotisch und biologisch handelt es sich aber nicht lediglich um eine terminologische 'Laxheit', die geflissentlich übersehen werden kann, sondern es handelt sich hierbei um eine erkenntnisund wissenschaftstheoretisch relevante Kontroverse bezüglich der Konstitution und Konstruktion wissenschaftlicher Forschungsgegenstände, die aber als solche gar nicht in den Fokus kritischer Reflexionen rückt, weil sie über den methodologischen Kurzschluss invisibilisiert wird:

„Wo der Unterschied zwischen [a]biotischen und biologischen Objekten nicht nur vernachlässigt, sondern absichtlich ignoriert wird, entwickelt bzw. hält sich die Auffassung, dass die Objekte der Biologie [und der Ökologie] 'naturgegeben` sind, wie man laienhaft Tiere und Pflanzen einer von Menschen unbewohnten Gegend als Gegenstände einer 'unberührten` Natur als natürlich gegeben annimmt. Hier (...) wird erkenntnistheoretisch übersehen, dass jedes mögliche, auch das geringste Wissen über das Lebendige immer aus menschlichen Eingriffen in das Vorgefundene, Vorhandene gewonnen wird - es sei denn, es ist kein Wissen, geschweige denn ein naturwissenschaftliches." (Gutmann \& Janich 2001a:332)

Das Ergebnis der Invisibilisierung ist die wirkmächtige, aber vor einem wissenschaftstheoretischen Hintergrund problematische Suggestion, dass es sich bei der Biodiversität um ein 'natürlich 'verfasstes Phänomen handeln würde, das mit den Mitteln der Biowissenschaften - über das Konzept der biologischen Vielfalt - adäquat und wirklichkeitsgetreu beschrieben und erfasst werden kann. Insbesondere die letztgenannte Suggestion, so wurde bereits in der Grundlagenreflexion aufgezeigt, kann mit guten Gründen bezweifelt werden. Aber auch die erstgenannte Suggestion, dass man es bei 
der Biodiversität mit einem natürlichen Phänomen zu tun hat, ist - so die hier vorgebrachte These - ist zu bezweifeln. Denn über die kritische Reflexion des methodologischen Kurzschlusses wird überhaupt erst die Problematik der Auffassung verdeutlicht, dass die naturwissenschaftliche Erkenntnis nicht als eine beobachterunabhängige Erfassung der natürlichen Wirklichkeit angesehen werden kann. Stattdessen wird anhand des methodologischen Kurzschlusses aufgezeigt, dass alle Wissenschaften teilnehmende Praxen der Gegenstandskonstitution und -konstruktion sind, weil sich die Konstitution der Referenzobjekte (biotische und abiotische Vielfalt) bereits vorwissenschaftlichen Erfahrungen verdankt und weil sich die Forschungskonzepte (Artenvielfalt, genetische Vielfalt, ökosystemare Viefalt zur Erfassung) zudem immer aus methodologischen und theoretischen Entscheidungen von WissenschaftlerInnen im Rahmen ihrer Forschungsaktivitäten ergeben, so dass die Forschungskonzepte konstruiert sind.

Eben diese erkenntnis- und wissenschaftstheoretische Perspektive auf die Konstitution und Konstruktion der Biodiversität als Forschungsprogramm, die erst über die kritische Reflexion des methodologischen Kurzschlusses überhaupt ersichtlich wird, kam bisher bei der Betrachtung der Biodiversität als Grenz- und Hybridkonzept zu kurz. Schaut man dabei etwas tiefer, dann zeigt sich, dass die wesentlichen Gründe für den methodologischen Kurzschluss in dem, für moderne Naturwissenschaften und somit auch die Biowissenschaften üblichen, realistischen Wissenschaftsverständnis liegen, nach dem die Naturwissenschaften in der Lage sind die "menschenunabhängige Natur" (Janich \& Gutmann 2000:67) und die „natürlich gegebene Wirklichkeit“ (ebd., 68) aus der Distanz eines passiven Beobachters objektiv und wahrheitsgemäß zu beschreiben. Gegen ein solches realistisches Wissenschaftsverständnis, das von vielen ProtagonistInnen in der Biodiversitätsdebatte - zumeist unintendiert, unthematisiert und damit auch unreflektiert - vertreten wird, können indes zahlreiche Argumente ins Feld geführt werden. Praktisch relevant ist vor allem der, dass die Standards für die 'Normalität' natürlicher Umgebungen nicht problemlos den biowissenschaftlichen Beschreibungen entnommen werden können, da ansonsten aus biowissenschaftlichen Beschreibungszusammenhängen auf Handlungsanweisen zum Schutz, zur nachhaltigen Nutzung und zur gerechten Verteilung der Biodiversität geschlossen wird (siehe 2.3).

Allerdings ergeben sich noch andere, methodologische sowie erkenntnistheoretische und wissenschaftstheoretische Facetten der Problematik des methodologischen Kurzschlusses, anhand derer sich verdeutlichen lässt, dass das Forschungsprogramm Biodiversität zu wenig als geistes- und ideengeschichtlich konstituiertes, methodisch-theoretisch konstruiertes sowie deskriptiv-normatives Hybridkonzept betrachtet, analysiert und kritisch reflektiert wird. Um eben diesen Ausblendungen konstruktiv zu begegnen, werden in Abschnitt III der Arbeit die verschiedenen Ebenen des methodologischen Kurzschlusses beleuchtet: 
a) Zum einen werden die wissenschaftstheoretischen Grundlagen bzw.

Voraussetzungen des methodologischen Kurzschlusses dargelegt, wofür die grundlegenden Annahmen des wissenschaftlichen Realismus idealtypisch herausgestellt werden müssen.

b) Zum weiten werden die theorieexternen als auch theorieinternen Kritikpunkte am wissenschaftlichen Realismus herausgestellt und es wird aufgezeigt, inwiefern diese Kritikpunkte auch für Biodiversität als Grenz- und Hybridkonzept relevant sind.

c) Zum dritten wird dargelegt, wie sich das Verständnis von Biodiversität verändert, wenn die kulturellen Konstitutionsbedingungen des Forschungsprogramms Biodiversität hinsichtlich der geistes- und ideengeschichtlichen Konstitutionsideen hinter den Referenzgegenständen der biotischen und abotischen Vielfalt und hinsichtlich der methodologischen Konstruktionsbedingungen der Forschungskonzepte der biologischen Vielfalt rekonstruiert werden.

Indem folglich auf der inhaltlichen Ebene die These aufgestellt und überprüft wird, dass es über den methodologischen Kurzschluss zu einer systematischen Ausblendung der kulturellen Konstitutions- und Konstruktionsbedingungen des Forschungsgegenstandes Biodiversität kommt und indem diese hinsichtlich der geistes- und ideengeschichtlichen Konstitutionsideen hinter den Referenzgegenständen der biotischen und abotischen Vielfalt einerseits und hinsichtlich der methodologischen Konstruktionsbedingungen der Forschungskonzepte der biologischen Vielfalt andererseits rekonstruiert werden, wird die inhaltliche Ebene der Biodiversität als Grenz- und Hybridkonzept als hinreichend bestimmt angesehen, so dass auf die normative Ebene übergegangen werden kann.

\subsection{2 normative Ebene: Die problemindizierte Neuausrichtung der Umwelt- und Naturschutzethik hin zu einer problem- und anwendungsorientierten Biodiversitätsethik}

Ausgehend von dem Umstand, dass das Wissen darum, was Biodiversität ist und ausmacht, nicht ausreichend ist, um auch überzeugend begründen und rechtfertigen zu können, warum die Biodiversität bewahrt und gefördert werden sollte, wird im Anschluss an die wissenschaftstheoretische Auseinandersetzung auf die normative Ebene übergegangen. Hier geht es um die ethische Reflexion auf die im wissenschaftlichen, politischen und umwelt- und naturschutzethischen Kontext zugleich relevanten Motive, Intuitionen und Beweggründe sowie Argumente und Begründungen für die Bewahrung und Förderung der Biodiversität. Hierbei wird von vornherein berücksichtigt, dass die Umwelt- und Naturschutzethik hinsichtlich ihrer Erkenntnisgegenstände, ihrer Verfahren und Vorgehensweisen grundlagentheoretisch ausgerichtet ist, dass sie als 
anwendungsorientierte Ethik aber dennoch die praktische Zielstellung verfolgt, möglichst lebensnahe Orientierungsleistungen im Umgang mit Natur und Umwelt im Allgemeinen sowie Biodiversität im Speziellen zu liefern (siehe 1.2.2). Bei dem Versuch, zu einer anwendungsorientierten Biodiversitätsethik zu gelangen, die speziell auf die drei, mit der Bewahrung und Förderung verbundenen Zielstellungen, zugeschnitten ist, ergeben sich, so die auf der normativen Ebene relevanten These, drei eigenständige, aber ineinandergreifende Detailproblematiken (a. Fokussierung auf die Inklusionsproblematik, b. Fokus auf Schutzpflichtbegründungen, c. Fokussierung auf normative Akzeptabilitätskriterien), die im Zuge einer ethischen Reflexion auf die Bewahrung und Förderung der Biodiversität als eigenständige Problemkomplexe ersichtlich werden und mit denen konstruktiv umgegangen werden muss, wenn zum einen die Logik der Ethik als interessenlose Prüfung aller Standpunkte nicht unterlaufen werden soll (siehe 2.4), sprich Ethik nicht bereits funktionalisiert und instrumentalisiert werden soll und wenn zum anderen das Ziel möglichst lebensnahe Orientierungsleistungen im Umgang mit der Biodiversität zu geben, als Aufgabenstellung ernst genommen werden soll:

a) Die erste Problematik ergibt sich daraus, dass am Anfang und am Ende nahezu aller umwelt- und naturschutzethischen Beschäftigungen immer die Klärung der Inklusionsproblematik steht. Dies macht Angelika Krebs, stellvertretend für viele andere, ähnlich gelagerte Charakterisierungen der Umwelt- und Naturschutzethik, deutlich:

„Ob die Natur nur von Wert für den Menschen sei oder ob ihr auch ein eigener Wert zukomme, ist die Grundfrage der Naturethik. Man kann diese Frage auch anders formulieren: Ist Naturschutz nur etwas, was wir den von der Natur abhängigen, der Natur bedürftigen Menschen schulden, oder ist er auch etwas, was wir der Natur selbst schulden? Haben wir - Kantisch gesprochen - nur Pflichten in Ansehung von oder auch Pflichten gegenüber der Natur? Hat nur der Mensch eine Würde, oder gebührt auch der Natur, der Erde, den Meeren, den Wäldern, den Flüssen, den Pflanzen, den Tieren Ehrfurcht?“ (Krebs 1996:31)

Bei der Inklusionsproblematik geht es folglich auf der axiologischen Ebene um die Klärung der werttheoretischen Frage, ob und wenn ja, in welchen Hinsichten die außermenschliche Natur 'um ihrer selbst willen`als wertvoll gelten kann und auf der moralphilosophischen Ebene um die Klärung speziell der pflichtenethischen Frage, wem gegenüber Menschen Pflichten haben und welchen Entitäten ggf. welche Rechte zukommen (siehe 2.3.2). Je nachdem wie die Grundfrage nach dem Eigenwert sowie den moralischen Pflichten gegenüber oder in Ansehung der Natur beantwortet wird, hat man es, so die 'Lagerdifferenzierung' in der Umwelt- und Naturschutzethik, entweder mit einer anthropozentrischen (anthropos = Mensch) oder mit einer physiozentrischen (physis = Natur) Position zu tun (vgl. insb. Krebs 1997; Ott 2010). Hierüber wird indes hintergründig immer wieder der Dualismus zwischen Mensch und Natur repliziert, was 
aber dazu führt, dass die Perspektive auf die Auslotung der gesellschaftlichen Naturverhältnisse (siehe 1.2.1), sprich auf das komplexe Wechselspiel zwischen Mensch, Natur und Gesellschaft, das für den ökosystemaren Ansatz der Bewahrung und Förderung der Biodiversität und mithin für das Innovationspotenzial des Leitbildes der Bewahrung und Förderung der Biodiversität zentral ist (siehe 2.2.1), zu stark ausgeblendet wird. Allerdings kann hier eingewandt werden, dass weniger um die Entscheidung gehen sollte, ob nun primär der Mensch oder die Natur im Zentrum der umwelt- und naturschutzethischen Überlegungen stehen sollte, sondern um die Auslotung der individuellen und kollektiven menschlichen Naturverhältnisse. Bezüglich der ersten Problematik besteht die zentrale Herausforderung insofern darin, den hintergründigen Dualismus zwischen Mensch und Natur, den die Umwelt- und Naturschutzethik über ihre Fokussierung auf das Inklusionsproblem immer wieder repliziert, zugunsten einer relationalen Sichtweise auf die menschlichen Naturverhältnisse zu durchbrechen. Der Mehrwert der relationalen Sichtweise liegt dabei darin, dass sie den Fokus von der Inklusionsproblematik zu anderen Problematiken der individuellen und gesellschaftlichen Naturverhältnisse verschiebt. In diesem Sinne geht es hier um eine, im Lakatoschen Sinne, „progressive Problemverschiebung“ (siehe Abschnitt III, im Besonderen 3.2.2).

b) Zweitens stellt sich selbst, wenn man eine relationale Perspektive zugrunde gelegt, immer noch das Problem, dass der primäre Fokus der Umwelt- und Naturschutzethik zumeist nur auf Schutzpflichtbegründungen unter Vernachlässigung von Nutzungs- und Verteilungsaspekten liegt. Genau dies führt aber dazu, dass die, für das Leitbild der Bewahrung und Förderung der Biodiversität so entscheidende Integrativität der Zieltrias vor allem in den physiozentrischen Ethiken, aber auch in den anthropozentrischen Ansätzen - nicht ausreichend beachtet wird. ${ }^{43}$ Der Startpunkt, um dieser zweiseitigen Problematik auf den Schutzaspekt einerseits und auf Pflichtbegründungen anderseits konstruktiv zu begegnen, ist bereits darüber getan, dass dem Gedanken Raum gegeben wird, dass es neben der Klärung des Inklusionsproblems auch noch andere wichtige Fragen hinsichtlich des menschlichen Umgangs mit der Biodiversität gibt. Diesbezüglich muss der Aufgabenstellung Rechnung getragen werden, die Konrad Ott bereits vor fast 20 Jahren formuliert hat:

43 Vor allem in den physiozentrischen Ethiken ist die Problematik bereits systematisch indiziert, weil es hier um die Begründung direkter Schutzpflichten gegenüber der Natur/Biodiversität um ihrer selbst willen geht. Dies macht es von vornherein fraglich, ob und ggf. wie Biodiversität überhaupt genutzt und/oder verteilt werden darf. Aber auch in den anthropozentrischen Ethiken geht es zumeist nur um Biodiversitätsschutz, was dann, wenn Biodiversität dabei zudem synonym mit Artenschutz oder Naturschutz gesetzt wird, nicht nur die anvisierte Integrativität der Zieltrias der Bewahrung und Förderung vernachlässigt, sondern auch die begrifflich-konzeptionellen Besonderheiten der Biodiversität zu wenig beachtet. 
„Es geht nicht um eine "Zentrierung", sondern vielmehr um eine Auffächerung der Argumente in praktischer Absicht, um sie auf verschiedene Typen von Naturschutzkonflikten beziehen zu können." (Ott 1996:99;SL)

Die geforderte Auffächerung der Argumente in praktischer Absicht bedeutet hier, dass in Bezug auf die Grundfrage der Umwelt- und Naturschutzethik wieder ein entscheidender Schritt zurückgegangen werden muss, indem erst einmal ganz allgemein danach gefragt wird, wie Menschen sich in Bezug auf die Biodiversität verhalten sollten und wie sie in konkreten Situationen unter Berücksichtigung der Biodiversität handeln sollten. Über die Fokussierung auf diese praktischen Fragen kann dann zur ethischen Frage im Speziellen übergegangen wer, ob es gute Gründe ${ }^{44}$ dafür gibt, die Biodiversität möglichst weitreichend zu schützen, möglichst nachhaltig zu nutzen und die Nutzen und Lasten aus der Erhaltung aber auch der Zerstörung der Biodiversität möglichst gerecht zu verteilen. Hier muss es folglich um einen biodiversitätsethischen Argumentationsraum ${ }^{45}$ gehen, der die Zieltrias im Ganzen beleuchtet. Dabei kann auf einen bereits bestehenden Ansatz zurückgegriffen werden, den Uta Eser, Ann-Kathrin Neureuter und Albrecht Müller in ihrer, für das Bundesamt für Naturschutz erstellten, Studie »Klugheit, Glück, Gerechtigkeit - ethische Argumentationslinien in der Nationalen Strategie zur biologischen Vielfalt» entwickelt haben.

Hierüber wird das komplexe Argumentationsfeld zwischen Schutz-, Nutzungs- und Verteilungszielen aus Klugheits-, Glücks- und Gerechtigkeitsgründen dargelegt, wobei wiederum die zweite 'Seite' des Problems, die Fokussierung auf Schutzpflichtbegründungen relevant wird: Dieses Problem führt auf eine allgemeinere Ebene zurück und liegt darin begründet, dass die allgemeinen praktischen Fragen der Ethik, wie man sich verhalten bzw. handeln soll, sowohl in der allgemeinen Ethik als auch in den Bereichsethiken unter einen stark deontischen, sprich einem auf Pflichten ausgerichteten Fokus beantwortet werden, so dass die praktischen Fragen zumeist auf die speziell pflichtenethische Frage verengt wird, welche moralischen Pflichten und Rechte wir uns gegen-

44 Der Frage nach den Gründen kommt bei praktischen Fragen zwar eine zentrale Rolle zu, es muss aber von vornherein berücksichtigt werden, dass es einen bedeutsamen Hiat zwischen den Handlungsgründen einerseits und den darauf basierenden Handlungen gibt (bspw: Auch wenn viele Menschen gute Gründe dafür haben, sich rein vegetarisch zu ernähren, tun sie dies nicht immer konsequent). Gerade deshalb sollten die mit der praktischen Frage verbundenen Entscheidungsaspekte, wie Menschen tatsächlich handeln, nicht in reine Erkenntnisfragen nach dem Grund ihres Handelns aufgelöst werden, sondern Erkenntnis und Entscheidung sollten als sich gegenseitig bedingende Aspekte der praktischen Frage selbst angesehen werden.

45 Wenn hier von "Argumentationsraum» die Rede ist, dann wird damit eine wenig offensichtliche, aber weitreichende Grundentscheidung bezüglich der Ausrichtung der eigenen Argumentation getroffen, die bereits an anderer Stelle erwähnt wurde (siehe 1.2.2): Mit Konrad Ott soll davon ausgegangen werden, dass es sinnvoll ist, die Umwelt- und Naturschutzethik weder über das Konzept der Natur, noch über das Interessenkonzept zu fundieren, sondern in der Argumentationspraxis von Menschen über ihre je individuellen und kollektiven Zugänge zur Natur zu verankern (vgl. zusammenfassend Ott 2010). 
seitig schulden und welche Pflichten und Rechte in Bezug auf die Natur/Biodiversität begründet werden können. Antworten auf die Frage nach den Pflichten und Rechten von Menschen untereinander und in Bezug auf die Natur/Biodiversität decken aber immer nur einen Aspekt von Gründen für die Bewahrung und Förderung der Biodiversität ab, weil hierbei auch pragmatische Fragen (Was mache ich, um Ziel x zu erreichen? Welches Ziel sollte ich wählen, wenn mir bestimmte Mittel zur Verfügung stehen?) und eudaimonistische Fragen (Was macht ein gutes Leben aus und welche Rolle spielt dabei die Natur/Biodiversität?) eine entscheidende Rolle spielen. Um diesem Problem wiederum zu begegnen, muss der Versuch unternommen werden, den biodiversitätsethischen Argumentationsraum in einem mehrdimensionalen Ethikverständnis zu fundieren, das sowohl die Frage nach den moralischen Pflichten, nach dem guten Leben und der klugen Lebensführung gleichermaßen berücksichtigt, ohne das eine in dem anderen aufgehen zu lassen.

c) Zum dritten ergibt sich das Problem, dass in der ethischen Kommunikation über $\mathrm{Na-}$ tur- und Umweltschutzanliegen im Allgemeinen und bezüglich der Bewahrung und Förderung der Biodiversität im Speziellen oftmals zu wenig beachtet wird, dass die Ausweisung 'guter Gründe' nicht lediglich eine Frage der normativen Akzeptabilität ${ }^{46}$ der Gründe ist, sondern immer auch eine Frage ihrer faktischen Akzeptanz. ${ }^{47}$ Obgleich der qualitative Unterschied zwischen faktischer Akzeptanz und normativer Akzeptabilität nicht negiert werden soll, wird problematisiert, dass es im umwelt- und naturschutzethischen Kontext zu einseitig nur um die normativen Akzeptabilitätsbedingungen für Umwelt- und Naturschutzanliegen geht - oftmals unter Ausblendung der Bedingungen ihrer faktischen Akzeptanz - während es im umwelt- und naturschutzpolitischen Kontext primär um die faktischen Akzeptanzen für Umweltschutzziele und -maßnahmen in der Bevölkerung geht - hier oftmals unter Ausblendung der Akzeptabilitätsbedingungen dieser Ziele und Maßnahmen. Zwar wird von Seiten der EthikerInnen immer wieder (zu Recht) betont, dass Akzeptanz und Akzeptabilität nicht aufeinander reduzierbar sind, weil man nicht vom Vorliegen faktischer Akzeptanzen für etwas auf dessen normative Akzeptabilität schließen kann, andererseits ist es aber aus der Sicht einer anwendungsorientierten Ethik unzureichend, bei der Behandlung gesellschaftspolitischer

46 In der Ethik wird Akzeptabilität zumeist synonym mit Richtigkeit gesetzt. Etwas ist in diesem Sinne akzeptabel, wenn - und nur wenn - es die Eigenschaft des Richtigseins erfüllt. Welche Kriterien (inhaltliche oder formale) dabei in der Ethik für Richtigkeit von Handlungsmotiven, Handlungen und Handlungsfolgen herangezogen werden, ist selbst ein metaethischer und ethischer Disput und daher nicht einheitlich beantwortbar.

47 Akzeptanz bezieht sich auf „kognitive, emotionale und behaviorale Elemente des positiven Umgangs mit Elementen der Welt, dergestalt, dass diese Elemente handlungsrelevant sind und nicht zur Abstoßung, zur Missachtung, zur Auflehnung oder dergleichen führen." (Wolkenstein 2014:226) Damit ist nicht gesagt, dass Akzeptanz auch schon bedeutet, etwas Gutzuheißen und/oder sein Handeln danach auszurichten (ebd., 226-227). Des Weiteren ist damit nicht gesagt, dass all das, was man gemeinhin akzeptiert auch akzeptanzwürdig, d.h. akzeptabel ist (siehe vorherige FN). 
Problematiken, wie die Bewahrung und Förderung der Biodiversität, bei der Beleuchtung der normativen Akzeptabilitätskriterien stehen zu bleiben. Denn die Bewahrung und Förderung der Biodiversität ist hinsichtlich ihrer Umsetzung im gesellschaftspolitischen Raum immer auch auf die Findung und Schaffung sowie den geschickten Umgang mit faktischen Akzeptanzen in der Bevölkerung angewiesen. Da Akzeptanz und Akzeptabilität metaphorisch gesprochen als zwei Seiten derselben Medaille angesehen werden können, wenn es um das Überzeugen Anderer geht, werden die Ansätze, Strategien und Instrumente der lebensstilorientierten Naturschutzkommunikation, ${ }^{48}$ der es um eine zielgruppengemäße Analyse und Ansprache der sozialen Milieus geht, als Schnittstelle zwischen Umweltethik und Umweltpolitik verortet. Diese kann dazu dienen, die Akzeptabilitäts- und Akzeptanzbedingungen für Umwelt- und Naturschutzanliegen gleichermaßen zu thematisieren und darzulegen.

Die drei Problematiken und der jeweils anvisierte Umgang mit ihnen, münden in einer Neuausrichtung der Umwelt- und Naturschutzethik hin zu einer problem- und anwendungsorientierten Biodiversitätsethik, die in Abschnitt IV der Arbeit einerseits hergeleitet und andererseits aufgezeigt wird. Hierüber wird die normative Ebene der Biodiversität als Grenz- und Hybridkonzept als hinreichend bestimmt angesehen, so dass auf die politische Ebene übergegangen werden kann.

\subsection{3 politische Ebene: Die 'blinden Flecken` beim Versuch der theoretischen Explikation und Beurteilung der "Good Biodiversity Governance»}

Ausgehend von dem Umstand, dass sich selbst dann, wenn man plausibel darlegen kann, welche Klugheits-, Glücks- und Gerechtigkeitsargumente es für die Bewahrung und Förderung der Biodiversität gibt, noch immer die Fragen stellen, wie bestehende und aufkommende Interessenskonflikte bezüglich des Umgangs mit Biodiversität prozedural bewältigt werden (können), wer daran beteiligt werden sollte und wie Klugheits-, Glücks- und Gerechtigkeitserwägungen in Bezug auf Schutz-, Nutzungs- und Verteilungsfragen demokratisch entschieden werden können und sollen und sich die Antworten auf diese Fragen im Kontext der politikstrategischen Ausgestaltung der Bewahrung und Förderung der Biodiversität bewegen (siehe 1.2.3), muss von der normativen auf die politische Ebene übergegangen werden. Als zentraler Bezugspunkt zur Klärung dieser Fragen wurde die NBS und die in ihr formulierte Vorstellung bestimmt, wie die Strategie und mithin die Ziele und Maßnahmen zur Bewahrung und Förderung der

48 Die lebensstilorientierte Naturschutzkommunikation fungiert hier als Oberbegriff für diejenigen psychologischen und empirischen Kommunikationsstrategien, die auf eine Akzeptanzforschung und -förderung ausgerichtet sind (vgl. Schuster 2008; Lichtl 2009). Das hierbei im Fokus stehende soziologische Lebensstilkonzept stellt dabei den theoretischen und empirischen Rahmen dar, auf dessen Basis Werte- und Normvorstellungen in Verbindung zu sozioökonomischen Lebensverhältnissen gesetzt werden (die sogenannten Sinus-Milieus), die dann in Verbindung zu Kommunikations- und Marketingstrategien gesetzt werden. 
Biodiversität von der Politik kommuniziert und geregelt werden sollen. Noch einmal zur Erinnerung (siehe auch 1.2.3):

„Die nationale Strategie spricht (...) alle gesellschaftlichen Akteure [an]. Ziel der Strategie ist es, alle gesellschaftlichen Kräfte zu mobilisieren und zu bündeln. Weiteres Ziel ist es, dass Deutschland seiner Verantwortung für eine weltweit nachhaltige Entwicklung verstärkt gerecht wird. (...) Eine nachhaltige Entwicklung kann nicht einfach vom Staat verordnet werden. Nur wenn die Akteure in Wirtschaft und Gesellschaft das Thema zu ihrer eigenen Sache machen, kann die Strategie erfolgreich umgesetzt und können die darin enthaltenden Ziele erreicht werden." (BMUB 2011:17)

Diese, als Good Biodiversity Governance (GBG) charakterisierte, im Umweltbereich populäre, Vorstellung von einer politischen Gestaltung und Umsetzung umweltpolitischer Zielstellungen durch eine kooperative Koordination zwischen Politik und Gesellschaft, in der der Staat eine dialogische und verhandelnde Vermittlerrolle einnimmt und politische Akteure darauf hinarbeiten, dass Zielsysteme wie Nachhaltigkeit und die Bewahrung und Förderung der Biodiversität in die Gesellschaft eingehen und dort vorangetrieben werden, bleibt solange vage und inhaltlich unbestimmt, wie nicht der Versuch unternommen wird, deren theoretische Grundlagen zu explizieren. Diesbezüglich lässt sich feststellen, dass alle Forschungsstränge, die etwas zur Explikation der GBG beitragen könnten, in bestimmten Hinsichten 'blinde Flecken ' haben. Eben dies lässt sich als spezifische Detailproblematik auf der politischen Ebene festhalten:

Zwar gibt es in der Politikwissenschaft eine ganze Reihe an Arbeiten zur Umweltpolitik, -governance und -demokratie, allerdings sind Arbeiten, die auch einen expliziten und systematischen Biodiversitätsbezug haben, ${ }^{49}$ zumeist im Bereich der /Internationalen Beziehungen « angesiedelt und haben daher einen primären Fokus auf die internationale politische Ebene. Bei diesen Ansätzen spielen demokratie- und legitimationstheoretische Fragen eine lediglich untergeordnete Rolle, da es zumeist nur um die spezielle Frage geht, inwieweit sich Demokratie und demokratische Legitimation auch postnational denken lassen und weniger darum, die governance- demokratie- und legitimationstheoretischen Überlegungen systematisch miteinander zu verbinden.

Auf Seiten der Umwelt- und Naturschutzethik wird zwar anerkannt, dass „eine politisch abstinente Umweltethik (...) nur gelehrte und erbauliche Schöngeisterei [wäre] “ (Ott 2010:21) - die Umwelt- und Naturschutzethik also durch politische Philosophie sowie

49 Hier sei insbesondere auf die einzelnen und gemeinsamen Arbeiten von Ulrich Brand und Christoph Görg (vgl. Görg et al. 1999a; Görg \& Brand 2000; Görg 2001; Brand \& Görg 2003; Görg 2003; Brand 2010a) bzw. weitere Arbeiten mit Biodiversitätsbezug verwiesen, die in Zusammenarbeit mit Beiden entstanden sind (vgl. insb. Flitner, Görg \& Heins 1998; Görg et al. 1999b). Selbst diese Arbeiten erfassen nicht die Biodiversität als Ganzes, sondern fokussieren vornehmlich auf die genetische Vielfalt und berücksichtigen hauptsächlich die Nutzungs- und die Verteilungsdimension. 
politische Theorie und Empirie flankiert werden muss (ebd., 23), es lässt sich aber festhalten, dass die Umwelt- und Naturschutzethik die politikwissenschaftliche Theorie und Empirie - vor allem hinsichtlich governance- und policytheoretischer Überlegungen - bislang zu wenig beachtet (vgl. Eser 2014). Dass dies indes notwendig ist, zeigt sich vor allem daran, dass die, auch in der Umweltethik betriebene Suche nach innovativen und alternativen Politikmodellen zur parlamentarisch-repräsentativen Praxis „über einen manchmal charmanten, manchmal abwegigen, gelegentlich sogar gefährlichen Wildwuchs an Entwürfen nicht hinausgelangt [ist]." (Ott 2010:195)

In der Biodiversitätsforschung wiederum, bei der der direkte Bezug auf Biodiversität bereits gegeben ist, ist insgesamt zu konstatieren, dass politiktheoretische Fragestellungen - genau wie ethische Fragestellungen - stark unterrepräsentiert sind, da hier vor allem biowissenschaftliche und ökonomische Arbeiten die Forschungslandschaft dominieren. Allerdings gibt es vor allem von Seiten des wissenschaftlichen und administrativen Umwelt- und Naturschutzes einige Beiträge, die sich mit policy-, governance-, demokratie- und legitimationstheoretischen Fragen in Bezug auf Umwelt- und Naturschutz im Allgemeinen und der Bewahrung und Förderung der Biodiversität im Besonderen beschäftigen. ${ }^{50}$ In Bezug auf diese ist indes anzumerken, dass der Fokus hier zumeist auf ganz konkreten Regelungen, Gesetzen, Verordnungen, Verwaltungsrichtlinien etc. liegt. Die grundlegende Frage, welchem Modell oder Modus die politische Regelung der Umsetzung der Biodiversitätsziele und -maßnahmen folgt bzw. folgen sollte, auf welchen theoretischen Grundlagen diese basieren und wie diese hinsichtlich ihrer Chancen und Risiken zu beurteilen sind, spielt hier - wenn überhaupt - nur unsystematisch und sporadisch eine Rolle.

Inwiefern diese jeweiligen 'blinden Flecken' in Bezug auf die Explikation, Bewertung und Beurteilung der GBG relevant sind, wird deutlich, wenn man sich vergegenwärtigt, dass die Aufgaben- und Zielstellung der Bestimmung der theoretischen Grundlagen der GBG im Kontext der bei weitem nicht nur in der Politikwissenschaft relevanten ReTheoretisierung des Staates steht: Dabei ist entscheidend, dass der moderne Staat, für den mittlerweile zahlreiche Attribuierungen wie bspw. 'reflexiver Staat', 'kooperativer Staat', 'Gewährleistungsstaat' usw. gefunden wurden, sich durch ein Spannungsverhältnis zwischen staatlicher Aufgabensteigerung und staatlichen Autoritätsverlusten gekennzeichnet ist, das zwar innerhalb und außerhalb der Staatstheorie theoretisches

50 Hier sei zum einen auf die Berichte des Sachverständigenrats für Umweltfragen (SRU) verwiesen, der sich in seinen Gutachten immer auch mit politisch-strategischen, verwaltungspolitischen und rechtlichen Fragen des Umwelt- und Naturschutzes beschäftigt (vgl. insb. SRU 1994, 1998, 2002, 2004, 2007, 2012). Zum anderen sei hier auf einige Forschungsvorhaben aus der Reihe "Naturschutz und Biologische Vielfalt« des Bundesamtes für Naturschutz (BfN) verwiesen sowie die dazugehörigen Publikationen zu »Musterlösungen im Naturschutz« (vgl. Brendle 1999), »Natur und Staat« (vgl. Frohn \& Schmoll 2006) sowie zum »Verwaltungshandeln im Naturschutz« (vgl. Benz 2008). 
Gemeingut ist und als solches gar nicht mehr erwähnenswert wäre, wenn nicht zugleich die Konsequenzen dieser Entwicklung - sowohl in analytischer als auch normativer Hinsicht - so umstritten wären. Diese finden ihre 'Klammer' nämlich in der These über den kooperativen Wandel der Staatlichkeit (Kleger 1995: 93), über die eine ganze Reihe an weiteren wichtigen Aspekten vermittelt wird: Unter dem hierbei so wichtigen und entscheidenden Begriff der Kooperation wird ein Steuerungs- bzw. Regelungsmodus des Staates verstanden, der sich gegenüber der klassischen, etatistischen Steuerung und dem damit verbundenen hierarchischen Verhältnis zwischen Staat und Gesellschaft insofern abgrenzt, als dass kooperative Bemühungen darauf abzielen mit anderen Akteuren zu verhandeln bzw. zusammenzuarbeiten (Stichwort: Verhandlung). Das bedeutet, dass auch dort, wo (verfassungs-)rechtlich ein direkter Steuerungsdurchgriff staatlicher Stellen möglich ist und die Folgebereitschaft der Adressaten erzwingbar wäre, auf eine solche Ausübung verzichtet und stattdessen für eine freiwilllige Erfüllung geworben wird (Stichwort: Selbstregulation), dass neben den professionellen Politikakteuren auch andere gesellschaftliche Gruppen zu Teilhabern an der staatlichen Macht werden (Stichwort: Partizipation und Deliberation) und dass der Staat zunehmend auf prozedurale Steuerung umlenkt, bei der der Staat Vermittlungs- und Garantieleistungen erbringt (Stichwort: Gewährleistungsstaat), um zu gesamtgesellschaftlich verbindlichen Regelungen zu gelangen, die dem Gemeinwohl dienen (Voigt 1995:42).

Dieser Zusammenhang um den kooperativen Wandel der Staatlichkeit verweist zum einen auf die, mit steigender Regelungsdichte wachsende, staatliche Abhängigkeit von komplexen Akteurs- und Interessensgeflechten im Rahmen der europäischen und internationalen, aber auch subnationalen Mehrebenenpolitik (Multi-Level-Governance) sowie nicht zuletzt auf die Möglichkeiten, aber auch Beschränkungen staatlicher Regulierung durch 'Hierarchie` und 'Kooperation` (vgl. Mayntz \& Streeck 2003, Mayntz \& Scharpf 2005, Scharpf 2006, Mayntz 2008, 2009a), was bedeutet, dass trotz der „neuen Architektur des Staates“ (Grande 1993) notwendigerweise nach Möglichkeiten zur Durchsetzung gesamtgesellschaftlicher und gemeinwohlorientierter Politikentwürfe gesucht werden muss. Da Biodiversitätspolitik als Querschnittsaufgabe viele andere Politikfelder berührt, innerhalb derer sich sowohl Synergien als auch Konflikte ausmachen lassen, kann es nicht nur darum gehen, durch die intelligente (Aus-)Wahl der richtigen Governancemodi und -instrumente, die Synergien zu nutzen und die Konflikte so zu regeln, sondern der Erfolg politischer Regulierung hängt immer auch davon $a b$, ob die gewählten Modi und Instrumente für den jeweiligen institutionellen Kontext, die damit verbundenen Akteurskonstellationen und die darin möglichen Interaktionsformen adäquat sind (Jänicke 2002:66-69). Hier geht es folglich aus policy- und governancetheoretischer Perspektive darum, die institutionellen und prozeduralen Bedingungen der Good Biodiversity Governance auszumachen. 
Die Policy-Analyse (Politikfeldanalyse) liefert die dafür notwendigen Einsichten und Analysen auf die Institutionen, Organisationsstrukturen und Akteurskonstellationen sowie deren Interaktionsformen. Die Governanceforschung komplementiert diese um die Formen der sozialen Handlungskoordination innerhalb und oberhalb des Nationalstaats. Unter das Phänomen "Governance ${ }^{51}$ fallen zahlreiche Aspekte moderner Politik (bspw. Komplexitätssteigerung, Vernetzung, Binnendifferen-zierung), bei denen es nach wie vor und der Diskussion um Global Governance zum Trotz - kein funktionales Äquivalent für nationale Regierungen gibt, zumindest was ihre Sichtbarkeit für die BürgerInnen, ihre Legitimation, ihre Fachkompetenz oder ihre materiellen Ressourcen angeht (Jänicke 2003:10). Denn es sind in aller Regel nationale Regierungen, die - im Kollektiv - die internationale Politik formulieren und dass sie dabei von wirtschaftlichen und gesellschaftlichen Akteuren beeinflusst werden, ist kein Spezifikum der globalen Ebene oder von Governance (ebd.). Daher bleibt der Nationalstaat auch in der Governancediskussion ein zentraler Faktor, wenn auch in modifizierter Form, da es hier darauf ankommt, die „neue Architektur der Staatlichkeit“ zu theoretisieren.

Zum anderen geht es beim 'kooperativen Wandel der Staatlichkeit' aber immer auch um die Herausforderungen und Anforderungen der gesellschaftlichen Partizipation, die in Form von pluralistischen, sprich zwischen Politik und Zivilgesellschaft stattfindenden oder korporatischen, d.h., zwischen Politik und Wirtschaft angesiedelten, Kooperationen realisiert wird. Für die, in der NBS formulierte, Vision einer kooperativen, verhandelnden und dialogischen Regulierung der Bewahrung und Förderung der Biodiversität bedeutet dies, dass aus dem Zwang zur Kooperation einerseits neue demokratische Formen und andererseits besondere Anforderungen an die Legitimation des kooperativen Staates erwachsen: Kooperative Staatstätigkeit ist deutlich stärker an den Formen der Verhandlungsdemokratie als an den klassischen mehrheitsdemokratischen Routinen orientiert, um die Potenziale der unterschiedlichen Akteure zu mobilisieren und um politische Schwerpunkte für die Gestaltung der Zukunft zu setzen (Voigt 1995: 76)..$^{52}$ Daher gilt es auszuloten, wann und warum die Ergebnisse von Verhandungen

51 "Governance» ist ein schillernder und „anerkannt uneindeutiger Begriff“ (Blumenthal 2005:1150), bei dem mit Detlef Sack bereits in kritischer Absicht davon ausgegangen werden muss, dass ,in der Governancedebatte 'alte` theoretisch-konzeptionelle, empirische und normative Auseinandersetzungen einen neuen 'Aufguss` erfahren" (Sack 2011:26). Zudem gibt es die Governanceforschung nicht, sondern lediglich verschiedene, in der Politikwissenschaft vertretene Analyseperspektiven auf Governance, die jeweils ganz unterschiedliche Zugänge und Antworten auf die Frage anbieten, auf welchen speziellen theoretischen Grundannahmen das Konzept der Governance fußt, was dabei als Funktion und Aufgabe des Politischen angesehen wird und welche (positiven und negativen) Veränderungen von Staatlichkeit und Demokratie über die Etablierung von Governancearrangements zu erwarten sind. Auf diese verschiedenen Analyseperspektiven innerhalb der politikwissenschaftlichen Governanceforschung wird ebenfalls an späterer Stelle genauer eingegangen (siehe 5.3.1).

52 Als verhandlungsdemokratisch lassen sich all die institutionellen Muster charakterisieren, bei denen die Machtteilungen sowohl innerhalb als auch außerhalb des Regierungssystems sowie innerhalb pluralistisch verfasster Gesellschaften dazu führen, dass „wesentliche Entscheidungen durch Aushan- 
erfolgreich bzw. nicht erfolgreich sind, da der de facto vorhandene Zwang zur Kooperation in kooperativen Staaten nicht mehr vor dem Hintergrund bewertet werden kann, dass es bessere, effektivere Wege der politischen Problemlösung gibt, nämlich mehrheitlich zu entscheiden und hoheitlich zu entscheiden, statt zu verhandeln. Unter diesen Bedingungen kann sich die politische Legitimation nicht mehr ausschließlich und alleinig, wie dies im klassischen Staatsparadigma der Fall war, aus der demokratischen Grundordnung, also aus der Verfassung und den in ihr enthaltenen Prinzipen herleiten. Vielmehr bedarf es unter verhandlungsdemokratischen Bedingungen einer Vielzahl legitimationstheoretischer Kriterien, die sich einerseits auf den Input, d.h. auf die in den politischen Prozess eingehenden gesellschaftlichen individuellen Interessen und kollektiven Präferenzen, beziehen und zum anderen die Ergebnisse des politischen Prozesses, d.h. die politischen Leistungen (Output) und die Wirkungen dieser Politikergebnisse (Outcomes), berücksichtigen. Hier geht es folglich darum aus einer demokratieund legitimationstheoretischen Perspektive darauf zu schauen, welche demokratischen Grundannahmen hinter dem Verweis auf einen verhandelnden, kooperativen und dialogischen Politikmodus stehen und zu prüfen, ob diese zu besseren politischen Bilanz in Bezug auf die Bewahrung und Förderung der Biodiversität führen.

Die Demokratie- und Legitimationstheorien liefern hierfür die normativen Standards zur Beurteilung der Grenzen und Möglichkeiten der GBG. Dies tut sie indes nicht einheitlich, da es verschiedenste Ansätze und Varianten moderner Demokratietheorien gibt, die eine je unterschiedliche Auffassung davon vertreten, was Demokratien ausmacht, welche Aspekte dabei im Besonderen relevant sind und womit und worüber sich Demokratien legitimieren. Allerdings lässt sich die Fülle an demokratietheoretischen Ansätzen anhand dieser Unterscheidungsmerkmale in drei Gruppen einteilen entscheidungszentrierte, kontextbezogene und prozeduralistische Demokratietheorien (vgl. Lösch 2005) $)^{53}$ - die verschiedenartige demokratie- und legitimationstheoretische Beurteilungen erlauben, was die Stärken und Schwächen eines politischen Modus Vivendi sind, der auf Kooperation, Verhandlung und Dialog setzt. Dies ist für die Auslotung der 'Politik der Biodiversität' nicht nur in theoretischer, sondern auch in praktischer Hinsicht relevant, weil hinter der Vision einer stärker kooperativen, dialogischen und verhandelnden Politik wiederum die, in der Umweltpolitik weit verbreitete Hoffnung steht, dass die Umweltpolitik im Allgemeinen und die Biodiversitätspolitik im

deln getroffen werden. " (Czada 2000:1) Insofern herrscht in Verhandlungsdemokratien zwangsläufig eine Politik der gegenseitigen Absprachen, der Kooperations- und Koordinierungsbemühungen sowie der Verständigungsversuche vor, die je nachdem, ob es sich dabei um pluralistische oder korporative Verhandlungsnetzwerke oder innerpolitische Koordinationsversuche handelt, ganz unterschiedliche Verhandlungsformen eröffnen (Scharpf 2006:313-318).

53 Was genau in dieser Hinsicht entscheidungszentrierte, kontextbezogene und prozeduralistische Demokratietheorien ausmacht und welche Demokratiekonzeptionen jeweils unter das Cluster fallen, wird erst an späterer Stelle deutlich gemacht (siehe 5.1.2). 
Speziellen durch einen stärker kooperativen, dialogischen und verhandelnden Politikmodus mehr Rückhalt erfährt, im Idealfall, wie in der NBS postuliert, durch diese getragen wird und politische Probleme und Interessenskonflikte dadurch zugleich legitim und effektiv und effizient bewältigt und/oder gelöst werden können.

Zusammenfassend zeigt sich folglich angesichts der 'blinden Flecken', sprich den Umständen, dass in der Politikwissenschaft spezielle Arbeiten mit Biodiversitäts- und nationalem Governancebezug 'Mangelware` sind, der Umwelt- und Naturschutzethik die policy- und governancetheoretischen Grundlagen für ihre alternativen Politikentwürfe fehlen und in der Biodiversitätsforschung generell grundlagenreflektorische Arbeiten mit sozial- und geisteswissenschaftlichem Bezug unterrepräsentiert sind, dass es in der politikwissenschaftlichen Detailreflexion in Abschnitt $V$ der Arbeit darum gehen muss, die binnenkomplexen theoretischen Grundlagen der, in der NBS anvisierten, auf Kooperation, Verhandlung und Dialog hin angelegten, politisch-strategische Ausgestaltung der deutschen Biodiversitätspolitik vor den Hintergründen der Policy-Analyse zur Explikation des Politikfeldes Biodiversität, der Governanceforschung zur Berücksichtigung der Transformationsprozesse des Politischen sowie der normativen Demokratieund Legitimationstheorie zur Beurteilung und Bewertung der Grenzen und Möglichkeiten kooperativer, verhandelnder und dialogischer Formen der demokratischen Beteiligung und Teilhabe zu bestimmen, um heraustellen, vor welchen Anforderungen, Chancen und Risiken eine Biodiversitätspolitik im Modus der GBG steht. 


\title{
ABSCHNITTE III, IV UND V
}

\author{
DIE DETAILREFLEXIONEN AUF DER \\ INHALTLICHEN, NORMATIVEN UND \\ POLITISCHEN EBENE
}




\section{WISSENSCHAFTSTHEORETISCHE DETAILREFLEXION: Die kulturellen Konstitutionsbedingungen des Forschungsprogrammes Biodiversität}

"Biologists can often decide how to save biodiversity even when they cannot define it. For many purposes, the term 'works' to guide action because they (...) act cooperatively on the assumption that: 'we know it, when we see it'."

(Norton 2006:56-57)

Startpunkt der ersten Detailreflexion - der wissenschaftstheoretischen Reflexion auf der inhaltlichen Ebene - ist das bereits aufgezeigte Problem des methodologischen Kurzschlusses der Gleichsetzung von Forschungsobjekt (abiotische und biotische Vielfalt) und Forschungskonzept (biologische Vielfalt) über die Kurzform Biodiversität. Damit wird die Beantwortung der Frage relevant, wie mit dieser Problematik konstruktiv umgegangen werden soll. Dieser Frage wird - unter Berücksichtigung der verschiedenen Ebenen der Problematik des methodologischen Kurzschlusses (siehe 2.4.1) - in drei Schritten nachgegangen:

Im ersten Schritt wird auf das hinter dem methodologischen Kurzschluss stehende realistische Wissenschaftsverständnis eingegangen (3.1). Hierfür wird dargelegt, warum überhaupt davon ausgegangen werden kann, dass der Biodiversitätsdiskurs auf einem realistischen Wissenschaftsverständnis basiert und welche Annahmen mit einem solchen realistischen Wissenschaftsverständnis idealtypisch verbunden sind. Darauf aufbauend geht es - in einem zweiten Schritt - um die kritische Reflexion des wissenschaftlichen Realismus (3.2). Hierbei wird einerseits aus einer externen, d.h. konstruktivistischen und wissenssoziologischen, Perspektive auf die pragmatischen Kritikpunkte am wissenschaftlichen Realismus als Grundlage für den Forschungsgegenstand der Biodiversität eingegangen (3.2.1). Hierbei wird argumentiert, dass das realistische Verständnis die soziale und kulturelle Praxis der Gegenstandskonstitution und -konstruktion von Forschungsprogrammen zu wenig beachtet, womit aber viele Aspekte, die für die Entgrenzung und Hybridisierung der Biodiversität relevant sind, nicht in ausreichendem Maße berücksichtigt werden können. In Anschluss daran wird der wissenschaftliche Realismus aus einer innertheoretischen Perspektive kritisiert, indem auf die realistischen Annahmen bezüglich der Wahrheitskonzeption und der Zielstellung wissenschaftlichen Handelns eingegangen wird (3.2.2). Hier wird argumentiert, dass sich die Begründung des wissenschaftlichen Realismus an theorieentscheidenden Stellen in Zirkelschlüsse verfängt, womit genau das bereits vorausgesetzt wird, was zu beweisen versucht wird. Die mit dem wissenschaftlichen Realismus verbundene Zielstellung, möglichst wahre und der Wirklichkeit entsprechende Theorien zu produzieren, ist damit zu hoch gegriffen, weil das theoretische Fundament, auf dem dieser Anspruch ba- 
siert, nicht tragfähig genug ist, um diesen Anspruch auch plausibel begründen zu können. Daher wird in Anschluss an Imre Lakatos "Methodologie der wissenschaftlichen Forschungsprogramme« (vgl. Lakatos 1982) eine alternative Zielbestimmung wissenschaftlicher Praxis dergestalt vorgenommen, dass das Ziel wissenschaftlicher Praxis in einer „progressiven Problemverschiebung“, d.h. einem theoretischen und empirischen Gehaltüberschuss gegenüber vorangegangenen Forschungsprogrammen gesehen wird. Außerdem wird die Lakatossche Unterscheidung zwischen den zwei Ebenen von Forschungsprogrammen - dem sogenannten "Schutzgürtel“ und dem „harten Kern" genutzt, um daran die kulturellen Konstitutionsbedingungen des Forschungsprogramms Biodiversität zu rekonstruieren (3.3). Dafür werden einerseits die methodologischen Konstruktionsbedingungen des Forschungskonzeptes der biologischen Vielfalt, die im Schutzgürtel des Forschungsprogramms lokalisiert sind, mittels des methodologischen Konstruktivismus herausgestellt (3.3.1). Andererseits werden die geistes- und ideengeschichtlichen Konstitutionsideen der Forschungsobjekte der abiotischen und biotischen Vielfalt, die im harten Kern des Forschungsprogramms lokalisiert sind, mittels des Kulturkonstitutionalismus beleuchtet (3.3.2). Über beide Ausführungen soll überprüft werden, ob die dabei zur Anwendung kommenden Ansätze zu einer progressiven Problemverschiebung, d.h. zu einem theoretischen und empirischen Gehaltsüberschuss gegenüber der realistischen Betrachtung der Biodiversität, führen. Die wichtigsten Ergebnisse der Analyse werden abschließend in einem Fazit zusammengefasst, welches zugleich eine Überleitung auf die normative Ebene sowie die ethische Reflexion der wissenschaftlichen, politischen Gründe und Begründungen für die Bewahrung und Förderung der Biodiversität ermöglicht (3.4)

\subsection{Das realistische Wissenschaftsverständnis hinter dem Biodiversitätsdiskurs}

Grundlegend wird mit Peter Janich, Mathias Gutmann und Susanne Hiekel davon ausgegangen, dass dem Biodiversitätsdiskurs, qua biowissenschaftlicher Dominanz, ein realistisches Wissenschaftsverständnis zugrunde liegt (Janich 1996:78-79; Gutmann \& Janich 2001a:298, 310, 346; Hiekel 2012:12-23). Der hierbei relevante Zusammenhang zwischen wissenschaftlichem Realismus und Biologie als Naturwissenschaft lässt sich mit Susanne Hiekel, die sich in ihrer Arbeit zu den "Grundbegriffen der grünen Gentechnik« (vgl. Hiekel 2012) und den hinter den Begriffen stehenden Wissenschaftsverständnissen beschäftigt, wie folgt charakterisieren:

„Das, was wir in der Biologie wissen, kann als das Repertoire angesehen werden, das jemandem zu den biologischen Gegenständen zur Verfügung steht (...). Üblicherweise wird dabei ein realistisches Verständnis des wissenschaftlichen Wissens vorausgesetzt. (...) So werden Theorien (...) hinsichtlich von Wahr-Falsch Un- 
terscheidungen beurteilt und es wird versucht, möglichst viele Beweise für die Wahrheit einer Theorie zu sammeln, die dann, wenn sie empirisch ausreichend belegt scheint, zumindest annäherungsweise als Widerspiegelung der Wirklichkeit gilt." (ebd., 15-16)

In den Biowissenschaften wird folglich zumeist - wenn auch nicht zwingend oder systematisch - ein Wissenschaftsrealismus vertreten. ${ }^{54}$ Dieser basiert auf der Annahme, dass es eine theorieunabhängige physische Wirklichkeit gibt, die den intendierten Gegenstandsbereich naturwissenschaftlicher Theorien umfasst (Suhm 2005:48). Dahinter stehen wiederum die Annahmen, dass wissenschaftliche Theorien ein direktes Wissen über die Welt enthalten, das der Wahrheit über die Welt entspricht, weil die Welt als subjektunabhängig existierend angenommen wird (Hiekel 2012:15), so dass die Gegenstände der Biologie als 'naturgegeben 'angenommen und Natur selbst als Gegenstand betrachtet wird, der durch die naturwissenschaftliche Forschung objektiv abgebildet werden kann. Eben dies wurde als Kerngedanke des methodologischen Kurzschlusses gekennzeichnet (siehe 2.1). Damit liegt dem methodologischen Kurzschluss - zumeist stark implizit und selten rational begründet (Tschepke 2003:xv) - ein realistisches Wissenschaftsverständnis zugrunde, was sich vor allem daraus erklärt, dass sich im Alltagsgeschäft von BiologInnen und ÖkologInnen selten die Frage nach ihrem eigenen Wissenschaftsverständnis stellt (Hiekel 2012:16), und weil es sich bei der realistischen Position um eine 'Common-Sense' Position zum Zusammenhang zwischen der Welt und unseren Wissen über sie handelt (Suhm 2005:10):

„Der moderne, aufgeklärte Naturforscher nimmt seinen Realismus aus folgender Überlegung: Weil die Naturwissenschaften einen unbestrittenen prognostischen, explikativen und technischen Erfolg haben, muss da etwas sein, wovon sie handeln und was ihre Sätze wahr macht. Dieser Realismus von Naturwissenschaftlern nimmt zuweilen drastische und naive Formen an (...) - nicht nur mit der theoretischen Konsequenz, dass ja nur entdeckt werden könne, was es da zu entdecken gäbe, sondern auch mit der praktischen Konsequenz, dass Absichten, Interessen und Politik, deren Einfluss auf den Gang der Forschung nicht bestritten werden brauche, nicht den Inhalt der Forschungsergebnisse berühren könne (...) Die Korrespondenz von Aussagen und Wirklichkeitsstücken bleibe vorläufig, auch wenn für den Realisten die Wirklichkeit freundlicherweise immer gerade mindestens solange stillhält, wie er benötigt, um die Korrespondenz seiner Theorien zur Wirklichkeit etwas zu verbessern.“ (Janich 1996:78-79)

54 Nach Keller und Golley (2000) vertreten die meisten Ökologen (und Biologen) einen abgeschwächten wissenschaftlichen Realismus ("mitigated scientific realism") (Keller \& Golley 2000:11), den sie wie folgt charakterisieren: “( $T$ )here are patterns or regularities in nature existing independently of human perceivers, and these patterns, to some extent, are objectively knowable by humans. Ecology attempt to discern these patterns." (ebd., 12) 
Demgegenüber werden Wissenschaftsverständnisse innerhalb der Wissenschafts- und Erkenntnistheorie kritisch reflektiert. Bevor allerdings auf die Kritik am realistischen Wissenschaftsverständnis in Bezug auf den Forschungsgegenstand Biodiversität eingegangen werden kann, muss es erst einmal darum gehen, den wissenschaftlichen Realismus als Position idealtypisch ${ }^{55}$ zu charakterisieren und herauszustellen, welche zentralen Annahmen damit verbunden sind. Die sicherlich unterkomplexe Idealtypisierung ist in diesem Fall notwendig, denn unter dem Oberbegriff des Realismus findet sich eine Vielzahl an Thesen, Annahmen und Argumenten über den Zusammenhang zwischen unseren Vorstellungen und Überzeugungen von der Realität und der Beschaffenheit der physischen Wirklichkeit. Ein erster Ansatzpunkt für die Erschließung des umfangreichen und binnendifferenzierten Spektrums des Realismus bzw. der realistischen Positionen stellt daher deren gemeinsame Intuition dar (Tschepke 2003:2). Diese lässt sich mit Thomas Nagel als „die Existenz vom menschlichen Bewusstsein unabhängiger Phänomene in der realen Welt" (Nagel 1986:90) bezeichnen. Crispin Wright differenziert diese allgemein formulierte Intuition und weist darauf hin, dass es für die Reichweite und Tiefe der realistischen Positionen einen grundlegenden Unterschied macht, ob diese an der bescheidenen ontologischen Annahme ansetzen, dass es eine von unseren Erkenntnisfähigkeiten unabhängige Wirklichkeit gibt - was nur von wenigen und im Übrigen auch nur von wenigen Realismusgegnern bestritten wird - oder ob an der 'gehaltvollen' und darüber hinaus epistemisch relevanten Annahme angesetzt wird, dass Menschen dazu in der Lage sind, diese Wirklichkeit so zu erkennen, wie sie ist (Wirklichkeit als Realität) und dass wir über die Erschließung der Realität auch die Wahrheit erkennen können (Wright 1992:1-2).

Der wissenschaftliche Realismus transferiert diese Intuitionen in eine Erkenntnis- und Wissenschaftstheorie und baut dabei auf der gehaltvolleren Intuition auf, nach der die Wissenschaft uns in die Lage versetzt, die von uns unabhängig existierende Wirklichkeit so zu erfassen und zu beschreiben, wie sie ist, so dass wir darüber zu 'wahrhaftiger Erkenntnis` gelangen können (Tschepke 2003:11). Diesbezüglich integriert der wissenschaftliche Realismus (a) die ontologisch relevanten Annahmen, dass (a1) eine erkennbare Wirklichkeit existiert, (a2) die unabhängig vom menschlichen Denken ist sowie (b) die epistemisch relevanten Annahmen, dass die Bestätigung einer wissenschaftlichen Theorie die Annahme begründet, dass diese Wirklichkeit so aussieht, wie wissenschaftliche Theorien es besagen (Suhm 2005:48):

55 Dies wird über die Methode des idealtypischen Verfahrens gemacht. Weber beschreibt den Idealtypus dabei als „einseitige Steigerung eines oder einiger Gesichtspunkte“ (Winckelmann 1988:191). In diesem Sinne kann die Idealtypisierung als heuristisches Mittel verstanden werden, mit dessen Hilfe die Begriffe, Modelle und Konzepte der sozialen Realität abstrahiert von nahezu allen Feinheiten und Relativierungen, im Kern dargestellt werden können (ebd., 190), womit sie sich dann aber als radikalere Positionen darstellen, als dies in der Realität oftmals der Fall ist. 
a) Die ontologisch-realistische These besteht dabei aus der zweistelligen Annahme, dass die Gegenstände, deren Existenz qua wissenschaftlicher Theorien postuliert wird, in der Wirklichkeit existieren (a1: Existenzthese) und dass diese Wirklichkeit unabhängig von unserer Erfahrung, unserem Denken und unserer Sprache existiert (a2: Unabhängigkeitsthese) (Tschepke 2003:4):

\author{
Existenzthese (ET): \\ Entitäten existieren real und besitzen bestimmte Eigenschaften, \\ die sich anhand bestimmter Gesetze zeigen. \\ Unabhängigkeitsthese (UT): \\ Die Wirklichkeit existiert unabhängig von unserer Erfahrung, \\ unserer Sprache und unserem Denken.
}

Diejenigen ontologischen Positionen, die die Unabhängigkeit der Wirklichkeit von der menschlichen Existenz hervorheben, zeichnen sich i.d.R. dadurch aus, dass die ontologischen Thesen auch mit einer referentiellen Semantik verknüpft werden, nach der sich die sprachliche Bedeutung eines Ausdrucks (Wörter, Sätze, Propositionen, Satzgefüge) aus dem realen Objekt, das inm zugeordnet ist, ergibt (Suhm 2005:49). Daraus folgt eine, die ontologischen Thesen erweiternde, semantische These (Tschepke 2003:8):

\title{
Semantische These (sT):
}

Was ein sprachlicher Ausdruck bedeutet, erschöpft sich darin, worauf er sich bezieht. Die spezifizierten sprachlichen Ausdrücke haben Wahrheitswerte, d.h., sie sind wahr oder falsch und zwar unabhängig von menschlichen epistemischen Mitteln zum Erkennen dieser Wahrheitswerte. ${ }^{56}$

Die ontologischen Thesen der Existenz und der Unabhängigkeit werden hierbei semantisch interpretiert: In Bezug auf die Existenzthese enthält die semantische These eine Festlegung darauf, dass die Begriffe einer Theorie reale Referenzobjekte besitzen, so dass Theorien als wirklichkeitsgetreue Abbildungen dieser betrachtet werden. In Bezug auf die Unabhängigkeitsthese wird die Annahme vertreten, dass die physische Wirklichkeit unabhängig von den wissenschaftlichen Theorien und methodischen Annahmen zu ihrer Erschließung gedacht werden kann, so dass die Wahrheit wissenschaftlicher Theorien durch die physische Wirklichkeit selbst festgelegt wird, da die wissenschaftlich gewonnenen Wahrheiten davon abhängen, ob die Objekte die ihnen zugeschriebenen Eigenschaften tatsächlich besitzen.

56 Die semantische These wird häufig dadurch eingeschränkt, dass „die fraglichen Sätze wörtlich zu nehmen oder buchstäblich zu verstehen“ (Tschepke 2003:8) sind. Hierbei liegt der Akzent darauf, dass die Theorie bzw. die Menge an sprachlichen Ausdrücken, die diese bilden, insgesamt - und damit ausdrücklich auch in ihren unbeobachtbaren Teilen - wörtlich zu nehmen ist. Dies soll unerwünschte Interpretationen der sprachlichen Ausdrücke ausschließen und den Spielraum für mögliche Reduktionen und Paraphrasierungen durch andersartige sprachliche Ausdrücke einschränken, die den 'eigentlichen` Gehalt oder die 'tatsächliche` Bedeutung der Ersteren beanspruchen (ebd., 13). 
Die in der semantischen These formulierte Annahme, dass die spezifizierten sprachlichen Ausdrücke Wahrheitswerte besitzen, berührt einen zentralen und weitestgehend eigenständigen Punkt, der über die semantische These zu den ontologischen Thesen hinzutritt und diese ergänzt. Er führt auf die Frage nach einer Wahrheitstheorie und eventuell nach einer Bedeutungs- und Referenztheorie für die fraglichen sprachlichen Ausdrücke, innerhalb derer die semantischen Eigenschaften und Relationen der Sätze charakterisiert und erläutert werden können. Dabei soll klar werden, was die Relation zwischen einem sprachlichen Ausdruck und seinem Wahrheitswert unabhängig von menschlichen Erkenntnismöglichkeiten bedeutet (ebd., 7). ${ }^{57}$ Der hierbei zur Anwendung kommende nicht-epistemische Wahrheitsbegriff zeichnet sich dadurch aus, dass sprachliche Ausdrücke auch dann Wahrheitswerte besitzen, d.h., auch dann wahr oder falsch sind, wenn niemand über epistemische Mittel verfügen würde, die es (wenigstens im Prinzip) ermöglichen würden, herauszufinden, ob die Sätze wahr oder falsch sind. Wahrheit wird dementsprechend als Übereinstimmung gedanklicher Vorstellungen mit der Wirklichkeit verstanden. Dies wird als Korrespondenztheorie der Wahrheit bezeichnet: Wissenschaftliche Aussagen über Gegenstände, Zusammenhänge, Prozesse und Zustände sind dem zufolge genau dann wahr, wenn sie mit den Tatsachen in der objektiven Welt übereinstimmen. In diesem Sinne wird angenommen, "dass sich eine sprachlich fundierte wissenschaftliche Theorie in genau der Weise auf die Welt bezieht, wie es die ontologischen Thesen unterstellen." (Bartels 2007:201) Die sprachlichen Ausdrücke einer wissenschaftlichen Theorie, denen bestimmte Wahrheitswerte unterstellt werden, beziehen sich folglich exakt so auf die Objekte und Eigenschaften der realen Welt, dass die Wahrheit bzw. Falschheit von Aussagen einer wissenschaftlichen Theorie von der Beschaffenheit der durch die Theorie beschriebenen Welt abhängt.

b) Neben diesen, für den wissenschaftlichen Realismus grundlegenden, ontologischen und semantischen Thesen lassen sich zwei epistemische Thesen anführen. Diese fallen insofern aus dem Rahmen der bisherigen Ausführungen, als dass sie nicht bei den potenziellen Erkenntnis- und Wahrheitsbedingungen ansetzen, sondern bei den bereits de facto vorhandenen wissenschaftlichen Theorien und ihren de facto Wahrheitsgehalten (ebd., 201). In diesem Sinne stehen die epistemischen Thesen in keinem direkten logischen Zusammenhang mit der semantischen und den ontologischen Thesen. Allerdings gehören die Thesen inhaltlich zusammen, da sie die von Wright herausge-

57 Bedeutungs- und Referenztheorien (verifikationistische Theorien, logischer Positivismus, Kontexttheorien, Hybridtheorien) sind, wie Tschepke herausstellt, wichtig für den wissenschaftlichen Realismus, weil sie zum Inhalt einer realistischen, respektive der sie kritisierenden antirealistischen, Position gehören (wenn sie die semantische These berühren) und weil sie bei der Argumentation für und gegen den wissenschaftlichen Realismus eine entscheidende Rolle spielen (Tschepke 2003:29). Hier sollen sie nur dann aufgegriffen werden, wenn Letzteres relevant wird. 
stellten Ebenen der realistischen Intuition konkretisieren: Die semantische These konkretisiert den 'bescheidenen ' Gedanken, der in den ontologischen Thesen der Existenz und der Unabhängigkeit metaphysisch stark und umfangreich eingeholt wird, während die epistemischen Thesen den 'gehaltvollen ' Gedanken konkretisieren, dass Menschen trotz der Unabhängigkeit der Wirklichkeit und trotz des nicht-epistemischen Wahrheitsverständnisses dennoch Wissen von der Welt haben können (Tschepke 2003:8).

Die epistemischen Thesen beziehen sich konkret gesprochen auf die Annahme, dass wissenschaftliche Theorien de facto wahre Beschreibungen der von innen postulierten Entitäten liefern. Hierbei wird kann mit Christian Suhm zwischen einer kriteriologischen und einer wissenschaftshistorischen These unterschieden werden kann (Suhm 2005:77), die mit Andreas Bartels wie folgt formuliert werden können (Bartels 2007:202):

kriteriologische These (kT):

Anhand logischer und methodologischer Kriterien kann entschieden werden, ob Theorien mit hinreichender Genauigkeit die Elemente der Wirklichkeit und ihrer Beschaffenheit repräsentieren und damit wahr sind. Diejenigen Theorien, die den logisch-methodologischen Kriterien am besten genügen, sind wahre, weil explanatorisch erfolgreiche, Theorien.

wissenschaftshistorische These der Konvergenz (wT):

Zumindest die Theorien der 'reifen ' wissenschaftlichen Disziplinen (gemeint sind damit insbesondere die Physik und die Mathematik) sind wahr. Jüngere Theorien kommen der Wirklichkeit dabei näher als ältere Theorien, da sie gegenüber diesen einen wissenschaftlichen Fortschritt darstellen.

Der Fokus dieser beiden Thesen, die aufs Engste miteinander verwoben sind, liegt sowohl auf den beobachtbaren als auch auf den unbeobachtbaren Entitäten. ${ }^{58}$ Das heißt, dass Theorien in allen Teilen als wahr postuliert werden (Tschepke 2003:13). Damit wird die empiristische Unterscheidung zwischen Beobachtungsbegriffen, die sich dem Namen nach auf beobachtbare Entitäten beziehen und theoretischen Begriffen, die sich auf Unbeobachtbares beziehen, zurückgewiesen (Bartels 2007:199). Da der Status von unbeobachtbaren Entitäten und Eigenschaften sowie Theorien über sie für den wissenschaftlichen Realismus und seine ontologischen und semantischen Annahmen deutlich problematischer ist als Theorien über beobachtbare Entitäten und Eigenschaf-

58 Anzumerken ist an dieser Stelle der wichtige Hinweis, dass Vertreterlnnen des wissenschaftlichen Realismus, die die Gültigkeit der ontologischen und epistemischen Thesen annehmen, die prinzipielle Fehlbarkeit menschlicher Erkenntnisfähigkeiten und -verfahren, sprich die Fehlbarkeit der Wissenschaft und wissenschaftlicher Methoden, problemlos anerkennen können: „Ein Realist, der bestimmte Überzeugungen für tatsächlich wahr hält, die bei sorgfältigem Einsatz für den fraglichen Bereich geeigneter epistemischer Mittel und Verfahren gewonnen wurden, hält im allgemeinen die zur Gewinnung eingesetzten Mittel und Verfahren nicht für unfehlbar, d.h. er schließt nicht aus, dass ihr 'Output' in Einzelfällen zu korrigieren sein könnte (Tschepke 2003:9). 
ten, finden sich gerade in Bezug auf die epistemischen Thesen eine Reihe von Abschwächungen und Modifizierungen, die auch für die Weiterentwicklung des wissenschaftlichen Realismus verantwortlich sind. ${ }^{59}$

Ergänzt werden die bisherigen Thesen durch eine - oftmals nur implizit mitlaufende axiologische These (Tschepke 2003:13). Diese bezieht sich nur vage auf die realistische Intuition, ist aber bedeutsam für die Wissenschaftspraxis: ${ }^{60}$ Die axiologische These ergänzt die anderen Thesen um die motivationale Angabe eines regulativen Ziels wissenschaftlichen Handelns. In einer realistischen Lesart steht hinter der Wissenschaft das Ziel, „die Welt zu zeigen, wie sie der Wirklichkeit nach beschaffen ist“ (Hiekel 2012:10):

axiologische These (aT):

Das Ziel der Wissenschaft ist die Produktion wahrer Theorien.

Für das weitere Vorgehen ist es an dieser Stelle ausreichend, aus dem bisher Ausgeführten eine idealtypische Position des wissenschaftlichen Realismus abzuleiten: Wissenschaftliche RealistenInnen gehen grundlegend davon aus, dass es eine Wirklichkeit gibt, die sich anhand bestimmter Sachverhalte und Gesetze zeigt (ET). Die Wirklichkeit existiert dabei unabhängig von unserer Erfahrung, unserer Sprache und unserem Denken (UT). Die Entitäten, Eigenschaften, Sachverhalte und Gesetze der physischen Welt können über wissenschaftliche Theorien sprachlich erfasst und wirklichkeitsgetreu beschrieben werden. Was ein sprachlicher Ausdruck bedeutet, erschöpft sich darin, worauf er sich bezieht (sT). Die spezifizierten sprachlichen Ausdrücke besitzen Wahrheitswerte, d.h., sie sind wahr oder falsch und zwar gemäß der UT unabhängig von menschlichen epistemischen Mitteln zum Erkennen dieser Wahrheitswerte. Auf Grundlage dieses Fundaments wird davon ausgegangen, dass anhand logisch-methodologischer Kriterien entschieden werden kann, dass im Mindesten die grundlegenden und allgemein akzeptierten Theorien 'reifer' empirischer Wissenschaften wahr sind (kT). Indem die Theorien sich darum bemühen, dass sie wahre Beschreibungen der Wirklichkeit liefern, sind die Theorien einer Disziplin in der Lage, sich im Laufe der Zeit immer stärker der Wirklichkeit anzunähernd (wT). Das 'Ziel' der Wissenschaft ist es somit, die Welt zu zeigen, wie sie in Wirklichkeit beschaffen ist (aT).

59 Diese Variationen sollen hier nicht weiter ausgeführt werden, da sie für die weitere Argumentation keine direkte Relevanz besitzen. Sie kommen nur dann stellenweise zum Tragen, wenn es im nächsten Kapitelabschnitt um die theoretische Kritik am wissenschaftlichen Realismus geht (siehe 3.2).

60 Konrad Ott führt in »Ipso Facto« aus, dass der Begriff der Praxis pragmatisch die Aussicht auf Gelingen impliziere und dass dieses Gelingen in Bezug auf Wissenschaft als Praxis in der realistischen Lesart darin gesehen wird, zu einer Wahrheitsannäherung bzw. zu einem Erkenntnisgewinn zu gelangen (Ott 1997:105). Eben diese Zielbestimmung wird vor allem von Seiten konstruktivistischer und wissenssoziologischer TheorieanhängerInnen bestritten bzw. hinsichtlich der Differenzierung von Zielen und Zwecken der Wissenschaft relativiert (siehe 3.2.1). 


\subsection{Die kritische Reflexion des wissenschaftlichen Realismus}

Im Anschluss an die Darlegung der idealtypischen Position des wissenschaftlichen Realismus wird diese im Folgenden kritisch reflektiert. Dies wird so geschehen, dass zuerst auf die 'externen 'Kritikpunkte am wissenschaftlichen Realismus eingegangen wird, die von Seiten konstruktivistischer und soziologischer Wissenschaftstheorien vorgebracht werden (3.2.1). Hierbei geht es um die theorieexternen Kritikpunkte am wissenschaftlichen Realismus, die gegen diesen, als wissenschaftstheoretische Grundlage für die Betrachtung der Biodiversität, vorgebracht werden. Hierüber wird im Kern dargelegt, dass durch das realistische Wissenschaftsverständnis die Konstitutionsbedingungen von Wissenschaft als kultureller und sozialer Praxis der Wahrheitsfindung nicht konsequent genug beachtet werden, die aber gerade für einen Forschungsgegenstand wie Biodiversität als Grenz- und Hybridkonzept im Besonderen relevant sind. Da diese externen Kritikpunkte indes nicht hinreichend sind, um den wissenschaftlichen Realismus als solchen substanziell zu hinterfragen, da alle Theorien seitens konkurrierender Ansätze Kritik ausgesetzt sind, wird im Anschluss daran auf die 'internen 'Kritikpunkte, sprich die innertheoretische Kritik am wissenschaftlichen Realismus eingegangen (3.2.2). Über diese Ausführungen soll gezeigt werden, dass der Realismus als Wissenschafts- und Erkenntnistheorie auf zu anspruchsvollen Annahmen hinsichtlich seiner Wahrheitskonzeption (epistemische Thesen) und letztlich auch seiner Zielb-stimmung wissenschaftlicher Praxis (axiologische These) basiert, so dass im Anschluss daran der Vorschlag unterbreitet wird, die Zielbestimmung wissenschaftlichen Handelns gemäß der "Methodologie wissenschaftlicher Forschungsprogramme" nach Imre Lakatos als „progressive Problemverschiebung“, d.h. nicht als theoretische Korrespondenz mit der Wahrheit und der Wirklichkeit, sondern als theoretischen und empirischen Erkenntnisüberschuss gegenüber vorangegangen Theorien zu bestimmen.

\subsubsection{Die externe Kritik am wissenschaftlichen Realismus: Die Ausblendung der kulturellen Konstitutionsbedingungen der Biodiversitätskonzeption}

Ausgehend von dem, in der Problemselektion (siehe 2.4.1) festgestellten und dargelegten methodologischen Fehlschluss der Gleichsetzung von Forschungsobjekten (biotische und abiotische Vielfalt) und Forschungskonzepten (biologische Vielfalt), wird im Folgenden auf Basis des sogenannten Erlanger Konstruktivismus ${ }^{61}$ - der darauf fokus-

61 Der Erlanger Konstruktivismus ist ein methodenkritischer Ansatz in der Wissenschaftstheorie: „In einem Satz zusammengefasst versucht der Erlanger Konstruktivismus einen begründeten und zirkelfreien Aufbau der Wissenschaftssprachen und damit der Wissenschaften selbst zu leisten." (Zitterbarth 1991:79) Alle Schulen des Erlanger Konstruktivismus fokussieren auf die rational nachvollziehbare Rekonstruktion von Begriffen (und Argumenten), mit dem Ziel begriffliche Unklarheiten in der Wissenschaft zu erkennen, begründete Alternativen dazu zu erarbeiten und auf diesem Wege Miss- 
siert, dass es mit Hilfe der Sprach- und Wissenschaftsmethodik möglich ist, die realistische Auffassung von der Wirklichkeit und dem 'naiven Vorfinden und Beobachten der Welt'zu überwinden und durch eine methodische Erkenntnis- und Wissenschaftskonstruktion zu ersetzen (Pörksen 2011:21-25) - argumentiert, dass das realistische Wissenschaftsverständnis keine geeignete Basis für die Analyse des Forschungsgegenstandes Biodiversität darstellt, weil das Verständnis von Erfahrung und Beobachtung in den Biowissenschaften, genau wie in allen Naturwissenschaften, nach wie vor primär rezeptorisch verstanden wird. Das bedeutet, dass Naturgegenstände als 'Ausdruck' der Wirklichkeit verstanden werden, auch wenn ein solcher 'Ausdruck' technisch im Labor oder im Experiment provoziert und erzeugt worden ist. Ein solches Verständnis von Erfahrung und Beobachtung führt aber dazu, dass die Konstitutionsbedingungen von Wissenschaft als kultureller und sozialer Praxis nicht konsequent genug beachtet werden, die aber gerade für den Forschungsgegenstand der Biodiversität als Grenz- und Hybridkonzept im Besonderen relevant sind. Maßgeblich ist dabei die Orientierung an den BeobachterInnen als TeilnehmerInnen, d.h. als die, die Wirklichkeit konstituierenden und Realitäten konstruierenden Instanzen, womit gleichzeitig die realistische Vorstellung einer nicht-epistemischen Wahrheitskonzeption und einer empirischen Objektivität verabschiedet werden, weil der Beobachter nicht als unabhängig von der Erkenntnis angesehen wird. Dementsprechend liegt der Fokus der Kritik nicht auf den ontologischen Thesen, d.h. dem 'Wesen der Dinge' (ontologische Was-Fragen), sondern auf den epistemischen Thesen, sprich dem Prozess und der Entstehung von Erkenntnissen (epistemologische Wie-Fragen). Kern der Kritik ist dabei die Plausibilisierung der Einsicht, dass jedes Unterscheidungskriterium von Erkennen und Nicht-Erkennen notwendigerweise einen normativen Charakter hat, so dass jeder Versuch metasprachliche Wahrheitskriterien von Aussagen als Behauptungen und nicht als Normen zu interpretieren, auf einen infiniten Regress hinausläuft (Janich 1996:148-149). Genau auf diesen Punkt läuft die externe Kritik am wissenschaftlichen Realismus hinaus:

verständnisse im wissenschaftlichen Austausch zu verringern. In einem engem Zusammenhang mit dieser, auf Pragmatik als Grunddisziplin beruhenden, Sprachtheorie steht die Konsensustheorie der Wahrheit: „Ein Satz soll als wahr gelten, wenn ihm in einem unvoreingenommenen Diskurs, einer idealen Sprechsituation, jeder Sachkundige und Gutwillige zustimmen kann. Ein solcher unvoreingenommener Diskurs ist u.a. dadurch gekennzeichnet, dass er von allen Teilnehmern undogmatisch, zwanglos und nicht persuasiv geführt wird: Zwischen allen Teilnehmern soll absolute Symmetrie herrschen. Wenn eine wissenschaftliche Theorie in diesem Sinne als begründet gelten soll, so müssen die ersten Sätze dieser Theorie, die ja ihrerseits nicht wieder aus anderen Sätzen abgeleitet werden können, in einem solchen Diskurs Zustimmung finden können. In schrittweisem Aufbau sollen dann aus diesen ersten Sätzen begründete Theorien entstehen, wobei bei jedem Schritt nur auf solche Hilfsmittel zurückgegriffen werden darf, die entweder bei diesem Aufbau bereits erarbeitet wurden oder die uns aus der vorwissenschaftlichen Praxis bereits zur Verfügung stehen. In diesem Sinne bildet die vorwissenschaftliche Praxis ein lebensweltliches Apriori, auf welches nicht nur jede wissenschaftliche Bemühung aufbauen muss, sondern dass auch von keiner Wissenschaft hintergangen werden kann." (ebd., 81) 
Obwohl auch RealistInnen nicht bestreiten würden, dass naturwissenschaftliche Praxen durch das Handeln von WissenschaftlerInnen zustande kommen, dass diese auch an anderen Zielen als der Wahrheitsfindung orientiert sein können, dass Wissenschaften und wissenschaftliches Handeln bestimmte Ziele verfolgen (Wahrheitsfindung bzw. Adäquanz), dass WissenschaftlerInnen in ihren Lehrmeinungen und Forschungsmethoden historisch wandelbar sind und dass von wissenschaftlicher Naturerkenntnis nur dort die Rede sein kann, wo sich diese sprachlich artikuliert, d.h., wo sie in Form von Techniken, Theorien und Methoden angewandt werden kann (Janich 1992b:14), ${ }^{62}$ wird unter einer realistischen Perspektive zu stark vernachlässigt, dass sich die Konstitution und Konstruktion wissenschaftlicher Gegenstände lebensweltlich-sozialen Praxen verdankt (ebd., 181), weil zu stark ausgeblendet wird, dass Wissenschaften nicht nur regulative Ziele, sondern auch applikative Zwecke besitzen (Ott 1997:442):

Bezogen auf den Aspekt der lebensweltlichen Konstitution und Konstruktion wissenschaftlicher Gegenstände, wird vor allem von Seiten konstruktivistischer und soziologischer WissenschaftstheoretikerInnen angeführt, dass selbst wenn RealistInnen die Theoriebeladenheit der Beobachtungssprache, sprich den Umstand, dass Beobachtungen immer einer Theorie folgen, als Faktum anerkennen, ihnen nach wie vor die Konsequenz aus der Einsicht schwerfällt, dass WissenschaftlerInnen selbst eine entscheidende Rolle bei der Konstitution und Konstruktion ihrer eigenen Forschungsgegenstände spielen: Wenn Wissenschaftlerlnnen nicht als passive und rezeptive Beobachterlnnen verstanden werden können, „denen sich die Naturphänomene (vermeintlich) qua Naturgesetzen aufdrängen“ (Janich 1992b:17), dann bedeutet dies auf einer abstrakteren Ebene, dass die realistischen Annahmen über die Wirklichkeit, die mit einem deskriptivistisch-naturalistischen Verständnis von Beobachtungs- und Erfahrungswissen einhergehen, relativiert bzw. aufgegeben werden müssen, weil dabei nicht beachtet wird, dass auch Beobachtungssätze methodisch-pragmatische Geltungskriterien haben. $^{63}$

62 Hier sei vor allem auf die wissenschaftstheoretische Betrachtungsweise des »Konstruktiven Realismus« (KR) Friedrich Wallners verwiesen, der in weiten Teilen die Kritik des Erlanger Konstruktivismus teilt. Das zentrale Anliegen des KR ist die Einsicht in die Konstruiertheit wissenschaftlicher Erkenntnisse und damit verbunden die Abkehr von der klassischen realistischen Auffassung, dass Wissenschaft zu verbindlichen Aussagen über die Wirklichkeit kommen könne (Klünger 2011:118-125). Stattdessen konstruiert Wissenschaft Realitäten, indem sie von ungeprüften und kulturell bedingten Vorannahmen ausgeht (ebd., 87-88).

63 Selbst der KR, der ja eine 'realistische Grenzposition ` hin zum Konstruktivismus darstellt, lehnt die instrumentalistischen und pragmatischen Annahmen ab, da hier die Gefahr gesehen wird, dass Phänomene 'verschleiert, ausgeschaltet oder verändert' werden, wo es den Wissenschaften doch um die 'interessenfreie Erklärung` der Phänomene gehen sollte. Dabei wird indes übersehen, dass jede Erklärung als solche mit dem Geltungsanspruch einhergeht, dass sie als Erklärung akzeptiert werden will, so dass im Bereich der Wissenschaft wiederum Kriterien dafür zu nennen sind, warum dies der Fall sein sollte (Janich 1996:131). 
Dies wiederum resultiert darin, einen Unterschied zwischen Wirklichkeit als dem vor aller menschlichen Auseinandersetzung mit ihr Vorhandenen und Gegebenen und Realitäten als den von Menschen durch Handeln und Erkennen geschaffenen Konstruktionen der Wirklichkeit als Unterschied zwischen der „Welt mit der wir leben“ (Wirklichkeit) und der „Welt in der wir leben“ (Realität) zu machen (Wallner 1990:68). Wird dies getan, dann wird schnell klar, dass die Naturforschung keine bloße Beobachtung der Wirklichkeit ist, sondern eine teilnehmende Praxis der Konstruktion von Realitäten, indem sich NaturforscherInnen für ihre Handlungen selbst Zwecke setzen, an deren Erreichen oder Verfehlen sich ihre Erfahrung und Erkenntnis festmacht. Der Unterschied zwischen Beobachtung und Teilnahme ist daher zentral:

„Wollte man unter den gängigen methodologischen Begriffen denjenigen auswählen, der am wenigsten verzichtbar ist für die Erklärung der Möglichkeiten von Erfahrungswissen, so müsste die Wahl wohl auf den Begriff der Beobachtung fallen. Für die Naturwissenschaften dürfte sich nur schwer eine Beschreibung, eine Analyse, eine Rekonstruktion oder sonst ein wissenschaftlicher Annäherungsversuch finden, in denen es nicht an hervorgehobener Stelle um Beobachtungen ginge. Dabei scheinen Beobachtungen nicht nur die erkenntnistheoretische Nahtstelle zwischen der Außenwelt und dem erkennenden Subjekt zu markieren, sie bilden auch den Aufhängepunkt pathetisch-empiristischer Bekenntnisse zu Aufgeschlossenheit und Unvoreingenommenheit der modernen Erfahrungswissenschaften, ja zu selbstgewissen Bekenntnissen, sich als moderner Erfahrungswissenschaftler jederzeit durch neue Beobachtungen belehren zu lassen, d.h. die Welt so zu sehen, wie sie nun einmal wirklich ist, anstatt sie so zu beschreiben, wie man sie gerne hätte." (Janich 1992b: 162)

Im Verständnis objektiver Beobachtung als Mittel der Erkenntnisgewinnung manifestiert sich folglich das (naive) Beobachtungsverständnis in den Naturwissenschaften: Objektive Beobachtung soll die Wissenschaft gegen dogmatische, ideologische, apriorische, metaphysische und andere, mit anderen Worten der Wissenschaftlichkeit abträgliche oder auch entgegenstehende Vorurteile immunisieren. Allerdings ist ein solches Verständnis von objektiver Beobachtung illusionär:

„Das Bild von der Beobachtung als Fenster zur Welt, durch das ehrfürchtig staunend, der ahnungslos entdeckende, der allein auf Wahrheit und Kontrollierbarkeit ausgerichtete Forscher hindurch blickt, jeder praktischen Anwendbarkeit seiner Resultate skeptisch gegenüberstehend - dieses Bild ist ein Ammenmärchen mit allen Widersprüchen, die sich nur Märchen erlauben können.“ (ebd., 181)

Demgegenüber soll hier der Umstand betont werden, dass das Beobachten Teil eines rational organisierten Handelns ist, sprich Beobachten ist kein außerhalb der Erkenntnis- und Forschungsgegenstände befindlicher Prozess, sondern ein sie bereits konsti- 
tuierender und konstruierender, womit dem Umstand Rechnung getragen wird, dass unser Wissen von der Welt immer schon sozial konstituiert und konstruiert ist. Entscheidend ist an dieser Stelle, dass sich auf der Basis einer konstruktivistischen Epistemologie die Auffassung über den Status naturwissenschaftlichen Wissens verändert: Biologie und Ökologie können demnach gar nicht die Aufgabe haben, die Welt des Lebendigen, wie sie naturgegeben ist oder sich uns natürlicherweise darstellt, möglichst wirklichkeitsgetreu zu beobachten, zu erfassen und zu analysieren, weil Wissenschaft eben keine wirklichkeitsgetreue und wahre Annäherung an einen (natürlichen) Gegenstand oder dessen Abbildung in der Sprache sein kann. Vielmehr sind die Biowissenschaften - und mit ihnen der Forschungsgegenstand der Biodiversität - ganz spezifische Herangehensweisen an spezifisch erfasste Problemzusammenhänge sowie spezifische Repertoires an Problemlösungsansätzen und Verfügungswissen, wobei die dabei formulierten Probleme, Ansätze und Ziele selbst kulturellen Zwecksetzungen unterliegen (Gutmann \& Janich 2001a:347).

An dieser Stelle wird der zweite Aspekt - die unzureichende Berücksichtigung der Zweckdimension wissenschaftlicher Praxis - relevant. Diesbezüglich kann mit Konrad Ott angeführt werden, dass Wissenschaften sowohl regulative Ziele (Wahrheitsfindung, Adäquanz, "know-that" als Faktenwissen) als auch applikative Zwecke (Anwendung, Technologie, Nutzen, »know-how « als Prozesswissen) besitzen, die weder in ihrer Identifikation (Nützlichkeit zeigt sich an der Wahrheit bzw. Wahrheit zeigt sich an der Nützlichkeit) noch als Gegensätzlichkeit (Zwecksetzung auf Kosten der Zielsetzung bzw. Zielsetzung auf Kosten der Zwecksetzung) adäquat begriffen werden können (Ott 1997:444). Das Verhältnis beider Aspekte müsse vielmehr als „historisch wie systematisch komplementär" (ebd., 442) ${ }^{64}$ verstanden werden, so dass die Berücksichtigung und Betonung der Zielkomponente (siehe axiologische These in 3.1) bei gleichzeitiger Ausblendung der Zweckkomponente „eine szientistische Abstraktion“ (ebd., 442) sei, die dazu führe, dass „eine Wissenschaftstheorie, die von der Zweckdimension absehen möchte, zunehmend anachronistisch werde." (ebd.) Denn damit würde zwar berücksichtigt, dass die Wissenschaft hinsichtlich ihrer Zieldimension einzig ihren eigenen Standards der Prüfung und Rechtfertigung unterworfen ist, es würde aber zugleich ausgeblendet, dass Wissenschaften hinsichtlich ihrer Zweckdimension zu etwas dienlich sind und - hier wird der erste Aspekt wieder aufgenommen - dass es kulturelle und soziale Zwecksetzungen sind, die mit (vor-)wissenschaftlichen Interessen und Werten verbunden sind.

64 Die sich daran anschließende Frage, ob die Zweckdimension ein Pendant zum internen Wahrheitsethos der Zieldimension enthält bzw. enthalten muss (Ott 1997: 443), wird von Ott selbst bejaht und begründet (ebd., Kap. VI), da er annimmt, dass „[j]e fortgeschrittener eine Wissenschaft in ihrer Zieldimension ist, (...) umso vielfältiger erweisen sich die technologischen und professionellen Möglichkeiten. Je 'reifer' die Wissenschaft wird, desto 'nützlicher' wird sie." (ebd., 444) 
Werden diese kulturellen und sozialen Zwecksetzungsprozesse in der Auseinandersetzung mit biowissenschaftlichen Theorien und Begriffen nicht explizit beachtet und wird zugleich davon ausgegangen, dass sich ohne Beobachtungsfehler die Natur an sich zeige, wie sie nun einmal sei ${ }^{65}$ - übersehend, dass diese sie sich nur relativ zu technisch gelingenden Beobachtungs- und Experimentalmethoden, also relativ zu künstlichen Maßnahmen zeigt (Gutmann \& Janich 2001a:333), „so läuft man Gefahr, die schlichte Tatsache zu übersehen, dass Wissenschaftler selbst [qua wissenschaftlicher Praxis] in ein 'kultürliches Unternehmen' verwickelt [sind], das aus ihren eigenen Handlungen, ihren eigenen Zwecksetzungen, ihren eigenen Methoden(aus)wahlen und ihren eigenen Wissenschaftsverständnissen mit bestimmten Meinungen über Wissenschaftlichkeit, Kausalität, Erklärung, Natur usw. besteht.“ (ebd., 332). Eben dies ist auf der konkreten Ebene der Fall, wenn Biodiversität zugleich für (a)biotische und biologische Vielfalt stehen kann und wenn nicht explizit zwischen den Referenzobjekten (abiotische und biotische Vielfalt) und Forschungskonzepten (biologische Vielfalt) unterschieden wird, sondern ganz im Gegenteil über die meisten Definitionen von Biodiversität nur wiedergegeben wird, was die Referenzobjekte sind, die unter Biodiversität subsumiert werden, nicht aber, was dann biologische Vielfalt als Forschungskonzept bedeutet. Die immer wieder vorgenommene Gleichsetzung der Begriffe Biodiversität und biologische Vielfalt verschleiert damit die wissenschafts- und erkenntnistheoretisch relevante Einsicht, dass die biologische Vielfalt eben nicht die [a]biotische Vielfalt und damit kein 'natürlicher Gegenstand' ist, der mit den Mitteln der Biowissenschaften 'wirklichkeitsgetreu ' beschrieben und erfasst werden kann. In diesem Sinne sind die Gegenstandsbereiche der Biowissenschaften nur angemessen verstehbar, wenn sie nicht einfach als 'natürlich' gegeben hingenommen und als generalisierte Abbilder der Natur verstanden werden, die aus empirischen Einzelbeobachtungen entstanden sind und daraufhin mittels Theorien als Wirklichkeit formuliert werden. Vielmehr werden die Gegenstände der Wissenschaft in Abhängigkeit von den sozialen Rahmenbedingungen ausgewählt und interpretiert. Daher sind sowohl die Referenzobjekte (abiotische und biotische Phänome), als auch die biowissenschaftlichen Begriffe, Modelle, Konzepte und Theorien (der biologischen Vielfalt) Produkte der menschlichen Wahrnehmung und des menschlichen Handelns:

„Pflanzen und Tiere, ja ganze Landschaften werden zu Kulturgegenständen, ungeachtet der Tatsache, dass menschliche Eingriffe nicht Beliebiges an ihnen hervorbringen können, sondern empirisch die Grenzen des Machbaren auszuloten haben. (...) Es sind die speziellen Erkenntnisziele des Paläontologen, des Taxonomen, des Physiologen, des Evolutionsbiologen, des Ethologen, des Genetikern und des Mole-

65 Insbesondere die in den Naturwissenschaften übliche Sprechweise, dass nicht akzeptierte Beobachtungsresultate bzw. Beobachtungsfehler als 'Artefakte', d.h. als menschlich erzeugte Gegenstände benannt werden, zeigt die Tiefe der hier aufgezeigten Problematik. 
kularbiologen, der sich handelnd und redend einer vorfindlichen Natur bemächtigt und sie nach seinen Zwecken ordnet und gestaltet." (ebd., 337)

In diesem Sinne kommt es auf die je spezifische Konstruktion der Begriffe, Modelle, Konzepte und Theorien an, die für den Forschungsgegenstand der Biodiversität von Relevanz sind, da die für die Biodiversität so zentralen biologischen Begriffe wie Lebewesen, Organismus, Art, Gen und Ökosystem eben keine „materiellen Gegenstände (sind), auf die sinnbildlich mit dem Finger gezeigt werden kann und so ein begreifbarer und handhabbarer Unterschied zu anderen Gegenständen eingeführt, festgelegt, bestimmt und daraufhin wiedererkannt werden könnte." (ebd., 339) Vielmehr muss bereits die Gegenstandskonstitution als menschliche Kulturleistung begriffen werden, was wiederum heißt, dass die biowissenschaftlichen Verfahren der Begriffs-, Modell- und Theoriebildung ebenso wie die Verfahren des Beobachtens, Messens und Experimentierens in eine differenzierte Theorie über das Verhältnis der Naturwissenschaften zur übrigen Kultur gestellt werden müssen, so dass sich hierüber thematisieren lässt, wie kulturell bedingte Ideen sowie lebensweltlich erworbene Zwecksetzungen in die Naturwissenschaften hinein wirken und dort in Gestalt naturwissenschaftlicher Theorien reformuliert und ausdifferenziert werden (Kirchhoff 2009:54).

Gerade ein solches wechselseitiges Verhältnis von Naturwissenschaft und Kultur ermöglicht die kritische Einsicht in eine nicht-reduktionistische und nicht-dualistische Theorie der gesellschaftlichen Naturverhältnisse. Denn auf Basis dieser wird überhaupt erst einsichtig, dass es nach wie vor (problematische) Versuche gibt, die menschliche Kultur naturalistisch als bloßen Teilbereich der Natur zu erklären, so dass ein geltungstheoretisch problematisches reduktionistisch-naturalistisches Bild der gesellschaftlichen Naturverhältnisse gezeichnet wird, mit dem versucht wird, über die Beschaffenheit der Natur gesellschaftliche Normen zu begründen. Da genau dies über die moralisch-epistemische Hybridisierung geschieht und darüber eine Vielzahl der im Problemaufriss dargelegten Problematiken hervor gerufen wird (vgl. 2.4), bieten die konstruktivistischen und soziologischen wissenschaftstheoretischen Perspektiven auf Biodiversität eine Alternative zur realistischen Sichtweise, da die Erforschung der Biodiversität hinsichtlich der Zweckdimension wissenschaftlicher Praxis hier von vornherein als eine konstruktive Praxis verstanden wird. Gemäß diesen alternativen, konstruktivistischen und wissenssoziologischen Perspektiven auf Biodiversität wird es im übernächsten Abschnitt (3.3) darum gehen, die kulturellen Konstitutionsbedingungen des Forschungsgegenstandes Biodiversität zu rekonstruieren. Dies kann allerdings erst dann plausibel geschehen, wenn auch auf die innertheoretische Kritik am wissenschaftlichen Realismus eingegangen wird, die noch einen weiteren, gewichtigeren Aspekt beleuchtet, warum die an Wahrheit und Wirklichkeit orientierte Zielbestimmung wissenschaftlicher Praxis, wie sie der wissenschaftliche Realismus anbietet, zu kurz greift. 


\subsubsection{Die innertheoretische Kritik am wissenschaftlichen Realismus: Die defizitäre Verteidigung der Korrespondenztheorie der Wahrheit und der Zielbestimmung wissenschaftlicher Praxis}

Im Folgenden wird - unabhängig davon, dass der wissenschaftliche Realismus von einem externen Standpunkt aus betrachtet bereits zahlreichen Kritikpunkten ausgesetzt ist - aus einer innertheoretischen Perspektive dafür argumentiert, dass sich die Begründung des wissenschaftlichen Realismus an theorieentscheidenden Stellen in Zirkelschlüsse verfängt, womit genau das bereits vorausgesetzt wird, was zu beweisen versucht wird. Daran wiederum wird ersichtlich, dass die, mit dem wissenschaftlichen Realismus implizit verbundene, axiologische Zielstellung, die als motivationales Element die realistischen Intuitionen vieler WissenschaftlerInnen abstützt, zu hoch gegriffen ist, weil das theoretische Fundament, auf dem dieser Anspruch basiert, nicht tragfähig genug ist, um diesen Anspruch auch plausibel begründen zu können. Kerngegenstand der innertheoretischen Kritik ist daher die Korrespondenztheorie der Wahrheit mitsamt dem objektiven, nicht-epistemischen Wahrheitsbegriff, der unabhängig von Konventionen der Erkenntnisleistungen gedacht ist und allein auf der Übereinstimmung mit der Wirklichkeit beruhen soll:

„Der realistische Ansatz der Wissenschaftstheorie hat zu anspruchsvolle Prämissen, die nicht ausreichend explizier- und rechtfertigbar sind. (...) Insbesondere die dem wissenschaftstheoretischen Realismus zugrunde liegende korrespondenztheoretische Wahrheitskonzeption ist mit erheblichen Schwierigkeiten versehen: Wenn Wissenschaft danach strebt, die Wahrheit über die Realität zu finden, muss entweder eine andere Wahrheitskonzeption gefunden werden oder aber dieses Ziel als nicht erreichbar verworfen werden." (Hiekel 2012:26)

Hierbei wird das Problem der unterstellten kognitiven Unabhängigkeit physischer Objekte und Eigenschaften vom menschlichen Denken und der Sprache in ersten Ansätzen ersichtlich:

„Die dem Realismus inhärente ontologische Prämisse, dass die Welt unabhängig von uns existiert, macht die Objekte bzw. die Erkenntnis von diesen 'rätselhaft', da der Wahrheitsbegriff radikal nicht epistemisch aufgefasst wird und demnach nicht von empirischer Bestätigung oder dem rationalen Gerechtfertigtsein abhängt, sondern von der physischen Wirklichkeit selbst und ihrer Beschaffenheit." (ebd., 20)

Hiekel fasst das damit einhergehende Problem daher wie folgt zusammen:

„Der wissenschaftliche Realismus [muss] ein Spannungsverhältnis auflösen, das darin besteht, dass eine von Erkenntnisleistungen unabhängige Wirklichkeit er- 
kannt werden können muss und dass die Wissenschaft hier den Anspruch erhebt, die Wahrheit über diese Wirklichkeit zu ermitteln. Die Bedingungen und Möglichkeiten von Erkenntnis über eine unabhängige Wirklichkeit müssen durch den [wissenschaftlichen] Realismus aufgeklärt werden, damit diese Spannung aufgelöst werden kann." (ebd., 18)

Da es hier nicht detailliert um die wissenschaftstheoretische Widerlegung der korrespondenztheoretischen Wahrheitskonzeption gehen kann und soll, wird der Argumentationsstrang der antirealistischen Kritik nur insofern von Belang sein, als dass die Probleme deutlich werden sollen, die sich für die wissenschaftliche Arbeit mit dem Forschungsgegenstand Biodiversität aus eben einer solchen realistischen Perspektive ergeben. Dabei setzen die Ausführungen am Standardargument des wissenschaftlichen Realismus zur Verteidigung und Explizierung des Korrespondenzprinzips der Wahrheit an. Dies ist das sogenannte abduktive Argument der besten Erklärung ${ }^{66}$ (Bartels 2007: 203-205). Über das Argument der besten Erklärung versuchen die VertreterInnen des wissenschaftlichen Realismus zu erklären, wie die Wahrheit durch die physische Welt festgelegt werden kann, wenn die physische Wirklichkeit und mithin die Theorien über sie als voneinander unabhängig gedacht sind. Das Argument baut auf allen Thesen der idealtypischen Position auf und besagt, dass die Korrespondenz von Wahrheit und Wirklichkeit am besten über den explanatorischen Erfolg von Theorien erklärt werden kann. Dementsprechend soll der explanatorische Erfolg von Theorien, den bestimmte, gut bestätigte Theorien (bspw. die Evolutionstheorie) real aufweisen, einen Beweis für unseren Glauben an die Wahrheit liefern (ebd., 203). Diesem Argument folgend kann man zur Wahrheit lediglich indirekt über den explanatorischen Erfolg wissenschaftlicher Theorien gelangen, so dass der explanatorische Erfolg wissenschaftlicher Theorien zum Garant für die Korrektheit der realistisch gedachten Beziehung zwischen Wahrheit und Wirklichkeit wird (ebd., 204).

Um auf die zentralen Einwände nachvollziehbar eingehen zu können, die gegen das abduktive Argument der besten Erklärung vorgebracht werden, ist es hilfreich sich dessen argumentative Struktur genauer vor Augen zu führen (ebd., 205):

(P1) Theorie T ist explanatorisch erfolgreich

(P2) Die Wahrheit von T ist die beste Erklärung für den Erfolg von T

66 Die Abduktion ist neben der Deduktion und der Induktion ein dritter Schlusstypus in der formalen Logik. Nach Charles Sanders Peirce ist die Abduktion "the process of forming an explanatory hypothesis." (Peirce 1931:171) Peirce beschreibt die Logik der Abduktion wie folgt: "The surprising fact, C, is observed; But if $A$ were true, $C$ would be a matter of course, Hence, there is reason to suspect that $\mathrm{A}$ is true. Thus, A cannot be abductively inferred, or if you prefer the expression, cannot be $a b-$ ductively conjectured until its entire content is already present in the premiss, »If A were true, C would be a matter of course." (ebd., 189) 
(P3) Wenn T erfolgreich ist und die Wahrheit von T die beste Erklärung für den Erfolg von $\mathrm{T}$ ist, dann ist T wahr

(K) T ist wahr

Die Einwände gegen die Konklusion, dass explanatorisch erfolgreiche Theorien auch wahre Theorien sind, richten sich hauptsächlich gegen die zweite und dritte Prämisse: Der zweiten Prämisse (P2) steht insbesondere der von Larry Laudan vorgebrachte Einwand der pessimistischen Metainduktion entgegen (ebd., 206). Diesem Einwand nach kann der Schluss auf die beste Erklärung weder theoretisch noch praktisch ein gutes Argument für die annähernde Wahrheit von Theorien sein, weil es in der Geschichte nachweislich immer wieder wissenschaftliche Theorien gab, die zwar explanatorisch erfolgreich waren, die sich aber im Nachhinein als nicht allgemeingültig oder als falsch herausgestellt haben (bspw. die »Newtonsche Mechanik»). Diesem Einwand nach gibt es folglich zu viele unerklärte Ausnahmen der Regel, so dass es nicht gerechtfertigt erscheint, die Wahrheit von Theorien über deren Erfolg erklären zu wollen. Diesbezüglich konstatiert Bartels:

„Der Erfolg einer Theorie ist demnach nicht nur nicht die beste Erklärung für die Wahrheit einer Theorie, sie ist gar keine Erklärung" (ebd., 206). ${ }^{67}$

Abgesehen von diesen allein schon schwerwiegenden Problemen zeigt die Problematisierung der dritten Prämisse (P3), dass hier eine weitere entscheidende Schwachstelle des abduktiven Arguments der besten Erklärung vorliegt: Zwar ist es „aus Rationalitätsgründen (...) durchaus einsichtig, die beste Erklärung vorzuziehen, wenn denn eine solche verfügbar ist. Keineswegs folgt daraus aber, dass die beste Erklärung auch wahr sein muss." (ebd., 205) Die hier formulierte Kritik geht in zwei Richtungen: Zum einen setzt die Kritik am Argumentationstyp an, da sich aus abduktiven Argumenten keine logisch zwingenden Schlüsse ergeben, weil diese keine zwingenden Beweise für die Richtigkeit ihrer Konklusionen liefern können (Hiekel 2012:24). ${ }^{68}$ Daher kann aus dem ex-

67 Im Anschluss an diesen Einwand wurde insbesondere mittels des epistemischen Strukturrealismus (eSr) versucht (vgl. Worral 1989), das ontologische Fundament des wissenschaftlichen Realismus zu bewahren, indem die Reichweite der ontologischen Verpflichtung reduziert wird (Bartels 2007:217). Aufbauend auf der Unterscheidung von inhaltlichem und strukturalen Wissen, versucht der eSr dem Einwand der pessimistischen Metainduktion zu entgehen, indem er zeigt, dass wissenschaftliche Theorien inhaltlich falsch sein können, während sie in Bezug auf ihre formale Struktur (annähernd) wahre Aussagen machen können (ebd., 218). Ob dieses 'Ausweichmanöver' auch tragfähig genug ist, um ein universelles Kriterium für jeden historischen Fall der Theorieablösung anzugeben, wurde des Öfteren bestritten (vgl. bspw. van Fraassen 1992), so dass der eSr - obschon als Ausweg aus dem Dilemma angelegt - hinsichtlich seiner ontologischen Verpflichtung nur recht vage bleibt (Bartels 2007:217). Damit muss die Annahme der Kongruenz von Wahrheit und explanatorischem Erfolg (P2) weiterhin als problematisch angesehen werden, weil sie - trotz der Relativierungen, die der eSr vornimmt - keine wirklich tragfähige Prämisse für den wissenschaftlichen Realismus abgibt.

68 Hiekel und Bartels machen beide deutlich, dass der abduktive Argumentationstyp (siehe FN 66) zwar ein in der wissenschaftlichen Praxis gebräuchliches und im Alltagsdenken gut verankertes logisches Prinzip darstellt, aber es liefert keine ausreichend tragfähige Basis für die Fundamentierung des wis- 
planatorischen Erfolg, die bestimmte Theorien de facto besitzen, aus formalen Gründen nicht logisch zwingend auch auf ihre Wahrheit geschlossen werden. Und selbst wenn das abduktive Argument in eine deduktive Schlussfigur verwandelt wird, was theoretisch möglich ist, dann würde das Argument auf diesem Weg zirkulär, da jede realistische Deutung einer Theorie bereits vom Prinzip des Schlusses auf die beste Erklärung Gebrauch machen muss, wenn der explanatorische Erfolg als Beleg für die Wahrheit der Theorie verwendet wird (Fine 1986:161). In diesem Sinne wird die Gültigkeit des Schlusses auf die beste Erklärung bereits vorausgesetzt. ${ }^{69}$

Ein weiterer Ansatz zur Kritik richtet sich gegen die, im realistischen Verständnis, unterstellte Beziehung zwischen explanatorischem Erfolg und Wahrheit. Die hier formulierte Kritik basiert insbesondere auf dem von Pierre Duhem und Willard Van Ormam Quine formulierten Einwand der theoretischen Unterbestimmtheit (Hiekel 2012:23). Diesem grundlegenden Einwand nach, der für den wissenschaftlichen Realismus im Ganzen ähnlich schwerwiegend ist, wie der Einwand der pessimistischen Metainduktion, da er ebenfalls auf das Fundament und die damit einhergehende ontologische Verpflichtung 'durchschlägt' (Bartels 2007:217), kann nicht vom explanatorischen Erfolg einer Theorie auf deren Wahrheit geschlossen werden, weil wissenschaftliche Theorien niemals völlig mit der Wirklichkeit übereinstimmen können. Der Einwand der theoretischen Unterbestimmtheit richtet sich somit sowohl gegen die in der kriteriologischen These unterstellte „naive Abbildrelation“ von Theorie und Wirklichkeit (Hiekel 2012:22) als auch gegen die dabei unterstellte Annahme, dass es ausreichend zwingende logisch-methodologische Gründe gibt, bestimmte Theorien für wahr zu halten und andere nicht. Demgegenüber besagt das Unterbestimmheitsargument, dass es zu jeder

senschaftlichen Realismus, da er keinen logisch zwingenden (deduktiven) Schluss auf die Richtigkeit seiner Konklusionen zulässt (Bartels 2007:204; Hiekel 2012:24).

69 An dieser Stelle ergibt sich ein Anschlusspunkt für eine ganze Reihe von Begründungsstrategien, die das Explanandum der 'explanatorisch erfolgreichen Theorien ' weitergehend spezifizieren, um weiterhin am Schluss auf die beste Erklärung festhalten zu können (Tschepke 2003:Kapitel 9, 185-290). Die Grundidee dieser Argumentationsstrategien (u.a. von Hilary Putnam, Richard Boyd, Martin Carrier, John Worrall) liegt darin, dass der Erfolg der Wissenschaften nach einer Erklärung verlangt, es sei denn, man glaubt an Wunder (»miracle argument« nach Putnam). Tschepke resultiert im Anschluss an seine sehr detaillierten Erörterungen der 'Wunderargumente', dass diese Begründungsstrategien i.d.R. daran 'kranken', dass nicht expliziert wird, „in welchem Sinne von 'erklären ' das Explanandum der verschiedenen Versionen des Wunderarguments durch das zugehörige Explanans erklärt wird.“ (ebd., 219) Zudem kann gegen das Wunderargument ganz grundsätzlich eingewandt werden, dass es zumindest ebenbürtige Konkurrenten für die realistische Erklärung für den 'Erfolg der Wissenschaften ' gibt. So zeigt van Fraassens 'evolutionäre Erklärung des Erfolgs reifer Wissenschaften', dass die realistische Erklärung für den Erfolg von Theorien keineswegs zwingend ist: „Wer sich realistischer Annahmen enthält, muss den Erfolg der Wissenschaft nicht als Wunder betrachten. (...) Der Realist muss daher zugestehen, dass der Realismus eine der möglichen Hypothesen zur Erklärung des Erfolgs von Theorien (bzw. der Wissenschaft) ist, und dass für die Auswahl dieser metatheoretischen Hypothese dieselben Kriterien gelten (...) wie für die Auswahl von Hypothesen auf der Ebene der wissenschaftlichen Theorien selbst. Der wissenschaftliche Realismus ist damit selbst eine metawissenschaftliche These." (Bartels 2007:206) 
Theorie eine alternative, empirisch genauso adäquate Theorie geben kann, die aber andere theoretische Erklärungen anbietet und dennoch zu denselben beobachtbaren empirischen Ergebnissen gelangt (Bartels 2007:209). Daher gibt es keine ausreichenden Gründe, warum die eine Theorie für wahr gehalten werden sollte, nicht aber die andere.

Um auf diese Einwände zu reagieren, wird von den Verfechtern des wissenschaftlichen Realismus der Begriff der approximativen Wahrheit eingeführt (Hiekel 2012:23): Die Rede von 'annähernder Wahrheit' ist dabei als eine deutliche Abschwächung gegenüber einem semantisch fundierten wissenschaftlichen Realismus zu verstehen, der wissenschaftliche Theorien für buchstäblich und ohne Einschränkung wahr hält. Entscheidend ist hier, dass der Begriff der approximativen Wahrheit eine Modifikation der epistemischen These sowie des abduktiven Arguments der besten Erklärung nach sich zieht: Indem davon ausgegangen wird, dass zumindest die grundlegenden und allgemein akzeptierten Theorien 'reifer' Wissenschaften annähernd wahr sind und damit davon ausgegangen werden muss, dass wissenschaftliche Theorien die Elemente der physischen Wirklichkeit nur ansatzweise repräsentieren können, ergibt sich die modifizierte Annahme, dass selbst die 'reifen ' wissenschaftlichen Disziplinen nur annähernd wahr sein können. Die Modifikation des Schlusses auf die beste Erklärung führt folglich auch zu einer Relativierung der epistemischen Thesen, die aber relevant für die Verteidigung der ontologischen Basis des wissenschaftlichen Realismus sind.

Um die kritischen Überlegungen zum abduktiven Argument der besten Erklärung an dieser Stelle zusammenfassend abzuschließen, kann mit Hiekel konstatiert werden, dass sich, trotz der inhaltlichen Veränderungen, die mit der approximativen Wahrheit einhergeht, eine Sache nicht verändert hat:

„Die approximative Wahrheit kann bislang nur unter eine [wie auch immer geartete] abgeschwächte korrespondenztheoretische Wahrheitskonzeption fallend gedacht werden. Nur der empirische Erfolg wissenschaftlicher Theorien gibt nach wie vor den Anhaltspunkt für ihre approximative Wahrheit. Das 'annähernde Wahrsein` kann also als eine außertheoretische Relation zwischen Theorien und der Welt aufgefasst werden, die aber wiederum nur durch die Annahme der realistischen Thesen gestützt wird.“ (ebd., 23)

Indem also die Wahrheit der Aussagen über die Korrespondenz mit der Wirklichkeit über den explanatorischen Erfolg wissenschaftlicher Theorien begründet wird, liegt dem explanatorischen Erfolg - in einem realistischen Verständnis - der Begriff der approximativen Wahrheit bereits zugrunde, da dieser die Korrespondenz von Wahrheit und Wirklichkeit bereits voraussetzt. Beim Versuch den ersten Zirkelschluss aufzulösen, wird folglich ein zweiter Zirkelschluss begangen: 
„Zur Fundierung des Realismus wird der Begriff der approximativen Wahrheit eingeführt, der aber selbst nur durch realistische Grundannahmen gestützt werden kann. Weiter wird vom Erfolg von Theorien auf deren annähernde Wahrheit geschlossen und über die Wahrheit wiederum auf den Erfolg von Theorien." (ebd.)

In diesem Sinne manövriert sich der wissenschaftliche Realismus in eine theoretische Sackgasse, weil die Verteidigung des abduktiven Arguments der besten Erklärung nicht überzeugend genug gelingt, so dass insgesamt von einem wissenschafts- und erkenntnistheoretisch sparsameren Wahrheits- und Wirklichkeitsverständnis ausgegangen werden sollte, was auch bedeutet, dass sich darüber das Zielverständnis wissenschaftlicher Praxis verändert, das der wissenschaftliche Realismus anbietet. Konkret meint das, dass die Zielbestimmung wissenschaftlicher Praxis, die im Realismus darin gesehen wird, zu wahren bzw. zu wirklichkeitsübereinstimmenden Theorien zu gelangen, reformuliert werden muss, so dass diese nicht auf Wahrheit und Wirklichkeit rekuriert.

\subsection{Die Rekonstruktion der kulturellen Konstitutionsbedingungen des Forschungsprogramms Biodiversität}

Eine solche Alternativkonzeption der Zielbestimmung wissenschaftlicher Praxis, die auch die externen Kritikpunkte am wissenschaftlichen Realismus aufnimmt, liefert Imre Lakatos "Methodologie der wissenschaftlichen Forschungsprogramme» (vgl. Lakatos 1982). ${ }^{70}$ Mit Lakatos kann davon ausgegangen werden, dass alle wissenschaftlichen Theorien, Konzepte und Modelle (und somit auch die für sie spezifischen Begriffe) als Forschungsprogramme betrachtet werden können (ebd., 4), die hinsichtlich ihrer Zielbestimmung „mit Poppers oberster heuristischer Regel [verbunden sind]: Erfinde Vermutungen, die höheren empirischen Gehalt besitzen als ihre Vorläufer.“ (ebd., 47) Mit Lakatos lässt sich der Fortschritt der Wissenschaft folglich nicht als eine kontinuierliche Annäherung an die Wahrheit oder Wirklichkeit verstehen, womit auch die Zielbestimmung wissenschaftlichen Handelns nicht in der Übereinstimmung mit der Wahrheit oder Wirklichkeit verstanden wird, sondern als eine Reihe „progressiver Problemverschiebungen":

„Wissenschaft lässt sich durch eine Reihe von aufeinander bezogenen Theorien kennzeichnen, wobei jedes neue Glied dadurch entsteht, dass man der voran-

70 Imre Lakatos versucht in seiner Methodologie wissenschaftlicher Forschungsprogramme die Probleme aufzulösen, die sich aus Poppers kritischen Rationalismus ergeben. Aufbauend auf Kuhn und dessen Kritik am Falsifikationismus, aber in Abgrenzung zur Kuhnschen Theorie wissenschaftlicher Revolutionen entwickelt Lakatos mit dem raffinierten methodischen Falsifikationismus einen eigenen Weg, indem er versucht die Kuhnsche und Poppersche Wissenschaftstheorie zu verbinden, wobei er aber mit Popper und gegen Kuhn an der Annahme eines rationales Fortschritts in der Wissenschaft festhält: „Bei genauem Hinsehen entpuppen sich sowohl Poppers experimenta crucis als auch Kuhns Revolutionen als Märchen, gewöhnlich ist es so, dass voranschreitende Forschungsprogramme an die Stelle von degenerierten treten." (Lakatos 1982:6) 
gegangenen Theorie bestimmte Hilfsklauseln hinzufügt (oder sie semantisch uminterpretiert), um sie an eine reale Anomalie anzupassen, wobei jede Theorie einen Gehalt besitzt, der dem nicht widerlegten Gehalt des Vorgängers entweder gleicht oder ihn übertrifft." (ebd., 33) ${ }^{71}$

Zu progressiven Problemverschiebungen kommt es Lakatos zu Folge immer dann, wenn neue Theorien einen epistemologischen und empirischen Gehaltsüberschuss gegenüber den vorangegangenen Theorien haben. Diese werden darüber erreicht, indem der sogenannte Schutzgürtel des Forschungsprogramms, der aus bestimmten Regeln besteht, wie das Forschungsprogramm entwickelt werden sollte (positive Heuristik) und was dabei zu vermeiden ist (negative Heuristik) (ebd., 46), durch Anomalien und adhoc-Modifikationen substanziell verändert wird, so dass die Heuristiken den harten Kern, der aus empirisch nicht begründbaren Prämissen über die allgemeine Beschaffenheit der Untersuchungsgegenstände besteht, gegen auftretende Anomalien immunisieren (ebd., 49) und vor der Falsifikation schützen kann (ebd., 47). Der harte Kern, der die zentralen Grundannahmen enthält, die das Forschungsprogramm charakterisieren und es von anderen Forschungsprogrammen unterscheiden (ebd. sowie Foucault (1971) 1991:11; Kuhn (1962) 1993:47), wird von den ForscherInnen als unverzichtbarer Kern der wissenschaftlichen Theorien, Konzepte und Modelle und Begriffe angesehen, da er die Grundüberzeugungen enthält, die nach Hesse konzeptioneller Art sind, weil sie sich auf die allgemeine Beschaffenheit der zu untersuchenden Gegenstände beziehen und bestimmen, welche Art von Fragen überhaupt gestellt und wie diese gelöst werden sollen (Hesse 2002:122).

Entscheidend für die folgenden Ausführungen ist dabei, dass sowohl die konzeptionellen Grundentscheidungen im harten Kern als auch die methodologischen Regeln im Schutzgürtel kulturspezifisch ausdifferenziert sind, da diese nicht zu allen Zeiten und unter allen Umständen gleich gesetzt werden können, sprich abhängig vom Stand der Erkenntnis, der Technologie, der Institutionalisierung wissenschaftlicher Praxen etc. sind (Eisel 2002:130; Hesse 2002:119, 122-125). In diesem Sinne befinden sich im harten Kern aller Forschungsprogramme die kulturellen Konstitutionsideen, die „den kulturellen Rahmen dafür liefern, was als vernünftige Weise der Bestimmung (natur)wissenschaftlicher Gegenstände gedacht und angestrebt werden kann" (Kirchhoff 2009: 47; Eisel 2002:131), die wiederum Teil einer rational rekonstruierbaren Ideengeschichte sind (Kirchhoff 2009:46). Im Schutzgürtel der Forschungsprogramme befinden sich wiederum die ebenfalls kulturell, weil lebensweltlich bedingten, methodologischen Konstruktionsbedingungen, die Regeln und Prinzipien für den Aufbau sowie die Arbeit mit und in den Forschungsprogrammen bereithalten.

71 In diesem Sinne können einzelne Theorien auch nie isoliert, sondern immer nur als Teile größerer Theoriensysteme und Methodologien beurteilt werden (Lakatos 1982:34). 
Dabei wird gemäß der externen Kritik am wissenschaftlichen Realismus (siehe 3.2.1) davon ausgegangen, dass wissenschaftliche Praxen auf lebensweltlichen Praxen beruhen und dass sich aus diesen die grundlegenden Unterscheidungen, die vor- und außerwissenschaftlich entwickelten Techniken sowie die Bedürfnisse entwickeln, auf welche die Ziel- und Zwecksetzungen der biowissenschaftlichen Forschungen bezogen sind. Eben diese kulturellen Konstitutionsbedingungen werden im Folgenden hinsichtlich der methodologischen Konstruktionsbedingungen für das Forschungskonzept der biologischen Vielfalt als auch hinsichtlich der geistes- und ideengeschichtlichen Konstitutionsideen für die Forschungsobjekte der abiotischen und biotischen Vielfalt detailliert dargelegt, um im Anschluss daran abschätzen zu können, ob die dabei zur Anwendung kommenden Ansätze zu einer progressiven Problemverschiebung in Bezug auf das Forschungsprogramm der Biodiversität führen. Dazu bieten sich zwei spezifische Rekonstruktionsansätze an: Zum einen der methodische Kulturalismus, über den die methodologischen Konstruktionsbedingungen, sprich das Forschungskonzept Biodiversität expliziert werden kann (3.3.1). Zum anderen können über den sogenannten Kulturkonstitutionalismus die geistes- und ideengeschichtlichen Konstitutionsideen, d.h. die Forschungsobjekte der Biodiversität, expliziert werden (3.3.2). Auf beide Ansätze, deren jeweilige Anwendung sowie deren Potenziale zu einer progressiven Problemverschiebung, wird im Folgenden eingegangen.

\subsubsection{Die Erschließung der methodologischen Konstruktionsbedingungen der biologischen Vielfalt}

Der aus dem Erlanger Konstruktivismus entwickelte "methodische Kulturalismus" (kurz mK) (vgl. insb. Janich, Kambartel \& Mittelstrass 1974; Janich 1992a,b; 1995; 1996; 1997; Gutmann 1998; Weingarten 1998; Janich \& Weingarten 1999; Janich 2000, 2006) ist für das Vorhaben die methodologischen Konstruktionsbedingungen der Konzeption der biologischen Vielfalt zu rekonstruieren ein im Besonderen geeigneter Ansatz, da dieser bereits auf die Biodiversität angewandt wurde (vgl. Barthlott \& Gutmann 2000; Janich \& Gutmann 2000; Gutmann \& Janich 2001a,b; Janich, Gutmann \& Prieß 2001). Allerdings ist er auch ein im Detail oft schwer verständlicher und in Bezug auf seine Ergebnisse recht abstrakt bleibender Ansatz: ${ }^{72}$

72 Da sich der methodische Kulturalismus $(m K)$ gegen die naturalistischen (szientistischen, biologistischen und/oder reduktionistischen) Tendenzen moderner Naturwissenschaften richtet, „um selbst die schwächste Form naturalistischer Thesen (...) als problematisch zu erweisen“ (Janich 1992b:9), müsste der mK eigentlich auch eine klare Definition dessen anbieten, was genau er dabei unter Naturalismus bzw. naturalistisch versteht. Dies ist jedoch der theoretische Schwachpunkt des mK, denn der Verweis darauf, dass „die Formen, in der dieser Naturalismus auftritt, vielfältig [sind] und ein breites Spektrum von naiven und plumpen bis hin zu höhst raffinierten und feinsinnigen Versuchen ab[decken], Natur und Naturerkenntnis nach demselben Muster zu beschreiben" (ebd., 11) reicht dafür nicht aus. Auch der Verweis darauf, dass „die Popularisierung und Aufbereitung moderner wissenschaftlicher Naturerkenntnis für den interessierten Laien ebenso naturalistisch erfolgt wie die Beschreibung von Naturerkenntnis durch solche Strömungen, die aus ökologischen, sozialpoliti- 
Unter der zugrundeliegenden Annahme, dass die vorherrschenden Strömungen und Ansichten in den Naturwissenschaften, aber auch der Philosophie deskriptivistischnaturalistisch ausgerichtet sind (Janich 1996:16, 2006:10), entwirft der mK ein kulturalistisches Verständnis von Wissenschaft, so dass auch die Naturwissenschaften als Kulturleistungen, d.h. als menschliches Handeln nach Zwecken und Zielen unter kulturellhistorisch bedingten Umständen, betrachtet werden (Janich 1992b:7). ${ }^{73}$ Dem mK geht es vor allem darum aufzeigen, dass sich die Naturforschung und damit die naturwissenschaftlichen Begriffe, Modelle, Methoden und Theorien aus den methodologischen Vorentscheidungen sowie den Zielsetzungen und Zweckbestimmungen wissenschaftlicher ProtagonistInnen ergeben (Gutmann \& Janich 2001a:329, 332). Dabei wird davon ausgegangen, dass die Begriffs- und Theoriebildung in den (Bio-)Wissenschaften als nach Erfolg und Misserfolg zu behandelnde zweckrationale Handlungen im Zusammenhang empirischer Forschung zu betrachten sind, die auf bestimmten lebensweltlichen Praxen beruhen, aus denen heraus sich die grundlegenden Unterscheidungen, die vor- und außerwissenschaftlich entwickelte Techniken sowie die Bedürfnisse entwickeln, auf welche die Ziel- und Zwecksetzungen der (bio-)wissenschaftlichen Forschungen bezogen sind (ebd., 298). Damit werden die (bio-)wissenschaftlichen Begriffe, Theorien, Konzepte und Modelle reflexiv in ein Zweck-Mittel-Schema gestellt, mit dem dann gefragt wird, zu welchen Zwecken und mit welchen Mitteln über Natur gesprochen wird, damit diese dann in ethischer Perspektive einem Rechtfertigungsdiskurs überantwortet werden können (Janich 1996:65). ${ }^{74}$

schen oder moralischen Gründen nach Alternativen in unserer naturwissenschaftlich-technischen Zivilisation suchen“ (ebd.) ist zwar hilfreich zur Verortung des Umfangs der 'Naturalisierung', sagt aber noch nichts darüber aus, was diesen ausmacht. Mit Holm Tetens kann davon ausgegangen werden, dass Naturalismus und Kulturalismus sich vor allem hinsichtlich der moralischen Frage gegenüberstehen, ob Menschen komplizierte, physikalische Maschinerien sind oder moralisch verantwortliche Vernunftwesen (Tetens 1992:115). Tetens stellt heraus, dass dieser Gegensatz auf der (vermeintlich) dualistischen Auffassung von Rede und technischer Intervention basiert, die aber keineswegs sich diametral gegenüberstehende oder kontradiktorische Auffassungen sein müssen: „Solange der Naturalismus nur behauptet: (a) Es gibt nur physische Verursachungen; (b) auch die Wirksamkeit symbolischer Interaktionen macht davon keine Ausnahme und solange der Kulturalismus nur behauptet: (a) Der Mensch wird zu dem, was er ist, durch Teilnahme an einer Kultur; (b) bei der Wirksamkeit kultureller Symbole kommt es auf deren Semantik an; (c) in den meisten Fällen können Rede und symbolische Interaktion nicht durch neurotechnische Interventionen ersetzt werden, so lange sind beide Positionen miteinander verträglich." (ebd., 118-119) Naturalistisch bzw. kulturalistisch sind also nur die Positionen zu nennen, die - in die eine oder andere Richtung - über diese Behauptungen hinausgehen.

73 Janich führt aus, dass auch in der Wissenschafts- und Erkenntnistheorie realistische Positionen vorherrschend sind, „die in ihren ontologisch-metaphysischen Resten derart fest etabliert sind, dass selbst sogenannte instrumentalistische Positionen innerhalb dieser Tradition noch realistisch-naturalistische Züge aufweisen. Sie verhindern dadurch, dass der Instrumentalismus der methodischen Konstruktivisten überhaupt adäquat verstanden wird." (Janich 1996:13)

74 Der $m K$ überschreitet damit, genau wie der Erlanger Konstruktivismus, die Grenzen einer bloß theoretischen, analytischen Philosophie und versucht mit den Mitteln der praktischen Philosophie das Wahrheitsproblem in den Wissenschaften mit dem Rechtfertigungsproblem von Handlungsvollzügen und -folgen zu verknüpfen (Janich 1996:65). 
Hierbei wird zugleich mitverhandelt, was ein korrekter Vollzug von Beobachtung als Handlung ist, was das Ziel und der Zweck einer Beobachtungshandlung ist, worin genau ihr Handlungscharakter liegt und was eine erfolgreiche oder gelingende Beobachtung von einer erfolglosen oder misslingenden unterscheidet (Janich 1992b:172): Beobachtungen sind in diesem Ansatz teilnehmende Herstellungshandlungen (poietische Handlungen). Solche Handlungen sind gegenüber dem Verhalten durch die Bestimmung präzisiert, dass über Handlungen analytische Zwecke und synthetische Ziele verfolgt werden (Janich 1996:33). Darüber wird zwischen zwei Sorten von Erfahrung unterschieden: Bei analytisch zweckbestimmten Handlungen lässt sich nur nachträglich (nämlich anhand der Handlungsresultate) von einer Erfahrung sprechen, da das Gelingen der Handlung davon abhängt, ob das Handlungsschema hinreichend korrekt ausgeführt worden ist. Im Fall synthetisch zielbestimmter Handlungen macht sich die Erfahrung daran fest, ob der Handlungsvollzug soweit kontrolliert werden kann, dass er beherrschbar, sprich frei von äußeren Störungen ist (ebd., 34-36). Damit geht sowohl die Unterscheidung zwischen dem Gelingen/Misslingen einer Beobachtung als Handlungsresultat und dem korrekten/nicht korrekten Durchführen einer Beobachtung als Handlungsvollzug einher (Janich 1992b:171) als auch bestimmte Prinzipien der methodischen Ordnung, da Herstellungshandlungen, um erfolgreich sein zu können, in einer nicht beliebigen Reihenfolge vorgenommen werden müssen (Janich 1996:36-38).

Ohne weiter auf diese Unterscheidung eingehen zu wollen, sind diese für den mK notwendig, um darzulegen, wie WissenschaftlerInnen in Abhängigkeit von einer vor- und außerwissenschaftlichen lebensweltlichen Praxis ihre Forschungskonzepte konstruieren (Gutmann \& Janich 2001a:336). Um die Gegenstandskonstruktion zu erschließen, wird im mK eine protobiologische, d.h. begriffliche Rekonstruktion (Gutmann 1998:69) derjenigen biologischen Theoriestücke, vollzogen, deren unklarer methodologischer Status zahlreiche der identifizierten Probleme mit dem Begriffs- und Konzeptverständnis der Biodiversität verursachen. Dabei wird die biotheoretische Diskussion im mK um eine wissenschafts- und erkenntnistheoretische Reflexion ergänzt (Janich \& Weingarten 1999:24). Über diese soll die Reichweite und die Geltung biowissenschaftlicher Resultate für die Praxis bestimmt werden, indem die Adäquatheit, Vollständigkeit und Legitimität der Naturbeschreibung anhand ihrer praktischen Verwertbarkeit bewertet wird (Janich \& Gutmann 2000:71). Dabei wird ein wissenschaftstheoretischer „Anfang von unten" gewählt, aus dem sich drei Ebenen der methodischen Ordnung ergeben die Ebene der Gegenstandskonstitution, die Ebene der Gegenstandskonstruktion und die Ebene der Gegenstandsreflexion (vgl. ausführlich Janich 1995): ${ }^{75}$

75 Auf Friedrich Kambartel geht die Unterscheidung zwischen Wissenschaftstheorie »von oben « oder „von unten « zurück (Janich, Kambartel \& Mittelstrass 1974:90-94). Eine wissenschaftstheoretische Annäherung "von oben « setzt bei den jeweils jüngsten Resultaten in Form wissenschaftlicher Theorien an und unterstellt deren historisch-relative Geltung. Eine Annäherung »von unten« versucht 
Zur Gegenstandskonstitution gehört die Ausweisung, dass die Gegenstände der Wissenschaften durch vor- und außerwissenschaftliche, also lebensweltliche Praxen konstituiert werden, während bei der Gegenstandskonstruktion darauf geschaut wird, wie diese verwissenschaftlicht werden, sprich welche Terminologien, Methoden, Theorien und Konzepte daraus entwickelt werden. Die Reflexion - als Reflexion zweiter Ordnung und damit als Begründungsreflexion (siehe 1.1) - fokussiert schließlich darauf, welche Ansprüche FachwissenschaftlerInnen jeweils an die Wissenschaftlichkeit ihrer Produkte und Resultate stellen und welche Geltungsansprüche dabei erhoben werden. Die Ergebnisse der Gegenstandsreflexion, die hier terminologisch als Rekonstruktion gefasst wird, bleiben, wie gesagt, recht abstrakt, auch wenn sie mit Bezug auf Biodiversität eine gewisse Konkretisierung erfahren. Diese sollen hier nur noch einmal prägnant mit Bezug auf die Biodiversität zusammengefasst werden, da viele Aspekte bereits im Vorhergehenden, in Bezug auf die externe Kritik am wissenschaftlichen Realismus, (3.2.1) ausgeführt wurden:

"Die Bestandsaufnahme der Behandlung des Themas "Biodiversität» in den mit diesem Phänomen primär befassten Biowissenschaften fördert zunächst eine erhebliche Divergenz an Wahrnehmungen, Begriffen und theoretischen Systematisierungen zu Tage. Im Interesse der praktischen Ziele einer Politik der Erhaltung der Vielfalt des Lebendigen kann man diese Divergenzen nicht einfach auf sich beruhen lassen. Vielmehr gilt es, Unklarheiten in Bezug auf die adäquate Beschreibung der mit »Biodiversität» gemeinten (...) Güter durch interdisziplinäre Anstrengungen zu überwinden, um der politischen und rechtlichen Diskussion eine ausreichende Grundlage zu geben. Diesbezügliche wissenschaftliche Unternehmen stehen erst am Anfang ihrer Entwicklung." (Janich 2001:XXIX)

Allerdings muss dabei in Rechnung gestellt werden, dass die Meinungsverschiedenheiten und Kontroversen innerhalb der Biowissenschaften nicht wesentlich auf innerbiologische Fragen bezogen sind, die etwa empirisch im Labor, im Freilandversuch oder in unberührten Naturstücken entscheidbar wären, sondern diese lassen sich vor allem auf methodologische Differenzen zurückführen (Gutmann \& Janich 2001a:329):

„Das grundlegende Problem ist - unabhängig von der Zahl der beteiligten Disziplinen - kein 'biologisches'. Es wird nicht von 'der Natur' oder 'den Lebewesen', sondern den beteiligten Forschern durch die Wahl von Fragen und die Verfahren ihrer Beantwortung entschieden." (Gutmann \& Janich 2001b:8)

dagegen, aus vor- und außerwissenschaftlichen und in diesem Sinne lebensweltlichen Verhältnissen einen Aufbau bis zum heutigen Kenntnisstand methodisch zu rekonstruieren (Janich 1996:53-54). Auffälligster Unterschied zwischen beiden Herangehensweisen ist, dass bei der logischen Analyse bereits klar ist, was das zu Analysierende und Reformulierende ist, während im methodischen Kulturalismus gerade dies zum Problem gemacht wird, indem gefragt wird, „ob das historisch Vorfindliche auch das methodisch Begründete und damit das Rekonstruierbare ist, oder ob sich darin kritikbedürftige Teile finden." (ebd., 59) 
In diesem Sinne lässt sich zusammenfassend festhalten, dass die Begriffsbestimmungen in allen Wissenschaften und damit auch in den Biowissenschaften nicht eindeutig gefasst werden können, weil dahinter unterschiedliche Ziel- und Mittelvorstellungen der ForscherInnen stehen. Es kommt daher auch nicht zufällig zu den erheblichen 'Spielräumen' bei der Modellierung und Theoretisierung sowie Operationalisierung und Messung der Biodiversität, was wiederum Spielräume für maßgebliche Differenzen bei der Bewertung und Beurteilung der Relevanz der biologischen Vielfalt lässt. Da die zentralen biowissenschaftlichen Forschungsgegenstände der Biodiversitätsdebatte (genetische Vielfalt, Artenvielfalt und Ökosystemvielfalt) zudem keine natürlichen Gegenstände sind, wird erst über eine wissenschafts- und erkenntnistheoretische Reflexion dieser Untersuchungsgegenstände ersichtlich, dass die deskriptivistisch-naturalistischen Sprachgebräuche immer dann ein pragmatisches und zugleich geltungstheoretisches Problem mit sich führen, wenn aus einer realistischen Perspektive heraus entschieden werden soll, welche der zahlreichen biowissenschaftlichen Erklärungsansätze nun die beste und damit wahrste Erklärung für die Zustände, Eigenschaften und Prozesse der biologischen Vielfalt liefert. Da es in allen wissenschaftlichen Disziplinen immer konkurrierende, aber gleichsam wissenschaftlich exakte Erklärungsansätze und Theorien gibt, die Erklärungen für Zusammenhänge und Zustände anbieten, können diese über das Argument der besten Erklärung nicht gegeneinander ausgespielt werden, weil die physische Wirklichkeit zugleich mit all diesen (Teil-)Phänomenen annähernd übereinstimmen kann und damit alle diese Theorien als annähernd wahr gelten können. Hierbei wird übersehen, dass auch für den Entdeckungszusammenhang in den Naturwissenschaften die Leistung zweckrationalen menschlichen Handelns konstitutiv ist, weil transsubjektive Aussagen über die natürliche und die künstliche Wirklichkeit immer auf die normative Bereitstellung und kulturelle Akzeptanz sprachlicher wie nicht-sprachlicher Mittel angewiesen sind (Janich 1996:16-17).

Konstruktiv betrachtet, lassen sich daraus drei methodologische Regeln im Umgang mit dem Forschungskonzept der biologischen Vielfalt gewinnen (Gutmann \& Janich 2001b: 7-8), über deren Beachtung sich viele der in der Grundlagenreflexion dargelegten begrifflich-konzeptionellen Problematiken (siehe 2.1) konstruktiv angehen lassen, so dass die Anwendung des $\mathrm{mK}$ auf das Forschungskonzept der biologischen Vielfalt zu einer progressiven Problemverschiebung führt, weil der Umgang mit dem Forschungskonzept der biologischen Vielfalt geltungstheoretisch adäquater gestaltet werden kann:

1) Zum einen müssen kontrollierbare Maßstäbe der Unterscheidbarkeit lebender Organismen entwickelt werden, denn wenn „»etwas« (...) von »jemandem« unter Verwendung bestimmter »Mittel« (Kriterien) nach Maßgabe bestimmter "Zwecke« (Unterscheidungsabsichten) als von etwas anderem "variierend" (verschieden) bestimmt" (ebd., 7) werden soll, dann muss auch deutlich gemacht werden, wer mit welchen Mit- 
teln und Zielen wie und warum, welche Unterscheidungen trifft. Nur auf diesem Weg kann nachvollziehbar und kontrollierbar überhaupt von Diversität gesprochen werden.

2) Zum anderen müssen Adäquatheitskriterien für die jeweiligen Unterscheidungen und Beschreibungen der genetischen Vielfalt, der Artenvielfalt und der Ökosystemvielfalt entwickelt werden, denn nur wenn deutlich wird anhand welcher Standards die vorgenommenen Unterscheidungen und Beschreibungen korrekt bzw. unkorrekt bzw. die Beschreibungsergebnisse gültig bzw. ungültig sind, erlauben diese auch die Abschätzung möglicher Maßnahmen zur Bewahrung und Förderung der Biodiversität.

3) Dazu bedarf es drittens (auch) von Seiten der BiowissenschaftlerInnen eines offenen und kritischen Umgangs damit, dass die üblichen Definitionen der biologischen Einheiten zumindest unvollständig sind, denn erst dann können alle Akteure der Biodiversitätsdebatte sich dem Umstand stellen, dass es keine einheitliche Biodiversitätskonzeption und kein einheitliches biowissenschaftliches Grundlagenwissen zur Anwendung der Biodiversitätskonzeption in anderen wissenschaftlichen Disziplinen sowie gesellschaftspolitischen Zusammenhängen gibt. Hierüber steigen indes die Chancen, dass Biodiversität auch substanzielle epistemische Gehalte erlangt.

\subsubsection{Die Erschließung der geistes- und ideengeschichtlichen Konstitutionsideen der biotischen und abiotischen Vielfalt}

Selbst wenn diese drei Regeln indes in Bezug auf das Forschungskonzept der biologischen Vielfalt beachtet werden, bleibt nach wie vor unklar, worauf genau sich die methodologischen Regeln beziehen, sprich was die konstitutionellen Grundideen im harten Kern des Forschungsprogramms Biodiversität sind. Eben diese versucht der »Kulturkonstitutionalismus« (kurz Kk) aufzuzeigen. ${ }^{76}$ Dieser ist, was die Beleuchtung der geistes- und ideengeschichtlichen Aspekte der kulturellen Konstitution angeht, ein geeigneter, weil oftmals auf Umwelt- und Naturschutzthematiken bezogener (vgl. insb. Eisel 1992, 1997, 2002; Körner \& Eisel 2003; Eisel 2004, 2007, 2009a, 2009b) sowie speziell auf Biodiversität fokussierender (Kirchhoff \& Trepl 2001; Kirchhoff 2005; Kirchhoff \& Haider 2009), methodisch und begrifflich aber (leider) oftmals nicht sonderlich elaborierter Ansatz. Auch deshalb wird im Folgenden wieder erst einmal auf den Ansatz eingegangen, bevor dessen Anwendung auf die abiotische und biotische Vielfalt dargelegt wird. Auch hier wird es wieder darum gehen, das Potenzial des Ansatzes für eine progressive Problemverschiebung abzuschätzen:

76 Was hier Kulturkonstitutionalismus (Kk) genannt wird, wird bei dessen Vertreterlnnen selber als 'konstitutionstheoretische Position ' bezeichnet, was aber in Anbetracht der Gefahr der Verwechslung mit der transzendentalphilosophischen Konstitutionstheorie Kants irreführend ist, da es ihnen nicht um die Bedingungen der Möglichkeit von Erkenntnis überhaupt sondern nur um die spezifischen Bedingungen wissenschaftlicher Erkenntnis als sozialkultureller Praxis geht. Daher soll hier von Kulturkonstitutionalismus die Rede sein. 
Unter der zugrundeliegenden Annahme des Kk, dass sich in der Biologie und Ökologie geistes- und ideengeschichtliche Analogien politischer Philosophien zu den Organismen und Systemen und deren intra- und interspezifische Beziehungen finden lassen, wird die Begriffs- und Theoriebildung in den Biowissenschaften im Kk (immer auch) auf bestimmte Konstitutionsideen über das Wesen des Menschen und seine Vergesellschaftung zurückgeführt, die in politischen Philosophien artikuliert werden und die auf das Verständnis von Organismen und ökologischen Systemen übertragen werden. Damit wendet der Kk sich der Erklärung des Umstandes zu, dass kulturell bedingte Ideen, Ideale, Motivationen, Norm- und Wertevorstellungen in die Naturwissenschaften hineinwirken und dort in Gestalt naturwissenschaftlicher Theorien reformuliert werden, so dass diese dann wechselseitig die kulturellen Ideen und Zwecksetzungen beeinflussen, die über die naturwissenschaftlichen Forschungen abgestützt und legitimiert werden (Kirchhoff 2009:54; Eisel 2002:140). ${ }^{77}$ Ausgehend von der dauerhaften Existenz konkurrierender Schulen in verschiedenen Teildisziplinen der Biowissenschaften vertritt der Kk die These, dass es solange kein Ende der grundsätzlichen Kontroversen in den Biowissenschaften geben wird, solange es konkurrierende kulturelle Konstitutionsideen in Hinblick auf die Bestimmung der zentralen Forschungsobjekte gibt (Kirchhoff 2009:6, 21). Dem Kk geht es folglich darum aufzuzeigen, dass es in Form kultureller Konstitutionsideen Analogien zwischen politischen Philosophien und biowissenschaftlichen Theorien und Modellannahmen gibt und dass diese wiederum helfen können, biowissenschaftliche Begriffe, Theorien und Konzepte zu klassifizieren und typisieren.

Was im Kk unter kulturellen Konstitutionsideen genau verstanden wird, erschließt sich am besten ideologietheoretisch: ${ }^{78}$

77 Da es auch hier nicht um eine detaillierte kritische Auseinandersetzung mit dem Kulturkonstitutionalismus gehen kann und soll, soll an dieser Stelle lediglich angemerkt werden, dass die im Kk postulierte „Inspiration der Wissenschaft durch Weltbilder“ (Eisel 2002:130) darüber hergestellt wird, dass Analogien zwischen den Auffassungen biowissenschaftlicher Theorien und politischer Philosophien aufgezeigt werden, die dann für die Typisierung biologischer und ökologischer Theorien verwandt werden (Voigt 2009:332). Dabei ist dann indes entscheidend, wie genau das Verhältnis zwischen biowissenschaftlichen Theorien und politischen Philosophien bestimmt wird. Dies geschieht im Kk nicht immer einheitlich und bleibt oft unklar, was bedauerlich ist, da derartige Erklärungsansätze weder neuartig sind, noch entbehren sie einer rationalen Grundlage, wie vor allem die von Marx herausgestellten Parallelen zwischen Liberalismus und Darwinismus zeigen (vgl. Marx \& Engels (1862) 1974: 249).

78 Entgegen den weit verbreiteten pejorativen Verständnissen von Ideologie als blinde Irrationalität, pathologisch verzerrte Kommunikation, Illusion, Verzerrung oder Mystifikation (Eagleton 2000:9) werden Ideologien, wenn sie denn in erster Linie analytisch-funktional betrachtet werden, wie das in den soziologischen Ideologietheorien durchgehend der Fall ist, nicht als Gegensatz zur Rationalität aufgefasst, sondern ganz im Gegenteil als rationale (wenn auch nicht immer intendierte) Gedankenkonstrukte verstanden, die Erfahrung konstituieren und zugleich das Ergebnis dieser ist (Freeden 1998: 4). Damit erfüllen Ideologien spezifische soziale Funktionen: Sie sollen Andere überzeugen, motivieren, mobilisieren und Ordnung und Übersicht angesichts komplexer und zumeist unüberschaubarer Verhältnisse schaffen und sind damit automatisch mit Wert- und Machtfragen verbunden (ebd., 6). Über diese „Denkstile“ (vgl. Fleck 1980) wird folglich festgelegt, was innerhalb eines Denkkollektivs überhaupt als wissenschaftliches Problem, evidentes Urteil oder angemessene Methode gilt. Auch 
Ideologien können dabei mit Seliger als eine Menge von Ideen verstanden werden, „durch die man Mittel und Ziele organisierter gesellschaftlicher Handlungen [u.a. Wissenschaft] postulieren, erklären und rechtfertigen kann." (Seliger 1976:11) Insofern haben kulturelle Konstitutionsideen ein ideologisches Moment, da sie sich aus unterschiedlichen Menschen-, Gemeinschafts-, Natur- und Gesellschaftsbildern sowie Welt-, Wert- oder Wissenschaftsverständnissen zusammensetzen, die als solche keine widerspruchsfreien, aber dennoch theoretisierte Ideenzusammenhänge darstellen (Eisel 2002:132), die sich aus intersubjektiven Problemformulierungen historisch entwickelt und ausdifferenziert haben (ebd., 132). In diesem Sinne wird im Kk auf das gemeinsame Moment von Ideologien und Theorien verwiesen: Theorien und Ideologien verfolgen gleichsam das Ziel die immanenten Widersprüche und Anomalien der eigenen Weltanschauung und Überzeugung respektive der eigenen Theorien, Disziplinen oder Schulen progressiv zu verschieben, wenn sie dies auch mit unterschiedlichen Mitteln tun und in unterschiedlichem Maße erreichen. Insofern bestehen sowohl Ideologien als auch wissenschaftliche Theorien aus inkommensurablen Paradigmen als auch im Paradigma miteinander vereinbare Ansätze (ebd., 132), die sich aus den kulturell geprägten Vorstellungen von der Natur, dem Menschen und seiner Stellung in der Welt sowie seinen Verhältnissen zu anderen Menschen und der Umwelt ergeben. Diese Denkstile sind indes nicht beliebig, sondern kulturhistorisch geprägt und rational zugänglich, da sie geistes- und ideengeschichtlich sowie naturphilosophisch rekonstruiert werden können (Kirchhoff 2009:46).

Werden diese rekonstruiert, dann erfolgt dabei eine nicht ausschließlich terminologische Verschiebung von der Analyse der Vielfalt der belebten Natur, sprich einer biowissenschaftlich aber auch wissenschaftstheoretisch geprägten Analyse der Biodiversität hin zur Analyse der Vieldeutigkeit der belebten Natur, d.h. einer geistes- und ideengeschichtlichen Analyse der Variationsbreite der Ideen, die hinter Biodiversität stehen. Diesbezüglich stellen vor allem Thomas Kirchhoff und Sylvia Haider in ihrem Artikel "Globale Vielzahl und lokale Vielfalt - zur kulturellen Ambivalenz von 'Biodiversität`“ und Annette Voigt in ihrem Artikel „ 'Wie sie ein Ganzes bilden ' - Analoge Deutungsmuster in ökologischen Theorien und politischen Philosophien der Vergesellschaftung " heraus, dass sich die unterschiedlichen Verständnisse davon, was eine biotische Ge-

was als Wahrheit gilt, hängt vom Denkstil ab und kann nur in der stilgemäßen Auflösung von Problemen bestimmt werden: „Wahrheit (...) ist nicht "relativ« oder gar "subjektiv« im populären Sinne des Wortes. Sie ist immer oder fast immer, innerhalb eines Denkstils, vollständig determiniert. Man kann nie sagen, derselbe Gedanke sei für A wahr und für B falsch. Gehören A und B demselben Denkkollektiv an, dann ist der Gedanke für beide entweder wahr oder falsch. Gehören sie aber verschiedenen Denkkollektiven an, so ist es eben nicht derselbe Gedanke, da er für einen von ihnen unklar sein muss oder von ihm anders verstanden wird." (Fleck 1980:131) In diesem Sinne können Begriffe wie Weltbild, Denkstil, Leitbild oder aber Ideologie als analog zu den kulturellen Konstitutionsideen verstanden werden. 
meinschaft ist und was sie ausmacht, auf unterschiedliche ideengeschichtlich auffindbare Auffassungen darüber zurückführen lassen, wie sich organismische 'Gesellschaften 'bilden, welcher 'Zweckbestimmung' sie unterliegen und wie sich organismische (biotische) und systemische (abiotische) Verhältnisse entwickeln. Indem unterschiedliche Auffassungen darüber das Verständnis von Organismen und ökologischen Systemen prägen, lässt sich idealtypisch zwischen a) einem Ganzheitlichkeitsverständnis und b) einem Partikularverständnis der (a)biotischen Vielfalt unterscheiden. Beide Verständnisse werden im Folgenden als Ansatzpunkte möglicher geistes- und ideengeschichtlicher Biodiversitätsverständnisse dargelegt:

a) Das Ganzheitlichkeitsverständnis ergibt sich den VertreterInnen des Kk zu Folge aus einer Auffassung, die sich, so Kirchhoff und Eisel, ideengeschichtlich auf das konservative Weltbild und mithin die Monadologie von Leibniz zurückführen lässt, die wiederum für die gegenaufklärerische Kulturtheorie und Geschichtsphilosophie Herders von besonderer Relevanz ist (vgl. Eisel 1992, 1997; Kirchhoff 2005, 2009). Vor diesen Hintergründen führen sie aus, warum und inwiefern die monadologische Individualitätsund Systemauffassung die primäre ideengeschichtliche Grundlage für die moderne Auffassung vom einzelnen Organismus in der Biologie bildet, „die wohl paradigmatisch für alle anderen biologischen Theorien organischer Systeme ist, so sehr diese auch im Einzelnen abweichen mögen." (Kirchhoff 2009:500) Zugleich liefert die monadologische Theorie, so die Auffassung des Kk, ein Modell für biologische Theorien überorganismischer Einheiten (ebd.). Dieser Auffassung nach sind Individuen notwendigerweise in Gemeinschaften eingebunden, in denen sie in funktionalen, zumeist obligatorischen Abhängigkeiten zueinander stehen (Voigt 2009:336). Die universelle Ordnung, in der alle Geschöpfe miteinander verbunden sind, beruht dabei auf der Individualität jedes Einzelnen. Die abgegrenzte und in diesem Sinne eigenständige Individualität bildet dabei die gemeinsame Verknüpfung, so dass die biotische Gemeinschaft aus einer emergenten Vielfalt unterschiedlicher Geschöpfe (Monaden) besteht, die zusammen ein harmonisches Ganzes und eine überindividuelle Ganzheit bilden (Kirchhoff \& Haider 2009:322). Diese Denkfiguren lassen sich, so Eisel, Kirchhoff und Voigt unisono, aus den Annahmen des politischen Konservatismus ableiten, der davon ausgeht, dass Individuen notwendigerweise ungleich seien, dass aber der Zweck dieser Ungleichheit darin liegt, dass aus dieser Unterschiedlichkeit unterschiedliche Aufgaben in und für die Gemeinschaft erwachsen (Voigt 2009:336).

In der Ökologie erscheinen diese Zusammenhänge vor allem in organizistisch-synökologischen Theorien (ebd., 332), die den Fokus auf den Umstand legen, dass sich Organismen wechselseitig ihre Existenz ermöglichen, insofern sie alle zur Bildung von Funktionseinheiten des Systems beitragen. Ökologische Systeme werden daher als funktionale Ganzheiten betrachtet, da sie zu ihrer Entwicklung und Selbsterhaltung auf die 
Existenz der in ihnen vorkommenden Art oder jeder funktionalen Artengruppe sowie auf deren eigene Zusammensetzung und Ordnung angewiesen sind (ebd., 340). Da die Organismen zueinander in obligatorischer Abhängigkeit stehen und konstitutiv für das System sind, bringt sich das System selbst hervor, erhält, entwickelt und grenzt sich autopoietisch ab. Ihre Sukzession verläuft 'zielgerichtet' und stadienhaft, indem frühere Stadien die Bedingungen für die nachfolgenden Stadien schaffen, so dass sich das System kontinuierlich immer besser an seine Umwelt anpasst (ebd., 340). Die abiotische und biotische Vielfalt wird vor diesem Hintergrund folglich als harmonische Einheit gedacht und stellt zugleich ein qualitatives $M a \beta$ dafür dar, wie einzigartig das Leben in lokale, überindividuelle Lebenseinheiten differenziert ist und wie sehr diese Einheiten wiederum intern, sprich individuell, differenziert sind (Kirchhoff \& Haider 2009:325). Das spezifische Wesen des Ganzen wird daher über seine Eigenart realisiert und herausgestellt, die sich wiederum daraus ergibt, dass die Individuen Zwecke in der Gemeinschaft für die Gemeinschaft erfüllen, die ihnen die Existenz ermöglichen, so dass das Ganze selbst als einzigartig und selbstzweckhaft betrachtet wird (Voigt 2009:336).

Übertragen auf die Biodiversität bedeutet dies, dass über die Ganzheitlichkeitskonzeption die biotische Vielfalt als organismische Gemeinschaft verstanden wird (Kirchhoff \& Haider 2009:325). Für eine solche organismische Gemeinschaft gilt, dass jede Komponte einen absolut notwendigen Beitrag zur Reproduktion des Gesamtsystems leistet und dass jedes Individuum sich nur als Komponente des Systems reproduzieren kann (Kirchhoff 2009:427). Insofern sind organismische Gemeinschaften funktional und hierarchisch strukturiert und bestehen aus einer nicht beliebigen Vielfalt von zweckmäßig angeordneten Organismen. Als substanzielles Einzelnes, sprich als Organismus, zu existieren bedeutet dann, sich dem Wesen nach von anderen Organismen zu unterscheiden und doch mit ihnen wesensmäßig verbunden zu sein (ebd., 473). Erst das spezifische Zusammenspiel aller Komponenten bringt folglich biotische Vielfalt hervor, da diese sich selbst erhalten und entwickeln und voneinander abgrenzen. In diesem Sinne kann nur das als Entwicklung realisiert werden, was in der Gemeinschaft und in ihren äußeren Bedingungen als Möglichkeit und Ziel angelegt ist (Voigt 2009:337). Die Bewahrung und Förderung der gesamten abiotischen und biotischen Vielfalt erscheint hier vor allem deshalb erstrebenswert, weil dies der Aufrechterhaltung einer harmonischen Einheit dient (Kirchhoff \& Haider 2009:325), so dass alle Elemente der biotischen Vielfalt aufgrund ihrer Funktion für das Ganze 'um ihrer selbst willen`erhalten werden sollen, weil deren Einzigartigkeit und gegenseitige obligatorische Abhängigkeit das System als solches erst ermöglichen und hervorbringen.

b) Das Partikularverständnis ergibt sich, so die Darlegungen der VertreterInnen des Kk, demgegenüber aus einer der liberalistischen Auffassung, die sich ideengeschichtlich auf die Kosmologie von Ockham zurückführen lässt (Kirchhoff 2009:321). Eisel, Kirch- 
hoff und Voigt führen diesbezüglich aus, dass die bestehende Welt, folgt man Ockham, eine in Umfang und Zusammensetzung beliebige Menge wesentlich individueller und in ihrem Wesen voneinander unabhängiger Geschöpfe ist, in der alles Individuelle nichts außer sich selbst repräsentiert, weil eine universelle Ordnung nicht existiert (Kirchhoff \& Haider 2009:321). Da den Individuen ihre Eigenschaften prizipiell unabhängig von anderen Individuen zukommen, ist die Struktur des Ganzen durch die Interaktionen der Individuen bestimmt. Diese Interaktionen sind im Wesentlichen durch die Konkurrenz um knappe Ressourcen charakterisiert (Voigt 2009:335). Dieses kompetitive und exploitative Verhältnis der Individuen zueinander, lässt sich aus den Annahmen des politischen Liberalismus (präziser! Libertarismus) ableiten, der davon ausgeht, dass Individuen egoistische Nutzenmaximierer sind, die nur deshalb Kooperationen eingehen, weil sie ihre eigene Stellung im System und ihre Nutzung von Ressourcen verbessern wollen. Dieser Auffassung nach ist das Ganze nur aus der Perspektive des Einzelnen nützlich und hat keinen Zweck an sich (ebd., 335). Daher kann es anders als im konservativen Weltbild auch keine unmittelbaren oder mittelbaren kausalen Beziehungen zwischen den einzelnen Komponenten und dem System selbst geben, die seine Zusammensetzung verursachen (Kirchhoff 2009:90), so dass alle Elemente eines Systems nur dadurch aufeinander bezogen werden, dass sie parallel zueinander existieren.

In der Ökologie erscheinen diese Zusammenhänge vor allem in Gestalt individualistisch -synökologischer Theorien (Voigt 2009:338). Individualistisch-synökologische Ansätze gehen davon aus, dass die Einzelorganismen nicht von anderen Organismen oder übergeordneten Einheiten abhängig sind, auch wenn die Ansprüche der einzelnen Organismen von der Umwelt, zu der ja auch andere Organismen gehören, erfüllt werden müssen (ebd., 339). Da jeder Organismus im Rahmen der Möglichkeiten seiner individuellen Eigenschaften agiert, reagieren diese lediglich auf äußere Bedingungen und Umstände und 'streben ' danach ihre Selbsterzeugung und -erhaltung zu 'optimieren`. Die Systembildung entsteht lediglich abgeleitet durch notwendige und nicht vermeidbare Interaktionen zwischen den Einzelorganismen durch Konkurrenz, die Nutzung anderer Individuen als Ressource (Beweidung, Prädation oder Parasitismus) sowie symbiotische Nutzenbeziehungen (Mutualismen) (ebd., 339). Das System ist folglich darüber charakterisiert, welche und wie viele Organismen es 'beherbergt', ob und wie es deren Erfordernisse erfüllt und letztendlich welche Organismen sich in der Konkurrenz durchsetzen können (ebd.). Die Sukzession wird dabei als offen und unabgeschlossen angesehen, da jederzeit veränderbar ist, welche Organismen interagieren und welche Ressourcen sie jeweils nutzen können. Vielfalt ist in diesem Kontext folglich die bloße Aggregation einzelner Organismen und stellt zugleich ein rein quantitatives $M a \beta$ dar, wie einzelne Organismen auf der Basis zufälliger Ähnlichkeiten in einen nominalistischen Ordnungszusammenhang gebracht werden (Kirchhoff \&Haider 2009:325). 
Übertragen auf Biodiversität bedeutet dies, dass die biotische Vielfalt hier als die quantitative Anzahl der lokalen, regionalen und global vorhandenen Arten verstanden wird (ebd., 325). Die Auffassung von Vielfalt als Vielzahl entspricht dabei einer elementaristischen Sichtweise, derzufolge die Welt kein überindividuelles System ist, sondern eine beliebig zusammengesetzte Menge von Elementen, so dass jederzeit Elemente zu ihr hinzukommen oder entfernt werden können (ebd.). Die Bewahrung und Förderung der abiotischen und biotischen Vielfalt ist in diesem Zusammenhang vor allem deshalb erstrebenswert, weil die (a)biotische Vielfalt als Fülle an menschlichen Spielräumen und Handlungsmöglichkeiten sowie als materielle, kognitive, spirituelle und/oder kulturelle Ressourcen zu bewahren und zu fördern sind, wobei es hierbei vor allem um die Bewahrung des Nutzens der Biodiversität für den Menschen geht. Zusammenfassend kann auf Grundlage dieser sicherlich nur stichpunktartigen Ausführungen zu den beiden idealtypisch unterschiedenen Verständnissen der abiotischen und biotischen Vielfalt, die der Kk anbietet, konstatiert werden, dass über die hier aufgezeigten konservativen und liberalistischen Denkfiguren die abiotische und biotische Vielfalt entweder als ganzheitlicher Sinnzusammenhang oder aber als bloßes Aggregat einzelner, miteinander nicht kausal verbundener Elemente zu einer Vielzahl erscheint. Auf Basis dieser Darlegungen lässt sich zwischen einem weitem und einem engen Verständnis der Biodiversität unterscheiden:

Das weite Biodiversitätsverständnis folgt dem konservativen Ganzheitlichkeitsverständnis und führt darauf, dass die biotische Vielfalt hier sowohl die Vielfalt der Lebensformen als auch um die Vielfalt ihrer Lebensbedingungen umfasst, weil beides zusammen eine Einheit bildet, sprich die biotische Vielfalt nicht ohne die Vielfalt der abiotischen Lebensbedingungen betrachtet werden kann. Das enge Biodiversitätsverständnis hingegen ergibt sich aus dem liberalen Partikularverständnis und impliziert, dass die biotische Vielfalt auch unabhängig von den abiotischen Lebensbedingungen betrachtet werden kann, da hier nur die Vielzahl der Lebensformen als solche relevant ist. Je nachdem, ob das enge oder das weite Verständnis biotischer Vielfalt - sowohl für das Konzept der biologischen Vielfalt als auch in Bezug auf Anwendungsfragen im Umweltund Naturschutz - zugrunde gelegt wird, hat dies Auswirkungen sowohl auf die Art und Weise, wie Biodiversität als Forschungskonzept konstruiert wird als auch darauf welche Ziele und Maßnahmen seitens des Umwelt- und Naturschutzes für sinnvoll und zielführend erachtet werden und welche Gründe, Argumente und Begründungsansätze angeführt werden müssen, um die Ziele und Maßnahmen zu plausibilisieren.

Je nachdem welcher Deutungshorizont folglich hinter dem Verständnis biotischer Vielfalt steht, ergeben sich ganz unterschiedliche Betrachtungen und Bewertungen der Zustände, Prozesse und Eigenschaften lebender Systeme. Insofern kann hier festgehalten werden, dass die geistes- und ideengeschichtliche Rekonstruktion der kulturellen Kon- 
stitutionsbedingungen der Biodiversität, die der Kk vornimmt, durchaus zu einer progressiven Problemverschiebung führen kann, da die geistes- und ideengeschichtlichen Konstitutionsideen von entscheidender Bedeutung sind, um zu verstehen, wie die biotische Vielfalt ausgedeutet werden kann und auf welche - eben gerade nicht biowissenschaftlich-theoretischen Kontroversen - diese Diversität immer auch zurückgeführt werden muss. Allerdings muss dabei beachtet werden, dass die Zwischenformen zwischen den idealtypischen ganzheitlichen und partikularen Konstititutionsideen, die der Kk herausstellt, für das Verständnis der biotischen Vielfalt fast wichtiger sind: So macht zwar der idealtypische Vergleich der Partikularkonzeption und der Ganzheitlichkeitskonzeption gewisse Spannungen deutlich, die als prägend für das Verständnis der abiotischen und biotischen Vielfalt angesehen werden müssen. Hieran zeigt sich aber auch, dass eine geistes- und ideengeschichtliche Analyse deutlich differenzierter, vor allem auf Seiten der politischen Philosophien vorgehen müsste, da sowohl konservative als auch liberale Denkfiguren eine deutlich größere Variationsbreite, Spannbreite und Binnendifferenzierung aufweisen, als dies im Kk in Rechnung gestellt wird. Thomas Potthast macht dies deutlich, indem er aufzeigt, dass liberale und konservative Denkstile in Bezug auf das Verständnis biotischer Vielfalt oftmals vermischt werden. Gerade daran, so Potthast, zeige sich die, der Biodiversität innewohnende, „dialektisch anmutende Verbindung“ (Potthast 1999:150) „alter neuer Ideen“ (Potthast 2007a: 76):

Potthast führt exemplarisch aus, dass die neuzeitliche, im liberalen Bild verhaftete, Vorstellung von der stetigen und dynamischen Veränderlichkeit der Natur der Debatte um Biodiversität partiell wieder aufgehoben wird, indem eine über menschliche Zeithorizonte weit hinausgehende evolutionäre Zeitspanne für die Veränderungsprozesse in der Natur zugrunde gelegt wird, die den Menschen und alle anderen Lebewesen in einen größeren Gesamtzusammenhang stellt, der aber als solcher eine Mischung aus Denkstilelementen des Liberalismus und des Konservatismus ist (Potthast 1999:150): Unter Zugrundelegung einer solchen evolutionären Zeitspanne erscheinen die abiotische und biotische Vielfalt sowohl als Bedingung als auch als Ergebnis eines fortwährenden evolutionären Wandels, der alle Lebewesen gleichermaßen betrifft (Potthast 2007a:75). Damit wird die konservative Vorstellung von einem „kontinuierlichen Netz der Wesen“ aufgegriffen und mit dem liberalistischen Bild vom „dynamischen Gradualismus der Veränderlichkeit" verbunden (Potthast 1999: 148), indem die konservativen Assoziationen der Vielfalt des Lebendigen als 'wohlgeordnete Fülle' mit den liberalen Assoziationen der Knappheit und der Selektion über die genetische Verknüpfung aller Lebewesen zusammengebracht werden (Potthast 1999: 150). In diesem Sinne werden die positiven Aspekte der 'Vielfalt als Fülle', die zahlreiche Möglichkeiten und Funktionen für den Menschen birgt, gegen den darwinistischen Kampf ums Dasein und der zunehmenden Knappheit der natürlichen Ressourcen gesetzt. 


\subsection{Fazit und Überleitung zum nächsten Kapitel}

Ziel der wissenschaftstheoretischen Reflexion auf den Forschungsgegenstand der Biodiversität war es, einen konstruktiven Zugang zu dem in der Problemselektion aufgezeigten methodologischen Kurzschluss der Gleichsetzung von Forschungsobjekt und Forschungskonzept aufzuzeigen, weil darüber die Rede von der Biodiversität, der Biodiversitätskrise aber auch der Bewahrung und Förderung der Biodiversität ganz automatisch und ganz systematisch die Tendenz mit sich führt, dass es zur Begehung von naturalistischen und Sein-Sollen Fehlschlüssen kommt. Denn wenn die (a)biotische Vielfalt mit biologischer Vielfalt gleichgesetzt wird und Biodiversität damit bereits im Ansatz naturalisiert wird, dann wird Vielfalt dabei per se als etwas Gutes und Erstrebenswertes betrachtet, so dass auch der Schutz dieser Vielfalt ganz automatisch als ein gutes und erstrebenswertes Ziel angenommen wird und sei es nur, weil dessen Gegenteil, nämlich die Vernichtung der Vielfalt als dumm, sinnlos und in Bezug auf andere als ungerecht betrachtet wird. Eben daraus ergeben sich aber vielfältige und weitreichende, nicht nur theoretisch relevante, sondern auch praktische Problematiken, die sich vor allem an der Entgrenzung und Hybridisierung der Biodiversität aufzeigen lassen (siehe Abschnitt II). Um mit diesen, mit der Entgrenzung und Hybridisierung verbundenen Problematiken konstruktiv umzugehen, wurde auf die theorieexternen und -internen Kritikpunkte am wissenschaftlichen Realismus eingegangen, der als theoretische Hintergrundfolie hinter dem methodologischen Kurzschluss verortet wurde. Die wichtigsten Erkenntnisse aus dieser kritischen Reflexion sind:

1) Erstens und grundlegend muss im Einzelfall immer genau geschaut werden, was mit dem Begriff Biodiversität genau gemeint ist: Die abiotische und biotische Vielfalt als Forschungsgegenstand, wobei dann relevant ist, welche Auffassungen über das Verhältnis von Individuen (Organismen) und Gemeinschaften (ökologische Systeme) es sind, die jeweils dahinterstehen oder biologische Vielfalt als Forschungskonzept, wobei dann wiederum relevant ist, welche biologischen und ökologischen Theorien hinter der jeweiligen Konzeptualisierung stehen und inwiefern deren Annahmen vor dem Hintergrund methodologischer Rekonstruktionen Geltung beanspruchen können.

2) Je nachdem, wie das Verhältnis von abiotischer und biotischer Vielfalt vor dem Hintergrund geistes- und ideengeschichtlicher Annahmen konstituiert wird (und hier gibt es deutlich mehr Variationen als nur die Partikular- und Ganzheitlichkeitskonzeption), hat man es mit unterschiedlichen Vorstellungen davon zu tun, was die abiotische und biotische Vielfalt als Referenzgegenstände der Biodiversitätsforschung ausmacht und welche Ansätze menschlicher Naturverhältnisse dabei eine Rolle spielen.

3) Dabei muss immer darauf geachtet werden, dass die Zustandsbeschreibungen der abiotischen und biotischen Vielfalt - sei es nun in ihrem weiten oder engen Verständnis 
als ganzheitliche Vielfalt oder als aggregierte Vielzahl, als wohlgeordnete Fülle oder als bedrohte Knappheit - nicht auf natürliche Eigenschaften des Lebens bzw. des Lebendigen zurückgeführt werden, sondern als sozial und kulturell konstituierte Vorstellungen von den Naturen und den menschlichen Verhältnissen zu diesen betrachtet werden.

4) Damit berücksichtigt werden kann, dass auch Wissenschaften soziale und kulturelle Praxen (aber spezielle Praxen der progressiven Problemverschiebung!) sind, müssen immer auch die methodologischen Konstruktionsbedingungen der Forschungskonzepte (und hier genauer der biologischen Vielfalt) berücksichtigt werden. Erst wenn klar ist, wie diese konstituiert, konstruiert und in Geltung gebracht werden, kann auch abgeschätzt werden, ob die jeweiligen Annahmen, Theorien, Modelle, Konzepte und Begriffe zu einem theoretischen und empirischen Gehaltsüberschuss gegenüber vorangegangen wissenschaftlichen Erkenntnissen führen.

5) Erst wenn diese vier Aspekte explizit berücksichtigt und thematisiert werden, kann die Biodiversitätskrise nicht nur terminologisch als Krise der gesellschaftlichen Naturverhältnisse betitelt, sondern auch dementsprechend gehandhabt werden, weil dann auch ersichtlich wird, welche Naturauffassungen sowie Handlungsweisen des Menschen es genau sind, die in die 'Krise' geführt haben und darüber selber in die Krise geraten sind. Daher wurde in diesem Kapitel gezeigt, welche geistes- und ideengeschichtlichen Konstitutionsbedingungen sowie methodologischen Konstruktionsbedingungen das Forschungsprogramm der Biodiversität prägen.

6) Die Beantwortung der Frage, für wen, inwiefern und warum es angeraten sein soll, die abiotische und biotische Vielfalt (und gerade nicht die biologische Vielfalt!) möglichst umfassend zu schützen, nachhaltig zu nutzen und gerecht zu verteilen, ist keine biowissenschaftliche und auch keine wissenschaftstheoretische, sondern eine ethische Aufgabenstellung, weil das Wissen darum, was Biodiversität und wie sie erfasst, analysiert und gemessen werden kann, nicht ausreichend ist, um auch begründen zu können, warum die Biodiversität bewahrt und gefördert werden sollte.

Insbesondere der letztgenannte Aspekt verweist darauf, dass zur Beantwortung der öko-sozialen Frage sowie für einen konstruktiven Umgang mit den Metaproblematiken des Biodiversitätsproblems im Anschluss an die hier erfolgte wissenschaftstheoretische Auseinandersetzung auf der inhaltlichen Ebene auf die normative Ebene übergegangen werden muss. Eben dies wird im nächsten Kapitel der Fall sein, wobei der Fokus der folgenden ethischen Analyse speziell auf der Reflexion der vielfältigen Gründe, Argumente und Begründungen für die Bewahrung und Förderung der Biodiversität aus der umwelt- und naturschutzethischen Perspektive liegt. 


\title{
IV. ETHISCHE DETAILREFLEXION: \\ Die theoretische Neuausrichtung der Umwelt- und Naturschutzethik hin zu einer anwendungsorientierten Biodiversitätsethik
}

\author{
"Wie viel Philosophie braucht die Ökologie? \\ Soviel, wie jeder Mensch braucht, um sein Leben sinnvoll zu gestalten!"
}

(Deppert 1998:62)

Ausgangspunkt der folgenden, sehr umfangreichen und binnenkomplexen, ethischen Detailreflexion ist die Frage, welche Gründe für die Bewahrung und Förderung der Biodiversität sowohl im politischen Kontext als auch in der Umwelt- und Naturschutzethik angebracht werden und wie diese jeweils begründet werden. Dieser Fragestellung wird in mehreren Schritten nachgegangen:

Im ersten Teil wird in die argumentationstheoretischen Grundlagen der Rede von den 'guten Gründen' eingeleitet (4.1). Daraufhin werden in einer Art 'Bestandsaufnahme' (4.2) zum einen die, im politischen Kontext, relevanten Gründe für die Erhaltung der Biodiversität in der NBS (4.2.1) als auch die umwelt- und naturschutzethischen Begründungsansätze für die Erhaltung und speziell den Schutz der Biodiversität dargelegt (4.2.2). Dies wiederum macht eine umfangreichere Reflexion der Wertdebatte (4.2.2.1) sowie der physiozentrischen Begründungsansätze für den Schutz der Biodiversität um ihrer selbst willen (4.2.2.2) notwendig: Um die Bandbreite und die Spezifika der möglichen Eigenwertannahmen darzulegen, wird zuerst auf die instrumentellen Werte eingegangen (4.2.2.1.1), da diese als Abgrenzung zu den Eigenwerttypen fungieren, worunter eudaimonistische Eigenwerte (4.2.2.1.2), moralische Selbstwerte (4.2.2.1.3) und objektive Naturwerte (4.2.2.1.4) fallen. Im Anschluss daran wird die Reflexion der Wertedebatte in einem Zwischenfazit bilanziert (4.2.2.1.5). Aufbauend darauf werden die umwelt- und naturschutzethischen Begründungsansätze reflektiert, die über die Eigenwertannahme versuchen, direkte Pflichten des Menschen zu begründen. Hier werden, in Rückgriff auf die wissenschaftstheoretische Reflexion, diejenigen physiozentrischen Ethiken selektiert, die sich für die Begründung von Schutzpflichten gegenüber der Biodiversität um ihrer selbst willen entweder auf die Gesamtheit aller Lebewesen (enges Verständnis von Biodiversität) oder auf alle Lebewesen und ihre Lebensbedingungen (weites Verständnis von Biodiversität) beziehen. Das weite Verständnis erfordert holistische (4.2.2.2.1) oder ökozentrische (4.2.2.2.2) Begründungsansätze, während das enge Biodiversitätsverständnis im Mindesten nach einem biozentrischen Begründungsansatz verlangt (4.2.2.2.3). In einem sich daran anschließenden Zwischenfazit wird deutlich gemacht, warum die Ergebnisse der kritischen Reflexion für eine Neuausrichtung der Umwelt- und Naturschutzethik sprechen (4.2.2.2.4). 
Aufbauend darauf und in Rückgriff auf die drei in der Problemselektion herausgestellten Detailproblematiken auf der normativen Ebene (siehe 2.4.2), die sich ergeben, wenn die Umweltethik sich den Ansprüchen der Anwendungsorientierung stellt, wird im zweiten Teil die anvisierte theoretische Neuausrichtung der Umwelt- und Naturschutzethik anhand der drei Detailproblematiken expliziert (4.3). Dabei sind alle Argumentationen, die hierunter fallen Analyse, kritische Reflexion und Fazit zugleich. Eine solche Synergie wird erreicht, indem mit Bezug auf die erste Problematik - dem zu starken Fokus auf die Inklusionsproblematik und der damit einhergehenden Replizierung der Gegenüberstellung von Mensch und Natur - auf die relationale Perspektive und die Ansätze einer inklusiven Umwelt- und Naturschutzethik eingegangen wird (4.3.1). Hierüber kann zwar die Fokussierung der Umwelt- und Naturschutzethik auf die Inklusionsproblematik zugunsten einer pragmatischen Fokussierung auf die Auslotung der individuellen und gesellschaftlichen Naturverhältnisse überwunden werden, es muss dabei aber geklärt werden, welche Annahmen mit einer solchen relationalen Perspektive einhergehen. Daher wird zum einen auf die Relationen zwischen Mensch, Natur und Gesellschaft eingegangen sowie die wesensmäßige Naturbeziehung als Grundlage der relationalen Perspektive dargelegt (4.3.1.1). Zum anderen wird auf die Relevanz eudaimonistischer Ansätze für die relationale Umwelt- und Naturschutzethik sowie die Bedeutung der Biodiversität für ein gutes Leben eingegangen (4.3.1.2). Mit Bezug auf die zweite Detailproblematik, d.h. dem zu starken Fokus der Umwelt- und Naturschutzethik auf Schutzplichtbegründungen, wird ein spezieller, auf die Biodiversitätszieltrias ausgerichteter Argumentationsraum zur Begründung der Bewahrung und Förderung der Biodiversität dargelegt (4.3.2). Dieser Ansatz wird dargelegt und kritisch reflektiert, wobei bezogen auf den ersten Problemaspekt, nämlich das Ansinnen der Integrativität der Biodiversitätszielstellung gerecht zu werden, ein konzeptionelles Problem des Ansatzes herausgestellt wird (4.3.2.1). Dieser Konzeptionsproblematik wird konstruktiv begegnet, indem die diskurstheoretischen Fundamente der Argumentationstrias rekonstruiert werden (4.3.2.2), so dass auf die pragmatischen (4.3.2.2.1), eudaimonistischen (4.3.2.2.2) und moralischen (4.3.2.2.3) Gebrauchsweisen der praktischen Vernunft eingegangen wird. Bezogen auf den zweiten Problemaspekt, nämlich den zu einseitigen Fokus auf Pflichtbegründungen, werden die drei Argumentationstypen wiederum in einem mehrdimensionalen Ethikverständnis verortet (4.3.2.3). Abschließend wird der dritten Problematik begegnet, indem die Akzeptanz- und Akzeptabilitätsbedingungen für die Kommunikation und Aushandlung der Bewahrung und Förderung der Biodiversität herausgestellt werden (4.3.3). Eben diese Ausführungen ermöglichen wiederum eine Überleitung auf die politische Ebene und in die politikwissenschaftliche Detailreflexion (4.4). 


\subsection{Die argumentationstheoretischen Grundlagen der Rede von guten Gründen}

Wenn nach Gründen für die Bewahrung und Förderung der Biodiversität gefragt wird, dann empfiehlt es sich Ursachenerklärungen, epistemische Begründungen und normative Begründungen voneinander zu unterscheiden, da die Ethik sich primär mit normativen Begründungen beschäftigt:

„Theoretische Begründungen beantworten in objektiver Hinsicht Warum-Fragen, die sich auf die Veränderungen von Dingen, Ereignissen und Zuständen in der realen Welt beziehen; solche objektiven `Gründe` (rationes) nennen wir im Unterschied zur metaphysischen Tradition sUrsachen (causae) und die >Begründung، aus Ursachen nennen wir `Erklärungく (...). Die subjektiv-theoretischen Begründungen beantworten die Fragen, warum es sinnvoll oder geraten ist, von der Existenz bestimmter Dinge, Ereignisse oder Zustände überzeugt zu sein; man nennt sie allgemein epistemische Begründungen (...). Antworten auf Warum-Fragen in praktischen Zusammenhängen, die sich auf objektive Gründe dafür beziehen, dass etwas zu tun oder zu unterlassen sei, kann man normative Begründungen nennen." (Schnädelbach 2006:484)

Wer etwas begründen will, und dabei ist es erst einmal irrelevant, welche Art von Begründung dabei zum Tragen kommt, der muss Argumente ${ }^{79}$ dafür finden und liefern, warum er meint, dass die von ihm getroffenen Feststellungen wahr (bei Erklärungen), plausibel (bei epistemischen Begründungen), richtig (bei normativen Begründungen) sein sollen (ebd., 484). Er muss folglich auf einer reflexiven Ebene zeigen, warum es gerechtfertigt sein soll, seine Gründe als Gründe anzuerkennen und warum diese Gründe anderen vorzuziehen sind, denn durch Argumente versuchen wir andere - aber auch uns selbst - von der Gültigkeit bestimmter Aussagen zu überzeugen, indem wir Argumente miteinander verknüpfen und diese gegeneinander abwägen. Wird in der Ethik argumentiert, so geht es dabei immer darum, dass von den Prämissen auf die Konklusion geschlossen wird und dies so, dass die Richtigkeit einer Konklusion aus der Richtigkeit der Prämissen geschlussfolgert werden kann (Tetens 2004:142). Der Clou und gleichzeitig die Crux des schlussfolgernden Argumentierens besteht dabei darin, dass über die Richtigkeit der Prämissen die Richtigkeit der Konklusion aufgezeigt werden soll, was immer dann der Fall ist, wenn sich zeigen lässt, dass die Prämissen richtig sind und die Konklusion logisch aus den Prämissen folgt (ebd., 25).

79 Als Argument soll hier im formalen Sinne eine Folge von aufeinander bezogenen Aussagen bezeichnet werden, von denen die sogenannten Prämissen $(P)$ als Gründe für den Schluss aus diesen, der sogenannten Konklusion ( $K$ ) ausgegeben werden. Der Übergang zwischen $\mathrm{P}$ und $\mathrm{K}$ wird als Schlussfolgerung (S) bezeichnet. In diesem Sinne setzen Argumente eine bestimmte Struktur voraus, so dass unvollständige Aussagesysteme hier nicht als Argumente sondern als Enthymene bezeichnet werden sollen (Føllesdal, Walløe \& Elster 1988:243). 
Ist dies der Fall, dann muss die Konklusion richtig sein, ohne dass die Richtigkeit der Konklusion gesondert überprüft werden muss, weil mit jedem normativen Argument auf der Metaebene zwei aufeinander bezogene Behauptungen aufgestellt werden: Zum einen wird behauptet, dass die Prämissen richtig sind, und zweitens, dass auch die Konklusion richtig ist, weil die Konklusion aus den Prämissen logisch folgt. Wenn folglich die Richtigkeit der Prämissen gezeigt werden kann und wenn das Argument einer logisch gültigen Schlussregel folgt, so hat der Argumentierende die Richtigkeit der Konklusion gesichert und das Argument ist als schlüssig zu bezeichnen (ebd., 23).

Die Crux des schlussfolgernden Argumentierens, einmal ganz davon abgesehen, was genau unter Richtigkeit ${ }^{80}$ zu verstehen ist, lässt sich am Begriff der Schlüssigkeit verdeutlichen. In der Logik wird der Ausdruck Schlüssigkeit in einem doppelten Sinn verwendet. Schlüssigkeit kann sich entweder ausschließlich auf die Form des Arguments beziehen und/oder auf die Inhalte der Aussagen: Wird die Schlüssigkeit auf die Form bezogen, so ist zumeist von formaler oder logischer Gültigkeit die Rede. Logisch gültig sind Argumente immer dann, wenn man die in einer Aussage vorkommenden nichtlogischen Wörter durch andere nicht-logische Wörter ersetzen kann. ${ }^{81}$ Für Logikerlnnen können Argumente folglich unabhängig von der konkreten Sachverhaltsbeschreibung der Aussagen logisch gültig sein, da die Gültigkeit eines Arguments nur von den Bedeutungen der logischen Ausdrücke eines Arguments abhängig ist (Føllesdal, Walløe \& Elster 1988:246-247), da deren Bedeutungen innerhalb der Logik eindeutig festgelegt sind (vgl. Tetens 2004:284-290). Über solche festgelegten logischen Ausdrücke lassen sich allgemeingültige, unter allen Umständen gültige Schlussregeln aufstellen, die garantieren, dass von der Richtigkeit der Prämissen auf die Richtigkeit der Konklusion geschlossen werden kann. Weil kein logisch gültiges Argument richtige Prämissen und eine falsche Konklusion besitzen kann, wird das Argument als gültig bezeichnet, weil der Schluss von den Prämissen auf die Konklusion logisch gerechtfertigt ist. Die Standardform eines solch logisch schlüssigen Arguments ist der deduktive Syllogismus. $^{82}$

80 Die Frage, warum eine Aussage für richtig gehalten werden kann und woran sich dies erkennen lässt, gehört zu den zentralen und damit auch umstrittensten Fragen der Philosophie (siehe auch FN 46). Diese soll hier nicht entschieden werden.

81 Hierbei gibt es indes ein paar wichtige Regeln zu beachten (Føllesdal, Walløe \& Elster 1988:247: 1): Wenn ein logisches Wort durch ein anderes ersetzt wird, dann muss dieses Wort überall ersetzt werden, wo es im Argument vorkommt. 2) Jedes Wort bzw. jeder Ausdruck der ersetzt wird, muss durch ein Wort bzw. ein Ausdruck derselben semantischen Kategorie (generelle Terme, singuläre Terme, Sätze) ersetzt werden (ebd., 215-224). 3) Es dürfen ausschließlich nicht-logische Wörter und Ausdrücke gegeneinander ausgetauscht werden. Logische Wörter und Ausdrücke hingegen sind in ihrer Bedeutung durch die formale Logik eindeutig festgelegt.

82 Hier wird deshalb von Standardform gesprochen, weil es zwar viele andere Formen schlüssiger Argumente gibt, der deduktive Syllogismus ist aber nach wie vor die Argumentationsform, die am häufigsten verwandt wird. 
Hilfreich ist dabei zwischen evaluativen und präskriptiven Aussagen zu unterscheiden, weil evaluative Aussagen etwas als gut bewerten (u.a. auch als moralisch gut!), während präskriptive Aussagen etwas vorschreiben oder zumindest anraten (und zwar nicht nur in moralischer Hinsicht!). Sowohl evaluative als auch präskriptive Aussagen sollten dabei von Tatsachenbehauptungen abgrenzt werden, ${ }^{83}$ da bei normativen Argumenten eine wichtige Regel hinzutritt, die bei deskriptiven Argumenten nicht von Relevanz ist (siehe 2.3.1): Normative Konklusionen brauchen dem Humeschen Gesetz zufolge mindestens eine normative Prämisse, auf die sie logisch gültig zurückgeführt werden können, da i.d.R. davon ausgegangen wird, dass die Wertung, ob etwas gut ist sich nicht schon aus der Beschreibung des Ist-Zustandes ergibt (Moores naturalistischer Fehlschluss) und ein Sollen nicht ausschließlich über ein Sein gerechtfertigt werden kann (Humes "Sein-Sollen Fehlschluss«). In beiden Fällen kann das Argument als logisch ungültig eingestuft werden, weil die Konklusion logisch ungültig ist, obwohl das Argument als solches (rein inhaltlich betrachtet) schlüssig sein kann. ${ }^{84}$ Dies wiederum verweist darauf, dass die logische Gültigkeit der Form des Arguments von der Stichhaltigkeit der inhaltlichen Aussagen zu unterscheiden ist, da sich die logische Gültigkeit eben nicht auf die Inhalte bezieht, die in den Prämissen oder der Konklusion zum Ausdruck gebracht werden (Føllesdal, Walløe \& Elster 1988:256-257). Eben die inhaltlichen Unterschiede sind es aber, die deskriptive von normativen Aussagen unterscheiden, so dass es hier nicht nur um Aspekte formaler Schlüssigkeit gehen kann.

Werden diese Grundlagen beachtet, dann kann differenziert herausgestellt werden, welche Positionen es bezüglich Tatsachenbehauptungen, evaluativen Annahmen und präskriptiven Urteilen gibt und es kann verhindert werden, dass normative Fragen auf bloß suggestive Weise beantwortet werden, indem in die Tatsachenbehauptungen bereits evaluative Annahmen einfließen, durch die wiederum bestimmte präskriptive Urteile impliziert werden. Werden die Differenzierungen indes beachtet, dann wird der Fokus darauf gelegt, wie evaluative Annahmen und präskriptive Urteile begründet werden können. Dabei muss indes mit der Schwierigkeit umgegangen werden, dass man es in der Alltagssprache oftmals mit unvollständigen Argumenten (Enthymemen) zu tun hat. Diese sind unvollständig, weil entweder die 'trivialen' Voraussetzungen, die für alle Beteiligten offensichtlich und/oder unproblematisch sind, weggelassen werden

83 Die Begründung der Tatsachenbehauptungen fällt dabei eigentlich aus dem genuinen Kompetenzbereich der normativen Ethik heraus, weil es der Ethik um die Fragen geht, wie Menschen sich verhalten und handeln sollten. Gerade wenn es aber um anwendungsorientierte Ethiken geht, wird der Graubereich zwischen Tatsachenbehauptungen und normativen Aussagen mehr als deutlich, denn es kann prinzipiell auch der Ethik nicht egal sein, ob die Faktenlage unklar ist und ob die Tatsachenbehauptungen unbegründbar sind, da es anwendungsorientierten Ethiken immer auch um Beiträge zur Lösung konkreter praktischer Probleme geht (siehe 1.2.2).

84 Als Beispiel ließe sich folgendes prominentes Argument anführen, das hier zur Verdeutlichung vereinfacht dargestellt wird: P1: (Arten-)Vielfalt führt zu Stabilität; P2 Ökosystemare Stabilität ist gut und wichtig; S: (Arten-)Vielfalt sollte erhalten werden! 
(ebd., 244, 267) oder es wird auf die Benennung der normativen Prämisse absichtlich verzichtet, weil diese zu kontrovers erscheinen. Enthymeme wie:

$\mathrm{P}_{\text {deskriptiv: }}$ Durch den Bau der B96 werden Naturflächen zerschnitten.

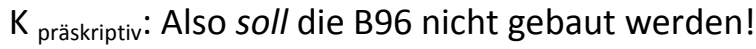

müssen, wenn die dahinterstehenden Kontroversen lediglich invisibilisiert werden, obwohl sie de facto existieren, rekonstruiert werden. Erst dann können sie zum einen als vollständige Argumente gelten und der normative Schluss kann hergestellt werden. Dies kann bspw. wie folgt geschehen:

$P_{\text {deskriptiv: }}$ Durch den Bau der B96 werden Naturflächen zerschnitten.

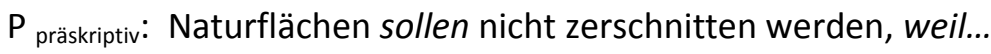

$\mathrm{P}_{\text {explanativ: }}$ Biodiversität ist wichtig, weil....

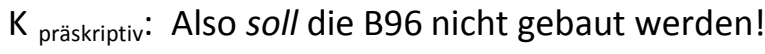

Hieran zeigt sich indes bereits deutlich, dass das Grundmodell des praktischen Syllogismus der Komplexität normativer Fragestellungen oft nicht gerecht werden kann, da die Überführung von einfachen normativen Aussagen in komplexe Zielstellungen die gängigen normlogischen Systeme überfordert (Ott 2004:176). ${ }^{85}$ Dies wird bereits daran ersichtlich, dass selbst scheinbar einfache normative Aussagen komplexe Argumentationsketten nach sich ziehen, falls die normative Prämisse in Frage steht, weil normative Prämissen immer über andere normative Argumente plausibilisiert werden müssen, die ihrerseits normative Prämissen besitzen, die begründungsbedürftig sind und begründungsfähig sein müssen. ${ }^{86}$ Eben dies soll im Folgenden über die kritische Bestandsaufnahme der gängigen Gründe und Begründungen für die Erhaltung und insbesondere den Schutz der Biodiversität expliziert werden, damit deutlich wird, welche Aussagen, Argumente und Begründungen für die Bewahrung und Förderung der Biodiversität überhaupt im gesellschaftspolitischen Raum stehen.

85 Hier bedarf es folglich entweder eines normlogisch tragfähigen Schlusssegments, das angesichts der zahlreichen Begründungslücken und Unschärfen normativer Argumente dennoch Plausibilisierungen ermöglicht und dabei die Übergänge zwischen Tatsachenbehauptungen, evaluativen Aussagen und präskriptiven Aussagen erlaubt oder es muss anerkannt werden, dass ethische Überlegungen selten eine syllogistische Struktur haben und dass deswegen entsprechende argumentationstheoretische Einsichten nur begrenzt weiterhelfen. Die Frage, wie hier zu entscheiden ist soll nicht weiter erörtert werden.

86 Da die Schlüssigkeit normativer Aussagen und Argumente im Allgemeinen davon abhängt, ob es andere normative Aussagen gibt, die faktisch nicht mehr hinterfragt werden (können), ist es eine zentrale Aufgabe der (allgemeinen) Ethik, solche in ihrer Geltung allgemein akzeptable normative Aussagen, die als Prämissen für die Richtigkeit anderer Prämissen herangezogen werden können, zu finden und zu begründen. Sie werden als oberste Prinzipien oder Universalnormen bezeichnet. Ob es möglich ist, diese ebenfalls zu begründen, ist eine nach wie vor strittige und mitnichten bereits entschiedene Frage innerhalb der Philosophie (Friesen \& Berr 2004:234), die hier weder thematisiert noch entschieden werden kann. 


\subsection{Kritische Reflexion der gängigen Gründe und Begründungen für die Erhaltung der Biodiversität}

Im Folgenden werden zum einen die ökologischen, ökonomischen, soziokulturellen und ethischen Gründe für die Erhaltung der Biodiversität reflektiert, die sich in der NBS finden lassen (4.2.1) als auch die umwelt- und naturschutzethischen Begründungsansätze für den Eigenwert und die direkten Schutzpflichten gegenüber der Natur genauer analysiert (4.2.2). Dass hierbei von Erhaltung bzw. von Schutz (und gerade nicht von Bewahrung und Förderung) die Rede ist, ist beabsichtigt, da sowohl im politischen als auch im umwelt- und naturschutzethischen Kontext das Leitbild der Bewahrung und Förderung der Biodiversität (problematischerweise) nicht in Gänze zum Tragen kommt.

\subsubsection{Die 'Argumente'der NBS: ökologische, ökonomische, soziokulturelle und ethische Gründe für die Erhaltung der Biodiversität}

Die NBS nennt im Kapitel Ausgangslage einige zentrale Gründe für die Erhaltung der Biodiversität und unterscheidet dabei zwischen ökologischen, ökonomischen, sozialen und kulturellen sowie ethischen Gründen. Für eine bessere Übersicht werden diese hier tabellarisch festgehalten:

Biodiversität soll aus ökologischen Gründen erhalten werden (auch wenn ihre jeweiligen Funktionen im Naturhaushalt noch nicht erkannt sind), weil

...für die biologische Vielfalt das Vorsorgeprinzip gilt (BMUB 2011:10).

...,,die Entwicklungsmöglichkeiten zukünftiger Generationen

gewährleistet" werden sollen (ebd., 10).

...ein großer Teil der Arten und Ökosysteme, die in Mitteleuropa vorkommen, speziell an die hiesigen Umweltbedingungen angepasst ist und hier ihren weltweiten Verbreitungsschwerpunkt haben (ebd.).

...intakte Ökosysteme dazu beitragen, Katastrophen zu vermeiden bzw. deren Ausmaß zu vermindern (ebd., 11).

Biodiversität soll aus ökonomischen Gründen erhalten werden (auch wenn ihr Nutzen für die Menschen nicht in allen Details bekannt ist), weil

...Biodiversität ein „unersetzlicher Bestandteil des Naturkapitals“ ist (ebd., 12).

...der „geschätzte jährliche Nutzen der gesamten Ökosysteme der

Welt zwischen 16 und 64 Billionen US \$“ liegt (ebd.). 
...die Natur Leistungen „liefert, die mit erheblichem Aufwand und zu sehr hohen Kosten technisch gelöst werden müssten oder technisch bisher gar nicht reproduzierbar sind." (ebd.)

...viele Einkommen und Arbeitsplätze direkt oder indirekt von Natur und Landschaft abhängen (ebd.).

...in Deutschland ca. $50 \%$ der heute gebräuchlichen Arzneimittel auf Heilpflanzen bzw. auf deren Inhaltsstoffen basieren (ebd.).

...die pharmazeutische Industrie die biologische Vielfalt nutzt und Deutschland der größte Importeur dieser Stoffe in Europa ist (ebd.).

...die Tourismuswirtschaft zu den wirtschaftlich am stärksten wachsenden Branchen gehört und diese auf "schöne und intakte Natur und Landschaft" angewiesen ist (ebd., 13).

\section{Biodiversität soll aus sozialen und kulturellen Gründen erhalten werden, weil}

...Naturerfahrung und -erlebnisse wichtige Aspekte der Persönlichkeitsentwicklung sind: „Positive Naturerfahrungen stärken das Lebensgefühl, schulen die sinnliche Wahrnehmung und das ästhetische Empfinden, vermindern Aggressivität, fördern Aufmerksamkeit, Konzentration und Wahrnehmungsfähigkeit sowie die Ausbildung motorischer Fähigkeiten." (ebd.)

..., die ungestaltete freie Natur" dem Gestaltungsdrang des Menschen besser entgegenkommt „,als alles vom Menschen künstlich Hergestellte.“ (ebd.)

...Naturerfahrungen und -erlebnisse unverzichtbar bei der Gestaltung der Freizeit und für die Erholung sind (ebd.).

...Natur und Landschaft zur Stärkung der regionalen Identität beitragen und das Heimatgefüh/ prägen (ebd.).

...die Nähe zur Natur ein wichtiger Aspekt der Lebensqualität ist (ebd.).

...die historisch gewachsene, als harmonisch empfundene Kulturlandschaft in Deutschland ,ein Ausdruck regionaler Vielfalt und gleichzeitig auch ein erhaltenswertes Kulturgut ist." (ebd., 14)

...es für den Großteil der Bevölkerung sehr wichtig oder wichtig ist, dass das Aussterben von Tier- und Pflanzenarten verhindert wird (ebd.).

...es für den Großteil der Bevölkerung sehr wichtig oder wichtig ist, dass für einen verbesserten Naturschutz gesorgt wird (ebd.). 
Biodiversität soll aus ethischen (besser normativen) Gründen erhalten werden, weil

...naturschutzrechtlich Natur und Landschaft auch „auf Grund ihres eigenen Wertes" zu erhalten sind (ebd., 15).

...aus den umweltethischen Positionen und Begründungen eine Verpflichtung resultiert, „möglichst die gesamte noch vorhandene biologische Vielfalt zu erhalten, es sei denn, es sprechen existenzielle Gründe dagegen.“ (ebd.)

Die einzelnen Aussagen, die unter die jeweiligen Typen von Gründen fallen, bilden die Grundlage für die weitere Reflexion der im politischen Kontext gegebenen Begründungen für die Bewahrung und Förderung der Biodiversität. Der Umstand, dass in der NBS überhaupt Gründe für die Erhaltung der Biodiversität aufgeführt werden, wird so interpretiert, dass ihr keine rein informativen Ziele zugrunde liegen, sondern dass über die NBS auch das Ziel verfolgt wird, möglichst viele Bürger, Unternehmen und Organisationen von der Wichtigkeit der Anliegen zu überzeugen, da sich an die gesellschaftspolitische Aufgabenstellung der Bewahrung und Förderung weitreichende Maßnahmen anschließen, die tiefgreifende Veränderungen der privaten Lebensrealität und Lebensqualität vieler Menschen zur Folge haben können, so dass diese einer Legitimation bedürfen. ${ }^{87}$ Daher ist es folgerichtig, dass man sich auch in der NBS darum bemüht hat, Gründe bereit zu halten, warum die Bewahrung und Förderung der Biodiversität wichtig für alle Menschen auf der Erde, sowohl innerhalb ihrer gesellschaftlichen Kontext als auch für ihre individuelle Lebensqualität ist, warum die Bewahrung und Förderung der Biodiversität eine zentrale politische Aufgabe darstellt, die angesichts anderer politischer Aufgabenstellungen, wichtig und berechtigt sein sollte und warum die Bevölkerung aktiv an der Erreichung der politischen Zielstellungen mitarbeiten muss. Eben vor diesem Hintergrund wird im Folgenden argumentiert, dass sich die NBS über die Art ihrer 'Begründung` für die Bewahrung und Förderung der Biodiversität in eine Reihe von vermeidbaren Begründungsproblematiken verstrickt, die dazu führen, dass die NBS ihr selbstgesetztes Ziel, möglichst viele Menschen für ihre Anliegen zu gewinnen, nicht in vollem Maße realisieren kann, da die gegebenen Gründe nur diejenigen zu überzeugen vermögen, die dies ohnehin schon sind:

Schaut man sich die aufgelisteten Aussagen an, dann fällt dabei als übergeordnete Problemlage auf, dass die NBS ein systematisches Argumentations- und Begründungsdefizit aufweist, da alle Aussagen, die in der NBS angeführt werden, rein formal betrach-

87 Obgleich oftmals lediglich von Legitimation gesprochen wird, muss hier bereits festgehalten werden, dass es nicht nur einen sprachlichen, sondern auch einen bedeutsamen inhaltlichen Unterschied zwischen Legitimation und Legitimität gibt, der analog zur Unterscheidung von Akzeptanz und Akzeptabilität verläuft bzw. sich sogar auf diesen beruft (genauer 4.3.3). 
tet, keine wirklichen Gründe im Sinne von Argumenten, sondern nur enthymemische Aussagen sind. ${ }^{88}$ Dies liegt daran, dass in der NBS (mehr oder weniger gut nachvollziehbare und vermeintlich rein deskriptive Tatsachen-)Aussagen als Gründe für die Wichtigkeit und Relevanz der Erhaltung der Biodiversität angebracht werden, aber für sämtliche dieser Behauptungen Argumente und Begründungen fehlen, warum ausgerechnet diese Gründe relevant sein sollen und auf Basis welcher Annahmen die Gründe überhaupt als Gründe fungieren sollen. Dass die systematisch angelegte und weitreichende Vernachlässigung der Argumentations- und Begründungsleistungen indes sowohl praktisch als auch theoretisch problematisch ist, lässt sich anhand der folgenden drei Aspekte deutlich machen:

Erstens zeigt sich, dass viele der als Gründe deklarierten Aussagen ohne weitere Erläuterungen als Gründe gar nicht verständlich und einsichtig sind, da die meisten Aussagen mit bestimmten Begriffen operieren oder auf theoretische Zusammenhänge zurückgreifen, die aber nicht weiter expliziert werden (1). Zweitens zeigt sich, dass in der NBS primär eine an Fakten und Tatsachen orientierte Argumentationslogik zur Anwendung kommt, mit der versucht wird, die Erhaltung der Biodiversität möglichst anhand 'harter' und in diesem Sinne sachlicher, objektiver und wertfreier Gründe zu rechtfertigen. Dies führt zu einer Verdrängung der kulturellen, sozialen, emotionalen vermeintlich 'weichen', aber motivational relevanten Argumente aus dem Umwelt- und Naturschutz, was wiederum ein entscheidender Grund für das oftmals beklagte Akzeptanzdefizit des deutschen Natur- und Umweltschutzes ist (2). Drittens zeigt sich, dass über die angewandte Argumentationslogik auch ein oftmals gar nicht erkanntes und refletiertes Akzeptabilitätsdefizit entsteht. Da auch die normativen Voraussetzungen der 'Gründe' in der NBS implizit bleiben, kann erst auf der reflexiven Ebene deutlich werden, dass ein Großteil der in der NBS angeführten Gründe auch in normativer Hinsicht hochgradig voraussetzungsreich ist, so dass die damit einhergehenden normativen Kontroversen nicht thematisiert werden, was vor allem dann problematisch ist, wenn hierbei bestehende praxisrelevante Konfliktpotenziale ausgeblendet werden (3). Auf alle drei Aspekte wird im Folgenden ausführlicher und stellenweise auch in noch einmal in Rückgriff auf die Grundlagenreflexion (siehe Abschnitt II) eingegangen:

88 Dabei muss sicherlich von vornherein eingeräumt werden, dass die NBS keine wissenschaftliche Arbeit sondern ein politisches Strategiepapier ist. Insofern wäre es verfehlt, wenn ihr das für die Ethik so zentrale Begründen und Argumentieren als Defizit ausgelegt werden würde, da dies gar nicht das Anliegen der NBS ist und sein kann. Vielmehr sollen die einzelnen Problematiken hier bereits mit Blick auf die Möglichkeiten und Grenzen einer anwendungsorientierten Umwelt- und Naturschutzethik ausgeführt werden, da sie es ist, die nach normativen Argumenten und Begründungen den Umgang mit der (belebten) Natur sucht. Insofern es also in den Aufgabenbereich der Umwelt- und Naturschutzethik fällt, die Argumentations- und Begründungsdefizite der NBS zu reflektieren und möglichst auch konstruktiv zu rekonstruieren, wird die Auseinandersetzung mit den Begründungsproblematiken in der NBS hier als Ansatzpunkt für die theoretische Reflexion in der Umwelt- und Naturschutzethik aufgefasst. 
1) In Bezug auf den ersten Problemaspekt stellen sich die konkreten Fragen, was genau es bedeuten soll, dass für die Biodiversität das sogenannte Vorsorgeprinzip gilt, dass Biodiversität ein unersetzbarer Teil des Naturkapitals ist, dass Naturerfahrung und Erlebnisse wichtige Aspekt der Persönlichkeitsentwicklung sind oder dass Natur und Landschaft aufgrund ihres eigen Wertes zu erhalten sind: Im Glossar der NBS wird ausgeführt, dass das Vorsorgeprinzip (siehe im Detail auch Problemlage 3) ein „Grundsatz der Umweltpolitik [ist], nach dem staatliche Maßnahmen so getroffen werden sollen, dass von vornherein möglichst sämtliche Umweltgefahren vermieden werden“ (BMUB 2011: 174). Im Anschluss daran stellen sich aber immer noch die Fragen, was genau 'Umweltgefahren' sind und was darunter alles fällt und ob es legitim ist, möglichst sämtliche Umweltgefahren zu vermeiden, wenn darunter bspw. auch die Vermeidung von Bevölkerungswachstum fällt. Des Weiteren kann eingewandt werden, wie dies berechtigterweise gefordert werden kann, wenn die Abläufe in der Natur und deren Wechselwirkungen mit menschlichen Handlungen als solche zum einen nicht klar abgrenzbar sind (was sind natürliche und was sind anthropogene Veränderungen?) und wenn diese zum anderen nicht klar prognostizierbar sind.

In Bezug auf die Kennzeichnung der Biodiversität als unersetzbarer Teil des Naturkapitals sucht man selbst im Glossar vergebens nach einer Erläuterung, was genau unter dem Begriff Naturkapital zu verstehen ist. Der Verweis in der NBS, dass in der neoklassischen Theorie und im Konzept "schwacher Nachhaltigkeit» die Ansicht vertreten wird, dass Naturkapital ließe sich prinzipiell durch menschengemachtes Kapital ersetzen, wohingegen sich immer mehr die Erkenntnis durchsetzt, dass Biodiversität, nach dem Konzept »starker Nachhaltigkeit« zum unersetzbaren Naturkapital gehöre (ebd., 12), hilft hier nicht sonderlich weiter, da die gerechtigkeitstheoretischen Grundlagen beider Nachhaltigkeitskonzepte unklar bleiben. Aber auch bei den soziokulturellen Gründen werden weitreichende anthropologische und psychologische Vorannahmen getroffen, wenn konstatiert wird, dass Naturerfahrungen und -erlebnisse wichtige Aspekte der Persönlichkeitsentwicklung und unverzichtbar bei der Gestaltung der Freizeit sind sowie dass die ungestaltete 'freie Natur' dem Gestaltungsdrang des Menschen besser entgegenkommt als alles vom Menschen künstlich Hergestellte. Hier wird bereits ein ganz bestimmtes Menschenbild vorausgesetzt, das vor dem Hintergrund der Lebensrealität vieler Menschen, die in Städten auf-gewachsen sind und dort (gut und zufrieden) leben, die ihren Gestaltungsdrang zu einem großen Teil 'artifiziell' ausleben, ohne von der Natur 'entfremdet`zu sein, zumindest erklärungsbedürftig ist.

Am entscheidendsten für den hier relevanten Kontext ist indes die fehlende Darlegung der Voraussetzungen und theoretischen Zusammenhänge beim Verweis der NBS auf den eigenen Wert der Biodiversität als explizit normativer (in der NBS ethischer) Grund für deren Erhaltung. Zwar ist der Schutz von Natur und Landschaft auf Grund ihres 
eigenen Wertes auch im Bundesnaturschutzgesetz festgelegt, aber es stellt sich doch die Frage, was mit dem Begriff Eigenwert genau gemeint ist, was einen solchen Eigenwert überhaupt ausmacht, sprich auf welchen Annahmen er basiert und welche ethischen Konsequenzen sich ggf. hinsichtlich einer daraus abzuleitenden Schutzpflicht ergeben. In diesem Sinne ist es bezeichnend, dass die NBS hier weder im Glossar noch anderswo eine ausführlichere Erklärung gibt, obwohl andere und vor allem biowissenschaftliche Fachtermini wie Sukzession, Taxon oder Trophie im Glossar ausgeführt werden (ebd., 173) und andere Begriffe wie Naturhaushalt zumindest in Ansätzen erläutert werden. In Bezug auf den Eigenwert stellt die NBS lediglich den Zusammenhang zwischen Wert und Ethik her, wobei über das dabei transportierte Verständnis von Ethik unterschlagen wird, dass es bei Ethik um verallgemeinerbare Begründung von Werten und Normen geht (siehe im Detail auch Problembereich 3), wenn die NBS ausführt, dass es „sehr unterschiedlich [ist] und wahrscheinlich vom Geschlecht, Alter oder Lebensstiltyp bzw. sozialem Milieu ab[hängt], [w]elcher Wert der Natur beigemessen wird“ (ebd., 13), da die Entscheidung „[w]elche ethischen Werte vertreten werden, von der jeweiligen Gesellschaft ab[hängt], in der sie entwickelt wurden und die sie dann auch prägen." (ebd., 15)

2) Die Art und Weise, wie die Gründe in der NBS kommuniziert werden, macht des Weiteren deutlich, dass sich die Erhaltung der Biodiversität möglichst anhand 'harter' sachlicher, objektiver und wertfreier Gründe erweisen soll. Abgesehen von den empirischen (siehe 2.2) und theoretischen Schwierigkeiten (siehe 2.3.1) solcher Argumentationsfiguren, bleiben dabei auch die Motive unangesprochen, die Menschen zur Erhaltung der Biodiversität bewegen können. Da vor allem die ästhetisch motivierten und heimatschutzbezogenen Argumente nach dem 2. Weltkrieg aus dem Natur- und Umweltschutz zugunsten biowissenschaftlicher und ökonomischer Argumente verschwunden sind (vgl. Piechocki 2010), tut sich eine Diskrepanz zwischen den oftmals unartikulierten, individuellen Motivlagen einerseits und den möglichst sachlichen, wertfreien, objektiv-wissenschaftlichen Begründungen für Natur- und Umweltschutzanliegen andererseits auf. Die auch in der NBS nicht beachtete Diskrepanz zwischen den Motiven und Gründen für die Bewahrung und Förderung der Biodiversität ist ein wesentlicher Grund für das oftmals beklagte Akzeptanzdefizit des deutschen Naturund Umweltschutzes (siehe 2.2), wobei die in der Fachliteratur nach wie vor intensiv geführte kritische Diskussion zu den Zielen und Aufgaben des Umwelt- und Naturschutzes sowie deren Begründung als Ausdruck der Reflexion dieses Akzeptanzdefizites verstanden werden kann (vgl. Körner \& Eisel 2003:6). Dass hierbei gerade auch auf das Konzept der Biodiversität geschaut werden muss, zeigt sich vor allem daran, dass die Vernichtung der Biodiversität und ebenso deren Bewahrung und Förderung oftmals mehr als technisches, denn als gesellschaftliches Problem des Naturumgangs verstan- 
den wird (ebd., 12). Dies ist indes eine kontraproduktive Reduktion, da eine auf Objektivität, Sachlichkeit und Wertfreiheit festgelegte Beschreibungssprache gerade deshalb angewandt wird, um die Biodiversitätszielstellungen besser abstützen und legitimieren zu können. Dies gelingt aber nicht, da der Versuch die Erhaltung der Biodiversität möglichst faktisch zu begründen in eine paradoxe Situation führt:

So führt bspw. ein Artenschutz, der über ökologische Funktionen bspw. zur Aufrechterhaltung der Stabilität argumentiert wird, nicht auf den Schutz der Arten, um den es vielen NaturschützerInnen eigentlich geht (Haber 2009: 27). Auf der einen Seite stehen die seltenen, repräsentativen, schönen und gefährdeten Arten, die den NaturschützerInnen und vielen Menschen emotional und ästhetisch am Herzen liegen, die aber hinsichtlich ihrer ökologischen Funktionen zumeist keine messbaren Effekte aufweisen. Auf der anderen Seite stehen die Arten, die solche funktionalen Effekte nachweisbar und in Fülle haben, aber bei den NaturschützerInnen und in der Bevölkerung keinerlei positive Emotionen wecken, weil gerade die Bodentiere und Insekten, die bei vielen Menschen eher Abneigung und Ekel auslösen, von enormer funktionaler Wichtigkeit sind. An solchen Stellen passiert es dann, dass die NaturschützerInnen Arten schützen wollen, weil sie deren Schönheit und Repräsentativität wertschätzen, dafür aber ökologische Gründe suchen (Piechocki 2010:12). Dies zeigt, dass ein Großteil der eigentlichen Motivationen, die Menschen zum Schutz der Natur und zur Bewahrung und Förderung der Biodiversität haben (können), über eine solche Argumentationslogik gar nicht angesprochen werden, womit in der NBS wichtige Aspekte für die Akzeptanz des Naturschutzes in der Gesellschaft ausblendet und vernachlässigt werden.

3) Über die faktische Argumentationslogik bleiben allerdings nicht nur die Motive für den Umwelt- und Naturschutz unangesprochen, die normativen Prämissen der Aussagen werden ebenfalls nicht genannt und zur Diskussion gestellt. Dies ist insbesondere für das oftmals gar nicht als Problem erkannte Akzeptabilitätsdefizit relevant, weil für die präskriptive Schlussfolgerung (Biodiversität soll erhalten werden) in der Regel Aussagen angeführt werden, die darlegen, was der Fall ist und nicht was der Fall sein sollte (Ausnahme ist hier lediglich die 'ökologische' Aussage, dass die Entwicklungsmöglichkeiten zukünftiger Generationen gewährleistet werden sollen). Insofern lässt sich an nahezu allen in der NBS angeführten, ökologischen, ökonomischen, sozio-kulturellen und ethischen (besser normativen) Gründen für die Erhaltung der Biodiversität zeigen, dass diese zwar präskriptiv gehaltvolle Schlussfolgerungen zugunsten der Erhaltung der Biodiversität implizieren, sie sind aber, im formalen Sinne betrachtet, keine wirklichen Argumente und Begründungen, die auch als Rechtfertigung dieser Schlussfolgerungen fungieren könnten. Daran wird ersichtlich, dass es der NBS in erster Linie um die faktische Akzeptanz für die Ziele und Maßnahmen geht, nicht aber auch um die Ausweisung der Akzeptanzfähigkeit der von ihr vorgebrachten Aussagen. Insofern bleibt hier 
der Umstand unreflektiert, dass selbst dann, wenn die Biodiversitätsziele und -maßnahmen in der Bevölkerung breit akzeptiert sind (was sich mittels empirischer Sozialforschung erheben und über Marketingstrategien fördern lässt), dies kein Garant dafür ist, dass diese auch akzeptabel sind. Dies zeigt sich vor allem an den beiden soziokulturellen Aussagen zu den Einstellungen der Menschen zum Umwelt- und Naturschutz sowie an den 'ethischen`Aussagen:

Nur weil es für den Großteil der Bevölkerung sehr wichtig oder wichtig ist, dass das Aussterben von Tier- und Pflanzenarten verhindert wird oder dass für einen verbesserten Naturschutz gesorgt wird (Ebene der faktischen Akzeptanz), sagt dies noch nichts darüber aus, ob es auch rechtfertigbar oder richtig ist, bestimmte Ziele und Maßnahmen anzuvisieren und zu implementieren (Ebene der normativen Akzeptabilität). Die Verwechselung von Akzeptanz und Akzeptabilität, sprich die Verwechselung der tatsächlichen individuellen und gesellschaftlichen Befürwortung von bestimmten Zielen und Maßnahmen und die an Gründen, Argumenten und Begründungen orientierte Rechtfertigbarkeit solcher Ziele und Maßnahmen andererseits (siehe 2.2), zeigt auch hier, dass die politischen Gründe für die Erhaltung der Biodiversität und ebenso die präskriptive Zielsetzung der Bewahrung und Förderung der Biodiversität nicht ohne eine ethische Begründung auskommen. Hier kommt dann das Problem zum Tragen, dass die NBS mit ihrer Einteilung in ökologische, ökonomische, soziokulturelle und ethische Gründe den Eindruck erweckt, als würden lediglich die ethischen Gründe normativer Vorannahmen bedürfen (Eser, Neureuther \& Müller 2011:15). Dass dies mitnichten der Fall ist, kann vor allem an den ökologischen Gründen deutlich gemacht werden, da vor allem diese den Eindruck erwecken, sie wären rein beschreibend, wertfrei und sachlich. Ein kritischer Blick darauf zeigt indes, dass sowohl das Vorsorgeprinzip als auch der Verweis auf die Entwicklungsmöglichkeiten zukünftiger Generationen, die als ökologische Gründe angeführt werden, keine ökologischen, als vielmehr normative Aussagen sind:

Hinter dem Verweis auf die Entwicklungsmöglichkeiten zukünftiger Generationen steht die Annahme, dass wir als jetzt lebende Menschen für Zukünftige eine Verantwortung tragen. Sicherlich ist dies für viele Menschen unstrittig und auch das Grundgesetz legt in Art. 20a fest, dass „(d)er Staat auch in Verantwortung für die künftigen Generationen die natürlichen Lebensgrundlagen (...) schützt." Eine solche Verantwortung, wenn sie denn als Verpflichtung verstanden wird, kann indes nicht ökologisch gerechtfertigt werden, sondern immer nur ethisch, was aber nur dann geschehen kann, wenn der vermeintlich ökologische Grund so rekonstruiert wird, dass dessen normative Prämissen ersichtlich werden. Dasselbe gilt, wenn auch schwieriger zu erkennen, für das Vorsorgeprinzip. Das normative Grundprinzip, auf dem es basiert, lässt sich besonders gut anhand der bereits im ersten Problembereich angesprochenen »Angewiesenheitsargu- 
mentation« deutlich machen, die in der NBS der eigentlichen Ausführungen der Gründe sogar vorangestellt wird, als ginge es hierbei erst gar nicht um einen zu rechtfertigen Grund, sondern um eine unbestreitbare, allen Menschen augenblicklich einleuchtende Tatsache. So führt die NBS aus, dass die:

„[b]iologische Vielfalt eine existenzielle Grundlage für das menschliche Leben [ist]: Pflanzen, Tiere, Pilze und Mikroorganismen sind Träger des Stoffkreislaufs sie reinigen Wasser und Luft, sorgen für fruchtbare Böden und angenehmes Klima, sie dienen der menschlichen Ernährung und Gesundheit und sind Basis und Impulsgeber für zukunftsweisende Innovationen. Nur eine intakte Natur ermöglicht heutigen und zukünftigen Generationen eine hohe Lebensqualität." (BMUB 2011:9-10;SL)

Diese (und ähnliche) Aussagen zur existenziellen Bedeutung der Biodiversität zielen darauf ab, den umstandlosen Schutz der gesamten Biodiversität als schiere Notwendigkeit zur Sicherung menschlichen Lebens zu begründen. Die (vermeintliche) Alternativlosigkeit der Erhaltung der Biodiversität soll sich wiederum anhand der (vermeintlich) ökologischen Gründe erweisen, auch wenn dieser Zusammenhang in der NBS nicht explizit dargelegt wird. Die Aufschlüsselung des Existenzarguments zeigt indes, dass sowohl an der tatsächlichen Alternativlosigkeit Zweifel angebracht sind, weil sich jeder Argumentationsschritt kritisch hinterfragen lässt, als auch an der Zuordnung der dabei in Anschlag gebrachten Aussagen als ökologische Aussagen. Eben dies lässt sich an der Rekonstruktion der Vorsorgeargumentation beispielhaft deutlich machen (vgl. Eser, Neureuther \& Müller 2011:10; SL):

„P1: Biodiversität ist eine Existenzgrundlage [allen?] menschlichen Lebens.

P2: Wir kennen nicht alle Funktionen aller Arten.

P3: Es ist möglich, dass wir jede einzelne Art [und auch alle Individuen einer Art?] [wofür genau?] brauchen.

P4: Wir haben uns in unserer Umweltpolitik dem Vorsorgeprinzip verpflichtet [weil?].

S: $\quad$ Aus Gründen der Vorsorge sollen wir möglichst alle Arten erhalten!“

Einmal abgesehen von der schwierigen Beantwortung dieser Nachfragen, wird anhand der Rekonstruktion bereits ersichtlich, dass das spezifisch ökologische am Vorsorgeprinzip lediglich ist, dass es „die Komplexität ökosystemarer Zusammenhänge bei der Aufstellung von handlungsleitenden Prinzipien berücksichtigt." (ebd., 10) Erst wenn etwas gegen die unterstellte existenzielle Bedeutung der Biodiversität eingewandt wird (bspw. über die kritischen Nachfragen, welchen Stellenwert diese Existenzgrundlage zu anderen Existenzgrundlagen hat und ob dazu die gesamte belebte Natur notwendig ist oder nur die, die eine existenzsichernde Funktion besitzen), zeigt sich die normative Grundlage des Vorsorgeprinzips. Dieses Prinzip lautet: „Irre lieber auf der sicheren Sei- 
te" (ebd., 10), womit wiederum das Risikovermeidungsprinzip zum Ausdruck kommt, dass bei Entscheidungen unter Unsicherheit die Option gewählt werden sollte, die mit den geringsten Risiken für alle Betroffenen behaftet ist. Auch dieses genuin normative Grundprinzip ist nicht ökologisch begründbar, sondern nur ethisch zu rechtfertigen.

Zusammenfassend kann auf Basis der hier aufgeführten Problematiken und ihres Zusammenspiels konstatiert werden, dass die benannten Probleme sich nicht rein zufällig ergeben, sondern sich systematisch aus der in der NBS geäußerten und nicht weiter begründeten Prämisse ableiten, dass alle Menschen existenziell auf eine 'gesunde', 'funktionsfähige 'und 'stabile' Natur angewiesen sind. Über den Verweis auf die existenzielle Bedeutung der Biodiversität, die den Ausführungen der Gründe in der NBS bezeichnenderweise vorgelagert ist, wird der (problematische) Eindruck erweckt, als ob es selbstevident wäre, die Biodiversität zu erhalten. Des Weiteren wird über die nach Disziplinen gruppierte Systematisierung der Gründe suggeriert (nicht ganz zu unrecht, aber problematischweise), dass sich die Ethik auf die Begründung der Eigenwertintuition sowie und der sogenannten Inklusionsproblematik ${ }^{89}$ reduzieren ließe. Dieser Kontext verweist wiederum auf ein weiteres Problem, dass den Kontext der weiteren Untersuchungen liefert: Wenn davon ausgegangen wird, dass die Erhaltung der Biodiversität existenziell notwendig und damit bereits aus ganz eigennützigen Motiven heraus für alle Menschen evident ist und wenn die Ethik gleichzeitig auf die Ausweisung des Eigenwertes sowie auf die theoretische Lösung der Inklusionsproblematik reduziert wird, dann wird darüber der Eindruck erweckt, als sei das "Angewiesenheitsargument" bereits eine ausreichende (Letzt-)Begründung für alle in der NBS dargelegten Gründe. Vor diesem Hintergrund ist es dann auch verständlich (nichtsdestotrotz aber problematisch), dass die von EthikerInnen notorisch gestellte Frage, ob diese Gründe auch gute oder aber akzeptable Gründe seien, bei vielen überzeugten Natur- und Umweltschützerlnnen als nicht notwendig erachtet wird bzw. immer wieder auf Entrüstung und Empörung stößt, da sie der Auffassung sind, dass die Fakten, die ja seit Jahrzehnten bekannt seien, doch für sich sprechen würden. In diesem Sinne ist die von Wolfgang Erz, dem langjährigen Präsidenten des Bundesamtes für Naturschutz (BfN), zitierte Aussage paradigmatisch, dass:

„[k]ein Mensch auf die Idee [käme], sich begründen zu lassen, warum Raffaels Sixtinische Madonna für alle Zeiten zu bewahren ist. Genauso sollte es mit der Natur sein! Der Schutz der Natur ist ebenso einleuchtend und ebenso wichtig, dass er keiner weiteren Begründung bedarf!“ (Erz zitiert in Piechocki 2010:7)

89 Noch einmal zur Erinnerung (siehe 2.4.2): Bei der Inklusionsproblematik geht es einerseits auf der axiologischen Ebene um die Klärung der werttheoretischen Frage, in welchen Hinsichten die außermenschliche Natur als wertvoll gelten kann und andererseits auf der moralphilosophischen Ebene um die Klärung der pflichtenethischen Frage, wem gegenüber Menschen Pflichten haben und welchen Entitäten Rechte zukommen. 
In einem eben solchen Gestus wird auch in der NBS der Eindruck erweckt, als seien die Begründungsleistungen vernachlässigbar, weil bereits das Angewiesenheitsargument (vermeintlich) als alleinige Begründung ausreiche und weil die restlichen Gründe zum anderen für sich selbst sprechen würden oder aber die Begründungen dafür schlichtweg zu trivial seien, um sie explizit ausführen zu müssen. Allerdings wird seitens des administrativen Umwelt- und Naturschutzes, stellvertretend durch Beate Jessel, der derzeitigen Präsidentin des BfN, mittlerweile anerkannt, dass:

"die ethische Betrachtung nicht eine Argumentationslinie unter vielen für das 'Für und Wider' naturschutzpolitischer Anliegen darstellt. Ethische Argumente sind nicht gegen Argumente anderer Art auszuspielen. Vielmehr handelt es sich um die Einnahme eines Blickwinkels, unter dem alle naturschutzrelevanten Argumente geordnet und abgewogen werden können und unter dem versucht wird, Gründe für den Schutz der biologischen Vielfalt zu formulieren, die allgemein anerkennungswürdig sind." (Jessel in Eser, Neureuther \& Müller 2011:7).

Selbst wenn dies anerkannt wird, dann wird wiederum eingeworfen, dass die breite Zustimmung zu den umwelt- und naturschutzpolitischen Ziele in der deutschen Bevölkerung $^{90}$ und der Nachweis der rechtlichen Unbedenklichkeit ausreichend seien, um die staatlichen Handlungen zum Schutz der Natur bzw. zur Erhaltung der Biodiversität zu rechtfertigen. Im Gegensatz dazu betonen vor allem EthikerInnen immer wieder, dass die faktische Akzeptanz von politischen Zielen und Maßnahmen nicht auch bedeutet, dass diese auch akzeptabel sind und dass rechtliche Normen ihren Usprung in moralischen Normen haben. Daher, so die Auffassung in der Ethik, muss unabhängig davon, was faktisch akzeptiert und rechtlich vorgegeben ist, immer auch geprüft werden, ob es Gründe dafür gibt, dass das Akzeptierte und Normierte auch akzeptabel und in diesem Sinne richtig ist.

In diesem Sinne wird im Folgenden auf die, in der NBS unter dem Abschnitt 'ethische' (besser normative) Gründe vorgelegte Tatsachenbehauptung fokussiert, dass die Biodiversität einen Eigenwert hat und dass aus den ethischen Begründungen Verpflichtungen abgeleitet werden können, möglichst die gesamte noch vorhandene Biodiversität zu erhalten. Um diese beiden Aussagen - auch hinsichtlich ihrer (problematischen) Kombination - kritisch zu reflektieren, werden sich die folgenden Ausführungen mit den umwelt- und naturschutzethischen Begründungsansätzen für den Eigenwert sowie den Verpflichtungen zur Erhaltung der Biodiversität auseinandersetzen.

90 Umfragewerte wie die, dass für 75\% der Bevölkerung die Erhaltung der Biodiversität zu den prioritären gesellschaftlichen Aufgaben zählt oder dass für mehr mehr als $80 \%$ der Befragten die Biodiversität eine unmittelbare Bedeutung für ihre Lebensqualität hat, lassen sich bspw. aus den bisherigen Naturbewusstseinsstudien entnehmen (vgl. BMU 2010, 2012, 2014, 2016) und dienen hier als Argumente. 


\subsubsection{Die umwelt- und naturschutzethischen Begründungsansätze: Der Eigenwert und die direkten (Schutz-)Pflichten}

In der Umwelt- und Naturschutzethik stand von Anfang an die Kontroverse um die Inklusionsproblematik im Fokus (siehe 2.4.2). Die AnthropozentrikerInnen versuchen hierbei über ganz verschiedene Argumente ${ }^{91}$ zu begründen, dass Menschen nur gegenüber anderen (jetzt lebenden und ggf. auch zukünftigen) Menschen direkte moralische Pflichten besitzen, indem sie aufzeigen, warum Menschen, nicht aber anderen, nichtmenschlichen Entitäten, eine moralische Berücksichtigungswürdigkeit um ihrer selbst willlen zukommen sollte. Daraus schlussfolgern sie, dass Menschen zwar direkte moralische Pflichten gegenüber jetzigen und zukünftigen Menschen haben, die Natur ist dabei aber insofern von Relevanz, als dass sie dem Menschen Mittel zum Zweck ist, so dass Menschen indirekte und in diesem Sinne abgeleitete Pflichten in Ansehung der Natur haben. Die PhysiozentrikerInnen argumentieren dagegen, indem sie ebenfalls über eine Vielzahl an Argumenten ${ }^{92}$ zu begründen versuchen, dass der Natur bzw. bestimmten Teilen der Natur eine moralische Berücksichtigungswürdigkeit 'um ihrer selbst willen` eingeräumt werden sollte. Gemeinsam ist allen Physiozentrikern, dass sie daraus schlussfolgern, dass Menschen direkte moralische Pflichten gegenüber (bestimmten Bereichen) der Natur haben sollten: Pathozentriker begründen die moralische Berücksichtigungswürdigkeit dabei auf der Gegenstandsebene für alle Wirbeltiere und argumentieren mit der Bewusstseinsfähigkeit bzw. der Befähigung zu Schmerz-, Leid- und Lustempfindungen, die nicht nur Menschen sondern auch viele Wirbeltiere haben. Biozentriker begründen die moralische Berücksichtigungswürdigkeit auf der Gegenstandsebene wiederum für alle Lebewesen und argumentieren dabei teleologisch, indem sie herausstellen, dass nicht nur das Wohl der Menschen sondern das Wohl aller Lebewesen darin besteht, dass diese ein 'Streben zum Leben ' haben. Ökozentriker begründen die moralische Berücksichtigungswürdigkeit auf der Gegenstandsebene, genau wie Holisten, auch für überindividuelle Ganzheiten wie Arten oder ökosystemare Gemeinschaften. Die herbei angeführten Argumente nehmen dabei aber andere Intuitionen und Begründungsansätze auf.

91 Anthropozentrische Argumente sind (nach Ott 2010:18): 1. Angewiesenheitsargumente auf Natur, 2. Gesundheits- und Wohlbefindensargumente 3. naturästhetische Argumente, 4. Heimat-Argumente 5. 'transformative-value' Argument 6. Biophilie-Hypothese 7. Differenzargument 8. Menschenrecht auf Natur Argumente 9. Pflichten gegenüber zukünftigen Generationen Argumente bezogen auf 1-8. Diese verschiedenen Argumente sollen hier erst einmal nur aufgezählt werden. Auf sie wurde stellenweise bereits eingegangen (Angewiesenheitsargument siehe 4.2.1), auf andere, wie bspw. die naturästhetischen Argumnte oder die Wohlbefindensargumente wird später noch ausführlicher eingegangen (siehe 4.3.2).

92 Physiozentrische Argumente sind (nach Ott 2010:18): 1. sentientistische oder pathozentrische Argumente, 2. biozentrische Argumente, 3. ökozentrische Argumente, 4. holistische Argumente und 5. naturphilosophische Argumente. Diese sollen hier ebenfalls erst einmal nur genannt werden, da zumindest auf die biozentrischen, ökozentrischen und holistischen Argumente später genauer eingegangen wird (siehe 4.2.2.2). 
Diese Auseinandersetzungen zwischen anthropozentrischen sowie physiozemtrischen (pathozentrischen, biozentrischen, ökozentrischen und holistischen) Positionen um das Inklusionsproblem sind nach wie vor ungeklärt. Anstatt dieser eine weitere Position hinzuzufügen, geht es im Folgenden darum, zum einen die, für die Inklusionsproblematik zentrale und auch für den politischen Umwelt- und Naturschutz akzeptanzfördernde Eigenwertintuition kritisch zu reflektieren (4.2.2.1) und zum anderen sollen diejenigen umwelt- und naturschutzethischen Begründungsansätze reflektiert werden, die das Eigenwertargument für die Begründung einer moralischen Schutzpflicht in Bezug auf die Biodiversität nutzen (4.2.2.2). Damit soll einerseits die Problematik in den Fokus gerückt werden, dass der Eigenwert selbst in der ethischen Debatte oftmals lediglich negativ bestimmt wird, indem eine rein instrumentelle Wertigkeit der Natur verneint wird (Norton 1987:153), was im Umkehrschluss bedeutet, dass selten präzisiert wird, auf welchen Annahmen ein solcher Eigenwert überhaupt basiert und was für ein Typus von Eigenwert hier genau gemeint ist. Andererseits wird, ausgehend von den unklaren Eigenwertbestimmungen, auf die Problematik eingegangen, dass selten gesonderte Begründungen dafür angeführt werden, warum der Wert der Natur, selbst wenn dies ein Eigenwert ist, bereits ein Grund dafür sein sollte, dass Menschen auch moralische Handlungspflichten gegenüber der Natur haben. Denn die Rede davon, dass etwas aus - erstmal aus noch nicht-moralischen Gründen - um seiner selbst willen wertzuschätzen ist, erlaubt nicht notwendigerweise auch, dass sich daraus eine moralische Verpflichtung den entsprechenden Gegenständen gegenüber ableitet. ${ }^{93}$ Indem beide Problematiken im Fokus der weiteren Untersuchungen stehen, wird erneut eine problemfokusssierte Reflexion vorgenommen, an die sich wiederum eine konstruktive Lösung der dabei herausgestellten Problematiken anschließen wird (4.3).

\subsubsection{Die Reflexion der Wertedebatte}

Nahezu alle Abhandlungen, die auf die Wertedebatte näher eingehen, weisen darauf hin, dass der Eigenwert eine Entgegensetzung zu den instrumentellen Werten ist, weil

93 Wenn hier angeführt wird, dass es problematisch ist von einem - wie auch immer gearteten - Eigenwert auf moralische Schutzpflichten zu schließen, dann wird dies deshalb als Problem verstanden, weil es sich beim Eigenwert der Natur/Biodiversität um ein axiologisches, nicht-moralisches Werturteil handelt, das sich, anders als ein moralisches Werturteil, weder direkt noch indirekt auf (menschliche) moralische Handlungen bezieht (Birnbacher 2007:47). Da indes auch axiologische Werturteile moralrelevant sind, weil die beurteilten Zustände, Güter oder Ereignisse durch menschliches Handeln herbeigeführt oder verhindert werden können und weil bis auf wenige Ausnahmen (bspw. streng deontologische Moraltheorien) alle Ethiken die Beurteilung der moralischen Qualität von Handlungen auch unter Berücksichtigung der nicht-moralischen Qualität der zu erwartenden oder beabsichtigten Handlungsfolgen abschätzen, sind axiologische Werturteile in fast allen Ethiken für moralische Handlungsurteile relevant. Entscheidend ist dabei allerdings, dass nur bestimmte Ethiken (nämlich teleologische) moralische Handlungsurteile (ausschließlich) von axiologischen Werturteilen abhängen lassen (ebd., 48). In allen anderen Fällen bedarf es einer gesonderten Begründung, wie von der axiologischen Ebene der Bestimmung und Begründung des Eigenwertes zum moralischen Grund der Handlungsverpflichtung gelangt werden soll. 
die zentrale Eigenwertintuition darin besteht, dass die Natur/Biodiversität gerade nicht nur instrumentell wertgeschätzt wird, sondern 'um ihrer selbst willen`Berücksichtigung finden soll. In diesem Sinne handelt es sich beim Eigenwert - ex negativo - um einen nicht-instrumentellen Wert (vgl. Krebs 1996; Eser \& Potthast 1999; Eser, Neureuther \& Müller 2011:79). Um im Folgenden über eine solche Negativ-Bestimmung von Eigenwert hinaus zu gehen, wird in Rückgriff auf Angelika Krebs, aber stellenweise abweichend von der bei ihr verwandten Terminologie, herausgestellt, dass über den Verweis auf den Eigenwert in der Regel drei unterschiedliche Intuitionen bedient werden:

Der ersten Intuition nach, kann die Angabe, dass etwas/jemand 'um seiner selbst willlen`wertvoll ist, sich auf ein Gut beziehen, das von Menschen aufgrund seines glückskonstitutiven Beitrags zum 'guten Leben' (Eudaimonie) wertgeschätzt wird, so dass von einem eudaimonistischen Eigenwert die Rede sein soll. Der zweiten Intuition nach, bezieht sich die Eigenwertigkeit auf bestimmte intrinsische Eigenschaften, die als moralisch relevant angesehen werden und aufgrund derer der Natur/Biodiversität oder Teilen von ihr eine moralische Berücksichtigungswürdigkeit um ihrer selbst willen zugeschrieben wird. Dieser Intuition entspricht der moralische Selbstwert. Der dritten Intuition nach gibt es in der Natur Werte 'an sich', die unabhängig von der Existenz des Menschen und seiner Betrachtung und Wahrnehmung, d.h., objektiv gültig sind. Diesen Eigenwerttypus kennzeichnet Krebs als absolute Werte, abweichend davon wird im Folgenden von objektiven Naturwerten die Rede sein, da darüber die dahinterstehende Eigenwertintuition direkt zum Ausdruck kommt.

Krebs macht diese Differenzierung auf, führt aber nur in Ansätzen aus, was das jeweils Spezifische der drei Werttypen ist. Eben dies ist aber für das Verständnis der Gemeinsamkeiten und Unterschiede der drei unterschiedenen Eigenwerttypen von Relevanz, weil sich erst hieran zeigen lässt, um welchen Typus von Eigenwerten es in der Umwelt- und Naturschutzethik primär geht und weil eine spezifischere Differenzierung der Eigenwerttypen die nachfolgende Reflexion der physiozentrischen Begründungsansätze (siehe 4.2.2.2) enorm erleichtert. Dazu muss indes erst einmal geklärt werden, was den Werttypus der instrumentellen Werte - als Entgegensetzung zu Eigenwerten - ausmacht (4.2.2.1.1). Daraufhin kann geschaut werden, worin die jeweiligen Spezifika eudaimonistischer Eigenwerte (4.2.2.1.2), moralischer Selbstwerte (4.2.2.1.3) und objektiver Naturwerte (4.2.2.1.4) bestehen.

\subsubsection{1 instrumentelle Werte}

Instrumentelle Werte beziehen sich auf Güter (materieller oder immaterieller Art), die als nützliche, brauchbare oder funktionale Mittel zum Erreichen bestimmter Zwecke wertgeschätzt werden, so dass hierbei oftmals von Nutzwerten bzw. funktionalen Werten (Ott 2010:82) die Rede ist. Dabei kann das Charakteristikum instrumenteller Werte 
anhand von drei Aspekten festgemacht werden, die auch im weiteren Verlauf der Untersuchung der Wertedebatte von besonderer Bedeutung sein werden, weil anhand dieser Aspekte die Unterscheidung zur Eigenwertigkeit präzisiert werden kann:

Erstens ist speziell für instrumentelle Werte der Aspekt relevant, dass diesen je nach Grad ihrer Nützlichkeit, Brauchbarkeit oder Funktionalität mehr oder weniger Wertigkeit zukommen kann. Das bedeutet, dass die Wertigkeit instrumenteller Werte nicht absolut sondern (nur) relativ ist, weil die Wertigkeit je nach Kontext durch ihre Nützlichkeit, Brauchbarkeit und Funktionalität zu- bzw. abnehmen kann ${ }^{94}$ und darüber hinaus auch gänzlich verschwinden kann, wenn die Funktionen auch anderweitig erbracht bzw. die Funktion als solche ersetzbar (substituierbar) ist. ${ }^{95}$

Ein zweiter wichtiger Aspekt ist, dass instrumentelle Werte immer extrinsische Werte sind, weil deren Wertigkeit sich nicht aus den jeweiligen Gegenständen selbst heraus ergibt bzw. in ihnen selbst liegt (ihnen intrinsisch ist), sondern sich erst aus ihrer Relation und Beziehung zu anderen ergibt. Mit anderen Worten: Ein instrumenteller Wert entsteht dadurch, dass etwas/jemand für jemanden anderen nützlich, brauchbar oder funktional ist.

Damit zusammenhängend, aber zugleich davon zu unterscheiden, ist der dritte Aspekt, dass instrumentelle Werte immer abhängige Werte sind, da es hier jemanden geben muss, der beurteilt, ob etwas nützlich, brauchbar oder funktional ist. Die Vorstellung, dass etwas 'an sich ' nützlich ist, obwohl es niemand dafür hält, ist zwar im potenziellen Sinne plausibel, bspw. indem man sagt, etwas könnte nützlich werden, auch wenn diese Nützlichkeit jetzt noch nicht vorhanden ist, aber es bleibt der Fakt, dass Nützlichkeit immer von Jemanden erkannt, zugeschrieben und beurteilt werden muss.

Unter Berücksichtigung dieser drei Aspekte, die den Typus der instrumentellen Werte auf der allgemeinen, abstrakten Ebene kennzeichnen, wird behauptet, wenn von der instrumentellen Wertigkeit der Natur/Biodiversität gesprochen wird, dass die Biodi-

94 Man stelle sich zur Illustration einen Kiefernwald vor. Dieser hat, als Ökosystem, zahlreiche Funktionen sowohl für die in ihm lebenden Organismen als auch für die Menschen. Insofern kann davon gesprochen werden, dass der Wald für jemanden/etwas instrumentell wertvoll ist. Allerdings ist diese Pauschalisierung bereits problematisch, denn man stelle sich vor, ein Teil des Waldes würde durch einen Waldbrand niederbrennen. Dies wäre für bestimmte Menschen, die bspw. aus diesem Flurstück ihr Brenn- oder Bauholz bezeogen haben von Nachteil, so dass der Wald an Nutzen und damit auch an instrumenteller Wertigkeit für diese verliert, obgleich der Waldbrand für andere nichtmenschliche 'Nutzer' des Waldes, bspw. bestimmte Insekten, Pflanzen oder Vogelarten an 'Nützlichkeit' gewinnt. Auch kann man sich vorstellen, dass es durch die Verknappung der lokalen Holzressourcen in diesem Gebiet nicht nur 'Verlierer', sondern auch 'Gewinner' gibt: Diejenigen, deren Waldgebiet nicht vom feuer betroffen war, sind möglicherweise einige Konkurrenten losgeworden, so dass selbst der abgebrannte Wald ihnen einen Nutzen beschehrt hat.

95

Bezüglich der Substitution sei nur das klassische Beispiel der Ersetzung echter Bäume durch täuschend echt aussehende Plastikbäume genannt, das Laurence Tribe in seinem Ausatz „Was spricht gegen Plastikbäume« (vgl. Tribe 1980) kritisch diskutiert. 
versität wertvoll ist, weil sie mehr oder weniger nützlich, brauchbar und funktional ist. Der Aspekt der funktionalen Werthaftigkeit der Biodiversität ist indes in zweifacher Hinsicht relevant - zum einen in Hinblick auf die Funktionen der (a)biotischen Vielfalt und zum anderen in Hinblick auf die Funktionen, die das Konzept der biologischen Vielfalt innehat (Jax 2003:149). Beide Betrachtungsweisen auf die Funktionen der Biodiversität sind voneinander zu unterscheiden, dennoch aber abhängig voneinander, da die Funktionen der (a)biotischen Vielfalt vor dem Hintergrund der verschiedenartigen Ansätze der Konzeptualisierung der Biodiversität dargelegt werden, während die Behandlung der Funktionen der biologischen Vielfalt ihrerseits die lebensweltlich geprägten Betrachtungen der (a)biotischen Vielfalt voraussetzen. Sowohl deren Unterschiedlichkeit als auch deren Bezüge aufeinander werden bei der Rede von den Funktionen der Biodiversität nicht hinreichend reflektiert, wenn die Frage nach dem instrumentellen Wert der Biodiversität auf die ökologische Funktion der (a)biotischen Vielfalt reduziert wird, so dass es hierbei um die Funktionen der genetischen Vielfalt, der Artenvielfalt und der ökosystemaren Vielfalt füreinander bzw. den Menschen sowie um die Bedrohung ihrer Funktionsfähigkeit geht. Vor diesem Hintergrund muss eingewandt werden, dass das, was hier jeweils mit Funktion gemeint ist, auf ganz unterschiedliche Sachverhalte und Kontexte verweisen kann, so dass sich damit auch das verändert, was als instrumentell wertvoll gekennzeichnet wird (ebd., 154-168): Von einem instrumentellen Wert kann nur dann stichhaltig die Rede sein, wenn die funktionalen Zusammenhänge nicht rein beschreibend gemeint sind, da ansonsten der Übergang von der Ebene der Deskription auf die Wertungsebene unklar bleibt. Dies disqualifiziert indes all die Funktionsbeschreibungen, in denen es um korrelative oder kausale Prozesse oder um die Interaktion zwischen verschiedenen Funktionsträgern geht (ebd., 154155), da der Fokus instrumenteller Werte auf dem Nutzen für jemanden oder etwas liegt, so dass Funktion bzw. funktionieren hier immer schon normativ als Leistung verstanden wird, die „einem System gemäß seinem Nutzen zugewiesen wird“ (ebd., 156):

„[E]s geht also, obwohl zunächst ganz wertfrei nur von 'Prozessen` als solchen gesprochen wird, de facto doch um spezielle Prozesse, nämlich solche, die typisch für das System sind, damit es nicht nur als (irgendein) Ökosystem, sondern als und hier gewinnt das Wort normative Bedeutung - 'funktionierendes`ökosystem angesehen wird." (ebd., 163)

Damit stellt sich indes die Aufgabe, Kriterien für den Nutzen und für die Leistungen von Ökosystemen, Arten und Genen zu definieren und außerdem einen Referenzzustand anzugeben, zu dessen Erhaltung Biodiversität einen Beitrag liefert. Dabei muss deutlich werden, dass es sich hierbei um individuelle und gesellschaftliche Zweckbestimmungen handelt, die einer kontroversen Diskussion, gerade weil es Zwecksetzungen sind, offen stehen müssen, auch wenn manche davon - wie die Angewiesenheit von Men- 
schen von der Biodiversität - als selbstverständlich und unhintergehbar ausgewiesen werden (siehe 4.2.1). Da genau dies selten explizit genug geschieht, werden die, rein deskriptiv zu erfassenden, Interaktionsmuster und Wirkungen der Komponenten der Biodiversität aufeinander nicht von den bereits normativ imprägnierten 'Funktionen ' unterschieden. Daher wird selten zwischen den ökosystemaren Prozessen als solchen und den Leistungen der Natur/Biodiversität (bspw. als Nahrungsgrundlage, als Arztneimittel, als Rohstoff, als Forschungsgegenstand, als Erholungsraum, als Quelle spiritueller oder ästhetischer Erfahrungen etc.) unterschieden (Baumgärtner 2002:74-78), auf die nicht-menschliche Lebewesen und Menschen (mehr oder weniger stark) angewiesen sind. Gelegentlich werden daher auch die Leistungen der Biodiversität in einer naturalistischen Art und Weise als naturgegeben bzw. als Normalzustand einer gesunden Natur interpretiert, obwohl schon die Art der Frage 'nach der Funktion von etwas für etwas` eine wertende Entscheidung voraussetzt, die begründet werden muss.

Das zeigt summa summarum, dass es für die Rede davon, dass die Biodiversität instrumentell wertvoll sei, nicht ausreichend ist, dass das instrumentelle an diesen Werten mit den Prädikaten funktional, nützlich oder brauchbar betitelt wird, weil genau diese Charakterisierungen zwangsläufig zu den Präzisierungen zwingen, von wem und für wen hier ein Wert postuliert wird, worauf sich der Wert beziehen soll, worin dieser genau besteht und warum dieser als wertvoll angesehen wird. Wird dies geleistet, dann sind es weniger die Spezifika instrumenteller Werte, die strittig sind, als vielmehr die darauf basierende Beurteilung der ökonomischen, institutionellen und politischen Verhältnisse, die aus der instrumentellen Wertigkeit der Natur/Biodiversität resultieren sollen. Gerade weil dies der Fall ist, muss bei instrumentellen Werten im Besonderen darauf geachtet werden, dass hinter den 'ökologischen Gründen ' und Argumenten für die Erhaltung der Biodiversität - die (problematischerweise) als natürlich gegeben angenommen und zugleich von ökonomischen, soziokulturellen aber auch normativen Gründen und Argumenten unterschieden werden, die als 'subjektiv' klassifiziert werden - als Leistungen interpretierte Zwecksetzungen stehen, die von den Ökosystemprozessen und ihrer Untersuchung unterschieden werden sollten (ebd., 161). Zudem sollte präziert werden, ob sich die instrumentellen Werte und die daran abschließenden Beurteilungen auf die (a)biotische Vielfalt oder auf die biologische Vielfalt beziehen, denn wenn diesbezüglich nicht die instrumentellen Werte der (a)biotischen Vielfalt sondern der biologischen Vielfalt als Konzept gemeint sind, steht hierbei eine anders gelagerte Fragestellung im Fokus, wo es darum geht, ob die biologische Vielfalt als Mittel zum Zweck brauchbar ist, um Menschen für Natur- und Umweltschutzanliegen zu motivieren und um die unterschiedlichen Stränge, Ausrichtungen und Sichtweisen innerhalb des Umwelt- und Naturschutzes zu integrieren. 


\subsubsection{2 eudaimonistische Eigenwerte}

Der eudaimonistische Eigenwert stellt ein Gut dar oder bezieht sich auf eines, das von Menschen aufgrund seines glückskonstitutiven Beitrags zum 'guten Leben' (Eudaimonie) wertgeschätzt wird. Hierbei wiederum stehen die antiken, aristotelischen Fragen im Vordergrund, was ein wahrhaft glückliches, gelingendes oder eben gutes Leben ist, was es ausmacht und wie es erreicht werden kann, die in der gegenwärtigen philosophischen Diskussion eine erstaunliche Renaissance erleben und zu einer Vielzahl an neoaristotelischen Ansätzen führen (Steinfath 1998:7-9). Selbiges gilt auch für den eudaimonistischen Eigenwert: Als Eigenwerttypus lässt er sich in gewisser Weise als Kontrapunkt zu den - in der Umwelt- und Naturschutzdebatte - stärker etablierten Werttypen (instrumentelle Werte, moralische Selbstwerte und objektive Naturwerte) verstehen, da auch er ein Produkt der derzeitigen Renaissance der Theorien des guten Lebens und speziell der Glücksgütertheorien ist. ${ }^{96}$ Diese zeichnen sich vor allem dadurch aus, dass sie die Voraussetzungen und Bedingungen von 'Glück' und 'Wohl' als inhärente Werte postulieren, wobei inhärente Werte zwischen extrinsischen und intrinsischen Werten verortet werden, weil angenommen wird, dass allen glückskonstitutiven Dingen ein eigener Wert zugeschrieben wird, diese Werte aber stets an den obersten Wert des Glücks gekoppelt sind und daher mit dem zusammen fallen können, was auch unter der instrumentellen Perspektive als mögliches Erleben von Glück thematisiert werden kann. Insofern muss aber der Status solcher inhärenter Werte als Eigenwerte hinterfragt werden. Eben dies lässt sich am besten konkret am Beispiel der Charakterisierung eudaimonistischer Eigenwerte der Biodiversität aufzeigen:

Bezogen auf Biodiversität wird bei den eudaimonistischen Eigenwerten die Intuition bedient, dass die Biodiversität einen ganz besonderen, weil einzigartigen und unersetzbaren Beitrag zum gelingenden Leben von Menschen leistet (Eser, Neureuther \& Müller 2011:81). Dass auch hier die Rede von Leistung ist, zeigt bereits, dass auch eudaimonistische Eigenwerte einer Zwecksetzung unterliegen und damit instrumentell

96 Die klassische Variante einer solchen Glücksgütertheorie ist die Theorie der Eudaimonie bei Aristoteles. In der Neuzeit sind diese Theorien auf fundamentale Kritik gestoßen. Insbesondere Kant prägte den Begriff „Eudämonismus“ für alle ethischen Lehren, in denen das Streben nach Glückseligkeit und nicht die Pflicht als maßgeblicher Grund des sittlichen Handelns gilt. Kants 'Verdammungsurteil' beeinflusste die moderne Rezeption der antiken Konzepte stark, wobei sich durch die neueren Diskussionen um die 'Glücksphilosophien`zumindest teilweise eine Rehabilitierung des antiken Gedankenguts abzeichnen. Im Anschluss daran sind in den letzten 20 Jahren wieder neuere Glücksgütertheorien entwickelt worden (etwa von James Griffin, Amartya Sen und Martha Nussbaum). Vor dem Hintergrund, dass die Überlegungen zum 'gelingenden Leben ' in der derzeitigen Phase nicht nur unabgeschlossen sind, sondern besonders in anwendungsorientierten Ethiken oftmals in einer negativen oder affirmativen Kritik an der modernen, kantisch geprägten Moraltheorie verharren (Steinfath 1998:12), wird der eudaimonistische Eigenwert auch in der Umwelt- und Naturschutzethik selten ausreichend genug bestimmt, so dass oftmals nicht deutlich wird, welche Aspekte des 'gelingenden Lebens' hierbei in den Vordergrund gestellt werden (Glück, Sinn, Tugenden etc.) und wie gutes Leben dabei genau verstanden wird (als das gute Leben, als Liste von bestimmten Grundgütern, als Summe aller glück-lichen Erlebnisse und Taten etc.). 
sind. Diese Zwecksetzung ist aber, so die zentrale Intuition hinter den eudaimonistischen Eigenwerten, eine qualitativ andere als dies bei instrumentellen Werten der Fall ist, da hierbei von rein funktionalen Erwägungen ganz bewusst abgesehen wird, indem auf den - über der unmittelbare Funktionalität stehenden - einzigartigen und unersetzbaren Wert von etwas für das gute Leben von jemanden abgehoben wird. ${ }^{97}$ Darunter fallen, so Krebs, vor allem ästhetisch-kontemplative Naturerlebnisse der schönen und erhabenen Natur (vgl. Seel 1997), so dass hier speziell von einem ästhetischen Eigenwert gesprochen wird. Des Weiteren fallen darunter aber auch identitätsstiftende und individualitätskonstitierende Naturbeziehungen, die Krebs mit „Heimatwert“ betitelt sowie sinnlich-spirituelle Naturerfahrungen der „Heiligkeit der Natur" oder Argumente eines aus der Natur ablesbaren „Sinn des Lebens" (Krebs 1996:35): Der ästhetische Eigenwert wird dabei von Krebs als der wichtigste Typ eudaimonistischer Eigenwerte eingestuft. ${ }^{98}$ Unter der Annahme, dass es sich bei der Praxis der ästhetischen Kontemplation um eine vollzugsorientierte Form der Wahrnehmung handelt (Seel 1997:312), deren Ausübung 'um ihrer selbst willen' angestrebt wird und genau deshalb glückskonstitutiv sei, ergibt sich, so Seel, ein doppelter Wertbezug sowohl auf die Wahrnehmungshandlung als auch auf das Wahrgenommene. ${ }^{99}$ Wenn die Natur/Biodiversität im

97 Exemplarisch kann die dahinterstehende Intuition und deren Problematik am Beispiel eines T-Shirts dargelegt werden: Wenn ein T-Shirt gut geschnitten, schön, bequem und kontextuell passend ist, dann ist es in vielerlei Hinsicht instrumentell wertvoll für denjenigen, der es besitzt. Ist das T-Shirt indes alt und verwaschen, hat überall Löcher, reißt entzwei und lässt sich nicht mehr reparieren oder aber anderweitig verwenden (bspw. als Flicken), dann ist es nutzlos geworden, es hat keine unmittelbare Funktion und damit keinerlei instrumentelle Wertigkeit mehr. Das bedeutet aber nicht, dass ein funktions- und nutzloses (aber eben niemals gänzlich zweckloses) T-Shirt nicht dennoch wertvoll für jemanden sein kann, wenn an diesem (funktions- und nutzlosen) T-Shirt bspw. noch der Duft einer geliebten Person haftet oder wenn jemand mit genau diesem Shirt ein ganz bestimmtes Erlebnis verbindet, das er als bedeutsam für seinen Lebensweg und seine Ich-Werdung ansieht. Ein kaputtes TShirt, was man aus solchen Gründen aufbewahrt, bewahrt man auf, weil es besonders gegenüber allen anderen Shirts ist. Das, was man also daran wertschätzt, ist, dass genau dieses T-Shirt es einem ermöglicht, in besonderer Art und Weise an den geliebten Menschen oder an bestimmte Situationen zu denken. Insofern hat auch das nutz- und funktionslose T-Shirt noch einen Zweck und dieser ist nicht relativ zur unmittelbaren Nützlichkeit, Brauchbarkeit oder Funktionalität, sondern stellt für den Betrachter aufgrund seiner Besonderheit, Einzigartigkeit und Unersetzbarkeit einen Selbstzweck und damit einen Eigenwert dar.

98 Oftmals werden eudaimonistische Eigenwerte und ästhethische Eigenwerte synonym oder aber in Bezug aufeinander verwendet, wobei dann aber nicht deutlich gemacht wird, wie beide sich zueinander verhalten bzw. wie die Ästhetik sich zu den Theorien des guten Lebens verhält. Ob es gerechtfertigt ist, sie synonym zu verwenden, wird hier angezweifelt, da für eine Subsumierung der ästethischen Eigenwerte unter den Typus der eudaimonistischen Eigenwerte spricht, dass die Möglichkeit zur ästhetischen Wahrnehmung (sowohl als Fähigkeit zu lustvollen Erlebnissen als auch als Fähigkeit seine Sinne zu benutzen) nur eine von vielen Möglichkeiten ist, ein gelingendes Leben zu führen (Nussbaum \& Sen 1993:339-340).

99 Nach Franz von Kutschera sind ästhetische Urteile „solche, mit denen wir den Gehalt ästhetischer Erfahrungen beschreiben." (Kutschera 1998:90) Dabei formuliert er die Hypothese, dass eine Erfahrung dann eine ästhetische Erfahrung ist, wenn diese interessenlos ist (ebd., 73). Genau darauf bezieht sich auch bei Seel der Begriff der Kontemplation, indem er als „interessenlose Aufmerksamkeit“ charaktersiert wird, bei der „von jeder Wichtigkeit der Dinge für das Erkennen oder Handeln abge- 
Ganzes oder aber auch in Teilen als Objekt ästhetischer Kontemplation angesehen, erfahren und erlebt werden kann, dann hätte sie deshalb einen ästhetischen Eigenwert, weil sie den Menschen aufgrund ihrer „Selbstständigkeit und Veränderlichkeit ihrer Gestaltungen, wegen der ungelenkten Fülle der Erscheinungen, die sie unseren Sinnen darbietet“, die Erfahrung einer „nicht vom Menschen bewirkten Prozessualität“ ermöglicht (ebd., 315). Dementsprechend führt auch Anne Kemper in ihrem Buch »Unverfügbare Natur« (vgl. Kemper 2001) aus, dass die Eigenwertigkeit der Natur aus ihrer „ästhetisch konstitutierten Unverfügbarkeit“ (ebd. 34) erwächst, so dass der spezifisch ästhetische Erfahrungsmodus dazu führt, die Natur/Biodiversität als Dasein um ihrer selbst willen wertzuschätzen:

„Wo immer Natur als 'freie` auf den Menschen zukommt, (...) ist eine ästhetische Erfahrung gemacht worden. Die Eigenwertigkeit der Natur ist dem ästhetischen Erfahrungsmodus insofern inhärent, als Natur hier freigelassen wird in ein 'Dasein um ihrer selbst willen` und gerade in dieser unvermeßbaren Selbsttätigkeit uns berührt und anspricht. Der ästhetisch erfahrene Naturgegenstand lässt sich nicht herauslösen aus einer Erscheinungsweise, in der weder die Natur als über den Menschen verfügend, noch der Mensch als über die Natur verfügend erlebt wird, sondern in der Natur dem Menschen auf eine Weise begegnet, das sie ihn in ihrer ästhetisch konstituierten Unverfügbarkeit anspricht.“ (Kemper 2001:34)

In Krebs Worten "färbt" damit der ästhetische Eigenwert auf die Naturobjekte ab, die sich für eine solche Kontemplation besonders eignen (Krebs 1997:371). Zu denken wäre hier an den Horizont des Meeres, gewaltige Wasserfälle, bizarre Felsformationen, idyllische Täler oder schöne Blumen (Krebs 1996:36). Auf Basis ihrer Eignung für ästhetische Kontemplationen wird wiederum auf einen ästhetischen Eigenwert der Natur/ Biodiversität geschlossen aufgrund der Eigenwertigkeit, die den ästhetisch-kontemplativen Naturerfarungen zugesprochen wird:

„Im (...) ästhetischen Fall gewinnt etwas einen Eigenwert zugleich mit dem Eigenwert, den seine Wahrnehmung für etwas gewinnt." (Seel 1997:318)

Diesbezüglich wird dann oftmals darauf verwiesen, dass insbesondere die Erhabenheit der Natur und das Naturschöne (anders als das Kunstschöne), als das nicht vom Menschen Gemachte oder Geschaffene, „ästhetische Attraktionen“ darstellen, die Kunstwerke prinzipiell nicht bieten und nicht bieten können (Krebs 1996:38) und dass ästhe-

sehen" wird (Seel 1991:39). Hierbei ist indes mit Meyer einzuwenden, dass die Interessenlosigkeit im Sinne einer Abwesenheit der die Erfahrung begleitenden Motive weder eine notwendige noch eine hinreichende Bedingung für ästhetische Erfahrungen darstellt (vgl. Meyer 2003:93-96). Wenn dies so ist, dann bleibt unklar, was genau die ästhetische Erfahrung von der nicht-ästhetischen Er-fahrung unterscheiden soll und warum dann davon ausgegangen werden kann, dass ästhetische Erfahrungen um ihrer selbst willen angestrebt werden, während dies bei nicht-ästhetischen Erfahrungen nicht der Fall ist. 
tische Naturerlebnisse eine wichtige, weil einzigartige, besondere und unersetzbare 'Glücksmöglichkeit' des Menschen darstellen. Warum indes davon ausgegangen werden kann, dass dies so ist, wird selten ausreichend genug plausibilisiert. Vielmehr werden hier zahlreiche Aussagen, Argumente und Beispiele dafür angeführt, inwiefern Natur schön und erhaben ist und warum die ästhetische Kontemplation als solche eigenwertig ist, es wird aber nicht ausreichend genug plausibilisiert, was die ästhetische $\mathrm{Na}$ turerfahrung von der nicht-ästhetischen unterscheidet und warum die ästhetische Kontemplation für Menschen wichtig für das gute Leben sein sollte. Genau dies müsste aber geschehen, wenn der ästhetische Eigenwert zum einen als Sonderfall eudaimonistischer Eigenwerte plausibilisiert werden soll und wenn das ästhetische Argument zum anderen im Kontext des Umwelt- und Naturschutzes angewandt wird, denn selbst wenn zugestanden werden kann, dass die ästhetische Kontemplation eine Wahrnehmungs- und Erfahrungsform ist, an der einem 'um ihrer selbst willen ' liegt - was verlangen würde, das Besondere und Eigenwertige ästhetischer Erfahrungen gegenüber nicht-ästhetischen Erfahrungen deutlich zu machen - kann eingewandt werden, dass über das ästhetische Naturschutzargument gerade kein ästhetischer Eigenwert, sondern nur einen ästhetisch-instrumenteller Wert zugeschrieben wird, da die Natur/ Biodiversität hierbei Mittel zum Zweck für die ästhetische Kontemplation ist:

„In dem Moment, in dem man die Natur für schützenswert erklärt und erhalten will, erklärt man ein Interesse an der Existenz der Natur aus einem Interesse an der Möglichkeit ihrer ästhetischen Betrachtung. (...) Der Schutz der Natur würde dann aber nicht wegen ihres Eigenwertes, sondern wegen des instrumentellästhetischen Wertes erfolgen, den ihre Betrachtung für den menschlichen Beobachter hat. (...) Damit wird sie aber nicht mehr um ihrer selbst willen geschützt und ihr wird auch kein Eigenwert zugeschrieben." (Meyer 2003:123)

So schwach die Fundamente des ästhetischen Eigenwertes hinsichtlich des Spezifikums ästhetischer Wahrnehmung sind und so wenig sich die Natur/Biodiversität über das ästhetische Argument im Umwelt- und Naturschutzkontext als nicht-instrumenteller Eigenwert ausweisen lässt, so nachvollziehbar und unhintergehbar scheint doch die hinter dem ästhetischen Eigenwert stehende Intuition zu sein, dass es einen qualitativen Unterschied macht, wie man die Natur/Biodiversität - egal ob im Ganzen, in Gestalt bestimmter Gruppierungen oder in Gestalt ganz bestimmter Entitäten - wahrnimmt, wie man sie erlebt, wie man mit ihr 'in Beziehung tritt'. Gerade die bewusste Absehung von rein nutzenbezogenen, funktionalen, auf Brauchbarkeit reduzierten Erwägungen zugunsten anderer Motive, die als 'glückskonstitutiv' verstanden werden, scheint hier das Entscheidende zu sein. Sobald der Natur/Biodiversität also von Jemandem nicht-instrumentelle Qualitäten zugeschrieben werden, wird sie von diesem behandelt, als ob sie einen Eigenwert hätte, da die Natur/Biodiversität für diesen Men- 
schen einen besonderen, einzigartigen, unersetzbaren Status in Hinblick auf die Realisierung seines guten Lebens hat.

Genau in dieser Hinsicht sind auch die beiden anderen eudaimonistischen Werttypen der Heimatwert und der sinnlich-spirituelle Wert - relevant, auf die Krebs jedoch nur am Rande näher eingeht: Der Heimatwert ergibt sich bei Krebs daraus, dass die Natur/ Biodiversität - und hier vor allem in Form bestimmter Kulturlandschaftsbilder - von vielen Menschen als wichtiger Aspekt ihrer eigenen Individualität verstanden wird. Das Gefühl der 'Beheimatung', das weitaus mehr umfasst als die Vertrautheit mit einer bestimmten Gegend sowie die Wertschätzung dieser als 'natürliche Heimat', verweist darauf, dass es bei allen Menschen bestimmte Orte, Gegenden oder Naturerlebnisse gibt, die mit der eigenen Lebensführung (positiv sowie negativ) verbunden sind:

„Wo Natur als Heimat Teil menschlicher Individualität ist, geht der eudaimonistische Eigenwert von Individualität über auf ihren Teil: Natur. Denn es macht keinen Sinn, dem, was einen Teil der Individualität ausmacht, nur einen instrumentellen Wert für die Individualität zuzuschreiben." (Krebs 1996:39)

Hierbei tritt indes das Problem zutage, dass 'Heimat' ganz unterschiedlich verstanden werden kann und nur in einer ganz bestimmten Passform auch in den Umwelt- und Naturschutzkontext passt: Wenn Menschen bspw. große Städte als Heimaten empfinden oder Heimat mit primär negativen, möglicherweise auch identitätsgefährdenden Erfahrungen verbinden, dann verschwimmt zum einen der Bezug von Heimat zur Natur/ Biodiversität und zum anderen wird dann unklar, warum der Heimat ein Wert zugeschrieben werden sollte, wenn diese auch als abträglich für das gute Leben verstanden werden kann. Des Weiteren kann genau wie beim ästhetischen Eigenwert eingewandt werden, dass die Heimat hierbei ebenfalls nur Mittel zum Zweck ist, um sich in seiner eigenen Identität zu vergegenwärtigen oder seine Individualität zu erfahren und herauszustellen, weil die Eigenwertigkeit einer identitätsstiftenden Heimat nur abgeleitet über die Eigenwertigkeit der menschlichen Individualität zustande kommt.

Meyer versucht diese Lücken des Heimatwertes über den Umweg der „Liebe zur (schönen) Natur" (vgl. Meyer 2003:124-129) zu schließen, kommt dabei aber nur zu dem Ergebnis, dass sich die gefühlte Eigenwertigkeit der Naturerfahrung über die Thematisierung der emotionalen Bindungen vieler Menschen zur Natur/Biodiversität intuitiver einfangen lässt als über das Konzept der ästhetischen Kontemplation oder der natürlichen 'Beheimatung' (ebd., 125-126). In diesem Sinne argumentiert Meyer dafür, dass sich der eudaimonistische Eigenwert der Natur/Biodiversität nicht direkt aus der Schönheit der Natur oder der Natur als Heimat herleiten lässt, sondern nur über den „Umweg der Liebe“ zur Natur/Biodiversität (ebd., 127). Hierbei ist indes entscheidend, dass nur eine bestimmte Form der Liebe überhaupt geeignet ist, um davon zu spre- 
chen, dass die Liebe 'um ihrer selbst willen 'vollzogen wird. Dies ist, so Harry Frankfurt, nur die „aktive Liebe“, bei der dem Liebenden selbstlos an dem liegt, was er liebt, weil er das Objekt seiner Liebe um 'seiner selbst willen` liebt:

"In active love, the lover cares selflessly about his beloved. It is important to him for its own sake that the object of his love flourishes; he is disinterestedly devoted to his interests and ends." (Frankfurt 1999:135)

Die Frage, die sich hier indes stellt, ist, ob die aktive Liebe zur Natur/Biodiversität, die um ihrer selbst willen als wertvoll für das gute Leben verstanden wird, auch der Natur/ Biodiversität selbst zukommt bzw. sich analog zur ästhetischen Kontemplation auf diese übertragen lässt, da hier eingewandt werden kann, dass die Natur/Biodiversität nur Mittel zum Zweck ist, um eine solche Liebe überhaupt zu erleben oder ausüben zu können. Selbst wenn diesem Einwand begegnet werden kann, kann des Weiteren bezweifelt werden, ob sich die Natur/Biodiversität als Ganzes überhaupt als Objekt einer solchen aktiven Liebe eignet. ${ }^{100}$ Darüber hinaus scheinen Menschen, die die Natur/Biodiversität lieben, sie eher nicht aktiv als vielmehr passiv zu lieben, was heißt, dass die Beziehung zur Natur/Biodiversität angestrebt wird, weil die Begegnung mit ihr bei vielen Menschen, bewusst oder unbewusst, durch Erwartungen von positiven Gefühlen motiviert wird. Meyer macht dies am Heimatgefühl indirekt deutlich:

„Die emotionale Verbundenheit oder Liebe zur Natur kann man auch als Heimatgefühl bezeichnen. (...) Die emotionale Bindung an eine Gegend kann daraus resultieren, dass man sie schon lange kennt und viele Erinnerungen an sie knüpft. (...) Menschen lieben häufig die Landschaft, aus der sie kommen, in der sie aufgewachsen sind und die ihnen daher sehr vertraut ist. Viele Menschen tendieren dazu, positive Gefühle gegenüber der Landschaft zu entwickeln, in der sie einige Zeit ihres Lebens gelebt haben." (Meyer 2003:127-128)

Diese Form der passiven Liebe scheint aber nicht nur auf das Heimatgefühl beschränkt zu sein, sondern trifft vielmehr für alle Kriterien zu aufgrund derer die Natur/Biodiversität von Menschen geliebt wird: Sowohl die Schönheit und Erhabenheit der Natur, die Vertrautheit mit der typischen Eigenart bestimmter Landschaftsbilder, die vom Menschen unabhängige 'Andersartigkeit' nicht-menschlicher Lebewesen als auch die 'Wildheit , 'Heiligkeit' oder 'Sinnbildlichkeit' der Natur, bei all diesen Aspekten sind die positiven Gefühle, Stimmungen, Erwartungen, Selbstbilder etc. ausschlaggebend, die durch das Erleben und die Erfahrung nicht menschengemachter Natürlichkeit hervorgerufen

${ }^{100}$ Wenn NaturliebhaberInnen bspw. bestimmte Schmetterlingsarten 'lieben', ihren Urlaub damit zubringen Babyschildkröten ins Meer zu geleiten oder aber Bäume zu umarmen, dann ist dies zweifelsohne auf eine emotionale Bindung zur Natur zurückzuführen. Das Problem dabei scheint aber zu sein, dass das was mit 'aktiver Liebe' gemeint ist, jemanden ganz bestimmten (besonderen, einzigartigen, unsetzlichen) zukommt und es NaturliebhaberInnen sowohl im Fall der Schmetterlinge, der Bäume als auch der Schildkröten nicht um einzelne Entitäten als solchen geht. 
werden. Insofern kann im Ganzen konstatiert werden, dass die Liebe zu einer schönen und erhabenen, vertrauten und typischen sowie heiligen und sinnhaften Natur - womit zugleich alle von Krebs benannten 'Kandidaten ' für eudaimonistische Eigenwerte benannt sind - nicht bedeuten muss, dass dabei der Natur/Biodiversität selbst auch ein eudaimonistischer Eigenwert zukommt: Zum einen sind es vornehmlich die hierbei ausgezeichneten Praxen des Wahrnehmens, Erfahrens und Erlebens von Natur/Biodiversität (kontemplativ und aktiv liebend), die hierbei als eigenwertig klassifiziert werden. Zum anderen wird nicht plausibel, warum die Eigenwertigkeit auf die Natur/Biodiversität im Ganzen übertragen werden sollte, denn sobald der postulierte ästhetische Eigenwert (der Kontemplation) und der Heimatwert (der aktiven Liebe) in den übergeordneten Kontext des guten Lebens gestellt werden, was notwendig ist, um auszuweisen, warum diese glückskonstitutiv sein sollen, wird die Natur/Biodiversität Mittel zum Zweck für die ästhetische Kontemplation und die aktive Liebe zur Natur/Biodiversität. Dies wiederum macht deutlich, dass die oftmals pauschal postulierte Gegensätzlichkeit zwischen instrumentellen Werten und Eigenwerten zumindest im Fall eudaimonistischer Eigenwerte nicht wirklich zu überzeugen vermag. Vielmehr scheint es hier (lediglich) einen qualitativen Unterschied zu geben, indem über bloße Nützlichkeitserwägungen hinaus Gründe der Wertschätzung angegeben werden:

Indem sich die Wahrnehmung, die Erfahrung, das Erleben bzw. die Beziehung zur Natur/Biodiversität nicht relativ zu dessen Nützlichkeit, Funktionalität oder Brauchbarkeit ergibt, ist die Wertigkeit dieses Gutes auch nicht relativierbar oder substituierbar über Erwägungen der Nützlichkeit, Funktionalität oder Brauchbarkeit. Da sie vielmehr in der Besonderheit, der Einzigartigkeit und der Unersetzbarkeit für jemanden im Rahmen seines guten Lebens liegt, handelt es sich um einen nicht relativierbaren und nicht-substituierbaren Wert, weil etwas entweder besonders, unersetzlich oder einzigartig ist oder eben nicht. ${ }^{101}$ Die Eigenwertigkeit ergibt sich hier folglich nicht daraus, dass der eudaimonistische Eigenwert kein instrumenteller Wert wäre, denn das ist er in Hinsicht auf die Zwecksetzung der Realisierung des guten Lebens auf jeden Fall. Sie ergibt sich vielmehr daraus, dass die relativierbare und substituierbare Wertigkeit von etwas/jemanden für Jemanden bestritten wird, so dass hierüber zum Ausdruck gebracht wird, dass etwas/jemand auch 'um seiner selbst willen ' für Jemanden relativ zu dessen Betrachtungen des guten Lebens wertvoll sein kann. Daraus ergibt sich wiederum, dass instrumentelle Werte und eudaimonistische Eigenwerte mehr gemeinsame als diver-

${ }^{101}$ Das, was für den einen aufgrund seiner ganz persönlichen Lebensgeschichte besonders, einzigartig und unersetzlich ist, kann für den Anderen nichts davon sein und dies wird für den anderen auch erst dann nachvollziehbar, wenn derjenige ihm Einblicke in seine Lebensgeschichte gewährt. Daher ist das, was das betreffende Gut für den einen Menschen eudaimonistisch eigenwertig macht, untrennbar mit dem Menschen und seiner persönlichen Lebensgeschichte verwoben, was indes nicht ausschließt, dass es auch intersubjektive Bewertungen bestimmter Güter als eudaimonistisch wertvoll geben kann, die nicht nur rein subjektiv wertvoll sind. 
gierende Charakteristika aufweisen: Genau wie bei den instrumentellen Werten liegt der Grund für die Wertschätzung nicht in den Objekten selber sondern bleibt diesen extrinsisch, weil es auch hier darum geht, dass diese einen bestimmten Zweck haben und dieser Zweck ist es eben, an den sich die Wertschätzung haftet. Des Weiteren bleibt auch hier die Wertzuschreibung davon abhängig, ob es Menschen gibt, die die Besonderheit, Einzigartigkeit und Unersetzbarkeit der Natur/Biodiversität erkennen, anerkennen und für wertvoll halten.

In diesem Sinne kann zusammenfassend festgehalten werden, dass die zwei zentralen, hinter den eudaimonistischen Eigenwerten stehenden Intuitionen, dass es bestimmte, wie auch immer geartete eigenwertige Praxen der Wahrnehmung, der Erfahrung und des Erlebens der Natur/Biodiversität gibt, die dazu führen, dass man die Natur/Biodiversität so wahrnimmt, erlebt und behandelt, als ob diese einen Eigenwert hätte, intuitiv nachvollziehbar sind und von vielen Menschen auch nachvollzogen werden können. Diese Annahmen sind aber vor allem in der Tiefe klärungsbedürftig. Da eudaimonistische Eigenwerte genau wie instrumentelle Werte einen Zweck haben und ihnen die Momente der intrinsischen Werthaftigkeit sowie der Unabhängigkeit von der Zuschreibung durch Menschen fehlen, die der Intuition nach von vielen für entscheidend bezüglich der Annahme einer Eigenwertigkeit gehalten werden, scheint die Frage von Kirsten Meyer berechtigt zu sein, ob der eudaimonistische Eigenwert tatsächlich einen Eigenwert darstellt und ob die Annahme eines Eigenwerts der Natur/ Biodiversität für den Menschen überhaupt Sinn macht (Mayer 2003:120)

„Ist es sinnvoll von einem Eigenwert für den Menschen zu reden? Ist dies nicht ein innerer Widerspruch, da es beim Eigenwert der Natur gerade darum gehen soll, dass die Natur einen vom Menschen unabhängigen Wert hat?“ (ebd.)

Anhand dieser Zweifel zeigt sich deutlich, dass der eudaimonistische Eigenwert vielen VertreterInnen von Eigenwerten der Natur/Biodiversität nicht weit genug geht bzw. bestimmte für den Eigenwert entscheidende Intuitionen nicht bedient. Daher plädieren viele (und vor allem überzeugte NaturschützerInnen) eher für diejenigen Eigenwerttypen, hinter denen die Intuition steht, dass der Eigenwert der Natur/Biodiversität a) unabhängig von allen menschlichen Zwecksetzungen ist und/oder b) unabhängig davon ist, ob dieser Wert von Menschen in der Natur entdeckt oder zugeschrieben wird. Da hierbei jeweils entscheidend ist, was dabei genau unter unabhängig verstanden wird und in welchen Kontext dieses Unabhängig-Sein gestellt wird, wird im Folgenden auf die moralischen Selbstwerte und die objektiven (bei Krebs absoluten) Naturwerte eingegangen, da diese zugleich - aber auf je andere Weise - auf das für die Eigenwertigkeit zentrale Moment der Unabhängigkeit abheben. 


\subsubsection{3 moralische Selbstwerte}

Über den moralischen Selbstwert wird die ziemlich binnenkomplexe und als solche auch erst einmal zu erläuternde Intuition bedient, dass der Natur/Biodiversität unabhängig von allen menschlichen Zwecksetzungen ein eigener Wert zukommt, weil a) die Natur/ Biodiversität nicht nur Mittel zum Zweck sondern ein Selbstzweck ist und weil b) davon ausgegangen wird, dass mit einer solchen Selbstzweckhaftigkeit eine unbedingte bzw. kategorische Geltung korrespondiert, die c) wiederum mit der Pflicht der Achtung vor der Natur/Biodiversität verbunden wird, so dass auch nicht-menschliche Entitäten aufgrund d) bestimmter moralisch relevanter Eigenschaften, die ihnen intrinsisch sind, e) um 'ihrer selbst willen' zu berücksichtigen sind. Bei einem solchen, auf mehrere Aspekte und ihre Verbindung aufbauendem Verständnis moralischer Selbstwerte drängen sich erst einmal die Fragen auf, welche Annahmen und Verweisungsbezüge hinter der Rede von der Selbstzweckhaftigkeit und unbedingter bzw. kategorischer Geltung stehen und welche Aspekte und Kennzeichen das Moralische hierbei haben soll und inwieweit diese Annahmen auch auf nicht-menschliche Entitäten übertragen werden können. Auf diese Fragen wird eingegangen (1), damit der moralische Selbstwert dann als Werttypus, auch in Abgrenzung zu den instrumentellen und eudaimonistischen Werten, charakterisiert werden kann (2).

1) Der Grund, weswegen das Attribut moralisch bei diesem Eigenwerttypus von vornherein in Klammern gesetzt werden sollte, liegt daran, dass über diese Terminologie unklar bleibt, ob es sich dabei um moralische Werturteile $e^{102}$ handelt, die sich auf (menschliche) Einstellungen sowie Handlungsmotive und -absichten beziehen oder um axiologische und in diesem Sinne gerade nicht-moralische Werturteile, ${ }^{103}$ die sich auf die

102 Moralische Werturteile beziehen sich auf die moralische Qualität von Motiven, Absichten, Einstellungen und Charakterzügen von Personen sowie auf auf die moralische Qualität von Personen selbst. Moralische Werturteile machen daher nur dort Sinn, wo berechtigterweise auch von moralischen Handlungen gesprochen werden kann und dies scheint - zumindest vor dem Stand der derzeitigen Forschung - nur bei Menschen der Fall zu sein. Das bedeutet indes nicht, dass die Natur/Biodiversität nicht zum indirekten Gegenstand moralischer Werturteile gemacht werden kann, indem es um die moralische Bewertung menschlicher Motive, Absichten, Einstellungen in Bezug auf die Natur/Biodiversität geht. Dabei müssen, so Birnbacher, mindestens drei Arten moralrelevanter Motive unterschieden werden (vgl. Birnbacher 2007:282-285): Moralische Motive im eigentlichen Sinn (bspw. Moralität, Gewissenhaftigkeit, Prinzipientreue, Achtung, Gerechtigkeitssinn) verweisen darauf, das moralisch Richtige um seiner moralischen Richtigkeit willen zu tun, also moralisch zu handeln, weil es moralisch ist, so zu handeln (ebd., 283) Moralische Motive im uneigentlichen Sinn (bspw. Mitleid, Nächstenliebe, Gemeinsinn, Solidarität, Ehrlichkeit Hilfsbereitsschaft) beziehen sich dagegen nicht auf das spezifisch Moralische sondern auf diejenigen Aspekte, die zu moralischem Verhalten motivieren. Hierbei geht es folglich um Tugenden im moralischen Sinne (ebd.). Moraldienliche Motive (bspw. Ordnungsliebe, Pünktlichkeit, Höflichkeit) werden demgegenüber als Sekundärtugenden bezeichnet, da sie dem Subjekt bei der Verfolgung seiner moralischen Zwecke nützlich sind, die aber von sich aus nicht die Moralität der Zwecke garantieren (ebd., 284).

103 Axiologische Werturteile beziehen sich auf Güter, Gegenstände, Zustände, Sachen und Sachverhalte. Da Axiologien (Wertlehren) i.d.R. danach klassifiziert werden, welche inneren (psychischen) Eigenschaften und äußeren (Welt-)Zustände von ihnen als an sich wertvolle Zustände d.h. intrinsische 
Bewertung bestimmter moralisch relevanter Eigenschaften in der nicht-menschlichen Umwelt und damit auch auf Natur/Biodiversität beziehen. Beide Arten von Werturteilen werden i.d.R. vermischt, wenn vom moralischen Selbstwert die Rede ist. Einerseits handelt es sich um ein axiologisches Werturteil, da der Selbstwert der Natur/ Biodiversität auf nicht-menschliche Entitäten bezogen wird, die als an sich wertvolle Zustände, d.h intrinsische Werte postuliert werden. Andererseits zielen die moralischen Selbstwerte aber zugleich auf moralische Werturteile ab, weil es dabei immer auch darum geht, aufzuzeigen, welche moralischen Motive im eigentlichen und uneigentlichen Sinne sowie moraldienliche Motive Menschen für eine moralische Berücksichtigung der Natur/ Biodiversität um ihrer selbst willen haben sollten. Vor diesem Hintergrund kann präzisiert werden, dass es beim moralischen Selbstwert einerseits - im Sinne eines axiologischen Werturteils - um die Plausibilisierung der Annahme geht, dass etwas/jemand als 'Zweck an sich selbst' und in diesem Sinne als Selbstzweck verstanden wird, was wiederum notwendigerweise bedeutet, dass sich dessen Wert nicht extrinsisch über den Zweck ergibt, den dieser für andere hat oder haben könnte, sondern dass dessen Wert diesem intrinsisch ist (Birnbacher 2007:242). Andererseits wird - als moralisches Werturteil - behauptet, dass die Geltung intrinsischer Werte nicht relativ zu bestimmten Zwecksetzungen und damit bedingt oder hypothetisch sein kann, sondern dass die Geltung kategorisch ist, weil sie nicht auf die Interessen, Bedürfnisse und Wünsche des Akteurs zurückgeht, sondern mit Bezug auf davon unabhängige moralische Standards beurteilt werden kann (Krijnen 2006:551).

Als basale Idee sowie hinsichtlich des terminologischen Gebrauchs geht dieser Gesamtzusammenhang zwischen der Existenz intrinsischer Werte sowie der unbedingten, kategorischen Geltung dieser Werte auf Immanuel Kant zurück, da dieser als erster die Idee begründete, dass alle Vernunftwesen ${ }^{104}$ für sich und alle anderen Vernunftwesen als unbedingte notwendige Zwecke an sich selbst und in diesem Sinne als würdehafte Wesen existieren und deshalb nicht zu instrumentalisieren seien (Selbstzweckformel), ${ }^{105}$ d.h., nicht auf den Status von Dingen oder bloßen Ressourcen für die Ziele anderer reduziert werden dürfen, während alle vernunftlosen Wesen nur Mittel zum Zweck sind und lediglich einen abgeleiteten, extrinsischen Wert haben:

Werte postuliert werden, sind extrinsische Werte demgegenüber diejenigen Eigenschaften oder Zustände, die intrinsische Werte bedingen, sprich Bedingungen oder Mittel für die Erreichung oder Realisierung intrinsischer Werteigenschaften (Birnbacher 2007:242).

${ }^{104}$ Kant grenzt würdehafte Wesen von vornherein auf Vernunftwesen ein. Insofern ist die Selbstwertund Würdekonzeption an den Begriff und das Verständnis von Vernunft gekoppelt, so dass die Herausforderung bei der Verwendung (moralischer) Selbstwerte im umwelt- und naturschutzethischen Kontext darin besteht, den moralischen Status (wenn er denn auf nicht-menschliche Lebewesen übertragen werden soll) ohne das Postulat der Vernunft zu begründen (siehe 4.2.2.2).

105 Kants Selbstzweckformel ist in Form eines kategorischen Imperativs, gefasst: "Handle so, dass du die Menschheit sowohl in deiner Person, als auch in der Person eines jeden andern zugleich als Zweck, niemals bloß als Mittel brauchest." (Kant (1786) 1983:246) 
"Nun sage ich: der Mensch und überhaupt jedes vernünftige Wesen existiert als Zweck an sich selbst, nicht bloß als Mittel zum beliebigen Gebrauche für diesen oder jenen Willen, sondern muss in allen seinen sowohl auf sich selbst, als auch auf andere vernünftige Wesen gerichteten Handlungen jederzeit zugleich als Zweck betrachtet werden. (...) Die Wesen, deren Dasein zwar nicht auf unserm Willen, sondern der Natur beruht, haben dennoch, wenn sie vernunftlose Wesen sind, nur einen relativen Wert, als Mittel und heißen daher Sachen, dagegen vernünftige Wesen Personen genannt werden, weil ihre Natur sie schon als Zwecke an sich selbst, d. i. als etwas, das nicht bloß als Mittel gebraucht werden darf, auszeichnet." (Kant (1786) 1983: 244-245)

Wenn der moralische Selbstwert im Kontext der Umwelt- und Naturschutzethik verwandt wird, dann geht es darum, genau diese Annahme Kants, dass Menschen einen intrinsischen Wert haben, während natürlichen Entitäten nur ein extrinsischer Wert zukommt, in Bezug auf die lediglich extrinsische Wertzuschreibung natürlicher Entitäten zu bestreiten, indem versucht wird, den intrinsischen Wert auch auf nicht-menschliche Entitäten auszudehnen (Extensionsstrategie). ${ }^{106}$ Das zentrale Problem dabei liegt indes in der hinter den intrinsischen Werten stehenden Verbindung der Ideen der Vernunft, der Würde und der kategorische Geltung, auf die im Fall der Extension des intrinsischen Wertes auf nicht-menschliche Entitäten in dieser Form nicht rekurriert werden kann. Die Selbstzweckformel, mit der Kant die Vereinbarkeit von Handlungen mit dem Selbstzweckcharakter des Menschen zum Ausdruck bringen will, beruft sich eben auf „die spezifisch kantische Annahme, dass der Mensch (als Person) an einem metaphysischen Bereich jenseits der empirisch zugänglichen Welt teilhat und dass die jedem Menschen eigene Vernunftfähigkeit gewissermaßen die Brücke darstellt, auf der er sich Zugang zu diesem 'intelligiblen` - d.h. nur gedanklich zugänglichen - Bereich verschaffen kann." (Birnbacher 2007:142) Nur aufgrund seiner Vernunftfähigkeit kommt dem Menschen als Vernunftwesen eine besondere Würde bzw. ein (moralischer) Selbstwert zu, der die Instrumentalisierung zu fremden Zwecken verbietet.

Hieraus ergibt sich wiederum auch die Annahme, moralische Selbstwerte würden eine unbedingte bzw. kategorische Geltung nach sich ziehen, wobei das, was dabei mit kategorischer Geltung gemeint ist, nicht frei von problematischen Zweideutigkeiten ist: Alle moralischen Handlungsurteile, Handlungsaufforderungen und Handlungsbeurtei-

${ }^{106}$ Wenn auch nicht-menschlichen Entitäten eine Würde in Form eines Selbstwerts zugesprochen werden soll, dann muss begründet werden können, dass diese Träger von intrinsisch werthaften und unbedingt bzw. kategorisch geltenden Werten sind, ohne dass dabei auf das Konzept der vernunftbasierten Würde Bezug genommen wird (Ott 2010:101). Eben dies geschieht in den Ansätzen der physiozentrischen Ethiken, auf die im Rahmen der Reflexion der Inklusionsdebatte näher eingegangen wird (4.2.2.2). Diesbezüglich stellen sich die Fragen, was den moralischen Status von nicht-menschlichen Entitäten ausmachen soll und warum davon ausgegangen werden sollte, dass nicht-vernunftbasierten Selbstwerten ebenfalls eine kategorische Geltung zukommt, so dass Träger solcher Werte auch direkt moralisch zu berücksichtigen sind. 
lungen (kategorische Imperative) sind immer dann kategorisch, wenn die jeweilige Handlung nicht mit Bezug auf die Interessen, Bedürfnisse und Wünsche des Akteurs, sondern mit Bezug auf davon unabhängige Standards gefällt und beurteilt werden. Nicht mehr und nicht weniger. Der Begriff kategorisch ist in diesem Sinne davon abhängig, als was genau diese unabhängigen Standards verstanden werden. Da Kant dazu tendierte, kategorisch mit moralisch und berechtigt sowie Imperativ mit streng und unbedingt gleichzusetzen, wird erstens oftmals davon ausgegangen, dass Kategorizität im oben stehenden Sinne auch ein hinreichendes Kennzeichen moralischer Urteile ist, was indes nicht stimmt, da es lediglich ein notwendiges Kennzeichen ist (ebd., 20-24). Zweitens wird davon ausgegangen, dass nur moralische Urteile und Forderungen kategorisch seien, was indes auch nicht stimmen kann, denn auch rechtliche, kulturelle oder ästhetische Urteile bzw. Forderungen können ebenfalls unbedingt sein (ebd., 51-53). Drittens wird oftmals davon ausgegangen, dass moralische Urteile und Forderungen per se berechtigte Urteile und Forderungen sind, weil sie kategorisch sind, bei näherem Hinsehen zeigt sich aber, dass Kategorizität im oben stehenden Sinne auch auf unberechtigte moralische Imperative zutreffen kann (ebd., 137). Viertens wird oftmals davon ausgegangen, dass Imperative einen unbedingten Gehorsam verlangen, weil sie als strenge moralische Forderungen und Urteile in allen Fällen Vorrang haben. Aber selbst wenn man der Auffassung ist, dass moralische Forderungen und Urteile in allen Konfliktfällen Vorrang vor nicht-moralischen Forderungen und Urteilen haben, folgt dieser Vorrang nicht schon aus der Kategorizität (ebd., 40-43).

Insofern ergibt sich, im Anschluss an die Problematik der Zweideutigkeiten der kategorischen Geltung moralischer Imperative, das Problem, dass die kategorische Geltung von moralischen Selbstwerten nicht gleichbedeutend mit dem ist, was Kant unter dem Kategorischen Imperativ im Besonderen als das oberste, allgemeine, monistische Prinzip meint, aus dem sich alle gültigen moralischen kategorischen Imperative ableiten lassen sollen, die moralisch erlaubt sind. Dies wiederum zeigt sich daran, dass es eine große Zahl von kategorischen Imperativen gibt, unter die moralische kategorische Imperative als Sonderfall fallen. Einer davon ist die bei Kant auf starke metaphysische Annahmen gestellte Selbstzweckformel der Beachtung der kategorischen Geltung des menschlichen Selbstwertes, die indes nicht denselben Status hat, wie der Kategorische Imperativ als das oberste, allgemeine, monistische Prinzip, aus dem sich alle gültigen moralischen kategorischen Imperative ableiten lassen sollen, die moralisch erlaubt sind. Obwohl Kant mehrere Varianten der "goldenen Regel« formulierte, entspricht nur die erste Formulierung ${ }^{107}$ dem von Kant selbst angestrebten rein formalen Verfah-

107 Die erste Formulierung der "goldenen Regel“ ist als Filter der Verallgemeinerung der Maxime einer Handlung angelegt: „Handle nur nach denjenigen Maximen, die du zugleich wollen kannst, dass sie ein allgemeines Gesetz werde." (Kant (1786) 1983:236) 
ren zur Überprüfung der moralischen Zulässigkeit vorgeschlagener Maximen und wird damit Kants Grundannahme gerecht, dass sich moralische Urteile mithilfe der reinen Vernunft auf ihre Geltung prüfen lassen, (ebd., 141-154). Daher muss angesichts der starken metaphysischen Voraussetzungen der beiden anderen Formulierungen (Selbstzweckformel und Autonomieformel) ${ }^{108}$ bezweifelt werden, dass diese in derselben Weise mithilfe der reinen Vernunft als zutreffend und berechtigt anerkannt werden können, wie Kant dies für alle drei Forderungen beansprucht, da dies nur dann der Fall wäre, wenn die Wahrheit der kantischen Metaphysik ihrerseits aus reinen Vernunftgründen einsehbar wäre. Da genau dies (vor allem von Seiten vieler physiozentrischer Umwelt- und NaturschutzethikerInnen) bestritten, gleichzeitig aber Kants Idee der Selbstzweckhaftigkeit und der kategorischen Geltung intrinsischer Werte beibehalten wird, müssen die VertreterInnen physiozentrischer Ansätze zeigen, warum berechtigterweise davon ausgegangen werden sollte, dass nicht-vernunftbasierte Selbstwerte, den Menschen auch auf kategorische moralische Handlungen gegenüber der Natur/ Biodiversität verpflichten, sprich sie müssen zeigen, wie sie von der metaethischen Begründung des (moralischen) Selbstwertes zum ethischen Grund der moralischen Verpflichtung gelangen (siehe 4.2.2.2).

2) Auf dieser Grundlage, sprich die Erschließung des binnenkomplexen Gefüges der einzelnen Aspekte des moralischen Selbstwertes, kann im Folgenden herausgestellt werden, was den moralischen Selbstwert als Werttypus sowohl gegenüber den instrumentellen Werten als auch den eudaimonistischen Eigenwerten der Natur/Biodiversität auszeichnet. Um dies deutlich zu machen, muss hier allerdings erneut weiter ausgeholt werden: In Bezug auf die Unterscheidbarkeit von instrumentellen Werten, eudaimonistischen Eigenwerten und moralischen Selbstwerten kann bis dato festgehalten werden, dass über die moralischen Selbstwerte, genau wie über die eudaimonistischen Eigenwerte, und zugleich gegen die instrumentellen Werte postuliert wird, dass die Wertigkeit von etwas/jemanden nicht-relativierbar sein kann. Zugleich wird aber auch über den eudaimonistischen Eigenwert hinausgehend postuliert, dass sich der Eigenwert nicht relativ zu bestimmten Zwecken ergibt, d.h., dass auch nicht-menschliche Entitäten 'an sich', d.h., intrinsisch wertvoll sind. Intrinsisch ist dabei so bestimmt worden, dass etwas/ jemand als Selbstzweck postuliert wird, dem zugleich eine unbedingte bzw. kategorische Geltung zugeschrieben wird, die unabhängig davon ist, ob etwas/ jemand ein Mittel für die Erreichung oder Realisierung bestimmter Ziele oder Zwecke ist. Der zentrale Unterschied liegt folglich darin, dass eudaimonistische Eigenwerte genau wie instrumentelle Werte - extrinsisch sind, während der moralische Selbstwert

108 Die Autonomieformel leitet sich aus der Selbstzweckformel ab und ist eher eine Feststellung als eine Forderung, indem sie den Menschen als zur Selbstgesetzgebung fähig auszeichnet (Kant (1786) 1983: 248-250). 
als intrinsischer Wert ausgezeichnet wird. Um diesen Unterschied auf der theoretischen Ebene deutlich zu machen, kann mit Dieter Birnbacher auf den Unterschied zwischen objektivistischen und subjektivistischen Positionen bezüglich axiologischer Werte verwiesen werden. Birnbacher führt diesbezüglich aus, dass die Begriffe Objektivismus und Subjektivismus "verwirrend vieldeutige Termini“ sind und dass sich das Begriffspaar objektiv/subjektiv in mindestens drei verschiedenen Bedeutungen auf zwei verschiedenen Ebenen interpretieren lässt (Birnbacher 2007:251):

Auf der ersten Ebene geht es um die Lokalisierung des von einer Axiologie postulierten Werts und betrifft die werttheoretische Frage, ob ein Wert intrinsisch oder extrinsisch ist (ebd., 252). Hierbei kann je nach Art der Antwort auf diese Frage zwischen den Positionen des axiologischen Objektivismus und des axiologischen Subjektivismus unterschieden werden. Über den axiologischen Subjektivismus wird behauptet, „dass ausschließlich subjektive Zustände bewusstseinsfähiger Wesen intrinsisch wertvoll sind, während über die entsprechende Variante des axiologischen Objektivismus behauptet wird, dass es einen intrinsischen Wert gibt, der nicht der Wert eines subjektiven Zustands ist." (ebd.) Davon zu unterscheiden ist die ebenfalls werttheoretische Frage nach der Instanz, die darüber entscheidet, welche Wertigkeit den Werten zugesprochen wird, da es hierbei - lediglich im Anschluss an den axiologischen Subjektivismus - „um das Ausmaß [geht], in dem das Subjekt selbst aus seiner eigenen Perspektive darüber befinden kann und muss, welcher Wert seinen inneren Zuständen zukommt." (ebd.) Auch hier kann je nach Antwort auf die Frage zwischen einen Wertungssubjektivismus und einem Wertungsobjektivismus unterschieden werden. Wenn ein Wertungssubjektivismus vertreten wird, dann wird nicht nur behauptet, dass ausschließlich subjektive Zustände bewusstseinsfähiger Wesen werthaftig sind, sondern es wird darüber hinaus behauptet, dass die Wertigkeit dieser Zustände allein davon abhängt, wie das jeweilige Individuum diese Zustände bewertet. Im Falle eines Wertungsobjektivismus wird dagegen behauptet, dass die Wertigkeit nicht ausschließlich davon abhängt, wie das jeweilige Subjekt diesen Bewusstseinszustand bewertet, sondern dass es auch andere Instanzen gibt, die dafür entscheidend sind (bspw. die Gesellschaft, Traditionen, Macht etc.).

Auf der zweiten Ebene geht es um die Wertquelle und die metaethische Frage woher einer Eigenschaft ein bestimmter Wert zukommt (ebd., 251). Hier unterscheidet Birnbacher zwischen einem metaethischen Objektivismus und einem metaethischen Subjektivismus. Die Antwort des metaethischen Subjektivisten lautet, dass die Wertquelle im Bewertenden selbst liegt, so dass Wertzuschreibungen nur von einer menschlichen Wertperspektive aus Sinn machen. Anders fällt die Antwort des metaethischen Objektivisten aus, der behaupten kann, dass es Werte 'an sich ' gibt, auch ohne dass es einen (menschlichen) Betrachter solcher Werte gibt, weil er/sie nicht davon ausgeht, dass Werte den menschlichen Betrachtern immer schon vorgegeben sind. 
Alle drei Deutungen sowie die Ebenenunterscheidung sind relevant für die Differenzierung der hier relevanten Werttypen und werden im Folgenden - zumindest in Bezug auf die bisher eingeführten Werttypen (instrumentelle, eudaimonistische und moralische Werte) - so sparsam wie möglich und so ausführlich wie nötig dargelegt:

Bezüglich der Lokalisierung des von einer Axiologie postulierten Werts und die werttheoretische Frage, ob ein Wert intrinsisch oder extrinsisch ist, können objektivistische und subjektivistische Axiologien durchaus darin übereinstimmen, welche Werte als extrinisisch anerkannt werden. Sie unterscheiden sich aber fundamental darin, welche Werte als intrinsisch angesehen werden. So können bspw. PathozentrikerInnen, die die Leidensfähigkeit als intrinsischen Wert postulieren und damit allen höheren Tieren einen Selbstwert zuschreiben, dies auf Basis einer subjektivistischen Axiologie tun, weil sie - zumindest nach dem neuesten Stand der Forschung - anführen können, dass alle höheren Wirbeltiere, genau wie Menschen, bewusstseinsfähige Wesen sind, so dass deren subjektive Zustände auch als intrinsisch wertvoll verstanden werden können. BiozentrikerInnen, ÖkozentrikerInnen und HolistenInnen können eben dies nicht, da diese qua ihrer Gegenstandsbereiche (alles was lebt bzw. alles was existiert) darauf rekurieren müssen, dass es intrinsische Werte gibt, die nicht aus subjektiven Zuständen resultieren, sprich ihre Argumentation auf Basis einer objektivistischen Axiologie aufbauen müssen. Da wiederum nur biozentrische, ökozentrische und holistische Positionen zur Begründung intrinsischer Werte speziell der Biodiversität in Frage kommen, da Begründungen in Bezug auf das weite Biodiversitätsverständnisses (Vielfalt der Lebensformen und Vielfalt der Lebensbedingungen) nur durch ökozentrische oder holistische Begründungsansätze geleistet werden können und Begründungen in Bezug auf das enge Biodiversitätsverständnis (Vielfalt der Lebensformen) im Mindesten nach einem biozentrischen Begründungsansatz verlangt, kann folglich davon ausgegangen werden, dass der intrinsische Wert der Biodiversität, den biozentrische, ökozentrische und holistische Ethiken zu begründen versuchen, auf einer objektivistischen Axiologie basiert.

Hinsichtlich der Unterscheidung zwischen moralischen Selbstwerten auf der einen Seite und eudaimonistischen Eigenwerten sowie instrumentellen Werten auf der anderen Seite bedeutet dies wiederum, dass hinter den instrumentellen Werten und den eudaimonistischen Eigenwerten der Natur/Biodiversität, die als extrinsische Werte gekennzeichnet wurden, eine subjektivistische Axiologie steht, während hinter dem moralischen Selbstwert der Biodiversität, der als intrinsischer Wert gekennzeichnet wurde, eine objektivistische Axiologie stehen muss. Das muss nun - wie bereits am eudaimonistischen Eigenwert aufgezeigt wurde - nicht heißen, dass eudaimonistische Eigenwerte, die auf der Basis einer subjektivistischen Axiologie argumentiert werden, nur relativierbare oder gar substituierbare Wertigkeiten annehmen, wie das bei instrumentellen Werten der Fall ist, da hinsichtlich der werttheoretischen Frage, von welchen 
Umständen eine subjektive Bewertung abhängt, wiederum zwischen wertungssubjektivistischen und -objektivistischen Positionen unterschieden werden muss: Wenn folglich im Fall eudaimonistischer Eigenwerte eine nicht relativierbare Wertigkeit postuliert wird, dann nur im Sinne eines Wertungsobjektivismus, d.h. vor dem Hintergrund überindividueller Standards (bspw. gesellschaftliche Konventionen, Regeln sozialer Milieus, Machthierarchien), da eudaimonistische Eigenwerte auf der Basis einer subjektivistischen Axiologie als relativ zu ganz bestimmten Bewusstseinszuständen verstanden werden und damit extrinsisch sind und sich wiederum von instrumentellen Werten dadurch unterscheiden, dass sie allen glückskonstitutiven Dingen eine nicht-relativierbare Wertigkeit zusprechen. In diesem Sinne basieren sowohl instrumentelle Werte als auch eudaimonistische Eigenwerte auf einer subjektivistischen Axiologie mit dem kleinen aber feinen Unterschied, dass eudaimonistische Eigenwerte auf der Basis eines Wertungsobjektivismus eine nicht-relativierbare Wertigkeit postulieren, während dies bei instrumentellen Werten nicht der Fall ist.

Von eudaimonistischen Eigenwerten unterscheiden sich nun moralische Selbstwerte wiederum dadurch, dass hier die subjektivistische Axiologie gegen eine objektivistische Axiologie eingetauscht wird, womit wiederum auf die Begründung der intrinsischen Werthaftigkeit der Natur/Biodiversität abgehoben wird. Das Spezifikum moralischer Selbstwerte besteht folglich darin, dass der axiologische und in diesem Sinne gerade nicht-moralische intrinsische Wert von einem objektiv-axiologischen Standpunkt aus als intrinsischer Wert anerkannt wird. Insofern kann das, was in der umwelt- und naturschutzethischen Wertedebatte oftmals unspezifisch als intrinsischer Wert bezeichnet wird, hier als moralischer Selbstwert betitelt werden. Obgleich das, was (nicht nur in der umwelt- und naturschutzethischen Wertedebatte) unspezifisch als intrinsischer Wert bezeichnet wird, auch darüber hinausgehen kann, was hier mit Selbstwert gemeint ist: Da der intrinsische Wert (sowohl in der politischen als auch in der umweltund naturschutzethischen Debatte) zumeist als Überbegriff für Eigenwert schlechthin benutzt wird, kann damit das gesamte Spektrum an Eigenwerten gemeint sein. Da indes der eudaimonistische Eigenwert oftmals als inhärenter Wert bezeichnet wird, der wiederum gegen den intrinsischen Wert abgegrenzt wird, werden unter dem intrinsischen Wert de facto sowohl moralische Selbstwerte als auch absolute (oder wie hier objektive) Naturwerte verhandelt, wobei es bei letzteren um die andersgelagerte und auf der theoretischen Ebene auch anders begründete Intuition geht, dass es Werte in der Natur gibt, die unabhängig von der menschlichen Existenz, Betrachtung und Bewertung sind. 


\subsubsection{4 objektive Naturwerte}

Der Eigenwerttypus unabhängiger Naturwerte - so wurde gesagt - nimmt die Intuition auf, dass der Wert von etwas/jemanden nicht erst durch eine wertschätzende Bezugnahme des Menschen entsteht, sondern dass es auch Werte 'an sich ' unabhängig von der Existenz des Menschen, menschlicher Betrachtung und Bewertung (in der Natur) geben kann. Das bedeutet wiederum, dass diese Annahme als Spezifikum eines eigenen Eigenwerttypus verstanden werden kann, der der Krebsschen Typologie folgend, gemeinhin als absoluter (hier objektiver) Wert betitelt wird. Zur Charakterisierung des objektiven Naturwerts werden im Folgenden dessen Spezifika herausgestellt, bevor anund abschließend die Problematiken dieses Werttypus diskutiert werden:

Indem bei den objektiven Naturwerten die Intuition im Vordergrund steht, dass es Werte an sich gibt, auch wenn es keine Menschen gäbe, die eine solche Wertigkeit erkennen, zuschreiben oder anerkennen würden, grenzt sich der objektive Naturwert sowohl von den eudaimonistischen Eigenwerten als auch von den moralischen Selbstwerten ab und dies auf einer ganz fundamentalen Ebene: VertreterInnen objektiver Naturwerte verwerfen das metaethisch-subjektivistische Fundament, das allen drei bisher vorgestellten Werttypen zugrunde liegt, indem sie für einen metaethischen Objektivismus argumentieren. Das bedeutet, zunächst auf der exemplarischen Ebene, dass VertreterInnen objektiver Naturwerte fordern, man solle die menschliche Wertperspektive transzendieren und die Natur als 'das Andere' akzeptieren, da Werte nicht erst mit den Menschen in die Welt gekommen seien, sondern schon immer da waren. Das Paradebeispiel für eine solche Intuition ist das sogenannte "Gedankenexperiment der letzten Person«:

„Der letzte Mensch (oder die letzte Person), die den Kollaps der weltlichen Ordnung überlebt, beginnt um sich zu schlagen und eliminiert, sofern es ihm möglich ist, jedes lebende Wesen, ob Tier, ob Pflanze (meinethalben auch schmerzfrei, wie in den besten Schlachthäusern). Was diese Person tut ist völlig unproblematisch im Kontext des [menschlichen] Chauvinismus, doch aus einer Umweltperspektive sind diese Handlungen falsch." (Routley 2003)

Um zu verstehen, warum begründeterweise davon ausgegangen werden sollte, dass die Eliminierung alles Seienden falsch ist, vorausgesetzt es wird nicht einfach unbegründet angenommen, dass es falsch ist, muss danach gefragt werden, was es bedeuten soll, dass dies aus einer Umweltperspektive falsch sein soll. Dies verweist wiederum auf zwei miteinander verbundene Annahmen: Die erste Annahme besteht darin, die Unabhängigkeit der Werte von der menschlichen Existenz anzunehmen, so dass davon ausgegangen wird, dass es Werte 'an sich ' gibt, die sich anhand objektiver Eigenschaften oder Kriterien festmachen lassen. Dies zieht die ontologische Frage nach sich, was für Eigenschaften und Kriterien diese Werte wertvoll machen. Die zweite damit zu- 
sammenhängende Annahme besteht darin, die Unabhängigkeit der Werte speziell von der menschlichen Wahrnehmung, Betrachtung und Bewertung anzunehmen. Dies wiederum verweist auf die ebenfalls ontologische Frage, woher Werte kommen, sprich wer oder was die Quelle oder der Ursprung von Werten ist und auf die pragmatische Frage, was es bedeuten soll die menschliche Wertperspektive zugunsten einer 'Umweltperspektive`zu transzendieren:

Beide Fragen verweisen nun auf der theoretischen Ebene auf die Unterscheidung zwischen metaethisch-subjektivistischen und metaethisch-objektivistischen Positionen. Die Antwort des metaethischen Subjektivisten auf die beiden Fragen lautet, dass die Wertquelle im Bewertenden selbst liegt, so dass Werte - aufgrund bestimmter Eigenschaften und Kriterien - von Menschen zugeschrieben werden. Anders fällt die Antwort des metaethischen Objektivisten aus, der behaupten kann, dass es Werte gibt, auch ohne dass es einen Betrachter solcher Werte gibt, weil er/sie nicht davon ausgeht, dass Werte den menschlichen Betrachtern immer schon vorgegeben sind. Entscheidend bezüglich der Charakterisierung objektiver Naturwerte ist nun vor allem der Umstand, dass es notwendig und wichtig ist, zwischen metaethisch-objektivistischen und axiologischobjektivistischen Positionen zu unterscheiden, da sich beide Positionen nicht bedingen (Birnbacher 2006:96). Vielmehr kann eine axiologisch-objektivistische Position auch mit einem metaethischen Subjektivismus „friedlich koexistieren“, genau wie der metaethische Objektivismus sowohl mit einem axiologischen Objektivismus als auch mit einem axiologischen Subjektivismus vereinbar ist. Die Differenzierung zwischen dem metaethischen Objektivismus und dem axiologischen Objektivismus führt, zugschnitten auf die Differenzierung zwischen moralischen Selbstwerten und objektiven Naturwerten, zu folgendem Schluss:

„Diese - zunächst reichlich akademisch anmutende - Unterscheidung ist gerade im Zusammenhang mit Werten wie Natürlichkeit von Bedeutung. (...) Redeweisen wie die vom 'Eigenwert' der Natur bzw. davon, dass der Natur 'von sich aus' Wert zukommt, [legen] den Schluss nahe, eine Anerkennung von nicht auf den Menschen, sondern auf die außermenschliche Natur bezogenen Werten impliziere zwangsläufig eine Verpflichtung auf den metaethischen Objektivismus, nach dem Werte unabhängig von menschlichen Wertsetzungen bestehen. Dies ist aber nicht der Fall. Dass eine Naturethik intrinsische Werte auch außerhalb der Sphäre des Menschen anerkennt, verpflichtet sie nicht darauf, diese Werte auch für [metaethisch] objektiv, d.h. vorgängig zu allen menschlichen Bewertungen für gegeben zu halten." (Birnbacher 2006:95)

Das wiederum bedeutet, dass zwischen moralischen Selbstwerten, die auf einem axiologischen Objektivismus basieren, und objektiven Naturwerten, die auf einem metaethischen Objektivismus basieren, differenziert werden muss, denn wenn dies nicht 
geschieht, geht damit die wichtige Ebenen-Unterscheidung zwischen der axiologischobjektivistischen Annahme, dass es „einen intrinsischen Wert gibt, der nicht der Wert eines subjektiven Zustandes ist" (Birnbacher 2007:252) und der metaethisch-objektivistischen Annahme verloren, dass es eine objektive Geltung von Wertaussagen gibt, indem angenommen wird, dass es Werte gibt, „die jeder Wertzuschreibung selbst vorgegeben sind." (ebd., 277) Auf eben diese Annahme, dass es Werte gibt, die jeder Wertzuschreibung selbst vorgegeben sind, wird dann abgehoben, wenn bestimmte Eigenschaften oder Kriterien angeführt werden, an denen sich die vom Menschen und seiner Existenz, Wahrnehmung und Bewertung unabhängige Eigenwertigkeit der Natur zeigen soll. Krebs führt einige dieser Eigenschaften der Natur auf, die in und außerhalb der Umwelt- und Naturschutzethik als objektive Naturwerte gehandelt werden:

„Nach dieser Auffassung habe alles, was existiert oder lebt oder komplex / (bio)divers / stabil / wohlgeordnet / harmonisch / systemisch / integer / gesund / alt / ehrgeizig / selten / wild / schön / bewusst / empfindend / teleologisch / autonom ist, absoluten [bzw. objektiven] [Natur-]Wert." (Krebs 1997:358)

Die speziell im Biodiversitätskontext wichtigsten Kriterien sind hierbei sicherlich die Eigenschaften der Vielfalt oder Diversität, der Komplexität und der Stabilität. Meyer führt diesbezüglich aus, dass gerade über das Kriterium der Vielfalt oftmals eine generelle und für sich selbst sprechende und in diesem Sinne objektive normative Geltung beansprucht wird (Meyer 2003:36). Dass dies indes, zumindest nicht derartig einfach, plausibilisierbar ist, wird schon anhand der kontraintuitiven Implikationen einer solchen Annahme ersichtlich: Sollte es stimmen, dass Vielfalt 'an sich 'wertvoll ist, dann müsste „eine Welt in der es PräsidentInnen und Witwenverbrennung gibt eine wertvollere, weil vielfältigere Welt [sein] als eine, in der es nur PräsidentInnen gäbe.“ (Krebs 1996:46) Dass viele von uns diese Schlussfolgerung verneinen würden, zeigt, dass entweder die Eigenschaft der Vielfalt lediglich eine Ausnahme darstellt, es aber durchaus andere Eigenschaften gibt, die den 'Intuitionen-Test' bestehen oder dass, wie Krebs dies glaubt, die ontologischen Annahmen des metaethischen Objektivismus bezüglich objektiver Naturwerte als solche unplausibel sind (ebd.).

Bezüglich der ersten Annahme würde es ausreichen andere Kriterien wie bspw. die Eigenschaft der Komplexität, der Stabilität, der Natürlichkeit, der Seltenheit oder der Eigenart als geeignetere 'Kandidaten ' für objektive Naturwerte herauszustellen. Einmal davon abgesehen, dass sich auch bei diesen Kriterien leicht kontraintuitive Implikationen aufzeigen ließen, ${ }^{109}$ kann anhand von Kirsten Meyers Ausführungen zum »Wert

${ }^{109}$ Gegen Komplexität ließe sich bspw. einwenden, dass Atomwaffen komplexer sind als Steinschleudern. Sind sie deswegen wertvoller? Gegen Stabilität ließe sich einwenden, dass Beton stabiler ist als Gras oder Blumen, ist Beton deshalb wertvoller? Gegen Natürlichkeit kann eingewandt werden, dass der Großteil der für den Menschen gefährlichen Bakterien und Viren 'natürliche` Organismen sind, 
der Natur« (vgl. Meyer 2003) gezeigt werden, dass bestimmte Eigenschaften bereits als werthaft diskutiert werden und dass diese Werthaftigkeit dann als Grund für die Schutzwürdigkeit der Natur herausgestellt wird. Damit wird aber die dabei eigentlich relevante Fragestellung umgangen, wie hierbei a) der Sprung von der Ebene der Eigenschaften auf die Ebene der Werte geleistet werden kann und b) warum diese dann zudem eine Schutzwürdigkeit implizieren sollen, die moralischer Natur ist:

a) In Bezug auf die erste Frage kann konstatiert werden, dass es sich bei der Feststellung, dass Natur bspw. die Eigenschaft der Vielfältigkeit aufweist erst einmal nur eine Beschreibung davon ist, was sie ist und wie etwas ist. Daher stellt sich die Frage, warum sich aus der Annahme, dass etwas so und nicht anders ist, schon ergeben soll, dass es einen Wert hat. Um von der Beschreibungsebene auf die Wertebene zu gelangen, muss vielmehr die ontologische Annahme begründet werden, dass die Natur selbst als Quelle von Werten in Frage kommt. Wird diese Annahme gar nicht oder nur unzureichend begründet, ist der Sprung von der Beschreibungs- auf die Wertebene nicht geleistet. Hier kann indes nur ontologisch-realistisch oder ontologisch-naturalistisch argumentiert werden. Gegen den ontologischen Realismus kann eingewandt werden, dass von Seiten der RealistInnen erst einmal gezeigt werden muss, "dass es empirische Phänomene gibt, die man nicht korrekt beschreiben kann, ohne eo ipso zu erkennen, dass etwas Wertvolles oder gar etwas Verpflichtendes vorliegt" (Ott 2010:74), da Werte durch empirisch fassbare Eigenschaften prinzipiell unterdeterminiert werden. Gegen die Grundannahmen des ontologischen Naturalismus kann wiederum eingewandt werden, was bereits in der Grundlagenreflexion auf Biodiversität (siehe 2.3) und darüber hinaus auch in der wissenschaftstheoretischen Reflexion (Abschnitt III) an mehreren Stellen angeklungen ist: Die Natur als solche ist weder moralisch noch unmoralisch, sie ist schlicht amoralisch. Daher ist nicht klar, wie man von deskriptiven Sätzen über die Natur zu normativen Sätzen in Bezug auf die Natur gelangen soll, solange nicht die Differenz zwischen deskriptiven Aussagen, evaluativen und präskriptiven Aussagen als solche aufgegeben wird. Das wiederum bedeutet summa summarum, dass die Annahme objektiver Naturwerte dazu zwingt, anzugeben, warum der Sein-Sollen und der naturalistische Fehlschluss als Fehlschlüsse abzulehnen sind. Gelingt eine solche Plausibilisierung nicht, ist und bleibt fraglich, ob es sich bei den objektiven Naturwerten überhaupt um einen Werttypus handelt, denn solange nicht gezeigt werden kann, dass die Natur selbst als Quelle von Werten in Frage kommt, ist der Sprung von der Beschreibungsebene auf die Wertebene nicht getan.

während viele Nahrungsmittel 'künstlich ' hergestellt werden. Was ist besser? Gegen Seltenheit ließe sich einwenden, dass Diktaturen in Europa immer seltener werden, während Demokratien mittlerweile in der Mehrzahl sind. Sind Diktaturen deswegen besser als Demokratien? Gegen Eigenart ließe sich einwenden, dass auch Kinderschänder, Mörder und Betrüger ihre ganz eigene Art zu leben haben. Mit anderen Worten: Nur weil etwas einzigartig ist, muss dies nicht per se gut sein. 
b) Selbst wenn plausibel dafür argumentiert werden könnte, dass es in der Natur objektive Werte gibt, bleibt weiterhin unklar, warum sich aus diesen auch eine moralische Berücksichtigungswürdigkeit 'um ihrer selbst willen` ergeben sollte. Denn, so argumentiert Katrin Reuter treffend,

„könnte [man] umgekehrt auch argumentieren, dass gerade weil diese Werte unabhängig vom Menschen existieren, nicht ersichtlich ist, warum der Mensch eine direkte moralische Verpflichtung ihnen gegenüber haben sollte. Verwendet man den Begriff des intrinsischen Wertes im Sinne der Aussage, dass ein Gegenstand selbst Quelle seines Wertes ist (...), wird zunächst einmal etwas über die entsprechenden Gegenstände und ihre Natur gesagt, und noch nichts darüber, wie sie zu behandeln sind." (Reuter 2014:43)

In Rückgriff auf Krebs spricht des Weiteren auch ein pragmatisches Argument gegen die Bezugnahme auf den Werttypus der objektiven Naturwerte zur Ausweisung der Berücksichtigungswürdigkeit der Natur 'um ihrer selbst willen` (Krebs 1997:359). Krebs argumentiert, dass selbst wenn der Natur/Biodiversität objektive Werte zukommen würden, so müssten diese trotz allem danach beurteilt werden, ob sie für den Menschen bzw. aus dessen Perspektive gut oder schlecht sind, so dass man wieder auf die menschliche Wertperspektive zurückverwiesen wäre. In diesem Fall macht es sowohl in theoretischer als auch in pragmatischer Hinsicht wenig Sinn zu fordern, man solle die anthropozentrische Perspektive transzendieren, sprich den metaethischen Subjektivismus zugunsten eines metaethischen Objektivismus überwinden, da die menschliche Perspektive, vor allem in pragmatischer Hinsicht, unhintergehbar ist.

\subsection{Zwischenfazit}

Die folgende Tabelle fasst die bisher dargelegten Erkenntnisse hinsichtlich der jeweiligen Problematiken der Werttypen, der wertcharakterisierenden Aspekte, der Lokalisierung der Werte, der Wertungsebene und der Quelle der Werte tabellarisch zusammen und ermöglicht so einen besseren Überblick über die reflektierten Werttypen:

\begin{tabular}{|c|l|l|l|l|}
\hline Werttypen: & $\begin{array}{l}\text { instrumentelle } \\
\text { Werte }\end{array}$ & $\begin{array}{l}\text { eudaimonistische } \\
\text { Eigenwerte }\end{array}$ & $\begin{array}{l}\text { moralische } \\
\text { Selbstwerte }\end{array}$ & $\begin{array}{l}\text { objektive } \\
\text { Naturwerte }\end{array}$ \\
\hline Problematik & Eigenwert? & $\begin{array}{l}\text { moralischer } \\
\text { Wert? }\end{array}$ & Wert? \\
\hline $\begin{array}{c}\text { funktionale } \\
\text { tertcharak- } \\
\text { Aspekte: }\end{array}$ & $\begin{array}{c}\text { Wertigkeit ist } \\
\text { relativierbar, } \\
\text { Perspelationale }\end{array}$ & $\begin{array}{c}\text { moralische } \\
\text { Wertigkeit nicht } \\
\text { relativierbar }\end{array}$ & $\begin{array}{c}\text { Perspektive } \\
\text { Wertigkeit nicht } \\
\text { relativierbar } \\
\text { perspektive }\end{array}$ & $\begin{array}{c}\text { Wertigkeit nicht } \\
\text { relativierbar }\end{array}$ \\
Wextrinsisch & $\begin{array}{c}\text { Wert ist inhärent } \\
\text { - extrinsisch }\end{array}$ & $\begin{array}{c}\text { Wert ist } \\
\text { intrinsisch und } \\
\text { gilt kategorisch }\end{array}$ & $\begin{array}{c}\text { Wert ist } \\
\text { unabhängig von } \\
\text { menschlicher } \\
\text { Existenz }\end{array}$ \\
\hline
\end{tabular}




\begin{tabular}{|c|l|l|l|l|}
\hline $\begin{array}{c}\text { Lokalisierung } \\
\text { des Wertes: }\end{array}$ & $\begin{array}{l}\text { axiologischer } \\
\text { Subjektivismus }\end{array}$ & $\begin{array}{l}\text { axiologischer } \\
\text { Subjektivismus }\end{array}$ & $\begin{array}{l}\text { axiologischer } \\
\text { Objektivismus }\end{array}$ & ? \\
\hline $\begin{array}{l}\text { Wertungs- } \\
\text { ebene: }\end{array}$ & $\begin{array}{l}\text { Wertungs- } \\
\text { subjektivismus }\end{array}$ & $\begin{array}{l}\text { Wertungs- } \\
\text { objektivismus }\end{array}$ & $\begin{array}{l}\text { Wertungs- } \\
\text { objektivismus }\end{array}$ & ? \\
\hline \multirow{2}{*}{$\begin{array}{c}\text { Quelle des } \\
\text { Wertes: }\end{array}$} & $\begin{array}{l}\text { anthropo- } \\
\text { relational }\end{array}$ & $\begin{array}{l}\text { anthropo- } \\
\text { relational }\end{array}$ & $\begin{array}{l}\text { anthropo- } \\
\text { relational }\end{array}$ & metaethischer \\
\cline { 2 - 5 } & Subjektivismus & $\begin{array}{l}\text { metaethischer } \\
\text { Subjektivismus }\end{array}$ & $\begin{array}{l}\text { metaethischer } \\
\text { Subjektivismus }\end{array}$ & Objektivismus \\
\hline
\end{tabular}

An der Tabelle zeigt sich noch einmal anschaulich, dass die oftmals vorgenommene negative Bestimmung von Eigenwerten in Abgrenzung zu den instrumentellen Werten eher verwirrend als klärend ist, weil immer expliziert werden muss, welcher Eigenwerttypus jeweils gemeint ist:

Wenn instrumentelle Werte funktionsabhängige, in ihrer Wertigkeit relativierbare und sogar substituierbare, extrinsische und anthroporelationale Werte sind, dann unterscheiden sich diese von eudaimonistischen Eigenwerten nur durch den Aspekt der Relativierbarkeit und ggf. auch Substituierbarkeit der funktional bedingten Wertigkeit, wohingegen die Aspekte der extrinsischen Wertzuschreibung und der Abhängigkeit von der menschlichen Wertperspektive beibehalten werden.

In diesem Sinne sind eudaimonistische Eigenwerte nach wie vor Mittel zum Zweck, dies aber aus anderen Motiven heraus. Diese Motive sind nicht nutzenorientiert oder funktional wie bei den instrumentellen Werten, sondern referieren auf glückskonstitutive Beiträge der Natur/Biodiversität für das gute Leben von Menschen. Hier wird folglich (nur) die relative Wertigkeit durch eine nicht-relativierbare Wertigkeit ersetzt, was wiederum auf der theoretischen Ebene den Sprung vom Wertungssubjektivismus zum Wertungsobjektivismus verdeutlicht.

Der Aspekt der extrinsischen Wertzuschreibung wird wiederum erst über den moralischen Selbstwert bestritten, indem bei diesem Werttypus darauf abgehoben wird, dass intrinsisch werthafte Entitäten immer auch als 'Zweck an sich selbst' und damit als Selbstzweck anerkannt werden und zu achten sind und dass eine solche Achtung moralische Pflichten gegenüber solchen selbstwerthaften Entitäten nach sich zieht. Damit ist hier wiederum der Sprung von einer subjektivistischen Axiologie auf eine objektivistische Axiologie getan, indem angenommen wird, dass intrinsische Werte, die als solche eine nicht-relativierbare Wertigkeit sowie eine kategorische Geltung haben auch unabhängig von subjektiven Zwecken zugestanden werden können.

Vor dem Hintergrund, dass sowohl die nicht-relativierbare Wertigkeit als auch die intrinsische Werthaftigkeit der Natur/Biodiversität bereits über die eudaimonistischen Eigenwerte und die moralischen Selbstwerte argumentiert werden können, ohne dass 
dabei die menschliche Wertperspektive hinterfragt werden muss, indem wiederum der Schritt von einem metaethischen Subjektivismus auf einen metaethischen Objektivismus getan wird, schafft der Werttypus der objektiven Naturwerte keinerlei 'Mehrwert' für die umwelt- und naturschutzethische Debatte, es sei denn, es wird ganz bewusst darauf abgezielt, die menschliche Wertperspektive zu transzendieren. Wird dies getan, dann muss man sich aber der Konsequenzen eines solchen Schritts bewusst sein, die sich aus den metaethisch-objektivistischen Annahmen einer solchen Position ergeben, so dass gezeigt werden muss, warum überhaupt davon ausgegangen werden soll, dass objektive Naturwerte Werte sind und warum diese Menschen zu etwas verpflichten sollen. Da in dieser Arbeit grundlegend davon ausgegangen wurde, dass die Natur amoralisch ist und in diesem Sinne keine Quelle von Werten sein kann, macht es m. E. in Bezug auf die Frage, welche Eigenwerttypen in Bezug Natur/Biodiversität unterschieden werden können, keinen Sinn von objektiven Naturwerten als Eigenwerttypus zu reden, da diese keine Werte sind.

Schließlich wird gerade hieran im Besonderen ersichtlich, dass die oftmals dichotomisierte Unterscheidung zwischen anthropozentrischen und physiozentrischen Ansätzen zumindest in Bezug auf die Wertedebatte irreführend ist, da auch der Werttypus der moralischen Selbstwerte, um dessen Begründung es in den physiozentrischen Ethiken vornehmlich geht, zumindest hinsichtlich seines metaethischen Fundaments anthroporelational ist. Im Anschluss an die Reflexion der Wertedebatte und die hierbei vorgenommenen Charakterisierungen und Unterscheidungen der Werttypen, werden im Folgenden die diejenigen umwelt- und naturschutzethischen Begründungsansätze reflektiert, denen es um die Begründung einer direkten (Schutz-)Pflicht der Biodiversität 'um ihrer selbst willen' geht. Gegenstand der Untersuchung ist dabei die jeweilige, idealtypische Position zum Inklusionsproblem, sprich die darauf abzielende Argumentation, dass aus der Begründung der Eigenwertigkeit der Natur/Biodiversität darauf geschlossen wird, dass Menschen direkte moralische (Schutz-)Pflichten gegenüber der Natur/Biodiversität haben. Dabei ist entscheidend, dass rein theoretisch alle Eigenwerttypen gemeint sein können, wenn vom Schutz der Biodiversität 'um ihrer selbst willen` die Rede ist. Legt man indes die Extensionsstrategie als Unterscheidungsmerkmal der verschiedenen Positionen zum Inklusionsproblem zugrunde und berücksichtigt, dass eben diese auch in Bezug auf die Spezifizierung der moralischen Selbstwerte von Relevanz ist, dann zeigt sich, dass es zumindest dem Ansinnen nach um die Begründung moralischer Selbstwerte zu gehen scheint, auch wenn dies nicht in allen Fällen deutlich gemacht wird. Im Folgenden wird daher der konstruktive Umgang mit der zweiten Problematik im Vordergrund stehen, wie direkte moralische (Schutz-)Pflichten gegenüber der Biodiversität begründet werden und wie dabei der Sprung vom Eigenwert zum ethischen Grund der Verpflichtung vollzogen wird. 


\subsubsection{Die Reflexion der physiozentrischen Begründungsansätze} für den Schutz der Biodiversität 'um ihrer selbst willen'

Wenn es um die umwelt- und naturschutzethischen und speziell physiozentrischen Begründungen direkter moralischer (Schutz-)Pflichten des Menschen gegenüber der Biodiversität geht, dann muss dabei grundlegend berücksichtigt werden, dass dies eine Art 'Superargumention' darstellt, bei der das Begründungsniveau extrem hoch ist, da es darum geht, prima facie (Schutz-)Pflichten aller Menschen gegenüber allen Lebewesen (und ihren abiotischen Lebensbedingungen) zu begründen, die, solange sich keine gegenteiligen Evidenzen ergeben, unter allen Umständen gelten sollen (siehe 4.2.1). Des Weiteren muss berücksichtigt werden, dass sich die Begründungen entweder auf die Gesamtheit aller Lebewesen, sprich deren Vielzahl und Vielfältigkeit (enges Verständnis von Biodiversität) oder auf alle Lebewesen und ihre Lebensbedingungen (weites Verständnis von Biodiversität) beziehen können (siehe 3.3.2): Werden die Begründungen auf das enge Verständnis bezogen, so verlangt dies im Mindesten nach einem biozentrischen Begründungsansatz, ${ }^{110}$ da dieser auf der Gegenstandsebene alle Lebewesen umfasst. Der Bezug auf das weite Verständnis von Biodiversität macht indes ökozentrische oder holistische Begründungsansätze notwendig. Für die folgende Reflexion wird ein 'Weg von außen' gewählt, so dass erst einmal bei der umfassendsten Position zum Inklusionsproblem angefangen wird. ${ }^{111}$ Dies ist der (pluralistische) Holismus (4.2.2.2.1), der allem was existiert (egal ob belebte oder unbelebte Entitäten oder überindividuelle Ganzheiten wie Arten, Ökosysteme oder die Biosphäre im Ganzen) eine moralische Berücksichtigungswürdigkeit um ihrer selbst willen zuspricht. Darauf folgt die Reflexion der ökozentrischen Position zum Inklusionsproblem (4.2.2.2.2), da es diesem auf der Gegenstandsebene ebenfalls um die moralische Berücksichtigungswürdigkeit überindividueller Ganzheiten um ihrer selbst willen geht. Abschließend wird die biozentrische Position zum Inklusionsproblem reflektiert (4.2.2.2.3), über die die moralische Berücksichtigungswürdigkeit für alles Lebendige gerechtfertigt wird.

110 Thorsten Galert, der einen Überblick über die naturethischen Positionen bietet, die sich direkt oder indirekt mit Biodiversität beschäftigen, diskutiert die biozentrischen Ethiken nicht, da er davon ausgeht, dass nur ökozentrische oder holistische Naturethiken den Schutz der Arten um ihrer selbst willlen begründen können (vgl. Galert 1998:60,96). Da hier zwischen einem engen und einem weiten Verständnis der Biodiversität differenziert wird und für das enge Verständnis sowohl die Vielzahl der einzelnen Lebewesen als auch deren Vielfältigkeit von Bedeutung ist (siehe 3.3.2), können und müssen biozentrische Ethiken hier ebenfalls reflektiert werden.

111 Die Darstellung der jeweiligen Positionen zum Inklusionsproblem kann und wird nicht umfassend erfolgen können. Vielmehr geht es darum, die jeweiligen Positionen idealtypisch herauszustellen (siehe FN 55). Dies bedeutet eine Reduktion der oftmals sehr umfangreichen und binnenkomplexen ethischen Argumentationen auf wenige Teilaspekte, die der eigentlichen Begründungstiefe der Ansätze gar nicht gerecht werden kann. Da es hierbei indes um die fokussierte Herausstellung der Begründungsproblematiken geht und hierbei die in den Ansätzen oftmals vorweggenommenen und mitgedachten Erwiderungen mit ausgeführt werden können, wird eine Reduktion auf idealtypische Argumente der einzelnen Positionen als angemessen betrachtet. 
Bei allen Reflexionen wird anhand repräsentativer Argumentationsausschnitte zuerst die Begründungsstruktur idealtypisch deutlich gemacht. Im Anschluss daran wird dann fokussiert auf die Begründungsproblematiken eingegangen, hinsichtlich der Fragen, a) welches Verständnis von Eigenwert dabei jeweils zum Tragen kommt und was den postulierten Eigenwert überhaupt zum Eigenwert machen soll und b) wie von der metaethischen Ebene der Begründung des Eigenwertes auf die pflichtenethische Ebene gelangt wird, sprich wie direkte moralische (Schutz-)Pflichten gegenüber der Biodiversität 'um ihrer selbst willen` begründet werden.

\subsection{Reflexion der holistischen Position zum Inklusionsproblem}

Der (pluralistische) Holismus ${ }^{112}$ ist auf der Gegenstandsebene die umfassendste Position aller physiozentrischen Umwelt- und Naturschutzethiken, weil hier zu begründen versucht wird, dass die Moralgemeinschaft alle individuellen sowie überindividuellen Entitäten umfassen sollte, die existieren. Der prominenteste deutsche Vertreter einer solchen pluralisch-holistischen Position ist Martin Gorke, von dessen Konzeption hier ausgegangen werden soll. Folgende Argumentationsauszüge aus dem Gorkeschen Gesamtwerk verdeutlichen seinen im Grundsatz gut nachvollziehbaren und bisweilen auch 'einfach-genialen', aber in der Tiefe nicht immer konsistenten und damit nicht in Gänze plausiblen holistischen Begründungsansatz:

„Der moralische Kern der [holistischen] Argumentation, der aus dem Unrecht besteht, eine bestimmte Tier- oder Pflanzenart weiter zu gefährden oder gar auszurotten, ist nicht über einen theoretischen Gedankengang abgeleitet, sondern unmittelbar einsichtig." (Gorke 2000:99)

„Die holistische Umweltethik schreibt der gesamten biologischen Vielfalt einen Eigenwert zu. Sie ist die einzige ethische Konzeption, die den Schutz von Biodiversität direkt, d.h. ohne Bezug auf irgendwelchen Nutzen für den Menschen, begründen kann.“ (Gorke 2003:94)

„Sobald wir bereit sind, unsere Egozentrik zu relativieren und uns als teilhafte Wirklichkeit in einem absoluten Horizont begreifen, 'erwachen' wir zu der Einsicht, dass andere Objekte - genauso wie wir selbst - eigenständige Subjekte und damit auch Gegenstände moralischer Verantwortung sind.“ (Gorke 2000:102)

„Von den zahlreichen Faktoren, die in die ökologische Krise geführt haben, ist einer der grundlegendsten zweifellos die in der Neuzeit bei weitem vorherrschende instrumentelle Sicht von Natur. (...) Die holistische Umweltethik vollzieht hier einen grundlegenden Einstellungswandel. Indem sie der gesamten Natur einen

112 Alle holistischen Ansätze, die nicht pluralistisch sind, sprich nicht individuelle und überindividuelle Ganzheiten behandeln, sind in Bezug auf den Gegenstandsbereich keine holistischen Ethiken, sondern ökozentrische Ethiken, die im nächsten Abschnitt behandelt werden (siehe 4.2.2.2.2). 
moralischen, also tatsächlichen Eigenwert zubilligt, relativiert und transzendiert sie diese Ressourcenperspektive. Tiere, Pflanzen, Mineralien, Wälder und Meere werden vom Menschen zwar auch weiterhin als Ressourcen genutzt werden (müssen), aber sie erschöpfen sich nun nicht mehr darin. Sie werden unter holistischer Perspektive zu Subjekten mit einer eigenen, d.h. nicht nur auf den Beobachter bezogenen Wirklichkeit. Sie werden zu 'Gegenübern', die aus sich heraus Rücksicht und Respekt verdienen." (Gorke 2000:97-98)

„Für all diejenigen, die sich für den moralischen Standpunkt entschieden haben (...), ist die Frage nach der Reichweite [der Moralgemeinschaft] im Grunde schon entschieden. Nehmen sie die Moral ernst, dürften sie gar nicht wählerisch sein. Denn wären sie wählerisch, würden sie genau jene Attitüde der Macht an den Tag legen, die sich als Kennzeichen des Egoismus herausgestellt hat: Ich bestimme, wem gegenüber ich mich wann und unter welchen Umständen rücksichtsvoll verhalte." (ebd., 92)

„Ich darf (...) nicht aus meiner egoistischen Perspektive heraus den Kreis derer festlegen, die zu achten sind. Dies wäre der 'Weg der Macht' und nicht der 'Weg der Moral’. Deshalb kann die Alternative zum Egoismus nur lauten: Rücksicht auf beliebiges anderes. (...) (Gorke 1999:250)

„Aufgrund des universellen Charakters der Moral bedeutet moralische Rücksicht zumindest prima facie a) immer und b) auf alle. Konsequent zu Ende gedacht, führt der moralische Standpunkt somit zum Holismus." (Gorke 2003:96)

„Da der moralische Standpunkt - zumindest in der ersten Annäherung - universal ist, muss nicht der Holist zeigen, warum welche Naturwesen aufgrund welcher Eigenschaften moralisch berücksichtigungswürdig sind. Es ist vielmehr an den Vertretern nicht-universaler Ethikkonzepte, plausibel zu machen, warum eine ganz bestimmte Eigenschaft - und nicht eine beliebige andere! - für die Aufnahme in die Moralgemeinschaft unabdingbar ist. Sie müssen zeigen, welchen Grund es gibt, vom egalitären Prinzip der universalen Berücksichtigung abzuweichen und bestimmte Teile der Natur aus der Moral auszuschließen." (Gorke 2000:93)

a) In Bezug auf die Frage, welcher Eigenwerttypus hierbei zum Tragen kommt, spricht Gorke, genau wie viele anderen physiozentrischen EthikerInnen, erst einmal davon, dass „alle natürlichen Wesen einen nutzenunabhängigen Wert haben und somit um ihrer selbst willen Schutz verdienen." (Gorke 2007:125) In einem zweiten Schritt macht er aber ganz deutlich, dass er einen „moralischen Eigenwert“ (Gorke 2000:97) meint, da er der Natur/Biodiversität den Status von „Subjekten mit einer eigenen, d.h. nicht nur auf den Beobachter bezogenen Wirklichkeit“ (ebd., 98) zuschreibt und somit einen axiologischen Objektivismus vertritt. Damit meint Gorke gerade keine metaethisch-objektivistische Position, da er selbst kritisiert, dass zu oft aus dem methodischen Reduk- 
tionismus der Naturwissenschaften ein ontologischer Reduktionismus abgeleitet wird (ebd., 101). Ein solcher ontologischer Reduktionismus sei, so Gorke, „letztendlich nicht mehr als eine metaphysische Behauptung, die aufgrund des indeterministischen Charakters biologischer Systeme prinzipiell nicht verifizierbar ist." (ebd., 103) An die Stelle des ontologischen Reduktionismus setzt Gorke den Universalismus des moralischen Standpunkts (Gorke 1999:247): Gorke argumentiert dafür, dass wenn man sich auf den moralischen Standpunkt einlasse, dieser automatisch dazu führe, dass man prima facie immer und auf alles beliebig Andere Rücksicht nehmen muss. In diesem Sinne versucht er sowohl den moralischen Selbstwert als auch die Ausweitung der Moralgemeinschaft über den universalen Charakter des moralischen Standpunkts zu begründen.

An dieser Stelle kann und muss indes eingewandt werden, dass Gorke hier genauer als er es tut, darauf eingehen müsste, was den von ihm benannten „tatsächlichen moralischen Eigenwert" (ebd., 97) ausmachen soll. Denn Gorke führt diesbezüglich nur aus, dass der Begriff des Eigenwertes „gar nicht befriedigend expliziert werden [kann]“ (Gorke 1999:250) und dass hierbei „die Übereinstimmung mit unseren elementaren Intuitionen letztlich das einzige Kriterium [sei], das uns zur Verfügung steht.“ (Gorke 2000:90) Des Weiteren argumentiert er, dass die moralische Eigenwertintuition weit verbreitet und bereits rechtlich kodifiziert ist und dass eine der „Intuition ständig zuwiderlaufende Argumentation langfristig auch die Intuition und damit den primären moralischen Impuls für das Handeln schwächt." (ebd., 99) Daher geht Gorke davon aus, dass "ein Verharren von Ethik und Naturschutz im anthropozentrischen Paradigma [sich nur schlecht] mit den heutigen wissenschaftlichen Einsichten über die Stellung des Menschen im Kosmos (...) vereinbaren [lässt]." (ebd.) Wie indes über die Rekonstruktion der verschiedenen Eigenwerttypen gezeigt wurde, ist die generelle 'Verabschiedung anthropozentrischer Positionen bei gleichzeitigem Eintreten für einen moralischen Selbstwert irreführend, da auch dieser ein anthroporelationales Fundament hinsichtlich der metaethischen Annahmen beibehält und zudem scheint es ungenügend zu sein, darauf zu verweisen, dass der Eigenwertbegriff nicht befriedigend expliziert werden könne, zumal wenn eben dies in dieser Arbeit doch zumindest ansatzweise versucht wurde.

b) Dies ist sicherlich eine kleinere Schwachstelle in Gorkes Argumentation. Um ein vielfaches entscheidender ist aber der Umstand, dass Gorke den Moralbegriff zum Ausgangspunkt seiner Begründung macht, aber nicht begründet, was das Moralische an der moralischen Eigenwertintuition sowie am moralischen Standpunkt ausmachen soll und warum man den moralischen Standpunkt überhaupt einnehmen sollte: Zwar räumt er zu Recht ein, dass „jede Begründung von irgendetwas ausgehen muss; da es kein voraussetzungsfreies Begründen gebe" (ebd., 92) und er führt an, dass eine Haltung, die sich verallgemeinerbaren ethischen Prinzipien zu unterwerfen bereit ist, ge- 
meinhin als moralischer Standpunkt bezeichnet wird. Aber die Frage ist doch, welche Aspekte dabei unter den moralischen Standpunkt fallen, sprich den moralischen Standpunkt zum moralischen Standpunkt machen. Gorke verweist hierbei (lediglich) auf das Prinzip der Willkürfreiheit, womit er zum Ausdruck bringen möchte, dass eine moralische Haltung umso willkürlicher ist, umso aufwendiger sie hinsichtlich ihrer ontologischen und metaphysischen Prämissen ist (ebd., 93). Diesbezüglich verweist Gorke auf das Prinzip der ontologischen Sparsamkeit (Gorke 2003: 97), über das er in Rückgriff auf Ernst Tugendhat zu der Schlussfolgerung gelangt, dass der moralische Standpunkt und dessen egalitäre Interpretation von »alle« am voraussetzungsärmsten ist:

„Tugendhat $(1994,374)$ hat im Zusammenhang mit Fragen der Gerechtigkeit auf den formalen Grund der Beweislastumkehr hingewiesen (...): ,Es ist falsch, wenn häufig versucht wird, die egalitäre Position in einen primären Begründungszwang zu bringen. Die egalitäre Position bedarf an und für sich keiner Begründung'(...).“ (Gorke 2000:93)

Hierbei stellt sich indes die Frage, warum eine egalitäre Berücksichtigung keiner weiteren Begründung bedarf und warum eine ontologisch sparsamere einer ontologisch gehaltvolleren Ethikposition moralisch vorzuziehen ist. Erst wenn diese Fragen substanziell beantwortet werden würden, wäre der moralische Standpunkt nicht mehr nur formal sondern auch inhaltlich bestimmt. Des Weiteren drängt sich bei Gorkes Argumentation der Verdacht auf, dass das, was hierbei moralisch genannt wird, von Gorke bereits in ganz bestimmten Hinsichten verstanden wird, indem er auf die Kriterien der Kategorizität und der Allgemeingültigkeit moralischer Urteile, Normen und Prinzipien abhebt. Was aber bei Gorke zu wenig beachtet wird, ist indes der Aspekt, dass im Mittelpunkt moralischer Bewertungen Handlungsmotive und im Mittelpunkt moralischer Urteile, Normen und Prinzipien Handlungen stehen und dass es eine notwendige Bedingung genuin moralischer Bewertungen und Urteile ist, dass sie ausschließlich menschliche Handlungsmotive und menschliches Handeln beurteilen, da mit moralischen Bewertungen oder Urteilen an die Einsicht und das Urteilsvermögen des Adressaten appelliert wird, die indes bei allen nicht-menschlichen Entitäten nicht in ausreichendem Maße gegeben sein dürfte, so dass man sie zwar als Bezugpunkt moralischen Handelns nicht aber auch als Adressaten moralischer Bewertungen und Urteile anerkennen kann. Ob diese Diskrepanz zwischen dem 'Empfängerstatus ohne Adressatenstatus', die charakteristisch für die Anwendung der Moral auf nicht-menschliche Entitäten ist, wiederum problematisch für die bei Gorke bereits in Rechnung gestellten Kriterien der Kategorizität und der Allgemeingültigkeit moralischer Bewertungen und Urteile ist, muss hier ungeklärt bleiben. Allein das Problem weist schon darauf hin, dass es mitnichten selbstverständlich und unproblematisch ist, davon auszugehen, dass der moralische Standpunkt eine notwendige oder gar eine hinreichende Bedingung für die 
Vergabe intrinsischer Werte an nicht-menschliche Entitäten ist. Die Schlussfolgerung daraus ist, dass die ethische Forderung des Holismus 'alles moralisch zu berücksichtigen ' bei Gorke über einen inhaltsleeren moralischen Standpunkt begründet wird, so dass hier offen bleibt, was genau dabei unter moralisch zu verstehen ist und was das moralisch Relevante an alle sein soll. Nur wenn dies klar wäre, wäre auch Gorkes Schlussfolgerung plausibel, dass man alles auch prima facie berücksichtigen sollte.

\subsection{Reflexion der ökozentrischen Position zum Inklusionsproblem}

Der Ökozentrismus (manchmal auch Ökologismus oder ökologische Ethik) kann genau wie der Holismus für den Schutz der Biodiversität im weiten Verständnis argumentieren, da es auch hier auf der Gegenstandsebene um überindividuelle Ganzheiten und speziell um Ökosysteme geht. Anders als beim pluralistischen Holismus ist der Ökozentrismus aber eher eine Art 'Sammelbecken ' für recht unterschiedliche und oftmals auch nicht primär ethische, sondern vielmehr diverse ökonomie- und industrialisierungskritische, gesellschafts-, gender und politikkritische sowie religiös-spirituelle Positionen. ${ }^{113}$ Was all diese recht diversen Ansätze im Kern gemeinsam haben, ist deren Kritik an der dualistischen Ontologie von Mensch und Natur, die von den meisten ÖkozentrikerInnen als die zentrale Ursache für die Umweltproblematiken gesehen wird. Zur Überwindung dieser dualistischen Geisteshaltung werden ganz unterschiedliche 'Therapien der gestörten Mensch-Natur Verhältnisse' anvisiert, wie bspw. mystische Einheitserfahrungen mit der Natur, naturreligiöse, buddhistische oder feministische Philosophien, systemtheoretische Natur- und Menschenverständnisse etc. All diese 'Therapieformen` sollen dazu führen, dass „der Mensch als Teil des Systems Natur, als Mitglied der natürlichen oder biotischen Gemeinschaft, als eins mit der Natur“ (Krebs 1997:362) gedacht und verstanden wird, denn es wird davon ausgegangen bzw. kritisiert, dass das Überleben von Menschen aber auch dessen gutes Leben nicht auf Kosten der Natur angestrebt werden könne, dass die Selbstverwirklichung des Menschen Hand in Hand gehe, mit der Selbstverwirklichung des Ganzen in der Natur und dass der Mensch nur für sein gutes Leben sorgen könne, wenn er auch für das Ganze der Natur sorgt (ebd.). Dies zeigt schon, dass es keinen repräsentativen ökozentrischen Begründungsansatz gibt, den man als solchen darlegen könnte. Vielmehr gibt es eine ganze Reihe an typischen Aussagen, die als repräsentativ für ökozentrische Positionen betrachtet werden können:

"The form of ecologism offered here (...) is science-oriented and naturalistic. It sets great store by the findings of the non-human, the way in which human acti-

113 Die ökozentrischen Ethiken sind vor allem in der angloamerikanischen Debatte vorherrschend, während sie in der deutschen Debatte nur eine marginale Stellung einnehmen. Da aber viele ökozentrische Statements auch in der deutschen Debatte zum 'Allgemeingut' gehören, ist die Reflexion dieser Ansätze trotz allem wichtig und notwendig. 
vity may be damaging the environment. Its naturalism - the attempt to understand and explain human values as produced by natural, biological, processes leads it to reject dualism between humans and nature." (Baxter 1999:8)

"A thing is right when it tends to preserve the integrity, stability and beauty of the biotic community." (Leopold (1949) 1970:262)

"The good of the community as a whole serves as a standard for the assessment of the relative value and ordering of its constitutive parts (...). The biosperic perspective does not exempt Homo Sapiens from moral evaluation in relation to the well-being of the community of nature taken as a whole. The preciousness of individual deer, as of any other specimen, is increasingly proportional to the population of the species. Environmentalists, however reluctantly and painfully, do not omit to apply the same logic to their own kind. (...). The extent of misanthropy in modern environmentalism thus may be taken as a measure of the degree to which it is [ecocentric]." (Callicott 1980:323)

„1.The well-being and flourishing of human and nonhuman life on Earth have value in themselves. These values are independent of the usefulness of the nonhuman world for human purposes. 2. Richness and diversity of life forms contribute to the realization of these values and are also values in themselves. (...)" (Devall \& Sessions 1985:70)

Die Popularität dieser und ähnlicher Aussagen in der Umwelt- und Naturschutzdebatte steht ganz außer Frage. Auch die dahinterstehenden Erwägungen und Intuitionen zur Interdependenz und den wechselseitigen Abhängigkeiten zwischen Menschen und der Natur sind mittlerweile Allgemeingut. Die Relevanz dieser Aussagen als ethische Argumente ist hingegen sehr viel fragwürdiger, was sich, einmal abgesehen von konkreten inhaltlichen Problematiken, schon allein daran zeigt, dass die ökozentrischen Ethiken von vielen professionellen EthikerInnen gar nicht als ernst zu nehmende Ethiktheorien angesehen werden, so dass sie diese nicht selten als 'unwürdig' für eine ernsthafte philosophische Diskussion erachtet werden (Krebs 1997:362). Obwohl diese Einschätzung hier geteilt wird und es sich als schwierig darstellt, derart 'ethikferne' Morallehren überhaupt ethisch zu reflektieren, sollen die ökozentrischen Positionen dennoch nicht außer Acht gelassen werden, weil sich hier viele Intuitionen und Ansätze wiederfinden, die in der Biodiversitätsdebatte eine entscheidende Rolle spielen und daher zumindest inhaltlich reflektiert werden müssen, weil viele ökozentrische Annahmen mit misanthropischen Ten-denzen einhergehen, des Weiteren die Gefahr 'ökofaschistischer Tendenzen` hier am stärksten ausgeprägt ist und nicht zuletzt, weil sich über die Ansätze oftmals nicht nur rein terminologische Schwierigkeiten ergeben, was hinter der Rede von der Integrität, Stabilität und Schönheit der ökosystemaren oder biotischen Gemeinschaften sowie dem Wohlergehen der Natur steht. 
a) Bezüglich der Frage, welcher Eigenwerttypus in den ökozentrischen Morallehren zum Tragen kommt und woraus sich die Annahme der Eigenwertigkeit ergibt, gibt es keine einheitliche Antwort. Da nur wenige ökozentrische Positionen überhaupt eine Bestimmung (geschweige denn eine axiologische Bestimmung) der von ihnen verwendeten Werte vornehmen, wird i.d.R. nicht deutlich, welcher Eigenwerttypus jeweils gemeint ist. Selbst wenn davon die Rede ist, dass die ökosystemaren und biotischen Gemeinschaften einen 'Wert in sich selber haben', der unabhängig von der Nützlichkeit für Menschen ist und dass Reichtum und Vielfältigkeit 'Werte in sich selbst' sind, kann die Reflexion bei wenig ansetzen. Allerdings kann mit Birnbacher festgehalten werden, dass gerade innerhalb der ökologischen Ethiken hier eine Vielzahl an objektivistischen Axiologien entwickelt worden ist, „die sich aus der Vielfalt der normativen Theorien und Engagements ergeben, denen diese Axiologien als Fundament dienen.“ (Birnbacher 2007:275) Dabei ist indes nicht immer eindeutig, ob die objektivistischen Positionen nur auf der axiologischen oder auch auf der metaethischen Ebene vertreten werden, denn oftmals wird ebenfalls pauschal behauptet, dass es vom Menschen unabhängige Werte in der Natur gibt.

Zu konstatieren ist an dieser Stelle daher lediglich, dass in ökozentrischen Ansätzen die Wertfrage oftmals nicht angemessen behandelt wird, weil in vielen Ansätzen einfach davon ausgegangen wird, dass die Natur einen Eigenwert hat, so dass hier im Grunde genommen alle Schwierigkeiten und Probleme auftauchen können, die für die jeweiligen Eigenwerttypen benannt worden sind, nur das diese selten auch als solche erkannt oder thematisiert werden.

b) Daher stellt sich bei den ökozentrischen Ansätzen in zweiter Instanz die viel entscheidendere Frage, was genau es bedeuten soll, dass davon ausgegangen wird, dass 'der Mensch Teil der Natur ist' oder 'eins mit der Natur werden soll'. Hier macht Krebs deutlich, dass es zwei unterschiedliche Deutungsvarianten geben kann: Wenn der ontologische Dualismus zwischen Mensch und Natur negiert wird, dann wird demgegenüber oftmals pauschal von dessen Gegenteil, nämlich der ontologischen Identität ausgegangen (Krebs 1997:362). Dies wiederum würde aber bedeuten, dass es bspw. hinsichtlich der Erscheinungsformen oder aber Wesenseigenschaften von Menschen, Zebras und Steinen keine seinsmäßigen Unterschiede gäbe, was nicht wirklich zu überzeugen vermag, zumal man sich hier in recht spekulative spirituelle und metaphysische Deutungen hineinwagt. Vielmehr scheint damit gemeint zu sein, dass es immer schon bestimmte Gemeinsamkeiten, graduelle Übergänge und wechselseitige Abhängigkeiten zwischen Menschen und nicht-menschlichen Entitäten gab und nach wie vor gibt. Dies ist allerdings weder eine ontologische Annahme, noch ist dies ein tragfähiges Fundament, um die 'Sonderrolle' des Menschen zu bestreiten oder gar zu negieren, da hier eingewandt werden kann, dass Menschen als Natur- und Kulturwesen gleicher- 
maßen immer nur partiell 'als Teil der Natur' verstanden werden können. Darüber hinaus hat eine solche, rein beschreibende Interdependenzannahme keinerlei Konsequenzen hinsichtlich der Zuerkennung eines Eigenwertes oder eines moralischen Status der Natur/ Biodiversität.

Die zweite Deutungsvariante nimmt die Intuition auf, dass menschliches Wohlergehen nur zusammen mit dem 'Wohlergehen der Natur' zu haben ist. Diese Deutung wird von Krebs als eudaimonistische Harmoniethese bezeichnet (ebd., 363). Hierbei stellt sich indes die Frage, worin genau das 'Wohlergehen der Natur' besteht, inwieweit Menschen überhaupt in der Lage sind ein solches Wohlergehen zu befördern und inwieweit Menschen für ein solches Wohlergehen Sorge tragen sollten. Hierbei muss des Weiteren, wie bereits einleitend ausgeführt, grundlegend in Rechnung gestellt werden, dass der Mensch als handelndes Wesen immer schon auf Eingriffe in die Natur festgelegt ist, deren Abfolge und Konsequenzen er aber weder vollends steuern noch kontrollieren kann, denn Menschen müssen Natur kultivieren, verringern und partiell immer auch zerstören, um selbst (über-)leben und gut leben zu können. Wird dies nicht berücksichtigt, dann läuft die Behauptung eines „friedlichen Zusammenstimmens des guten Lebens aller in der Natur" Gefahr auf einem "falschen Harmonismus" zu basieren (ebd., 363).

Wird dies hingegen in Rechnung gestellt, dann stellt sich wiederum nicht die Frage nach dem Wohlergehen der Natur, sondern nach dem Wohlergehen des Menschen und wieviel Naturzerstörung dazugehört. Die Thematiken des 'maßvollen Umgangs mit der Natur' sowie die Idee und das Konzept der Nachhaltigkeit, die eben dies fokussieren, stehen indes nur selten im Mittelpunkt ökozentrischer Morallehren. Ganz im Gegenteil, sie stehen im Zentrum anthropozentrischer Umwelt- und Naturschutzethiken, die der Ökozentrismus allerdings zu überwinden versucht. Daher wird die, für die ökozentrischen Positionen eigentlich ebenfalls relevante Nachhaltigkeitsperspektive von den VertreterInnen der Harmoniethese nicht eingenommen, was aber auch bedeutet, dass die hierbei zu beantwortenden Fragestellungen als solche gar nicht erkannt und aufgegriffen werden. Stattdessen wird die Richtigkeit der These oftmals einfach vorausgesetzt, womit dann aber - zumindest im ökozentrischen Rahmen - die Argumente fehlen, um die eudaimonistische Harmoniethese abstützen zu können. Darüber hinaus können, wenn lediglich festgestellt wird, dass der Mensch zum Überleben und zu seinem guten Leben irgendwie abhängig von Naturressourcen ist, lediglich für die instrumentellen Werte und ggf. für den eudaimonistischen Eigenwert der Natur argumentiert werden, nicht aber für einen moralischen Selbstwert oder einen unabhängigen Naturwert. 


\subsection{Reflexion der biozentrischen Position zum Inklusionsproblem}

Der Biozentrismus ist die exklusivste Position zur Begründung direkter moralischer (Schutz-)Pflichten gegenüber der Biodiversität (in ihrem engen Verständnis), weil hier versucht wird, die moralische Berücksichtigungswürdigkeit für alle Lebewesen (nicht aber auch für die abiotische Natur) 'um ihrer selbst willen`zu rechtfertigen. Gleichzeitig ist sie wohl die, sowohl innerhalb als auch außerhalb der Umwelt- und Naturschutzethik, populärste und zugleich plausibelste und nachvollziehbarste ethische Position, was sicherlich daran liegt, dass sich der Biozentrismus, genau wie der pluralistische Holismus, auf seine einzelnen Argumentationsschritte herunterbrechen lässt, so dass hier, anders als in vielen ökozentrischen Ansätzen, von einer, im formalen Sinne, logischen Argumentation gesprochen werden kann. Des Weiteren ist die hinter dem Biozentrismus stehende Intuition, dass Lebewesen, gerade weil sie leben und danach streben ihr Leben zu erhalten, auch moralisch zu berücksichtigen sind, für viele Menschen intuitiv eingängig, so dass man es erst einmal mit dem aussichtsreichsten Kandidaten für eine physiozentrische Begründung des Eigenwerts der Biodiversität sowie direkter moralischer Pflichten gegenüber der Biodiversität zu tun hat. In Anlehnung an Krebs und in Rückgriff auf Paul Taylor wird der biozentrische Begründungsansatz hier in vier Argumentationsschritte aufgeschlüsselt (vgl. Krebs 2000:70-71):

1) Nicht nur Menschen haben bestimmte Ziele, die sie verfolgen, sondern auch alle anderen Lebewesen tun dies. Gemeinsam sind ihnen die inneren und damit intrinsischen Ziele oder Zwecke (Telos) der Selbsterhaltung, der Selbstorganisation und Reproduktion. In diesem Sinne sind sie ihre eigenen Ursachen und können sich durch sich selbst realisieren.

2) Seine Ziele verfolgen zu können und die wichtigsten davon zu erreichen, ist ein wesentlicher Bestandteil des guten Lebens. Traditionellerweise (aristotelisch) lässt sich Moral als Respekt vor dem (menschlichen) Wohl oder als Schutz dieses Wohls verstehen und Ethik als Reflexion auf das Gute.

3) Da auch nicht-menschliche Lebewesen Zwecke haben, gehört das Verfolgen und Erreichen dieser Zwecke zu ihrem Wohl. Da ihnen die Zwecke und Ziele intrinsisch sind und deren Verfolgung und Erreichung das Wohl eines Lebewesens ausmacht, gilt auch für den damit verbundenen Wert, dass dieser intrinsisch ist. In diesem Sinne, besitzen alle Lebewesen einen intrinsischen Wert.

4) Nicht-menschliche Lebewesen von moralischem Respekt auszunehmen ist willkürlich und ungerechtfertigt, da alle nicht-menschlichen Lebewesen aufgrund ihres intrinsischen Wertes inhärent wertvoll sind und ihr Wohl moralisch um ihrer selbst berücksichtigt werden muss. Nicht-menschliche Lebewesen gehören auch zur Moralgemeinschaft. Speziezismus ist daher moralisch genauso verwerflich wie Sexismus oder Rassismus. 
a) In Bezug auf die Frage, welcher Eigenwerttypus im biozentrischen Begründungsansatz zum Tragen kommt, muss erst einmal etwas weiter ausgeholt werden, da Taylor selbst mit den Begriffen »inherent worth « und »inherent value " operiert, die indes erst einmal in die vorgelegte Typologie eingeordnet werden müssen: Das was Taylor »inherent worth « nennt und was in den deutschen Übersetzungen seiner Texte mit intrinsischer Wert übersetzt wird, bezieht sich bei ihm auf den Gedanken, dass alle Lebewesen ein eigenes Wohl (»the good«) haben, das ihren Selbstwert, unabhängig vom Nutzen für andere Entitäten ausmacht, so dass Taylor (zumindest hier) für einen moralischen Selbstwert argumentiert:

"To say that an entity $X$ has inherent worth is to assert the following: A state of affairs in which the good of $X$ is realized is better than on otherwise similar state of affairs in which it is not realized (or not realized to the same degree), (a) independently of X's being valued, either intrinisically or instrumentally by some human valuer, and (b) independently of $X$ 's being in fact useful in furthering the ends of a conscious being or in furthering the realization of some other being's good, human or nonhuman, conscious or nonconscious." (Taylor 1986:75)

Das, was Lebewesen in diesem Sinne für sich selbst wertvoll macht und gleichsam dessen Wohl bestimmt, sind die Fähigkeiten zur Selbstorganisiertheit, zur Selbsterhaltung sowie zur Selbstreproduktion (Taylor 1997:129). Die Annahme, dass Lebewesen sich aus sich selbst heraus und durch sich selbst realisieren, führt wiederum dahin innen die Verwirklichung innerer Zwecke oder Ziele (Telos) zu unterstellen, so dass Lebewesen nach Taylor als teleologische Zentren von Leben verstanden werden können:

"We conceive the organism as a teleological center of life, striving to preserve itself and realize its own good in its own unique way. To say it is a teleological center of life is to say that its internal functioning as well as its external activities are all goal-oriented (...). It is the coherence and unity of these functions of an organism, all directed towards the realization of its good, that make it one teleological center of activity." (Taylor 1986:121)

Da angenommen wird, dass es sich bei den 'Strebungen zum Leben ' um intrinsische, also in der Natur des Lebendigen selbst befindliche Eigenschaften handelt, wird darauf geschlossen, dass Lebewesen auch ein eigenes innen ebenfalls intrinsisches Wohl haben, das Lebewesen zu realisieren anstreben. Das Wohl eines Lebewesens ist das, was gut für eben dieses Lebewesen als spezielle Lebensform ist. Dabei wird angenommen, dass die Berücksichtigung dieses Wohl moralisch relevant ist, weil sowohl die Lebensform als auch die 'Strebungen zum Leben 'natürlichen Normen unterliegen:

„Es ist ein Kennzeichen des Lebendigen, dass es natürlichen Normen unterliegt und es liegt im Begriff der Norm, dass das, was unter sie fällt, in einer bestimmten 
Weise sein soll. Dass etwas auf eine bestimmte Weise sein soll, bedeutet, dass es in dieser bestimmten Hinsicht gut [oder eben defizitär] sein kann, da man sonst nicht angeben könnte, ob und inwiefern es in dieser Hinsicht [nicht] so ist, wie es sein soll[te]. Diese Bedeutung von 'gut' bezieht sich auf die natürliche Qualität von Lebewesen (...) und diese ist ihnen intrinsisch. Wenn etwas gut ist, heißt das auch, dass es auf irgendeine Weise einen Zweck oder einen Wert verkörpert, und wenn der Zweck oder das Gute einem Lebewesen intrinsisch ist, so gilt das auch für den mit diesem Zweck oder Guten verbundenen Wert. Bei Lebewesen fallen das Gute [ihr Wohl] und ihr [Selbst-]Wert zusammen, beides ist ihnen intrinsisch." (Reuter 2014:78)

b) Aufbauend darauf und im Übergang zur Begründung einer unbedingten moralischen Haltung gegenüber der Natur, die Taylor „Achtung gegenüber die Natur“ (respect for nature) nennt (Taylor 1997:111), ist bei Taylor auch die Rede vom »inherent value levant, der in den deutschen Übersetzungen i.d.R. mit dem Begriff des inhärenten Wertes übersetzt wird. Hierbei ist der Gedanke zentral, dass die Unterstützung anderer Lebewesen in ihrem Streben nach ihrem eigenen Wohl selbst einen Eigenwert besitzt, woraus sich wiederum ergeben soll, dass alle moralisch Handelnden die moralische Pflicht haben, diese als Wesen mit einem intrinsisches Wert und einem intrinsischen Wohl, um ihrer selbst willen zu erhalten. Der inhärente Wert ergibt sich bei Taylor daher aus einer Kombination aus dem intrinsischen Wert aller Lebewesen und der Forderung intrinsisch wertvolle Lebewesen moralisch zu berücksichtigen:

„Kombinieren wir diese beiden Prinzipien [Prinzip des intrinsischen Wertes und Prinzip der moralischen Berücksichtigung], können wir definieren, was es für ein Lebewesen oder eine Gruppe lebendiger Wesen bedeutet, einen inhärenten Wert zu besitzen. Von etwas zu behaupten, es besitze einen inhärenten Wert, bedeutet, dass alles Wohl dieses Etwas die Berücksichtigung und Beachtung aller moralisch Handelnden verdient und dass der Verwirklichung seines Wohls ein intrinsischer Wert zukomme, der als Zweck an sich und um der Entität willen, um deren Wohl es geht, verfolgt werden soll." (ebd., 117)

Welchem Typus von Eigenwert lässt sich der inhärente Wert nun also zuordnen? Das Problem hierbei ist, dass bei Taylor nicht deutlich wird, ob es sich dabei um einen eudaimonistischen Eigenwert oder um einen moralischen Selbstwert handeln soll, denn Taylor führt lediglich aus, dass der inhärente Wert ein von der menschlichen Wertperspektive abhängiger Wert ist, der in Absehung jeglicher praktischer Nutzen für Andere ein Eigenwert ist:

„Obwohl es Menschen sind, die die Entität wertschätzen müssen, ist der ihnen beigemessene Wert gemäß der Ethik der Achtung gegenüber der Natur kein menschlicher Wert. Das heißt, es ist kein Wert, der aus Erwägungen, die das men- 
schliche Wohlergehen oder die menschlichen Rechte betreffen, hervorginge. Es ist ein Wert, der nichtmenschlichen Lebewesen selbst beigemessen wird, unabhängig von dem, was Menschen für ihr eigenes Wohl als förderlich erachten." (ebd., 122)

Dafür, dass Taylor hier keinen eudaimonistischen sondern einen moralischen Eigenwert meint, obgleich dies bei der Kennzeichnung als inhärenter Wert naheliegen würde, spricht, dass Taylor zur Begründung der moralischen Berücksichtigungswürdigkeit aller Lebewesen auf die Haltung der „Achtung gegenüber der Natur" verweist, die „auf dem Gebiet der Humanethik dem Topos der Achtung gegenüber Personen entspricht." (ebd., 118) Entscheidend dabei ist, dass die Haltung der „Achtung gegenüber der Natur" bei Taylor so konzipiert ist, dass sie analog zu Kants moralischen Motiv der Achtung aufgebaut ist und dass Taylor mittels der bereits benannten und für die Begründung moralischer Selbstwerte typischen Extensionsstrategie versucht, die moralische Berücksichtigung aller Menschen auf alle Lebewesen auszudehnen:

„Die Achtung gegenüber der Natur ist keine Haltung, die in einer anderen, allgemeineren oder grundlegenderen begründet wäre. Sie selbst steckt den Rahmen für unsere Verantwortung gegenüber der natürlichen Welt ab. (...) [I]hre Begründung kann nicht in einem Rückgriff auf eine allgemeinere Haltung oder ein grundlegenderes normatives Prinzip bestehen. Zweitens ist diese Verpflichtung eine moralische, da sie nicht als eine Frage persönlicher Interessen, sondern als eine Frage des Prinzips verstanden wird. Dadurch unterscheidet sie sich von jener Vielfalt von Gefühlen und Stimmungen, die in der Liebe zur Natur ihren Ausdruck finden. (...) Achtung gegenüber der Natur ist eine Einstellung, die unserer Überzeugung nach jeder moralisch Handelnde schlicht als moralisch Handelnder an den Tag legen sollte, unabhängig davon, ob er nun ein Naturliebhaber ist oder nicht. (...) Kantisch gesprochen: (...) Es gilt, diesen Standpunkt kategorisch einzunehmen." (ebd., 118-119)

So plausibel dieser gesamte Gedankengang auf den ersten Blick ausschaut, so problematisch ist (auch) dieser im Detail. Im Kern setzt die Kritik an der Ambiguität des TelosBegriffes und der moralischen Überhöhung des Wohlbegriffes an: Bezüglich der Ambiguität des Begriffes Telos ist zu sagen, dass der Zweckbegriff (im Mindesten) doppeldeutig ist (Krebs 2000:71). ${ }^{114}$ Krebs unterscheidet dabei zwischen praktischen und funktionalen Zwecken. Zwecke im praktischen Sinn „sind die Zustände, die Handelnde

${ }^{114}$ Krebs unterscheidet zwischen teleonomischen von teleologischen Zweckbeschreibungen, führt dann aber auf Seiten der funktionalen Organisation (Teleonomie) eine weitere Unterscheidung zwischen mechanischer Organisation und Selbstorganisation ein (Krebs 2000:73). Dies wiederum entspricht der Dreifachunterscheidung, die bspw. Katrin Reuter in Rückgriff auf Boris Hennig (vgl. Hennig 2006) vornimmt, indem sie zwischen nicht-teleologischen (bei Krebs: mechanischer Organisation), teleologischen (bei Krebs: Selbstorganisation) sowie intentionalen Bewegungen (bei Krebs: teleologisch) unterscheidet (Reuter 2014:72-74). 
mit ihren Handlungen hervorbringen wollen" (ebd., 72), wohingegen Zwecke im funktionalen Sinn „bestimmte Zustände eines ereignishaften Geschehens" sind (ebd.). Während bei praktischen Zwecken teleologisch, handlungstheoretisch und moralisch argumentiert werden kann, ist dies, so Krebs, bei funktionalen Zwecken nicht der Fall, da es hier lediglich um deren Funktionalität sowie um deren kausale Struktur geht, was Krebs mit dem Begriff der Teleonomie zu fassen versucht. Das Entscheidende an dieser Unterscheidung zwischen Teleologie und Teleonomie ist, dass solange nicht-menschliche Lebewesen rein funktionale Zwecke verfolgen, diese Zweckverfolgung nicht moralisch relevant ist, da funktionale Zwecke keinen Wert implizieren (ebd., 73). In diesem Sinne ist die Rede davon, dass ein Lebewesen bestimmte Eigenschaften aufweist, die ihm intrinsisch sind sowie die Rede von einem darauf basierenden intrinsischen Wohl nicht ausreichend, um daraus schlussfolgern zu können, dass diese intrinischen Eigenschaften werthaft sind und moralisch zu berücksichtigen wären. Denn in beiden Fällen handelt es sich lediglich um eine Beschreibung dessen, was Lebewesen ihrer Natur nach sind. Aus dieser Beschreibung geht zwar hervor, wie Lebewesen gemäß ihrer speziellen Natur sind und wonach sie streben (Reuter 2014:103). Dieses 'Strebevermögen` impliziert aber solange kein moralisches Sollen, wie nicht begründet wird, warum die intrinsischen Eigenschaften der Selbstorganisiertheit, des Selbsterhaltungsdrangs sowie die Fähigkeit zur Reproduktion (die Krebs als Autonomiefähigkeit charakterisiert), die Lebewesen ohne Frage aufweisen, auch als Kriterien für eine moralische Berücksichtigungswürdigkeit angesehen werden sollten:

„Mit dem Verweis auf die Autonomie der Natur kommt (...) ein (...) moralisches Kriterium ins Spiel, das eigens zu begründen wäre. Aber wie? (...) Ist ein Krebsgeschwür dadurch, dass es von selbst entsteht und wächst, irgendetwas Gutes an sich? Dass Autonomie als Nicht-vom-Menschen-Gemachtsein moralischen Eigenwert haben soll, leuchtet nicht ein." (Krebs 2000:75)

In diesem Sinne kann die Rede von der Autonomie der Lebewesen zwar dazu benutzt werden, um zu beschreiben, was Lebewesen ihrer Natur nach sind, aber damit ist erst einmal nur gesagt, dass Lebewesen ein teleonomisches Strebevermögen haben. Wenn aus diesem teleonomischen Strebevermögen dann auf das Wohl von Lebewesen geschlossen und dieses wiederum als moralisch zu berücksichtigendes Wohl verstanden wird, dann wird der Wohlbegriff damit moralisch überhöht, da über den inhärenten Wert die Frage, ob Lebewesen einen intrinsischen Wert und ein Wohl haben, bereits mit der Frage nach dem moralischen Status von Lebewesen vermengt wird:

"Intrinsic value involves a thing's good. It needn't be a moral good. (...) In contrast with intrinsic value, moral standing is a direct moral matter. To know $\mathrm{x}$ has moral standing is to know that we must take $x$ 's interests into account as a matter of right that is a matter of moral concern." (O 'Neil 1997:47) 
Krebs wendet darüber hinaus ein, dass eine solche moralische Überhöhung auch ganz praktisch gesehen, zu absurden Konsequenzen führen würde:

„Moralischer Respekt ist Respekt für das, woran anderen liegt, für ihr subjektiv gutes Leben. Wäre dieser Satz falsch, dann müssten wir die funktionalen Zwecke von Thermostaten, Computern und Autos, auch da, wo sie unseren Zwecken zuwiderlaufen, um ihrer selbst willen respektieren." (Krebs 2000, 73).

Krebs vermutet daher, dass die BiozentrikerInnen dem Ansinnen nach zwar einen moralischen Selbstwert für alles Lebendige begründen wollen, aber eigentlich einen ästhetischen Eigenwert meinen, da in der ästhetischen Betrachtung der Natur die Autonomie der Lebewesen von Bedeutung ist, weil Menschen für ihr Selbstverständnis vom guten Leben auch Dinge brauchen, die keine Funktion haben oder die man nicht primär unter der 'funktionalen Brille' zu betrachten braucht:

„Mein Verdacht ist, dass Philosophen, die (...) das teleologische Argument unter Verweis auf die Autonomie der Natur verteidigen einer Verwechselung aufsitzen. Sie verwechseln moralischen Eigenwert mit ästhetischem Eigenwert. Ästhetischer Eigenwert hat die Natur für den Menschen, für sein gutes Leben. (...) In der ästhetischen Betrachtung ist die Autonomie in der Tat bedeutsam. Wir schauen gern Dinge an, die keine Funktion haben für uns oder zu haben scheinen." (Krebs 2000:75)

Das wiederum hat aber zur Folge, dass der Schutz der ästhetisch attraktiven Natur/ Biodiversität zwar den (menschlichen) Betrachtern moralisch geschuldet ist, aber nicht der Natur selbst, denn das gute Leben der Betrachter hat hierbei moralischen Eigenwert, während es fraglich ist, ob dem Wohl nichtmenschlicher Lebewesen ebenfalls ein solcher Eigenwert zukommt (siehe 4.2.2.1.2). Wenn es also zutreffend ist, dass Lebewesen eudaimonistische Eigenwerte zukommen, dann bleibt im Biozentrismus nicht nur der Übergang vom ersten zum zweiten Argumentationsschritt unbegründet, dass jeder Organismus als ein teleologisches Zentrum von Leben einen intrinischen Wert habe, sondern zugleich auch der letzte und entscheidende Argumentationsschritt, dass alle Lebewesen eine moralische Berücksichtigung verdienen, die sich aus ihrem inhärenten Wert ergäbe, so dass alle Lebewesen Teil der Moralgemeinschaft seien.

\subsection{Zwischenfazit}

Zusammenfassend zeigen die vorangegangenen Ausführungen, dass alle in Frage kommenden physiozentrischen Ansätze zur Begründung von (Schutz-)pflichten gegenüber der Biodiversität auf ihre je eigene Weise mit Begründungsproblematiken und Argu- 
mentationsdefitiziten einhergehen. ${ }^{115}$ Diese Problematiken und Defizite ergeben sich zumeist aus der vorschnellen und im Einzelnen nicht separat begründeten Ableitung direkter moralischer (Schutz-)Pflichten aus der ebenfalls oftmals unklaren Eigenwertigkeit der Natur/Biodiversität. Daraus lassen sich zwei Schlussfolgerungen ziehen:

Die erste Schlussfolgerung aus den Begründungsproblematiken und Argumentationsdefitiziten der physiozentrischen Ansätze zum Inklusionsproblem ist, dass die in der NBS unterbreitete Suggestion es gäbe ethisch plausible Begründungen zur Ausweisung von (Schutz-)Verpflichtungen gegenüber der Biodiversität um ihrer selbst willen, die sich wiederum dafür anführen lassen, möglichst die gesamte noch vorhandene Biodiversität zu erhalten, kein tragfähiges Fundament besitzt, insofern es bis dato kein ethisches 'Superargument' für den Schutz der Biodiversität um ihrer selbst willen gibt (Ott 2007:109). Statt des 'großen Wurfes', den Schutz der gesamten Biodiversität als moralische Pflicht aller Menschen auszuweisen, kann die Alternative nur darin bestehen, den „Weg über die Dörfer“ (ebd., 109) zu gehen, der ein ganzes 'Sammelsurium ' an Gründen eröffnet, die in ihrer Gesamtheit mehr oder weniger für den Schutz aber auch die nachhaltige Nutzung und die gerechte Verteilung vieler einzelner Segmente der Biodiversität sprechen, so dass man auf diesem Weg zu selektiven und zumeist kontextabhängigen Begründungen gelangt, die eine unterschiedliche Schutzreichweite haben und unterschiedliche Verbindlichkeiten nach sich ziehen. So naheliegend diese Schlussfolgerung ist, so problematisch sind sicherlich die damit verbundenen Konsequenzen für viele Natur- und UmweltschützerInnen, denn in diesem Fall gibt es eben nicht das alles entscheidende Argument, das den Schutz der gesamten Biodiversität begründen kann. Insofern ist der „Weg über die Dörfer“ zumindestens der Sache nach kein Problem und vielleicht sogar ratsam. Er widerspricht aber der Motivation vieler BiodiversitätsprotagonistInnen. Denn einen solchen Weg zu beschreiten, bedeutet, das mühsam erkämpfte Schutzziel, um das es vielen BiodiversitätsprotagonistInnen in erster Linie geht, zumindest hinsichtlich der möglichst umfassenden Schutzreichweite und der prioritären Stellung des Schutzziels zu relativieren. Dies ist für viele Umwelt- und NaturschutzaktivistInnen sicherlich ein zweischneidiges Schwert, da sie ihre Botschaft relativieren müssen. Für EthikerInnen gibt es dazu aber keine ernsthafte Alternative, da das Gegenteil davon, die Priorität der Zielstellung des möglichst umfassenden Schutzes der gesamten Biodiversität um ihrer selbst willen bereits voraussetzen und die Ethik funktionalistisch dafür einzuspannen, um dieses Ziel erreichen (siehe 2.3), deutlich problematischer ist.

\footnotetext{
115 Zu dieser Konsequenz gelangen auch Thorsten Galert (vgl. Galert 1998:102-103) und Katrin Reuter (vgl. Reuter 2014:117-119). Galert zieht daraus indes keine Schlussfolgerung und Reuter andere, als die, die hier im Folgenden gezogen werden.
} 
Die zweite Schlussfolgerung aus den Begründungsproblematiken und Argumentationsdefitiziten der physiozentrischen Ansätze zum Inklusionsproblem besteht darin, dass die im Folgenden anvisierte theoretische Neuausrichtung der Umwelt- und Naturschutzethik zur Begründung der Bewahrung und Förderung der Biodiversität wegen der dargelegten Begründungsproblematiken auf der Basis einer anthropozentrischen Position zum Inklusionsproblem argumentiert wird. Diese beiden Konsequenzen, die im metaphorischen Sinne sicherlich eine 'bittere Pille' für alle überzeugten Naturschützerlnnen darstellen muss, wird von diesen sicherlich nur dann 'geschluckt' werden, wenn im Gegenzug dazu von Seiten der EthikerInnen praxisrelevante ethische Überlegungen für die Bewahrung und Förderung der Biodiversität angeboten werden und wenn diese aufzeigen, wie und mit welchen Argumenten die Bewahrung und Förderung der Biodiversität nicht nur im ethischen, sondern auch gesellschaftlichen Kontext überzeugend kommuniziert werden kann. Diesem Anspruch der Anwendungsorientierung bzw. Praxisrelevanz stehen indes die drei Detailproblematiken entgegen, die bereits in der Problemselektion dargelegt wurden (siehe 2.4.2). Zur Erinnerung handelt es sich dabei um folgende Problematiken:

1) Über die Leitunterscheidung zwischen anthropozentrischen und physiozentrischen umweltethischen Ansätzen wird immer wieder der hintergründige Dualismus zwischen Mensch und Natur repliziert, was aber dazu führt, dass dabei die Perspektive auf die Auslotung der gesellschaftlichen Naturverhältnisse, die für die Bewahrung und Förderung der Biodiversität sowohl auf der inhaltlichen, auf der normativen als auch auf der politischen Ebene zentral ist, zu stark verloren geht.

2) Der primäre Fokus der Umwelt- und Naturschutzethik liegt zumeist auf Schutzpflichtbegründungen unter Vernachlässigung von Nutzungs- und Verteilungsbegründungen. Genau dies führt dazu, dass die für die Bewahrung und Förderung der Biodiversität so entscheidende Integrativität der Zieltrias zu wenig beachtet wird und dass nicht der gesamte 'Raum ` praktischer Gründe für den Schutz, die Nutzung und die Verteilung der Biodiversität berücksichtigt wird.

3) In der ethischen Kommunikation über Natur- und Umweltschutzanliegen im Allgemeinen und bezüglich der Bewahrung und Förderung der Biodiversität im Speziellen wird oftmals zu wenig beachtet, dass die Ausweisung 'guter Gründe` nicht lediglich eine Frage der normativen Akzeptabilität der Gründe ist sondern immer auch eine Frage ihrer faktischen Akzeptanz.

Um diesen drei Problematiken zugleich zu begegnen und um auf diesem Weg zu einer anwendungsorientierten Biodiversitätsethik zu gelangen, bedarf es - so die im nächsten Abschnitt vertretene und zu plausibilisierende These - einer theoretischen Neuausrichtung der Umwelt- und Naturschutzethik. 


\subsection{Die theoretische Neuausrichtung der Umwelt- und Naturschutzethik hin zu einer anwendungsorientierten Biodiversitätsethik}

Die theoretische Neuausrichtung der Umwelt- und Naturschutzethik wurde anhand der kritischen Reflexionen auf die gängigen Gründe und Begründungen für die Erhaltung der Biodiversität plausibilisiert und wird im Folgenden anhand der drei aufgezeigten Detailproblematiken und ihrer Lösungen konkretisiert. Daher sind alle nun folgenden Ausführungen Analyse, kritische Reflexion und Fazit zugleich. Eine solche Synergie wird erreicht, indem mit Bezug auf die erste Detailproblematik auf die relationale Perspektive und damit auf die Ansätze und Schwierigkeiten einer inklusiven Umwelt- und Naturschutzethik eingegangen wird (4.3.1). Hierüber soll die Fokussierung der Umweltund Naturschutzethik auf die Inklusionsproblematik zugunsten einer pragmatischen Fokussierung auf die Auslotung der individuellen und gesellschaftlichen Naturverhältnisse verschoben werden. Daher werden zum einen die, für das Konzept der gesellschaftlichen Naturverhältnisse relevanten Relationen zwischen Mensch, Natur und Gesellschaft fokussiert sowie die wesensmäßige Naturbeziehung als umweltsoziologische Grundlage der relationalen Perspektive dargelegt (4.3.1.1). Zum anderen wird auf die Relevanz eudaimonistischer Ansätze für die relationale Umwelt- und Naturschutzethik sowie die Bedeutung der Biodiversität für ein gutes Leben eingegangen (4.3.1.2). Mit Bezug auf die zweite Detailproblematik, dem zu starken Fokus der Umwelt- und Naturschutzethik auf Schutzplichtbegründungen, womit zum einen der Aspekt der Nichtbeachtung der Integrativität der Zieltrias und zum anderen der zu starke deontische Fokus auf Pflichtenargumente fokussiert wird, wird ein spezieller, auf die Biodiversitätszieltrias ausgerichteter Argumentationsraum aus Klugheits-, Glücks- und Gerechtigkeitsargumenten zur Begründung der Bewahrung und Förderung der Biodiversität dargelegt (4.3.2). Dieser Ansatz wird dargelegt und kritisch reflektiert, indem bezogen auf den ersten Problemaspekt, nämlich das Ansinnen der Integrativität der Biodiversitätszielstellung gerecht zu werden, das konzeptionelle Problem des Ansatzes dargelegt wird (4.3.2.1). Diesem Konzeptionsproblem wird konstruktiv begegnet, indem die diskurstheoretischen Fundamente der Argumentationstrias aus Klugheit, Glück und Gerechtigkeit rekonstruiert werden (4.3.2.2), so dass die pragmatischen (4.3.2.2.1), eudaimonistischen (4.3.2.2.2) und moralischen (4.3.2.2.3) Gebrauchsweisen der praktischen Vernunft dargelegt werden. Bezogen auf den zweiten Problemaspekt, den zu einseitigen deontischen Fokus, werden die drei Argumentationstypen wiederum in einem mehrdimensionalen Ethikverständnis verortet (4.3.2.3). Abschließend wird der dritten Problematik begegnet, indem die Akzeptanz- und Akzeptabilitätsbedingungen für die Kommunikation und Aushandlung der Biodiversitätsziele und -maßnahmen herausgestellt werden (4.3.3). Diese Ausführungen ermöglichen wiederum eine Überleitung in die politikwissenschaftliche Detailreflexion (4.4). 


\subsubsection{Die relationale Perspektive: Die Ansätze und Schwierigkeiten einer inklusiven Umwelt- und Naturschutzethik}

Diejenigen Umwelt- und Naturschutzethiken, die dafür argumentieren, dass die bis dato unentschiedene Debatte um das Inklusionsproblem zugunsten einer pragmatischen Position in Hinblick auf die Auslotung der individuellen und gesellschaftlichen menschlichen Naturverhältnisse verlassen werden sollte, wurden von Thomas Potthast und Uta Eser als inklusive Umwelt- und Naturschutzethik charakterisiert (vgl. Eser \& Potthast 1999; Potthast 2000; Eser, Neureuther \& Müller 2011). Diese Position, die um Missverständnisse hinsichtlich der Inklusionsdebatte zu vermeiden, ${ }^{116}$ hier als relationale Perspektive charakterisiert wird, wird zwar mittlerweile von vielen UmweltethikerInnen eingenommen, es stellt sich aber die Frage, was diese Position im Detail ausmacht:

Definitorisch führen Eser et al. diesbezüglich aus, dass über die relationale Perspektive, genau wie im Ökozentrismus, die enge Verbundenheit von Mensch und Natur betont wird, andererseits verbleibt die relationale Perspektive aber im Rahmen der Anthropozentrik, indem sie „in Zweifelsfällen den Bezug auf menschliche Bedürfnisse anerkennt" (Eser, Neureuther \& Müller 2011:114). ${ }^{117}$ So wird im Rahmen der relationen Perspektive kritisiert, dass es auch in vielen physiozentrischen Ansätzen bei näherem Hinsehen, trotz der anderweitigen Formulierungen und Ambitionen, um die menschliche Wertschätzung der Natur im Allgemeinen und der Biodiversität im Speziellen geht. Dabei steht oftmals das Ansinnen im Fokus der Natur/Biodiversität einen über das rein Gebräuchliche hinausgehenden Status zu geben, was, Eser et al. als eine durchaus berechtigte und nachvollziehbare Intuition ansehen (ebd., 69). Problematisch erscheint ihnen das Ansinnen indes immer dann, wenn dabei der Umstand vergessen oder vernachlässigt wird, dass Natur immer nur im Kontext der zugrundeliegenden Mensch-Natur Relation denkbar ist, was aber durch die Zentrierung auf die Natur (und gegen den Menschen) nicht in ausreichendem Maße berücksichtigt wird (ebd. 68).

${ }^{116}$ Da die Debatte um die Begründung der moralischen Berücksichtigungswürdigkeit der Natur/Biodiversität um ihrer selbst willen als Inklusionsproblematik bezeichnet wird, liegt hier das Missverständnis nahe, dass eine inklusive Position sich mit eben dieser Frage beschäftigt. Eben dies ist aber nicht der Fall. Daher wird hier von einer relationalen Perspektive gesprochen.

117 Der naheliegende und berechtigte Einwand, dass der bloße Verweis auf die individuellen und gesellschaftlichen Naturverhältnisse (siehe 1.2.1) nicht die Notwendigkeit der Klärung der Inklusionsfrage suspendiere, nötigt dazu, auch innerhalb der relationalen Perspektive zu einer begründeten Position zum Inklusionsproblem zu gelangen, auf der aufbauend die anvisierte Fokusverschiebung vollzogen wird: Zwar wird die inklusive Ethik von Eser und Potthast in den Rahmen der anthropozentrischen Ethik gestellt (vgl. Eser \& Potthast 1999; Potthast 2000; Eser, Neureuther \& Müller 2011), allerdings geschieht diese Verortung weniger begründet als notwendig wäre. Im Rahmen dieser Art wurde daher bereits auf die physiozentrischen Positionen zum Inklusionsproblem reflektiert (siehe 4.2.2.2), woraufhin der Schluss gezogen wurde, dass keiner dieser Begründungsansätze ausreichend ist (siehe 4.3), so dass hier im Sinne eines Ausschlussverfahrens begründet auf der Basis eines anthropozentrischen Ansatzes weiter argumentiert wird. 
Andersherum, so die weitere Kritik, wird aber auch in vielen anthropozentrischen Ansätzen das Missverständnis bedient, dass es um Konflikte zwischen Menschen und der Natur und weniger um Konflikte zwischen Menschen bezüglich ihrer Handlungsweisen im Umgang mit der Natur geht, die mehr oder weniger zweckdienlich, sinnvoll oder richtig sein können. Dies wird bereits daran ersichtlich, dass nicht alle Konflikte in Bezug auf die Natur/Biodiversität automatisch auch zugunsten des menschlichen Wohls gelöst werden müssen, denn Menschen verzichten sehr wohl zugunsten 'der Natur' auch auf ihre eigenen Vorteile und Nutzen und tun dies auch dann, wenn es sie dies nicht aus einer Pflicht anderen gegenüber oder für die Natur um ihrer selbst willen tun. Eben diese Kategorie von Handlungen in Bezug auf die Natur/Biodiversität, die als freiwillige Selbstverpflichtungen von den GegnerInnen anthropozentrischer Positionen abgetan werden, da diese als rein beliebige, weil freiwillige Möglichkeiten angesehen werden, stehen im Fokus der relationalen Perspektive.

Demnach zeichnet sich die relationale Perspektive vor allen dadurch aus, dass sie darauf hinweist, dass es bei der umwelt- und naturschutzethischen Bewertung und Beurteilung aller menschlichen Handlungen in Bezug auf die Natur/Biodiversität nicht darum gehen kann und sollte, das Wohl des Menschen gegen das Wohl der Natur abzuwägen und umgekehrt. Vielmehr geht es unter dieser Perspektive um die Qualität der vielschichtigen und heterogenen Beziehungen zwischen Menschen und der Natur. Hierbei ist die Annahme zentral, dass der Fokus auf die individuellen und gesellschaftlichen Naturverhältnisse über die (Anthropo- und Physio-)'Zentriken ' notorisch versperrt wird (ebd., 68), da der polarisierende und die Zwischentöne verdeckende Streit zwischen Anthropozentrismus und Physiozentrismus um die Frage, ob nun der Mensch oder die Natur im Fokus der ethischen Begründung stehen sollte, weder ausreichend noch zielführend ist, um zu einer anwendungsorientierten Umwelt- und Naturschutzethik im Allgemeinen und zu einer Biodiversitätsethik im Speziellen zu gelangen:

"Gerade in Konfliktfällen, in denen menschliches und nicht-menschliches Wohlergehen sich ausschließen, ist eine Konfliktlösung ohne Bezugnahme auf menschliche Bedürfnisse und Rechte nicht überzeugend und widerspruchsfrei möglich. Gleichwohl bleibt ein Unbehagen an der [anthropo-]'zentrischen' Sicht. So sehr es einleuchtet, dass Konflikte zwischen dem Wohl der Natur und dem Wohl von Menschen nur im Rahmen der Anthropozentrik widerspruchsfrei zu lösen sind, so wenig leuchtet es ein, dass damit Menschen im Mittelpunkt der Betrachtung stehen, wie es der Begriff der Zentrik suggeriert. Geht es nicht, so möchte man fragen, vielmehr darum, herauszufinden, wo jeweils die Grenzen der Berücksichtigung von Mensch und Natur liegen? (...) Durch die 'zentrische` Einengung können Fragen danach, was denn genau menschliches Wohlergehen ausmacht und in wie vielfältiger Weise es von Biodiversität abhängt, nicht mehr angemessen in den Blick geraten.“ (ebd., 67-68) 
In diesem Sinne werden über die relationale Perspektive sowohl die physiozentrischen als auch die anthropozentrischen Ansätze kritisiert und es wird auf Basis einer anthropozentrischen Grundposition zum Inklusionsproblem versucht eine Vermittlungsposition zwischen den umwelt- und naturschutzethischen 'Lagern' zu finden, indem die Auslotung der gesellschaftlichen Naturverhältnisse sowie die eudaimonistische Frage nach dem guten Leben und dem Beitrag der Natur/Biodiversität zu diesem ins Zentrum der Aufmerksamkeit gerückt wird. Wenn Eser et al. folglich dazu aufrufen „ex-zentrisch zu denken“ (ebd., 67), dann geht es auf der grundlegenden begrifflichen Ebene um die Auslotung der Relationen zwischen Mensch, Natur und Gesellschaft und auf der umweltsoziologischen Ebene um die Klärung der Frage, auf welcher beziehungsmäßigen Grundlage zur Natur/Biodiversität Menschen agieren, die eine relationale Perspektive einnehmen (4.3.1.1). Damit korrespondiert wiederum, dass es auch auf der ethiktheoretischen Ebene um die Beantwortung der Frage geht, ob und inwiefern die Natur im Allgemeinen und die Biodiversität im Speziellen einen besonderen, glückskonstitutiven Beitrag zum guten Leben liefern und wenn dies der Fall ist, was daraus in normativer Hinsicht für die Bewahrung und Förderung der Biodiversität folgt (4.3.1.2). Alle drei Ebenen und die damit verknüpften Fragen sind zugleich wichtig für das Verständnis der relationalen Perspektive, so dass diese im Folgenden expliziert werden.

\subsubsection{Die Relationen zwischen Mensch, Natur und Gesellschaft und die wesensmäßige Naturbeziehung in der relationalen Perspektive}

Auf der begrifflichen Ebene setzt die relationale Perspektive mit ihrem Fokus auf die gesellschaftlichen Naturverhältnisse zum 'Schulterschluss' mit der Sozialen Ökologie an, so dass an dieser Stelle auch auf bestimmte begriffliche und methodische Operationen, die die Soziale Ökologie vornimmt, verwiesen werden kann (siehe 1.2.1):

„Gesellschaftliche Naturverhältnisse zu denken heißt, methodisch in Relationen statt in Substanzen zu denken, auf Differenzen statt auf Identität zu achten, Prozesse statt Strukturen zu untersuchen." (Becker \& Jahn 2006:26)

Zum Objekt des Wissens, der Bewertung und Beurteilung werden damit vor allem die Interaktionsmuster zwischen Mensch, Natur und Gesellschaft, die sich wiederum im Kontext kulturell und historisch geprägter Unterscheidungen bewegen. Dies ist der Fall, weil die drei, für die Theorie der Naturverhältnisse zentralen Begriffe Natur, Gesellschaft und Mensch seit jeher eine komplizierte und aufeinander bezogene Begriffsgeschichte haben, die an spezifische historische Konstellationen gebunden ist (Becker 2006:35): Die Bedeutung von Natur hat sich zwar seit der Antike immer wieder verändert, sie wird aber bis heute fast durchgängig als unbestimmter Begriff für das Andere von Kultur und Gesellschaft gebraucht. Gesellschaft hingegen ist als Begriff erst mit der Moderne entstanden und ist ein genuin politischer Begriff, der zumeist synonym ge- 
setzt wird mit ganz unterschiedlich gelagerten und eingebetteten Begriffen, wie der sozialen Sphäre, mit der Gesamtheit aller Bürger in einem Staat, einer sozialen Gemeinschaft oder dem Aggregat aller Individuen zu einem kollektiven Ganzen. Hierüber wird Natur begrifflich 'entpolitisiert', obgleich dieser, speziell auf dieser Ebene vorhandene Dualismus zwischen Natur und Gesellschaft über die Rede vom Menschen relationiert wird, denn was das Wesen des Menschen ist und wie Menschen sich verhalten, wird wechselseitig mit Bezug auf beide Begriffe, also Natur und Gesellschaft, festgemacht. In diesem Sinne werden unsere Vorstellungen von den menschlichen und gesellschaftlichen Beziehungen zur Natur durch unser Denken über das Verhältnis vom Mensch, Natur und Gesellschaft konstituiert, so dass die Beziehungen zwischen Menschen und ihren unterschiedlichen Umwelten sowie die Art und Weise ihres Handelns in Bezug auf diese Umwelten einen Einfluss auf gesellschaftliche Veränderungsprozesse des Natürlichen haben (vgl. Jahn \& Wehling 1998; Weingarten 1998; Schramm 1999; Eser \& Potthast 1999; Potthast 1999; Becker \& Jahn 2003, 2006; Becker, Hummel \& Jahn 2011; Eser 2014).

Hierüber taucht die alte philosophische Frage nach dem Weltverhältnis in einem 'neuem Gewand 'wieder auf, weil es hierbei um die aufeinander bezogenen Probleme der gesellschaftlichen Handlungskoordination, der individuellen Lebensführung und der ökologischen Zusammenhänge geht (Potthast 2000:58). Dadurch wird das, in der Umwelt- und Naturschutzethik hintergründig immer mitlaufende Gegensatzpaar 'Mensch oder Natur' sowohl begrifflich als auch konzeptionell suspendiert, indem die Perspektive auf die Relationen, Differenzen und Prozesse zwischen Mensch und Natur und zugleich auf die zutiefst gesellschaftliche Verfasstheit sowohl menschlicher als auch außermenschlicher Naturen gelenkt wird (Potthast 2000:131): Menschen wird als Vernunftwesen eine Sonderstellung unter den natürlichen Entitäten eingeräumt, die sich aber nur darauf beschränkt, dass Menschen die einzigen Wesen sind, die ihr Verhalten und Handeln nicht bloß als das Ergebnis kausaler Gesetzmäßigkeiten erfahren, sondern diese auch beschreiben, bewerten und beurteilen können, so dass aus dieser 'beschränkten Sonderrolle' Fragen der individuellen und kollektiven Lebensführung des und der Menschen in Bezug auf die Natur/Biodiversität erwachsen. Darüber werden Menschen unbestreitbar als Teil der Natur angesehen und es wird berücksichtigt, dass Menschen von der Natur/Biodiversität in vielerlei Hinsicht und mehr oder weniger stark abhängig sind. Diese Abhängigkeit ist in einigen Fällen durchaus existenzieller $\mathrm{Na}-$ tur (bspw. im Falle indigener Bevölkerungsgruppen), in der Regel (gerade in den westlichen Industrienationen) ist diese 'Abhängigkeit' aber deutlich weniger greifbar und durch etwas gänzlich anderes über das Überleben hinausgehendes bestimmt. Hierbei geht es nicht um das Überleben sondern um die Möglichkeit der Gestaltung eines guten und gelingenden Lebens, wozu, so die Annahme, die auch der relationalen Perspek- 
tive unterliegt, es die Möglichkeiten geben sollte, dass Menschen zu einer nicht rein instrumentellen sondern wesensmäßigen Mensch-Naturbeziehung gelangen können (vgl. Eser, Benzing \& Müller 2013:Kap. 5). Dahinter steht die Annahme, dass jeder Mensch eine spezielle nicht-instrumentelle Form der Beziehung zur Natur/Biodiversität aufbauen kann, die wiederum seine Erfahrung mit sich und anderen Menschen beeinflusst und dass dies letztendlich einen wichtigen, weil glückskonstitutiven Beitrag für ihr gutes und gelingendes Leben leistet. Wie leicht zu sehen ist, hat dies zugleich psychologische als auch ethische Implikationen.

In Rückgriff auf die Beziehungsphilosophie von Martin Buber und dem Gegensatzpaar zwischen 'Ich-Es' als Raum der Erfahrung und 'Ich-Du' als Raum der Beziehung (vgl. Buber \& Casper 1995) ${ }^{118}$ unterscheiden Eser et al. zwischen gebräuchlichen Naturerfahrungen und wesensmäßigen Naturbeziehungen: Die gebräuchliche Naturerfahrung resultiert darin, dass man andere als von sich selbst getrennte Gegenüber, als Objekt der Erfahrung oder als Mittel zum Zweck betrachtet. Um ein Ich-Bewusstsein zu entwickeln bedarf es indes der dialogischen Relationalität der Beziehung zu Anderen, denn hierüber wird das Ich überhaupt erst konstituiert. Wer andere - und hierbei ist es egal, ob es sich dabei um menschliche oder nicht-menschliche Entitäten handelt - folglich nur „gebräuchlich“ erfährt, höhlt das Fundament aus, auf dem das Wesen der Beziehung und damit des Ichs basiert (Eser, Neureuther \& Müller 2011: 98-99). Daher wird die wesensmäßige Beziehung (zwischen Mensch und Natur) als konstituierender und identitätsstiftender Aspekt des Ichs verstanden. Denn das 'in Beziehung treten ' steht in einem untrennbaren Zusammenhang damit, dass Menschen sich selbst in ihrer Ich-Werdung und Ich-Identität erfahren und reflektieren können und eben dies bildet wiederum eine wichtige Grundlage dafür, diejenigen Fähigkeiten und Fertigkeiten auszuprägen, die ein gutes Leben ausmachen. Mit anderen Worten: Nur diejenigen, die eine solche wesensmäßige Beziehung erlernen und eingehen können, sind demnach in der Lage eine stabile Ich-Identität aufzubauen. Dies wiederum ist eine hinreichende, wenn auch keine ausreichende Bedingung dafür, dass Menschen sich die Frage stellen, was sie selbst und ihr Leben aus-macht, was sie für gut und schlecht bzw. richtig und falsch halten und was sie glücklich und zufrieden macht.

118 Buber hebt über den Gegensatz zwischen Ich-Es und Ich-Du den Wert und das Primat der Beziehung (Ich-Du) hervor: „Die Welt ist dem Menschen zwiefältig nach seiner zwiefältigen Haltung. Die Haltung des Menschen ist zwiefältig nach der Zwiefalt der Grundworte, die er sprechen kann. (...) Das eine Grundwort ist das Wortpaar Ich-Du. Das andere Grundwort ist das Wortpaar Ich-Es; wobei, ohne Änderung des Grundwortes, für Es auch eins der Worte Er und Sie eintreten kann. (...) Die Welt als Erfahrung gehört dem Grundwort Ich-Es zu. Das Grundwort Ich-Du stiftet die Welt der Beziehung. (...) Im Anfang ist die Beziehung. (...) Man suche den Sinn der Beziehung nicht zu entkräften: Beziehung ist Gegenseitigkeit. (...) Nur dadurch, dass das Du gegenwärtig wird, entsteht Gegenwart." (Buber \& Casper 1995:9-25) 
Wenn eine wesensmäßige Beziehung zu sich und anderen folglich als glückskonstitutiv, sprich als essentieller Bestandteil eines guten Lebens ausgewiesen werden kann, dann wäre es unter einem normativen Standpunkt ratsam, gut und richtig für sich selbst und andere die Haltung der Verbundenheit und Achtsamkeit ${ }^{119}$ anzunehmen, d.h., einen Lebensstil zu wählen, bei der die Verbundenheit und Achtsamkeit mit anderen Menschen, nicht-menschlichen Lebewesen sowie Dingen, Zuständen und Orten konstitutiv für die Identität der eigenen Person ist. Vor dem Hintergrund der bisherigen Ausführungen ist allerdings zu berücksichtigen, dass es hier nicht um eine internalisierte moralische Pflicht geht, die zur (Selbst-)Verpflichtung wird, weil diese aus dem (einzigen) moralischen Motiv erwächst, nämlich das zu tun, was richtig ist. Anders als im kanntisch-pflichtenethischen Kontext muss die wesensmäßige Naturbeziehung als eine $\mathrm{Hal}-$ tung im tugendethischen Sinne verstanden werden, bei der es um bestimmte Dispositionen von Personen geht, die diese dazu motivieren sich seinen Überzeugungen entsprechend zu verhalten (Wils 2006:534). In diesem Sinne geht es gerade nicht um eine allgemeine, verbindliche Pflicht anderen gegenüber, denn die Haltung der Verbundenheit und das Motiv der Achtsamkeit müssen hierbei als moralische Motive im uneigentlichen Sinn bzw. als Primärtugenden verstanden werden, die keine „in der festen Gesinnung begründete Übereinstimmung des Willens mit der Pflicht" (Kant (1797) 2013: $\mathrm{VI}$ ) begründen. Mit anderen Worten: Menschen können nicht darauf verpflichtet bzw. gezwungen werden, solche Haltungen und Tugenden einzunehmen. Vielmehr ergibt sich die wesensmäßige Beziehung zu sich und anderen aus einer freiwilligen Haltung heraus, die aber erlernt, eingeübt und weitergegeben werden kann. In diesem Sinne betonen auch Eser et al., dass die wesensmäßige Naturbeziehung „weder naturgegeben noch notwendig [ist], sondern eine freie Entscheidung des Einzelnen." (Eser, Benzing \& Müller 2013:100)

\subsubsection{Die Relevanz eudaimonistischer Ansätze für die relationale Umwelt- und Naturschutzethik und die Bedeutung der Biodiversität für das gute Leben}

Indem über die relationale Perspektive die Bedeutsamkeit wesensmäßiger Naturbeziehungen verdeutlicht wird und diese wiederum eine eudaimonistische Argumentation der normativen Relevanz der Natur/Biodiversität nach sich zieht, bedeutet dies, dass die komplexen Zusammenhänge zwischen dem guten Leben von Menschen (jetzt und in Zukunft) und dem Beitrag der Natur/Biodiversität zum menschlichen Wohlergehen geklärt werden müssen. Daher führen Eser et al. den Bezug auf das menschliche Wohl-

\footnotetext{
${ }^{119}$ Diese Haltung der Verbundenheit und Achtsamkeit ist in ihren vielen Facetten bspw. in den buddhistischen Philosophien dargelegt, die den „Weg der Achtsamkeit“ (Satipatthana) als eins ihrer höchsten Ziele ausweisen. Auf diese soll und kann nicht näher eingegangen werden. Wichtiger sind hier die ethiktheoretischen Implikationen einer solchen Haltung der Verbundenheit und Achtsamkeit.
} 
ergehen und die, über Nützlichkeitsaspekte hinausgehende Betrachtung von Natur/ Biodiversität an, um die Relevanz eudaimonistischer Ansätze für die relationale Umwelt- und Naturschutzethik zu verdeutlichen: ${ }^{120}$

„Insofern könnte die Begründung für die moralische Berücksichtigung der Natur gerade darin gesucht werden, (...) dass menschliches Leben nur dann gelingt, wenn nicht eine der beiden Seiten die andere völlig unterwirft. Im Begriff des gelingenden menschlichen Lebens ist einerseits der Bezug auf menschliches Wohlergehen gewahrt, auf den die Anthropozentrik zu Recht insistiert. Gleichzeitig ist der kommunikative Raum eröffnet, was Natur für Menschen - jenseits ihrer Nützlichkeit - bedeutet." (Eser, Neureuther \& Müller 2011:69)

Eser et al. lassen diese Bezüge weitestgehend offen, da sie sich im Rahmen ihrer Studie nicht um deren Spezifizierung bemühen (können). Dies leisten jedoch andere Schriften, die den Schutz der Natur und Umwelt bzw. die Erhaltung der Biodiversität vor dem Hintergrund der Sicherung minimaler Voraussetzungen eines guten Lebens für jetzige und zukünftige Generationen zu begründen versuchen. Aurelié Halsband hat dies mit speziellem Fokus auf die Biodiversität expliziert: Anhand von Martha Nussbaums Fähigkeitenansatz zeigt sie auf, dass der Natur/Biodiversität (a) hinsichtlich der Sicherung existenzieller Grundbedürfnisse, (b) als inspirierende und erholsame Naturerfahrung, (c) als Erfahrung der Verbundenheit mit sich und anderen und (d) als Möglichkeit zur Entwicklung und Reflexion eines eigenen Lebensplans eine in sich wertvolle und stellenweise auch unsubstituierbare Relevanz für das gute Leben von Einzelnen zukommt (vgl. Halsband 2016:Teil II, Kap. 3). ${ }^{121}$ Halsband argumentiert, dass diese Möglichkeiten

${ }^{120}$ Eudaimonie ist ein Begriff der antiken Philosophie. Hier bezeichnete er eine gelungene Lebensführung (siehe FN 96), in heutigen Kontexten wird er auch oft mit gutem Leben, Glück oder Glückseligkeit übersetzt. In den antiken Philosophien galt, dass man ein gelungenes Leben nicht durch 'äußere' Faktoren erreichen kann, sondern es in sich selbst findet, indem man sich richtig verhält. Benötigt und erarbeitet wurden daher Regeln für eine Lebensweise, die Eudaimonie ermöglichen sollte. Was dies auf der axiologischen Ebene in Bezuf auf die eudaimonistischen Eigenwerte bedeutet, wurde bereits ausgeführt (siehe 4.2.2.1.2). Auf diese Ausführungen wird im Folgenden, wenn es um die ethiktheoretischen Implikationen eudaimonistischer Positionen geht, zurückgegriffen.

${ }^{121}$ Aurélie Halsband konzipiert verschiedene Schwellen bzw. Stufen des guten Lebens, anhand derer sie wiederum im Hinblick auf zukünftige Generationen die schwierigen Fragen beantwortet, warum Gegenwärtige gegenüber Zukünftigen überhaupt eine Verpflichtung bezüglich der Sicherung ihrer minimalen Voraussetzungen des guten Lebens haben sollten, wie sich intragenerationelle und intergenerationelle Pflichten und die damit verbundenen Konzeptionen der Gerechtigkeit in Konfliktfällen zueinander verhalten, wie diese Pflichten der Gerechtigkeit im Hinblick auf den Schutz der Biodiversität inhaltlich bestimmt werden können und wie bestimmt werden kann, wie weit die Pflichten reichen, die Gegenwärtige gegenüber Zukünftigen haben sollten (Halsband 2016:Teil III). Dazu nimmt sie vorab eine Kategorisierung der Nussbaumschen Fähigkeitenliste vor, die im folgenden genutzt werden kann: Die, der ersten Kategorie der Sicherung der Grundbedürfnisse (a) zugeordneten Fähigkeiten (Fähigkeiten 1,2 und 3) sind von essentieller Bedeutung, weil sie im Gegensatz zu den anderen Fähigkeiten „für jede Konzeption des Guten eine notwendige Voraussetzung darstellen.“ (ebd., 5) Die, den anderen drei Aspekten ( $c$, b und d) zugeordneten Fähigkeiten (Fähigkeiten 4 bis 9) sind demgegenüber subordinativ, da es hierbei um - im weitesten Sinne - ästhetische und damit für das Über- 
für alle erhalten und bereitgestellt werden sollten, auch wenn nicht alle Menschen diese Möglichkeiten tatsächlich nutzen wollen und werden. Eben diese Bezüge und die damit verbundenen Argumente für die Erhaltung der Biodiversität werden im Folgenden, indes lediglich pointiert, dargelegt, um zu zeigen, welch binnenkomplexe Argumente mit dem Verweis auf das „gelingende menschliche Leben“ einhergehen:

a) Bezüglich der Relevanz der Biodiversität zur Sicherung existenzieller Grundbedürfnisse kann grundlegend auf die Ökosystemfunktionen und ihre Relevanz für das Überleben aller Menschen verwiesen werden (vgl. Neßhöver et al. 2007). Hierbei muss indes spezifiziert werden, inwiefern und inwieweit die Biodiversität einen entscheidenden Beitrag für die Funktionsfähigkeit der Ökosysteme leistet. In diesem Sinne kann zwar darauf verwiesen werden, dass natürliche Ressourcen wie bspw. Wasser, Sauerstoff, Nahrung, Böden, Baurohstoffe, Arzneimittelpflanzen etc. elementar zur Befriedigung menschlicher Grundbedürfnisse beitragen, es bleibt aber zu klären, inwiefern diese Ökosystemleistungen und ihr Beitrag zur Sicherung existenzieller Grundbedürfnisse wiederum von der Erhaltung der Biodiversität abhängen (Halsband 2016:3-4). Vor dem Hintergrund, dass es immer (nur) um ganz bestimmte Funktionen geht, um die, mit der Grundbedürfnissicherung verbundenen Fähigkeiten ${ }^{122}$ zu ermöglichen und dass es zur Aufrechterhaltung dieser wiederum nur ganz bestimmter Bereiche der Biodiversität bedarf, lässt sich über den Verweis auf die existenziellen Grundbedürfnisse des Menschen argumentieren, dass die Biodiversität so umfassend geschützt werden soll, dass alle Menschen in die Lage versetzt werden, die natürlichen Ressourcen zur Grundbedürfnisbefriedigung (nachhaltig!) zu nutzen. Darüber hinaus muss das Erhaltungsmaß der Biodiversität so groß sein, dass überhaupt die Möglichkeit besteht, dass alle Menschen Zugang zu denjenigen natürlichen Ressourcen haben, die ihre Grundbedürfnisse abdecken. Falls dies nicht der Fall ist, müssen diese gerecht (um-)verteilt werden können. Zieht man dabei in Betracht, dass es bislang keine gesicherten Erkenntnisse darüber gibt, welche Bereiche, Aspekte und Funktionszusammenhänge der Natur/Biodiversität für die Bereitstellung der grundbedürfnissichernden Ökosystemleistungen erforderlich sind (und eine solche Angabe voraussichtlich auch nie exakt gemacht wer-

leben nicht essentiell notwendige, aber für das gute Leben entscheidende Aspekte von Natur/Biodiversität geht.

122 Halsband ordnet der Grundbedürfnissicherung die Fähigkeiten 1 bis 3 zu (siehe vorherige FN), da diese die grundlegenden und existenziell notwendigen Voraussetzungen erfassen, um Menschen die Möglichkeit eines guten Lebens zu eröffnen (Halsband 2016:1): „1. Leben: Fähig zu sein, bis zum Ende eines menschlichen Lebens normaler Länge zu leben; nicht vorzeitig zu sterben oder bevor das Leben so reduziert ist, dass es nicht mehr lebenswert ist. 2. Körperliche Gesundheit: Fähig zu sein, eine gute Gesundheit zu haben, einschließlich der reproduktiven Gesundheit; angemessen ernährt zu sein; angemessene Unterkunft zu haben. 3. Körperliche Unversehrtheit: Fähig zu sein, sich frei von einem Ort zum anderen zu bewegen; geschützt zu sein vor gewalttätigen Angriffen, sexuelle und häusliche Gewalt eingeschlossen; Möglichkeiten zu haben zu sexueller Befriedigung und zur Wahl in Fragen der Fortpflanzung." (Nussbaum 2003:19-20) 
den kann), dann kann - über diesen Umweg - ein nahezu umfassendes Erhaltungsmaß argumentiert werden. Paradoxerweise hängt das Erhaltungsmaß dann aber davon ab, dass neue Erkenntnisse eher eine Verringerung denn eine Erhöhung der Schutzreichweite begründen könnten. Zudem hängt die Einschätzung, wieviel Schutz notwendig und geboten ist, immer auch davon ab, wie risikofreudig bzw. risikoavers die jeweiligen Entscheidungsträger sind (ebd., 4).

b) Bezüglich der Relevanz der Biodiversität für inspirierende und erholsame Naturerfahrungen lässt sich anführen, dass die Begegnung mit Natur/Biodiversität den Gebrauch der Sinne, Phantasie und Argumentationsfähigkeit unterstützt, dass sie die Erfahrung von ästhetischen Erlebnissen befördert und angenehme und erholsame Erfahrungen ermöglicht (ebd., 6). ${ }^{123}$ Hier verweist Halsband auf das Aisthesis-Argument, das auf der Annahme beruht, dass die Begegnung mit Natur eine Quelle von vielfältigen, sowohl positiven als auch negativen, sinnlichen und emotionalen Eindrücken ist (ebd., 7). ${ }^{124}$ Die Besonderheit dieser sinnlich-emotionalen Naturerfahrung liege erstens in der Vielzahl und zweitens in der Intensität an Gefühlen, die sie auslösen, was sich wiederum positiv auf die Fähigkeiten auswirken soll „seine Sinne und seine Phantasie zu gebrauchen, zu denken und zu argumentieren." (Nussbaum 2003:20) Allerdings gilt es auch hier zu spezifizieren, ob und inwiefern die Natur und speziell die Biodiversität dies auf eine besondere Weise ermöglicht (Halsband 2016:7). Halsband zeigt anhand ihrer Ausführungen, dass die vermuteten Zusammenhänge zwischen der ästhetischen Kontemplation und den dadurch hervorgerufenen positiven Emotionen und Sinneseindrücken bisher nur unzureichend nachgewiesen werden konnten, zugleich betont sie aber, dass es nicht von der Hand zu weisen sei, dass die Natur, vor allem wenn sie biodivers ist, sich im Besonderen als Projektionsfläche für simultane Sinneseindrücke und starke emotionale Reaktionen eignet (ebd., 7-8). In diesem Sinne sind es gerade die Aspekte der Vielfältigkeit und Variabilität, die eben jene Simultanität und Intensität der Eindrücke maßgeblich befördern (ebd., 16).

${ }^{123}$ Halsband fokussiert hinsichtlich der inspirierenden und erholsamen Naturerfahrungen die Fähigkeiten 4 und 9 (Halsband 2016:6): „4. Wahrnehmung, Vorstellungskraft und Denkvermögen: Fähig zu sein, seine Sinne und seine Phantasie zu gebrauchen, zu denken und zu argumentieren und diese Dinge auf eine , wirklich menschliche' Art und Weise zu tun, geschult und kultiviert durch eine angemessene Erziehung, eingeschlossen, aber keinesfalls beschränkt auf die Fähigkeit, lesen und schreiben zu können sowie grundlegende mathematische und wissenschaftliche Kenntnisse. Fähig zu sein, Vorstellungskraft und Denkvermögen zum Erleben und Produzieren von religiösen, literarischen, musikalischen u.ä. Werken und Ereignissen eigener Wahl einzusetzen. Fähig zu sein, seinen Verstand in einer Weise einzusetzen, die durch Garantien der politischen und künstlerischen Meinungsfreiheit und die Religionsfreiheit geschützt ist. Fähig zu sein, angenehme Erfahrungen zu machen und unnötigen Schmerz zu vermeiden. (...) 9. Spiel: Fähig zu sein, zu lachen, zu spielen und erholsame Tätigkeiten zu genießen." (Nussbaum 2003:20-21)

${ }^{124}$ Die emotionalen Bezüge zur Natur/Biodiversität wurden bereits im Kontext der Reflexion auf die eudaimonistischen Eigenwerte ausgeführt (siehe 4.2.2.1.2). Hierbei wurde dargelegt, dass es vornehmlich die Praxen des Wahrnehmens, erfahrens und Erlebens sind, die hierbei als eigenwertig ausgewiesen werden können, nicht aber die Natur/Biodiversität selbst. 
Analog dazu verfährt Halsband in Bezug auf die Fähigkeiten zu Denken und zu Argumentieren: Auch hier sind es die Eigenschaften der Variabilität und Vielfältigkeit, die kombiniert mit der Eigenschaft des 'Nicht vom Menschen gemacht sein', die Fähigkeiten zu Denken und zu Argumentieren gerade dadurch stimulieren und anregen, dass die Erfahrungen aus der Begegnung mit der Natur (auch schon lange bevor die schulische Bildung einsetzt), ein unerschöpfliches Repertoire dafür bietet, die spielerischen, imaginativen, sprachlichen, intellektuellen aber auch die handwerklichen, künstlerischen und technischen Fertigkeiten auszubilden und zu befördern (ebd., 10-13). In diesem Sinne fungiert Natur in ihrer Vielfältigkeit, Veränderlichkeit und Andersartigkeit als wichtiger und zumindest im Ganzen auch unsubstituierbarer Gegenstand wissenschaftlicher Erklärungen und technischer Innovationen, handwerklicher und künstlerischer Schöpfungen sowie als Inspirationsquelle von Imaginationen.

Im Hinblick auf die Fähigkeiten sich zu erholen und angenehme Erfahrungen zu machen, legt Halsband anhand der Natur als „Raum der Kontemplation“ sowie als „Ort der Korrespondenz" dar, ob und inwiefern die Begegnung mit biodiverser Natur eine für das gute Leben relevante Erfahrung ist (ebd., 14-16). Dabei stellt sie heraus, dass wenn Natur als Ganzes kontempliert wird, dann setzt dies die Distanz des Betrachtenden von seinem Gegenstand voraus (ebd., 14). Gerade dieses 'Abstand von sich selbst Nehmen`ermögliche die Erfahrung der Erholung und Befreiung vom Alltag und von der uns umgebenden Zwängen und Restriktionen. Letzteres ist wiederum entscheidend, wenn Natur als Ort der Korrespondenz erfahren wird (ebd., 15). Natur ist dabei ein „sinnfremder" Raum, der abseits von allen gesellschaftlichen Konventionen und Sinnzuschreibungen liegt, so dass es in der Natur zu einer „befreienden Unterbrechung der Teilnahme am Leben unter den Menschen“" kommt (Seel 1991:315 in Halsband 2016: 15). Die Tendenz vieler, vor allem erwachsener Menschen, sich bevorzugt in der Natur zu erholen und die Selbstvergessenheit, vor allem vieler Kinder, beim Spielen in der Natur, können dabei als Indizien für die Möglichkeit interpretiert werden, dass kontemplative und korrespondierende Erfahrungen mit Natur/Biodiversität wichtige Aspekte des guten Lebens sind.

c) Bezüglich der Relevanz der Biodiversität für die Erfahrung der Verbundenheit mit sich und anderen lässt sich wiederum anhand von Halsbands Ausführungen aufzeigen, dass sich die Fähigkeiten zu sozialer Zugehörigkeit, d.h. zu Interaktion, Freundschaft, Anteilnahme und gegenseitigem Respekt (Fähigkeit 7) und die damit verbundenen Emotionen der Liebe, Anteilnahme, Trauer, Wut, Sehnsucht und Dankbarkeit (Fähigkeit 5), auch in der Begegnung mit Natur/Biodiversität eröffnen (Fähigkeit 8). ${ }^{125}$ Halsbands

125 Der Möglichkeit der Erfahrung der Verbundenheit mit sich und anderen ordnet Halsband die Fähigkeiten 8, 5 und 7 zu (Halsband 2016:18): "8. Andere Arten: Fähig zu sein, mit Rücksicht auf und in Beziehung zu Tieren, Pflanzen und der Natur zu leben.“ (...) 5. Gefühle: Fähig zu sein, Beziehungen zu 
These dabei ist, dass nicht nur die Verbundenheit zwischen Menschen sondern auch die Verortung des Menschen in der Welt des Natürlichen alle drei Fähigkeiten durchädringt (ebd., 18). Allerdings kann diese Verortung sehr unterschiedliche Formen annehmen, die von der bloßen Zugehörigkeit zur Gruppe der Lebewesen bis hin zu einer 'im Einklang mit der Natur' stehenden Lebensweise reichen kann (ebd., 20-22). Daraus schlussfolgert sie, dass sich über dem Verweis auf die Verbundenheit und die Erfahrungen der Zugehörigkeit zur Natur keine direkte Begründung für die Erhaltung der Biodiversität ergibt (ebd., 22), da alle vorstellbaren naturnahen Lebensweisen, individuellen Identifikationen mit einer Landschaft als Heimat sowie Wertschätzungen eines Naturgegenstandes als Teil des kulturellen Erbes primär das Ergebnis biographischer Ereignisse sind (ebd., 21). Schützenswert ist daher nicht in erster Linie die Natur/Biodiversität selbst, sondern die Möglichkeiten zu bestimmten Formen identitätsbildener und -beeinflussender Beziehungen zur Natur/Biodiversität (siehe 4.3.1.1). Hieraus lässt sich, so Halsband, zumindest die allgemeine Forderung ableiten, „für Menschen Möglichkeiten der Rücksichtnahme und der Beziehung zu Tieren, Pflanzen und der Natur als Ganze offen zu halten.“ (ebd., 19) Aber nicht nur die Zugehörigkeit und Verbundenheit mit der Natur/Biodiversität, sondern auch das Gefühl der Begrenztheit durch die Natur sowie die Erfahrung und Wahrnehmung der Andersartigkeit des Menschen von anderen Lebewesen sind in dieser Hinsicht relevant (ebd. 22-26). Diesbezüglich verweist Halsband erneut auf Martin Seel und dessen Annahmen bezüglich der Natur als Raum der Kontemplation und der Korrespondenz (ebd., 24). In diesem Kontext unterstreicht sie, dass die Begegnung mit der Natur immer zugleich ein Gefühl der Zugehörigkeit und der Begrenzung vermittelt, woraus wiederum die Erfahrung von Natur als einem zugleich sinngebenden und sinnfreien Raum erwächst, zu dem Menschen als Lebewesen zugehörig sind und von dem sie sich zugleich mittels sozialer (und moralischer) Normen abgrenzen (ebd., 30).

d) Bezüglich der Relevanz der Biodiversität für die Entwicklung und Reflexion eines eigenen Lebensplans führt Halsband aus, dass die Fähigkeit der Menschen ihr Leben aktiv zu gestalten, die Möglichkeit der Wahl zwischen unterschiedlichen Optionen, d.h.,

anderen Personen und zu Dingen einzugehen; diejenigen zu lieben, die uns lieben und für uns sorgen, über ihre Abwesenheit traurig zu sein; ganz allgemein zu lieben, zu trauern, Sehnsucht, Dankbarkeit und gerechtfertigten Ärger zu verspüren. Und dass die emotionale Entwicklung nicht durch Angst und Schrecken verhindert wird. (...) 7. Soziale Zugehörigkeit: (a) Fähig zu sein, mit anderen zusammenzuleben, an ihrem Leben Anteil zu nehmen, sich auf verschiedene Formen sozialer Interaktion einzulassen; fähig zu sein, sich in die Situation anderer hineinzudenken und mitzufühlen; die Fähigkeit zu Gerechtigkeit und Freundschaft zu haben. (...) (b) Die sozialen Grundlagen der Selbstachtung und der Nicht-Demütigung zu haben; fähig zu sein, als ein würdevolles Wesen behandelt zu werden, dessen Wert mit dem anderer gleich ist. Dies umfasst den Schutz vor Diskriminierung aufgrund von Rasse, Geschlecht, sexueller Orientierung, Religion, Kaste, ethnischer Abstammung oder nationaler Herkunft." (Nussbaum 2003:20-21) 
die Befähigung zur praktischen Vernunft voraussetzt. ${ }^{126}$ Auch hier verweist Halsband auf Seel und dessen Konzeption des Guten in der ästhetischen Begegnung mit Natur, wobei sie herausstellt, dass Natur bei Seel erstens ein „Ort der beglückenden Instanz zum tätigen Handeln“, zweitens die „korrespondierende Vergegenwärtigung der eigenen Lebenssituation" und drittens die "imaginative Deutung des Seins der Welt" ist (ebd., 31-32). Hierüber entsteht zu den je individuellen Vorstellung des guten Lebens und dem Beitrag der Natur/Biodiversität zu diesen (ebd. 32): Über die erste Dimension (Natur als Raum der Kontemplation) entsteht die Möglichkeit Abstand zu nehmen von der Sinnzuschreibung im Alltag und frei von sozialen Konventionen über die eigene Konzeption des Guten nachzudenken (ebd.). In der zweiten Dimension (Natur als Ort der Korrespondenz) erfolgt die Entwicklung einer Vorstellung des Guten über die Erfahrung der Verbundenheit mit der Natur und der Begrenzung durch die Natur (siehe c). Natur wird darin als etwas wahrgenommen, das einerseits sinngebend und andererseits sinnfrei ist, so dass die individuellen Vorstellungen des Guten sich gerade dadurch schärfen, dass wir uns in unserer Gleich- und Andersartigkeit zu anderen Lebewesen und Dingen wahrnehmen (ebd.). In der dritten Dimension (Natur als imaginierter Ort) ist die Natur Inspirationsquelle für unterschiedliche Vorstellungen des Guten, wodurch die Betrachtenden Anregungen für mögliche Inhalte des Guten, wie z.B. das Harmonische, das Starke oder das Komplexe finden (ebd.). In dieser dreidimensionalen Begegnung mit Natur/Biodiversität, so argumentiert Halsband in Rückgriff auf Martin Seel, eröffnen sich unersetzliche Möglichkeiten, eine individuelle Vorstellung des guten Lebens zu entwickeln und zu reflektieren (ebd., 33). In diesem Sinne erwächst aus der Nussbaumschen Zielstellung, für alle Menschen die grundlegenden Voraussetzungen eines guten Lebens bereitzuhalten, die Forderung, die dazu erforderliche Natur/Biodiversität zu erhalten, weil „die Vernichtung des Naturschönen (...) eine Liquidation der nicht-instrumentellen Beziehung zur lebensweltlichen Natur“, eine „Zerstörung positiver Kontingenz, ästhetischer Differenz, realer Freiheit und erfüllter Zeit" sowie eine „Beschneidung nichtrelativer Lebensmöglichkeiten und des Bewusstseins der Möglichkeit gelingenden Lebens" wäre (Seel 1991:343 in Halsband 2016:34).

Zusammenfassend zeigt sich anhand dieser Ausführungen, dass es gute Argumente dafür gibt, sich auf eine eudaimonistische Argumentation der Erhaltung der Biodiversität auf der Grundlage einer relationalen Position einzulassen, denn hierüber können zahlreiche Motivationen, Intuitionen, Gründe und Begründungen für die Erhaltung der Biodiversität fokussiert werden, die bislang zu wenig beachtet wurden (siehe 4.2.1). Dies trifft im Kern bestimmte im umwelt- und naturschutzethischen Kontext moralrelevante

${ }^{126}$ Der Möglichkeit zur Entwicklung und Reflexion eines eigenen Lebensplans ordnet Halsband die 6. Fähigkeit zu (Halsband 2016:31): „6. Praktische Vernunft: Fähig zu sein, eine Vorstellung des Guten zu entwickeln und sich auf kritische Überlegungen zur eigenen Lebensplanung einzulassen." (Nussbaum 2003:21) 
Motive, die als Liebe zur Natur, als Achtung vor dem Lebendigen, als Respekt für die Natur, als Anerkennung ihrer Erhabenheit und Schönheit, als Mitleid mit nicht-menschlichen Wesen etc. betitelt werden, denn wer die Natur liebt, sie achtet, sie als schön und erhaben empfindet, der neigt dazu, mit der Natur durch sich selbst in Verbindung zu treten, achtsam mit Naturgegenständen und natürlichen Entitäten umzugehen sie nicht als ein 'Es' zu erfahren, sondern wie ein 'Du'zu erleben. Dies rechtfertigt es die relationale Perspektive als progressive Problemverschiebung auf dem Weg hin zu einer anwendungsorientierten Umwelt- und Naturschutzethik zu verstehen. Dabei darf indes nicht übersehen werden, dass ein solcher Ansatz, so lohnend er in pragamtischer Hinsicht sein mag, immer auch zahlreiche binnenkomplexe Fragen nach sich zieht, die sich in Anschluss an eine solche relationale Perspektive auf der abstrakteren, ethiktheoretischen Ebene stellen und die expliziert werden müssen. Wenn es folglich um die eudaimonistische Begründung der moralischen Berücksichtigungswürdigkeit der Natur/Biodiversität geht, fallen darunter bspw. die Fragen, inwiefern die Haltungen der Achtsamkeit und der Verbundenheit mit der Natur/Biodiversität bereits mit bestimmten Gerechtigkeitsvorstellungen verknüpft sind und welchen Status diese hinsichtlich des guten Lebens jetziger und zukünftiger Generationen haben.

Selbst wenn diese binnenkomplexen Fragen zufriedenstellend beantwortet werden können - was nicht Gegenstand dieser Arbeit sein wird und sein kann - so ist damit noch nichts zu der Problematik gesagt, dass nahezu alle Begründungsansätze in der Umwelt- und Naturschutzethik - und davon sind die eudaimonistischen auch nicht ausgenommen - primär auf den Schutz der Natur/Biodiversität fokussieren, wodurch indes das innovative Moment der Bewahrung und Förderung der Biodiversität, nämlich die Integrativität der Zielstellungen, nicht in ausreichendem Maße berücksichtigt wird. Zudem wird über die relationale Perspektive mit ihrem Fokus auf die moralrelevanten Motive der Verbundenheit und Achtsamkeit sowie die Tugenden einer gelingenden Lebensführung das Problem noch einmal deutlicher, dass es in der Umwelt- und Naturschutzethik vornehmlich um Begründungen für Pflichten und Rechte in Ansehung bzw. gegenüber der Natur/Biodiversität geht. Das heißt, dass der deontische Fokus der Umwelt- und Naturschutzethik die Einsicht darauf versperrt, dass es bei der Bewahrung und Förderung der Biodiversität auch um andere Typen von Handlungen und Begründungen geht und gehen muss, die über die Rede von Pflichten und Rechte sowie über Schutzpflichtbegründungen nicht ausreichend abgedeckt sind. Dieser, aus zwei Aspekten zusammengesetzten, Detailproblematik (Abschnitt 2.4.2 sowie 4.3) kann nur ein speziell auf die Biodiversitätszieltrias ausgerichteter Argumentationsraum begegnen, der zugleich die Vielzahl an Argumenten für und prinzipiell auch gegen die Bewahrung und Förderung der Biodiversität nicht auf Pflichtbegründungen reduziert. 
Ein solcher, in allen drei Punkten geeigneter Ansatz, ist bereits von Uta Eser, AnnKathrin Neureuther und Albrecht Müller mit ihrer Studie »Klugheit, Glück, Gerechtigkeit - ethische Argumentationslinien in der Nationalen Strategie zur biologischen Vielfalt« (vgl. Eser, Neureuther \& Müller 2011) vorgelegt worden. Im Folgenden werden daher die konzeptionellen Neuerungen, aber auch die Probleme dieses Ansatzes bei der Begründung der Bewahrung und Förderung der Biodiversität herausgestellt.

\subsubsection{Der biodiversitätsethische Argumentationsraum zur Integration der Biodiversitätszieltrias und zur Überwindung des deontischen Fokus der Umwelt- und Naturschutzethik}

Ausgehend von der Grundfrage, welche guten Gründe angeführt werden können, um Menschen von der Wichtigkeit und Richtigkeit der Ziele und Maßnahmen zum Schutzes zur nachhaltigen Nutzung und zur gerechten Verteilung der Biodiversität zu überzeugen (Eser, Neureuther \& Müller 2011:9), fragen Eser, Neureuther und Müller zum einen „wie eine angemessene Kommunikation über Biodiversität aussehen könnte“ (ebd., 10), bei der es nicht nur um Gründe sondern auch um Motive für Umwelt- und Naturschutzanliegen geht und zum anderen wie aus einer ethischen Perspektive dafür argumentiert werden kann, dass die Biodiversität geschützt, die natürlichen Ressourcen nachhaltig genutzt und die Nutzen und Lasten aus der Erhaltung aber auch der Vernichtung der Biodiversität g-recht verteilt werden (ebd., 27). ${ }^{127}$ Um eben diese beiden Fragen nach dem 'wie' und dem 'warum' zu beantworten, werden die in der NBS genannten Gründe (siehe 4.2.1) von ihnen neu strukturiert und daraufhin ethisch reflektiert. Hierfür schlagen Eser et al. drei Argumentationstypen vor. Zur allgemeinen Bezeichnung dieser drei Argumentationstypen wählen sie die Begriffe Klugheit, Glück und Gerechtigkeit (ebd.), da ihnen diese sowohl zur Einholung des oben anvisierten Ziels einer „angemessenen Kommunikation“ sowie „zur Strukturierung der vorgefundenen Argumente geeignet scheint" (ebd., 12) als auch zur Ausweisung 'guter Gründe` für die Bewahrung und Förderung der Biodiversität:

Die Zuordnungen zu den Klugheits-, Glücks- und Gerechtigkeitsargumenten, die Eser et al. sowohl in ihrer Auftakt- als auch in ihrer Folgestudie vornehmen (vgl. Eser, Neu-

127 Die Auftaktstudie "Klugheit, Glück, Gerechtigkeit - ethische Argumentationslinien in der Nationalen Strategie zur biologischen Vielfalt« (vgl. Eser, Neureuther \& Müller 2011) sowie die darauf basierende Folgestudie zu »Gerechtigkeitsfragen im Naturschutz« (vgl. Eser, Benzing \& Müller 2013) resultieren aus einem vom BfN ausgeschriebenen Forschungsgutachten. Hierbei lag der Fokus auf der ethischen Reflexion der politischen Argumentationen für den Schutz der Biodiversität in den Strategiepapieren zur biologischen Vielfalt (NBS) und zum Klimawandel (DAS). Dabei ging es Ihnen darum, zur kommunikativen Bewältigung von Naturschutzkonflikten im Allgemeinen beizutragen, indem sie die „normativen Bezüge solcher Konflikte ausdrücklich adressieren.“ (Eser, Benzing \& Müller 2013:11) Insofern soll es hier nicht als Kritik an den Studien selbst verstanden werden, wenn im Folgenden insbesondere die theoretischen Grundlagen der von Ihnen vorgeschlagenen Argumentationstrias einer vertiefenden Untersuchung unterzogen werden. 
reuther \& Müller 2011; Eser, Benzing \& Müller 2013), können in Anlehnung an Konrad Otts Darlegung des umweltethischen Argumentationsraums (vgl. (Ott 2010:18) hier als biodiversitätsethischer Argumentationsraum verstanden werden. Dieser ist, auf Grundlage der drei Argumentationstypen, wie folgt ausgestaltet:

\section{Argumentationraum der Biodiversitätethik}

b. Klugheitsargumente (...weil es in unserem eigenen Interesse liegt)

1. Funktionsargumente

a. Biodiversität liefert Basisleistungen

b. Biodiversität liefert Regulationsleistungen

c. Biodiversität liefert Versorgungsleistungen

d. Biodiversität liefert kulturelle Leistungen

2. Vorsorgeargumente

a. es gibt nur unvollständige Kenntnisse bezüglich der ökologischen Funktionen

b. es bestehen Ungewissheiten bezüglich künftiger sozial-ökologischer Entwicklungen

b. Glücksargumente (...weil es zu unserem guten Leben beiträgt)

1. Argumente des Naturerlebens

a. Bedeutung der Biodiversität für die Gesundheit, die Erholung und das Wohlergehen von Menschen

b. Bedeutung speziell der Vielfalt für das Engagement von Menschen

im Umwelt- und Naturschutz

2. Argumente der Naturästethik

a. Naturschönheit

b. Selbsterfahrung durch Natur

3. Argumente der Naturbeziehung

a. wesensmäßige Verbundenheit mit Tieren, Pflanzen und der Natur im Allgemeinen

c. Gerechtigkeitsargumente (...weil es moralisch verpflichtend ist)

1. soziale Gerechtigkeitsargumente

a. Verteilung von Umweltlasten und Umweltnutzen innerhalb der Gesellschaft

b. Auswirkungen auf Arbeit, Einkommen, Bildung, Status etc.

2. globale Gerechtigkeitsargumente

a. Verteilung der Kosten und Gewinne aus dem Schutz und der Nutzung der

Biodiversität zwischen den Staaten

b. Auswirkungen auf transnationale Kooperationen, Transferleistungen,

Weltwirtschaftsordnung etc.

3. Zukunftsgerechtigkeitsargumente

a. Verteilung der Pflichten zur Erhaltung der Biodiversität und der Rechte auf

Biodiversität zwischen den Generationen

b. Auswirkungen auf allgemeine Handlungsoptionen sowie Zugänge zu Biodiversität

[4. ökologische Gerechtigkeitsargumente] ${ }^{128}$

128 Die ökologischen Gerechtigkeitsargumente werden bei Eser et al. nur dem Namen nach unter die Kategorie der Gerechtigkeitsargumente geornet, da es ihnen um die Auslotung dessen geht, „wie weit menschliche Bedürfnisse zur Begründung verstärkter Naturschutzmaßnahmen reichen, wenn man sie denn konsequent und umfassend berücksichtigt." (Eser, Benzing \& Müller 2013:35) In der ersten Studie führen sie gegen die ökologischen Gerechtigkeitsargumente ins Feld, dass diese aus Gründen der Widerspruchsfreiheit sowie aus Gründen ungewollter praktischer Konsequenzen für inakzeptabel als Gerechtigkeitsargumente erachtet werden (ebd., 66). 
Ohne die jeweiligen Argumente im Einzelnen reflektieren zu wollen und auch ohne, dass bereits auf die theoretischen Grundlagen der drei Argumentationstypen eingegangen wird (siehe 4.3.2.1 - 4.3.2.3), wird anhand des Argumentationsraumes bereits klar, dass es hier im Kern nicht nur darum geht, die umwelt- und naturschutzpolitischen Gründe neu zu strukturieren, sondern dass es innen auch und vor allem darum geht, die möglichen Antworten auf die Frage auszuweisen, warum die Biodiversität geschützt, nachhaltig genutzt und gerecht verteilt werden soll: Nach dem Typus Klugheit kann die Warum-Frage damit beantwortet werden, dass die Bewahrung und Förderung der Biodiversität in unserem eigenen Interesse liegt; dem Typus Glück zufolge ist die Bewahrung und Förderung der Biodiversität begründbar, indem Natur/Biodiversität als wesentliche und unsubstituierbare Bestandteile eines gelingenden menschlichen Lebens ausgewiesen werden und nach dem Typus Gerechtigkeit lässt sich die Warum-Frage beantworten, indem darauf verwiesen wird, dass wir dazu moralisch verpflichtet sind (Eser, Neureuther \& Müller 2011:27). ${ }^{129}$

Indem Eser et al. damit sowohl die gesamte Bandbreite an möglichen Argumenten einfangen, die im politischen und umwelt- und naturschutzethischen Kontext gebräuchlich sind als auch eine reflexive und differenzierte ethische Kommunikation über diese ermöglichen, ohne dabei die normativen Imprägnierungen all dieser Antworten zu vernachlässigen, selbst wenn diese sich selbst als 'nicht-normativ' ausweisen (siehe 2.3 sowie 4.2.1), ist es Eser et al. gelungen, einen für die Praxis des Umwelt- und Naturschutzes geeigneten und lebensnahen ethischen Argumentationsraum für die Bewahrung und Förderung der Biodiversität vorzulegen, denn Eser et al. haben in ihren Studien deutlich gemacht, welche Aspekte bei der Begründung der Bewahrung und Förderung einerseits mehr Beachtung finden müssen als dies bisher der Fall ist und andererseits machen sie deutlich, wie die Biodiversitätsziele und -maßnahmen überzeugend kommuniziert werden können und wie darüber zu einer argumentativen Schlichtung von Umwelt- und Naturschutzkonflikten beigetragen werden kann. Aufgrund dieser 'praktischen Verdienste', die ganz im Sinne der hier vorliegenden Arbeit sind, wird die Argumentationstrias aus Klugheit, Gerechtigkeit und Glück hier zur Grundlage der weiteren Ausführungen gemacht. Gerade deshalb muss hinsichtlich der Verwendung des Ansatzes von Eser et al. dem konzeptionellen Problem begegnet werden, dass die Differenzierungen zwischen Klugheit, Glück und Gerechtigkeit, die Eser et al. vornehmen, nicht so klar sind, wie dies den Anschein hat.

${ }^{129}$ Eser et al. verweisen darauf, dass die drei Begriffe Klugheit, Glück und Gerechtigkeit in einem bewusst alltagssprachlichen Verständnis gemeint sind, auch wenn sie in einer Fußnote darauf verweisen, dass die Dreiteilung der Systematik von prudentiellen (Klugheit), pflichtenethischen (Gerechtigkeit) und tugendethischen (Glück) Ansätzen entspricht (Eser, Neureuther \& Müller 2011:27). Dieser Zusammenhang wird an späterer Stelle noch einmal relevant, wenn es um die Integration des Argumentationsraumes in ein mehrdimensionales Ethikverständnis geht (siehe 4.3.2.3). 
Diese Problematik ergibt sich vor allem dadurch, dass sich die Konzeptualisierung des Argumentationsraumes auf zwei unterschiedlichen Ebenen bewegt. Zum einen auf der analytischen Ebene, auf der es Eser et al. darum geht, die Aussagen in der NBS danach umzustruktrieren und neu zu typologisieren, ob es sich bei Ihnen um Aussagen handelt, die eher dem Typus Klugheit, dem Typus Gerechtigkeit oder dem Typus Glück zuzuordnen und zum anderen auf der Begründungsebene, auf der es Eser et al. darum geht „gute Argumente für die Bewahrung und Förderung der Biodiversität" auszuweisen, „die Menschen von der Richtigkeit und Wichtigkeit dieser Aufgabe überzeugen" (ebd., 9). Das Problem besteht nun darin, dass Eser et al. nicht ausreichend zwischen diesen beiden Ebenen differenzieren und damit der Tendenz verfallen, dass sie Fragen, die sich auf der einen Ebene stellen mit Antworten auf der anderen Ebene zu beantworten und umgekehrt. Eben diese konzeptionelle Problematik wird im Folgenden erst einmal anhand konkreter Ausführungen dargelegt und expliziert (4.3.2.1). Dieser wird im Anschluss daran konstruktiv begegnet, indem die, immer nur implizit anklingenden, diskurstheoretischen Fundmente der Argumentationstrias rekonstruiert werden, da dies eine systematische Differenzierung der drei Argumentationstypen auf beiden Ebenen ermöglicht (4.3.2.2).

\subsubsection{Das konzeptionelle Problem des biodiversitätsethischen Argumentationsraumes}

Eser et al. versuchen mit ihrer Argumentationstrias aus Klugheit, Glück und Gerechtigkeit verschiedene Anliegen einzuholen. Auf der analytischen Ebene - quasi in Form einer Reflexion erster Ordnung - geht es ihnen um die Plausibilisierung der Annahme, dass eine explizite Kommunikation der Klugheits-, Gerechtigkeits- und Glücksaspekte zu einer vor allem im strategischen Sinne angemesseneren Kommunikation über die Bewahrung und Förderung der Biodiversität führt. Eben dieses Anliegen steht im Vordergrund der Studie und diesem wird von den AutorInnen systematisch nachgegangen. Weniger systematisch verfolgt zugleich aber genauso relevant für die Konzeptualisierung des Argumentationsraumes ist das Anliegen, im ethischen Sinne gute Argumente für die Bewahrung und Förderung der Biodiversität auszuweisen. Hierbei geht es nicht darum, Klugheits-, Glücks- und Gerechtigkeitsargumente voneinander zu unterscheiden und ihnen klassische umwelt- und naturschutzethische Argumente zuzuordnen, sondern hier geht es auf der Begründungsebene - also als Reflexion zweiter Ordnung darum zu plausibilisieren, warum die jeweils zugeordneten Argumente auch im ethischen Sinne gute Argumente sind. Wenn Eser et al. davon sprechen, dass die Argumente und Gründe für die Bewahrung und Förderung der Biodiversität 'gut' sind (ebd., 2024), dann tun sie dies vor dem Hintergrund der Differenzierung zwischen überzeugenden Argumenten im Sinne von rhetorisch erfolgreichen, d.h. überredenden, werbenden und auf die Herbeiführung bestimmter Handlungs- und Verhaltensweisen aus- 
gelegten strategischen Argumenten einerseits sowie sach- und wertangemessenen, argumentativ logischen, widerspruchsfreien und verständigungsorientierten ethischen Argumenten andererseits (ebd., 20-21). Der Standard guter Argumente bei Eser et al. ist dabei das Überzeugen im ethischen Sinn:

"Im Unterschied zu solcher Werbung gehen die Gutachter(inn)en und ihre Auftraggeber davon aus, dass es allgemein anerkennungswürdige, in diesem Sinne also gute Gründe gibt. (...) Diese Gründe in der Sache zu finden und zu benennen, ist Aufgabe des vorliegenden Ethikgutachtens." (ebd., 22)

An dieser Stelle ist nun wiederum entscheidend, dass die Aufgabenstellung, gute Gründe für die Bewahrung und Förderung der Biodiversität zu finden und zu benennen bereits voraussetzt, dass gewusst wird, welche Argumente allgemein anerkennungswürdig sind und warum. Aus diesem Grund führen Eser et al. relativ detailliert aus, was genau sie unter „allgemein anerkennungswürdig“ verstehen und wie sie prüfen wollen, was gute Gründe von schlechten Gründen unterscheidet: Erstens führen sie an, dass Argumente grundsätzlich nur dann überzeugen können, wenn sie nachvollziehbar und stichhaltig sind (ebd., 11). Dies ist sowohl in Bezug auf strategische als auch für ethische Argumente notwendig. Anders als indes bei strategischen Argumenten folgen ethische Argumente formalen Regeln (siehe 4.1), weil bei ethischen Argumenten versucht werden muss, die Prämissen der Argumente möglichst vollständig darzulegen, so dass die daraus gezogenen Konklusionen nachvollziehbar und diskutierbar werden (ebd., 16). Zweitens haben ethische Argumente immer auch inhaltliche Regeln, da es beim ethischen Argumentieren darum geht, die Richtigkeit der Prämissen und die Gültigkeit der Schlussfolgerungen auszuweisen (ebd., 20). Hierbei begnügen sich Eser et al. mit „bescheidenen Voraussetzungen“130 und beschränken sich auf zwei Mindestkriterien (ebd., 22-23): Das erste Kriterium ist das der „internen Widerspruchsfreiheit", weil Argumente nur dann überzeugend sind, wenn man sich innerhalb der Argumentation nicht selbst widerspricht. Als zweites Kriterium wird, ohne weitere Erläuterungen, die „Übereinstimmung mit anerkannten Praktiken und Prinzipien“ benannt.

${ }^{130}$ Hierbei verweisen sie, ohne auf den Aspekt der Gültigkeit der Schlussfolgerungen einzugehen (siehe hierzu 4.1), darauf, dass es in der Philosophie verschiedene Definitionen des Richtigen gibt (siehe FN 46) und dass hier von Richtigkeit unter der Voraussetzung gesprochen wird, dass das Richtige überhaupt erkennungsfähig ist (kognitivistische Position), dass Widerspruchsfreiheit ein notwendiges, wenn auch kein hinreichendes Kriterium des Richtigen ist (kohärentistische Position) und dass sich das, was richtig ist, über die intersubjektive Anerkennungswürdigkeit ergibt (konsenstheoretische Position). Relevant ist hierbei insbesondere das letzte Kriterium, denn damit stellen sich Eser et al. in den Kontext einer diskurstheoretischen und -ethischen Position (siehe FN 14). Dies 'schillert' an mehreren Stellen in den Studien auch immer wieder durch, ohne dass es indes theoretische Rückbezüge darauf gibt. Dieser fehlende Rückbezug macht sich vor allem anhand des Problems bemerkbar, dass bei Eser et al. nicht in Gänze klar wird, was Klugheit, Gerechtigkeit und Glück als Argumentationstypen einerseits und als gute Argumente andererseits ausmacht. Hier hätte der explizite Rückbezug auf Habermas' Explikation der Gebrauchsweisen der praktischen Vernunft (vgl. Habermas 1991a) mehr Klarheit gebracht. Dies wird im Laufe der Arbeit nachgeholt (siehe 4.3.2.2). 
Inwiefern das Kriterium selbst problematisch ist, muss hier offenbleiben. Entscheidend ist an dieser Stelle vielmehr, dass Eser et al. beide Kriterien einführen, um die Plausibilität von Gründen zu bestimmen, so dass diese Kriterien auch rekursiv darauf verwandt werden müssten, wenn herausgestellt werden soll, dass Klugheits-, Glücks- und Gerechtigkeitsargumente gute Argumente für die Bewahrung und Förderung der Biodiversität sind. Genau dies passiert allerdings bei Eser et al. nur ein einziges Mal und zwar, bezeichnenderweise, in Bezug auf die ökologischen Gerechtigkeitsargumente (ebd., 57-69). An dieser Stelle drängt sich daher der Verdacht auf, dass das, was im Rahmen der Studie mit 'guten Argumenten' eigentlich gemeint ist, unterhalb der ethischen Prüfung um die Richtigkeit der Prämissen liegt, da bei Eser et al. im Endeffekt (nur) deutlich gemacht wird, dass die vorgeschlagene Typlogisierung gegenüber der klassischen Aufteilung in ökologische, ökonomische, sozialkulturelle und ethische Gründe besser kommunizierbar ist, weil hierüber deutlich wird, welche sach- und wertbezogenen Aspekte üblicherweise aus der Diskussion um Biodiversität und dessen Bewahrung und Förderung ausgeklammert werden, die aber präzisiert und explizit berücksichtigt werden müssen, wenn es darum gehen soll, Menschen hinsichtlich praktischer Fragen und Problemstellungen ethisch zu orientieren und um konkrete Naturschutzkonflikte in all ihren Dimensionen zu adressieren.

Insofern zeigen Eser et al. zwar den Mehrwert der Dreiteilung von Klugheits-, Gerechtigkeits- und Glücksargumenten in kommunikativer Hinsicht und unter strategischen Gesichtspunkten auf, was definitiv eine lohnenswerte und wichtige Aufgabe angesichts der kommunikativen Schwierigkeiten der Umwelt- und Naturschutzethik mit anderen Bereichen der Wissenschaft und mit Praxisakteuren ist. Es bleibt aber im Laufe der gesamten Studie unklar, warum überhaupt davon ausgegangen werden kann, dass die von ihnen dargelegten Klugheits-, Gerechtigkeits- und Glücksargumente auch gute Argumente im ethischen Sinne sind, denn der Standard guter Argumente ist bei Eser et al. wie bereits gesagt das Überzeugen im ethischen Sinn. Wenn Eser et al. folglich das Ziel haben, gute Argumente für alle drei Biodiversitätsziele darzulegen und diese nicht nur im strategischen sondern auch im ethischen Sinne als überzeugend zu kennzeichnen, dann dürfen die Fragen, wie das Verhältnis der drei Argumentationstypen zueinander bestimmt wird und warum überhaupt davon ausgegangen werden kann, dass Klugheits-, Glücks- und Gerechtigkeitsargumente allesamt auch ethische Argumente sind, nicht ausgeblendet werden: ${ }^{131}$

${ }^{131}$ Der ersten Fragestellung bezüglich des Verhältnisses der drei Argumentationstypen zueinander wird über die Rekonstruktion der diskurstheoretischen Fundamente der Argumentationstrias nachgegangen (4.3.2.2), indem die pragmatischen (4.3.2.2.1), die eudaimonistischen (4.3.2.2.2) und die moralischen (4.3.2.2.3) Gebrauchweisen der praktischen Vernunft expliziert werden. Der Frage, ob Klugheits-, Glücks- und Gerechtigkeitsargumente allesamt auch ethische Argumente sind, wird aufbauend darauf nachgegangen, wenn es um die Darlegung des mehrdimensionalen Ethikverständnisses als Grundlage der gesamten Argumentationstrias geht (4.3.2.3). 
Bezüglich der ersten Frage, wie das Verhältnis der drei Argumentationstypen zueinander bestimmt wird, ist insbesondere das Verhältnis zwischen Klugheitsargumenten und Gerechtigkeitsargumenten bei Eser et al. näher zu beleuchten:

Auf der analytischen Ebene, wo es, wie bereits gesagt, um die Zuordnung der einzelnen Argumente zu den Argumentationstypen der Klugheit, des Glücks und der Gerechtigkeit geht, stellen Eser et al. heraus, dass unter die Kategorie der Gerechtigkeitsargumente all die Aussagen und Argumente fallen, in denen es um moralische Pflichten und Rechte geht. Da moralische Pflichten und Rechte ihrem Anspruch nach für alle Menschen gelten sollen, wird unter der Überschrift Gerechtigkeit folglich nach Gründen gesucht, warum die Maßnahmen zur Bewahrung und Förderung der Biodiversität Handlungen sein sollten, auf die man andere verpflichten kann (ebd., 36) oder die man von anderen zu recht abverlangen kann (ebd., 39). Hierunter verorten sie alle Bezüge, die in der NBS bezüglich intragenerationaler und intergenerationaler Pflichten und Rechte gemacht werden, aber auch die im umwelt- und naturschutzethischen Kontext so relevante Inklusionsfrage bezüglich der Begründung direkter Pflichten gegenüber der $\mathrm{Na}$ tur/Biodiversität. Unter die Kategorie der Klugheitsargumente fallen demgegenüber all diejenigen Aussagen in der NBS, die sich darauf beziehen, die Biodiversität im eigenen Interesse zu bewahren und zu fördern, weil diese eine Vielzahl an wichtigen ökosystemaren Funktionen und relevanten Leistungen für den Menschen bereitstellt. Insofern verorten sie unter den Typus der Klugheitsargumente sowohl die existenziellen Aussagen über Biodiversität (Angewiesenheitsargumente) als auch ein Großteil der damit verbundenen ökologischen und ökonomischen Aussagen. Damit fallen unter den Typus der Klugheit bei Eser et al. die Gründe, Argumente und Begründungen, die üblicherweise für die instrumentellen Werte der Biodiversität angebracht werden nur mit dem Unterschied, dass hier nicht gefragt wird, warum Biodiversität instrumentell wertvoll ist, sondern warum die Bewahrung und Förderung der Biodiversität zweckdienlich und nützlich sein sollte. Insofern basieren die Klugheitsargumente bereits auf den Annahmen, dass die Biodiversität zahlreiche instrumentelle Werte besitzt, deren Aufrechterhaltung wichtig für das Wohlergehen von vielen Menschen ist, so dass hier geschlussfolgert wird, dass es im Interesse aller und somit ein generelles Gebot der Klugheit bzw. ein Rationalitätsgebot ist, wenn jeder Mensch durch Schutz und nachhaltige Nutzung das zu erhalten versucht, was er zum Leben braucht. Daher steht im Zentrum der Klugheitsargumente die Handlungsaufforderung, im eigenen Interesse an der Nutzung der Funktionen und Leistungen der Biodiversität, diese zu bewahren und zu fördern, so dass Eser et al. mit den Klugheitsargumenten die praktische Lebensweisheit einfangen, nach der es 'dumm ist, den Ast abzusägen, auf dem man sitzt' (ebd., 28).

Über die bei Eser et al. erfolgte Reflexion der Debatte um die Ökosystemdienstleistungen (ebd., 31-34) und das Vorsorgeprinzip (ebd., 34-36) lässt sich exemplarisch her- 
ausstellen, unter welchen Bedingungen Klugheitsargumente überzeugend, d.h., als Klugheitsargumente plausibel sind: Ausgehend von der Diagnose, dass in den Strategiepapieren vor allem Argumente vom Typ Klugheit verwandt werden (ebd., 28), kritisieren Eser et al. die damit einhergehende Annahme, dass die Klugheitsargumentation die plausibelste Begründungsstrategie zur Bewahrung und Förderung der Biodiversität darstellt, weil hierbei lediglich Eigeninteressen vorausgesetzt werden müssten (ebd.). Dem halten sie entgegen, dass dieser Eindruck nur dadurch entsteht, dass Argumente vom Typ Klugheit häufig in der 'Wir-Form 'verwandt werden, womit indes deren Leistungsfähigkeit überschätzt und deren Plausibilitätsbedingungen unterschätzt werden, weil das pauschale 'Wir' selten entschlüsselt wird. Daher sei es für die Beurteilung der Überzeugungskraft von Argumenten vom Typ Klugheit notwendig, darauf zu schauen, wer jeweils mit dem pauschalen wir argumentiert und welche Interessen- und Nutzungskonflikte dahinter stecken bzw. versteckt werden (ebd., 29):

“'Wir' müssen Natur schützen, weil 'wir' auf ihre Nutzung angewiesen sind. Betrachten wir genauer, wer mit 'wir' gemeint ist, dann fällt schnell auf, dass das erste 'wir' häufig andere Personen bezeichnet als das zweite. (...) Die suggestive Metapher 'man sägt den Ast nicht ab, auf dem man sitzt' hat einen entscheidenden Haken: Bei genauer Betrachtung konkreter Situationen ist es allzu häufig so, dass die Einen sägen, und die anderen fallen." (ebd., 30, 38)

Aufbauend darauf führen Eser et al. aus, dass jeder Klugheitsappell, wenn er als Rationalitätsgebot plausibel sein soll, zweier wichtiger Präzisierungen bedarf:

"Um mehr als trivial zu sein, müssen die (...) Aussagen voraussetzen, dass Klugheitsargumente keine individuelle Perspektive einnehmen, sondern eine kollektive und mit Eigeninteressen nur wohlverstandene Eigeninteressen gemeint sein können, keineswegs aber beliebige." (ebd., 29; SL)

Einmal davon abgesehen, dass in der Studie nicht deutlich gemacht wird, was genau unter „wohlverstandenem Eigeninteresse" zu verstehen ist und worin und warum sich dieses von anderen Interessen abgrenzt, wird - zumindest in der ersten Studie - nicht präzisiert, was genau hier mit einer „kollektiven Perspektive“ gemeint ist. Im Folgegutachten findet sich hierzu indes ein Hinweis, der gleichzeitig auch ansatzweise erhellt, was mit „wohlverstandenen Eigeninteresse“ gemeint sein könnte, indem die von Julian Nida-Rümelin eingeführte Unterscheidung zwischen kollektiven Interessen (Alle ${ }_{k}$ ) und distributiven Interessen (Alle ${ }_{d}$ ) aufgegriffen wird (Eser, Benzing \& Müller 2013:43-44):

"Auf der Verwechselung von Alle $e_{k}$ und Alle $e_{d}$ beruht die vielleicht wirkmächtigste Ideologie der Gegenwart. Das kollektive Interesse aller ist nicht identisch mit dem distributiven Interesse aller. (...) Wenn jeder für sich selbst sorgt, ist für alle ge- 
sorgt - das stimmt nur distributiv. (...) Alle $\mathrm{k}_{\mathrm{k}}$ haben ein Interesse daran, dass sich nicht jeder lediglich um sich selbst sorgt." (Nida-Rümelin 2011:74)

Wird die Unterscheidung von Alle $\mathrm{k}_{\mathrm{k}}$ und Alle $\mathrm{e}_{\mathrm{d}}$ auf die oben genannten Plausibilitätsbedingungen von Klugheitsargumenten rückbezogen, dann kann hier präzisiert werden, dass die "kollektive Perspektive“, die hinter plausiblen Klugheitsargumenten stehen soll, zweideutig ist und dass beide Bedeutungen in Konflikt miteinander geraten können: So können zwar Alle im kollektiven Sinne (Alle ${ }_{k}$ ) ein Interesse daran haben, dass es bestimmte Regeln gibt, die allgemeinverbindlich sind (bspw. Regelungen der sozialen Sicherungssysteme wie Gesundheitsvorsorge oder Rentenkassen), während Alle im distributiven Sinne (Alle $e_{d}$ ) ein partiales aber durchaus nicht weniger berechtigtes Interesse daran haben können, diesen Regeln (bewusst oder unbewusst!) zuwider zu handeln (bspw. indem sie risikoreiche Sportarten betreiben oder keine Kinder kriegen). Aber auch im Biodiversitätskontext zeigt sich der fundamentale Unterschied beider Perspektiven die nach Eser et al. vor allem hinsichtlich des motivationalen Gehalts der Klugheitsargumente zu wenig Beachtung findet: Zwar wollen alle im kollektiven Sinne $\left(\right.$ Alle $\left._{k}\right)$, dass die Biodiversität möglichst umfassend geschützt wird, weil niemand die Stabilität der Ökosysteme gefährdet sehen will und weil prinzipiell alle einsehen, dass jeder Mensch dasselbe Anrecht auf eine intakte Natur hat, aber nicht alle im distributiven Sinne $\left(\right.$ Alle $\left._{d}\right)$ haben auch ein Interesse daran, dass Sie selber die Biodiversität schützen, weil die Nutzung der natürlichen Ressourcen ihnen Vorteile einbringt, die sie ansonsten nicht hätten. Werden folglich Alle $_{k}$ und Alle $e_{d}$ nicht auseinandergehalten, dann führt dies zum einen dazu, dass die im Fall der Klugheitsargumente so entscheidenden Divergenzen zwischen den kollektiven und distributen Interessen innerhalb der Gesellschaft nicht in ausreichendem Maße berücksichtigt werden (können), gleichzeitig können aber auch nicht diejenigen Interessen als "wohlverstandene Eigeninteressen" selektiert werden, in denen distributive Interessen sich an kollektiven Interessen orientieren. In diesem Sinne halten Eser et al. mit Bezug auf die Biodiversität fest:

„Selbstverständlich gibt es - im konkreten Einzelfall - immer Handlungsoptionen, die individuell vorteilhafter sind als Schutzmaßnahmen oder nachhaltige Nutzungsformen, die im kollektiven Interesse liegen. (...) Schutz und nachhaltige Nutzung sind also nur dann 'klug', wenn man eine hinreichend weite zeitliche und räumliche Perspektive einnimmt. Dies zu tun bedarf freilich der (moralischen) Vorentscheidung, im Handeln nicht nur eigennützige, sondern auch fremdnützige Aspekte zu berücksichtigen." (Eser, Neureuther \& Müller 2011:30)

Über die Präzisierungen, was überzeugende, d.h., plausible und in diesem Fall eben gute Klugheitsargumente auszeichnet, zeigt sich, dass oftmals mit Argumenten vom Typ Klugheit argumentiert wird nicht aber auch guten Klugheitsargumenten, weil die beiden wichtigen Schritte von der rein individuellen Perspektive auf die distributive Per- 
spektive sowie vom Eigeninteresse auf das wohlverstandene Eigeninteresse selten Beachtung finden. Vergleicht man diese Präzisierungen kluger Klugheitsargumente aber nun mit den von Eser et al. vorgenommenen Differenzierungen zwischen Klugheitsund Gerechtigkeitsargumenten, dann - so der im Folgenden entscheidende und aufzuzeigende Punkt - stellt sich die Frage, was gute Klugheitsargumente von Gerechtigkeitsargumenten unterscheidet, da es bei beiden offensichtlich darum geht, nicht nur eigennützige sondern auch fremdnützige Interessen zu berücksichtigen, was gemeinhin als die Einnahme des moralischen Standpunkts (»moral point of view») beschrieben wird, vor dem beurteilt wird, welche Pflichten und Rechte Menschen begründeterweise voneinander abverlangen können. Eben dieser - für das herauszustellende konzeptionelle Problem des Ansatzes - entscheidende Punkt, bedarf einer Erläuterung:

Eser et al. führen das zur Differenzierung von Klugheits- und Gerechtigkeitsargumenten Beispiel an, dass es unkluges aber eben nicht moralisch verwerfliches bzw. unmoralisches Verhalten ist, wenn jemand am Abend vor einer Prüfung feiert und trinkt und deswegen am nächsten Tag nicht fit ist und die Prüfung vermasselt (ebd., 36). Wenn indes die Folgen dieser Handlungen andere betreffen (bspw. wenn man sich im betrunkenen Zustand ans Steuer setzt und einen Unfall baut), dann sei dies ganz klar eine Frage der Gerechtigkeit und eben nicht der Klugheit (ebd., 38). Daraus kann abgeleitet werden, dass solange die negativen Folgen der Handlungen nur einen selbst betreffen, die Handlungen dem Typus der Klugheit zuzuordnen sind (ebd., 36), sobald aber die negativen Folgen der Handlungen Andere betreffen, sind diese dem Typus der Gerechtigkeit zuzuordnen. Dies wiederum würde bedeuten, dass Klugheitshandlungen keinerlei (negative) soziale und 'natürliche`Auswirkungen für andere haben dürfen, da es sich sonst bereits um Gerechtigkeitshandlungen handelt. Insofern muss aber konstatiert werden, dass es unter Berücksichtigung dieses Kriteriums (der negativen Folgen) im Kontext des Umwelt- und Naturschutzes gar nicht so viele Handlungen vom Typ Klugheit geben kann, da jede soziale Handlung (wenn man den Bezugsrahmen der Handlung nur weit genug macht) mit negativen Folgen für jemanden oder etwas einhergehen kann. An dieser Stelle lassen sich zwei Rückschlüsse ziehen: Entweder dieses spezielle Unterscheidungskriterium zwischen Klugheit und Gerechtigkeit ist als solches problematisch, woraufhin sich dann aber die Frage stellt, wie und anhand welcher Aspekte Klugheit und Gerechtigkeit unterschieden werden sollten oder aber es ist zutreffend, was dann aber die Frage nach sich zieht, warum Klugheitsargumente im Kontext des Umwelt- und Naturschutzes überhaupt eine Rolle spielen sollten, wenn diese kontextabhängig immer auch als Gerechtigkeitsfragen adressiert werden können.

Insofern kann festgehalten werden, dass die Differenzierung zwischen Klugheits- und Gerechtigkeitsargumenten auf der analytischen Ebene durchaus Sinn macht - nämlich als Unterscheidung zwischen zwei unterschiedlichen Argumentationstypen, die sich ei- 
nerseits auf pragmatische (Klugheit) und andererseits auf moralische (Gerechtigkeit) Aspekte beziehen. Eben diese Differenzierbarkeit verschwimmt aber auf der Begründungsebene, wenn es darum geht, die Plausibilitätsbedingungen der einzelnen Argumentationstypen auszuweisen. Hier liegt die Unterscheidbarkeit darin, dass gute Klugheitsargumente distributive Interessen zum Gegenstand haben, mit denen Forderungen einhergehen, die zwar nicht universalisierbar sein müssen, sprich aus denen sich keine Pflichten und Rechte ableiten lassen, die sich aber zugleich im Rahmen von universalisierbaren Kollektivinteressen bewegen müssen, um plausibel zu sein. Das bedeutet, dass der Perspektivwechsel von der analytischen Betrachtung der Klugheitsargumente, sprich vom Eigeninteresse, das durchaus rein egoistisch sein kann, auf die Begründungsebene der Plausibilitätsbedingungen der Klugheitsargumente, wo das wohlverstandene Eigeninteresse im Fokus steht, bei dem man seine eigenen Interessen bereits im Kontext der Interessen anderer betrachten muss, für die Konzeption der Klugheitsargumente problematisch ist. Dies ist der Fall, weil die Bereitschaft im Handeln nicht nur eigene sondern auch fremdnützige Interessen zu berücksichtigen, i.d.R. als die Einnahme eines moralischen Standpunkts bezeichnet wird, der wiederum von vielen Ethiken für die Ausweisung und Begründung von Pflichten und Rechten herangezogen wird, die aber für den Typus der Gerechtigkeitsargumente konstitutiv sein sollen. Der Knackpunkt ist folglich, dass Eser et al. den Gerechtigkeitsbegriff rein formal und nicht inhaltlich bestimmen, da sie den Typus der Gerechtigkeitsargumente auf der analytischen Ebene darüber bestimmen, dass er sich auf Pflichten und Rechte bezieht und damit alle Aussagen umfasst, „die ein Sollen behaupten“ (Eser, Benzing \& Müller 2013:22), welches wir „mit guten Gründen einander abverlangen können“ (Eser, Neureuther \& Müller 2011:39) und gleichzeitig die Plausibilitätsbedingungen der Gerechtigkeitsargumente auf der Annahme basieren lassen, dass das moralisch geboten sein sollte und damit gerecht ist, was „wir uns gegenseitig schulden.“ (Mazouz 2006)

Das Problem an dieser rein formalen Bestimmung dessen, was gerecht ist, ist aber, dass unter dieser Bestimmung dann auch Klugheitsargumente gerecht sind, weil auch Klugheitsargumente ein Sollen behaupten - nämlich ein pragmatisches Sollen: Handle klug! - und dass wir dieses Rationalitätsgebot unter bestimmten Umständen, nämlich genau dann, wenn Klugheitsargumente plausibel sind, was der Fall ist, wenn es sich dabei um wohlverstandene Eigeninteressen handelt, auch mit guten Gründen voneinander verlangen können. Mit anderen Worten: Wenn einerseits all diejenigen Argumente unter die Kategorie der Klugheit fallen sollen, die sich darauf beziehen, die Biodiversität im eigenen Interesse zu bewahren und zu fördern, wobei gute Klugheitsargumente erfordern, die eigenen Interessen bereits im Kontext der Interessen anderer zu betrachten. Und wenn andererseits all diejenigen Argumente unter die Kategorie der Gerechtigkeit fallen sollen, die Pflichten und Recht betreffen, wobei gute Gerechtigkeits- 
argumente nur diejenigen Argumente sind, die begründeterweise von anderen verlangt werden können, dann wird die von Eser et al. auf der analytischen Ebene vorgenommene Differenzierung zwischen guten Klugheitsargumenten und guten Gerechtigkeitsargumenten unscharf, da beide Argumentationstypen auf der Begründungsebene auf den moralischen Standpunkt verweisen, der aber nur in deskriptiver nicht aber auch in normativer Hinsicht spezifiziert wird:

Wenn der moralische Standpunkt sowohl für die Klugheitsargumente als auch für die Gerechtigkeitsargumente von Relevanz ist, dann vornehmlich in seiner deskriptiven Interpretation, nach der die Einnahme des moralischen Standpunkts immer dann erfolgt, wenn man sich in die Situation all derjenigen versetzt, die von einer Handlung (potenziell) betroffen sind und sich fragt, ob man an ihrer Stelle die Ausführung der Handlung wünschen könnte (Misselhorn 2006:431). Für die dabei entscheidende Fragen, warum man den moralischen Standpunkt einnehmen sollte, ist die auf das wohlverstandene Eigeninteresse verweisende Argumentation, dass die uneingeschränkte Vorrangigkeit eigennütziger Motive zu einem gesellschaftlichen Zustand führen würde, an dem niemand ernsthaft interessiert sein kann (ebd., 433), nur ein Argument unter vielen. Insofern müsste man, um die Unterscheidbarkeit von Klugheits- und Gerechtigkeitsargumenten beibehalten zu können, präzisieren, wie der moralische Standpunkt bestimmt wird, sprich, ob er vor dem Hintergrund utilitaristischer, kantischer, rawlsianischer, diskursethischer, tugendethischer etc. Annahmen ausgedeutet wird und wie in diesen Theorien das Verhältnis zwischen Klugheit und Moral bestimmt wird (ebd., 432-433). Die von Eser et al. angeführten Aspekte der Unparteilichkeit, der Universalisierbarkeit und der Reziprozität, über die sie den moralischen Standpunkt auszeichnen, helfen hier nicht weiter, da diese für eine Vielzahl an unterschiedlichen ethiktheoretischen Ausdeutungen des moralischen Standpunkts sprechen können (ebd., 432).

Zusammenfassend wird an den bisherigen Ausführungen zum Verhältnis von Klugheitsund Gerechtigkeitsargumenten ersichtlich, dass es in der Studie nicht lediglich um ein analytisches Schema zur (Neu-)Strukturierung der in der NBS gegeben Gründe geht, sondern dass hier auch eine ethiktheoretische Neuausrichtung der Umwelt- und Naturschutzethik forciert wird, über die allerdings auch weitreichende theoretische Setzungen vorgenommen werden, die in der Studie nicht in ausreichendem Maße expliziert und eingeholt wurden (konnten). Diese Setzungen sind pragmatisch betrachtet - wie bereits gesagt - voll und ganz im Sinne der hier vorliegenden Arbeit. Theoretisch betrachtet ist es indes unbefriedigend, dass dabei bereits die Modellierung des Ansatzes hinsichtlich der Differenzierung und Verhältnisbestimmung der Argumentationstypen unscharf bleibt. Auch ist nach wie vor die Frage ungeklärt, warum überhaupt davon ausgegangen werden kann, dass Klugheits-, Glücks- und Gerechtigkeitsargumente allesamt auch ethische Argumente sind. 
Beiden Problematiken soll im Folgenden konstruktiv begegnet werden, indem zum einen die diskurstheoretischen Fundamente der Argumentationstrias rekonstruiert werden, die an vielen Stellen in der Studie durchscheinen, aber selten explizit gemacht werden (4.3.2.2) und indem zum anderen ein mehrdimensionales Ethikverständnis als Grundlage der gesamten Argumentationstrias expliziert wird (4.3.2.3).

\subsubsection{Die Rekonstruktion der diskurstheoretischen Fundamente der Argumentationstrias}

Vor dem Hintergrund der aufgezeigten konzeptionellen Problematik stellt sich die Frage, auf welchen theoretischen Fundamenten die Argumentationstrias aus Klugheit, Glück und Gerechtigkeit steht. Diesbezüglich wird (erst) im Folgegutachten angemerkt, dass die Argumentationstrias ausweisen soll, ob und warum der Schutz, die nachhaltige Nutzung und die gerechte Verteilung der Biodiversität instrumentell klug, axiologisch wünschenswert und moralisch geboten ist (Eser, Benzing \& Müller 2013:21). ${ }^{132}$ Hinter diesem Ansinnen steht die bei Eser et al. nicht explizit herausgestellte aber aus dem Kontext herauslesbare diskurstheoretisch begründete Annahme, dass die Ethik dann einen substanziellen Beitrag zur Lösung praktischer Fragen und Probleme leisten kann, wenn die praktischen Fragen und Problemstellungen entweder unter einem moralischen Blickwinkel, wie zu handeln richtig ist oder unter einem eudaimonistischen Blickwinkel, wie zu leben gut ist oder unter einem pragmatischen Blickwinkel, wie zu verfahren zweckdienlich ist, behandelt werden. Jürgen Habermas hat eben diese Dreiteilung, die analog zur Eserschen Argumentationstrias aus Gerechtigkeitsargumenten, Glücksargumenten und Klugheitsargumenten ist, in seinen »Erläuterungen zur Diskursethik» (vgl. Habermas 1991a) und speziell in seinem Artikel »Zum moralischen, ethischen ${ }^{133}$ und pragmatischen Gebrauch der praktischen Vernunft ${ }^{134}$ (vgl. Habermas 1991b) ausgewiesen:

132 Hierbei berufen sich Eser et al. auf Konrad Ott, der in seinem Beitrag "Zur ethischen Begründung des Schutzes von Biodiversität“ (vgl. Ott 2007) untersucht, „ob und warum der Schutz von Biodiversität instrumentell klug, axiologisch wünschenswert und moralisch geboten sein könnte." (Ott 2007:89) Damit zeichnet er selber in Rückgriff auf Habermas die drei verschiedenen Zugänge vor, die Eser et al. mit ihren drei Typen Klugheit, Glück und Gerechtigkeit aufnehmen allerdings mit dem bewusst in Kauf genommenen 'Defizit', dass er sich nur auf den Schutz und nicht auch auf die Nutzung und die Verteilung der Biodiversität fokussiert (Ott 2007:89).

133 Habermas redet selbst von einer ethischen Gebrauchsweise der praktischen Vernunft in allen Fällen, wo diese auf »das Gute« abzielt. Davon soll hier terminologisch vor allem deshalb abgewichen werden, weil Ethik nicht wie bei Habermas philosophiegeschichtlich mit Fragen des guten Lebens identifiziert wird, sondern umfassender verstanden wird (siehe auch 4.3.2.3).

134 Indem Habermas davon ausgeht, dass sich die Vernunft allein in der kommunikativen, intersubjektiven Verständigung zeigt (siehe FN 14), setzt Habermas den Begriff der praktischen Vernunft mit dem Begriff der „kommunikativen Vernunft" gleich (Habermas 1981a:30). Daher spricht nichts dagegen, wie dies Habermas auch selbst tut, von praktischer Vernunft zu sprechen und das Konzept der kommunikativen Vernunft als deren Fundament zu meinen. 
„Die klassische Ethik geht, ebenso wie die modernen Theorien, von der Frage aus, die sich dem orientierungsbedürftigen Einzelnen aufdrängt, wenn er in einer bestimmten Situation unschlüssig einer praktisch zu bewältigenden Aufgabe steht: wie soll ich mich verhalten, was soll ich tun? (...) In dieser Hinsicht mag der Name Diskursethik ein Missverständnis nahegelegt haben. Die Diskurstheorie bezieht sich in je anderer Weise auf moralische, ethische und pragmatische Fragen. (...) Je nach Problemstellung gewinnt also die Frage "Was soll ich tun? eine pragmatische, ethische oder moralische Bedeutung. In allen Fällen geht es um die Begründung von Entscheidungen zwischen alternativen Handlungsmöglichkeiten. Sie alle erfordern einen anderen Typus von Handlungen, die entsprechenden Entscheidungen einen anderen Typus von Begründungen." (ebd., 101, 108)

Vor diesem Hintergrund werden praktische Fragen hier als Reflexions- und Entscheidungsfragen in Bezug auf unser Verhalten und Handeln betrachtet, die an einen selbst oder an sndere gerichtet sein können. Wenn sie an andere gerichtet sind, dann gehen diese mit bestimmten Handlungsaufforderungen (Imperative) derart einher, dass man anderen rät oder von ihnen verlangt, wie sie sich zu verhalten haben oder wie sie handeln sollten. Das hierbei relevante imperative 'Sollen' behält dabei solange „einen unspezifischen Sinn, solange nicht das Problem näher bestimmt ist und der Aspekt unter dem es gelöst werden soll“ (ebd., 101), weil unter den Aspekten des Zweckmäßigen, des Guten und des Gerechten jeweils andere Leistungen von der praktischen Vernunft erwartet werden. Über die Darlegung dieser Leistungen, so die hier angebrachte Argumentation, kann ein substanzieller Beitrag für die, im Eserschen Ansatz bisher zu kurz kommende Frage geleistet werden, welche Annahmen hinter den einzelnen Argumentationstypen jeweils stehen und was das Spezifische am pragmatischen (4.3.2.2.1), eudaimonistischen (4.3.2.2.2) und moralischen (4.3.2.2.3) Gebrauch der praktischen Vernunft bezüglich der Entscheidung praktischer Fragen und der Lösung praktischer Probleme ist. Hierüber wird in konstruktiver Weise dem ersten Aspekt der zweiten Detailproblematik, nämlich der Integrativität der Zieltrias auch konzeptionell gerecht zu werden, begegnet, indem herausgestellt wird, dass die Argumentationstypen einerseits mit unterschiedlichen Reflexionsleistungen einhergehen und andererseits mit unterschiedlichen Sollenstypen operieren, die hinsichtlich ihres Sollgeltungsanspruchs voneinander unterschieden werden müssen, weil dabei das Verhältnis zwischen Sollen, Wollen und Können jeweils anderen Bedingungen unterliegt. Eben diese Spezifizierungen führen auf alternative systematische Verhältnisbestimmungen und Differenzierungen zwischen den Argumentationstypen. Dies ist im Weiteren von Bedeutung, wenn es im Anschluss darum geht, dem zweiten Aspekt der Detailproblematik nämlich dem zu starken deontischen Fokus auf Pflichtenargumente zu begegnen, indem der biodiversitätsethische Argumentationsraum in den Kontext eines mehrdimensionalen Ethikverständnis gestellt wird (4.3.2.3). 


\subsection{Zum pragmatischen Gebrauch der praktischen Vernunft}

Wenn die allgemeinen praktischen Fragen unter dem pragmatischen Aspekt betrachtet werden, wie zu verfahren sinnvoll und was zu tun zweckdienlich ist, dann macht man von der praktischen Vernunft in zweckrationaler Hinsicht Gebrauch. Bei pragmatischen Überlegungen und Erwägungen wird daher im Speziellen danach gefragt, was das Zweckdienliche, das Nützliche, Effiziente, das Effektive etc. für den einzelnen oder für viele ist, wobei es im Allgemeinen immer darum geht, welche Mittel zweckdienlich sind, um bestimmte Ziele zu erreichen oder welche Ziele geeignet sind, um bestimmte Mittel einsetzen zu können (Ott 1993:103). Wichtig ist hierbei, dass es entweder um eine Entscheidung zwischen verschiedenen Mitteln geht, die alle auf dasselbe Ziel führen, wenn die Zielstellung als solche feststeht oder dass es darum geht, zwischen unterschiedlichen Zielen abzuwägen, für deren Erreichung die Mittel bereits feststehen (Habermas 1991b: 102). Daraus ergibt sich, dass sich die Reflexionsleistung ausschließlich auf die Zweck-Mittel Relation bezieht, weil die Frage, ob die Ziele- oder die Mittel als solche gut oder richtig sind bei pragmatischen Reflexionen keine Rolle spielt (Ott 1993:103). Dennoch geht es um Handlungsaufforderungen (Imperative), so dass auch der pragmatische Gebrauch der praktischen Vernunft mit einem bestimmten Sollgeltungsanspruch einhergeht. Um diese beiden Aspekte deutlich zu machen, kann auf Charles Taylors Unterscheidung zwischen schwachen und starken Wertungen (vgl. Taylor 2006) verwiesen werden. Hierbei kann anhand der schwachen Wertungen a) präzisiert werden, um welche Art von Reflexionsleistung es sich beim pragmatischen Gebrauch der praktischen Vernunft handelt und b) welcher Sollgeltungsanspruch mit dem pragmatischen Sollenstyp einhergeht. Dafür muss Taylors Argumentation bezüglich der Unterscheidbarkeit schwacher und starker Wertungen nachgezeichnet werden:

a) Taylor führt in Rückgriff auf Harry Frankfurt aus, dass die Unterscheidung zwischen "Wünschen erster Ordnung" und "Wünschen zweiter Ordnung" (vgl. Frankfurt 1971) wesentlich zur Charakterisierung eines menschlichen Akteurs bzw. menschlichen Handelns im Vergleich zu nicht-menschlichen Lebewesen und Verhalten ist:

"Menschen sind nicht die einzigen Lebewesen, die Bedürfnisse und Motive haben oder Wahlen treffen. Sie teilen dies mit den Mitgliedern bestimmter anderer Spezies, von denen einige sogar Überlegungen anstellen oder überlegte Entscheidungen treffen. Es scheint jedoch ein spezifisches Charakteristikum des Menschen zu sein, daß er imstande ist, Wünsche zweiter Ordnung auszubilden." (ebd., 6 in Taylor 2006:1)

Diesbezüglich führt Taylor aus, dass davon ausgegangen werden kann, dass (zumindest höhere) Tiere Wünsche erster Ordnung haben, da sie „zwischen Wünschen wählen oder zumindest manche Wünsche zugunsten anderer zu hemmen vermögen." (ebd., 
1), während die Fähigkeit „Wünsche zu bewerten, manche als wünschenswert und andere als nicht wünschenswert zu betrachten" (ebd.), spezifisch menschlich zu sein scheint. Dies, so Taylor in Rückgriff auf Frankfurt, „ist der Grund dafür, daß kein anderes Tier als der Mensch (...) die Fähigkeit zu reflektierender Selbstbewertung zu haben scheint, die sich in der Ausbildung von Wünschen zweiter Ordnung manifestiert." (ebd. mit Bezug auf Frankfurt 1971:7) Im Anschluss daran führt Taylor die Unterscheidung zwischen schwachen und starken Wertungen als unterschiedliche Modi der Bewertung von Wünschen ein. Deren Unterschied macht Taylor, nach einigen Spezifizierungen, was diese nicht sind, wie folgt deutlich:

„Bei der Unterscheidung zwischen beiden Arten von Wertung geht es nicht einfach um die Differenz von quantitativer und qualitativer Bewertung oder um die Anwesenheit oder Abwesenheit von Bedürfnissen zweiter Stufe. Es handelt sich eher darum, ob die Wünsche sich hinsichtlich ihres Wertes unterscheiden. Und hierfür können wir zwei miteinander verknüpfte Kriterien aufstellen: (1) Im Falle schwacher Wertungen genügt es, daß etwas gewünscht wird, damit es als gut beurteilt wird, während starke Wertungen eine Verwendung von "gut" oder eines anderen evaluativen Ausdrucks erfordern, für die ein Gewünschtsein allein nicht ausreicht (...). Hieraus folgt, daß wenn (2) im Falle einer schwachen Wertung auf einen der alternativen Wünsche verzichtet wird, so nur aufgrund seiner kontingenten Unvereinbarkeit mit einer stärker angestrebten Alternative. (...) Im Falle starker Wertungen jedoch ist dies nicht notwendig der Fall. Ein angestrebtes Ziel würde nicht deshalb aufgegeben, weil es mit einem anderen Ziel unverträglich ist, zumindest nicht aufgrund einer zufälligen Unvereinbarkeit.“ (Taylor 2006:4)

Bereits hierüber lassen sich Präzisierungen bezüglich des pragmatischen Gebrauchs der praktischen Vernunft anführen: ${ }^{135}$ Die Differenzierung zwischen schwachen und starken Wertungen ist in Bezug auf die Reflexion des pragmatischen Gebrauchs der praktischen Vernunft relevant, weil, in Rückgriff auf das erste Kriterium, diese sich dadurch auszeichnen lassen, dass es hierbei, genau wie bei schwachen Wertungen, nicht zu einer Unterscheidung der Wünsche im Hinblick auf die Werte kommt, die hinter den Wünschen stehen. Insofern genügt es bei pragmatischen Entscheidungen, in Bezug auf das zweite Kriterium, dass auf einen alternativen Wunsch verzichtet wird, weil dieser in einer kontingenten Unvereinbarkeit mit einer anderen Alternative steht, da die Alternativen unter dem Gesichtspunkt ihrer Erwünschtheit bewertet werden (ebd., 5). Im Kontrast dazu, spielt bei starken Wertungen die nicht-kontingente Unvereinbarkeit von Alternativen eine zentrale Rolle:

135 Anhand der starken Wertungen lassen sich auch die Präzierungen des eudaimonistischen Gebrauchs der praktischen Vernunft ausführen, was aber erst im nächsten Abschnitt geschehen wird (4.3.2.2.2). 
„Es ist kein Zufall, daß es sich hier um eine nicht-kontingente Inkompatibilität handelt. Denn starke Wertungen entfalten eine Sprache wertender Unterscheidungen, in der Wünsche als edel oder gemein, als integriert oder fragmentiert, als mutig oder feige, als umsichtig oder blind usw. beschrieben werden. Dies bedeutet jedoch, daß sie kontrastiv charakterisiert sind. Bei sämtlichen aufgeführten Begriffspaaren läßt sich jeder Begriff nur im Verhältnis zu einem Gegenbegriff verstehen." (ebd., 4-5)

Taylor führt folglich an, dass die Entscheidungen zwischen Alternativen bei starken Wertungen kontrastiv beschrieben werden müssen, wenn zum Ausdruck kommen soll, was an der bevorzugten Alternative wirklich wünschenswert ist. Demgegenüber ist ein Subjekt, das nur schwach wertet „ein bloß Alternativen abwägendes Subjekt“, das:

„in einem minimalen Sinne reflektiert, indem [es] die Handlungsverläufe bewertet und manchmal imstande ist, entsprechend dieser Wertung, anstatt unter dem Eindruck unmittelbarer Wünsche zu handeln. Das bloß abwägende Subjekt verfügt über Überlegung, Wertung und Willen. Aber ihm fehlt im Gegensatz zum stark wertenden Subjekt etwas, das wir oft durch die Metapher der 'Tiefe` umschreiben." (ebd., 8)

Das hierbei entscheidende Moment der Tiefe starker Wertungen besteht darin, so Taylor, dass man über die Sprache wertender Kontrastierungen verfügt und diese zur Anwendung bringt, um eine Entscheidung zwischen Handlungsalternativen zu fällen. Starke Wertungen gehen tiefer als schwache Wertungen, da es hierbei darum geht, die Motivation(en) hinter den Wünschen zu erfassen, zu reflektieren und zu beschreiben und weil es sich dabei nicht lediglich um eine Abwägung zwischen verschiedenen Mitteln oder Zielen handelt, sondern von Wünschen oder Neigungen „in Kategorien der Art von Lebensqualität“ (ebd., 9) gesprochen wird, die über die Entscheidung zwischen Wünschen zum Ausdruck gebracht und aufrechterhalten wird:

„Während es für das schwach wertende Subjekt um die Erwünschtheit unterschiedlicher Ziele geht, die durch seine De-facto-Wünsche definiert werden, untersucht das Nachdenken des stark wertenden Subjekts auch die verschiedenen möglichen Seinsweisen des Handelnden. Motivationen oder Wünsche zählen nicht nur aufgrund der Anziehungskraft der Ziele, sondern auch aufgrund der Lebensweise und des Subjekttypus, denen diese Wünsche eigentlich entsprechen. Diese zusätzliche Dimension jedoch, so kann man sagen, fügt eine Tiefendimension hinzu, da wir nunmehr über unsere Wünsche unter dem Gesichtspunkt der Frage nachdenken, welche Art von Wesen wir sind, wenn wir diese Wünsche haben oder realisieren. Während ein Nachdenken darüber, was wir als den stärkeren Wunsch empfinden - was alles ist, was ein bloß abwägendes Subjekt beim Bewerten von Motivationen tun kann -, uns weiter an der Oberfläche festhält, führt 
ein Nachdenken darüber, welche Art von Wesen wir sind, uns direkt ins Zentrum unserer Existenz als Handelnde. Starke Wertung ist nicht nur eine Bedingung dafür, Präferenzen artikulieren zu können, sondern beinhaltet auch Fragen der Lebensqualität, der Art von Existenz, die wir führen und führen wollen." (ebd., 9-10)

Bezogen auf den pragmatischen Gebrauch der praktischen Vernunft bedeutet dies zusammenfassend, dass prudentielle Entscheidungen „bei der nichtartikulierbaren Erfahrung [enden], daß A attraktiver ist als B“ (ebd. 8), d.h., wo es um eine Entscheidung zwischen kontrastiven und damit inkommensurablen Alternativen geht, so dass die Wahl nur zwischen dem besteht, was vor dem Hintergrund kontingenter Erfahrungen und Neigungen (im Augenblick) besser oder schlechter ist, ohne dass es dabei auch schon um die Reflexion dessen geht, warum das eine oder andere als besser oder schlechter angesehen, erfahren oder empfunden wird. Hier beginnen die starken Wertungen, die Wünsche in einer 'tieferen ' Dimension betrachten, worauf allerdings erst bezüglich des eudaimonistischen Gebrauchs der praktischen Vernunft eingegangen wird (4.3.2.2.2).

b) Gemäß der oben vorgenommenen Charakterisierung pragmatischer Überlegungen und Erwägungen anhand schwacher Wertungen muss bezüglich der Charakterisierung des pragmatischen Sollgeltungsanspruchs das Verhältnis zwischen Wollen, Können und Sollen untersucht werden. Bezüglich des Verhältnisses von Wollen und Können kann hier - aufbauend auf den Charakterisierungen des pragmatischen Handlungstypus anhand schwacher Wertungen - festgehalten werden, dass das pragmatische Handeln an der Willkür des Subjekts ausgerichtet ist, dem hinsichtlich dessen, was Subjekte können wollen, kaum Grenzen gesetzt sind: Da jeder Mensch prinzipiell auch Dinge wünschen und/oder bevorzugen kann, die er/sie derzeit nicht realisieren kann oder sogar niemals realisieren können wird (bspw. ein berühmter Sänger zu werden, auch wenn man sich noch so anstrengt, dies zu erreichen) und umgekehrt Dinge tun kann, die er/sie aus pragmatischen Gründen nicht will (mit etwas Übung, den geeigneten Anleitungen und Materialen könnte jeder bspw. eine Nagelbombe bauen), ist das pragmatische Wollen nicht durch das Können bedingt und umgekehrt. Insofern kann davon gesprochen werden, dass das pragmatische Wollen vom Können relativ unabhängig ist, da die Grenze des Wollens in Richtung des Könnens erst dann erreicht ist, wenn es um eine prinzipiell unüberbrückbare Diskrepanz zwischen Wollen und Können geht (bspw. ist das Unterfangen aussichtslos, Magie wirken zu können oder durch die Zeit zu reisen, auch wenn einige Menschen dies gerne könnten). Solange Wollen und Können also prinzipiell in einem Verhältnis der gegenseitigen (potenziellen) Einlösbarkeit stehen, sind dem Individuum kaum Machbarkeitsgrenzen für sein pragmatisches Handeln gesetzt. 
Allerdings sind dem pragmatischen Handeln andere Grenzen gesetzt, wenn es um das Verhältnis von Sollen zu Wollen und Können geht. Diesbezüglich kann festgehalten werden, dass pragmatische Handlungsaufforderungen abhängig von den tatsächlichen oder potenziellen, individuell verschiedenen, situationsspezifischen und kontingenten Mitteln und Zielen des Adressaten sind (Habermas 1991b:103). Da hier nur in einem rein subjektiven Sinn von gut und schlecht, besser oder schlechter, richtig und falsch die Rede ist (schwache Wertungen), da das, was man tun soll, immer schon an dem orientiert ist, was man selbst unter gegebenen Umständen tun will bzw. an dem, was andere unter gegebenen Umständen tun wollen (Ott 1993:103), kann man sich selbst oder anderen das Rationalitäts(an)gebot nahelegen, möglichst diejenigen Mittel zur Erreichung eines bestimmten Zieles zu ergreifen, die dafür am Geeignetsten sind und diejenigen Ziele anzustreben, die den größten (Gesamt-)Nutzen für einen selbst haben. Daher können einem pragmatische Imperative nur anraten, welches Ziel effektiv ist, wenn man bestimmte Mittel anwenden will oder wie man effizient verfahren soll, wenn man bestimmte Ziele realisieren möchte. ${ }^{136}$ Insofern sind pragmatische Ratschläge auch nur schwach präskriptiv, da man niemanden im moralisch-kategorischen Sinne von Pflicht darauf verpflichten kann, so und nicht anders zu handeln: ${ }^{137}$

Wenn ich es bspw. für vernünftig halte, im Biosupermarkt einkaufen zu gehen, weil ich gern regionale und nachhaltig produzierte Lebensmittel einkaufen möchte und meine, dass, wenn dies alle täten, es für alle günstiger und einfacher werden würde Biolebensmittel zu erwerben, dann kann ich eben dies auch anderen anraten. Ich kann aber niemanden dazu zwingen, ebenfalls so zu handeln. Vielmehr kann ich anderen nur nahelegen, meinen Ratschlag als Alternative zu ihrem bisherigen Handeln in Erwägung zu ziehen. Dieser kann, muss aber nicht, von anderen beherzigt werden, denn andere können diesen Ratschlag auch vor dem Hintergrund ihrer eigenen Ziele und Mittel modifizieren (indem sie bspw. nicht in den Bioladen gehen, sondern ihre Biolebensmittel im Discounter kaufen), den Ratschlag für nicht anwendbar halten (weil sie bspw. nicht genug Geld haben, um überhaupt Biolebensmittel einzukaufen) oder vom Prinzip her ablehnen (weil sie bspw. der Auffassung sind, dass Biolebensmittel und konventionelle Lebensmittel qualitativ gleichwertig sind).

${ }^{136}$ Die Grundvoraussetzung dabei ist indes, dass ich selbst und/oder andere bereit sind, das Nutzenmaximierungsprinzip als Gebot der Klugheit auch anzuerkennen. Erst wenn dies der Fall ist, handelt derjenige dumm bzw. unvernünftig, wenn es effektivere Ziele bzw. effizientere Handlungsabläufe gegeben hätte, die man ungenutzt gelassen hat.

137 An dieser Stelle ist die Unterscheidung zwischen einer Pflicht im pragmatischen und hypothetischen Sinn, als das, was getan werden sollte, um ein bestimmtes Ziel zu erreichen und einer Pflicht im moralischen und kategorischen Sinn, als das, was verpflichtend gegenüber allen anderen getan werden muss (siehe 4.2.2.1.4), unabhängig von unseren persönlichen Interessen und Zielen, relevant. 
Auf der allgemeinen Ebene heißt dies wiederum, dass der Sollgeltungsanspruch des pragmatischen Sollens rein hypothetisch ist, weil er an die Ziele und Mittel aber auch an die Lebensumstände, Möglichkeiten und Zwänge desjenigen gebunden ist, dem ein solcher Ratschlag gegeben wird. Hiermit geht wiederum einher, dass der Sollgeltungsanspruch pragmatischer Imperative immer schon durch andere pragmatische Imperative aufgrund seiner kontingenten Unvereinbarkeit mit einer anderen Alternative relativiert und suspendiert werden kann: ${ }^{138}$ Wenn bspw. jemand vor der Wahl steht, entweder noch schnell einkaufen zu gehen bevor die Läden zumachen, weil er etwas kochen möchte oder schnell nach Hause zu fahren, weil er das Fußballspiel nicht verpassen will, dann wird er sich, wenn es für ihn annehmbare Alternativen zum Einkaufen gibt (Tankstelle), aber keine zum Fußballschauen (weil das Spiel Live übertragen und nicht wiederholt wird), für das Fußballspiel entscheiden. Ist dies umgekehrt (er muss einkaufen, weil zwei Feiertage anstehen, er das Spiel aber problemlos auch aufzeichnen und später schauen kann), dann wird er dieser Variante den Vorzug geben.

Entscheidend ist, dass die Wahl, welcher Variante der Vorzug gegeben wird, von den jeweils gegebenen Umständen und Möglichkeiten abhängt, seine Ziele auch tatsächlich zu realisieren bzw. bestimmte Mittel überhaupt zu ergreifen. Ob andere einen pragmatischen Ratschlag annehmen und ihm Folge leisten, hängt folglich davon ab, welche Ziele und Mittel die Adressaten des Ratschlags jeweils verfolgen und welche Lebensumstände, Möglichkeiten und Zwänge sie haben. Plausible pragmatische Ratschläge sind daher immer nur solche, die diese je individuellen Konstellationen berücksichtigen und diese in Bezug auf den Ratschlag einkalkulieren. Dies ist indes etwas gänzlich anderes, als zu behaupten, man müsse für plausible Klugheitsargumente eine distributive Perspektive einnehmen, da man sich für einen pragmatischen Ratschlag (lediglich) in diejenigen Menschen und deren Situation hinein versetzen muss, denen man den Ratschlag erteilt, nicht aber auch schon prüfen muss, ob der Ratschlag als solcher auch verallgemeinerbar ist: Wenn ein Energieberater einem Hotel nahelegt, sie könnten doch zwischen 22 Uhr und 7 Uhr morgens die Sauna abschalten, um so einen erheblichen Teil der Energiekosten zu sparen, das Hotel aber eine 24 Stunden geöffnete Sauna anbieten muss, um den vierten Stern zu behalten, dann ist dieser Ratschlag für das Hotel, das den vierten Stern behalten muss, um wettbewerbsfähig zu bleiben, schlicht nicht umsetzbar und damit kein plausibler, sprich kluger Ratschlag.

\footnotetext{
138 Wenn von einem hypothetischen Sollgeltungsanspruch die Rede ist, dann heißt dies, dass die Zulässigkeit der Handlungsaufforderung bzw. der Handlungsgründe abhängig von den (tatsächlichen oder möglichen) Zielen und Mitteln des Akteurs ist (Birnbacher 2007:20). Darüber hinaus ist der Sollgeltungsanspruch pragmatischer Imperative auch durch eudaimonistische und moralische Imperative und deren Sollgeltungsansprüche eingeschränkt. Warum dies der Fall ist und was daraus folgt, soll allerdings erst anhand des eudaimonistischen und moralischen Gebrauchs der praktischen Vernunft deutlich gemacht werden (4.3.2.2.2 und 4.3.2.2.3).
} 


\subsection{Zum eudaimonistischen Gebrauch der praktischen Vernunft}

Wenn die allgemeine praktische Frage, wie man sich verhalten soll und was zu tun ist, unter dem eudaimonistischen Aspekt betrachtet wird, dann - so Habermas zentrale Annahme - verlässt man das 'Terrain` der pragmatischen Gebrauchsweise der praktischen Vernunft: Unter der eudaimonistischen Perspektive wird danach gefragt, was ein gutes Leben ausmacht. Hierbei steht weder die pragmatische Frage im Vordergrund, wie Menschen ihr Leben gestalten sollten, sprich welche Ziele sie wählen und welche Mittel sie einsetzen sollten, um ein gutes Leben zu erreichen oder führen zu können, noch die moralische Frage, welchen moralischen Grundregeln, Prinzipien, Maximen und Geboten ein gutes Leben folgen sollte (Hübenthal 2006:82). Hierbei geht es vielmehr um die spezielle Frage, wie das (menschliche) Leben bzw. die Art und Weise der Lebensführung überhaupt als gut bewertet und beurteilt werden kann, welche Haltungen und Handlungsweisen 'glückskonstitutiv` sind und welche (Grund-)Güter, Befähigungen und Ermöglichungen es braucht, damit davon die Rede sein kann, das ein Leben gelingend ist. Insofern es geht es hier um die Art und Weise der Lebensführung, der authentischen Selbstinterpretation und der spezifischen Identität eines Menschen, Hierbei geht es folglich nicht um schwache Wertungen, wie beim pragmatischen Gebrauch der praktischen Vernunft, aber auch nicht um moralische Wertungen von Absichten und Motiven bzw. moralischen Beurteilungen von Handlungen als richtig, sondern es geht hier um starke Wertungen, $d$.h. die qualitative Bewertung der Ziele selbst. Hieran wird ausgeführt, a) um welche Art von Reflexionsleistung es sich beim eudaimonistischen Gebrauch der praktischen Vernunft handelt und b) welcher Sollgeltungsanspruch mit dem eudaimonistischen Sollstyp einhergeht:

a) Über starke Wertungen wird, wie bereits ansatzweise bei der Darstellung schwacher Wertungen ausgeführt, eine "Sprache wertender Unterscheidungen“ entfaltet, die mit kontrastiven Beschreibungen arbeitet (Taylor 2006:4). Da sich Begriffe starker Wertungen nur im Verhältnis zu ihren Gegenbegriffen verstehen lassen (bspw. fleißig/ faul, mutig/feige, integriert/fragmentiert, tief/oberflächlich), kann immer nur in Kontrast zu dem, was unerwünscht ist, zum Ausdruck gebracht werden, was an der bevorzugten Alternative wirklich wünschenswert ist (ebd., 11). Hierüber wird ein nicht nur sprachlich bedeutsamer Unterschied zu den schwachen Wertungen deutlich: Taylor stellt heraus, dass der entscheidende Punkt bei starken Wertungen in der Gegenüberstellung der unterschiedlichen Arten des Selbst besteht, mit denen sie jeweils verknüpft sind. Taylor versucht über die starken Wertungen plausibel zu machen, dass Menschen Wesen sind, deren authentische Wertungen qualitativer Natur sind (ebd., 8). Wenn im Fall starker Wertungen gerade nicht zwischen kontingenten, voneinander unabhängigen Alternativen in einem bloß quantitativen Sinne abgewogen wird, sondern es darum geht, zwischen nicht-kontingenten, kontrastiven Alternativen zu unter- 
scheiden und zu entscheiden, ${ }^{139}$ dann weil es bei starken Wertungen darum geht, was Menschen als handelnde Wesen ausmacht, auf Basis welcher Motivationen sie handeln und inwiefern hinter unseren Motivationen und Handlungen authentische Selbstinterpretationen und bestimmte Vorstellungen von Lebensqualität stehen:

"Es geht [bei starken Wertungen] um die Frage, worin tatsächlich unsere Motivation besteht, wie wir in Wahrheit die Bedeutung der Dinge für uns beschreiben sollten. Es handelt sich um einen Konflikt der Selbstinterpretationen. (...) Einen Wunsch oder eine Neigung als wertvoller, edler oder ausgeglichener als andere zu bezeichnen heißt, von ihm in Kategorien der Art von Lebensqualität zu sprechen, die er ausdrückt und aufrechterhält. (...) Während es für das schwach wertende Subjekt um die Erwünschtheit unterschiedlicher Ziele geht, die durch seine De-facto-Wünsche definiert werden, untersucht das Nachdenken des stark wertenden Subjekts auch die verschiedenen möglichen Seinsweisen des Handelnden. Motivationen oder Wünsche zählen nicht nur aufgrund der Anziehungskraft der Ziele, sondern auch aufgrund der Lebensweise und des Subjekttypus, denen diese Wünsche eigentlich entsprechen. (...) [D]a wir nunmehr über unsere Wünsche unter dem Gesichtspunkt der Frage nachdenken, welche Art von Wesen wir sind, wenn wir diese Wünsche haben oder realisieren (...), führt ein Nachdenken darüber, welche Art von Wesen wir sind, uns direkt ins Zentrum unser Existenz als Handelnde." (ebd., 7, 8,9,10)

Im Anschluss daran stellt Taylor prägnant heraus, dass Motivationen und/oder Wünsche nicht nur aufgrund der Anziehungskraft der Ziele zählen (schwache Wertung), sondern diese sind immer auch aufgrund der Lebensweise und des Subjekttypus relevant, denen diese Wünsche und/oder Motivationen eigentlich entsprechen (ebd., 9). Insofern sieht Taylor die Aneignung und Erlernung einer stark wertenden Sprache als Bedingung dafür, die eigenen Präferenzen besser artikulieren zu könne, sprich zu einer besser artikulierbaren Reflexion seiner Selbst imstande zu sein (ebd.). Da unsere Identität als selbst-reflektives, handelndes, wertendes und beurteilendes Individuum durch starke Wertungen definiert wird, bedeutet die Ablehnung der Relevanz starker Wertungen, sich selbst nicht authentisch wahrnehmen zu können, da starken Wertungen

139 Ein Beispiel: Wenn man vor der Wahl steht, ob man seinen freien Abend allein zuhause verbringt, weil man nach einer langen Arbeitswoche Ruhe braucht oder ob man ausgeht und sich mit Freunden trifft, weil man diese schon seit Wochen nicht gesehen hat, wählt man unter pragmatischen Gesichtspunkten diejenige Alternative aus, die seinen momentanen Bedürfnissen bzw. den jeweiligen Sachlagen am ehesten entspricht. Im Falle starker Wertungen steht man indes vor der Alternative, ob man sich selbst eher als einen geselligen oder 'eigenbrödlerischen 'Typ sieht und wenn man sich aus seinem Selbstverständnis als geselliger Typ mit der Freundin trifft, dann heißt dies im Umkehrschluss, dass man die Alternative den Abend alleine zu Hause zu verbringen grundsätzlich ablehnt, weil dies schlicht nicht dem eigenen Selbstverständnis entspricht. In einem vergleichbaren Fall, würde man daher nicht zuhause bleiben, wenn die Freunde nicht können, sondern man würde allein ausgehen und ggf. neue Menschen kennenlernen. 
eine 'tiefe' Reflexion darüber vorausgeht, was wir für Menschen sein wollen. Insofern sind starke Wertungen und die Vorstellung von Identität eng miteinander verwoben, da unsere Identität durch unsere fundamentalen Wertungen definiert wird:

„Handelnde, die starke Wertungen vornehmen, [können] als tief bezeichnet werden (...), weil das, was für sie ausschlaggebend ist, nicht nur die gewünschten Ziele, sondern auch ihre Lebensweise, ihre Natur als Handelnde umfaßt. Dies ist eng mit der Vorstellung von Identität verknüpft. Mit "Identität" meine ich diejenige Verwendungsweise des Begriffs, von der wir Gebrauch machen, wenn wir davon sprechen, 'seine Identität zu finden` oder eine 'Identitätskrise`zu durchleben. (...) In jedem Falle ist das Konzept der Identität verknüpft mit dem bestimmter starker Wertungen, die untrennbar mit mir verbunden sind. Dies ist entweder der Fall, weil ich mich selbst aufgrund meiner starken Wertungen als jemand begreife, der diese Überzeugungen aufgrund seines Wesens besitzt, oder aber weil ich erkenne, daß bestimmte meiner sonstigen Eigenschaften nur möglich sind aufgrund einer Art starker Wertung durch mich selbst, weil diese Eigenschaften so entscheidend das berühren, was mich als Handelnden ausmacht, das heißt, als ein stark wertendes Wesen, daß ich sie nicht allesamt vollständig zurückweisen kann. Denn dann würde ich mich selbst zurückweisen, wäre innerlich zerrissen und folglich unfähig zu wirklich authentischer Wertung." (ebd., 16-17)

Um indes seine eigene authentische Identität zu finden bzw. wiederherzustellen, muss man sich oftmals zwischen inkommensurablen Alternativen im Sinne eines kognitiven, emotionalen und motivationalen 'Streits' bezüglich der eigenen Selbstinterpretation entscheiden. Die Befähigung sich für eine dieser Seiten begründet zu entscheiden, ist daher eine wesentliche Bedingung der (Wieder-)Herstellung der Kommensurabilität bzw. Authentizität, wobei nach Taylor die entscheidende Frage hierbei ist, „welches die wahrere, authentischere, illusionsfreiere Interpretation ist und welche auf der anderen Seite zu einer Verzerrung der Bedeutung führt, die die Dinge für mich besitzen“ (ebd. 11). Dabei ist indes zu beachten, dass die Art der Formulierung starker Wertungen innerhalb eines kontrastiven Vokabulars dazu führt, dass hierüber unser Verständnis dafür geformt wird, was wir wünschen oder in einer bestimmten Weise für wichtig und erstrebenswert erachten (ebd. 18). Daher sind starke Wertungen essentiell für unsere Selbstinterpretationen, die wiederum für unsere Erfahrungen konstitutiv sind: ${ }^{140}$

${ }^{140}$ Die Feststellung, dass die Selbstinterpretationen erfahrungskonstitutiv sind, sagt noch nichts darüber aus, wie der Wandel sowohl der Beschreibungen unseres Selbst als auch der Erfahrung zustandekommt (Taylor 2006:19), da die bloße Feststellung dieses Zusammenhangs Interpretationen in beide Richtungen zulässt: Einerseits kann man seine Erfahrungen dadurch verändern, indem man zu neuen Einsichten über sich selbst, sprich zu einer anderen Selbstinterpretation gelangt. Andererseits prägen die Erfahrungen, die man macht auch das Bild von einem selbst und das Selbstverständnis. 
„Wir können sagen, daß unsere Selbstinterpretationen teilweise für unsere Erfahrung konstitutiv sind. Es kann nämlich sein, daß eine veränderte Beschreibung unserer Motivationen untrennbar mit einer Veränderung dieser Motivation selbst verknüpft ist. Die Behauptung dieses Zusammenhangs bedeutet nicht, eine kausale Hypothese aufzustellen: sie besagt nicht, daß wir unsere Beschreibungen ändern und daß sich dann als Resultat unsere Situationserfahrung ändert. Es handelt sich eher darum, daß bestimmte Erfahrungsmodi ohne bestimmte Selbstbeschreibungen nicht möglich sind." (ebd. 19)

Wenn unsere Selbstbeschreibungen auf bestimmten Erfahrungsmodi basieren und eine veränderte Beschreibung unserer Motivationen zu einer Veränderung dieser führen kann, dann bedeutet dies wiederum, dass starke Wertungen - anders als schwache Wertungen - nicht vollkommen willkürlich sein können, ${ }^{141}$ denn es ist nicht nur für einen selbst ersichtlich, dass es mehr oder weniger adäquate, mehr oder weniger aufrichtige, eher zur Selbsterkenntnis oder zur Selbst-Illusionierung tendierende Interpretationen der eigenen Identität gibt. Insofern ist das Vokabular starker Wertungen etwas, das Menschen miteinander teilen, gerade weil es hierbei um das Selbstverständnis von Menschen als Menschen geht (Habermas 1991b:103-104). Dies wiederum bedeutet, rückgekoppelt an den eudaimonistischen Zugang zur praktischen Vernunft, dass die Fragen des guten Lebens, die mit starken Wertungen korrespondieren, einer intersubjektiven Überprüfbarkeit zumindest prinzipiell zugänglich sind, denn wir alle würden sagen, dass es in Bezug auf unser Lebensglück im Prinzip besser ist, großzügig zu sein als gierig und knauserig, dass Toleranz besser als Intoleranz und Lieben besser als Hassen ist. Auch haben wir alle eine ungefähre wenn auch nicht unbedingt deckungsgleiche Vorstellung davon, was damit jeweils gemeint ist und was nicht.

b) Gemäß der oben vorgenommenen Charakterisierung eudaimonistischer Überlegungen und Erwägungen anhand starker Wertungen, muss auch hier bezüglich der noch vorzunehmenden Charakterisierung des Verbindlichkeitsgrades eudaimonistischer Imperative wiederum das Verhältnis zwischen Wollen und Können und deren Verhältnis zum Sollen genauer untersucht werden, um wiederum den Sollgeltungsanspruch eudaimonistischer Handlungsaufforderungen bestimmen zu können: Anders als beim pragmatischen Handeln, bei dem ein recht simples weil nicht aufeinander verweisendes Verhältnis von Wollen und Können festgestellt wurde (das Wollen ist bis auf we-

${ }^{141}$ Ein Beispiel: Wenn jemand sagt, dass ein Urlaub auf den Malediven besser ist als Urlaub am Nordkap, dann kann er dafür zwar Gründe anführen, dass man auf den Malediven bspw. tolle Strände hat, exotische Früchte, tolles Wetter usw. und gegen das Nordkap anführen, dass es da immer kalt ist und man nur Schnee sieht usw. Jemand anderes könnte dagegen einwenden, dass man nur am Nordkap das Wetterleuchten sieht und nur hier in einem Eishotel übernachten kann und dass zu viel Sonne schädlich für die Haut ist und er gegen alle exotischen Früchte allergisch ist. Hier ist folglich keine gemeinsame Basis vorhanden, auf der die Bewertung stattfindet, da jeder seine ganz individuelle Sicht auf beide Alternativen hat. 
nige prinzipielle Ausnahmen nahezu unabhängig vom Können und das Können beeinflusst ebenfalls nicht die Art unserer Ziele und Wünsche), sind die Machbarkeitsgrenzen des eudaimonistischen Wollens sehr viel difiziler. Aufgrund der engen Kopplung zwischen bestimmten Erfahrungsmodi, Identitätsbildungsprozessen und darauf basierenden Selbstinterpretationen ist die gewollte Überbrückung des 'Grabens' zwischen dem, wie man (geworden) ist, und dem, was man für sich selbst will, zumeist schwer zu erreichen. Um dies exemplarisch zu verdeutlichen, stelle man sich folgende, sicherlich arg trivialisierte und 'küchenpsychologische' Szenerie vor:

Ein Schwerverbrecher, der die ersten dreißig Jahre seines Lebens damit zugebracht hat zu stehlen, zu kämpfen, andere zu verletzen und umzubringen, lernt eine Frau kennen, in die er sich verliebt und durch die er den Impuls bekommt, ein besserer Mensch werden zu wollen. Nach einer gewissen Zeit beginnt er allerdings, geprägt durch seine oftmals negativen Erfahrungen mit anderen Menschen, ihr zu misstrauen, weil er sich nicht vorstellen kann, dass eine so freundliche und liebevolle Frau, die alles ausmacht, was er selbst gerne wäre, ihn, der das alles nicht hat, wirklich lieben kann. Geplagt von Selbstzweifeln und nicht fähig, diese zu artikulieren, streitet er sich immer öfter mit ihr, macht ihr Vorwürfe und beleidigt sie. Daraufhin fängt sie an, ihn ebenfalls zu kritisie-ren. Das bestätigt inn wiederum darin, dass er ein schlechter und sie ein guter Mensch ist. Frustriert über diese 'selbstproduzierte' Einsicht, fällt er partiell in alte Gewohn-heiten zurück. Es kommt zu noch mehr Streit, der Vorwurf ihrerseits steht im Raum, dass er ein Lügner ist, weil er sich doch ändern wollte. Er wiederum fühlt sich zu-rückgewiesen und unverstanden, sucht Sicherheit in noch mehr alten Gewohnheiten und die Beziehung zerbricht langsam aber sicher, so dass er erneut mit der Erfahrung konfrontiert ist, dass er nun mal ein schlechter Mensch und der Liebe von anderen nicht würdig ist, so dass er erneut zu der Schlussfolgerung gelangt, dass er andere auch nicht zu lieben, zu achten und zu respektieren braucht.

An vielen derartigen Beispielen ließe sich exemplarisch aufzeigen, dass der Weg hin zu einem besseren Selbst keine momentane und spontane Angelegenheit wie bei pragmatischen Entscheidungen sondern eine langwierige und stetige Aufgabe ist, bei der man allein nur bedingt Chancen auf Erfolg hat, weil es eben auch immer andere Menschen braucht, die einem helfen zu beurteilen, was gut und schlecht ist und ob und wann man der Mensch ist, der man sein will. Des Weiteren zeigt sich daran, dass alle Versuche zu einem besseren Selbst zu gelangen, mit identitätsgefährdenden Momenten verbunden sind, da man selbst nie von dem abstrahieren kann, wie man (geworden) ist, sprich, einem selbst nur der oftmals unsichere Weg offensteht, auf Basis der alten neue Erfahrungen, Sichtweisen und Selbstinterpretationen zuzulassen und zu internalisieren, was eine - nicht spezifisch therapeutisch gesehene - tiefenpsychologische Analyse des eigenen Ichs verlangt. 
Bezogen auf das Verhältnis von Wollen und Können zum Sollen bedeutet die beim eudaimonistischen Gebrauch der Vernunft deutlich engere Kopplung von Wollen und Können, dass man anderen, genau wie bei pragmatischen Ratschlägen, nur das Angebot machen kann, auf alternative Perspektiven für das gute Leben zu reflektieren. Indem der eudaimonistische Gebrauch der praktischen Vernunft die Handlungsaufforderung enthält, dass man bestimmten Motivationen nachgehen, bestimmte Verhaltensweisen kultivieren und bestimmte Handlungen vollziehen soll, um von sich selbst als 'guter Mensch' zu sprechen, wird über den eudaimonistischen Sollstyp aufgezeigt, welche Werte und Güter für einen selbst und für andere wichtig für ein gutes Leben sind sowie welche Tugenden es auszuprägen, zu kultivieren und zur Anwendung zu bringen gilt, um dem Ideal des guten Lebens nahe zu kommen. Dabei ist das eudaimonistische Sollen genau wie das pragmatische Sollen ein vom Wollen abhängiges Sollen, da auch dieses abhängig von den Zielen und Präferenzen ist, wie Menschen sich ihr gutes Leben vorstellen (Ott 1993:103). Andererseits ist das eudaimonistische Sollen anders als das pragmatische Sollen aufgrund der dabei vorgenommenen starken Wertungen nicht von kontingenten Zielen und Mitteln abhängig, da die Ziel- und Mittelwahl hier in einem größeren Kontext bzw. auf einer 'tieferen` Reflexionsebene beurteilt wird. Während die Zweckdienlichkeit von pragmatischen Handlungsweisen an situations- und kontextspezifischen Wünschen bzw. Einzelhandlungen oder Handlungstypen festgemacht werden kann, ist für die Zuschreibung von Authentizität und der 'Güte' eines Menschen mehr als die Kenntnis isolierter Einzelhandlungen erforderlich. Denn die Frage, ob sich ein Mensch gemäß seiner Selbstinterpretation verhält und ob diese Selbstinterpretation der eines guten Menschen entspricht, verlangt mehr als die Betrachtung eines einzigen Augenblicks. Insofern appelliert man bei eudaimonistischen Ratschlägen an das Selbstverständnis von Menschen, bei denen wir die Grenzen der Einsicht rückbeziehen auf ein Urteil über sie als gute oder schlechte Menschen im moralischen Sinne (Taylor 2006:20). ${ }^{142}$

Was hierbei zentral zu sein scheint, ist der Gedanke, dass starke Wertungen bezüglich unserer Selbstinterpretation stets anfechtbar sind und dass uns daher auch die moralische Verantwortung zukommt, diese als revidierbar zu betrachten, so dass man sich

${ }^{142}$ Auch dies kann beispielhaft expliziert werden: Taylor führt hier das Beispiel eines (religiösen) Fanatikers an, der in einer Sekte aufgewachsen ist und die dort vermittelten Inhalte unhinterfragt übernimmt, so dass er gar nicht sieht, was er sich selbst und anderen mit seiner Sicht auf die Welt antut: „Diese früheren Erfahrungen sind für den Zustand ihrer gegenwärtigen Erfahrung verantwortlich, in der diese Probleme als trügerisch und bedeutungslos erscheinen, und dieser gegenwärtige Zustand macht es ihnen unmöglich, die Einsichten zuzulassen, die wir ihnen nahezubringen versuchen. Sie können sie nicht anerkennen, ohne dass ihre ganze Haltung den Dingen gegenüber zerbröckelte, und diese Einstellung ist für sie möglicherweise von größter Wichtigkeit." (Taylor 2006:20) Obgleich wir also berücksichtigen, dass Menschen nicht ohne Grund sind, wie sie sind, würden wir einen Fanatiker dennoch als schlechten Menschen bezeichnen, da wir ihn für moralisch verantwortlich halten, sich für Revisionen seiner Selbstinterpretation offen zu halten. 
selbst immer wieder fragen muss, wer man sein will und was ein gutes Leben wirklich ausmacht. Wer sich hierbei alternativen Lebensentwürfen kategorisch versperrt und seine eigenen Ansichten für 'die ganze Wahrheit' hält, der hat eben den Sinn dieser Verantwortung nicht verstanden. Dies zeigt bereits, dass beim eudaimonistischen Sollen, ebenfalls im Unterschied zum pragmatischen Sollen, nicht in einem rein subjektiven Sinn von gut oder schlecht, besser oder schlechter, richtig oder falsch die Rede ist, da das eudaimonistische Sollen nicht ausschließlich an der Willkür des Subjekts ausgerichtet ist: Ob man selbst ein guter Mensch ist, ist nicht allein eine Frage meiner persönlichen Selbsteinschätzung und -beurteilung, sondern aufgrund der intersubjektiven Zugänglichkeit starker Wertungen in hohem Maße davon abhängig, ob andere meinen, dass man selbst wirklich derjenige ist, der man vorgibt zu sein. Eben dieses Moment der 'Selbstoffenheit' ist bei allen Lebensstilen universalisierbar: Wenn man jemanden nahelegt, er solle aufhören immer gleich das Schlimmste zu erwarten und er dies zurückweist mit der Begründung, dass er noch nie Glück hatte im Leben, dass alles was er anpacken würde immer schief ginge und dass er gar nicht wisse, wie es sei wirklich glücklich zu sein und man ihm daraufhin aufzeigt, welche Dinge bisher in seinem Leben gut gegangen sind, wann er Glück hatte und wann er sich, seinem eigenen Vernehmen nach, glücklich gefühlt hat, er dies aber abstreitet, weil es zu seinem Selbstbild gehört, dass er das 'Opfer' ist, dann versperrt er sich kategorisch für alle gegenteiligen Evidenzen, obwohl ihm die Möglichkeit offen stehen würde, diese zu revidieren.

Zusammenfassend zeigt sich hier folglich, dass eudaimonistische Ratschläge einen deutlich präskriptiveren Gehalt haben als pragmatische Ratschläge, da man prinzipiell alle Menschen darauf verpflichten kann, auf ihre Selbstinterpretation und ihre Erfahrungen zu reflektieren, weil wir eben davon ausgehen, dass Menschen prinzipiell dazu in der Lage sind und in der Lage sein sollten, auf sich selbst zu reflektieren, auch wenn wir anerkennen, dass es weitreichende Machbarkeitsgrenzen solcher Selbstveränderungen gibt, die in manchen Fällen (bspw. bei Menschen mit schweren Traumata) dem Weg zu einem besseren Menschen rigide entgegenstehen können. Das ist auch der Grund dafür, weswegen man, obwohl hier von Verpflichtung die Rede ist, niemanden dazu zwingen kann, selbstreflektiv zu sein. Das Argument, dass sich nur diejenigen Lebensstile und Selbstinterpretationen als gelungen und wahrhaft gut auszeichnen lassen, bei denen mit Gründen und anhand von Motivationen darlegt werden kann, warum man sich so und nicht anders entschieden hat zu leben und zu sein, ist lediglich eine Minimalbedingung für die Plausibilität eudaimonistischer Erwägungen und Ratschläge: Wer seine 'Innenansichten `vor sich selbst und vor anderen plausibel darlegen und ausweisen kann, der erfüllt zwar die Minimalbedingung der Authentizität für die Anerkennung seines Lebensstils, er sichert damit aber nicht schon hinreichend ab, dass er sich auch gemäß seiner 'Innenansichten `verhält. 
Einmal abgesehen von den schwierigen Fragen, wie die Brücke zwischen Einsichten und Handeln geschlagen werden kann und inwiefern nicht das tatsächliche Handeln und nicht die hehren Absichten ausschlaggebend für die Beurteilung des guten Lebens ist, zeigt sich an den bisherigen Ausführungen, dass plausible eudaimonistische Ratschläge, genau wie das bei pragmatischen Ratschlägen der Fall ist, immer nur solche sein können, die sowohl die eigenen Lebensumstände, Möglichkeiten und Zwänge als auch die der anderen berücksichtigen und diese einkalkulieren, aber mit der entscheidenden Eingrenzung, dass man hierbei bereits voraussetzt, dass nur diejenigen Lebensstile wirklich gut sind, bei denen prinzipiell die Möglichkeit besteht, dass diese auch für Revisionen und Veränderungen offen sind. Insofern hat der Sollgeltungsanspruch des eudaimonistischen Sollens, obgleich das eudaimonistische Sollen genau wie das pragmatische Sollen bedingt und damit nicht-kategorisch ist, ein universalistisches Moment. Dies klingt erst einmal paradox, weil in der Regel die Bedingtheit des Sollens und das Hypothetische des Sollgeltungsanspruchs einerseits und die Unbedingtheit des Sollens und die Kategorizität des Sollgeltungsanpruchs andererseits über die Differenzierung zwischen moralisch und deshalb universalisierbar und nicht-moralisch und deshalb nicht-universalisierbar einander gegenübergestellt werden. Die Verbindung zwischen Bedingtheit und Universalisierbarkeit, die hier für den eudaimonistischen Sollgeltungsanspruch als charakteristisch herausgestellt wird, lässt sich aber plausibilisieren, wenn man mit Birnbacher und (gegen Kant) annimmt, dass man kategorische Imperative nicht bereits mit moralisch berechtigten Imperativen gleichsetzen muss. ${ }^{143}$ Eben diese Annahme ist auch, und im Besonderen, relevant, wenn es im Folgenden um den moralischen Gebrauch der praktischen Vernunft geht.

\subsection{Zum moralischen Gebrauch der praktischen Vernunft}

Wenn die allgemeinen praktischen Fragen unter dem Aspekt des moralischen Gebrauchs der praktischen Vernunft betrachtet werden, dann steht die Frage im Raum, wie zu handeln falsch (verboten oder pflichtenwidrig), akzeptabel (erlaubt) und richtig (geboten oder pflichtgemäß) ist. ${ }^{144}$ Wenn man diese Fokusverschiebung erst einmal nur rein begrifflich betrachtet, verlässt man damit das 'Terrain` der eudaimonistischen Reflexion, weil es hierbei nicht mehr darum geht, was das Gute ist, sondern um die

${ }^{143}$ Birnbacher führt aus, dass kategorische Urteile Handlungen unabhängig davon bewerten, ob diese den Zwecken des Akteurs entsprechen (Birnbacher 2007:20). In diesem Sinne müssen kategorische Imperative nicht auch schon allgemeinverbindlich und universalisierbar sein, da sich beide Kriterien weder aus der Kategorizität selbst ergeben (ebd., 23) noch aus dem Wesen des Imperativs als Handlungsaufforderung (siehe auch 4.2.2.1.3), da Imperative, wie hier gezeigt wird, sowohl pragmatischer, eudaimonistischer und moralischer Natur sein können.

144 Den allgemein akzeptierten begrifflichen Bestimmungen nach, ist etwas genau dann erlaubt, wenn es nicht geboten ist, den Sachverhalt A nicht herbeizuführen bzw. wenn es nicht geboten ist, die Herbeiführung von $\mathrm{A}$ zu unterlassen. Etwas ist demgegenüber genau dann verboten, wenn es nicht geboten ist, den Sachverhalt A oder dessen Herbeiführung zu unterlassen (Ricken 2003:89). 
Auslotung dessen, was das Falsche/Akzeptable/Richtige ist. Damit verbunden ist die Frage, welche Regeln und Prinzipien allgemeinverbindlichen gelten und welche Pflichten und Rechte Menschen gegenüber allen anderen Moralsubjekten (sowohl gegenwärtige als auch zukünftige Menschen und ggf. auch nicht-menschliche Entitäten) haben sollten. Insofern zeichnen sich moralische Erwägungen vor allem dadurch aus, dass hierbei die Beachtung des moralischen Status aller Moralsubjekte maßgeblich ist. Dabei geht es einerseits um die moralische Bewertung der Motive, Absichten, Einstellungen und Charakterzüge von Personen, die danach bewertet werden, ob und inwieweit diese sich bereits moralisch bewährt haben oder Aussichten darauf begründen, sich bei passender Gelegenheit moralisch zu bewähren. ${ }^{145}$ Andererseits geht es aber auch und vor allem - um die moralische Beurteilung der Handlung und ihrer Folgen hinsichtlich ihrer Verallgemeinerbarkeit. Hierbei ist entscheidend, dass moralische Bewertungen und Beurteilungen mit dem Anspruch einhergehen, dass alle Menschen diese prinzipiell als gültig anerkennen können, auch wenn sie davon nicht selbst betroffen sind (bspw. wenn es um moralische Fragen in Bezug auf zukünftige Menschen geht) oder sich nur schwer bzw. unmöglich in die Lage derjenigen versetzen können, für die solche Regelungen gelten sollen (bspw. im Fall der Anwendung auf nicht-menschliche Entitäten). In Aufnahme dessen wird auch hier ausgeführt, a) welche Art von Reflexionsleistung der moralische Gebrauch der praktischen Vernunft erbringt und b) welcher Sollgeltungsanspruch mit dem moralischen Sollen einhergeht:

a) Das Spezifikum der moralischen Gebrauchsweise der praktischen Vernunft zeigt sich an der Prüfung der eigenen Maximen mit der Vereinbarkeit der Maximen anderer. ${ }^{146}$ Insofern es bei der moralischen Reflexion folglich um die Prüfung der Verallgemeinerbarkeit geht, wird bei der moralischen Betrachtungsweise der praktischen Fragen konsequent mit der egozentrischen Perspektive gebrochen, die für die pragmatischen und eudaimonistischen Erwägungen gleichermaßen als konstitutiv herausgestellt wurde (auch wenn dargelegt wurde, dass man sich dabei durchaus auch in andere hinein-

${ }^{145}$ Betrachtet man für den moralischen Gebrauch der praktischen Vernunft lediglich den Aspekt der moralischen Bewertungen (siehe 4.2.2.1.3), dann bestünde zwischen dem moralischen und dem eudaimonistischen Zugang zu praktischen Fragen kein wesentlicher Unterschied, weil das, was hier mit moralischen Wertungen gemeint ist, das ist, was im Eudaimoniekontext unter dem Begriff der starken Wertung eingeführt wurde, allerdings mit dem wesentlichen Unterschied, dass es im Fall moralischer Wertungen stärker um den Aspekt geht, welche Handlungsmotive verallgemeinerbar und universalisierbar sind, während es bei starken Wertungen im Eudaimoniekontext um den Aspekt geht, welche Wertungen einen Einfluss auf die Identitätsbildung und -konstitution haben, woraufhin Rückschlüsse gezogen werden, welche Seinsweisen auch auf gute Lebensstile führen.

146 Maximen können hier (anders als allgemeine Prinzipien) als situationsnahe Handlungsregeln verstanden werden, nach denen sich die Praxis des Handelns des Einzelnen gewohnheitsmäßig ausrichten. Sie regeln den Tagesablauf, den Umgangsstil, die Art und Weise Probleme anzugehen, Konflikte zu lösen usw. Maximen bilden damit die Schnittstelle zwischen eudaimonistischen und moralischen Überlegungen und Erwägungen, weil sie gleichzeitig unter strebensethischen und pflich-enethischen Gesichtspunkten beurteilt werden können (vgl. Habermas 1991b:106-107). 
versetzen muss), da es hier darum geht, was man selbst für zweckdienlich oder gut erachtet. Der moralische Standpunkt verlangt indes die grundlegende Abstraktion vom eigenen Selbst, die Einnahme einer überindividuellen Bewertungs- und Beurteilungsperspektive und einen unparteiischen Standpunkt, weil es unter moralischen Gesichtspunkten darum geht, welche Pflichten und Rechte allen moralischen Subjekten gleichermaßen zukommen sollten. Entscheidend dabei ist, dass moralische Imperative (bspw. die Forderung, dass kein Mensch einen anderen töten sollte) weder von spezifischen Situationen (wie bspw. Armut) oder persönlichen Befindlichkeiten (wie bspw. Rache) abhängig sind und darüber relativiert oder gar neutralisiert werden können (Ott 1993:104). Sobald man nämlich den Anspruch an sich selbst und an andere stellt, dass das eigene und das fremde Handeln verallgemeinerbar sein soll, wird das Sollen mit Bezug auf das, was alle Moralsubjekte unabhängig von ihren konkreten Zielen, die sie erreichen wollen sowie den Mitteln, die ihnen zur Verfügung stehen, bestimmt (Habermas 1991b:106-108).

In diesem Sinne sind moralische Imperative, im genauen Gegenteil zu pragmatischen, unbedingt und zugleich kategorisch, da sie alle Moralsubjekte anweisen, dass sie diejenigen Maximen verfolgen und in ihrem Handeln berücksichtigen sollen bzw. beim Handeln der anderen einfordern können, die gerecht sind. Ob das, was dabei jeweils als gerecht tituliert und ausgewiesen wird, auch tatsächlich gerecht ist, so dass berechtigterweise alle wollen sollten, dass daraus Pflichten und Rechte erwachsen, die für alle gleichermaßen gelten, zeigt sich indes erst anhand der Überprüfung der Gerechtigkeitsvorstellungen auf ihre tatsächliche Verallgemeinerbarkeit. ${ }^{147}$ Erst wenn ausgewiesen werden kann, dass es sich bei den moralischen Imperativen, die kategorisch gelten sollen, auch um moralisch berechtigte, sprich tatsächlich geltende Imperative handelt und wenn diesen berechtigten moralischen Imperativen dann zuwider gehandelt wird, dann ist dies keine bloß unkluge oder dem guten Leben abträgliche sondern eine unmoralische Handlung, da man sich für ein solches Handeln nicht nur vor sich selbst sondern auch vor anderen verantworten muss.

In diesem Sinne gibt es zahlreiche kategorische Imperative (du sollst nicht töten, auch wenn du jemanden noch so sehr hasst; du sollst nicht stehlen, auch wenn du es dringend brauchst; du sollst andere nicht körperlich oder verbal verletzten, auch wenn andere dasselbe mit dir getan haben etc.). ${ }^{148}$ Die hierbei relevante Frage, warum man

147 Die anknüpfende Frage, wie eine solche Verallgemeinerungsregel als oberste Moralregel selbst begründet werden kann, ist keine, im allgemeinen Sinne moralische Frage, sondern bereits eine 'metamoralische'. Ich vermeide hier bewusst den Terminus metaethisch, weil ich aus später nachvollziehbaren Gründen nicht möchte, dass Ethik einseitig auf Prinzipien- und Pflichtenethik beschränkt wird (siehe 4.3.2.3).

${ }^{148}$ Kategorizität bedeutet indes nicht Objektivität (siehe 4.2.2.1.3), sprich, dass sich moralische Regeln 'im luftleeren Raum ' und in Abstraktion von allen Umständen aufstellen lassen, da erst anhand der jeweiligen Umstände (zu denen auch die Absichten und Gründe des Handelnden und die Folgen der 
sich aus moralischen Gründen so und nicht anders verhalten sollte, sprich warum es falsch/akzeptabel/richtig sein sollte, so zu handeln, so dass derartige Maximen zu verbindlichen Pflichten und Rechten für alle werden sollten, steht im Vordergrund der moralischen Reflexion. Was folglich die moralische Gebrauchsweise der praktischen Vernunft - auch gegenüber pragmatischen und eudaimonistischen Gebrauchsweisen auszeichnet, ist die für den Moralkontext spezifische Reflexion darauf, ob Handlungsmotive und Handlungen verallgemeinerbar sind, unabhängig davon, ob die daraus erwachsenden Pflichten und Rechte im Interesse einzelner liegen. Dies schließt nicht aus, dass auch pragmatische oder eudaimonistische Imperative im Prinzip verallgemeinerbar sein können, obgleich es sich hierbei um bedingte, d.h. an die Ziele, Zwecke und Interessen von Individuen gebundene, Bewertungen und Beurteilungen handelt. Vielmehr ist es so, dass sich die Verallgemeinerbarkeit im Fall moralischer Imperative bereits aus der Einnahme des moralischen Standpunkts, der spezifisch für die moralische Gebrauchsweise der praktischen Vernunft ist und das moralische Handeln kennzeichnet, ergeben soll.

b) Wenn die für die moralische Gebrauchsweise der praktischen Vernunft spezifischen Momente des moralischen Standpunkts, der Kategorizität moralischer Imperative und Handlungsbeurteilungen sowie die Prüfung auf Verallgemeinerbarkeit auf die Bestimmung des Sollgeltungsanspruchs des moralischen Sollens angewendet wird, dann gelangt man wiederum zu einer spezifischen Bestimmung der Verhältnisse von Wollen, Können und Sollen. Bezüglich des Verhältnisses von Wollen und Sollen ist festzuhalten, dass die Geltung moralischer Imperative auch dann eingefordert wird, wenn diese dem Handelnden nicht nützlich im Rahmen seiner individuellen Interessenverfolgung sind und analog dazu werden unmoralische Handlungen auch missbilligt, wenn sie dem Handelnden zweckdienlich sind. Insofern wird das moralische Sollen zu einem vom individuellen Wollen unabhängigen muss: Anders als das pragmatische und eudaimonistische Sollen, bei denen sich der Sollenstyp als Ratschlag, als Appell an die Vernunft bzw. an den Sinn darstellt, geht es hierbei um eine Pflicht im moralischen und kategorischen Sinn nicht als das, was getan werden sollte, um ein bestimmtes Ziel zu erreichen (Pflicht im hypothetischen Sinn), sondern als das, was verpflichtend gegenüber allen anderen getan werden muss unabhängig von persönlichen Interessen und Zielen. Insofern ist das Sollen - genau umgekehrt zum pragmatischen Sollenstyp - unabhängig vom Wollen. Vielmehr ist es hier so, dass sich das Wollen immer an dem orientieren, aus dem ergeben und dem Rechnung tragen soll, was moralisch gesollt wird.

Handlung zählen) bestimmt werden kann, was in konkreten Handlungssituationen praktisch notwendig und gesollt ist (Ricken 2003:136). Insofern ist die verbreitete Annahme, dass moralische Fragen unter objektiven Gesichtspunkten beurteilt werden könnten, irreführend (ebd., 90). 
In Bezug auf das Verhältnis von Können zum Sollen ist des Weiteren zu sagen, dass das moralische Sollen grundsätzlich dadurch begrenzt wird, das nur das gesollt werden kann, was auch gekonnt wird. Daher haben moralische Imperative entscheidende und komplexe Machbarkeitsgrenzen, denn moralische Beurteilungen von Handlungsmotiven, Handlungen und Handlungsfolgen sind nur dann möglich, wenn Handelnde die Möglichkeit und die Freiheit haben, auch anders zu handeln, als sie es beabsichtigt bzw. getan haben: Wer bspw. unter Zwang handelt (töte ihn, sonst töte ich dich), wer falsch handelt, aber dennoch davon überzeugt ist, richtig gehandelt zu haben (dem Patienten aus Versehen die falschen Pillen zu geben, die zu einer Verschlechterung seines Gesundheitszustandes führen), wer richtig handelt und dabei anderen schadet (den Verletzten von der Fahrbahn zerren und ihm dabei das Genick brechen), wer falsch handelt, ohne dass dies in seiner Absicht lag (als Zugfahrer jemanden überfahren, weil derjenige vor den Zug gesprungen ist), wer anderen unabsichtlich schadet, dies aber in Kauf nimmt (zu schnell fahren und dabei jemanden überfahren) (etc.), dessen Handlungsmotive, Handlungen und Handlungsfolgen sind zweifellos Gegenstand moralischer Beurteilungen. Die im Moralkontext relevante Frage, ob der Handelnde die Verantwortung für sein Handeln tragen muss und warum, zeigt, wie diffizil die Machbarkeitsgrenzen moralischer Imperative und mithin des moralischen Sollenstypus sind.

Zusammenfassend zeigt sich hier folglich, dass moralische Imperative, die eine kategorische Verpflichtung zum Ausdruck bringen, das zu unterlassen, was falsch ist und das zu tun, was akzeptabel und richtig ist, einen wiederum deutlich präskriptiveren Gehalt haben als eudaimonistische und pragmatische Ratschläge. Auch wenn es - genau wie bei den eudaimonistischen Imperativen - weitreichende Machbarkeitsgrenzen kategorischer moralischer Imperative gibt, die in vielen Fällen gegen eine generelle, d.h. von allen Umständen absehende und zu allen Zeiten und an allen Orten gültige, Verpflichtung sprechen, ist hier dennoch von einer kategorischen Art und Weise der Verpflichtung die Rede, weil die Pflicht unabhängig von persönlichen Wünschen, Neigungen, Eignungen, Interessen etc. besteht. Insofern zeigt sich an den bisherigen Ausführungen, dass plausible moralische (Handlungsan-)Weisungen - anders als pragmatische und eudaimonistische Ratschläge, bei denen die Wollens-Grenzen eine entscheidende Rolle spielen - nur diejenigen sein können, bei denen die jeweiligen Machbarkeitsgrenzen berücksichtigt und einkalkuliert werden. Wenn bspw. die moralische Forderung im Raum steht, man solle keine Lebewesen verletzen oder gar töten unabhängig davon, ob es sich dabei um menschliche oder nicht-menschliche Lebewesen handelt, dann muss hierbei nicht nur ausgewiesen werden, warum dies kategorisch gesollt ist, sondern immer auch, wie sich diese Forderung im realen Alltag bewerkstelligen lässt. $\mathrm{Ob}$ andere einer moralischen Forderung entsprechen können, hängt folglich davon ab, ob einerseits plausibel dafür argumentiert werden kann, dass man dieser Weisung 
unabhängig von allen persönlichen Interessen die dagegenstehen, dennoch folgen sollte und andererseits auf Basis des derzeitigen Erkenntnisstandes folgen kann. ${ }^{149}$ Für plausible Gerechtigkeitsargumente im Kontext der Bewahrung und Förderung der Biodiversität ist daher nicht nur entscheidend, dass die Begründungen für moralische Pflichten und Rechte gegenüber der Natur bzw. in Ansehung der Natur explizit ausgewiesen werden, worauf Eser et al. fokussieren, sondern immer auch, dass auf diejenigen Bedingungen verwiesen wird, die dies ermöglichen bzw. erschweren oder gar verhindern, dass so und nicht anders gehandelt wird.

Die bisherigen Ausführungen zum pragmatischen, eudaimonistischen und moralischen Gebrauch der praktischen Vernunft zeigen, dass diese zum einen verschiedene Ebenen der Handlungsreflexion betreffen und dass zum anderen zwischen pragmatischen, eudaimonistischen und moralischen Sollenstypen unterschieden werden kann, die mit je anderen Sollgeltungsansprüchen einhergehen und je andere Begründungstiefen verlangen. Daher lässt sich die kategorische Trennung zwischen Klugheits-, Glücks- und Gerechtigkeitsargumenten weniger dadurch rechtfertigen, dass man darauf verweist, dass es sich beim pragmatischen, eudaimonistischen und moralischen Handeln um dem Sinn nach unterschiedliche Handlungstypen handelt. Vielmehr kann hier eingewandt werden, dass sich die einzelnen Handlungstypen in konkreten Situationen keineswegs ausschließen müssen (Luckner 2006:207-208). Eine adäquatere Begründung für deren Differenzierung scheint vielmehr darin zu liegen, dass es sich bei der pragmatischen und eudaimonistischen Frage, was zu tun zweckdienlich ist und wie Menschen leben sollten, damit ihr Leben und ihre Lebensführung als gut bezeichnet werden können sowie der moralischen Frage, wie wir in Bezug auf andere handeln sollten, um dem Sinn nach verschiedene ethische Fragen handelt (Steinfath 1998:9-10). Diese Annahme macht indes nur Sinn, wenn Klugheits-, Glücks- und Gerechtigkeitsargumente auch als Teilaspekte eines gemeinsamen Ethikverständnisses verstanden werden (können), innerhalb dessen die Relationen zwischen moralinternen und moralexternen Erwägungen nicht schon von vornherein aufgrund der Konzeptualisierung der ethischen Theorie entschieden sind. Eben dies ist aber nicht so selbstverständlich wie man meinen könnte, denn in der Ethik wird zumeist versucht, alle drei Aspekte der praktischen Frage über einen einzigen Ansatz zu erschließen und zu begründen:

149 In konkreten Fällen ist es durchaus schwierig zu entscheiden, ob Menschen etwas tatsächlich nicht können oder lediglich nicht wollen. Das, was gekonnt wird, ist abhängig von den je individuellen Voraussetzungen der Menschen, dem Wissensstand der Gesellschaft und der Auffassungen darüber, was Menschen an Einschränkungen zugemutet werden kann. So hängt bspw. die in der Tierrechtsdebatte relevante Frage, ob alle Menschen sich über längere Zeit vegetarisch oder vegan ernähren können, ohne dass dies negative Auswirkungen auf ihre Gesundheit hat, immer auch davon ab, ob es individuelle Faktoren gibt, die dagegen sprechen (wie bspw. das Vorliegen einer Schwangerschaft, in der von medizinischer Seite häufig davon abgeraten wird), ob adäquate Ersatzprodukte für bspw. tierische Eiweiße entwickelt werden können oder ob es kulturell anerkannt oder verpönt ist, eine Kultur des Verzichts und der gegenseitigen Rücksichtsname auf andere zu leben. 
Vor allem in deontologischen und konsequenzialistischen Ethiken steht die Begründung von Verpflichtungsgründen für Handlungen und Handlungsfolgen im Fokus. Fragen der klugen und vernünftigen Lebensführung sowie Fragen des guten Lebens sind hierin zweitrangig. Dies mag theoretisch begründbar sein, praktisch führt es indes dazu, dass auch die Umwelt- und Naturschutzethik oftmals „pflichtenzentriert“ operiert und damit in Bezug auf die praktischen Fragen weniger Orientierung gibt, als sie dies rein theoretisch könnte. Wenn die Umwelt- und Naturschutzethik indes anwendungsorientiert sein und einen substanziellen Beitrag zur Orientierung von Menschen leisten will, dann wirft dies rückwirkend Fragen bezüglich des grundlegenden Ethikverständnisses auf. Wenn gesagt wird, dass Ethik die Reflexionstheorie der Moral sei und Moral dabei aber bereits in einem engen, auf Pflichten und Rechte verengten, Verständnis verstanden wird, dann ist dies ein spezieller, an vielen Stellen problematischer und eben nicht zwingend notwendiger Zugang zur ethischen Reflexion auf die praktische Frage, wie Menschen sich in Bezug auf die Biodiversität verhalten sollten und wie zu handeln klug, gut und richtig ist. Daher wird im Folgenden auf den zweiten Aspekt den deontischen Fokus der Umwelt- und Naturschutzethik - konstruktiv eingegangen. Dazu wird ein mehrdimensionales ${ }^{150}$ Ethikverständnis zumindest in seinen Grundzügen dargelegt, über das die klassische Differenzierung zwischen moralisch und ethisch auf der einen Seite und nicht-moralisch und nicht-ethisch auf der anderen Seite hinterfragt wird, indem moralinterne (moralische Gebrauchsweisen der praktischen Vernunft) sowie moralexterne (eudaimonistische und pragmatische Gebrauchsweisen der praktischen Vernunft) zugleich in das 'Haus der Ethik' integriert werden.

\subsubsection{Ein mehrdimensionales Ethikverständnis als Grundlage der gesamten Argumentationstrias}

Im Folgenden wird mit Hans Krämer dafür argumentiert, dass es sich bei einem solchen mehrdimensionalen (er nennt es integrativen) Ethikverständnis um einen qualitativ anderen und mit Krämers eigenen Worten „vervollständigten, nicht-restriktiven und nicht reduktionistischen Begriff von Ethik [handelt], (...) der Probleme, die bisher zwischen Ethischem und Außerethischem bestanden, als ethikinterne Probleme thematisierbar macht“ (Krämer 1992:9):

Einsteigend ist zu sagen, dass sich das gegenwärtige und allgemein geläufige Verständnis von Ethik auf einen ganz bestimmten Begriffsinhalt bezieht, nach dem Ethik die philosophische Reflexionstheorie der Moral (bzw. der Moralen) ist. Hierbei wird Moral aber zumeist in einem engen und bereits inhaltlich stark voraussetzungsreichen Sinne

${ }^{150}$ Hier wird allgemein von einem mehrdimensionalen Ethikverständnis gesprochen, obwohl rein terminologisch auch von „,integrativer Ethik“ (vgl. Krämer 1992) die Rede sein könnte, da diese dem Ansinnen und der Absicht nach deckungsgleich mit dem hier postulierten Verständnis einer mehrdimensionalen Ethik ist. 
verstanden, was nur das umfasst, was alle Personen in sozialen Bezügen verbindlich zu tun bzw. zu unterlassen haben. Dies macht Thomas Scanlon prägnant deutlich, indem er herausstellt, dass Moralität in einem engen Sinne die moralphilosophische Frage betrifft, was wir uns gegenseitig schulden (Scanlon 2000:173). Das Problem hierbei ist nun, dass Ethik im Allgemeinen oftmals mit Pflichtenethik im Speziellen gleichgesetzt wird (Krämer 1992:9), was immer dann eine unzulängliche Reduktion darstellt, wenn dabei andere nicht primär auf moralische Pflichten bezogene Ethikansätze wie die Strebensethik oder Tugendethik (mit ihrem Fokus auf das gute Leben und die Tugenden, die es dazu braucht) oder die prudentielle Ethik oder Klugheitsethik (mit ihrem Fokus auf die individuelle oder kollektive strategische Lebensführung und die Güter, die es dazu braucht) „entweder explizit aus der philosophischen Ethik ausgebürgert oder ins Abseits des Irrelevanten verdrängt" (ebd., 10) werden.

Demgegenüber kann und wurde immer wieder mit guten Gründen darauf verwiesen, dass die Ethik nicht nur allgemeine Regeln und moralische Pflichten und Rechte für den Bereich des Sozialen zum Gegenstand haben kann, sondern sich mit menschlichen Handlungen im Allgemeinen beschäftigen muss, auch da, wo es um das Verhältnis von Menschen zu sich selber sowie unterhalb der Ebene von allgemeinen Regeln stehende Handlungssituationen geht. Auch hier macht Scanlon - allerdings selber in Konzentration auf eine Theorie der Moral im engeren Sinne - deutlich, dass die Unterscheidung zwischen moralisch und nicht-moralisch komplexer ist und differenzierter geführt werden muss, als dies oftmals der Fall ist:

"[W] hat seems to me most important is to recognize the distinctness of the various values (...) and their complex relation to 'what we owe to each other'. Once the nature and motivational basis of these values is recognized, it does not matter greatly how broadly or narrowly the label 'moral' is applied. (...) [E]ven if these values go beyond what 'we owe to each other' (as is shown by the fact that one cannot arrive at an understanding of them just by thinking about what principles others could not reasonably reject), they are, as I have tried to show, related to this part of morality in complex ways. There is therefore no point in exaggerating the difference by calling one 'moral' and the others 'nonmoral'." (Scanlon 2000:173, 176)

Auch Krämer nimmt den Aspekt der Verweisungszusammenhänge zwischen Werten und Pflichten auf, indem er darauf verweist, dass Pflichtenethik, Tugendethik und Klugheitsethik unter einem einheitlichen Funktionszusammenhang betrachtet werden können, unter dem sie komplementär aufeinander verweisen (Krämer 1992:37):

„Was die Koordination und Kooperation der (...) Ethiktypen angeht, so ist zunächst davon auszugehen, dass sie einander auf der Theorieebene komplementär ergänzen, also die Leerräume des jeweils (...) anderen abdecken. Daraus ergibt 
sich eine wechselseitige Problemstimulierung und Schärfung des Selbstverständnisses im Blick auf die jeweilige Partialität und Ergänzungsbedürftigkeit und auf ein wechselseitiges Bedingungsverhältnis." (ebd., 123)

Dies wiederum legt eine Aufgabenverteilung innerhalb der Ethik zwischen Pflichtenethik, Tugendethik und Klugheitsethik nahe, die mit Krämer (zumindest grob) wie folgt bestimmt werden kann: ${ }^{151}$

„Eine vollständige Integrative Ethik muss (...), wenn sie sich nur an die Phänomene halten will, mehrgleisig operieren und Moralphilosophie und Strebensethik als heterogene und autonome Zweige der Ethik in einem teils konkurrierenden, teils kooperativen Nebeneinander belassen. (...) Gerade auf der Theorieebene kann realistischerweise nur eine Integrative Ethik in der wohldefinierten Bedeutung angestrebt werden, dass sie die (...) Teildisziplinen in relativer Autonomie und Eigenständigkeit erhält und eben dadurch ihre Kooperation erst möglich und sinnvoll macht. Das so verstandene Programm einer Integrativen Ethik bedeutet heute für die ethische Theoriebildung primär die Reetablierung der StrebensSelbst- und Glücksethik, aber nicht ohne und auch nicht gegen die Moralphilosophie, sondern als deren notwendiges Komplement und Pendant.“ (ebd., 120, 122)

Dieses wechselseitige Ergänzungsverhältnis, von dem Krämer hier spricht, ist in die eine Richtung schauend derart, dass die Pflichtenethik den Rahmen für die Klugheitsethik und die Tugendethik liefert. Das, was dann Moral genannt wird, bzw. das, was als genuin moralisch gekennzeichnet und von Pflichtenethiken expliziert und begründet wird, wird dadurch zu einem „rein sozialen Phänomen nach Genesis, Geltung und Exekution." (ebd., 19) Alles, was dagegen das Selbstinteresse, die Selbstverwirklichung, die Selbstachtung, die Eigenverantwortung etc. betrifft, fällt in den moralexternen, zugleich aber moralisch relevanten und moralrelativen Bereich der Tugend- und Klugheitsethiken, deren Aufgabe es ist, Ratschläge für das gute Leben von einzelnen oder Gruppen sowie für situationsadäquates Verhalten und zweckdienliches Handeln bereitzustellen. $^{152}$

${ }^{151}$ Krämer spricht von Moralphilosophie einerseits und Strebensethik andererseits und meint damit auf Seiten der Moralphilosophie die Pflichtenethik und auf Seiten der Strebensethik sowohl die Tugendethik als auch die prudentielle Ethik. Stellvertretend für viele Aussagen dieser Art: „Funktionsanalytisch betrachtet ist die Moralphilosophie streng genommen nur dort gefragt und bedeutungsvoll, wo Konflikte zwischen den eigenen und den fremden Interessen auftreten, während die Strebensethik für alle Aporien in der Realisierung des Eigeninteresses zuständig ist." (Krämer 1992:118)

152 Exemplarisch zeigt dies insbesondere der Terminus der 'Selbstgerechtigkeit', der in besonderem Maße auf den Hiat zwischen moralinternen (moralischen) und moralexternen (prudentiellen und eudaimonistischen) Phänomenen einerseits hinweist, andererseits zeigt sich an ihm aber auch, dass moralische, prudentielle und eudaimonistische Erwägungen nicht unabhängig voneinander sind: Gerechtigkeit ist der formalen Bestimmung nach ein moralisches Phänomen, da es das erfasst, „was wir uns gegenseitig schulden“ (Mazouz 2006:371) bzw. „mit guten Gründen voneinander verlangen können. “ (Eser, Benzing \& Müller 2013:22) Das, was man mit guten Gründen von sich selbst verlangen kann, ist demgegenüber zwar erwünscht, kann aber nicht eingefordert werden, da solche 'Pflichten 
Der Pflichtenethik kommt folglich die Funktion zu, die verallgemeinerbaren moralischen Regeln und Pflichten in der sozialen Sphäre zu formulieren, die quasi das äußere Gerüst für das 'Haus der Ethik' bilden und an denen sich alle Menschen im Umgang mit anderen orientieren können, wenn es um zwischenmenschliche oder um Mensch-Natur Verhältnisse geht. Denn ohne das basale und grundlegende Verständnis von allgemeinen Pflichten und Regeln in der sozialen Sphäre drohen die auf individuelle bzw. partikulare Handlungs- und Verhaltensweisen zugeschnittenen Klugheits- und Tugendethiken wie 'Wolken in der Luft' zu schweben, da diese ohne allgemeine Orientierungspunkte völlig subjektivistisch werden. Erst im Rahmen der Pflichtenethik werden aus der Klugheits- und Strebensethik überhaupt 'Zimmer' im Haus der Ethik.

In die andere Richtung schauend, macht Krämer anschaulich deutlich, dass die pflichtenethischen Orientierungspunkte immer dann nicht ausreichend orientierend sind und inhaltsleer werden, wenn diese nicht durch tugend- und klugheitsethische Überlegungen und Erwägungen komplementiert werden, da der Befund, dass Handelnde immer selbst für die Folgen ihres Handelns (sowohl als Tun als auch als Zulassen) aufkommen müssen, keine Sache moralischer Verantwortung sondern einer vorausschauenden Lebensplanung und -führung ist (ebd., 16-22): Wenn es daher um Anforderungen bzw. Anstrengungen geht, die Akteure an sich selbst stellen und von sich selbst fordern, die aber jederzeit zurückgenommen werden können, wie bspw. wenn man sich vornimmt, weniger Fleisch zu konsumieren oder mehr Sport zu treiben, weil man gesünder oder bewusster leben will oder wenn es um die Rücksichtnahme auf bzw. Achtsamkeit der eigenen Person gegenüber geht, wenn man sich bspw. vornimmt, dieses Jahr nicht so viel zu arbeiten, mehr auf seine Gesundheit zu achten und nicht alles zu machen, was man rein theoretisch machen könnte, dann fällt dies in den Bereich der Klugheits- und Tugendethik. In beiden Fällen „artikuliert sich die Stimme des wahren Selbstinteresses und das Streben nach Selbstverwirklichung und -erhaltung im Ganzen, d.h., dessen, was ich eigentlich will, gegenüber weniger wichtigen, peripheren Bestrebungen." (ebd., 17) Aber auch wenn es um die (Selbst-)Verpflichtungen in Ansehung anderer geht, die i.d.R. dem pflichtenethischen Kontext zugeordnet werden, zeigt sich, dass es auch hier im eigentlichen Sinne um „eine Selbstaufklärung auf dem Boden der richtigen Lebensführung geht" (ebd.), bspw. wenn man sich zusammen mit seinem Partner darauf verpflichtet, genauso viel Zeit mit der Familie zu verbringen

gegen sich selbst' keine Frage der Gerechtigkeit sondern der klugen und guten Lebensführung bzw. der Vorstellung von einer solchen sind (Mazouz 2006:371). Dies soll nicht bedeuten, dass solche 'Pflichten gegen sich selber' moralisch irrelevant sind, denn dies sind sie keineswegs. Wären sie dies, dann wäre gar nicht einsichtig, warum wir, wenn wir im Alltag von Selbstgerechtigkeit reden, bereits in Abrede stellen, dass ein selbstgerechter Mensch einen moralisch richtigen Umgang mit Anderen pflegt, da erst vor dem Hintergrund eines, auf Reziprozität basierenden, Gerechtigkeitsverständnisses deutlich wird, dass ein selbstgerechtes Denken, Verhalten und Handeln auf der unzulässigen Verallgemeinerung eigener Wünsche, Bedürfnisse, Überzeugungen und Vorstellungen basiert. 
und sich genauso wie der Partner um die alltäglichen Dinge der Lebensführung wie Einkaufen, Putzen oder Aufräumen zu kümmern, weil man sich gegenseitig auf ein modernes Familienbild verständigt hat, indem nicht einer allein der Erwerbsarbeit und der andere der unbezahlten Hausarbeit nachgeht.

Die relative Autonomie der Teilethiken, von der Krämer spricht, bedeutet vor dem Hintergrund des wechselseitigen Bedingungsverhältnisses nichts anderes, als dass die Beantwortung der Frage nach dem moralischen und mithin richtigem Handeln, die von der Pflichtenethik übernommen wird, sich nicht auch schon durch die eudaimonistische Grundfrage der Strebensethik, wie Menschen leben sollen, sowie die pragmatische Grundfrage der Klugheitsethik, wie zweckdienlich zu verfahren sei, beantworten lässt. Sicherlich ist unbestritten, dass die drei Grundfragen unter bestimmten Bedingungen zur Konvergenz gebracht werden können - genau dies versuchen viele Pflichten-, Tugend- und Klugheitsethiken zu machen - und dass es zwangsläufig bestimmte Überschneidungspunkte zwischen diesen drei Teilethiken gibt. Entscheidend ist hier aber, dass die moralische Antwort auf die eudaimonistische und die strategisch-pragmatische Frage nicht die einzige und nicht einmal die in erster Linie gesuchte Antwort auf die eudaimonistische Frage darstellt, genau wie dies auch bei der pragmatischen Antwort auf eudaimonistische und moralische Fragen und eudaimonistische Antworten auf moralische und pragmatische Fragen der Fall ist. Vielmehr geht es hierbei um die Auslotung, welche pragmatischen, eudaimonistischen und moralischen Überlegungen und Erwägungen in Bezug auf die praktischen Fragen jeweils relevant sind, weil allen drei Aspekten der praktischen Frage innerhalb einer mehrdimensionalen Ethik auf je eigene Weise entsprochen werden muss.

Wie schnell ersichtlich wird, zieht die Mehrdimensionalität der Ethik indes eine Reihe gewichtiger Fragen nach sich. Zu den hier relevanten Fragen in Bezug auf die Bewahrung und Förderung der Biodiversität zählt die bislang unbeantwortete Gewichtungsfrage wie die drei Argumentationstypen der Klugheit, des Glücks und der Gerechtigkeit innerhalb eines mehrdimensionalen Ethikverständnisses gewichtet werden und was das wiederum für das Schutz-, Nutzungs- und Verteilungsziel bedeutet. Konkreter gefragt: Sind alle drei Argumentationstypen gleichwertig oder gibt es zwischen diesen eine Hierarchie und warum? Spricht eine Hierarchie wiederum dafür, dass es auch bezüglich der Zieltrias und der damit verbundenen Maßnahmen eine Vorrangregelung gibt oder geben sollte und impliziert eine Gleichwertigkeit der Argumentationstypen wiederum eine Gleichwertigkeit der Biodiversitätsziele und der damit verbundenen Maßnahmen? So wichtig und relevant die Beantwortung dieser Fragen für ein mehrdimensionales Ethikverständnis und die Ausbuchstabierung des biodiversitätsethischen Argumentationsraumes sind, so entscheidend ist an dieser Stelle in pragmatischer Hinsicht, dass diese Fragen, wenn sie ausschließlich im Rahmen der Ethik gestellt und 
beantwortet werden, in der Endkonsequenz auf bislang unabgeschlossene und nach wie vor kontrovers geführte metaethische und ethische Debatten zurückführen, die hier weder geführt, noch entschieden werden können und sollen.

Vielmehr soll an dieser Stelle daran erinnert werden, dass es in der Arbeit primär um problem- und anwendungsorientierte Reflexionen geht und dass die Gewichtungsfrage als solche unterschiedlich angegangen werden kann. Wenn nämlich berücksichtigt wird, dass die Gewichtungsfrage nicht kontextlos ist, da diese sich bei jeder konkreten Problematik, je nach den kontextuellen Rahmenbedingungen, in anderer Weise stellt, je nachdem wie konkrete Personen sich in konkreten Situationen auf konkrete Ziele unter Zuhilfenahme konkreter Mittel mit anderen Personen verständigen oder nicht verständigen können, welche Ziele und Mittel am geeignetsten sind, um das konkrete Problem zu begreifen, anzugehen und zu lösen, erscheint eine kontextlose durch die Philosophie quasi 'von außen`entschiedene Gewichtung nicht angemessen zu sein. Vielmehr wird hieran die Relevanz der letzten Detailproblematik deutlich, auf die im Folgenden, die theoretische Neuausrichtung der Umwelt- und Naturschutzethik abschließend, eingegangen wird. Hierüber wird sowohl die Problematik als auch die konstruktive Lösung der dritten Detailproblematik, sprich, der zu einseitigen Fokussierung auf die normativen Akzeptabilitätsbedingungen bezüglich der ethischen Begründung der Bewahrung und Förderung der Biodiversität verdeutlicht, was wiederum einen Übergang auf die politische Ebene ermöglicht, so dass den nun folgenden Ausführungen lediglich ein kurzes Fazit angeschlossen wird (4.4), über das die Überleitung in die poli-tikwissenschaftliche Detailreflexion realisiert wird.

\subsubsection{Die Akzeptabilitäts- und Akzeptanzbedingungen für die Kommunikation und Aushandlung der Biodiversitätsziele und -maßnahmen}

Der, sich aus der Mehrdimensionalität der Ethik ergebende, interne Zusammenhang zwischen der Gewichtungsfrage und den Anforderungen an eine anwendungsorientierte Ethik kann, basierend auf Michael Walzers Ausführungen zu den verschiedenen Wegen der philosophischen Gesellschaftskritik, dargelegt werden. Walzer differenziert in seinem Band über »Kritik und Gemeinsinn« (vgl. Walzer 1993) drei verschiedene Wege der philosophischen Gesellschaftskritik, die er als „Pfad der Entdeckung“, als „Pfad der Erfindung“ und als „Pfad der Interpretation“ bezeichnet:

Nach dem Selbstverständnis des 'Entdeckers' haben die Normen und Prinzipien einen objektiven Status. „Es gibt sie gewissermaßen dort - in der moralischen Welt - und sie warten darauf, in Kraft gesetzt zu werden.“ (ebd., 15) Einen solchen objektiven Status erhalten Werte und Normen indes nur dadurch, so Walzer, dass sich ihre 'Entdecker' außerhalb der von ihnen vorgefundenen Gesellschaft befinden, was insofern problematisch ist, als dass Menschen als Teil der Gesellschaft die Gesellschaft nicht von 
einem externen Punkt aus erfassen können (ebd.). Dem 'Erfinder' geht es demgegenüber darum, ein Konstruktionsverfahren zu entwickeln, d.h., eine „Methodologie der Moral zu erschaffen, die eine moralische Übereinstimmung aller in der Gesellschaft garantiere." (ebd., 18) Die Normen und Prinzipien der Gesellschaft beurteilende Vernunft liege, so Walzer, nicht wie beim Pfad der Entdeckung außerhalb der Gesellschaft, sondern wird hier in die Verfahrensregeln integriert, auf die sich die Gesellschaftsmitglieder einigen sollen (ebd., 19). In einer solchen rationalistischen Vorstellung seien die Menschen aber ihrer eigenen Lebensweise beraubt, da das Zusammenleben von Menschen vor dem Hintergrund von Konstrukten wie dem Rawlsschen 'Schleier des Nichtwissens' oder aber der Habermaschen 'unverzerrten Sprechsituation ' gedacht wird (ebd., 21). Seinen eigenen Ansatz versteht Walzer als Methode der Interpretation. Moralisches Argumentieren bedeutet hier eine Bestandsaufnahme der bereits existierenden Moralvorstellungen, „die sich als Produkt von Zeit, von Zufällen, äußeren Einflüssen sowie als Ergebnis politischer Kompromisse, fehlbarer und partikularistischer Absichten herausgebildet hat (ebd., 30). Übersetzt man den von Walzer gewählten und priorisierten "Pfad der Interpretation" daher in eine Sozialpraktik, so erscheinen die praktischen ethischen Fragen wiederum im Anschluss an die soziopragmatische Position von John Dewey als „das praktische Problem von Menschen, die in Verbindung miteinander leben“ (Dewey 2001:41). Daher sind ethische und politische Prioritätensetzungen etwas, was sich nicht vornehmlich an TheoretikerInnen, WissensexpertInnen oder professionelle Politikeliten richtet. Nach Walzer existiert folglich das, was als klug, beglückend oder gerecht angesehen wird bereits in einer Vielzahl an Konzepten und Vorstellungen in sozialen Gemeinschaften und muss (lediglich) interpretiert werden (Walzer 1992a:20).

Über diese lediglich stichpunktartigen Bestimmungen des interpretatorischen Wegs der philosophischen Gesellschaftskritik soll deutlich werden, dass Walzer einen Zusammenhang zwischen der Gewichtungsfrage anwendungsorientierter Ethik und demokratischer Aushandlungsprozesse schafft und dass das von ihm proklamierte Philosophieverständnis einen demokratischen Grundgehalt innehat: Kein Mensch kommt umhin, sich in seinem eigenen Moralverständnis zu befragen und zu hinterfragen, so "dass sich die Beratung [von PhilosophInnen] in Wirklichkeit auf die Bedeutung desjenigen konkreten moralischen Lebens bezieht, an dem die Diskussionsteilnehmer bereits teilnehmen." (Walzer 1993:33) In diesem Kontext zählt das Wort der PhilosophInnen nicht mehr als das aller anderen DiskussionsteilnehmerInnen:

"Democray has no claims in the philosophical realm, and philosophers have no special rights in the political community. In the world of opinion, truth is indeed another opinion, and the philosopher is only another opinion-maker." (Walzer 1981:397) 
Das wiederum bedeutet in Bezug auf die Gewichtungsfrage, dass diese zwar mit Hilfe von metaethisch und ethisch begründeten Vorrangregeln entschieden werden könnte, dass aber alle hierbei vorgebrachten Entscheidungsregeln Gefahr laufen, kontextlos zu bleiben und zu abstrakt zu sein und letztlich, egal wie die Vorrangregelungen ausfallen und wie plausibel diese begründet sind, es immer auch andere nicht weniger plausible Antworten auf die Gewichtungsfrage gibt. Wenn dies aber von vornherein klar ist, dann kann die Gewichtungsfrage auch gleich in praktischer Absicht gestellt werden: Das bedeutet, dass die Aushandlung der Wichtigkeit der Motive, Gründe, Argumente und Begründungen für die Ziele und Maßnahmen der Bewahrung und Förderung der Biodiversität in den Kontext ihrer politisch-demokratischen Aushandlung und Ausgestaltung gestellt wird. Wenn dies der Fall ist und wenn dabei die vorpolitischen Erfahrungen der Menschen als unverzichtbare Grundvoraussetzungen und Gelingensbedingungen für Demokratie brücksichtigt werden, dann können nicht nur die normativen Akzeptabilitätsbedingungen hinsichtlich der Begründung von Zielen und Maßnahmen von Relevanz sein, auf die sich die Umwelt- und Naturschutzethik vornehmlich fokussiert, sondern dann spielen immer auch die faktischen Akzeptanzen innerhalb der Bevölkerung eine entscheidende Rolle.

Mit anderen Worten: Wenn das praxisrelevante und anwendungsorientierte Wissen, was die Ethik in Bezug auf praktische Fragen einbringen kann, immer schon im Kontext demokratischer Aushandlungsprozesse steht, weil die Methoden der Gesellschaftskritik, die PhilosophInnen vorbringen können, sich nicht neutral zur politischen Verfasstheit einer Gesellschaft verhalten, dann muss in der ethischen Kommunikation über Natur- und Umweltschutzanliegen folglich auch stärker beachtet werden, dass die Ausweisung 'guter Gründe ' für die Bewahrung und Förderung der Biodiversität nicht lediglich eine Frage der normativen Akzeptabilität der Gründe ist sondern immer auch eine Frage ihrer faktischen Akzeptanz. Wenn indes die zu einseitige Fokussierung der Ethik auf die Akzeptabilitätsbedingungen erweitert werden soll, dann muss erst einmal geklärt werden, welche Rolle die faktische Akzeptanz für die normative Akzeptabilität von Entscheidungen spielt. Andreas Wolkenstein geht im Kontext der Sicherheitsethik eben dieser Fragestellung nach, indem er die Spannbreite von Akzeptanzverständnissen aufzeigt und daraufhin darlegt, wie deren Verhältnisse zu Akzeptabilitätskriterien jeweils bestimmt werden können (Wolkenstein 2014:226). Er differenziert dabei zwischen drei verschiedenen Kontexten, in denen Akzeptanzfragen eine Rolle spielen und in denen Akzeptanz jeweils anders verstanden wird (ebd., 226-228): ${ }^{153}$

153 Wolkenstein differenziert genau genommen zwischen vier Bereichen, allerdings ist die Unterscheidung zwischen Akzeptanz als Objekt empirischer Untersuchungen und als Objekt deskriptiver Beschreibungen unnötig, da er damit nur zum Ausdruck bringt, dass Akzeptanz mal als zu erklärende (abhängige) und mal als erklärende (unabhängige) Variable genutzt werden kann. Beides fällt in einer gröberen Betrachtung unter den Kontext sozialwissenschaftlicher Akzeptanzforschung. 
Zum einen identifiziert er den politischen Kontext, in dem Akzeptanz mit Rechtfertigung für politisches Handeln identifiziert wird (Akzeptanz als Legitimation). Davon unterscheidet er den strategisch-ökonomischen Kontext, in dem Akzeptanz als ein zu erreichender Zielzustand verstanden wird, den es mit bestimmten Mitteln zu schaffen, zu halten oder zu erhöhen gilt (Akzeptanz als Marketing). Schließlich verortet er Akzeptanz im wissenschaftlichen Kontext, in dem Akzeptanz entweder als abhängige oder als unabhängige Variable untersucht wird (Akzeptanz als Untersuchungsgegenstand).

Der in diesem Kontext wichtigste Aspekt bezüglich der Unterscheidung der drei Akzeptanzformen ist, dass sich die Kritik der Ethik zumeist gegen die Formen der ökonomisch -strategischen Akzeptanzbeschaffung als Marketing nicht aber zugleich auch gegen Akzeptanz als Legitimation oder als Untersuchungsgegenstand richtet. Die hier vorgebrachte Kritik basiert dann zumeist auf der Annahme, dass Forschungsergebnisse zu faktischen Akzeptanzen dazu verwendet werden, Urteile und Beurteilungen von Menschen dadurch zu beeinflussen, dass diese als repräsentative oder gerade nicht repräsentative Mehrheits- oder Minderheitsmeinung innerhalb sozialer Gruppen dargestellt werden, in die Menschen sich einordnen oder von denen sie sich abgrenzen. Die Kritik richtet sich aber auch daran aus, dass es beim Marketing immer auch darum geht, wie etwas argumentiert, dargestellt und kommunikativ 'verpackt' werden muss, damit der Inhalt akzeptiert wird. Hier wird kritisiert, dass die auf diesen Wegen hergestellte 'oberflächliche`Akzeptanz keine 'echte' inhaltliche Akzeptanz ist, da sie auf 'Tricks`zur Überredung und Beeinflussung beruhen, so dass Menschen entgegen ihrem (vermeintlich unverfälschten) Willen zur Akzeptanz gebracht werden.

An dieser Stelle kann und muss den Kritikern indes selbst entgegnet werden, dass sie von der unrealistischen Grundannahme ausgehen, es gäbe unverfälschte bzw. unverzerrte Präferenzen, die vom Einfluss anderer Personen oder von situativ, räumlich und kulturell-kontingenten Bedingungen unabhängig wären und dass nur die 'wahren` bzw. 'echten` Präferenzen und Überzeugungen ein geeignetes Richtmaß für Akzeptanz darstellen würden. Außerdem scheint hierbei eine einseitige und negativ konnotierte Sichtweise auf die strategische Kommunikation durch, die sich daraus ergibt, dass die strategische Kommunikation in der Habermaschen Diskursethik über die Teilung von System und Lebenswelt als Gegenpol zur lebensweltlich-verständigungsorientierten Kommunikation gesehen wird und weil die ethische Kommunikation oftmals selbst als eine solche auf Verständigung ausgerichtete Kommunikation verstanden wird (Eser, Neureuther \& Müller 2011:21). Wenn strategische Kommunikation daher als Synonym für eine, den anderen unter Missachtung seiner eigenen Präferenzen und Ansichten überredende, unter Vorhaltung falscher oder selektiver Informationen täuschende und zu seinem eigenen Nutzen manipulierende Kommunikationsweise (fehl-)interpretiert wird, dann stellt sich erst gar nicht die praktisch-normative Frage, wie strategisch kom- 
muniziert werden sollte, sondern eher die grundsätzliche Frage, ob dies überhaupt zulässig ist. Dies wiederum ist aber in einer Welt, in der Verständigung immer auch massenmedial vermittelt ist und dies wiederum strategischer Planung bedarf, an der Realität vorbei gedacht und hierbei wird übersehen, dass es auch in der verständigungsorientierten Kommunikation zahlreiche strategische Aspekte gibt, da das 'sich miteinander Verständigen ` kein zielloser und planloser Prozess ist, so dass darüber nachgedacht werden muss, wie und auf welchen Wegen verständigungsorientiert miteinander umgegangen werden kann.

Da die individuellen Präferenzen vielfältig, nicht immer kohärent, zuweilen widersprüchlich und darüber hinaus beinflussbar und wandelbar sind, ist dies auch die Akzeptanz. Wenn also konkret danach gefragt wird, ob und inwiefern die Meinungsforschung und die mit ihren Ergebnissen arbeitenden Kommunikations- und Marketingstrategien, wie bspw. die lebensstilorientierte Naturschutzkommunikation und die ethische Naturschutzkommunikation dazu beitragen können, die Bewahrung und Förderung der Biodiversität zu bestärken, indem sie andere von der Wichtigkeit und der Relevanz der Biodiversitätsziele und -maßnahmen überzeugen, dann ist das, was im strategischen Sinne überzeugungsfördernd ist und das, was im ethischen Kontext als zustimmungswürdig angesehen wird, im sprichwörtlichen Sinne zwei Seiten derselben Medaille, auf die sowohl die Ethik als auch die strategische Naturschutzkommunikation jeweils (oftmals unter mangelnder Berücksichtigung der jeweils anderen Seite) schaut. Da es aber in beiden Kontexten darum geht überzeugende Positionen auszumachen, sollte es stärker als bisher um die Auslotung der Möglichkeiten gehen, wie die strategischen und die ethischen Aspekte der Kommunikation über die Bewahrung und Förderung der Biodiversität sinnvoll zusammengebracht werden können, ohne dass dabei negiert wird, dass diese unterschiedliche Eigenlogiken haben, die sich indes nicht ausschließen müssen, sondern auch ergänzen können:

Wie bereits eingangs ausgeführt (siehe 4.1) wird in der Ethik der Aspekt betont, dass die Überzeugungskraft von Handlungsgründen davon abhängt, ob diese Gründe von allen akzeptiert werden könnten, so dass hier die Qualität der Argumente im Sinne ihrer Richtigkeit oder Geltung ausschlaggebend ist. Was hierbei indes vernachlässigt wird, ist das eine solche Prüfung nicht kontextlos ist, denn die Überzeugungskraft von Gründen ist in hohem Maße davon abhängig, welche emotionalen, motivationalen sowie kognitiven Hintergründe Menschen haben und welche räumlich, zeitlich und kulturell kontingenten Rahmenbedingungen gegeben sind. Insofern ist das Wissen um die in der Gesellschaft vorfindbaren Wert- und Normvorstellungen der Menschen nicht nur für die lebensstilorientierte, sondern auch für die ethische Naturschutzkommunikation relevant, weil die Ausweisung nachvollziehbarer Werte und Normen (im Umgang mit der Biodiversität) ansonsten zu kontextlos bleibt. 
An dieser Stelle ist vor allem das Konzept der Lebensstile geeignet, um die in der Gesellschaft vorfindbaren Wert- und Normvorstellungen der Menschen geclustert nach sozialen Milieus, aufzuzeigen und in ihrer Veränderlichkeit verständlich zu machen. ${ }^{154}$ Die Kenntnis der sozialen Milieus und ihrer Eigenheiten ist für die lebensstilorientierte Naturschutzkommunikation in strategischer Hinsicht wichtig, weil es ihr darum geht, „das Thema Natur und Naturschutz positiv in das Wahrnehmungsfeld der einzelnen Zielgruppen zu tragen" (Schuster 2008:24), so dass ihre Botschaften gehört, verstanden, für überzeugend erachtet, behalten und umgesetzt werden (ebd., 24). Insbesondere unter der Berücksichtigung, dass politische Inhalte fast ausschließlich massenmedial kommuniziert werden und dass dies der zentrale Weg der Informationsbeschaffung und -übermittlung ist, müssen die relevanten Informationen nicht nur aus strategischer Hinsicht zur Akzeptanzgewinnung, sondern auch aus demokratietheoretischer Sicht als Bedingung der Möglichkeit überhaupt informiert zu partizipieren, möglichst zielgruppenspezifisch vermittelt werden. Dies gelingt indes nur dann, wenn die Aufmachung, die Ansprache und die Darstellung der Informationen zielgruppengemäß ist, um bei der massenmedialen Kommunikation zumindest die Aufmerksamkeits-, Aufnahme- und Verstehensschwelle zu überwinden (ebd., 26): Die Anwendungsschwelle, sprich die Internalisierung der Informationen in eigene Handlungsschemata, gelingt indes immer erst dann, wenn Informationen auch als persönlich bedeutsam wahrgenommen werden und diese in Einklang mit den persönlichen Zielen von Personen und den in ihrem sozialen Umfeld stehen. Insofern ist der Anspruch der lebensstilorientierten Naturschutzkommunikation, die „Naturschutzthemen mit persönlichen Meinungen und Handlungen sowie Wissensbeständen und Gefühlen zu verbinden - kurz mit dem, was eine Person für wichtig hält, womit sie sich identifiziert und worüber sie sich nach innen und außen definiert" (ebd., 13), auf jeden Fall strategisch. Ein solcher Anspruch ist aber einer verständigungsorientierten Kommunikation keineswegs abträglich, da es hierbei im Wesentlichen um die Fragen geht, wie die Naturschutzkommunikation gestaltet wird, welche stilistischen Elemente dabei eingesetzt werden und über welche Medien die Botschaften und Argumente verbreitet werden können, um die Zielgruppen anzusprechen (Lichtl 2009:32).

154 Das Konzept der Lebensstile (siehe FN 48) beruht auf empirisch gewonnenen Milieutypologien, die mit Einstellungsmustern in Verbindung gebracht werden. Die dabei unterschiedenen sozialen Milieus (vgl. insbesondere die Sinus-Milieus und die Studien zur Veränderung dieser) beschreiben soziale Gruppen, deren Wertorientierungen, Lebensziele und Lebensweisen ähnlich sind, so dass diese sich durch jeweils charakteristische 'harte' (bspw. Einkommen, Alter, Haushaltsgröße, Beziehungsstatus usw.) und 'weiche' Merkmale (wie bspw. Wertorientierungen, Lebensziele, Arbeitseinstellungen, Freizeitmotive, alltagsästhetische Neigungen usw.) in ihrer soziokulturellen Identität auszeichnen. Gerade weil hierüber bestimmte Konsumorientierungen, aber auch Wahlverhalten gut erklärt und prognostiziert werden können, wird das Lebensstilkonzept vor allem in der Wahlforschung und in der Marktforschung aufgegriffen. In der Ethik wird weniger das Konzept der Lebensstile kritisch betrachtet als vielmehr dessen Verwendung und Nähe zum Produkt- und Konsummarketing (Eser, Neureuther \& Müller 2011:21). 
Die Relevanz dieser Fragen wird vor allem dann ersichtlich, wenn man sich vor Augen führt, dass es in der Bevölkerung verschiedene Zugänge zu Natur- und Umweltthematiken gibt, die die wissenschaftliche Debatte oftmals gar nicht in der Form aufgreifen und handhaben kann, wie sie in der Gesellschaft vorherrschen. Während es in der Wissenschaft vor allem die Erforschung der komplexen biowissenschaftlichen, ökonomischen, ethischen, politischen, rechtlichen etc. Zusammenhänge geht, die in Gänze nur schwer in die Gesellschaft kommunizierbar sind, ohne dass es dabei zu entscheidenden Informationsverlusten und zu Trivialisierungen kommt, können die im Alltagserleben vieler Menschen wichtigen heimatlichen und ästhetischen Aspekte von Natur/Biodiversität, die eine hohe Motivationskraft für das umwelt- und naturschützerische Handeln haben, von der lebensstilorientierten Naturschutzkommunikation in Form von Bildern, Farben, Tönen und Stimmungen aufgegriffen und thematisiert werden (ebd., 33). Die hierbei im Vordergrund stehende emotionale Ansprache der Adressatinnen erhöht die Wahrscheinlichkeit, dass massenmediale Botschaften die einzelnen Aufmerksamkeits- und Verarbeitungsschwellen passieren können (Schuster 2008:26). Dies wiederum ist wichtig, weil die Aufmerksamkeit für Natur- und Umweltthemen in Konkurrenz zu anderen Themen steht. Deshalb bedarf es einer Berücksichtigung und Beantwortung der Fragen, an wen genau sich die Naturschutzkommunikation richten soll, wie Botschaften kommuniziert werden können, damit sie bei den Adressatinnen auch ankommen ${ }^{155}$ und welche Intentionen dahinter stehen (bloßes Informieren, Aufmerksamkeit erregen, Spenden einwerben, Appelle aussprechen etc.). Andererseits müssen solche Kommunikationskampagnen nicht nur strategische Anforderungen sondern auch normativen Standards genügen:

Zum einen sollte die Kommunikation zum Umwelt- und Naturschutz, die im Wesentlichen von zivilgesellschaftlichen und staatlichen Behörden in Auftrag gegeben und bezahlt wird, nicht soweit vereinheitlicht werden, dass in der Umwelt- und Naturschutzkommunikation nur bestimmte soziale Milieus angesprochen werden (bspw. das dem Naturschutz besonders nahe stehende sozial-ökologische Milieu oder aber das dem Naturschutz besonders ferne Milieu der Hedonisten). Vielmehr muss es darum gehen, alle Bevölkerungsgruppen gleichermaßen zu berücksichtigen und anzusprechen. Hierbei bietet es sich aus der strategischen Perspektive an, die Vielfalt als zentrales Leitmotiv der Kommunikation über Biodiversität herauszustellen (vgl. Lichtl 2009:11), um diese mit dem persönlichen Alltag und dem eigenen Handeln in Verbindung zu bringen

155 Damit dies gelingt, müssen verschiedene Schwellen überwunden werden. Gemäß dem klassischen Kommunikationsansatz 'Gesagt bedeutet nicht gehört (Aufmerksamkeitsschwelle), gehört bedeutet nicht behalten (Aufnahmeschwelle), behalten bedeutet nicht verstanden (Verstehensschwelle), verstanden bedeutet nicht akzeptiert (Akzeptanzschwelle), akzeptiert bedeutet nicht internalisiert (Internalisierungsschwelle), internalisiert bedeutet nicht danach gehandelt (Anwendungsschwelle)', bedarf es auf jeder dieser prototypischen Schwellen anderer Ansätze (vgl. Schuster 2008:24-31). 
(ebd., 11-12). Dabei darf das Motiv der Vielfalt aber nicht nur auf der inhaltlichen Ebene adressiert werden, sondern muss auch auf der sprachlichen und inhaltlichen Ebene berücksichtigt werden, was wiederum gegen eine Vereinheitlichung und Reduzierung der Kommunikation auf eine bestimmte 'Corporate Identity' spricht, da die Kommunikation nicht bereits auf bestimmte Inhalte reduziert werden sollte, bei denen erwartbar ist, dass diese besonders akzeptanzfähig sind. In diesem Sinne sollte die Umweltund Naturschutzkommunikation nicht einseitig darauf ausgerichtet werden, dass Natur im Allgemeinen und Biodiversität im Speziellen eine monetär wichtige sowie existenziell notwendige (materielle) Ressource für jeden von uns ist, weil dabei die immateriellen, ästhetischen und kulturellen Werte, die funktionalen Grundbedingungen der Natur bzw. Biodiversität zur Erfüllung unserer jeweiligen Vorstellungen vom guten Leben sowie die mit dem Zugang zu Natur verbundenen Gerechtigkeitsmotive oftmals unartikuliert bleiben. Werden diese Bezüge indes vernachlässigt, dann werden lediglich ganz bestimmte Auffassungen, Haltungen und Positionen vermittelt und reproduziert, so dass gesellschaftliche Wandlungsprozesse hin zu anderen Naturvorstellungen aber auch zu alternativen Mensch-Natur Beziehungen gar nicht abgebildet und damit nicht in die gesellschaftliche Sphäre hineingetragen werden.

Zum Zweiten dürfen aber auch die, die Umwelt- und Naturschutzdebatten ebenfalls prägenden Dissense und Konfliktpunkte nicht ausgeblendet und verschwiegen werden (bspw. die Problematiken um den Biomasseanbau zum Klimaschutz oder aber Betretungs- und Nutzungsverbote in bestimmten Naturschutzgebieten). Zwar wird eine „positive Kommunikation, die konkrete Handlungsmöglichkeiten und Erfolgsbeispiele aufzeigt von vielen Zielgruppen bevorzugt“ (ebd., 34), während „(e)in moralischer Zeigefinger dagegen (...) meist abstoßend wirkt" (ebd., 35), aber das bedeutet nicht, dass die Naturschutzkommunikation nicht auch Konflikte und Problematiken adressieren kann. Diese müssen indes in einen Kontext gestellt werden, durch den Menschen sich emotional davon angesprochen fühlen und den sie in ihr eigenes alltägliches Leben auch übertragen können. Werden Konflikte und Probleme indes ausgeblendet und damit ein 'Schönwetterbild' des Umwelt- und Naturschutzes gezeichnet, läuft dieses Gefahr, die Glaubhaftigkeit der Darstellungen, Botschaften und Appelle des Umwelt- und Naturschutzes zu gefährden, sobald diese auf anderen Wegen ins Bewusstsein der Menschen treten und dort ihre Lebensumstände berühren. Gerade weil die Natur bzw. die Biodiversität ein in weiten Teilen öffentliches Gut darstellt, über das auch öffentlich geredet, verhandelt und gestritten wird und werden muss, geht es hierbei nicht nur um ganz konkrete Konfliktsituationen, wie bspw. die geplante Elbvertiefung zum Hamburger Hafen, die Ausweisung eines Nationalparks im Schwarzwald oder aber der Straßenausbau durch bislang noch weniger zerschnitte Gebiete sondern immer auch um abstrakte Deutungskonflikte zwischen verschiedenen Auffassungen, Haltungen, Ein- 
stellungen und Überzeugungen zur Bewahrung und Förderung der Biodiversität, die kommunikativ und das bedeutet in unseren Gesellschaften und in unserer Zeit vor allem massenmedial adressiert werden müssen. Hierfür ist es wichtig, dass nicht nur die Willensbildungsprozesse, sondern auch die Deutungskonflikte in der Gesellschaft prozedural gerecht ausgetragen werden können.

\subsection{Fazit und Überleitung zum nächsten Kapitel}

Ziel der ethischen Reflexion auf Biodiversität als Wert- und Normbegriff war es zum einen die gängigen Gründe und Begründungen, die für die Bewahrung und Förderung der Biodiversität angeführt werden, kritisch zu reflektieren und zum anderen die theoretische Neuausrichtung der Umwelt- und Naturschutzethik hin zu einer anwendungsorientierten Biodiversitätsethik anhand der konstruktiven Lösung der drei in der Problemselektion aufgezeigten Detailproblematiken aufzuzeigen.

Zum einen wurde postuliert, dass über die zu einseitige Fokussierung auf das Inklusionsproblem und damit die Leitunterscheidung zwischen anthropozentrischen und physiozentrischen Ansätzen in der Umwelt- und Naturschutzethik immer wieder der hintergründige Dualismus zwischen Mensch und Natur repliziert wird. Dies führt aber dazu, dass die Perspektive auf die Auslotung der gesellschaftlichen Naturverhältnisse, die für die Bewahrung und Förderung der Biodiversität sowohl auf der inhaltlichen, auf der normativen als auch auf der politischen Ebene zentral ist, zu stark ausgeblendet wird. Diesbezüglich wurde darauf verwiesen, dass es in pragmatischer Hinsicht weniger um die Entscheidung gehen sollte, ob nun primär der Mensch oder die Natur im Zentrum der umwelt- und naturschutzethischen Überlegungen steht, sondern dass es gerade wenn es um die Bewahrung und Förderung der Biodiversität geht um die Auslotung der individuellen und kollektiven menschlichen Naturverhältnisse gehen muss. Daher wurde im Sinne einer progressiven Problemverschiebung vorgeschlagen, den hintergründigen Dualismus zwischen Mensch und Natur, den die Umwelt- und Naturschutzethik immer wieder über ihre Fokussierung auf das Inklusionsproblem repliziert, zugunsten einer relationalen Sichtweise auf die menschlichen Naturverhältnisse zu durchbrechen.

Zum zweiten wurde der primäre Fokus auf Schutz-(pflicht)begründungen problematisiert, da dieser dazu führt, dass die Umwelt- und Naturschutzethik auf Pflichten und Rechte beschränkte Begründungen für den Schutz der Biodiversität anbietet, dabei aber die Nutzungs- und Verteilungsziele sowie nicht genuin moralische Argumente und Begründungen vernachlässigt, womit die für die Bewahrung und Förderung der Biodiversität so entscheidende Integrativität der Zieltrias zu wenig beachtet wird. Dieser aus zwei Aspekten zusammengesetzten, zweiten Detailproblematik wurde über die Dar- 
stellung und Reflexion eines speziell auf die Biodiversitätszieltrias ausgerichteten biodiversitätsethischen Argumentationsraumes begegnet, der die Vielzahl an Argumenten im politischen und umwelt- und naturschutzethischen Kontext nach Klugheits-, Glücksund Gerechtigkeitsargumenten klassifiziert. Obgleich der Argumentationsraum in pragmatischer Hinsicht alle Anforderungen erfüllt, die sich über die Neuausrichtung der Umwelt- und Naturschutzethik ergeben, wurde herausgestellt, dass der Ansatz mit einem grundlegenden konzeptionellen Problem sowie bestimmten, für die Konzeptualisierung aber wichtigen, Auslassungen behaftet ist, die wiederum dazu führen, dass die anvisierten konstruktiven Lösungen der beiden Aspekte der zweiten Detailproplematik (die Integration der gesamten Zieltrias in einem Begründungsansatz sowie die Erweiterung des deontischen Fokusses) nicht in ausreichendem Maße durch den Ansatz realisiert werden können. Daher wurden die, in der Studie immer nur implizit anklingenden diskurstheoretischen Fundamente der Argumentationstrias aus Klugheit, Glück und Gerechtigkeit rekonstruiert, so dass auf der Grundlage der Spezifizierungen der pragmatischen, eudaimonistischen und moralischen Gebrauchweisen der praktischen Vernunft sowohl die Integration der Zieltrias eine Verhältnisbestimmung der Argumentationstypen zueinander als auch eine systematische und begründete Differenzierung der drei Argumentationstypen erfolgen konnte. Des Weiteren wurden die drei Argumentationstypen innerhalb eines mehrdimensionalen Ethikverständnisses verortet, so dass sowohl moralinterne (moralische Gebrauchsweisen der praktischen Vernunft) sowie moralexterne aber moralrelevante und moralrelative (eudaimonistische und pragmatische Gebrauchsweisen der praktischen Vernunft) zugleich in das 'Haus der Ethik' integriert werden konnten.

Drittens wurde speziell hinsichtlich der anvisierten Anwendungsorientierung vorgebracht, dass in der ethischen Kommunikation über Natur- und Umweltschutzanliegen im Allgemeinen und bezüglich der Bewahrung und Förderung der Biodiversität im Speziellen zu wenig beachtet wird, dass die Ausweisung 'guter Gründe ' nicht lediglich eine Frage der normativen Akzeptabilität der Gründe ist sondern immer auch eine Frage ihrer faktischen Akzeptanz. Diesbezüglich wurden unterschiedliche Akzeptanzverständnisse voneinander differenziert, um darzulegen, dass der Fokus der Ethik auf die normativen Akzeptabilitätsbedingungen keine reines Erkenntnisinteresse ist, sondern dass in der Ethik auch und vor allem dann eine wertende (und keine rein analytische) Unterscheidung zwischen faktischer Akzeptanz und normativer Akzeptabilität gemacht wird, wenn Akzeptabilität vor dem Hintergrund der Theorie des kommunikativen Handelns ausgedeutet wird. Dies, so wurde argumentiert, ist indes selbst eine problematische Verknüpfung, denn in diesem Kontext stellt sich erst gar nicht die praktischnormative Frage welchen Kriterien eine, auch im normativen Sinne gute strategische Naturschutzkommunikation unterliegen sollte. Daher wurde aufgezeigt, inwieweit die 
lebensstilorientierte Naturschutzkommunikation dazu beitragen kann, die gesellschaftlichen Akzeptanzbildungsprozesse im Umwelt- und Naturschutzbereich einerseits besser zu verstehen und andererseits die gesellschaftliche Meinungs- und Willensbildung zu gesellschaftlichen Umwelt- und Naturschutzanliegen gerechter zu gestalten.

Über die Lösung dieser drei Problematiken wurde der Zielstellung zu einer anwendungsorientierten Biodiversitätsethik zu gelangen entsprochen und zugleich das anvisiert, was Konrad Ott die „praktische Dimension der Umweltethik“ (Ott 2010:8) nennt. Im Anschluss daran stellt sich bezogen auf die Bewahrung und Förderung der Biodiversität allerdings nun die Frage, wie die ethisch begründeten Ziele und Maßnahmen zur Bewahrung und Förderung der Biodiversität umgesetzt werden sollen und wie eine solche Umsetzung politisch-strategisch ausgestaltet werden kann. Diese Fragestellung geht über die Entwicklung zielgruppenspezifischer Kommunikations- und Umsetzungsstrategien hinaus, denn hier stellt sich die Frage, wie die in der NBS anvisierte Good Biodiversity Governance (siehe 1.2.3) umgesetzt werden kann. 


\title{
V. POLITIKWISSENSCHAFTLICHE DETAILREFLEXION: Die theoretischen Grundannahmen, Anforderungen sowie Chancen und Risiken der Good Biodiversity Governance
}

\begin{abstract}
„Die Frage nach der Gestaltung gesellschaftlicher Naturverhältnisse muss sich zentral mit der Rolle des Politischen im weiteren Sinne und der Funktion der politischen Institutionen im engeren Sinne beschäftigen. Diese Zielsetzung (...) hat nichts mit der Erwartung zu tun, dass der Staat besonders geeignet sei für die Lösung sozialer wie ökologischer Probleme. In gewisser Weise ist genau das Gegenteil der

Fall. Da der Staat aber den zentralen Fokus für gesellschaftliche Konflikte und das Austragen von Interessensgegensätzen darstellt, muss diese Ebene selbst dann genau untersucht werden, wenn die Zweifel an seinen Gestaltungskompetenzen gewachsen sind."
\end{abstract}

(Görg 2003:22)

Das Eingangszitat von Christoph Görg macht bereits deutlich, dass die ökosoziale Frage nicht umhin kommt, nach der politikstrategischen Ausgestaltung der Bewahrung und Förderung der Biodiversität, der Rolle der Politik sowie nach den Funktionen der politischen Institutionen und Akteure zu fragen. Dies ist ganz im Sinne sowohl der Fokussierung nicht nur auf die Bewahrung, sondern auch auf die Förderung der Biodiversität (siehe 1.1) als auch gemäß der Präliminarien der Arbeit (siehe 1.2.3), wo die in der NBS anvisierte auf Kooperation, Verhandlung und Dialog ausgerichtete politikstrategi-sche Ausgestaltung der Bewahrung und Förderung der Biodiversität als Good Biodiver-sity Governance (GBG) charakterisiert worden ist. Wenn es unter Beachtung der in Abschnitt 2.3.4 aufgezeigten Kontexte des kooperativen Wandels der Staatlichkeit sowie der 'blinden Flecken' hinsichtlich der Explizierung der theoretischen Grundlagen der GBG im Folgenden um die theoretisch relevante Zielstellung gehen soll, die policy-, governance-, demokratie- und legitimationstheoretischen Grundlagen der GBG zu bestimmen, als auch um die praktische Zielstellung die Anforderungen, Chancen und Risiken zu benennen, die mit einem, an Kooperation, Verhandlung und Dialog orientierten Politikstil einhergehen, dann bedarf dies der Beantwortung folgender Fragstellungen:

a) Welche Annahmen von politischer Steuerung und Regulierung sowie demokratischer Beteiligung und Verantwortung können der GBG zugrunde liegen?

b) Welche Anforderungen ergeben sich an die deutsche Biodiversitätspolitik vor dem Hintergrund der theoretischen Annahmen der GBG?

c) Vor welchen Defiziten und Entwicklungsmöglichkeiten steht die deutsche Biodiversitätspolitik vor dem Hintergrund der Anforderungen, die sich über die GBG ergeben? 
Die Beantwortung der ersten Fragestellung visiert die Einholung der theoretischen Zielstellung an und erfordert eine ganze Reihe an abstrakten theoretischen Überlegungen, die im Grunde genommen auch gänzlich unabhängig von ihrem Biodiversitätsbezug angestellt werden können und sogar müssen, da es sich um in sich geschlossene und stellenweise hoch abstrakte Überlegungen zu den 'Grundbausteinen 'von Staatlichkeit und Demokratie handelt, die auch geeignet wären ein alternatives theoretisches Verständnis und Grundgerüst für Good Governance im Allgemeinen darzustellen. Die zweite und dritte Fragestellung, die wiederum die praktische Zielstellung der Arbeit anvisieren, die Anforderungen, Chancen und Risiken der GBG für die deutsche Biodiversitätspolitik auszumachen, dienen daher dazu, die allgemeinen und sehr abstrakten theoretischen Überlegungen an die Biodiversitätsdebatte zurückzubinden und im Endeffekt zu praxisrelevanten Erkenntnissen für die Umsetzung und politikstrategische Ausgestaltung der Bewahrung und Förderungen der Biodiversität zu gelangen: ${ }^{156}$

Erst einmal müssen die theoretischen Grundlagen des kooperativen, dialogischen und verhandlungsbasierten strategischen Politikmodus erarbeitet werden (5.1), der in Abschnitt 1.2.3 als Good Biodiversity Governance charakterisiert worden ist. Dazu muss zum einen das Politikfeld Biodiversität als spezieller Bereich der Umweltpolitik hinsichtlich seiner strukturellen Charakteristika dargelegt werden (5.1.1). Dies geschieht, indem das Mehrebenensystem erst einmal hinsichtlich seiner vertikalen Koordination zwischen den politischen Ebenen (5.1.1.1) sowie hinsichtlich seiner horizontalen Koordination speziell auf der nationalstaatlichen Ebene (5.1.1.2) beleuchtet wird. Im Anschluss daran werden die für die Beurteilung der Performanz umweltpolitischer Maßnahmen so entscheidenden umweltpolitischen Prinzipien und Instrumente dargelegt (5.1.1.3). Zum anderen muss zur Vorbereitung der für die erste Fragstellung relevanten Analyse der theoretischen Annahmen der GBG das in dieser Arbeit zur Anwendung kommende kooperative, dialogische und verhandlungsbasierte Verständnis von Good Governance gegen das entwicklungspolitische Standardkonzept abgegrenzt werden (5.1.2). Dazu wird erst einmal auf das Standardkonzept und dessen Problematiken eingegangen (5.1.2.1) und im Anschluss daran werden die relevanten Unterschiede zwi-

156 Die Rückbindung an die Biodiversitätsthematik geschieht durch den hohen Abstraktionsgrad der Ausführungen zur Beantwortung der ersten Fragestellung nicht zwischendrin sondern am Anfang und am Ende: Da die GBG nicht im 'luftleeren Raum ' konzipiert und theoretisiert werden kann, werden vorab ihre politikfeldspezifischen Grundlagen dargelegt (Abschnitt 5.1.1). Erst daraufhin ist die Vielzahl an komplexen und aufeinander bezogenen governance-, policy-, demokratie- und legitimationstheoretischen Überlegungen zur Beantwortung der ersten Frage 'eingebettet' (Abschnitte 5.1.2 5.3.3.3). Die praktischen Desiderate der theoretischen Überlegungen in Bezug auf die Bewahrung und Förderung der Biodiversität können demgegenüber nur als theoretisch begründete Abschätzung erfolgen, so dass über die Darlegung Anforderungen an die deutsche Biodiversitätspolitik, die sich aus den theoretischen Überlegungen ergeben sowie die Darlegung der Defizite und Entwicklungsmöglichkeiten, die sich wiederum aus den Anforderungen ergeben (Abschnitt 5.4), erst ganz am Ende die Rückbindung an die Biodiversitätsthematik erfolgt. 
schen den Good Governance Konzeptionen anhand der verschiedenen Varianten moderner Demokratietheorien erarbeitet und dargelegt (5.1.2.2). Diesbezüglich wird aufgezeigt, dass das Standardkonzept auf einem entscheidungszentrierten Demokratieverständnis fußt, während das anvisierte verhandlungsbasierte, kooperative und dialogische Verständnis von Good Governance durch seine theoretische Nähe zu den kontextbezogenen und prozeduralistischen Demokratietheorien gekennzeichnet ist. Daher wird das Good Governance Konzept anschließend anhand der Annahmen der kontextbezogenen und prozeduralistischen Demokratietheorien sowie der daran anknüpfenden partizipativen und deliberativen Governancemodelle expliziert (5.2). Dafür werden vor dem Hintergrund kontextbezogener Demokratietheorien einerseits die Zusammenhänge zwischen Good Governance und demokratischer Selbstbestimmung erarbeitet (5.2.1), indem das theoretische Grundgerüst der kontextbezogenen Demokratietheorien skizziert (5.2.1.1) und dann beispielhaft auf das Modell der »Empowered Participatory Governance» eingegangen wird (5.2.1.2). Andererseits werden vor dem Hintergrund der Ansätze der prozeduralistischen Demokratietheorie die Zusammenhänge zwischen Good Governance und diskursiver Verständigung sowie deliberativer Politik aufgezeigt (5.2.2). Auch hier wird erst der theoretische Hintergrund skizziert (5.2.2.1), bevor auf das Modell »deliberativer Governance» eingegangen wird (5.2.2.2). In einem Zwischenfazit (5.2.3) werden die bis dato erbrachten Überlegungen zusammengefasst und die Ansätze werden hinsichtlich ihrer Stärken und Schwächen zur Beantwortung der drei eingangs genannten Analysefragen bilanziert. Dabei wird aufgezeigt, warum es einer stärkeren Berücksichtigung governancethereotischer Erkenntnisse bedarf.

In einem nächsten Schritt wird Good Governance daher vor dem Hintergrund governancetheoretischer Überlegungen spezifiziert (5.3). Hierfür muss erst einmal auf den Begriff der Governance und die theoretischen Gehalte der politikwissenschaftlichen Governanceforschung eingegangen werden (5.3.1), damit aufgezeigt werden kann, wie der Übergang von Government zu Governance und mithin von Steuerung zu Regelung auf der theoretischen Ebene vollzogen wird (5.3.2). Dabei wird sowohl auf die theoretischen Verschiebungen (5.3.2.1) als auch auf die Kontinuitäten zwischen Steuerungsund Governancetheorie eingegangen (5.3.2.2). Die sich darüber ergebenden konzeptionellen und theoretischen Problematiken bei der Explizierung von Governance werden über den Ansatz des «akteurszentrierten Institutionalismus " geschlossen (5.3.2.3). In einem sich daran anschließenden Zwischenfazit (5.3.2.4) wird festgestellt, dass sich unter Zugrundelegung der so explizierten governancetheoretischen Annahmen auch das Erscheinungsbild der Demokratie wandelt. Daher werden die governancetheoretischen Überlegungen in einem nächsten Schritt speziell um demokratie- und legitimationstheoretische Überlegungen mit Bezug auf das Modell der Verhandlungsdemokratie erweitert. Dies - so die im weiteren Verlauf der Analyse relevante These - leistet 
die Theorie der »komplexen Demokratie« von Fritz Scharpf. Daher wird diese zugleich als Analyseinstrument als auch als Antwort auf die erste der eingangs gestellten Fragen nach der Ausbuchstabierung der GBG herangezogen und bildet damit zugleich das Fazit der Ausführungen zur theoretischen Konzeptualisierung der GBG (5.3.3). Dieses Fazit ist indes selbst mehrstufig, denn die komplexe Demokratietheorie muss, was ihre Modifikationen unter dem Einfluss der zunehmenden Internationalisierung der Staaten und der Globalisierung der Wirtschaft angeht, erst einmal rekonstruiert werden: Ausgehend von der Grundlegungsschrift "Demokratietheorie zwischen Utopie und Anpassung " (5.3.3.1) werden die von Scharpf vorgenommenen Erweiterungen der komplexen Demokratietheorie (5.3.3.2) herausgearbeitet, indem auf die Kriterien guter Politik (5.3.3.2.1) und die Kriterien legitimer Politik (5.3.3.2.2) unter verhandlungsdemokratischen Bedingungen eingegangen wird. Abschließend wird die komplexe Demokratietheorie hinsichtlich der aus ihr resultierenden Grundannahmen für die GBG zusammengefasst (5.3.3.3). Hiermit wird die Einholung der theoretischen Zielstellung der Arbeit als abgeschlossen betrachtet. Daher kann aufbauend darauf die praktische Zielstellung eingeholt werden, indem aufgezeigt wird, welche Anforderungen, Chancen und Risiken sich aus der GBG für die deutsche Biodiversitätspolitik ergeben (5.4). Dies geschieht, indem die Anforderungen an die deutsche Biodiversitätspolitik expliziert werden, die sich aus den theoretischen Annahmen der GBG ergeben (5.4.1) und indem die Defizite und Entwicklungsmöglichkeiten dargelegt werden, vor denen die deutsche Biodiversitätspolitik vor dem Hintergrund dieser Anforderungen steht (5.4.2). Über die letzten drei Abschnitte der Arbeit werden die allgemeinen Überlegungen zu Good Governance an die Biodiversitätsthematik zurückgebunden.

\subsection{Die Grundlagen und Bedingungen der Good Biodiversity Governance}

Ausgehend von der in Abschnitt 1.2.3 dargelegten Charakterisierung der Good Biodiversity Governance als kooperativem, dialogischem und verhandlungsbasiertem strategischen Politikmodus und der in Abschnitt 2.4.3 konstatierten 'blinden Flecken ' innerhalb der Politikwissenschaft, der Umwelt- und Naturschutzethik sowie der Biodiversitätsforschung werden im Folgenden zum einen das Politikfeld Biodiversität und dessen strukturelle Charakteristika und zum anderen das zur Beantwortung der ersten Frage nach den 'theoretischen Grundpfeilern` der GBG relevante, alternative Verständnis von Good Governance expliziert. Beides schafft die Grundlage für die weiteren dann bereits deutlich tiefergehenden theoretischen Betrachtungen. 


\subsubsection{Das Politikfeld Biodiversität und dessen Charakteristika}

Umweltpolitik ist ein Paradebeispiel für eine integrative Mehrebenenpolitik (MultiLevel-Governance) sowohl in vertikaler als auch in horizontaler Hinsicht. Der politische Bezugsrahmen der deutschen Biodiversitätspolitik umfasst dabei internationale, europäische und nationale Regelungen und Prinzipien, in denen sich die Akteure in den Fraktionen sowie aus den Ministerien und Ämtern des Bundes und der Ländern bewegen. Dabei ist für die deutsche Umweltpolitik zudem die föderale Struktur innerhalb der Bundesrepublik Deutschland von Bedeutung. Daher ist es für die Darlegung der Charakteristika des Politikfeldes Biodiversität analytisch sinnvoll vom Allgemeinen zum Besonderen zu gehen, indem zuerst die vertikale Koordination zwischen den politischen Ebenen (5.1.1.1), dann die horizontale Koordination speziell auf der nationalstaatlichen Ebene (5.1.1.2) und anschließend die für die Beurteilung der Performanz umweltpolitischer Maßnahmen so entscheidenden umweltpolitischen Prinzipien und Instrumente (5.1.1.3) beleuchtet werden. ${ }^{157}$

\subsubsection{Vertikale Koordination im Mehrebenensystem}

Vertikale Koordination meint die Behandlung von Politikfeldgrenzen überschreitenden Querschnittsaufgaben über die verschiedenen politischen Ebenen hinweg (Jänicke \& Jörgens 2004:318; Meijers \& Stead 2004:10). Martin Jänicke macht deutlich, dass es im Laufe der umweltpolitischen Entwicklung eine zunehmende Auslagerung regelungsrelevanter Materien auf höhere politische Ebenen gab und gibt, so dass sich darüber ein zwar nicht-hierarchisches aber strukturelles Mehrebenensystem entwickelt hat (Jänicke 2008:158). Vertikale Koordination ist innerhalb eines solchen Mehrebenensystem immer dann erfolgreich, wenn effiziente und legitime politische Problemlösungen auf der Ebene gefunden werden können, für die sie gebraucht werden, ohne dass es darüber zu vermeidbaren Externalitäten für die anderen politischen Ebenen kommt (Subsidiaritätsprinzip). Für die Politik der Biodiversität sind dabei - auch stellenweise wiederholend (siehe 2.2.1 und 2.2.2) - die folgenden Regelungsstrukturen relevant:

157 Dies ist nicht vergleichbar mit einer detaillierten Politikfeldanalyse, die eine Analyse der institutionellen Rahmenbedingungen, eine Analyse der interdependenten Organisations- und Koordinationsstrukturen, d.h. der Vernetzungen der politischen Akteure zwischen den politischen Ebenen (vertikale Koordination) sowie der genuin politischen und kollektiven und korporativen Akteuren wie Verbände, Gremien, Lobbygruppen, Unternehmen, NGO's, etc. innerhalb der politischen Ebenen (horizontale Koordination) und letztendlich der auf den jeweiligen politischen Ebenen einsetzbaren Policy-Instrumente vorsehen müsste (siehe vergleichend und verweisend insb. Aden 2012; Böcher \& Töller 2007, 2012). Dies kann und soll, schon allein aufgrund des Umfangs einer solchen Analyse, nicht Ziel dieser Darlegung sein. Hier werden im Folgenden nur die wichtigsten Aspekte dargelegt. Auf diese Aspekte wird dann immer wieder, auch vor dem Hintergrund der Darlegungen zur Good Governance (5.1.2), referiert, ohne dass diese noch einmal ausgeführt werden müssen. 
Auf der internationalen Ebene besteht die Regelungsstruktur primär aus den völkerrechtlich verbindlichen UN-Abkommen über die biologische Vielfalt (CBD), die Aktionspläne des "Washingtoner Artenschutzabkommens (CITES) «, der »Bonner Konvention zu wandernden Arten (CMS)« und über das »Übereinkommen über Feuchtgebiete (Ramsar-Konvention)«, da diese einen direkten Biodiversitätsbezug haben. Hinter diesen Konventionen steht eine ebenso komplexe organisatorische Struktur: Finanziert werden die internationalen Aktivitäten durch die von der Weltbank ins Leben gerufene »Globale Umweltfazilität (GEF)«. Dieser Umweltfond wird vor allem von den Industrienationen getragen und soll die Finanzierung von Biodiversitätsprojekten in den Entwicklungsländern unterstützen. Das wichtigste Entscheidungsgremium ist die zweijährig stattfindende »Vertragsstaatenkonferenz (COP)«, wo sich die Delegierten der Vertragsstaaten treffen, um die oftmals allgemeinen Aussagen des Konventionstextes zu konkretisieren und an einer gemeinsamen Umsetzung zu arbeiten. Hierbei sind im Mindesten VertreterInnen des "Bundesministeriums für Umwelt, Naturschutz, Bau und Reaktorsicherheit (BMUB)« als hauptsächlich zuständiges Ministerium und VertreterInnen anderer Ministerien, wie bspw. das »Bundesministerium für wirtschaftliche Zusammenarbeit und Entwicklung (BMZ)« dabei. Zudem gibt es den »Clearing-House Mechanismus (CHM)«, der als Informations-, Kommunikations- und Kooperationssystem der CBD dafür sorgt, dass die wissenschaftliche und technologische Zusammenarbeit der Vertragsstaaten gefördert und auf internationaler und zwischenstaatlicher Ebene der Austausch von Daten und Informationen ermöglich wird. Zuarbeitend sind hier der »wissenschaftlich-technische Ausschuss (SBSTTA)«, der den Stand der globalen Biodiversitätsvernichtung zu erfassen versucht, die "Arbeitsgruppe zur Überprüfung der Umsetzung der CBD (WGRI)« sowie der nicht in der CBD-Struktur enthaltene »Weltbiodiversitätsrat (IPBES)«, der als zwischenstaatliches Gremium zur wissenschaftlichen Politikberatung den politischen Entscheidungsträgern Informationen über den Zustand der Ökosystemleistungen zur Verfügung stellt.

Auf der europäischen Ebene sind die EU-Kommission, der Europäische Rat und das Europäische Parlament die drei zentralen Gesetzgebungsorgane. In der Regel macht das Exekutivorgan, sprich die Kommission, Vorschläge, die dann von den Staats- und Regierungschefs der Mitgliedsstaaten im Rat und durch die Abgeordneten im Parlament diskutiert, abgelehnt, modifiziert und/oder ratifiziert werden. Die Grundlagen der europäischen Umweltgesetzgebung ${ }^{158}$ bilden die »Fauna-Flora-Habitat-Richtlinie (FFH)« mit ihren Naturschutzbestimmungen zum »Natura-2000«-Schutzgebietssystem sowie die »Vogelschutzrichtlinie», die »Wasserrahmenrichtlinie» und die »Rahmenrichtlinie der

\footnotetext{
158 Die wichtigsten EU-Regelungen sind Verordnungen und Richtlinien: Verordnungen werden unmittelbar in den Mitgliedstaaten gültig. Richtlinien geben ein Ziel vor und es bleibt den Mitgliedstaaten überlassen, sie in eigenes Recht umzusetzen.
} 
Meeresstrategie«. Obwohl das Schutzgebietsnetz mit rund 27.000 Schutzgebieten bereits rund 19 Prozent der EU-Landfläche einnimmt und andere Schutzmaßnahmen der Wasserrahmenrichtlinie und der Rahmenrichtlinie zur Meeresstrategie begonnen wurden, scheiterte die EU mit ihrem anspruchsvollen 2010-Ziel, den Verlust der Biodiversität zu stoppen. Daher hat die »Europäische Kommission« nach der 10. Vertragsstaatenkonferenz 2010 in Japan eine »Europäische Biodiversitätsstrategie» (EBS) mit dem Ziel beschlossen, den Verlust an biologischer Vielfalt bis 2020 zu stoppen. Die EBS umfasst sechs Handlungsfelder: 1) Die Erhaltung und Wiederherstellung der Natur durch die vollständige Umsetzung der Vogelschutz- und FFH-Richtlinie, 2) die Erhaltung und Verbesserung der Ökosysteme und ihrer Leistungen durch „grüne Infrastrukturmaßnahmen“ zur Erhaltung und Verbesserung von fragmentierten Ökosystemen, 3) die Sicherstellung einer nachhaltigen Landwirtschaft, Forstwirtschaft und Fischerei durch die Ausdehnung genutzter Flächen, die von biodiversitätsbezogenen Maßnahmen profitieren, 4) die Bekämpfung invasiver gebietsfremder Arten sowie die stärkere Kontrolle ihrer Einschleppungspfade, 5) die Bewältigung der globalen Biodiversitätskrise durch eine bessere Umsetzung des bereits existierenden Umweltrechts in der EU sowie 6) die Verkleinerung des „Biodiversitätsfußabdrucks“ der EU als Handelsmacht und Unterstützung von Entwicklungsländern bei ihren Bemühungen um den Schutz der Biodiversität und ihrer nachhaltigen Nutzung (vgl. EU COM 2010). Der WWF konstatierte im Mai 2016 quasi als Zwischenbilanz, dass es entweder gar keine signifikanten Fortschritte gibt (Handlungsfeld 3 und 5) oder diese zu langsam passieren, um das 2020Ziel überhaupt noch erreichen zu können (vgl. WWF 2016).

Auf der nationalen Ebene ist das Bundesministerium für Umwelt, Naturschutz, Bau und Reaktorsicherheit (BMUB) der zentrale Akteur auf der Bundesebene. Das BMUB vertritt die Bundesrepublik Deutschland in seinen Politikbereichen auch auf der europäischen und internationalen Ebene und hat darüber hinaus auch die Aufgabe, mit den anderen Ministerien in umweltrelevanten Fragen zu kooperieren und umweltrelevante Informationen zu kommunizieren. Zum Geschäftsbereich des BMUB gehören fünf Bundesämter - das Umweltbundesamt (UBA), das Bundesamt für Naturschutz (BfN), das Bundesamt für Bauwesen und Raumordnung (BBR), das Bundesamt für Strahlenschutz (BfS) sowie das Bundesamt für kerntechnische Entsorgungssicherheit (BfE). Für das Politikfeld Biodiversität sind vor allem die ersten beiden Ämter maßgeblich. Die Programme zum Schutz und zur nachhaltigen Nutzung der Biodiversität werden dabei schwerpunktmäßig vom BfN bearbeitet und betreut. Das UBA übernimmt wiederum die Umsetzung, Weiterentwicklung und empirische Erfassung der in der NBS enthaltenen Umweltqualitäts- und Umwelthandlungsziele und nimmt an den, das Verteilungsprinzip bestimmenden, internationalen Verhandlungen zum ABS-Mechanismus teil (siehe 2.2.2). 
Als rechtlicher Regelungsrahmen fungiert das "Bundesnaturschutzgesetz (BNatSchg)«, welches unter Berücksichtigung internationaler Abkommen und Entwicklungen im Umweltrecht sowie im Rahmen der Umweltgesetzgebung innerhalb der Europäischen Union bereits mehrfach modifiziert wurde (siehe 2.2.2). 2010 ist es aufgrund der 2006 in Kraft getretenen Föderalismusreform nochmals novelliert worden. Entscheidend dabei ist, dass das Naturschutzrecht damit von der Rahmen- in die konkurrierende Gesetzgebung überführt wurde. Das bedeutet, dass der Bund nun die gesetzgeberische Kompetenz zur vollständigen und umfassenden Regelung des Naturschutzes und der Landschaftspflege hat, worunter auch der Schutz der Biodiversität fällt. Den Ländern ist es jedoch gestattet vom Bundesrecht abweichende Regelungen zu schaffen (Abweichungskompetenz), wenn das Bundesrecht zu der beabsichtigten Bestimmung keine Regelungen enthält (Regelungslücke), wenn eine Öffnungs- oder Unberührtheitsklausel vorliegt, die die beabsichtigte Vorschrift umfasst, wenn eine abweichende Gesetzgebungskompetenz des Landes vorliegt oder wenn es sich um eine Zuständigkeits- und Verfahrensvorschrift handelt (vgl. NABU 2010:7-8, 11). Es gibt indes auch „abweichungsfeste Inhalte“ des BNatSchg, wozu die allgemeinen Grundsätze des Naturschutzes (die ebenfalls novelliert wurden), der Artenschutz und der Meeresnaturschutz gehören (ebd., 6). Während also vorher für eine Materie entweder der Bundes- oder nur der Landesgesetzgeber zuständig war, kann nun Bundes- und Landesrecht aufeinandertreffen. Auch die Lösung solcher „Normenkollisionen“ wurde im Zuge der Föderalismusreform verändert. Wo vorher der Geltungsvorrang des Bundesrechts gegolten hat, geht nun das jeweils spätere Gesetz vor (ebd.). Das bedeutet, dass die Landesnaturschutzgesetze ebenfalls novelliert werden müssen und dass die Überführung in die konkurrierende Gesetzgebung eine noch stärkere Politikverflechtung im Umweltbereich zwischen und innerhalb der staatlichen Ebenen mit sich bringt. Diesbezüglich ist des Weiteren entscheidend, dass auch $\S 3$ des BNatSchg zu den Zuständigkeiten, Aufgaben und Befugnissen sowie zur Zusammenarbeit der Behörden neu geregelt wurde. So leitet §3, Abs. 4 dazu an, Landschaftspflegemaßnahmen zukünftig verstärkt durch Naturschutzorganisationen, Landschaftspflegeverbände und land- und forstwirtschaftliche Betriebe umsetzen zu lassen (ebd., 14). Abs. 5 bestimmt zudem, dass die Pflicht der Naturschutzbehörden zur frühzeitigen Einbindung und Information nicht nur gegenüber der Öffentlichkeit besteht, sondern auch gegenüber den Fachbehörden. Ins-gesamt (ver-)stärkt die Föderalismusreform die Notwendigkeit für eine horizontale Koordination im Mehrebenensystem im Allgemeinen und schafft Bedarfe für speziell kooperative, dialogische und verhandelnde Ansätze.

\subsubsection{Horizontale Koordination im Mehrebenensystem}

Horizontale Koordination meint die Behandlung von Querschnittsaufgaben über die Zuständigkeiten der jeweiligen politischen Ressorts hinweg (Meijers \& Stead 2004:10). 
Dass eine solche Koordination insbesondere im Umweltbereich nicht nur angezeigt sondern notwendig ist, liegt daran, dass sich die Umweltpolitik in ihrer spezifischen Problemstruktur von anderen Politikfeldern unterscheidet (Böcher \& Töller 2012:89): Zum einen durch die Persistenz und Irreversibilität bestimmter Umweltprobleme (Klimawandel, Zerstörung der Ozonschicht, Vernichtung der Biodiversität, Flächenversiegelung etc.), deren Verlauf und deren Folgen zumeist nicht genau prognostierbar sind sowie durch die selbst für langfristige Materien unübliche generationenübergreifende Zielperspektive, d.h. einem Umweltschutz, der auch im Interesse zukünftiger Generationen ist, und zum zweiten durch die Eigenschaft als redistributive Politik eines genuin öffentlichen Gutes, das aber privat angeeignet wird. Horizontale Koordination ist daher immer dann erfolgreich, wenn für diese systemimmanenten Probleme politisch-administrative Lösungen und Strukturen gefunden und etabliert werden können, um diese soweit abzumildern, dass sie einer effizienten und legitimen Lösung kollektiver verbindlicher Probleme nicht im Weg stehen (Planungs- und Integrationsprinzip):

Da die Langfrist- und Unsicherheitsproblematik in der Umweltpolitik dazu führt, dass die Wirkungen vieler umweltpolitischer Entscheidungen oftmals nur schwer abschätzbar sind und zudem erst Jahre oder Jahrzehnte später sichtbar werden, liegen in der Ungewissheit, Komplexität und Langfristigkeit des Politikfeldes Umwelt wesentliche Gründe, warum gerade hier die Versuche hoheitlich-staatlicher Steuerung so offensichtlich an ihre Grenzen stoßen und stattdessen das Kooperationsprinzip vordringlich wird: Die wachsende Komplexität und Kontingenz der Entscheidungsmöglichkeiten führt zu einem steigenden Bedarf an Informationen. Die Informationsverarbeitungskapazitäten sowie die im politischen System eingeübten Routinen zur politischen Entscheidungsfindung halten diesem Bedarf nicht Stand, so dass die staatlichen Akteure ohne die Kooperation mit anderen politischen Akteuren in anderen Politikfeldern bzw. mit außerstaatlichen Akteuren kaum noch handlungsfähig sind. Nach dem Grad der Informationsverarbeitungskapazitäten lassen sich drei verschiedene in ihrer Wirksamkeit nach unten abnehmende Regelungsmuster unterscheiden (Voigt 1995:41): Kodifizierung, wo auf Basis von hinreichendem Wissen und gesellschaftlich akzeptierten Werten eindeutige Rechtsvorschriften erlassen werden. Konfliktregulierung, wo es darum geht bestehende einer Problemlösung entgegenwirkende Interessenskonflikte friedlich zu verarbeiten und für alle Beteiligten annehmbare Zielsysteme auszuhandeln, da die Problematiken zwar bekannt sind, aber Uneinigkeit über die Lösungsmöglichkeiten besteht. Informationsverarbeitung zur Zielkonkretisierung und-realisierung, wo es auf Basis abstrakter Zielvorgaben sowie ungewisser Wirkungszusammenhänge erst einmal um die Erzeugung von konsensfähigem und anwendbarem Wissen geht, damit konkrete Probleme und deren Lösungen überhaupt erkennbar werden. 
Das zweite und dritte Regelungsmuster ist im Bereich der Umweltpolitik basal und beide gehen nicht selten dem ersten Regelungsmuster voraus, da umweltpolitischen Entscheidungen zumeist ein langer Prozess vorausgeht, was überhaupt als Problem angesehen wird (Gibt es überhaupt einen Klimawandel?), ob und wie dem Problem begegnet werden kann und soll (Primär technisch?, Durch einen kulturellen Wertewandel?, Durch neues Wissen und wenn ja welches?) und welche Maßnahmen dabei adäquat wären. Daher ist die Versuchung des Aufschubs (Abdiskontierung) von Entscheidungen und des 'Abwälzens ` der Folgen auf zukünftige Generationen extrem groß. Die Aufnahme solcher Thematiken in die politische Agenda ist damit mit dem enormen Risiko verbunden, dass keine oder aber nur sehr geringe, abgeschwächte, vielfach dem Problem gar nicht mehr angemessene politische Ergebnisse produziert werden (Böcher \& Töller 2012:92). Zudem besteht die Problematik, dass Zukunftsinteressen keine reguläre parlamentarische Stimme im politisch-repräsentativen System haben und dass der Vierbzw. Fünfjahreszyklus politischer Wahlen dafür sorgt, dass politische Parteien vornehmlich auf kurzfristige und schnell sichtbare Wahlerfolge hin orientiert sind (Zilleßen 2013:84-85). Hierzu gibt es indes gangbare Vorschläge:

Während wissensbasierte politische Beratungs- und Entscheidungsprozesse unter Beteiligung wissenschaftlicher Experten bereits an der 'Tagesordnung' sind und vor allem hinsichtlich der Beachtung des Vorsorgeprinzips (siehe 2.3.1, 4.2.1 und 5.1.1.3) gut in das politische System integriert wurden, laufen die Vorschläge zur „ökologischen und politischen Modernisierung der Demokratie“ (vgl. Jänicke 2013) wie bspw. Vorschläge zur Einführung eines Familien- und Kinderwahlrechts, bundesweite Volksabstimmungen zu Langfristthemen, die Einbindung von Zukunfts- und Minderheitenanwälten sowie die Einführung und Institutionalisierung von Beratungs- und Entscheidungsforen zur Konfliktlösung nach deliberativen Mustern oder dem Ralwsianischen Prinzip des 'Schleier des Nichtwissens' bis hin zu Überlegungen zum bedingungslosen Grundeinkommen und dessen 'ökologische' Wirkungen weitestgehend ins Leere. Gerade hinsichtlich der dabei relevanten partizipativen Erneuerungen ließen sich - so zumindest die These der BefürworterInnen - bisher ungenutzte Potenziale zur Abmilderung der Langfrist- und Unsicherheitsproblematik ausschöpfen. Ob dies wirklich der Fall ist und unter welchen Bedingungen, bleibt bisher nicht nur aufgrund des Umstandes, dass es wenige Realexperimente dazu gibt sondern auch auf Basis der theoretischen Überlegungen dazu ungewiss.

Als überwiegend redistributives Politikfeld produziert die Umweltpolitik zumeist einen diffusen Nutzen für die gesamte Gesellschaft, indem sie abgrenzbaren Gruppen Kosten auferlegt (Böcher 2007:255). Insofern ist es nicht verwunderlich sondern systemimmanent, dass die Umweltpolitik zu harten (inner-)politischen Konflikten führt, zumal es sich bei den Gegnern bzw. bei den Kostenträgern zumeist um gut organisierte, finanz- 
starke und zumeist vetomächtige Akteure (Wirtschafts- und Industrieverbände und Unternehmen) handelt, während die Befürworter häufig 'diffus' sind, auch wenn es auch hier mittlerweile gut organisierte und etablierte Akteure gibt (Umwelt- und $\mathrm{Na}$ turschutzverbände, Umweltstiftungen sowie wissenschaftliche Institute, Gremien und Beratungsorganisationen). ${ }^{159}$ Dennoch verlieren umweltpolitische Anliegen im politischen Prozess gegenüber anderen Interessen oftmals an Bedeutung, da es insbesondere die Wirtschaftsakteure mit ihren recht homogenen Interessen verstehen, ihre Ziele durch ihre Machtressourcen als Arbeitgeber durchzusetzen, wohingegen die eher diffusen Interessenlagen der Umwelt- und Naturschutzakteure sich weniger gut bündeln lassen und deren Vetomacht gegenüber den Wirtschaftsakteuren deutlich geringer ist (Böcher \& Töller 2012:91). Dass dies indes mitnichten zwangsläufig der Fall sein muss und gerade weil es auch umgekehrt schwierige Verhandlungen gibt, in denen die Umwelt- und Naturschutzakteure 'am längeren Hebel' sitzen, zeigt, dass es vor dem Hintergrund bestehender Konflikte zwischen 'Ökonomie' und 'Ökologie' - oder besser zwischen Nutzungs- und Schutzinteressen - oftmals um konkrete Verteilungsfragen geht und dass deren Entscheidung wiederum immer auch davon abhängig ist, welche Akteurskonstellationen sich in Verhandlungen ergeben und welche Interaktionsformen und -beziehungen diese miteinander haben oder ausprägen. Mit anderen Worten: Form und Inhalt bedingen sich gegenseitig und der 'Inhalt' ist im umweltpolitischen Kontext wesentlich davon abhängig, welchen Prinzipien die Umweltpolitik folgt und welche politischen Instrumente zur Anwendung kommen können, um sowohl innerpolitische Konflikte als auch gesellschaftspolitische Konflikte zu entscheiden.

159 Zahlreiche nichtstaatliche Akteure versuchen auf allen politischen Ebenen Einfluss auf die Umweltpolitik zu nehmen. Zum Lager der Vetoplayer zählen i.d.R. Wirtschaftsunternehmen und -Verbände. $\mathrm{Zu}$ den wichtigsten Wirtschaftsverbänden in Deutschland gehören u.a. der »Bundesverband der Deutschen Industrie e.V. (BDI)« und der »Verband Deutscher Automobilhersteller (VDA)«. Große Wirtschaftsunternehmen, die sich mit ihren finanzstarken Lobbygruppen (zum Teil auch versteckt) gegen umwelt- und naturschutzpolitische Maßnahmen stellen, sie zu verhindern, abzuschwächen oder zu unterlaufen versuchen, sind bspw. Energiekonzerne wie »Vattenphal« oder »RWE«, große Chemie- und Lebensmittelkonzerne wie "Monsanto« oder Nestle» sowie Transport- und Automobilhersteller wie »Cargo", "Lufthansa« oder »Volkswagen«. Auf der anderen Seite gibt es zahlreiche Verbände und Stiftungen, die sich für mehr Umwelt- und Naturschutz einsetzen. Zu den wichtigsten gehören u.a. der »Naturschutzbund Deutschland (NABU)«, der »Bund für Umwelt und Naturschutz Deutschland (BUND)«, der »Word Wild Wide Fund for Nature (WWF Deutschland), der »Bund Heimat und Umwelt (BHU)« der »Bundesverband beruflicher Naturschutz (B.B.N)«, der deutsche Alpenverein (DAV), der deutsche Naturschutzring (DNR), die »Deutsche Umwelthilfe (DUH)« sowie die »Deutsche Bundesstiftung Umwelt (DBU)«. Darüber hinaus gibt es wissenschaftliche Institute, Gremien bzw. Beratungsorganisationen, die Umwelt- und Naturschutzanliegen durch ihre Expertise (indirekt) unterstützen. Hier sind in Deutschland vor allem das »Max-Planck-Institut für Meteorologie», das »Potsdam Institut für Klimafolgenforschung (PIK)«, das »Wuppertal Institut», das »Freiburger Öko-Institut« und das Frankfurter »Institut für sozialökologische Forschung (ISOE)« zu nennen. Zu den wissenschaftlich-politischen Beratungsgremien gehören schließlich der »Wissenschaftliche Beirat globale Umweltveränderungen (WBGU)«, der Sachverständigenrat für Umweltfragen (SRU)« sowie der »Rat für Nachhaltige Entwicklung (RNE)«. 


\subsubsection{Prinzipien und Instrumente der Umweltpolitik}

Sowohl innerhalb der europäischen Union als auch im deutschen Umweltrecht sind das Vorsorgeprinzip, das Verursacherprinzip und das Kooperationsprinzip verankert. Diese Prinzipien sind die zentralen Leitlinien, nach denen sich auch die Biodiversitätspolitik in Deutschland ausrichtet:

Auf das Vorsorgeprinzip wurde bereits an verschiedenen Stellen in der Arbeit unter verschiedenen Blickwinkeln eingegangen (siehe 2.3.1, 4.2.1). Hier geht es primär um dessen politische Anwendung. Diese wird über die Mitteilung der Europäischen Kommission festgelegt (vgl. EU KOM 2000). Hier wurde bestimmt, dass die Anwen-dung des Prinzips auf einer möglichst umfassenden wissenschaftlichen Bewertung beruhen soll, in der auch das Ausmaß der wissenschaftlichen Unsicherheit ermittelt wird. Bevor folglich politische Entscheidungen und Maßnahmen getroffen werden, die die Umwelt, den Zustand der Natur oder auch die Gesundheit von Menschen betreffen, „sind die mit einem Tätigwerden oder Nichttätigwerden verbundenen Vor- und Nachteile gegeneinander abzuwägen. Diese Abwägung sollte eine wirtschaftliche Kosten-NutzenAnalyse umfassen (...) auch andere Analysemethoden z. B. zur Ermittlung der Wirksamkeit und der sozioökonomischen Auswirkungen der möglichen Optionen kommen in Frage." (ebd., 23)

Das Verursacherprinzip (eng: "polluter pays principle«) besagt, dass diejenigen, die Umweltschäden verursachen, auch die Kosten zur Vermeidung und Beseitigung von Umweltverschmutzungen tragen sollen. Es dient damit, anders als das Vorsorgeprinzip, nicht in erster Linie der Vermeidung von Umweltverschmutzungen sowie dem gesamtwirtschaftlich sparsamen Einsatz der natürlichen Ressourcen sondern der Zurechnung von Kosten. Damit soll auf Basis der Antizipation der durch Umweltnutzung entstehenden Kosten eine volkswirtschaftlich sinnvolle und schonende Nutzung der Natur und Umwelt erreicht werden. Am Verursacherprinzip orientieren sich solche umweltpolitischen Maßnahmen wie Umweltabgaben, Umweltauflagen in Form von Verfahrens- oder Produktnormen und freiwillige Maßnahmen wie Branchenabkommen oder Öko-Audits, die einen hohen Aufwand an Überwachung durch die Behörden erfordern. Bei der Durchsetzung des Prinzips treten zudem Zurechnungs- und Anwendungsprobleme auf. In Fällen, wo das Verursacherprinzip nicht oder nicht vollständig durchgesetzt werden kann, wird das Verursacherprinzip nach dem Gemeinlastprinzip so angewandt, dass die Allgemeinheit die Kosten für notwendige 'Reperaturmaßnahmen` trägt, d.h., die 'öffentliche Hand` für den Ausgleich der Umweltschäden aufkommt.

Das Kooperationsprinzip ist auf eine möglichst integrative und gemeinschaftliche Verwirklichung umweltpolitischer Ziele gerichtet und besagt, dass Umweltschutzaspekte in allen Politikfeldern berücksichtigt werden müssen und dass dafür sowohl staatliche 
als auch gesellschaftliche Kräfte bei der Findung und Durchsetzung der Umweltschutzziele mitwirken sollen (Politikintegration). Hierbei sollen politische Lösungen auf den politischen Ebenen gesucht und gefunden werden sollen, wo sie gebraucht werden (Subsidiaritätsprinzip). Davon verspricht man sich sachkundige Anregungen, eine Intensivierung der Umweltschutzanstrengungen aller Betroffenen und eine Stärkung des Umweltbewußtseins. Anwendung findet das Prinzip sowohl in der Umweltbildung und Umweltinformation, in Selbstverpflichtungserklärungen sowie bei der Einbindung der gesellschaftlichen Gruppen in die Weiterentwicklung der Umweltpolitik. Das Kooperationsprinzip steht aber auch dem Zusammenwirken von Bund, Ländern und Kommunen durch interministerielle Arbeitsgruppen im Umweltschutz vor oder kommt bei Vereinbarungen zwischen öffentlichen und privaten Akteuren beim Umweltmanagement sowie beim Erlass von Gesetzen und Verordnungen zum Tragen. Gesetzlich festgelegt ist dies bspw. für einen Teil der genehmigungsbedürftigen Anlagen nach dem Bundesimmissionsschutzgesetz, wo in der Planungsphase umweltbeeinträchtigender Vorhaben wie beim Straßen- oder Industriebau die 'beteiligten Kreise' anzuhören sind. Hier kommen VertreterInnen von Wissenschaft, betroffenen BürgerInnen, der beteiligten Wirtschaft und der Behörden zusammen. Durch die breite Beteiligung aller gesellschaftlichen Kreise hofft man die Akzeptanz und Umsetzung des Umweltrechts zu steigern. Die zentrale Schwierigkeit besteht hierbei jedoch im Ungleichgewicht der gesellschaftlichen Interessengruppen hinsichtlich ihrer Fähigkeit zum Agenda-Setting und zur politischen Einflussnahme.

Alle politischen Instrumente, die in der Umweltpolitik zum Einsatz kommen, müssen direkt oder indirekt zur Erfüllung dieser Prinzipien beitragen, obgleich die Instrumente selbst dazu dienen, konkretere politische Ziele zu erfüllen (Böcher \& Töller 2007:305). Sie stellen damit gewissermaßen den 'Werkzeugkoffer' politischer Akteure dar. Dieser lässt sich sowohl nach dem jeweiligen Koordinationsmechanismus der Instrumente als auch nach dem Ausmaß der politischen Intervention, die die Instrumente entfalten, klassifizieren. Die daraus entstehende Typologie besteht aus fünf instrumentellen Hauptgruppen: ordnungsrechtliche, ökonomisch-anreizbasierte, prozedurale, kooperative und informativ-persuasive Instrumente (vgl. Böcher \& Töller 2012:74-82):

Unter die regulativen Instrumente fallen klassischerweise Gesetze, Verordnungen und Richtlinien, aber auch verbindlich festgelegte Grenzwerte und rechtlich geregelte Bewilligungsverfahren für Produkt- und Prozessstandards (ebd., 74). Damit ist das Feld der regulativen Instrumente sehr breit gesteckt und es bietet sich an, zwischen direkten und indirekten regulativen Instrumenten zu unterscheiden: Direkte regulative Instrumente sind Gesetze, Verordnungen und Richtlinien, die sowohl die Handlung als auch das Ergebnis der Handlung normieren. Indirekte regulative Instrumente sind verbindlich festgelegte Grenzwerte und rechtlich geregelte Bewilligungsverfahren für Pro- 
dukt- und Prozessstandards, die zwar den Rahmen rechtlich normieren, die Handlungen und deren Ergebnisse aber selbst unbestimmt lassen. Regulative Maßnahmen im engeren Sinne sind somit alle ordnungsrechtlichen Instrumente. Diese bedürfen als Koordinationsmechanismus der Hierarchie des Staates, über den die Einhaltung verbindlicher Anweisungen durch Zwang und Sanktionen durchgesetzt werden kann. Dahinter steht (mindestens) die normativ relevante Vorstellung einer (Letzt-)Verantwortung des Staates, sprich, dass dem Staat innerhalb demokratischer Grenzen die Verantwortung für die Regelung und Lösung gesellschaftlicher Angelegenheiten zukommt (Stichwort: Gewährleistungsstaat). ${ }^{160}$ Vor diesem Hintergrund entfalten ordnungsrechtliche Instrumente durch hoheitlich-staatliche Steuerung den höchsten Grad an Intervention, indem sie die zulässigen Alternativen reduzieren und aufzeigen, welche Handlungsoptionen sozial erwünscht sind und welche nicht. Zu ihren Vorzügen zählt daher, dass sie - vor allem bei Krisen - schnell und treffsicher einsetzbar sind, dass dies aber um den Preis komparativer Kostenvorteile erkauft wird, dass reaktives Ordnungsrecht keine Anreize schafft, nach Innovationen zu suchen und dass das ordnungsrechtliche Ansätze mit der rasant fortschreitenden technischen Entwicklung überfordert sind (Böcher \& Töller 2012:77). Dazu gesellen sich praktische Probleme: Die Durchsetzung ordnungsrechtlicher Maßnahmen kann mit erheblichen Konflikten seitens der Adressaten verbunden sein. Ein primär durch ordnungsrechtliche Instrumente geschaffener Regulierungsrahmen für Umweltprobleme tendiert zudem im Laufe der Zeit ganz automatisch dazu immer komplizierter und differenzierter zu werden, so dass er für die BürgerInnen und stellenweise selbst für die Vollzugsbehörden schwer durchschaubar wird. Die bis dato beklagten Verwaltungs- und Vollzugsdefizite in der deutschen Umweltpolitik (vgl. insb. SRU 2007, 2012) sind Symptome dieser Problematik. Hierfür sollen sowohl ökonomisch-anreizbasierte als auch kooperative, prozedurale und informativ-persuasive Instrumente Abhilfe schaffen.

${ }^{160}$ Im Zuge der Kritik an den regulativen Instrumenten wird zuweilen auch eine Entbürokratisierung und Privatisierung der Wahrnehmung der Umweltschutzaufgaben gefordert, was jedoch aus verfassungsrechtlichen Gründen nur bis zu einem gewissen Punkt überhaupt zur Debatte stehen kann (SRU 2007:188). Die dem Staat aus den Grundrechten obliegenden Schutzpflichten sowie Artikel 20 a GG verpflichten den Staat letztverantwortlich das Gemeinwohl zu sichern. Daher ist der Staat bei nicht eigener Aufgabenwahrnehmung mindestens dazu verpflichtet, „die fachliche Rahmensetzung und Kontextsteuerung in Form von geeigneten Regelungsstrukturen zur Verfügung zu stellen, da diese die Erfüllung öffentlicher Aufgaben durch private Akteure erst ermöglichen, für den Fall der Nicht- oder Schlechterfüllung einer öffentlichen Aufgabe durch einen privaten Akteur eine subsidiäre Aufgabenerfüllung zu ermöglichen sowie die Folgen der privatwirtschaftlichen Aufgabenerfüllung abzumildern, falls diese zu einer defizitären Aufgabenerfüllung führen“ (ebd.). Dem Staat kommt insofern eine „Gewährleistungsverantwortung“ für die Erfüllung der Staatsaufgabe Umweltschutz zu (vgl. dazu allg. Schuppert 2005 und insb. Hoffmann-Riem 2005; Proeller \& Schedler 2005). Kritisch wird hiergegen vor allem seitens der Governanceforschung eingewendet, dass dem Staat zwar unzweifelhaft diese (Letzt-)Verantwortung zukommt, dass der Staat aber immer weniger in der Lage ist, dieser Verantwortung auch effektiv nachzukommen. Auf diese grundsätzliche Problematik wird vor allem in Abschnitt 5.3 genauer eingegangen. 
Zu den ökonomisch-anreizbasierten Instrumenten gehören Umweltabgaben in Form von Steuern und handelbare Emissionsrechte aber auch Anreizmechanismen wie Subventionen und finanzielle Förderprogramme (Böcher \& Töller 2012:74). Sie kommen zumeist dort zum Einsatz, wo ordnungsrechtliche Instrumente durch zu viel Widerstand scheitern würden oder wo es darum geht, durch finanzielle Anreize zu einer für die Adressaten möglichst flexiblen und dezentralen Lösung zu kommen, so dass der Spielraum für individuelle Entscheidungen so weit wie möglich verbleibt. Der hier wirksame Koordinationsmechanismus ist somit der Preis bzw. die Kosten für ein bestimmtes Verhalten, auf das der Staat u.a. in Form von Steuern oder Subventionen einen Einfluss ausübt, um die Adressaten zu gewünschten Verhaltensänderungen zu bewegen. Hierüber lassen sich Umweltstandards einholen, bei denen es keine Auflagen gibt, wie diese erreicht werden sollen, nur dass sie erreicht werden müssen (bspw. das 2 Grad Ziel in der Klimapolitik). Es handelt sich folglich um indirekte regulative Instrumente, da entweder im Falle negativer externer Effekte eine vorgeschriebene Abgabe dazu führt, dass es zu einer Internalisierung dieser externen Kosten kommt oder im Falle begrenzter Ressourcen bzw. schädlicher Produktionsmengen eine rechtlich bindende Höchstmarke festgelegt wird, deren Preis sich aber erst auf dem Markt bildet (Böcher \& Töller 2012:79). Dahinter steht die normativ relevante Vorstellung, dass der Markt und der Preismechanismus besser geeignet sind (d.h. effizienter und effektiver) als der Staat und das Ordnungsrecht, um gesellschaftliche Angelegenheiten zu regulieren. ${ }^{161}$ Vor diesem Hintergrund entfalten ökonomische Instrumente ein Lenkungsprinzip, da sie einen Anreiz schaffen, die umweltbelastenden Tätigkeiten einzuschränken, solange die Kosten und Nutzen der Bemühungen darum geringer sind als die zu entrichtende Abgabe. Ihr Vorteil besteht somit darin, dass sie eine eigendynamische und motivationale Wirkung entfalten, solange sie Anreize bieten, Umweltbelastungen anhand komparativer Kosten-, Nutzenvorteile einzuschränken. Entscheidende Nachteile sind indes, dass es oftmals schwer ist, bestimmte Umweltgüter konsistent 'einzupreisen', dass oftmals die Kosten für die Einschränkung der Umweltbelastungen kurzfristig höher sind als die Nutzen und diese sich erst mittel- und langfristig amortisieren und dass der Einsatz ökonomischer Instrumente, entgegen der eigentlichen Lehrbuchmeinung, häufig mit hohen politischen Widerständen und Konflikten verbunden ist, gerade weil darüber frühzeitig transparent gemacht wird, auf wen welche Kosten zukommen (ebd., 80). Insbesondere der letztgenannte Aspekt der realen geringeren politischen Durchsetzbarkeit solcher Instrumente, gerade wenn es um Umweltabgaben geht, relativiert die aus ökonomischer Sicht bestehenden Vorteiler marktwirtschaftlicher Instrumente.

${ }^{161}$ Einwenden ließe sich hier, dass der dabei postulierte Markt-Staat Antagonismus zu unterkomplex ist und dass Effizienzargumente zwar bedeutsam, mitnichten aber ausreichend sind, um die Wirksamkeit politischer Instrumente zu bewerten. Auf diese Problematik kann in der Arbeit nur vereinzelt eingegangen werden. Wichtiger ist der Aspekt, dass politische Instrumente nicht lediglich als administrativ-technische Mechanismen verstanden werden können. 
Vor dem Hintergrund dieser Problematiken stellen die prozeduralen Instrumente eine Alternative zu den ökonomischen Instrumenten dar. Zu den wenigen prozeduralen Instrumenten in der Umweltpolitik gehören der freiwillige "Öko-Audit« für Unternehmen, Organisationen und Behörden sowie die seit 1990 in Deutschland rechtlich geregelte »Umweltverträglichkeitsprüfung (UVP) « ${ }^{162}$ und deren ergänzende Richtlinie über die "Strategische Umweltprüfung (SUP)«, die die projektbezogene UVP auch auf Pläne und Programme ausdehnt (ebd., 74). Sie kommen insbesondere dort zum Einsatz, wo es Unternehmen, Organisationen oder Behörden darum geht ihre 'Öko-Bilanz' (auch aus repräsentativen oder wettbewerblichen Gründen) zu verbessern oder wenn es um die Planung und Verwirklichung von Großprojekten (bspw. Flussvertiefungen, Bau von Industrieanlagen, Flughäfen, Energiegewinnungsanlagen) geht, die einen Einfluss auf den Zustand der Natur und Umwelt haben (ebd., 80). Der hier wirksame Koordinationsmechanismus ist die "dezentrale Kontextsteuerung" (vgl. Teubner \& Willke 1984), die auf der systemtheoretischen Überlegung basiert, dass es durch politische Interventionen immer nur bedingt gelingen kann, in die Funktionslogik komplexer, eigenwilliger Systeme einzugreifen, so dass es bestimmter Ansätze bedarf, Firmen und Planungsverfahren mit ihren eigenen Umwelteffekten zu konfrontieren, so dass diese in die Lage versetzt werden, darauf mit ihren eigenen Mitteln zu reagieren. Weil dies vornehmlich durch Verfahrensvorgaben und deren behördliche Einhaltung und Überprüfung geschieht, handelt es sich um prozedurale Instrumente, die dennoch als indirekte regulative Instrumente verstanden werden können, da die Verfahrensvorgaben rechtlich geregelt sind, aber nicht normieren, wie das Ergebnis einer solchen Verfahrensprüfung auszusehen hat (Böcher \& Töller 2012:81). Verfahrensvorgaben dienen somit dazu, „die im materiellen Recht angelegte Konfliktlösung im Einzelfall durchzusetzen und eine rechtlich einwandfreie sowie sachlich gerechtfertigte Entscheidung herbeizuführen." (SRU 2007:153) Hinter den prozeduralen Instrumenten steht dabei die normativ relevante Vorstellung, dass Verfahrensfragen immer auch Machtfragen sind und dass der Staat über die behördliche Kontrolle der Verfahrensvorschriften Einfluss auf Bereiche nehmen kann und soll, die ihm ansonsten sowohl durch das Ordnungsrecht als auch durch ökonomische Anreizmechanismen verschlossen bleiben würden. Neben diesem Umstand besteht der größte Vorteil prozeduraler Instrumente darin, dass sie einen integrativen und sektorübergreifenden Umwelt- und Naturschutz ermöglichen (ebd., 36). Andererseits besteht die Schwierigkeit solcher Instrumente nach wie vor darin, dass sie mit dem stark sektoralisierten Umweltrecht nur bedingt kompatibel sind

\footnotetext{
162 Nach § 2, Abs. 1, Satz 2 UVPG beinhaltet die Umweltverträglichkeitsprüfung die Ermittlung, Beschreibung und Bewertung der unmittelbaren und mittelbaren Auswirkungen der Projekte auf Mensch, Flora und Fauna, Boden, Wasser, Luft, Klima und Landschaft, Sachgüter und kulturelles Erbe sowie der Wechselwirkungen zwischen diesen Faktoren. Dabei haben die für die Durchführung der UVP zuständigen Behörden umfassende ökosystemare Beurteilungen vorzunehmen, um genaue Erkenntnisse über die potenziellen Umweltauswirkungen zu gewinnen (SRU 2007:37).
} 
(Böcher \& Töller 2012:81) und dass es zuweilen unverhältnismäßiger Anstrengungen bedarf, medienübergreifende Umweltbewertungen durchzuführen, da wegen der sektoriell ausgerichteten Verwaltungsstrukturen eine Kooperation mit anderen Behörden oder anderen Staaten, deren umweltbezogener Aufgabenbereich von einem Vorhaben betroffen ist, zwar zwingend geboten nicht zugleich aber einfach realisierbar ist (SRU 2007:37). Dies verschärft sich noch einmal im Zusammenhang mit der strategischen Umweltprüfung von Plänen und Programmen, da diese bereits auf der Planungsebene weitsichtige prognostische und abwägende Entscheidungen verlangen (ebd.).

Eine Alternative hierzu stellen wiederum die kooperativen Instrumente dar. Kooperative Instrumente sind zumeist situativ oder formalisiert in Kraft tretende Verhandlungsformen wie runde Tische, Dialogforen und Mediationsverfahren (Böcher \& Töller 2012:74). Hier kommen politische Regelungseingriffe durch Koordinations- und Kooperationsversuche zwischen öffentlichen und privaten Akteuren zustande, wobei sich diese zumeist durch Freiwilligkeit, einen geringen Institutionalisierungsgrad sowie ein hohes Maß an Freiheit der Adressaten bei der Umsetzung auszeichnen (ebd., 81). Als politisches Instrument kommen Verhandlungen daher auch immer dort zum Einsatz, wo eine Problemlösung die Beteiligung vieler unterschiedlicher Akteure verlangt, weil dies entweder - wie in der Umweltpolitik - geboten ist (Kooperationsprinzip) oder weil die Politik die Entscheidungsfindung 'delegieren ' kann, da sich die Akteure Vorteile von einer gemeinsamen Abstimmung und Regelung erwarten (freiwillige Selbstregulation). Der hier wirksame Koordinationsmechanismus ist die Verhandlung, was je nach Situation zwischen den beiden Extrempolen ergebnisoffener, konsensualer Verständigung einerseits und (mehrheitlicher) Abstimmung bereits feststehender nicht mehr zur Debatte stehendender alternativer Ergebnisse ausgestaltet sein kann. Der Klassifizierung nach gehören kooperative Instrumente zu den nicht-regulativen Instrumenten, auch wenn Verhandlungen oftmals „im Schatten der Hierarchie“ stattfinden (Börzel 2008: 118-119), sprich, daran entweder VertreterInnen staatlicher oder weitestgehend politischer Institutionen teilnehmen. Diese können im Zweifelsfall auf ihre hierarchische Weisungsgewalt zurückgreifen, so dass hinter einer Einigung entweder die Drohkulisse staatlicher Regulierung steht oder das Koordinations- und Sanktionspotenzial des Staates explizit angerufen wird, weil freiwillige Beschlüsse Gefahr laufen durch Kooperationsverweigerungen oder 'Trittbrettfahren 'zu scheitern. Hinter den kooperativen Instrumenten steht, wenn erst einmal akzeptiert wird, dass diese keine regulativen Instrumente sind, die normativ relevante Annahme, dass diese zu legitimeren und problemadäquateren Lösungen als regulative Instrumentarien führen, da die von einer Entscheidung Betroffenen bereits in den Prozess der Entscheidungsfindung eingebunden werden können und weil auf diesem Weg gemeinwohlorientierte Lösungen gefunden werden können, indem der Dialog und der Austausch zwischen den Verhandelnden da- 
zu führen, dass individuelle und damit erst einmal egoistische und auf das eigene Wohl bezogene Interessen sich zu kollektiven und damit andere Interessen antizipierenden, sozialen Präferenzen wandeln. ${ }^{163}$ Neben diesen empirisch nur zum Teil bestätigten Erwartungen (vgl. Bäckstrand et al. 2010 gelten das schnelle und situative Zustandekommen, die große Flexibilität und Bandbreite von Verhandlungsformen und ihre vielseitigen Einsatzmöglichkeiten als entscheidende Vorteile. Deutliche Nachteile liegen hingegen in ihren mit steigender Problemkomplexität anwachsenden zeitlichen und strukturellen Anforderungen an die Verhandlungsteilnehmer, der motivationalen Grundlage der Gewinnerwartung durch Kooperation sowie der Vielzahl an Verhandlungsdysfunktionalitäten und -blockaden durch ungleiche Macht- und Ressourcenverteilungen, was sich an den zum Teil beachtlichen Informationsasymmetrien zwischen den Verhandlungsteilnehmern zeigt. Wenigstens letzteres kann durch informative Instrumente abgemildert werden.

Zu den informativ-persuasiven Instrumenten gehören Umweltbildungs- und Informationsmaßnahmen sowie staatlich regulierte und überprüfte Produktkennzeichnungen (Böcher \& Töller 2012:74). Hierbei handelt es sich um politische Regelungseingriffe, die auf formaler Ebene versuchen, Handlungen durch neue Erkenntnisse, Lernprozesse, Überzeugungen sowie Appelle zu beeinflussen. Der Koordinationsmechanismus der Information, im Sinne wichtiger und wissenswerter Neuigkeiten, soll auf niederschwelliger Ebene bewirken, dass VerbraucherInnen schnell und einfach Orientierung erhalten und BürgerInnen, Unternehmen, soziale Träger etc. Wissen über Umweltgefahren erhalten und überhaupt erst einmal in die Lage versetzt werden, sich dazu eine Meinung zu bilden. Insbesondere die Aufklärungs- und Berichterstattungsinstrumente, wozu in Deutschland die vom Umweltbundesministerien (BMUB) veröffentlichten »Daten zur Umwelt« sowie die vom Bundesamt für Naturschutz (BfN) veröffentlichten »Daten zur Natur« gehören, sollen der Aufklärung der BürgerInnen sowie zur Früherkennung dienen und überdies hinaus dazu beitragen, dass die Adressaten über ihren Umgang mit

163 Speziell die Governanceforschung beschäftigt sich mit den kooperativen Instrumenten, da ihr Fokus auf der Erforschung der sozialen Handlungskoordination durch die Kooperation öffentlicher und privater Akteure in formalisierten Verhandlungssystemen oder Netzwerken jenseits hierarchischer Formen des Regierens liegt (Börzel 2008:119). Die dabei oftmals im Fokus stehenden «New Modes of Governance«, die in der Regel mit partiziativen, deliberativen und diskursiven Regelungsweisen in Verbindung gebracht werden, gelten als funktionale Äquivalente zur hierarchischen Steuerung sowohl diesseits als auch jenseits des Nationalstaats. Die hierüber zum Ausdruck kommende (einseitige) Fokussierung der Governancedebatte auf kooperative Instrumente wird später noch einmal aufgegriffen und hinsichtlich der Problematik der theoretischen Grundlagen der Governancedebatte spezifiziert (siehe 5.3.2). An dieser Stelle genügt der Verweis darauf, dass kooperative Instrumente zumeist als nicht-regulative Instrumente verstanden werden und dass sich eben daraus ein problematisches Paradox für die Governanceforschung ergibt, welches Tanja Börzel wie folgt formuliert: „Die Ineffektivität hierarchischer Steuerung erfordert den Einsatz nicht-hierarchischer Formen der sozialen Handlungskoordination, deren Effektivität (und Legitimität) wiederum vom Schatten der Hierarchie abhängt, der weder auf der internationalen Ebene noch in sogenannten 'Räumen begrenzter Staatlichkeit’ gegeben ist.“ (ebd., 119-120) 
Natur und Umwelt nachdenken, diesen ggf. überdenken und ändern. Hinter den informativ-persuasiven Instrumenten steht damit die normativ relevante Annahme, dass BürgerInnen, aber auch Unternehmen und Organisationen zu wissensbasierten und überlegtem Handeln befähigt werden sollen, da, so die Hoffnung, informierte Entscheidungen eher dazu beitragen die Ziele des Umwelt- und Naturschutzes zu erreichen. Die übergeordnete Zielstellung solcher Aufklärungs- und Berichterstattungsinstrumente ist es damit, das Thema Natur und Naturschutz (positiv) in das Wahrnehmungsfeld verschiedener Zielgruppen zu tragen. Gerade weil es hierbei darum geht, Adressaten auf möglichst niederschwelliger Ebene und ohne Eingriff in ihre Handlungsoptionen zu beeinflussen, sind die informativen Instrumente nicht regulativ, selbst wenn das "Umweltinformationsgesetz« Behörden vorschreibt, Daten über den Zustand der Umweltmedien sowie umweltrelevante Daten über öffentliche und private Projekte auf Anfrage der Öffentlichkeit zugänglich zu machen und bestimmte Unternehmen bzw. Betriebe nach dem Kreislaufwirtschaftsgesetz dazu verpflichtet sind, Emissionsberichte oder Abfallbilanzen für genehmigungspflichtige Anlagen zu erstellen, deren Qualität aufgrund fehlender Standards aber sehr unterschiedlich ist (Böcher \& Töller 2012: 82). Die Vorteile informationeller Instrumente sind ihre leichte Ein- und Umsetzung, die Nachteile sind ihre, selbst bei zielgruppenspezifischer Einsetzung, nicht abschätzbare Wirksamkeit. Zudem gilt hier, dass ein 'Mehr' an Informationen, Berichterstattungen und Siegeln dazu führt, dass die Aufklärungs- und Orientierungsleistung solcher Instrumente abnimmt, da es den Adressaten schwer fällt zu entscheiden, welche Informationen wichtig bzw. wichtiger sind.

Einmal abgesehen von der hier dargelegten Klassifizierung und Charakterisierung der Instrumente qua Koordinationsmechanismus und Ausmaß der Intervention kann die Beurteilung umweltpolitischer Instrumente nach unterschiedlichen Kriterien erfolgen Effektivität, Effizienz und politische Durchsetzbarkeit (Böcher \& Töller 2012:74):

Bei der Effektivitätsnalyse geht es um die schwierige empirische Analyse, ob die eingesetzten Instrumente die mit ihnen verbundenen Zielstellungen wirklich erreichen können. Unter diesem Maßstab betrachtet, lässt die Diskussion um die 'neuen', nicht regulativen Instrumente oder Steuerungskonzepte (»New Modes Of Governance«) in der Umweltpolitik zuweilen den Eindruck entstehen in der Umweltpolitik müsse mit nicht regulativen Instrumenten gearbeitet werden, weil nur diese den Prinzipien der Umweltpolitik gerecht werden können. Hierbei lässt sich indes einwenden, dass auch ökonomische, prozedurale und kooperative Instrumente eine indirekte regulative Komponente haben und zudem ist es nach wie vor so, dass der wesentliche Teil der umweltpolitischen Regularien aus ordnungsrechtlichen Ge- und Verboten sowie Grenzwerten und Genehmigungungsverfahren besteht, was auch - und besonders - für die Umweltpolitik auf der europäischen Ebene gilt (vgl. Jacob \& Jörgens 2011:6). 
Bei der Effizienzanalyse geht es demgegenüber darum, ob der Einsatz der Mittel und die Erreichung der Ziele in einem (kosten-)günstigen Verhältnis zueinander stehen und welche Instrumente dasselbe Ziel unter der Maßgabe eines geringeren Ressourceneinsatzes und geringerer Transaktionskosten erfüllen können. Gerade hinsichtlich dieses Kriteriums wird über umweltpolitische Instrumentarien viel diskutiert. ${ }^{164}$ Hierbei ließe sich wiederum einwenden, dass diese Beurteilungsform „die Illusion einer rein technokratisch-sachrationalen Problemlösung nach Abwägung technischer Eigenschaften“ internalisiert (Böcher \& Töller 2012:75), was indes zu kurz greift, weil die strukturellen Bedingungen eine entscheidende Rolle bezüglich der Beurteilung sowohl der Effektivität als auch der Effizienz von Instrumenten haben und weil politische Instrumente immer auch bestimmte Annahmen bezüglich der Rolle des Staates und des demokratischen Modells enthalten, so dass die Beurteilung eben diese Aspekte auch berücksichtigen muss (Böcher \& Töller 2012:75).

Eben dies berücksichtigt das Kriterium der politischen Durchsetzbarkeit, da es „nach den tatsächlichen politischen Anwendungsmöglichkeit und dem Einfluss politischer Prozesse [fragt]“ (ebd.) und weil über dieses Kriterium geschaut wird, ob und warum sich bestimmte Instrumente eher etablieren können als andere. Hier finden sich u.a. Erklärungen, warum Effektivität und Effizienz oftmals gar nicht die ausschlaggebenden Kriterien bei der Wahl bestimmter Instrumente oder 'Instrumenten-Mixe' sind und warum die horizontale Intergration in der Umweltpolitik vorwiegend additiv und situativ geschah und geschieht (Jänicke 2008:157), da es zumeist darum geht, die Effekte anderer politischer Maßnahmen (für Biodiversität sind insbesondere verkehrs-, agrarund handelspolitische Effekte relevant; Böcher \& Töller 2012:93) durch verschiedenartige Instrumente einzudämmen. Zum anderen zeigt sich hierrüber, dass die Diskussion um die Bedeutung und Wirkung bestimmter Instrumente solange unterkomplex und kontextlos bleibt, solange nicht zugleich beachtet wird, dass der Erfolg und Misserfolg bestimmter Instrumente immer auch durch die institutionellen Rahmenbedingungen und das politische Gefüge bestimmt wird, d.h., dass Effizienz- und Effektivitätskriterien immer auch im Lichte ihrer strukturellen und prozeduralen Kontextbedingungen betrachtet werden müssen. Eben diese strukturellen und prozeduralen Kontextbedingungen gilt es aber ersteinmal zu bestimmen. Zu diesen Kontextbedingungen zählt u.a. das den weiteren Untersuchungen als Grundlage dienende Verständnis von Good Governance, welches sowohl die hier dargelegten Prinzipien als auch die Instrumente 'einbettet'.

164 Unter Effizienzaspekten werden vor allem die ordnungsrechtlichen Instrumente in Zweifel gezogen. Hier wird stattdessen für marktwirtschaftliche Instrumente argumentiert. Aber auch von anderen, vor allem kooperativen und informativen Instrumenten erhofft man sich neue Impulse und eine verbesserte Effizienzbilanz. 
Im Folgenden wird daher gezeigt, warum das Good Governance Konzept in seiner entwicklungspolitisch geprägten 'Standardkonzeption' weder ein methodisch adäquater, noch ein hinreichend komplexer, noch ein inhaltlich passender Ansatz zur Beantwortung der eingangs gestellten Frage ist, welche Vorstellungen und Annahmen von politischer Steuerung und Regulierung sowie demokratischer Beteiligung und Verantwortung der GBG zugrunde liegen können.

\subsubsection{Good Governance als Leitbild des Regierungshandelns und als normative Grundlage für die GBG}

Nachdem die Charakteristika des Politikfeldes Biodiversität umrissen wurden, muss nun als inhaltlich-theoretischer Einstieg in die Debatte um die Good Biodiversity Governance das Verständnis und die theoretische Konzeptualisierung von Good Governance als Leitbild des Regierungshandelns geklärt werden. Hierbei ist entscheidend, dass der von der "Weltbank« und dem »Internationalen Währungsfond" entwickelte Terminus Technicus Good Governance sowohl in inhaltlicher als auch in theoretischer Hinsicht von dem abweicht, was hier zur Basis der anvisierten Good Biodiversity Governance gemacht werden soll. Um dies zu zeigen, wird erst einmal auf das Standardkonzept von Good Governance und dessen Problematiken eingegangen (5.1.2.1) und im Anschluss daran werden die relevanten Unterschiede zwischen den Good Governance Konzeptionen anhand der Varianten moderner Demokratietheorien erarbeitet und dargelegt (5.1.2.2).

\subsubsection{Das Standardkonzept von Good Governance und dessen Problematiken}

Roland Czada führt in seinem Übersichtsartikel über "Good Governance als Leitkonzept für Regierungshandeln « (vgl. Czada 2010) aus, dass das Konzept der Good Governance, anders als das Konzept der Governance, wo es sehr verschiedenartige Begriffsvariationen und theoretische Hintergründe gibt (siehe 5.3.1), weniger ein analytisches als ein „normativ-praxeologisches Konzept [ist], das auf entwicklungspolitische Erfahrungen der 1970er und 80er Jahre zurückgeht." (ebd., 201) Die 1989 von der Weltbank veröffentlichte Studie über Subsahara Afrika und dessen Weg zu nachhaltigem Wachstum (vgl. Weltbank 1989) sowie das später veröffentlichte Policy Paper von 1992 über "Governance and Development " (vgl. Weltbank 1992) gelten als wichtigste 'Agenda Setting Agency` für die Etablierung des Good Governance Konzeptes (Druwe 1992:58). Dahinter stand und steht ein spezifischer Normenkatalog, „der die Teilnahme an politischen Kooperationsbeziehungen mit bestimmten Verhaltenserwartungen verbindet.“ (Czada 2010:201) Dieser Normenkatalog ist analog zu den generellen Verwendungen des Begriffes Governance neoinstitutionalistisch begründet und integriert einen persistenten neoliberalen Kern: 
Der neoinstitutionalistische Impetus zeigt sich vor allem daran, dass es beim Good Governance Konzept um „die institutionelle Koordination von sozialen, politischen und ökonomischen Austauschbeziehungen“ (ebd., 203-204) geht und dass der Vorteil und der Nutzungen von Institutionen und Strukturen darin gesehen wird, dass diese zur wechselseitigen Erwartungssicherheit beitragen und so die Rationalitätsgrundlagen individuellen Handelns verbessern als auch die Sicherung von kollektiven Langfristinteressen sicherstellen sollen (Stegmüller 1979:51-55). Der persistente neoliberale Kern der Good Governance Agenda zeigt sich wiederum darin, dass unter der Maßgabe effizienter und effektiver öffentlicher Dienstleistungen „alte Programme der Liberalisierung und globalen Marktschaffung im neuen Gewand (...) fortgesetzt" (Czada 2010: 211) werden und dass Programme der Entstaatlichung und der Privatisierung von Leistungen der Daseinsvorsorge weiterhin eine große Priorität genießen, auch wenn Prinzipien wie Rechtsstaatlichkeit, Transparenz, Fairness und die Gleichheit politischer Beteiligung und pauschal hohe ethische Verhaltensstandards für eine gute Regierungsführung ebenfalls eingefordert werden (vgl. OECD 1995).

Czada ist nicht nur vor dem Hintergrund skeptisch, dass das wirtschaftsliberal ausgerichtete Good Governance Konzept „lediglich technokratischen Reformforderungen“ anbringt (Czada 2010:211), sondern er wirft auch ein, dass hierbei ein mittlerweile bereits von der politischen Theorie überholtes Verständnis von einem dualistischen und antagonistischen Staat-Gesellschafts- und Marktverhältnis durch den neoliberalen Impetus immer wieder aufs Neue repliziert wird:

„Wie gut oder schlecht Regieren im Sinne der Fähigkeit ist, kollektive Problemlösungen zu finden, zu formulieren und umzusetzen, hängt überwiegend davon ab, wie das Interaktionsverhältnis zwischen staatlichen und gesellschaftlichen Akteuren beschaffen ist. (...) Die Betonung des Gegensatzes von staatlicher Souveränität und gesellschaftlichem Gruppeneinfluss hat die staats- und politikwissenschaftliche Forschung so nachhaltig und holzschnittartig geprägt, dass die Feinheiten von Staat-Gesellschaftsbeziehungen häufig nur verzerrt wahrgenommen werden. (...) Man gelangt in dieser Perspektive leicht zur Vorstellung einer einfachen, das Verhältnis von Staat und Gesellschaft bestimmenden politischen Kräftemechanik. Ihr liegt die Überlegung zugrunde, dass Autonomiegewinne der einen Seite notwendig mit Autonomieverlusten der anderen Seite verknüpft sind. Eine wohl organisierte, mit großen politischen Einflusspotenzialen ausgestattete Gesellschaft erscheint dann als Herrschaft der Verbände und drohende 'Kolonialisierung` des Staates (...), ein starker, autonomer Staat als Hemmnis zur die Entfaltung der Organisationsgesellschaft.“ (ebd., 219, 218)

Obgleich das auch dem Good Governancekonzept unterliegende simple GesellschaftsPolitik Modell von der politischen Theorie überholt ist und vor allem in der Gover- 
nanceforschung immer wieder in Frage gestellt und retheoretisiert wird, hat sich das entwicklungspolitische Good Governance Konzept vor allem dahingehend dieser theoretischen Neuausrichtung angepasst, als dass es auf eine aktive Einbringung und Einbindung privater, zivilgesellschaftlicher Akteure zur Verbesserung gesellschaftlicher und ökonomischer Entwicklungen und Potenziale beharrt (ebd., 217). Hier nimmt das Good Governance Konzept durchaus die empirischen Befunde der Neokorporatismusforschung und der politischen Ökonomie sowie die theoretischen Anleihen der modernen Demokratietheorie auf, die zeigen, dass gerade das unterhalb der parlamentarisch-demokratischen Repräsentation befindliche Subsystem der politischen Interessenvermittlung durch Verbände, Nichtregierungsorganisationen, Lobbygruppen, Firmen, Politikberatungsinstitute oder zivilgesellschaftliche Einzelakteure den für das alltägliche Regieren notwendigen Handlungskonsens hervorbringt und politische Entscheidungen vorbereitet und deliberiert. Der nicht mehr zivilgesellschaftliche aber auch noch nicht in Gänze politische und in diesem Sinne vorpolitische Zwischenbereich zwischen der institutionalisierten und professionalisierten Politik und der Zivilgesellschaft erhält somit über das Governancekonzept auf der analytischen Ebene und über das Good Governance Konzept auf der normativen Ebene eine besondere Relevanz. Good Governance hängt demnach immer auch davon ab, wie gut der Staat gesellschaftliche Akteure integrieren und koordinieren kann, wie gut er mit diesen kooperieren kann und zugleich wie gut gesellschaftliche Akteure sich mit ihren jeweiligen Expertisen, Möglichkeiten aber auch Vetopositionen einbringen und partizipieren können.

In diesem Kontext ist entscheidend, dass die von Czada konstatierte normativ-praxeologische Handhabung des Good Governance Konzepts (ebd. 202) dazu führt, dass die Prinzipien einer guten Regierungsführung - und somit auch die kooperativen, dialogischen und verhandlungsbasierten Momente - bereits als effizient, effektiv, sozial gerecht, fair, transparent und/oder demokratisch ausgedeutet werden und hierbei aber nicht im Sinne normativer Wissenschaft die Geltungsgründe der zugrundeliegenden Normen hinterfragt werden, sondern diese lediglich hinsichtlich ihrer Wirksamkeit bei der Lösung praktischer Probleme relevant sind (ebd.). Vor der Maßgabe normativer Wissenschaft kann daher nicht per se und pauschal davon ausgegangen werden, dass all die Prinzipien, die gemeinhin als kennzeichnend für eine gute Regierungsführung angeführt werden, auch tatsächlich erstrebenswert sind und dass ein 'Mehr' bspw. an Effizienz, Effektivität, Marktwirtschaftlichkeit, Transparenz, Rechtsstaatlichkeit und Demokratie im Endeffekt zu einer immer besseren Regierungsführung führt. ${ }^{165}$

165 Für viele (aber eben nicht alle) dieser Prinzipien lassen sich plausible und stichhaltige Argumente anführen, warum diese unverzichtbar für eine gute Regierungsführung sind. Dazu zählen Kriterien wie Rechtsstaatlichkeit, Beachtung der Menschenrechte und Transparenz. Ein generelles 'umso mehr, umso besser' lässt sich aber nicht problemlos argumentieren. So steht bspw. in Frage, ob ein prinzipielles 'Mehr' an demokratischer Teilhabe der BürgerInnen per se besser ist, als ein kontrolliertes 
Zudem steht hier in Frage, ob es bei einer guten Regierungsführung immer nur um das Ziel der Lösung praktischer Probleme geht. Einwenden ließe sich hier die eigentlich altbekannte Tatsache, dass die Stabilität und die Qualität eines politischen Systems nicht nur davon abhängt, was es für einen 'Output' und ferner einen 'Outcome' produziert ${ }^{166}$ sondern auch davon, welche 'Inputs' es bekommt und wie diese im politischen Prozess verarbeitet und zu politischen Entscheidungen umgewandelt werden (vgl. Easton 1971): Das politische System ist als Integrations- und Steuerungsinstanz auf verschiedenartige Inputs angewiesen: Ganz grundlegend braucht es ein Mindestmaß an ideeller Unterstützung, um überhaupt als politisches System anerkannt zu werden. Zweitens bedarf jedes politische System der Zufuhr zahlreicher materieller und immaterieller Ressourcen bspw. in Form von Steuern, Humankapital aber auch Wissen, Zeit, Werte und Normen sowie von Ideen und Impulsen, aus denen sich die politische Agenda konstituiert. Drittens bedarf es der Beteiligung (zivil-)gesellschaftlicher kollektiver und korporativer Akteure, um die ungleiche Verteilung von regelungsrelevanten Informationen, Steuerungs- und Expertenwissen, 'Know How und Regelungsressourcen auf Seiten des politischen Systems einerseits und den realen Betroffenheiten, den gefühlten Dringlichkeiten, dem 'Know That' und den vorfindbaren Kontextbedingungen und Ressourcen auf Seiten der gesellschaftlichen Sender und Adressaten andererseits zumindest abmildern und ausgleichen zu können. Hierfür bedarf es wiederum spontaner und dauerhafter Austauschbeziehungen politischer Akteure mit gesellschaftlichen Akteuren und Gruppen sowie einer über Wahlen hinausgehende Partizipation dieser Akteure an der politischen Entscheidungsfindung und Regelsetzung. Insbesondere der letztgenannte Aspekt geht über den Rahmen des entwicklungspolitischen Standardkonzepts von Good Governance hinaus. Warum dies der Fall ist, lässt sich anhand der verschiedenen Varianten moderner Demokratietheorien zeigen. Auch lassen sich hierüber die relevanten Unterschiede zwischen der Standardkonzeption von Good Governance und der hier anvisierten, alternativen Good Governance Konzeption darlegen, die auf Kooperation, Verhandlung und Dialog basieren soll.

\subsubsection{Die alternative Good Governancekonzeption}

Demokratietheorien unterscheiden sich grundsätzlich darin, was als das Spezifikum des Politischen angesehen wird, welche Rolle die politische Öffentlichkeit und mithin die (Zivil-)gesellschaft einnimmt und wie die Interaktion zwischen der privaten und der öffentlichen Sphäre verstanden wird (Lösch 2005:107). Besonders deutlich werden die

Maß durch Wahlen oder Volksentscheide bei ganz bestimmten Entscheidungen. Und es ist zu fragen, für wen dies besser ist und warum.

166 Policy-Outputs sind das materielle Ergebnis des politischen Entscheidungsprozesses, sprich, Gesetze, Verordnungen, neue administrative Strukturen und so weiter. Policy-Outcomes sind hingegen das, was solche Outputs in der realen Welt tatsächlich bewirken. 
Differenzierungen vor allem daran, welche normative Aufladung des Begriffes Zivilgesellschaft in den Theorien jeweils vorgenommen wird und wie wesentlich diese normativen Implikationen wiederum für andere bspw. legitimationstheoretische oder organisationstheoretische Aspekte der jeweiligen Demokratietheorie sind, da die mit der Zivilgesellschaft verbundenen 'Heilserwartungen`erst vor dem Hintergrund der damit verbundenen Befürchtungen einer Fern- und Fremdbestimmung verständlich werden (Kleger 1994:306). Insofern lassen sich die Verständnisse von politischer Teilhabe und die Verfahren und Praxen der politischen Entscheidungsfindung und Policygenese über die Austauschbeziehungen zwischen Politik und Gesellschaft (Stichwort: Deliberation $^{167}$ ) mit Heinz Kleger idealtypisch danach klassifizieren, ob die damit verbundenen Erwartungen und Befürchtungen:

- dem liberalistischen Bild einer wirtschaftlich aktiven, auf die Privatsphäre bezogenen Bürgergesellschaft entsprechen, die gegen die hypertrophe Staats- und Rechtsgesellschaft gesetzt wird, was Kleger als „Paradigma der Handelsgesellschaft" (ebd.) kennzeichnet;

- in das radikaldemokratische Bild einer sitten- und kulturgeschichtlich zusammengeführten, auf die Gemeinschaftssphäre bezogenen Bürgergesellschaft passt, die gegen die hypertrophe Stadt- und Weltgesellschaft konzipiert wird, was Kleger als „Paradigma der Volksgemeinschaft“ (ebd.) kennzeichnet oder

- aus dem deliberativen und diskursiven Bild einer aktiven, auf die Öffentlichkeitssphäre bezogenen Bürgergesellschaft erwachsen, die gegen die systemisch agierende Wirtschafts- und Technikgesellschaft gestellt wird, was Kleger als „Paradigma der Diskursgesellschaft“ (ebd.) kennzeichnet.

167 Deliberation ist im angloamerikanischen Sprachgebrauch ein alltagssprachlicher Begriff, der in etwa Abwägung, Überlegung und/oder gegenseitiges Beraten bedeutet (Lösch 2005:10). Deliberation umfasst dabei zwei verschiedenartige aber zusammenhängende Tätigkeiten: Einerseits individuelles Abwägen und Bedenken andererseits gemeinsames Beratschlagen durch Dialoge, Diskussionen und Debatten. Insbesondere über letzteres ergibt sich der sinngemäße Zusammenhang von deliberativ, partizipativ und diskursiv, der sich aber nur dann in vollem Maße erhellt, wenn diskursiv nicht nur alltagssprachlich verstanden wird, sondern wenn diskursiv im Sinne des sprachphilosophischen Diskursbegriffes benutzt wird. Gemäß der Habermaschen Diskurstheorie und -ethik soll Diskurs im Folgenden als „Schauplatz kommunikativer Rationalität" verstanden werden, in dem über die Wahrheit von Behauptungen und die Richtigkeit bzw. Legitimität von Normen mittels Argumenten vernünftig verhandelt werden kann (Habermas 1981a:114). Damit ist der Begriff des Diskurses in den theoretisch anspruchsvollen und binnenkomplexen Kontext der "Theorie des kommunikativen Handelns" eingebettet (vgl. Habermas 1981a, 1981b). Insofern Deliberation damit einerseits auf die Kommunikations- und Beteiligungsprozesse innerhalb der Bürger- bzw. Zivilgesellschaft abhebt und andererseits auf die Vermittlungsprozesse zwischen Zivilgesellschaft und Staat, stehen damit immer auch die demokratietheoretisch relevanten Fragen im Fokus, was die Bürger- bzw. Zivilgesellschaft in normativer Hinsicht ausmachen bzw. auszeichnen soll, welche Rolle der Zivilgesellschaft in der Demokratie zukommt und wie ihr Verhältnis zur staatlichen, aber auch zur ökonomischen Sphäre zu bestimmen ist. 
Diese verschiedenen idealtypischen Möglichkeiten der Antwort auf die Frage, was eine (Zivil-)gesellschaft ist und ausmachen soll, lassen sich wiederum bestimmte Kategorien moderner Demokratietheorien zuordnen: ${ }^{168}$

Auf das Paradigma der Bürgergesellschaft als Handelsgesellschaft heben vor allem liberale, liberalistische, empiristische, elitentheoretische, pluralistische und ökonomistische sowie zu einem gewissen Grad auch systemtheoretische Modellierungen von Demokratie ab, die Bettina Lösch als entscheidungszentrierte Demokratietheorien kennzeichnet (vgl. Lösch 2005:122-133), da ihre Gemeinsamkeit darin besteht, dass sie sich an der als universalistisch postulierten Rationalität eigennutzmaximierender Akteure orientieren, so dass wiederum auf ein Verständnis von Zivilgesellschaft als Aggregat individueller Akteure fokussiert wird, die möglichst frei von äußeren Zwängen und Einschränkungen sein sollte, so dass die politische Öffentlichkeit primär als 'Drohkulisse für demokratische Abstimmungs- und Entscheidungsprozesse fungiert und Demokratie als Mechanismus zum Zweck der Erzielung kollektiver Entscheidungen und zur Lösung kollektiver Problematiken verstanden wird.

Auf das Paradigma der Bürgergesellschaft als Volksgemeinschaft beziehen sich demgegenüber vor allem republikanische, kommunitaristische, partizipative und postmoderne Demokratietheorien, die Lösch als kontextbezogene Demokratietheorien zusammenfasst (ebd., 134-149), da diese sich dadurch auszeichnen, dass sie ein individualistisches, partikulares Vernunftverständnis inkludieren, worüber die Zivilgesellschaft als kulturell-, territorial-, sozial- und wertgebundene politische Gemeinschaft erscheint, so dass die politische Öffentlichkeit gleichbedeutend mit der Sphäre der Zivilgesellschaft ist, von der aus sich ableitet, wie Demokratie verstanden wird, was demokratisch verhandelt und entschieden wird und wie dies vonstatten gehen soll. Die Zivilgesellschaft ist folglich für das Gelingen und Scheitern demokratischer Teilhabe relevant, die in dieser Modellierung dem Ideal der demokratischen Selbstregierung folgt.

Auf das Paradigma der Bürgergesellschaft als Diskursgesellschaft heben schließlich die deliberativen Demokratietheorien ab, wobei hier zwischen einer liberalen und einer diskursiven Variante unterschieden wird, die Lösch zusammenfassend als prozeduralistische Demokratietheorien einstuft (ebd., 150-169), da in diesen von einem ebenfalls als universalistisch postulierten, verständigungsorientierten Vernunftgebrauch kommunikativer Abwägung und Einigung ausgegangen wird, so dass die Zivilgesellschaft hier als Diskursgemeinschaft konzipiert wird und die Meinungs- und Willensbildungs-

168 Bettina Lösch, die sich in ihrem Buch kritisch mit den verschiedenen Konzeptionen von Öffentlichkeit, Demokratie und politischer Partizipation beschäftigt (vgl. Lösch 2005), weicht bewusst von der üblichen Unterteilung in normative oder klassische versus empiristische und realistische Demokratietheorien ab (vgl. ebd. 101-121). Stattdessen entwickelt sie über die verschiedenen Auffassungen der Zivilgesellschaft eine dreiteilige Kategorisierung zwischen entscheidungszentrierten, kontextbezogenen und prozeduralistischen Demokratietheorien, die hier zur Anwendung kommt (siehe 2.4.3). 
prozesse innerhalb der politischen Öffentlichkeit sowie ihre 'Weitergabe' in die Sphäre der institutionalisierten und professionalisierten Politik im Vordergrund der demokratietheoretischen Betrachtung stehen.

Nun lässt sich vor dem Hintergrund der Differenzierung zwischen den Demokratietheorien aufzeigen, dass das Standardkonzept von Good Governance auf einem entscheidungszentrierten Demokratieverständnis fußt, während das hier anvisierte kooperative, dialogische und verhandlungsbasierte Verständnis von Good Governance durch seine theoretische Nähe zu den kontextbezogenen und prozeduralistischen Demokratietheorien gekennzeichnet ist:

Lösch führt aus, dass sich die entscheidungszentrierten Demokratietheorien dadurch auszeichnen, dass sie „die Regelung politischer Abstimmungs- und Entscheidungsprozesse sowie die Präferenzgenese in den Mittelpunkt der Betrachtung" (Lösch 2005: 122) stellen, die sich über den institutionalisierten, professionalisierten politischen Entscheidungsprozesses via Wahlen, Parteien, Parlamenten, Ausschüssen, Kammern, Behörden etc. ergibt (ebd. 128). Dies geht wiederum mit einem grundlegenden Skeptizismus gegenüber der Ausweitung partizipatorischer Möglichkeiten der Bürgerlnnen einher (ebd. 124), weil Partizipation sowohl in den entscheidungszentrierten Demokratietheorien als auch im entwicklungspolitischen Good Governance Konzept lediglich als Schutzmechanismus vor antidemokratischen Tendenzen interpretiert wird, was aber bereits als erreicht angesehen werden kann, wenn Partizipation durch Wahlen und pluralistische Interessensvertretungen abgedeckt ist (ebd., 74). Obgleich also das Good Governance Konzept die Ausweitung partizipatorischer Einflussmöglichkeiten fordert, geschieht dies vor dem Hintergrund von Überlegungen und Annahmen, die sich auch im Rahmen entscheidungszentrierter Demokratietheorien wiederfinden. In beiden Fällen geht es auf der analytischen Ebene primär um die Beleuchtung der Ausgabe-Seite des politischen Prozesses und auf der normativen Ebene um die Output- und Outcomeoptimierung sowie die Outputlegitimation hinsichtlich einer guten Regierungsführung im Sinne der Erzielung kollektiver Entscheidungen. Demokratische Verfahren sind hier lediglich in einem rein funktionalistischen Sinn relevant, sprich als Instrumente zur Erzielung kollektiver Entscheidungen und zur Lösung kollektiver Probleme (ebd., 122), so dass auch eine gute Regierungsführung am Maßstab dieser Output- und Outcomeorientierung gemessen wird. Vor diesem Hintergrund lassen sich die aus dem klassischen Good Governance Konzept ableitbaren Reformvorschläge als „'top-down '-Ansät$z e^{\prime \prime}$ (Walk 2008: 16,73-75) verstehen, da hier die institutionellen Arrangements, d.h. „die Strukturen und Prozesse repräsentativer (Kollektiv-)Akteure wie Parlamente, Parteien, Nationalstaaten, Länder und Verbände“ im Fokus stehen, die „ihren Beitrag zur Verbesserung der Problemlösungsfähigkeit und Effektivität von Entscheidungen beitragen." (ebd.,73) 
Für das in dieser Arbeit anvisierte Good Governance Konzept würde dies recht eingeschränkte Verständnisse von Kooperation, Dialog und Verhandlung nach sich ziehen, denn die entscheidungszentrierte Ausbuchstabierung vernachlässigt sowohl die sozialen, gesellschaftlichen und kulturellen Voraussetzungen demokratischer Beteiligung und Teilhabe, die Menschen überhaupt dazu bewegen sich politisch zu engagieren oder zu professionalisieren und übersieht des Weiteren die in ausdifferenzierten Gesellschaften vorfindbaren Notwendigkeiten und Möglichkeiten einer auf Verhandlung und Verständigung ausgerichteten rationalen, kommunikativen Abwägung der verschiedenartigen Interessen innerhalb pluralistischer Gesellschaften. Mit anderen Worten: Wenn das entwicklungspolitische Standardkonzept von Good Governance hier zur Grundlage der weiteren Überlegungen gemacht werden würde, dann fallen dabei die Austauschbeziehungen zwischen Zivilgesellschaft und der politische Sphäre (Stichwort: Deliberation) aus dem Fokus sowie die in den kontextbezogenen Demokratietheorien hervorgehobenen Aspekte der emanzipatorischen Bedeutung der Beteiligung der 'NormalbürgerInnen` am politischen Prozess (Stichwort: zivilgesellschaftliche Partizipation) und der in den prozeduralistischen Demokratietheorien herausgestellte Aspekt der zugleich inputlegitimierenden als auch outputlegitimierenden kollektiven Suche nach der Lösung gesellschaftlicher und politischer Problematiken durch den rationalen Austausch von Argumenten (Stichwort: rationaler Diskurs).

Würden diese Aspekte indes berücksichtigt, dann würde auf der analytischen Ebene die Input-Seite des politischen Prozesses und auf der normativen Ebene die Inputoptimierung und Inputlegitimation hinsichtlich einer guten Regierungsführung stärker beleuchtet. Auch würden sich dann aus dem Good Governance Konzept andere Reformvorschläge ableiten lassen, die sich als „'bottom-up 'Ansätze“ betiteln ließen (ebd., 16, 74-75): Hierbei stünden ganz explizit die Möglichkeiten der Maximierung von Teilhabe, Beteiligungs- und Teilnahmechancen im Fokus, welche nicht nur einen funktionalistischen sondern vor allem einen normativ relevanten Stellenwert erhalten. Hierbei wird davon ausgegangen, dass einerseits die politischen Kompetenzen der 'NormalbürgerInnen `gefördert werden müssen, wenn die erwünschte bürgerliche Emanzipation erlangt werden soll, die als oberster Wert hinter der Demokratie als Regierungs- und Staatsform steht (ebd., 74), indem die politisch-öffentlichen Kompetenzen sowie die „Empowerment-Funktionen der Zivilgesellschaft“ gestärkt werden (ebd., 16). Andererseits wird hierüber auch auf die Etablierung und Stärkung deliberativer und diskursiver Verfahren und Prozesse fokussiert, da diese dazu führen sollen, dass die politischen Entscheidungen von Beginn an durch einen breiten gesellschaftlichen Konsens getragen werden, was Implementierungsdefiziten vorbeugen soll und hierüber die Leistungsfähigkeit des politischen Systems steigert (Blatter 2007:277-278). 
Da es im Folgenden zur Bestimmung der Good Biodiversity Governance, wie in Abschnitt 2.4.3 dargelegt, um eine Integration von Demokratie-, Legitimations- und Policy- und Governancetheorie unter der Fragestellung geht, welche Vorstellungen und Annahmen von politischer Steuerung und Regulierung sowie demokratischer Beteiligung und Verantwortung der GBG zugrunde liegen können, werden im Folgenden die hier hergeleiteten partizipatorischen, deliberativen und diskursiven Aspekte der New Modes of Governance fokussiert. Genaue Abgrenzungen sind dabei oftmals nur schwer möglich, da alle drei Aspekte zumeist unscharf, zugleich und/oder bezogen aufeinander verwandt werden (vgl. insb. Fung \& Wright 2001; Grote \& Gbikpi 2002; Nanz \& Steffek 2004; Walk 2008; Geissel 2009; Sørensen \& Triantafillou 2009; Dryzek 2012). Allerdings sind gewisse Fokusunterschiede auszumachen: Partizipative Governance bezieht sich vor allem auf die durch direktdemokratische Elemente beförderte aktive Mitbestimmung und Mitgestaltung zivilgesellschaftlicher Akteure am politischen Prozess sowie die dezentrale Umstrukturierung politischer Institutionen (Walk 2008:1416). Deliberative Governance fokussiert wiederum stärker auf prozedurale Mechanismen im Austausch zwischen (Zivil-)Gesellschaft und Politik, so dass es auf der Prozessund der Akteursebene um die Analyse aber auch die Beurteilung der Beziehungsmuster zwischen diesen sowie um die Instrumente und Ressourcen zu deren Koordinierung geht (Bäckstrand et al. 2010:3). Diskursive Governance wiederum betont, dass es bei Governancearrangements um eine nicht-strategische, verständigungsorientierte Interdependenzbewältigung politischer Komplexität geht (bzw. gehen sollte) (Viehöver 2013: 219-220).

Der Fokus auf alle drei Varianten ist nicht nur theoretisch sondern auch praktisch relevant, da hinter diesen die selten so klar geäußerte Annahme steht, dass über ein Mehr an partizipativen, deliberativen und diskursiven Elementen die durch die Globalisierung, die zunehmende politische Vernetzung und die Zunahme globaler Problematiken entstandene Gefahr der Handlungsunfähigkeit demokratischer Staaten abgemildert werden kann (Walk 2008:20) und dass sich trotz oder gerade aufgrund der weitreichenden, irreversiblen und sowohl inner- als auch oberhalb der Nationalstaaten wirksamen Transformationsprozesse des Politischen neue Möglichkeiten zur demokratischen Aushandlung sowie zur Erzeugung kollektiver politischer Güter ergeben. Dies ist, so formulieren Karin Bäckstrand und KollegInnen pointiert, das hoffnungsvolle Versprechen hinter den „New Modes of Environmental Governance“ (Bäckstrand et al. 2010:6). Daher werden im Folgenden die kontextualistischen und prozeduralistischen demokratietheoretischen Überlegungen zu Good Governance als kooperativem, verhandlungsbasierten und dialogischen Politikstil im Allgemeinen und zu den partizipativen, deliberativen und diskursiven New Modes of Governance im Speziellen dargelegt. 


\subsection{Die Spezifizierung von Good Governance vor dem Hintergrund demokratietheoretischer Überlegungen zu Partizipation, Deliberation und Diskurs}

Für die nun anstehende Explikation der theoretischen Grundlagen der GBG müssen die demokratietheoretischen Hintergründe der partizipativ, deliberativ und diskursiv ausbuchstabierten Good Governance Konzeptionen herausgearbeitet werden. Hierbei werden gemäß der vorgenommenen demokratietheoretischen Differenzierung einerseits die Zusammenhänge zwischen Good Governance und demokratischer Selbstregierung vor dem Hintergrund kontextbezogener Demokratietheorien erarbeitet (5.3.1), indem zuerst das theoretische Grundgerüst der kontextbezogenen Demokratietheorien skizziert (5.3.1.1) und dann exemplarisch auf das Modell der „Empowered Participatory Governance» (vgl. Fung \& Wright 2001) von Archon Fung und Erik Olin Wright eingegangen wird (5.3.1.2). Zum anderen werden die Zusammenhänge zwischen Good Governance und diskursiver Verständigung vor dem Hintergrund prozeduralistischer Demokratietheorien aufgezeigt (5.3.2). Auch hier wird zuerst der theoretische Hintergrund skizziert (5.3.2.1), bevor dann exemplarisch auf die Modellierung »deliberativer Governance« (vgl. Dryzek 2012) nach John Dryzek eingegangen wird (5.3.2.2). Abschließend werden die Ausführungen zusammengefasst und die Stärken und Schwächen der partizipatorischen und deliberativen Governancekonzeption zur Ausbuchstabierung der Good Biodiversity Governance bilanziert (5.3.3).

\subsubsection{Partizipative Governance vor dem Hintergrund kontextbezogener Demokratietheorien}

Die kontextbezogenen Demokratietheorien, die oftmals auch unter partizipatorischen oder kommunitaristischen Demokratietheorien firmieren, bestimmt Bettina Lösch zum einen über deren aktive Abgrenzung von den entscheidungszentrierten Demokratietheorien, über ihren Fokus auf die politisch-demokratische Selbstbestimmung der Bürgerlnnen sowie die konstitutiven (vor-)politischen Voraussetzungen von demokratischer Politik (Lösch 2005:133). Dabei zeigt sie auf, dass die kontextbezogenen Demokratietheorien eine universalistische, abstrakte Betrachtungsweise des Politischen ablehnen und stattdessen von partikularen und kulturspezifischen Erfahrungen der Menschen sowie von der tatsächlichen individuellen Handlungspraxis von Menschen in heterogenen, komplexen Gemeinschaften sowie von einem individualistischen, kulturspezifischen und partikularen Vernunftverständnis selbstbestimmten Handelns ausgehen, so dass sie sich der demokratietheoretischen Grundfrage zuwenden, welche Voraussetzungen und Bedingungen erfüllt sein müssen, damit Menschen zu einer Demokratie als Lebensform gelangen können (ebd. 121). Die Befürworterlnnen kontextbezogener Demokratietheorien fokussieren daher insbesondere auf den (vor-)politischen Pro- 
zess der Meinungs- und Willensbildung innerhalb der Zivilgesellschaft. Diesen sehen weder als Mittel zum Zweck kollektiver Problemlösungen, wie dies entscheidungszentrierte Demokratietheorien tun, noch betrachten sie diesen als Mittel zur Legitimationsbeschaffung oder als Verfahren der Wahrheitsfindung, wie dies prozeduralistische Demokratietheorien (siehe 5.2.2) tun, weil sie an diesen kritisieren, dass die prozeduralistischen Theorien „in ihrer universellen Ausrichtung und philosophischen Abstraktheit den konkreten Lebens- und Problemkontexten der Menschen nicht gerecht wird.“ (ebd. 134) Um im Folgenden den zwischen diesen Abgrenzungen liegenden 'Kern ` kontextbezogener Demokratietheorien verständlich zu machen und vor diesem Hintergrund zu spezifizieren, was mit partizipativer Governance gemeint ist, wird das theoretische Grundgerüst der kontextbezogenen Demokratietheorien skizziert (5.2.1.1) und danach wird auf das Governancemodell der »Empowered Participatory Governance» von Fung und Wright eingegangen (5.2.1.2).

\subsubsection{Das theoretische Grundgerüst der kontextbezogenen Demokratietheorien}

Die VertreterInnen kontextbezogener Demokratietheorien versuchen eine „gehaltvolle“ Demokratietheorie zu entwerfen, die sich in erster Linie gegen die in ihren Augen „mageren“ Demokratieverständnisse abgrenzen soll, wie sie in den entscheidungszentrierten Demokratiemodellen mit ihrer Betonung des Individualismus und der starken Gewichtung privater Interessen vertreten wird (Barber 1994:32). ${ }^{169}$ Die Kritik richtet sich damit vornehmlich an den elitären und ökonomistischen Verständnissen der Demokratie aus. Diese würden, neben all den Vorzügen, die diese als liberale Demokratiemodelle haben, die von den kontextbezogenen Demokratietheorien auch weitestgehend anerkannt werden, dazu beigetragen, dass sich Individuen in erster Linie durch ihre Privatsphäre und ihren Besitz definieren, „aber unfähig zur Selbstbestimmung sind." (ebd. 70) Vor diesem Hintergrund stehen in der Kommunitarismusdebatte die sich als Weiterentwicklung sowie gleichzeitig als eigenständige Abgrenzung zum politischen Liberalismus verstehen lässt (Honneth 1993:7) die Fragen im Vordergrund, „bis zu welchem Maße liberal-demokratische Gesellschaften auf einen gemeinsamen Wertehorizont angewiesen sind" (Lösch 2005:135, SL) und welche soziomoralischen Ressourcen notwendig sind, um ein modernes, ausdifferenziertes Gemeinwesen am Leben zu erhalten (Honneth 1993:15). Diesbezüglich wenden sich die Vertreterlnnen

169 Das entscheidungszentrierte Modell der Demokratie krankt aus kommunitaristischer Sicht vor allem daran, dass es einem zu starken elitären Denken zuneige, den normativen Gehalt partizipatorischer, emanzipatorischer und direkter Demokratie verkenne und unterschätze und damit für die gesellschaftlichen Pathologien des 20. und 21 Jahrhunderts mitverantwortlich sei (Lösch 2005: 33). Denn diese bleiben immer dann eine Antwort schuldig bzw. diese erweist sich als wirkungslos, wenn es darum gehe, Angriffe auf die Gemeinschaft, die Gerechtigkeit, die Bürgerschaft oder die Bürgerbeteiligung zu verhindern (ebd.). Insofern wird das liberale Denken als 'mager' angesehen, da es lediglich darauf abhebe, alle Angriffe auf das Individuum, d.h., auf seine Privatsphäre, sein Eigentum, seine Interessen und seine Rechte abzuwehren. 
kontextbezogener Demokratietheorien in erster Linie „gegen eine elitäre Vereinseitigung der Demokratie, wie sie zumeist von (wirtschafts-)liberaler Seite vertreten wird.“ (Lösch 2005:133) ${ }^{170}$ Das Spannungsverhältnis zwischen liberalen und kommunitaristischen Ansätzen findet dabei in zwei unterschiedlichen Annahmen ihren Ausdruck, die sich als „unterschiedliche Strategien der Artikulation und Thematisierung ein und derselben demokratischen Grundproblematik lesen [lassen] " (Buchstein \& Schmalz-Bruns 1992:376). Lösch formuliert diese wie folgt:

"Während die liberale Vorrangthese auf die Priorität eines universalistisch begründeten Rechts setzt und die Autonomie des Individuums und die Sicherung der Freiheit fokussiert, behauptet die kommunitaristische Vorrangthese die Priortät der Gemeinschaft, des Gleichheitspostulats und des demokratischen Meinungs- und Willensbildungsprozesses." (Lösch 2005:135)

Hiermit wird der Vorrang der lebensweltlichen, kontextbezogenen Praxis vor einer abstrakten, universalistischen Theorie angenommen, womit sich die BefürworterInnen kontextbezoger Demokratietheorien einerseits gegen den „objektivistischen und elitären, paternalistischen Charakter [stellen], den ein universalistisches Recht gegenüber den Willensbildungsprozessen und den Wertüberzeugungen der BürgerInnen annehme." (ebd.) Andererseits wenden sie sich damit aber auch gegen VertreterInnen prozeduralistischer Demokratiekonzeptionen (siehe 5.2.2), da sie an diesen kritisieren, sie würden an der Unfähigkeit scheitern, die kulturellen Bedingungen der Reproduktion einer gerechten Gesellschaft hinreichend zu berücksichtigen (Honneth 1993:15). Insofern wird Demokratie in den kontextbezogenen Demokratietheorien - in beide Richtungen abgrenzend - als Sphäre der Selbstregierung der Bürgerlnnen verstanden (Barber 1994:146). In Anlehnung an Carole Patemens "partizipatorische Demokratie« (vgl. Pateman 1970) wird dabei sowohl von der Lernfähigkeit des Menschen als auch von der

170 In elitären Demokratietheorien, wie bspw. von Joseph Schumpeter, wird davon ausgegangen, dass es den BürgerInnen zumeist an den entscheidenden Qualitäten zur Findung und Umsetzung politischer Entscheidungen mangelt. Bei Schumpeter bspw. macht sich dies an einer nicht für alle entscheidungszentrierten Demokratietheorien verallgemeinerbaren, vernunftskeptischen Ansicht gegenüber den politischen Fähigkeiten der BürgerInnen fest: So fehlt es, Schumpeter zufolge, den meisten BürgerInnen selbst bei lokalen öffentlichen Angelegenheiten an Fähigkeiten, die relevanten Tatsachen zu erkennen, nach ihnen zu handeln und sich dafür auch verantwortlich zu zeigen (Schumpeter 1942:415 in Lösch 2005:124). Der typische Bürger „argumentiert und analysiert auf eine Weise, die er innerhalb seiner Sphäre seiner wirklichen [d.h. privaten] Interessen bereitwillig als infantil anerkennen würde. Er wird wieder zum Primitiven. Sein Denken wird assoziativ und affektmäßig." (Schumpeter 1942:416 in ebd.) Dementsprechend finden sich in vielen entscheidungszentrierten Demokratietheorien ablehnende bis zuweilen sogar abschätzige Haltungen gegenüber politischen Partizipationsrechten der 'NormalbürgerInnen', die über das Wahlrecht bzw. über die Abstimmung von Führungspositionen hinausgehen. Hier offenbart sich dasselbe Bild eines politisch unmündigen und damit gefährlichen Bürgers, wie es auch in den psychologischen Studien von Gustave Le Bon (vgl. LeBon 1982) oder José Ortega y Gasset (vgl. Ortega y Gasset, 1989) zum Ausdruck kommt, die auch heute noch als 'Schreckensbilder` gegen die Unvernunft der „Massen“ und die "Massendemokratie“ herangeführt werden. 
Möglichkeit politischer Bildungsprozesse im Verlauf der demokratischen Meinungsund Willensbildung ausgegangen, obgleich diese nicht lediglich unterstellt sondern als Aufgabenstellung angesehen werden. Hierbei kommt ein gesamtgesellschaftlicher Ansatz von Demokratie und Demokratisierung zum Tragen, bei dem Demokratie als „Idee des Gemeinschaftslebens selbst“" (Barber 1994:101) verstanden wird, obgleich das politische oder demokratische Leben - anders als in republikanischen Ansätzen - auch nicht als die Lebensform schlechthin betrachtet wird (Lösch 2005:143). Der 'homo politicus' von dem hierbei ausgegangen wird, ist unter demokratischen Bedingungen ein besserer Mensch:

„(...) abhängig, doch unter demokratischen Verhältnissen im Besitz der Selbstbestimmung, unzulänglich und unwissend, doch unter demokratischen Verhältnissen belehrbar, egoistisch, doch, unter demokratischen Verhältnissen kooperativ, eigensinnig und solipsistisch, unter demokratischen Bedingungen jedoch schöpferisch und zu echter Selbstveränderung fähig." (Barber 1994:101-102)

Benjamin Barber hebt damit hervor, dass nicht primär die politischen Institutionen, sondern zuallererst der demokratische aber in diesem Sinne auch vorpolitische Meinungs- und Willensbildungsprozess das Herzstück der Demokratie darstellt. Genau wie in einem Organismus, in dem das Herz nicht ohne das Funktionieren anderer Organe schlagen kann, ist auch die demokratische Meinungs- und Willensbildung an das Vorhandensein und Funktionieren anderer demokratischer Aspekte (wie bspw. ein Rechtssystem, Gewaltenteilung, Wahlen oder andere plebiszitäre Elemente, Parteien und Verbände institutionelle Ausgestaltung demokratischer Praktiken und Prozesse etc.) gebunden, aber ohne die demokratischen Meinungs- und Willensbildungsprozesse würden die anderen demokratischen Aspekte ihre eigentliche Essenz verlieren. Sie wären, wieder in Analogie zum Herzen, dann zwar immer noch wichtige 'Organe' des Gesamtorganismus, aber es würde ihnen an 'Leben ' fehlen. Was in einer demokratischen Sphäre der Selbstregierung der BürgerInnen daher am wichtigsten sei, ist die 'vitale' Diskussion, die allerdings nach bestimmten Spielregeln verlaufen muss, um auch eine demokratische zu sein. Martin Walzer führt diesbezüglich aus, dass „der Bürger, der das überzeugendste Argument vorträgt - d.h. der mit seiner Argumentation die meisten anderen Bürger überzeugt - sich durchsetzt. Er kann dabei aber weder Gewalt anwenden, noch seinen Rang ausspielen, noch Geld verteilen, sondern er muss über die anliegenden Probleme sprechen." (Walzer 1992a:430) Erst wenn er das tut, ist er Teil der Bürgerschaft und nimmt ernsthaft an der Gemeinschaft teil. Der Titel des Bürgers und der Bürgerschaft ist daher nichts Gegebenes sondern etwas Aufgegebenes, da weder das 'Volk' bereits die Bürgerschaft bildet noch die Vorstellung von der Selbstregierung der Massen zutreffend sei: 
„Massen machen Lärm, Bürger beratschlagen, Massen verhalten sich, Bürger handeln, Massen stoßen zusammen und überschneiden sich, Bürger engagieren sich, teilen etwas miteinander und leisten einen Beitrag." (Barber 1994:152).

Durch diese im Laufe der individuellen und gesellschaftlichen Sozialisation entwickelten Qualitäten konstituieren sich Personen folglich erst als BürgerInnen und bilden darüber erst eine politische Gemeinschaft. Erst wenn 'Massen' beginnen sich zu beratschlagen, überlegt zu handeln und etwas zum Wohle der Gemeinschaft beizutragen, sprich deliberieren, werden sie zur Bürgerschaft. Gemeinschaft, Partizipation und Deliberation sind daher notwendige Aspekte ein und derselben sozialen Daseinsweise, nämlich der Bürgerschaft innerhalb einer Demokratie als Lebensform:

"Gemeinschaft ohne Partizipation rationalisiert lediglich den Kollektivismus, verleiht ihm die Aura der Legitimität. Partizipation ohne Gemeinschaft rationalisiert nur den Individualismus und verleiht ihm die Aura der Demokratie.“ (ebd., 153)

Was in Bezug auf die Voraussetzungen bürgerschaftlicher Partizipation, Deliberation und Vergemeinschaftung auf den ersten Blick betrachtet unisono zur Theorie kommunikativen Handelns von Habermas klingt (siehe 5.2.2.1) und zumindest was die Voraussetzungen angeht, wie eine Dopplung der deliberativen Demokratietheorie erscheint, ist, auf den zweiten Blick betrachtet ihre partielle Erwiderung. Denn in den kontextbezogenen Demokratietheorien wird insofern sowohl der Rawlsche Entwurf einer vertragstheoretischen Urgesellschaft als auch die Habermasche Theorie des kommunikativen Handelns und speziell das Modell der idealen Sprechsituation als Grundlage vorpolitischer und politisch-demokratischer Prozesse zurückgewiesen. Vielmehr konzipiert Walzer in expliziter Abgrenzung zur Habermaschen Diskursethik seine Vorstellung von Diskurs und Deliberation als "real talk" (Walzer 1990:182, SL). Damit betont er, dass ein konsensuales Verhandlungsergebnis in Demokratien schwieriger zu erlangen sei, als in anderen (bspw. autoritären) politischen Systemen, da die Resultate eines demokratischen Abstimmungsprozesses selbst für die Beteiligten unvorhersehbar sind und am Ende durchaus widersprüchlich sein können (ebd., 193). Demokratische Debatten sind für Walzer zudem stark von den rhetorischen Kompetenzen der Beteiligten abhängig, zumeist chaotisch, ohne rechten Beginn und absehbares, klar festlegbares Ende, die nicht selten ohne jeden Effekt (Output und Outcome) betrieben werden (ebd., 195). Daher ist der 'real talk' nicht immer vernünftiges Abwägen sondern „more radical than ideal speech.“ (ebd.)

Die hier dargelegten Eckpunkte kontextbezogener Demokratietheorien bilden die demokratietheoretischen Grundpfeiler partizipatorischer Governancemodelle, von denen bisher weniger formuliert wurden als gemeinhin anzunehmen ist. Statt auf universale Prinzipien, „einem im Niemandsland gelegenen Idealstaat oder ein überall anwendba- 
res philosophisches Ideal“ (Walzer 1992a:19) setzen die VertreterInnen partizipativer Governancemodelle ihre Hoffnung auf partikulare Praktiken, indem sie den Fokus von der Ebene der Begründung universeller Gerechtigkeitsprinzipien auf die Ebene kontextgebundener Anwendungsfragen verschieben, die sie wiederum in unterschiedliche gesellschaftliche Handlungsbereiche und Verteilungssphären unterteilen, die über ihre je eigenen Handlungs- und Verteilungslogiken verfügen (Lösch 2005:138) Diese partikulare Herangehensweise zeichnet sich durch drei Aspekte aus: Erstens, die Konzentration auf sehr spezifische und greifbare Probleme (praktische Orientierung); zweitens, dass dabei möglichst auch die 'NormalbürgerInnen` sowie die direkt involvierten politischen, ökonomischen und administrativen Funktionsträger als entscheidende Akteure der Politik berücksichtigt werden (Inklusion und Kooperation), und drittens wird eine auf die Ermöglichung partizipativer Potenziale ausgerichtete Reorganisation und Umstrukturierung staatlicher Institutionen gefordert, so dass deliberative Prozesse initiiert, gefördert und effizient umgesetzt werden können (Deliberation) (Walk 2008:71). Alle drei Aspekte finden sich im Ansatz der „Empowered Participatory Governance“ von Archon Fung und Erik Olin Wright wieder.

\subsubsection{Das Modell der »Empowered Participatory Governance« nach Fung und Wright}

Die drei benannten Kernaspekte der Orientierung auf praktische Probleme, auf die Inklusion zivilgesellschaftlicher Akteure sowie deren Kooperation mit politischen Akteuren sowie die deliberative Lösung gesellschaftlicher Probleme nutzen Fung und Wright zur Beschreibung ihres Modells der »Empowered Participatory Governance» (vgl. Fung \& Wright 2001):

"[Empowered Participatory Governance] attempts to advance three currents in social science and democratic theory. First, (...) it extends the application of deliberation from abstract questions over value conflicts and principles of justice to very concrete matters such as street paving, school improvement, and habitat management. It also locates deliberation empirically, in specific organizations and practices, in order to marshal social experience to deepen understandings of practical deliberation and directions for its improvement. Second, (...) it attempts to understand, and by doing so demonstrate, the importance of civic life and nongovernmental organization to vigorous and effective democracy. It builds upon this insight by exploring whether the reorganization of formal state institutions can stimulate democratic engagement in civil society and so form a virtuous circle of reciprocal reinforcement. Finally, it is part of a broader collaboration to discover and imagine democratic institutions, that are at once more participatory and effective than the familiar configuration of political representation and bureaucratic administration. (...) We thus begin, (...) by laying out three general principles: (1) a focus on specific, tangible problems, (2) involvement of ordinary people 
affected by these problems and officials close to them, and (3) the deliberative development of solutions to these problems." (ebd. 17, SL)

Fung und Wright versuchen ausgehend von der Analyse von fünf verschiedenen deliberativ-partizipatorischen Realexperimenten (wie bspw. die »Neighborhood governance councils in Chicago", die "Wisconsin Regional Training Partnerships" oder das "participatory budget of Porto Alegre, Brazil«) partizipatorisch-deliberative Reformstrategien aufzeigen. Hinter diesen Reformstrategien steht der Kerngedanke, dass die Stabilität und Qualität einer Demokratie nicht nur von den demokratischen Qualitäten der formalen Institutionen sondern ganz entscheidend von den demokratischen Kompetenzen der BürgerInnen sowie ihrem Vermögen hinsichtlich kommunikativer Problemlösungsstrategien abhängen. In der von Fung und Wright entworfenen »Empowered Participatory Governance« werden daher Deliberationsprozesse fokussiert und priviligiert, die gegen expertokratische, plebiszitäre und verhandelnde Formen der sozialen Interaktion und Kommunikation abgegrenzt werden (ebd. 20-21):

"(d)eliberative decision making can be contrasted with three more familiar methods of social choice: command and control by experts, aggregative voting, and strategic negotiation. In the first mode, power is vested in managers, bureaucrats, or other specialists who are charged to act in the common weal and are capable of acting effectively by dint of their training, knowledge, and normative commitments. While such experts may engage in deliberative practices among themselves, their discussions are insulated from popular participation. (...) Aggregation is a second familiar method of social decision making in which a group's choice results from combining the preferences of the individual participants that make it up. Voting - over issues, proposals, or candidates - is perhaps the most common procedure of aggregative social choice. In voting, participants begin by ranking alternatives according to their desires. Then an algorithm such as majority rule selects a single option for the whole group. (...) Strategic bargaining and negotiation is a third contrasting method of social choice. As with aggregation but distinct from deliberation or most varieties of command, parties in strategic bargaining use decision-making procedures to advance their own unfettered selfinterest backed by resources and power they bring to the table. By comparison, voting procedures typically attempt to equalize such power differentials through provisions like 'one person one vote'. Collective bargaining between large unions and employers captures this difference; each brings different sources of authority and force to the encounter, and each uses them to secure the best (not necessarily the fairest) deal that it can for its side. Unlike purely deliberative interacttions, parties typically do so through the use of threats, differential power, misrepresentation, and 'strategic talk'." (ebd. 20-21, SL) 
Im Unterschied zu diesen expertokratischen, plebiszitären und verhandelnden Formen der sozialen Interaktion zielt eine deliberative Entscheidungsfindung darauf ab, dass Experten sich nicht nur untereinander sondern zusammen mit Laien, sprich 'NormalbürgerInnen' beratschlagen. Hierbei wird die politische Freiheit der 'NormalbürgerInnen ' nicht (lediglich) darin gesehen, dass sie sich ihren Interessen nach zwischen konkurrienden Gruppen von politischen Eliten und ExpertInnen entscheiden können, sondern es wird ganz grundsätzlich in Frage gestellt, warum es ausschließlich den Politikprofis und ExpertInnen vorbehalten sein sollte, in Politiknetzwerke, private-public partnerships oder andere Governancearrangements eingebunden zu sein und die Geschicke der Mehrheit zu bestimmen und zu lenken. Selbst wenn, was im Governancekontext grundsätzlich immer geschieht, die Integration von nicht-staatlichen Akteuren in den politischen Prozess gefordert wird, so geschehe dies nur unter der Prämisse, dass die in den politischen Prozess integrierbaren Kooperationspartner erfahren in politischen Verhandlungs- und Aushandlungsprozessen, sprich, keine politischen Laien oder 'einfache' Bürger sein sollten sondern 'Profis' bzw. Experten auf ihrem Gebiet (Funktionseliten).

Obgleich auch von Fung und Wright ein gewisses Maß an Professionalisierung als notwendig angesehen wird, schließen sie sich der Kritik an, dass Partizipation in den governancetheoretischen Kontexten zu eng gefasst wird, da 'NormalbürgerInnen' hier nach wie vor lediglich zu „'Stand-by-Citizens` verniedlicht“ werden (Demirović \& Walk 2011:13) und weil kein Raum dafür gesehen und geschaffen wird, dass BürgerInnen gestaltend in den politischen Prozess eingreifen können, da sie lediglich als „demokratiepolitische Drohkulisse“ (ebd.) gesehen werden. Insofern ist es vor allem die zum Ausdruck kommende, normativ gehaltvolle Forderung der Inklusion der 'NormalbürgerInnen ' in die politische Entscheidungsfindung und Regelung vor Ort, die die partizipatorischen Governancemodelle auszeichnet (Walk 2008:71). Zwar wird genau wie bei den deliberativen und diskursiven Governancemodellen (siehe 5.2.2.2) darauf verwiesen, dass bei deliberativen Entscheidungsfindungen im Unterschied zu bloßen Wahlentscheidungen nicht lediglich Eigeninteressen verhandelt werden, sondern dass dabei die Begründbarkeit, Fairness und Akzeptabilität für andere mitbedacht werden muss. Aber damit dies gewährleistet werden kann und sich nicht diejenigen mit der größten Macht bei Verhandlungen durchsetzen, wird nicht auf abstrakte Normen und Prinzipien wie den „zwanglosen Zwang des besseren Arguments“ (sensu Habermas) gebaut sondern auf institutionelle Reformen in Richtung Dezentralisierung, Subsidiarisierung und Vernetzung:

"Since EDD [Empowered Deliberative Decisionmaking] targets problems and solicits participation localized in both, issue and geographic space, its institutional reality requires the commensurate reorganization of the state apparatus. It en- 
tails the administrative and political devolution of power to local action units such as neighborhood councils, personnel in individual workplaces, and delineated ecosystem habitats - charged with devising and implementing solutions and held accountable to performance criteria. The bodies in the reforms below are not merely advisory, but rather creatures of a transformed state endowed with substantial public authority. (...) Although they enjoy substantial power and discretion, local units do not operate as autonomous, atomized sites of decision making in EDD. Instead, each of the cases features linkages of accountability and communication that connect local units to superordinate bodies. These central offices can reinforce the quality of local democratic deliberation and problem solving in a variety of ways: coordinating and distributing resources, solving problems that local units cannot address by themselves, rectifying pathological or incompetent decision making in failing groups, and diffusing innovations and learning across boundaries. (...) EDD reforms attempt to remake official institutions along these principles. This formal route potentially harnesses the power and resources of the state to deliberation and popular participation and thus to make these practices more durable and widely accessible." (Fung \& Wright 2001:22-23)

Insofern sind es vor allem zwei institutionelle Voraussetzungen, die gegeben sein oder aber erst geschaffen werden müssen, damit partizipativ-deliberative Formen der Entscheidungsfindung verwirklicht werden können: 1) Die Dezentralisierung und Subsidiarisierung der Entscheidungsstrukturen auf lokale Einheiten sowie 2) die Herausbildung von „central offices“, um die dezentralen Problemlösungsanstrengungen vor Ort zu unterstützen, so dass diese die Koordination und die Verteilung entscheidungsrelevanter Ressourcen übernehmen und überwachen, die einzelnen lokalen Entscheidungsinstanzen mit anderen vernetzen, die Problemlösung im Falle von Konflikten zwischen diesen vorantreiben und diese mit Wissen und Informationen versorgen können. Diese institutionellen (An-)Forderungen wiederum ergeben sich konsequenterweise aus dem Verständnis von zivilgesellschaftlicher Partizipation als Emanzipation der Bürger mit dem Ziel einer möglichst demokratischen Selbstregierung.

\subsubsection{Deliberative Governance vor dem Hintergrund prozeduralistischer Demokratietheorien}

Die prozeduralistischen Demokratietheorien beschäftigen sich genau wie die kontextualistischen Demokratietheorien mit den Voraussetzungen demokratischer Gesellschaften sowie der Inputseite des politischen Prozesses. Dabei gehen sie aber von anderen Grundannahmen aus, so dass sich in den prozeduralistischen Demokratietheorien auch eine andere demokratietheoretische Schlüsselfrage stellt: Wie ist Herrschaft zu legitimieren und wie kann ihre Ausübung an einem diskursiven Verständigungsprozess der BürgerInnen gebunden werden? (Lösch 2005:122) Der primäre Fokus dieser 
Demokratietheorien liegt daher auf den Verfahren der gesellschaftlichen, (vor-)politischen Meinungs- und Willensbildung im Allgemeinen sowie auf diskursiven Verfahren und deliberativen Beratungsprozessen in der politischen Öffentlichkeit im Speziellen. Dabei orientieren sich die BefürworterInnen prozeduralistischer Demokratietheorien an ganz bestimmten als universell postulierten rationalitäts- und kommunikationstheoretischen Prinzipien und Normen, die den diskursiven und deliberativen Verfahren der gesellschaftlichen Meinungs- und Willensbildung sowie der deliberativen Demokratietheorie unterliegen. In Bezug auf diese Prinzipien und Normen sowie deren Begründung lassen sich zwei Varianten unterscheiden, die beide an Kants Theorie der öffentlichen Vernunft anknüpfen (ebd.):

Dies ist einerseits - und vornehmlich - die von Jürgen Habermas entworfene in die diskursethische Theorie des kommunikativen Handelns eingebettete verständigungsorientierte, kommunikative, diskursiv gebildete Vernunft, bei der sich der in rationalen Diskursen vorherrschende „zwanglose Zwang des besseren Arguments“ mit der „administrativen Macht des Staates" zu Verfahren deliberativ-demokratischer Politik vereinen soll. Andererseits ist hier die liberal-konstitutionalistische Konzeption eines öffentlichen Vernunftgebrauchs sowie die Möglichkeit der Deliberation moralischer Fragen und Kontroversen unter dem „Schleier des Nichtwissens" (vgl. Rawls 2003) zu nennen, bei der es ebenfalls, allerdings unter anderen Vorzeichen und in Betonung anderer Aspekte, um die Rationalität von Verfahren sowie um ein Modell prozeduraler Öffentlichkeit geht:

"In our century, this idea [of deliberative democracy] has been developed by John Rawls and Jürgen Habermas, from very different perspectives. The Rawlsian idea of reflective equilibrium presupposes the possibility of moral deliberation. In Habermas, the ideal speech situation is intended to permit deliberation about ends as well as about means. (...) Yet the arguments advanced by Habermas and Rawls do seem to have a common core: political choice, to be legitimate, must be the outcome of deliberation about ends among free, equal, and rational agents." (Elster 1998b:5)

Ohne hier $\mathrm{n}$ auf die Gemeinsamkeiten und Unterschiede zwischen der Habermaschen und der Rawlschen Konzeption deliberativer Politik eingehen zu wollen und zu können, sollen folgende Punkte festgehalten werden: Beide Ansätze betonen die prozedurale Rationalität der Demokratie und verstehen die Legitimität politischer Macht und die Kontrolle von politischen Institutionen als einen öffentlichen Prozess, in den die Bürgerlnnen eingebunden sein sollen (Benhabib 1995a:18). Beide Theorien können daher als paradigmatische Konzeptionen prozeduraler Demokratie angesehen werden, obgleich die Unterscheidung zwischen diskursiven (nach Habermas) und konstitionalistischen (nach Rawls) Konzeptionen deliberativer Demokratie angeraten ist (Dryzek 
2000:162). Nach Seyla Benhabib gibt es drei zentrale Unterschiede zwischen beiden Theorien, die diese Differenzierung rechtfertigen: Zum einen der Unterschied bezüglich derjenigen Materien, auf die sich der öffentliche Vernunftgebrauch bzw. deliberative Verfahren beziehen; zum zweiten der Unterschied bezüglich der Rahmenbedingungen und Regeln deliberativer Verfahren und zum dritten der Unterschied bezüglich der Differenzierung zwischen öffentlichen und politischen sowie privaten und unpolitischen Angelegenheiten (Benhabib 1995a:18).

Der öffentliche Vernunftgebrauch ist bei Rawls „der Vernunftgebrauch gleicher Bürger (...), die als ein Kollektiv in letzter Instanz politische Zwangsgewalt übereinander ausüben, indem sie Gesetze erlassen und Verfassungsänderungen vornehmen." (Rawls 2003:314) Damit beziehen sich die für den öffentlichen Vernunftgebrauch auferlegten Begrenzungen auf das, „was wir 'wesentliche Verfassungsinhalte' nennen können und auf elementare Fragen der Gerechtigkeit.“ (ebd.) Für Rawls liegt die politische Öffentlichkeit und mithin öffentlich-politische Fragen im Staat und in seinen Organisationen, vor allem im Bereich des Rechts und seinen Institutionen, die von den Bereichen der Zivilgesellschaft und mithin den Bereichen des „nicht-öffentlichen Vernunftgebrauchs" (ebd., 321) abgegrenzt werden. Das Habermasche Deliberationsmodell hinterfragt demgegenüber diese Grenzziehungen, indem Diskurse auch darüber geführt werden sollen, wo die Trennlinien zwischen dem Öffentlichen und dem Privaten verlaufen (Benhabib 1995a:19). Der öffentliche, politische Bereich umfasst damit auch die pluralen Netzwerke und Assoziationen innerhalb der Zivilgesellschaft, die bei Rawls in Gegenüberstellung zur öffentlichen politischen Kultur als ein Teil der „Hintergrundkultur“ (Rawls 2003:321) angesehen werden.

Obgleich die Rawlsche Variante viele Aspekte in sich trägt, die es theoretisch lohnenswert machen würden, diese genauer in Bezug auf deliberative Governance zu untersuchen, eignet sich das Habermasche Deliberationsmodell mit seiner Fokussierung auf den intermediären Bereich zwischen Zivilgesellschaft und professionalisierter Politik, den er als politische Öffentlichkeit bezeichnet, eher zur Anwendung bzw. zur Übernahme in den Governancekontext, da hier oftmals die Netzwerkbeziehungen zwischen privaten und öffentlichen Akteuren im Vordergrund stehen. Daher werden sich die weiteren Ausführungen vornehmlich auf die diskursiven Konzeptionen der deliberativen Demokratietheorie fokussieren. Diesbezüglich wird auch hier erst einmal allgemein auf das theoretische Grundgerüst der kontextualistischen Demokratietheorie in ihrer diskurstheoretischen Variante eingegangen (5.2.2.1) Anschließend wird dann spezifischer auf das diskursive Modell deliberativer Governance nach John Dryzek eingegangen (5.2.2.2). 


\subsubsection{Das theoretische Grundgerüst der kontextbezogenen Demokratietheorien in ihrer diskursiven Variante}

Die Habermasche Demokratietheorie ist von dem Ansinnen motiviert, diese auf einer Theorie der Vernunft basieren zu lassen, die einerseits von der liberaldemokratischen Vorstellung rein nutzenmaximierender Individuen als 'homo politicus' abweicht, die lediglich eine aus Individuen aggregierte Gesellschaft bilden, ohne den BürgerInnen andererseits per se eine Gemeinsinnorientierung zu unterstellen und sie darüber zu einer gemeinschaftlichen politischen Einheit zu verschmelzen, wie dies in republikanischen Demokratietheorien der Fall ist (Palazzo 2002:21). Der in der diskurstheoretisch untermauerten deliberativen Demokratietheorie anvisierte Mittelweg besteht darin, „das eigenorientierte Handeln von Individuen als Kennzeichen einer freiheitlichen Ordnung zu begrüßen und zugleich [zu] behaupten, dass sich das Handlungspotenzial von Menschen nicht in dieser Kategorie erschöpfe, sondern daneben immer auch eine genuine Gemeinschaftsorientierung möglich bleibe.“ (ebd.) Im Mittelpunkt der diskursiv-deliberativen Demokratietheorie steht daher die öffentliche Kommunikation von BürgerInnen und die Annahme, dass diese skeptisch gegenüber Fremdbestimmung und zu autonomen Handeln befähigt sind und dass sie die Mitgestaltung ihrer Gesellschaft als konstitutiven Aspekt ihrer Freiheit begreifen.

Die Grundidee und der normative Anspruch dieses Demokratiemodells ist es daher, „dass nur diejenigen Normen, d.h. die allgemeinen Handlungsregeln und institutionellen Regelungen, gültig genannt werden, denen alle von ihren Konsequenzen Betroffenen zustimmen können." (Benhabib 1995a:9). Diese Zustimmung wird auf Basis eines Abwägungsprozesses entschieden, der durch folgende Merkmale gekennzeichnet ist: Die Teilnahme an einem Deliberationsprozess soll durch Normen der Gleichheit und der Symmetrie bestimmt sein, d.h., alle BürgerInnen sollen gleichermaßen die Möglichkeit und das Recht haben, an der Debatte über Sach-, Wert- und Normfragen teilzunehmen, sich selbst zu (hinter-)fragen und andere zu befragen (ebd., 9-10). Das durch diese Normen abgesicherte Abwägungsverfahren der Deliberation erzeuge, so Benhabib, nicht nur demokratische Legitimität, sondern gewährleiste auch einen gewissen Grad an praktischer Rationalität (ebd., 11), da erst der deliberative Prozess dazu führe, dass sich die Individuen qua Eigen- und Fremdreflexion auf ihre Ansichten und Meinungen über die Konsequenzen ihrer Interessen und Präferenzen klar werden (ebd.). Zusätzlich sieht das Deliberationsmodell bestimmte Vorkehrungen für den Fall vor, dass das deliberative Verfahren missverstanden, falsch angewendet oder missbraucht wird. Auf der Ebene der Diskurse können missverständliche, missbräuchliche und falsche Anwendungen reklamiert und angefochten werden, da alle Beteiligten grundsätzlich das Recht haben, eine reflexive Diskussion über die Regeln des Verfahrens und die Art und Weise seiner Anwendung und Ausführung zu eröffnen (ebd., 9-10, 13). 
Im Gegensatz zu solchen Diskursen wird Deliberation zumeist als eine Form der öffentlichen Konversation und gegenseitigen Beratschlagung über öffentliche Angelegenheiten verstanden, „whereby individuals speak and listen sequentially, before making a collective decision." (Gam-betta 1998:19) Da die öffentlichen Konversationen und Beratschlagungen darauf abzielen, zu kollektiven Entscheidungen zu gelangen, müssen dafür in modernen, demokratischen Gesellschaften i.d.R. drei Wege bzw. Verfahren, nämlich "voting", "arguing" und "bargaining" beachtet werden (Elster 1998b:6). Die deliberative Demokratietheorie diskursiver Prägung bezieht zu allen drei Wegen - analog zu den kontextualistischen Demokratietheorien - Stellung: Zum einen wendet sie sich gegen die Ansicht, dass Wahlen ("voting") als eine hinreichende Form kollektiver Entscheidungsfindung betrachtet werden können, zum zweiten strebt sie eine kollektive Entscheidungsfindung durch diskursiv-argumentative Verfahren (»arguing") an und zum dritten lehnt sie Verhandlungslösungen, die durch strategisches Verhandeln (»bargaining") zu Stande kommen vor allem aufgrund ihrer negativen Implikationen für die Legitimität von Entscheidungsfindungsprozessen ab (Gambetta 1998:19). Diese drei Aspekte werden von nahezu allen VertreterInnen der diskursiv-deliberativen Demokratietheorie mehr oder weniger explizit hervorgehoben. Stellvertretend für viele andere führt bswp. John Dryzek bezüglich seinem Demokratieverständnis an:

„Democracy (...) is not just about the making of decision through the aggregation of preferences. Instead, it is also about processes of judgment and preference formation and transformation within informed, respectful, and competent dialogue. Democratic legitimacy is sought in the participation in consequential deliberation of those subjects to a decision (or their representatives)." (Dryzek 2012: 3)

Guido Pallazo stellt die Gründe für den Fokus auf diese drei Aspekte klar heraus, indem er in Rückgriff auf Habermas und Joshua Cohen auf die zentrale Überlegung hinter der deliberativen Demokratietheorie vor allem in Abgrenzung zu den entscheidungszentrierten Demokratietheorien verweist:

„[Entscheidungszentrierte Demokratietheorien] können zwischen einer manipulierten und einer freien Zustimmung bzw. Übereinstimmung der Bürgerinnen und Bürger nicht unterscheiden. Der Legitimitätsglaube allein bedeutet mithin noch keine Legitimität. Diese funktionalistische, rein an faktischer Geltung und Stabilität orientierte Erklärung politischer Herrschaft bleibt unbefriedigend. Durch die Aggregation von Mehrheitsmeinungen lässt sich im Prinzip jede Form der Herrschaft legitimieren (...). Sobald jedoch normative Differenzen nicht einfach durch Mehrheitsentscheidungen erstickt werden, tritt der Anspruch öffentlicher Auseinandersetzung hinzu, der über den reinen Legitimitätsglauben hinausgeht. (...) Eine Demokratietheorie, die nur die gegenwärtige demokratische Realität beschreibt und sie auf Wahlen, Parteienkonkurrenz und Mehrheitsregel reduziert, greift hier 
zu kurz. Sie verfehlt sie Möglichkeit, die Bedingungen zu bestimmen, welche den Institutionen selbst Akzeptanz und Glaubwürdigkeit verleihen.“ (Palazzo 2002:19)

Hieran wird deutlich, dass die deliberative Demokratietheorie in erster Linie eine Theorie über die Bedingungen und Verfahren zur Herstellung demokratischer Legitimität ist (Dryzek 2012:21). ${ }^{171}$ Die Chancen zur Erfüllung der Bedingungen, die allen Dimensionen des Politischen - gemein sind die politischen Institutionen ("polity«), Verfahren (»politic») und Entscheidungen (»policy«) - Legitimität, d.h. Anerkennungswürdigkeit, verleihen, werden innerhalb der deliberativen Demokratie nur solchen kommunikativen Verfahren zugeschrieben, die anders als Wahlen keine singulären für sich abgeschlossenen Prozesse darstellen, bei denen es um die reine Aggregation von individuellen Interessen zu kollektiven Interessen geht. Vielmehr sollen die kommunikativen Prozesse die Möglichkeit eröffnen, zu einer gemeinschaftlichen Transformation individueller Interessen in kollektive, gemeinwohlorientierte Präferenzen zu gelangen (Elster 1998b:5-6). Dazu bedarf es allerdings verständigungsorientierter Verfahren, die auf bestimmten institutionellen und rechtlichen Voraussetzungen (Rechtsstaatlichkeit) und bestimmten Kommunikationsnormen (der idealen Sprechsituation) basieren, deren Grundlagen maßgeblich über die Habermasche Diskurstheorie und -ethik abgestützt sind und die auf den öffentlich-politischen Bereich übertragen dazu führen sollen, dass es zu einem deliberativen Beratungsprozess innerhalb der Zivilgesellschaft und der politischen Öffentlichkeit kommt, sprich einem „diskursiven Zuarbeitungsprozess der BürgerInnen zum politischen System." (Lösch 2005:157) Hieran wird u.a. ersichtlich, dass Legitimität und Legitimation im Kontext der Habermaschen Diskursethik auf eine bestimmte Weise zusammengebracht werden: Indem die Rechtsmäßigkeit (Legitimität qua Legalität) politischer Institutionen, Verfahren und Entscheidungen durch die Prinzipien des Rechtsstaat gesichert werden und indem die Anerkennungswürdigkeit (Legitimität qua Akzeptabilität) politischer Personalien, Prozesse und Entscheidungen durch demokratisch-deliberative Beratungsprozesse sichergestellt werden soll, wird wiederum die Wahrscheinlichkeit erhöht, dass diejenigen, die an den Diskursen beteiligt sind, deren Ergebnisse auch anerkennen bzw. akzeptieren können (Legitimation).

Bezüglich dieser Legitimitäts-Legitimations-Schleife entwirft Habermas das sogenannte Schleusenmodell demokratischer Politik (vgl. Habermas 1992b:Kap. VIII), das auf der Grundunterscheidung zwischen den verständigungsorientierten, auf Solidarität bzw. Gemeinschaftlichkeit basierenden „wilden Kommunikationen in den nichtorganisierten Öffentlichkeiten“ (ebd. 142) der Zivilgesellschaft, den „formell geregelten, über administrative Macht abgesicherten Beratungs- und Entscheidungsprozessen“ (ebd.) in

${ }^{171}$ Die im angloamerikanischen Raum gebräuchliche Verwendung »legitimate« kann im Deutschen in zweierlei Hinsicht übersetzt werden: zum einen als Legitimation und zum anderen als Legitimität. Zum bereits explizierten Bezug der beiden Begriffe aufeinander bzw. zu deren Abgrenzung siehe Abschnitt 4.3.3. 
den politisch-staatlichen Kerninstitutionen wie Parlamenten, Behörden, Gerichten usw. sowie den „auf Eigennutzoptimierung und persönlichen Gewinn ausgerichteten strategischen Kommunikationen der Wirtschaft" (ebd.) aufbaut. Entscheidend dabei ist, dass dahinter eine System-Lebenswelt-Differenz steht, womit Gesellschaften, anders als in den materialistischen Gesellschaftstheorien, nicht durch den ökonomisch induzierten Konflikt zwischen gesellschaftlichen Klassen sondern über die widerstreitenden Integrationsmechanismen des politischen und ökonomischen Systems und der Lebenswelt gekennzeichnet sind. ${ }^{172}$

Je weniger die sozialen Interaktionen in der Gesellschaft durch soziale Integrationsmechanismen sowie durch lebensweltliche Homogenität, sprich eine gemeinsame Kultur, gemeinsame Traditionen, Vergemeinschafts und Sozialisierungsprozesse, d.h. im Ganzen durch eine gemeinsame „Hintergrundkultur“, abgesichert sind, desto größer wird der Verständigungsbedarf (Habermas 1981a:108). Gleichzeitig wird die soziale Gültigkeit von Normen (die Geltung) fragwürdig, so dass diese diskursiv erzeugt werden muss, indem die "Lebensform normativ reflektiert" wird (Benhabib 1995b:55). Im Endeffekt bedeutet diese normative Reflexion der Lebensform, dass Menschen verständigungsorientiert handeln (müssen!), weil nur die Verständigungsorientierung dazu führt, dass gesellschaftliche Übereinstimmungen hergestellt werden können (Habermas 1981b:139). Kommunikatives Handeln im lebensweltlichen, verständigungs-

172 Das Handeln von Individuen in einer Gesellschaft geschieht im Rahmen dessen, was Habermas in Rückgriff auf John Dewey die Lebenswelt nennt. Diese besteht einerseits aus kulturellen Gewissheiten und andererseits aus ,individuellen Fertigkeiten, dem intuitiven Wissen, wie man mit einer Situation fertig wird, und aus sozial eingelebten Praktiken, dem intuitiven Wissen, worauf man sich in einer Situation verlassen kann, nicht weniger als aus den trivial gewussten Hintergrundüberzeugungen." (Habermas 1984:593) Dieses Set an unproblematischen Überzeugungen bildet den Kontext gesellschaftlicher Interaktionen (Habermas 1981b:191). In modernen Gesellschaften fächert sich die Lebenswelt in die drei Strukturkomponenten der Kultur bzw. Tradition, der Gemeinschaft und der Identität bzw. Persönlichkeit und gleichsam in drei verschiedene Weltbezüge auf (objektiv-Kultur, sozial-Gemeinschaft, subjektiv-Persönlichkeit), die in vormodernen Gesellschaften eng miteinander verzahnt waren und so die normativen Regeln des Zusammenlebens ergaben (Habermas 1981a:85). Im Zuge der Moderne ergeben sich aber nicht nur unterschiedliche lebensweltliche Weltbezüge, sondern Habermas berücksichtigt, in Anlehnung an die Luhmannsche Systemtheorie, auch die Abkopplung von Systemen bzw. Systemlogiken aus der Lebenswelt, die sich zur Bewältigung bestimmter gesellschaftlicher Aufgaben mit hochspezialisierten Handlungsweisen und kodierten Handlungslogiken ausgebildet haben. Hier nennt Habermas in erster Linie die Ökonomie und die Politik, die sich als ausdifferenzierte Funktionssysteme von der Logik lebensweltlicher Kommunikation entfernt hätten und deren Wirksamkeit beschränken (Habermas 1981b:180). Diese haben aber genau dadurch einen doppelten Effizienzvorteil, da Handlungen und Entscheidungen zum einen von moralischen und ethischen Erwägungen abgekoppelt werden, deren Berücksichtigung angesichts der hohen Komplexität mit denen entscheidungsfällende Systeme umzugehen haben, zum Strukturproblem werden würden. Zum anderen erfolgt die Koordination von Handlungen in Systemen unabhängig von den unmittelbaren Handlungsintentionen einzelner Akteure auf Basis einer systemischen Kodierung und einer funktionalen Reproduktion (ebd., 226-228). Für die Lebenswelt bedeutet die Ausdifferenzie-rung systemischer Steuerungsmedien daher einerseits eine Entlastung andererseits aber auch eine Belastung, da die strategischen Handlungen und die Systemlogiken auf die Lebenswelt und ihre verständigungsorientierten und solidarischen Integrationsmechanismen destruktiv zurückwirken. 
orientierten Sinne ist damit, so Habermas Ansicht, in der Moderne für die Moral alternativlos und zudem die einzig verbleibende Möglichkeit bzw. die einzige Quelle für die Erzeugung, Regeneration und Revitalisierung von Solidarität, auf die alle demokratischen Gesellschaften fundamental angewiesen sind, die sich aber durch politisch-institutionelle Maßnahmen nicht herstellen durchaus aber sicherstellen lässt (Habermas 1990:37). ${ }^{173}$

Insofern sind die Begriffe Lebenswelt und ihr Komplementärbegriff des kommunikativen Handelns die zentralen Verbindungsstellen zwischen der Habermaschen Diskursethik und seiner deliberativen Demokratietheorie: Die Zivilgesellschaft, wie Habermas sie bestimmt, unterscheidet sich damit einerseits von der Vorstellung der Öffentlichkeit als rein ökonomischer Handlungs- bzw. Handelssphäre (wie dies in wirtschaftsliberalen und libertären Demokratietheorien angenommen wird) aber auch von der politischen Öffentlichkeit reduziert auf das politische System bzw. den Staat im engeren Sinne (Lösch 2005:158). Vielmehr verortet Habermas die Zivilgesellschaft zwischen Wirtschaft und Staat und verbindet sie gleichsam über intermediäre, beide Sphären überlappende Bereiche der politischen Öffentlichkeit (ebd.). Diese intermediären Bereiche der politischen Öffentlichkeit sind die für Habermas entscheidenden Sphären der Deliberation, die einerseits durch die Prinzipien des Rechtsstaats (und partiell auch des Sozialstaats) geprägt werden, die für Habermas grundlegend sind, damit „die anspruchsvollen Kommunikationen einer demokratischen Mei-nungs- und Willensbildung institutionalisiert werden können." (Habermas 1992b: 361) Andererseits sind diese aber auch durch die lebensweltlich geprägte „höherstufige Intersubjektivität von Verständigungsprozessen [gekennzeichnet], die sich über demokratische Verfahren oder im Kommunikationsnetz politischer Öffentlichkeiten bilden." (ebd., 362) Da die Prozesse der Deliberation folglich „von der Institutionalisierung entsprechender Verfahren und Kommunikationsvoraussetzungen sowie vom Zusammenspiel der institutionalisierten Beratungen mit informell gebildeten öffentlichen Meinungen“ (ebd.) abhängig gemacht werden, wird demokratische Politik weder vom Vorhandensein einer kollektiv handlungsfähigen Bürgerschaft abhängig gemacht, noch wird diese an die anonyme Herrschaft der Gesetze über konkurrierende Einzelsubjekte gebunden.

${ }^{173}$ Dort wo die systemischen Mechanismen die Formen der sozialen Integration verdrängen, die in ihrer verständigungsorientierten Handlungskoordination nicht systemisch substituiert werden können und dürfen, spricht Habermas von einer „Kolonialisierung der Lebenswelt" (ebd., 293). Hierbei wird das strategische Handeln als parasitär zum verständigungsorientierten Handeln verstanden, obgleich strategische Handlungen angesichts der Modernisierung der Gesellschaft als notwendig und innerhalb eines bestimmten rechtlichen und institutionellen Rahmens von Habermas selbst als legitim angesehen werden. Habermas Kritik setzt folglich da ein, wo die Systemlogiken ungebremst und ungeschützt in die Lebenswelt einwirken und die Reproduktionsbedingungen der sozial-kulturellen Identitätsbildung subtil entmachten können. Innerhalb der System-Lebenswelt-Differenz wird daher der Vorrang der Lebenswelt und des kommunikativen Handelns vor den Systemen und dem strategischen Handeln postuliert und begründet. 
Vielmehr setzt Habermas auf die „Prozeduralisierung der Volkssouveränität“ (ebd) und damit die Rückbindung des politischen Entscheidungssystems an die politische Öffentlichkeit, was wiederum in dem Bild einer dezentralisierten Politik und Gesellschaft mündet, in der „subjektlose Kommunikationen“ sich zu „Arenen der öffentlichen Deliberation“ verdichten, „, in denen eine mehr oder weniger rationale Meinungs- und Willlensbildung über gesamtgesellschaftlich relevante und regelungsbedürftige Materien stattfinden kann." (ebd.) ${ }^{174}$ Insofern besteht der Zusammenhang von Demokratie, Deliberation und Diskurs bei Habermas darin, dass Verfahren und Prozesse der öffentlichen Meinungs- und Willensbildung sowie gegenseitigen Beratschlagung institutionalisiert werden müssen, über die sich der in rationalen Diskursen vorherrschende 'zwanglose Zwang des besseren Arguments' mit der administrativen Macht der Politik zu einem komplexen Zusammenspiel deliberativ-demokratischer Entscheidungsfindung vereinen kann, und dass eben dieser Weg zugleich die an der Beteiligung und Berücksichtigung der Präferenzen der BürgerInnen bemessene (Input-)Legitimität als auch die an der Erreichung von politischen Entscheidungen, Ergebnissen und Gütern zu ermessende Effizienz und Effektivität gewährleistet (Habermas 1992a:20).

Vor diesen Hintergründen betrachtet, hat die deliberative Demokratietheorie diskurstheoretische, demokratietheoretische und legitimationstheoretische Aspekte (Elster 1998a:6). Während sich nahezu alle VertreterInnen der deliberativen Demokratietheorie im Grundsatz über die diskurs- und demokratietheoretischen Aspekte einig sind, gehen vor allem die legitimationstheoretischen Verständnisse der Deliberation auseinander, da diese entweder - oder aber zugleich - über die Voraussetzungen der Verfahren (den Input), das Verfahren selber (den Throughput) oder ihre erwartbaren Ergebnisse (den Output oder Outcome) bestimmt werden kann und bestimmt wird:

"There is a robust core of phenomena that counts as deliberative democracy (...). All agree (...) that the notion includes collective decision making with the participation of all who will be affected by the decision or their representatives: this is

174 Die Annahme der Prozeduralisierung der Volkssouveränität ergibt sich aus Habermas Überlegungen zur „Gleichursprünglichkeit“ der Ideen der Volkssouveränität und der Menschenrechte, die er in seiner Schrift zum »internen Zusammenhang von Rechtsstaat und Demokratie« darlegt (vgl. Habermas 1996b). Um diese zentrale Intuition die Habermas zur Folge sowohl vom Republikanismus als auch vom Liberalismus zu Unrecht vereinseitigt wurde gerecht zu werden, „empfiehlt es sich, das demokratische Verfahren, daß dem Prozess der Rechtssetzung unter Bedingungen des gesellschaftlichen und weltanschaulichen Pluralismus erst seine legitimitätserzeugende Kraft verleiht, unter diskurstheoretischen Gesichtspunkten zu betrachten“ (ebd., 299). Demnach dürfen „genau die Regelungen Legitimität beanspruchen (...), denen alle möglicherweise Betroffenen als Teilnehmer an rationalen Diskursen zustimmen könnten. Wenn nun Diskurse und Verhandlungen - deren Fairness wiederum auf diskursiv begründeten Verfahren beruhen - den Ort bilden, an dem sich ein vernünftiger politischer Wille bilden kann, dann muss sich jene Vermutung auf Vernünftigkeit, die das demokratische Verfahren begründen soll, letztlich auf ein kunstvolles kommunikatives Arrangement stützen: es kommt auf die Bedingungen an, unter denen die für eine legitime Rechtssetzung notwendigen Kommunikationsformen ihrerseits rechtlich institutionalisiert werden können." (ebd.) 
the democratic part. Also, all agree that it includes decision making by means of arguments offered by and to participants who are committed to the values of rationality and impartiality: this is the deliberative part. (...) The intensions diverge widely, however, especially with respect to what counts as deliberation. (...) The object of deliberation may be defined by its outcomes or ultimate ends, by features of the process or by the settings as a set of institutional conditions that promote rationality and impartiality." (Elster 1998b:8-9)

Insofern ist entscheidend, wie genau der Begriff der «Deliberation « jeweils verstanden wird. Wenn Deliberation lediglich auf den diskursiv-deliberativen und demokratischen Aspekt bezogen wird, was bspw. daran deutlich wird, wenn Deliberation unspezifisch als „öffentliche Kommunikation über politische Fragen“, als ein Verfahren „über politische Fragen informiert zu werden und informiert zu sein" (Arenhövel 1998:124) bzw. als Verfahren zur „Präferenzreinigung oder -läuterung“ (Forst 1996:188) verstanden wird, dann wird zwar die Idee der diskursiven Transformation von individuellen Interessen zu kollektiven Präferenzen aufgegriffen, der legitimationstheoretische Aspekt der Deliberation wird aber vernachlässigt. Exakt an diesen Punkt finden sich zumeist - trotz der Komplexität und Tiefe der prozeduralistischen Demokratietheorien vereinfachende und verkürzte Verständnisse. John Dryzeks Konzeption deliberativer Demokratie und seine Weiterentwicklung hin zu deliberativer Governance bieten demgegenüber sogar ein erweitertes Deliberationskonzept, welches auch noch weitere theoretische Kontexte wie die Hegemonietheorie und die Theorie der reflexiven Moderne berücksichtigt (vgl Dryzek 2012: 34), und vor diesem Hintergrund ein recht umfassendes Deliberationsverständnis ermöglicht.

\subsubsection{Die Modellierung »deliberativer Governance» nach John Dryzek}

John Dryzek, der mittlerweile einer der wichtigsten US-amerikanischen Vertreter der „deliberative democracy“ ist, entwickelt sein Modell deliberativer Governance (vgl. Dryzek 2012) vornehmlich in Kritik an den liberal-konstitutionalistischen Annahmen von Rawls und auf Basis der späten Diskurs- und Öffentlichkeitstheorien von Habermas, die er wiederum mit dem stark partizipatorisch geprägten deliberativen Demokratiemodell von Amy Gutmann und Dennis Thompson kombiniert (vgl. Gutmann \& Thompson 1996). Dryzek begreift dabei das Entstehen von politischen Netzwerkstrukturen über die politischen Ebenen hinweg („multi-level governance) und speziell kooperativen Governancearrangements zwischen politischen und gesellschaftlichen Akteuren („network governance“) als spezifische Herausforderung für die Demokratietheorie im Allgemeinen und die deliberative Demokratietheorie im Speziellen. ${ }^{175}$

175 Governance wird von Dryzek zum einen ganz klassisch als Entgegensetzung zu Government verstanden (Dryzek 2012:120), da Dryzek davon ausgeht, dass "when government is missing, we can however still speak of processes of governance." (Dryzek, Norgaard \& Schlosberg 2013:93) Andererseits 
Dieser Herausforderung begegnet er, indem er (als „reexamination of the foundations of deliberative democracy“) zum einen die theoriespezifischen Problematiken aufzeigt und analysiert, die sich ergeben, wenn die deliberative Demokratietheorie auf den Governancekontext angewandt wird. Darunter fallen bspw. Überlegungen bzgl. der legitimationstheoretischen Grundannahmen der deliberativen Demokratietheorie, bzgl. des Verhältnisses von Argumentation und Rhetorik in deliberativen Prozessen sowie bzgl. der starken Konsensorientierung der Diskurstheorie. Zum anderen problematisiert er (als "frontiers of deliberative democracy") eine Reihe an nicht-theoriespezifischen Problematiken, die aber wiederum theorierelevante Auswirkungen haben. Darunter fällt u.a. der im Governancekontext relevante Umstand, dass Demokratietheorien nach wie vor von souveränen Staaten ausgehen (Palazzo 2002:202-203): “A state with no rivals when it comes to exercising legitimate authority over the people within its jurisdiction, with the authorization of that people." (Dryzek 2012:119) Eben diese Vorstellung souveräner staatlicher Gebilde wird sowohl durch das Entstehen von überstaatlichen Governancearrangements oberhalb der Nationalstaaten (Global Governance) als auch durch die Zunahme von Politiknetzwerken über alle politischen Ebenen hinweg (Multi-Level-Governance) sowie die Anhäufung kooperativer Governancearrangements innerhalb der Nationalstaaten in Frage gestellt.

Alle drei Entwicklungen führen, so Dryzek, dazu, dass die nationalen Grenzen und mithin die Vorstellung souveräner Staatlichkeit als Grundlage von Demokratie und Demokratietheorie transzendiert wird (ebd., 120). Vor diesem Hintergrund merkt Dryzek an, dass Governancearrangements vor allem vor dem Hintergrund liberaler Freiheitsvorstellungen und Demokratietheorien problematisch erscheinen, dass sich aber andere bspw. republikanische Freiheitsvorstellungen und Demokratietheorien viel besser in den Governancekontext, so wie Dryzek ihn versteht, eingliedern lassen (ebd., 123). Daher kann und sollte die Anerkennung des Umstandes, dass es zu einem Anwachsen von Governancearrangements gekommen ist, nicht automatisch zu der innerhalb der liberalen Demokratietheorie verbreiteten Schlussfolgerung führen, "that governance is always and necessarily bad from a democratic point of view." (ebd.) Vielmehr sollte, so Dryzek indirekt, daraus geschlussfolgert werden, dass es eines postliberalen, postelektoralen und postnationalen demokratischen Denkens bedarf (ebd. 124). ${ }^{176}$

versteht Dryzek Governance spezifischer als Netzwerk im Sinne eines „broader concept that allows for more fluid, informal, and transnational arrangements, though it can also include governments as conventionally definded." (ebd., 93) Ein solches Verständnis von Netzwerkgovernance baut auf einem speziellen auf horizontale Eigenkoordination fokussierten Verständnis von Governance auf, das später als enges Governanceverständnis charakterisiert wird (siehe 5.3.3.1)

176 Diesbezüglich kann mit Guido Palazzo festgehalten werden, dass die diskursiv-deliberativen Demokratietheorien zwar von Beginn an darauf abzielten, ein postliberales und postelektorales Verständnis von Demokratie zu entwickeln und zu etablieren, dass indes das Ansinnen auch eine postnationale Demokratietheorie vorzulegen, erst deutlich später hinzugetreten ist (Palazzo 2002:212). So argumentiert bspw. Habermas in seiner Schrift zur »postnationalen Konstellation« (vgl. Habermas 
Bezüglich aller drei Momente argumentiert Dryzek dafür, dass die Prinzipien und Ansätze der deliberativen Demokratietheorie die adäquateste Grundlage für Governancearrangements sind (ebd., 124), da die basale Grundidee der Deliberation kollektive Entscheidungen durch gemeinsame und gegenseitige Beratungen und diskursive Verständigung zu erzielen auf nahezu alle möglichen institutionellen politischen Settings anwendbar ist, „including those that do not contain legislatures, political parties, citizen forums, or elections." (ebd., 11) Dryzek stellt und beantwortet daher die Fragen, „how governance networks can be interpreted as deliberative systems, and how democratic standards can therefore be applied in the evaluation of such governance." (ebd., 15) Die Antworten, die Dryzek auf beide Fragen gibt, zielen im Wesentlichen auf die Reformulierung der theoretischen Grundlagen der deliberativen Demokratietheorie ab, die Dryzek sehr ausführlich und umfangreich darlegt. Da dies hier nicht im Ganzen nachgezeichnet werden kann und soll, werden sich die folgenden Ausführungen darauf beschränken, bestimmte Aspekte dieser Modifikationen deutlich zu machen:

Bezüglich der ersten Frage, inwiefern sich Governancenetzwerke als deliberative Systeme charakterisieren lassen, schlägt Dryzek ein allgemeines Analyseschema bestehend aus fünf Komponenten vor (vgl. ebd. 11-12): Zum einen sind dies strukturelle Komponenten, wie Habermas sie auch über sein Schleusenmodell beschreibt, nämlich das Vorhandensein einer (zivil-)gesellschaftlichen Sphäre („public space“) sowie einer institutionell-stabilisierten politischen Sphäre („empowered space“) und dazwischen einem intermediären Bereich der politischen Öffentlichkeit („transmission from public to empowered space"). Bezüglich dieser ersten drei Komponenten und der in der deliberativen Demokratietheorie legitimationstheoretisch relevanten Unterscheidung zwischen der zivilgesellschaftlichen und der politischen Sphäre stellt Dryzek heraus, dass beide Sphären in Netzwerken ineinander verlaufen, da diejenigen, die an Governancearrangements zur Findung kollektiver Positionen und Entscheidungen beteiligt sind, über ihre Beteiligung und die gemeinsame Aufgabe eine Lösung kollektiver Problematiken zu finden, nur noch eine geringe kritische Distanz zu den Ausübenden politischer Macht und staatlicher Gewalt haben (ebd., 131). Das bedeutet, dass die Art und

1998), dass eine politische Gestaltung der Globalisierung vor der Aufgabe stünde, den demokratischen Prozess aus seiner nationalstaatlichen Monopolisierung zu lösen und eine genuin politische Antwort auf die „postnationale Konstellation“ zu finden (ebd., 95). Dabei merkt er an, dass die Relativierung des Territorialprinzips veränderte Formen demokratischer Selbststeuerung erfordern würde (ebd., 134). Habermas deterritorialisiert daraufhin sein territorial verfasstes Schleusenmodell zum Modell einer „Weltinnenpolitik ohne Weltregierung“ (ebd., 163), indem er fordert, dass es einer transnationalen Politik bedürfe, die mit einer transnationalen politischen Öffentlichkeit verbunden werden müsse, welche ihrerseits aus dem Engagement einer transnationalen Zivilgesellschaft („Weltbürgergesellschaft") hervorgehen müsse (ebd.). Nur eine aus der Weltbürgergesellschaft hervorgehende transnationale Politik könne, so Habermas (ziemlich optimistische) Hoffnung, der globalisierten Ökonomie einerseits auf Augenhöhe andererseits mittels legitimer und effektiver Politikproduktion entgegentreten und entgegenwirken. 
Weise der Transmission zwischen diesen Sphären in besonderem Maße von Relevanz ist. Da viele Governancearrangement eine „single-issue-agenda“ aufweisen und durch einen „single discourse" geprägt sind, so dass bestimmte Aspekte der Thematik von vornherein ausgeblendet und marginalisiert werden und daran nur bestimmte Interessensvertreter beteiligt sind, die für ihre Mitglieder-, Verbands- oder Organisationsinteressen eintreten, bergen Governancearrangements für Dryzek die Gefahr, dass die dabei entstandenen Entscheidungen auf Kosten des Wohls aller gehen, deren Interessen dann nicht ausreichend bedacht und einkalkuliert werden. Desweiteren hält Dryzek aber auch fest, dass die von der Demokratietheorie eben wegen dieser legitimatorischen Defizite oftmals negativ beäugten Governancenetzwerke hinsichtlich ihrer Potenziale und Möglichkeiten für dialogische Koordinationsformen wichtige Überschneidungen zum Konzept der diskursiven Deliberation haben, „because to excert influence, an actor has to persuade others in the network." (ebd., 125)

Wenn es aber auch im Rahmen deliberativer Governance immer schon darum geht, sich und andere kommunikativ von etwas zu überzeugen, dann ist wiederum relevant, dass Rhetorik und Deliberation und mithin 'bargaining 'und 'arguing' sowie grundlegend strategische und verständigungsorientierte Kommunikation nicht in einem generellen und vor allem normativen Oppositionsverhältnis zueinander stehen und stehen sollten (ebd., 71). ${ }^{177}$ Vielmehr betont Dryzek den Umstand, dass es in allen repräsentativen und deliberativen Systemen rhetorischer Kommunikationsformen und Kompetenzen bedarf (ebd., 74), obgleich es dabei zur Charakterisierung deliberativer Systeme einen qualitativen Unterschied zwischen bindenden („,bonding“) und überbrückenden (,bridging") Rhetorikformen gibt:

„[B]onding is associated with people who are similar in social background, bridging is associated with pluralism. (...) In this light, bonding rhetoric is (...) is likely to deepen divisions with out-groups, to invoke dangerous emotions, to mobilize passions, and to move groups to extremes. (...) In contrast, bridging rhetoric takes seriously the outlooks (ideally, the discursive psychology) of an intended audience that is different in key respect from the speaker - and from the kind of people or discourses the speaker represents. Bridging rhetoric is hard work for the speaker, because he or she must strive to understand an audience whose dispositions are different, as well as hold on to his or her own side. The idea of discursive representation shows how this can be done. The trick here is not to represent a group in its entirety to an audience in its entirety. Instead, the idea is to represent

177 Die bereits festgestellte Fokussierung der deliberativen Demokratietheorie auf 'arguing 'wird bei Dryzek durch den Verweis auf die wichtige Rolle rhetorischer Stilmittel, die gemeinhin eher dem 'bargaining zugeordnet werden, relativiert. Dryzek geht inm darum, Deliberationen danach zu bewerten und zu evaluieren, „how closely it involves a reflective quest for mutual understanding, not opposed to strategic bargaining among self-interest participants, but within the field of deliberation that includes bridging rhetoric." (Dryzek 2012:125) 
a discourse on one's own side that has some compatibility with a discourse on the other side. (...) This very difference in cognitive demands may invoke greater reflection and reflexivity on the part of the bridging speaker (...)." (ebd. 77)

Die überbrückende Rhetorik mit ihrer Reflexions- und Reziprozitätsanforderungen ist für Dryzek ganz klar der kommunikative Orientierungspunkt deliberativer Politik, auch wenn sich Deliberationen bei weitem nicht darin erschöpfen. Dies zeigt sich schon allein daran, dass Dryzek zwar die Unterscheidung zwischen bindenden und überbrückenden Rhetoriken aufmacht, um das Spektrum diskursiver Verständigung aufzuzeigen. Er relativiert diese Unterscheidung aber zugleich hinsichtlich der generellen normativen Vorzugwürdigkeit überbrückender Rhetoriken, indem er aufzeigt, dass die Unterscheidung zwar hilft wichtige Aspekte deliberativer Systeme zu kennzeichnen, sie selber aber keinen normativen Standard liefert:

„(T)he bonding-bridging distinction does not produce a sufficiently clear evaluative standard. (...) [It] is ultimately limited because while it pays some attention to the systematic aspects of rhetoric, it does not pay enough attention to the consequences for the larger deliberative system in which the communication in question is occurring. Now, there are many things we might want a deliberative system to do, and so want rhetoric to contribute toward. (...) But whatever we want a deliberative democracy to accomplish (...) we can take a step back and ask: does the rhetoric in question help create and constitute an effective deliberative system joining competent and reflective actors?" (ebd. 80-81)

Bezüglich dieser Fragestellung zeigt Dryzek über eine längere Strecke auf, dass nur diejenigen (rhetorischen) Kommunikationsformen deliberativ zu nennen sind, die zu einer authentischen, inklusiven und konsequenten Entscheidungsfindung durch Verhandlungen und Diskurse führen:

"A system can be said to possess deliberative capacity to the degree it has structures to accommodate deliberation that is authentic, inclusive, and consequential. To be authentic, deliberation ought to be able to induce reflection upon preferences in noncoercive fashion, and involve communicating in terms that those who do not share one's point of view can find meaningful and accept (it is what Gutmann and Thompson call 'reciprocity' (...)). To be inclusive, deliberation requires the opportunity and ability of all affected actors (or their representatives) to participate. To be consequential, deliberation must somehow make a difference when it comes to determining or influencing collective outcomes. Such outcomes might include laws and other explicit and codified puplic policy decisions, international treaties, the more informal outcomes reached by governance networks, or even cultural change." (ebd., 10, SL) 


\subsubsection{Zusammenfassung und Zwischenfazit}

Die letzten beiden Kapitel hatten die Spezifizierung von Good Governance vor dem Hintergrund demokratietheoretischer Überlegungen zu Partizipation, Deliberation und Diskurs zum Gegenstand. Dabei stand die Frage im Vordergrund, welche konzeptionellen Grundpfeiler demokratische Systeme aufweisen müssen, damit von guter Regierungsführung die Rede sein kann. Partizipation, Deliberation und Diskurs wurden dabei von Beginn an als wesentliche Grundpfeiler bestimmt, die auch für eine Good Biodiversity Governance eine tragende Rolle spielen sollten. Diese Grundpfeiler galt es indes genauer zu spezifizieren, was im Allgemeinen über die Explizierung der theoretischen Grundlagen der kontextbezogenen und der prozeduralistischen Demokratietheorien sowie im Speziellen über die pointierte Darlegung zweier, daran anknüpfender Governancemodelle erfolgt ist. Hierüber lassen sich zusammenfassend bestimmte Stärken und Schwächen der jeweiligen Demokratie- und Governancekonzeptionen bezüglich der Explikation der Good Biodiversity Governance darlegen:

Zu den Stärken der kontextbezogenen Demokratietheorien sowie des darauf basierende partizipatorischen Governancemodells zählt, dass die Rolle der Zivilgesellschaft die sich aus der bürgerschaftlichen Aufgabenstellung der Partizipation ergibt als zentrales, vitales und demokratieermöglichendes und -förderliches Element postuliert wird. Beide Schlüsselbegriffe - Zivilgesellschaft und Partizipation - haben daher einen normativ relevanten Status, da das, was hier mit Bürger- bzw. Zivilgesellschaft gemeint ist, ein, „gegenüber den institutionellen Realitäten westlicher Konkurrenzdemokratien und gegen die Modellierungen entscheidungszentrierter Demokratietheorien abgehobenes, abstraktifiziertes normatives Konzept" (Dubiel 1994:94) darstellt, an denen sich die westlichen Demokratien im Allgemeinen und die GBG im Speziellen insofern messen lassen müssen, als dass die Debatte um die Fundamente der Demokratie vor allem über die Debatte um eine zivile Gesellschaft geführt wird (Lösch 2005:102). Dabei zeigen insbesondere die kontextbezogenen Demokratietheorien auf, dass sich das, was mit Zivilgesellschaft gemeint ist und was sich darin verwirklichen soll, nicht lediglich darin erschöpfen kann und soll, dass zivilgesellschaftliche Akteure als Drohkulisse gegen antidemokratische Tendenzen oder Entscheidungen als turnusmäßig auf den Plan tretendes Wahlvolk oder aber als diffuses Themenbecken für das politische Agenda Setting begriffen wird, sondern dass es essentiell ist, dass ihre Rolle sehr viel umfassender und basaler gefasst wird: Zivilgesellschaft und Partizipation sind die Basis und die Grundvoraussetzungen demokratischer Ordnungen. Ohne sie macht die Rede von Demokratie aber auch von Good Governance gar keinen Sinn.

Damit geht wiederum einher, dass die Funktion demokratischer Prozesse noch aus einem anderen Blickwinkel betrachtet wird als in den anderen Demokratietheorien. Hierbei steht weniger die Produktion politischer Entscheidungen (Outputs) bzw. die 
Veränderung gesellschaftlicher, ökonomischer, kultureller und 'natürlicher' Prozesse durch politische Entscheidungen (Outcomes) im Vordergrund der Betrachtungen, wie dies in den entscheidungszentrierten Demokratietheorien der Fall ist, und auch nicht die legitimatorische Funktion der Zivilgesellschaft und ihrer Deliberationen und Diskurse für politische Entscheidungen (Inputs und Throughputs), sondern vornehmlich der Selbstzweck der Orientierung an und die Erfüllung des Ideals der zivilgesellschaftlichen Selbstregierung. Hierbei spielen Prinzipien wie Subsidiarität und die Orientierung an und in der sozialen Bezugsgruppe bei gleichzeitiger Kontextsensibilität und Offenheit für andere Gruppen, Werte, Konventionen und Regeln eine wesentliche Rolle, da diese Prinzipien das Miteinander, aber auch den Streit um Entscheidung innerhalb zivilgesellschaftlicher Eigenkoordination ausmachen sollen. Dabei bleibt der Fokus politischer Entscheidungsfindungen stets auf alltägliche Situationen und Probleme gerichtet, die Menschen im Umgang miteinander haben - sowohl innerhalb ihrer sozialen Bezugsgruppen als auch zwischen. Abstrakte Regeln und Prinzipien, die universalistisch für alle gelten, ungeachtet der jeweiligen Konventionen, kulturellen Hintergründe und Werteorientierungen der jeweiligen sozialen Gruppen der Gesellschaft, gibt es nur insofern, als dass sich alle Mitglieder der Gesellschaft grundsätzlich auf eine möglichst gewaltfreien und toleranten Umgang miteinander verständigen, der aber eingeübt, gelehrt, gelernt und dann als gelebte Praxis weitergegeben werden muss.

Insofern wird das, was das zivilgesellschaftliche Miteinander und Gegeneinander ausmacht, zum „kontrafaktischen Normbestand“ (Dubiel 1994:94), der gleichzeitig Grundlage, Mittel und Ziel demokratischer Prozesse ist. Kritisch betrachtet stellt sich dabei natürlich die Frage, wie eine in diesem Sinne nicht schon vollends demokratische (also nicht am Ideal der Selbstregierung operierende) Gesellschaft in eine solche überführt werden soll, ohne gleichzeitig diejenigen demokratischen Prinzipien zu missachten, die dadurch etabliert und gefördert werden sollen. Des Weiteren ist kritisch anzumerken, dass sowohl die kontextbezogenen Demokratietheorien als auch die partizipativen Governancemodelle nicht plausibel genug zeigen, wie das Ideal der Selbstregierung in ausdifferenzierten und pluralistischen Gesellschaften aber vor allem auch korporatistischen Beziehungsgeflechten zwischen Politik und Wirtschaft realisiert werden kann und soll. In Fällen, wo Probleme aus der Koordination räumlich heterogene und zeitlich verschobener sozialer Interaktionen resultieren, sprich, die sich nicht aus dem direkten Umgang miteinander als aus Externalitäten und Folgeerscheinung der Entscheidungen anderer Gruppen ergeben, die aber auch nicht innerhalb dieser sozialen Gruppen gelöst werden können, bedarf es gesellschafts- und staatsübergreifender Strukturen, Prozesse und Entscheidungen. Eben diese Ebene wird hier zu stark vernachlässigt, was aber für die Good Biodiversity Governance von besonderer Bedeutung ist. 
Des Weiteren bleiben die Schlüsselwörter der Partizipation und der Zivilgesellschaft in den kontextbezogenen Demokratietheorien hinsichtlich ihrer Verwendungsweisen und Funktionen innerhalb des Governancekontextes notorisch vieldeutig (Holtkamp, Bogumil \& Kissler 2006:21): Die in den kontextbezogenen Demokratietheorien vorherrschenden Verständnisse von Partizipation als Mittel und Weg zur Ein- und Ausübung demokratischer Lebensformen sowie der Zivilgesellschaft als Sphäre demokratischer Selbstregierung dienen im Governancekontext (vornehmlich) zur Beschreibung ganz bestimmter Formen von "Self-Governance«, worunter wiederum ganz konkrete Formen der aktiven Bürgerbeteiligung wie bspw. konstatierte Aktionen, runde Tische, Bürgerinitiativen, Bürgerforen, Selbsthilfegruppen oder politische Gesprächskreise fallen. Diese einseitige Fokussierung auf solche Formen der zivilgesellschaftlichen Selbstorganisation und der aktiven Bürgerbeteiligung führt aber dazu, dass partizipative Governancekonzepte lediglich die horizontale (Selbst-)Koordination der BürgerInnen in den Blick nehmen, womit Governance aber in einem eng gefassten Sinne verstanden (siehe 5.3.2.2) und einseitig hinsichtlich der selbstregulativen Prozesse (Jessop 2002: 163) und der in ihr wirksamen deliberativen Rationalität beleuchtet und untersucht wird (Kronsell \& Bäckstrand 2010: 28). Selbst eine partizipativ fundierte GBG erschöpft sich aber nicht in selbstregulativen Prozessen, weil ein Großteil umweltpolitischer Regulierung nach wie vor „im Schatten der Hierarchie“ stattfindet (siehe 5.1.1) und insbesondere hier effektiv ist (Töller 2012: 412). Daher sind die kontextualistischen und partizipativen Ansätze zwar wichtig und hinreichend, um zu spezifizieren, welche Vorstellungen und Annahmen von demokratischer Beteiligung der GBG zugrunde liegen könnten, die Ansätze sind aber nicht ausreichend, um auch zu spezifizieren, welche Vorstellungen und Annahmen von politischer Steuerung und Regulierung sowie von demokratischer Verantwortung sich daraus für die GBG ergeben, so dass im Endeffekt auch die Substanz fehlt, um darlegen zu können, welche Veränderungen sich für die Politik der Biodiversität vor dem Hintergrund der theoretischen Gehalte der GBG ergeben (Frage 2) und vor welchen Defiziten und Entwicklungsmöglichkeiten die deutsche Biodiversitätspolitik vor dem Hintergrund der Anforderungen steht, die sich über die GBG ergeben (Frage 3).

Ähnliches gilt, wenn auch aus anderen Gründen und stärker bezogen auf Diskurs und Deliberation, für die deliberativen Governancemodelle und die dahinterstehenden prozeduralistischen Demokratietheorien. Die zu berücksichtigenden theoretischen Stärken der prozeduralistischen Demokratie- und deliberativen Governancemodelle liegen vor allem darin, dass das Verhältnis zwischen Zivilgesellschaft und Politik anhand des Schleusenmodells fokussiert wird, wobei auf die zentrale Rolle des intermediären Bereichs der politischen Öffentlichkeit verwiesen wird. Eine weitere Stärke der prozeduralen Demokratietheorien liegt darin, dass hier die zentrale und entscheidende Rolle 
der Kommunikation für demokratische Aushandlungs- und Entscheidungsprozesse fokussiert wird. Indem Kommunikation zum Basismedium gesellschaftlicher und damit auch politischer Aushandlungs- und Entscheidungsprozesse modelliert wird, die deliberative Demokratietheorie aber zugleich in den Rahmen der Theorie des kommunikativen Handelns eingebettet ist, die als Gesellschaftstheorie und -ethik zahlreiche theoretische Bezugspunkte eröffnet und ermöglicht, wird ein sehr großer und komplexer Kontext für die Ausbuchstabierung der GBG eröffnet. Ein entscheidender Bezugspunkt ist dabei der legitimationstheoretische Aspekt. Insbesondere auf diesen muss genauer eingegangen werden, da sich hierüber sowohl Stärken als auch Schwächen der prozeduralistischen Demokratietheorie sowie der deliberativen Governancemodellierung hinsichtlich der Explikation der GBG zeigen:

Fokussiert auf demokratische Legitimität, die in den prozeduralistischen Demokratietheorien als normative Grundlage der Demokratie betrachtet wird, wird die Auffassung vertreten, dass Legitimität und Rationalität in einem Gemeinwesen nur dann erreicht werden können, „wenn die Institutionen dieses Gemeinwesens und ihre ineinandergreifenden Beziehungen so angeordnet sind, dass das, was als Gemeinwohl (...) aufgefasst wird, sich aus einem rational und fair geführten Prozess der kollektiven Deliberation unter freien und gleichen Individuen ergibt." (Benhabib 1995a:9) Eben diese Verbindung aus praktischer Rationalität gebunden an institutionalisierte deliberative Verfahren, woraus sich die deliberative Verfahrensrationalität ergibt, ist für die deliberative Demokratietheorie in legitimationstheoretischer Hinsicht zentral, da das wichtigste Gut einer demokratischen Gesellschaftsordnung in der Organisation und Institutionalisierung einer freien, ungezwungenen und vernünftigen Deliberation gleichberechtigter BürgerInnen zur Legitimation politischer Macht gesehen wird, die ihren Ausgangspunkt in einer starken politischen Kultur und Öffentlichkeit und einer lebendigen Zivilgesellschaft hat. Diese drei Komponenten - politische Kultur, politische Öffentlichkeit und Zivilgesellschaft - konkretisieren somit die politische Dimension einer rationalen und reflexiven Lebenswelt, aus der heraus die Anwendung und der Einsatz politischer Macht gerechtfertigt werden muss. Legitimität wird auf diesem Weg zum zentralen Begriff einer aus der Lebenswelt heraus argumentierenden diskursiv-deliberativen Demokratietheorie.

Die Crux hierbei besteht nun indes darin, dass die rational geführte Deliberation sowie die rational-diskursive Problemlösung autonomer Individuen zwar normativ relevante Aspekte demokratischer Politik sind, dass aber die Aushandlung und Verhandlung sozialer Konflikte, ohne dass diese konsensual gelöst werden könnten, politischer Alltag sind. Dies verweist auf einen theorieimmanenten und eigentlich altbekannten Kritikpunkt an der deliberativen Demokratietheorie, der aber vor dem Hintergrund der prozeduralistischen und deliberativen Explikation der Governance von Relevanz ist: Im 
Kern geht es um die Kritik, dass das deliberative Legitimationskonzept hinsichtlich der damit verbundenen Konsensorientierung zu anspruchsvoll ist (Palazzo 2002:241). Über die im Moralkontext sicherlich unproblematischere Annahme, es gebe ein universalistisches Vernunftsmoment, nämlich das der kommunikativen Rationalität, abstrahieren die prozeduralistischen Demokratietheorietheorien zu stark von den konkreten Motivationen, Umständen und Befähigungen der Menschen, die sie zur politischen Meinungs- und Willensbildung sowie zur Teilnahme an politischen Entscheidungsfindungen bewegen, wo die kontextualistischen Demokratietheorien wiederum einen starken Punkt haben. Denn nicht allein die Verständigungsorientierung und mithin das gemeinsame vernünftige Argumentieren zur Lösung gesamtgesellschaftlicher Problematiken, sondern auch andere, ebenfalls rationale, aber eben nicht auch per se vertändigungsorientierte Beweggründe, wie bspw. Leidenschaften, Langeweile, Routinen, Prestige, Alternativlosigkeit, Machtbewusstsein oder Konkurrenzkampf sind entscheidende Motivationen, die politische und soziale Aktivitäten kennzeichnen.

Zum anderen lässt sich mit Blick auf die Pluralisierung der Gesellschaft einwenden, dass die Vorstellung zu optimistisch ist, es ließe sich über Deliberationen eine weitreichende Übereinstimmung über gesamtgesellschaftlich relevante aber strittige Fragen erzielen, die wiederum als stabile Basis politischer Entscheidungen genutzt werden können. Vielmehr geht es im politischen Kontext darum die Pluralität der Assoziations-, Koordinations- und Verhandlungsformen anzuerkennen und deren normative und praktische Implikationen zu untersuchen (ebd.). Dies bleibt der prozeduralen Demokratietheorie aber weitestgehend verschlossen, solange die real existierenden Notwendigkeiten zu Bargaining, Tauschgeschäften und Konzessionen nicht als legitime demokratische Verfahren anerkannt werden. Eben dies fällt der deliberativen Demokratietheorie schwer, da diese in den Bereich des strategischen Handelns fallen und damit nicht als Formen legitimer, da verständigungsorientierter Verhandlung begriffen werden (können). Diese Vereinseitigung kann, wie im Rahmen des deliberativen Governancemodells von Dryzek gezeigt (siehe 5.2.2.2), durchaus theorieimmanent relativiert und partiell aufgehoben werden, bereits die Notwendigkeit einer solchen Relativierung macht aber deutlich, dass die deliberative Demokratietheorie das kommunikative Handelns, die Verständigungsorientierung und mithin das zivilgesellschaftliche Steuerungsmedium der Solidarität systematisch überfordert.

Des Weiteren ergibt sich die notwendige Relativierung des deliberativen Legitimationskonzeptes auch aus der Berücksichtigung der „raumzeitlichen Entschränkung der Globalisierung" (ebd., 242) und der zunehmenden Internationalisierung der Politik. Da das demokratische Schleusenmodell, das als Verbindung der politischen Kultur zwischen zivilgesellschaftlicher Lebenswelt und dem politischen System angelegt ist, dort aufhört, wo sich das politische Engagement zivilgesellschaftlicher Akteure gegen Akteurskon- 
stellationen, Institutionen und Organisationen richtet, die außerhalb der territorialen Grenzen des Nationalstaats verortet sind, folgt das deliberative Demokratiemodell in seiner Verhältnisbestimmung von Lebenswelt, Ökonomie und Politik einer genuin nationalstaatlichen Logik (ebd., 202) und gerät dort unter Druck, wo die Entgrenzung des Raumes diese Logik durchkreuzt (ebd., 203). Auf legitimationstheoretische Überlegungen angewandt, bedeutet dies, dass die Entgrenzung, die vor allem durch Globalisierungsprozesse geschieht und vorangetrieben wird, den Anspruch einer legitimatorischen Verbindung zwischen Staat und Zivilgesellschaft unscharf werden lässt, da ein Teil des zivilgesellschaftlichen Engagements auf Zusammenhänge verweist, welche die Steuerungsfähigkeit und den Legitimationsbedarf des Rechts- und Sozialstaats überschreiten (ebd., 242). Die Veränderungen der Staatlichkeit unter den Bedingungen der Globalisierung verweisen dann entweder auf ein transnationales politisches System, welches allerdings erst in Ansätzen und bei weitem nicht nationalstaatsanalog etabliert ist oder sie führen zurück auf den Nationalstaat und die subnationalen politischen Ebenen und die Frage, wie diese die Legitimationsdefizite, die sowohl oberhalb und innerhalb der Nationalstaaten produziert werden, aufgefangen und beseitigt werden können. Die von Habermas selbst vorgeschlagene Stärkung zivilgesellschaftlicher Beteiligungsmöglichkeiten durch die konsequentere Einbeziehung transnational agierender NGO's in die internationalen und nationalen politischen Entscheidungsprozesse ( $\mathrm{Ha}$ bermas 1998:161) ist hierbei sicherlich nur eine gangbare praktische Möglichkeit.

Wichtiger sind hierbei die theorieimmanenten Problematiken, die sich aus und für die deliberative Demokratietheorie ergeben: Der zunehmende Einfluss der Globalisierung auf alle Lebens- und Systembereiche macht deutlich, dass sich Legitimität nicht nur auf das Verhältnis des politischen Systems zur Lebenswelt beziehen kann. Auch wenn das ökonomische System dem lebensweltlichen Legitimationsdruck in der deliberativen Demokratietheorie nicht unmittelbar ausgesetzt wird (Palazzo 2002:238), geht Habermas davon aus, dass ökonomische Entscheidungen dann als legitim angesehen werden können, wenn sie sich in jenem rechtlichen Rahmen bewegen, den die Politik ihr in Rückkoppelung an die Meinungs- und Willensbildung innerhalb der Zivilgesellschaft setzt (ebd.) Dies hat zur Folge, dass die Politik nicht nur die Legitimität ihrer Entscheidungen sondern auch die Effektivität ihrer Leistungen sicherstellen muss (Habermas 1992b:466). Eben dieser Ansatz ist ein wichtiges und wesentliches legitimationstheoretisches Element der deliberativen Demokratietheorie, welches ihr den Anschluss an die Governancedebatte einerseits sichert und worüber sich aber andererseits auch aufzeigen lässt, dass die Vorstellung von politischer Legitimität in der deliberativen Demokratietheorie bezogen auf die lediglich mittelbare Anwendung auf das ökonomische System zu eng und andererseits bezogen auf die mit dem Verständnis der Legitimität einhergehende Konsensorientierung zu anspruchsvoll gefasst ist: 
Da es bei der Bewertung von Governancearrangements in zunehmendem Maße nicht mehr nur darum geht, Governance als Problemlösungsansatz für die Ineffektivität politischer Regelungen zu postulieren, sondern weil es vor dem Hintergrund der politischen Einbindung demokratisch nicht gewählter Akteure in Governancenetzwerke immer auch darum geht, dass die Legitimitätsproblematiken moderner, zumeist transnationaler Politiken diskutiert werden (vgl. Papadopoulos \& Benz 2006), kann die deliberative Demokratietheorie hier wichtige Impulse und Einsichten liefern. Andererseits ist die in der deliberativen Demokratietheorie verankerte Auffassung von der unmittelbaren Legitimationsfreiheit des ökonomischen Systems unter den durch Globalisierung geprägten Bedingungen nicht mehr zeitgemäß, was sich an der notwendigen Modifikation drei relevanter Grundannahmen zeigt: Zum einen kann wirtschaftliches Handeln nicht mehr problemlos dem Raum der bürgerlich-freiheitlichen Privatsphäre zugeordnet werden. Vielmehr zeigen gerade Umwelt- und Naturzerstörungen im globaIen Ausmaß, dass die Externalitäten ökonomischer Entscheidungen von ebenso kollektiver Wirkung wie politische Entscheidungen sind (ebd.). Zum zweiten kann die Lebenswelt nicht nur über das Recht auf das ökonomische System einwirken und drittens ist es legitimationstheoretisch ungenügend, dass ökonomische Handlungen, wenn diese in erster Linie private Handlungen sind, keiner kollektiven Rechtfertigung bedürfen.

Alle drei Annahmen „bringen das legitimationstheoretische Grundgerüst der deliberativen Demokratietheorie ins Wanken“ (Palazzo 2002:239), denn die Verschiebung der „Freiheitsgefahren“ vom Staat zum privatwirtschaftlichen Handeln sei, gerade wenn die Ökonomie transnational und globalisiert agiere, zur primären Gefahr politischer und bürgerlicher Freiheiten geworden (ebd.). Zwar sieht Habermas diese Gefahr, Palazzo weist aber pointiert darauf hin, dass die liberale Vorstellung, dass nicht der Besitz legitimiert werden muss sondern der Versuch, auf das private Eigentum einen politischen Einfluss auszuüben, bei Habermas auch dort noch die Argumentation beeinflusst, wo die für liberale Ansätze typische Dualität von Gesellschaft und Staat bereits durch das dreiteilige Modell von Staat, Ökonomie und Zivilgesellschaft ersetzt worden ist (ebd.). Zusammenfassend gelangt Palazzo daher zu dem Ergebnis, dass wenn ökonomische Handlungen private Entscheidungen mit kollektiven Wirkungen verbinden und darüber einerseits die bürgerlichen Freiheiten ausgedehnt gleichzeitig aber deren Wirkungen potenziell freiheitsbedrohend werden, „dann besteht unter diesen Bedingungen die Notwendigkeit, den Begriff der politischen Legitimation neu zu bestimmen." (ebd.) Das wiederum bedeutet konkreter, dass Legitimität sich nicht allein auf das Verhältnis von Zivilgesellschaft zum Staat beziehen kann, sondern als eine Kategorie subpolitischen Handelns schlechthin verstanden werden muss. Eine Theorie deliberativer Demokratie muss sich daher auch mit dem bis dato legitimationstheoretisch vernachlässigten Bereich der Ökonomie widmen und dessen Verhältnis zur Lebenswelt 
neu bestimmen, wobei darauf geachtet werden muss, dass ein solches Verhältnis auch nicht einseitig negativ ist, da „gerade dieser unerwünschten kollektiven Wirkung ökonomischer Handlungen (...) die Politisierung lebensweltlicher Kommunikation und das Entstehen einer Vielzahl zivilgesellschaftlicher Assoziationen zu verdanken ist", die ja für die deliberative Demokratietheorie so relevant sind.

Unterm Strich ist folglich zu bilanzieren, dass polyzentrische Gesellschaften polyzentrischer Legitimitätsquellen bedürfen (ebd., 241), was wiederum mit einer Relativierung der legitimationstheoretischen Ansprüche an die Zivilgesellschaft sowie deren Konsensfähigkeiten verbunden ist. Konkret bedeutet dies, dass sich die Legitimität kommunikativen und solidarischen Handelns als das Ergebnis akkumulierter Deliberationserfahrungen zeigt, die eine eher diffuse Unterstützung des politischen Systems bedeuten, nicht aber für einzelne Diskursergebnisse auch konkret abgeprüft werden können (ebd.). Wenn es aber darum gehen muss und soll, die Qualität und Güte deliberativer Verhandlungs- und Verständigungsprozesse zur Lösung kollektiver Probleme zu bestimmen, dann bedarf es einer an konkreten Ansprüchen der Effektivität und Effizienz sowie der demokratischen Partizipation orientierten legitimationstheoretischen Betrachtung, die die deliberative Demokratietheorie sowie die auf ihr basierenden Governancemodellierungen aufgrund ihrer Überschätzung des verständigungsorientierten Modus sowie der zu geringen Beachtung empirischer und policytheoretischer Überlegungen nicht in Gänze leisten können, auch wenn sich anhand der prozeduralistischen und deliberativen Ansätze zahreiche wichtige Aspekte hinsichtlich der Explikation der GBG bezüglich der Annahmen von demokratischer Beteiligung und Verantwortung ausweisen lassen.

Zentrales Manko ist hier indes, dass die Globalisierung der Wirtschaft und die zunehmende Internationalisierung des Politischen auf Wandlungsprozesse des territorial verfassten Verwaltungs-, Steuer-, Rechts- und Sozialstaats verweisen, was wiederum zu einem Wandel staatstheoretischer Annahmen führt, dem die deliberative Demokratietheorie bisher nur unzureichend nachgekommen ist, so dass die deliberativen Demokratie- und Governancemodelle ein unterkomplexes Verständnis von politischer Steuerung und Regulierung inkludieren, obgleich es ihnen mithilfe des Schleusenmodells gelingt, das Verhältnis von Gesellschaft und Staat als konzentrisches und multizentrisches System zu begreifen, das sich aus einer Kombination aus hierarchischen und heterarchischen Elementen zusammensetzt. Da die den einzelnen Bereichen zugeordneten Kommunikationsformen aber weder der Komplexität des politisch-administrativen Systems noch der Binnendifferenzierung der Gesellschaft gerecht werden, bedarf es im Folgenden weiterführender Überlegungen zu den policy- und governancetheoretischen Aspekten der politischen Interaktion in einem internationalen Mehrebenensystem. 


\subsection{Die Spezifizierung von Good Governance vor dem Hintergrund governancetheoretischer Überlegungen}

An den aufgezeigten Problematiken der Spezifizierung von Good Governance vor dem Hintergrund demokratietheoretischer Überlegungen zu Partizipation, Deliberation und Diskurs zeigt sich die Notwendigkeit, Good Governance auch vor dem Hintergrund governance- und policytheoretischer Überlegungen zu spezifizieren. Bevor indes erneut mit der Explikation der theoretischen Grundlagen der GBG begonnen werden kann, muss erst einmal genauer auf das schwierige und zumeist auch nicht eindeutige Konzept der Governance eingegangen werden (5.3.1). Das Problem hierbei ist, dass es rein theoretisch viele in Frage kommende Governancekonzepte gibt, die aber nicht alle auch auf einer tragfähigen theoretischen Basis aufbauen. Vor diesem Hintergrund wird das aus der Steuerungstheorie hervorgegangene Verständnis von Governance als kooperative Regelung ausgeführt und herangezogen (5.3.2). Dieses wird dargelegt, indem einerseits die theoretischen Verschiebungen beim Übergang von Government zu Governance und von Steuerung zu Regelung aufgezeigt werden (5.3.2.1) und indem andererseits auf die Kontinuitäten zwischen Steuerungs- und Governancetheorie eingegangen wird (5.3.2.2). Die sich dabei ergebenden theoretischen Problematiken bei der Explizierung von Governance werden anschließend über den Ansatz des wakteurszentrierten Institutionalismus« geschlossen, der als theoretische Hintergrundfolie zur Explizierung von Governance herangezogen wird (5.3.2.3).

Im anschließenden Zwischenfazit wird darauf eingegangen unter welchen Bedingungen die dargelegte Governancekonzeption um demokratie- und legitimationstheoretische Überlegungen erweitert werden kann (5.3.2.4). Hierbei wird deutlich gemacht, dass sich unter Zugrundelegung der explizierten governancetheoretischen Annahmen auch die gängigen Demokratieverständnisse verändern (müssen), wenn von Good Governance die Rede ist. Eben dies - so die im weiteren Verlauf relevante These - leistet die Theorie der »komplexen Demokratie« von Fritz Scharpf: Indem Scharpf für seine Demokratietheorie, aufbauend auf den Erkenntnissen der empirischen Demokratieforschung, als reale Hintergrundfolie ein verhandlungsdemokratisches Modell wählt, bei dem ausgehend von steuerungs- und governancetheoretischen Überlegungen kontextualistische und prozeduralistische aber auch entscheidungszentrierte demokratietheoretische Ansätze und Aspekte zugleich berücksichtigt werden, können, anders als dies unter der rein demokratietheoretischen Perspektive auf die partizipativen, deliberativen und diskursiven Aspekte der GBG möglich war, in konsistenter Weise Antworten auf alle drei der eingangs gestellten Fragen (siehe Abschnitt $\mathrm{V}$ ) gegeben werden, so dass die Ausführungen zur komplexen Demokratietheorie als sicherlich in sich komplexes und mehrstufiges - Fazit der politikwissenschaftlichen Detailreflexion gelesen werden können. 
Allerdings muss die komplexe Demokratietheorie, gerade was ihre Modifikationen unter dem Einfluss der zunehmenden Internationalisierung der Staaten und der Globalisierung der Wirtschaft angeht, erst einmal rekonstruiert werden (5.3.3): Ausgehend von der 1970 erschienenen Grundlegungsschrift »Demokratietheorie zwischen Utopie und Anpassung " (5.3.3.1) werden die bei Scharpf nur in einzelnen Artikeln ausgeführten und damit als Ganzes unzusammenhängenden Überlegungen zur 'Erweiterung 'der komplexen Demokratietheorie systematisch herausgearbeitet (5.3.3.2), indem sowohl die Kriterien guter Politik (5.3.3.2.1) als auch die Kriterien legitimer Politik (5.3.3.2.2) unter verhandlungsdemokratischen Bedingungen dargelegt werden. Abschließend werden die Ausführungen zur komplexen Demokratietheorie hinsichtlich der Spezifizierung der Good Biodiversity Governance zusammengefasst (5.3.3.3). Dies wiederum erlaubt Antworten auf die die praktische Zielstellung der Arbeit anvisierende Frage, welche Anforderungen, Chancen und Risiken sich aus der GBG für die deutsche Biodiversitätspolitik ergeben (5.4). Dies wird expliziert, indem zum einen die Anforderungen an die deutsche Biodiversitätspolitik dargelegt werden, die sich aus den theoretischen Annahmen der GBG ergeben (5.4.1) und indem die Defizite und Entwicklungsmöglichkeiten aufgezeigt werden, vor denen die deutsche Biodiversitätspolitik vor dem Hintergrund dieser Anforderungen steht (5.4.2).

\subsubsection{Der Begriff der Governance und die theoretischen Gehalte der politikwissenschaftlichen Governanceforschung}

Wird der oben dargelegte Weg eingeschlagen, die Governancetheorie zur Grundlage der Spezifizierung einer Good Biodiversity Governance zu machen, dann besteht die zentrale Herausforderung erst einmal darin, spezifizieren zu müssen, was genau mit dem Begriff der Governance gemeint sein soll und zudem muss angegeben werden, worin die spezifischen theoretischen Gehalte der politikwissenschaftlichen Governanceforschung liegen. Beides ist keineswegs so einfach, wie man auf den ersten Blick meinen mag: Governance ist ein schillernder und "anerkannt uneindeutiger Begriff“ (Blumenthal 2005:1150), bei dem mit Detlef Sack bereits in kritischer Absicht davon ausgegangen werden kann, dass „'alte` theoretisch-konzeptionelle, empirische und normative Auseinandersetzungen schlicht einen neuen 'Aufguss' erfahren" (Sack 2011: 26). Dies wiederum ist der Fall, weil die Verwendung des Governancebegriffes zumeist nicht theorieinduziert erfolgt, sondern aus dem Bedürfnis heraus erwächst, „verkrustete theoretische Zusammenhänge aufzubrechen, neue Perspektiven zu eröffnen und neue Phänomene und Entwicklungen des Politischen erkennbar werden zu lassen." (Schuppert 2008:13-14) Daher stellt sich die Frage, was die spezifischen theoretischen Gehalte der Governanceforschung sind. Wenn man sich vergegenwärtigt, dass es weder den Governancebegriff noch die Governanceforschung gibt sondern verschiedene, in der Politikwissenschaft vertretene Analyseperspektiven auf Governance, die jeweils 
ganz unterschiedliche Zugänge und Antworten auf die Frage anbieten, auf welchen speziellen theoretischen Grundannahmen das Konzept der Governance fußt, was dabei als Funktion und Aufgabe des Politischen angesehen wird und welche (positiven und negativen) Veränderungen von Staatlichkeit und Demokratie über die Etablierung von Governancearrangements zu erwarten sind, dann lassen sich mit Detlef Sack vier verschiedene Analyseperspektiven innerhalb der politikwissenschaftlichen Governanceforschung unterscheiden: ${ }^{178}$

1) Der advokatorisch-modernisierungstheoretische Diskussionsstrang ist auf die Analyse und die programmatische Etablierung von Good Governance ausgerichtet. Hier werden bestimmte als 'universal' verstandene Prinzipien einer guten Regierungsführung und Verwaltung aufgestellt und propagiert. Beides geschieht vor einem für das entwicklungspolitische Verständnis von Good Governance typischen neoinstitutionalistischen, neoliberalen und normativ-praxeologischen Hintergrund (siehe 5.1.2).

2) Im netzwerkfokussierten Diskussionsstrang stehen die verschiedenen Formen sozialer Ordnung (Markt, Staat, Gemeinschaft usw.) im Fokus der empirischen Analysen im Rahmen organisationssoziologischer Untersuchungen der Politikfeldforschung, der Europaforschung und der Internationalen Beziehungen. Die in diesen Zusammenhängen auftauchenden Begriffe des Netzwerkes und der Verhandlungssysteme werden dabei oft als Synonym für Governance verwandt. Sie sind aber genauso 'schillernd wie der Governancebegriff selbst.

3) Der politikökonomische Diskussionsstrang kann als kritische Variante des netzwerkfokussierten Governancestranges verstanden werden. Hier wird auf Überlegungen zur Relation und Regulation von Ökonomie, Staat und (Zivil-)Gesellschaft fokussiert, wobei deren Verhältnisse vornehmlich unter regulations- und machttheoretischen Gesichtspunkten analysiert werden. Die zunehmende Relevanz von Governancearrangements wird hier als Aspekt einer umfassenden Transformation der Regulationsweise und der gesellschaftlichen Regulierungsprozesse verstanden, wobei die für den Governanceansatz stehenden Koordinationsformen im Kontext des materialistischen Institutionalismus und der Regulationstheorie dekontextualisiert und in einen spezifischen, kapitalismuskritischen, neomarxistischen Theoriezusammenhang gebracht werden.

\footnotetext{
178 Diese nach Analyseperspektiven aufgegliederte Übersicht über die politikwissenschaftliche Governanceforschung ist besser geeignet als die übliche Differenzierungen zwischen deskriptiver, empirisch-analytischer und normativer oder normativ-programmatischer Governanceforschung (vgl. Rhodes 1997; Blumenthal 2005): Die übliche Differenzierung zwischen deskriptiver und normativer Governanceforschung suggeriert, dass sich die empirisch-analytische klar von der normativ-programmatischen Governanceforschung abgrenzen ließe. Diesbezüglich muss indes kritisch angemerkt werden, dass die (vermeintlich) 'saubere` Trennung zwischen analytischen und normativen Aspekten im Umgang mit Governance nicht gegeben ist und dass es diese auch nicht geben kann, da sich analytische und normative Aspekte bereits in den Konzeptionen, Fragestellungen und Methoden durchdringen und überlagern (Sack 2011:21).
} 
4) Der deliberative Diskussionsstrang geht von den demokratietheoretischen Narrativen und Prinzipien aus, die für Demokratie als Ideal der Selbstregierung und das Regieren als Argumentieren zentral sind. Hier stehen speziell die 'neuen` auf Verhandlung, Deliberation, Partizipation und Diskurs ausgerichteten Governancemodi im Vordergrund der Analyse (siehe 5.1.2.2 sowie 5.2.2.1 und 5.2.2.2). Hierbei steht anders als bei den anderen Diskussionssträngen kein institutionalistischer sondern ein demokratietheoretischer Ansatz dahinter, was indes dazu führt, dass zumeist kein eigenständiger Governancebegriff entwickelt wird sondern entweder auf Governance als Netzwerk zurückgegriffen wird oder unspezifisch von Governance als Platzhalter für 'neue Politik' die Rede ist. Dabei entsteht jedoch das Problem, dass im ersten Fall ein eigentlich deskriptiv gemeinter Begriff normativiert wird, ohne dass diese Normativierung theoretisch eingeholt und begründet wird. Im zweiten Fall bleibt die Rede von Governance schlicht oberflächlich und inhaltsleer.

Trotz dieser analytisch sinnvollen Aufgliederung der Governanceforschung in verschiedene Diskussionsstränge ist es zumindest so, dass die Governanceforschung bei aller Differenzierung eine Art 'gemeinsamen kleinsten Nenner' besitzt. Dies ist ihr thematischer Fokus, denn in erster Linie fokussiert die Governanceforschung auf die weitreichenden Transformationen des Politischen, des Ökonomischen und des Sozialen, die zugleich Ursache als auch das Ergebnis der zunehmenden Internationalisierung des Staates, der Globalisierung der Wirtschaft und der Mediatisierung kultureller und sozialer Gemeinsamkeiten und Unterschiede sind (Grande 2012:565). Allerdings erhält die Governanceforschung über diesen Zugang kein theoretisches Alleinstellungsmerkmal innerhalb und außerhalb der Politikwissenschaft, weil sich auch andere Disziplinen, wie bspw. die vergleichende Demokratieforschung, die politische Ökonomie oder die politische Soziologie ebenfalls mit diesen Transformationsprozessen des Politischen beschäftigen. Daher muss man noch spezifischer schauen: Die politikwissenschaftliche Governanceforschung richtet ihren thematischen Fokus auf die Transformationen des Politischen in den verschiedenen politischen Dimensionen. Dabei geht es in erster Linie um die Transformationen der politischen Strukturen und Institutionen (Polity) wie dies bei Multi-Level-Governance, Network Governance oder Global Governance vornehmlich der Fall ist, die Transformationen der politischen Prozesse und Verfahren (Politics) wie diese bei Analysen zu Self-Governance, deliberativer Governance oder partizipativer Governance im Vordergrund stehen oder die Transformationen einzelner Politikfelder und Politikbereiche (Policy) wie etwa der environmental governance, der economic governance oder der media governance.

So unterschiedlich diese Studien sind, in ihnen wird immer auf bestimmte Aspekte abgehoben, die mit Edgar Grande zur thematischen Bestimmung der politikwissenschaftlichen Governancedebatte herangezogen werden können (Grande 2012:566-567): 
Unter dem bereits erwähnten thematischen Fokus auf die Transformation des Politischen unter den Bedingungen der zunehmenden Internationalisierung des Staates und der Globalisierung der Wirtschaft wird erstens auf die empirisch beobachtbare Zunahme an Interdependenzen zwischen gesellschaftlichen Teilsystemen, territorialen Handlungsebenen sowie verschiedenen Politikfeldern verwiesen, was zum einen mit der Etablierung politischer Handlungsebenen oberhalb und unterhalb des Staates als auch mit der Auflösung der klaren Grenzen und Zuständigkeiten der jeweiligen politischen Handlungsebenen einhergeht (Stichwort: Interdependenz und Komplexität). Zweitens ergibt sich daraus die Betonung nicht-hoheitlicher, nicht-hierarchischer, nicht-etatistischer Formen der politischen Regelung sowie die Forderung der Einbindung gesellschaftlicher und wirtschaftlicher Akteure in den politischen Prozess (Stichwort: kooperative Regelung). Damit geht wiederum der Verweis auf die Relevanz der Etablierung und Einübung alternativer Regelungsformen und Steuerungsinstrumente (Stichwort: New Modes of Governance) einher (siehe 5.1.2.2).

Im Folgenden stehen insbesondere die beiden Sinnzusammenhänge "Interdependenz und Komplexität" sowie "kooperative Regelung" im Vordergrund, da dahinter zwei Wandlungsprozesse stehen, die das Spezifikum der politikwissenschaftlichen Governanceforschung ausmachen (siehe auch 1.2.3): Zum einen der Fokus auf den Wandlungsprozess von Government und Governance und zum anderen der Fokus auf den Wandlungsprozess von staatlicher Steuerung zu kooperativer Regelung ${ }^{179}$ (Benz et al. 2007:10). Beide Wandlungsprozesse werden obgleich sie unterschiedliche theoretische Anknüpfungspunkte und Foki haben innerhalb der Governanceforschung in Bezug zueinander gesetzt, indem die empirisch-institutionalistische Analyse von Governancearrangements (Übergang von Government zu Governance) mit normativ-präskriptiven Aussagen über den Niedergang staatlicher Steuerungsfähigkeit sowie die wünschenswerten Alternativen zu staatlicher Steuerung (kooperativ-prozedurale Regelung statt hierarchische Steuerung) verbunden wird:

Der begriffliche Wandel von Government zu Governance, der für die Governanceforschung insbesondere in Bezug auf das Konzept der Global Governance von besonderer Bedeutung ist, resultiert aus der empirischen Feststellung, dass sich eine mit Internationalisierungs- und Globalisierungsprozessen konfrontierte Politik vor allem auf den politischen Ebenen oberhalb der Nationalstaaten, nicht mehr mit den üblichen Begriffen wie bspw. Regierung, Staatlichkeit, Staatstätigkeit, Steuerung und Hierarchie, sprich nach Maßgabe von nach innen und außen souveränen, hierarchisch strukturier-

179 Der begriffliche Wandel von Steuerung (»Steering") zu Regelung (»Governance») lässt sich anhand des Übergangs von Steuerungs- zur Governancetheorie nachzeichnen (siehe 5.3.2). Entscheidend hierbei ist, dass dies der für die Bestimmung der GBG entscheidende Wandlungsprozess ist, da es hier im Wesentlichen um die Grundlagen für die New Modes of Governance, d.h. die Voraussetzungen für den Wandel der politischen Prozesse und Verfahren, geht. 
ten Staaten (Government), begreifen und beschreiben lässt, da sich hier eine „Governance without Government“ (vgl. Rosenau \& Czempiel 1992) herausgebildet hat. Insofern also Global Governance als überstaatliche, regierungslose, netzwerkförmige und diffuse da nicht hierarchisch gestaltete, politische Ordnung verstanden wird, bei der der Nationalstaat nicht mehr als der vorherrschende Produzent öffentlicher Güter und damit „als Gewaltmonopolist, als Rechtsgarant, zentrale Legitimationsinstanz und universelle Wohlfahrtssicherung" (Mayntz 2008:43) angesehen wird, was sich u.a. an der zunehmenden Privatisierung und Liberalisierung öffentlicher Infrastrukturen und dem Kompetenztransfer auf supranationale Institutionen wie die EU, die UN oder andere internationale Regime wie das Kyoto-Protokoll zeigt (Grande 2012:572), hat der Wandel von Government zu Governance auch einen Einfluss auf den Wandel von Steuerung zu Regelung, da hierüber insbesondere die Steuerungsproblematik moderner, vernetzter Staaten sowie die Leistungsfähigkeit nationaler Politiken angesprochen ist (vgl. insb. Jänicke 1986). Beide Übergänge werden vornehmlich im Rahmen des netzwerk- und koordinationsfokussierten Governancestranges untersucht, so dass es dabei um die empirische Analyse der Zunahme an Interdependenzen zwischen gesellschaftlichen Teilsystemen sowie zwischen den territorialen Handlungsebenen und Politikfeldern geht. Durch ein solches Forschungsprogramm sind vor allem die Analysen zu Global Governance, European Governance und überschneidend dazu Multi-Level-Governance sowie die Policy-Forschung gekennzeichnet.

Über die Verbindung der beiden Wandlungsprozesse kommt es nun zu einer normativen Imprägnierung der sich selbst als empirisch-analytisch verstehenden Governanceforschung, die eine der größten theoretisch-konzeptionellen Hypotheken der politikwissenschaftlichen Governanceforschung darstellt (Blumenthal 2005:1168): Wenn auf den Umstand verwiesen wird, dass kollektive Entscheidungen in modernen Gesellschaften zunehmend in kooperativen Formen der Zusammenarbeit zwischen staatlichen und privaten Akteuren zustande kommen (ebd., 14), dann wird Governance als eine notwendige Anpassung an die tiefgreifenden und irreversiblen Internationalisierungs- und Globalisierungsprozesse verstanden, deren Auswirkungen sich auf allen politischen Ebenen in nahezu allen Subsystemen der Gesellschaft sowie in zahlreichen Politikfeldern abzeichnen. Zumeist wird es dabei allerdings nicht belassen, da es in der Governanceforschung immer auch darum geht, die Veränderungsprozesse zur Plausibilisierung eines fundamentalen Wandels von Staatlichkeit heranzuziehen (Blumenthal 2005: 1167). Geschieht dies, dann wird Governance nicht nur rein beschreibend als Oberbegriff für alle gesellschaftlichen Koordinationsformen (typischerweise Markt, Staat, Gemeinschaft) und -mechanismen (von staatlich-hierarchischer Steuerung bis hin zu Mechanismen gesellschaftlicher Selbstregulierung) verwandt, sondern auch als erstrebenswerte politische Modernisierungsstrategie betrachtet (Benz et al. 2007:11-14). 
Dies wiederum betrifft den Wandel von Steuerung zu Regelung, indem davon ausgegangen wird, dass „bekannte Formen, von denen man gemeinhin die Lösung kollektiver Probleme in der modernen Gesellschaft erwartet hat" (also vom Staat bzw. vom Hierarchiemodell in der Politik) „problematisch geworden sind“ (ebd., 9). Daher wird konstatiert, dass von den 'alten', etatistischen (hierarchischen, staatlichen, top-down) Formen der Gesellschaftssteuerung abgerückt wird (und werden kann und sollte), so dass diese gegen 'neue', kooperative Regelungsformen ersetzt werden (können und sollten), bei denen der Staat als Interdependenzmanager, Mediator und Verhandlungspartner in eigener Sache auftritt. Wird so argumentiert, dann wird nicht nur sprichwörtlich aus der Not eine Tugend gemacht und die Tugend dann durch Notwendigkeit gerechtfertigt, indem die kooperativen Regelungsformen nicht nur als die einzig verbleibenden sondern auch als die bestmöglichen Alternativen zur staatlichen Steuerung verstanden werden, womit bereits das vorausgesetzt wird, was eigentlich erst erwiesen werden muss (Benz et al. 2007:9). Durch einen solchen Modus Vivendi fehlt aber die theoretische Grundlage dafür, um substanziell darüber urteilen zu können, vor welchen Herausforderungen und Schwierigkeiten Governancearrangements im Allgemeinen und die kooperative, dialogische und verhandlungsbasierte Ausgestaltung der Biodiversitätspolitik, die als Good Biodiversity Governance bezeichnet worden ist, im Speziellen steht. Daher muss im Folgenden der Frage nachgegangen werden, wie der normativ relevante Wandel von staatlicher Steuerung zu kooperativer Regelung auf der theoretischen Ebene vollzogen werden kann.

\subsubsection{Der Wandel von staatlicher Steuerung hin zu kooperativer Regelung: Von der Steuerungstheorie zur Governancetheorie}

Wenn es darum geht, den normativ so relevanten Wandel von staatlicher Steuerung hin zu kooperativer Regelung theoretisch zu untermauern, dann ist es sinnvoll sich den Übergang von der Steuerungstheorie hin zur Governancetheorie anzuschauen. ${ }^{180} \mathrm{Hier}$ bei besteht die zentrale Herausforderung darin, die problematisch gewordenen Grundannahmen der Steuerungstheorie gegen neue, speziell governancetheoretische Annahmen zu ersetzen. Dies betrifft in erster Linie die Abgrenzung vom Paradigma der staatlichen Steuerung, das seinerseits im Kontext der Abgrenzung von den Planungstheorien der 1960er und frühen 1970er Jahre gesehen werden muss:

${ }^{180}$ Um die Verbindungen aber auch die Abgrenzungen von Steuerungs- und Governancetheorie sowie politischer Steuerung und kooperativer Regelung herauszuarbeiten, kann auf eine ganze Reihe an Arbeiten und Schriften von Renate Mayntz zurückgegriffen werden, die zusammen mit Fritz Scharpf am Berliner Max-Planck Institut für Gesellschaftsforschung eine der zentralen Akteurlnnen war, die das steuerungstheoretische Paradigma entwickelt und (selbst-)kritisch zu Governance weiterentwickelt haben (vgl. insb. Mayntz \& Scharpf 1975, 2005; Mayntz 2009c, 2010). 
Während die Planungstheorien sowie die Untersuchungen zur Planungsorganisation noch in einem „Klima politischen Reformwillens" und einem weitgehenden „Planungsoptimismus" stattfanden und explizit der Politikberatung dienten, wurde der noch in den 1960er Jahren gebräuchliche Terminus der Planung in den 1970er Jahren verstärkt durch den Begriff der Steuerung ersetzt, als die Planungssemantik zusammen mit der Planungseuphorie verschwand (Mayntz 2001:18). Damit geht einerseits ein Wechsel von den präskriptiv ausgerichteten Planungstheorien hin zu einer sich als analytisch verstehenden, empirisch ausgerichteten Steuerungstheorie einher (Mayntz 2008:44), der es um die Beurteilung der Erfolgs- und Misserfolgsbedingungen für die Implementationsprozesse der politischen Entscheidungen ging (Mayntz 2009b:15). Das Paradigma der Steuerungstheorie ist das einer aktiven Politik:

„)Aktive Politik« meint eine Politik, die kurzfristig und elastisch reagieren, langfristig planen und gestalten, beherzt verteilen und umverteilen kann und sich durch all dies von einer bloß reagierenden Politik, einer "reaktiven Politik», vorteilhaft abhebt.“ (Schmidt 2003:155)

Damit wurde im Kontext der Steuerungstheorie davon ausgegangen, dass „eine im Sinne ihrer Ziele erfolgreiche Einwirkung der Politik auf gesellschaftliche Strukturen und Prozesse schwierig aber (gegen die systemtheoretisch ausgerichteten soziologischen Steuerungsverständnisse) ${ }^{181}$ nicht grundsätzlich ausgeschlossen und unter bestimmten Bedingungen sogar erfolgreich sei." (Mayntz \& Scharpf 2005:236) Andererseits wird bereits im Rahmen der Steuerungstheorie im Zuge ihrer Auseinandersetzung mit den Planungstheorien die Kritik am bürokratischen Staatsverständnis sowie an der Hierarchie als alleinigem Koordinationsmechanismus des Politischen laut, wenn auch nicht mit der Folge der dekompensatorisch anmutenden Betonung der Wichtigkeit aller nicht-hierarchischen Regelungsinstrumente, wie dies zuweilen in der Governancedebatte geschieht. Dabei lässt sich in Anlehnung an Mancur Olsons Unterscheidung der Interaktionsformen zwischen dem Staat und den organisierten Interessen der Gesellschaft, die auch als Steuerungsmuster interpretiert werden können, zwischen etatis-

${ }^{181}$ Bezüglich der Steuerungssemantik gibt es einen beachtenswerten Unterschied zwischen soziologischen und politikwissenschaftlichen Ansätzen: In der politikwissenschaftlichen Policyforschung standen Akteure und ihr Handeln im Vordergrund, wodurch sich ein akteurszentriertes Steuerungsverständnis herausgebildet hat, bei dem Steuern als ein zielgerichtetes Handeln verstanden wurde, das von der Wirkung der Handlung analytisch zu unterscheiden ist (Mayntz 2009c:42), weil davon ausgegangen wurde, dass das (staatliche) Steuerungshandeln von (staatlichen) Steuerungssubjekten auf (gesellschaftliche) Steuerungsobjekte sich gerade nicht am Steuerungserfolg (Outcome) sondern an den Steuerungswirkungen (Output) bemisst, weil die Steuerungsfähigkeit der Akteure und die Steuerbarkeit der Adressaten zwei unterscheidbare Aspekte sind (ebd.) In der Soziologie wird der Begriff Steuerung hingegen systemtheoretisch ausgedeutet. Dieses Verständnis fand im Gefolge der Luhmannchen Systemtheorie auch in die Politikwissenschaft Eingang. Dies führte dazu, dass sich die politikwissenschaftliche Steuerungstheorie einerseits dagegen abgegrenzt, andererseits aber auch weiterentwickelt hat, vor allem was die Sicht auf die Eigenständigkeit der Steuerungsobjekte und die Grenzen der Steuerbarkeit der Gesellschaft angeht (vgl. Mayntz \& Scharpf 2005). 
tisch-hoheitlicher Steuerung, heterarchisch-kooperativer Regelung, freiwilliger Selbststeuerung und inkrementaler Verselbstständigung ${ }^{182}$ unterscheiden (vgl. Olson 1968):

Die etatistisch-hierarchische Steuerung zeichnet sich dadurch aus, dass die gesellschaftliche Ebene der Interessensvermittlung pluralisiert aber die staatlich-administrative Problemlösungsebene zentralisiert ist (Voigt 1995:39). Der Steuerungsmodus der Hierarchie basiert dabei auf einem Über- und Unterordnungsverhältnis und im Vordergrund steht der Anspruch des Staates, von ihm autonom gesetzten Steuerungsimperative durchzusetzen. Dazu bedarf es eines 'starken Staats' an der Spitze der Hierarchie, der seine Legitimation alleinig aus der Legalität, sprich der verfassungsmäßigen Ordnung, gewinnt und diese gerade nicht aus den Verhandlungen mit den gesellschaftlichen Interessensgruppen oder aus der Zufriedenheit der BürgerInnen mit den Ergebnissen des staatlichen Handelns zieht.

Davon unterscheidet sich die heterarchisch-kooperative Regelung, da hier organisierte Interessensvermittlung und politisch-administrative Problemverarbeitung gleichzeitig integrativ sind (ebd.). Der Steuerungsmodus der Kooperation basiert darauf, dass Verfahrensregeln für den Konfliktaustragungsprozess zwischen unterschiedlichen Interessen bestimmt wird und nicht im Vorhinein festgelegt wird, wie das Ergebnis der Verhandlungsprozesse inhaltlich auszusehen hat. Legitimiert werden kooperative Steuerungsversuche über die diffuse Zufriedenheit und Akzeptanz, d.h. die "Massenloyalität“ (vgl. Offe 1975), der BürgerInnen, die sich wiederum entweder darüber ergeben kann, dass sie als Beteiligte und Betroffene in den Prozess der Interessensvermittlung und Entscheidungsfindung eingebunden sind (Inputlegitimierung) oder aber indem sie mit den staatlichen Leistungen (bspw. bürokratisch-administrative Strukturen, soziale Leistungen, Krisenbewältigung etc.) zufrieden sind (Outputlegitimierung).

Freiwillige Selbststeuerung zeichnet sich dadurch aus, dass die Interessensvermittlung integrativ, die politisch-administrativen Problemlösungskapazitäten aber fragmentiert sind (Voigt 1995:39). In diesem Fall werden Problemlösungen pluralistisch ausgehandelt, in der Folge jedoch häufig privatisiert, so dass hier nicht im eigentlichen Sinne von politischer Steuerung die Rede sein kann. Wenn staatlich-politische Akteure ihre Handlungsintentionen indes darüber zu realisieren oder durchzusetzen versuchen, indem sie pluralistische Verhandlungen initiieren, begleiten oder deren Ergebnisse absichern,

182 Inkrementale Verselbstständigung zeichnet sich als Steuerungsmodus dadurch aus, dass sowohl die gesellschaftliche Interessensvermittlung, als auch die politisch-administrativen Problemlösungsmechanismen fragmentiert sind und wenn überhaupt eher aus der spontanen Koordination resultieren, so dass es hierbei um die Zufälligkeit sowie die Verselbstständigung von Entwicklungen geht (Voigt 1995:39). Sowohl die etatistische als auch die kooperative Steuerung können unter ungünstigen Umständen in inkrementale Verselbständigung umschlagen. Kennzeichnend hierfür ist dann eine Politik der kleinen Schritte, der Minimalkonsense und des 'Durchwurschtelns'. Gesellschaftliche Krisen und große gesellschaftliche Problemlagen sind dann nicht mehr lösbar (ebd., 40). 
dann ist die Rede von „Verhandlungen im Schatten der Hierarchie“ (Scharpf 1993a:71), die dann aber dem Typus der kooperativen Steuerung zuzurechnen wären (ebd., 40).

Mit Blick auf den Umstand, dass dem mit der hierarchischen Steuerung verbundenen klassischen Staatsverständnis zunehmend der Boden entzogen wird, dass die hierarchische Steuerung zudem vielfältige Politikblockaden produziert, dass eine zunehmende Vernetzung zwischen den politischen Ebenen zu beobachten ist sowie eingedenk der gestiegenen Notwendigkeiten der Inkorporation wirtschaftlicher und zivilgesellschaftlicher Akteure und wissenschaftlicher Experten an der Politikformulierung und -durchsetzung, rückt der Steuerungsmodus der Kooperation zunehmend in den Mittelpunkt des steuerungstheoretischen Interesses. Am Ende der Modifikationen und Erweiterungen des steuerungstheoretischen Paradigmas stand daher konsequenterweise das Modell des „kooperativen Staates“ (vgl. Ritter 1979), in dem die kontrollierte Enthierarchisierung des Regierens als eine „angemessene Reaktion auf die sozialen Differenzierungsprozesse“ (Mayntz \& Scharpf 2005:236) betrachtet wurde. Im Zuge dessen wurde auch der bis dato eingegrenzte Fokus auf den Staat bzw. das politisch-administrative System partiell erweitert (Mayntz 2009b:17), indem die Mitwirkung gesellschaftlicher Akteure an der Entwicklung und Implementation staatlicher Politik in multiplen Verhandlungsnetzwerken, die Differenzierung des politischen Systems in Mehrebenensysteme sowie die gesellschaftlichen Selbstregulierungspotenziale mit in die Analyse einbezogen wurden (Mayntz 2009c:43).

Spätestens hier sind die Parallelen zu der sich in dieser Zeit ebenfalls entwickelnden Governanceforschung offensichtlich, allerdings mit dem Unterschied, dass im Rahmen der Steuerungstheorie - anders als im Rahmen der Governanceforschung, wo es stellenweise zu einer dekompensatorisch anmutenden Überbetonung kooperativer und selbstregulativer Steuerungsmodi kommt - immer wieder darauf verwiesen wurde, dass es sich bei den Steuerungsmodi der Kooperation und der Hierarchie um „zwei eigenständige Koordinationsmechanismen von prinzipiell gleicher normativer Dignität [handelt]." (Scharpf 1992:100) Aufgrund dieser Paralle bei gleichzeitiger Binnendifferenz stellt sich die Frage, wie sich Steuerungs- und Governancetheorie und mithin die Leitbegriffe Steuerung, Governance, Regierung und Regelung zueinander verhalten: Annäherungsweise kann diesbezüglich erst einmal auf die ambivalente Nutzung der Begrifflichkeiten verwiesen werden. So werden über die Leitbegriffe einerseits die gleichen Thematiken adressiert, weil alle drei Begrifflichkeiten dazu genutzt werden, um komplexe und interdependente, über und unter dem Staat befindliche kooperative Koordinationsformen und -mechanismen sowie deren Wirkungen zu erfassen, zu analysieren und zu beurteilen (Mayntz 2009c:41). Andererseits zeigt sich aber auch immer wieder, dass bewusst zwischen den Begriffen unterschieden wird. Ein Indiz dafür ist, dass Governance nicht einfach zu Steuerung eingedeutscht wird, sondern ent- 
weder weiterhin von Governance oder aber von Regelung gesprochen wird (Mayntz \& Scharpf 1995a:16). Vor allem bei Global Governance ist dies der Fall, da Governance hier deutlich macht, dass sich die auf das eigene Territorium bezogene Handlungsfähigkeit der Nationalstaaten durch die ökonomischen, politischen sowie kulturellen Wandlungsprozesse in Folge der Globalisierung verändert wird. Daher wird vor allem durch Global Governance in Frage gestellt, ob man oberhalb des Nationalstaats sinnvollerweise überhaupt noch von Regierung oder politischer Steuerung reden kann. Vor allem hier hat das Governancekonzept gegenüber dem Konzept der politischen Steuerung daher auch einen begrifflichen Vorteil, weil das Governancekonzept die Veränderungsprozesse des Politischen oberhalb der Nationalstaaten bereits begrifflich erfasst. Dadurch dass Governance folglich nicht mehr auf den Nationalstaat fokussiert, sondern ein differenziertes Geflecht miteinander verbundener Akteure meint, unterscheidet sich die Governance- von der Steuerungsperspektive vor allem hinsichtlich ihres Staatsverständnisses und damit in ihrem Fokus und ihrer Reichweite:

„Die Steuerungstheorie ist von ihrer Genese her an eine kontinentaleuropäische Staatsvorstellung geknüpft. Bei ihr steht das handelnde Steuerungssubjekt im Vordergrund, bei der Governance-Theorie dagegen die Regelungsstruktur (...). Die Steuerungstheorie hat nie die Nabelschnur zu "Politik» in einem relativ engen Sinn des Begriffs gekappt, sie lässt sich nicht von dem lösen, was von Anfang an mitgedacht wurde: Gesellschaftsgestaltung durch dazu legitimierte politische Instanzen. (...) Der den Perspektivenwechsel anzeigende, semantische Umschlagpunkt liegt dort, wo die Politikwissenschaft beginnt, sich intensiv mit den politisch zunehmend bedeutsamen Vorgängen auf der europäischen und der internationalen Ebene zu beschäftigen, jenen Ebenen also, auf denen ein "Steuerungssubjekt« nur noch in generalisierter und zugleich fragmentierter Form erscheint. Der Begriffswechsel von Steuerung zu Governance verweist damit auch auf Veränderungen in der politisch relevanten Wirklichkeit, die eben diese neue Sichtweise »realitätsgerechter« erscheinen lassen." (Mayntz 2009c:47-48)

Trotz des Umstandes, dass die Governanceperspektive mit ihrem Fokus auf die Übergänge von Government zu Governance sowie politischer Steuerung zu Regelung eine den veränderten Umständen angemessene Forschungs- und Analyseperspektive bietet, die sich stärker auf die Strukturen und Institutionen als auf Akteure und Prozesse fokussiert, ist sie keine in Gänze neue Perspektive auf die politischen Koordination. Dies konstatiert Renate Mayntz, indem sie ausführt, dass die Governancetheorie keineswegs einen Paradigmenwechsel anzeigt (ebd., 42). Vielmehr stellt der Perspektivenwechsel auf Governance einerseits eine Weiterentwicklung der Steuerungstheorie und andererseits eine Fokusverschiebung dar (Blumenthal 2005:1175). Dies hat theoretische Verschiebungen (5.3.2.1) aber auch Kontinuitäten zwischen Steuerungs- und Governancetheorie zur Folge (5.3.2.2). 


\subsubsection{Die theoretischen Verschiebungen beim Übergang von Government zu Governance und von Steuerung zu Regelung}

Der entscheidende Bruch zwischen Steuerungs- und Governancetheorie besteht nach Mayntz vor allem darin, dass das Konzept der Governance primär institutionstheoretisch bestimmt wird (Mayntz 2009c:47): Wenn der Fokus auf der Beteiligung gesellschaftlicher Akteure am politischen Prozess liegt, sprich auf der Koordination und der Kooperation zwischen öffentlichen und privaten Akteuren, dann kann nicht mehr von einem durch staatliche Akteure verursachten und verantwortbaren Steuerungshandeln die Rede sein, da die für das Konzept politischer Steuerung konstitutive Unterscheidung von Staat, Steuerungssubjekt und Steuerungshandeln auf der einen Seite und Gesellschaft, Steuerungsadressaten und Steuerungswirkung auf der anderen Seite unscharf wird, sobald die Adressaten der Steuerung selbst zu Steuerungssubjekten werden (Mayntz 2009c:42-43). Daher muss konsequenterweise die Rede von der politischen Steuerung in ihrem handlungstheoretischen Kontexten aufgegeben und gegen eine stärker subjektlose Rede von »Regelung« eingetauscht werden. Rein begrifflich ist dies geschehen, da Governance als "subjektloser Begriff“ (Offe 2008:61) fungiert. Auf der theoretischen Ebene ist der Übergang von der akteurszentrierten Institutionstheorie, die der Steuerungstheorie unterliegt, hin zur rein institutionstheoretischen Analyse komplexer, dynamischer Regelungsstrukturen indes nicht systematisch vollzogen worden, was sich vor allem daran zeigt, dass die für die Governancekonzeption so zentrale Rede von den nicht-hierarchischen, kooperativen Formen der Regelung uneindeutig ist und dies oftmals auch bleibt:

Dies ist der Fall, weil im Kontext der Governancetheorie fraglich ist und oftmals auch bleibt, was genau unter 'kooperativer Regelung' zu verstehen ist. Eben diese Frage stellt sich im Rahmen der Steuerungstheorie nicht, da »kooperativ« hier im handlungstheoretischen Kontext gegen die hierarchischen Formen der staatlichen Steuerung abgegrenzt ist und sich auf Kooperationen zwischen staatlichen und privaten sowie staatlichen und wirtschaftlichen Akteuren sowie innerpolitischen Interdependenzen, d.h. auf staatlich-kooperative Steuerung, bezieht. Die im Governancekontext so zentralen Übergänge von Government zu Governance führen aber dazu, dass Governance und mithin Regelung etwas anderes sein muss als staatliche Steuerung, die im steuerungstheoretischen Kontext sowohl die hierarchische top-down Steuerung als auch die kooperativ-staatliche Steuerung umfasst. Dementsprechend kann Kooperation im Governancekontext sowohl als Gegenbegriff zu staatlich-hierarchischer Steuerung als auch als Gegenbegriff zu staatlich-kooperativer Steuerung angelegt sein. Daraus ergibt sich, dass man es entweder mit einem auf Formen und Mechanismen der gesellschaftlich-freiwilligen Selbstregulierung reduzierten Governanceverständnis zu tun hat, wenn der Begriff der Steuerung als solcher ad acta gelegt wird, womit kooperative Regelung 
sowohl als Gegensatz zu staatlich-kooperativer als auch staatlich-hierarchischer Steuerung verstanden wird (enges Governancekonzept) oder aber mit einem umfassenderen Governancekonzept, welches den Begriff der Steuerung weiterhin in Form der staatlich-kooperativen Steuerung inkludiert, sich aber speziell gegen staatlich-hierarchische Steuerungsformen abgrenzt (weites Governancekonzept). Beide Konzeptionen haben ihre jeweiligen Vor- und Nachteile und ihre ganz eigenen 'Fallstricke', aber letztendlich, so wird im Folgenden aufgezeigt, hat nur das weite Governanceverständnis eine hinreichend komplexe Grundlage, um das Phänomen Governance einzufangen, auch wenn gerade hierbei die Übergänge von Government zu Governance und von Steuerung zu Regelung theoretisch nicht eindeutig eingeholt werden:

In der engen Fassung ist Governance zwar eindeutig verschieden zu staatlicher Steuerung, allerdings bringt die Exklusion aller Formen staatlicher Steuerung aus dem Governancekonzept den entscheidenden Nachteil mit sich, dass die für die Politikwissenschaft eigentlich entscheidende Frage nach dem Zusammenwirken staatlich-hierarchischer, staatlich-kooperativer und selbstregulativer Koordinationsformen und -mechanismen weitestgehend ausgeblendet wird (Schuppert 2008:24), womit einem solchen Governancekonzept kaum noch analytischer Gehalt zukommt, schlicht weil die Realität nach wie vor so aussieht, dass von gesellschaftlicher Selbstregulierung nur ganz partiell die Rede sein kann und weil die freiwillig-selbstregulativen Koordinationsmechanismen zumeist im „Schatten hierarchischer Autorität“ (Scharpf 1993a:71) operieren, um Kooperationen herbeizuführen und Regelungen zu erzielen (Töller 2012:412).

Der Nachteil der weiten Governancekonzeption ist, dass die Differenzierung zwischen kooperativer Regelung und politischer Steuerung unscharf wird, da immer dann, wenn von Governance als Übergriff für alle Formen der kooperativer Regelung die Rede ist auch von staatlich-kooperativer Steuerung die Rede sein muss, so dass die theoretisch relevanten Übergänge von Government zu Governance sowie von Steuerung zu Regelung nicht eingeholt werden. Der Vorteil demgegenüber ist, dass genau diese Aspekte des Zusammenspiels der möglichen Koordinationsformen und -mechanismen untersucht werden können, wenn Governance als Oberbegriff für alle möglichen Regelungsstrukturen und -prozesse verstanden wird, „in denen öffentliche und private, hierarchische und kooperative Formen der Regelung zusammenwirken." (Mayntz 2009c:41) Wie bedeutsam dies ist, wird erst in Gänze offensichtlich, wenn berücksichtigt wird, dass die Governancetheorie in inhaltlicher Sicht nicht mit der Steuerungstheorie bricht und brechen kann.

\subsubsection{Die Kontinuitäten zwischen Steuerungs- und Governancetheorie}

Wenn es um die Herausstellung der Kontinuitäten zwischen Governance- und Steuerungstheorie geht, dann besteht die wichtigste Parallele darin, dass die Governance- 
theorie, genau wie die Steuerungstheorie, annimmt, dass es das primäre Ziel bzw. die Funktion der Politik sei, „zur Lösung gesellschaftlicher und globaler Probleme zu gelangen." (Mayntz 2001:19) Insofern trifft der für die Steuerungstheorie von Mayntz selbstkritisch konstatierte „Problemlösungsbias" (ebd., 18) auch für die Governancetheorie zu, allerdings unter geänderten Vorzeichen und ohne dass dieser im Rahmen der Governancetheorie kritisch reflektiert wird (vgl. Grande 2012:581-582; Haus 2010:459):

Der Problemlösungsbias besteht im Kern darin, dass „Politik nicht als Herrschaft qua Macht über andere, als Nutzung von Macht im eigenen Interesse oder dem Interesse einer Klasse, sondern als Handeln im öffentlichen Interesse verstanden wird", so dass „Steuerung demnach die konzeptionell orientierte Gestaltung der Gesellschaft durch dazu demokratische legitimierte - politische Instanzen“ begriffen wird (Mayntz 2009c: 43). Indem auch in der Governanceforschung davon ausgegangen wird, dass Governancearrangements die Funktion haben, „kollektive Sachverhalte zu regeln, Kollektivgüter bereitzustellen und politische Entscheidungen herbeizuführen“ (Risse 2008:152), wird die theoretische Problematik aufgeworfen, inwiefern die intentionale Sprechweise von Zielen, Orientierungen und Interessen sowohl inhaltlich als auch formal berechtigt sein sollte, wenn im Mittelpunkt der Governanceforschung - anders als im Kontext der Steuerungstheorie - nicht mehr konkrete Akteure, sondern Strukturen und Institutionen stehen. Denn wenn Governancearrangements über die Angabe des Zwecks oder des Ziels von emergenten Formen der Ordnungsbildung abgegrenzt werden, dann muss dargelegt werden, woher dieser Zweck kommt. Die naheliegende und zumeist gegebene Antwort darauf ist, dass die „Intentionalität in den institutionalisierten Regelungsstrukturen selbst abgelagert ist, so dass Governance nicht davon abhängt, ob individuelle Akteure in ihren Motivationen immer und ständig am Gemeinwohl bzw. der Bereitstellung kollektiver Güter orientiert sind." (ebd., 152)

Dies führt indes dazu, dass die Intentionalitätsproblematik lediglich zur Funktionalitätsproblematik modifiziert wird, indem nicht mehr nach der Absicht von steuernden Akteuren, sondern vom Zweck politischer Institutionen und Strukturen die Rede ist. Fragt man aber nach dem Zweck von politischen Institutionen und Strukturen, wird wiederum die Antwort gegeben, dass politische Institutionen dazu da sind, dass diese zur wechselseitigen Erwartungssicherheit beitragen und so die Rationalitätsgrundlagen individuellen Handelns verbessern, um die Wahrscheinlichkeit zu erhöhen, dass politische Akteure in die Lage versetzt werden, kollektive Sachverhalte zu regeln und politische (Kollektiv-)Güter bereitzustellen (Stegmüller 1979:51-55). Damit wiederum wird vorausgesetzt, dass politische Macht kein Selbstzweck ist, sondern ein Mittel zur Funktionserfüllung politischer Systeme darstellt. Dabei gerät indes aus dem Blick, dass PolitikerInnen nicht nur agieren, um kollektive Probleme zu lösen und öffentliche Güter wie Sicherheit, funktionierende Bildungs- und Gesundheitssysteme und andere Dimen- 
sionen sozialer Wohlfahrt bzw. des Gemeinwohls hervorzubringen und für alle BürgerInnen zu gewährleisten (Weichart 2008:230), sondern, dass nicht selten gerade durch Governancearrangements die Tür für die „Anhäufung politischer Macht zum eigenen Nutzen“ geöffnet wird (ebd., 231). Daher muss der durch die institutionalistische Herangehensweise bewirkte und in der Regel als unproblematisch akzeptierte Problemlösungsbias kritisch reflektiert werden:

Wenn Machtaspekte bzw. die Asymmetrien der Abhängigkeits- und Dominanzverhältnisse in der Governanceforschung zu wenig Beachtung finden, indem macht- und ressourcendiverse 'Akteure' wie Staaten, Institutionen, Organisationen und Unternehmen umstandslos in die 'black-box Verhandlungsnetzwerk' zusammengefügt werden, ohne dass dabei deren innewohnende interne Hierarchien, Autoritäts-, Abhängigkeits- und Dominanzverhältnisse sowie die unterschiedlichen Handlungsmotivationen der daran beteiligten Akteure bedacht werden, dann gerät aus dem Blick, dass Governancearrangements, die in der Governanceforschung in erster Linie und zu einseitig als Modernisierungsstrategie der Ordnungspolitik verstanden werden, immer auch als Indikator dafür betrachtet werden müssen, dass die quantitative Zunahme an "Governance« selbst als eine Ursache der zu lösenden Probleme angesehen werden muss, weil die Möglichkeiten zur Beteiligung, Teilhabe und Teilnahme an Governancenetzwerken nicht für alle Akteure gleich sind, denn die verfahrensbestimmenden Akteure und Institutionen haben einen großen Einfluss darauf, wer überhaupt in ein Netzwerk aufgenommen wird (Demirović \& Walk 2011:11,12). So konstatiert vor allem Bob Jessop wiederholt, dass „die Verantwortlichen in Politik und Verwaltung keineswegs nur auf die Forderungen der mit dem Markt- und Staatsversagen unzufriedenen gesellschaftlichen Kräfte reagiert [haben], vielmehr haben sie selbst diese Form der Governance als Ergänzung zu oder als Ersatz für traditionelle Formen des top-down Regierens aktiv vorangetrieben." (Jessop 2011:50) Dies haben sie in der Art und Weise einer „selektiven Strukturkonservativität" betrieben, da der Staat über die Wahl der politischen Verfahren und Prozesse diejenigen Interessen priorisiert und privilegiert (hat), die mit der Reproduktion der bereits vorhandenen Strukturen kompatibel waren (ebd., 65). Daher stellt Jessop heraus, dass sich der Staat das Recht vorbehält, „Governance nicht nur mit Blick auf bestimmte Funktionen sondern auch von Standpunkt parteiischer oder allgemeiner politischer Vorteile aus zu beginnen, sie zu beenden, mit ihr zu jonglieren oder sie zu reartikulieren" (ebd., 66):

„Regierungen geben die Grundregeln für Governance und den regulatorischen Rahmen vor, in dem und durch den Governance-PartnerInnen ihre Ziele verfolgen können, sie sichern die Kompatibilität und Kohärenz verschiedener GovernanceMechanismen und -Regime ab; sie schaffen Dialogforen oder initiieren Dialoge zwischen politischen Interessengruppen; sie entfalten ein relatives Monopol or- 
ganisationaler Intelligenz und Information mit dem Ziel, kognitive Erwartungen zu formen; sie fungieren als ein 'Berufungsgericht' bei Streitfragen, die innerhalb von Governance und über die aufkommen; sie versuchen Machtdifferentiale und strategische Bias in Regimen durch die Stärkung schwächerer Kräfte und Systeme im Interesse der Systemintegration oder der sozialen Kohäsion auszugleichen; sie ergreifen materielle oder symbolisch (sic!) Maßnahmen, um Formen der Koordination zu flankieren und zu unterstützen, die als nützlich, aber instabil angesehen werden, sie subventionieren die Produktion öffentlicher Güter; sie organisieren Neben-Einkommen für diejenigen, die sich für effektive Koordination einsetzen; sie tragen zur Verzahnung von kurz-, mittel- und langfristigen Zeithorizonten und zeitlichen Rhythmen bei, die verschiedene Orte, Ebenen und Akteure übergreifen (...), sie versuchen, das Selbstverständnis der individuellen und kollektiven Akteure von ihren Identitäten, strategischen Kapazitäten und Interessen in verschiedenen strategischen Kontexten zu modifizieren und somit deren Implikationen für die bevorzugten Strategien und Taktiken abzuändern, (...) und sie übernehmen außerdem im Fall von Governance-Versagen in Bereichen jenseits des Staates als letzte Instanz die politische Verantwortung.“ (ebd., 63-64)

Hierdurch wird im Besonderen ersichtlich, dass Governance mitnichten die Abwesenheit von Government und auch nicht die Abwesenheit von staatlicher Steuerung bedeuten kann, sondern dass gerade Regierungen und mithin ihre Ziele, Absichten und Maßnahmen eine entscheidende Rolle sowohl für das Gelingen als auch für das Scheitern von Governancearrangements spielen. Eben dies macht Detlef Sack deutlich:

„Die aus der Parteienforschung bekannte Aussage 'Verfahrensfragen sind Machtfragen ' bringt es auf den Punkt! Wettbewerbliche, kooperative, gemeinschaftliche und hierarchische Governance-Modi weisen nicht alleine bestimmte Dysfunktionalitäten, also 'governance failure` auf. (...) Diese haben ihre Ursache oftmals in der strategischen Selektivität der Verfahren. (...) Die spezifische Selektivität eines Verfahrens ergibt sich über den Ein- und Ausschluss bestimmter AkteurInnen, über den damit einhergehenden Ressourcenaufwand, die Spezifität der einzusetzenden Mittel und nicht zuletzt über die milieuspezifische Befähigung zur Akzeptanz von Interaktionen. D.h. auch, dass aufgrund des ermöglichenden oder einschränkenden Charakters von Governance-Modi narrative und kalkulierende Strategien ebenso beeinflusst werden, wie Politikergebnisse. (...) Zudem [zeigt sich], dass Governance-Modi, gerade weil sie selektiv sind, ihrerseits zum Gegenstand politischer Konflikte werden." (Sack 2011:35)

Mit anderen Worten: Governance kann konzeptionell und inhaltlich nicht auf ein enges Governanceverständnis reduziert werden, auch wenn hierbei die theoretischen Übergänge von Government zu Governance und von Steuerung zu Regelung exakter und systematisch vollzogen werden. Denn wenn es speziell darum gehen soll, die auf Ko- 
operationen, Verhandlungen und Dialog basierende Good Governance, die eine weitere an sich nicht zwangsläufige Ausdehnung des politischen Prozesses in den nur vermeintlich unpolitischen (zivil-)gesellschaftlichen Raum hinein bewirkt, zu explizieren, dann geht es immer auch darum, welche Konflikte und welche möglichen gesellschaftlichen Krisenprozesse diese hervorgebracht haben und wiederum hervorbringen. Zudem können Governancearrangements auch nicht darauf reduziert werden, wie und welche politischen Probleme gelöst werden, sprich, wie effektiv und effizient Governancearrangements sind, da sowohl die Strukturen als auch die Prozesse von und innerhalb der Governancenetzwerke Auswirkungen auf das Kräftegleichgewicht haben, das wiederum die Ergebnisse netzwerkbasierter Koordination beeinflussen. Erst wenn berücksichtigt wird, dass das Ziel von Governance, nämlich soziale Handlungskoordination innerhalb politischer Mehrebenensysteme, nicht frei von jeglichen Einwirkungen in die institutionalisierten Regelungsstrukturen 'eingelagert' ist, sondern durch Akteure aktiv beeinflusst werden kann, wird ein Reflexionsschritt zurück und zugleich ein theoretischer Schritt nach vorn getan, indem „die intentionale Gestaltung von institutionellem Wandel nicht auf die kausale Wirkungsweise von Institutionen reduziert wird." (Haus 2010:461) Vielmehr werden Institutionen dann selbst als komplexe Regelsysteme verstanden, die das Ergebnis bestimmter Problemdefinitionen und Lösungsmöglichkeiten sind, die erst durch kollektive und oftmals umkämpfte Prozesse hervorgebracht werden (ebd., 458). Erst wenn die Aspekte des selektiven Einsatzes von Wissen, Macht, Zwang, Hegemonie und Rationalität explizit berücksichtigt werden und davon ausgegangen wird, dass diese Selektivitäten in das Governancekonzept, sprich, in die Konstellation der daran beteiligten Akteure sowie deren Interaktionsmodi selbst eingeschrieben sind, ist die Gefahr gebannt, dass der Problemlösungsbias 'blinde Flecken` hinsichtlich der Macht- und Dominanzlogiken in der politikwissenschaftlichen Governanceforschung produziert.

Erst wenn folglich die Akteure ihre Handlungsorientierungen, ihre Interaktionsformen und -konstellationen mit in die Analyse einbezogen werden, wird ersichtlich, dass Governance mehr ist als ein institutionelles Design. In diesem Sinne ist die Angrenzungsproblematik aber auf der inhaltlichen Ebene nicht auflösbar wohl aber auf der analytischen Eben. Beides - die analytische Lösung der Differenzierungsproblematik als auch die Beachtung der Randbedingungen und 'Fallstricke' der Funktionalitätsproblematik kann über die theoretische Fundierung der Governanceforschung durch den policytheoretischen Ansatz des "akteurszentrierten Institutionalismus" (vgl. Mayntz \& Scharpf 1995b; Scharpf 2006) realisiert werden, da dieser institutionen- und akteurszentrierte Ansätze zur Explizierung von Governance miteinander kombiniert und zugleich analytisch auseinanderhält. 


\subsubsection{Der akteurszentrierte Institutionalismus als theoretische Hintergrundfolie zur Explizierung von Governance}

Der akteurszentrierte Institutionalismus ist ein von Renate Mayntz und Fritz Scharpf entwickelter Ansatz, der nicht als inhaltliche Theorie sondern als mehrdimensionale Forschungsheuristik verstanden werden muss. Mit anderen Worten: Über den akteurszentrierten Institutionalismus lässt sich herausstellen, welche Aspekte bei Governancearrangements in analytischer Hinsicht von Belang sind und wie diese in Beziehung zueinander stehen. Dabei wird zwischen systemischen, institutionenzentrierten und strukturellen Aspekten einerseits und handlungs-, akteurszentrierten und prozeduralen Aspekten anderseits unterschieden, die aber dennoch in wechselseitigen Verhältnissen zueinander stehen:

Auf der systemischen bzw. strukturellen Ebene stehen im akteurszentrierten Institutionalismus die institutionellen Kontextbedingungen im Fokus, wobei Institutionen innerhalb dieses Ansatzes im Sinne von sich wandelbaren, kontextgebundenen Regelungssystemen verstanden werden, die einer Gruppe von Akteuren offenstehende Handlungsverläufe strukturieren, ohne sie zu determinieren. ${ }^{183}$ Solche Reg-lungssysteme organisieren und ermöglichen die Arenen der sozialen Handlungskoordination, teilen Akteuren Kompetenzen und Ressourcen zu. Über sie wird der Zugang zu politischen Entscheidungsarenen reguliert und sie beeinflussen die Handlungsorientierungen von Akteuren. Institutionelle Faktoren bilden damit einen stimulierenden, ermöglichenden, restringierenden aber eben nicht determinierenden Handlungskontext und fungieren sowohl als abhängige und unabhängige Variable für die Konstellation und das Handeln der Akteure (vgl. Mayntz \& Scharpf 1995b:45-48). Hierüber können folglich auch die institionellen Komponenten von Governance expliziert werden.

Die andere handlungs- und prozessorientierte Ebene bilden die Akteure mitsamt ihren vielfältigen durch den Regelungsrahmen beeinflussten individuellen Handlungsorientierungen und strategischen Interaktionsorientierungen, den ebenfalls durch den Regelungsrahmen beeinflussten Akteurskonstellationen, wenn sie in Interaktion miteinander treten, sowie den verschiedenen vom Organisationsgrad der Entscheidungsare-

${ }^{183}$ Der Begriff der Institution ist vielgestaltig: Oftmals werden die Begriffe Institution, Organisation und kollektiver Akteur synonym verwandt (Scharpf 2006:77). Scharpf und Mayntz trennen diese begrifflich voneinander: Akteure sind im Unterschied zu Institutionen und Organisationen handlungsfähig, obgleich institutionalisierte Regelsysteme Akteure konstituieren und in wichtigen Merkmalen prägen. Sowohl Institutionen als auch Organisationen sind abstrakte, normierende, konstituierende Regelungssysteme, obgleich Organisationen nicht immer Institutionen sein müssen, da sie je nach Organisationsgrad auch lediglich strukturierte kollektive Akteure sein können, denen die 'Qualitäten' von Institutionen fehlen. Mit anderen Worten: Der Begriff der Institution verbleibt auf der strukturellen Ebene, während kollektive Akteure auf der prozeduralen Seite des Ansatzes verortet werden. Organisationen werden je nach Organisationsniveau entweder als kollektive Akteure oder als Institutionen verstanden. 
nen beeinflussten Interaktionsformen, die die strategische Interaktion zwischen den Akteuren kennzeichnen (vgl. ebd., 49-65). Hierüber können folglich die Prozesskomponenten von Governance expliziert werden.

Dieses interdependente und intervenierende Geflecht aus struktrellen und prozeduralen Aspekten lässt sich aufgeschlüsselt nach den einzelnen Komponenten wie folgt darstellen: Im Rahmen einer Mehrebenenpolitik (Multi-Level-Governance) richten sich die Governanceaerrangements auf die Lösung von Koordinations-, Kollektivgut- und Redistributionsproblemen (Scharpf 2006: 125-126). All diese Problematiken verlangen nach einem Ansatz der strategischen Interaktion und die möglichen Lösungen dieser Probleme haben sowohl strukturelle und prozedurale Aspekte. Die Trennung zwischen strukturellen und prozeduralen Aspekten hinsichtlich der strategischen Interaktion wird durch die analytische Differenzierung zwischen den durch den Regelungsrahmen beeinflussten Akteurskonstellationen auf der einen Seite (vgl. ebd., 128-148) und dem durch den Organisationsgrad beeinflussten Interaktionsmodus der Akteure untereinander auf der anderen Seite (vgl. ebd., 90-94 sowie Kap. 5, 6, 7 und 8) entsprochen:

Die durch institutionalisierte Regelungssysteme beeinflusste Akteurskonstellation repräsentiert die strukturellen Aspekte bei der Betrachtung sozialer Handlungskoordination. Diese Modellierung geschieht im »akteurszentrierten Institutionalismus» spieltheoretisch, ${ }^{184}$ indem die kognitiven und motivationalen Handlungsorientierungen der Akteure (übersetzt in 'Strategien'), ihre Wahrnehmungen und Bewertungen der erreichbaren Ergebnisse in einer gegebenen Situation (übersetzt in 'Auszahlungen') und das Ausmaß der Kompatibilität bzw. Inkompatibilität untereinander (übersetzt in 'Aus-

${ }^{184}$ Die Spieltheorie ist ein theoretisches System von abstrakten mathematischen Modellen (Spiele) für interaktive Entscheidungssituationen, in denen es um interpersonelle Konfliktaustragung (kompetitive Spiele), Kooperationen (kooperative Spiele) oder auch beides zugleich (gemischte Spiele) gehen kann. Sie ist im Grunde genommen eine mathematisch-formalisierte Form einer interaktiven oder interdependenten Entscheidungstheorie, die anders als in den Wirtschaftswissenschaften üblich lediglich als Heuristik verstanden werden sollte, die auf speziellen nicht unbedingt allgemeingültigen, Prämissen basiert. Die Spieltheorie ist dabei - und auch dies wird in den Wirtschaftswissenschaften zu wenig beachtet - immer auch eine normative Theorie, die jedem einzelnen Entscheider in einer interaktiven Entscheidungssituation aufzuzeigen versucht, wie er seine eigenen (egoistischen) Interessen in dieser Situation rationaler Weise am besten verfolgen kann. Zudem sind die spieltheoretischen Modellierungen zumeist nur dann aussagekräftig und als Instrumentarium zur schematischen Darstellung und Verallgemeinerung von Interaktionskonstellationen geeignet, wenn nur wenige Aspekte dabei berücksichtigt werden. Umso mehr Variablen darin eingehen, sprich, umso realistischer die Modelle werden (bspw. wenn der Anstieg der Anzahl diverser Akteure, unterschiedlich verteilte Interaktionsressourcen, über die Zeit auftretende Lerneffekte, unterschiedliche Motivationen zur Kooperation oder Nichtkooperation, die über die Gewinnmaximierung hinausgehen etc. berücksichtigt werden), umso differenzierter und damit auch schwieriger und weniger leicht nachvollziehbar werden die Modelle. Aus diesen Gründen, so Scharpf, sollten die Spielmatrixen vor allem als Möglichkeit gesehen werden, die „relativ komplexen Präferenzkonstellationen mit Hilfe relativ einfacher Spielmatrixen zu analysieren“, wobei nicht vergessen werden darf, dass „diese Methode nur als Werkzeug zur Unterstützung und Explikation unseres eigenen Verständnisses [eingesetzt werden kann] und nicht als automatisiertes Verfahren, das mangelnde Verständnis ersetzen könnte.“ (ebd., 148) 
zahlungswünsche') erfasst wird (Scharpf 2006:27). Entscheidend ist, dass die Akteurskonstellation und mit ihr die unterschiedlichen sich hierüber ergebenden archetypischen Spielkonstellationen (wichtig sind hier vor allem symmetrische und assymetrische Konflikt- und Koordinationsspiele sowie 'Mixed Motive Games ' wie bspw. das Gefangenendilemma, das 'Battle of the Sexes', das 'Assurance-Game' oder das 'Chicken Play； vgl. ebd., 129-148) immer nur die Logik der Situation beschreiben, in der sich die Akteure befinden und gerade nicht die tatsächlichen Handlungen, die im politischen Prozess stattfinden. Die Spieltheorie selbst bietet bei der Identifikation der Strategien, der erwartbaren Ergebnisse und bei der Ermittlung des Konfliktniveaus keinerlei Hilfe. Sie ist lediglich eine Heuristik, um die empirische und theoretische Arbeit der kleinteiligen und fallspezifischen Politikfeldanalyse systematisch darzulegen, generalisierend zu vereinfachen und um daran eventuelle Trends und wiederkehrende Muster aufzuzeigen (ebd., 24-31). In sie geht folglich das verfügbare Wissen über die an politischen Interaktionen beteiligten Akteure ein und darüber wird das Konflikt- bzw. Kooperationsniveau zwischen diesen modelliert.

Das bedeutet, dass die Modellierung der Akteurskonstellation bereits Informationen darüber enthält, wie Konflikte verarbeitet werden - nämlich in Form der Strategien. Dafür muss wiederum der strategische Interaktionsmodus zwischen den Akteuren modelliert werden, über den die prozedurale Ebene der sozialen Handlungskoordination in den Blick genommen wird. Mayntz und Scharpf unterscheiden hierbei zwischen unterschiedlichen Interaktionsformen (einseitiges Handeln, wechselseitige Anpassung, Kooperation, Abstimmung, hierarchische Steuerung), die als unterschiedliche 'Schwellen` der Interaktion zu verstehen sind (Mayntz \& Scharpf 1995b:61). Diese verschiedenen 'Schwellen' ergeben sich zum einen über die organisatorischen Kontextbedingungen $^{185}$ (anarchische Felder, minimale Institutionen, Netzwerke, Regimestrukturen und Zwangsverhandlungssysteme), die diese ermöglichen und zugleich begrenzen. Da der Organisationsgrad dazu führt, dass anspruchsvolle Formen der Interaktion einerseits nicht möglich andererseits aber weniger anspruchsvolle Interaktionsformen nicht ausgeschlossen sind, stehen die Interaktionsformen und die strukturellen Kontextbedingungen der Entscheidungsarenen in einem gegenseitigen Bedingungsverhältnis:

185 Anarchische Felder sind durch das Fehlen jeglicher Organisationsstrukturen gekennzeichnet. Solche Felder gibt es nur noch bei wirklichen Innovationen, wo noch nicht einmal Eigentumsrechte geregelt sind. Sind diese und andere basale Strukturen vorhanden, handelt es sich um minimale Institutionen. Treten hier semipermanente Beziehungsmuster hinzu, die auf Ressourcenaustausch und gegenseitige Unterstützung hin angelegt sind, bei denen der Austritt aber jederzeit möglich ist, dann handelt es sich um dydische Netzwerkstrukturen. Wenn ein absichtsvoll geschaffener normativer Bezugsrahmen hinzutritt, der die Kooperationen steuert, dann kann von Regimestrukturen gesprochen werden. Ist der Austritt aus solchen Netzwerken und Regimen nur um den Preis möglich, dass die aufgebauten und etablierten freiwilligen Beziehungen zerbrechen oder ist de jure und/oder de facto kein Austritt möglich, handelt es sich um Zwangsverhandlungssysteme. 
Anarchische Felder ermöglichen lediglich einseitiges Handeln. Minimale Institutionen lassen einseitiges Handeln und wechselseitige Anpassung zu. Netzwerke garantieren die Möglichkeit zu einseitigem Handeln, zur Anpassung aneinander und zur Kooperation miteinander. Regime ermöglichen einseitiges Handeln, Anpassung, Kooperation und zudem majoritäre Abstimmungen. Zwangsverhandlungssysteme können rein theoretisch über alle allen Interaktionsformen verfügen, es bedarf hier aber de facto hierarchischer Steuerung und zugleich negieren sie die Möglichkeit zu einseitigem Handeln (Scharpf 2006:244).

Zum anderen werden die Interaktionsformen, die in verschiedenen Akteurskonstellationen zur Lösung von Problemen möglich sind, von der Art der Akteure (einzeln, aggregiert, kollektiv, korporativ) ${ }^{186}$ bestimmt und von ihren Handlungsorientierungen im Allgemeinen ${ }^{187}$ sowie im Speziellen von den jeweiligen Interaktionsorientierungen derjenigen in Interaktion miteinander treten (Scharpf 2006:90-94). Die unter die motivationalen Handlungsorientierungen fallenden Interaktionsorientierungen sind dabei typisierte Interpretationen der möglichen strategischen Beziehungen zwischen Akteuren (Mayntz \& Scharpf 1995b:57). Hier unterscheiden Mayntz und Scharpf zwischen Egoismus, wo es nur um die Vor- und Nachteile für den je einzelnen Akteur ungeachtet der Vor- und Nachteile für die anderen geht (Scharpf 2006:152). Solidarität, wo es darum geht, dass die Akteure einen gemeinsamen Nutzen aus der Koordination ziehen, selbst wenn sie dies unter Inkaufnahme eigener Verluste tun (ebd.). Wettbewerb, wo es um

186 In Bezug auf die Kategorie der Akteure verläuft das Kontinuum von Individualakteuren über aggregierte Akteure, zu kollektiven Akteuren bis hin zu korporatistischen Akteuren (vgl. Scharpf 2006:95122): Aggregierte Kategorien werden benutzt, um die parallel verlaufenden aber nicht aufeinander abgestimmten Handlungen von mehreren Individuen (bspw. WählerInnen, KonsumentInnen, ArbeitnehmerInnen etc.) zu beschreiben (ebd., 98-100). Kollektive Akteure - nochmals unterteilt in Koalitionen (ebd., 102), Clubs (ebd., 103), soziale Bewegungen (ebd., 103-104) und Verbände (ebd., 104) zeichnen sich dadurch aus, dass „die beteiligten Individuen die Absicht haben, ein gemeinsames Produkt zu schaffen oder ein gemeinsames Ziel zu erreichen." (ebd., 101) Kollektive Akteure zeichnen sich zudem dadurch aus, dass sie von den Präferenzen ihrer Mitglieder abhängig sind und von diesen kontrolliert werden (Bottom-Up-Organisationen). Korporative Akteure grenzen sich davon ab, indem die Strategieentscheidungen von den Präferenzen der Mitglieder abgekoppelt sind (hierarchische Top-Down-Organisationen) und indem sie „über ein hohes Maß an Unabhängigkeit von den letztendlichen Nutznießern ihres Handelns verfügen und [dass] deren Aktivitäten von Arbeitnehmern ausgeführt werden, deren eigene Interessen durch Arbeitsverträge neutralisiert werden." (ebd.)

${ }^{187}$ Zum einen lassen sich kognitive und motivationale Aspekte der Handlungsorientierung unterscheiden (vgl. Mayntz \& Scharpf 1995b:52-58 und Scharpf 2006: 110-122): Kognitive Orientierungen betreffen die Wahrnehmung der Handlungssituation und ihrer kausalen Struktur sowie der verfügbaren Handlungsoptionen und ihrer erwartbaren Ergebnisse. Diesbezüglich gehen Mayntz und Scharpf nicht von allwissenden Akteuren aus, sondern postulieren, dass „Akteure mit unterschiedlichen Aufgaben oder Rollenpflichten und unterschiedlichen Interessen auch unterschiedliche Ausschnitte der Wirklichkeit mit unterschiedlicher Aufmerksamkeit wahrnehmen." (Mayntz \& Scharpf 1995b:53) Die motivationalen Orientierungen (Präferenzen) werden von Mayntz und Scharpf in Form eines Stufenmodells erfasst und nicht lediglich auf egoistische Präferenzen reduziert. Hierzu gehören nicht relative Eigeninteressen (Scharpf 2006:117-118), normative Rollenerwartungen (ebd., 118-119), identitäre Erwartungen (ebd., 119-122) sowie die für strategische Interaktionen besonders wichtigen Interaktionsorientierungen (ebd., 148-165). 
die Maximierung der eigenen Gewinne vor dem Hintergrund der negativen Bewertung der Differenz zwischen eigenem und fremden Gewinn geht (ebd., 153). Altruismus, wo es nicht in erster Linie um den eigenen Gewinn, sondern um die Gewinne der anderen geht, die dann als positives Ergebnis für einen selbst bewertet werden (ebd.) und Feindschaft, wo die eigenen Gewinne irrelevant sind, da die Verluste der anderen als eigener Gewinn bewertet werden (ebd.).

Auf Basis dieser verschiedenen Komponenten auf den einzelnen Analyseebenen des "akteurszentrierten Institutionalismus" lässt sich nun zusammenfassend mit Bezug auf das Governanceonzept folgendes festhalten: Governance kann als Modus der politischstrategischen Handlungskoordination im Sinne der weiten Governancekonzeption verstanden werden. Als solche können Governancearrangements hinsichtlich ihrer intervenierenden Struktur- und Prozesseigenschaften systematisch analysiert werden. Auf der Strukturebene erscheinen die Governanceformen dabei als institutionalisierte Regelungssysteme zur Lösung kollektiver Probleme, die durch die je verschiedenen Konstellationen der Akteure zueinander immer wieder neu konstituiert werden. Auf der Prozessebene erscheinen die Governanceformen als Koordinations- und Interaktionsprozesse komplexer Akteure mit ganz unterschiedlichen Fähigkeiten, Ressourcen und Orientierungen, so dass die Ergebnisse der Koordinations- und Kooperationsbemühungen davon abhängen, welche Interaktionsformen und -orientierungen in diesen Konstellationen jeweils vorherrschen.

\subsubsection{Zwischenfazit}

Wenn Governance sowohl als insitutionalisiertes Regelungssystem als auch als Koordinations- und Interaktionsprozess verstanden wird und dies wiederum die theoretische Grundlage dafür liefert, um das Phänomen der Governance analytisch und theoretisch zu fassen, dann hat dies Rückwirkungen auf das damit verbundene Demokratieverständnis: Dass Politik sowohl innerhalb als auch oberhalb des Nationalstaats in zunehmenden Maße durch machtverteilende Mechanismen und Strukturen wie pluralistische Politiknetzwerke ${ }^{188}$ und (neo-)korporatistische Verhandlungssysteme ${ }^{189}$ gekennzeichnet ist und dass politische Ergebnisse in zunehmenden Maße durch verhandelte Interessenausgleiche auf und zwischen den politischen Ebenen (Mehrebenenpolitik) sowie zwischen politischen und privaten Akteuren (Private-Public Partnerships) durch prozedural-kooperative Regelung zustande kommen, führen zu substanziellen Verän-

${ }^{188}$ Der Begriff der pluralistischen Politiknetzwerke wird hier benutzt, um die Interaktionen zwischen zivilgesellschaftlichen kollektiven Akteuren (siehe FN 186) und politischen Akteuren und Institutionen zu charakterisieren (vgl. Scharpf 2006:101-104).

189 Der Begriff der (neo-)korporatistischen Verhandlungssysteme bezeichnet die Interaktionen zwischen wirtschaftlich orientierten, korporativen Akteuren (siehe FN 186) und politischen Akteuren und Institutionen. 
derungen des demokratischen Erscheinungsbildes. Wenn Hierarchie als klassischer staatlicher Steuerungsmodus in einer vernetzten Welt überall dort an Grenzen stößt, wo gesellschaftlich, wirtschaftlich und politisch mächtige Interessen einer direkten Umsetzung staatlicher Steuerungsintentionen entgegenstehen, so dass deren Zustimmung bzw. die Aufgabe ihres Widerstandes und ggf. sogar ihre aktive Mitwirkung ausgehandelt werden muss, dann muss zugleich berücksichtigt werden, dass die klassischen politischen Koordinationslogiken, wie der demokratische Parteienwettbewerb und das Mehrheitsprinzip zunehmend durch Verhandlungslösungen mit dem Ziel des Interessensausgleiches erweitert werden. Eben dies kennzeichnet verhandlungsdemokratische Settings, ${ }^{190}$ die in der Demokratietheorie indes stärker zur Kenntnis genommen werden müssen als dies bisher der Fall ist, denn solange dies nicht geschieht, kann sich die Demokratietheorie nicht „auf die Realität der vielfach vernetzten und durch Verhandlungen geprägten Politik einstellen." (Scharpf 1992:107)

Daher bedarf es der Erweiterung der governancetheoretischen Grundannahmen um verhandlungsdemokratische Überlegungen. Dabei dürfen weder die strukturellen Kontextbedingungen politischer Abstimmungs- und Entscheidungsprozesse vernachlässigt werden, was in den kontextualistischen Demokratietheorien und partizipativen Governancemodellen oftmals der Fall ist (siehe 5.2.1), noch dürfen Macht und Dominanzaspekte sowie die strategischen Interaktionen zwischen den Akteuren aus der Betrachtung ausgeklammert werden, wie dies in den prozeduralistischen Demokratietheorien und deliberativen Governancemodellen oftmals der Fall ist (siehe 5.2.2). Eben hier haben die in der Analyse bisher gar nicht weiter beachteten entscheidungszentrierten und insbesondere die pluralistischen Demokratietheorien ihr 'starkes Moment', denn diese stellen, wie bereits an anderer Stelle ausgeführt (siehe 5.1.2.2), den Wettbewerb der politischen Intereressen und die Regelung politischer Abstimmungs- und Entscheidungsprozesse in den Mittelpunkt ihrer demokratietheoretischen Betrachtung (Lösch 2005:122). Der entscheidende Nachteil pluralistischer Demokratiekonzeptionen ist indes, dass sie den politischen Prozess „nicht anders zu begreifen [vermögen] als einen Prozess der Aggregation individueller Präferenzen." (ebd., 128). Damit kommen sie zwar zu durchaus adäquaten Beschreibungen der politischen Prozesse, in normativer Hinsicht schaffen es diese Demokratietheorien aber nicht den Kern dessen abzubilden, was Demokratie ausmacht. So konstatiert Fritz Scharpf als ein Vertreter der pluralistischen Demokratietheorie durchaus selbstkritisch:

190 Was genau Verhandlungsdemokratien sind (siehe FN 52), was diese ausmacht und welche governance-, policy-, demokratie- und legitimationstheoretischen Annahmen damit verbunden sind, wird über die Darlegung der "Theorie der komplexen Demokratie» von Fritz Scharpf deutlich gemacht (siehe 5.3.3) 
„Die empirischen Untersuchungen der Gruppen-, Meinungs- und Partizipationsforschung stützen zwar einerseits die Pluralismustheorie in der Hinsicht ab, dass der Einfluss konkurrierender Interessenorganisationen auf Entscheidungsprozesse im Bereich der Gesetzgebung, der Exekutive und der Verwaltung und sogar in der Rechtsprechung bestätigt wurden (...) - ein Befund der bis heute gültig ist und mit dem Begriff Lobbyismus gefasst wird. Andererseits zeigten die empirischen Untersuchungen aber auch, dass das Pluralismusmodell als Beschreibung der Wirklichkeit nicht länger auf der normativen Annahme einer allgemeinen politischen Beteiligung und demokratischen Orientierung der Gruppenmitglieder aufbauen kann, weil die Befunde eher dafür sprechen, dass das pluralistische System mit einer sehr kleinen Minderheit politischer Aktivistinnen auskommen und mit einer Mehrheit von Bürgerinnen operieren kann, die sich nicht beteiligen will und kann (...). Die Quintessens ist daher eine Elitendemokratie, in der eine geringe politische Beteiligung als Indiz für die generelle Zufriedenheit mit dem politischen System gewertet wird, während ihr umgekehrt die Politisierung breiter Schichten als Krisensymptomatik erscheint." (Scharpf 1975:32-33,40)

Habermas äußert in »Faktizität und Geltung" die gleiche Kritik und kommt ebenfalls zu dem Schluss, dass sich der Blick der entscheidungszentrierten Demokratietheorien zu einseitig auf den Output des politischen Prozesses verschiebt (und zwangsläufig verschieben muss), um der normativen Erklärungsnot zu entgehen (Habermas 1992b: 402). Dies lässt „vom normativen Gehalt der Demokratie nicht mehr übrig als die alternierende Machtverteilung auf Regierung und Opposition, womit ein abgemagertes Zerrbild des Sinn und Zwecks demokratischer Ordnung gezeichnet wird." (ebd., 404) Dies ist nicht nur für einen Vertreter einer explizit normativen Demokratietheorie nicht hinnehmbar. Vielmehr zeigen die nun folgenden Ausführungen zur »Theorie der komplexen Demokratie" von Fritz Scharpf, dass es auch einem Vertreter einer pluralistischen und dem Anspruch nach 'realistischen` Demokratietheorie gelingt, die Gräben zwischen einseitiger Input- und Outputfixierung, empirischer und normativer Demokratietheorie, zwischen der einseitigen Orientierung entweder an der Legitimität oder an der Effektivität sowie zwischen governance- und demokratietheoretischen Aspekten über einen 'Mittelweg`zu schließen.

\subsubsection{Die «komplexe Demokratietheorie» als demokratietheoretisches Pendant der Governancedebatte}

Scharpfs komplexe Demokratietheorie ist, so die nun folgende Argumentation, ein geeignetes demokratietheoretisches Pendant für die Governancedebatte, da Scharpf die Veränderungsprozesse des Politischen durch die Internationalisierung der Staaten und die Globalisierung der Wirtschaft auch demokratie- und legitimationstheoretisch untersucht. Dies geschieht auf der Grundlage, dass Scharpf die Gräben zwischen der go- 
vernancetheoretischen Fokussierung auf die Effektivität und die in den normativen Demokratietheorien vorherrschende Fokussierung auf Legitimität zu schließen versucht, indem er sowohl (zivil-)gesellschaftliche Einflussmöglichkeiten auf den politischen Prozess zu dessen Inputlegitimierung als auch die strukturellen und prozeduralen Bedingungen für eine effektive Politikproduktion berücksichtigt. Für Scharpf muss eine sowohl realistische als auch normativ anspruchsvolle Demokratietheorie in der Lage sein, sowohl den Input als auch den Output politischer Ordnung zu erfassen, zu rationalisieren und zu optimieren. Hierbei geht es ihm einerseits darum, die politische Realität möglichst genau wiederzugeben, „ihr Realismus kann aber andererseits auch nicht in der bloßen Anpassung an eine jeweilige Realität liegen." (Scharpf 1975: 93) Daher ist für Scharpf die Realisierbarkeit der durch die Demokratietheorie verfolgten Ziele und Werte nicht per se das wichtigste Kriterium für die Relevanz der Theorie. Zugleich ist Scharpf aber auch der Ansicht, dass normative Demokratietheorien nicht als Utopie konzipiert werden dürfen, da es der „Formulierung von Zielen [bedarf], die unter den gegebenen Umständen verfolgt werden können.“ (ebd.) Daher zielen Scharpfs Überlegungen darauf $a b$ :

„zu einer empirisch informierten, normativen Demokratietheorie zu gelangen, die darauf verzichtet, einen einzigen Zielwert zu maximieren, und sich stattdessen darum bemüht, eine Mehrzahl jener Zielvorstellungen, die in unserer westlichen Demokratietradition als relevant anerkannt werden, in einem komplexen Modell zu akkommodieren. Gewiss kann auch eine komplexe Demokratietheorie kein Puzzlespiel sein, bei dem sich am Ende nur alle scheinbar disparaten Elemente zu einem harmonischen Gesamtbild zusammenfügen. Auch ihr Modell des politischen Systems muss auf widersprüchliche Anforderungen reagieren und kann Zielkonflikte nicht ausschließen.“ (ebd., 92)

Damit nimmt die komplexe Demokratietheorie eine demokratietheoretische Vermittlungsposition ein, da sie sowohl in empirisch-analytischer als auch in normativer Hinsicht die Input- und Outputseite des politischen Prozesses beleuchtet, womit Scharpf wichtige Elemente der entscheidungszentrierten, der kontextbezogenen und der prozeduralistischen Demokratietheorien zugleich aufzunehmen versucht. Der Name des Ansatzes ist folglich 'Programm '. Bemerkenswert ist vor dem Hintergrund dieser Liste an 'Pluspunkten ' indes, dass die komplexe Demokratietheorie selbst in der Politikwissenschaft wenig Beachtung findet und außerhalb der Politikwissenschaft nahezu unbekannt ist. Dies liegt vor allem daran, dass Scharpfs aktuelle demokratietheoretische Überlegungen bisher nicht in einem Standardwerk zusammengefasst wurden, sondern lose und unverbunden in zahlreichen Artikeln verstreut vorliegen. Daher ist die systematische Darlegung der komplexen Demokratietheorie bereits für sich genommen eine lohnenswerte Aufgabenstellung, so dass die folgenden Ausführungen als Rekon- 
struktion und Einbindung eines „bislang noch nicht gebührend gewürdigten Schatz[es]“ (Schmidt 2003:151) betrachtet werden können, „den Scharpf mehr als Neben- denn als Hauptprodukt seiner umfangreichen politikwissenschaftlichen Arbeiten und bisweilen auch eher im Vorbeigehen als in systematischer Absicht entwickelt hat." (ebd., 152)

Die Rekonstruktion der komplexen Demokratietheorie beginnt aufbauend auf Scharpfs demokratietheoretischer 'Grundsteinlegung' "Demokratietheorie zwischen Utopie und Anpassung" (vgl. Scharpf 1975) (5.3.3.1). Hierüber wird das Grundgerüst der komplexen Demokratietheorie herausgearbeitet. Anschließend werden dann die 'Erweiterungen` der komplexen Demokratietheorie erarbeitet, die sich durch Scharpfs Arbeiten zur Globalisierung der Wirtschaft und zur Internationalisierung des Staates ergeben (5.3.3.2). Hierbei wird aufgezeigt, dass es in modernen Verhandlungsdemokratien einerseits besondere Kriterien guter Politik hinsichtlich der Politikproduktion und des politischen Kampfes um die Verteilungsdimension gibt und dass Verhandlungslösungen andererseits besondere Kriterien demokratischer Legitimation genügen müssen. Auf die Kriterien guter Politik unter verhandlungsdemokratischen Bedingungen (5.3.3.2.1) wird eingegangen, indem die Effektivitäts- und Effizienzbedingungen der prototypischen Verhandlungsformen (5.3.3.2.1.1) in ihren jeweiligen strukturellen Kontextbedingungen (5.3.3.2.1.2) dargelegt werden. Auf die Kriterien legitimer Politik (5.3.3.2.2) wird wiederum eingegangen, indem Scharpfs Überlegungen bezüglich der Inputlegitimation und hier genauer der »Herrschaft des Volkes" (5.3.3.2.2.1) und der »Herrschaft durch das Volk« (5.3.3.2.2.2) sowie der Outputlegitimation, d.h. der »Herrschaft für das Volk« (5.3.3.2.2.3), dargelegt werden. Abschließend wird die komplexe Demokratietheorie zusammengefasst und hinsichtlich der theoretischen Grundannahmen hinter der GBG spezifiziert (5.3.3.3). Hiermit wird die Einholung der theoretischen Zielstellung der Arbeit als abgeschlossen betrachtet. Daher kann aufbauend darauf auf-gezeigt werden, welche Anforderungen, Chancen und Risiken sich aus der GBG für die deutsche Biodiversitätspolitik ergeben (5.4). Dies geschieht, indem zum einen die Anforderungen an die deutsche Biodiversitätspolitik expliziert werden, die sich aus den theoretischen Annahmen der GBG ergeben (5.4.1) und indem zum anderen die Defizite und Entwicklungsmöglichkeiten aufgezeigt werden, vor denen die deutsche Biodiversitätspolitik vor dem Hintergrund dieser Anforderungen steht (5.4.2).

\subsubsection{Demokratietheorie zwischen Utopie und Anpassung}

Den 'Startpunkt' zur Scharpfs demokratietheoretischen Überlegungen markiert seine 1972 erstmals im Universitätsverlag veröffentlichte und 1975 dann auch als Taschenbuch erschienene Schrift "Demokratietheorie zwischen Utopie und Anpassung" (vgl. Scharpf 1975), die eine überarbeitete und erweiterte Fassung seiner 1969 gehaltenen Antrittsvorlesung an der Universität Konstanz ist. Hierin legt Scharpf das Grundgerüst 
seiner Demokratietheorie dar. Dabei geht es Scharpf darum deutlich zu machen, dass „Demokratie wie kaum ein anderer Begriff der politischen Theorie zum Signalwort für positive Wertungen in der Sprache der Politik geworden ist" (ebd., 8), dass dieser aber auch wie kein anderer politischer Begriff in seinen Bedeutungen 'schillert und daher mehr der Auseinandersetzung als der Verständigung dient (ebd.). Scharpf vertritt daher die Ansicht und den Anspruch, dass:

„(...) die Demokratietheorie sich der doppelten Herausforderung des Wirklichkeitsbezuges und der normativen Komplexität nicht entziehen kann, wenn sie ihre Relevanz als Bezugsrahmen der empirischen Politikforschung und als Anleitung der politischen Praxis behaupten will.“ (ebd., 93)

Um diesen Anspruch einzulösen, steigt Scharpf mit einer Kritik an normativ abstinenten Demokratietheorien ein, die versuchen eine „wertfreie Demokratietheorie als politische Soziologie der westlichen Demokratien zu entwickeln und sich dabei auf die empirische Untersuchung und Systematisierung ihrer ökonomischen, sozialen und kulturellen Voraussetzungen und ihrer funktionalen Interdependenzen zu beschränken.“ (ebd., 8) Gegen diese demokratietheoretischen Entwürfe wendet Scharpf ein, dass ein nicht willkürlicher und nicht inhaltsleerer Rahmen für die kritische Diskussion über konkurrierende Demokratietheorien nur innerhalb der relevanten politischen Wertungen einer politischen Kultur geschehen kann (ebd., 18). Dies impliziert für Scharpf eine notwendige Komplexität auf der normativen Ebene, woraus er wiederum schlussfolgert, dass der kritische Maßstab einer solchen Diskussion „die normative Komplexität der zu prüfenden Theorie [ist], das heißt ihre Fähigkeit, nicht nur einen einzelnen Zielwert, sondern einen weiteren normativen Kontext zu erfassen und zu berücksichtigen.“ (ebd.) Scharpf geht es folglich darum „die Demokratiediskussion über die wissenschaftlich kaum noch ergiebige Auseinandersetzung zwischen konkurrierenden eindimensionalen Zielmodellen hinauszuführen und die Konturen eines normativ komplexeren Demokratiemodells wenigstens im Ansatz sichtbar zu machen." (ebd., 7) Dies geschieht, indem er sich mit den dualistischen Perspektiven auseinandersetzt, die charakteristisch für die Demokratietheorien sind:

"[D]ie eigentümliche Dichotomie der Perspektiven, unter denen die politische Ordnung beurteilt wird (...) erscheint in wechselnder Terminologie zwischen demokratischer Gleichheit und bürgerlicher Freiheit, zwischen Identität und Repräsentation, zwischen Mehrheitsprinzip und gewaltenteilendem Rechtsstaat oder zwischen universeller Partizipation und Elitenkonkurrenz." (ebd., 21)

Bezüglich dieser Dichotomien stellt Scharpf die These auf, dass sich Demokratietheorien grundlegend danach unterscheiden lassen, ob sie den Input oder den Output politischer Ordnungen zu rationalisieren und zu optimieren versuchen (ebd.). Die outputorientierten Demokratietheorien normieren, so Scharpf, „eine wünschbare Qualität po- 
litischer Leistungen und bestimmen dann von diesem Punkt her die weiteren Anforderungen an die Strukturen des politischen Systems." (ebd.) Hierunter verortet er die liberalen und systemtheoretischen Demokratiekonzeptionen (ebd., 21-23). Demgegenüber üben die inputorientierten Demokratietheorien, „die das politische System ausgehend von den, in den politischen Prozess eingehenden, Willensäußerungen (Präferenzen) zu optimieren versuchen, Kritik an der technologischen Leistungsorientierung und versuchen die politisch-demokratische Rationalität aufzuwerten." (ebd., 25) ${ }^{191}$ Konsequenterweise wenden sich diese 'radikaldemokratischen' Demokratiekonzeptionen, unter die Scharpf die republikanischen und partizipatorischen Demokratiekonzeptionen fallen lässt, der Frage zu, wie das politische System eingerichtet werden müsste, damit politische Entscheidungen aus den Präferenzen der BürgerInnen hervorgehen können (ebd.). Entgegen derjenigen republikanischen Konzeptionen, die Demokratie als Aufhebung des Herrschaftssystems begreifen, indem sie postulieren, dass es einen wahren Volkswillen gebe (volonté générale), der mit dem empirischen Mehrheitswillen (volonté de tous) in Übereinstimmung gebracht werden kann (ebd., 26-27) und zugleich entgegen denjenigen liberalen Konzeptionen, die „die technologische Rationalität fokussieren, diese aber nicht als Reaktion auf das manifeste Anspruchsniveau moderner Gesellschaften verstehen" (ebd., 24), hält Scharpf die Annahmen der pluralistischen Demokratietheorie, da ihr Ausgangspunkt weder das "undifferenzierte Volk“ noch das „abstrakte Individuum“, sondern die durch soziale Interaktionen definierte Gruppen sowie deren Präferenzen und Interessen sind (ebd., 29). Entscheidend dabei ist, dass Scharpf die pluralistische Demokratietheorie zwar auf der Beschreibungsebene nicht aber auf der normativen Ebene für überzeugend erachtet. Vielmehr konstatiert er nach einer Reihe an Überlegungen zum Pluralismus (ebd., 30-53), dass die zentrale Problematik weniger auf der empirischen als auf der normativen Ebene liege:

„Solange sie [die Pluralismutheorie] ihren Anspruch aufrecht erhält, die Wirklichkeit westlicher Demokratien mit demselben Modell zugleich erklären und rechtfertigen zu wollen, kann sie auf empirisch begründete Einwendungen (...) nur mit einer weiteren Verkürzung ihrer normativen Anforderungen in Anpassung an die Wirklichkeit reagieren. (...) Darum kann es wenig überraschen, daß die normativ reduzierte Pluralismustheorie zunehmend auf eine Kritik trifft, die sich in ähnlich einseitiger Weise an der Geltung der dort vernachlässigten demokratischen Werte orientiert." (ebd., 54-55)

Diese an demokratischen Werten orientierte Pluralismuskritik wird zumeist durch „radikaldemokratische oder neomarxistische Prämissen definiert (ebd., 55). Zu deren Prämissen zählt, dass es ein fundamentales menschliches Interesse an Selbstbestimmung

191 Scharpf berücksichtigt hier noch nicht die deliberative Demokratietheorie. Wenn er dies täte, dann würde diese in Scharpfs Systematik unter die inputlegitimierenden Demokratietheorien fallen. 
gibt und dass es möglich sei die Herrschaft von Menschen über Menschen prinzipiell aufzuheben, womit sie die Intentionen und Prämissen der älteren inputorientierten Demokratietheorien wieder aufgreift (ebd.). Insofern erkennt Scharpf es als konsequent an, wenn „Autoren dieser Richtung sich weigern, die Frage nach der Realisierbarkeit der eigenen Ziele als Kriterium für ihre Relevanz und als Maßstab bestehender Verhältnisse anzuerkennen." (ebd.) Allerdings wendet er kritisch ein, dass diese Berechtigung als kritische Theorie die Ansätze nicht gegen die Nachfrage immunisieren kann, „ob und auf welche Weise ein politisches System auf der Maxime universeller Beteiligung aufgebaut werden könnte.“ (ebd., 56) Scharpf konstatiert hier, dass die Beantwortung eben dieser Frage erhebliche Schwierigkeiten aufwirft (ebd.), weil es (zumindest 1970!) an ausgearbeiteten Modellen für eine auf Partizipation gegründete Organisation zentraler Entscheidungsprozesse fehlt, an denen die konkrete Kritik ansetzen könnte (ebd., 57). Insofern beschränkt sich Scharpf hier auf eine kritische Überprüfung der Prämissen, „,von denen jedes Partizipationsmodell - unabhängig von seinen jeweiligen Strukturempfehlungen - notwendigerweise ausgehen muss." (ebd.) Abgesehen von den üblichen und mittlerweile altbekannten Einwänden, dass die „postulierte Partizipation aller Bürger am diskutant aufzuhellenden politischen Entscheidungsprozess (...) nicht nur sehr kleine politische Einheiten [voraussetzt], sondern auch eine äußerste Beschränkung auf jeweils wenige politisch zu entscheidende Fragen [impliziere]“ (ebd., 58), besteht für Scharpf der Knackpunkt aller partizipativen Demokratiemodelle in der Frage nach der Motivation der BürgerInnen, die sich unabhängig von der konkreten Ausgestaltung des jeweiligen Partizipationsmodells stelle:

„Wenn Spezialisierung in der politischen Partizipation unvermeidlich ist, dann bedarf, vom einzelnen her gesehen, auch jedes spezialisierte Engagement einer besonderen, positiven Begründung und konkurriert insoweit auf gleicher politischer Ebene mit einer Vielzahl anderer relevanter Interessen. (...) Gerade wer menschliche Selbstbestimmung als obersten Wert postuliert, könnte den absoluten Vorrang der politischen Partizipation nur dann behaupten, wenn dadurch für den einzelnen und sein 'Streben nach Glück' generell die wichtigsten Bedingungen zu beeinflussen wären." (ebd., 59-60)

Trotz der prinzipiellen Anerkennung der Prämissen der individuellen Selbstbestimmung und der aktiven Gestaltung der konkreten Lebensverhältnisse kritisiert Scharpf die Annahme, politische Partizipation sei die einzige oder sogar beste Möglichkeit zur Selbstbestimmung oder zur aktiven Gestaltung der konkreten Lebensverhältnisse. Er wendet ein, dass es eine Vielzahl anderer, viel wirksamerer Gestaltungsoptionen gibt, wie die berufliche Arbeit, der bewusste Konsum, die Freizeitgestaltung oder das soziale 'Arbeiten `im unmittelbaren Nahbereich, um die individuellen Lebensverhältnisse zu gestalten (ebd., 62). Am Ende seiner Überlegungen steht für Scharpf daher fest, 
„daß vom Standpunkt des einzelnen Bürgers die Motivation zu aktiver politischer Beteiligung keineswegs selbstverständlich und universell vorausgesetzt werden kann. Empirisch erscheint das politische Engagement der wenigen viel eher erklärungsbedürftig als das relative Desinteresse der vielen, das nur selten über die passive Aufnahme politischer Informationen und die Beteiligung an politischen Wahlen hinausgeht." (ebd., 63)

Deshalb ist es für Scharpf zwar lohnenswert, dass es der Partizipationstheorie gelungen ist, Modelle für die demokratische Selbstbestimmung in kleinen Organisationen (Betrieben, Vereinen, Verwaltungen etc.) zu entwerfen (ebd.), was den partizipatorischen Demokratiemodellen der Neuzeit indes (für Scharpf auch aus nachvollziehbaren Gründen) nicht gelungen sei, sind einigermaßen überzeugende Entwürfe für die Organisation zentraler politischer Entscheidungsprozesse auf der Basis universeller Partizipation in modernen, ausdifferenzierten Gesellschaften mit binnenkomplexen politischen Systemen vorzulegen. Daher bliebe den partizipatorischen Demokratietheorien nichts anderes übrig, als auf die möglichst breite Partizipation in möglichst vielen Bereichen zu setzen und auf deren mögliches Übertragungspotenzial zu hoffen (ebd. 64). Scharpf geht es daher vor allem darum, die Relevanz des Partizipationspostulates für die normative Demokratietheorie auf der Input-Seite beizubehalten, ohne das Postulat selber zu einem Strukturprinzip für die Entscheidungsprozesse innerhalb der Politik zu machen (ebd., 66). Für zu entwickelnde Zielstellungen kommt es ihm vielmehr darauf an „seine Anwendungsbereiche differenzierter zu bestimmen“ (ebd.):

„[U]nter Partizipationsaspekten kommt es zunächst darauf an, das Gewicht der Wahlentscheidung im politischen Prozess zu erhöhen und den Menschen die Chancen zu geben, Einfluss auf die Gestaltung ihrer Lebensverhältnisse zu nehmen. Zum zweiten kommt es darauf an, dass diese Chancen prinzipiell allen, die fähig und bereits sind, sich aktiv zu engagieren, in der gleichen Weise zugänglich gemacht werden. (...) Zum dritten kommt es darauf an, dass solche Chancen, soweit wie irgend möglich ausgeschöpft werden." (ebd.)

Gemäß dieser drei Anforderungen ist es für Scharpf die wichtigste Herausforderung moderner Demokratietheorien, wie dem Einzelnen die Chance geboten werden kann die für demokratische Prozesse benötigte Kooperationsbereitschaft und Zusammenarbeit zu erlernen, um an politischen Entscheidungsprozessen mitwirken zu können. Hierbei muss aufgezeigt werden, wie Meinungsverschiedenheiten fair und ergebnisorientiert im Sinne aller oder aber möglichst vieler austragen zu können (ebd., 71). Auf die erste Forderung bezüglich der Aufwertung von Wahlentscheidungen geht Scharpf nur stichpunktartig ein, indem er auch heute noch diskutierte Vorschläge anführt, wie bspw. dass Familien für ihre Kinder zusätzliche Wahlstimmen bekommen und dass über bestimmte besonders relevante politische Regelungen mit langfristigen, gesell- 
schaftlich breiten und konfliktären Konsequenzen Volksabstimmungen durchgeführt werden. Scharpfs Augenmerkt liegt vor allem auf den Voraussetzungen für die zweite und dritte Forderung:

Diese sieht Scharpf in einer „Ausdehnung des Begriffs des Politischen weit über den staatlichen Bereich hinaus auf alle sozialen Beziehungen, in denen Herrschaft ausgeübt wird. ${ }^{192}$ Hierbei soll es, so Scharpf, aber nicht um die „formale Übertragung der Prinzipien der politischen Demokratie auf unpolitische Lebensbereiche" gehen. Vielmehr geht es ihm „um die inhaltliche Veränderung jener sozialen Beziehungen, denen sich der einzelne am wenigsten entziehen kann, um den qualitativen und quantitativen Abbau unnötiger Schranken menschlicher Selbstentfaltung und um die systematische Ausweitung des Raumes individueller und kollektiver Selbstbestimmung." (ebd., 68) Da den Möglichkeiten der mitgliedschaftlichen Selbstbestimmung in modernen, ausdifferenzierten Gesellschaften enge Grenzen gezogen sind, kommt es Scharpf darauf an „den Spielraum der Organisation so weit wie möglich in Freiheitsräume der Organisationsmitglieder umzuwandeln." (ebd., 69) ${ }^{193}$ Auch ist Scharpf daran gelegen für eine bessere Vertretung von „Unterschicht-Interessen“ in den Entscheidungsprozessen der pluralistischen Demokratie zu sorgen (ebd., 71), denn erst wenn in den 'Unterschichten ' die besondere Befähigung und das Interesse für die aktive politische Beteiligung geweckt und ausgebildet wird, kann sich eine gleichmäßigere Partizipationsstruktur ausbilden. Die Voraussetzungen dafür sieht Scharpf in der frühzeitigen Einübung und Vermittlung politischer Handlungs- und Organisationsfähigkeiten in der Schule, am Arbeitsplatz und in Vereinen (ebd., 72), da diese elementar sind, um die Rekrutierungsbasis des pluralistischen Elitensystem zu verbreitern (ebd., 73). Bezogen auf die Inputseite der Demokratie sowie den Zusammenhang von Pluralismustheorie und Partizipationspostulat konstatiert Scharf daraufhin abschließend:

„Verlangt wird also zugleich eine höhere Entscheidungsfähigkeit und ein höheres Wertberücksichtigungspotenzial, als es in einem rein pluralistischen Modell vorausgesetzt werden kann (...). Erst wenn diese Voraussetzungen gegeben sind,

192 Gerade hier ist Scharpf in hohem Maße anschlussfähig an das von Gramsci und Poulantza vertretene erweiterte Staatsverständnis sowie den strategisch-relationalen Ansatz von Bob Jessop, da hier die Zivilgesellschaft in den Raum des Politischen inkludiert und als Sphäre vermachteter sozialer Verhältnisse verstanden wird (siehe 5.3.2.2).

193 Scharpf merkt diesbezüglich an, dass der Abbau hierarchischer Koordination und Disziplinierung nur dann nicht zu ungeregelten Konflikten und unlösbaren Koordinationsproblemen führt, wenn im gleichen Maße nicht-partikulare und in diesem Sinne universalisierbare Ziele und längerfristige Loyalitäten von den Mitgliedern der Organisationen internalisiert werden (ebd., 69-70). Auch ist eine breite Partizipation der Organisationsmitglieder wesentlich eine Frage der Zwecksetzung der Organisation und der Verfügbarkeit materieller und immaterieller Ressourcen, denn für Organisationen, die eine externe Zwecksetzung besitzen oder nur über knappe Ressourcen verfügen und damit nur wenig Spielräume für die Befriedigung der Partizipationsbedürfnisse ihrer Mitglieder haben, besteht die Gefahr einer manipulierten 'Pseudo-Beteiligung', die im Ergebnis repressiver sein kann als die Fortdauer einer explizit hierarchischen Gliederung und einer top-down Steuerung (ebd., 70). 
kann auch das Pluralismusmodell als Grundstruktur einer demokratischen Gesellschaft mit besseren Gründen verteidigt werden. Erst dann brauchen seine unbestreitbaren Vorzüge - die Mäßigung der Machtausübung und die institutionalisierte Suche nach dem breiten Konsens gesellschaftlicher Gruppen im politischen Prozeß - nicht mehr im gleichen Maße mit der Vernachlässigung der Interessen der Unterschicht erkauft werden. Das Partizipationspostulat, das für sich allein genommen keinen praktikablen Entwurf der politischen Systeme tragen könnte, gewinnt hier also eine eminente praktische Bedeutung als normativ unerlässliche Korrektur und Ergänzung der realistischen Pluralismusmodelle.“ (ebd., 75, 73-74)

Diese Voraussetzungen auf der Inputseite reichen Scharpf aber nicht aus, da „[a]uch die breitere Partizipation in Betrieben und Organisationen (...) nichts daran änder[t], dass bestimmte Interessen - und gerade die [unter kapitalistischen Bedingungen] am meisten Benachteiligten (...) - kaum oder nur unter erheblichen Bedingungen wirkungsvoll zu organisieren sind. Hier ist für Scharpf daher der Punkt erreicht, wo er den Blick von der Inputseite auf die Outputseite lenkt, da es hier darum geht die Anforderungen an das politische System ausgehend von den Leistungen zu definieren, die die Politik erfüllen soll und kann. Diesbezüglich hält Scharpf an dem liberalen Postulat fest, dass über den pluralistisch-partizipativen Strukturen eine politisch-staatliche Ebene vorhanden sein muss, „auf der Entscheidungen in relativer Unabhängigkeit von den Pressionen der organisierten Interessengruppen und ihrer Verbündeten in den politischen Institutionen beschlossen und durchgesetzt werden können." (ebd., 75) Hierbei kommt es darauf an eine zentrale politische Entscheidungsinstanz mit einer von den pluralistischen und korporativen Gruppen unabhängigen Legitimationsbasis zu etablieren, so dass Entscheidungen auch gegen den Widerstand einzelner Gruppen durchgesetzt werden können (ebd., 76). ${ }^{194}$

Gleichzeitig bedarf es seitens der professionalisierten politischen Akteure eines Sensoriums für diejenigen Interessen, die politisch oft unartikuliert bleiben, sprich die Interessen von organisations- und ressourcenschwachen Minderheiten (wie bspw. alleinerziehenden Vätern), Großgruppen (bspw. arbeitenden Müttern) sowie Issue-Groups (wie bspw. Umwelt-, Natur- und TierschützerInnen). Nur so können latente Unzufriedenheiten, politisch verdrängte Probleme und Konflikte und politisch nicht perzipierte Möglichkeiten und Chancen der Politik wahrgenommen werden (ebd., 84). Hier sieht Scharpf Möglichkeiten über die Einrichtung „heuristischer Stäbe oder von gemeinsa-

1941970 favorisiert Scharpf diesbezüglich noch ein Modell der strikten Zwei-Parteien-Konkurrenz (vgl. ebd. 76-83). Von dieser einfachen Modellierung weicht Scharpf im Laufe der Zeit, „geprägt durch die Sensibilität für die Möglichkeiten und die engen Grenzen politischer Steuerung, gehärtet durch den wissenschaftlichen Streit mit dem Steuerungsagnostizismus in Luhmanns Systemtheorie (...) und mit geschärftem Blick für die Unterscheidung von Verhandlungs- und Mehrheitsdemokratie" (Schmidt 2003:158) ab und differenziert seine Diagnosen je nach vorliegender Organisations- und Entscheidungsstruktur (vgl. insb. Scharpf 1993b). 
men 'Suchkommissionen' des Parlaments und der Regierung." (ebd.) Dabei gibt er aber zu bedenken, dass die „politische Artikulation von Bedürfnissen und Interessen (...) nur innerhalb eines relativ engen Zeithorizonts voll wirksam werden kann“ (ebd., 85) und dass „die aktive Öffentlichkeit, die sich - ohne unmittelbar in den eigenen Interessen betroffen zu sein - für ein ihnen wichtiges Problem engagiert, oft der einzige Fürsprecher gerade für die am meisten vernachlässigten gesellschaftlichen Bedürfnisse und ungenutzten Möglichkeiten [ist]." (ebd., 88) Daher geht Scharpf von davon aus, dass die aktive politische Öffentlichkeit zwar vereinzelt in der politischen Öffentlichkeit eine Resonanz findet, dass diese aber den Arenen der politischen Entscheidungsfindung zumeist fern bleibt, da hinter ihr zumeist Einzelpersonen und keine Verbände und Organisationen stehen, für die sie stellvertretend agieren (ebd., 88-89). Hier sieht Scharpf Möglichkeiten darin, an einer breiten sozialwissenschaftlichen Ausbildung des Verwaltungsnachwuchses zu arbeiten und den personellen Austausch zwischen Wissenschaft, Wirtschaft und Verwaltung zu erleichtern (ebd., 90-91).

Zusammenfassend sind damit die entscheidenden Charakteristika von Scharpfs komplexer Demokratietheorie dargelegt: Für Scharpf ist der gemeinte Sinn oder das Bezugsproblem von Demokratie die Ermöglichung kollektiver Selbstbestimmung im Sinne einer absichtsvollen Beeinflussung eines gemeinsamen Schicksals (Scharpf 1998b:83), womit er ein normativ-instrumentelles Demokratieverständnis vertritt. Komplex ist sein Demokratieverständnis dabei hinsichtlich des Ziels, eine Integration der Vielzahl anerkennungswürdiger normativ-demokratischer Anliegen zu leisten. Für Scharpf ist es weder ausreichend auf die möglichen Rationalisierungs- und Optimierungspotenziale gesellschaftlicher Partizipation zu setzen, noch hält er es für ausreichend, dass Partizipation lediglich als Aggregation individueller Präferenzen angesehen wird und damit vornehmlich auf das politische System und dessen Regeln und Leistungen geschaut wird. Zur Optimierung der Demokratie gehört für Scharpf daher sowohl ein dosiertes Mehr an politischer Beteiligung als auch eine intelligente politisch-administrative Planung und Steuerung, da Scharpf davon ausgeht, dass sich „die Arbeitsteilung zwischen dem wäh-lenden und mitunter demonstrierenden Souverän und dem Verwaltungsapparat, der für die »Umsetzung" des Volkswillens zuständig [bleibt], (...) allenfalls in der Utopie, nicht aber in der wirklichen Welt aufheben ließe." (Mayntz \& Streeck 2003:11) Für Scharpf folgt daraus der Grundsatz, dass eine möglichst breite Beteiligung nur dann erstrebenswert ist, soweit diese möglich und effektiv ist. Seine langjährige Kollegin Renate Mayntz konstatiert überspitzt, dass für Scharpf Demokratie „nichts wert ist, wenn sie nichts zustande bringt“, demokratische Werte für inn vor allem dann bedeutsam sind, wenn sie einen Weg zu ihrer Verwirklichung finden" und Demokratie dann Unterstützung findet, „wenn der vom demokratischen Souverän geformte und zum Ausdruck gebrachte politische Wille Ergebnisse zeitigt.“ (Mayntz \& Streeck 2003:10-11) 
Gegenüber den in den 1970er Jahren dominierenden Debatten um Partizipation (vgl. bspw. die im selben Jahr publizierte Schrift zur "partizipativen Demokratie« von Carole Pateman (vgl. Pateman 1970) stehen Scharpfs Arbeiten insofern auch in einem krassen Gegensatz (Mayntz \& Streeck 2003:11): Scharfps demokratietheoretischer Ansatz, der demokratische Partizipation weder als Selbstzweck noch als „Weg zur vollen Entfaltung der allseits gebildeten Persönlichkeit“ (Scharpf 1998b:83) versteht, kann als „Ausdruck eines kühlen Realitätsprinzips“ (Mayntz \& Streeck 2003:11) gewertet werden, da Scharpf Demokratie nicht als Eigenwert demokratischer Selbstbestimmung, durchaus aber als Organisationsprinzip zivilgesellschaftlicher Interaktionen oder als Ausdruck der expressiven und/oder persönlichkeitsbildenden Bedeutungen demokratischer Partizipation versteht. Scharpf merkt diesbezüglich an, dass „gegen die kommunitaristische Wiederentdeckung eines zwischen Markt und Staat liegenden Bereichs des freiwillig gemeinsamen Handelns ebenso wenig einzuwenden [ist], wie gegen die Vermutung, dass in der Zivilgesellschaft noch ungenutzte Problemlösungspotenziale mobilisiert werden könnten, welche in bestimmten Bereichen die abnehmende Handlungsfähigkeit des Staates kompensieren könnten." (Scharpf 1998b:83) In diesem Sinne versteht Scharpf Partizipation zwar als wichtiges und für eine Demokratie essentielles, aber zugleich nur begrenzt einsetzbares Mittel zum Zweck, über das Menschen Einflussmöglichkeiten generieren können, die es wiederum wahrscheinlicher machen, dass politische und gesellschaftliche (Organisations-)Strukturen sich auch nach den Bedürfnissen ihrer Mitglieder ausrichten. Für Scharpf ist diese Begrenzung weder theoretisch noch praktisch ein Problem, da es ihm nicht um Partizipation als Selbstzweck, sondern um die hinter dem Partizipationspostulat stehenden normativ-funktionalen Prinzipien der Selbstbestimmung geht.

Auch in seinen späteren Schriften geht es Scharpf daher um die Erweiterung der Beteiligungsmöglichkeiten über den Ist-Zustand hinaus, er macht aber immer wieder deutlich, dass er nicht sieht, was die „positiv eingeschätzten Veränderungen in den Interaktionen zwischen Eltern und Kind, zwischen Managern und Mitarbeitern, in Selbsthilfegruppen und in den transnational vernetzten Projekten (...), [die] als 'radikale Demokratisierung' interpretiert werden, (...) gegen den 'globalen Kapitalismus' ausrichten [sollen].“ (Scharpf 1998b:101) Scharpfs Blick richtet sich vielmehr auf die „normative Funktionsbasis der Demokratie“ (Scharpf 2005:707), die für ihn in einem „gesellschaftlich geteilten Legitimationsglauben politischer Herrschaft" besteht (Scharpf 1998a:84). Eben dieser bewirkt, dass demokratische Entscheidungen freiwillig, d.h. nicht erst unter Kontrolle und Zwangsausübung, von den BürgerInnen akzeptiert werden. Scharpfs normativ-instrumentelles Grundverständnis von Demokratie besteht folglich darin, dass er Demokratie als ein funktionales Prinzip zur Legitimierung staatlicher Herrschaft versteht, wobei er den „Wirklichkeitsbezug der Theorie [betont], (...) ihre 
historisch-empirische Verankerung in der genauen Erkundung von Fortdauer und Wandel der Verfassungswirklichkeit demokratischer Systeme [verlangt] und (...) auf demokratietheoretische und demokratiepraktische Unternehmungen [drängt], die machbar sind und die Nebenfolgen der globalisierten Politik minimieren." (Schmidt 2003:154)

\subsubsection{Die 'Erweiterungen 'der komplexen Demokratietheorie unter den Voraussetzungen der zunehmenden Internationalisierung der Staaten und der Globalisierung der Wirtschaft}

Die frühesten systematischen Rückbezüge zu Scharpfs komplexer Demokratietheorie finden sich erst in den 1990er Jahren. Obgleich Scharpf hier oftmals gar nicht mehr von komplexer Demokratietheorie redet, sondern entlang seiner Auseinandersetzung mit der zunehmenden Internationalisierung des Staates und der Globalisierung der Wirtschaft - manchmal eher implizit, manchmal ganz explizit - demokratie- und legitimationstheoretische Überlegungen entwickelt, können diese an sein früheren Überlegungen zurückgebunden werden. Die Grundlagen für Scharpfs 'Erweiterungen` der komplexen Demokratietheorie finden sich dabei im Wesentlichen in seinen Aufsätzen über die "Handlungsfähigkeit des Staates am Ende des 20. Jahrhunderts" (vgl. Scharpf 1992), den "Versuch über Demokratie im verhandelnden Staat« (vgl. Scharpf 1993b), "Demokratie in der transnationalen Politik» (vgl. Scharpf 1998a), "Demokratische Politik in der internationalisierten Ökonomie « (vgl. Scharpf 1998b), »Regieren in Europa« (vgl. Scharpf 1999b), "Legitimationskonzepte jenseits des Nationalstaats" (vgl. Scharpf 2005) sowie in seinem Buch »Interaktionsformen« (vgl. Scharpf 2006), wo zahlreiche Überlegungen und Aspekte der einzelnen Artikel noch einmal gebündelt und stellenweise auch ausführlicher dargelegt werden.

In all diesen Schriften, die sich mehr oder weniger systematisch auch mit demokratieund legitimationstheoretischen Thematiken befassen, weist Scharpf - unter je anderen Aspekten und mit je anderen Bezügen - auf den Umstand hin, dass die Akteure der nationalen Politik am Ende des 20. Jahrhunderts immer weniger 'selbstbestimmt' und in zunehmendem Maße von den Auswirkungen der Entscheidungen anderer betroffen sind, wobei alle Staaten derartige Externalitäten produzieren, wenn es ihnen um die Regelung ökonomischer, gesellschaftlicher und ökologischer Anliegen geht, die die Grenzen des Nationalstaats überschreiten (Scharpf 1998a:235). In zahlreichen Aufsätzen betont Scharpf daher, dass moderne Politik „verpflochten“ (Scharpf 1992:108) ist und zu einem erheblichen und weiterhin zunehmenden Grad aus einem Geflecht aus innerstaatlichen, transnationalen und internationalen Kooperationen und Verhandlungen hervorgeht (Scharpf 1992:93-94, 1993b:25, 1998a:230, 1998b:81-83, 1999b:13, 2005:705). Dies heißt für Scharpf aber nicht, dass die klassische hierarchische Steuerung sowie die, für die Mehrheitsdemokratien charakteristischen, majoritären Abstim- 
mungsstrukturen keine Rolle mehr spielen. Ganz im Gegenteil:

„Bei allen Varianten des verhandelnden Staates wird ein Großteil effektiver Politik nicht im verfassungsmäßigen Standardmodus hierarchischer und durch majoritäre Verantwortlichkeit legitimierter Staatsgewalt produziert, sondern vielmehr in Verbänden und durch Verhandlungen mit oder zwischen Organisationen, die formal Teil der Selbstorganisation der Zivilgesellschaft und nicht des Entscheidungssystems des Staates sind (...). Sofern diese Verbände die Probleme ihrer Mitglieder effektiv lösen, mag ihre Regelungskompetenz legitimiert sein. Wenn dies jedoch nicht der Fall ist, dann ist die Fähigkeit der Verbände, zur Problemlösungsfähigkeit der Gesellschaft insgesamt beizutragen, vor allem darauf zurückzuführen, dass sie diese Funktionen 'im Schatten des Staates' ausüben: (...) Die Tatsache, dass die Einhaltung der zwischen den wirtschaftlichen und gesellschaftlichen Interessensgruppen getroffenen Verhandlungskompromisse mit Hilfe der staatlichen Gewalt und Verwaltung garantiert werden kann, erzeugt eine Regulierungsfähigkeit, die weit über das hinausgeht, was durch ausschließlich selbstorganisierte private Interessenregierung erreicht werden kann. Allerdings kann nichts von alledem als 'hierarchische Koordination' (...) bezeichnet werden. Die politischen Entscheidungen werden nicht durch die einseitigen Handlungsvollmachten einer hierarchischen Autorität getroffen, sondern sie werden weitgehend durch Verhandlungen bestimmt, wobei der Umstand, dass diese Verhandlungen in eine hierarchische oder majoritäre Struktur eingebettet sind, einen großen Einfluss auf die Tendenz dieser Interaktionen hat und auf die Ergebnisse, die diese produzieren." (Scharpf 2006:334-335)

Auf der theoretischen Ebene bedeutet dies, dass sich die wenigsten politischen Gebilde - und schon gar nicht die oberhalb der Nationalstaaten befindlichen politischen Verhandlungssysteme - vor dem Hintergrund des klassischen Modells eines nach außen souveränen und nach innen hierarchisch gegliederten Staates verstehen lassen, welches auf der klaren Unterscheidbarkeit von Staat und Gesellschaft aufbaut. Dies ist gemeinhin akzeptiert. Dass aber eben diese klassische Staatsvorstellung in vielen Demokratietheorien 'konserviert' ist, ist für Scharpf ein Problem. Um hier eine 'realistische' Grundlage zu haben, geht Scharpf, ausgehend von der durch Arend Lijphart eingeführten Unterscheidung zwischen Mehrheits- und Konkordanzdemokratie (vgl. Lijphart 1980, 1984) sowie der durch George Tsebelis vorgeschlagenen Klassifizierung politischer Systeme nach der Zahl der in ihnen institutionalisierten 'Vetospieler' (vgl. Tsebelis 2002) davon aus, dass demokratische Systeme sich danach klassifizieren lassen, ob diese eher dazu neigen Macht zu konzentrieren oder aber diese zu verteilen: 
„Am einen Extrem dieser Skala finden sich die quasi-monokratischen, machtzentrierenden Strukturen des idealtypischen Westminster-Modells ${ }^{195}$ - mit unitarischem Staatsaufbau, Hierarchie als dominantem Steuerungsmodus, Einkammerparlamenten, Zweiparteiensystemen und einer Dominanz des Regierungschefs im Kabinett und in der Mehrheitsfraktion. Das andere Extrem bilden konkordanzdemokratische Modelle, ${ }^{196}$ die zu einer Pluralisierung der Macht tendieren, eine föderale Gewaltenteilung zwischen Zentral- und Gliedstaaten aufweisen, Zweikammer-Parlamente besitzen, Koalitionsregierungen ausbilden und von der Regierung unabhängige Notenbanken haben.“ (Scharpf 2005:713)

Scharpf stellt heraus, dass die meisten real existierenden politischen Systeme irgendwo zwischen diesen beiden Extremen liegen, denn weder haben sie den Parteienwettbewerb in dem Maße neutralisiert, wie dies in der Allparteienregierung der Schweiz der Fall ist, noch haben sie die Verantwortlichkeit einer einzigen Partei in dem Maße institutionalisiert, wie dies im britischen Westminster-Modell möglich ist (Scharpf

195 Das Westminster-Modell ist eine parlamentarische Regierungsform nach dem Vorbild der britischkonstitutionellen Demokratie und bildet den Prototypen einer majoritären Konkurrenzdemokratie (Schmidt 2000:325-326): Dessen Kernelemente sind zwei sich konträr gegenüberliegende Parteien, von denen eine die Regierung und eine die Opposition innehat. Ein solches kompetitives Zweiparteiensystem, das durch ein absolutes Mehrheitswahlrecht stabilisiert ist, maximiert die politische Verantwortlichkeit der Regierung gegenüber den BürgerInnen, da die Regierung die volle Kontrolle über den Regierungs- und Verwaltungsapparat besitzt. Ihre Handlungsfähigkeit wird weder durch die Notwendigkeit beschränkt eine Koalitionsregierung zu bilden, noch wird sie durch föderale Institutionen oder unabhängige Notenbanken eingehegt. Sie verfügt folglich über uneingeschränkte hierarchische Autorität gegenüber allen Mitgliedern des Staatsapparats (außer der Gerichtbarkeit), so dass die Regierung die volle politische Verantwortung für alle erzielten politischen Ergebnisse übernehmen kann und muss. Dabei stellt diese Modellierung nahezu keine Anforderungen an die Wähler, bis auf den Umstand, dass sie wählen gehen müssen, damit sie repräsentiert werden. Hierbei ist lediglich entscheidend, beide Parteien im Prinzip gleich große Chancen haben müssen, die Wahlen zu gewinnen und keine der beiden Parteien strukturell so dominant ist, um den Wahlsieg der anderen zu verhindern. Diese Annahmen werden von Scharpf spieltheoretisch übersetzt, wobei das Verhältnis von Regierung und Opposition als nicht-kooperatives, asymmetrisches Nullsummenspiel und das Verhältnis zwischen politischen Akteuren und Wählern als sequenzielles Spiel modelliert wird (vgl. Scharpf 2006:303-309 und zu den noch recht einfachen spieltheoretischen Modellierungen siehe S.129 sowie $185-192)$.

196 In seiner prototypischen Fassung hat das Konkordanzmodell nur in der Schweiz bis heute Bestand. Es wird dadurch charakterisiert, dass die gesellschaftlichen Interessen gut organisiert und als solche auch in das Parteien- und Regierungssystem integriert sind. Darüber hinaus können unzufriedene Gruppen mithilfe von Referenden Regierungsentscheidungen leicht anfechten. Auf diese Weise ist der Parteienwettbewerb als Mechanismus zur Sicherung demokratischer Verantwortlichkeit weitestgehend außer Kraft gesetzt. Vorausgesetzt, dass alle wichtigen gesellschaftlichen Interessen tatsächlich an den multilateralen Verhandlungen beteiligt oder darin repräsentiert sind, entspricht die letztlich getroffene parlamentarische Entscheidung nicht nur dem Willen der Mehrheit, sondern einem 'Konsens', was nicht bedeutet, dass die Entscheidungen einstimmig getroffen werden müssen und alle Parteien ein formelles Vetorecht haben. Vielmehr kommt hierbei die abgestimmte Sichtweise der Entscheidungseliten zum Ausdruck, wobei die Kartellbildung dadurch begrenzt wird, dass benachteiligte Minderheiten via Referendum ihr Veto zum Ausdruck und die Verhandlungen zum Scheitern bringen können. Diese Grundannahmen übersetzt Scharpf wiederum spieltheoretisch und bezieht diese auf kooperative Spiele, die er wiederum mit den transaktionsökonomischen Annahmen des Coase-Theorems verbindet (vgl. Scharpf 2006:310-312 sowie zu den recht anspruchsvollen spieltheoretischen Modellierungen S.197-249). 
2006:313). Folglich sind die politischen Prozesse in den meisten Demokratien sowohl von Wettbewerb und politischen Mehrheiten als auch von Verhandlungen zwischen politisch unabhängigen Akteuren und deren Kooperationsstrukturen gekennzeichnet, was aber dazu führt, dass die Zentralisierung von Macht innerhalb des politischen Zentrum immer stärker dezentralisiert und multizentrisch wird. Eben für diese multizentrischen Zwischenformen sowie die Kombination aus Wettbewerb und Verhandlungen wählt Scharpf den Begriff der Verhandlungsdemokratie (Scharpf 2006:313-318) und stellt in Anknüpfung an die theoretischen und empirischen Erkenntnisse der Policy-Forschung, der Pluralismus-, Korporatismus- und Föderalismusforschung sowie der theoretischen Hintergrundfolie des akteurszentrierten Institutionalismus (siehe 5.3.2.3) heraus, dass es besondere Herausforderungen gibt, denen Verhandlungsdemokratien gerecht werden müssen:

Scharpf stellt heraus, dass die Analyse von Verhandlungsdemokratien eine engere Verknüpfung zwischen 'positiven ' und normativen Fragestellungen erfordert, als dies normalerweise in der Demokratietheorie üblich ist, da diese zum Verständnis und zur Verbesserung der Bedingungen beitragen soll, welche die Politik in die Lage versetzen, effiziente, effektive und legitime Lösungen für politische Probleme zu finden (Scharpf 2006:36). Scharpf räumt selbst ein, dass alle drei Kriterien evaluativ sind, dennoch weist er darauf hin, dass die Kriterien der Effizienz und Effektivität einer rein positiv ausgerichteten Forschung eher zugänglich sind, während das Kriterium der Legitimität rein normativ ist (ebd.). Zugleich verweist er auf das wechselseitige Verhältnis der Kriterien untereinander: „[D]ie Gültigkeit einer Norm [hängt] nicht nur von der richtigen Ableitung aus höherrangigen Normen ab, sondern auch von der positiven Frage, ob sie effektiv in die Praxis umgesetzt werden kann. Um ein politisches Programm als effektiv einzustufen, benötigt man umgekehrt nicht nur Informationen über seine empirischen Folgen, sondern auch normative Annahmen darüber, was als Problem angesehen werden soll und was als gute Lösung gelten würde." (ebd.) Obgleich auch für Scharpf klar ist, „dass wir das eine nicht mit dem anderen verwechseln dürfen, können wir nicht hoffen, normative Fragen dadurch zu vermeiden, dass wir uns ausschließlich auf die 'positiven`Aspekte eines politischen Gegenstandes konzentrieren.“ (ebd., 37)

Scharpfs Weg mit normativen Fragen umzugehen und sich zugleich an die „Maxime 'wissenschaftlicher Objektivität' oder wenigstens 'Neutralität “ (ebd., 38) zu halten, ist es, zwischen den Kriterien guter Politik (Effizienz und Effektivität) einerseits und der Legitimation des politischen Entscheidungssystems (auf der Input- und Outputseite) andererseits zu unterscheiden (ebd.): Die dabei relevanten Aspekte, nämlich der in Verhandlungsdemokratien qualitativ veränderte politische Prozess zur Generierung effizienter und effektiver politischer Ergebnisse und die für Verhandlungslösungen geltenden eigenen Legitimationsgrundlagen, sprechen Scharpfs Ansicht nach dafür, dass 
die Bedingungen und Ergebnisse moderner Politik an den Maßstäben der Verhandlungsdemokratie als eigenständigem Demokratietypus gemessen werden sollten (Scharpf 1998a:236). Um als gut charakterisiert zu werden, müssen Verhandlungen möglichst effizient zu Entscheidungen führen und die effektive Implementation dieser Entscheidungen muss garantiert werden. Aus legitimatorischen Gründen muss wiederum sicher gestellt werden, dass all diejenigen die von kollektiven Entscheidung negativ betroffen sein könnten die Möglichkeit erhalten an den Verhandlungen beteiligt zu sein oder sich interessenwirksam repräsentieren zu lassen (Inputseite), um von den Leistungen politischer Entscheidungen zu profitieren oder im Extremfall angemessen entschädigt zu werden, wenn die Entscheidung zu partiellen Sonderopfern führt (Outputseite). Daher will Scharpf aufzeigen, dass das Modell der Verhandlungsdemokratie einerseits an besondere Kriterien guter Politik hinsichtlich der Politikproduktion und des politischen Kampfes um die Verteilungsdimension gebunden ist und dass es andererseits auch besonderer Kriterien für die demokratische Legitimation bedarf.

Auf ersteres, d.h. die Kriterien guter Politik unter verhandlungsdemokratischen Bedingungen (5.3.3.2.1), wird im Folgenden eingegangen, indem zum einen die Effizienzund Effektivitätskriterien (1) der prototypischen Verhandlungsformen sowie zum anderen (2) deren strukturelle Kontextbedingungen herausgestellt werden. Auf letzteres, sprich die Kriterien legitimer Politik vor dem Hintergrund der realen Besonderheiten verhandlungsdemokratischer Settings (5.3.3.2.2) wird eingegangen, indem Scharpfs Überlegungen bezüglich der Inputlegitimation und hier genauer (a) der »Herrschaft des Volkes« und (b) der "Herrschaft durch das Volk» sowie der Outputlegitimation, d.h. der (c) »Herrschaft für das Volk«, dargelegt werden.

\subsection{Die Kriterien guter Politik}

Wie bereits dargelegt, sieht Scharpf die Aufgabe der Demokratie darin, einen funktionalen Beitrag zur Selbstorganisation der Gesellschaft zu leisten (siehe 5.3.3.1). Verhandlungsdemokratien tun dies im Wesentlichen dadurch, dass politische Entscheidungen auf dem Weg von Verhandlungen und innerpolitischer Koordinationen zustande kommen und dass eben diese einen Beitrag dazu leisten das Gemeinwohl aller zu steigern. ${ }^{197}$ Dabei unterscheidet Scharpf zwischen verschiedenen Koordinationstypen (Verhandlungen, Mehrheitsentscheidungen und hierarchische Steuerung), die er danach untersucht, ob und wie diese in der Lage sind, Entscheidungen zu produzieren (Effizienzaspekt) und diese wirksam zu implementieren (Effektivitätsaspekt). Da die An-

197 Die Gemeinwohlthematik wird bei Scharpf sowohl unter wohlfahrtsökonomischen als auch unter Gerechtigkeitsaspekten behandelt. Dennoch wird diese hier als explizit normative Thematik eingestuft und damit, wie Scharpf dies auch selber macht, unter legitimationstheoretischen Aspekten diskutiert (siehe 5.3.3.2.2). 
tizipation von Implementationsproblemen bereits einen gewichtigen Einfluss auf den Prozess der Entscheidungsfindung hat und die Art und Weise der Entscheidungsfindung wiederum einen Einfluss darauf hat, wie leicht oder schwer Entscheidungen implementiert werden können, müssen Effizienz und Effektivität in ein gegenseitiges Bedingungsverhältnis gestellt werden. Unter verhandlungsdemokratischen Bedingungen, wo sowohl der politische Prozess der Entscheidungsfindung als auch die Implementationsphase nicht mehr alleinig durch die hierarchische Regulierung des Staates und durch Mehrheitsentscheidungen realisiert werden können, geraten die strategischen Interaktionen zwischen unabhängigen Akteuren in den Fokus der Aufmerksamkeit. Eben diese werden von Scharpf vor dem Hintergrund spieltheoretischer Annahmen zu verschie-denen Verhandlungsformen modelliert. Bevor diese dargelegt werden, muss indes noch auf deren spieltheoretischen Grundannahmen eingegangen werden:

In der Spieltheorie gilt der grundlegende Lehrsatz, dass alle möglichen Koordinationsformen auf die Form eines nicht-kooperativen Spiels reduziert werden können. Für die politische Forschung ist ein solcher in der Mathematik üblicher Reduktionismus indes wenig hilfreich, da die unterschiedlichen Formen der gesellschaftlichen und politischen Koordination (Hierarchie, Abstimmung, Verhandlung) ja jeweils eigene, sie charakterisierende Stärken und Schwächen haben (Scharpf 2006:171). Entscheidend ist innerhalb des spieltheoretischen Kontexts daher vielmehr, dass unabhängig von den konkreten Strukturen, in deren Rahmen die Akteure agieren, diese immer die Möglichkeit haben (und sei sie nur rein theoretisch, widerrechtlich, mit hohen Transaktionskosten oder um den Preis der sozialen Diskreditierung) auf einseitiges, die anderen Akteure und deren Interessen und Ressourcen ignorierendes, Handeln zurückzugreifen. Im politischen Kontext ist des Weiteren entscheidend, dass alle Formen von wechselseitiger Anpassung aneinander bestimmter insitutioneller und organisatorischer Voraussetzungen bedürfen. Basal sind hier auf der strukturellen Ebene politische Rechte und Freiheiten sowie administrative Organisationsstrukturen, die die Verbindlichkeit ausgehandelter Vereinbarungen sicherstellen. Scharpf nennt diese minimale Instititionen.

Aufbauend auf diesen basalen Grundgedanken formuliert Scharpf die innerhalb des verhandlungsdemokratischen Kontextes relevanten und durch spieltheoretische Überlegungen angeleiteten normativen Annahmen, dass Verhandlungen notwendig werden, wenn hierarchische Steuerung und Mehrheitsentscheidungen nicht möglich sind oder adäquat erscheinen und dass die Verhandlungsteilnehmer solange versuchen sich selbstorganisiert auf eine Lösung zu einigen, solange sie die Erwartung hegen, dass sie dadurch ihren Status Quo verbessern. Dies müssen keine materiellen Gewinne sein. Die Motivation der verhandlungsteilnehmer kann auch darin liegen, überhaupt beteiligt zu sein oder in seinen Interessen antizipiert zu werden (ebd., 204). Entscheidend dabei ist desweiteren, dass die Ergebnisse solcher Verhandlungen nicht vorgegeben 
sind, sondern in vielen Fällen erst erdacht und erarbeitet und vor dem Hintergrund differierender Interessenlagen ausgehandelt werden müssen (Produktionsdimension). Das bedeutet i.d.R. auch, dass alle Teilnehmer zusammen eine für alle akzeptable Antwort auf die Frage finden müssen, wie die Nutzen und Kosten jeweils verteilt werden sollen (Verteilungsdimension). Scharpf macht diesbezüglich in Rückgriff auf die analytischen Kategorien des akteurszentrierten Institutionalismus (siehe 5.3.2.3) deutlich, dass je höher der Organisations- und Institutionalisierungsgrad über die minimalen Institutionen hinaus ist (in Form von Netzwerken, Regimen und Zwangsverhandlungssystemen), umso mehr Interaktionsformen (einseitiges Handeln, wechselseitige Anpassung, Kooperation, Mehrheitsentscheidungen und hierarchische Steuerung) stehen für die Aufgabenerfüllung in der Produktions- und Verteilungsdimension zur Verfügung. Dabei werden aber zugleich auch die strategischen Relationen zwischen den Verhandlungsteilnehmern immer komplexer und binnendifferenzierter. Dies hat wiederum Rückwirkungen auf die Ursachen potenzieller Differenzen und der Faktoren, die eine Einigung ermöglichen und ist damit für die Abschätzung der Effizienz und Effektivität der unterschiedlichen Verhandlungsformen von Relevanz (ebd., 313). Um diese Effizienz- und Effektivitätskriterien darzulegen, werden im Folgenden zuerst 1) die prototypischen Verhandlungsformen und dann 2) die strukturellen Kontextbedingungen dieser Verhandlungsformen dargelegt.

\section{1) Die prototypischen Verhandlungsformen}

Scharpf unterscheidet zwischen a) Tauschgeschäften, wo primär die Produktionsdimension im Zentrum steht, b) lösungsorientierten Kooperationen, wo primär die Produktionsdimension im Zentrum steht und schließlich c) positiven Koordinationen, wo die Produktions- und die Verteilungsdimension zugleich gelöst werden müssen:

a) Bei Tauschgeschäften (Bargaining) ist die Bedeutung der Verteilungsdimension hoch, wogegen die Bedeutung der Produktionsdimension niedrig ist, denn hier können die von einem bestimmten Projekt profitierenden Akteure die Zustimmung und Mitwirkung der durch das Projekt benachteiligten Parteien mit Hilfe von Ausgleichszahlungen oder anderen (nicht monetären) Entgegenkommen 'erkaufen`. Dabei ist entscheidend, dass das Projekt von den Verhandlungsteilnehmern nicht als solches in Frage gestellt wird, ein Mindestmaß an Vertrauen zwischen den Verhandlungsteilnehmern herrscht und dass die Teilnehmer solcher Tauschverhandlungen eine reale Vetomacht besitzen, d.h., dass sie die Verhandlungen durch ihren Austritt zum Stillstand bringen können (ebd., 214). ${ }^{198}$

\footnotetext{
${ }^{198}$ Dies ist zum Beispiel immer dann der Fall ist, wenn VertreterInnen der Pharmaindustrie, mit Kranken-
} kassen und staatlichen Gesundheitsbehörden über Patentauflösungen für Medikamente oder Zulas- 
Unter diesen Bedingungen entstehen effiziente Entscheidungsfindungsprozesse immer dann, wenn alle Beteiligten davon profitieren, d.h., wenn die gefundene Entscheidung keinen schlechter stellt als der Status Quo, den sie auch ohne die Entscheidung hätten oder wenn diejenigen Parteien, die davon mehr als andere profitieren, ihren Gewinn mit denjenigen teilen, deren Nutzen nicht vorhanden oder geringer sind, ohne dass dies seine Gewinne wiederum schmälert (ebd., 216). In solchen Fällen haben alle Beteiligten etwas von der Einigung und jeder der Teilnehmer hat zudem ein Interesse daran, dass es detaillierte und sanktionierende Implementationsvorschriften gibt, um das Ergebnis der Einigung festzuhalten und auch in Zukunft nicht zu gefährden. In einem solchen Fall zieht eine effiziente Einigung auch eine effektive Implementation nach sich. Der entscheidende 'Knackpunkt' ist hier allerdings, dass überhaupt erst einmal Tauschobjekte gefunden werden müssen, die von anderen als Gewinne betrachtet werden. Hier gibt es große Asymmetrien, da gerade mächtige und finanzstarke Akteure einen deutlichen Vorteil haben, was die Vielseitigkeit ihrer Angebote angeht, wohingegen einige Akteure nur sehr spezialisierte Angebote machen können, die außerhalb ihres eigenen Bereiches keine Relevanz für andere haben. Das wiederum führt zu einer systematischen Fokussierung auf monetäre oder handelbare Güter, was wiederum bestimmte Interessen automatisch privilegiert, die über diese verfügen. ${ }^{199}$

Da es in der Praxis nicht selten auch solche Fälle von Tauschverhandlungen gibt, wo Ausgleichszahlungen nicht realisierbar sind, normativ für nicht akzeptabel gehalten werden $^{200}$ oder es sich dabei um Fragen handelt, die nicht oder nur sehr schwer quantifiziert sind oder materiell aufgewogen werden können (wieviel ist der Schutz bzw. die Ausrottung einer Art wert und für wen?), kommen in solchen Fällen zumeist Koppelgeschäfte oder Paketlösungen zum Tragen (ebd., 217-220):

Bei Koppelgeschäften geht es darum, dass jede Partei der anderen etwas anbietet, was deren Status Quo verbessert, um sich auf ein Projekt zu einigen. ${ }^{201}$ Das können schwer

sungen als Impfstoffe verhandeln und es von Seiten der staatlichen Behörden keine rechtlichen Mittel gibt, eine solche Regelung auch anderweitig zu veranlassen.

199 Dies ist erst einmal die Feststellung, dass Tauschgeschäfte diese Tendenz generieren, es ist aber noch keine normative Wertung, ob dies legitim ist oder nicht. Zur legitimatorischen Betrachtungen dieser Analysen wird an späterer Stelle übergegangen (siehe 5.3.3.2.2).

${ }^{200}$ Zu denken wäre hier zum Beispiel an gesellschaftlich brisante Themen, wie Abtreibung, Flüchtlingsunterbringung, Kinderversorgung und Naturschutz etc. Selbst wenn es hier primär um Finanzierungsfragen ginge, gibt es beachtliche Unterschiede zwischen unterschiedlichen Kulturen, Ländern und sozialen Milieus: Allein der Versuch einer Tauschverhandlung zum gegenseitigen Nutzen kann auf Widerstand stoßen, was dann aber weniger praktisch-rational als vielmehr emotional und moralisch motiviert ist, was aber bei Tauschverhandlungen eben auch mitbedacht werden muss, weil dies eben dazu führen kann, dass solche Verhandlungen abbrechen oder zu Ergebnissen führen, die dann im Nachhinein in einem größeren Kontext betrachtet zu Problemen führen.

201 Als Beispiel: Für die Zustimmung der Landwirte, ihre Gebiete extensiver zu bewirtschaften, kann ein Naturschutzverband bspw. anbieten, diesen Prozess durch Experten kostenlos zu begleiten und das Umweltministerium kann ihnen bspw. Ausgleichszahlungen für die, durch die Umstellung entstande- 
zugängliche Informationen und Expertisen sein, das Versprechen zur Mitwirkung in einer anderen Sache oder auch die Zusage, bei dem verhandelten Projekt auf seinen Gewinn zu verzichten, wenn die andere Partei dies bei einem anderen Projekt ebenso macht. Analog funktioniert dies bei gegenseitig angedrohten Schädigungen, die keiner der Verhandlungsparteien in Kauf nehmen möchte oder über die Androhung von Seiten der Regierung rigidere Vorschriften zu erlassen, um die VertreterInnen der Interessensverbände zu freiwilligen Verhandlungen zu bewegen (ebd., 218). Knackpunkt ist hier, dass die Bedingungen solcher Koppelgeschäfte zumeist informell sind und dass die Bedingungen der Einigung zumeist nicht selbst Teil von Verträgen werden, so dass darüber zwar Blockade- und Vetopositionen durchbrochen werden können, es aber dadurch zu recht hohen (meist unsichtbaren) Transaktionskosten kommen kann und dass die Effektivität der Implementation eher dem Vertrauen oder gegenseitigen Abhängigkeiten geschuldet ist, als das diese in den Verhandlungen formell abgesichert werden kann. Zudem erfordern solche Koppelgeschäfte Verhandlungen auf 'Gipfelebene', wo die beteiligten Akteure autorisiert sind, auf unterschiedliche Ressourcen zurückzugreifen und Tauschgüter anzubieten.

Paketlösungen müssen diese Voraussetzungen ebenfalls erfüllen, kommen demgegenüber aber immer dann zum Einsatz, wenn sich keine tauschbaren Güter finden oder inhaltlich keine Kompromisse erzielen lassen (bspw. weil es um grundsätzliche Fragen oder Formulierungen geht oder weil Parteien keinen Kompromissspielraum haben oder sehen), die Einigung aber dennoch bereits einen (Prestige-)Gewinn für alle Beteiligten darstellt (ebd., 219). Sie zielen daher darauf ab, dass jeder Verhandlungsteilnehmer einen für ihn wichtigen Aspekt uneingeschränkt einbringen kann, so dass am Ende ein 'großes Paket mit lauter unzusammenhängenden Einzelteilen darin' geschnürt wird. Viele internationale Verträge, wie die Klimarahmenkonvention oder auch die CBD sind derartige Paketlösungen. Im Ergebnis ist auch hier der Entscheidungsstau erst einmal aufgehoben. Die gefundenen Lösungen sind aber anfällig für Detailproblematiken und müssen in der Regel ständig nachverhandelt werden, wo sich immer wieder aufs Neue die Problematik stellt, dass das Verhandlungsdilemma verhindert werden muss. Dies führt dazu, dass die Effizienz von Paketlösungen immer geringer wird. Zudem tendiert die Effektivität der Implementation immer dann gegen Null, wenn die Ratifikation nicht nur durch das Plenum des Parlaments geschieht, sondern funktional spezialisierte Ausschüsse das entscheidende Wort haben oder wenn einige Teile des Pakets von denen implementiert werden müssen, gegenüber denen die 'Gipfelakteure`keine direkte hierarchische Autorität besitzen (ebd., 220).

nen Kosten anbieten. Wenn die Landwirte dadurch nicht einmal mittelfristig einen Nutzen sehen würden, dann würden auch solche Koppelgeschäfte hinfällig werden. 
b) Bei lösungsorientierten Kooperationen stehen der Prozess des gemeinsamen Problemlösens und damit die Nutzenproduktion im Vordergrund, wohingegen die Verteilungsdimension (erst einmal) von untergeordneter Bedeutung ist. Hier besteht das vordergründige Interesse der Verhandlungsteilnehmer darin, neue Möglichkeiten auszuloten, andere Wege zu suchen oder über Innovationen nachzudenken, die sie alleine nicht realisieren könnten. Solche Konstellationen sind typisch für Joint Ventures oder die Entscheidungsfindung innerhalb der Ministerialbürokratie (ebd., 221). Um diesen gemeinsamen Handlungsspielraum indes überhaupt zu eröffnen bzw. nutzen zu können, müssen die einzelnen Akteure nicht nur ihre eigenen Ressourcen und Handlungsoptionen kennen und ausloten, sondern sich auch gegenseitig darüber in Kenntnis setzen, um die Interaktionseffekte zwischen diesen Ressourcen und Optionen sondieren zu können (ebd., 223). Hierfür ist ein in dieser Arbeit als deliberativ und diskursiv spezifizierter Kommunikations- und Verhandlungsmodus erforderlich: Problemlösen ist am ehesten dann erfolgreich, wenn die Akteure in der Lage sind, sich mit Hilfe von Argumenten über die Beschaffenheit des Problems und die bestmögliche Lösung sowie die bestmögliche Art und Weise, diese Lösungen zu erreichen, verständigen und dies in der Annahme tun, dass etwaige Lösungen keine Verteilungsprobleme aufwerfen, die sich nicht ebenfalls kooperativ lösen lassen. (ebd.). ${ }^{202}$

Diese verständigungsorientierte Suche wird auf der interpersonalen Ebene unterstützt durch gegenseitiges Vertrauen, gute Kommunikations- und Argumentationsfähigkeiten, die Offenheit und Bereitschaft aller Teilnehmer auch die anderen Positionen gelten zu lassen beziehungsweise, wenn es daran mangelt, die Einrichtung einer Mediationsstelle. Diese soll garantieren, dass Vetopositionen nicht zur Verhinderung von Entscheidungen gebraucht werden und dass zu wenig beachtete Möglichkeiten eingebracht werden, selbst wenn die Position derjenigen, die diese in die Verhandlungen einbringen, schwach ist (ebd.) Unter diesen durchaus anspruchsvollen Bedingungen haben lösungsorientierte Verhandlungen einen positiven Einfluss auf die Effizienz der Entscheidungsfindung und auf die Effektivität der Implementation, da das Richtungsziel solcher Verhandlungen von vornherein klar ist und deren Ausgang davon abhängt, wie kooperativ die Verhandlungsteilnehmer im Einzelnen sind. Indes muss der Erfolg solcher Verhandlungen nicht notwendigerweise auch vom Vorhandensein solidarischer

202 Scharpf merkt hierzu an, dass das Problemlösen als Interaktionsmodus, in dem sie Akteure die Maximierung der gemeinsamen Interessen aller Beteiligten anstreben, in der Definition nach Habermas (vgl. Habermas 1992b:204-206) nicht als rationaler Diskurs gelten kann, da hierbei auch Formen der Kommunikation eine Rolle spielen, in denen akteursspezifische Interessen und unterschiedliche Grade der Verhandlungsmacht eine Rolle spielen (Scharpf 2006:223). Da hier aber kein primär normativer sondern analytischer Unterschied zwischen Bargaining und Problemlösen gemacht wird und damit die scharfe, dualistische Unterscheidung zwischen systemischer und verständigungsorientierter Kommunikation verschwimmt, kann im Falle lösungsorientierter Verhandlungen von einem charakteristischen diskursiven und deliberativen Kommunikationsmodus gesprochen werden, der weder distributiven Tauschverhandlungen, noch der positiven Koordination so zu eigen ist. 
Interaktionsorientierungen abhängen, da selbst unter kompetitiven Bedingungen lösungsorientierte Kooperationen von Vorteil sein können, wenn äußere Rahmenbedingungen, institutionelle Arrangements und Anreizsysteme geschaffen werden bzw. vorhanden sind, die die Entscheidungen über die zu realisierenden Ergebnisse vom individuellen oder sogar organisatorischen Eigeninteresse abkoppeln. ${ }^{203}$ Wenn solche Bedingungen geschaffen werden können, dann können Verhandlungen im Modus des Problemlösens stattfinden, auch ohne dass auf solidarische Interaktionsorientierungen als einziger Garant dafür gebaut werden muss.

c) Im Modus der positiven Koordination müssen sich die Verhandlungsteilnehmer zugleich mit Verteilungs- und Produktionsproblemen auseinandersetzen und diese lösen, sprich sie müsen zugleich distributives Bargaining und lösungsorientiertes Problemlösen betreiben. Unter den Umständen, dass sie dabei erfolgreich sind, koordinieren sie sich positiv, ist dies nicht der Fall oder aber nicht nötig, spricht Scharpf hingegen von negativer Koordination (ebd., 225). Um deutlich zu machen, was es mit der positiven Koordination auf sich hat, ist es sinnvoll zuerst ihr Gegenteil, d.h. die negative Koordination genauer anzuschauen: Der prozedurale Gegenpart von Verhandlungen sind Mehrheitsentscheidungen, denn hier werden sowohl die Schwierigkeiten der Produktionsdimension als auch der Verteilungsdimension, und dies nicht weil sie inhaltlich keine Rolle spielen, durch den Modus der Interaktion neutralisiert (ebd., 212). ${ }^{204}$ Ein wesentliches Element der prozeduralen Besonderheit von Mehrheitsentscheidungen als Abstimmungsmodus ist ja gerade die Erwartungshaltung, dass selbst komplexe Entscheidungen auf ein Votum für oder gegen etwas reduziert werden können und dass Mehrheitsentscheidungen durch eine Abstimmung zwischen Alternativen schneller und reibungsloser getroffen werden können, als durch langwierige Verhandlungen mit einstimmigen oder konsensualen Abstimmungsmodus, vor allem da, wo die Verhandlungskoordination durch die Anzahl der teilnehmenden unabhängigen Parteien schwieriger wird (ebd., 251). Dies ist der Fall, weil Mehrheitsentscheidungen nicht durch einseitiges Handeln und noch nicht einmal durch den Widerstand Vieler (je nach Qualifizierung der Mehrheit) blockiert werden können, obgleich die Möglichkeit zu einseitigem Handeln auf der strukturellen Ebene die Bedingung der Möglichkeit für die Auswahl zwischen negativer und positiver Koordination ist.

${ }^{203}$ Hier nennt Scharpf Beispiele wie den Umstand, dass der Wettbewerbsdruck von außen höher ist als der Wettbewerbsdruck zwischen den Verhandlungsteilnehmern (Kontextfaktor), dass es vorab Einigungen über den Verteilungsmodus gibt (institutionelles Arrangement) oder das gefundene Lösungen, die für alle von Vorteil sind, extra und individuell prämiert werden (Anreizsysteme) (Scharpf 2006:224-225).

${ }^{204}$ Klassisches Beispiel hierfür sind Wahlen, bei denen der Wähler zwischen Parteien entscheiden kann, aber auch die Nichtwahl eine Option ist sowie Vorschläge von Vermittlungsausschüssen im Parlament, die von den Parteien entweder angenommen oder abgelehnt werden, nicht aber inhaltlich diskutiert werden können oder internationale und intergouvernementale Verträge, bei denen sich nur noch die Frage stellt, ob sie ratifiziert werden oder nicht (ebd.). 
Positive Koordination bezeichnet demgegenüber die Verbindung aus Tausch- und Problemlösungsverhandlungen. Scharpf stellt heraus, dass die basalen Voraussetzungen dafür die gar nicht immer so selbstverständliche Anerkennung der gleichzeitigen Existenz und Wichtigkeit der Lösung beider Bereiche ${ }^{205}$ sowie die Orientierung an Gemeinwohlnormen (siehe 5.3.3.2.2) ist. Erst wenn alle Verhandlungsparteien zu dem Resultat kommen, dass keine Seite dazu im Stande ist eine Problemlösung allein zu entwickeln, d.h. einseitig zu handeln, und den Verteilungskampf ausschließlich zu ihrem eigenen Vorteil entscheiden kann, so dass alle Teilnehmer von ihren Maximalforderungen abrücken müssen, kann die Suche nach den, für alle Teilnehmer vorteilhaften Lösungen beginnen (ebd., 228). Mit anderen Worten: „Solange nicht explizit anerkannt wird, dass jede akzeptable Lösung auch eine faire Verteilung von Nutzen und Kosten beinhalten muss, können politische Verhandlungen nicht positiv, sondern nur negativ koordiniert werden." (ebd., 227) Insofern unterscheidet sich der Modus der positiven Koordination qualitativ von den beiden erstgenannten Verhandlungsformen. Dadurch, dass die Verhandlungsteilnehmer sowohl das Ziel haben kooperativ zu einer Entscheidung zu gelangen, ihnen aber nicht die Option offen steht, auf einseitiges Handeln umzuschwenken und alle Verhandlungsteilnehmer zugleich darauf aus sind, eine möglichst günstige Verteilungsrelation für sich selbst zu erzielen, entsteht ein für die positive Koordination typisches "Verhandlungsdilemma“:

„Die erfolgreiche gemeinsame Suche nach besseren Gesamtlösungen erfordert Kreativität, Verständigung und gegenseitiges Vertrauen, während der Erfolg im Verteilungskampf von der strategischen und sogar opportunistischen Kommunikation oder Zurückhaltung der zur Verfügung stehenden Informationen abhängt und von einem gehörigen Misstrauen gegenüber potenzieller Fehlinformationen. Das bedeutet nicht nur, dass die solidarische Interaktionsorientierung, die in der Produktionsdimension dem gemeinsamen Lernen zuträglich ist, psychologisch inkompatibel ist mit der wettbewerblichen Orientierung, die den Erfolg in der Verteilungsdimension begünstigt, sondern auch, dass die Partei, die am meisten zur Suche nach gemeinsamen Lösungen beiträgt, sich der Gefahr aussetzt, von einer Partei, die sich auf die Verteilungsdimension konzentriert, ausgebeutet zu werden." (ebd., 211)

205 Scharpf führt hier das Beispiel an, dass bei politischen Gesprächen um Richtlinien und Standards bspw. in der Telekommunikation ausschließlich technische Argumente zur Effizienz und zur Qualität alternativer Lösungen als legitim erachtet werden, wohinter sich aber handfeste ökonomische Interessen verbergen, die aber als solche nicht offen diskutiert werden, so dass es oftmals weniger die technischen Details als die ungelösten Verteilungsfragen sind, die einer Einigung entgegenstehen (Scharpf 2006:227-228). Analoges findet sich in vielen Politikbereichen, wo Verteilungsfragen aufgrund ihrer Brisanz nicht offen diskutiert werden, diese aber einen starken Einfluss auf die gefundenen Kompromisse oder aber das Scheitern der Verhandlungen haben. Dass der strittigste Punkt bei den Verhandlungen der CBD der ABS-Mechanismus war und dass eben dieser am wenigsten konkret ausgeführt worden ist (siehe 2.2.1), zeigt die Relevanz dieser Beobachtung auch im Biodiversitätskontext. 
Scharpf hält diesbezüglich fest, dass es sich hierbei um ein Faktum handelt und dass sich die daran anschließenden normativen Wertungen nicht pauschal gegen Verhandlungen bzw. verhandlungsdemokratische Settings als solche richten, da positive Koordinationen zwar anspruchsvoll sind, aber weder sind sie immer notwendig noch existieren immer die strukturellen Bedingungen, damit überhaupt positiv koordiniert werden kann. Um im Folgenden zu zeigen, welche dies sind, müssen die strukturellen bzw. Kontextbedingungen der drei Verhandlungsformen dargelegt werden.

2) Die strukturellen Kontextbedingungen der Verhandlungsformen

Für die drei aufgeführten Verhandlungsformen sind in je unterschiedlichem Maße die strukturellen Rahmenbedingungen von a) selbstorganisatorischen Netzwerken, b) normativen Regimestrukturen sowie von c) Zwangs- bzw. Kollektivverhandlungssystemen mitsamt der durch sie ermöglichten Interaktionsformen relevant: ${ }^{206}$

a) Netzwerkbeziehungen, die sich durch mehrmalige freiwillige Interaktionsbeziehungen zwischen Akteuren auszeichnen, tragen dazu bei zwischen den Akteuren eine gewisse Erwartungssicherheit qua schwacher und starker Bindungen zu generieren (ebd., 233-234). Schwache Bindungen bedeuten die Erwartung, dass die von den Akteuren mitgeteilten Informationen über ihre eigenen Optionen und Präferenzen wahrheitsgemäß sind und nicht absichtlich irreführend eingesetzt werden und dass die Akteure ihren abgesprochenen Verpflichtungen nachkommen (ebd.). Weiter gewährleisten diese, dass die Akteure sich untereinander kleinere Gefallen erweisen und auf kleinere Vorteile verzichten, die für andere große Verluste mit sich bringen würden (ebd.). Dies sind schon einmal wichtige Minimalvoraussetzungen für effiziente Tauschverhandlungen, aber auch für Problemlösungsverhandlungen, da beide auf gegenseitigem Vertrauen in die Integrität und die grundsätzliche Bereitschaft zur Zusammenarbeit der anderen Verhandlungspartner basieren. Verstetigen und erweitern sich solche Netzwerkbeziehungen auch auf andere Bereiche, dann kommt es zur Ausbildung starker Bindungen (ebd.). Hierfür ist kennzeichnend, dass die Verhandlungsteilnehmer sich gut kennen und in mehreren Bereichen erfolgreich zusammenarbeiten. Das wiederum verstärkt die Erwartung, dass die Akteure Strategieoptionen vermeiden, die zwar für sie selber attraktiv wären, die Interessen der anderen aber in fundamentaler Weise verletzten würden. Mit anderen Worten: Starke Bindungen schwächen das Risiko egoistischer und feindschaftlicher Interaktionsorientierungen ab und wirken in Richtung solidarischer Orientierungen (ebd.).

${ }^{206}$ Anarchische Felder und minimale Institutionen scheiden hierbei aus der Betrachtung aus, da Verhandlungen im Mindesten semipermanente Beziehungsmuster erfordern, die auf Ressourcenaustausch und gegenseitige Unterstützung hin angelegt sind, bei denen der Austritt aber jederzeit möglich sein muss. Eben dies geschieht erst auf der Interaktionsschwelle der Kooperation. 
Dies ist vor allem für gemeinsame Problemlösungen ein effizienz-und effektivitätsmächtiger Vorteil, da unter der solidarischen Orientierung die Vorteile der anderen wie die eigenen Vorteile interpretiert werden (ebd., 152) und damit von Vornherein ein möglichst offener Raum der Kooperationsmöglichkeiten entsteht. Zugleich stellen starke Bindungen für Tauschverhandlungen einen Effektivitätsvorteil dar, da sie alle Verhandlungsteilnehmer davor schützen, dass Ausbeutungsstrategien, wie im Gefangenendilemma zum Einsatz kommen und man sich hinsichtlich der Implementation vor diesen nicht extra schützen oder gegen diese absichern muss (ebd., 234). Aber auch auf der Effizienzseite wirken die starken Bindungen vorteilhaft. Da der Grundmodus bei Tauschverhandlungen, durch deren Fokussierung auf die Verteilungsrelation, wettbewerbliche Interaktionsorientierungen sind, bewirken die starken Bindungen, dass die Verluste die im Einzelfall zu tragen sind durch die generelle Erwartung zukünftiger Vorteile in anderen Verhandlungen oder anderen Bereichen aufgewogen werden, so dass es schneller zu Einigungen kommt. Dies gilt zum Teil auch für die positive Koordination. Hier schwächen starke Bindungen das Verhandlungsdilemma insofern ab, als dass die reale Gefahr der Ausbeutung derjenigen, die Zugeständnisse in der Verteilungsrelation machen, um für alle Beteiligten zu einer möglichst guten Lösung zu gelangen abgeschwächt wird, indem kooperatives und vertrauenvolles Verhalten mittelund langfristig eher honoriert wird. Indes ist die Erlangung des Status als vertrauenswürdiger und kooperativer Verhandlungspartner insofern kostspielig, als dass nicht alle potenziell möglichen Gewinne, die im egoistischen Interaktionsmodus mitgenommen werden könnten, abgeschöpft werden können und der Status ist zudem fragil, da er durch einmalige Enttäuschung leicht wieder zerstört werden kann (ebd., 235). Aus diesen Gründen ist die Zahl der Netzwerkbeziehungen, die sich Akteure in Tauschverhandlungen oder im Modus der positiven Koordination 'leisten` können, begrenzt. Der umgekehrte Fall gilt für Problemlösungsverhandlungen. Umso mehr Netzwerkbeziehungen ein Akteur unterhält, umso mehr vertrauensvolle und enge Verbindungen besitzt er zu anderen Akteuren und dies wiederum verschafft ihm Vorteile in Hinblick auf Informationen, Einflussnahme und Koalitionsbildungen innerhalb von Problemlösungsverhandlungen. Paradoxerweise führen also die durch Netzwerke ausgebildeten solidarischen Interaktionsorientierungen dazu, dass Informations- und Vertrauensasymmetrien zwischen den Akteuren entstehen, so dass bestimmte Koalitionen wahrscheinlicher werden als andere und sich ggf. sogar Konstellationen bilden, wo bestimmte Akteure mit Extrempositionen zum 'Zünglein an der Waage' zwischen den größeren Koalitionen werden (ebd., 236-238).

b) Unter den Bedingungen von Regimestrukturen, sprich absichtsvoll geschaffener normativer Bezugsrahmen zur Regelung von Interaktionen zwischen einer formell festgelegten Anzahl von Akteuren, zeichnen sich Verhandlungsregime dadurch aus, dass sie 
das Verhalten und Handeln beteiligter Akteure insofern 'steuern', als dass Mitglieder von Regimen sich explizit (zumeist vertraglich) dazu verpflichten, bestimmte Interessenspositionen zu respektieren, bestimmte Ziele gemeinsam zu verfolgen und bestimmte Verfahren zu beachten (ebd., 241). ${ }^{207}$ Daher gelten grundsätzlich dieselben Bedingungen wie bei Netzwerken, denn die sachlichen Entscheidungen und Ergebnisse innerhalb von Regimen werden nicht durch das Regime selbst bestimmt (genauso wie das Vertragsrecht nicht den Inhalt von Verträgen festlegt), sondern durch die Interaktionen derjenigen, die sich dazu verpflichtet haben, die Regeln des Regimes zu befolgen. Dies hat indes, je nach Regulierungsniveau, bestimmte Konsequenzen:

Die Etablierung und Aufrechterhaltung des Regimes ist anders als bei Netzwerken nicht nur vom Vertrauen der Mitglieder untereinander abhängig, sondern ganz wesentlich von der Möglichkeit bestimmt, dass Verstöße gegen die Regeln des Regimes intern wirksam zu sanktioniert werden können (ebd., 242). Hierbei hängt die Einhaltung der Regimeregeln sowohl vom je individuellen Interesse der einzelnen Mitglieder als auch von der Bereitschaft des Kollektivs ab, Regelverletzungen zu sanktionieren. Dabei sollte zwischen der Versuchung zum 'Trittbrettfahren` bei der Herstellung kollektiver Güter und der expliziten Verletzung der vertraglichen Verpflichtungen unterschieden werden: Im ersten Fall haben alle Parteien ein Eigeninteresse daran, nicht durch andere übervorteilt zu werden, so dass hierfür Regeln der Verhandlungsführung etabliert und ggf. bei wiederholt unsolidarischem Verhalten gegenüber den anderen Mitgliedern des Regimes mit 'Blaming und Shaming 'sowie notfalls mit dem 'Rausschmiss' aus dem Regime gedroht werden kann. Vor allem bei stark asymmetrischen Machtverteilungen zwischen den Mitgliedern des Regimes ist entscheident, ob die Mehrheit der Mitglieder des Regimes stark genug sind, mögliche 'Trittbrettfahrer' wieder auf 'Linie' zu bringen bzw. bereit sind ggf. die Kosten für das unsolidarische Verhalten einzelner zu übernehmen. Denn wenn dies nicht geschieht, besteht die Gefahr, dass nach und nach auch andere Mitglieder zu unsolidarischem Verhalten tendieren, so dass das gesamte Regime nach und nach destabiliert wird. Bei der expliziten Verletzung von Vertragsregelungen kommt es wiederum darauf an, dass das Regime über einen intern institutionalisierten Mechanismus verfügt, der solche Regelverstöße ahndet. Regime mit nicht vorhandenen oder lediglich unzureichenden Sanktionsmechanismen laufen immer Gefahr den

207 Das Regimekonzept entstammt der Theorie der Internationalen Beziehungen (vgl. Krasner 1983), wo es benutzt wird, um das Internationale politische System zu charakterisieren, was unterhalb der Ebene hierarchischer Staatsstrukturen operiert, zugleich aber mehr ist, als eine freiwillige Netzwerkstruktur. Scharpf enthebt diese spezielle Organisationsstruktur ihrem eigentlichen theoretischen Rahmen und verweist darauf, dass einerseits auch das Privatrecht und das Zivilprozessrecht als Modelle hoch entwickelter Verhandlungsregime angesehen werden können (Scharpf 2006:241), dass Beispiele wie kommunales Management, technische Normen sowie manche Regelungen des Zivilund Handelsrechts, aber auch des internationalen Zahlungsverkehrs oder des Zugangs zum Internet, Regimecharakter haben, weil es hier weniger darauf ankommt, wie die jeweiligen Regelungen definiert werden, als das alle Beteiligten sich an diese halten (Scharpf 2005:708). 
zusätzlichen Effizienz- und Effektivitätsvorteil, der grundsätzlich durch gemeinsame Regeln und Richtlinien geschaffen wird, wieder zu 'verspielen', da die Nichtahndung einzelner Regelverstöße dazu führt, dass die Mitglieder einen Vorteil darin sehen, die Vorschriften und ihre ausgehandelten Verpflichtungen zu umgehen oder zu verletzten, was ebenfalls, wenn auch auf anderen Wegen als im ersten Fall, dazu dazu führt, dass das Regime erodiert (ebd.).

Wenn dies nicht der Fall ist, sprich wenn eine starke Mehrheit existiert, die sich gegenüber den anderen Regimemitgliedern solidarisch verhält und wenn es wirksame Sanktionsmechanismen gegen Regelverstöße gibt, dann werden durch Regimestrukturen die kooperativen Momente der Interaktion durch die Ausrichtung und die Verpflichtung auf die gemeinsamen Regeln zugleich erleichtert und begrenzt: Erleichtert werden sie durch die Möglichkeit, dass einseitig positive Ausbeutungsstrategien verhindert werden können und die Verhandlungsführung durch die Orientierung an gemeinsam aufgestellten Reglungen erleichtert wird. Insofern internalisieren Regimestrukturen einen deutlichen Effizienz- und Effektivitätsvorteil, der allen drei Verhandlungsformen in unterschiedlichem Maße zugute kommt: Tauschverhandlungen sind unter Regimebedingungen immer dann effizient und effektiv, wenn sie von einem Regelungsrahmen profitieren können, der nicht-kooperative Interaktionsorientierungen ahndet und der zudem die Einhaltung der kooperativ ausgehandelten Ergebnisse absichert. Auch Problemlösungsverhandlungen, die in einem bereits als unstrittig und akzeptabel anerkannten normativen und rechtlichen Rahmen stattfinden, sind effizienter, weil die Entscheidungsalternativen minimiert, aber nur insoweit begrenzt werden, als dass nicht immer wieder von neuem ein gemeinsamer Status Quo ausgehandelt werden muss. In Fällen positiver Koordination wirken diese Vorteile zusammen, bewirken aber einen Wechsel des Modus Vivendi:

Unter Regimebedingungen bietet sich die Möglichkeit Verfahrensvorschriften zu etablieren, die darauf ausgerichtet sind, in Frage kommende Handlungsoptionen grob zu selektieren, diese dann hinsichtlich der dabei jeweils in Frage kommenden Verteilungsrelationen zu analysieren und darüber abzustimmen, welche dieser Optionen zum Vorteil aller ist. Durch diesen, erst auf der Ebene von Regimestrukturen möglichen Wechsel von der positiven zur negativen Koordination (siehe 1c) sinken die Transaktionskosten selbst bei komplexen und schwierigen Entscheidungen. Allerdings ist hierbei zu beachten, dass Mehrheitsentscheidungen unter den strukturellen Bedingungen von Regimestrukturen nur dann zu effizienten und effektiven Ergebnissen führen, wenn es sich dabei um Projekte in der "Zone gemeinsamer Attraktivität“ handelt (ebd., 214). ${ }^{208}$

\footnotetext{
208 Scharpf verdeutlicht diese Gedanken anhand umfangreicher spieltheoretischer Darlegungen zum sogenannten "Concorcet-Arrow-Paradox« bezüglich zyklisch instabiler Mehrheiten (vgl. ebd., 261-265), das er wiederum in den Kontext der »strukturinduzierten Gleichgewichte» (vgl. ebd., 265-269) stellt.
} 
Ist das Projekt für alle Beteiligten mehr oder weniger von Vorteil (was der Fall ist, wenn deren Status Quo nicht negativ verändert wird) und lässt dieses den Parteien zugleich genug Freiheiten, wie sie mit dem Ergebnis der Entscheidung umgehen können (geringere Implementationsvorschriften sind ja durchaus auch ein gewichtiger Vorteil) so kommt es zumeist auf sehr effizienten Wegen zur Abstimmung, was aber auf der Ebene der Effektivität bedeuten kann, dass die Implementation nur solange vorangetrieben wird, wie sich keine lohnenswertere Alternative zu der einstigen Entscheidung ergibt. Geht es indes um Projekte von denen einige klar profitieren, andere nicht direkt betroffen und einige absehbar negativ betroffen sein werden, so werden sowohl die Profiteure als auch die möglichen Verlierer alles daran setzen, dass die Abstimmung zu ihren Gunsten ausfällt, womit dem eigentlichen Modus der Mehrheitsentscheidung dann doch (unkoordinierte) Tausch- und Problemlösungsverhandlungen vorausgehen, die auf der Effizienzebene wiederum zu deutlich höheren Transaktionskosten führen und überdies hinaus schmälert dies auch die Effektivität, da die Verlierer der Mehrheitsentscheidung nach Möglichkeiten suchen werden, die Abstimmung auf anderen Wegen zu hintergehen, bei den nächsten Verhandlungen respektive Abstimmungen ihren Vorteil auf Kosten der einstigen Gewinner zu maximieren oder aber dazu neigen, die Regeln des Regimes zu brechen oder aus ihm auszutreten.

Dies wiederum hat Rückwirkungen sowohl auf die Stabilität als auch die Effizienz- und Effektivitätskriterien des Regimes: Da sich bei Verträgen über den Austausch von Gütern und Leistungen, wo nicht alle zugleich profitieren können, die Teilnehmenden von Mehrheitsentscheidungen an die gemeinsam getroffenen Entscheidungen halten müssen, auch wenn diese ihren ursprünglichen Interessen zuwiderlaufen, ist die Erzielung als auch die Implementation ausgehandelter Vereinbarungen durch Mehrheitsentscheidungen von der Existenz (endogener und im Zweifelsfall auch exogener) Implementations- und Sanktionsmechanismen abhängig. ${ }^{209}$ Diese können durch Anreize, d.h. durch das Versprechen von Belohnungen, oder durch die Androhung von Strafe sowie im Nachhinein durch Sanktionen wirken: Die auf Belohnung basierende Macht ist in jedem Fall kostspielig, denn sie muss im Falle ihrer Ankündigung auch gewährleistet werden, wenn die Glaubhaftigkeit solcher Mechanismen keinen Schaden nehmen soll. Die Androhung von Strafe hat gegenüber solchen positiven Anreizen den bedeutsamen

Diese Überlegungen können und sollen hier nicht im Einzelnen nachvollzogen werden. Entscheidend dabei ist mit Blick auf die Kriterien guter Politik, dass Mehrheitsentscheidungen nur unter sehr restriktiven Voraussetzungen überhaupt effiziente und effektive Ergebnisse produzieren (siehe die weiteren Ausführungen im Text) und dass dies, so Scharpf, der Grund dafür ist, warum die Mehrheitsregel eine nicht utilitaristische normative Begründung braucht, mit der sich Scharpf im Zuge seiner legitimationstheoretischen Überlegungen (siehe 5.3.3.2.2) auseinandersetzt.

${ }^{209}$ Wenn es sich um exogene Sanktionsmenchanismen handelt, spricht Scharpf von „Selbstorganisation im Schatten des Staates" (Scharpf 2006:327-329), was bereits des Öfteren unter dem Stichwort der 'Verhandlungen im Schatten der Hierarchie' angesprochen wurde. 
Vorteil, dass es oft ausreicht, wenn diese glaubhaft angedroht wird (ebd., 253), was im Fall von endogenen Mechanismen wirkungslos sein kann, denn unter bestimmten Umständen ist ein Austritt aus dem Regime die individuell bessere Option. ${ }^{210}$ Nachträgliche Sanktionierungen können indes noch viel kostspieliger sein als Anreize, denn Sanktionen bergen immer das Risiko, dass sie schnell zu Widerstand führen, da die Betroffenen dann eher zu kompetitiven und feindlichen als zu kooperativen und solidarischen Interaktionsorientierungen neigen (ebd., 253-254). An diesem Punkt steigen die Transaktionskosten für weitere Verhandlungen und Abstimmungen an. Handelt es sich zu guter Letzt um Abstimmungen zu Projekten, die allen mehr oder weniger missfallen, dann werden alle Teilnehmer dazu neigen, das Problem einfach nach hinten zu verschieben (Stichwort: Abdiskontierung), auch wenn sich die Möglichkeiten effiziente und effektive Lösungen zu finden dabei für alle verschlechtern (Stichwort: Abwärtsspiralen).

All dies zeigt, dass Mehrheitsentscheidungen auf den zweiten Blick betrachtet deutlich ineffizienter und ineffektiver sind, als es erstmal den Anschein hat. Die Anforderungen aber auch die Restriktionen an den Mechanismus der Mehrheitswahl werden noch einmal höher, umso weniger klar ist, wer genau von Entscheidungen wie betroffen sein wird, wenn der Kreis derjenigen, die eine Entscheidung treffen nicht deckungsgleich mit dem Kreis derjenigen ist, die von einer Entscheidung betroffen sind und wenn ein Austritt aus dem Verhandlungsregime de jure oder de facto nicht real möglich ist, ${ }^{211}$ was in den sogenannten Zwangsverhandlungs- bzw. Kollektiventscheidungssystemen der Fall ist, die Scharpf als letzte, komplexeste Stufe der strukturellen Organisation kennzeichnet (ebd., 260).

c) Zwangsverhandlungs- bzw. Kollektiventscheidungssysteme sind in Scharpfs Systematik dadurch charakterisiert, dass die beteiligten Parteien aus realen, rechtlichen oder institutionellen Gründen nicht mehr dazu in der Lage sind, ihre Ziele durch einseitiges Handeln zu erreichen, da kollektives Handeln von der einstimmigen bzw. konsensualen

${ }^{210}$ Scharpf räumt ein, dass es sich hierbei um graduelle Unterschiede zwischen Freiwilligkeit und Zwang handelt: So kann das Verlassen eines Regimes, in das man einst freiwillig eingetreten ist so kostspielig sein, dass ein Austritt nicht realisierbar erscheint. Umgekehrt kann die Verweigerung zu kooperativem Verhalten in Zwangsverhandlungssystemen einem Austritt aus diesen gleichkommen, auch wenn de facto oder de jure gar keine Austrittsoption besteht (Scharpf 2006:244).

${ }^{211}$ Zu denken ist hier an bspw. an Orgisationen, die vom Staat geschaffen werden um bestimmte wirtschaftliche und/oder gesellschaftliche Problemfelder eigenständig zu regulieren (bspw. selbstverwaltete Versicherungskörperschaften im Bereich der Altersvorsorge wie die deutsche Rentenversicherung, im Bereich der Gesundheitsvorsorge wie die Krankenkassen oder der Arbeitslosenunterstützung wie die Jobcenter oder die Agenturen für Arbeit). Hier geben gesetzliche Regelungen die institutionellen Rahmenbedingungen vor, in denen Verhandlungen stattfinden, was auch bedeutet, dass diese Körperschaften sich dazu verflichten müssen Regelungen im Sinne der Allgemeinheit zu finden, denn ansonsten sanktioniert diese der Staat, kassiert die Regelung ein, ersetzt die Teilnehmer durch andere oder hat im drastischsten Fall die Option die 'entliehene` Regelungskompetenz zurück zu nehmen. 
Billigung aller Beteiligten abhängig ist (ebd., 244). ${ }^{212}$ Solche Konstellationen können durch funktionale und räumliche Interdependenzen entstehen oder sich dadurch bilden, dass Netzwerke und Regime in Kollektiventscheidungssysteme transformieren, indem diese Einstimmigkeits- und/oder Konsensregeln institutionalisieren. Entscheidend hierbei ist, dass einmal getroffene Regelungen auch nur mit der Zustimmung aller wieder verändert werden können, so dass Zwangsverhandlungs- bzw. Kollektiventscheidungssysteme systembedingt dazu tendieren, dass der erreichte Status Quo 'zementiert ' und substanzielle Reformen anfällig für Blockaden sind, da die Nutznießer des Status Quo alle Reformen blockieren oder exorbitante Ausgleichszahlungen für ihre Zustimmung zu Veränderungen verlangen können (ebd., 245). ${ }^{213}$ Entscheidend ist daher zum einen, dass sich die in Netzwerken und Regimen möglichen Lösungsmöglichkeiten wesentlich von denjenigen in Zwangsverhandlungs- bzw. Kollektiventscheidungssystemen unterscheiden: Sowohl Netzwerk- als auch Regimestrukturen sind dem Sinn und Zweck nach selbstregulative Koordinationsstrukturen, da es sich hierbei um freiwillige Kooperationen handelt, bei denen die potenziell gleichgerichteten Interessen der Beteiligten nach Kooperation und gemeinsamer Regelung verwirklicht werden (Scharpf 2005:739). Das bedeutet, dass sowohl der Eintritt als auch der Austritt in und aus Netzwerken und Regimen von rationalen Erwägungen des persönlichen Gewinns und des Verlusts abhängig sind und dass sich selbstregulative Strukturen immer nur dann ausbilden, wenn alle Verhandlungspartner sich davon (materielle und immaterielle) Vorteile versprechen. Umgekehrt erodieren diese, wenn es darüber zu einer unverhältnismäßigen Beschränkung der individuellen Handlungsmöglichkeiten und/oder Ressourcen kommt oder sie transformieren, wenn die Koordinationsstrukturen für die Teilnehmer noch eine Funktion erfüllen (Scharpf 1992:97).

212 Die Etablierung von Einstimmigkeits- oder Konsensregeln ist bei vielen Joint-Ventures zwischen Unternehmen und Regierungskoaltionen über Tarifverhandlungen etabliert, sie findet in Konstellationen des 'Divided Government' Anwendung, wo Regierung und Opposition gezwungenermaßen zusammen arbeiten müssen und sie kommt in föderalen Strukturen und in der Europäischen Union zur Anwendung, wo Entscheidungen sowohl de jure als auch de facto von der einstimmigen Billigung der Länder bzw. der Mitgliedsstaaten abhängen (Scharpf 1999b:112).

${ }^{213}$ Diese Tendenzen von Zwangsverhandlungs- bzw. Kollektiventscheidungssystemen zur Blockade lassen sich bspw. an föderalen Strukturen aufzeigen (Scharpf 1993b:36). Scharpf hat zur Charakterisierung dieser Konstellation den Begriff der „Politikverflechtung“ und die Diagnose der „Politikverflechtungsfalle“ geprägt (vgl. Scharpf, Reissert \& Schnabel 1976, Scharpf 1999c): Politikverflechtung liegt vor, wenn wesentliche öffentliche Aufgaben nicht autonom von Akteuren der jeweiligen politischen Ebenen, sondern nur in Zusammenarbeit von mehreren Akteuren über die Ebenen hinweg wahrgenommen werden können und wenn neben den hierarchischen Beziehungen zwischen den verschiedenen Ebenen informelle Formen der horizontalen und vertikalen Kooperation zur Abstimmung von Entscheidungen notwendig sind. Die Politikverflechtungsfalle ist wiederum, anders als in vielen populärwissenschaftlichen Arbeiten verwendet, keine Bezeichnung für eine Blockade durch Vetospieler innerhalb verpflochtener Politikbereiche, sondern tritt ein, wenn zusätzlich zu einer Blockadesituation in Sachentscheidungen auch die institutionellen Änderungen einer schrittweisen Entflechtung unmöglich sind (Benz 2003:220). Hiermit wird folglich die systemisch bedingte Unfähigkeit des politischen Systems bezeichnet, institutionelle Änderungen zur Auflösung von Entscheidungsblockaden herbeizuführen. 
Dabei ändert sich jedoch der Interaktionsmodus: Die Teilnehmer von Kollektiventscheidungssystemen müssen sich auf Regeln einigen, die ihnen das Recht zum Austritt verweigern und die ihnen die Möglichkeit zu einseitigem Handeln kategorisch verweigern (Scharpf 2006:245). Darüber wird die Möglichkeit verhindert in den Modus der negativen Koordination zu wechseln, um Verhandlungsblockaden zu verhindern und die Transaktionskosten zu senken. Allerdings können hier andere Ressourcen eingesetzt werden, um andere dazu zu bewegen und ggf. dazu zu zwingen gegen ihre eigenen Präferenzen zu handeln. Das können Ressourcen recht unterschiedlicher Art sein, wie 'Blaming und Shaming' wenn Loyalität eine internalisierte Norm ist, Vertrauensentzug oder sozialer Ausschluss wenn soziale Normen Konformität verlangen, Arbeitsplatzverlust oder der Entzug von Leistungen wenn Regeln verletzt oder verbindliche Ansprüche nicht erfüllt werden bis hin zu administrativen und strafrechtlichen Sanktionen wenn Folgebereitschaft geboten und Zuwiderhandlungen nicht erlaubt sind. Insbesondere was die 'harten ' Zwangsmittel wie administrative und strafrechtliche Sanktionen seitens des Staates angeht, macht Scharpf deutlich, dass dabei oftmals vergessen wird, dass auch diese Sanktionen nur dann ihre Wirksamkeit entfalten, solange den Akteuren diese Sanktionen nicht egal sind und sie sich diesen nicht auf anderen Wegen entziehen können. Das bedeutet mit anderen Worten: Die hierarchische Steuerung ist in eine größere strukturelle Konstellation eingebettet, welche die Möglichkeit einseitiger Anordnung einerseits ermöglicht und andererseits auch begrenzt, da diejenigen, die über hierarchische Autorität verfügen bzw. diese einsetzen können, um andere zur Konformität zu zwingen, selbst keine einseitigen Handlungsoptionen mehr besitzen. Auch staatliche Akteure müssen daher, obgleich sie über die ultimativen Zwangsmittel verfügen, kooperative und verhandelnde Interaktionsmodi annehmen und einüben und der Einsatz von Zwangsmitteln sollte auch bei ihnen gut überlegt sein und strategisch abgewogen werden (ebd., 284).

Trotz all der offenkundigen Nachteile und Probleme der Kollektiventscheidungssysteme gegenüber den Netwerken und Regimen muss beachtet werden, dass diese ab einem bestimmten Punkt funktionale Notwendigkeiten sind: Selbstregulative Interaktionsformen (Netzwerke und Regime) können mit ihrem Modus der Freiwilligkeit zwar politisch orientierte Tausch- und Problemlösungsverhandlungen hervorbringen und zumindest Regime können sich auch eines, an sich demokratischen Abstimmungsmodus (Mehrheitsentscheidungen) bedienen, rein strukturell betrachtet bleiben selbstregulative Koordinationsstrukturen aber unterhalb der Schwelle genuin politischer, und das heißt hier hierarchischer, Verhandlungs- und Koordinationsstrukturen. Dies ist der Fall, weil in der Politik auch dann Entscheidungen getroffen werden müssen, wenn die Kosten-Nutzen-Relation für die Entscheider nicht klar ist (im übrigen auch dann, wenn die Folgen der Entscheidungen unsicher und ungewiss sind), wenn es um die Lösung 
von (re-)distributiven Problematiken geht, die über den Kreis derjenigen Akteure hinaus gehen, die befugt sind, eine Entscheidung darüber treffen und wenn die zur Abstimmung respektive zur Verhandlung stehenden Regelungen nicht von relativem Nutzen für alle Beteiligten sind, sprich wenn es um Verteilungsproblematiken geht, die den Status Quo bestimmter Parteien kurz-, mittel- und langfristig gefährden. Politische Herrschaft zieht ihre Berechtigung und Legitimation (siehe 5.3.3.2.2) aus der Kombination diese drei Umstände: Es braucht den Staat, staatliche Intervention und Hierarchie, weil alle anderen gesellschaftlichen und sozialen Koordinationsformen wie bspw. der Markt und die Gemeinschaft in ganz bestimmten Fällen nicht ausreichend sind, um zu kollektiv verbindlichen Regelungen zu gelangen, die alle Beteiligten effektiv binden. Diese Fälle treten immer dann ein, wenn die Art des zu lösenden Problems als Kollektivproblematik angesehen wird und wenn es dabei zugleich um zu lösende Verteilungsprobleme geht, von denen nicht alle zugleich profitieren können. Da eine kollektiv verbindliche und zudem gemeinwohlorietierte und sozial gerechte Lösung von Kollektivproblematiken auf freiwilliger Basis nur unter ganz bestimmten Bedingungen wahrscheinlich ist, nämlich dann, wenn alle Beteiligten davon in irgendeiner Weise profitieren, müssen Kollektiventscheidungen und distributiven Regelungen, wenn dies nicht der Fall ist, kollektive Entscheidungen aber dennoch von Nöten sind, durch die Anwendung staatlicher Macht initiiert und im Zweifelsfall hierarchisch 'erzwungen ' werden (Scharpf 1993b:36). ${ }^{214}$

Zwangsverhandlungs- bzw. Kollektiventscheidungssysteme sind bezüglich der in ihnen möglichen Anwendung von Hierarchie - analog zu den Regimen und den in ihnen möglichen Mehrheitsentscheidungen - einerseits auf der strukturellen Ebene strategischer Interaktion (als hierarchische Organisationen) und andererseits auf der prozeduralen Ebene in Bezug auf den Interaktionsmodus (als hierarchische Steuerung) definiert. ${ }^{215}$ Hierarchische Organisation bedeutet, dass man sich über die Präferenzen anderer Akteure hinwegsetzen kann. ${ }^{216}$ Dabei ist zu beachten, dass selbst innerhalb verflochtener

${ }^{214}$ Dies zeigt, dass hierarchische Steuerung immer gegenüber denjenigen gerechtfertigt, d.h. politisch verantwortet und legitimiert, werden muss, die eine Nicht-Einmischung des Staates einklagen und die die Nichtregelung der staatlich 'erzwungenen` Regelung vorgezogen hätten (zu den Legitimationskriterien siehe 5.3.3.2.2). Dabei ist im Rahmen einer verhandlungsdemokratischer Modellierung wiederum entscheidend, wie kollektiv verbindliche Entscheidungen im Rahmen der Interaktionen zwischen den aktiv am Prozess der Entscheidungsfindung beteiligten Akteuren zustande kommen und wie diese Interaktionen innerhalb der politisch-staatlichen Sphäre verknüpft werden (Scharpf 2006:282).

${ }^{215}$ Zur Erinnerung: Auf der prozeduralen Ebene wurde hierarchische Steuerung bereits so definiert, dass diese auf einem Über- und Unterordnungsverhältnis basiert und dass autonom gesetzte Steuerungsimperative auch autonom durchgesetzt werden können (siehe 5.3.2.1).

${ }^{216} \mathrm{Ob}$ dies auch legitim, gerecht, verantwortbar oder gemeinwohlförderlich ist und warum, ist genau wie bei Mehrheitsentscheidungen, wo eine Minderheit von 49,99\% von einer Mehrheit von 51, 01\% überstimmt werden kann, eine normative Frage und wird mit Bezug auf die Legitimationskriterien behandelt (siehe 5.3.3.2.2). 
hierachischer Organisationen unterschiedliche Interdepenzformen auftreten, die unterschiedliche Formen hierarchischer Koordination erfordern und damit die dargelegten Verhandlungsformen in je unterschiedlicher Weise notwendig machen (Scharpf 2006: 288-301):

Erstens gibt es die Form der gepoolten Interdependenz, wo mehrere Akteure auf einen gemeinsamen Ressourcenpool zugreifen. Diese Interdepenzform kann, analog zu den Regimen, durch gemeinsame Regeln bewältigt werden. Können solche Regeln gemeinschaftlich gefunden werden, dann ist hierarchische Koordination als solche effizient und effektiv. Dies ist sie aber nur solange, wie sich der Ressourcenpool nicht substanziell verändert, da dies zu einer Anpassung bzw. Änderung der Regeln zwingt. Wenn dies eintritt, dann müssen Umverteilungen realisiert werden, sprich es müssen Tauschverhandlungen geführt werden. Da es unter den Bedingungen des Kooperations- und Koordinationszwanges und eines einstimmigen oder konsensualen Abstimmungsmodus hierbei trategisch für jeden Beteiligten von Vorteil ist, wenn sie Vorschläge und Positionen einbringen, die ihre Maximalforderungen beinhalten und wenn sie bei der Aushandlung solange keine Kooperationsbereitschaft erkennen lassen, bis diejenigen, die an einer Einigung stärker interessiert sind als andere 'einknicken 'und Konzessionen anbieten, ist es an dieser Stelle effizienzsteigernd, wenn die hierarchischen Akteure auf Koppel- und Paketgeschäfte zurückgreifen, selbst wenn diese hinsichtlich ihrer Effizienz und Effektivität beschränkt sind (1a).

Bei der sequenziellen Interdependenz wiederum, bei welcher der Output des einen Akteurs zum Input des anderen wird, ist eine sorgfältige, aber nicht zu unflexible Planung der einzelnen Arbeitsschritte erforderlich, damit jeder Akteur seine Aufgaben selbstständig erfüllen kann. Lassen sich solche arbeitsteiligen Strukturen etablieren, ist die hierarchische Koordination effizient und effektiv. Sie ist dies aber nur solange, bis Probleme auftauchen, die mit den vorhandenen Strukturen nicht mehr zufriedenstellend oder gar nicht gelöst werden können. In solchen Fällen müssen Problemlösungsverhandlungen geführt werden. Auch hierbei entsteht durch den Zwang zur Einstimmigkeit bzw. zum Konsens die Tendenz, dass sich die Lösungsvorschläge schrittweise in Richtung derjenigen opponierenden Partei verschieben, die die Verhandlungen am stärksten blockiert. Da diese nur sehr schwer zur Mitarbeit und zur Zustimmung zu bewegen ist, steigen auch hier die Transaktionskosten für alle Verhandlungsteilnehmer an und die dabei entstehenden Ergebnisse tendieren entweder zu substanzloser Symbolpolitik oder zu inhaltlichen Kompromissen auf dem kleinsten gemeinsamen Nenner, die keiner Partei 'schmecken ' und für die keiner die politische Verantwortung übernehmen will. Wirkliche Strukturreformen lassen sich daher nur dann erzielen, wenn Kommunikations- und Verhandlungsmodi etabliert werden, die die Akteure in die Lage versetzen, sich mit Hilfe von Argumenten über die Beschaffenheit des Problems und 
die bestmögliche Lösung zu verständigen (siehe 1b). Solche Deliberationsregeln sind aber selbst recht anspruchsvoll und binden Zeit und Ressourcen, da sie ein festes Netzwerk, Vertrauen zwischen den Akteuren und die kommunikativen Fähigkeiten und Fertigkeiten seitens der Akteure benötigen.

Letztendlich gibt es noch die reziproke Interdependenz, bei der die Entscheidungen und Arbeitsschritte wechselseitig voneinander abhängen, was eine dauerhafte, direkte Interaktion der beteiligten Akteure erfordert, sprich wo zugleich Probleme gelöst als auch Verteilungen ausgehandelt werden müssen, so dass positiv koordiniert werden muss (siehe 1c). Hier - und wohl bemerkt erst hier! - tritt das Verhandlungsdilemma in vollem Maße auf, da selbst ein Mindestmaß an Kooperationsbereitschaft, das aber in Form von gegenseitigem Aufeinandereinlassen und nicht rein strategischem Argumentieren für die Produktionsdimension notwendig ist, in dieser Konstellation bestraft wird. Daher geht es hier darum konstruktiv mit dem Verhandlungsdilemma umzugehen. Scharpf schlägt hierfür eine prozedurale Trennung von Verteilungs- und Produktionsfragen vor, um die tendenzielle Inkompatibilität von 'Argumentieren 'und 'Bargaining` zu überbrücken (ebd., 228-229), denn wenn Rahmenbedingungen für die gemeinsame Suche nach produktiven Lösungen geschaffen werden können, dann kann auch eine separate Einigung über die Verteilung von Nutzen und Kosten angestrebt werden.

Im nächsten Abschnitt wird, aufbauend auf den hier dargelegten Verhandlungsformen und deren institutionelle Rahmenbedingungen, darauf eingegangen, welche verschiedenen Legitimationsformen und -argumente Scharpf in die Debatte um die Verhandlungsdemokratie einbringt und wie er diese theoretisch begründet. Dabei werden die oberhalb selbstregulativer Verhandlungsnetzwerke liegenden Verhandlungsformen immer wieder aufgegriffen und hinsichtlich ihrer Legitimationsbedarfe auf der Input- und Outputseite spezifiziert.

\subsection{Die Kriterien legitimer Politik}

Da Scharpf den Anspruch einer an realen Umständen orientierten Demokratietheorie nach wie vor verfolgt, stellt sich für ihn auch in legitimationstheoretischer Hinsicht die Frage, was aus der, die Politik legitimierenden Rhetorik von der demokratischen Selbstbestimmung auf der Inputseite und der politischen Verantwortung der Regierenden für eine gute Politik auf der Outputseite wird, wenn das Modell des nach außen souveränen und nach innen hierarchisch gegliederten Staats aufgrund der quantitativen Zunahmen und dem qualitativen Bedeutungszuwachs politischer Verhandlungssysteme innerhalb aber vor allem oberhalb der Nationalstaaten im Mindesten relativiert werden muss (Scharpf 1993b:25). Scharpfs geht diesbezüglich davon aus, dass Staatlichkeit unabwendbar Hierarchie bedeutet, dass aber demokratische Selbstbe- 
stimmung als auch politische Verantwortlichkeit auf institutionelle Arrangements angewiesen sind, die ein zirkuläres Verhältnis zwischen den Regierenden und den Regierten herstellen (Scharpf 2006:300). In modernen Demokratien wird dieses Verhältnis in erster Linie durch Wahlmechanismen erzeugt, in zweiter Linie - und zunehmend relevant - durch Verhandlungslösungen zwischen politischen und gesellschaftlichen Akteuren. Eingedenk der zunehmenden Wichtigkeit von Verhandlungslösungen will Scharpf zeigen, dass diese unter verhandlungsdemokratischen Bedingungen eigene Legitimationsquellen und -grundlagen haben.

Dabei bleibt er seiner bereits 1970 eingeschlagenen Linie im Grundsatz treu, indem er nach wie vor einen additiven Ansatz vertritt (siehe 5.3.3.1). Für Scharpf ist die Qualität demokratischer Verfahren zur Herstellung kollektiv verbindlicher Entscheidungen vor allem auf der Inputseite von wesentlicher Bedeutung (Scharpf 2005:711), während sie auf der Outputseite lediglich eine instrumentelle Bedeutung hat, da es hier darum geht, ob und inwiefern Verhandlungslösungen, Mehrheitsentscheidungen und demokratisch kontrollierte hierarchische Entscheidungen geeignet sind, eine allgemeinwohlorientierte Politik hervor zu bringen (Scharpf 2006:256). Die Verbindung zwischen Input- und Outputlegitimation ergibt sich hierbei darüber, dass alle Legitimationsargumente auf Sachverhalte verweisen, „welche die moralische Verpflichtung begründen, Gebote und Verbote auch dann zu befolgen, wenn diese den eigenen Interessen zuwiderlaufen“ (Scharpf 2005:705), so dass politisches Handeln für Scharpf immer (erst) dann legitimationsbedürftig ist, wenn dadurch die kollektiven Präferenzen und/ oder die individuellen Interessen der von politischen Entscheidungen Betroffenen berührt oder verletzt werden (ebd., 711). Mit anderen Worten: In einem Gemeinwesen, in dem alle BürgerInnen eine solidarische Gemeinschaftsorientierung besitzen, es eine kollektive Präferenz auf ein ganz bestimmtes, von allen akzeptiertes Gemeinwohl gibt und in dem jeder Einzelne das bekommt und realisieren kann, was er will und damit zugleich niemandem schadet, braucht es keine Legitimation, da alles legitim ist. Da die Realität anders aussieht - gerade im Kontext des wirtschaftlichen Drucks auf das Sozialstaatsprinzip und der politisch gewollten, aber innerstaatlich nur schwer zu verarbeitenden, Auslagerung politischer Entscheidungen auf die Ebenen oberhalb des Nationalstaats - spielen sowohl die inputorientierten als auch die outputorientierten Legitimationsargumente zur Rechtfertigung politischer Herrschaft, politischer Entscheidungsfindungen und politischer Ergebnisse eine zentrale Rolle.

Daher ist es für Scharpf nicht nur theoretisch sondern auch praktisch problematisch, wenn nur eine ganz bestimmte Quelle der Legitimation fokussiert wird, obgleich alle Legitimationsquellen - mal mehr, mal weniger und anhängig vom politischen Setting von entscheidender Bedeutung sind. Daher insistiert Scharpf darauf, dass die unterschiedlichen Legitimationsweisen nicht gegeneinander ausgespielt werden dürften, 
sondern als zusammengehörig gedacht werden müssen (Scharpf 1998b:84). Da der Status bzw. der Stellenwert der politischen Legitimation bei Scharpf zudem umso wichtiger wird, je mehr die Internationalisierung des Staates, die Globalisierung der Wirtschaft und damit die Mehrebenenpolitik (Multi-Level-Governance) an Gewicht gewinnen, geht es ihm darum, wie die real vorfindlichen Formen staatlicher und überstaatlicher Herrschaft gerechtfertigt und begründet werden können, gerade weil er darauf insistiert, dass Legitimation auch unter diesen Umständen die „unhintergehbare normative Funktionsbasis der Demokratie“ und damit "die funktionale Voraussetzung der Möglichkeit von zugleich effizienter und liberaler Herrschaft“ ist (Scharpf 2005:707).

Dabei macht Scharpf - in Rückgriff auf Abraham Lincolns wohl berühmteste Rede (die "Gettysburg Address") - drei verschiedene Legitimationsweisen aus: Die »Herrschaft des Volkes", die "Herrschaft durch das Volk» und die "Herrschaft für das Volk«. Die ersten beiden Legitimationsweisen sind inputorientierz und die letzte ist outputorientiert. Auf der Inputseite des politischen Prozesses geht es darum, dass politische Entscheidungen, aber auch die politischen Institutionen und Prozesse wirksam aus den kollektiven Präferenzen der Mitglieder des Gemeinwesens abgeleitet werden können (ebd., 709), so dass sich die Legitimität (die Akzeptabilität) der politischen Ordnung letztlich aus der Zustimmung (der faktischen Akzeptanz) der Regierten zu konkreten politischen Entscheidungen ableitet (Scharpf 2006:255). Dabei macht es indes einen Unterschied, ob man von der Existenz einer kollektiven Identität wie bei der Legitimationsweise der »Herrschaft des Volkes« ausgeht oder annimmt, dass solche kollektiven Identitäten erst geschaffen werden müssen und divers sind, wie im Falle der »Herrschaft durch das Volk«. Zugleich geht es auf der Outputseite des politischen Prozesses darum, „dass die Ausübung der Herrschaft die Interessen der Mitglieder des Gemeinwesens wirksam fördern soll“ (Scharpf 2005:711), so dass „politische Programme Legitimität beanspruchen können, wenn sie dem Allge-meinwohl dienen und den Kriterien der sozialen Gerechtigkeit genügen." (Scharpf 2006:255) Mit Scharpfs eigenen Worten:

„Unter nicht lediglich theoretischen, sondern realen politischen Bedingungen betont die inputorientierte Perspektive die Herrschaft durch das Volk. Politische Entscheidungen sind demnach legitim, wenn sie von den authentischen Präferenzen der Mitglieder einer Gemeinschaft abgleitet werden können. Im Unterschied dazu stellt die outputorientierte Perspektive den Aspekt der Herrschaft für das Volk in den Vordergrund. Danach sind politische Entscheidungen legitim, wenn und weil sie auf wirksame Weise das allgemeine Wohl im jeweiligen Gemeinwesen fördern. Obwohl beide Argumente komplementär verwendet werden, sind sie analytisch zu unterscheiden und sie beruhen bei separater Betrachtung auf höchst unterschiedlichen Vorbedingungen." (Scharpf 1999b:16) 
Um diese Vorbedingungen der drei Legitimationsweisen darzulegen, werden im Folgenden zuerst die beiden Formen der Inputlegitimation, d.h. a) die Herrschaft des Volkes und b) die Herrschaft durch das Volk, dargelegt, da deren Abgrenzung im Rahmen der komplexen Demokratietheorie entscheidend ist. Dann wird auf die Bedingungen der Outputlegitimation, sprich c) der Herrschaft für das Volk, eingegangen.

\section{a) Die Herrschaft des Volkes}

Das klassische demokratietheoretische Argument für die Inputlegitimation ist die Legitimation durch die Herrschaft des Volkes. Es entstammt den republikanischen Demokratietheorien, die sich nur auf die nationalstaatliche Konstellation beziehen (mussten und konnten). In diesen Demokratietheorien geht es, in je unterschiedlicher Ausformulierung, vor allem um die Souveränität des Volkes durch die Aspekte der kollektiven Identität, der direkten Partizipation und der Entscheidungsfindung durch Konsens (Scharpf 1999b:17). Dieses sind bis heute zentrale Elemente auch in vielen modernen Demokratietheorien. Scharpf interessieren deren realpolitische Entsprechungen: Direkte Partizipation und Konsens sind für ihn plausibel, wenn der empirische Schwerpunkt auf lokalen Problemen liegt, bei denen die von einer politischen Entscheidung betroffenen Personen oder deren demokratisch legitimierte VertreterInnen zur Beratung über Problemlösungen zusammen kommen, die im gemeinsamen Interesse aller liegen und denen deshalb alle, auf die eine oder andere Weise zustimmen können (ebd.). Die Überzeugungskraft dieser Ausformulierung der „Partizipationsformel“ schwindet jedoch in dem Maße, wie sich die Distanz zwischen den von einer Entscheidung direkt betroffenen Personen und ihren VertreterInnen vergrößert und die „Konsensformel“ versagt immer dann, wenn Lösungen zum Nutzen aller durch alle nicht möglich sind und demzufolge Mehrheitsentscheidungen getroffen werden müssen (ebd.) Daher muss unter theoretischen als auch praktischen Gesichtspunkten bei der Legitimationsweise der Herrschaft des Volkes und in Bezug auf den Topos der Volkssouveränität in erster Linie die Rechtfertigung der Mehrheitsregel in Bezug auf verhandlungsdemokratische Annahmen untersucht werden: ${ }^{217}$

Hinter der Volkssouveränität steht, so Scharpf, die Vorstellung, dass die Art des Gemeinwesens und dessen Regeln jederzeit zur Disposition des Souveräns stehen müsse, „da ihre Geltung von ihrer immer erneuerten impliziten oder expliziten Anerkennung des Volkes abhängig bleiben." (Scharpf 1999a:682) Dahinter steht wiederum die Norm,

217 Scharpf stellt heraus, dass die Legitimität der Mehrheitsregel zwar „eine historisch plausible Annahme für Staaten [war], in denen die demokratische Verantwortlichkeit absolutistischen oder diktatorischen Regimen abgerungen werden musste, die Grenzen majoritärer Legitimation werden jedoch immer dann offensichtlich, wenn man Gemeinwesen mit tiefen ethischen, religiösen oder ideologischen Konfliktlinien betrachtet oder politische Gebilde wie die EU oder die UN, die keinen hohen politischen Integrationsgrad erreichen." (Scharpf 2006:260) 
dass der 'Wille des Volkes' zu geschehen habe, es sei denn, dieser habe sich selbst konstitutionellen Beschränkungen unterworfen." (ebd.) Eine der wichtigsten konstitutionellen Beschränkungen in modernen Demokratien ist die Mehrheitsregel (siehe unter Effizienz- und Effektivitätsgesichtspunkten 2b). Damit die Mehrheitsregel normativ vertretbar ist, braucht es das Vertrauen, dass die Mehrheit ihre Macht nicht zur Schädigung der Minderheit einsetzen wird, was wiederum die begründete Unterstellung erfordert, dass „die Präferenzfunktion jedes einzelnen Mitglieds des Gemeinwesens die Wohlfahrt aller Mitglieder bereits als ein Argument enthält." (Scharpf 1999b: 18) Mit anderen Worten: Die Akzeptanz der persönlichen 'Einbußen', die dem Einzelnen im Namen der Allg-meinheit aufgebürdet werden, setzt eine kollektive Identität voraus, die sich in der Regel „auf präexistente geschichtliche, sprachliche, kulturelle oder ethnische Gemeinsamkeiten gründet." (ebd.) ${ }^{218}$

Scharpf versucht dieses Moment analytisch einzufangen: Unter den Bedingungen, dass es eine Kongruenz zwischen dem Kreis der an Prozessen Beteiligten und dem Kreis der von den Entscheidungen Betroffenen gibt (Scharpf 1998a: 235), d.h. entweder unter Bedingungen einer politischen Welt, die aus souveränen, untereinander nicht reziprok vernetzten Nationalstaaten bestand, in der die Rede vom Volk und deren Souveränität konstituierend für das Verständnis von Staatlichkeit und Demokratie war oder aber moderner gefasst, unter den Bedingungen normativer Regimestrukturen, wo die Mitglieder nur für diejenigen Regelungen erlassen, die Teil des Regimes sind, die aber austreten können, wenn sie davon keinen Nutzen mehr haben, ist die Mehrheitsregel bereits auf Grundlage einer solchen Kongruenzannahme legitim.

Eine so begründete Legitimität der Mehrheitsregel qua Volkssouveränität und/oder gemeinsamem Nutzen, die beide hinter der Legitimationsweise der »Herrschaft des Volkes" stehen können, wird indes immer dann fraglich, wenn die Kongruenzannahme nicht mehr ohne weiteres haltbar ist. Eben dies ist ganz offensichtlich auf den Ebenen oberhalb des Nationalstaats der Fall. Zudem verweist Scharpf auf den Umstand, dass der Gemeinsamkeitsglaube auch innerhalb der Nationalstaaten unter den Bedingungen prekär wird, in denen die regelungsbedürftigen gesellschaftlichen Anliegen die Grenzen des Nationalstaates überschreiten (Scharpf 1999a: 673). Scharpf insistiert daher darauf, dass die Legitimationsweise der Herrschaft des Volkes zwar eine wichtige

\footnotetext{
${ }^{218}$ Vielen Varianten inputorientierter Demokratietheorien liegt die Annahme einer kollektiven Identität zugrunde (Scharpf 1999b:18). Sowohl die klassischen republikanischen Entwürf, als auch die modernen Varianten deliberativer und diskursiver Demokratietheorie lassen nur die Berücksichtigung von verallgemeinerungsfähigen Interessen zu, so dass alles von der vorausgesetzten Identität der solidarischen Gemeinschaft abhängt (ebd.). Entscheidend hierbei ist, dass die Grenzen solcher Gemeinschaften nicht fixierbar sind und dass in diesen Varianten der Demokratietheorie eine präexistente kollektive Identität umso wichtiger wird, je öfter nicht-verallgemeinerungsfähige Interessen geopfert werden müssen, sprich wenn partielle Sonderopfer für bestimmte Bevölkerungsgruppen anfallen, die weder verhindert noch kompensiert werden können.
} 
ist, diese aber nur auf Regimestrukturen sowie kleine politische Gemeinwesen mit einer recht homogenen Bevölkerung unter den Bedingungen eines Minimalstaats anwendbar ist, der fast ausschließlich mit der Sicherung der inneren und äußeren Sicherheit befasst ist (Scharpf 2006:259). Da der Nationalstaat indes selbst einen Wandlungsund Veränderungsprozess erfährt, indem die Nationalstaaten politische Entscheidungen auf supranationale Ebenen verlagern und diese wiederum Externalitäten für die nationale Politik produzieren, hat dies theoretische und praktische Konsequenzen für die Anwendbarkeit inputorientierter Legitimationsargumente bei Zugrundlegung verhandlungsdemokratischer Settings:

Zum einen merkt Scharpf an, dass die auf der Idee der Volkssouveränität basierende Legitimationsweise der Herrschaft des Volkes nur eine verzerrte Bewertung von Verhandlungs/ösungen zulässt. Dies ist der Fall, weil verhandlungsdemokratische Strukturen innerhalb und oberhalb der Nationalstaaten die für die Volkssouveränität konstitutive Annahme gefährden, dass sich die Legitimität des Politischen aus der „unverfälschten Durchsetzung des jeweiligen internen 'Mehrheitswillens' in jeder der beteiligten Einheiten“ ergibt (Scharpf 1992:95). ${ }^{219}$ Diese Verknüpfung zwischen Kongruenzannahme, Mehrheitsregel und demokratischer Legitimation führt dazu, dass „die real existierenden intra- und transnationalen Abhängigkeiten und Verhandlungszwänge nur als Regression oder Verfallserscheinungen beklagt und kritisiert werden [können]“ (ebd., 103) und dass jegliche Formen von Verhandlungslösungen als „freilich unvermeidliche, aber defizitäre Notlösungen“ (Scharpf 1993b:34) angesehen werden. Die politischen Abhängigkeiten und Verhandlungszwänge sind jedoch schlicht Fakten, mit denen man konstruktiv umgehen muss (Scharpf 1998a:236). Scharpf plädiert daher dafür, dass Verhandlungslösungen nicht lediglich „als coupierte Varianten einer allein legitimationskräftigen Mehrheitsdemokratie“ (ebd.) verstanden werden sollten, denn die politische Legitimation ergibt sich nicht allein daraus, dass klassische Mehrheitsregierungen den Wählern im strengen Sinne direkt verantwortlich sind und dass diese souverän entscheiden können. Daher ist es für Scharpf problematisch, dass viele Demokratietheorien den politisch Verantwortlichen weit mehr Souveränität und Ereignisbeherrschung zuschreiben, als diese selbst unter günstigen Umständen haben können und dass Verhandlungszwänge und -blockaden, die nun einmal zum notwendigen poli-

219 Dabei ist für Scharpf von vornherein klar, dass es keine normativ überzeugenden inputbezogenen Legitimationsargumente geben kann, die versuchen die „ungefilterten, wahren Präferenzen“ der BürgerInnen umzusetzen (scharpf 2005:710). Vielmehr geht Scharpf grundlegend davon aus, dass es bereits als politische Aufgabenstellung angesehen werden muss, Mechanismen und Prozesse innerhalb der Gesellschaft zu etablieren, zu installieren und zu fördern, die auf eine möglichst „informierte und reflektierte Präferenzbildung“ zur Bildung eines „Gemeinsinns“ führen. Dies ist neben anderen Gründen ein weiterer wichtiger Aspekt, warum Scharpf sich vornehmlich mit der inputorientierten Legitimationsweise der Herrschaft durch das Volk beschäftigt, da eben diese Annahme hier konstitutiv ist (siehe b). 
tischen Alltag in vernetzten Verhandlungsdemokratien gehören, vorschnell als Demokratiedefizite bewertet werden (Scharpf 1992:103). Das Hauptaugenmerk von Scharpf Kritik richtet sich daher auf die mit der Legitimationsweise der Herrschaft des Volkes verbundenen Souveränitätsunterstellungen sowohl in Hinblick auf den Staat als auch in Hinblick auf die Souveränität des Volkes:

Der moderne Nationalstaat ist zwar nach wie vor souverän in dem Sinne, dass er das Recht besitzt, gesetzliche und andere Regelungen in seinem Territorium aufzustellen und seine Politik daran auszurichten, Scharpf weist aber darauf hin, dass die in vielen Demokratietheorien unhinterfragte Souveränitätsunterstellung zwei 'realistischer ' Korrekturen bedarf: Zum einen geht es dabei um „die Anerkennung exogener Restriktionen moderner Staatlichkeit, die sich aus der gesteigerten internationalen Mobilität von ökonomischen Faktoren und Akteuren ergeben." (Scharpf 1999a: 683) Zum anderen geht es ihm um die Anerkennung der wechselseitigen Abhängigkeiten der politischen Akteure von den Entscheidungen anderer Akteure und Staaten. Beide Arten von Abhängigkeit verletzen, so Scharpf, „die staatlichen Omnipotenz- und Souveränitätsphantasien der Demokratietheorie.“ (ebd.) Im Zuge dessen weist er auf den Umstand hin, dass die Theorie kooperativer Staatlichkeit bereits mit einer notwendigen Relativierung der Vorstellung des souveränen Staates verbunden ist und dass eben diese Relativierung auch in die Modelle der Mehrebenenpolitik (Multi-Level-Governance) eingegangen ist, so dass es hierfür geeignete Vorlagen gäbe.

Auf die postulierte Souveränität des Volkes schauend, merkt Scharpf zudem an, dass Wahlentscheidungen nur in sehr seltenen Fällen überhaupt als Entscheidungen über materielle politische Fragen bzw. als Mechanismus zur Herstellung kollektiv verbindlicher Entscheidungen interpretiert, sprich diese nur in einem sehr beschränkten Sinne überhaupt als Ausdruck der Souveränität des Volkes verstanden werden können. Vielmehr dienen diese in erster Linie der allgemeinen Legitimation des politischen Systems und ferner als regelmäßige demokratische Kontrolle der hierarchischen Regierungsgewalt (Scharpf 2006:281). Bei Verhandlungen, bei denen es gerade nicht darum gehen kann und soll, immer alle BürgerInnen zugleich an Entscheidungen zu beteiligen, sondern wo es aus staatlicher Sicht um eine gezielte und informierte Auswahl derjenigen Akteure geht, die zu der anstehenden Entscheidungsfindung einen substanziellen Beitrag leisten können, können und sollten nicht nur diejenigen beteiligt werden, die sowieso partizipieren wollen und können, sondern auch die herangezogen und eingebunden werden, die von den verhandelten Entscheidungen möglicherweise negativ betroffen sind oder sich gegen eine kooperative Lösung sträuben, sprich Vetospieler sind. Dabei macht Scharpf auf die normativ-empirische Ambivalenz hinsichtlich des Einsatzes der Mehrheitsregel und hierarchischer Autorität innerhalb solcher Verhandlungskonstellationen aufmerksam (Scharpf 2006:283): 
Aus der Perspektive des normativen Individualismus, von dem Scharpf grundlegend ausgeht, muss selbst eine durch kollektive Identität bzw. gemeinsamen Nutzen abgestützte Mehrheitsregel und eine demokratisch legitimierte und 'eingehegte' hierarchische Autorität seitens staatlicher Akteure als Anwendung substanziell überlegender, einseitiger Macht verstanden werden, die die Entscheidungsfreiheit der Akteure einschränkt oder sogar gänzlich beseitigen kann, so dass es vollkommen rational ist, wenn diese versuchen, jede unerwünschte Form der Fremdbestimmung und Einmischung in ihren Handlungsspielraum möglichst zu unterbinden oder zu umgehen. Vor dem Hintergrund empirischer Analysen hingegen erscheint der gezielte Einsatz von Mehrheitsentscheidungen und Hierarchie für alle Beteiligten von freiwilligen und selbstregulativen Verhandlungssystemen als grundsätzlich erstrebenswert, da solche, den Lösungsfindungsprozess verkürzende und den Verteilungskampf einhegende, Abstimmungen als auch der kontrollierende und gewährleistende 'Schatten der Hierarchie' die Transaktionskosten kooperativen Handelns verringern (ebd., 323-329).

Scharpf legt auf Grundlage dieser Überlegungen die Relativierung und Neuverortung der Mehrheitsregel innerhalb verhandlungsdemokratischer Settings dar: Politische Entscheidungen innerhalb kooperativer Staaten und politischer Mehrebenensysteme werden sowohl durch eine indirekte aber möglichst inklusive Partizipation der Bürgerlnnen (bei Wahlen) als auch durch eine direkte, aber exklusive Partizipation (in Verhandlungen) legitimiert, wobei die Durchsetzung und Einhaltung politischer Entscheidungen von einem Staatsapparat als Letztgarant überwacht, ermöglicht und gewährleistet wird, der über die überlegene Fähigkeit zu hierarchischer Weisung und über die Möglichkeit zur Anwendung von Zwangsmitteln verfügt. Vor diesem Hintergrund gelangt Scharpfs zu seiner durch empirische Studien gestützten aber dennoch normativen Hintergrundthese, dass verhandlungsdemokratisch geprägte Staaten die Funktion haben, durch das gezielte Arrangieren der vorfindbaren Akteurskonstellationen und das Moderieren zwischen den dabei beteiligten Einzelinteressen, die partikularen Handlungsstrategien der Akteure in gesamtgesellschaftlich verbindliche und gemeinwohlorientierte Entscheidungen zu transformieren (Scharpf 1992:95). Dabei geht Scharpf davon aus, dass die Legitimationsargumente dazu dienen, politische Entscheidungen auch dann zu akzeptieren, wenn sie den individuellen Bedürfnissen der BürgerInnen zuwiderlaufen. Daher ist das letztlich entscheidende Moment der Inputlegitimation für ihn, dass die Politik die Grundlagen und Bedingungen schafft, damit sich überhaupt so etwas wie ein 'Gemeinsinn ' ausbilden und ausprägen kann. Eben dies ist für Scharpf die basale Grundlage der Inputlegitimation, die sich aber nicht im klassischen Sinne über eine Herrschaft des Volkes herbeiführen lässt, sondern nur durch eine Herrschaft durch das Volk (Scharpf 1992:95, Scharpf 1998a:235, Scharpf 2006:270-278). 
b) Die Herrschaft durch das Volk

Bei der Legitimationsweise der Herrschaft durch das Volk geht es um die Bedingungen und um die Schaffung kollektiver Identitäten. Scharpf nennt diese je nach Kontext „,solidarische Interaktionsorientierungen ", ${ }^{220}$ wenn es um Betrachtungen auf der individuellen Ebene der Akteure geht (Scharpf 2005:738) oder Gemeinsinn, ${ }^{221}$ wenn es um Interaktionen auf der kollektiven Ebene des sozialen und politischen Handelns geht (Scharpf 1993b:25). Analog zu seiner, bereits in den 1970er Jahren vertretenen, Position insistiert Scharpf darauf, dass die Politik dafür Sorge tragen müsse, dass sich solidarische Orientierungen und gemeinsinnige Handlungsweisen in der Gesellschaft im Laufe der Sozialisation entwickeln (ebd., 26). Hier nimmt Scharpf einen grundlegenden Aspekt der kontextualistischen Demokratietheorien auf (siehe 5.2.1), indem er davon ausgeht, dass die Befähigung zur Bürgerschaft nicht einfach unterstellt, sondern aktiv gefördert, ausgebildet und unterstützt werden muss und dass diese im Laufe der individuellen Sozialisation zu entwickelnden Qualitäten wesentlich dafür sind, dass sich Personen als BürgerInnen fühlen und eine politische Gemeinschaft bilden (ebd.). Da Identität und Solidarität, psychologisch und empirisch betrachtet, indes nur auf der Ebene von Primärgruppen universell vorausgesetzt werden können und alles darüber hinausgehende von Prozessen und Strategien der Identifikation und der Identitätsbildung abhängig ist, verlangen Solidarität und Gemeinschaftsorientierung, so Scharpf in Rückgriff auf Norbert Elias, die Möglichkeit der Entwicklung von exklusiven „Ich-Identitäten“ zu inklusiven, die Mitglieder der Gemeinschaft einschließenden „Wir-Identitäten“, die genutzt werden können, um für jeweils verschiedene Zwecke sowohl das gemeinsame Interesse, die Normen der Solidarität als auch die, für die Outputlegitimation wichtigen, Normen der sozialen Gerechtigkeit (siehe c) abzugrenzen (Scharpf 2006:271). Das impliziert erneut eine ganze Reihe bedeutsamer Annahmen, die zur Charakterisierung der Legitimationsweise der Herrschaft des Volkes angeführt, aber auch noch einmal zur Abgrenzung von der Legitimationsweise der Herrschaft des Volkes herangezogen werden können:

Zum einen bedeutet diese 'konstruktivistische' und gerade nicht organisch-ontologische Sichtweise auf kollektive Identität, dass es nicht das eine kollektive Interesse, sondern eine Vielzahl kollektiver Präferenzen innerhalb politischer Gemeinwesen gibt. Dabei geht Scharpf davon aus, dass deren Bildung durch vorhandene Ähnlichkeiten oder

${ }^{220}$ Zur Erinnerung: Solidarische Interaktionsorientierungen (siehe 5.3.2.3) sind bei Scharpf durch uneingeschränkte Kooperationsbereitschaft gekennzeichnet, was heißt, dass ein Vorteil für den einen genauso positiv bewertet wird, wie ein Vorteil für den anderen und dass es unter dieser Orientierung dazu kommen kann, dass Ego Verluste hinnimmt, solange diese durch größere Verluste für Alter gerechtfertigt sind (vgl. Scharpf 2006:152-153).

${ }^{221}$ Gemeinsinn meint hier im Folgenden die Bereitschaft der sozial und politisch Handelnden, sich an dem Ideal des Gemeinwohls (siehe c) zu orientieren und dieses durch solidarische Verhaltens- und Handlungsweisen wirksam werden zu lassen (Münkler\&Fischer 2001:9). 
Gemeinsamkeiten angeleitet wird, so dass jeder Mensch als Besitzer variabler, exklusiver Ich-Identitäten auch inklusive Wir-Identitäten bspw. als Familienmitglied, als KonsumentIn, als Arbeitnehmerln, Arbeitgeberln, als Mitglied in sozialen Gruppen oder Vereinen, als Zugehöriger bestimmter religiöser, sozialer und/oder ethnischer Gruppen annehmen und entwickeln kann und dass diese durch gemeinsame Regeln, Konventionen, Routinen, Rituale etc. gefestigt werden (ebd., 271-272). Das bedeutet aber mitnichten, dass diese Gruppenidentitäten universalisierbar seien oder dazu führen würden, dass viele Gruppenidentitäten sich schlicht zu einer großen gesellschaftlichen Kollektividentität aufsummieren ließen. Vielmehr zeigen die sozialpsychologischen Untersuchungen im Rahmen der "Theorie der sozialen Identität « immer wieder, dass Gruppenidentitäten sich nicht nur durch Binnenprozesse bilden, sondern auch dadurch charakterisieren lassen, dass sie sich vergleichen und von anderen 'Fremdgruppen' abgrenzen. Gerade in ihrer kollektiven Identität nicht verfestigte Gruppen sowie Gruppen, die sehr rigide Regeln und Konventionen haben, neigen dazu, sich gegen andere Gruppen und deren Identität abgrenzen, was umso vehementer geschieht, umso stärker diese in Konkurrenz zueinander stehen oder sich in ihrer Identität bedrohen. Dennoch gibt es in jeder Gesellschaft Regeln und Mechanismen, die im abstrakten gesellschaftlichen Kontext einen Gemeinsinn und im konkreten Interaktionszusammenhang solidarische Orientierungen ermöglichen. Solche politischen, sozialen und rechtlichen Freiheiten und Beschränkungen können indes nur eine Grundlage schaffen, sie können die soziomoralischen Ressourcen zur Bildung überpartialer kollektiver Identitäten aber nicht garantieren. Daher merkt Scharpf, dem Böckenförde Dilemma folgend ${ }^{222}$ an, dass zur Förderung des Gemeinsinns nur der Weg offen stünde, diejenigen gesellschaftlichen und politischen Rahmenbedingungen zu schaffen, die die Reproduktion der soziomoralischen Ressourcen zumindest wahrscheinlich machen (Scharpf 1993b:27).

Zum zweiten ist damit klar, dass die Normen der Solidarität nicht nur von politischen und rechtlichen Regelungen abhängen, die es ermöglichen, dass sich die sozialen Gruppen entfalten können und zugleich vor Schädigungen durch andere Gruppen bewahrt werden, sondern der 'soziale Friede' hängt auch von den politischen Ergebnissen und hierbei ganz zentral von den Lösungen gesellschaftlicher Verteilungsfragen und -probleme ab (siehe c). In praktischer Hinsicht bedeutet dies - und hier sucht Scharpf den

${ }^{222}$ Ernst-Wolfgang Böckenförde stellt in dem nach inm benannten Böckenförde-Dilemma heraus, dass „[d]er freiheitlich-säkularisierte Staat (...) von Voraussetzungen [lebt], die er selbst nicht garantieren kann. Das ist das große Wagnis, das er, um der Freiheit willen, eingegangen ist. Als freiheitlicher Staat kann er einerseits nur bestehen, wenn sich die Freiheit, die er seinen Bürgern gewährt, von innen her, aus der moralischen Substanz des einzelnen und der Homogenität der Gesellschaft, reguliert. Anderseits kann er diese inneren Regulierungskräfte nicht von sich aus, das heißt mit den Mitteln des Rechtszwanges und autoritativen Gebots zu garantieren suchen, ohne seine Freiheitlichkeit aufzugeben und - auf säkularisierter Ebene - in jenen Totalitätsanspruch zurückzufallen, aus dem er in den konfessionellen Bürgerkriegen herausgeführt hat." (Böckenförde 1991:60) 
Anschluss an die deliberative Demokratietheorie - dass es bei der Inputlegitimation in Form der Herrschaft durch das Volk vor allem um die Ermöglichung dessen geht, was Habermas die „Einbeziehung des Anderen“ nennt (vgl. Habermas 1996a): ${ }^{223}$ Wenn kollektive Wir-Identitäten nicht einfach vorhanden, sondern geschaffen, befördert und ermöglicht werden müssen, wenn dies nur zum Teil eine politische Aufgabe sein kann und darf und wenn die Normen der Solidarität je nach Kontext verschiedentlich ausgedeutet und unterschiedlich relevant sind, dann ist für Scharpf die kritische Auseinandersetzung mit der, von Habermas im Rahmen seines Schleusenmodells (siehe 5.2.2.1) eingebrachten, „diskursiven 'Veredelung' (...) der freien Präferenzbildung“ (Scharpf 2005:710) zentral. Scharpf nimmt hier einige Aspekte der deliberativen Demokratietheorie auf, weicht aber zugleich in anderen Punkten davon ab und hebt wieder andere Aspekte hervor, die vor allem dann über den Rahmen der deliberativen Demokratietheorie hinausgehen, wenn es ihm um verhandlungsdemokratische Strukturen oberhalb des Nationalstaates geht:

Scharpf sind die philosophischen Entwürfe der deliberativen Demokratietheorie zu abstrakt und normativ zu stark überfrachtet, obgleich er viele Ansatzpunkte der deliberativen Demokratietheorie auf der Inputseite des politischen Prozesses teilt und diese auch nicht in Abrede stellt. Aber es ist bezeichnend, dass er kurz nach der Veröffentlichung der deliberativen Demokratietheorie durch Habermas darauf verweist, dass „eine der verflochtenen Politik angemessene normative Theorie der demokratischen Partizipation und der politischen Verantwortlichkeit, an der sich die öffentliche Diskussion orientieren könnte, nirgendwo in Sicht [ist]“, obgleich diese „ganz oben auf der politiktheoretischen Traktandenliste stehen [sollte]." (Scharpf 1992:108) Dies ist nicht als Herabwürdigung der deliberativen Demokratietheorie zu verstehen, sondern als (berechtigte) Kritik Scharpfs, dass die deliberative Demokratietheorie unlösbar an bestimmte Prämissen gebunden ist - nämlich das Vorhandensein einer kollektiven Identität, solidarische Interaktionsorientierungen und die Gemeinwohlorientierung der BürgerInnen (Scharpf 2006:270-276) - und dass eben diese dazu führen, dass:

„wir es mit einer praktischen Inkompatibilität zwischen dem konsensuellen Ideal der deliberativen Demokratie einerseits und den notwendigen Folgen der Wettbewerbsdemokratie andererseits zu tun [haben]. Zumindest unter normalen Bedingungen ist die Erwartung, dass die Regierungspartei und die Opposition sich auf diejenigen politischen Maßnahmen einigen, die dem Gemeinwohl am zuträg-

${ }^{223}$ Indem die deliberative Demokratietheorie (siehe 5.2.2.1) versucht über den Austausch von Informationen und Argumenten zu möglichst konsensfähigen gesellschaftlich-politischen Problemlösungen zu gelangen und damit die Leistungsfähigkeit des soziopolitischen Systems zu steigern (Blatter 2007: 277-278), wird in der deliberativen Demokratietheorie ebenfalls der Übergang von der input- zur outputorientierten Perspektive vollzogen. Daher ist speziell die deliberative Demokratie, neben ihrer Eignung wichtige Aspekte für die Legitimationsweise der Herrschaft durch das Volk zu liefern und trotz aller Kritik, die Scharpf daran äußert, besonders interessant für ihn (Scharpf 1999:674). 
lichsten sind (...) unrealistisch (...). Die institutionellen Rollen beider Seiten und die mit diesen Rollen verbundenen Anreize schließen kooperatives Problemlösen normalerweise aus." (ebd., 278)

Analytisch - und das heißt bei Scharpf immer auch spieltheoretisch - betrachtet, beruht der, in der deliberativen Demokratietheorie angestrebte diskursive Problemlösungsmodus auf den empirisch 'wacheligen` Prämissen, dass es eine richtige, d.h. für alle Beteiligten akzeptable Lösung von Problemen geben muss, dass die Akteure aufrichtig und unabhängig voneinander für diese Lösung stimmen und dass jeder Einzelne diese Lösung als wahre und richtige Lösung erkennen kann (ebd., 258). Die spieltheoretische Rekonstruktion dieser Annahmen verdeutlicht aber, dass diese Annahmen nur auf der Grundlage reiner Koordinationsspiele Sinn machen (vgl. ebd., 130), womit die Bedeutung von sozialen Konflikten ignoriert wird (ebd., 258). Der diskursive Modus scheitert dementsprechend bei Konstellationen, wo es sich um Multi-Akteur-Konstellationen handelt, bei denen mehrere Ergebnisse möglich sind, die sich zudem in ihren Verteilungsfolgen unterscheiden oder wo Nullsummenkonflikte ausgehandelt werden müssen (vgl. ebd., 131-148).

Daher grenzt sich Scharpf gegen die Versuche der deliberativen Demokratietheorie ab, die Vielzahl der Verhandlungsformen, den starken Einfluss der Hierarchie darauf, den politischen Wettbewerb und die kompetitiven Interaktionsorientierungen zugunsten von selbstorganisierten Netzwerkstrukturen durch öffentliche Diskurse zwischen Zivilgesellschaft und Politik, kooperativen Problemlösungsverfahren und kontrafaktisch unterstellten solidarischen Gemeinwohlorientierungen der BürgerInnen zu neutralisieren (ebd., 269-278). Zwar stimmt Scharpf den VertreterInnen der deliberativen Demokratietheorien durchaus zu, dass sich die meisten BürgerInnen des Unterschieds zwischen ihren privaten Interessen und den öffentlichen kollektiven Präferenzen bewusst seien und dass die philosophisch angemessene Lösung des Politischen eine prozedurale ist, bei der das Wählen im Endeffekt „nur die letzte Stufe eines organisierten öffentlichen Diskurses [ist], in dem individuelle Präferenzen und Wahrnehmungen im Hinblick auf kollektive Ergebnisse entwickelt, kommuniziert, konkretisiert, kritisiert, verteidigt und verändert werden“ (ebd., 270), er wendet aber zugleich ein, dass diejenigen, die für öffentliche Diskurse mit Hilfe von wahren und richtigen Argumenten unter den Grundbedingungen einer idealen Sprechsituation plädieren, zu stark die policytheoretischen Aspekte ihrer demokratietheoretischen Modellierungen vernachlässigen.

Für Scharpf bedeutet dies in erster Linie, dass es nicht allein um prozedurale, sondern auch um strukturelle Aspekte geht und dass es nicht um die Legitimität eines ganzen politischen Systemim Allgemeinen, sondern um die Legitimität ganz bestimmter Strukturen und Verfahren gehen muss, in deren Rahmen politische Entscheidungen gefällt und politische Maßnahmen erarbeitet werden (ebd., 39). Daher ist es für ihn essentiell, 
dass die strukturellen Bedingungen der Verhandlungsformen, die spezifischen Interaktionsmodi von öffentlichen Diskursen, die in realen politischen Verhandlungen zugehörigen Kämpfe um Verteilungsfragen sowie die dabei vorherrschenden kompetitiven Interaktionsorientierungen auch in der Deliberation mehr Beachtung finden. Daher kann Scharpfs Anschluss an die deliberative Demokratietheorie nur selektiv sein:

Er hält sowohl an seinem normativ-instrumentellen Verständnis von Demokratie als auch an seinem pluralistisch-individualistischen Ansatz fest, bei dem er den Menschen in aller erster Linie eigeninteressiertes Handeln unterstellt. Dieses, so sieht es Scharpf ebenfalls, muss im politischen Raum gebändigt und auf Gemeinschaftsorientierungen hin trainiert werden. Zwar spricht auch Habermas davon, dass es prozeduraler Vorkehrungen bedarf, die „den Fluss der diskursiven Meinungs- und Willensbildung so regulieren, dass ihre falliblen Ergebnisse die Vermutung der Vernünftigkeit für sich haben" (Habermas 1992a:291), Scharpf merkt dazu aber an, dass sich der Maßstab der Vernünftigkeit bei Habermas auf normative Diskurse und weniger auf Fragen der Realisierbarkeit und rechtlichen und ökonomischen Restriktionen bezieht (Scharpf 1999a: 689). Insofern können auch in normativen Diskursen die externen Voraussetzungen nicht ausgeblendet werden, wenn man davon ausgehen will, dass ein moralisches Sollen immer auch ein Können implizieren muss (ebd.). Scharpf fokussiert daher vornehmlich auf die realen Möglichkeiten der Deliberation in der politischen Öffentlichkeit. Dazu gehört, dass die Politik die öffentlichen Debatten, wenn diese es in den Fokus der medialen Aufmerksamkeit schaffen, so oder so als relevante Inputs wahrnimmt und behandelt, ungeachtet dessen, ob deren Ergebnisse nun dem Anspruch der Vernünftigkeit gerecht werden können oder nicht (ebd., 690). Vielmehr gibt Scharpf zu bedenken, dass ungeachtet des zwanglosen Zwangs des besseren Arguments sowohl strukturelle (siehe 5.3.3.2.1) als auch machtbasierte Faktoren darüber entscheiden, welche Positionen und Meinungen sich in der Politik im Allgemeinen und in Verhandlungen im Besonderen durchsetzen können und welche nicht (ebd., 684-686 sowie Schmidt 2000: 264 und ausf. 265-282). Des Weiteren relativiert Scharpf die, für die deliberative Demokratietheorie so entscheidende Annahme, dass politische Beteiligungen, Deliberationen und Diskurse auf Basis idealer Sprechsituationen zu einer besseren oder gar zur besten, d.h. wahren und richtigen, Lösung führen, denn dies ist für Scharpf weniger eine Frage des Wollens als des Könnens:

Selbst wenn es möglich wäre, nur die für die deliberative Demokratietheorie so entscheidende Verhandlungsform des Problemlösens zu betrachten, gehen auch auf Probleme und deren Lösung fokussierte Verhandlungsformen in zunehmendem Maße aus multilateralen Verhandlungen hervor. Bei denen steht in Frage, ob es den einzelnen VerhandlungspartnerInnen, die ja zumeist VertreterInnen staatlicher, verbandlicher und/oder korporativer Interessen sind, überhaupt möglich ist, eine kooperative Pro- 
blemlösung anzustreben, die im Sinne aller ist, sprich ob die Vertreterlnnen sich ein konsensfähiges Verhalten überhaupt leisten können, ohne dadurch ihren Status als delegierte VerhandlungsführerInnen zu verlieren (Scharpf 1993b:41). Wenn man also Problemlösungsverhandlungen nicht in den engen normativen Grenzen rationaler Diskurse versteht und hierbei akteursspezifische Eigeninteressen, strategische Kommunikationsformen und unterschiedliche Grade der Verhandlungsmacht einbezieht, wird deutlich, dass politische Problemlösungsverhandlungen zumeist 'Spitzentreffen 'sind, so dass sich kooperative und solidarische Handlungs- sowie Kompromiss- und Konsensorientierungen erst einmal nur zwischen den Verhandlungsführerlnnen ausprägen können, die diese dann gegenüber ihrer mitgliedschaftlichen Basis oder ihrer Geschäftsführung vertreten und verteidigen müssen. Dabei kann es immer zu dem 'Trade off' kommen, dass die VerhandlungsfüherInnen sich entscheiden müssen, ob sie sich an ihren internen Zielen orientieren und ihre Positionen und Ziele 'durchboxen 'oder sich um kooperative Strategien und für alle Beteiligten zumindest annähernd vorteilhafte, Kompromisse bemühen. Wenn sie sich ausschließlich oder vornehmlich an den internen Zielen orientieren, dann können die Spielräume der Kooperation nicht in Gänze ausgeschöpft werden. Andererseits ist es für Scharpf aber auch problematisch, dass die Suche nach einer effizienten und effektiven kooperativen Verhandlungslösung dem Ziel der demokratischen Repräsentation der internen Interessen geopfert wird.

Daher verweist er auf die Notwendigkeit, aber auch die Schwierigkeiten der doppelten Orientierung sowohl an der authentischen Repräsentation bzw. Vertretung der internen Interessen und Positionen einerseits als auch der Effizienz und Effektivität der kooperativ zu findenen Verhandlungsergebnisse andererseits. Dies bezeichnet Scharpf wiederum abstrahiert auf den verhandlungsdemokratischen Kontext als „EffektivitätsAuthentizitäts-Dilemma" (Scharpf 1999b:12): In Verhandlungsdemokratien ringen die Ziele der Wahrnehmung demokratischer Integration, Partizipation und Inklusion mit den Zielen der effizienten und effektiven Entscheidungsfindung sowie der Schaffung von Wohlfahrtseffizienz und sozialer Gerechtigkeit. Scharpf macht damit deutlich, dass ein Mehr des einen nicht automatisch ein Mehr des anderen nach sich zieht, wie dies in der deliberativen Demokratietheorie suggeriert wird, wenn davon ausgegangen wird, dass über den Austausch von Informationen und Argumenten zu möglichst konsensfähigen Problemlösungen gelangt werden kann, worüber zugleich die Leistungsfähigkeit des soziopolitischen Systems gesteigert wird. Vielmehr zeigt er auf, dass zwischen beiden Zielen eine unauflösbare Spannung besteht, was sowohl für die Möglichkeiten der Inputlegitimation als auch der Outputlegitimation relevant ist (siehe c).

Zu guter Letzt zeigt Scharpf inwiefern das Authentizitäts-Effektivitäts-Dilemma auch für die Mehrebenenpolitik sowie die europäische und internationale Politik und deren demokratische Bewertung und Beurteilung relevant ist: Scharpf bemängelt die Ver- 
suche der VertreterInnen inputorientierter Demokratietheorien der europäischen und internationalen Politik per se ein Demokratie- und Legitimationsdefizit aufgrund des, im Vergleich zu den Nationalstaaten, Mangels an demokratischer Repräsentation zu attestieren, da er dies für ein viel zu kurz gegriffenes und pauschales Urteil hält. Ohne Zweifel ist es so, dass es auf der europäischen und internationalen Ebene weder präexistenzielle kollektive Identitäten gibt noch kann in internationalen und europäischen Verhandlungen auf quantitativ und qualitativ ausgeprägte und etablierte Wir-Identifikationen zurückgegriffen werden, über die solidarische Normen und Gemeinsinn vermittelt werden (Scharpf 1999b:12). Daher merkt Scharpf auch immer wieder kritisch an, dass die Voraussetzbarkeit realer und tragfähiger Wir-Identitäten jenseits des $\mathrm{Na}$ tionalstaats „zur Achillesferse“ aller inputorientierten Legitimations- und Demokratietheorien wird (Scharpf 1998a:235). Die Versuche der VertreterInnen der deliberativen Demokratietheorie, die nationale Demokratie auf Basis kollektiver Identitäten in eine postnationale Diskursgemeinschaft zu überführen, erscheinen Scharpf daher auch „in die falsche Richtung gedacht." (ebd.)

Scharpf verweist vielmehr auf eine alternative Sichtweise der Problematik europäischer und internationaler Mehrebenenpolitik: Für Scharpf bestehen die Problematiken nicht nur und auch nicht primär darin, dass auf der europäischen und internationalen Ebene Politikergebnisse produziert werden, die nicht oder zu wenig aus der authentischen Willensbildung der BürgerInnen hervorgehen. Vielmehr entstehen für ihn gravierende aber von den inputorientierten Demokratietheorien zu wenig beachtete Problematiken auf der Outputseite dieser politischen Systeme: Wenn solidarische Orientierungen und Gemeinsinn nur spärlich oder gar nicht vorhanden sind, dann steigt die Wahrscheinlichkeit, dass politische Entscheidungen gar nicht erst zustande kommen, weil sie entweder einseitig blockiert (in Zwangsverhandlungssystemen) oder wegen Unrentabilität in die Zukunft verschoben werden (in freiwilligen Verhandlungssystemen). Wenn diese doch zu Stande kommen (weil einige 'Mächtige` ein Interesse daran haben eine Regelung zu finden und die weniger Mächtigen zur Kooperation zwingen), haben derartige Vereinbarungen oftmals den Charakter von symbolischen Kompromissen, weil Lösungsvorschläge auf 'den kleinsten gemeinsamen Nenner' hinauslaufen, Verteilungsproblematiken in Form von Koppelgeschäften oder Paketlösungen ausgehandelt werden und nicht zuletzt weil positive Koordinationen eben jene solidarischen und gemeinsinnigen Ressourcen erfordern, die nur bedingt vorhanden sind.

Handelt es sich dann doch einmal um substanzielle Ergebnisse, sprich kommt es dazu, dass inhaltlich weitreichende Lösungen gefunden, Verteilungsproblematiken angegangen und Umverteilungsregelungen gefunden bzw. positive Koordinationen funktionieren, dann besteht immer noch das Problem, dass der Kontroll- und Sanktionsaufwand zur Einhaltung dieser Entscheidungen in hohem Maße prohibitiv ist, was wiederum ins- 
gesamt zu einer in hohem Maße ineffizienten und ineffektiven Politik führt (Scharpf 1998b:84). Ineffizienz und Ineffektivität sind keine Problematiken, die sich auf der Inputseite ergeben, sondern diese schmälern die Bilanz der Outputlegitimation (siehe c). Daher verweist Scharpf darauf, dass es für die Beurteilung der institutionellen Praxen supranationaler politischer Gebilde und ferner für die Bewertung und Beurteilung ihrer verhandlungsdemokratischen Qualitäten problematisch ist und zu kurz greift, wenn diese vor dem Hintergrund inputorientierter Kriterien bewertet und beurteilt werden, weil diese nur vor dem Hintergrund nationaler Standards Sinn machen.

Vielmehr verweist Scharf darauf, dass die Ineffizienz und Ineffektivität der politischen Ergebnisse auf der europäischen und internationalen Ebene Rückwirkungen auf die Legitimationsbilanzen der Nationalstaaten haben: Die politischen Ergebnisse, die auf diesen Ebenen getroffen werden, bedürfen der Bereitschaft und Fähigkeit nationaler politischer Akteure, ihre eigenen, viel breiteren Legitimationsquellen und -ressourcen für die Rechtfertigung europäischer und internationaler politischer Entscheidungen zu benutzen (Scharpf 1999b:31). Dies ist indes nicht so ohne weiteres möglich, da sowohl die Neuverhandlung als auch die nachträgliche Korrektur der Ergebnisse in der Regel ausgeschlossen ist, womit den damit befassten Parlamenten oder Stimmbürgern in der Regel nur die Wahl zwischen unveränderter Akzeptanz oder einer Ablehnung bleibt (Scharpf 1999a:687). Die schlichte Wahl zwischen Akzeptanz, auch ohne dass man die Ergebnisse für akzeptabel hält, oder offener Ablehnung und Auflehnung führt dazu, dass die supranationlen Effizienz- und Effektivitätsdefizite in den nationalen Legitimationsbilanzen wiederhallen (ebd., 680). Die Folgeprobleme müssen von den nationalen Politikern verarbeitet werden, sei es durch die Entwicklung eigenständiger Problemlösungen ohne Rückgriff auf die supranationalen Ebenen oder durch die Einbeziehung europäischer Entscheidungen in den Verantwortungszirkel der nationalen Demokratie (ebd., 681). Die Problematik hierbei besteht Scharpfs Ansicht gar nicht so sehr darin, administrative Problemlösungen für die Folgeprobleme der europäischen und internationalen Integration zu entwickeln, sondern darin die politische Legitimation für die durch sie selbst vorangetriebene Auslagerung politischer Entscheidungen auf die supranationalen Ebenen zu beschaffen (ebd., 682). Die Schwierigkeit besteht dabei vor allem darin, dass die nationale Politik effiziente und effektive Lösungen für gesellschaftliche Probleme aufzeigen muss, die sich in einigen Bereichen nur noch oberhalb der Nationalstaaten finden lassen und gleichzeitig darf die nationale Politik nicht aus dem Auge verlieren, dass es dabei unter bestimmten Umständen zu sozialen Umverteilungsmaßnahmen kommen kann, die eigentlich nur auf der Inputseite des politischen Prozesses zu legitimieren sind.

Im Endeffekt ergibt sich darüber das eigentliche Dilemma der Mehrebenenpolitik und die zu beachtende Schwierigkeit verhandlungsdemokratischer Settings innerhalb die- 
ser: Die nationalstaatlichen Regierungen, obgleich sie die Auslagerung politischer Entscheidungen auf die Ebenen oberhalb der Nationalstaaten aktiv anstreben und dies angesichts gemeinsamer Problematiken auch tun müssen, geraten zunehmend unter Druck, die europäischen und internationalen Regulierungen in gemeinwohlförderliche und verteilungsgerechte Politikergebnisse auf der nationalen Ebene zu überführen. Welche programmatischen Vorschläge Scharpf anbringt, damit dies gelingen kann, welche institutionellen und strukturellen Anforderungen diese 'übertragene Legitimation` erfordert, was genau dies für die nationale Politik und ihre demokratische Praxis bedeutet und inwiefern diese ihre eigenen Legitimationsquellen und -ressourcen überhaupt einsetzen können und warum sie dies trotz aller Schwierigkeiten auch sollten, wird im Folgenden Abschnitt genauer geklärt. Hierbei geht es dann nicht mehr um die Güte und die Qualität der demokratischen Gestaltung von politischen Entscheidungsfindungsprozessen, sondern, wie bereits im Vorbeigehen öfter angeklungen, um die Ergebnisse solcher Prozesse und um die Leistungen des politischen Systems, sprich um die Legitimationsweise der Herrschaft für das Volk.

c) Die Herrschaft für das Volk

Bei der Legitimationsweise der Herrschaft für das Volk geht es darum, „dass die Ausübung der Herrschaft die Interessen der Mitglieder des Gemeinwesens wirksam fördern soll." (Scharpf 2005:711) Hierbei wird die Legitimität, wie bereits im letzten Abschnitt kurz angedeutet, von der Fähigkeit des politischen Systems zur Lösung kollektiver und distributiver Probleme abgeleitet, ${ }^{224}$ deren Lösungen immer auch eine dauerhafte und multifunktionale politische Einheit erfordert (Scharpf 1999b:20). Die Anforderungen an eine solche politische Einheit sind unter der 'Brille`der Outputlegitimation deutlich geringer als die Voraussetzungen, die sich bei der Inputlegitimation stellen. Denn hierbei geht es, wie bereits ausgeführt, nicht um die Ausbildung kollektiver Präferenzen, wozu die Ressourcen der Solidarität und des Gemeinsinns erforderlich sind, sondern hier reicht es aus, wenn es einen Bestand an gemeinsamen Interessen gibt, um institutionelle Arrangements für kollektives Handeln zu rechtfertigen (ebd., 22). Daher haben die outputorientierten Legitimationsweisen eine höhere Toleranz für schwach ausgeprägte und etablierte kollektive Identitäten, zugleich sind die Mechanismen der Outputlegitimation aber kontextabhängiger und in ihrer Reichweite begrenzter:

Unter den Bedingungen der Verfassungsstaatlichkeit, der Sozialstaatlichkeit und nicht zuletzt der Anforderungen an den kooperativen Staat geht es bei der Herrschaft für das Volk um die Berücksichtigung einer größeren Zahl legitimierender Mechanismen,

\footnotetext{
${ }^{224}$ Zu erinnern ist hierbei, dass Scharpf sowohl empirische als auch normative Standards zur Beurteilung politischer Ergebnisse anwendet. Im Folgenden liegt der Fokus nicht mehr auf den empirischen Aspekten (siehe 5.3.3.2.1) sondern explizit auf den normativen Aspekten.
} 
die jeweils von bestimmten kontextuellen Faktoren abhängig sind. Zu den wichtigsten Mechanismen gehören die Verhinderung staatlicher Willkür und die Gewährleistung des demokratischen Verantwortungszirkels sowie die Absicherung sozialstaatlicher Standards und die Gemeinwohlorientierung politischer Akteure (ebd., 20). Hierbei geht es im Wesentlichen um die Gewährleistungspflichten des demokratischen Verfassungsstaats, die eine negative und eine positive Stoßrichtung haben (Scharpf 2005:710):

In negativer Hinsicht richten sich die Gewährleistungspflichten gegen die Versuchungen des Macht- und Amtsmissbrauchs für eigene oder parteiische Zwecke, wofür es in jedem demokratischen Staat verfassungsrechtliche Regelungen, Mechanismen und Vorkehrungen für den Fall gibt, dass die über hierarchische Autorität verfügenden Akteure sich an ihrem egoistischen Eigeninteresse orientieren und dass Konflikte mit Blick auf Verteilungsgerechtigkeit, zu Lasten aller anderen, zum eigenen Vorteil beigelegt werden (Scharpf 2006:298). Am wichtigsten sind hier die institutionellen Mechanismen der Gewaltenteilung (Stichwort: Checks and Balances) sowie die Institutionen des Rechtsstaats (Grundrechtekatalog, Prinzip der Gesetzmäßigkeit, unabhängige Gerichtbarkeit, freie Medien etc.), wobei diese lediglich einen Schutz vor Verstößen bieten, „die an den Rändern der Staatsgewalt stattfinden.“ (ebd., 300) Sie können nicht sicherstellen, dass politische Entscheidungen sich im Einzelnen am Gemeinwohl und der Verteilungsgerechtigkeit orientieren. Zudem gehört zu den negativen Gewährleistungspflichten zumindest unter den Bedingungen der Sozialstaatlichkeit ein grundsätzliches Verschlechterungsverbot bezüglich der Einschränkungen bzw. des Abbaus sozialstaatlicher Leistungen. In positiver Hinsicht richten sich die Gewährleistungspflichten auf das Gebot gesellschaftliche und zunehmend auch globale Problematiken politisch zu regeln, was unter den Bedingungen der Sozialstaatlichkeit immer auch heißt, dass solche Regelungen darauf bedacht sein müssen den gemeinsamen Nutzen des Volkes bzw. das Gemeinwohl aufrecht zu erhalten und zu mehren. Auf eben diese sich gegenseitig beeinflussenden Aspekte bzw. Mechanismen der Outputlegitimation muss genauer eingegangen werden:

Was die negative Auslegung der zu erbringenden Gewährleistungspflichten vor allem hinsichtlich des Verschlechterungsverbots sozialstaatlicher Standards und Leistungen angeht, sind die industriell hochentwickelten europäischen Sozialstaaten durch die Globalisierung stark herausgefordert. Dies zeige sich, so Scharpf, vor allem daran, dass die Entwicklung politischer Problemlösungen, die unter den Bedingungen der europäischen und internationalen Standortkonkurrenz Bestand haben, zu stellenweise massiven Einschnitten und Eingriffen in die Strukturen der nationalen Beschäftigungs- und sozialen Sicherungssysteme führen. Der zunehmende Verlust an Beschäftigungszahlen, der die Ausgaben der Sozialstaaten ansteigen lässt, während gleichzeitig die sozialen Finanzierungslasten als Beeinträchtigung der Wettbewerbsfähigkeit immer schärfer 
kritisiert werden, führt zu einem Anstieg an Regelungen, die mit schwer zu kompensierenden bzw. nicht kompensierbaren Sonderopfern für einzelne Bevölkerungsgruppen einhergehen (Scharpf 1999a:686). Daher gebe es „überall heftige Anstrengungen zur Einschränkung sozialer Leistungen und zur Senkung der Sozialbeiträge, die aber einerseits gegen massiven po-litischen Widerstand kaum vorankommen und andererseits immer wieder durch die Kosten steigender Arbeitslosigkeit sabotiert werden." (Scharpf 1998a:243) Für Scharpf ergibt sich aus der diesem Trend unterliegenden Interaktion zwischen "entgrenzter Ökonomie" einerseits und "beschränkter Politik" andererseits (Scharpf 1998b:81) das sogenannte „Paradox der Stärke“ (Scharpf 1998a:244):

„In der Interaktion zwischen Politik und Marktkräften manifestiert sich (...) ein 'Paradox der Stärke`: (...) Das Paradox der Stärke kennzeichnet die Lage, in der Politik und Gewerkschaften nicht mehr in der Lage sind, die Marktkräfte zu beherrschen, aber doch noch stark genug sind, um die automatische Anpassung der Verteilungsrelationen zu verhindern. Es wird dann zum demokratischen Dilemma, wenn Politik und Gewerkschaften gezwungen sind, entweder den weiteren Anstieg der Massenarbeitslosigkeit zu begünstigen oder aber durch aktives politisches Handeln die verteilungspolitischen Konsequenzen der neuen ökonomischen Lage selbst durchzusetzen." (ebd., 244-245) 225

Scharpf sieht folglich, und dies ist auch unter Bezugnahme auf die positive Stoßrichtung der Gewährleistungspflichten relevant, keine andere Möglichkeit, als dass sich die Politik an die neuen Bedingungen anpasst und die daraus resultierenden Beschränkungen an die BürgerInnen kommunizieren muss. Dabei ist es essentiell, dass sich sowohl die Regierenden als auch die Regierten darüber im Klaren sind bzw. von den Regierenden darüber aufgeklärt werden, dass die Steuerungsleistungen der nationalen Politiken unter den Bedingungen der Globalisierung und der transnationalen politischen Verflechtungen immer voraussetzungsvoller und insgesamt auch schwieriger werden (Scharpf 1993b:26). Diese Schwierigkeiten, so Scharpf, sind in nahezu allen ge-

225 Das Paradox der Stärke wird in der Literatur zumeist in drei Varianten interpretiert und beurteilt (vgl. Scharpf 1998b:82-83): Die einen sind der Ansicht, dass die Entgrenzung der Ökonomie als unhintergehbar und unabwendbar anzusehen sei und dass eben diese Entgrenzung vielmehr gefördert als bekämpft werden solle, indem staatliche Hindernisse (noch weiter) abgebaut werden. In den beiden anderen Varianten ist die ökonomische Entgrenzung kein Garant von Freiheit und Wohlstand. Deren VertreterInnen interpretieren den ebenfalls als unvermeidlich angesehenen Verlust staatlicher Steuerungspotenziale durch die Marktkräfte entweder als das 'Ende der Demokratie 'oder setzen auf die Hoffnung der Ausweitung der Demokratie auf transnationale Ebenen. Scharpf hat bei allen drei Interpretationen Vorbehalte: „Mir scheint die erste Antwort normativ defizitär. Sie unterschätzt (...) die wohlfahrtstheoretische Bedeutung marktbeschränkender und marktkorrigierender Staatsfunktionen. (...) Meine Einwände gegen die zweite und dritte Antwort sind empirischer Art: Die zweite erscheint mir zu pessimistisch, weil sie den Spielraum für nationale Lösungen unterschätzt, die auch gegenüber dem verschärften internationalen Wettbewerb robust sind. Die dritte Antwort erscheint mir zu optimistisch, weil sie nicht nur die faktischen, sondern auch die legitimatorischen Schranken der europäischen - und erst recht der internationalen - Politik unterschätzt.“ (ebd., 83) 
genwärtigen westlichen Demokratien offensichtlich und trotzdem kann und sollte daraufhin nicht "der politisch leichtere Weg“ (Scharpf 1998a:246) eingeschlagen werden, dass sich die nationale Politik schlicht als abhängig oder noch perfider als 'Opfer' supranationaler politischer Regulierungen darstellt:

„Der Bereich der wählbaren Ziele (...) wird immer durch die jeweiligen Restriktionen bestimmt und in dem Maße, wie das Verhältnis zwischen exogenen Sachzwängen und Wahlmöglichkeiten ungünstiger wird, schrumpfen nicht nur die Handlungsspielräume, sondern es verändert sich auch der Charakter der Politik. An die Stelle der öffentlichen Auseinandersetzung um das Wünschbare, bei der legitimerweise alle mitreden können, tritt der Expertenstreit um das Notwendige oder Unmögliche und die Herstellung von Akzeptanz für das Unvermeidliche. Demokratie reduziert sich dann auf die Delegation oder Verweigerung unkontrollierbaren Führungsvertrauens - was unter Krisenbedingungen auf Zeit ertragen werden mag, als Dauerzustand aber den demokratischen Verantwortungszirkel außer Kraft setzt.“ (Scharpf 1993b:29-30)

„Zum Demokratie-Problem (...) wird diese Entwicklung jedenfalls in den hochentwickelten europäischen Sozialstaaten, weil sie nicht nur die Interessen der breiten Mehrheit verletzt, sondern auch das moralische Selbstverständnis von Gesellschaften in Frage stellt, die sich selbst am Anspruch der sozialen Gerechtigkeit zu messen gelernt haben. (...) Kurz: Die sozialen Folgeprobleme der entgrenzten Ökonomie sind geeignet, das Vertrauen in die demokratische Politik überhaupt zu untergraben." (Scharpf 1998a:229)

Über beide Zitate wird ein für Scharpf und seine komplexe Demokratietheorie programmatisch entscheidender Aspekt deutlich: Trotz oder gerade wegen der enormen Effektivitätsgewinne durch eine verflochtene Politik und Wirtschaft, die aber politische Bürden und Hürden mit sich bringt, können die Folgeprobleme der Globalisierung nur dann politisch be- und verarbeitet werden, wenn die durch die Internationalisierung der Staaten und die Globalisierung der Wirtschaft hervorgerufenen Krisensymptome nicht als unumstößliche Zwangslage für die nationalen Politiken und die Demokratie gedeutet werden. Denn wenn die nationale Politik sich selbst als abhängig oder als 'Opfer' supranationaler politischer Regulierungen empfindet und jenes gegenüber den BürgerInnen auch so darstellt und somit selbst die Handlungsspielräume 'liegen 'lässt, die noch gegeben sind, dann wird über die Betonung externer Zwänge und Restriktionen sowie aufgrund der in Reaktion darauf zur Anwendung gebrachten TINA-Logik $^{226}$ der demokratische Verantwortungszirkel zu oft ausgehebelt.

${ }^{226}$ Die sogenannte TINA (There Is No Alternative)-Logik kommt in der Politik immer dann zur Anwendung, wenn vermeintlich nicht abwendbare Probleme, Hindernisse oder politische Regelungen aufgeschoben oder gerechtfertigt werden. Der slowenische Philosoph Slavoj Žižek schrieb über die Selektivität der TINA-Logik im Kontext der Bankenkrise von 2008 pointiert: „Bedrohte Arten retten, den 
Mit anderen Worten: Die Antizipation der Abhängigkeit, des Kontrollverlustes und der schwindenden politischen Handlungs- und Steuerungsmacht werden dann mittel- und langfristig tatsächliche Realität, da hierüber die negativen Gewährleistungspflichten des Staates bezüglich der Verhinderung des Missbrauchs von öffentlicher Macht latent gefährdet werden. Scharpf, der diese Gefahren als Herausforderungen für die Demokratie sowie seine komplexe Demokratietheorie versteht, kommt hier zu einem recht nüchternen, pragmatischen Ergebnis. Seiner Ansicht nach ist ein normativ begründbarer und pragmatisch adaquater Umgang mit dem Paradox der Stärke nur dann möglich, wenn die Folgen der politischen und ökonomischen Integration, die ja politisch gewollt und vorangetrieben worden sind, nicht als "unabwendbares Schicksal“ (ebd., 246) verstanden werden, das die Politik schlichtweg als solches hinzunehmen und nur noch zu verwalten habe. Die Herausforderung moderner Politik besteht für ihn vielmehr im proaktiven Handeln: Auch wenn Scharpf selber das ein oder andere Mal zugespitzt zum Ausdruck bringt, dass die westlichen Demokratien in einer tiefen „Malaise“ stecken (ebd., 228), deren Grund vor allem die „seit den siebziger Jahren zunehmende transnationale Integration der Wirtschaft [sei], welche die in den Nachkriegsjahrzehnten entwickelte Fähigkeit der nationalen Politik zur 'demokratischen Domestizierung des Kapitalismus` wieder beseitigt hat" (ebd.), haben die 'Polit-Profis', d.h. Berufspolitiker und Verwaltungsbeamte, die Aufgabe die politische Handlungsspielräume trotz aller Restriktionen zu identifizieren. Daher geht Scharpf, in Analogie zur Politik als dem zu steuernden Schiff, davon aus, dass sich „auch mit dem Segelboot (...) selbstgesetzte Ziele erreichen [lassen]." (Scharpf 1993b:29)

„Der Staat muss nicht befehlen können, um die nur ihm zugänglichen Funktionen zu erfüllen. Auch wenn die staatlichen Akteure selbst als Partner unter anderen in konsensabhängigen Verhandlungssystemen involviert sind, darf die Bedeutung ihrer spezifischen Handlungsorientierung und Handlungspotenziale nicht unterschätzt werden. Im Inland wird es sich dabei nach wie vor oft um 'Verhandlungen im Schatten der Hierarchie' handeln, denn auch der auf Verhandlung angewiesene Staat hat ja seine Rechtsetzungskompetenz und sein Sanktionspotenzial keineswegs verloren." (Scharpf 1992:106)

Scharpf betont folglich, dass „nichts gegen die Möglichkeit einer expliziten und demokratisch legitimierten Politik der aktiven Anpassung an die neuen ökonomischen Rahmenbedingungen spricht." (Scharpf 1998a:246) Vielmehr macht er immer wieder deutlich, dass, gerade unter den realen Voraussetzungen des Verlusts staatlicher Steuerungspotenziale und externer Zwänge, die Inputlegitimierung durch die Herrschaft

Planeten vor der Erderwärmung retten, AIDS-Kranke retten, Patienten retten, die sterben müssen, weil es kein Geld für teure Behandlungen und Operationen gibt, verhungernde Kinder retten - all das kann noch ein bisschen warten, aber der Ruf 'Rettet die Banken` ist ein bedingungsloser Imperativ, der unmittelbare Taten fordert und sie auch erhält." (Žižek 2008) 
durch das Volk, an Bedeutung gewinnt. Auch wenn er einräumt, dass der Idealzustand einer „effektiven Selbstbestimmung politisch relevante Wahlmöglichkeiten voraussetzt und Demokratie in den Ländern keine Chance hat, die ganz zum Spielball äußerer Einflüsse geworden sind" (ebd.), zeigt er auf, dass die westeuropäischen Sozialstaaten, die ja nach wie vor zu den reichsten Ländern der Welt gehören, von dieser Grenze der völ-ligen Ohnmacht weit entfernt sind. Vielmehr geht es hier quasi als Bedingung der Mög-lichkeit von Sozialstaatlichkeit und als Gebot der positiven Gewährleistung des Staates um politische Entscheidungen zum Wohle der Allgemeinheit, d.h. um kollektiv verbind-liche Entscheidungen zur lösungsorientierten Schaffung von Gemeinwohl:

Scharpf insistiert darauf, dass die demokratische Outputlegitimation innerhalb derjenigen Staaten, die auch Sozialstaaten sind, unauflöslich an die normative Orientierung am Gemeinwohl gebunden ist (ebd., 245) ${ }^{227}$ Diese zentrale Annahme unterstützen auch Herfried Münkler und Karsten Fischer, die herausstellen, dass „die etatistische Tradition des Gemeinwohltopos in Deutschland ungebrochen [ist] " (Münkler \& Fischer 2002b: 14), was bedeutet, dass der Staat sowohl in der Theorie als auch in der Praxis eine gemeinwohlverpflichtete Institution ist. Zudem machen sie deutlich, dass Gemeinwohlrhetoriken „ein für nahezu alle gesellschaftlichen Interessensgruppen gleichsam attraktives und nur jeweils unwesentlich variiertes Strategem ist, um in Interessenskonflikten die eigene Position zu legitimieren." (ebd.) Dabei zeige sich, so Münkler und Fischer, immer wieder, dass Gemeinwohlrh-toriken eine „nachgewiesene Selbstbindungswirkung“ entfalten (ebd.), d.h. dass „eine strategisch intendierte Gemeinwohlrhetorik eine tatsächliche Gemeinwohlorientierung erzwingt." (ebd., 15) Dies liegt daran, dass Gemeinwohl und Gemeinsinn (siehe b) als aufeinander bezogene Konzepte verstanden werden müssen (ebd., 9):

Das Gemeinwohl ist der normative Orientierungspunkt sozialen und politischen Handelns auf der Outputseite. Der Gemeinsinn wiederum ist die Bereitschaft der sozial und politisch Handelnden, sich an diesem normativen Ideal tatsächlich zu orientieren und diesen Anspruch in ihrem Verhalten und Handeln umzusetzen. Insofern gibt das Gemeinwohlideal an, wieviel Gemeinsinn in Anspruch genommen werden soll und fordert dies ein. Umgekehrt ist aber erst das Vorhandensein von Gemeinsinn die motivationale Voraussetzung dafür, dass überhaupt die Bereitschaft zur Orientierung am Ge-

${ }^{227}$ Sowohl in der Politik als auch in der Politikwissenschaft wird das Gemeinwohlpostulat nach wie vor als wichtiger, wenn nicht sogar zentraler Bezugspunkt für die Legitimation des Politischen verwandt (vgl. Münkler \& Fischer 2002a). Bezüglich der Schwierigkeiten zu bestimmen, was das Gemeinwohl ist und ausmacht, stellt Claus Offe in seinem Artikel "Wessen Wohl ist das Gemeinwohl" heraus, dass wer vom Gemeinwohl redet, zusätzlich zu den Kriterien der legalen Korrektheit und der strategischen Erfolgssicherheit des öffentlichen Handelns immer auch angeben muss, woran, wie und für wen sich eigenes und fremdes Handeln bewähren muss, wenn es dem allgemeinen Wohl genügen soll (Offe 2002: 64). Eben über die Beantwortung dieser Fragen ergeben sich die Schwierigkeiten und Aporien beim Versuch den Allgemeinwohltopos fassbar zu machen. 
meinwohl ent- und besteht (ebd., 10). ${ }^{228}$ Hierbei darf indes der partikulare Charakter des Gemeinwohls nicht verkannt werden, indem man über das Gemeinwohl im Singular spricht (Offe 2002:55). Jede Deutung des Gemeinwohls ist insoweit nur eine, „die sich selbst als eine unter mehreren weiß und sich mit rivalisierenden Deutungen auseinandersetzen muss", denn Prosperität, Vollbeschäftigung, Bildung, Gesundheitsfürsorge, Friedenssicherung, Sicherheit, Nachhaltigkeit, etc. sind allesamt Zielformeln des Gemeinwohls unter denen es weder eine Hierarchie noch eine Verrechnungseinheit gibt (ebd., 71). Daher muss es notwendigerweise viele Varianten des Gemeinwohlbegriffes geben, je nachdem, wie diese Aspekte zueinander in Beziehung gesetzt werden. Auch Scharpf hat für die Aporien und Schwierigkeiten des Gemeinwohlbegriffes keine Lösung. Er versucht der Bestimmungsproblematik dennoch analytisch zu begegnen, indem er darauf verweist, dass das Gemeinwohlpostulat unter sozialstaatlichen Bedingungen einen doppelten normativen Bezug voraussetzt - zum einen die Gewährleistung wohlfahrtsstaatlicher Effizienz und zum anderen die Schaffung von sozialer Gerechtigkeit (Scharpf 1992:96, 1998a:243). Diese zentrale Überlegung ergibt sich wie folgt:

Ausgehend von der spieltheoretisch begründbaren Annahme, dass freiwillige Verhandlungen immer nur dann zustande kommen und selbst Verhandlungen unter den Bedingungen von Zwangsverhandlungssystemen (siehe 5.3.3.2.1) nur dann Ergebnisse zeitigen, wenn alle Beteiligten von der Kooperation in irgendeiner Weise profitieren, geht Scharpf davon aus, dass Verhandlungsergebnisse danach bewertet und untereinander verglichen werden können, welchen Gesamtnutzen diese produzieren (Scharpf 2006: 159). Die wohlfahrtstheoretische Dimension, als Summe der produzierten politischen Ergebnisse und Leistungen, ist damit vom Grundsatz her utilitaristisch und wohlfahrtsökonomisch definiert. Scharpf legt indes selbst dar, dass utilitaristische Kriterien aufgrund ihrer normativen Prämissen hochgradig strittig sind und dass es zudem in der Wohlfahrtsökonomie konzeptionelle Schwierigkeiten gibt, verschiedene Nutzenarten, die zu unterschiedlichen Zeiten und an unterschiedlichen Orten auftreten und unterschiedlichen Personen/Personengruppen zu Gute kommen zu vergleichen (ebd., 160). Daher begnügt sich die Wohlfahrtsökonomie mit dem normativen Kriterium des Pareto-Optimums (ebd.). ${ }^{229}$ Dieses favorisiert Ergebnisse, die den Nutzen in einer Wohl-

${ }^{228}$ Hier sei lediglich angemerkt, dass unglaubwürdige Gemeinwohlrhetoriken destruktive Wirkungen auf die soziomoralischen Ressourcen des Gemeinsinns haben können (vgl. Münkler, Fischer \& Bluhm 2001).

${ }^{229}$ Auf der individuellen Akteursebene bedeutet das Pareto-Optimum, dass Verhandlungen dem normativen Grundsatz folgen, dass durch Kooperationen „niemand schlechter und mindestens einige besser gestellt werden als der Status quo." (Scharpf 2005:711) Dies zeigt schon, dass das Pareto-Kriterium für sich genommen ein plausibles und normativ unproblematisches Kriterium für individuelle und gesellschaftliche Entscheidungen ist, denn es befürwortet alle Veränderungen, die einigen nützen und niemandem schaden. Normativ problematisch wird es jedoch, wenn die so definierte Optimalität der einzige Aspekt für die Gemeinwohlorientierung der Politik ist, da das Pareto-Kriterium normativ nicht ausreichend und als alleiniger Parameter nicht überzeugend ist (ebd., 739). 
fahrtsdimension (bspw. Umweltschutz) erhöhen, ohne dass die anderen für die staatliche Wohlfahrt relevanten Nutzendimensionen (bspw. Energieversorgung, Steuerabgaben, Arbeitsplätze etc.) dadurch verringert werden (Scharpf 2005:711). Sobald indes diese für niemanden problematischen Win-Win-Konstellationen und deren 'kostenlose' Vorteile ausgeschöpft sind, geht es um die Bereiche der Politik, wo substabzielle Verteilungsproblematiken gelöst werden müssen.

Wenn es um politisch zu lösende Verteilungsprobleme geht, von denen nicht alle zugleich profitieren können, d.h. wo es um nicht-paretooptimale Lösungen geht, ist für die Abschätzung der wohlfahrtsstaatlichen Effizienz der interpersonale Vergleich zwischen unterschiedlichen Arten von Nutzen und Kosten erforderlich, die gegeneinander abgewogen werden müssen (Scharpf 2006:160). ${ }^{230}$ Hier schlägt Scharpf als normativen Maßstab das sogenannte Kaldor-Hicks-Kriterium vor, welches auf der Idee einer interpersonellen Kompensation bei gegenläufigen Wohlstandsänderungen beruht. ${ }^{231}$ Demgemäß sind politische Entscheidungen immer dann akzeptabel, „wenn die Nutzen der Gewinner mindestens so groß sind, dass sie die Kompensation aller Verlierer ermöglichen." (Scharpf 2005:712; 2006:161) Gemäß dieser 'Formel entsteht ein normatives Legitimationsargument für diejenigen Verhandlungssituationen, in denen es zu Austritten der 'Verlierer' aus den Verhandlungsrunden, einseitigen Verhandlungsblockaden in Zwangsverhandlungssystemen, Kämpfen um die Verteilungsrelation auf Kosten der Produktionsdimension oder aber den Einsatz politischer Macht zur Aufrechterhaltung der Verhandlungen und das 'Erzwingen 'von kooperativen Verhandlungsergebnissen kommt: Gelingt eine solche Kompensation, d.h. kann man sich darauf einigen, warum überhaupt kompensiert werden muss, in welcher 'Währung' die Kompensation erfolgen soll (Geld, Informationen, Beteiligung, Vorteile in anderen Bereichen etc.), wie hoch die Kompensation sein soll, wer an wen kompensiert und wie lange, dann lassen

${ }^{230}$ Eben dieser interpersonale Vergleich von Kosten und Nutzen ist, so Scharpf, „das Hauptproblem, zu dem die moderne Wohlfahrtsökonomik keine Stellung beziehen will.“ (Scharpf 2006:160) Die Begründungen hierfür seitens der Wohlfahrtsökonomik, nämlich, dass diese eine wertfreie Wissenschaft sei, die keinerlei Aussagen zu Verteilungsfragen machen will und kann, wird von Scharpf mit dem Argument zurückgewiesen, dass das Pareto-Kriterium selbst auch nicht wertfrei ist, denn es postuliert, dass alle bestehenden Nutzenoptionen geschützt werden sollen und dass Verteilungsfolgen zu ignorieren seien, was genauso wertbeladen ist, wie die explizite Präferenz für ein bestimmtes Verteilungsergebnis (ebd., 161). Überdies hinaus wird in der Wohlfahrtsökonomik postuliert, dass interpersonale Nutzenvergleiche per se unmöglich seien, was Scharpf wiederum mit dem Argument zurückweist, dass auf der Basis von indirekten Vergleichen, d.h. von der antizipierten Vorstellung man selbst befinde sich in der Situation des anderen, durchaus nachvollziehbare Kosten- und Nutzenvergleiche möglich sind (ebd.).

231 Anders als das Pareto-Kriterium bei welchem die Änderungen einer ökonomischen Situation unter Wohlstandsgesichtspunkten nur dann beurteilt werden können, wenn keine gegenläufigen individuellen Wohlstandsänderungen auftreten, können über das Kaldor-Hicks-Kriterium auch gesamtgesellschaftliche Wohlfahrtsänderungen bewertet werden, bei denen die Wohlfahrt einzelner Individuen steigt während die anderer sinkt, indem Wohlstandsgewinne und Wohlstandsverluste gegeneinander aufgerechnet werden (Scharpf 2006:162). 
sich auch inhaltlich schwierige Umverteilungsmaßstaben verhandeln und es gelingt zugleich, den politischen Umgang mit Verteilungskonflikten in Richtung derjenigen zu legitimieren, die „durch den Oktroy in ihren Interessen relativ benachteiligt werden.“ (Scharpf 2005:708)

Zu beachten ist hierbei jedoch, dass auch das Kaldor-Hicks-Kriterium nichts über die inhaltliche Dimension der Verteilung aussagt, sprich wer, wie, an wen und warum verteilt. Diesbezüglich ist Scharpf klar, dass dies notwendigerweise den Bezug auf normative Standards der Gerechtigkeit verlangt und dass diese inhaltliche Dimension des Gemeinwohltops als Fragen der sozialen Gerechtigkeit diskutiert und entschieden werden müssen (Scharpf 2006:163,270-271): Einmal abgesehen von der Vielzahl der möglichen Auslegungen des Gerechtigkeitsbegriffs (bspw. als Gleichberechtigung, Verfahrensgerechtigkeit, politische Gerechtigkeit, Tauchgerechtigkeit, Geschlechtergerechtigkeit Generationengerechtigkeit, Umweltgerechtigkeit etc.) und den Kriterien, Grundsätzen und Prinzipien, die diese begründen können (u.a. qua Gleichheit, Fairness, Rechtmäßigkeit, Bedürftigkeit, Leistung, Zufall, Nachhaltigkeit etc.), zeigt sich, dass sowohl die Gerechtigkeitsbegriffe als auch deren Kriterien in partiellem Konflikt miteinander liegen, zumal dann, wenn man nicht auf ihre räumliche, zeitliche und dimensionale Einschränkung verzichtet (Offe 2002:71). Angesichts dieser Schwierigkeiten, die Scharpf weder lösen will noch kann, weist er darauf hin, dass empirische Studien zeigen, dass „normale Bürger, Richter, Bürokraten und Politiker in der Lage sind, normativ gerechtfertigte Lösungen für Probleme der Verteilungsgerechtigkeit zu finden (Scharpf 2006: 164) und dass es daher auch weniger darum geht, dass Politikwissenschaftler „diese Arbeit übernehmen und sozusagen 'wissenschaftlich 'validierte Werturteile über bestimmte Verteilungsprobleme entwickeln." (ebd., 163) Vielmehr geht es aus der politikwissenschaftlichen Perspektive darum, anzugeben, welche Spiel- und Akteurskonstellationen welchen Legitimationsbedarf erfordern.

Hiermit ist das zentrale Desiderat von Scharpfs legitimationstheoretischen Überlegungen benannt: Ausgehend von den Darlegungen der Verhandlungsformen sowie deren strukturellen Kontextbedingungen (siehe 5.3.3.2.1) und den sowohl für die Akteure als auch in Bezug auf die dafür erforderlichen strukturellen Ressoucen immer anspruchsvoller werdenden Grundformen der strategischen Interaktion (einseitiges Handeln, wechselseitige Anpassung, Kooperation, Mehrheitsentscheidungen und hierarchische Steuerung), macht Scharpf deutlich, dass je gravierender die politischen Eingriffe hinsichtlich der Zumutungen für Einzelne und Gruppen werden, umso stärker muss die Legitimationskraft der Argumente sein, mit dem diese gerechtfertigt werden, da der Legitimationsbedarf den die Regierenden in Demokratien haben je nach Schwere der potenziellen Verletzung der Intressen der BürgerInnen variiert (Scharpf 2005:707). 
Diesbezüglich lässt sich bei Scharpf, unter Bezugnahme auf unterschiedliche, spieltheoretisch modellierte Akteurskonstellationen, eine Stufenskala des Legitimationsbedarfs herauslesen und herausarbeiten: ${ }^{232}$

Auf der untersten Legitimationsstufe, d.h. den Bereichen mit dem wenigsten aufzubringenden politischen Legitimationsbedarf, geht es spieltheoretisch betrachtet, um die Koordination von symmetrischen Dilemmaspielen. Solche Spiele zeichnen sich dadurch aus, dass die Kooperationsbereitschaft bei allen Teilnehmern grundsätzlich vorhanden und pareto-optimale Lösungen möglich sind, die Einigung auf gemeinsame Regelungen erfolgt aber nur dann bzw. kann nur dann garantiert werden, wenn dahinter monetäre Anreizmechanismen oder administrative Sanktionsmechanismen stehen, die die Kooperationsbereitschaft belohnen und/oder den Austrittsgewinn übersteigen. Da unkooperatives Verhalten bei Dilemmaspielen für alle Beteiligten schädlich wäre, aber jeder für sich besser abschneiden würde, wenn er die von den anderen eingehaltenen Regeln verletzt, lassen sich hier durch den 'Schatten der Hierarchie' Kooperationen und pareto-optimale Lösungen erzielen. Hierbei finden die pluralistischen und neokorporativen Verhandlungsnetzwerke zwischen Verbänden, Unternehmen und dem Staat unter der Prämisse statt, dass das Rechtsetzungs- und Gewaltmonopol des Staates in Anspruch genommen werden kann, wenn es nicht gelingt, ein Problem allein durch freiwilliges Handeln zu lösen (Scharpf 1993b:36-37). Staatliche Organisationshilfen und die Androhung alleiniger, hoheitlicher Rechtsdurchsetzung bieten hier einen Ausweg, zumal wenn es im Interesse staatlicher Akteure ist, einzugreifen um externe Effekte solcher Verhandlungsrunden auf andere zu beschränken oder um absehbaren Implementationsproblemen vorzubeugen (ebd., 38). Selbst wenn die Verhandlungspartner indes erst einmal aktiv dafür werben müssen, dass sich der Staat als 'Hüter der Ordnung einmischt, sind Verhandlungen 'im Schatten der Hierarchie' solange legitimationstheoretisch unproblematisch, solange das Problem und seine Lösung nur die Gruppenmitglieder selbst betrifft (ebd., 37). Solange dies der Fall ist, geht es 'nur' darum, qua staatlicher Gewalt, das fehlende Koordinationspotenzial zur Kooperation zu schaffen oder das Sanktionspotenzial zu generieren, das erforderlich ist, damit die Regelungen auch von allen Beteiligten eingehalten werden.

\footnotetext{
${ }^{232}$ Hierbei geht es um genuin politische Konstellationen, sprich gerade nicht um freiwillige und selbstregulative Verhandlungsnetzwerke und Regime, bei denen es nicht um kollektiv verbindliche Entscheidungen sondern um gruppeninterne Regelungen geht und wo alle Verhandlungspartner ein gemeinsames und gleich gerichtetes Interesse an der Regelung von Sachverhalten haben und die Einhaltung der Regelungen auch intern garantiert werden kann (Scharpf bezeichnet diese als reine Kooperationsspiele). In solchen Fällen bedarf es keinerlei Intervention seitens der Politik. Vielmehr kann und muss sich der Staat sogar aus solchen Entscheidungsfindungsprozessen raus halten, auch wenn er die gesellschaftliche Selbstorganisation begünstigen kann, indem er bspw. zivilgesellschaftliche Strukturen durch materielle Förderung unterstützt und indem er klare und durchschaubare Richtlinien für die Wirtschaft erlässt (Scharpf 2006:327).
} 
Hierüber entsteht ein zwar geringer, aber dennoch vorhandener politischer Legitimationsbedarf, bei dem gerechtfertigt werden muss, warum es im Sinne aller sein soll, dass sich der Staat auf gerade diese Verhandlungen fokussiert und zeitliche, humane und finanzielle Ressourcen aufbringt, um die Verhandlungen zu unterstützen und/oder warum er sein Gewaltmonopol zur Sicherung dieser Regelungen einsetzt. Hier geht es folglich um outputseitige Legitimationargumente, die sich vor allem anhand der positiven Gewährleistungspflichten des Staates rechtfertigen lassen, gesellschaftliche und zunehmend auch globale Problematiken über die Etablierung expertokratischer Strukturen und die Beteiligung an intergouvernementalen sowie pluralistischen und korporatistischen Verhandlungsnetzwerken politisch zu regeln. Hierbei müssen die staatlichen Akteure indes besonders aufpassen, dass sie ihre negativen Gewährleistungspflichten nicht verletzten, sprich ihre politische Macht und ihre Position nicht für eigene oder parteiische Zwecke missbrauchen, sondern dass sie als Mediator und AgendaSetter (siehe 5.3.3.2.1) aufzutreten.

Auf der 'zweiten Stufe', d.h. der Ebene, wo ein höherer Legitimationsbedarf entsteht, geht es nicht mehr um die für niemanden wirklich problematischen Win-Win-Konstellationen, bei denen die Gewinn- und Verlusterwartungen der Beteiligten gleich gerichtet sind, so dass alle zu denselben (dominanten) Strategien neigen und bei denen anhand der positiven Gewährleistungspflichten des Staates die Gemeinwohlorientierung der Politik bzw. politischer Verfahren und Entscheidungen gerechtfertigt werden können. Auf der zweiten Stufe rangieren all die Bereiche der Politik, wo bereits zu Beginn der Verhandlungen absehbar ist, dass nicht alle Verhandlungsteilnehmer zugleich davon profitieren werden. Da sich hier folglich nicht zugleich alle gegenüber ihrem Status Quo verbessern können, ist eine Einigung auf gemeinsame Regelungen nur dann möglich, wenn alle Beteiligten dazu bereit sind auf ihren maximalen Gewinn für die Suche nach der Option mit dem größten Gesamtgewinn und dem geringsten Gesamtschaden für alle zu verzichten. Bei solchen Konstellationen, die Scharpf als asymmetrische Positivsummenspiele mit Verteilungskonflikt kennzeichnet, sind maximal kaldor-optimale Lösungen möglich, da bei all den Konstellationen, in denen Wohlfahrtsgewinne für die Gesamtheit nur erreicht werden können, wenn in bestehende Besitzstände eingegriffen wird, „der bloße Verzicht auf unnötige Nutzenvereitelung ein zu schwaches Wohlfahrtskriterium" ist (Scharpf 1992:97). Hierbei ist zu beachten, dass solche Umverteilungsregelungen häufig durch staatliche Macht initiiert und oftmals auch 'erzwungen ' werden müssen, was bedeutet, dass dies gegenüber denjenigen zu rechtfertigen ist, die die Nichtregelung der Regelung vorgezogen hätten. Entscheidend hierbei ist des Weiteren, dass die Nutznießer und Verlierer nicht schon von vornherein feststehen, sondern dass dies von der Wahl der Entscheidungsoption abhängig ist (Scharpf 2005: 708). So werden die Nutznießer und Verlierer je nach Entscheidungsoption wechseln, 
zugleich müssen aber alle zusammen an einer kooperativen Lösung arbeiten. Dadurch besteht immer die latente Gefahr, dass diejenigen Optionen innerhalb solcher Entscheidungsgremien favorisiert werden, wo die Benachteiligten extern und diffus sind, sprich dass Entscheidungen zu Lasten der Allgemeinheit (bspw. der Steuerzahler oder der Verbraucher) gefällt werden. Hier müssen die beteiligten staatlichen Akteure folglich besonders achtsam sein, dass die Willensbildung nicht einseitig von Partialinteressen zu Lasten des Gemeinwohls dominiert wird. Eben dies ist in all den Situationen der Fall, wo kritisch von einer „Herrschaft der Verbände“ (ebd.) die Rede ist, denn hier geht es im Kern um die Norm, dass „die staatliche Politik ihre Maßnahmen nicht an den Vorschlägen ihrer externen Partner orientieren darf, ohne damit ihre demokratische Verantwortung gegenüber der Allgemeinheit in Frage zu stellen." (ebd.) Daher ist es bei solchen Konstellationen die Aufgabe der staatlichen Akteure als 'Agenten der Allgemeinheit' aufzutreten und nur dienigen Entscheidungsoptionen zuzulassen, die einzelne oder viele Unbeteiligte nicht unnötig benachteiligen, ohne dass die Benachteiligungen möglichst verhindert oder aber, wenn diese unumgänglich sind, durch die Gewinne aus der Regelung kompensiert werden. Hier geht es folglich ebenfalls um outputseitige Legitimationargumente, die indes anhand von Gerechtigkeitsargumenten und -kriterien ausgewiesen werden müssen, da hierbei gerechtfertigt werden muss, warum und wie umverteilt werden soll und unter welchen Bedingungen die Umverteilung als gerecht angesehen werden kann. Solche Legitimationsargumente sind immer anfällig für Kritik und Gegenrede und hierbei kommt es darauf an, dass sich vor allem die von den Entscheidungen Benachteiligten verstanden und angesprochen fühlen. Hier können speziell die Ansätze der lebenstilorientierten Kommunikation (siehe Abschnitt 4.3.3) wichtige Hilfestellungen leisten, um zu antizipieren, welche sozialen Milieus von bestimmten Entscheidungen besonders betroffen sind, wie diese kommunikativ zu erreichen sind und welche Gerechtigkeitsargumente und -kriterien in den jeweiligen sozialen Milieus von besonderer Wichtigkeit sind.

Auf der 'dritten Stufe' steigt der politische Legitimationsbedarf noch einmal an bzw. transformiert sich sogar. Hier rangieren alle Verhandlungskonstellationen, bei denen „die Befriedigung der Interessen einer Gruppe nur auf Kosten der Interessen einer anderen Gruppe erfolgen kann“ (ebd., 709), was bei vielen politischen Regelungen im Umwelt- und Naturschutz aber auch im Bereich der Sozialpolitik der Fall ist. Solche Konstellationen kennzeichnet Scharpf als Nullsummenspiele. Wenn von vornherein klar ist, dass „Wohlfahrtsgewinne für die Gesamtheit nur erreicht werden können, wenn einem Teil der Betroffenen unkompensierte [bzw. unkompensierbare] Sonderopfer abverlangt werden“ (ebd., 711), dann müssen diese faktisch unkompensierten oder dem Sinn nach unkompensierbaren Sonderopfer politisch legitimiert und gerechtfertigt werden. Hier sind für Scharpf die Grenzen der outputorientierten Legitimation erreicht 
(ebd.). An dieser Stelle schließt sich bei Scharpf die 'Schleife`zwischen Input- und Outputlegitimation:

„Ausgeschlossen wären sozialpolitische Umverteilungen und alle anderen Regelungen, die nicht kompensierte ('solidarische') Sonderopfer verlangen. Für diese bleiben moderne politische Systeme ausschließlich auf inputorientierte Legitimationsargumente angewiesen. Nur die Bürger selbst sind frei in der Wahl ihrer kollektiven Identität und nur sie selbst können entweder gemeinwohlbezogene oder sogar altruistische Kriterien in die Spezifikation ihrer eigenen Präferenzen aufnehmen. Ohne die eine oder andere dieser Grundlagen aber könnte die Zumutung unkompensierter Sonderopfern nicht legitimiert werden.“ (ebd.)

Dabei macht Scharpf erneut deutlich, wie wichtig die Anerkennung und Vermittlung des Umstandes ist, dass auch eine gemeinwohlförderliche Politik nicht ohne „die Wahl zwischen unangenehmen Alternativen, die Hinnahme von Verlusten in anderen Bereichen oder die Auferlegung von individuellen und kollektiven [Sonder-]Opfern" (Scharpf 1993b:30) auskommen könne und dass diese vermeintlichen 'Fehlentwicklungen ' normaler politischer Alltag seien, die aber politisch verantwortet werden müssen. Sollen Nullsummenregelungen legitimiert werden, so bedarf es kollektivistischer Legitimationsargumente, was wiederum bezogen auf Scharpfs Legitimationstheorie bedeutet, dass eben dies nur durch eine Herrschaft durch das Volk geschehen kann, was wiederum bedeutet, dass solche Maßnahmen - aber eben auch nur solche Maßnahmen! - nicht staatlich verordnet, hoheitlich geregelt und politisch gesteuert, sondern vorab gesellschaftlich diskursiv erörtert und deliberiert werden müssen. Die Aufgabe staatlicher Akteure besteht hierbei vor allem darin, eben die Situationen und Fälle zu erkennen und zu selektieren, in denen deliberative Prozesse unumgänglich sind. Diese Prozesse haben sie, so Scharpf, anzustoßen, vorzubereiten, zu begleiten und finanziell und materiell zu befördern. Was am Ende das Ergebnis solcher deliberativen Prozesse ist und ob es dabei auch dazu kommt, dass die von der Entscheidung negativ betroffenen Gruppen die Regelungen gerechtfertigt finden und diese auch gegen ihren eigenen Vorteil akzeptieren, ist und bleibt dabei erst einmal ungewiss. Denn selbst innerhalb der Nationalstaaten, die ja über eine historisch gewachsene kollektive Identität sowie über zivilgesellschaftliche Strukturen mit vielen Möglichkeiten zur Ausprägung dauerhafter solidarischer Orientierungen verfügen, ist dies mitnichten selbstverständlich. Hier ist es aber zumindest möglich. Anders ist dies auf der europäischen und internationalen politischen Ebene. Wenn es, wie dies auf den supranationalen Ebenen der Fall ist, keinen Grund gibt von einer kollektiven Identität auszugehen, dann kann lediglich auf die solidarischen Orientierungen zwischen den Mitgliedern eines Gemeinwesens gehofft werden. Wenn indes auf die gegenseitige Solidarität der Mitglieder und ihren Gemeinsinn kein Verlass ist, dann gibt es keinen Grund anzunehmen, dass die Akteure 
gemeinwohlorientiert handeln würden (Scharpf 1999b:22), denn wo kein Gemeinsinn vorherrscht, da lässt sich auch schwerlich - es sei denn lediglich symbolisch - von einem Gemeinwohl bzw. einer Gemeinwohlorientierung sprechen. Daher beruhen die Möglichkeiten und Grenzen der Legitimation oberhalb der Nationalstaaten lediglich auf ganz bestimmten Mechanismen der Outputlegitimation, die zumindest innerhalb des institutionellen Settings der gegenwärtigen supranationalen politischen Systeme den potenziell widersprüchlichen Gewährleistungszielen dienen müssen, einerseits den Missbrauchs politischer Macht zu verhindern und andererseits expertokratische Strukturen und intergouvernementale Verhandlungen zur effektiven Problemlösung zu etablieren (ebd., 30). Obgleich also immer mehr Entscheidungen auf die supranationalen Ebenen delegiert werden und dies auch müssen (wegen globaler Umweltproblematiken, aber auch gemeinsamer Sicherheitspolitik, Regulierung des internationalen Finanzmarktes etc.) und auch hier Nullsummenspiele verhandelt werden, haben die supranationalen Ebenen nur eine enge Auswahl an Optionen und Ressourcen zur Outputlegitimation zur Verfügung. Wenn auf diesen Ebenen folglich Verteilungskonflikte und Nullsummenspiele verhandelt und entschieden werden, wie dies bei der Regulierung der Bewahrung und Förderung der Biodiversität durch die CBD im Bereich des gerechten Vorteilsausgleichs der Fall war und ist, dann müssen die dabei entstehenden 'negativen Legitimationsbilanzen' von der nationalen Politik aufgefangen werden. Gerade auf der letzten Legitimationsstufe ist dies mit den heutigen partizipativen und deliberativen Strukturen so gut wie unmöglich, so dass Scharpf hier den größten Entwicklungsbedarf im Rahmen seiner komplexen Demokratietheorie sieht.

\subsubsection{Zusammenfassung der komplexen Demokratietheorie mit Fokus auf die Konkretisierung der theoretischen Grundannahmen der GBG}

Wie in den vorangegangen Abschnitten gezeigt wurde, hat Scharpf über gut 35 Jahre ein zwar unzusammenhängendes, aber dennoch hoch interessantes Sammelsurium an steuerungs- und governancetheoretischen, demokratietheoretischen sowie legitimationstheoretischen Überlegungen zur komplexen Demokratietheorie zusammengetragen. Diese wurden vor dem Hintergrund der begründeten Annahme, dass das Konzept der Good Governance ausgehend von governancetheoretischen Überlegungen um verhandlungsdemokratische Überlegungen erweitert und expliziert werden sollte (siehe 5.2.3), systematisch unter den Titeln »Demokratietheorie zwischen Utopie und Anpassung« (5.3.3.1) sowie »Die Erweiterungen der komplexen Demokratietheorie» (5.3.3.2) rekonstruiert, expliziert und analysiert. Die Erkenntnisse dieser Analysen werden im Folgenden stichpunktartig zusammengefasst und in Bezug auf die Good Biodiversity Governance konkretisiert: 
1. Hinsichtlich der herausgearbeiteten Governancekonzeption lässt sich die GBG als kooperativer Modus der politisch-strategischen Handlungskoordination im Sinne der weiten Governancekonzeption verstehen, die zur Lösung von Koordinations-, Kollektivgut- und Redistributionsproblemen in politischen Mehrebenensystemen zur Anwendung kommt. Dabei ist die GBG selbst das Ergebnis bestimmter Problemdefinitionen und Lösungsmöglichkeiten bezüglich der Ansichten zum Problem der Vernichtung der Biodiversität, des Machtverlustes der Staaten und deren Internationalisierung, die selbst erst durch kollektive, umkämpfte Prozesse der Problemdefinition hervorgebracht werden.

2. Auf der Strukturebene ist die GBG dabei ein, an den normativen Prinzipien der Subsidiarität, der Politikintegration, der Verursachung und der Vorsorge orientiertes Regelungssystem zur Verminderung und Lösung der Problematik der Vernichtung der Biodiversität. Hierbei sind für die GBG die prototypischen Verhandlungsformen der Tauschgeschäfte, der Problemlösungsverhandlungen und der positiven Koordination zu unterscheiden, die unter den Bedingungen dyadischer Netzwerke, normativer Regimestrukturen sowie von Zwangs- oder Kollektiventscheidungssystemen je andere Effektivitäts- und Effizienzkriterien erfüllen müssen und deren Ergebnisse gegenüber denjenigen, die davon negativ betroffen sind, auch unterschiedlich zu rechtfertigen und zu legitimieren sind.

3. Auf der Prozessebene erscheint die GBG als strategischer Interaktionsprozess der Kooperation, der Verhandlung und des Dialogs, wobei die Ergebnisse der Interaktionen einerseits davon abhängig sind, welche Akteure mit welchen Fähigkeiten, Ressourcen und Orientierungen in diesen Konstellationen zusammenkommen und ob die hierüber gebildeten Interaktionsformen den normativen Prinzipien folgen, dass alle potenziell Betroffenen beteiligt werden müssen (Inputseite), dass sich aber niemand beteiligen und keiner zustimmen muss, wenn er sich dabei per Saldo schlechter stellt als bei einem Scheitern der Verhandlungen (Outputseite).

4. Unter diesen Bedingungen muss die GBG den doppelten Standard guter und legitimer Politik erfüllen. Die Beurteilung der Stabilität und Qualität der GBG hängt nicht nur davon ab, was für Outputs und Outcomes durch Verhandlungen produziert werden, sondern auch welche Inputs in diese eingehen und wie diese im politischen Prozess verarbeitet und zu politischen Entscheidungen umgewandelt werden. Das bedeutet einerseits, dass Verhandlungen möglichst effizient zu Entscheidungen führen sollen und dass die effektive Implementation dieser Entscheidungen garantiert werden muss. Andererseits muss aus legitimatorischen Gründen sicher gestellt werden, dass all diejenigen, die von kollektiven Entscheidung betroffen sein könnten, die Möglichkeit erhalten an den Verhandlungen beteiligt zu sein oder sich interessenwirksam repräsentieren zu 
lassen, um von den gemeinwohlorientierten Leistungen politischer Entscheidungen zu profitieren oder im Extremfall angemessen entschädigt zu werden, wenn die Entscheidung zu partiellen Sonderopfern führt.

5. Demokratietheoretisch betrachtet verweist die GBG dabei auf ein, an sich nicht neues, aber dennoch qualitativ anderes Demokratiemodell: Bei der GBG geht es sowohl um die Konturen als auch um die Details eines normativ komplexen verhandlungsdemokratischen Demokratiemodells, das sowohl als Bezugsrahmen der empirischen Politikforschung als auch als Anleitung der politischen Praxis fungieren kann, da die Orientierung an verhandlungsdemokratischen Grundannahmen Rückwirkungen auf die Beurteilung der Effizienz-, Effektivitätsund Legitimitätskriterien der Politik hat, die es zu beachten gilt.

6. Obgleich die GBG durch verhandlungsdemokratische Strukturen, kooperative Steuerungsbemühungen und Verhandlungslösungen zwischen politisch unabhängigen Akteuren und deren Koordinations- und Kooperationsstrukturen mit dem Ziel des gesellschaftlichen Interessensausgleiches charakterisiert ist, ist sie nach wie vor durch die klassischen politischen Koordinationslogiken, wie die staatlich-hoheitliche Regulierung und durch repräsentativ-demokratische Aspekte wie den demokratische Parteienwettbewerb und das Mehrheitsprinzip gekennzeichnet. Kooperative Staatlichkeit und Verhandlungsdemokratie bedingen sich im Modell der GBG gegenseitig.

7. Mit Blick auf die Inputseite politischer Prozesse wird über die GBG damit das Interesse an demokratischer Selbstbestimmung und Selbstorganisation anerkannt, was aber nicht gleichzeitig bedeuten kann, dass dies die Maxime universeller Beteiligung nach sich zieht. Vielmehr verweist die GBG darauf, dass Strukturen geschaffen und Verfahren etabliert werden müssen, um das Sensorium der politischen Entscheidungsstellen für das Problembewusstsein, die Kritik und die Empfehlungen der aktiven Öffentlichkeit erheblich zu verbessern. Hier ist der gezielte Einsatz der lebensstilorientierten Naturschutzkommunikation sowohl bezüglich der Auswahl der Agenda als auch der relevanten Akteure zur Identifizierung der Konsens- und Dissenspunkte für die GBG unerlässlich.

8. Mit Blick auf die Outputseite politischer Prozesse bedeutet dies wiederum, dass die GBG voraussetzt, dass über den pluralistisch-partizipativen Strukturen eine politisch-staatliche Ebene vorhanden sein muss, die in relativer Unabhängigkeit von den Pressionen der organisierten Interessengruppen agiert, so dass Entscheidungen auch gegen den Widerstand einzelner Gruppen durchgesetzt werden können. Diese Instanzen müssen gemeinwohlorientiert agieren und haben die Aufgabe ihre rechts- und sozialstaatlichen Gewährleistungspflichten zu erfüllen. 
Im Kontext der GBG bedeutet dies vor allem, dass das Verteilungsziel und die Verteilungsfragen bezüglich der Bewahrung und Förderung der Biodiversität nur solange ausgeblendet bleiben können, wie durch die Optimierung von Schutz- und Nutzungsansprüchen annähernd alle profitieren können. Da dies nur sehr selten überhaupt der Fall ist (nämlich dann, wenn es sich um reine Kooperationsspiele handelt), bedeutet eine proaktive Politik zur Förderung der Biodiversität, dass die Einholung der Biodiversitätsziele und -maßnahmen nicht ohne die Wahl zwischen unangenehmen Alternativen, die Hinnahme von Verlusten in anderen Bereichen oder die Auferlegung von individuellen und kollektiven Sonderopfern erfolgen kann. Diese sind indes keine politischen Fehlentwicklungen sondern normaler politischer Alltag.

9. Mit Fokus auf die Politik der Biodiversität und ihre vertikale und horizontale Koordination ist vor diesen Hintergründen zu beachten, dass bei der GBG nicht nur politikinterne Strukturen und Prozesse betrachtet werden können und müssen. Die Erweiterungen der politischen Sphäre oberhalb und innerhalb der Nationalstaaten sowie durch kooperative Steuerung und Verhandlungslösungen hat vielmehr relevante Rückwirkungen auf die für die GBG relevanten Prinzipien der Subsidiarität, der Politikintegration, der Vorsorge und der Verursachung:

a. Für das Subsidiaritätsprinzip ist relevant, dass die vertikale Koordination, vor allem oberhalb der Nationalstaaten, wo es keine vergleichbaren zivilgesellschaftlichen Strukturen wie innerhalb der Nationalstaaten gibt, alleinig durch expertokratische Verhandlungssysteme realisiert wird. Diese haben ihre eigenen Standards bezüglich guter und legitimer Politikproduktion, die nicht im Vergleich zur nationalstaatlichen Ebene beurteilt werden können und sollten. In Bezug auf die GBG heißt das, dass das Subsidiaritätsprinzip zwar anhand von Effektivitäts- und Effizienzkriterien beurteilt werden kann, nicht aber zugleich hinsichtlich politischer Beteiligung und Partizipation.

b. Für das Prinzip der Politikintegration ist damit wiederum relevant, dass die horizontale Koordination vor allem innerhalb der nationalstaatlichen Ebene durch die Koordinationsformen der gesellschaftlichen Selbstorganisation ergänzt und erweitert werden können und müssen. Hierbei geht es mit Bezug auf die GBG vor allem darum, dass langfristige Perspektiven und zukünftige Interessen stärker integriert werden, als dies bisher der Fall ist. Für die GBG ist des Weiteren entscheidend, dass auf die realen Möglichkeiten der Deliberation in der politischen Öffentlichkeit fokussiert wird und dass geschaut wird, welche formalen und machtbasierten Faktoren darüber entscheiden, welche Positionen und Meinungen sich in der Politik durchsetzen können und welche nicht. Schlussendlich kommt es hierbei darauf an, dass die Vertreterlnnen in 
Verhandlungen die Suche nach einer effektiven Verhandlungslösung nicht dem Ziel der innerdemokratischen Beteiligung opfern und umgekehrt und wie dies strukturell und prozedural gestaltet und bewerkstelligt werden kann.

c. In Bezug auf das Vorsorgeprinzip impliziert die GBG, dass alle mit der Bewahrung und Förderung der Biodiversität verbundenen Belange daraufhin geprüft werden müssen, ob diese mit unkompensierbaren Sonderopfern einhergehen. Hierbei ist entscheidend, dass durch gesetzliche Regelungen die institutionellen Rahmenbedingungen dafür geschaffen werden, dass die Risikobewertungen nicht nur auf Basis wissenschaftlicher Fakten oder ökonomischer Kosten-Nutzen Analysen durchgeführt werden, denn ansonsten ist das Risiko zu hoch, dass wichtige Aspekte wie die Argumente des guten Lebens und Gerechtigkeitsargumente ausgeblendet werden.

d. In Bezug auf das Verursacherprinzip wird schließlich über die GBG der Umstand anerkannt, dass eindeutig zurechenbare Verantwortungen für Umweltbelastungen und -schäden weder identifizierbar sind, noch klar und eindeutig zurechenbar gemacht werden können. Das bedeutet, dass das Gemeinlastprinzip, das in solchen Fällen zur Anwendung kommt, so ausgestaltet werden muss, dass die Kosten und Nutzen aus dem Schutz und der Nutzung der Biodiversität gerecht verteilt werden. Diesbezüglich weist die komplexe Demokratietheorie im Biodiversitätskontext darauf hin, dass es weniger um den Trade-off zwischen Schutz und Nutzung als vielmehr um Effizienz- und Effektivitätsfragen bei den Entscheidungsfindungs-prozessen sowie die dabei relevante Debatte um Verteilungsfragen geht.

10. Gerade in Bezug auf das Vorsorge- und Verursacherprinzip lässt sich anhand der Stufenskala für den Legitimationsbedarf auch für die GBG deutlich machen, in welchen Fällen hierarchische Koordination und Steuerung seitens des Staates dringend erforderlich sind, welche Spielräume zur Effizienz- und Effektivitätssteigerung dabei auf Basis der strukturellen Kontextbedingungen der Verhandlungsformen möglich sind, welche Instrumente dabei jeweils eingesetzt werden können und welche Legitimationsanforderungen sich unabhängig von den konkreten Ergebnissen bereits durch die strukturellen und prozeduralen Charakteristika der strategischen Interaktionsformen ergeben:

a. Reine Kooperationsspiele, dich sich dadurch auszeichnen, dass alle zugleich durch die Kooperation gewinnen und Regelungen freiwillig zustande kommen, verlangen unter der Bedingung, dass diese keine Externalitäten für Nicht-Mitglieder der Verhandlungen produzieren, keine hierarchische Koordination und Steuerung. Die Maßnahmen zur Effizienz- und Effektivitätssteigerung sind hier bereits durch die 
Kooperationslogik selbstregulativer Verhandlungsnetzwerke und normativer Regime angelegt, die eine Erwartungssicherheit qua schwacher und starker Bindungen sowie durch gemeinsam festgelegte Regeln und Normen generieren. Um diese zu aufrecht zu erhalten, müssen sich die staatlichen Akteure sogar aus den Verhandlungen heraus halten, da sie ansonsten die Kräfteverhältnisse einseitig verschieben oder aber dazu beitragen, dass die solidarischen Interaktionsorientierungen durch starke Bindungen und gemeinsam festgelegte Regelungen untergraben werden. Den staatlichen Akteure bleibt hier 'lediglich` der Einsatz persuasiv-informatorischer Policyinstrumente, die keinen direkten oder indirekten regulativen Charakter haben. Dazu zählen Umweltbildungs- und Informationsmaßnahmen, aber natürlich auch motivationales und vorbildwirksames Verhalten. Vor allem die Informationsmaßnahmen können, zumal wenn sie zielgruppenspezifisch ausgelegt sind, auf niederschwelliger Ebene bewirken, dass BürgerInnen, Unternehmen, soziale Träger etc. relevantes Handlungswissen erhalten und damit in die Lage versetzt werden, Entscheidungen zu treffen.

b. Im Falle von symmetrischen Dilemmaspielen, die sich dadurch auszeichnen, dass zwar alle durch Kooperation gewinnen können, der Gewinn aber für einige Wenige größer ist, wenn sie ihre Kooperation verweigern, ist der 'Schatten der Hierarchie' von besonderer Bedeutung, da pareto-optimale Lösungen zwar möglich sind, die Einigung auf gemeinsame Regelungen aber nur dann erfolgt bzw. garantiert werden kann, wenn dahinter monetäre Anreizmechanismen und/oder administrative Sanktionsmechanismen seitens des Staates stehen, die den Austrittsgewinn deutlich übersteigen. Auch hier sind die Maßnahmen zur Effizienz- und Effektivitätssteigerung durch die Kooperationslogik selbstregulativer Verhandlungsnetzwerke und normativer Regime angelegt, da unkooperatives Verhalten in einer solchen Konstellation für alle Beteiligten schädlich wäre, aber jeder für sich besser abschneiden würde, wenn er die von den anderen eingehaltenen Regeln verletzt. Staatliche Organisationshilfen und die Androhung hoheitlicher Rechtsdurchsetzung bieten hier Effizienz- und Effektivitätssteigerungen, zumal wenn es im Interesse staatlicher Akteure ist, einzugreifen um externe Effekte solcher freiwilliger Verhandlungsrunden auf Andere zu beschränken oder um absehbare Implementationsproblemen vorzubeugen. Dazu können die staatlichen Akteure sowohl auf prozedurale als auch auf kooperative Instrumente zurückgreifen, da beide zugleich geeignet sind, eine Problemlösung herbei zu führen, die die Beteiligung vieler unterschiedlicher Akteure verlangt, bei denen aber nicht sicher gestellt werden kann, dass es überhaupt zu Ergebnissen kommt. Hier können prozedurale Verfahrensvorschriften aber auch Dialog und der Austausch zwischen den Verhandelnden dazu führen, dass individuelle, erst einmal egoistische und auf das 
eigene Wohl bezogene Interessen sich zu kollektiven, andere Interessen antizipierenden und sich so dem Gemeinwohl annähernden sozialen Präferenzen wandeln. Solange dies unterstellt werden kann, ist der Einsatz hierarchischer Gewalt anhand der positiven Gewährleistungspflichten des Staates durch outputseitige Legitimationsargumente rechtfertigbar, obgleich die Politik sich um die Etablierung von zugleich praktisch anwendbaren und gerechten Verfahrensvorschriften bemühen und gegenüber der Allgemeinheit kommunizieren muss, warum sie bei der Vielzahl an Möglichkeiten gerade diese Verhandlungen durch zeitliche, humane und finanzielle Ressourcen unterstützt und/oder warum er sein Gewaltmonopol zur Sicherung dieser Regelungen einsetzt.

c. Bei assymetrischen Positivsummenspielen mit Verteilungskonflikt, wo sich nicht alle gegenüber ihrem Status Quo verbessern können, weil die Gewinn- und Verlusterwartungen ungleich verteilt sind, ist eine Einigung auf gemeinsame Regelungen überhaupt nur dann möglich, wenn kaldor-optimale Lösungen angestrebt werden, sprich wenn alle Beteiligten dazu bereit sind auf ihren maximalen Gewinn für die Suche nach der Option mit dem größten Gesamtgewinn und dem geringsten Gesamtschaden für alle zu verzichten. Da hierbei immer die latente Gefahr besteht, dass diejenigen Optionen favorisiert werden, wo die Benachteiligungen zu Lasten der Allgemeinheit gehen, müssen die staatlichen Akteure besonders achtsam sein, dass die Willensbildung nicht einseitig von Partialinteressen zu Lasten des Gemeinwohls dominiert wird. Daher ist es bei solchen Konstellationen die Aufgabe der staatlichen Akteure als 'Agenten der Allgemeinheit` aufzutreten und nur dienigen Entscheidungsoptionen zuzulassen, die einzelne oder viele Unbeteiligte nicht unnötig benachteiligen, ohne dass die Benachteiligungen möglichst verhindert, oder aber, wenn diese unumgänglich sind, durch die Gewinner der Regelung kompensiert werden. Die Maßnahmen zur Effizienz- und Effektivitätssteigerung sind hier durch die Kooperationslogik innerhalb von Zwangs- bzw. Kollektiventscheidungssystemen angelegt. Hier lassen sich Entscheidungsblockaden nur verhindern, indem Konsensregeln etabliert werden. Dies wiederum erhöht die Transaktionskosten, da die Findung eines Konsenses sehr viel Zeit und Ressourcen in Anspruch nimmt. Die Transaktionskosten lassen sich wiederum über den Einsatz eines Mediators verringern, der den Akteuren hilft eine einvernehmliche Lösung zu finden. Zudem schaffen hier ordnungsrechtliche und ökonomische Instrumente Abhilfe. Da ordnungsrechtliche Instrumente sowohl die Handlung als auch das Ergebnis der Handlung normieren, reduzieren sie die zulässigen Alternativen und zeigen auf, welche Handlungsoptionen sozial erwünscht sind und welche nicht. Ökonomische Instrumente wiederum schaffen Anreize um die Adressaten zu gewünschten Verhaltensände- 
rungen zu bewegen. Da beide Instrumentarien durch den Einsatz staatlicher Macht initiiert werden müssen, bedeutet dies, dass ihr Einsatz gegenüber denjenigen zu rechtfertigen ist, die eine Nichtregelung vorgezogen hätten. Hierfür reichen ebenfalls outputseitige Legitimationargumente, allerdings nur unter Bezug auf einen gerechten Verteilungsmaßstab, da hier argumentiert werden muss, dass die Gewinne aus der Regelung die partiellen Verluste der Verlierer kompensieren.

d. Diejenigen politischen Verhandlungen, die Regulierungen mit nicht kompensierbaren Sonderopfern für bestimmte Bevölkerungs- oder Interessensgruppen zum Ergebnis haben und damit Nullsummenspiele darstellen, können nicht mehr anhand outputorientierter Legitimationsargumente gerechtfertigt werden und hierbei greifen auch sämtliche Policy-Instrumente zu kurz. Diese können nur mit kollektivistischen, inputorientierten Legitimationsargumenten gerechtfertigt werden, was einen gesellschaftlichen Prozess der Diskussion und Deliberation über eben diese Problematiken erforderlich macht. Die staatlichen Akteure als Gewährleistungsinstanz des Gemeinwohls müssen hierbei die relevanten gesellschaftlichen Diskurse anregen, Deliberationsarenen eröffnen und für eine solidarische Umgangsweise miteinander sowie für eine solidarische Regelung werben. Ihre Aufgaben sind koordinierender, strukturschaffender, vorbildwirksamer und ermöglichender Art und zudem müssen die staatlichen Akteure sicher stellen, dass alle relevanten Interessen in den gesellschaftlichen Willensbildungsprozessen vertreten sind und dass die Ergebnisse auch Eingang in die staatlichadministrativen Prozesse finden. Zugleich sind solidarische Orientierungen je nach dem welche strukturellen Voraussetzungen vorherrschen mehr oder weniger wahrscheinlich. Wahrscheinlicher sind sie dort, wo sich partnerschaftliche Beziehungsmuster entwickeln können, sprich in Netzwerkbeziehungen idealerweise unterlegt mit normativen Regelungen. Ungünstiger sind sie innerhalb von Koordinationsstrukturen die Zwangsverhandlungssystemen gleichen. Die Ermöglichung netzwerkartiger Strukturen erfordert indes weniger konkrete als vielmehr grundlegende und basale Maßnahmen seitens der Politik. Solche Strukturen, die im abstrakten gesellschaftlichen Kontext einen Gemeinsinn und im konkreten Interaktionszusammenhang solidarische Orientierungen ermöglichen sollen, sind auf der Akteursebene mit enorm hohen kognitiven, motivationalen und emotionalen Ansprüchen an die Fähigkeiten und Fertigkeiten der Verhandlungsteilnehmer gebunden. Hierfür braucht es Lern- und Übungsprozesse, die es nur in einer 'lebendigen Demokratie' gibt. Eben hieran 'krankt' es in nahezu allen repräsentativen Demokratien, was aber auch bedeutet, dass diejenigen Maßnahmen, die eigentlich den höchsten politischen Legitimationsbedarf haben, am wenigsten legitimiert werden können. 


\subsection{Fazit: Die Anforderungen, Chancen und Risiken der deutschen Biodiversitätspolitik}

Bisher ging es, wie in Abschnitt 1.2.3 anvisiert, um die Erschließung der theoretischen Grundlagen einer auf Kooperation, Verhandlung und Dialog hin angelegten politischstrategischen Ausgestaltung der Biodiversitätspolitik, die als Good Biodiversity Governance charakterisiert wurde. Dabei hat die erste der zu Beginn der politikwissenschaftlichen Detailreflexion gestellten Frage, welche Vorstellungen und Modelle von politischer Steuerung und Regulierung sowie von demokratischer Beteiligung und Verantwortung der Good Biodiversity Governance (GBG) zugrunde liegen können, die gesamten bisherigen Untersuchungen geleitet. Da im letzten Abschnitt die theoretischen Grundannahmen der GBG expliziert worden sind, wird damit auch die theoretische Zielstellung der politikwissenschaftlichen Detailreflexion, nämlich die policy-, governance-, demokratie- und legitimationstheoretischen Grundlagen der GBG zu bestimmen, als eingeholt betrachtet. Daher kann nun anschließend die praktische Zielstellung anvisiert werden, indem erst einmal der Frage nachgegangen wird, welche Anforderungen sich an die deutsche Biodiversitätspolitik vor dem Hintergrund der theoretischen Annahmen der GBG ergeben (5.4.1). Danach wird abschließend dargelegt, vor welchen Defiziten und Entwicklungsmöglichkeiten die deutsche Biodiversitätspolitik vor dem Hintergrund dieser Anforderungen steht (siehe 5.4.2).

\subsubsection{Die Anforderungen an die deutsche Biodiversitätspolitik}

Da es bei der Abschätzung von Anforderungen immer auch darauf ankommt, wie der 'Stand der Dinge' ist, werden die empirischen Ergebnisse des »Politikbarometer zur Biodiversität in Deutschland « (vgl. Laws 2014) zur Explizierung herangezogen: ${ }^{233}$

${ }^{233}$ Das vom WWF Deutschland in Kooperation mit der Universität Lüneburg und dem dortigen Institut für Nachhaltigkeitssteuerung herausgegebene "Politikbarometer zur Biodiversität in Deutschland" (Laws 2014) ist eine empirische Studie, die ihre Ergebnisse aus zahlreichen leitfadengestützten Experteninterviews mit den für Biodiversität zuständigen VertreterInnen der fünf Bundesfraktionen CDU/CSU, SPD, Bündnis90/die Grünen (im weiteren nur die Grünen), die Linke, FDP - in der 17. Legislaturperiode (27.10.2009 bis 22.10.2013) sowie den für Biodiversität jeweils zuständigen VertreterInnen auf unterschiedlichen Funktionsebenen der Bundesministerien gewinnt. In der Studie wurden bis auf das Bundesministerium für Arbeit und Soziales (BMAS), für Justiz (BMJ) sowie für Familie, Senioren, Frauen und Jugend (BMFSFJ), die eine Befragung wegen mangelnder fachlicher Zuständigkeit abgelehnt haben und dem Bundeskanzleramt, wo eine Befragung ohne Gründe abgelehnt wurde, VertreterInnen des Auswärtiges Amtes (AA), des Bundesministeriums für Bildung und Forschung (im folgenden Forschungsministerium; BMBF), des Bundesministeriums für Ernährung, Landwirtschaft und Verbraucherschutz (im folgenden Landwirtschaftsministerium; BMELV [jetzt BMEL]), des Bundesministeriums der Finanzen (im folgenden Finanzministerium; BMF), des Bundesministeriums für Gesundheit (im folgenden Gesundheitsministerium; BMG), des Bundesministeriums des Inneren (im folgenden Innenministerium; BMI), des Bundesministeriums für Umwelt, Naturschutz und Reaktorsicherheit (im folgenden Umweltministerium; BMU [jetzt BMUB]), des Bundesministeriums für Verkehr, Bau und Stadtentwicklung (im folgenden Verkehrsministerium; BMVBS [jetzt BMVI]), des Bundesministeriums der Verteidigung (im folgenden Verteidigungsministerium; BMVg), des Bundesministeriums für Wirtschaft und Technologie [jetzt Bundesministerium für Wirtschaft und Energie] (im 
Die Studie untersucht anhand verschiedener Schlüsselindikatoren „wie Politik und Verwaltung auf Bundesebene mit dem Thema Biodiversität umgehen und wie im parlamentarischen und administrativen Alltag Biodiversitätspolitiktik betrieben wird." (Laws 2014:8) $)^{234}$ Da die Ergebnisse in der Studie lediglich deskriptiv dargelegt und anhand eines dreiteiligen Bewertungsschemas (Regen, bewölkt und sonnig) klassifiziert werden, sollen die Ergebnisse im Folgenden genutzt werden, um die Anforderungen zu beurteilen, die sich über die theoretischen Annahmen der GBG für die deutsche Biodiversitätspolitik ergeben. Dafür ist es indes nötig, dass die ursprüngliche Auswertungssystematik der Studie verändert wird: Der Fokus liegt im Folgenden nicht auf der Frage, welche handlungspraktischen Relevanzen das Thema Biodiversität in Exekutive und Legislative besitzt (ebd., 16), ${ }^{235}$ sondern es geht zum einen um strukturellen Aspekte der Integration, Planung, Steuerung und Umsetzung der Biodiversitätspolitik in den verschiedenen Ministerien, Parteien und Gremien und zum anderen um die Verfahrensweisen mit denen die Biodiversitätspolitik vorangetrieben wird und wie das Thema Biodiversität in die politischen Abläufe integriert wird.

Bezüglich der hier interessierenden strukturellen Aspekte der Integration, Planung, Steuerung und Umsetzung der Biodiversitätspolitik sind von den einzelnen, in der Studie untersuchten Teilindikatoren folgende von Relevanz: a) das Biodiversitätsverständnis der relevanten Akteure in den Ministerien und Parteien, wozu die darunter subsummierten Politikbereiche sowie die Ursachen und Lösungen für die Biodiversitätsvernichtung betrachtet werden; b) die vertikale Politikintegration und der Einfluss der

folgenden Wirtschaftsministerium; BMWi) und des Bundesministeriums für wirtschaftliche Zusammenarbeit und Entwicklung (im folgenden Entwicklungsministerium; BMZ) befragt.

${ }^{234}$ Die Schlüsselindikatoren werden durch max. 3 Teilindikatoren (in Klammern geschrieben) erfasst und sind: 1) strategische Planung (durch Beachtung der Langfristperspektive, des Machtfaktors und der Neuinstitutionalisierung); 2) der individuelle Faktor (durch Beachtung der Biodiversität in der Personalentwicklung und durch Führungspersonen); 3) Policy-Steuerung (durch die Wahl der Policy-Instrumente und die Integration der Biodiversitätsthematik in Planungsprozesse); 4) Umsetzung der Biodiversitätspolitik (durch die Beachtung der Umsetzungsprobleme, der Kontinuitäten und der Biodiversitätsstrategie); 5) die Biodiversitätsziele und ihre Evaluation (über die Biodiversitätszielmarken und die Evaluation der Arbeit zur Erreichung dieser); 6) die Wahrnehmung von Zielkonflikten (durch die Beachtung der Zielkonfilkte mit anderen Politikfeldern und Zielkonflikten im 'eigenen Haus'); 7) die Kooperation mit der Zivilgesellschaft (über die Kooperation mit nichtstaatlichen Akteuren, die Rolle gemeinsamer Bewertungsgremien und Intensität der Einflussnahme von Dritten); 8) die vertikale Politikintegration (über den Einfluss von EU und UN sowie die Berücksichtigung subnationaler Ebenen); 9) das Biodiversitätsverständnis (in den jeweiligen Politikfeldern sowie über die angeführten Gründe und Lösungen für die Biodiversitätsproblematik); 10) die horizontale Politikintegration (über die Koordination mit anderen Ressorts, das Engagement dieser in Biodiversitätsangelegenheiten und die Bedeutung der Interministeriellen Arbeitsgruppe Biodiversität), 11) die Bedeutung von Biodiversität (über die Konkurrenz zu anderen Themen) (vgl. Laws 2014: 22,59).

235 In der Studie werden die Ergebnisse nach Exekutive und Legislative aufgeteilt, d.h., die Schlüsselindikatoren werden einmal bezogen auf die Ministerien und einmal bezogen auf die Parteien ausgewertet. Für die hier interessierende Beurteilung werden die Teilindikatoren so aufgeteilt, dass sie Aussagen über die strukturellen und prozeduralen Defizite und Entwicklungsmöglichkeiten der GBG erlauben. Wie dies geschieht, wird jeweils am Anfang der Abschnitte 1 und 2 ausgeführt. 
supra- und subnationalen Ebenen auf die bundesdeutsche Biodiversitätspolitik; c) die Beurteilung der NBS als Grundlage der deutschen Biodiversitätpolitik unter Beachtung ihrer Zielmarken und Maßnahmen im administrativen Alltag, der strategischen Relevanz der Biodiversität und der Konkurrenz zu anderen politischen Themen sowie d) die Neuinstitutionalisierung, die Personalentwicklung und Führungsverantwortung für die Umsetzung der Biodiversitätspolitik:

a) Das Biodiversitätsverständnis der Befragten in den meisten Ministerien ist eher klassisch auf den Bereich des Natur- und Umweltschutzes ausgerichtet, ohne die gesamte Bandbreite der mit der Biodiversitätspolitik in Verbindung stehenden Politikbereiche aufzunehmen (vgl. ebd., 48). So werden überwiegend Bezüge zu Landwirtschaft, Landnutzung und Flächeninanspruchnahme hergestellt und nur vereinzelt werden Handels-, Außen-, Entwicklungs-, Verkehrs-, Finanz-, Forschungs-, Bildungs- und Klimapolitik sowie der Verbraucherschutz als integrative Bestandteile genannt (vgl. ebd., 49). Ausnahme ist hier das Umweltministerium (BMUB), das fast all diese Politikbereiche unter die Biodiversitätspolitik subsumiert (ebd.). Auch in den Parteien wird Biodiversität überwiegend mit Naturschutz und hier im Besonderen mit Schutzgebietsausweisungen assoziiert.Des Weiteren werden lediglich Landwirtschaft, Flächeninanspruchnahne und Verkehrspolitik von den Parteien vereinzelt benannt und lediglich bei den Grünen wurde auch die Sozial-, die Steuerpolitik genannt (vgl. ebd., 73).

Dieses eingeschränkte Biodiversitätsverständnis setzt sich auch im Verständnis der Gründe für die Biodiversitätsvernichtung sowie hinsichtlich der dafür angeführten Lösungsmöglichkeiten fort. Bezüglich der 'Gründe' werden überwiegend und in erster Linie die sogenannten „direkten Treiber" von den VertreterInnen der Ministerien genannt, die auch im Millennium Ecosystem Assassment (MEA) als Ursachen für den Biodiversitätsverlust aufgeführt werden (vgl. MEA 2005:64-70). Darunter fallen Habitatwandel durch Landnutzung, Flächenzerschneidung und Bebauung, Übernutzung und Verschmutzung durch Emissionen und Stoffeinträge. Die Verdrängung 'heimischer' durch 'gebietsfremde` Arten wurden nur einmal durch das Landwirtschaftsministerium (BMEL) erwähnt, der Klimawandel als einer der wichtigsten direkten Treiber wurde gar nicht erwähnt (vgl. ebd., 50). Von den im MEA aufgelisteten indirekten Ursachen (demographische, soziopolitische, ökonomische, technische, kulturell-religiöse Ursachen) wurden - wenn auch nur sehr selektiv - bis auf die technischen Ursachen alle mindestens einmal benannt. Das bedeutet, dass der technologische 'Fortschritt gar nicht als Hintergrundproblematik für die Vernichtung der Biodiversität angesehen wird und zudem ist auffällig, dass lediglich das Umweltministerium (BMUB) sowohl auf die direkten als auch die indirekten Treiber verweist, wohingegen alle anderen Ministerien in selektiver Weise diejenigen Ursachen benennen, die für den von ihnen geregelten Politikbereich jeweils relevant sind. Bei den Parteien ergibt sich ein ähnlich selektives 
Bild. Auch hier wurden in allen Parteien in erster Linie die direkten Treiber benannt. Die SPD, die Linke und die Grünen stellen aber - anders als CDU/CSU und FDP - auch die Verbindung zu den demographischen und ökonomischen Ursachen her. Soziopolitische, technologische und kulturell-relegiöse Faktoren werden hier indes gar nicht benannt (vgl. ebd., 74), so dass konstatiert werden muss, dass die Biodiversitätsvernichtung über die technologische Ausblendung hinaus in der Legislative auch nicht als gesellschaftliches und politisches Problem angesehen wird.

Eben diese selektiven Problemwahrnehmungsdefizite spiegeln sich auch in Bezug auf die Lösungsvorschläge und -präferenzen wieder, die von den für die Biodiversität zuständigen VertreterInnen der Parteien und Ministerien vorgebracht werden. Alle Parteien sind sich zumindest in einem Punkt einig, nämlich dass es wichtig ist, mehr Bewusstsein für Biodiversität zu schaffen (vgl. ebd., 76). Darüber hinaus gibt es in den Fraktionen ganz unterschiedliche Fokussierungen: Analog zu ihrer Ursachendiagnose präferieren die zuständigen VertreterInnen der CDU/CSU-Fraktion veringerte Flächennutzungen und die verstärkte Ausweisung von Schutzgebieten und weisen zudem auf zu schaffende gesetzliche und politische Vorgaben sowie verwaltungstechnische Regularien hin. Damit verbleiben sie aber im Lösungsbereich des klassischen Naturschutzes. Die FDP verweist wiederum lediglich auf individuelle Verhaltensänderungen im Konsum, womit die politische Verantwortung für das globale Problem der Vernichtung der Biodiversität gar nicht erst wahrgenommen wird (siehe auch 2b). Bei den Grünen, der SPD und den Linken ist - analog zu ihrer Ursachendiagnose - auch das Spektrum der Lösungsansätze breiter, wenn von mehr Umweltverträglichkeitsprüfungen (die Linke), von Inwertsetzungsstrategien (SPD, Grüne), der Veränderung des Wirtschaftssystems (die Linke), Abschied vom Wachs-tumsmodell (Grüne, die Linke) und ökologischer Industriepolitik (SPD) die Rede ist (vgl. ebd.). Genuin politische Lösungsvorschläge wurden nur hinsichtlich der stärkeren Nutzung der Richtlinienkompetenz seitens des Kanzleramtes (SPD), in Bezug auf die Internationalisierung der Politik (die Grünen) und einer stärkeren Koordination (Policy-Integration) zwischen den Fachgebieten (SPD) gemacht (vgl. ebd.). Ansätze für eine stärker partizipative, deliberative oder diskursive Politikformulierung und -gestaltung wurden kein einziges Mal genannt. Dies ist auch auf der ministeriellen Ebene der Fall (vgl. ebd., 52). Lediglich das Stichwort „gesellschaftliche Strukturen", was in lediglich einem Interview benannt wurde (ebd.), lässt auch hier darauf schließen, dass zivilgesellschaftliche Partizipation nicht vollständig aus dem Fokus der Politik fällt (siehe auch 2c). Einmal abgesehen davon, dass hier ebenfalls die Inwertsetzungsstrategie als auch die Internationalisierung noch vor Maßnahmen zur Bewusstseinsbildung sowie vor politischen Regularien präferiert werden, wird hier der Einsatz von Führungspersonal für Biodiversitätsbelange (vgl. ebd,. 51-52) als wichtiger Aspekt von mehreren MinisterialvertreterInnen benannt (siehe 1d). 
b) Die internationale und europäische Ebene, die zwar nicht hierarchisch aber strukturell der nationalen Ebene übergeordnet sind, interagieren im Umweltbereich im Prozess der Politikformulierung und -gestaltung mit der bundes- und landespolitischen Ebene (Mehrebenenpolitik) und üben einen Einfluss auf sie aus. Da für eine effiziente und effektive Biodiversitätspolitik auch Koordinationsmechanismen zwischen den Politikebenen erforderlich sind (vertikale Politikintegration), ist es von Bedeutung den tatsächlichen Einfluss von UN und EU auf die nationale Politik abzuschätzen: ${ }^{236}$ Hierbei ist zu beachten, dass sich zwar in vielen Ministerien Arbeits- und Austauschbeziehungen mit den anderen politischen Ebenen finden, hierbei ist aber entscheidend, dass ein Großteil der biodiversitätsrelevanten UN- und EU-Regelungen Richtlinien sind, d.h., sie geben ein Ziel vor und es bleibt den Mitgliedstaaten überlassen, sie in eigenes Recht umzusetzen. Das bedeutet wiederum, dass die mit der Internationalisierung und Europäisierung der Umweltpolitik verbundenen, bereits theoretisch dargelegten Chancen und Risiken (siehe 5.3.3.2.2) vor allem in den Ministerien angegangen, bearbeitet und aufgefangen werden müssen.

Der Einfluss der UN wird vor allem in denjenigen Ministerien betont, die mit den allgemeinen Regelungen der CBD beschäftigt sind (BMUB, BMEL, BMBF). Wenn es speziell um das unter die CBD fallende Nagoya-Protokoll zur Regelung des gerechten Vorteilsausgleiches geht, verweisen auch andere Ministerien (BMZ, BMG und AA) auf den Einfluss der internationalen Politikebene für ihre Arbeit (vgl. ebd., 46). (ebd.). Allerdings wird dem Einfluss der EU in all diesen und auch noch in den anderen Ministerien eine höhere Bedeutung für die ministerielle Praxis eingeräumt. Insbesondere die Ministerien, deren Regelungsmaterien im besonderen Maße von den Auswirkungen der Biodiversitätsvernichtung betroffen sind und die mit den Anpassungsmaßnahmen zum Schutzgebietmanagement und zu nachhaltigen Bewirtschaftungsweisen konfrontiert sind (BMUB, BMZ, BMEL, BMVI, BMWi, BMVg, BMI), veweisen auf den Einfluss der FFH-Richtlinie, der Vogelschutzrichtlinie, der Wasserrahmenrichtlinie, des Naturschutzgebietsnetzes Natura 2000 und der gemeinsamen Agrarpolitik (GAP) auf die innerministerielle Arbeit (vgl. ebd., 46). Die anderen Ministerien (BMBF, BMG, AA, BMF), die zu einem Großteil auch zu der 'aktiven Gruppe' gehören, konstatieren bezogen auf das Thema Biodiversität immerhin einen selektiven Einfluss der EU auf ihre Arbeit. Darüber lassen sich drei ministerielle Gruppen bilden:

Das Umwelt (BMUB)- das Entwicklungs (BMZ)- und das Landwirtschaftsministerium (BMEL) stehen an 'erster Front', wenn es um nationale und internationale Biodiver-

\footnotetext{
${ }^{236}$ Die vertikale Koordination wird hier unter die strukturellen Aspekte gefasst, da die Studie nicht abfragt, wie die Beziehungen zwischen den jeweiligen politischen Ebenen im Einzelnen ausgestaltet sind, d.h., ob und wie die Koordinations- und Kooperationsbeziehungen funktionieren, sondern nur, ob ein Einfluss supra- und subnationaler Ebenen vorhanden ist.
} 
sitätsbelange geht, da diese sowohl den Einfluss der UN als auch den der EU auf ihre Arbeit unmittelbar spüren bzw. diesen auch mitgestalten können. Folglich haben diese drei Ministerien die administrative Gestaltungshoheit aber zugleich auch die Hauptverantwortung für die Bewahrung und Förderung der Biodiversität auf der nationalen Ebene. Dahinter rangiert eine ganze Liste an 'aktiven`oder 'betroffenen 'Ministerien (BMBF, BMG, AA, BMI, BMVI, BMWi, BMVg), die eher selektiv von den Biodiversitätszielen, -maßnahmen und -regelungen betroffen sind bzw. diese, wenn es um deren Ressort geht, auch mitgestalten. Diesen Ministerien obliegt es nicht die Querschnittsaufgabe Biodiversität sowie die damit verbundene Politikintegration zu befördern und zu koordinieren, aber sie dürfen dieser auch nicht im Wege stehen bzw. diese behindern. Zu guter Letzt bleibt eine Gruppe an Ministerien, für die die Biodiversität ein eher peripheres Thema ist und bleibt (BMF, BMAS, BMJ, BMSFJ), so dass diesen keine nennenswerten Aufgaben und Pflichten bezüglich der Bewahrung und Förderung der Biodiversität zukommen.

Was die Berücksichtigung subnationaler Ebenen angeht, so ergeben sich die ministeriellen Arbeits- und Austauschbeziehungen zwischen Bund- und Ländern bereits aus der Zuständigkeitsverteilung über die konkurrierende Gesetzgebung im Naturschutz im Rahmen des Föderalismus. Hier stellt sich indes die Frage, wie intensiv diese Austauschbeziehungen jeweils geführt werden und ob sie sporadisch oder verstetigt sind. Diesbezüglich zeigen die Ergebnisse der Studie, dass sowohl das Umwelt (BMUB)- als auch das Verkehrsministerium (BMVI) die engsten Kooperationsbeziehungen zur Länderebene pflegen. Sicherlich auch, weil hier die Zuständigkeiten im Wesentlichen bei den Ländern liegt. Allerdings unterhält auch ein Großteil der enger mit Biodiversitätsthematiken befassten Ministerien (BMZ, BMEL, BMBF, BMI, BMVg, BMWi) punktuell oder thematisch eingegrenzte Beziehungen zu den Länderkollegen, wenn es um Biodiversitätsfragen geht. Lediglich das Gesundheitsministerium (BMG) sowie das Auswärtige Amt (AA) unterhalten keine nennenswerten institutionalisierten Beziehungen zur Landesebene, was aber zumindest in Bezug auf das AA damit zu erklären ist, dass die auswärtigen Angelegenheiten vornehmlich auf der Bundesebene reguliert werden.

c) Die NBS, als nationales Strategiepapier zur Umsetzung der Biodiversitätsziele und maßnahmen, wird in der Mehrzahl der Ministerien als „ausreichende Grundlage“ für einen „effektiven Schutz der Biodiversität" in Deutschland angesehen, wobei auffällig ist, dass gerade im Umweltministerium (BMUB), wo sowohl die Fachkompetenz sowie die primäre Zuständigkeit für die Umsetzung der deutschen Biodiversitätspolitik liegt, die Umsetzung der in der NBS anvisierten Ziele, Maßnahmen und Leuchtturmprojekte als „noch nicht ausreichend“ angesehen wird, die nicht vorhandene Priorisierung der Ziele als praktische Problemstellung angemahnt wird und darauf verwiesen wird, dass es der NBS aufgrund ihres Status als Strategiepapier, an Verbindlichkeit mangelt (vgl. 
ebd., 35). Vor dem Hintergrund der Annahme, dass ein problembewusster Umgang mit den Zielen und Maßnahmen der NBS immer auch auf Wissen und Handlungsexpertise basiert, ist es als positiv anzusehen, dass diese kritische Perspektive sich auch in den Gesprächen mit dem Forschungs (BMBF)- und Verkehrsministerium (BMVI) gezeigt hat.

Die Ergebnisse lassen sich besser einordnen, wenn die Ergebnisse auf die Fragen betrachtet werden, wie wichtig die Biodiversitätszielmarken in den jeweiligen Ministerien sind, sprich, welche Bedeutung ihnen im administrativen Alltag beigemessen werden. Hierbei wird deutlich, dass der Umsetzung der Biodiversitätsziele und -maßnahmen auch hier lediglich im Umweltministerium (BMUB) eine hohe Bedeutung im administrativen Tagesgeschäft zukommt. In allen anderen Ministerien spielt die Umsetzung der Biodiversitätsstrategie eine lediglich untergeordnete Rolle, wobei die VertreterInnen des Entwicklungs (BMZ)- des Landwirtschafts (BMEL)- und des Gesundheitsministeriums (BMG) angeben, dass zumindest immer wieder sporadisch auf die Biodiversitätszielmarken geachtet wird (vgl. ebd., 37).

Die geringe Beachtung der NBS erklärt sich wiederum darüber, dass das Thema Biodiversität nicht als Machtfaktor in der innerministeriellen und parteilischen Arbeit angesehen wird. Hiermit ist gemeint, ob und inwieweit das Thema Biodiversität strategisch eingesetzt wird bzw. eingesetzt werden kann, um eigene Politikvorstellungen besser vermitteln, durchsetzen und rechtfertigen zu können. Diesbezüglich stimmt erneut lediglich das Umweltministerium (BMUB) der Aussage zu, dass Biodiversität strategisch eingesetzt werden kann, da Biodiversität als „offener und weniger restriktiv“ sowie „als weniger bedrohlich“ als der Begriff Naturschutz wahrgenommen wird (vgl. ebd., 24). In den anderen, für die Umsetzung der Biodiversitätsziele ebenfalls relevanten Ministerien ( $\mathrm{BMZ}, \mathrm{BMEL}, \mathrm{BMG}, \mathrm{BMBF}, \mathrm{AA}, \mathrm{BMF}$ ) wird Biodiversität zumindest als Thema angesehen, „das in der politischen Diskussion eingeführt ist und mit dem sich in bestimmten Bereichen und bei bestimmten Personengruppen politische Wirkungen erzielen lassen." (ebd.) Innerhalb der Parteien, wo Machtfaktoren noch eine wesentlich größere Rolle für das Tagesgeschäft spielen, wird die in den Ministerien zumindest partiell geteilte Sichtweise auf die strategische Relevanz des Themas Biodiversität von keiner Partei - nicht einmal von den Grünen - vertreten (vgl. ebd., 60). Vielmehr wird hier darauf hingewiesen, dass „Biodiversität eine zu geringe politische Bedeutung besitzt“, „dass Biodiversität als Begriff nicht positiv genug besetzt ist“ und „dass die Begriffe Biodiversität und Naturschutz problematisch seien, da sie mit Verhinderung assoziiert werden." (ebd.) Ob dies zutreffend ist und ob die Einschätzungen der Parteien zur strategischen Relevanz der Biodiversitätsthematik richtig sind, ließe sich anhand der Ergebnisse der seit 2009 erhobenen »Naturbewusstseinsstudien« zur Einstellung der Bevölkerung in Bezug auf Natur und Biodiversität (vgl. BMUB 2010, 2012, 2014, 2016) durchaus kritisch hinterfragen. 
Ungeachtet dessen zeigen die Ergebnisse der Studie, wie schwer es das Thema Biodiversität hat, sich gegen andere Themen - sowohl auf der ministeriellen als auch auf der parlamentarischen Ebene durchzusetzen. So zeigt auch der Indikator Themenkonkurrenz, dass Biodiversität in keiner Partei zu den politischen Themen gehört, denen eine große Bedeutung beigemessen wird (vgl. Laws 2014:78). Nach Themen mit hoher politischer Bedeutung gefragt, nennen - wohlbemerkt die für Biodiversität zuständigen VertreterInnen in den Parteien - Themen wie die Finanzkrise, den Arbeitsmarkt und die Sozialpolitik und selbst bei den Grünen wird eingeräumt, dass Biodiversität „nicht unter dem ersten Drittel der zu behandelnden Themen zu finden ist." (ebd.) Diese Bilanz kann wiederum als Ursache dafür interpretiert werden, dass das Thema Biodiversität nicht genügend Aufmerksamkeit im Bundestag als dem zentralen Ort des politischen Agenda-Settings auf Bundesebene erfährt, so dass auch nicht weiter verwunderlich ist, dass diesem von allen Parteien kaum eine Relevanz in Bezug auf die Etablierung von biodiversitätsrelevanten Themen zugesprochen wird (vgl. ebd., 77-78). Auf der ministeriellen Ebene wird dem Thema Biodiversität - analog zur Einschätzung der strategischen Relevanz - eine größere Gesamtbedeutung beigemessen. Indes wird dem Thema nur im Entwicklungsministerium (BMZ) Priorität vor anderen Themen eingeräumt, was sich vor allem dadurch erklären lässt, dass dieses Ministerium federführend in den Verhandlungen um das Nagoya-Protokoll ist, was auf nahezu allen Vertragsstaatenkonferenzen Thema von Nachverhandlungen ist (siehe 1b). Allerdings wird Biodiversität auch in den anderen Ministerien, die sich an 'erster Front' mit den Fragen der nationalen Umsetzung der CBD beschäftigen (BMUB, BMEL) sowie einigen VertreterInnen der dahinterstehenden Gruppe an biodiversitätsrelevanten Ressorts (BMBF, BMG, AA, $B M V I)$ als ein Themenkomplex unter vielen betrachtet. Bemerkenswert ist hier zweierlei: Zum einen, dass sich das Auswärtige Amt in dieser Gruppe der Ministerien findet, obgleich kein Vertreter des AA an der Interministeriellen Arbeitsgruppe zur Umsetzung der Biodiversitätsstrategie beteiligt ist (siehe $2 \mathrm{~d}$ ) und zum anderen, dass im Verteidigungsministerium (BMVg) das Thema Biodiversität von untergeordneter Bedeutung ist, obgleich 50\% der unbebauten Flächen im Geschäftsbereich des BMVg ausgewiesene Natura-2000 Flächen sind (ebd., 46).

d) Was schlussendlich die biodiversitätsbedingte Einrichtung bzw. Anpassung ihrer institutionellen Strukturen sowie die Personalentwicklung und Führungsverantwortung für die Umsetzung der Biodiversitätspolitik anbelangt, zeigt die Studie, dass vor allem diejenigen Ministerien, die sowohl über eine enge vertikale Politikintegration mit der internationalen als auch der europäischen Ebene berichten (BMUB, BMZ, BMEL) auch eine als positiv wahrgenommene Umgestaltung ihrer Organisation bzw. ihrer Organisationsweisen aufweisen (vgl. ebd., 25). Hier ist anzumerken, dass zu dieser Gruppe auch das Verkehrs (BMVI)- und das Verteidigungsministerium (BMVg) gehören. Auch 
wenn daraus nicht geschlossen werden kann, dass eine Neuinstitutionalisierung eine effizientere oder effektivere Befassung mit dem Thema Biodiversität bedeutet, zumal keine Angaben darüber gemacht wurden, was genau in den Ministerien verändert oder angepasst wurde, ist auffällig, dass insbesondere die Ministerien strukturelle Veränderungen vorgenommen haben, die an 'erster Front' bei der nationalen Umsetzung der Biodiversitätsziele und -maßnahmen stehen (siehe 1b) und deren Arbeit durch die internationalen und europäischen Regelungen maßgeblich beeinflusst wird.

Sieht man dies zudem im Zusammenhang mit den Ergebnissen der Studie zur Personalentwicklung sowie der Frage, inwieweit es eine explizite Führungsverantwortung in den Ministerien und Parteien für die Biodiversitätspolitik gibt, dann wird auf der ministeriellen Ebene ersichtlich, dass dort, wo sich Führungspersonen aktiv für das Thema Biodiversität einsetzen (BMUB, BMZ, BMEL und BMBF) (vgl. ebd., 28) und dort, wo das Thema Biodiversität auch eine Rolle in der Personalentwicklung und Fortbildung der MitarbeiterInnen spielt (BMUB, BMZ, BMEL, BMVg und AA) (vgl. ebd., 27) insbesondere das Umwelt (BMUB)-, das Entwicklungs (BMZ)- und das Landwirtschaftsministerium (BMEL) eine Überschneidung in allen drei Indikatorbereichen aufweisen. Allerdings ist dabei anzumerken, dass selbst in diesen drei Ministerien keine systematischen Fortbildungen stattfinden, „deren Ziel die Förderung des Wissens um Interdepenzen von Biodiversität mit verschiedenen Nachhaltigkeitsdimensionen und/oder Wirkungszusammenhänge zwischen den jeweiligen Fachgebieten des Ministeriums und Biodiversität" sind (ebd., 26). Auch ist hierbei auffällig, dass ausgerechnet das für Forschung und Bildung zuständige Ministerium (BMBF) keinerlei Fortbildungen zum Thema Biodiversität angeboten hat, auch wenn hier von Führungsverantwortung für das Thema Biodiversität berichtet wurde. In Bezug auf die Parteien zeigt die Studie zudem, dass Biodiversität in der Personalentwicklung lediglich bei den Grünen eine Rolle spielt (vgl. ebd., 61), auch wenn alle Parteien angeben, dass das Führungspersonal in den Fraktionen grundsätzlich am Thema Biodiversität interessiert sei. Anhand der Interviews zeigt sich indes, dass Biodiversität in keiner Partei - und auch nicht bei den Grünen - als Profilierungsthema des Führungspersonals gesehen wird (vgl. ebd., 62).

Bezüglich der Verfahrensweisen mit denen die Biodiversitätspolitik vorangetrieben und das Thema Biodiversität in die politischen Abläufe integriert wird, sind von den Teilindikatoren der Studie folgende relevant: a) die strategische Planung und damit der Umgang mit Zielkonflikten zwischen Kurz- und Langfristigkeit bei gleichzeitiger Betrachtung der Präferenzwechsel in der Umweltpolitik sowie den Zielkonflikten innerhalb und zwischen den Ressorts; b) die Policy-Steuerung über das Vorhandensein von Planungsprozessen, die Identifikation von Umsetzungsproblematiken und deren Evaluation sowie den Vergleich zwischen der präferierten und der tatsächlichen Nutzung der PolicyInstrumente, c) die Kooperation mit der Zivilgesellschaft in Hinblick auf die Berück- 
sichtigung nicht-staatlicher Akteure, die Relevanz gemeinsamer institutionalisierter Beratungs- und Bewertungsgremien und die gesellschaftlichen Akteursgruppen und ihre Einflussnahme sowie d) die horizontale Politikkoordination über das Engagement der einzelnen Ressorts, deren Kooperationsbereitschaft und die Bedeutung der Interministeriellen Arbeitsgruppe zur Umsetzung der Biodiversitätsstrategie (IMA NBS).

a) Bei der Untersuchung der Integration der Biodiversitätsthematik in die strategische Planung steht im Vordergrund, ob Biodiversität in der Alltagspraxis der Ministerien und Parteien eher unter der Perspektive eines kurz- oder langfristigen Zeithorizonts behandelt wird, ob es Zielkonflikte zwischen Kurz- und Langfristigkeit sowie zwischen und innerhalb der Ressorts gibt und wie das Thema in Planungsprozesse integriert wird. Auf der ministeriellen Ebene zeigt sich, dass zumindest in einigen Ministerien (BMUB, BMZ, $B M B F, B M V g, B M G)$, die sich auf der strukturellen Ebene durch einen engeren Biodiversitätsbezug ausweisen ließen (siehe $1 \mathrm{~b}$ ), biodiversitätsbezogene Themen auch mit langfristigeren Planungsprozessen unterlegt sind (vgl. ebd., 23). ${ }^{237}$

Beachtet man hierbei den Indikator Präferenzwechsel, über den gefragt wird, ob „ein Regierungswechsel und/oder der Beginn neuer Legislaturperioden die Schwerpunktsetzungen in der Biodiversitätspolitik verändern“ (ebd., 43), dann wird deutlich, dass die deutsche Biodiversitätspolitik als in hohem Maße konstant angesehen werden kann (vgl. ebd.). Vor diesem Hintergrund und unter Beachtung des Umstandes, dass die Umsetzung der meisten in der NBS formulierten Biodiversitätsziele einen mittelund langfristigen Handlungshorizont erfordern, ist es bemerkenswert, dass das Landwirtschaftsministerium (BMEL), welches immerhin zu den 'Frontakteuren ' in der deutschen Biodiversitätspolitik gehört (siehe 1b), aber auch das Verkehrs (BMVI)-, das Wirtschafts (BMWi)- und das Innenministerium sowie das Auswärtige Amt (AA) keinen langfristigen Planungshorizont bezüglich biodiversitätsrelevanter Themen haben (vgl. ebd., 23). ${ }^{238}$

Die konkreten Schwierigkeiten langfristiger Planung in der ministeriellen Alltagspraxis zeigen sich deutlicher, wenn diese in Zusammenhang mit dem Teilindikator bezüglich der Zielkonflikte zwischen kurz- und langfristigen Erfordernissen betrachtet werden (vgl. ebd., 40-41). Während die Diskrepanz zwischen Kurz- und Langfristigkeit vor allem im Umwelt (BMUB)- und im Entwicklungsministerium (BMZ) bejaht und explizit als Pro-

${ }^{237}$ Eine ausschließliche Fixierung auf langfristige Themen, Projekte und Zeitrahmen ist augrund der Art der Arbeitsweise in politisch-administrativen Organisationen gar nicht möglich, da diese tagespolitische Aufgaben prioritär zu erfüllen haben. Bei der Analyse der Langfristorientierung geht es in der Studie daher darum herauszufinden, „ob eine Langfristorientierung ebenfalls einen Arbeitsrahmen darstellt, wie es bei Fragestellungen mit Biodiversitätsbezug notwendig erscheint." (Laws 2014:22)

${ }^{238}$ Im Fall des Auswärtigen Amtes (AA) ist sicherlich einzuräumen, dass in diesem Ministerium die Notwendigkeit auf kurzfristige Entwicklungen reagieren zu können einen großen Stellenwert einnimmt und sich auch in den administrativen Strukturen niederschlägt, so dass hier das Fehlen langfristigerer Planungsprozesse zumindest erklärbar ist. 
blemstellung angesprochen und einigen anderen Ministerien zumindest nicht verneint wird (AA, BMVI, BMI, BMVg, BMF, BMBF), ist auffällig, dass diejenigen Ministerien, die einen eher ökonomischen und nutzenbezogenen Schwerpunkt hinsichtlich der Bewahrung und Förderung der Biodiversität haben (BMG, BMEL, BMWi und BMF), keine Zielkonflikte auf der Zeitachse sehen bzw. diese explizit verneinen (vgl. ebd., 41). Die Studie verweist diesbezüglich darauf, dass es innerhalb dieser Ministerien im Wesentlichen „um die finanzielle Belastung von Unternehmen“ sowie „um konkrete Fragen des Flächenverbrauchs und der wirtschaftlichen Nutzung" geht (vgl. ebd.), woran sich zeige, dass es weniger um grundsätzliche und damit auch langfristigere Fragen der Organisation und Entwicklung des Wirtschaftssystems, als vielmehr um aktuelle und ganz konkrete Fragen der Nutzung bzw. der Verteilung von Nutzen und Kosten geht.

Dies wiederum geht 'Hand in Hand' mit den, durch die Ministerialvertreter benannten Zielkonflikte zwischen den Ressorts, wo sich zeigt, dass insbesondere die Diskrepanzen zwischen den ökonomischen und nutzenbezogenen sowie den schutzbezogenen Aspekten der Bewahrung und Förderung der Biodiversität zu Spannungsverhältnissen führen (vgl. ebd., 39). Obgleich alle befragten Ministerien Zielkonflikte zwischen ihrem und den anderen Ressort einräumen (vgl. ebd., 38), werden von den VertreterInnen der einzelnen Ministerien vor allem Zielkonflikte zwischen Wirtschaft und Biodiversität (BMUB, BMVI, AA), Energie und Biodiversität (BMUB, BMEL, BMVI), Verkehr und Biodiversität (BMVI) sowie Nutzung/Bewirtschaftung und Biodiversität (BMUB, BMEL, AA, $B M V g$ ) benannt (vgl. ebd.). Des Weiteren werden die finanziellen Belastungen, die sich aus der Bewahrung und Förderung der Biodiversität ergeben (BMVI, BMWi) sowie der Einfluss der Biodiversitätsvernichtung auf soziale Ungleichheiten und Entwicklungsfragen (BMZ, BMUB) als Dissenspunkte zwischen den einzelnen Ressorts identifiziert (vgl. ebd., 39).

Hierbei ist zu beachten, inwiefern es auch Zielkonflikte innerhalb der Ministerien zwischen den einzelnen Organisationseinheiten bezüglich biodiversitätsrelevanter Themen gibt (vgl. ebd., 39-40). Während das Wirtschafts (BMWi)-, das Innen (BMI)-, das Verteidigungs- (BMVg) und das Finanzministerium (BMF) keinerlei Konflikte bezüglich biodiversitätsbezogener Themen 'im eigenen Haus' sehen, werden diese in den anderen Ministerien (BMUB, BMEL, BMZ, BMBF, BMVI, AA) vor allem dann eingeräumt, wenn biodiversitätspolitische Ziele mit anderen Aufgaben des Ministeriums in Konflikt geraten. Dies ist bspw. bezogen auf Flächenkonkurrenzen vor allem im Landwirtschafts(BMEL)- und im Verkehrsministerium (BMVI) sowie bezüglich der Ausgestaltung der Energiewende im Umweltministerium (BMUB) der Fall. Der Umgang mit Zielkonflikten ist folglich vor allem im Umwelt (BMUB)- und im Verkehrsministerium (BMVI) von besonderer Relevanz, da diese beiden Ministerien sowohl auf zeitliche als auch auf sehr verschiedenartige inhaltliche Konfliktbereiche verarbeiten müssen, wohingegen die an- 
deren Ministerien eher auf ganz bestimmte, ihren Ressortaufgaben angepasste Zielkonflikte reagieren. Vor dem Hintergrund der Annahme, dass das Erkennen und die Einschätzung von Hindernissen - sowohl auf der Zeitachse, als auch inhaltlich - wichtige Grundbausteine bei der Erreichung einer effektiven und legitimen Biodiversitätspolitik sind, geht es darauf aufbauend immer auch um die Frage, ob diese Hindernisse auch in den administrativen Planungsprozessen berücksichtigt werden, was wiederum ein Aspekt der Policy-Steuerung ist.

b) Bezüglich der Policy-Steuerung muss zuerst auf das Vorhandensein von administrativen Planungsprozessen, die Identifikation der Umsetzungsprobleme sowie die Evaluation des Umsetzungsprozesses geschaut werden. Bezüglich der Frage, ob es ressortspezifische administrative Planungsprozesse zu biodiversitätsbezogenen Zielen und Vorhaben innerhalb der einzelnen Ministerien gibt, zeigt die Studie, dass es bis 2014 in keinem Ministerium verwaltungstechnische Vorgaben im Umgang mit den Biodiversitätszielen und -maßnahmen gab für das gesamte Ministerium gab (vgl. ebd., 31). Allerdings gibt es innerhalb des Umwelt (BMUB)-, Verkehrs (BMVI)-, Forschungs (BMBF)und Verteidigungsministeriums (BMVg) zumindest sektoral bestimmte Arbeitsbereiche für die es derartige Pläne gibt, was aber auch bedeutet, dass es in den restlichen Ministerien keinerlei Handlungsanweisungen und -pläne gibt, die explizit auf Biodiversität ausgerichtet sind und die über die Beteiligungs- und Mitzeichnungsvorschriften hinausgehen, die sich aus übergeordneten Vorgaben wie bspw. der allgemeinen Gesetzesfolgenabschätzung, aus Umweltverträglichkeitsprüfungen oder der gemeinsamen Geschäftsordnung der Bundesministerien ergeben (vgl. ebd., 32). Dies ist vor allem vor dem Hintergrund der Annahme relevant, dass die Wahrnehmung von Zielkonflikten (zwischen Kurz- und Langfristigkeit sowie zwischen und innerhalb der Ressorts, siehe 2a) Planungsprozesse erforderlich machen und dies umso stärker, je mehr Zielkonflikte dabei vorhanden sind. Dies trifft vor allem das Umwelt (BMUB)- und das Verkehrsministerium (BMVI), da diese beiden Ministerien sowohl auf zeitliche als auch auf sehr verschiedenartige inhaltliche Konfliktbereiche für das eigene Ministerium reagieren müssen (siehe 2a). Grundsätzlich ist dies aber auch für das Landwirtschafts- (BMEL) und das Entwicklungsministerium (BMZ) von Notwendigkeit, da beide Ministerien ebenfalls an 'erster Front' bei der Umsetzung der deutschen Biodiversitätspolitik stehen (siehe 1b). In Bezug auf das Landwirtschaftsministerium ist zudem anzumerken, dass das Erkennen von Zielkonflikten eine wesentliche Bedingung für die Aufstellung von Planungsprozessen ist, d.h., dass nur weil die VertreterInnen des BMEL keine Konflikte auf der Zeitachse identifizieren, heißt dies nicht, dass keine vorhanden sind.

Des Weiteren ist für die Policy-Steuerung die Identifikation der zentralen Umsetzungsproblematiken sowie die Evaluation des Umsetzungsprozesses relevant. Was die Umsetzungsproblematiken betrifft, werden im Wesentlichen Personalmangel und fehlen- 
de finanzielle Mittel sowie die Diskrepanz zwischen der grundsätzlichen Anerkennung und der konkreten Umsetzung der Ziele und Maßnahmen zur Bewahrung und Förderung der Biodiversität benannt (vgl. ebd., 37). Hier wird der eigentlich erfreuliche Umstand, dass die politischen Schwerpunkte in der deutschen Biodiversitätspolitik als in hohem Maße konstant angesehen werden können (siehe Indikator Präferenzwechsel), dadurch konterkariert, dass die von den MinisterialvertreterInnen benannten Umsetzungsproblematiken als langfristige Strukturprobleme angesehen werden müssen. Zur Behebung dieser Problematiken werden von den VertreterInnen in den einzelnen Ministerien indes nicht lediglich mehr Personal oder Finanzen sondern vielmehr konkretere, fallspezifischere Aufgabenstellungen eingefordert, welche Organisationseinheit bei welchen Zielen in welchem Zeitraum welche Aufgaben zu erfüllen haben, sprich, es werden die nicht bzw. nur in Ansätzen vorhanden administrativen Pla-nungsprozesse eingefordert. Diese müssen allerdings 'hausintern ' auch regelmäßig evaluiert werden, um das vorhandene (knappe) Personal und die zur Verfügung stehenden (nicht ausreichenden) Finanzmittel möglichst effektiv einzusetzen und um die Pläne den sich verändernden Gegebenheiten anzupassen, was aber bisher nur ansatzweise im Umwelt (BMUB)-, im Entwicklungs (BMZ)- und im Verkehrsministerium (BMVI) der Fall ist (vgl. ebd., 37-38).

Bezüglich der in Abschnitt 5.1.1 aufgeführten Policy-Instrumente lässt sich zur Bilanz der Policy-Steuerung schlussendlich anführen, dass die tatsächliche Instrumentennutzung und die Instrumentenpräferenz stellenweise sehr unterschiedlich in den einzelnen Ministerien und Parteien ausfallen. Obgleich sowohl das Umwelt (BMUB)-, das Landwirtschafts (BMEL)-, das Verkehrs (BMVI)- und das Wirtschaftsministerium (BMWi) angeben, auf einen Instrumentenmix aus ordnungsrechtlichen, marktwirtschaftlichen, fördernden und informatorischen Instrumenten zurückzugreifen, ${ }^{239}$ präferiert lediglich das Umweltministerium (BMUB) diesen Instrumentenmix auch (vgl. ebd., 30). Sowohl das Verkehrs (BMVI), das Landwirtschafts (BMEL)- als auch das Wirtschaftsministerin (BMWi) präferien hingegen hauptsächlich informatorische und marktwirtschaftliche Instrumente (ebd.). Auch wird in der Studie deutlich, dass obgleich marktwirtschaftliche Instrumente darüber hinaus auch beim Finanzministerium (BMF) präferiert werden, eben diese Instrumente in der ministeriellen Praxis kaum zum Einsatz kommen (ebd.). Demgegenüber wird der Einsatz von informatorischen Instrumenten von der Mehrzahl der Ministerien präferiert (AA, BMG, BMZ, BMEL, BMVI, BMWi, BMBF). Sie

${ }^{239}$ Die Studie unterscheidet zwischen ordnungsrechtlich-regulativen, marktwirtschaftlichen, fördernden und informativen Instrumenten (Laws 2014:29). Sie macht aber nicht deutlich, was genau unter die Kategorie der fördernden Instrumente fällt. Da marktwirtschaftliche Instrumente über den Einsatz von Anreizmechanismen ebenfalls einen stark fördernden Charakter haben, zugleich aber planerische und kooperative Instrumente nicht explizit benannt werden, obgleich die Auswertung der Ergebnisse dafür spricht, dass diese mit fördernden Instrumenten gemeint sind, wird hier auf die in Abschnitt 5.1.1 dargelegte Kategorisierung der Policy-Instrumente zurückgegriffen. 
kommen aber speziell beim Ausländischen Amt (AA), beim Gesundheits (BMG)- und beim Entwicklungsministerium (BMZ) zum Einsatz (ebd.). Der Einsatz von ordnungsrechtlichen Instrumenten wird neben den Ministerien, die auf einen breiten Instrumentenmix setzen (BMUB, BMEL, BMVI, BMWi) lediglich vom Verteidigungsministerium (BMVg) explizit genannt. Der Einsatz von planerischen und kooperativen Instrumenten steht schließlich im Bildungs (BMBF)-, im Innen (BMI)- und im Entwicklungsministerium (BMZ) im Fokus, obgleich das $B M Z$ die informatorischen Instrumente präferiert. Die hier ersichtlich werdende starke Präferenz für die informatorischen Instrumente, die sich auf der ministeriellen Ebene zeigt, findet sich auch auf der Parteienebene wieder (vgl. ebd., 62-63). Die Ergebnisse zeigen, dass alle Parteien informatorische Instrumente präferien (ebd., 63). In der Studie wird zudem der kritische Fokus der Grünen auf die marktwirtschaftlichen Instrumente angeführt und dass sowohl die Gesprächspartner der CDU/CSU-Fraktion als auch der SPD darauf hinweisen, dass „es einer Renaissance des ordnungsrechtlichen Denkens" bedarf (ebd.). Die FDP betont demgegenüber, dass ordnungsrechtliche Instrumente „nicht alle Aufgaben lösen können und marktwirtschaftliche Anreizsysteme geschaffen werden müssten.“ (ebd.) Bei diesen recht klassischen Positionen stellt sich indes die Frage, inwieweit die befragten Personen stärker an Aspekte des Naturschutzes im engeren Sinne als an die Bewahrung und Förderung der Biodiversität gedacht haben, wo es aufgrund der integrativen Zielstellung um ein sowohl als auch, sprich um die fallbezogene und je nach strukturellem Kontext auch unterschiedliche Anwendung eines Instrumentenmixes geht.

c) Bezüglich des Schlüsselindikators 'Kooperation mit der Zivilgesellschaft ' fragt die Studie nach der Intensität der Zusammenarbeit der politisch-staatlichen mit nicht-staatlichen Akteuren, ob Gremien zur gemeinsamen Bewertung und Weiterentwicklung der Biodiversitätspolitik von den Ministerien und Parteien genutzt werden und welche Interessensgruppen mit welchen Ministerien und Parteien kooperieren (vgl. ebd., 41-45). Über diese Indikatoren soll festgestellt werden, ob und inwiefern das Kooperationsprinzip im Aufgabenfeld Biodiversität tatsächlich erfüllt wird (siehe 5.1.1).

Diesbezüglich ist in erster Instanz wichtig, welche gesellschaftlichen Akteursgruppen auf welche Ministerien einen Einfluss ausüben. Hierbei unterscheidet die Studie zwischen Nutzer- und Wirtschaftsverbänden, Umwelt- und Naturschutzverbänden, Organisationen und Einzelakteuren aus Wissenschaft und Forschung sowie zivilgesellschaftlichen Akteursgruppen (vgl. ebd., 42). Die Umwelt- und Naturschutzverbände bilden laut MinisterialvertreterInnen die aktivste Gruppen, die Einfluss auf die staatliche Politikformulierung nehmen, gefolgt von den Nutzer- und Wirtschaftsverbänden (vgl. ebd., 45). Hierbei zeigt sich, dass alle gesellschaftlichen Akteure in ihren Versuchen der Einflussnahme sich vornehmlich an diejenigen Ministerien richten (BMUB, BMZ, BMEL, $B M V I, B M B F)$, die sowohl durch die internationalen als auch die innerstaatlichen Po- 
litikverflechtungen Einfluss auf die Gestaltung der Biodiversitätspolitik nehmen (siehe 1b) (vgl. ebd., 42,44). Schaut man demgegenüber auf die Ministerien und deren Kooperationsstrukturen mit den gesellschaftlichen Akteursgruppen, dann weisen die oben genannten Ministerien (BMUB, BMZ, BMEL, BMVI, BMBF) auch die engsten und dauerhaftesten Kooperationsbeziehungen zu den gesellschaftlichen Akteuren auf, während die anderen Ministerien nur teilweise, fallbezogen oder in unregelmäßigen Abständen mit gesellschaftlichen Akteuren zusammen arbeiten ( $A A, B M W i, B M V g$ ) bzw. keinerlei Kooperationsstrukturen und -beziehungen vorhanden sind (BMG, BMI, BMF), weil diese als kontraproduktiv für die administrative Arbeit betrachtet werden (vgl. ebd., 42). Zudem fällt auf, dass der Großteil der Ministerien, die über Kooperationsstrukturen mit gesellschaftlichen Akteuren verfügen, bestimmte Exklusionen haben bzw. Schwerpunkte in ihren Kooperationsbeziehungen setzen:

Lediglich das Umwelt (BMUB)- und das Verkehrsministerium (BMVI) kooperien mit allen gesellschaftlichen Akteuren (vgl. ebd., 44). Bei den anderen Ministerien fallen bestimmte Exklusionen auf. So exkludiert das Landwirtschaftsministerium (BMEL) die zivilgesellschaftlichen Akteursgruppen, wohingegen das Entwicklungs (BMZ)- und das Forschungsministerium (BMBF) mit der gesamten Bandbreite an Akteursgruppen außer den Nutzerverbänden koopieren (ebd.). Das Ausländische Amt (AA) kooperiert schwerpunktmäßig mit den Umweltverbänden sowie den Akteuren aus Wissenschaft und Forschung, das Wirtschaftsministerium (BMWi) mit den Wirtschafts- und den Umweltverbänden und das Verteidungsministerium (BMVg) lediglich mit Vertreterlnnen aus Wissenschaft und Forschung (ebd.). Hierbei ist auffällig, dass alle Ministerien mit den Umweltverbänden kooperieren und dass in fast allen Ministerien VertreterInnen aus Wissenschaft und Forschung an der Politikformulierung beteiligt sind (vgl. ebd., 45). Ähnlich ist das Bild auf der Parteienebene: Während alle Fraktionen mit den Umweltverbänden zusammen arbeiten, werden Akteure aus Wissenschaft und Forschung nicht bei SPD und Linken inkludiert und die zivilgesellschaftlichen Akteure finden sich bei CDU/CSU und FDP nicht stark genug repräsentiert. Wirtschafts- und Nutzerverbände werden demgegenüber lediglich bei der FDP als wichtige Kooperationspartner bei Fragen der Biodiversität benannt (vgl. ebd., 70).

Schaut man sich schlussendlich an, welche Ministerien und Parteien auch über gemeinsame Bewertungs- und Beratungsgremien verfügen, d.h., dass es auch institutionalisierte Beteiligungsforen für nicht-staatliche bzw. nicht parteizugehörige Akteure gibt, wird deutlich, dass lediglich das Umwelt (BMUB)-, das Entwicklungs (BMZ)- und das Forschungsministerium (BMBF) (vgl. ebd., 43) sowie die Grünen (vgl. ebd., 70) nicht nur rein informationelle Austauschbeziehungen mit gesellschaftlichen Akteuren zum Thema Biodiversität pflegen, sondern diese darüber hinaus auch an der Formulierung und Durchsetzung konkreter Ziele, Pläne und Projekte beteiligen (vgl. ebd., 43). Hier- 
über wird unter Beachtung der anderen Indikatoren indes deutlich, dass lediglich im Umweltministerium (BMUB) dem Kooperationsprinzip in Gänze nachgekommen wird und dass lediglich die Grünen alle Interessensgruppen auf substanzielle Art zur Meinungs- und Willensbildung in Biodiversitätsfragen politik einladen und heranziehen.

d) In Bezug auf die horizontale Politikkoordination ist schlussendlich - neben den Kooperationsbeziehungen zu den gesellschaftlichen Akteuren - auch die Koordination und Kooperation zwischen den politischen Ressorts sowie die Bedeutung der Interministeriellen Arbeitsgruppe Biodiversitätsstrategie (IMA NBS) bei der Umsetzung der Biodiversitätsziele und -maßnahmen relevant. Hierbei geht es im Wesentlichen um das jeweilig wahrgenommene Engagement der Ministerien sowie - vor dem Hintergrund der Zielkonflikte zwischen den Ministerien (siehe 2a) - darum, wo die Schaffung inhaltlicher Konvergenzen bzw. verstärkter Abstimmungen von Nöten sind, um zu einer kohärenten Biodiversitätspolitik zu gelangen. Positive Koordinationen zeichnen sich in der Studie dabei dadurch aus, dass die jeweiligen Ministerien als engagiert in Bezug auf Biodiversitätsfragen wahrgenommen werden und dass die Möglichkeit der Kooperation in wesentlichen Fragen bejaht wird, wohingegen negative Koordinationen dadurch charakterisiert sind, dass ein gemessen an der Zuständigkeit zu geringes Engagement von den anderen Ministerien attestiert wird und dass die Kooperationsbeziehungen als schwierig und/oder konfliktträchtig angesehen werden (vgl. ebd., 53). ${ }^{240}$

Was das Engagement der Ressorts für Biodiversitätsbelange angeht, zeigt sich, dass insbesondere die Ministerien (BMEL, BMWi, BMG, BMVI), die einen eher ökonomischen und nutzenbezogenen Schwerpunkt bezüglich der Bewahrung und Förderung der Biodiversität haben (siehe 2a), von den anderen Ministerien als weniger engagiert in Biodiversitätsbelangen wahrgenommen werden, wohingegen das Umwelt (BMUB)-, das Entwicklungs (BMZ)- und das Bildungs- und Forschungsministerim (BMBF) als besonders engagiert in Biodiversitätsangelegenheiten wahrgenommen werden (vgl. ebd., 54). Ein differenziertes Bild ergibt sich, wenn darauf geschaut wird, welche Kooperationsbereitschaft den einzelnen Ressorts bei der Umsetzung der Biodiversitätsziele und -maßnahmen von ihren KollegInnen in den anderen Ministerien attestiert wird: Hierbei wird lediglich dem Entwicklungsministerium (BMZ) eine hohe Kooperationsabilanz von den anderen Ministerien attestiert (vgl. ebd., 53). Alle anderen Ministerien werden in ihren Kooperationsbereitschaften differenziert bilanziert und hierbei ist eine Zweiteilung auffällig: Das Umwelt (BMUB)- und Forschungsministerium (BMBF) sowie das Auswärtige Amt (AA) werden überwiegend mit guten Kooperationsbeziehungen asso-

240 In der Studie wird angemerkt, dass „sich nicht alle Ministerien zu ihrer Einschätzung hinsichtlich der Qualität der Zusammenarbeit mit anderen Ministerien geäußert haben und von einigen Interviewpartnern diesbezüglich große Zurückhaltung zu erkennen war, (...) was die persönliche Einschätzung anderer Ministerien betrifft." (Laws 2014:53, 54) 
ziert, auch wenn von anderen Ministerien auf bestehende Konfliktpotenziale verwiesen wird (vgl. ebd.), die aber durch die Ministerien auch selbst gesehen und reflektiert werden (siehe 2a). Dem Landwirtschafts (BMEL)-, Gesundheits (BMG)- und Verkehrsministerium (BMVI) hingegen werden zugleich positive und negative Kooperationsbilanzen durch die anderen Ministerien attestiert (vgl. ebd.), sprich, diese haben sowohl konfliktträchtige als auch kooperative Koordinationsbeziehungen. Dies könnte daran liegen, dass es gerade in diesen Ministerien um ganz konkrete ökonomische Fragen und aktuelle Maßnahmen zur Anpassung geht, was eben auch bedeutet, dass immer wieder neue Allianzen 'geschmiedet' werden müssen, bei denen es immer auch um konkrete Verteilungsfragen geht, die nun einmal nicht ohne Konflikte beigelegt werden können. Den restlichen Ministerien (BMWi, BMI, BMF) wird eine überwiegend negative Kooperationsbilanz attestiert (vgl. ebd.), wobei bemerkenswert ist, dass hier die Ministerien benannt werden, die angegeben haben, kein Konfliktpotenzial bezüglich Biodiversitätsfragen 'im eigenen Haus' zu sehen (siehe 2a), was wiederum so interpretiert werden kann, dass 'in sich geschlossenen Positionen 'eher dazu führen, dass deren VertreterInnen weniger kompromiss- und kooperationsbereit sind.

In diesem Zusammenhang ist daher die Bedeutung der Interministeriellen Arbeitsgruppe zur Umsetzung der Biodiversitätsstrategie (IMA NBS) ${ }^{241}$ von besonderer Relevanz, da hiermit ist das einzige ressortübergreifende Gremium benannt ist, welches die Zusammenarbeit und den Austausch der Miniterien untereinander fördern und koordinieren kann. Schaut man sich hier die Ergebnisse der Studie an, dann wird indes deutlich, dass die IMA NBS eher ein informatorisches, denn ein koordinatives Gremium ist, welches zudem nur einen geringen Einfluss auf die alltägliche administrative Arbeit in den Ministerien hat (vgl. ebd., 55). Die Studie zeigt zudem, dass in einigen Ministerien überhaupt nicht über Vernetzung und Policy-Integration nachgedacht wird (vgl. ebd., 56). ${ }^{242}$ Stattdessen ist das klassische 'Ressortprinzip', nach dem die Ministerium sich lediglich um ihren eigenen Fachbereich kümmern und auf diesem Weg alle zusammen (additiv) zu einer Erfüllung der Ziele und Maßnahmen gelangen, in einigen Ministerien nach wie vor stark verbreitet. Angesichts dieser Ergebnisse ist die Wirkung der IMA NBS als Koordinationsgremiun der Bundesregierung nur als sehr gering einzuschätzen.

${ }^{241}$ Die IMA NBS wurde 2010 gegründet und ihre Leitung liegt in den Händen des Umweltministeriums. In ihr versammeln sich die für Biodiversität zuständigen VertreterInnen des BMAS, das an der Studie nicht teilgenommen hat, weil es keine inhaltlichen Berührungspunkte zur Biodiversitätsthematik gesehen hat (siehe FN 285) sowie VertreterInnen des BMBF, des BMEL, des BMF, des BMG, des BMI, des $B M V I$, des BMVg, des BMWi sowie des BMZ. Das AA, obgleich in der Studie durch die internationale Verpflechtung als wichtiges Ministerium für Biodiversitätsangelegenheiten ausgezeichnet und als Ansprechpartner für Biodiversitätsfragen seitens der gesellschaftlichen Akteure, hinsichtlich des Engagements für Biodiversitätsfragen sowie über seine Kooperationsbereitschaft mit den anderen Ministerien auch in die horizontale Koordination involviert, ist nicht Mitglied in der IMA NBS.

242 Hier macht die Studie aus Datenschutz- und Anonymitätsgründen keine Angaben, da diese Äußerungen recht eindeutig auf die jeweiligen Mitglieder der IMA NBS zurückführbar wären. 


\subsubsection{Die Defizite und Entwicklungsmöglichkeiten der deutschen Biodiversitätspolitik}

Bilanzierend lassen sich anhand der Ausführungen zu den strukturellen und prozeduralen Anforderungen an die deutsche Biodiversitätspolitik nun auch die Defizite und Entwicklungsmöglichkeiten der deutschen Biodiversitätspolitik aufzeigen. Die Beurteilungen erfolgen vor dem Hintergrund folgender empirischer und normativer Kriterien:

Zum einen muss die Materie des zu regulierenden Politikbereiches von den politischen Akteuren in ausreichendem Maße verstanden werden, da ansonsten die Grundlage dafür fehlt, die damit verbundenen Probleme und Lösungsmöglichkeiten zu identifizieren und anzugehen. Zum Zweiten ergibt sich aus der Intensität der vertikalen Politikintegration die jeweils zu erfüllenden Aufgaben auf der horizontalen Ebene. Mit anderen Worten: Parteien und Ministerien, die Regierungsübernahme und Zuständigkeit hauptverantwortlich für die Umsetzung der internationalen und eurpäischen Richtlinien zur Bewahrung und Förderung der Biodiversät sind, müssen den daran anknüpfenden Aufgaben auf der nationlen Ebene hinsichtlich ihrer Führungsverantwortung für das Thema Biodiversität als auch hinsichtlich der administrativen Aufgabenerfüllung nachkommen. Dazu gehört, dass die Parteien und Ministerien ihrer je vorhandenen Führungsverantwortung für die Umsetzung der Biodiversitätspolitik nachkommen, lösungsangemessene strukturelle Anpassungen vornehmen, ihr Personal adäqat schulen, über strategische Planungsprozesse im Umgang mit Zielkonflikten und Umsetzungsproblematiken und über Koordinations- und Kooperationsstrukturen verfügen, die der integrativen Zielstellung und dem Querschnittscharakter der Bewahrung und Förderung der Biodiversität angemessen sind sowie Beteiligungsstrukturen schaffen, um das Sensorium der politischen Entscheidungsstellen für das Problembewusstsein, die Kritik und die Empfehlungen der aktiven Öffentlichkeit zu verbessern. Hierbei muss es um Beteiligungsverfahren gehen, bei denen das Verteilungsziel bzw. die kritischen Verteilungsfragen bezüglich der Bewahrung und Förderung der Biodiversität nur solange ausgeblendet werden, wie durch die Optimierung von Schutz- und Nutzungsansprüchen annähernd alle profitieren können.

Erstens zeigen die Ergebnisse der Studie, dass in den meisten Ministerien aber auch Parteien ein unterkomplexes Biodiversitätsverständnis vorherrscht, das eher klassisch auf den Bereich des Umwelt- und Naturschutzes ausgerichtet ist und somit gar nicht die gesamte Bandbreite der mit der Biodiversitätspolitik verbundenen Politikbereiche aufnimmt. Diesbezüglich wird die Vernichtung der Biodiversität nach wie vor zu stark als 'ökologische' und ökonomische denn als soziokulturelle und politische Problematik betrachtet, die gesellschaftliche und politische Lösungen braucht. Politisches Personal, das nur wenig über Biodiversität weiß und nicht geschult und weitergebildet wird, zumal dann, wenn sie explizit dafür zuständig sind, kann schwerlich mit einer komplexen 
Materie wie der Bewahrung und Förderung der Biodiversität umgehen. Dies wiederum hängt aber wesentlich davon ab, ob das Führungspersonal das Thema Biodiversität auf die politische Agenda setzt. Insbesondere die Parteien sind hier zu vorsichtig und unterschätzen die Bedeutsamkeit des Natur- und Umweltschutz im Allgemeinen und der Biodiversitätsthematik im Speziellen, obgleich repräsentative Bevölkerungsumfragen immer wieder zeigen, dass der Schutz der Natur im Allgemeinen und der Biodiversität im Besonderen als eine wichtige und sogar vorrangige politische Aufgabe in nahezu allen Bevölkerungsgruppen angesehen wird (BMU 2015:75) und dass die Bevölkerung mehrheitlich mehr politische Anstrengungen im Umwelt- und Klimaschutz fordert (BMUB 2009:42; 2013:20). Wenn es das Thema Biodiversität indes nicht oder nur sehr selten auf die politische Agenda schafft, obgleich es als wichtig in der Bevölkerung angesehen wird, dann können die mit der Bewahrung und Förderung der Biodiversität verbundenden gesellschaftlichen Handlungspotenziale gar nicht aktiviert und ausgeschöpft werden. Zudem besteht hierbei die Gefahr, dass eine zu geringe Behandlung des Themas auf der 'politischen Bühne' negative Auswirkungen auf die bislang positive und stark ausgeprägte öffentliche Aufmerksamkeit für das Thema hat. Ein wesentliches Defizit ist es daher, dass die Integrativität der Biodiversitätszieltrias als solche weder in der Exekutive noch in der Legislative zur Kenntnis genommen wird und dass damit die Grundlage fehlt, dass die Biodiversität auch auf substanziellen Wegen zum Symbolgriff für einen modernen, umfassenden und integrativen Umwelt- und Naturschutz wird. Entwicklungsmöglichkeiten liegen daher vor allem in der Kompetenzerweiterung und der fachlichen Schulung der zuständigen Akteure sowie darin, dass die verantwortlichen Entscheidungsträger in Politik und Verwaltung die Biodiversitätszieltrias in ihren jeweiligen Zuständigkeitsbereich integrieren, um eine stärkere politische Profilierung des Themas Biodiversität gegenüber anderen politischen Themen zu erreichen.

Zweitens zeigt sich anhand der Ergebnisse der Studie, dass die NBS - obgleich diese explizit als politisches Strategiepapier verfasst ist - keine nennenswerte politische Strategie zur Umsetzung der Biodiversitätsziele und -maßnahmen inkludiert. In der NBS wird zwar deutlich, dass die Bewahrung und Förderung der Biodiversität eine Querschnittsaufgabe ist, die politische Integration und Kooperation erfordert. Die Ergebnisse der Studie zeigen aber an sehr vielen Stellen das nach wie vor stark wirkmächtige Ressortprinzip sowie die damit verbundenen Problematiken für die Bewahrung und Förderung der Biodiversität. Diese lassen sich differenzierter beurteilen, wenn berücksichtigt wird, dass sich über die vertikale Politikintegration drei funktional unterschiedlich stark verantwortliche ministerielle Hauptgruppen bilden, die ihren Aufgaben bezüglich der Umsetzung der Biodiversitätsziele und -maßnahmen auf der Ebene der horizontalen Politikintegration in unterschiedlichem Maße nachkommen müssen. Bei der in erster Linie verantwortlichen ministeriellen 'Hauptgruppe`aus dem Umwelt-, dem Entwick- 
lungs- und dem Landwirtschaftsministerium schneidet lediglich das BMUB bei allen untersuchten Indikatoren positiv ab. Was speziell die Indikatoren zur Führungsverantwortung für die Umsetzung der Biodiversitätsziele, problem- und lösungsorientierte institutionelle Anpassungen sowie Schulungen und Weiterbildungen des Personals anbelangt, haben alle drei Ministerien eine gute Bilanz. Allerdings haben sowohl das BMZ als auch das BMEL noch Nachholbedarf, was die Beachtung der Biodiversitätszielmarken in ihrer administrativen Alltagspraxis angeht, auch wenn die Biodiversitätsthematik im BMZ, zumindest was die Regelungen zum gerechten Vorteilsausgleich angeht, zeitweilig durchaus Priorität hat. Die Defizite des BMEL sind deutlich schwerwiegender und breiter gefächert. So gibt es im BMEL keinen langfristigen Planungshorizont bezüglich der Umsetzung der Biodiversitätsziele, was sicherlich auch daran liegt, dass die in diesem Ressort ebenfalls bestehenden Zielkonflikte zwischen kurz- und langfristigen Maßnahmen zu wenig beachtet werden. Hierüber ergeben sich allerdings bereits grundsätzliche Defizite hinsichtlich der strategischen Planung, wie die Biodiversitätsstrategie im eigenen Ressort bzw. in Zusammenarbeit mit den anderen Ressorts umgesetzt werden kann und wird.

Drittens zeigt sich anhand der Ergebnisse der Studie, dass alle Ministerien - einschließlich des BMUB - derartige Defizite hinsichtlich der Etablierung und Evaluation administrativer Planungsprozesse aufweisen auf, was umso problematischer ist bzw. wird, umso mehr Zielkonflikte innerhalb des eigenen Ministeriums sowie bei der Koordination und Kooperation mit den anderen Ministerien beachtet werden müssen. Die Notwendigkeit administrativer Planungsprozesse beruht hierbei vornehmlich auf dem Umstand, dass die bestehenden Zielkonflikte zwischen schutzbezogenen und nutzungsbezogenen Erwägungen in Bezug auf die Bewahrung und Förderung der Biodiversität nur über den Fokus auf Verteilungsfragen bzw. die Aushandlung und Verhandlung gerechter (Um-)Verteilungsprozesse gehandhabt werden können. Hierzu gehören sowohl Problemlösungsverhandlungen, um geeignete Optionen zu selektieren, die für alle Beteiligten/Betroffenen gangbar sind, als auch Tauschverhandlungen, wo es um die Aushandlungen der Verteilungen geht. Mit anderen Worten: Die Bewahrung und Förderung der Biodiversität macht immer auch positive Koordinierung erforderlich und diese verlangt Planung. Außerdem werden Planungsprozesse notwendig, weil es sowohl zwischen als auch innerhalb der Ministerien keine klaren Vorgaben und Zuweisungen gibt, wie, durch wen, in welchen Zeitraum und durch welche Maßnahmen die Ziele der Biodiversitätsstrategie umgesetzt werden sollen. Vor diesem Hintergrund liegt eins der wichtigsten Entwicklungspotenziale darin, die konkrete Umsetzung der Biodiversitätsziele und -maßnahmen durch eine auf die jeweiligen Ressorts angepasste aber dennoch integrative Policy-Strategie zu ergänzen. Eine solche Policy-Strategie könnte idealerweise durch die interministerielle Arbeitsgruppe zur Umsetzung 
der Biodiversitätsstrategie erarbeitet und abstimmt werden. Dazu ist es indes nötig, dass die IMA NBS als ständiges Koordinationsgremium und strategische Planungsstelle reinstitutionalisiert wird, was aber mehr Weisungsbefugnisse, mehr finanzielle Ressourcen sowie in Policy-Planung geschultes, auf Leitungsebene arbeitendes administratives Personal voraussetzt.

Viertens verweisen die Ergebnisse der Studie, auch vor dem Hintergrund der Defizite bezüglich der strategischen Planung, auf grundlegende Problematiken hinsichtlich der Policy-Steuerung: Obgleich sowohl die MinisterialvertreterInnen als auch die für Biodiversitätsangelegenheiten zuständigen VertreterInnen der Parteien mehrheitlich primär auf mehr Bewusstseinsbildung und Informationen zur Bewahrung und Förderung der Biodiversität setzen, geht die Bereitstellung von Informationen und die Wissensvermittlung (selbst wenn diese zielgruppenspezifisch aufbereitet sind) nicht weit genug. Denn diese werden die erforderlichen Verhaltensänderungen in der Bevölkerung nicht schnell genug bewirken und zudem sind diese 'weichen ' und nicht-regulativen Instrumente auch nicht ausreichend für eine effektive und legitime Policy-Steuerung, die proaktiv und zudem darauf angelegt ist, dass sowohl politische, zivilgesellschaftliche als auch wirtschaftliche Akteure die Bewahrung und Förderung der Biodiversität vorantreiben. Hierzu bedarf es des gezielten Einsatzes aller regulativen Instrumente. Gerade in Bezug auf die immer stärker zum Einsatz kommenden ökonomischen Instrumente ist die Inwertsetzungsstrategie zentral, bei der die zahlreichen, bisher 'kostenlosen` Ökosystemleistungen eingepreist werden. Dies ist keineswegs ein Allheilmittel und dient in vielen Fällen stärker der Bewusstseinsbildung als der Regulation, aber die gezielte Einpreisung wichtiger ökosystemarer Leistungen insbesondere für die Wirtschaft und die Industrie schafft Anreize, die wiederum der Erfüllung des Vorsorge- und des Verursacherprinzips entgegenkommen. Die planerischen Instrumente ermöglichen wiederum genauere dem strukturellen Kontext angepasste Vorgaben zur Umsetzung der Biodiversitätsziele und -maßnahmen, wobei hierbei darauf geachtet werden muss, dass das Subsidiaritätsprinzip beachtet wird. Außerdem müssen speziell die kooperativen Instrumente sehr viel intensiver genutzt werden, als dies bisher der Fall ist. Denn in der administrativen Alltagspraxis sind die New Modes of Governance bislang reine Rhetorik, obgleich sich theoretisch fundiert aufzeigen lässt, wie wichtig diese gerade im Bereich von Problemlösungsverhandlungen sowie zur Erfüllung des Kooperationsprinzips sind. Der Umstand, dass lediglich das Umweltministerium einen Policy-Mix sowohl anwendet als auch präferiert, zeigt, dass hier Entwicklungspotenziale für eine proaktive und kooperative Policy-Steuerung zur Umsetzung der Biodiversitätsziele und -maßnahmen vorhanden sind, aus denen sich wiederum Spielräume in Hinblick auf die Berücksichtigung und Einbeziehung nicht-staatlicher Akteure in die Meinungs- und Willensbildung sowie die Politikformulierung ergeben: Vor dem Hintergrund des Umstandes, 
dass lediglich das Umweltministerium das Kooperationsprinzip in Gänze erfüllt, geht es vornehmlich darum institutionalisierte Beteiligungsforen für nicht-staatliche Akteure zu schaffen, so dass die gesamte Bandbreite an gesellschaftlichen Akteuren an der Formulierung und Durchsetzung konkreter Ziele, Pläne und Projekte zur Bewahrung und Förderung der Biodiversität beteiligt werden kann.

Alles in allem können daraus fünf Entwicklungsmöglichkeiten abgeleitet werden:

\section{Die Bewahrung und Förderung der Biodiversität als Kompetenz}

Die mit der Vernichtung der Biodiversität und ihrer Bewahrung und Förderung verbundenen Aspekte sind komplex, vielschichtig und bedürfen einer Menge theoretischer und praktischer Expertise. Diese Expertise muss durch Wissensvermittlung sowie praktische Handlungsanleitungen in Form von Workshops sowie Fortbildungs- und Trainingsprogrammen in die Personalentwicklung integriert werden.

2. NBS durch administrative Pläne komplementieren

Die Nationale Biodiversitätspolitik ist bereits an den 2010-Zielen gescheitert und droht auch die bis 2020 gesetzten Ziele zu verfehlen. Wenn dies verhindert werden soll, dann bedarf es (im Mindesten) klar definierter, eindeutig zugeordneter und an die administrative Arbeitsrealität angepasster kurzfristiger, mittelfristiger und langfristiger Vorgaben für die jeweiligen ministeriellen Ebenen, wie genau und bis wann die Ziele und Maßnahmen umgesetzt werden. Dies muss regelmäßig für die Mitarbeiter evaluiert werden, d.h., es geht hierbei nicht um repräsentative Bilanzen, sondern darum, dem zu wenigen Personal den möglichst effizienten Einsatz ihrer knappen Ressorcen zu ermöglichen.

3. IMA NBS zum strategischen Koordinationsgremium umbauen Die Interministerielle Arbeitsgruppe kann ihre eigentlich angedachte Aufgabe als Koordinationsgremium für die Etablierung und Überwachung einer ressortübergreifenden Policy-Strategie nicht erfüllen, wenn sie vornehmlich als Austausch- und Informationsgremium genutzt wird. Die IMA muss daher als Koordinationsorgan aller Ministerien auf Abteilungsleiterebene umgebaut und mit weitereichenderen administrativen Befugnissen ausgestattet werden.

4. pro-aktive und kooperative Steuerung

Der Stellenwert des Themas Biodiversität hängt im Wesentlichen von den verantwortlichen Entscheidungsträgern ab. Diese sind gefordert biodiversitätsrelevante Zielstellungen in ihren Zuständigkeitsbereich zu integrieren und dort für deren Umsetzung zu sorgen. Dies ist gerade vor dem Hintergrund hierarchischer Organisationsstrukturen entscheidend, wobei es je anderer Formen von Führungskompetenz bedarf je nach dem, um welche Form von 
Interdependenz es sich dabei handelt. Außerdem hängt die Umsetzung der Biodiversitätspolitik von einem gezielten und die kontextuellen Faktoren beachtenden Einsatz der Policy-Instrumente ab. Dies erfordert indes ein umfangreiches Wissen um Interaktionsstrukturen und -prozesse, so dass die Entscheidungsträger nicht nur inhaltlich zum Thema Biodiversität sondern auch policy- und organisationstheoretisch geschult werden sollten.

5. Biodiversität braucht gesellschaftliche Mitgestaltung

Die Ziele aber auch die Maßnahmen und Leuchtturmprojekte der NBS können nur durch gemeinsame Anstrengungen und über die Beteiligung gesellschaftlicher Interessensgruppen realisiert werden. Hierfür müssen sowohl die Parteien als auch die Verwaltung institutionalisierte Beratungsstrukturen und -prozesse mit allen gesellschaftlich relevanten Akteursgruppen initialisieren und installieren. Hierbei geht es sowohl um gemeinsame Problemlösungsgespräche als auch darum, wie diese Lösungen umgesetzt werden können. Die Potenziale der Zivilgesellschaft und der Wirtschaft zur Selbstregulierung und -organisation sind hierbei so weit es geht auszuschöpfen. 


\section{ABSCHNITT VI}

SCHLUSSBETRACHTUNG 


\section{Schlussbetrachtung}

Gemäß der in der Einleitung benannten Zielstellung wurde die Arbeit auf das Leitbild der Bewahrung und Förderung der Biodiversität ausgerichtet, dessen Spezifikum darin besteht, Schutz-, Nutzungs- und Verteilungsaspekte gleichermaßen zu berücksichtigen, so dass von einer integrativen Zielstellung die Rede ist. Insofern ist die gesamte Arbeit auf die Beleuchtung der wechselseitigen Einflüsse von biowissenschaftlichen Erkenntnissen zur Biodiversität, normativen Werte- und Moralvorstellungen im Umgang mit der Biodiversität sowie politikstrategischen Fragen der Umsetzung der Biodiversitätsziele und-maßnahmen ausgerichtet gewesen. Grundlegend wurde dabei von der Annahme ausgegangen, dass die integrativen Ziele und Maßnahmen zur Bewahrung und Förderung der Biodiversität durch eine ganze Reihe von wissenschaftlich relevanten Problematiken mit dem vordergründigen Problem der Vernichtung der Biodiversität zu einer praktisch-theoretischen Herausforderung sowohl für die Wissenschaft als auch für die Politik werden. Vor diesen Hintergründen war es der Anspruch und die Zielstellung dieser Arbeit, eine interdisziplinäre und anwendungsorientierte Reflexion des Leitbildes der Bewahrung und Förderung der Biodiversität vorzunehmen.

Die Grundlage sowohl für die interdisziplinäre Herangehensweise an die Biodiversitätsthematik als auch für den anwendungsorientierten Fokus wurde geschaffen, indem Biodiversität als Grenz- und Hybridkonzept zur Erschließung gesellschaftlicher Naturverhältnisse verstanden und ausgedeutet wurde. Im Zuge dessen wurde die Biodiversität sowohl als biowissenschaftliches Konzept, als Wert- und Normbegriff sowie als politischer Symbolbegriff für einen modernen Natur- und Umweltschutz ausgezeichnet. Diese drei Facetten der Biodiversitätsthematik wurden vor dem Hintergrund der analytischen Trennung von Sach- und Wertungsebene unterschieden und zugleich über die Analyse, was Biodiversität als Grenz- und Hybridkonzept ausmacht, in Beziehung zueinander gesetzt: Die analytische Unterscheidung erfolgte, indem zwischen einer auf der Sachebene angesiedelten aber dennoch normativ 'imprägnierten 'inhaltlichen Ebene, auf der es um den Forschungsgegenstand der Biodiversität ging, einer explizit normativen mit Gründen und Begründungsfragen beschäftigen Ebene und einer wiederum auf der Sachebene liegenden aber ebenfalls mit normativen Fragen beschäftigten politischen Ebene differenziert wurde, bei der es um die gesellschaftspolitische Umsetzung der Biodiversitätsziele und -maßnahmen geht. Im Fall des Grenzobjektes geht es um die Bezüge zwischen der normativen und der politischen Ebene über die wissenssoziologisch relevante Entgrenzung der Wissenschaften, die die Biodiversität erforschen und im Fall des Hybridkonzeptes geht es um die Bezüge zwischen der normativen und der inhaltlichen Ebene über die wissenschaftstheoretisch relevante epistemisch-moralische Hybridisierung der unterschiedlichen Blickwinkel auf Biodiversität. 
Hierbei wurde herausgestellt, dass speziell der normativen Ebene eine besondere Relevanz zukommt, weil diese als 'Schnittstelle' zwischen der inhaltlichen und der politischen Ebene fungiert, so dass es notwendig war, darauf zu reflektieren, was Biodiversität als Gegenstand der Wissenschaft und Forschung bedeutet, da dies die Grundlage normativer Bewertungen und Beurteilungen bildet und welche Möglichkeiten zur politikstrategischen Umsetzung der ethischen Überlegungen es gibt und geben sollte. Um eben dieser über die normative Ebene vermittelten 'Dreigliedrigkeit' gerecht zu werden, die sich aus dem Grenz- und Hybridcharakter der Biodiversität ergibt, wurde dargelegt, auf welche speziellen Detailproblematiken auf der inhaltlichen, der normativen und der politischen Ebene die Arbeit eingehen wird und warum gerade diese speziellen Problematiken als relevant betrachtet werden, um die jeweiligen mit dem Grenz- und Hybridcharakter der Biodiversität verbundenen Metaproblematiken konstruktiv anzugehen. Folglich wurden auf jeder Ebene sowohl bestimmte Metaproblematiken als auch je eine spezifische, der Lösung dieser Metaproblematik entgegenstehende, Detailproblematik in der Grundlagenreflexion verdeutlicht, auf deren Grundlage dann die Detailreflexionen erfolgten.

Da postuliert wurde, dass sowohl die Meta- als auch die Detailproblematiken dem Ziel entgegenstehen, über die Bewahrung und Förderung der Biodiversität zu einer Praxis gelingender gesellschaftlicher Naturverhältnisse zu gelangen, ging es in dieser Arbeit trotz der oftmals stark theoretischen Reflexionsleistungen in der Grundlagen- und den Detailreflexionen immer auch darum, einen konstruktiven Beitrag dazu zu leisten, um das Leitbild der Bewahrung und Förderung der Biodiversität zu bekräftigen und zu bestärken. In der nun folgenden die Arbeit abschließenden Schlussbetrachtung, geht es darum, die bereits herausgestellten, analysierten und hinsichtlich ihrer theoretischen und praktischen Relevanz für die Bewahrung und Förderung der Biodiversität beurteilten Detailproblematiken auf der inhaltlichen, normativen und politischen Ebene noch einmal aufzugreifen, um die zentralen Ergebnisse der Detailreflexionen zusammenfassend und in der Zusammenschau sowie hinsichtlich der Zielstellung, einen konstruktiven Beitrag zur Stärkung Leitbildes der Bewahrung und Förderung der Biodiversität zu leisten, zu bilanzieren. Lediglich der Übersichtlichkeit halber wird hier erneut gegliedert.

\section{Bilanz der inhaltlichen Reflexionen}

In Bezug auf Biodiversität als biowissenschaftliches Konzept, wo die methodisch-theoretische Unterbestimmung der Biodiversitätskonzeption als bedeutsame Metaproblematik identifiziert wurde, wurde in Abschnitt III der Arbeit detailliert darauf reflektiert, was Biodiversität als Gegenstand der (bio-)wissenschaftlichen Beschäftigung bedeutet und bedeuten kann. Diesbezüglich wurde davon ausgegangen, dass sich Biodiversität 
als Forschungsprogramm zum einen auf die Referenzgegenstände der Biodiversitätsforschung, sprich, die Vielfalt der Lebewesen (biotische Vielfalt) und ihrer Lebensbedingungen (abiotische Vielfalt) beziehen kann. Zum anderen kann sich Biodiversität aber zugleich auch auf die Vielfältigkeit der wissenschaftlichen Zugänge und Perspektiven zur Beschreibung, Erfassung und Erschließung der biotischen und abiotischen Vielfalt beziehen, was wiederum auf die verschiedenen Forschungskonzepte verweist, die unter dem Oberbegriff biologische Vielfalt subsumiert werden. Vor diesem Hintergrund wurde der methodologische Kurzschluss, d.h. die Gleichsetzung der Begriffe der (a)biotischen Vielfalt und der biologischen Vielfalt, über die Kurzform Biodiversität als spezifische Detailproblematik auf der inhaltlichen Ebene identifiziert, da hierbei der methodologische Unterschied zwischen den Referenzobjekten (abiotische und biotische Vielfalt) und den Forschungskonzepten (biologische Vielfalt) der Biodiversitätskonzeption immer dann nicht beachtet wird, wenn Biodiversität synonym mit biologischer Vielfalt gesetzt wird und wenn die biologische Vielfalt wiederum als Konglomerat aus der genetischen Vielfalt, der Artenvielfalt und der ökosystemaren Vielfalt definiert wird, die aber nicht als biologische Beschreibungskonzepte ausgewiesen werden, sondern als Spektrum der biotischen und abiotischen Vielfalt definiert wird.

Diese, im Biodiversitätskontext bislang zu wenig beachtete wissenschaftstheoretische Perspektive auf den Forschungsgegenstand Biodiversität wurde in der ersten Detailreflexion in Abschnitt III fokussiert. Dabei wurde herausgestellt, dass es sich bei der umgangssprachlich zwar fest etablierten und als solcher auch nur selten hinterfragten Gleichsetzung von biotisch und biologisch nicht lediglich um eine terminologische 'Laxheit' sondern um eine Kontroverse bezüglich der Konstitution und Konstruktion wissenschaftlicher Forschungsgegenstände handelt, die aber als solche gar nicht ersichtlich wird, weil sie über den methodologischen Kurzschluss invisibilisiert wird. Daher wurde im Rahmen der wissenschaftstheoretischen Detailreflexion auf die wissenschaftstheoretischen Voraussetzungen des methodologischen Kurzschlusses reflektiert, die in einem realistischen Wissenschaftsverständnis mit naturalistischen, objektivistischen und deskriptivistischen Tendenzen verortet wurden, das von vielen ProtagonistInnen in der Biodiversitätsdebatte - zumeist unintendiert, unthematisiert und damit auch unreflektiert - vertreten wird, auch weil dieses Wissenschaftsverständnis, trotz aller Kritik daran, die Common-Sense` Position zum Zusammenhang zwischen der Welt und dem Wissen über sie widerspiegelt.

Hierbei wurden speziell die Annahmen kritisch hinterfragt, dass es sich bei der Biodiversität um ein 'natürlich verfasstes Phänomen handelt, welches mit den Mitteln der Biowissenschaften adäquat und wirklichkeitsgetreu beschrieben und erfasst werden kann und, auf einer allgemeineren Ebene, dass die Naturwissenschaften in der Lage wären, die natürlich gegebene Wirklichkeit aus der Distanz eines passiven Beobachters 
objektiv und wahrheitsgemäß zu beschreiben. Um die problematischen Aspekte dieser Annahmen speziell für das Biodiversitätsverständnis zu verdeutlichen, wurde demgegenüber die Ansicht plausibilisiert, dass alle Wissenschaften teilnehmende Praxen der Gegenstandskonstitution und -konstruktion sind, so dass sich die Forschungskonzepte (biologische Vielfalt) immer schon aus den methodologischen und theoretischen Entscheidungen von WissenschaftlerInnen im Rahmen ihrer Forschungsaktivitäten ergeben, dass sich aber auch die Selektion und Konstitution der Referenzobjekte der Biodiversitätsforschung (abiotische und biotische Vielfalt) bereits vorwissenschaftlichen Erfahrungen verdanken.

Diese grundlegenden Differenzen im Wissenschaftsverständnis wurden indes nicht nur als theoretisch relevant sondern auch als praktisch bedeutsam für das Grenz- und Hybridverständnis der Biodiversität charakterisiert. Die praktische Relevanz des methodologischen Kuzschlusses zeigt sich bspw. immer dann, wenn Standards für die 'Normalität’ bzw. für 'Störungen` natürlicher Umgebungen vermeintlich problemlos aus den biowissenschaftlichen Beschreibungen entnommen werden. Daher wurde gezeigt, dass über den methodologischen Kurzschluss auch diejenigen Problematiken ausgeblendet werden, die sich über die epistemisch-moralische Hybridisierung ergeben, sprich, wenn aus Beschreibungszusammenhängen auf Handlungsanweisen zum Schutz, zur nachhaltigen Nutzung und zur gerechten Verteilung der Biodiversität geschlossen wird. Da hierüber die Rede von der Biodiversität, der Biodiversitätskrise aber auch der Bewahrung und Förderung der Biodiversität bereits im Ansatz naturalisiert werden und damit systematisch die Gefahr naturalistischer Fehlschlüsse sowie von Sein-Sollen Feh/schlüssen provoziert wird, weil Biodiversität und damit Vielfalt per se als etwas Gutes und Erstrebenswertes betrachtet werden, wird auch der Schutz dieser Vielfalt ganz automatisch als ein gutes und erstrebenswertes Ziel angenommen, ohne dass hierfür eine gesonderte normative Begründung angeführt wird, obgleich diese notwendig ist. Um mit diesen sowohl theoretisch als auch praktisch relevanten Problematiken auf der inhaltlichen Ebene der Biodiversität als Grenz- und Hybridkonzept konstruktiv umzugehen, wurde die Notwendigkeit betont, dass im Einzelfall genauer geschaut werden muss, worauf genau mit dem Begriff Biodiversität referiert wird:

Wenn dabei auf die biologische Vielfalt als Forschungskonzept referiert wird, dann ist dabei relevant, welche biologischen und ökologischen Theorien hinter der jeweiligen Konzeptualisierung stehen und inwiefern deren Annahmen vor dem Hintergrund methodologischer Rekonstruktionen Geltung beanspruchen können. Hierbei müssen die methodologischen Konstruktionsbedingungen der unterschiedlichen Forschungskonzepte zur Erschließung der Biodiversität berücksichtigt werden, denn nur wenn abgeschätzt werden kann, ob die jeweiligen Annahmen, Theorien, Modelle, Konzepte und Begriffe zu einem theoretischen und empirischen Gehaltsüberschuss gegenüber voran- 
gegangen wissenschaftlichen Erkenntnissen führen, kann auch von wissenschaftlicher Erkenntnis gesprochen werden. Hierfür ist im Einzelnen wichtig, dass kontrollierbare Maßstäbe der Unterscheidbarkeit lebender Organismen entwickelt werden, was vor dem Hintergrund eines konstruktivistischen Wissenschaftsverständnisses immer auch bedeutet, dass dargelegt werden muss, wer mit welchen Mitteln und Zielen, wie und warum, welche Unterscheidungen trifft. Nur auf diesem Weg kann nachvollziehbar und kontrollierbar überhaupt von Diversität gesprochen werden. Des Weiteren müssen Adäquatheitskriterien für die jeweiligen Unterscheidungen und Beschreibungen der genetischen Vielfalt, der Artenvielfalt und der Ökosystemvielfalt entwickelt werden, denn nur wenn deutlich wird, anhand welcher Standards die vorgenommenen Unterscheidungen und Beschreibungen korrekt bzw. die Beschreibungsergebnisse gültig sind, können diese auch als empirische Grundlage zur Abschätzung möglicher Maßnahmen zur Bewahrung und Förderung der Biodiversität fungieren. Dazu bedarf es indes (auch) von Seiten der BiowissenschaftlerInnen eines offenen und kritischen Umgangs damit, dass die üblichen Definitionen der biologischen Einheiten zumindest unvollständig sind, denn erst dann können alle Akteure der Biodiversitätsdebatte sich dem Umstand stellen, dass es keine einheitliche Biodiversitätskonzeption und kein einheitliches biowissenschaftliches Grundlagenwissen zur Anwendung der Biodiversitätskonzeption in anderen wissenschaftlichen Disziplinen sowie gesellschaftspolitischen Zusammenhängen gibt.

Eben deshalb, so wurde aufgezeigt, ist es wiederum wichtig, auch auf die biotische und abitische Vielfalt als Forschungsgegenstände zu reflektieren. Hierbei ist nämlich relevant, welche Auffassungen über das Verhältnis von Individuen (Organismen) und Gemeinschaften (ökologische Systeme) jeweils hinter dem Verständnis von Vielfalt stehen und um erklären zu können, warum eben dieses so divers ist. Dabei muss indes immer darauf geachtet werden, dass die Zustandsbeschreibungen der biotischen und abiotischen Vielfalt - sei es nun in ihrem weiten oder engen Verständnis als ganzheitliche Vielfalt oder als aggregierte Vielzahl, als wohlgeordnete Fülle oder als bedrohte Knappheit - nicht bereits auf natürliche Eigenschaften zurückgeführt werden, sondern dass diese als sozial und kulturell konstituierte Vorstellungen von Natur und den menschlichen Verhältnissen zu dieser betrachtet werden. Je nach dem, wie die (a)biotische Vielfalt folglich vor dem Hintergrund geistes- und ideengeschichtlicher Annahmen konstituiert wird, hat man es mit unterschiedlichen Vorstellungen zu tun, was die abiotische und biotische Vielfalt als Referenzgegenstände der Biodiversitätsforschung ausmachen und welche Ansätze menschlicher Naturverhältnisse dabei eine Rolle spielen. Erst wenn diese explizit berücksichtigt und thematisiert werden, kann die Biodiversitätskrise nicht nur terminologisch als Krise der gesellschaftlichen Naturverhältnisse betitelt sondern auch dementsprechend gehandhabt werden, weil dann ersichtlich wird, 
welche Naturauffassungen sowie Handlungsweisen des Menschen es sind, die in die 'Krise' geführt haben und darüber selber in die Krise geraten sind. Daher wurde gezeigt welche möglichen - und stellenweise durchaus widersprüchlichen geistes- und ideengeschichtlichen Konstitutionsideen in das Biodiversitätskonzept eingehen. Diesbezüglich wurde idealtypisch zwischen einem Ganzheitlichkeitsverständnis und einem Partikularverständnis der (a)biotischen Vielfalt unterschieden. Beide Verständnisse wurden als Ansatzpunkte möglicher Biodiversitätsverständnisse dargelegt:

Das Ganzheitlichkeitsverständnis ist dadurch charakterisiert, dass alle Organismen notwendigerweise in Gemeinschaften eingebunden sind, in denen sie in funktionalen, zumeist obligatorischen Abhängigkeiten zueinander stehen, so dass die biotische Gemeinschaft aus einer emergenten Vielfalt unterschiedlicher Geschöpfe besteht, die zusammen ein harmonisches Ganzes und eine überindividuelle Ganzheit bilden. Diese Denkfiguren lassen sich aus den Annahmen des politischen Konservatismus ableiten, der davon ausgeht, dass Individuen ungleich seien, aber der Zweck dieser Ungleichheit darin liegt, dass aus dieser Unterschiedlichkeit unterschiedliche Aufgaben in und für die Gemeinschaft erwachsen. In der Ökologie erscheinen diese Zusammenhänge vor allem in organizistisch-synökologischen Theorien (ebd., 332), die den Fokus auf den Umstand legen, dass sich Organismen wechselseitig ihre Existenz ermöglichen, insofern sie alle zur Bildung von Funktionseinheiten des Systems beitragen. Das spezifische Wesen des Ganzen wird daher über seine Eigenart realisiert, die sich wiederum daraus ergibt, dass die Individuen Zwecke in der Gemeinschaft für die Gemeinschaft erfüllen, die ihnen die Existenz ermöglichen, so dass das Ganze selbst als einzigartig und selbstzweckhaft betrachtet wird. Die abiotische und biotische Vielfalt wird vor diesem Hintergrund folglich als harmonische Einheit gedacht und stellt zugleich ein qualitatives $M a ß$ dafür dar, wie einzigartig das Leben in lokale, überindividuelle Einheiten differenziert ist und wie sehr diese Einheiten wiederum intern differenziert sind. Übertragen auf das Biodiversitätsverständnis bedeutet dies, dass jede Komponte einen notwendigen Beitrag zur Reproduktion des Gesamtsystems leistet und dass jeder Organismus darin sich nur als Komponente des Systems reproduzieren kann. Die Bewahrung und Förderung der gesamten abiotischen und biotischen Vielfalt erscheint hier erstrebenswert, weil dies der Aufrechterhaltung einer harmonischen Einheit dient, so dass alle Elemente der (a)biotischen Vielfalt aufgrund ihrer Funktion für das Ganze 'um ihrer selbst willen`erhalten werden sollen, weil deren Einzigartigkeit und gegenseitige obligatorische Abhängigkeit das System als solches erst ermöglichen und hervorbringen. Dies wurde als weites Biodiversitätsverständnis charakterisiert, da Biodiversität hier notwendigerweise sowohl die Vielfalt der Lebensformen als auch die Vielfalt ihrer Lebensbedingungen umfasst. 
Das Partikularverständnis ist demgegenüber dadurch charakterisiert, dass das 'Ganze' nur aus der Perspektive des Einzelnen nützlich ist und keinen Zweck an sich hat, da es sich aus einer in Umfang und Zusammensetzung beliebigen Menge wesentlich individueller und in ihrem Wesen voneinander unabhängiger Geschöpfe ergibt. Die Struktur des Ganzen ist folglich nur durch die Interaktionen der Individuen bestimmt, wobei diese im Wesentlichen durch die Konkurrenz um knappe Ressourcen charakterisiert sind. Diese Denkfiguren lassen sich aus den Annahmen des politischen Libertarismus ableiten, der davon ausgeht, dass Individuen egoistische Nutzenmaximierer sind, die nur deshalb Kooperationen eingehen, weil sie ihre eigene Stellung im System und ihre Nutzung der Ressourcen verbessern wollen. (ebd., 335). In der Ökologie erscheinen diese Zusammenhänge vor allem in Gestalt individualistisch-synökologischer Theorien, die postulieren, dass die Einzelorganismen nicht von anderen Organismen oder übergeordneten Einheiten abhängig sind, auch wenn die Ansprüche der einzelnen Organismen von der Umwelt, zu der ja auch andere Organismen gehören, erfüllt werden müssen. Vielfalt ist in diesem Kontext folglich die bloße Aggregation einzelner Organismen und stellt zugleich ein rein quantitatives Maß dar, wie einzelne Organismen auf der Basis zufälliger Ähnlichkeiten in einen nominalistischen Ordnungszusammenhang gebracht werden. Übertragen auf Biodiversitätsverständnis bedeutet dies, dass die (a)biotische Vielfalt hier als die quantitative Vielzah/ der lokalen, regionalen und global vorhandenen Arten verstanden wird (ebd., 325). Die Bewahrung und Förderung der abiotischen und biotischen Vielfalt ist in diesem Zusammenhang erstrebenswert, weil die (a)biotische Vielfalt als Fülle an menschlichen Spielräumen und Handlungsmöglichkeiten sowie als materielle, kognitive, spirituelle und/oder kulturelle Ressourcen zu bewahren und zu fördern sind, wobei es hier vor allem um die Bewahrung des Nutzens der Biodiversität für den Menschen geht. Dies wurde als enges Biodiversitätsverständnis charaktersiert, weil die biotische Vielfalt hier auch unabhängig von den abiotischen Lebensbedingungen betrachtet werden kann und nur die Vielzahl der Lebensformen als solche relevant ist.

Je nachdem, welcher Deutungshorizont folglich hinter dem Verständnis (a)biotischer Vielfalt steht, ergeben sich ganz unterschiedliche Betrachtungen und Bewertungen der Zustände, Prozesse und Eigenschaften lebender Systeme. Dabei wurde darauf hingewiesen, dass auch die Zwischenformen zwischen den idealtypischen ganzheitlichen und partikularen Konstititutionsideen für das Verständnis der (a)biotischen Vielfalt wichtig sind, weil sich daran gewisse Spannungen innerhalb des Biodiversitätsverständnisses verdeutlichen lassen, die sich über die Zusammenführung widersprüchlicher Annahmen aus unterschiedlichen ideengeschichtlichen Kontexten ergeben. Insofern wurde festgehalten, dass die geistes- und ideengeschichtliche Rekonstruktion der kulturellen Konstitutionsbedingungen der Biodiversität durchaus zu einer progressiven Problem- 
verschiebung führen kann. Denn hierüber erfolgt eine nicht ausschließlich terminologisch relevante Verschiebung von der Analyse der Vielfalt der belebten Natur, sprich einer biowissenschaftlich aber auch wissenschaftstheoretisch geprägten Analyse der Biodiversität hin zur Analyse der Vieldeutigkeit der belebten Natur, $d . h$. einer geistesund ideengeschichtlichen Analyse der Variationsbreite der Ideen, die hinter Biodiversität stehen. Es wurde aufgezeigt, dass diese von entscheidender Bedeutung sind, um zu verstehen, wie die (a)biotische Vielfalt ausgedeutet werden kann und auf welche eben gerade nicht biowissenschaftlichtheoretischen Kontroversen - die Diversität der Biodiversitätsverständnisse zurückgeführt werden muss. Denn je nachdem, ob das enge oder das weite Verständnis (a)biotischer Vielfalt sowohl für das Konzept der biologischen Vielfalt als auch in Bezug auf Anwendungsfragen im Umwelt- und Naturschutz zugrunde gelegt wird, hat dies, so wurde argumentiert, Auswirkungen sowohl auf die Art und Weise, wie Biodiversität als Forschungskonzept konstruiert wird als auch darauf, welche Ziele und Maßnahmen seitens des Umwelt- und Naturschutzes für sinnvoll und zielführend erachtet werden, sowie darauf, welche Gründe, Argumente und Begründungsansätze aufgewendet werden müssen, um die Ziele und Maßnahmen zu plausibilisieren.

Insbesondere der letztgenannte Aspekt führte dazu, dass zur Beantwortung der ökozialen Frage sowie hinsichtlich des konstruktiven Umgangs mit den inhaltlichen Metaproblematiken des Biodiversitätsproblems auf die normative Ebene der Biodiversität als Grenz- und Hybridkonzept übergegangen werden musste. Denn die Beantwortung der Frage, für wen, inwiefern und warum es angeraten sein soll, die abiotische und biotische Vielfalt (und gerade nicht die biologische Vielfalt!) möglichst umfassend zu schützen, nachhaltig zu nutzen und gerecht zu verteilen, ist keine biowissenschaftliche und auch keine wissenschaftstheoretische sondern eine ethische Aufgabenstellung, weil das Wissen darum, was Biodiversität und wie sie erfasst, analysiert und gemessen werden kann, nicht ausreichend ist, um auch begründen zu können, warum sie bewahrt und gefördert werden sollte.

\section{Bilanz der normativen Reflexionen}

In Bezug auf Biodiversität als Wert- und Normbegriff, wo als entscheidende Metaproblematik festgehalten wurde, dass sich die normative Imprägnierung des Leitbildes der Bewahrung und Förderung der Biodiversität bereits über die Selektion bestimmter Elemente aus der gegebenen Vielzahl der Möglichkeiten ergibt, Biodiversität inhaltlich zu bestimmen, wurde im Anschluss an die wissenschaftstheoretische Auseinandersetzung auf die normative Ebene und damit in die ethische Detailreflexion übergegangen. Hier ging es um die bereits deutlich umfangreichere Reflexion auf die im wissenschaftlichen, politischen und umwelt- und naturschutzethischen Kontext zugleich relevanten Intui- 
tionen, Motive und Beweggründe sowie Argumente und Begründungen für die Bewahrung und Förderung der Biodiversität. Hierbei wurde davon ausgegangen, dass das 'Kerngeschäft' der Ethik zwar in der theoretischen Bestimmung und Begründung von Werten und Normen liegt, dass speziell die Umwelt- und Naturschutzethik aber nicht nur abstrakt theoretische Fragen über die Ermittlung des guten und richtigen Handelns in Bezug auf die Natur/Umwelt im Allgemeinen und die Biodiversität im Speziellen zum Gegenstand haben kann, sondern dass sie immer auch auf konkrete gesellschaftspolitische Fragen zum Umgang mit der Natur/Umwelt/Biodiversität reagieren muss. Daher wurde in der ethischen Detailreflexion - trotz der binnendifferenzierten und stellenweise stark theoretischen Abhandlungen - immer auch die praktische Zielstellung verfolgt, Orientierungsleistungen im Umgang mit der Natur/ Umwelt/Biodiversität zu liefern.

Dazu bedurfte es grundlegender Überlegungen und Darlegungen: Dies betraf zum einen die Darstellung der argumentationstheoretischen Grundlagen der Rede von 'guten Gründen`, schlicht weil die Art und Weise des Argumentierens in der Ethik, die in der ethischen Detailreflexion an vielen Stellen unerklärt zur Anwendung kam, für NichtPhilosophInnen nicht ohne weiteres verständlich ist und somit an vielen Stellen in der Arbeit zu unnötigen Verständnisproblemen geführt hätte. Zum anderen - und dies war inhaltlich relevant - musste vorab auf die gängigen Gründe und Begründungen für die Erhaltung der Biodiversität eingegangen werden, die sich sowohl im politischen als auch im umwelt- und naturschutzethischen Kontext finden, da eben hieran die kritische Reflexion ansetzen sollte und sich hierüber der Problemaufriss für die ethische Detailreflexion ergab. Diesbezüglich wurde zum einen auf die in der NBS angeführten ökologischen, ökonomischen, soziokulturellen und ethischen Gründe für die Erhaltung der Biodiversität reflektiert. Hierüber wurde das Problem deutlich gemacht, dass in der NBS suggeriert wird, alle Menschen wären existenziell auf eine 'gesunde', 'funktionsfähige 'und 'stabile' Natur/Umwelt angewiesen, so dass darüber indirekt auch auf die existenzielle Bedeutung der Biodiversität verwiesen wird, was bezeichnenderweise den eigentlichen Ausführungen zu den Gründen für die Erhaltung der Biodiversität vorgelagert wird. Damit wird indes der problematische Eindruck erweckt, als ob es selbstevident wäre, die Biodiversität zu erhalten. Des Weiteren wurde der Umstand problematisiert, dass in der NBS unter dem Abschnitt 'ethische' (besser normative) Gründe die Tatsachenbehauptung aufgestellt wird, dass die Biodiversität einen Eigenwert hat und dass aus den ethischen Begründungen Verpflichtungen abgeleitet werden können, möglichst die gesamte noch vorhandene Biodiversität zu erhalten. Hier wird wiederum suggeriert, dass sich die Umweltethik auf die Begründung des Eigenwerts sowie des Inklusionsproblems reduzieren ließe und dass es dabei keine strittigen Punkte gäbe. 
Um darzulegen, was die Problematik hinter der ersten Annahme ist und um zu zeigen, dass die zweite Annahme schlicht falsch ist, wurde genauer auf die umwelt- und naturschutzethischen Begründungsansätzen für den Eigenwert sowie die Verpflichtung zur Erhaltung der Biodiversität um ihrer selbst willen eingegangen. Dies machte eine umfangreichere Reflexion der Wertdebatte sowie der physiozentrischen Begründungsansätze für den Schutz der Biodiversität um ihrer selbst willen notwendig. Vor dem Hintergrund der Annahme, dass es in der Umwelt- und Naturschutzethik auf der axiologischen Ebene zum einen um die Klärung der werttheoretischen Frage geht, ob und wenn ja, in welchen Hinsichten die außermenschliche Natur 'um ihrer selbst willen `als wertvoll gelten kann und dass es auf der moralphilosophischen Ebene zum anderen um die Klärung speziell der pflichtenethischen Frage geht, wem gegenüber Menschen Pflichten haben sollten und welchen Entitäten welche Rechte zukommen, wurden einerseits die jeweiligen Problematiken der unterschiedlichen Werttypen anhand der wertcharakterisierenden Aspekte, der Lokalisierung dieser Werte, der Wertungsebene und der Quelle der Werte herausgearbeitet und anderseits wurde anhand der idealtypischen Begründungsstrukturen der holistischen, ökozentrischen und biozentrischen Ansätze deutlich gemacht, welche Verständnisse von Eigenwert dabei jeweils zum Tragen kommen und wie die direkten moralische (Schutz-)Pflichten gegenüber der Biodiversität um ihrer selbst willen begründet werden. Dabei wurde gezeigt, dass alle für die Begründung von (Schutz-)pflichten gegenüber der Biodiversität in Frage kommenden physiozentrischen Ansätze auf ihre je eigene Weise mit Begründungsproblematiken und Argumentationsdefitiziten einhergehen und dass sich diese Problematiken zumeist aus der vorschnellen und im Einzelnen nicht separat begründeten Ableitung direkter moralischer (Schutz-)Pflichten aus der ebenfalls oftmals unklaren Eigenwertigkeit der Biodiversität ergeben.

Daraus ergaben sich wiederum zwei für den weiteren Verlauf der Arbeit relevante Schlussfolgerungen: Die erste Schlussfolgerung war, dass die in der NBS unterbreitete Suggestion, es gäbe ethisch plausible Begründungen zur Ausweisung von (Schutz-)Verpflichtungen gegenüber der Biodiversität um ihrer selbst willen, die sich wiederum dafür anführen lassen, möglichst die gesamte noch vorhandene Biodiversität zu erhalten, kein tragfähiges Fundament besitzt. Diesbezüglich wurde die Alternative darin gesehen, ein 'Sammelsurium 'an Gründen zu eröffnen, die in ihrer Gesamtheit mehr oder weniger für den Schutz aber auch die nachhaltige Nutzung und die gerechte Verteilung vieler einzelner Segmente der Biodiversität sprechen, so dass man auf diesem Weg zu selektiven und zumeist kontextabhängigen Begründungen gelangt, die eine unterschiedliche Schutzreichweite haben und unterschiedliche Verbindlichkeiten nach sich ziehen. Die zweite Schlussfolgerung bestand darin, dass es einer theoretischen Neuausrichtung der Umwelt- und Naturschutzethik hin zu einer anwendungsorientierten Biodi- 
versitätsethik bedarf, bei der auf Grundlage der dargelegten Begründungsproblematiken auf der Basis einer anthropozentrischen Position zum Inklusionsproblem argumentiert wird. Um indes zu dieser Neuausrichtung zu gelangen, bedurfte es, so die Argumentation, der Lösung von drei eigenständigen aber aufeinander aufbauenden und ineinandergreifenden Problematiken, die als die Detailproblematiken der ethischen Reflexion ausgemacht wurden:

So wurde zum einen postuliert, dass über die zu einseitige Fokussierung auf das Inklusionsproblem und damit die Leitunterscheidung zwischen anthropozentrischen und physiozentrischen Ansätzen in der Umwelt- und Naturschutzethik immer wieder der hintergründige Dualismus zwischen Mensch und Natur repliziert wird, was aber dazu führt, dass dabei die Perspektive auf die Auslotung der gesellschaftlichen Naturverhältnisse, die für die Bewahrung und Förderung der Biodiversität sowohl auf der inhaltlichen, auf der normativen als auch auf der politischen Ebene zentral ist, zu stark ausgeblendet wird. Zum zweiten wurde der primäre Fokus auf Schutz-(pflicht)begründungen problematisiert, da dieser dazu führt, dass die Umwelt- und Naturschutzethik auf Pflichten und Rechte beschränkte Begründungen für den Schutz der Biodiversität anbietet, dabei aber die Nutzungs- und Verteilungsziele sowie nicht genuin moralische Argumente und Begründungen vernachlässigt, womit die für die Bewahrung und Förderung der Biodiversität so entscheidende Integrativität der Zieltrias zu wenig beachtet wird. Drittens wurde speziell hinsichtlich der anvisierten Anwendungsorientierung vorgebracht, dass in der ethischen Kommunikation über Natur- und Umweltschutzanliegen im Allgemeinen und bezüglich der Bewahrung und Förderung der Biodiversität im Speziellen zu wenig beachtet wird, dass die Ausweisung 'guter Gründe' nicht lediglich eine Frage der normativen Akzeptabilität der Gründe ist sondern immer auch eine Frage ihrer faktischen Akzeptanz.

Um diesen drei Detailproblematiken konstruktiv zu begegnen und damit zu der anvisierten Neuausrichtung der Umwelt- und Naturschutzethik hin zu einer problem- und anwendungsorientierten Biodiversitätsethik zu gelangen, wurden problemfokussierte Lösungsvorschläge erarbeitet (was aufgrund der Tiefe und des Umfangs der Problematiken stellenweise nur im Ansatz möglich war), die aufeinander aufbauen und sich gegenseitig bedingen:

In Bezug auf die grundlegende und damit auch umfassendste Detailproblematik der zu einseitigen Fokussierung auf das Inklusionsproblem wurde darauf verwiesen, dass es in pragmatischer Hinsicht weniger um die Entscheidung gehen sollte, ob nun primär der Mensch oder die Natur im Zentrum der umwelt- und naturschutzethischen Überlegungen steht, sondern dass es, gerade wenn es um die Bewahrung und Förderung der Biodiversität geht, um die Auslotung der individuellen und kollektiven menschlichen Naturverhältnisse gehen muss. Hierfür wurde im Sinne einer progressiven Problemverschie- 
bung vorgeschlagen, den hintergründigen Dualismus zwischen Mensch und Natur, den die Umwelt- und Naturschutzethik immer wieder über ihre Fokussierung auf das Inklusionsproblem repliziert, zugunsten einer relationalen Sichtweise auf die menschlichen Naturverhältnisse zu durchbrechen. Hierfür wurden bestimmte Charakteristika einer solchen relationalen Perspektive in Bezug auf die Natur/Biodiversität festgehalten: Bezüglich der für das Konzept der gesellschaftlichen Naturverhältnisse relevanten Auslotung der begrifflichen Relationen zwischen Mensch, Natur und Gesellschaft wurde festgehalten, dass es um die aufeinander bezogenen Probleme der gesellschaftlichen Handlungskoordination, der individuellen Lebensführung und der ökologischen Zusammenhänge geht. Dies verweist auf die Relationen, Differenzen und Prozesse zwischen Mensch und Natur und zugleich auf die zutiefst gesellschaftliche Verfasstheit sowohl menschlicher als auch außermenschlicher Naturen (siehe wiederum die Ergebnisse der wissenschaftstheoretischen Reflexion). Hierüber werden Menschen auch als Teil der Natur angesehen und es wird davon ausgegangen, dass sie von der Natur/Biodiversität in vielerlei Hinsicht, allerdings mal mehr und mal weniger stark, abhängig sind.

Diese Abhängigkeit kann in einigen Fällen durchaus existenzieller Natur sein, aber gerade in den westlichen Industrienationen geht es dabei nicht um das Überleben sondern um die Möglichkeit der Gestaltung eines guten und gelingenden Lebens. Dazu wiederum, so wurde herausgestellt, muss es Möglichkeiten geben, dass Menschen zu einer nicht rein instrumentellen sondern wesensmäßigen Mensch-Naturbeziehung gelangen können, da die wesensmäßige Beziehung zwischen Mensch und Natur als konstituierender, identitätsstiftender Aspekt des Ichs verstanden wird, bei dem das 'in Beziehung treten ' in einem untrennbaren Zusammenhang mit der Ich-Werdung und IchIdentität gesehen wird. Dies wiederum wurde als eine hinreichende wenn auch keine ausreichende Grundlage dafür angesehen, dass Menschen diejenigen Fähigkeiten und Fertigkeiten ausprägen können, die ein gutes Leben ausmachen. Dies bedeutete aber auch, dass im Rahmen der relationalen Perspektive die komplexen Zusammenhänge zwischen dem guten Leben von Menschen (jetzt und in Zukunft) und dem Beitrag der Natur/Biodiversität zum menschlichen Wohlergehen (in Ansätzen) geklärt werden mussten. Über Martha Nussbaums Fähigkeitenansatz wurde aufgezeigt, dass der Natur/Biodiversität hinsichtlich der Sicherung existenzieller Grundbedürfnisse, als inspirierende und erholsame Naturerfahrung, als Erfahrung der Verbundenheit mit sich und anderen und als Möglichkeit zur Entwicklung und Reflexion eines eigenen Lebensplans eine besondere sowohl instrumentell als auch eudaimonistisch eigenwertige Bedeutung für das gute Leben von einzelnen zukommt, woraufhin vorgebracht wurde, dass es bei der relationalen Position auch darum gehen muss, eudaimonistische Argumente und Begründungen aufzuzeigen, dass diese Möglichkeiten für alle Menschen (auch Zukünftige) zu erhalten und bereit zu stellen sind. 
Zugleich wurde aber auch verdeutlicht, dass die Ausweisung solcher Argumente und Begründungen, so lohnend die relationale Perspektive in pragmatischer Hinsicht sein mag, immer auch zahlreiche binnenkomplexe ethiktheoretische Fragen nach sich zieht und dass selbst dann, wenn diese Fragen zufriedenstellend beantwortet werden können, sich immer noch die Problematiken ergeben, dass nahezu alle Begründungsansätze - auch die eudaimonistischen - in der Umwelt- und Naturschutzethik primär auf den Schutz der Natur/Biodiversität fokussieren, wodurch das innovative Moment der Bewahrung und Förderung der Biodiversität, nämlich die Integrativität der Zielstellungen, nicht in ausreichendem Maße berücksichtigt wird. Zudem wird über die relationale Perspektive mit ihrem Fokus auf die moralrelevanten Motive der Verbundenheit und Achtsamkeit, den Tugenden einer gelingenden Lebensführung und ihren eudaimonistischen Argumentations- und Begründungsansätzen das Problem deutlich, dass die Umwelt- und Naturschutzethik über ihren Fokus auf das Inklusionsproblem auch unter einem deontischen Fokus operiert, sprich, primär auf Argumente und Begründungen abzielt, in denen es um Pflichten und Rechte in Ansehung bzw. gegenüber der Natur/ Biodiversität geht. Dies versperrt indes die Einsicht darauf, dass es bei der Bewahrung und Förderung der Biodiversität aufgrund der Integrativität der Zieltrias immer auch um andere Typen von Handlungen und Begründungen geht und gehen muss, die über Schutzpflichtbegründungen hinausgehen. Dieser aus zwei Aspekten zusammengesetzten zweiten Detailproblematik wurde über die Darstellung und Reflexion eines speziell auf die Biodiversitätszieltrias ausgerichteten biodiversitätsethischen Argumentationsraumes begegnet, der die Vielzahl an Argumenten im politischen und umwelt- und naturschutzethischen Kontext nach Klugheits-, Glücks- und Gerechtigkeitsargumenten klassifiziert und es sich zur Aufgabe gemacht hat, gute Argumente für den Schutz, die nachhaltige Nutzung und die gerechte Verteilung der Biodiversität auszuweisen:

Obgleich der Argumentationsraum in pragmatischer Hinsicht alle Anforderungen erfüllt, die sich über die Neuausrichtung der Umwelt- und Naturschutzethik ergeben, wurde herausgestellt, dass der Ansatz mit einem grundlegenden konzeptionellen Problem sowie bestimmten für die Konzeptualisierung aber wichtigen Auslassungen behaftet ist, die wiederum dazu führen, dass die aufgezeigten konstruktiven Lösungen der beiden Aspekte der zweiten Detailproplematik (die Integration der gesamten Zieltrias in einem Begründungsansatz sowie die Überwindung bzw. Erweiterung des deontischen Fokusses) nicht in ausreichendem Maße durch den Ansatz realisiert werden können. Die Auslassungen wurden darin bestimmt, dass den Fragen, wie das Verhältnis der drei Argumentationstypen zueinander bestimmt wird und warum überhaupt davon ausgegangen werden kann, dass Klugheits-, Glücks- und Gerechtigkeitsargumente allesamt ethische Argumente sind, nicht in ausreichendem Maße nachgegangen wurde (bzw. werden konnte). Das konzeptionelle Problem wiederum wurde darin bestimmt, 
dass sich die Konzeptualisierung des Argumentationsraumes auf zwei unterschiedlichen Ebenen bewegt - zum einen auf der analytischen Ebene, auf der es Eser et al. darum geht, die gängigen politischen Argumente den drei Argumentationstypen zuzuordnen und zum anderen auf der Begründungsebene, auf der es Eser et al. darum geht im ethischen Sinne gute Klugheits-, Glücks- und Gerechtigkeitsargumente für die Bewahrung und Förderung der Biodiversität auszuweisen, dass diese beiden Ebenen aber, die auch als Reflexion erster und zweiter Ordnung verstanden werden können (siehe 1.1), nicht ausreichend voneinander differenziert wurde, so dass Eser et al. der Tendenz verfallen, Fragen, die sich auf der einen Ebene stellen, mit Antworten auf der anderen Ebene zu beantworten und umgekehrt. Daher wurde in Frage gestellt, ob der biodivesitätsethische Argumentationsraum von Eser et al. nicht eigentlich und überwiegend ein biodiversitätsstrategischer Argumentationsraum ist, was indes für das Ansinnen der theoretischen Neuausrichtung der Umwelt- und Naturschutzethik hin zu einer anwendungsorientierten Biodiversitätsethik nicht ausreichend ist, zumal dann nicht, wenn dabei auch noch die Auslassungen beachtet werden.

Daher wurden zugleich mehrere 'Fliegen mit einer Klappe`geschlagen, indem sowohl den beiden Aspekten der zweiten Detailproblematik, den thematischen Auslassungen als auch der Konzeptionsproblematik konstruktiv begegnet wurde, indem zum einen die in der Studie immer nur implizit anklingenden diskurstheoretischen Fundamente der Argumentationstrias rekonstruiert wurden, so dass auf der Grundlage der Spezifizierungen der pragmatischen, eudaimonistischen und moralischen Gebrauchweisen der praktischen Vernunft sowohl die Integration der Zieltrias, eine Verhältnisbestimmung der Argumentationstypen zueinander als auch eine systematische und begründetet Differenzierung der drei Argumentationstypen erfolgen konnte. Diesbezüglich wurde herausgestellt, dass Klugheits-, Glücks- und Gerechtigkeitsargumente analytisch unterschieden werden können, da sie verschiedene Ebenen der Handlungsreflexion betreffen. Auf der Begründungsebene wiederum sind sie unabhängig davon danach zu unterscheiden, dass sie mit je anderen Sollenstypen einhergehen, die mit je anderen Sollgeltungsansprüchen verbunden sind und je andere Begründungstiefen verlangen. In diesem Sinne, so wurde aufgezeigt, lässt sich die das Verhältnis der Argumentationstypen zueinander dadurch bestimmen, dass es sich bei der pragmatischen und eudaimonistischen Frage, was zu tun zweckdienlich ist und wie Menschen leben sollten, damit ihr Leben und ihre Lebensführung als gut bezeichnet werden können sowie der moralischen Frage, wie wir in Bezug auf andere handeln sollten, um dem Sinn nach verschiedene ethische Fragen handelt. Indes wurde angemerkt, dass diese Verhältnisbestimmung nur dann Sinn macht, wenn Klugheits-, Glücks- und Gerechtigkeitsargumente auch zugleich als Teilaspekte eines gemeinsamen Ethikverständnisses verstanden werden können. 
An diesen Gedanken anschließend wurde zum anderen sowohl in konstruktiver Weise auf den zweiten Aspekt der Detailproblematik hinsichtlich der Erweiterung des deontischen Fokus als auch auf die bisher zu wenig beachtete Frage eingegangen, inwieweit Klugheits-, Glücks- und Gerechtigkeitsargumente allesamt ethische Argumente sind, indem auf ein mehrdimensionales Ethikverständnis verwiesen wird, über das die sowohl innerhalb auch außerhalb der Ethik gängige Verbindung und Differenzierung zwischen moralisch und ethisch auf der einen Seite und nicht-moralisch und nicht-ethisch auf der anderen Seite hinterfragt wird, indem moralinterne (moralische Gebrauchsweisen der praktischen Vernunft) sowie moralexterne, aber moralrelevante und moralrelative (eudaimonistische und pragmatische Gebrauchsweisen der praktischen Vernunft) zugleich in das 'Haus der Ethik' integriert werden. Dabei wurde sowohl die relative Autonomie, das wechselseitige Ergänzungsverhältnis als auch die Aufgabenteilung innerhalb der mehrdimensionalen Ethik expliziert, wohl wissend, dass dies lediglich einen Anfang und noch lange kein Ende der binnenkomplexen theoretischen Ausführungen zum mehrdimensionalen Ethikverständnis markiert. In pragmatischer Hinsicht ging es daher vielmehr darum, deutlich zu machen, dass auch, wenn es zwangsläufig bestimmte Überschneidungspunkte zwischen den Teilethiken gibt, die moralische Antwort auf die eudaimonistische und die strategisch-pragmatische Frage und umgekehrt nicht die einzige und nicht einmal die in erster Linie gesuchte Antwort ist. Im Rahmen der mehrdimensionalen Ethik geht es zudem, so wurde weiter argumentiert, um die an konkreten Fällen orientierte Auslotung, welche pragmatischen, eudaimonistischen und moralischen Überlegungen und Erwägungen jeweils relevant sind.

Dies bedeutet aber auch, so wurde weiter ausgeführt, eine fallspezifische Priorisierung der drei Argumentationstypen, was gerade in Bezug auf die Bewahrung und Förderung der Biodiversität relevant ist. Hierbei stellten sich die Fragen, ob diese gleichwertig sind bzw. ob es zwischen diesen eine Hierarchie gibt und was das Verhältnis der drei Argumentationstypen zueinander für die Zieltrias und die damit verbundenen Maßnahmen bedeutet. Bezüglich dieser sich aus der Mehrdimensionalität der Ethik ergebenden Gewichtungsfrage wurde darauf verwiesen, dass eine kontextlose, durch die Ethik 'von außen' entschiedene, grundsätzliche und die konkreten Gegebenheiten nicht beachtende Gewichtung der drei Argumentationstypen und der Biodiversitätsziele nicht angemessen ist. Vielmehr wurde speziell hierüber die Relevanz der dritten Detailproblematik deutlich gemacht, indem argumentiert wurde, dass das praxisrelevante und anwendungsorientierte Wissen, das die Ethik in Bezug auf die Bewahrung und Förderung der Biodiversität einbringen kann, immer schon im Kontext gesellschaftlich-demokratischer Aushandlungsprozesse steht. Dies wiederum verweist darauf, dass die Ausweisung ethisch guter Gründe für die Bewahrung und Förderung der Biodiversität, was im Endeffekt Ziel eines biodiversitätsethischen Argumentationsrau- 
mes ist, nicht lediglich eine Frage der normativen Akzeptabilität der Gründe sein kann, sondern immer auch eine Frage ihrer faktischen Akzeptanz ist, so dass die theoretische Neuausrichtung der Umwelt- und Naturschutzethik letztendlich nach einer Ausweisung der Akzeptabilitäts- und Akzeptanzbedingungen hinsichtlich der Kommunikation und Aushandlung der Biodiversitätsziele und -maßnahmen verlangt.

Diesbezüglich wurden unterschiedliche Akzeptanzverständnisse (Akzeptanz als Legitimation, Marketing und wissenschaftlicher Untersuchungsgegenstand) voneinander differenziert, um darzulegen, dass der Fokus der Ethik auf die normativen Akzeptabilitätsbedingungen kein reines Erkenntnisinteresse ist, sondern dass in der Ethik auch und vor allem dann eine wertende (und keine rein analytische) Unterscheidung zwischen faktischer Akzeptanz und normativer Akzeptabilität gemacht wird, wenn Akzeptabilität vor dem Hintergrund der Theorie des kommunikativen Handelns ausgedeutet wird. Denn hierbei wird die ethische Kommunikation selbst als eine auf Verständigung ausgerichtete Kommunikation verstanden, so dass unterschieden wird zwischen ethisch und auf Akzeptabilität fokussiert und strategisch und auf Akzeptanz fokussiert. Dass die ethisch-verständigungsorientierte Kommunikationen dabei als normativ vorzugswürdig und die strategischen Kommunikationen zur Akzeptanzbeschaffung als grundlegend problematisch angesehen werden, ist, so wurde argumentiert, selbst eine problematische Verknüpfung, denn in diesem Kontext stellt sich erst gar nicht die praktischnormative Frage, welchen Kriterien eine auch im normativen Sinne gute strategische Naturschutzkommunikation unterliegen sollte.

Demgegenüber wurde verdeutlicht, dass das, was im politischen und im ökonomischstrategischen Sinne überzeugungsfördernd ist und das, was im ethischen Kontext als zustimmungswürdig angesehen wird, im sprichwörtlichen Sinne zwei Seiten derselben Medaille sind. Denn es geht in beiden Kontexten darum, überzeugende Positionen auszumachen und zu kommunizieren, ohne dass dabei negiert wird, dass beide Kommunikationsformen unterschiedliche Eigenlogiken haben, die sich indes nicht ausschließen müssen sondern auch ergänzen können. So wurde argumentiert, dass die normative Überzeugungskraft von Gründen in hohem Maße davon abhängig ist, welche emotionalen, motivationalen sowie kognitiven Hintergründe Menschen haben und welche räumlich, zeitlich und kulturell kontingenten Rahmenbedingungen gegeben sind, so dass das Wissen darum, was in einer Gesellschaft für wichtig gehalten wird, nicht nur für die strategische, sondern auch für die ethische Naturschutzkommunikation relevant ist. Zudem muss sich auch die ethische Naturschutzkommunikation darum bemühen, dass ihre 'Botschaften 'zielgruppenspezifisch kommuniziert werden. Denn dies ist mitnichten nur in strategischer Hinsicht zur Akzeptanzgewinnung von Relevanz, sondern auch aus demokratietheoretischer Sicht als Bedingung der Möglichkeit überhaupt informiert zu partizipieren. Deshalb bedarf es einer Berücksichtigung und Beantwor- 
tung der Fragen, an wen genau sich die Naturschutzkommunikation richten soll und wie Botschaften kommuniziert werden können. Zugleich geht es aber nicht nur um strategische Anforderungen sondern auch um normative Standards. Daher wurde aufgezeigt, inwieweit die lebensstilorientierte Naturschutzkommunikation dazu beitragen kann, die gesellschaftlichen Akzeptanzbildungsprozesse im Umwelt- und Naturschutzbereich einerseits besser zu verstehen und andererseits die gesellschaftliche Meinungs- und Willensbildung zu gesellschaftlichen Umwelt- und Naturschutzanliegen gerechter zu gestalten.

Im Anschluss daran stellt sich allerdings die Frage, wie die ethisch begründeten Ziele und Maßnahmen zur Bewahrung und Förderung der Biodiversität gesellschaftspolitisch umgesetzt werden können. Diese Fragestellung geht indes über die Darlegung zielgruppenspezifischer Kommunikationsstrategien hinaus, denn dabei stellt sich vor dem Hintergrund der Ergebnisse der wissenschafttheoretischen und ethischen Detailreflexion aber auch bezogen auf governancetheoretische, policyanalytische, demokratietheoretische und legitimationstheoretische Überlegungen die Frage, wie bestehende und aufkommende Interessenskonflikte bezüglich des Umgangs mit Biodiversität prozedural bewältigt werden (können), wer daran beteiligt werden sollte und wie Klugheits-, Glücks- und Gerechtigkeitserwägungen in Bezug auf Schutz-, Nutzungs- und Verteilungsfragen demokratisch entschieden werden können.

\section{Bilanz der politischen Reflexionen}

In Bezug auf Biodiversität als politischer Symbolbegriff für einen modernen, umfassenden und integrativen Umwelt- und Naturschutz wurde die Metaproblematik festgehalten, dass die strategische Relevanz der Bewahrung und Förderung der Biodiversität hinsichtlich der Modernisierung des Umwelt- und Naturschutzes in Deutschland nicht eingeholt werden kann, da die NBS in den Kontext einer Naturschutzgesetzgebung gestellt wird. Hierüber wird indes erneut das statische Naturschutzdenken betont und der Grundfehler des klassischen Naturschutzes wiederholt, der eigentlich über die Zielstellung der Bewahrung und Förderung der Biodiversität behoben werden könnte. Daher ging es auf der politischen Reflexionsebene um die Analyse, Bewertung und Beurteilung einer am Leitbild nachhaltiger Entwicklung orientierten Biodiversitätspolitik, die unter den Kontextbedingungen einer internationalen Mehrebenenpolitik, den funktionalen Erfordernissen einer präventiven Langfrist- und integrativen Querschnittsorientierung gerecht werden soll, indem die politischen Akteure eine Vermittlerrolle zwischen den gesellschaftlichen Interessen einnehmen und die Beteiligung zivilgesellschaftlicher Akteure an der Formulierung und Umsetzung politischer Ziele anstreben und ermöglichen. 
Hierfür wurde genauer auf die NBS und die in ihr formulierte Vision von einer auf Koordination, Verhandlung und Dialog hin angelegten politisch-strategischen Ausgestaltung der Biodiversitätspolitik geschaut. Diese (vage) Vorstellung wurde anhand der damit sinnhaft assozierten Annahmen (in Bezug auf die Anerkennung der Notwendigkeit demokratischer Ordnungen sowie hinsichtlich normativer Annahmen zur Qualität des Regierungshandelns zur Regulation des Politikfeldes Biodiversität) als Good Biodiversity Governance (GBG) charakterisiert und es wurde herausgestellt, dass es sowohl policy-, governance-, demokratie- und legitimationstheoretischer Überlegungen zugleich bedarf, um diese hinsichtlich ihrer theoretischen Grundannahmen zu bestimmen. Hierbei ergibt sich, so wurde in der Problemselektion argumentiert, indes die Detailproblematik, dass es in den einzelnen Forschungssträngen, die etwas zur Explikation der GDB beitragen können, jeweils 'blinde Flecken ' gibt, da in der Politikwissenschaft spezielle Arbeiten mit Biodiversitäts- und nationalem Governancebezug fehlen, in der Umweltund Naturschutzethik die governance- und policytheoretischen Grundlagen zu wenig beachtet werden und in der Biodiversitätsforschung wiederum grundlagentheoretische Arbeiten mit sozial- und gesellschaftswissenschaftlichem Bezug unterrepräsentiert sind. Daher ging es im Rahmen der politikwissenschaftlichen Detailreflexion zum einen um das theoretisch relevante Ziel, die governance-, policy-, demokratie- und legitimationstheoretischen Grundlagen der GBG zu bestimmen als auch um die praktische Zielstellung darüber die Anforderungen, Chancen und Risiken eines auf Kooperation, Verhandlung und Dialog orientierten Politikstils auszuloten.

Um die theoretische Zielstellung einzuholen, bedurfte es einer ganzen Reihe an abstrakten Überlegungen zu den 'Grundbausteinen 'von Staatlichkeit und Demokratie, die auch gänzlich ohne Bezug zum Politikbereich Biodiversität hätten angestellt werden können, da es hierbei im Grunde genommen um die Darlegung eines alternativen theoretischen Grundgerüsts für Good Governance ging. Da es aber darum ging, speziell die theoretischen Annahmen einer Good Biodiversity Governance zu bestimmen, wurde vorab das Politikfeld Biodiversität hinsichtlich seiner strukturellen Charakteristika dargelegt, so dass die darauf folgenden Analysen zumindest kontextuell 'eingebettet' waren. Als inhaltliche Grundlage für die Spezifizierung der GBG musste zudem das in dieser Arbeit anvisierte kooperative, verhandlungsbasierte und dialogische Verständnis von Good Governance von der entwicklungspolitischen Standardkonzeption unterschieden werden. Dabei wurde aufgezeigt, dass das Standardkonzept praxelogisch ist, zu 'top-down' Reformvorschlägen kommt und auf einem entscheidungszentrierten Demokratieverständnis fußt, während das anvisierte Verständnis von Good Governance vor allem, wenn man dabei die New Modes of Governance und die damit verbundenen partizipativen, deliberativen und diskursiven Ansätze und Elemente betrachtet, explizit normativ ist, zu 'bottom-up 'Reformvorschlägen gelangt und durch seine theoretische 
Nähe zu den kontextbezogenen und prozeduralistischen Demokratietheorien gekennzeichnet ist. Daher wurde die GBG erst einmal anhand der kontextbezogenen und prozeduralistischen Demokratietheorien sowie der daran anknüpfenden partizipativen und deliberativen Governancemodelle mit dem Ziel expliziert, deren Stärken und Schwächen hinsichtlich der theoretischen Fundierung der GBG aufzuzeigen:

Die zentrale Stärke der kontextbezogenen und partizipativen Modellierung der GBG wurde in der Herausstellung der zentralen Rolle der Zivilgesellschaft als vitales, demokratieermöglichendes und -förderliches Element gesehen, da insbesondere die kontextbezogenen Demokratietheorien aufzeigen, dass sich das, was mit Zivilgesellschaft gemeint ist und was sich darin verwirklichen soll, nicht lediglich darin erschöpft, dass zivilgesellschaftliche Akteure als Drohkulisse gegen antidemokratische Tendenzen, als turnusmäßig auf den Plan tretendes Wahlvolk oder als diffuses Themenbecken für das politische Agenda Setting begriffen werden kann. Des Weiteren liegt die Stärke dieser Ansätze darin, dass sie auf die vorpolitischen Bedingungen für die politische Meinungsund Willensbildung sowie auf die soziomoralischen Kontextbedingungen für Demokratie reflektieren, die nicht lediglich als Regierungs- sondern als Lebensform begriffen wird. Die Kritik richtete sich indes darauf, dass die kontextualistischen Demokratieund die partizipativen Governancemodelle nicht plausibel genug zeigen (können), wie das Ideal der zivilgellschaftlichen Selbstregierung und -regulierung in ausdifferenzierten und pluralistischen Gesellschaften aber vor allem innerhalb korporatistischer Beziehungsgeflechte zwischen Politik und Wirtschaft realisiert werden kann und soll. Außerdem wurde angeführt, dass die Sinnzusammenhänge zwischen Partizipation, Zivilgesellschaft und Demokratie als Lebensform ganz automatisch zur Beschreibung von ganz bestimmten Formen von "Self-Governance» führen, womit die partizipativen Governancekonzepte indes lediglich die horizontale (Selbst-)Koordination der BürgerInnen in den Blick nehmen und Governance nicht im umfassenden Sinne als kooperative Regelung verstehen können. Daher, so wurde argumentiert, sind die kontextualistischen und partizipativen Ansätze zwar wichtig und hinreichend, um zu spezifizieren, welche Vorstellungen und Annahmen von Partiziaption der GBG zugrunde liegen können. Die Ansätze sind aber nicht ausreichend, um auch zu spezifizieren, welche Vorstellungen und Annahmen von politischer Steuerung und Regulierung sowie von demokratischer Verantwortung sich daraus für die GBG ergeben, so dass die Beurteilung der Chancen und Risiken der GBG hier zu einseitig erfolgen würde.

Ähnliches galt, wenn auch aus anderen Gründen und stärker bezogen auf Diskurs und Deliberation, für die deliberativen Governancemodelle und die dahinterstehenden prozeduralistischen Demokratietheorien. Die zu berücksichtigenden theoretischen Stärken liegen hier vor allem darin, dass das Verhältnis zwischen Zivilgesellschaft und Politik fokussiert und dabei insbesondere die zentrale Rolle der politischen Öffentlichkeit her- 
ausgestellt wird. Des Weiteren wurde die im Rahmen der GBG wichtige Rolle der Kommunikation für demokratische Aushandlungs- und Entscheidungsprozesse als Modus der politischen Koordination fokussiert. Hierüber wurde indes ein größerer theoretischer Kontext eröffnet, da die deliberative Demokratietheorie in den Rahmen der Theorie des kommunikativen Handelns eingebettet ist. Ein für die GBG entscheidender Bezugspunkt ist dabei der legitimationstheoretische Aspekt von Deliberationen und Diskursen, an dem sich sowohl Stärken als auch Schwächen der prozeduralistischen und deliberativen Modellierung der GBG zeigen. Es wurde aufgezeigt, dass die in den deliberativen Demokratietheorien und Governancemodellen fokussierte rational-diskursive Problemlösung, an der sich die Legitimität politischer Entscheidungen erweisen soll, zwar ein relevanter Aspekt demokratischer Politik ist, die real existierenden Notwendigkeiten zu Bargaining, Tauschgeschäften und Konzessionen, die in der deliberativen Demokratietheorie in den Bereich des strategischen Handelns fallen und damit gerade nicht als Formen legitimer verständigungsorientierter Verhandlung begriffen werden können, nehmen aber den größeren Raum des politischen Alltags ein.

Die Kritik richtete sich folglich darauf, dass das deliberative Legitimationskonzept hinsichtlich der damit verbundenen Konsensorientierung zu anspruchsvoll ist. Des Weiteren wurde kritisch angemerkt, dass das deliberative Demokratiemodell in seiner Verhältnisbestimmung von Lebenswelt, Ökonomie und Politik dort in 'Nöte' gerät, wo die Entgrenzung des Raumes diese Verhältnisbestimmung unscharf werden lässt, da die Prozesse der Globalisierung der Wirtschaft und der Internationalisierung der Politik zu einem Wandel staatstheoretischer Annahmen führen, dem die deliberative Demokratietheorie (indes nicht alleinig) bisher nur unzureichend nachgekommen ist. Zwar kann die deliberative Demokratietheorie mithilfe des Schleusenmodells das Verhältnis von Gesellschaft und Staat als konzentrisches und multizentrisches System begreifen, das sich aus einer Kombination aus hierarchischen und heterarchischen Elementen zusammensetzt, jedoch werden die, den einzelnen Bereichen (Staat, politische Öffentlichkeit und zivilgesellschaftliche Peripherie) zugeordneten Kommunikationsformen der Komplexität des politisch-administrativen Systems ebenso wenig gerecht wie der Binnendifferenzierung der Gesellschaft. Daher wurde dargelegt, dass die deliberativen Demokratie- und Governancemodelle ebenfalls ein unterkomplexes Verständnis von politischer Steuerung und Regulierung sowie ein normativ zu anspruchsvolles Ideal von politischer Verantwortung enthalten. Insofern ergab sich aus den Defiziten der demokratietheoretischen Explikation der an Kooperation, Verhandlung und Dialog orientierten GBG anhand partizipativer, deliberativer und diskursiver Elemente die Notwendigkeit einen 'anderen Weg eingeschlagen', so dass es auf der empirisch-analytischen Ebene weiterführender Überlegungen zu den policy- und governancetheoretischen Aspekten der politischen Interaktion in einem internationalen Mehrebenensystem bedurfte. 
Ausgehend von einer, erneut erst einmal einführenden, kritischen Reflexion des Governancekonzeptes und der politikwissenschaftlichen Governanceforschung wurde das aus der Steuerungstheorie hervorgegangene Verständnis von Governance als kooperative Regelung herangezogen. Dabei wurde festgehalten, dass die entscheidende theoretische Verschiebung von der Steuerungs- zur Governancetheorie darin liegt, dass das Konzept der Governance nur noch institutionstheoretisch vermittelbar ist, weil die Unterscheidung zwischen Steuerungssubjekten und Steuerungsadressaten unscharf wird. Eben dieser Übergang von der akteurszentrierten Institutionstheorie hin zur rein institutionstheoretischen Strukturanalyse, so wurde aufgezeigt, wird indes nicht systematisch innerhalb der Governanceforschung vollzogen. Dies zeigte sich vor allem daran, dass die für die Governancekonzeption so zentrale Rede von den nicht-hierarchischen, kooperativen Formen der Regelung uneindeutig ist und dies oftmals auch bleibt. Daher hat man es entweder mit einem auf Formen und Mechanismen der gesellschaftlich-freiwilligen Selbstregulierung reduzierten Governanceverständnis zu tun, wenn kooperative Regelung als Gegensatz zu staatlicher Steuerung verstanden wird (enges Governancekonzept) oder aber mit einem Governancekonzept, welches den Begriff der Steuerung weiterhin in Form der staatlich-kooperativen Steuerung inkludiert, sich aber speziell gegen staatlich-hierarchische Steuerungsformen abgrenzt (weites Governancekonzept).

Letztendlich, so wurde argumentiert, bietet nur das weite Governanceverständnis eine hinreichend komplexe Grundlage, um das Phänomen Governance einzufangen, da das Zusammenwirken staatlich-hierarchischer, staatlich-kooperativer und selbstregulativer Koordinationsformen und -mechanismen ansonsten ausgeblendet wird. Der Nachteil hier ist indes, dass die Differenzierung zwischen kooperativer Regelung und politischer Steuerung unscharf wird, da immer dann, wenn von Governance als Übergriff für alle Formen der kooperativer Regelung die Rede ist auch von staatlich-kooperativer Steuerung die Rede sein muss. Diese Abgrenzungsproblematik, so wurde argumentiert, ist auf der inhaltlichen Ebene nicht auflösbar, da Governancearrangements eine Ausdehnung des politischen Prozesses in den nur vermeintlich unpolitischen (zivil-)gesellschaftlichen Raum bewirken, so dass diese danach untersucht werden müssen, welche Konflikte und welche möglichen gesellschaftlichen Krisenprozesse diese hervorgebracht haben und wiederum hervorbringen. Daher wurde aufgezeigt, dass der Abgrenzungsproblematik nur analytisch begegnet werden kann. Dies kann, so wurde argumentiert, über die theoretische Fundierung der Governanceforschung durch den Ansatz des "akteurszentrierten Institutionalismus" geleistet werden, da dieser strukturelle, systemische und institutionenzentrierte sowie handlungs- und akteurszentrierte und prozedurale Ansätze zur Explizierung von Governance miteinander kombiniert: 
Auf Basis der verschiedenen Analyseebenen und Komponenten des Ansatzes wurde Governance als Modus der politisch-strategischen Handlungskoordination bestimmt, wobei Governancearrangements hinsichtlich ihrer intervenierenden Struktur- und Prozesseigenschaften systematisch analysiert werden können: Auf der Strukturebene erscheinen die Governanceformen dabei als institutionalisierte Regelungssysteme zur Lösung kollektiver Probleme, die durch verschiedene Akteurskonstellationen immer wieder neu konstituiert werden. Auf der Prozessebene erscheinen die Governanceformen als Koordinations- und Interaktionsprozesse komplexer Akteure mit ganz unterschiedlichen Fähigkeiten, Ressourcen und Orientierungen, so dass die Ergebnisse der Koordinations- und Kooperationsbemühungen davon abhängen, welche Interaktionsformen und -orientierungen in diesen Konstellationen vorherrschen. Dies, so wurde im Weiteren argumentiert, hat wiederum Rückwirkungen auf das damit verbundene Demokratieverständnis, da die klassischen politischen Koordinationslogiken, wie der demokratische Parteienwettbewerb und das Mehrheitsprinzip durch Verhandlungslösungen mit dem Ziel des Interessensausgleiches erweitert werden müssen, was wiederum verhandlungsdemokratische Bedingungen kennzeichnet. Dabei dürfen weder die strukturellen Kontextbedingungen politischer Abstimmungs- und Entscheidungsprozesse vernachlässigt noch die Macht und Dominanzfragen sowie die strategischen Interaktionen der Akteure ausgeklammert werden. Eben dies leistet die komplexe Demokratietheorie von Fritz Scharpf, da sie sowohl in empirisch-analytischer als auch in normativer Hinsicht die Input- und Outputseite des politischen Prozesses beleuchtet, wobei Scharpf dabei wichtige Elemente der entscheidungszentrierten, kontextbezogenen und prozeduralistischen Demokratietheorien zugleich aufnimmt. Da die komplexe Demokratietheorie selbst in der Politikwissenschaft wenig Beachtung findet und außerhalb der Politikwissenschaft nahezu unbekannt ist, wurde diese umfangreich und detailliert dargelegt und gerade was ihrer 'Erweiterungen 'unter den Bedingungen der Globalisierung der Wirtschaft und Internationalisierung der Politik angeht, rekonstruiert.

Das 'Grundgerüst' der komplexen Demokratietheorie wurde herausgearbeitet, indem Scharpfs Arbeit zur "Demokratietheorie zwischen Utopie und Anpassung" dargelegt wurde. Wichtiger waren indes die darauf aufbauenden, lediglich in zahlreichen Artikeln formulierten Annahmen zu den besonderen Herausforderungen, denen Verhandlungsdemokratien gerecht werden müssen. Diesbezüglich wurde herausgearbeitet, dass die Bedingungen und die Ergebnisse moderner Politik an den Bedingungen und Maßstäben der Verhandlungsdemokratie als eigenständigem Demokratietypus gemessen werden sollten, weil es in Verhandlungsdemokratien einerseits besondere Kriterien guter Politik hinsichtlich der Politikproduktion und des politischen Kampfes um die Verteilungsdimension gibt und weil aus legitimatorischen Gründen sicher gestellt werden muss, dass all diejenigen, die von kollektiven Entscheidung negativ betroffen sein 
könnten, die Möglichkeit erhalten an den Verhandlungen beteiligt zu sein oder sich interessenwirksam repräsentieren zu lassen (Inputseite), um von den Leistungen politischer Entscheidungen zu profitieren oder im Extremfall angemessen entschädigt zu werden, wenn die Entscheidung zu partiellen Sonderopfern führt (Outputseite). Die von Scharpf herausgestellten Kriterien guter Politik unter verhandlungsdemokratischen Bedingungen wurden aufgezeigt, indem auf die je spezifischen Effizienz- und Effektivitätskriterien der prototypischen Verhandlungsformen (Tauschverhandlungen, Problemlösungsverhandlungen und positive Koordination) unter den strukturellen Kontextbedingungen dyadischer Netzwerke, normativer Regime und von Kollektivverhandlungssystemen eingegangen wurde. Auf die Legitimationskriterien wurde eingegangen, indem Scharpfs Überlegungen bezüglich der Input- und Outputlegitimation vor dem Hintergrund der realen Besonderheiten verhandlungsdemokratischer Settings dargelegt wurden. Die hierbei diskutierten und dargelegten Aspekte wurden dann herangezogen, um die Good Biodiversity Governance hinsichtlich ihrer theoretischen Grundannahmen zu spezifizieren. Dabei wurden zehn Punkte festgehalten, unter die stellenweise mehrere Unterpunkte fielen:

Die GBG wurde hier als kooperativer Modus der politisch-strategischen Handlungskoordination im Sinne der weiten Governancekonzeption definiert, die zur Lösung von Koordinations-, Kollektivgut- und Redistributionsproblemen in politischen Mehrebenensystemen zur Anwendung kommt. Auf der Strukturebene wurde die GBG dabei als ein an den normativen Prinzipien der Subsidiarität, der Politikintegration, der Verursachung und der Vorsorge orientiertes Regelungssystem zur Verminderung und Lösung der Problematik der Vernichtung der Biodiversität verstanden, wobei unter den Bedingungen dyadischer Netzwerke, normativer Regimestrukturen sowie von Kollektiventscheidungssystemen je andere Effektivitäts- und Effizienzkriterien für die prototypischen Verhandlungsformen der Tauschgeschäfte, der Problemlösungsverhandlungen und der positiven Koordination gelten. Auf der Prozessebene wurde die GBG als strategischer Interaktionsprozess der Kooperation, der Verhandlung und des Dialogs verstanden, wobei die Ergebnisse der Interaktionen einerseits davon abhängig sind, welche Interaktionsformen und -orientierungen in diesen Kooperations-, Verhandlungs- und Dialogkonstellationen jeweils vorherrschen und ob die diese den normativen Prinzipien folgen, dass alle potenziell Betroffenen beteiligt werden müssen (Inputseite), dass sich aber niemand beteiligen und keiner zustimmen muss, wenn er sich dabei per Saldo schlechter stellt als bei einem Scheitern der Verhandlungen (Outputseite).

Vor diesen Hintergründen wurde mit Fokus auf die Politik der Biodiversität und ihre vertikale und horizontale Koordination angemerkt, dass bei der GBG nicht nur politikinterne Strukturen und Prozesse betrachtet werden können, denn die Erweiterungen der politischen Sphäre oberhalb und innerhalb der Nationalstaaten durch kooperative 
Steuerung und Verhandlungslösungen hat vielmehr relevante Rückwirkungen auf die für die GBG relevanten Prinzipien der Subsidiarität, der Politikintegration, der Vorsorge und der Verursachung: Für das Subsidiaritätsprinzip ist relevant, dass die vertikale Koordination, vor allem oberhalb der Nationalstaaten, wo es keine vergleichbaren zivilgesellschaftlichen Strukturen wie innerhalb der Nationalstaaten gibt, alleinig durch expertokratische Verhandlungssysteme realisiert wird, so dass das Subsidiaritätsprinzip sich nur anhand von Effizienz- und Effektivitätskritieren beurteilen lässt. Für das Prinzip der Politikintegration ist damit wiederum relevant, dass die horizontale Koordination vor allem innerhalb der nationalstaatlichen Ebene durch die Koordinationsformen der gesellschaftlichen Selbstorganisation ergänzt und erweitert werden können und müssen. Mit Bezug auf die GBG geht es hierbei vor allem um langfristige Perspektiven und zukünftige Interessen und dass geschaut wird, welche formalen und machtbasierten Faktoren darüber entscheiden, welche Positionen und Meinungen sich in der Politik durchsetzen können und welche nicht. In Bezug auf das Vorsorgeprinzip impliziert die GBG, dass alle mit der Bewahrung und Förderung der Biodiversität verbundenen Belange daraufhin geprüft werden müssen, ob diese mit unkompensierbaren Sonderopfern für bestimmte Bevölkerungsgruppen einhergehen. Hierbei ist entscheidend, dass durch gesetzliche Regelungen die institutionellen Rahmenbedingungen dafür geschaffen werden, dass die Risikobewertungen nicht nur auf Basis wissenschaftlicher Fakten oder ökonomischer Kosten-Nutzen Analysen durchgeführt werden, denn ansonsten ist das Risiko zu hoch, dass wichtige Aspekte, wie eben Argumente des guten Lebens und Gerechtigkeitsargumente ausgeblendet werden. In Bezug auf das Verursacherprinzip wurde schließlich über die GBG der Umstand anerkannt, dass das Gemeinlastprinzip, so ausgestaltet werden muss, dass die Kosten und Nutzen aus dem Schutz und der Nutzung der Biodiversität gerecht verteilt werden, wobei es hier vor allem um Effizienz- und Effektivitätsfragen bei den Entscheidungsfindungsprozessen sowie die dabei relevante Debatte um Verteilungsfragen geht.

Gerade in Bezug auf das Vorsorge- und Verursacherprinzip wurde anhand der Stufenskala für den Legitimationsbedarf für die GBG deutlich gemacht, in welchen Fällen hierarchische Steuerung seitens des Staates dringend erforderlich ist, welche Spielräume zur Effizienz- und Effektivitätssteigerung dabei auf Basis der strukturellen Kontextbedingungen der Verhandlungsformen möglich sind, welche Instrumente dabei jeweils eingesetzt werden können und welche Legitimationsanforderungen sich unabhängig von den konkreten Ergebnissen bereits durch die strukturellen und prozeduralen Charakteristika der strategischen Interaktionsformen ergeben: Reine Kooperationsspiele verlangen keine hierarchische Koordination und Steuerung. Die Maßnahmen zur Effizienz- und Effektivitätssteigerung sind hier bereits durch die Kooperationslogik selbstregulativer Verhandlungsnetzwerke und normativer Regime angelegt, die 
eine Erwartungssicherheit qua schwacher und starker Bindungen sowie durch gemeinsam festgelegte Regeln und Normen generieren. Um diese aufrecht zu erhalten, müssen sich die staatlichen Akteure sogar aus den Verhandlungen heraushalten, da sie ansonsten die Kräfteverhältnisse einseitig verschieben oder aber dazu beitragen, dass die solidarischen Interaktionsorientierungen untergraben werden. Hier bedarf es persuasiv-informatorischer Policy-Instrumente. Dazu zählen Umweltbildungs- und Informationsmaßnahmen aber natürlich auch motivationales und vorbildwirksames Verhalten. Vor allem die Informationsmaßnahmen können, zumal wenn sie zielgruppenspezifisch ausgelegt sind, auf niederschwelliger Ebene bewirken, dass BürgerInnen, Unternehmen, soziale Träger etc. relevantes Handlungswissen erhalten und damit in die Lage versetzt werden, gemeinsame Entscheidungen zu treffen. Im Falle von symmetrischen Dilemmaspielen ist der 'Schatten der Hierarchie' von besonderer Bedeutung, da paretooptimale Lösungen zwar möglich sind, die Einigung auf gemeinsame Regelungen aber nur dann erfolgt bzw. garantiert werden kann, wenn dahinter monetäre Anreizmechanismen und/oder administrative Sanktionsmechanismen seitens des Staates stehen, die den Austrittsgewinn deutlich übersteigen. Staatliche Organisationshilfen und die Androhung hoheitlicher Rechtsdurchsetzung bieten hier Effizienz- und Effektivitätssteigerungen. Dazu können die staatlichen Akteure sowohl auf prozedurale als auch auf kooperative Instrumente zurückgreifen, da beide zugleich geeignet sind, eine Problemlösung herbei zu führen, die die Beteiligung vieler unterschiedlicher Akteure verlangt, bei denen aber nicht sicher gestellt werden kann, dass es überhaupt zu Ergebnissen kommt, obgleich die Politik sich um die Etablierung von zugleich praktisch anwendbaren und gerechten Verfahrensvorschriften bemühen und gegenüber der Allgemeinheit kommunizieren muss, warum sie bei der Vielzahl an Möglichkeiten gerade diese Verhandlungen unterstützt und zeitliche, humane und finanzielle Ressourcen aufbringt, um die Verhandlungen zu unterstützen und/oder warum er sein Gewaltmonopol zur Sicherung dieser Regelungen einsetzt. Bei assymetrischen Positivsummenspielen mit Verteilungskonflikt ist eine Einigung auf gemeinsame Regelungen nur dann möglich, wenn kaldoroptimale Lösungen angestrebt werden, sprich, wenn alle Beteiligten dazu bereit sind, auf ihren maximalen Gewinn für die Suche nach der Option mit dem größten Gesamtgewinn und dem geringsten Gesamtschaden für alle zu verzichten. Daher ist es bei solchen Konstellationen die Aufgabe der staatlichen Akteure als 'Agenten der Allgemeinheit' aufzutreten und nur dienigen Entscheidungsoptionen zuzulassen, die einzelne oder viele Unbeteiligte nicht unnötig benachteiligen, ohne dass die Benachteiligungen möglichst verhindert oder aber, wenn diese unumgänglich sind, durch die Gewinne aus der Regelung kompensiert werden. Die Maßnahmen zur Effizienz- und Effektivitätssteigerung sind hier durch die Kooperationslogik innerhalb von Kollektiventscheidungssystemen angelegt. Hier lassen sich Entscheidungsblockaden nur verhindern, indem Konsensregeln etabliert werden. Dies wiederum erhöht die 
Transaktionskosten, wobei sich diese über den Einsatz eines Mediators verringern lassen, der den Akteuren hilft eine einvernehmliche Lösung zu finden. Zudem schaffen ordnungsrechtliche und ökonomische Instrumente hier Abhilfe, da ordnungsrechtliche Instrumente sowohl die Handlung als auch das Ergebnis der Handlung normieren, aufzeigen, welche Handlungsoptionen sozial erwünscht sind und damit die zulässigen Alternativen reduzieren. Ökonomische Instrumente wiederum schaffen finanzielle Anreize um die Adressaten zu gewünschten Verhaltensänderungen zu bewegen. Da beide Instrumentarien durch den Einsatz staatlicher Macht initiiert und oftmals auch 'erzwungen` werden müssen, bedeutet dies, dass ihr Einsatz gegenüber denjenigen zu rechtfertigen ist, die eine Nichtregelung der Regelung vorgezogen hätten. Hierfür reichen ebenfalls outputseitige Legitimationargumente allerdings nur unter Bezug auf einen gerechten Verteilungsmaßstab da hier argumentiert werden muss, dass die Gewinne aus der Regelung die partiellen Verluste der Verlierer kompensieren. Nullsummenspiele können nicht mehr anhand outputorientierter Legitimationsargumente gerechtfertigt werden und hierbei greifen auch sämtliche Policy-Instrumente zu kurz. Diese können nur mit kollektivistischen, inputorientierten Legitimationsargumenten gerechtfertigt werden, was es erforderlich macht, dass bevor politische Maßnahmen in Erwägung gezogen und politische Entscheidungen getroffen werden, es einen gesellschaftlichen Prozess der Diskussion und Deliberation über eben diese Problematiken gibt. Die staatlichen Akteure als Gewährleistungsinstanz des Gemeinwohls müssen hierbei die relevanten gesellschaftlichen Diskurse anregen, Deliberationsarenen eröffnen und für eine solidarische Umgangsweise miteinander und eine solidarische Regelung werben. Ihre Aufgaben sind koordinierender, strukturschaffender, vorbildwirksamer und ermöglichender Art. Zudem müssen die staatlichen Akteure sicher stellen, dass alle relevanten Interessen in den gesellschaftlichen Willensbildungsprozessen vertreten sind und dass die Ergebnisse auch Eingang in die staatlich-administrativen Prozesse finden. Zugleich sind solidarische Orientierungen je nach dem, welche strukturellen Voraussetzungen vorherrschen, mehr oder weniger wahrscheinlich. Wahrscheinlicher sind sie dort, wo sich partnerschaftliche Beziehungsmuster entwickeln können, sprich in Netzwerkbeziehungen, die idealerweise mit normativen Regelungen unterlegt sind. Ungünstiger sind sie innerhalb von Zwangsverhandlungssystemen. Die Ermöglichung netzwerkartiger Strukturen erfordert grundlegende und basale Maßnahmen seitens der Politik zur Ausprägung zivilgesellschaftlicher und solidarischer Strukturen und sind auf der Akteursebene mit hohen kognitiven, motivationalen und emotionalen Ansprüchen an die Fähigkeiten und Fertigkeiten der Verhandlungsteilnehmer verbunden. Hierfür braucht es Lern- und Übungsprozesse, die es nur in einer 'lebendigen Demokratie` gibt. 
Mit Blick auf die Inputseite politischer Prozesse wurde folglich festgehalten, dass über die GBG das Interesse an demokratischer Selbstbestimmung und -organisation anerkannt wird, wobei Strukturen und Verfahren geschaffen werden müssen, um das Sensorium der politischen Entscheidungsstellen für das Problembewusstsein, die Kritik und die Empfehlungen der aktiven Öffentlichkeit erheblich zu verbessern. Hier wurde auf den gezielten Einsatz der lebensstilorientierten Naturschutzkommunikation für die Auswahl der politischen Agenda, der relevanten Akteure als auch zur Identifizierung der Konsens- und Dissens-punkte verwiesen. In Bezug auf die Outputseite politischer Prozesse wurde wiederum deutlich gemacht, dass über den pluralistisch-partizipativen Strukturen eine politisch-staatliche Ebene vorhanden sein muss, die in relativer Unabhängigkeit von den Pressionen der organisierten Interessengruppen agiert, so dass Entscheidungen auch gegen den Widerstand einzelner Gruppen durchgesetzt werden können. Da diese Instanzen sich durch ihre Gemeinwohlorientierung legitimieren, kann das Verteilungsziel der Bewahrung und Förderung der Biodiversität und die damit in Verbindung stehenden Verteilungsfragen nur solange ausgeblendet werden, wie durch die Optimierung von Schutz- und Nutzungsansprüchen annähernd alle profitieren können. Die Beurteilung der Stabilität und Qualität der GBG hängt davon ab, was für Outputs und Outcomes durch Kooperationen, Verhandlungen und Dialoge produziert werden und welche Inputs in diese eingehen, wie diese im politischen Prozess verarbeitet und zu politischen Entscheidungen umgewandelt werden und welche strukturellen und prozeduralen Herausforderungen sich jeweils stellen.

Daher wurden abschließend anhand der empirischen Ergebnisse des »Politikbarometers Biodiversität" die Anforderungen benannt, die sich aus der theoretischen Konkretisierung der GBG ergeben. Diese wurden nach strukturellen und prozedualen Aspekten geclustert. Aufbauend darauf wurden die Defizite und Entwicklungsmöglichkeiten der deutschen Biodiversitätspolitik vor dem Hintergrund dieser Anforderungen herausgestellt. Hier ergaben sich fünf zentrale Punkte: 1) Die Stärkung der Bewahrung und Förderung der Biodiversität durch Expertise und Kompetenzerwerb, 2) die Komplementierung der NBS durch administrative Pläne, 3) den Umbau der IMA NBS zum strategischen Koordinationsgremium aller Ministerien auf Abteilungsleiterebene, 4) den Ausbau der pro-aktiven und kooperativen Steuerung seitens der verantwortlichen Entscheidungsträger und 5) die Förderung der gesellschaftliche Mitgestaltung der Ziele aber auch der Maßnahmen und Leuchtturmprojekte der NBS. 


\section{LITERATURVERZEICHNIS}

Gesetze/Konventionen/politische Protokolle/Berichte/Strategiepapiere (chronologisch):

1949. Grundgesetz der Bundesrepublik Deutschland (GG). Ausfertigungsdatum 23.05.1949. Zuletzt geändert am 23.12.2014 durch Art. 1

1987. Unsere gemeinsame Zukunft: Der Brundtland-Bericht. Weltkommission für Umwelt und Entwicklung (WCED). Genf

1989. Sub-Saharan Africa: From crisis to sustainable growth. Weltbank. Washington

1992. Convention on Biological Diversity (CBD). United Nations Conference on Environment and Development (UNCED). Vereinte Nationen (UN). Rio de Janeiro

1992. Governance and development. Weltbank. Washington

1994. Umweltgutachten: Für eine dauerhafte umweltgerechte Entwicklung. Sachverständigenrat für Umweltfragen (SRU). Stuttgart

1995. Participatory Development and Good Governance. Bericht der Organisation für wirtschaftliche Zusammenarbeit (OECD). Paris

1996. Entwicklungspolitisches Konzept der Bundesregierung. BMZ aktuell 72.

Bundesministerium für wirtschaftliche Zusammenarbeit und Entwicklung (BMZ). Berlin

1998. Umweltgutachten: Erreichtes sichern - Neue Wege gehen. Sachverständigenrat für Umweltfragen (SRU). Stuttgart

2000. Decision V/6. 5. Vertragsstaatenkonferenz der Mitglieder der Konvention über die Biologische Vielfalt (COP 5). Nairobi, 15 - 26. Mai 2000

2000. Die Anwendbarkeit des Vorsorgeprinzips. Mitteilung der Europäischen Kommission. Brüssel

2000. Umweltgutachten: Schritte ins nächste Jahrtausend. Sachverständigenrat für Umweltfragen (SRU). Stuttgart

2002. Umweltgutachten: Für eine neue Vorreiterrolle. Sachverständigenrat für Umweltfragen (SRU). Stuttgart

2004. Addis-Abeba-Prinzipien (AAP) für die nachhaltige Nutzung der Biodiversität. Sekretariat der CBD. Montreal

2004. Umweltgutachten: Umweltpolitische Handlungsfähigkeit sichern: Sachverständigenrat für Umweltfragen (SRU). Baden-Baden

2005. Ecosystems and Human Well-Being: Biodiversity Synthesis. A Report of the Millennium Ecosystem Assessment (MEA). Washington DC

2007. Sondergutachten: Umweltverwaltungen unter Reformdruck: Herausforderungen, Strategien, Perspektiven. Sachverständigenrat für Umweltfragen (SRU). Berlin

2009. Bundesnaturschutzgesetz (BNatschG). Ausfertigungsdatum 29.07.2009. Zuletzt geändert am 13.10.2016 durch Art. 19 
2009. Förderung von Good Governance in der deutschen Entwicklungspolitik. BMZ Konzepte 172. Bundesministerium für wirtschaftliche Zusammenarbeit und Entwicklung (BMZ). Berlin

2010. Nagoya-Protokoll: Protokoll über den Zugang zu genetischen

Ressourcen und die ausgewogene und gerechte Aufteilung der sich aus ihrer Nutzung ergebenden Vorteile. 10. Vertragsstaatenkonferenz der Mitglieder der Konvention über die Biologische Vielfalt (COP 10). Nagoya

2010. Naturbewusstsein 2009: Bevölkerungsumfrage zu Natur und biologischer Vielfalt. Bundesministerium für Umwelt, Naturschutz, Bau und Reaktorsicherheit (BMUB). Berlin

2010. Recht einfach: Das Bundesnaturschutzgesetz 2009. Naturschutzbund Deutschland (NABU). Berlin

2011. Nationale Strategie zur biologischen Vielfalt (NBS). 3. unveränderte Auflage nach der Erstauflage 2007. Bundesministerium für Umwelt, Naturschutz, Bau und Reaktorsicherheit (BMUB). Berlin

2012. Living planet report: biodiversity, biocapacity and better choices. World Wide Fund for Nature (WWF). Berlin

2012. Naturbewusstsein 2011: Bevölkerungsumfrage zu Natur und biologischer Vielfalt. Bundesministerium für Umwelt, Naturschutz, Bau und Reaktorsicherheit (BMUB). Berlin

2012. Our life insurance, our natural capital: An EU biodiversity strategy to 2020. Europäische Kommission. Brüssel

2012. Umweltgutachten: Verantwortung in einer begrenzten Welt. Sachverständigenrat für Umweltfragen (SRU). Berlin

2013. Umweltbewusstsein in Deutschland: Repräsentativumfrage zum Umweltbewusstsein und Umweltverhalten im Jahr 2012. Bundesministerium für Umwelt, Naturschutz, Bau und Reaktorsicherheit (BMUB). Berlin

2014. Indikatorenbericht zur Nationalen Strategie zur Biologischen Vielfalt. Bundesministerium für Umwelt, Naturschutz, Bau und Reaktorsicherheit (BMUB). Berlin

2014. Living Planet Report: Species and spaces, people and places. World Wide Fund for Nature (WWF). Berlin

2014. Naturbewusstsein 2013: Bevölkerungsumfrage zu Natur und biologischer Vielfalt. Bundesministerium für Umwelt, Naturschutz, Bau und Reaktorsicherheit (BMUB). Berlin

2016. Der Zustand der biologischen Vielfalt in Europa. World Wide Fund for Nature (WWF). Berlin

2016. Living planet report 2016: Risk and resilience in a new era. World Wide Fund for Nature (WWF). Berlin

2016. Naturbewusstsein 2015: Bevölkerungsumfrage zu Natur und biologischer Vielfalt. Bundesministerium für Umwelt, Naturschutz, Bau und Reaktorsicherheit (BMUB). Berlin 
A

Aden, Hartmut 2012. Umweltpolitik. VS Verlag für Sozialwissenschaften/Springer: Wiesbaden.

Arendt, Hannah \& Ludz, Ursula (Hg.) 2012a. Zwischen Vergangenheit und Zukunft: Übungen im politischen Denken I. Ungekürzte Taschenbuchausg. Piper: München

Arendt, Hannah \& Ludz, Ursula 2012b. In der Gegenwart: Übungen zum politischen Denken II. Ungekürzte Taschenbuchausg. Piper: München

Arenhövel, Mark 1998. Neubeschreibungen der Demokratie: Konflikte um Differenz und Integration, in Neumann, Franz (Hg.): Handbuch politische Theorien und Ideologien. (UTB, 1810) Leske + Budrich: Opladen, 115-158.

\section{B}

Bäckstrand, Karin et al. 2010. The Promise of New Modes of Environmental Governance, in Bäckstrand, Karin et al. (Hg.): Environmental Politics and Deliberative Democracy: Examining the Promise of New Modes of Governance. Edward Elgar: Cheltenham, 3-27.

Baumgärtner, Stefan 2002. Der ökonomische Wert der biologischen Vielfalt, in Bayrische Akademie für Naturschutz und Landschaftspflege (ANL) (Hg.): Laufener Seminarbeiträge, 73-90.

Barber, Benjamin R. 1994. Starke Demokratie: Über die Teilhabe am Politischen. Rotbuch Verlag: Hamburg.

Bartels, Andreas 2007. Wissenschaftlicher Realismus, in Bartels, Andreas \& Stöckler, Manfred (Hg.): Wissenschaftstheorie: Ein Studienbuch. Mentis: Paderborn, 199220.

Barthlott, Wilhelm \& Gutmann, Mathias 2000. Biodiversitätsforschung in Deutschland: Potentiale und Perspektiven. 2. unveränderte Aufl. (Graue Reihe). NeuenahrAhrweiler.

Bayertz, Kurt 1994. Praktische Philosophie als angewandte Ethik, in Bayertz, Kurt (Hg.): Praktische Philosophie: Grundorientierungen angewandter Ethik. (Rowohlts Enzyklopädie), Rowohlt: Hamburg, 7-47.

Bayertz, Kurt 2004. Zur Selbstaufklärung der angewandten Ethik, in Friesen, Hans \& Berr, Karsten (Hg.): Angewandte Ethik im Spannungsfeld von Begründung und Anwendung. (Praktische Philosophie kontrovers, Bd.2) Lang: Frankfurt am Main, 51-73.

Bechmann, Gotthard 2000. Das Konzept der "nachhaltigen Entwicklung" als problemorientierte Forschung: Zum Verhältnis von Normativität und Kognition in der Umweltforschung, in Brand, Karl-Werner (Hg.): Nachhaltige Entwicklung und Transdisziplinarität: Besonderheiten, Probleme und Erfordernisse der Nachhaltigkeitsforschung. (Angewandte Umweltforschung, 16) Analytica-Verlag: Berlin, 31-46.

Becker, Egon 2006. Sozial-ökologische Forschung - Konzepte und Probleme, in Glaeser, Bernhard (Hg.): Fachübergreifende Nachhaltigkeitsforschung: Stand und Visionen am Beispiel nationaler und internationaler Forscherverbünde (Edition Humanökologie, Bd. 4) Oekom-Verlag: München, 185-214. 
Becker, Egon; Hummel, Diana \& Jahn, Thomas 2011. Gesellschaftliche Naturverhältnisse als Rahmenkonzept, in Groß, Matthias (Hg.): Handbuch Umweltsoziologie. VS Verlag für Sozialwissenschaften: Wiesbaden, 75-96.

Becker, Egon \& Jahn, Thomas 2003. Umrisse einer kritischen Theorie gesellschaftlicher Naturverhältnisse, in Böhme, Gernot \& Manzei (Hg.): Kritische Theorie der Technik und der Natur. Fink: München, 91-112.

Becker, Egon \& Jahn, Thomas (Hg.) 2006. Soziale Ökologie: Grundzüge einer Wissenschaft von den gesellschaftlichen Naturverhältnissen. Campus Verlag: Frankfurt/Main.

Benhabib, Seyla 1995a. Ein deliberatives Modell demokratischer Legitimität. Deutsche Zeitschrift für Philosophie 43(1), 3-29.

Benhabib, Seyla 1995b. Selbst im Kontext: Kommunikative Ethik im Spannungsfeld von Feminismus, Kommunitarismus und Postmoderne. 1. Aufl., dt. Erstausg (Edition Suhrkamp Gender Studies, Bd. 1725) Suhrkamp: Frankfurt am Main.

Benhabib, Seyla 1996. Towards a deliberative Model of democratic legitimacy, in Benhabib, Seyla (Hg.): Democracy and difference: Contesting boundaries of the political. Princeton University Press: Princeton, 67-94.

Benz, Arthur 2003. Konstruktive Vetospieler in Mehrebenensystemen, in Mayntz, Renate \& Streeck, Wolfgang (Hg.): Die Reformierbarkeit der Demokratie: Innovationen und Blockaden: Festschrift für Fritz W. Scharpf. (Schriften aus dem Max-Planck-Institut für Gesellschaftsforschung, Köln, Bd. 45) Campus Verlag: Frankfurt/Main, 205-236.

Benz, Arthur et al. 2007. Einleitung, in Benz, Arthur et al. (Hg.): Handbuch Governance: Theoretische Grundlagen und empirische Anwendungsfelder. VS Verlag für Sozialwissenschaften:Wiesbaden

Benz, Arthur 2008. Verwaltungshandeln im Naturschutz: Herausforderungen und Folgen veränderter Rahmenbedingungen. (Naturschutz und biologische Vielfalt, Heft 66) Bundesamt für Naturschutz: Bonn-Bad Godesberg.

Benz, Arthur 2010. Multilevel Governance: Governance in Mehrebenensystemen, in Benz, Arthur \& Dose, Nicolai (Hg.): Governance: Regieren in komplexen Regelsystemen: Eine Einführung. VS Verlag für Sozialwissenschaften: Wiesbaden, 111-135.

Beyme, Klaus von 2000. Zivilgesellschaft: Von der vorbürgerlichen zur nachbürgerlichen Gesellschaft?, in Henkes, Christian \& Arenhövel (Hg.): Systemwechsel: Zivilgesellschaft und demokratische Transformation. Leske + Budrich: Opladen, 51-70.

Birnbacher, Dieter 1980. Sind wir für die Natur verantwortlich?, in Birnbacher, Dieter (Hg.): Ökologie und Ethik. (Bd. Nr. 9983) Reclam: Stuttgart, 103-159.

Birnbacher, Dieter 2006. Natürlichkeit. (Grundthemen Philosophie). De Gruyter: Berlin.

Birnbacher, Dieter 2007. Analytische Einführung in die Ethik. 2., durchgesehene und erweit. Aufl. (De Gruyter Studienbuch) De Gruyter: Berlin.

Blatter, Joachim 2007. Demokratie und Legitimation, in Benz, Arthur et al. (Hg.): Handbuch Governance: Theoretische Grundlagen und empirische Anwendungsfelder. VS Verlag für Sozialwissenschaften: Wiesbaden, 271-284. 
Blumenthal, Julia v. 2005. Governance - eine kritische Zwischenbilanz. Zeitschrift für Politikwissenschaft 15(4), 1149-1180.

Bobach, Reinhard 1999. Interdisziplinearität in der Transformation, in Umstätter, Walther \& Wessel, Karl-Friedrich (Hg.): Interdisziplinarität - Herausforderung an die Wissenschaftlerinnen und Wissenschaftler: Festschrift zum 60. Geburtstag von Heinrich Parthey. (Berliner Studien zur Wissenschaftsphilosophie \& Humanontogenetik, Bd. 15) Kleine: Bielefeld, 217-242.

Böcher, Michael \& Töller, Annette E. 2007. Instrumentenwahl und Instrumentenwandel in der Umweltpolitik: Ein theoretischer Erklärungsrahmen. PVS Sonderheft Politik und Umwelt, 299-322.

Böcher, Michael \& Töller, Annette E. 2012. Umweltpolitik in Deutschland: Eine politikfeldanalytische Einführung. Springer: Wiesbaden.

Böckenförde, Ernst-Wolfgang (1976) 1991. Staat, Gesellschaft, Freiheit: Studien zur Verfassungstheorie und zum Verfassungsrecht. Suhrkamp Verlag: Michigan.

Börzel, Tanja 2008. Der "Schatten der Hierarchie“ - Ein Governance-Paradox?, in Schuppert, Gunnar F. \& Zürn, Michael (Hg.): Governance in einer sich wandelnden Welt. (Politische Vierteljahresschrift. Sonderheft, 41) VS Verlag für Sozialwissenschaften: Wiesbaden, 118-131.

Brand, Ulrich (Hg.) 2010a. Globale Umweltpolitik und Internationalisierung des Staates: Biodiversitätspolitik aus strategisch-relationaler Perspektive. 1. Aufl. Westfälisches Dampfboot: Münster.

Brand, Ulrich 2010b. Internationale Umweltpolitik aus strategisch-relationaler Perspektive erforschen, in Brand, Ulrich (Hg.): Globale Umweltpolitik und Internationalisierung des Staates: Biodiversitätspolitik aus strategisch-relationaler Perspektive. Westfälisches Dampfboot: Münster, 15-26.

Brand, Karl-Werner \& Fürst, Volker 2002. Sondierungsstudie: Voraussetzungen und Probleme einer Politik der Nachhaltigkeit - Eine Exploration des Forschungsfeldes, in Brand, Karl-Werner (Hg.): Politik der Nachhaltigkeit: Voraussetzungen, Probleme, Chancen : eine kritische Diskussion. (Global zukunftsfähige Entwicklung Perspektiven für Deutschland, 3) Edition Sigma: Berlin, 15-110.

Brand, Ulrich \& Görg, Christoph 2003. Postfordistische Naturverhältnisse: Konflikte um genetische Ressourcen und die Internationalisierung des Staates. 1. Aufl. Westfälisches Dampfboot: Münster.

Brendle, Uwe 1999. Musterlösungen im Naturschutz: Politische Bausteine für erfolgreiches Handeln. Ergebnisse aus dem F+E-Vorhaben "Akzeptanzsteigerung im Naturschutz: Ermittlung von erfolgreichen und zukunftsweisenden naturschutzpolitischen Musterlösungen sowie Konfliktslösungs- und Vermittlungsstrategien". Landwirtschaftsverlag: Münster.

Buber, Martin \& Casper, Bernhard 1995. Ich und du. (Universal-Bibliothek, Bd. Nr. 9342) Reclam: Stuttgart.

Buchstein, Hubertus 2012. Umweltpolitik in Diktaturen und Demokratien: Neue Befunde zu einer alten Kontroverse, in Heinrich-Böll-Stiftung (Hg.): Braune Ökologen: Hintergründe und Strukturen am Beispiel Mecklenburg-Vorpommerns. (Schriften zur Demokratie, 26) Heinrich-Böll-Stiftung: Berlin, 51-61. 
Buchstein, Hubertus \& Schmalz-Bruns, Rainer 1992. Gerechtigkeit als Demokratie: Zur politischen Philosophie von Martin Walzer. Politische Vierteljahresschrift (PVS) 33(3), 375-398.

C

Callicott, J. B. 1980. Animal Liberation: A Triangular Affair. Environmental Ethics 2(4), 311-338.

Czada, Roland 2010. Good Governance als Leitkonzept für Regierungshandeln: Grundlagen, Anwendungen, Kritik: Kapitel 10, in Benz, Arthur \& Dose, Nicolai (Hg.): Governance: Regieren in komplexen Regelsystemen: Eine Einführung. ) VS Verlag für Sozialwissenschaften: Wiesbaden, 201-224.

D

Demirović, Alex \& Walk, Heike 2011. Einleitung, in Demirović, Alex \& Walk, Heike (Hg.): Demokratie und Governance: Kritische Perspektiven auf neue Formen politischer Herrschaft. Westfälisches Dampfboot: Münster, 7-17.

Deppert, Wolfgang 1998. Problemlösen durch Interdisziplinarität:

Wissenschaftstheoretische Grundlagen Integrativer Umweltbewertung, in Theobald, Werner (Hg.): Integrative Umweltbewertung: Theorie und Beispiele aus der Praxis. Springer: Berlin, 35-64.

Der Spiegel 1997: Die Wut der Waldler. Thema: Naturschutz: Nationalparks in Deutschland. Ausgabe 47

Devall, Bill \& Sessions, George 1985: Deep Ecology. Gibbs Smith: Layton.

Dewey, John (1927) 2001. Die Öffentlichkeit und ihre Probleme. hrsg. von Hans-Peter Krüger. Philothek: Berlin, Wien.

Dose, Nicolai 1997. Die verhandelnde Verwaltung: Eine empirische Untersuchung über den Vollzug des Immissionsschutzrechts. 1. Aufl. (Schriften zur Rechtspolitologie, Bd. 4) Nomos: Baden-Baden.

Downs, Anthony 1968. Ökonomische Theorie der Demokratie. (Die Einheit der Gesellschaftswissenschaften, Bd. 8) Mohr:Tübingen.

Druwe, Ulrich 1992. Studienführer Politikwissenschaft. 1. Aufl. (Politikwissenschaft aktuell: Reihe Lehre). Ars una: Neuried.

Dryzek, John; Norgaard, Richard \& Schlosberg, David 2013. Climate-challenged society. Oxford University Press: Oxford.

Dryzek, John S. 2000. Deliberative Democracy and beyond: Liberals, critics, contestations. Oxford University Press: Oxford.

Dryzek, John S. 2012. Foundations and frontiers of deliberative governance. Oxford University Press: Oxford.

Dubiel, Helmut 1994. Ungewissheit und Politik. (Edition Suhrkamp, Bd. 1891) Suhrkamp: Frankfurt a Main.

Düwell, Marcus \& Steigleder, Klaus 2003. Bioethik: Zu Geschichte, Bedeutung und Aufgaben, in Düwell, Marcus \& Steigleder, Klaus (Hg.): Bioethik: Eine Einführung. (Suhrkamp Taschenbuch Wissenschaft, 1597) Suhrkamp: Frankfurt a Main, 12-37. 
Eagleton, Terry 2000. Ideologie: Eine Einführung. Metzler: Stuttgart, Weimar.

Easton, David (1953) 1971. The Political System: An inquiry into the state of political science. Alfred A. Knopf: New York.

Ehrlich, Paul R. \& Ehrlich, Anne E. 1992. The value of biodiversity. AMBIO 21(3), 219226.

Eisel, Ulrich 1992. Individualität als Einheit der konkreten Natur: Das Kulturkonzept der Geographie, in Glaeser, Bernhard \& Teherani-Krönner, Parto (Hg.): Humanökologie und Kulturökologie: Grundlagen, Ansätze, Praxis. Westdeutscher Verlag: Opladen, 107-151.

Eisel, Ulrich 1997. Triumph des Lebens: Der Sieg christlicher Wissenschaft über den Tod in Arkadien, in Eisel, Ulrich \& Schultz, Hans-Dietrich (Hg.): Geographisches Denken. Urbs et regio Sonderband, 65)Universitätsverlag: Kassel, 39-160.

Eisel, Ulrich 2002. Das Leben ist nicht einfach wegzudenken, in Gnädinger, Johannes \& Lotz, Achim (Hg.): Wie kommt die Ökologie zu ihren Gegenständen?: Gegenstandskonstitution und Modellierung in den ökologischen Wissenschaften: Beiträge zur Jahrestagung des Arbeitskreises Theorie in der Ökologie in der Gesellschaft für Ökologie vom 21. - 23. Februar 2001 im Kardinal-Döpfner-Haus Freising (Bayern). (Theorie in der Ökologie, 7) Lang: Frankfurt a Main, 129-151.

Eisel, Ulrich 2004. Landschaftliche Vielfalt mit und ohne Sinn, in BfN (Hg.): Die Verwissenschaftlichung kultureller Qualität in der Landschaftsplanung und im Naturschutz. Tagungsdokumentation vom 04. bis 07. Oktober 2004 an der Internationale Naturschutzakademie Insel Vilm, BfN-Schriften Vertrieb im Landwirtschaftsverlag: Münster, 1-24.

Eisel, Ulrich 2007. Vielfalt im Naturschutz - Ideengeschichtliche Wurzeln eines Begriffs, in Potthast, Thomas (Hg.): Biodiversität - Schlüsselbegriff des Naturschutzes im 21. Jahrhundert? Erweiterte Ergebnisdukumentation einer Vilmer Sommerakademie. (Naturschutz und biologische Vielfalt, Heftnr. 48) BfN-Schriften Vertrieb im Landwirtschaftsverlag: Münster, 25-40.

Eisel, Ulrich 2009a. Landschaft und Gesellschaft: Räumliches Denken im Visier. 1. Aufl. (Raumproduktionen: Theorie und gesellschaftliche Praxis, Bd. 5) Westfälisches Dampfboot: Münster.

Eisel, Ulrich 2009b. Naturschutz als politisches Handeln. (Landschaft in einer Kultur der Nachhaltigkeit, Bd. 3) Universitätsverlag: Kassel

Elster, Jon (Hg.) 1998a. Deliberative democracy. Cambridge University Press: Cambridge.

Elster, Jon 1998b. Introduction, in Elster, Jon (Hg.): Deliberative democracy. Cambridge University Press: Cambridge, 1-18.

Eser, Uta 2001. Die Grenze zwischen Wissenschaft und Gesellschaft neu definieren: Boundary work am Beispiel des Biodiversitätsbegriffs, in Höxtermann, Ekkehard; Kaasch, Joachim \& Kaasch, Michael (Hg.): Berichte zur Geschichte und Theorie der Ökologie: Beiträge zur 9. Jahrestagung der DGGTB in Neuburg a.d. Donau. (Bd. 7) VWB:Berlin, 135-152. 
Eser, Uta 2003a. Der Wert der Vielfalt: Biodiversität zwischen Wissenschaft, Politik und Ethik, in Bobbert, Monika; Düwell, Marcus \& Jax, Kurt (Hg.): Umwelt, Ethik, Recht. (Bd. 13) A. Francke Verlag: Tübingen, 160-181.

Eser, Uta 2003b. Einschluss statt Ausgrenzung: Menschen und Natur in der Umweltethik, in Düwell, Marcus \& Steigleder, Klaus (Hg.): Bioethik: Eine Einführung. (Suhrkamp Taschenbuch Wissenschaft, 1597) Suhrkamp: Frankfurt a Main, 344353.

Eser, Uta 2004. Projektionsfeld fremde Arten: Soziale Konstruktionen des Fremden in ökologischen Theorien, in Fischer, Ludwig (Hg.): Projektionsfläche Natur: Zum Zusammenhang von Naturbildern und gesellschaftlichen Verhältnissen. (Veröffentlichungen des Forschungsprojekts "Natur im Konflikt - Naturschutz, Naturbegriff und Küstenbilder") Hamburg University Press: Hamburg, 165-192.

Eser, Uta 2007. "Biodiversität" und der Wandel im Wissenschaftsverständnis: Überarbeitung und Ergänzung eines Vortrags bei der Vilmer Sommerakademie 2002, in Potthast, Thomas (Hg.): Biodiversität - Schlüsselbegriff des Naturschutzes im 21. Jahrhundert? Erweiterte Ergebnisdukumentation einer Vilmer Sommerakademie. (Naturschutz und biologische Vielfalt, Heftnr. 48) BfNSchriftenvertrieb im Landwirtschaftsverlag: Münster, 41-55.

Eser, Uta 2009. Biodiversität - ein wissenschaftliches oder politisches Konzept? Denkanstösse - Biodiversität (7), 36-45.

Eser, Uta 2014. Umweltethik und Politische Ethik: Natur als Gegenstand von Interessenkonflikten, in Maring, Matthias (Hg.): Bereichsethiken im interdisziplinären Dialog. (Schriftenreihe des Zentrums für Technik- und Wirtschaftsethik am Karlsruher Institut für Technologie, 6) KIT Scientific Publishing: Karlsruhe, 221-238.

Eser, Uta, Benzing, Birgit \& Müller, Albrecht 2013. Gerechtigkeitsfragen im Naturschutz: Was sie bedeuten und warum sie wichtig sind. Gutachten im Auftrag des BfN. (Naturschutz und biologische Vielfalt, 130). Bundesamt für Naturschutz: Bonn-Bad Godesberg.

Eser, Uta \& Müller, Albrecht (Hg.) 2006. Umweltkonflikte verstehen und bewerten: Ethische Urteilsbildung im Natur- und Umweltschutz. Oekom-Verlag: München.

Eser, Uta; Neureuther, Ann-Kathrin \& Müller, Albrecht 2011. Klugheit, Glück, Gerechtigkeit: Ethische Argumentationslinien in der nationalen Strategie zur biologischen Vielfalt. Gutachten im Auftrag des Bundesamtes für Naturschutz. (Naturschutz und biologische Vielfalt, 107) Bundesamt für Naturschutz: Bonn-Bad Godesberg.

Eser, Uta \& Potthast, Thomas 1997. Bewertungsproblem und Normbegriff in Ökologie und Naturschutz aus wissenschaftsethischer Perspektive. Ökologie und Naturschutz(6), 163-171.

Eser, Uta \& Potthast, Thomas 1999. Naturschutzethik: Eine Einführung für die Praxis. 1. Aufl. Nomos-Verlag: Baden-Baden. 
Farnham, Timothy J. 2007. Saving nature's legacy: Origins of the idea of biological diversity. Yale University Press: New Haven.

Fine, Arthur 1986. Unnatural Attidudes: Realist an Instrumentalist Attachments to Science. Mind 95, 149-179.

Fleck, Ludwig 1980. Entstehung und Entwicklung einer wissenschaftlichen Tatsache: Einführung in die Lehre von Denkstil und Denkkollektiv. (Wissenschaftsforschung, 312) Suhrkamp: Frankfurt a Main.

Flitner, Michael; Görg, Christoph \& Heins, Volker (Hg.) 1998. Konfliktfeld Natur: Biologische Ressourcen und globale Politik. Leske + Budrich: Opladen.

Føllesdal, Dagfinn; Walløe, Lars \& Elster, Jon 1988. Rationale Argumentation: Ein Grundkurs in Argumentations- und Wissenschaftsheorie. (De-Gruyter-Studienbuch. Grundlagen der Kommunikation) De Gruyter: Berlin.

Forst, Rainer 1996. Kontexte der Gerechtigkeit: Politische Philosophie jenseits von Liberalismus und Kommunitarismus. (Suhrkamp-Taschenbuch Wissenschaft, 1252) Suhrkamp: Frankfurt am Main.

Foucault, Michel (1971) 1991. Die Ordnung der Dinge: Eine Archäologie der Humanwissenchaften. 12. Aufl. (Suhrkamp Taschenbuch Wissenschaft, 96) Suhrkamp: Frankfurt a Main.

Fraenkel, Ernst 1974. Deutschland und die westlichen Demokratien. 6. Aufl. Kohlhammer: Stuttgart.

Frankfurt, Harry G. 1971. Freedom of the will and the concept of a person. Journal of Philosophy 68(1), 5-20.

Frankfurt, Harry G. 1999. Necessity, volition, and love. Cambridge University Press: Cambridge.

Freeden, Michael 1998. Ideologies and political theory: A conceptual approach. Clarendon Press: Oxford.

Freudenthal, Gideon 1982. Atom und Individuum im Zeitalter Newtons: Zur Genese der mechanistischen Natur- und Sozialphilosophie. Suhrkamp: Frankfurt a Main.

Friesen, Hans \& Berr, Karsten 2004. Zur Vermittlung von Begründung und Anwendung: Versuch einer sozialphilosophischen Betrachtung, in Friesen, Hans \& Berr, Karsten (Hg.): Angewandte Ethik im Spannungsfeld von Begründung und Anwendung. (Praktische Philosophie kontrovers, Bd.2) Lang: Frankfurt a Main, 233-253.

Frohn, Hans-Werner \& Schmoll, Friedemann (Hg.) 2006. Natur und Staat: Staatlicher Naturschutz in Deutschland 1906 - 2006. (Naturschutz und biologische Vielfalt, Bd. 35) Bundesamt für Naturschutz: Bonn-Bad Godesberg.

Fukuyama, Francis 1992. Das Ende der Geschichte: Wo stehen wir? Kindler:München.

Fung, Archon \& Wright, Erik O. 2001. Deepening democracy: Innovations in Empowered Participatory Governance. Politics and Society 29(1), 5-41.

\section{G}

Galert, Thorsten 1998. Biodiversität als Problem der Naturethik: Literaturreview und Bibliographie. (Graue Reihe). Bad Neuenahr-Ahrweiler.

Geissel, Brigitte 2009. Participatory Governance: Hope or Danger for Democracy? A Case Study of Local Agenda 21. Local Government Studies 35(4), 401-414. 
Gethmann, Armin \& Mittelstraß, Jürgen 1992. Umweltstandards. Gaia (1), 16-25.

Görg, Christoph et al. (Hg.) 1999a. Zugänge zur Biodiversität: Disziplinäre Thematisierungen und Möglichkeiten integrierender Ansätze. (Ökologie und Wirtschaftsforschung, Bd. 34) Metropolis-Verlag: Marburg.

Görg, Christoph et al. 1999b. Die Biologische Vielfalt als Interdisziplinäres Forschungsfeld, in Görg, Christoph et al. (Hg.): Zugänge zur Biodiversität: Disziplinäre Thematisierungen und Möglichkeiten integrierender Ansätze. (Ökologie und Wirtschaftsforschung, Bd.34) Metropolis-Verlag: Marburg, 9-20.

Görg, Christoph 1999c. Erhalt der biologischen Vielfalt zwischen Umweltproblem und Ressourcenkonflikt: zuerst veröffentlicht in: Hummel et al. (1999): Konfliktfeld Biodiversität, S. 67-82, in Görg, Christoph et al. (Hg.): Zugänge zur Biodiversität: Disziplinäre Thematisierungen und Möglichkeiten integrierender Ansätze. (Ökologie und Wirtschaftsforschung, Bd. 34) Metropolis-Verlag: Marburg, 279-306.

Görg, Christoph 1999d. Gesellschaftliche Naturverhältnisse. (Einstiege, Bd. 7) Westfälisches Dampfboot: Münster.

Görg, Christoph 2001. Kulturelle und biologische Vielfalt: Stricke und Fallstricke in einem komplexen Verhältnis, in Spehl, Harald \& Held, Martin (Hg.): Vom Wert der Vielfalt: Diversität in Ökonomie und Ökologie. (Zeitschrift für angewandte Umweltforschung. Sonderheft, 13) Analytica-Verlag: Berlin, 106-120.

Görg, Christoph 2003. Regulation der Naturverhältnisse: Zu einer kritischen Theorie der ökologischen Krise. Westfälisches Dampfboot: Münster.

Görg, Christoph \& Brand, Ulrich 2000. Globale Umweltpolitik und nationalstaatliche Konkurrenz: Zur Regulation der biologischen Vielfalt, in Hein, Wolfgang \& Fuchs, Peter (Hg.): Globalisierung und ökologische Krise. Schriften des Deutschen ÜberseeInstituts: Hamburg, 231-264.

Gorke, Martin 1999. Artensterben: Von der ökologischen Theorie zum Eigenwert der Natur. Books on Demand: Norderstedt.

Gorke, Martin 2000. Was spricht für eine holistische Umweltethik? Natur und Kultur: transdisziplinäre Zeitschrift für ökologische Nachhaltigkeit 1(2), 86-105.

Gorke, Martin 2003. Die Vernichtung der biologischen Vielfalt als Herausforderung für eine holistische Ethiktheorie. Natur und Kultur: transdisziplinäre Zeitschrift für ökologische Nachhaltigkeit 4(2), 90-104.

Gorke, Martin 2007. Bewahrung der Biodiversität vom Standpunkt einer holistischen Ethik, in Potthast, Thomas (Hg.): Biodiversität - Schlüsselbegriff des Naturschutzes im 21. Jahrhundert? Erweiterte Ergebnisdukumentation einer Vilmer Sommerakademie. (Naturschutz und biologische Vielfalt, Heftnr. 48), BfNSchriftenvertrieb im Landwirtschaftsverlag: Münster, 125-144.

Grande, Edgar 1993. Die neue Architektur des Staates. Aufbau und Transformation nationaler Handungskapazität - untersucht am Beispiel der Forschungs- und Technologiepolitik, in Czada, Roland \& Schmidt, Manfred G. (Hg.): Verhandlungsdemokratie, Interessenvermittlung, Regierbarkeit: Festschrift für Gerhard Lehmbruch. Westdeutscher Verlag: Opladen, 51-71.

Grande, Edgar 2012. Governance-Forschung in der Governance-Falle?: Eine kritische Bestandsaufnahme. Politische Vierteljahresschrift (PVS) 53(4), 565-592. 
Grote, Jürgen R. \& Gbikpi, Bernard (Hg.) 2002. Participatory governance: Political and societal implications. Leske + Budrich: Opladen.

Gudo, Michael \& Steininger, Fritz F. 2001. Der Beitrag der Paläontologie zur Biodiversitätsdebatte, in Janich, Peter, Gutmann, Mathias \& Prieß, Kathrin (Hg.):

Biodiversität: Wissenschaftliche Grundlagen und gesetzliche Relevanz. (Schriftenreihe der Europäischen Akademie zur Erforschung von Folgen wissenschaftlich-technischer Entwicklungen, 10) Springer: Berlin, 31-114.

Gutmann, Amy \& Thompson, Dennis F. 1996. Democracy and disagreement. Harvard University Press: Harvard.

Gutmann, Mathias 1998. Methodologische und normative Aspekte der Umweltbewertung: Elemente einer rationalen Ökologie, in Theobald, Werner ( $\mathrm{Hg}$.): Integrative Umweltbewertung: Theorie und Beispiele aus der Praxis. Springer: Berlin, 65-92.

Gutmann, Mathias \& Janich, Peter 2001a. Methodologische Grundlagen der Biodiversität, in Janich, Peter, Gutmann, Mathias \& Prieß, Kathrin (Hg.): Biodiversität: Wissenschaftliche Grundlagen und gesetzliche Relevanz. (Schriftenreihe der Europäischen Akademie zur Erforschung von Folgen wissenschaftlich-technischer Entwicklungen, 10) Springer: Berlin, 281-353.

Gutmann, Mathias \& Janich, Peter 2001b. Überblick zu methodischen Grundproblemen der Biodiversität, in Janich, Peter, Gutmann, Mathias \& Prieß, Kathrin (Hg.): Biodiversität: Wissenschaftliche Grundlagen und gesetzliche Relevanz. (Schriftenreihe der Europäischen Akademie zur Erforschung von Folgen wissenschaftlich-technischer Entwicklungen, 10) Springer: Berlin, 3-27.

\section{H}

Haber, Wolfgang 2002. Biodiversität: Ein neues Leitbild und seine Umsetzung in der Praxis. Vortragsveranstaltung am 30. Oktober 2002 im Blockhaus Dresden. Sächsische Landesstiftung Natur und Umwelt Akademie, 8-56.

Haber, Wolfgang 2009. Biologische Vielfalt zwischen Mythos und Wirklichkeit. Denkanstösse - Biodiversität(7), 16-35.

Habermas, Jürgen 1973. Wahrheitstheorien, in Schulz, Walter \& Fahrenbach, Helmut (Hg.): Wirklichkeit und Reflexion: Walter Schulz zum 60. Geburtstag. Neske: Pfullingen, 211-265.

Habermas, Jürgen 1981a. Theorie des kommunikativen Handelns (Band 1): Handlungsrationalität und gesellschaftliche Rationalisierung. Suhrkamp: Frankfurt a Main.

Habermas, Jürgen 1981b. Theorie des kommunikativen Handelns (Band 2): Zur Kritik der funktionalistischen Vernunft. Suhrkamp: Frankfurt a Main.

Habermas, Jürgen 1984. Vorstudien und Ergänzungen zur Theorie des kommunikativen Handelns. Suhrkamp: Frankfurt a Main:.

Habermas, Jürgen 1985. Der philosophische Diskurs der Moderne: Zwölf Vorlesungen. (Suhrkamp Taschenbuch Wissenschaft, 749) Suhrkamp: Frankfurt a Main.

Habermas, Jürgen (1962) 1990. Strukturwandel der Öffentlichkeit: Untersuchungen zu einer Kategorie der bürgerlichen Gesellschaft. Suhrkamp: Frankfurt a Main.

Habermas, Jürgen (Hg.) 1991a. Erläuterungen zur Diskursethik. (Suhrkamp Taschenbuch Wissenschaft, 975). Suhrkamp: Frankfurt a Main. 
Habermas, Jürgen 1991b. Vom praktischen, ethischen und moralischen Gebrauch der Vernunft, in Habermas, Jürgen (Hg.): Erläuterungen zur Diskursethik. (Suhrkamp Taschenbuch Wissenschaft, 975) Suhrkamp, Frankfurt a Main, 100-118.

Habermas, Jürgen 1992a. Drei normative Modelle der Demokratie: Zum Begriff deliberativer Demokratie, in Münkler, Herfried \& Fetscher, Iring (Hg.): Die Chancen der Freiheit: Grundprobleme der Demokratie. (Serie Piper, Bd. 1545) Piper: München, 11-24 sowie in Habermas, Jürgen (Hg.): Die Einbeziehung des Anderen: Studien zur politischen Theorie. (Suhrkamp Taschenbuch Wissenschaft, 1444) Suhrkamp: Frankfurt am Main, 277-292.

Habermas, Jürgen 1992b. Faktizität und Geltung: Beiträge zur Diskurstheorie des Rechts und des demokratischen Rechtsstaats. Suhrkamp: Frankfurt a Main.

Habermas, Jürgen (Hg.) 1996a. Die Einbeziehung des Anderen: Studien zur politischen Theorie. (Suhrkamp Taschenbuch Wissenschaft, 1444) Suhrkamp: Frankfurt a Main.

Habermas, Jürgen 1996b. Über den internen Zusammenhang von Rechtsstaat und Demokratie in Habermas, Jürgen (Hg.): Die Einbeziehung des Anderen: Studien zur politischen Theorie. (Suhrkamp Taschenbuch Wissenschaft, 1444) Suhrkamp: Frankfurt a Main, 293-305.

Habermas, Jürgen 1998. Die postnationale Konstellation: Politische Essays. (Edition Suhrkamp, 2095) Suhrkamp: Frankfurt a Main.

Habermas, Jürgen (1989) 2009. Volkssouveränität als Verfahren: Ein normativer Begriff von Öffentlichkeit, in Habermas, Jürgen (Hg.): Politische Theorie. (Philosophische Texte, 4) Suhrkamp: Frankfurt a Main, 35-69.

Hacking, Ian 1999. The social construction of what? Harvard University Press: Harvard. Halsband, Aurelié 2016. Konkrete Nachhaltigkeit. Welche Natur wir für zukünftige Generationen erhalten sollten (Ethik in der Nachhaltigkeitsforschung, Bd. 3) Nomos: Baden-Baden.

Haus, Michael 2010. Governance-Theorien und Governance-Probleme: Diesseits und jenseits des Steuerungsparadigmas. Politische Vierteljahresschrift (PVS)(3), 457479.

Heins, Bernd 1997. Die Rolle des Staates für eine nachhaltige Entwicklung der Industriegesellschaft. (Angewandte Umweltforschung, 8) Analytica: Berlin.

Heiland, Stefan 2008. Zielgruppenanalysen in Naturschutz und Landschaftsplanung: Vorschlag einer praxisorientierten Vorgehensweise, in Erdmann, Karl-Heinz, Löffler, Jörg \& Roscher, Sabine (Hg.): Naturschutz im Kontext einer nachhaltigen Entwicklung: Ansätze, Konzepte, Strategien. (Naturschutz und biologische Vielfalt, 67) Landwirtschaftsverlag: Berlin, 231-256.

Hennig, Boris 2006. Naturteleologie, in Buchheim, Thomas et al. (Hg.): Philosophisches Jahrbuch 113 (2), 296-315.

Henrich, Károly 2003. Biodiversitätsvernichtung: Ökologisch-ökonomische Ursachenanalysen, kausalitätstheoretische Grundlagen und evolutorische Eskalationsdynamik. (Ökologie und Wirtschaftsforschung, Bd. 50) MetropolisVerlag: Marburg. 
Hertler, Christine 1999. Aspekte der historischen Entstehung von

Biodiversitätskonzepten in den Biowissenschaften, in Görg, Christoph et al. (Hg.): Zugänge zur Biodiversität: Disziplinäre Thematisierungen und Möglichkeiten integrierender Ansätze. (Ökologie und Wirtschaftsforschung, Bd. 34) MetropolisVerlag: Marburg, 39-52.

Hesse, Heidrun 2002. Zur Konstitution naturwissenschaftlicher Gegenstände, insbesondere in der Biologie, in Gnädinger, Johannes \& Lotz, Achim (Hg.): Wie kommt die Ökologie zu ihren Gegenständen? Gegenstandskonstitution und Modellierung in den ökologischen Wissenschaften Beiträge zur Jahrestagung des Arbeitskreises Theorie in der Ökologie in der Gesellschaft für Ökologie vom 21. - 23. Februar $2001 \mathrm{im} \mathrm{Kardinal-Döpfner-Haus} \mathrm{Freising} \mathrm{(Bayern).} \mathrm{(Theorie} \mathrm{in} \mathrm{der} \mathrm{Ökologie,}$ 7) Lang: Frankfurt a Main, 117-127.

Hiekel, Susanne 2012. Grundbegriffe der grünen Gentechnik: Wissenschaftstheoretische und naturphilosophische Grundlagen. (Graue Reihe)

Hoffmann, Andreas, Hoffmann, Sönke \& Weimann, Joachim 2005. Irrfahrt Biodiversität: Eine kritische Sicht auf europäische Biodiversitätspolitik. (Ökologie und Wirtschaftsforschung, Bd. 58) Metropolis-Verlag: Marburg.

Hoffmann-Riem, Wolfgang 2005. Das Recht des Gewährleistungsstaates, in Schuppert, Gunnar F. (Hg.): Der Gewährleistungsstaat: Ein Leitbild auf dem Prüfstand. (Schriften zur Governance-Forschung, Bd. 2) Nomos: Baden-Baden, 89-108.

Holtkamp, Lars; Bogumil, Jörg \& Kissler, Leo 2006. Kooperative Demokratie: Das demokratische Potenzial von Bürgerengagement. (Studien zur Demokratieforschung, Bd. 9) Campus Verlag: Frankfurt a Main.

Honneth, Axel 1993. Kommunitarismus: Eine Debatte über die moralischen Grundlagen moderner Gesellschaften. (Theorie und Gesellschaft, Bd. 26) Campus-Verlag: Frankfurt a Main.

Honneth, Axel 2000. Das Andere der Gerechtigkeit: Aufsätze zur praktischen Philosophie. (Suhrkamp Taschenbuch Wissenschaft, 1491) Suhrkamp: Frankfurt a Main.

Hübenthal, Christoph 2006. Eudaimonismus, in Düwell, Marcus, Hübenthal, Christoph \& Werner, Micha H. (Hg.): Handbuch Ethik. Metzler: Stuttgart, 82-94.

\section{J}

Jacob, Klaus \& Jörgens, Helge 2011. Wohin geht die Politikfeldanalyse? Eine Forschungsagenda für ein erwachsen gewordenes Politikfeld. FFU-Report: Berlin.

Jahn, Thomas \& Wehling, Peter 1998. Gesellschaftliche Naturverhältnisse - Konturen eines theoretischen Konzepts, in Brand, Karl-Werner (Hg.): Soziologie und Natur: Theoretische Perspektiven. (Soziologie und Ökologie, Bd. 2) Leske + Budrich: Opladen, 75-93.

Janich, Peter (Hg.) 1992a. Entwicklungen der methodischen Philosophie. (Suhrkamp Taschenbuch Wissenschaft, 979) Suhrkamp: Frankfurt am Main.

Janich, Peter 1992b. Grenzen der Naturwissenschaft: Erkennen als Handeln. (Beck'sche Reihe, 463) C.H. Beck: München. 
Janich, Peter 1995. Die methodische Konstruktion der Wirklichkeit der Wissenschaften, in Lenk, Hans \& Poser, Hans (Hg.): Neue Realitäten: Herausforderung der Philosophie. XVI. Deutscher Kongress für Philosophie, Berlin, 20.-24. September 1993, Vorträge und Kolloquien. Akademie Verlag: Berlin, 460-476.

Janich, Peter 1996. Konstruktivismus und Naturerkenntnis: Auf dem Weg zum Kulturalismus. (Suhrkamp Taschenbuch Wissenschaft, 1244) Suhrkamp: Frankfurt a Main.

Janich, Peter 1997. Kleine Philosophie der Naturwissenschaften. (Beck'sche Reihe, 1203) Beck: München.

Janich, Peter 2000. Was ist Erkenntnis? Eine philosophische Einführung. (Beck'sche Reihe, 1376) Beck: München.

Janich, Peter 2001. Zusammenfassung, in Janich, Peter, Gutmann, Mathias \& Prieß, Kathrin (Hg.): Biodiversität: Wissenschaftliche Grundlagen und gesetzliche Relevanz. (Schriftenreihe der Europäischen Akademie zur Erforschung von Folgen wissenschaftlich-technischer Entwicklungen, 10) Springer: Berlin, XXI-XXIX.

Janich, Peter 2006. Kultur und Methode: Philosophie in einer wissenschaftlich geprägten Welt. (Suhrkamp Taschenbuch Wissenschaft, 1773) Suhrkamp: Frankfurt a Main.

Janich, Peter \& Gutmann, Mathias 2000. Normative Grundlagen der Biodiversität, in Professor Dr. Carl Friedrich Gethmann (Hg.): Biodiversitätsforschung in Deutschland: Potentiale und Perspektiven. Graue Reihe. Neuenahr-Ahrweiler, 6673.

Janich, Peter; Gutmann, Mathias \& Prieß, Kathrin (Hg.) 2001. Biodiversität: Wissenschaftliche Grundlagen und gesetzliche Relevanz. (Schriftenreihe der Europäischen Akademie zur Erforschung von Folgen wissenschaftlich-technischer Entwicklungen, 10) Springer: Berlin.

Janich, Peter; Kambartel, Friedrich \& Mittelstrass, Jürgen 1974. Wissenschaftstheorie als Wissenschaftskritik. Aspekte Verlag: Frankfurt am Main.

Janich, Peter \& Weingarten, Michael 1999. Wissenschaftstheorie der Biologie: Methodische Wissenschaftstheorie und die Begründung der Wissenschaften. (Uni-Taschenbücher, 2033) Fink: München.

Jänicke, Martin 1986. Staatsversagen: Die Ohnmacht der Politik in der Industriegesellschaft. Piper: München.

Jänicke, Martin 2002. Vom instrumentellen und strategischen Ansatz: Umweltpolitische Steuerung im Lichte der Politikfeldanalyse, in UBA (Hg.): Perspektiven für die Verankerung des Nachhaltigkeitsleitbildes in der Umweltkommunikation: Chancen, Barrieren und Potenziale der Sozialwissenschaften. (Berichte des Umweltbundesamt 2001, 4) Erich Schmidt: Berlin, 63-79.

Jänicke, Martin 2003: Die Rolle des Nationalstaats in der globalen Umweltpolitik. Zehn Thesen. Aus Politik und Zeitgeschichte (APuZ) 27(B), 1-12

Jänicke, Martin 2006. Politikintegration im Mehrebenensystem: Das Beispiel der deutschen Umweltpolitik, in Mielke, Siegfried; Koch-Baumgarten, Sigrid \& Rütters, Peter (Hg.): Pluralismus und Demokratie: Interessenverbände, Länderparlamentarismus, Föderalismus, Widerstand: Siegfried Mielke zum 65. Geburtstag. Bund: Frankfurt am Main, 63-73. 
Jänicke, Martin 2008. Megatrend Umweltinnovation: Zur ökologischen Modernisierung von Wirtschaft und Staat. Oekom-Verlag: München.

Jänicke, Martin 2013. Ökologische und politische Modernisierung in entwickelten Industriegesellschaften, in Prittwitz, Volker v. (Hg.): Umweltpolitik als Modernisierungsprozess. VS Verlag für Sozialwissenschaften: Wiesbaden, 15-29.

Jänicke, Martin \& Jörgens, Helge 2004. Neue Steuerungskonzepte in der Umweltpolitik. Zeitschrift für Umweltpolitik und Umweltrecht 27(3), 297-348.

Jänicke, Martin \& Volkery, Axel 2001. Persistente Probleme des Umweltschutzes. Natur und Kultur(2), 45-49.

Jax, Kurt 2003. Die Funktion biologischer Vielfalt, in Körner, Stefan; Nagel, Annemarie \& Eisel, Ulrich (Hg.): Naturschutzbegründungen. Bundesamt für Naturschutz. BfNSchriftenvertrieb im Landwirtschaftsverlag: Bonn Bad-Godesberg, 149-174.

Jax, Kurt 2004. Haben Ökosysteme eine Eigenart? Gedanken zur Rolle des EigenartBegriffs in naturwissenschaftlich geprägten Naturschutzdiskussionen, in Fischer, Ludwig (Hg.): Projektionsfläche Natur: Zum Zusammenhang von Naturbildern und gesellschaftlichen Verhältnissen. (Veröffentlichungen des Forschungsprojekts "Natur im Konflikt - Naturschutz, Naturbegriff und Küstenbilder") Hamburg University Press: Hamburg, 135-163.

Jessop, Bob 2002. Governance and metagovernance.: On reflexivity, requisite variety, and requisite, in Bang, Henrik P. (Hg.): Governance as Social and Political Communication. Manchester University Press: Manchester, 142-172.

Jessop, Bob 2010. Vorwort, in Brand, Ulrich (Hg.): Globale Umweltpolitik und Internationalisierung des Staates: Biodiversitätspolitik aus strategisch-relationaler Perspektive. Westfälisches Dampfboot: Münster, 11-12.

Jessop, Bob 2011. "Regieren + Governance im Schatten der Hierarchie": Der integrale Staat und die Herausforderungen der Metagovernance, in Demirović, Alex \& Walk, Heike (Hg.): Demokratie und Governance: Kritische Perspektiven auf neue Formen politischer Herrschaft. Westfälisches Dampfboot: Münster, 43-72.

\section{K}

Kannetzky, Frank 1999. Methode und Systematik der Philosophie, in Raatz, Richard

(Hg.): Philosophieren über Philosophie. (Leipziger Schriften zur Philosophie, 10) Leipzig, 321-356.

Kant, Immanuel (1786) 1983. Grundlegung der Metyphysik der Sitten. Reclam: Leipzig.

Kant, Immanuel (1797) 2013. Die Metaphysik der Sitten. Reclam: Berlin

Katz, Christine et al. 1996. Biotechnologien für die "Dritte Welt": Eine entwicklungspolitische Perspektive? (Studien des Büros für TechnikfolgenAbschätzung beim Deutschen Bundestag, 2) Edition Sigma: Berlin.

Kaufmann, Arthur 1989. Prozedurale Theorien der Gerechtigkeit. (Sitzungsberichte der Bayerischen Akademie der Wissenschaften, Philosophisch-Historische Klasse, Jahrg. 1989, Heft 7). Verlag der Bayerischen Akademie der Wissenschaften: München.

Keller, David R. \& Golley, Frank B. 2000. The philosophy of ecology: From science to synthesis. University of Georgia Press: Athens.

Kemper, Anne 2001. Unverfügbare Natur: Ästhetik, Anthropologie und Ethik des Umweltschutzes. (Campus Forschung, Bd. 823) Frankfurt: Campus. 
Kettner, Matthias 2014. Wann haben wir ein moralisches Problem?, in Maring, Matthias (Hg.): Bereichsethiken im interdisziplinären Dialog. (Schriftenreihe des Zentrums für Technik- und Wirtschaftsethik am Karlsruher Institut für Technologie) KIT Scientific Publishing: Karlsruhe, 25-44.

Kirchhoff, Thomas 2005. Kultur als individuelles Mensch-Natur Verhältnis: Herders Theorie kultureller Eigenart und Vielfalt, in Weingarten, Michael (Hg.): Strukturierung von Raum und Landschaft: Konzepte in Ökologie und der Theorie gesellschaftlicher Naturverhältnisse. Westfälisches Dampfboot: Münster, 63-106.

Kirchhoff, Thomas 2009. Systemauffassungen und biologische Theorien: Zur Herkunft von Individualitätskonzeptionen und ihrer Bedeutung für die Theorie ökologischer Einheiten. (Beiträge zur Kulturgeschichte der Natur, Bd. 16) Lehrstuhl für Landschaftsökologie an der Technischen Universität München Freising. Graue Reihe

Kirchhoff, Thomas \& Haider, Sylvia 2009. Globale Vielzahl oder lokale Vielfalt: Zur kulturellen Ambivalenz von 'Biodiversität', in Kirchhoff, Thomas \& Trepl, Ludwig (Hg.): Vieldeutige Natur: Landschaft, Wildnis und Ökosystem als kulturgeschichtliche Phänomene. Transcript: Bielefeld, 215-330.

Kirchhoff, Thomas \& Trepl, Ludwig 2001. Vom Wert der Biodiversität: Über konkurrierende politische Theorien in der Diskussion um Biodiversität, in Spehl, Harald \& Held, Martin (Hg.): Vom Wert der Vielfalt: Diversität in Ökonomie und Ökologie. (Zeitschrift für angewandte Umweltforschung, Sonderheft, 13) AnalyticaVerlag: Berlin, 27-58.

Kleger, Heinz 1994. Reflexive Politikfähigkeit: Zur Verschränkung von Bürger- und Staatsgesellschaft, in Gebhardt, Jürgen \& Schmalz-Bruns, Rainer (Hg.): Demokratie, Verfassung und Nation: Die politische Integration moderner Gesellschaften. Nomos: Baden-Baden, 301-319.

Kleger, Heinz 1995. Verhandlungsdemokratie: Zur alten und neuen Theorie des kooperativen Staates, in Voigt, Rüdiger (Hg.): Der kooperative Staat: Krisenbewältigung durch Verhandlung? Nomos: Baden-Baden, 93-118.

Klünger, Gerhardt (Hg.) 2011. Wörterbuch des Konstruktiven Realismus: Aus Vorlesungen, Seminaren und Werken von Friedrich G. Wallner. Lang: München.

Körner, Stefan \& Eisel, Ulrich 2003. Naturschutz als kulturelle Aufgabe - theoretische Rekonstruktion und Anregungen für eine inhaltliche Erweiterung, in Körner, Stefan; Nagel, Annemarie \& Eisel, Ulrich (Hg.): Naturschutzbegründungen. Bundesamt für Naturschutz. BfN-Schriftenvertrieb im Landwirtschaftsverlag: Bonn, BadGodesberg, 5-49.

Kötzle, Markus 1999. Eigenart durch Eigentum: Die Transformation des christlichen Ideals der Individualität in die liberalistische Idee von Eigentum. (Beiträge zur Kulturgeschichte der Natur, Bd. 10). Freising, Weihenstephan. 28-29.

Krasner, Stephen D. 1983. International regimes. Cornell University Press: Ithaca.

Krämer, Hans 1992. Integrative Ethik. Suhrkamp: Frankfurt am Main.

Krebs, Angelika 1996. "Ich würde gern mitunter aus dem Hause tretend ein paar Bäume sehen": Philosophische Überlegungen zum Eigenwert der Natur, in Nutzinger, Hans G. (Hg.): Naturschutz - Ethik - Ökonomie: Theoretische Begründungen und praktische Konsequenzen. (Ökologie und Wirtschaftsforschung, Bd. 21) Metropolis-Verlag: Marburg, 31-48. 
Krebs, Angelika 1997. Naturethik im Überblick, in Krebs, Angelika (Hg.): Naturethik: Grundtexte der gegenwärtigen tier- und ökoethischen Diskussion. Suhrkamp: Frankfurt a Main, 337-379.

Krebs, Angelika 2000. Das teleologische Argument in der Naturethik, in Ott, Konrad \& Gorke, Martin (Hg.): Spektrum der Umweltethik. (Ökologie und Wirtschaftsforschung, Bd. 36) Metropolis-Verlag: Marburg, 67-80.

Krijnen, Christian 2006. Wert, in Düwell, Marcus, Hübenthal, Christoph \& Werner, Micha H. (Hg.): Handbuch Ethik. Metzler: Stuttgart, 548-553.

Kronsell, Annica \& Bäckstrand, Karin 2010. Rationalities and forms of governance: A framework for analysing the legitimicy of new modes of governance, in Bäckstrand, Karin et al. (Hg.): Environmental Politics and Deliberative Democracy: Examining the Promise of New Modes of Governance. Edward Elgar: Cheltenham, 28-46.

Kuhn, Thomas S. (1962) 1993. Die Struktur wissenschaftlicher Revolutionen. 13. Auflage. (Suhrkamp-Taschenbuch Wissenschaft, 25). Suhrkamp: Frankfurt a Main. Kutschera, Franz v. 1998. Ästhetik. (De-Gruyter-Studienbuch). De Gruyter: New York.

\section{$\mathbf{L}$}

Laclau, Ernesto \& Mouffe, Chantal 2006. Hegemonie und radikale Demokratie: Zur Dekonstruktion des Marxismus. Passagen-Verlag: Wien.

Lakatos, Imre 1982. Die Methodologie der wissenschaftlichen Forschungsprogramme. (Philosophische Schriften Band 1). Vieweg: Braunschweig.

Laufer, Heinz \& Münch, Ursula 1998. Das föderative System der Bundesrepublik Deutschland. Leske + Budrich: Opladen.

Laws, Norman 2014. Politische Vorfahrt für biologische Vielfalt: Politikbarometer zur Biodiversität in Deutschland. WWF Studie

LeBon, Gustave (1912) 1982. Psychologie der Massen. 15. Aufl. Kröner: Stuttgart.

Lenk, Hans 1993. Interpretationskonstrukte: Zur Kritik der interpretatorischen Vernunft. 1. Aufl. Suhrkamp: Frankfurt am Main.

Leopold, Aldo (1949) 1970. A Sand County almanac: With essays on conservation from Round River. Ballantine Books: New York.

Lichtl, Martin 2009. Leitmotive für eine moderne Kommunikation zur biologischen Viefalt. Ergebnisse des F+E-Vorhabens "Natur und Biodiversität in der politschen Kommunikation". (Naturschutz und biologische Vielfalt, 80) Bundesamt für Naturschutz: Bonn-Bad Godesberg.

Lijphart, Arend 1980. Democracy in plural societies: A comparative exploration. Yale University Press: New Haven.

Lijphart, Arend 1984. Democracies: Patterns of majoritarian and consensus government in twenty-one countries. Yale University Press: New Haven.

Litsch, Franz-Johannes 2002. Buddhismus und der Schutz der Mitwelt. Beitrag der Vertreter der Deutschen Buddhistischen Union zum "Orientierungsgespräch in Deutschland vertretener Religionen zur Umweltpolitik" am 6.- 7. Mai 2002 in Göttingen. Graue Reihe

Lönnig, Wolf-Ekkehard 1993. Artbegriff, Evolution und Schöpfung: Dokumentation u. Diskussion $d$. verschiedenen Auffassungen. Naturwissenschaftlicher Verlag: Köln. 
Lösch, Bettina 2005. Deliberative Politik: Moderne Konzeptionen von Öffentlichkeit, Demokratie und politischer Partizipation. Westfälisches Dampfboot: Münster.

Luckner, Andreas 2006. Klugheitsethik, in Düwell, Marcus, Hübenthal, Christoph \& Werner, Micha H. (Hg.): Handbuch Ethik. Metzler: Stuttgart, 206-217.

M

Macpherson, Crawford B. (1962) 2010. Political Theory of Possessive Individualism: Hobbes to Locke: Oxford University Press: Oxford.

Marx, Karl \& Engels, Friedrich (Hg.) (1862) 1974. Marx-Engels-Werke: Briefe von Marx an Engels, Band 30. Dietz Verlag: Berlin (DDR).

Massing, Peter 2003. Ernst Fraenkel, in Massing, Peter \& Breit, Gotthard (Hg.): Demokratie-Theorien: Von der Antike bis zur Gegenwart. Texte und Interpretationen. (Schriftenreihe der Bundeszentrale für Politische Bildung, Bd. 424) Bundeszentrale für politische Bildung: Bonn, 214-222.

Mayntz, Renate 2001. Zur Selektivität der steuerungstheoretischen Perspektive, in Burth, Hans-Peter \& Görlitz, Axel (Hg.): Politische Steuerung in Theorie und Praxis. (Schriften zur Rechtspolitologie, Bd. 12) Nomos: Baden-Baden, 17-28.

Mayntz, Renate 2008. Von der Steuerungstheorie zu Global Governance, in Schuppert, Gunnar F. \& Zürn, Michael (Hg.): Governance in einer sich wandelnden Welt. Wiesbaden: VS Verlag für Sozialwissenschaften. (Politische Vierteljahresschrift. Sonderheft, 41), 43-60.

Mayntz, Renate (Hg.) 2009a. Über Governance: Institutionen und Prozesse politischer Regelung. (Schriften des Max-Planck-Instituts für Gesellschaftsforschung, Bd. 62) Campus Verlag: Frankfurt a Main.

Mayntz, Renate 2009b. New Challenges to Governance Theory: (1998), in Mayntz, Renate (Hg.): Über Governance: Institutionen und Prozesse politischer Regelung. (Schriften des Max-Planck-Instituts für Gesellschaftsforschung, Bd. 62) Campus Verlag: Frankfurt a Main, 13-28.

Mayntz, Renate 2009c. Governance Theory als fortentwickelte Steuerungstheorie?: (2005), in Mayntz, Renate (Hg.): Über Governance: Institutionen und Prozesse politischer Regelung. (Schriften des Max-Planck-Instituts für Gesellschaftsforschung, Bd. 62) Campus Verlag: Frankfurt a Main, 41-52.

Mayntz, Renate 2010. Governance im modernen Staat, in Benz, Arthur \& Dose, Nicolai (Hg.): Governance: Regieren in komplexen Regelsystemen: Eine Einführung. VS Verlag für Sozialwissenschaften: Wiesbaden, 37-48.

Mayntz, Renate \& Scharpf, Fritz W. 1975. Policy-making in the German federal bureaucracy. Elsevier: New York.

Mayntz, Renate \& Scharpf, Fritz W. 1995a. Steuerung und Selbstorganisation in staatsnahen Sektoren, in Mayntz, Renate \& Scharpf, Fritz W. (Hg.): Gesellschaftliche Selbstregelung und politische Steuerung. (Schriften des Max-Planck-Instituts für Gesellschaftsforschung, Bd. 23) Campus Verlag: Frankfurt a Main, 9-38.

Mayntz, Renate \& Scharpf, Fritz W. 1995b. Der Ansatz des akteurzentrierten Institutionalismus, in Mayntz, Renate \& Scharpf, Fritz W. (Hg.): Gesellschaftliche Selbstregelung und politische Steuerung. (Schriften des Max-Planck-Instituts für Gesellschaftsforschung, Bd. 23) Campus Verlag: Frankfurt a Main, 39-72. 
Mayntz, Renate \& Scharpf, Fritz W. 2005. Politische Steuerung - Heute? Vortrag gehalten am 04.12.2004 anlässlich der Verleihung des 'Bielefelder Wissenschaftspreises`. Zeitschrift für Soziologie 34(3), 236-243.

Mayntz, Renate \& Streeck, Wolfgang 2003. Die Reformierbarkeit der Demokratie: Innovationen und Blockaden: Einleitung, in Mayntz, Renate \& Streeck, Wolfgang (Hg.): Die Reformierbarkeit der Demokratie: Innovationen und Blockaden: Festschrift für Fritz W. Scharpf. (Schriften aus dem Max-Planck-Institut für Gesellschaftsforschung, Bd. 45) Campus Verlag: Frankfurt a Main, 9-28.

Mazouz, Nadine 2006. Gerechtigkeit, in Düwell, Marcus, Hübenthal, Christoph \& Werner, Micha H. (Hg.): Handbuch Ethik. Metzler: Stuttgart, 371-376.

Meijers, Evert \& Stead, Dominic 2004. Policy integration: what does it mean and how can it be achieved? A multi-disciplinary review. Beitrag zur "Berlin Conference on the Human Dimensions of Global Environmental Change: Greening of Policies Interlinkages and Policy" Integration. Graue Reihe

Messner, Dirk 1998. Die Transformation von Staat und Politik im Globalisierungsprozess, in Messner, Dirk (Hg.): Die Zukunft des Staates und der Politik: Möglichkeiten und Grenzen politischer Steuerung in der Weltgesellschaft. (EINE Welt, Bd. 5) Dietz: Bonn, 14-44.

Meyer, Kirsten 2003. Der Wert der Natur: Begründungsvielfalt im Naturschutz. Mentis: Paderborn.

Mieth, Dietmar 1995. Ethische Evaluierung der Biotechnologie, in Schell, Thomas von (Hg.): Biotechnologie - Gentechnik: Eine Chance für neue Industrien. (Veröffentlichungen der Akademie für Technikfolgenabschätzung) Springer: Berlin, 505-530.

Misselhorn, Catrin 2006. Moral point of view, in Düwell, Marcus, Hübenthal, Christoph \& Werner, Micha H. (Hg.): Handbuch Ethik. Metzler: Stuttgart, 431-434.

Mol, Arthur; Lauber, Volkmar \& Liefferink, Duncan 2000. The voluntary approach to environmental policy: Joint environmental policy-making in Europe. Oxford University Press: New York.

Mouffe, Chantal 2000. The democratic paradox. Verso: London, New York.

Müller, Markus 2012. Justice as a framework for the Solution of Environmental Conflicts, in Kals, Elisabeth \& Maes, Jürgen (Hg.): Justice and conflicts: Theoretical and empirical contributions. Springer: Berlin, 239-250.

Münkler, Herfried, Fischer, Karsten \& Bluhm, Harald (Hg.) 2001. Gemeinwohl und Gemeinsinn 1: Historische Semantiken politischer Leitbegriffe. (Forschungsberichte der interdisziplinären Arbeitsgruppe Gemeinwohl und Gemeinsinn der BerlinBrandenburgischen Akademie der Wissenschaften). Akademie Verlag: Berlin.

Münkler, Herfried \& Fischer, Karsten (Hg.) 2002a. Gemeinwohl und Gemeinsinn 2: Rhetoriken und Perspektiven sozial-moralischer Orientierung. (Forschungsberichte der interdisziplinären Arbeitsgruppe Gemeinwohl und Gemeinsinn der BerlinBrandenburgischen Akademie der Wissenschaften). Akademie Verlag: Berlin

Münkler, Herfried \& Fischer, Karsten 2002b. Einleitung: Rhetoriken des Gemeinwohls und Probleme des Gemeinsinns, in Münkler, Herfried \& Fischer, Karsten (Hg.): Gemeinwohl und Gemeinsinn 2: Rhetoriken und Perspektiven sozial-moralischer Orientierung. (Forschungsberichte der interdisziplinären Arbeitsgruppe 
Gemeinwohl und Gemeinsinn der Berlin-Brandenburgischen Akademie der Wissenschaften). Akademie Verlag: Berlin, 9-17.

$\mathbf{N}$

Nagel, Thomas 1986. The view from nowhere. Oxford University Press: New York.

Nanz, Patrizia \& Steffek, Jens 2004. Global Governance, Participation and the Public Sphere. Government \& Opposition 39(2), 314-335.

Neßhöver, Carsten et al. 2007. Das Millennium Ecosystem Assessment - eine deutsche

Perspektive. Natur und Landschaft 82(6), 262-267.

Nida-Rümelin, Julian 1994. Begründung in der Ethik. Logos(3), 306-320.

Nida-Rümelin, Julian 2011. Die Optimierungsfalle: Philosophie einer humanen Ökonomie. Irisiana: München.

Norton, Bryan G. 1987. Why preserve natural variety? Princeton University Press: Princeton.

Norton, Bryan G. 2006. Toward a Policy-Relevant Definition of Biodiversity, in Goble, Dale D., Scott, J. M. \& Davis, Frank W. (Hg.): The Endangered Species Act at thirty: Conserving Biodiversity in Human-Dominated Landscapes. Island Press: Washington D.C., 49-58.

Nuschler, Franz 2009. Good Governance: Ein universelles Leitbild von Staatlichkeit und Entwicklung. INEF Report 96/2009: Duisburg.

Nussbaum, Martha \& Sen, Amartya 1993. The Quality of life. Oxford University Press: New York.

Nussbaum, Martha 2003. Frauen und Arbeit: der Fähigkeitenansatz. Zeitschrift für Wirtschafts- und Unternehmensethik 4(1), 8-37.

0

O`Neil, Rick 1997. Intrinsic value, moral standing, and species. Environmental Ethics 19, 45-52.

Offe, Claus 1975. Wohlfahrtsstaat und Massenloyalitat. Kiepenheuer und Witsch: Köln.

Offe, Claus 2002. Wessen Wohl ist das Gemeinwohl, in Münkler, Herfried \& Fischer, Karsten (Hg.): Gemeinwohl und Gemeinsinn 2: Rhetoriken und Perspektiven sozialmoralischer Orientierung. (Forschungsberichte der interdisziplinären Arbeitsgruppe Gemeinwohl und Gemeinsinn der Berlin-Brandenburgischen Akademie der Wissenschaften). Akademie Verlag: Berlin, 55-76.

Offe, Claus 2008. Governance - "Empty signifier" oder sozialwissenschaftliches Forschungsprogramm?, in Schuppert, Gunnar F. \& Zürn, Michael (Hg.): Governance in einer sich wandelnden Welt. (Politische Vierteljahresschrift. Sonderheft, 41) VS Verlag für Sozialwissenschaften: Wiesbaden, 61-76.

Ortega y Gasset, José 1989 (1958). Der Aufstand der Massen. Deutsche Verlagsansalt: Stuttgart.

Ott, Konrad 1993. Ökologie und Ethik: Ein Versuch praktischer Philosophie. (Ethik in den Wissenschaften, 4) Attempto Verlag: Tübingen.

Ott, Konrad 1996. Vom Begründen zum Handeln: Aufsätze zur angewandten Ethik.

(Ethik in den Wissenschaften, 8) Attempto-Verlag: Tübingen. 
Ott, Konrad 1997. Ipso facto: Zur ethischen Begründung normativer Implikate wissenschaftlicher Praxis. Suhrkamp: Frankfurt a Main.

Ott, Konrad 2004. Umweltethik zwischen Grundlagenreflexion und Politikberatung, in Friesen, Hans \& Berr, Karsten (Hg.): Angewandte Ethik im Spannungsfeld von Begründung und Anwendung. (Praktische Philosophie kontrovers, Bd. 2) Lang: Frankfurt am Main:, 173-195.

Ott, Konrad 2007. Zur ethischen Begründung des Schutzes von Biodiversität, in Potthast, Thomas (Hg.): Biodiversität - Schlüsselbegriff des Naturschutzes im 21. Jahrhundert? Erweiterte Ergebnisdukumentation einer Vilmer Sommerakademie. Bundesamt für Naturschutz. (Naturschutz und biologische Vielfalt, Heftnr. 18), BfNSchriftenvertrieb im Landwirtschaftsverlag: Münster, 89-124.

Ott, Konrad 2010. Umweltethik zur Einführung. (Zur Einführung, 377) Junius: Hamburg

Ott, Konrad 2013. Environmental Deliberative Democracy. Vortrag zur Sommerakademie der Internationalen Naturschutzakademie Vilm. Graue Reihe.

Ott, Konrad \& Döring, Ralf 2004. Theorie und Praxis starker Nachhaltigkeit. (Ökologie und Wirtschaftsforschung, Bd. 54) Metropolis-Verlag: Marburg.

$\mathbf{P}$

Palazzo, Guido 2002. Die Mitte der Demokratie: Über die Theorie deliberativer Demokratie von Jürgen Habermas. Nomos: Baden-Baden.

Papadopoulos, Yannis \& Benz, Arthur (Hg.) 2006. Governance and democracy: Comparing national, European and international experiences. Routledge: New York.

Parthey, Heinrich 1999. Persönliche Interdisziplinearität bei Wissenschaftlern, in Umstätter, Walther \& Wessel, Karl-Friedrich (Hg.): Interdisziplinarität Herausforderung an die Wissenschaftlerinnen und Wissenschaftler: Festschrift zum 60. Geburtstag von Heinrich Parthey. (Berliner Studien zur Wissenschaftsphilosophie \& Humanontogenetik, Bd. 15) Kleine: Bielefeld, 243-254.

Pateman, Carole 1970. Participation and democratic theory. Cambridge University Press: Cambridge.

Piechocki, Reinhard 2005. Biodiversität: Zur Entstehung und Tragweite eines neuen Schlüsselbegriffs. Denkanstösse - Thesen zur Biodiversität(2), 6-17.

Piechocki, Reinhard 2007. "Biodiversität" - Zur Entstehung und Tragweite eines neuen Schlüsselbegriffs, in Potthast, Thomas (Hg.): Biodiversität - Schlüsselbegriff des Naturschutzes im 21. Jahrhundert? Erweiterte Ergebnisdukumentation einer Vilmer Sommerakademie. (Naturschutz und biologische Vielfalt, Heftnr. 48) BfN Schriftenvertrieb im Landwirtschaftsverlag: Münster, 11-24.

Piechocki, Reinhard 2010. Landschaft - Heimat - Wildnis: Schutz der Natur - aber welche und warum? Beck'sche Reihe, 1711). Beck: München.

Pörksen, Bernhard 2011. Schlüsselwerke des Konstruktivismus: Eine Einführung. In: Pörksen, B. Hrsg: Schlüsselwerke des Konstruktivismus. VS Verlag für Sozialwissenschaften: Wiesbaden, 13-28.

Potthast, Thomas 1996. Inventing Biodiversity: Genetics, Evolution, and Environmental Ethics. Biologisches Zentralblatt 115(2), 177-185. 
Potthast, Thomas 1999. Die Evolution und der Naturschutz: Zum Verhältnis von Evolutionsbiologie, Ökologie und Naturethik. (Campus Forschung, 777) CampusVerlag: Frankfurt a Main.

Potthast, Thomas 2000. Wo sich Biologie, Ethik und Naturphilosophie treffen (müssen): Epistemologische und moralphilosophische Aspekte der Umweltethik, in Ott, Konrad \& Gorke, Martin (Hg.): Spektrum der Umweltethik. (Ökologie und Wirtschaftsforschung, Bd. 36) Metropolis-Verlag: Marburg, 101-146.

Potthast, Thomas 2005a. Umweltforschung und das Problem epistemisch-moralischer Hybride: Ein Kommentar zur Rhetorik, Programmatik und Theorie interdisziplinärer Forschung, in Baumgärtner, Stefan \& Becker, Christian (Hg.): Wissenschaftsphilosophie interdisziplinärer Umweltforschung. (Ökologie und Wirtschaftsforschung, Bd. 59) Metropolis-Verlag: Marburg, 87-100.

Potthast, Thomas 2005b. Was ist Biodiversität und warum soll sie erhalten werden?: Wissenschaftstheoretische und ethische Perspektiven. Denkanstösse - Thesen zur Biodiversität(2), 18-29.

Potthast, Thomas 2006. Naturschutz und Naturwissenschaft - Symbiose oder Antagonismus? Zur Beharrung und zum Wandel prägender Wissenschaftsformen vom ausgehenden 19. Jahrhundert bis in die Gegenwart, in Frohn, Hans-Werner \& Schmoll, Friedemann (Hg.): Natur und Staat: Staatlicher Naturschutz in Deutschland 1906 - 2006. (Naturschutz und biologische Vielfalt, Bd. 35) Bundesamt für Naturschutz: Bonn-Bad Godesberg, 343-444.

Potthast, Thomas 2007a. Biodiversität, Ökologie, Evolution - Epistemisch-moralische Hybride und Biologietheorie: erweiterte und überarbeitete Fassung eines Vortrags bei der Vilmer Sommerakademie 2002, in Potthast, Thomas (Hg.): Biodiversität Schlüsselbegriff des Naturschutzes im 21. Jahrhundert? Erweiterte Ergebnisdukumentation einer Vilmer Sommerakademie. (Naturschutz und biologische Vielfalt, Heftnr. 48) BfN Schriftenvertrieb im Landwirtschaftsverlag: Münster, 57-88.

Potthast, Thomas 2007b. Einleitung: "Biodiversität" und die Praxisrelevanz theoretischer Grundlagen reflexionen im Naturschutz, in Potthast, Thomas (Hg.): Biodiversität - Schlüsselbegriff des Naturschutzes im 21. Jahrhundert? Erweiterte Ergebnisdukumentation einer Vilmer Sommerakademie. (Naturschutz und biologische Vielfalt, Heftnr. 48) BfN Schriftenvertrieb im Landwirtschaftsverlag, 710.

Proeller, Isabella \& Schedler, Kuno 2005. Verwaltung im Gewährleistungsstaat, in Blanke, Bernhard et al. (Hg.): Handbuch zur Verwaltungsreform. VS Verlag für Sozialwissenschaften: Wiesbaden, 94-101.

$\mathbf{R}$

Raatz, Richard (Hg.) 1999. Philosophieren über Philosophie. Leipziger Schriften zur Philosophie.

Rawls, John 2003. Politischer Liberalismus. 1. Aufl. Suhrkamp Taschenbuch Wissenschaft, 1642. Suhrkamp: Frankfurt am Main. 
Renn, Ortwin 2001. Kooperative Verfahren zur Umsetzung einer nachhaltigen Entwicklung, in Fischer, Andreas \& Hahn, Gabriela (Hg.): Vom schwierigen Vergnügen einer Kommunikation über die Idee der Nachhaltigkeit. (Reihe Innovationen in den Hochschulen - nachhaltige Entwicklung, Bd. 3), VAS: Frankfurt a Main, 122-149.

Reuter, Katrin 2014. Ökologische Tugenden und gutes Leben: Der Schutz der Biodiversität im Zeitalter der ökologischen Krise und nachhaltiger Entwicklung. Oekom Verlag: München.

Rhodes, Rod 1997. Understanding Governance: Policy networks, Governance, Reflexivity, and Accountability. Open University Press: Buckingham, Philadelphia.

Ricken, Friedo 2003. Allgemeine Ethik. 4. überarbeitet und erweiterte Auflage. (UrbanTaschenbücher, 348) Kohlhammer: Stuttgart.

Risse, Thomas 2008. Regieren "in Räumen begrenzter Staatlichkeit": Zur Reisefähigkeit des Governance-Konzepts, in Schuppert, Gunnar F. \& Zürn, Michael (Hg.): Governance in einer sich wandelnden Welt (Politische Vierteljahresschrift. Sonderheft, 41) VS Verlag für Sozialwissenschaften: Wiesbaden, 149-170.

Ritter, Ernst-Hasso 1979. Der kooperative Staat: Bemerkungen zum Verhältnis von Staat und Wirtschaft. Archiv des öffentlichen Rechts (104), 389-413.

Rosenau, James \& Czempiel, Ernst O. 1992. Governance without Government: Order and change in world politics. Cambridge University Press: New York.

Routley, Richard 2003. Is there a need for a new, an environmental, ethic?: (Originalaufsatz von 1973), in Light, Andrew \& Rolston, Holmes (Hg.): Environmental ethics: An anthology (Blackwell philosophy anthologies, 19) Blackwell Pub: Malden, MA, 47-52.

\section{S}

Sack, Detlef 2011. Governance zwischen Gouvernementalität, Regierungsform und Regulation, in Demirović, Alex \& Walk, Heike (Hg.): Demokratie und Governance: Kritische Perspektiven auf neue Formen politischer Herrschaft. Westfälisches Dampfboot:Münster, 18-42.

Scanlon, Thomas 2000. What we owe to each other. Harvard University Press: New York.

Schaber, Peter 2006. Naturalistischer Fehlschluss, in Düwell, Marcus, Hübenthal, Christoph \& Werner, Micha H. (Hg.): Handbuch Ethik. Metzler:Stuttgart, 454-456.

Schark, Marianne 2005. Lebewesen versus Dinge: Eine metaphysische Studie. De Gruyter: Berlin.

Scharpf, Fritz W. 1975. Demokratietheorie zwischen Utopie und Anpassung. ScriptorVerlag: Kronberg.

Scharpf, Fritz; Reissert, Bernd; Schnabel, Fritz W. 1976. Politikverflechtung: Theorie und Empirie des kooperativen Föderalismus in der Bundesrepublik. Scriptor Verlag: Kronberg.

Scharpf, Fritz W. 1992. Die Handlungsfähigkeit des Staates am Ende des 20. Jahrhunderts, in Kohler-Koch, Beate (Hg.): Staat und Demokratie in Europa (18. wissenschaftlicher Kongress der Deutschen Vereinigung für politische Wissenschaft). Leske + Budrich: Opladen, 93-115. 
Scharpf, Fritz W. 1993a. Positive und negative Koordination in Verhandlungssystemen, in Héritier, Adrienne (Hg.): Policy-Analyse: Kritik und Neuorientierung (Politische Vierteljahresschrift. Sonderheft, 24). Westdeutscher Verlag: Opladen, 57-83.

Scharpf, Fritz W. 1993b. Versuch über Demokratie im verhandelnden Staat, in Czada, Roland \& Schmidt, Manfred G. (Hg.): Verhandlungsdemokratie, Interessenvermittlung, Regierbarkeit (Festschrift für Gerhard Lehmbruch). Westdeutscher Verlag: Opladen, 25-50.

Scharpf, Fritz W. 1998a. Demokratie in der transnationalen Politik, in Beck, Ulrich (Hg.): Politik der Globalisierung (Edition zweite Moderne). Suhrkamp: Frankfurt a Main, 228-253.

Scharpf, Fritz W. 1998b. Demokratische Politik in der internationalisierten Ökonomie, in Greven, Michael (Hg.): Demokratie - eine Kultur des Westens? (20. Wissenschaftlicher Kongress der Deutschen Vereinigung für Politische Wissenschaft). Leske + Budrich: Opladen, 81-104.

Scharpf, Fritz W. 1998c. Interdependence and Democratic legitimation. MPIfG Working Paper 98/2: Köln.

Scharpf, Fritz W. 1999a. Demokratieprobleme in der europäischen Mehrebenenpolitik, in Merkel, Wolfgang \& Busch, Andreas (Hg.): Demokratie in Ost und West. Festschrift für Klaus von Beyme (Suhrkamp Taschenbuch Wissenschaft, 1425). Suhrkamp: Frankfurt a Main, 672-694.

Scharpf, Fritz W. 1999b. Regieren in Europa. Effektiv und demokratisch? Campus Verlag: Frankfurt a Main.

Scharf, Fritz W 1999c. Föderale Politikverflechtung: Was muß man ertragen - was kann man ändern? MPIfG Working Paper 99/3: Köln.

Scharpf, Fritz W. 2000. Interaktionsformen: Akteurzentrierter Institutionalismus in der Politikforschung. VS Verlag für Sozialwissenschaften: Wiesbaden.

Scharpf, Fritz W. 2005. Legitimationskonzepte jenseits des Nationalstaats, in Schuppert, Gunnar F. et al. (Hg.): Europawissenschaft. Nomos: Baden-Baden, 705741.

Scharpf, Fritz W. 2009. Legitimität im europäischen Mehrebenensystem. Leviathan 37, 244-280.

Scharpf, Fritz W. 2012. Legitimacy Intermediation in the Multilevel European Polity and its Collapse in the Euro Crisis. MPIfG Discussion Paper 12/6.

Schmidt, Hermann 1963. Seinerkenntnis und Staatsdenken. Niemeyer: Tübingen.

Schmidt, Manfred G. 2000. Demokratietheorien: Eine Einführung. 3. überarbeitete und erweiterte Auflage (UTB, 1887). Leske + Budrich: Opladen.

Schmidt, Manfred G. 2003. Die »komplexe Demokratietheorie« nach drei Jahrzehnten, in Mayntz, Renate \& Streeck, Wolfgang (Hg.): Die Reformierbarkeit der Demokratie: Innovationen und Blockaden. Festschrift für Fritz W. Scharpf. (Schriften aus dem Max-Planck-Institut für Gesellschaftsforschung) Campus: Frankfurt a Main, 151172.

Schnädelbach, Herbert 1977. Reflexion und Diskurs: Fragen einer Logik der Philosophie. Suhrkamp: Frankfurt a Main.

Schnädelbach, Herbert 2006. Rationalität, in Düwell, Marcus, Hübenthal, Christoph \& Werner, Micha H. (Hg.): Handbuch Ethik. Metzler: Stuttgart, 480-486. 
Schramm, Engelbert 1999. Zum Problem der Interdisziplinearität in der Umweltforschung: Biodiversität als Herausforderung für Wissenschaft und Gesellschaft, in Görg, Christoph et al. (Hg.): Zugänge zur Biodiversität: Disziplinäre Thematisierungen und Möglichkeiten integrierender Ansätz. (Ökologie und Wirtschaftsforschung, Bd. 34) Metropolis-Verlag: Marburg, 21-37.

Schuppert, Gunnar F. (Hg.) 2005. Der Gewährleistungsstaat: Ein Leitbild auf dem Prüfstand (Schriften zur Governance-Forschung, Bd. 2). Nomos: Baden-Baden.

Schuppert, Gunnar F. 2008. Governance - auf der Suche nach Konturen eines "anerkannt uneindeutigen Begriffs", in Schuppert, Gunnar F. \& Zürn, Michael (Hg.): Governance in einer sich wandelnden Welt. (Politische Vierteljahresschrift. Sonderheft, 41) VS Verlag für Sozialwissenschaften: Wiesbaden, 9-40.

Schuster, Kai 2008. Gesellschaft und Naturschutz: Empirische Grundlagen für eine lebensstilorientierte Naturschutzkommunikation. (Naturschutz und biologische Vielfalt, 53) Bundesamt für Naturschutz: Bonn.

Seel, Martin 1991. Eine Ästhetik der Natur. Suhrkamp: Frankfurt a Main.

Seel, Martin 1997. Ästhetische und moralische Anerkennung der Natur, in Krebs, Angelika (Hg.): Naturethik: Grundtexte der gegenwärtigen tier- und ökoethischen Diskussion. Suhrkamp: Frankfurt a Main, 307-330.

Seliger, Martin 1976. Ideology and politics. Allen \& Unwin: London.

Solbrig, Otto 1994. Biodiversität: Wissenschaftliche Fragen und Vorschläge für die internationale Forschung. Rheinsicher Landwirtschaftsverlag: Bonn.

Sørensen, Eva \& Torfing, Jacob 2007. Introduction: Governance Network Research: Towards a Second Generation, in Sørensen, Eva \& Torfing, Jacob (Hg.): Theories of democratic network governance. Palgrave Macmillan: New York, 1-21.

Sørensen, Eva \& Triantafillou, Peter 2009. The politics of self-governance. Ashgate Pub: Burlington.

Stadler, Jutta 2007. Die Biodiversitätskonvention und ihr ökosystemarer Ansatz: Neue Anforderungen an die Forschung, in Potthast, Thomas (Hg.): Biodiversität Schlüsselbegriff des Naturschutzes im 21. Jahrhundert? Erweiterte Ergebnisdukumentation einer Vilmer Sommerakademie. (Naturschutz und biologische Vielfalt, Bd. 48) BfN Schriftenvertrieb im Landwirtschaftsverlag: Bonn, 175-184.

Stegmüller, Wolfgang 1979. Rationale Rekonstruktion von Wissenschaft und ihrem Wandel. Reclam: Stuttgart.

Steigleder, Klaus 2006. Kant, in Düwell, Marcus, Hübenthal, Christoph \& Werner, Micha H. (Hg.): Handbuch Ethik. Metzler: Stuttgart, 128-139.

Steinfath, Holmer 1998. Die Thematik des guten Lebens in der gegenwärtigen philosophischen Diskussion: Einführung, in Steinfath, Holmer (Hg.): Was ist ein gutes Leben? Philosophische Reflexionen. (Suhrkamp Taschenbuch Wissenschaft, 1323) Suhrkamp: Frankfurt am Main, 7-31.

Strasser, Stephan \& Redl, Jakob 2010. Verhandlungen und Positionsfindungen im Rahmen der CBD aus strategisch-relationaler Perspektive: Das Beispiel der europäischen Union, in Brand, Ulrich (Hg.): Globale Umweltpolitik und Internationalisierung des Staates: Biodiversitätspolitik aus strategisch-relationaler Perspektive. Westfälisches Dampfboot: Münster, 74-103. 
Suhm, Christian 2005. Wissenschaftlicher Realismus: Eine Studie zur Realismus-

Antirealismus-Debatte in der neueren Wissenschaftstheorie. Ontos Verlag: Frankfurt a Main

Syme, Geoffrey 2012. Justice and Environmental Decision Making, in Kals, Elisabeth \& Maes, Jürgen (Hg.): Justice and conflicts: Theoretical and empirical contributions. Springer: Heidelberg, 283-295.

\section{$\mathbf{T}$}

Takacs, David 1996. The idea of biodiversity: Philosophies of paradise. John Hopkins University Press: Baltimore.

Taylor, Charles 2006. Was ist menschliches Handeln? Erstpublikation in: Charles Taylor: Negative Freiheit. Zur Kritik des neuzeitlichen Individualismus. Suhrkamp: Frankfurt a Main 1999, 9-51.

Taylor, Paul W. 1986. Respect for nature: A theory of environmental ethics. Princeton University Press: New York.

Taylor, Paul W. 1997. Die Ethik der Achtung gegenüber der Natur, in Krebs, Angelika (Hg.): Naturethik: Grundtexte der gegenwärtigen tier- und ökoethischen Diskussion. Suhrkamp: Frankfurt a Main, 111-143.

Teubner, Gunther \& Willke, Helmuth 1984. Kontext und Autonomie: Gesellschaftliche Selbststeuerung durch reflexives Recht. Zeitschrift für Rechtssoziologie 5, 4-35.

Tetens, Holm 1992. Naturalismus und Kulturalismus: Reflexionen zur naturalistsichen Erforschung des Mentalen, in Janich, Peter (Hg.): Entwicklungen der methodischen Philosophie. (Suhrkamp Taschenbuch Wissenschaft, 979) Suhrkamp: Frankfurt a Main, 113-124.

Tetens, Holm 2004. Philosophisches Argumentieren: Eine Einführung. (Beck'sche Reihe 1607) Beck: München.

Theobald, Werner 1998. Umweltbewertung als inter- und transdisziplinärer Diskurs, in Theobald, Werner (Hg.): Integrative Umweltbewertung: Theorie und Beispiele aus der Praxis. Springer: Berlin, 7-18.

Theobald, Werner 2003. Mythos Natur: Die geistigen Grundlagen der Umweltbewegung. Wissenschaftliche Buchgesellschaft: Darmstadt.

Töller, Annette E. 2007. Die Rückkehr des befehlenden Staates?: Muster und Ursachen der Veränderung staatlicher Handlungsformen in der deutschen Abfallpolitik. Politische Vierteljahresschrift (PVS) 48(1), 66-96.

Töller, Annette E. 2012. Warum kooperiert der Staat? Kooperative Umweltpolitik im Schatten der Hierarchie. Nomos: Baden-Baden.

Trepl, Ludwig 1988. Leitwissenschaft Ökologie?, in Ingensiep, Hans W. \& Jax, Kurt (Hg.): Mensch, Umwelt und Philosophie: Interdisziplinäre Beiträge. Wissenschaftsladen: Bonn, 163-173.

Trepl, Ludwig 1999. Die Diversitäts-Stabilitäts-Diskussion in der Ökologie, in Görg, Christoph et al. (Hg.): Zugänge zur Biodiversität: Disziplinäre Thematisierungen und Möglichkeiten integrierender Ansätze. (Ökologie und Wirtschaftsforschung, Bd 34) Metropolis-Verlag: Marburg, 91-126.

Tribe, Laurence H. 1980. Was spricht gegen Plastikbäume?, in Birnbacher, Dieter (Hg.): Ökologie und Ethik. Reclam: Stuttgart, 20-71. 
Tschepke, Frank 2003. Wissenschaftlicher Realismus. Disserationsschrift. Universität Göttingen.

Tsebelis, George 2002. Veto players: How political institutions work. Princeton University Press: New York.

\section{V}

van Fraassen, Bas 1992. From Vicious Circle to Infinite Regress, and Back Again. Proceedings of the Biennial Meeting of the Philosophy of Science Association (PSA)(2), 6-29.

Viehöver, Willy 2013. Keep on Nano truckin', truck our blues away: Zur Rolle von Sprache und Narrativen in der diskuriven Governance der Wissensproduktion im Feld der Nanotechnologie, in Viehöver, Willy, Keller, Reiner \& Schneider, Werner (Hg.): Diskurs - Sprache - Wissen: Interdisziplinäre Beiträge zum Verhältnis von Sprache und Wissen in der Diskursforschung. Springer: Wiesbaden, 213-290.

Voigt, Rüdiger 1995. Der kooperative Staat: Auf der Suche nach einem neuen Steuerungsmodus, in Voigt, Rüdiger (Hg.): Der kooperative Staat: Krisenbewältigung durch Verhandlung? Nomos: Baden-Baden, 33-92.

Voigt, Annette 2009. 'Wie sie ein Ganzes bilden`: Analoge Deutungsmuster in ökologischen Theorien und politischen Philosophien der Vergesellschaftung, in Kirchhoff, Thomas \& Trepl, Ludwig (Hg.): Vieldeutige Natur: Landschaft, Wildnis und Ökosystem als kulturgeschichtliche Phänomene. Transcript: Bielefeld, 331-347.

W

Walk, Heike 2008. Partizipative Governance: Beteiligungsformen und Beteiligungsrechte im Mehrebenensystem der Klimapolitik. VS Verlag für Sozialwissenschaften: Wiesbaden.

Wallner, Fritz 1990. Acht Vorlesungen über den Konstruktiven Realismus. WUVL: Wien. Walzer, Michael 1981. Philosophy and Democracy. Political Theory 9(3), 379-399.

Walzer, Michael 1990. A Critique of Philosophical Conversation, in Kelly, Michael (Hg.): Hermeneutics and critical theory in ethics and politics. MIT Press: Cambridge, 182196.

Walzer, Michael 1992a. Sphären der Gerechtigkeit: Ein Plädoyer für Pluralität und Gleichheit. Campus Verlag: Frankfurt a Main.

Walzer, Michael 1992b. Zivile Gesellschaft und amerikanische Demokratie. Rotbuch Verlag: Berlin.

Walzer, Michael 1993. Kritik und Gemeinsinn: Drei Wege der Gesellschaftskritik. Fischer Taschenbuch Verlag: Frankfurt a Main.

Weichart, Peter 2008. Neoliberalism meets Political Economy - Politikversagen, Entdemokratisierung und die vergebliche Hoffnung auf Governance in der Zweiten Moderne, in Bruckmeier, Karl (Hg.): Ethik und Umweltpolitik: Humanökologische Positionen und Perspektiven. (Edition Humanökologie, 6) Oekom Verlag: München, 213-236.

Weingarten, Michael 1998. Die Krise der gesellschaftlichen Naturverhältnisse: Eine Annäherung an die kulturell konstituierte Differenzierung von Natur und Kultur, in Hartmann, Dirk \& Janich, Peter (Hg.): Die kulturalistische Wende: Zur Orientierung 
des philosophischen Selbstverständnisses. (Suhrkamp Taschenbuch Wissenschaft, 1391) Suhrkamp: Frankfurt a Main, 371-414.

Werner, Micha H. 2003. Hans Jonas` Prinzip Verantwortung, in Düwell, Marcus \&

Steigleder, Klaus (Hg.): Bioethik: Eine Einführung. (Suhrkamp Taschenbuch

Wissenschaft, 1597) Suhrkamp: Frankfurt a Main, 41-56.

Wils, Jean-Pierre 2006. Tugend, in Düwell, Marcus, Hübenthal, Christoph \& Werner, Micha H. (Hg.): Handbuch Ethik. Metzler: Stuttgart, 534-538.

Wilson, Edward O. (Hg.) 1989. Biodiversity. National Academy Press: Washington, DC.

Winckelmann, Johannes (Hg.) 1988. Max Weber: Gesammelte politische Schriften.

5. Aufl. Mohr: Tübingen.

Wittkämper, Gerhard W. 1993. Abschied vom Staat in der Umweltpolitik, in Voigt, Rüdiger (Hg.): Abschied vom Staat - Rückkehr zum Staat? Nomos: Baden-Baden, 285-306.

Wolfrum, Rüdiger 2001. Biodiversität: Juristische insbesondere völkerrechtliche Aspekte ihres Schutzes, in Janich, Peter, Gutmann, Mathias \& Prieß, Kathrin (Hg.):

Biodiversität: Wissenschaftliche Grundlagen und gesetzliche Relevanz.

(Schriftenreihe der Europäischen Akademie zur Erforschung von Folgen

wissenschaftlich-technischer Entwicklungen, 10) Springer: Berlin, 417-443.

Wolkenstein, Andreas 2014. Akzeptanz und Akzeptabilität im Kontext der

Angewandten Ethik, in Ammicht Quinn, Regina (Hg.): Sicherheitsethik. (Studien zur Inneren Sicherheit, 16) Springer: Wiesbaden, 225-240.

Wood, Paul M. 1997. Biodiversity as the Source of Biological Resources: A New Look at Biodiversity Values. Environmental values 6(3), 251-268.

Worral, John 1989. Structural Realism: The best of both worlds? Dialectica 43(12), 99124.

Wright, Crispin 1992. Truth and objectivity. Harvard University Press: New York.

\section{Z}

Zieschank, Roland 1999. Bodenschutz: Steuerungsfunktionen von Recht und Politik. FFU-Report: Berlin.

Zilleßen, Horst 1997. Umweltpolitik als Politik der Gesellschaft, in Mez, Lutz \& Weidner, Helmut (Hg.): Umweltpolitik und Staatsversagen: Perspektiven und Grenzen der Umweltpolitikanalyse. Festschrift für Martin Jänicke zum 60. Geburtstag. Edition Sigma: Berlin, 56-66.

Zilleßen, Horst 2013. Die Modernisierung der Demokratie im Zeichen der Umweltproblematik, in Prittwitz, Volker v. (Hg.): Umweltpolitik als Modernisierungsprozess. VS Verlag für Sozialwissensachaften: Wiesbaden, 81-92.

Zitterbarth, Walter 1991. Der Erlanger Konstruktivismus in seiner Beziehung zum Konstruktiven Realismus, in Peschl, Markus F. (Hg.): Formen des Konstruktivismus in Diskussion: Materialien zu den "Acht Vorlesungen über den Konstruktiven Realismus". WUV: Wien, 73-87.

Žižek, Slavoj 2008. Kapital fürs Kapital. Zeit Online, 12. Dezember. 
ANHÄNGE 


\section{Selbstständigkeitserklärung}

gemäß § 9 Abs. c der Promotionsordnung der Philosophischen Fakultät der Universität Göttingen von 2015

„Hiermit versichere ich, dass ich die Dissertation selbstständig verfasst und keine anderen als die angegebenen Quellen und Hilfsmittel benutzt habe. Ich versichere überdies, dass nicht anderweitig eine entsprechende Promotion beantragt wurde und hierbei die eingereichte Dissertation oder Teile daraus vorgelegt worden sind."

Silke Lachnit 